1.9. (1) (1).

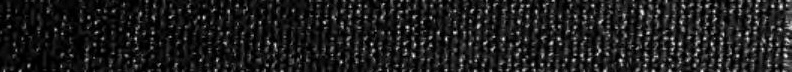

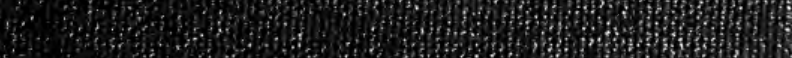
now

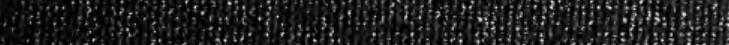
2. a

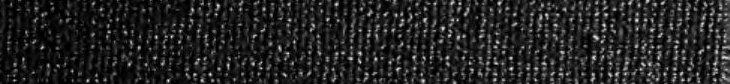
S.

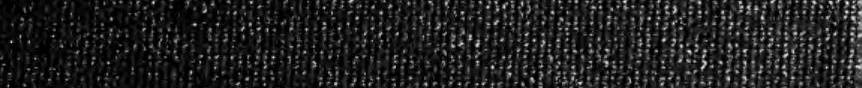

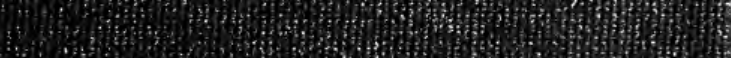

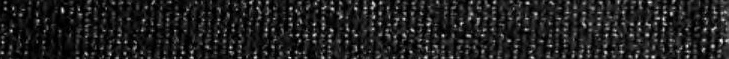
it W. What 2.

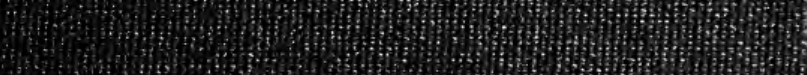
(3)

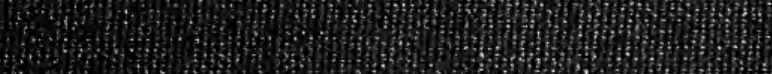
2.M

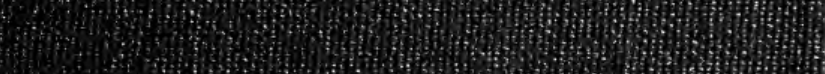

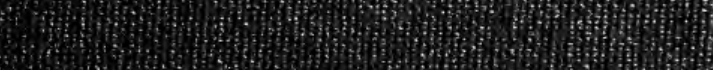

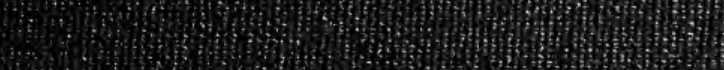

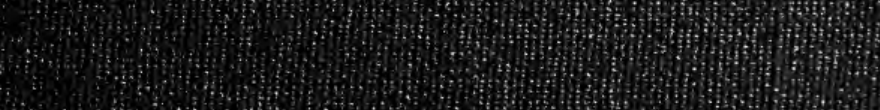
S. (1) (2) 6.4.

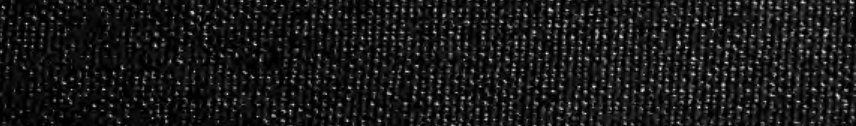


4 
5itho 

atro

stal. 
Nots appest 



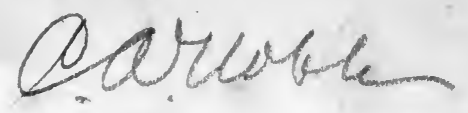

\title{
DIE
}

\section{ELLIPTISCHEN FUNKTIONEN}

\section{UND IHRE ANWENDUNGEN}

\author{
VON \\ DR. ROBERT FRICKE \\ PROFESSOR AN DER TECHNISCHEN HOCHSCHULE \\ IN BRAUNSCH WEIG
}

\section{ZWEITER TEIL}

DIE ALGEBRAISCHEN AUSFÜHRUNGEN

MIT 40 IN DEN TEXT AEDRUCKTEN FIGUREN

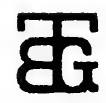

LEIPZIG UND BERLIN

VERLAG UND DRUCK VON B. G. TEUBNER 
MATH - STAT:

SCHUTZFORMEL FÜR DIE VEREINIGTEN STAATEN VON AMERIKA: COPYRIGHT 1922 BY B. G. TEUBNER IN LEIPZIG 


\section{Q4343 F7 V.2 MATH.- STAT. LURARY}

\section{DEM ANDENKEN \\ RICHARD DEDEKIND'S \\ GEWIDMET}




\section{Vorwort.}

Der vorliegende zweite Teil meines Buches „Die elliptischen Funktionen und ihre Anwendungen" behandelt die algebraischen Ausführungen, er umfabt also die Theoreme der Addition, Mnltiplikation und Division der elliptischen Funktionen, sowie die Transformationstheorie derselben. Das Interesse für diese Gegenstände ist während der langen Zeit der Entwicklung der Theorie der elliptischen Funktionen lebendig geblieben. Am 23. Dezember 1751 wurden Euler die Arbeiten Fagnano's zur Begutachtung vorgelegt und regten ihn zur Entdeckung der Additionstheoreme an, so daB Jacobi den genannten Tag als den Geburtstag der elliptischen Funktionen bezeichnete. Die Entdeckungen Abel's und die Schöpfung der Theorie der Modulfunktionen durch Klein sind wichtigste Marksteine der reichen Entwicklung, die noch bis in die jüngste Zeit hinein zu neuen Erkenntnissen führte. In letzterer Hinsicht scheint mir die Auffindung des "Klassenpolygons" nicht unwichtig. Es handelt sich hierbei um ein beziehungsreiches Gebilde, das 'die früh bemerkte Beziehung der elliptischen Funktionen zur Theorie der ganzzahligen binären quadratisehen Formen in klarster Weise darlegt und sich als ein wertvolles Mittel zur Behandlung der Transformationstheorie erwies.

Es bleibt mir übrig, der verehrten Verlagsbuchhandlung für die tatkräftige Förderung des Druckes meinen verbindlichen Dank auszusprechen und der Hoffnung Ausdruck zu geben, daB auch der letzte Teil, der die arithmetischen, geometrischen und mechanischen Anwendungen behandeln soll, in nicht zu ferner Zeit folgen möchte.

Bad Harzburg, den 15. September 1921.

Rohert Fricke. 


\title{
Inhaltsverzeichnis.
}

\section{Einleitung.}

Zusammenstelluug ron Sätzen aus der Algebra und Zahlentheorie.

\author{
I. Endliche Gruppen.
}

Seite

$\S$ 1. Begriff einer Gruppe endlicher Ordnung . . . . . . . . . . . . . . 1

$\S$ 2. Begriff der Untergruppe. . . . . . . . . . . . . . . . . . 4

$\S$ 3. Gleichberechtigte und ausgezeichnete Untergruppen . . . . . . . . . 6

$\S$ 4. Sätze über ausgezeichnete Untergruppen . . . . . . . . . . . . . 8

§ 5. Kompositionsreihe einer Gruppe $G_{m}$. . . . . . . . . . . . . . . . 12

$\S$ 6. Sätze über Abelsch Gruppen . . . . . . . . . . . . . . . . . 14

$\S$ 7. Permutationsgruppen ................... . 17

$\S$ 8. Transitivität und Primitivität der Permutationsgruppøn . . . . . . . 20

II. Algebraische Gleichungen.

$\S$ 1. Symmetrische Funktionen .. . . . . . . . . . . . . . . 23

§ 2. Tschirnhausentransformation. . . . . . . . . . . . . . 26

$\S$ 3. Hilfssatz über ganze Funktionen. . . . . . . . . . . . . . . 27

§ 4. Funktionen in Zahlkörpern . . . . . . . . . . . . . . . . . . 28

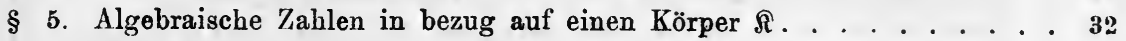

$\S$ 6. Gleichzeitige Adjunktion mehrerer algebraischer Zahlen . . . . . . . 34

$\S$ 7. Konjugierte Körper. Primitive und imprimitive Zahlen. . . . . . . . 38

8. Galoissche Körper und Galoissche Resolventen . . . . . . . . . . . 41

$\S$ 9. Die 'Transformationen eines Galoisschen Körpers in sich . . . . . . . 43

\$10. Die Galoissche Gruppe einer Gleichung $f(z)=0$. . . . . . . . . . . 46

§11. Untergruppen der Galoisschen Gruppe und zugehörige Zahlen . . . . 49

§ 12. Die rationalen Resolventen einer Gleichung $f(z)=0$. . . . . . . . . 52

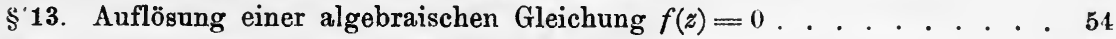

$\S 14$. Beispiel der Kreisteilungsgleichungen . . . . . . . . . . . . . . . 56

$\S$ 15. Zyklische Gleichungen . . . . . . . . . . . . . . . . . . . 58

\$ 16. Abelsche Gleichungen. . . . . . . . . . . . . . . . . . . . . . 61

§ 17. Algebraisch lösbare Gleichungen. . . . . . . . . . . . . . . . 62

III. Algebraische Funktionen.

$\S$ 1. Funktionen und Gleichungen in Funktionenkörpern . . . . . . . . . 64

§ 2. Algebraische Funktionen in bezug auf einen Körper $\Omega_{x}$. . . . . . . 67

§ 3. Gleichzeitige Adjunktion mehrerer algebraischer Funktionen . . . . . 68

$\S$ 4. Konjugierte Körper. Primitive und imprimitive Funktionen . . . . . 71

$\S$ 5. Galoissche Körper und Galoissche Resolventen . . . . . . . . . . . 72

$\S$ 6. Galoissche Gruppe einer Gleichung $f(z)=0$. . . . . . . . . . . . . 73

$\S$ 7. Auflösung einer algebraischen Gleichung $f(z)=0$. . . . . . . . . . 75

$\S$ 8. Monodromiegruppe einer Gleichung $f(z)=0$. . . . . . . . . . 76

IV. Algebraische Zahlen.

§ 1. Algebraische und ganze algebraische Zahlen. . . . . . . . . 78

\& 2. Ein algebraischer Hilfssatz . . . . . . . . . . . . . . . 80 
3. Folgerungen betreffs rationaler ganzer Zahlen

§ 4. Algebraische Zahlkörper . . . . . . . . . . . . . . . . 83

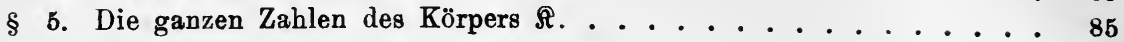

$\S$ 6. Teilbarkeit der Zahlen $\eta$ im Systeme e.............. 87

$\S$ 7. Begriff und Darstellung eines Ideals . . . . . . . . . . . . . . . 89

§ 8. Multiplikation der Ideale. . . . . . . . . . . . . . . . . . . . . 93

§ 9. Faktorenzerlegung eines Ideals . . . . . . . . . . . . . . . . . . 95

$\S$ 10. Die Basen eines Ideals a. . . . . . . . . . . . . . . . . . . . . 99

$\S 11$. Norm eines Ideals . . . . . . . . . . . . . . . . . . . . . . 101

§ 12. Äquivalenz der Ideale . . . . . . . . . . . . . . . . . . . . . 104

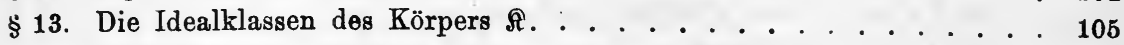

§ 14. Zerfällung der rationalen Primzahlen in Primideale. . . . . . . . . 107

§15. Sätze über Galoissche Zahlkörper . . . . . . . . . . . . . . . 110

§ 16. Beispiel der quadratischen Körper . . . . . . . . . . . . . . 112

$\S 17$. Gegen ein Ideal a teilerfremde Zahlklassen . . . . . . . . . . . 115

\$18. Satz über die zu einem gegebenen a teilerfremden Ideale. . . . . . 119

V. Quadratische Körper und Formen negativer Diskriminante.

$\S$ 1. Zweige und $\mathrm{Zweigideale} \mathrm{im} \mathrm{quadratischen} \mathrm{Körper} \AA . . . . . .121$

$\S$ 2. Zahlstrahlen im quadratischen Körper. . . . . . . . . . . . . 125

$\S$ 3. Zerlegung der Idealklassen von $\AA$ in Zweigklassen . . . . . . . . 129

$\S$ 4. Multiplikation und Äquivalenz der Zweigideale . . . . . . . . 131

$\S$ 5. Basen der Ideale und ebene Punktgitter. . . . . . . . . . . . . 135

$\$$ 6. Notizen über quadratische Formen negativer Diskriminante . . . . . 137

$\S$ 7. Beziehung zwischen den Zweigidealen $a_{n}$ und den quadratischen Formen 141

$\S 8$. Komposition der quadratischen Formen . . . . . . . . . . . 148

$\S$ 9. Einteilung dọr Formklassen in Geschlechter . . . . . . . . . . . . 151

Erster Abschnitt.

Die Additions-, Multiplikations- und Divisionssätze der elliptischen Funktionen.

Erstes Kapitel.

Die Additionssätze der elliptischen Funktionen.

$\S$ 1. Additionstheoreme der elliptischen Funktionen erster Stufe . . . . 157

§ 2. Invariante algebraische Gestalten der Additionsformeln . . . . . . . 161

\$ 3. Übergang zu den Additionsformeln der Jacobischen Funktionen . . . 164

$\S$ 4. Einführung einer Abelschen Gruppe $G_{256}$. . . . . . . . . . . . 166

$\S 5$. Die 256 dreigliedrigen Sigmarelationen . . . . . . . . . . . . . 171

$\S$ 6. Die Additionstheoreme der Jacobischen Funktionen . . . . . . . . . 175

$\S 7$. Additionssätze für mehrgliedrige Argumentsummen . . . . . . . . . 180

Zweites Kapitel.

Die Multiplikationssätze der elliptischen Funktionen.

§ 1. Multiplikationssätze der Funktionen erster Stufe . . . . . . . . . . 184

$\S$ 2. Partielle Differentialgleichung der Funktionen $\psi^{(n)}$. . . . . . . 190

$\S 3$. Berechnung yon $\wp(n u)$ durch ein Kettenbruchverfahren . . . . . . . 192

$\S$ 4. Ansatz der Multiplikationsformeln für sn, cn und dn . . . . . . 196

$\$$ 5. Weitere Beziehungen zwischen den Funktionen $G(z)$. . . . . . . . 199

$\S$ 6. Differentialgleichungen zur Berechnung der Funktionen $G(z)$. . . 205 


\section{Drittes Kapitel.}

\section{Die Divisionssätze der elliptischen Funktionen.}

§ 1. Die allgemeine Teilungsgleichung der $\wp$-Funktion . . . . . . . . . 210

§ 2. Die Monodromiegruppe der allgemeinen Teilungsgleichung . . . . . 214

§ 3. Zyklische Untergruppen $\operatorname{der} G_{n^{2}}$ und Kongruenzgruppen $n^{\text {ter }}$ Stufe . . 218

$\S$ 4. Elliptische Funktionen $n^{\text {ter }}$ Stufe . . . . . . . . . . . . . 225

§ 5. Lösung der allgemeinen Teilungsgleichung. . . . . . . . . . . . 231

§6. Divisionssätze der elliptischen Funktionen zweiter Stufe. . . . . . . 234

§ 7. Die Abelschen Relationen . . . . . . . . . . . . . . . . . . . 240

Viertes Kapitel.

\section{Die Teilwerte der elliptischen Funktionen.}

§ 1. Die Teilwerte $\wp_{\lambda \mu}, \wp_{\lambda \mu}^{\prime}$ und die speziellen Teilungsgleichungen . . . 244

$\S 2$. Kongruenzgruppen $n^{\text {ter }}$ Stufe in der Modulgruppe $\Gamma$. . . . . . . 249

§ 3. Die Galoisschen Resolventen der speziellen Teilungsgleichungen . . . 255

§ 4. Lösung der speziellen Teilungsgleichung. . . . . . . . . . . . . . 261

§ 5. Die Teilwerte der Funktionen sn, cn und dn. . . . . . . . . . 265

\section{Zweiter Abschnitt.}

\section{Die Transformationstheorie der elliptischen Funktionen.}

\section{Erstes Kapitel. \\ Die Transformation $n^{\text {ten }}$ Grades und die allgemeinen Transformationsgleichungen.}

§ 1. Aưfstellung des Transformationsproblems und Ansatz zur Lösung . . 270

§ 2. Die Repräsentanten der Transformationen $n^{\text {ten }}$ Grades . . . . . . . . 274

§ 3. Die allgemeine Transformationsgleichung der $\wp$-Funktion . . . . . . 278

$\S 4$. Transformation $n^{\text {ten }}$ Grades der Sigmafunktion . . . . . . . . . . . 284

§ 5. Transformation zweiten Grades der Thetafunktionen . . . . . . . . 286

§6. Transformation zweiten Grades der Funktionen sn, cn und dn. . . 290

$\S 7$. Transformation ungeraden Grades der Funktionen zweiter Stufe . . . 293

\section{Zweites Kapitel.}

Systeme ganzer elliptischer Funktionen dritter Art $n^{\text {ter }}$ Stufe.

$\S 1$. Teilwerte nnd Wurzeln der Diskriminante $4 . . . . . . . . . .297$

$\S$ 2. Einführung der ganzen elliptischen Funktionen dritter Art $n^{\text {t }}$ Ordnung $X_{\lambda}\left(u \mid \omega_{1}, \omega_{2}\right)$. . . . . . . . . . . . . . . 303

§ 3. Lineare Transformation der Funktionen $X_{\lambda}\left(u \mid \omega_{1}, \omega_{2}\right)$. . . . . . . 308

$\S 4$. Systeme von Modulformen für ungerade Stufen. . . . . . . . . . . $\$ 14$

§ 5. Ein weiteres System für Modulformen für ungerade Stufen . . . . . 316

$\S 6$. Mehrgliedrige Bilinearverbindungen der $X_{\lambda}$ und ihre lineare Transformation . . . . . . . . . . . . . . . . . 320

§ 7. Die Systeme der Funktionen $Y_{\lambda}$ und der Modulformen $y_{\lambda}$. . . . . 324

$\S 8$. Die Systeme der Funktionen $Z_{\lambda}$ und der Modulformen $z_{\lambda}$. . . . . . $\quad 327$

Drittes Kapitel.

Die speziellen Transformationsgleichungen erster Stufe.

$\S 1$. Die speziellen Transformationsgleichungen als Resolventen der speziellen Teilungsgleichungen. . . . . . . . . . . . . 
§ 2. Ansatz der speziellen Transformationsgleichungen. Geschichtliche Notizen $\mathbf{3 4 2}$

§ 3. Das Transformationspolygon $T_{n}$ und die Transformationsfläche $F_{n}$. $\quad 349$

$\S$ 4. Die erweiterte Gruppe $\Gamma^{(n)}$ und das Klassenpolygon $\mathbf{K}_{n}$. . . . . . . 357

$\S$ 5. Algebraische Methode zur Aufstellung der speziellen Transformationsgleichungen. . . . . . . . . . . . . . . . . 367

\section{Viertes Kapitel.}

\section{Anfstellung der Transformationsgleichungen erster Stufe fiir nielere Grade $n$.}

§ 1. Die Transformationsgrade 2, 4, 8, 16 und 32 . . . . . . . . . . . 371

§ 2. Die Transformationsgrade 3,9 und 27 . . . . . . . . . . . . . . 383

§ 3. Die Transformationsgrade 5, 25, 7 und 49 . . . . . . . . . . . . . . 389

$\S$ 4. Primzahlige Transformationsgrade der Gestalt $n=4 h+3$. . . . . 403

$\S$ 5. Primzahlige Transformationsgrade der Gestalt $n=4 h+1$. . . . . 424

§ 6. Zusammengesetzte ungerade Transformationsgrade . . . . . . . . 437

$\S$ 7. Zusammengesetzte gerade Transformationsgrade . . . . . . . . . 446

\section{Fünftes Kapitel.}

Die Gruppen der speziellen Transformationsgleichungeu und die drei Resolventen der Grade 5, 7 und 11.

$\S$ 1. Die Galoisschen Gruppen der speziellen Transformationsgleichungen .

$\$$ 2. Die Galoisschen imaginären Zahlen und die imaginäre Gestalt der $G_{\frac{1}{2} n\left(n^{2}-1\right)}$

§ 3. Zyklische Gruppen, metazyklische Gruppen und Diedergruppen in der $G_{\frac{1}{2} n\left(n^{2}-1\right)}$

$\S$ 4. Ansatz zur Aufstellung aller Untergruppen der $G$

5. Der Satz von Galois .

$\S$ 6. Die Resolventen fünften und siebenten Grades .

§ 7. Die beiden Resolventen elften Grades

\section{Sechstes Kapitel.}

\section{Die speziellen Transformationsgleichungen hïherer stufen.}

$\S$ 1. Wiederholte Landensche Transformation.

$\S$ 2. Die Jacobi-Sohnkeschen Modulargleichungen .

$\S$ 3. Die Schlaeflischen Modulargleichungen

$\S$ 4. Die Jacobischen Multiplikatorgleichungen

§ 5. Gruppentheoretische Grundlagen für die Resolventen fünften Grades zweiter Stufe .

§. Aufstellang der Resolventen fünften Grades zweiter Stufe.

\$ 7. Notizen über die Lösung der allgemeinen Gleichung fünften Grades durch elliptische Funktionen . . . . . . . . . . . . . . . . . 520

$\S$ 8. Notizen über irrationale Modulargleichungen. . . . . . . . . . . . 524

$\S$ 9. Notizen über Modularkorrespondenzen . . . . . . . . . . . . $\$ 27$

$\$ 10$. System der Modnlfunktionen sechster Stufe . . . . . . . . . . . 533

§ 11. Die Thetarelationen des dritten Transformationsgrades . . . . . . 538 


\section{Einleitung.}

\section{Zusammenstellung von Sätzen aus der Algebra und Zahlentheorie.}

Die Behandlung der Divisionssätze der elliptischen Funktionen und der Transformationstheorie dieser Funktionen setzt eine etwas tiefere Kenntnis der Algebra und Zahlentheorie voraus. In einer in fünf Teile zerlegten Einleitung werden zunächst die zur Verwendung kommenden Sätze aus den beiden genannten Gebieten entwickelt.

\section{Endliche Gruppen. ${ }^{1}$ )}

\section{§ 1. Begriff einer Gruppe endlicher 0rdnung.}

In I, $126 \mathrm{ff}^{2}$ ) betrachteten wir ein System von $m$ linearen Substitutionen einer komplexen Variablen $z$, von dem wir aussagten, es bilde „eine Gruppe $G_{m}$ endlicher Ordnung $m^{\prime \prime}$. Dabei war $m$ eine endliche Anzahl, die Substitutionen waren symbolisch durch $S_{0}, S_{1}, \ldots, S_{m-1}$ bezeichnet, und es bedeutete insbesondere die erste unter ihnen, $S_{0}$, die „identische Substitution“, die auch symbolisch durch 1 bezeichnet wurde.

Ein erstes Kennzeichen des Begriffs einer Gruppe bestand darin, daB die aufeinanderfolgenden Anwendungen zweier Substitutionen $S_{a}$ und $S_{b}$ der Gruppe $G_{m}$ eine gleichfalls in $\operatorname{der} G_{m}$ enthaltene Substitution lieferten. Genauer gesprochen: Wenn $z^{\prime}=S_{a}(z)$ und $z^{\prime \prime}=S_{b}\left(z^{\prime}\right)$ zwei unserer Substitutionen sind, so sollte auch $z^{\prime \prime}=S_{b}\left(S_{a}(z)\right)$ eine der Gruppe angehörende Substitution sein. Die in dieser Art aus $S_{a}$ und $S_{b}$,zusammengesetzte" Substitution bezeichneten wir symbolisch als "Produkt" $S_{b} \cdot S_{\alpha}$ von $S_{a}$ und $S_{b}$. Die Reihenfolge, in der die Substitutionen auf die Variable auszuüben sind, entspricht der von rechts nach links im Produkte $S_{b} \cdot S_{a}$ gelesenen Faktorenfolge. Das Produkt $S_{a} \cdot S_{b}$ erwies sich im allgemeinen

1) Von ausführlichen neueren Darstellungen sei genannt $\mathrm{H}$. Weber, „Lehrbuch der Algebra", 2. Aufl. (Braunschweig 1898 u. 1899), Bd. 2, S. 1 ff. und Bd. 1, S. $513 \mathrm{ff}$. Eine Zusammenstellung der Sätze findet man bei A. Loewy im Kap. III des „Pascalschen Repertoriums“, 2. Aufl. (Leipzig 1910), erste Hälfte, S. $168 \mathrm{ff.}$

2) Diese Angabe bedeutet "Seite $126 \mathrm{ff}$. in Band I des vorliegenden Werkes". 
als von $S_{b} \cdot S_{a}$ verschieden, d. h. für unsere symbolischen Produkte gilt das „kommutative Grundgesetz" der gewöhnlichen Multiplikation nicht. Dagegen gilt fürr dreigliedrige symbolische Produkte unserer Substitutionen das „assoziative Gesetz", wie in I, 127 näher ausgeführt ist.

Die nähere Beschaffenheit der einzelnen Gruppen gründet sich auf das Gesetz, nach dem sich die Zusammensetzung irgend zweier Substitutionen der Gruppe $G_{m}$ zu einer dritten gleichfalls in $G_{m}$ enthaltenen Substitution vollzieht. Dieses Gesetz ist für unsere Substitutionen $S$ durch die Gleichung (2) in I, 127 zum Ausdruck gebracht.

Es tritt uns nun die Idee einer ganz entsprechenden Gruppenbildung nicht nur bei linearen Substitutionen, sondern weiterhin bei vielen verschiedenen Gelegenheiten entgegen. Wir wollen daher hier gleich bei Aufstellung der allgemeinen Sätze über endliche Gruppen die Begriffe von der besonderen Einkleidung befreien, welche bei den Gruppen linearer Substitutionen vorliegen.

Es sei demnach jetzt irgendein System von $m$ gleichartigen Operationen oder $m$ gleichartigen analytischen Ausdrücken oder $m$ sonstwie gleichartig erklärten mathematischen Gebilden vorgelegt, die wir sogleich wieder durch $S_{0}, S_{1}, S_{2}, \ldots, S_{m-1}$ bezeichnen wollen und die $m$ „Elemente" nennen, um einen von der besonderen Einkleidung unabhängigen Namen für dieselben zu besitzen. Es soll ferner ein Gesetz der Zusammensetzung irgend zweier Elemente $S_{a}, S_{b}$ zu einem symbolisch als Produkt zu schreibenden, eindeutig bestimmten Ergebnis $S_{b} \cdot S_{a}$ bekannt sein. Wir stellen dann unter der Voraussetzung, daß $m$ eine endliche Anzahl ist, folgende Erklärung auf: Die $m$ Elemente $S_{0}, S_{1}, \ldots, S_{m-1}$ bilden eine „Gruppe" $G_{m}$ der endlichen „Ordnung" $m$, wenn folgende drei Bedingungen zutreffen:

1. Das Ergebnis der Zusammensetzung $S_{b} \cdot S_{a}$ irgend zweier Elemente $S_{a}, S_{b}$ ist stets wieder eines der Elemente $S$.

2. Für die nach 1. herstellbaren dreigliedrigen symbolischen Produkte gilt das assoziative Gesetz:

$$
S_{c} \cdot\left(S_{b} \cdot S_{a}\right)=\left(S_{c} \cdot S_{b}\right): S_{a} .
$$

3. Ist $S_{b} \neq S_{c}, d$.h. sind $S_{b}$ und $S_{c}$ irgend zwei verschiedene Elemente, und ist $S_{a}$ irgendein Element, so soll auch $S_{b} \cdot S_{a} \neq S_{c} \cdot S_{a}$ und $S_{a} \cdot S_{b} \neq S_{a} \cdot S_{c}$ gelten.

Ist $S_{a}$ irgendeines der $m$ Elemente, so sind die $m$ Elemente $S_{0} \cdot S_{a}$, $S_{1} \cdot S_{a}, S_{2} \cdot S_{a}, \ldots, S_{m-1} \cdot S_{a}$ alle voneinander verschieden, und da sie alle in $G_{m}$ enthalten sind, so stellen sie alle $m$ Elemente der Gruppe in irgendeiner Reihenfolge dar. Dasselbe gilt von den $m$ Produkten $S_{a} \cdot S_{0}, S_{a} \cdot S_{1}$, $S_{a} \cdot S_{2}, \ldots, S_{a} \cdot S_{m-1}$. Wir folgern hieraus, daB, wenn $S_{a}$ und $S_{d}$. willkürlich gewählt sind, stets ein und nur ein Element $S_{b}$ in $G_{m}$ enthalten 
ist, das die Gleichung $S_{b} \cdot S_{a}=S_{d}$ befriedigt; ebenso gibt es in $G_{m}$ ein und nur ein Element $S_{c}$, das die Gleichung erfüllt $S_{a} \cdot S_{c}=S_{a}$.

Insbesondere gibt es für ein vorgelegtes $S_{a}$ ein und nur ein etwa durch $S_{(a)}$ zu bezeichnendes Element, das die Gleichung $S_{(a)} \cdot S_{a}=S_{a}$ befriedigt und gleichfalls ein und nur ein Element $S_{(a)}^{\prime}$, für das $S_{a} \cdot S_{(a)}^{\prime}=S_{a}$ gilt. Aus diesen beiden Gleichungen ergeben sich für irgendwelche $S_{b}, S_{c}$ die folgenden:

$$
S_{(a)} \cdot\left(S_{a} \cdot S_{c}\right)=S_{a} \cdot S_{c} \cdot \quad\left(S_{b} \cdot S_{a}\right) \cdot S_{(a)}^{\prime}=S_{b} \cdot S_{a}
$$

Da nun durch geeignete Auswahl von $S_{c}$ und $S_{b}$ die Elemente $S_{a} \cdot S_{c}$ und $S_{b} \cdot S_{a}$ mit einem beliebigen Elemente $S_{e}$ von $G_{m}$ gleich werden, so gilt für jedes $S_{e}: \quad S_{(a)} \cdot S_{e}=S_{e}, \quad S_{e} \cdot S_{(\alpha)}^{\prime}=S_{e}$,

woraus wir die Folgerungen $S_{(e)}=S_{(x)}, S_{(e)}^{\prime}=S_{(a)}^{\prime}$ ziehen. Die Elemente $S_{(0)}, S_{(1)}, S_{(2)}, \ldots, S_{(m-1)}$ sind also einander gleich, ebenso die Elemente $S_{(0)}^{\prime}, S_{(1)}^{\prime}, S_{(2)}^{\prime}, \ldots, S_{(m-1)}^{\prime}$. Es gibt also ein und nur ein Element $S$ in $G_{m}$, welches für alle $S_{a}$ die Gleichung $S \cdot S_{a}=S_{a}$ befriedigt, und ebenso ein und nur ein $S^{\prime}$, das $S_{a} \cdot S^{\prime}=S_{a}$ erfüllt. Setzt man in die beiden letzten Gleichungen $S_{a}=S$ ein, so folgt $S \cdot S=S$ und $S \cdot S^{\prime}=S$, so daB aus $S \cdot S=S \cdot S^{\prime}$ nach dem Grundsatze 3. endlich $S^{\prime}=S$ erkannt wird. Das eine so gefundene Element $S$ spielt in den symbolischen Produkten die Rolle der Einheit; es wird demnach das "Einheitselement" genannt und auch symbolisch durch 1 bezeichnet. Wir wollen es hinfort an die erste Stelle setzen und also $S_{0}=1$ nehmen: In der Gruppe $G_{m}$ gibt es ein und nur ein "Einheitselement" $S_{0}=1$, das die Eigenschaft besitst, mit irgendeinem $S_{a}$ die Ergebnisse $S_{0} \cdot S_{a}=S_{a}$ und $S_{a} \cdot S_{0}=S_{a}$ zu liefern.

Weiter gibt es für irgendein Element $S_{a}$ aus $G_{m}$ zwei symbolisch durch $S_{(a)}^{\prime \prime}$ und $S_{(a)}^{\prime \prime \prime}$ zu bezeichnende Elemente, die die Gleichungen:

$$
S_{(a)}^{\prime \prime} \cdot S_{a}=S_{0}=1, \quad S_{a}^{\prime} \cdot S_{(a)}^{\prime \prime \prime}=S_{0}=1
$$

befriedigen. Aus $1=S_{(a)}^{\prime \prime} \cdot S_{a}$ folgt unter Anwendung des assoziativen Gesetzes mit Rücksicht auf die zweite Gleichung (2):

$$
S_{(a)}^{\prime \prime \prime}=1 \cdot S_{(a)}^{\prime \prime \prime}=S_{(a)}^{\prime \prime} \cdot\left(S_{a} \cdot S_{(a)}^{\prime \prime \prime}\right)=S_{(a)}^{\prime \prime} \cdot 1=S_{(a)}^{\prime \prime},
$$

so daB die beiden Elemente $S_{(a)}^{\prime \prime}$ und $S_{(a)}^{\prime \prime \prime}$ einander gleich sind. Wir führen für sie auch das Symbol $S_{a}^{-1}$ ein und nennen dies Element $S_{a}^{-1}$ das zu $S_{a}$,inverse" Element: $Z u$ jedem Elemente $S_{a}$ gibt es in $G_{m}$ ein eindeutig bestimmtes "inverses" Element $S_{a}^{-1}$, das mit $S_{a}$ zusammengesetzt, die Gleichungen $S_{a}^{-1} \cdot S_{a}=1$ und $S_{a} \cdot S_{a}^{-1}=1$ befriedigt; $z u S_{a}^{-1}$ ist umgekehrt $S_{a}$ invers.

Das kommutative Gesetz für die symbolischen Produkte gehörte nicht zu den Grundgesetzen, durch die wir den Begriff einer endlichen Gruppe erklärten. Es braucht demnach keineswegs immer $S_{b} \cdot S_{a}$ gleich $S_{a} \cdot S_{b}$ zu sein. Besteht indessen für zwei besondere Elemente $S_{a}$ und $S$ 
die Gleichung $S_{a} \cdot S_{b}=S_{b} \cdot S_{a}$, so heißen diese Elemente „vertauschbar" oder „kommutativ". Besteht für je zwei Elemente $S_{a}$ und $S_{b}$ von $G_{m}$ die Regel $S_{a} \cdot S_{b}=S_{b} \cdot S_{a}$, so wird $G_{m}$ eine „kommutative“ oder "Abelsche Gruppe genannt.

\section{§ 2. Begriff der Untergruppe.}

Eine Gruppe $G_{\mu}$, deren sämtliche Elemente in $G_{m}$ enthalten sind, heißt eine "Untergruppe" von $G_{m}$. Das Einheitselement $S_{0}=1$ bildet für sich eine Untergruppe $G_{1}$, und die Gesamtgruppe $G_{m}$ ist der Erklärung entsprechend auch zu ihren Untergruppen zu rechnen.

Wir bezeichnen die Elemente einer Untergruppe $G_{\mu}$ durch die Symbole $T_{0}=S_{0}=1, T_{1}, T_{2}, \ldots, T_{\mu-1}$. Bedeutet $U$ irgendein.Element von $G_{m}$, so bezeichnen wir das System der $\mu$ verschiedenen Elemente:

$$
T_{0} \cdot U=U, \quad T_{1} \cdot U, \quad T_{2} \cdot U, \ldots, T_{\mu-1} \cdot U
$$

symbolisch durch $G_{\mu} \cdot U$ und nennen es eine „Nebengruppe" von $G_{\mu}$. Die Nebengruppe $G_{\mu} \cdot U$ bleibt, abgesehen von der Anordnung ihrer Elemente, unverändert, wenn wir $U$ durch irgendein Element $U^{\prime}=T_{\alpha} \cdot U$ von $G_{\mu} \cdot U$ ersetzen. Dies folgt aus der Tatsache, daB die Produkte $T_{0} \cdot T_{\alpha}$, $T_{1} \cdot T_{\alpha}, T_{2} \cdot T_{\alpha}, \ldots, T_{\mu-1} \cdot T_{\alpha}$ wieder die $G_{\mu}$ bilden. Zwei Nebengruppen $G_{\mu} \cdot U_{1}$ und $G_{\mu} \cdot U_{2}$ sind entweder gleich, $G_{\mu} \cdot U_{1}=G_{\mu} \cdot U_{2}$, d. h. sie bestehen abgesehen von der Anordnung aus den gleichen Elementen, oder sie haben kein Element gemein. Haben sie nämlich ein gemeinsames Element $T_{\alpha} \cdot U_{1}=T_{\beta} \cdot U_{2}$, so ist $U_{2}=\left(T_{\beta}^{-1} T_{\alpha}\right) \cdot U_{1}$; also ist $U_{2}$ in $G_{\mu} \cdot U_{1}$ enthalten, so daß $G_{\mu} \cdot U_{2}=G_{\mu} \cdot U_{1}$ folgt. $\mathrm{Zu}$ den Nebengruppen gehört auch $G_{\mu}$ selbst; denn wir erhalten die $G_{\mu}$ im Systeme (1) wieder, sooft $U$ ein Element von $G_{\mu}$ ist. Alle von $G_{\mu}$ verschiedenen Nebengruppen sind keine Gruppen, da keine von ihnen das Einheitselement enthält.

Haben wir $s$ verschiedene Nebengruppen $G_{\mu}, G_{\mu} \cdot U_{1}, G_{\mu} \cdot U_{2}, \ldots$, $G_{\mu} \cdot U_{s-1}$ bereits gebildet, so sind in den $s \mu$ verschiedenen Elementen derselben entweder bereits alle $m$ Elemente von $G_{m}$ erschöpft, oder es gibt noch ein weiteres Element $U_{s}$ in $G_{m}$ und damit zugleich $\mu$ weitere Elemente, die eine neue Nebengruppe $G_{\mu} \cdot U_{s}$ bilden. Da $m$ endlich ist, so wird bei Fortsetzung dieses Prozesses die $G_{m}$ durch $t$ Nebengruppen erschöpft, wo die Anzahl $t$ aus der Gleichung $t \cdot \mu=m$ zu berechnen ist: Die Ordnung $\mu$ der Untergruppe $G_{\mu}$ erweist sich als ein Teiler von $m$; der Quotient $t=\frac{m}{\mu}$ heißt der "Index“ der Untergruppe $G_{\mu}$. Es gilt der Satz: Die Gesamtgruppe $G_{m}$ läßt sich der Untergruppe $G_{\mu}$ entsprechend in $t$ Nebengruppen zerlegen:

$$
G_{m}=G_{\mu}+G_{\mu} \cdot U_{1}+G_{\mu} \cdot U_{2}+\cdots+G_{\mu} \cdot U_{t-1},
$$


wo $1, U_{1}, U_{2}, \ldots, U_{t-1}$ geeignet gewählte Elemente von $G_{m}$ sind. Durch die Pluszeichen in (2) soll zum Ausdruck kommen, daß wir $G_{m}$ durch Zusammenfügung der Elemente aller $t$ Nebengruppen erhalten. Aus den vorausgehenden Betrachtungen folgt noch: Die $t$ Nebengruppen der $G_{\mu}$ sind, abgesehen von ihrer Anordnung, eindeutig bestimmt, d.h. unabhängig von der besonderen Auswahl der $U$.

Man kann die Bildung der Nebengruppen auch in der Art vollziehen, dab man an Stelle von (1):

$$
V \cdot T_{0}=V, \quad V \cdot T_{1}, \quad V \cdot T_{2}, \ldots, V \cdot T_{\mu-1}
$$

als eine Nebengruppe $V \cdot G_{\mu}$ erklärt, unter $V$ irgendein Element aus $G_{m}$ verstanden. Alle weiteren Schlüsse gestalten sich dann wie oben, und wir gelangen zu einer Zerlegung:

$$
G_{m}=G_{\mu}+V_{1} \cdot G_{\mu}+V_{2} \cdot G_{\mu}+\cdots+V_{t-1} \cdot G_{\mu}
$$

von $G_{m}$. Eine spezielle Zerlegung dieser Art können wir aus (2) in der folgenden Gestalt ableiten:

$$
G_{m}=G_{\mu}+U_{1}^{-1} \cdot G_{\mu}+U_{2}^{-1} \cdot G_{\mu}+\cdots+U_{t-1}^{-1} \cdot G_{\mu} .
$$

Da nämlich $G_{\mu}$ mit dem einzelnen Elemente $T_{\alpha}$ stets auch das inverse $T_{\alpha}^{-1}$ enthält und $\mathrm{zu} T_{\alpha} \cdot U_{\beta}$ offenbar $U_{\beta}^{-1} \cdot T_{\alpha}^{-1}$ invers ist, so besteht $U_{\beta}^{-1} \cdot G_{\mu}$ aus allen zu den Elementen von $G_{\mu} \cdot U_{\beta}$ inversen Elementen und mag demnach die zu $G_{\mu} \cdot U_{\beta}$ inverse Nebengruppe heißen. Nun geht jede Gruppe in sich selbst über, wenn man jedes ihrer Elemente durch sein inverses ersetzt. Es ist demnach auf der rechten Seite von (5) jedes Element von $G_{m}$ vertreten und jedes nur einmal, so dab wir durch die in (5) rechts stehende Summe tatsächlich die ganze $G_{m}$ erschöpfen. Wir folgern noch den Satz: Irgend zuei Zerlegungen (2) und (4) stehen in der Beziehung, daß bei der einen Zerlegung, abgesehen von der Anordnung, die inversen Nebengruppen der anderen Zerlegung als Summenglieder auftreten.

Zu einer einfachsten Art von Untergruppen führt folgende Überlegung: Mittelst eines beliebigen Elementes $S$ von $G_{m}$ bilden wir die Reihe der gleichfalls in $G_{m}$ enthaltenen Elemente:

$$
S, \quad S \cdot S=S^{2}, \quad S \cdot S \cdot S=S^{3}, \quad \ldots,
$$

die wir abgekürzt als Potenzen von $S$ schreiben. In dieser zunächst nicht abbrechenden Reihe können höchstens $m$ verschiedene Elemente auftreten, so dab unter den Potenzen (6) notwendig solche auftreten, die gleich sind, d. h. die das gleiche Element von $G_{m}$, darstellen. Es mag z. B. $S^{n}=S^{n+v}$ sein, wo $\nu>0$ ist, während die $S^{n+1}, S^{n+2}, \ldots, S^{n+v-1}$ noch alle von $S^{n}$ verschieden sein sollen. Dann gilt:

$$
S^{n} \cdot S^{\nu}=S^{n}, \quad S^{n} \cdot S^{\nu^{\prime}} \neq S^{n}, \quad 0<\nu^{\prime}<\nu,
$$


woraus hervorgeht, daB $S^{v}$ die niederste unter den Potenzen (6) ist, die das Einheitselement darstellt, $S^{\nu}=S_{0}=1$. Es ergibt sich weiter, daB keine zwei unter den Potenzen:

$$
S, S^{2}, S^{3}, \ldots, S^{v-1}, S^{v}=S_{0}=1
$$

einander gleich sind (da man sonst durch Wiederholung der Überlegung eine Gleichung $S^{\nu^{\prime}}=1$ mit $0<\nu^{\prime}<\nu$ fände), sowie daB $S$ und $S^{\nu-1}$ zu einander invers sind, desgleichen $S^{2}$ und $S^{\nu-2}, S^{3}$ und $S^{\nu-3}$ usw. Verstehen wir unter $S^{0}$ das Einheitselement und bezeichnen allgemein das zu $S^{n}$ inverse Element durch $S^{-n}$, so sind in der unendlichen Reihe:

$$
\ldots, S^{-3}, S^{-2}, S^{-1}, S^{0}=1, S, S^{2}, S^{3}, \ldots
$$

zwei Elemente stets und nur dann einander gleich, wenn ihre Exponenten $\bmod \nu$ kongruent sind. Die Reihe (8) enthält demnach nur $\nu$ verschiedene Elemente, als welche wir die Elemente (7) wählen können; bei Fortsetzung der Reihe (8) nach rechts und links hin tritt beständig periodische Wiederholung derselben Elemente in der gleichen Anordnung ein. Man

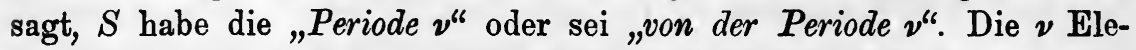
mente $(7), \mathrm{d}$. h. die verschiedenen Potenzen von $S$, bilden für sich eine Untergruppe $G_{v}$, die eine ,zyklische Gruppe genannt wird; $S$ heiBt das "erzeugende Element" dieser $G_{v}$, insofern die gesamten Elemente $\operatorname{der} G_{v}$ durch wiederholte Zusammensetzung von $S$ mit sich selbst erzeugt werden können.

\section{§ 3. Gleichberechtigte und ausgezeichnete Untergruppen.}

Sind $T$ und $U$ beliebige Elemente aus $G_{m}$, so ist auch $T^{\prime}=U \cdot T \cdot U^{-1}$ in $G_{m}$ enthalten. Man sagt, das Element $T^{\prime}=U \cdot T \cdot U^{-1}$ entstehe aus $T$ durch „Transformation" mit $U$ oder $T$ gehe in $T^{\prime}$ durch Transformation mit $U$ über. Da umgekehrt $T=U^{-1} \cdot T^{\prime} \cdot U$ ist, so geht $T^{\prime}$ in $T$ durch Transformation mit dem zu $U$ inversen Elemente $U^{-1}$ über. Das Einheitselement geht bei jeder Transformation nur in sich selbst über. Sind $T_{a}$ and $T_{b}$ verschieden, so sind auch:

$$
T_{a}^{\prime}=U \cdot T_{a} \cdot U^{-1}, \quad T_{b}^{\prime}=U \cdot T_{b} \cdot U^{-1}
$$

verschieden. Sind $T_{a}$ und $T_{b}$ irgend zwei Elemente, die durch Transformation mit $U$ in $T_{a}^{\prime}$ und $T_{b}^{\prime}$ übergehen, so wird $T_{b} \cdot T_{a}$ durch $U$ in $T_{b}^{\prime} \cdot T_{a}^{\prime}$ transformiert. Es gilt nämlich zufolge des Assoziationsgesetzes:

$$
T_{b}^{\prime} \cdot T_{a}^{\prime}=\left(U \cdot T_{b}^{\prime} \cdot U^{-1}\right) \cdot\left(U \cdot T_{a} \cdot U^{-1}\right)=U \cdot\left(T_{b} \cdot T_{a}\right) \cdot U^{-1}
$$

Bilden die $T_{0}=1, T_{1}, T_{2}, \ldots, T_{\mu-1}$ eine Untergruppe $G_{\mu}$ ron $G_{m}$, so bezeichnen wir mit $U \cdot G_{\mu} \cdot U^{-1}$ das System der $\mu$ durch $U$ transformierten Elemente $T_{\alpha}^{\prime}=U \cdot T_{\alpha} \cdot U^{-1}$. Diese $\mu$ Elemente $T_{\alpha}^{\prime}$ sind alle roneinander verschieden, und man erkennt aus den eben ausgesprochenen Sätzen leicht, daß das System $U \cdot G_{\mu} \cdot U^{-1}$ alle Kennzeichen einer Gruppe 
$G_{\mu}^{\prime}=U \cdot G_{\mu} \cdot U^{-1}$ der Ordnung $\mu$ hat. Wir sagen, diese Gruppe $G_{\mu}^{\prime}$ gehe aus $G_{\mu}$ durch Transformation mit $U$ hervor; umgekehrt ist natürlich wieder $G_{\mu}=U^{-1} \cdot G_{\mu}^{\prime} \cdot U$. Zwei Untergruppen dieser Art $G_{\mu}, G_{\mu}^{\prime}$, die durch Elemente von $G_{m}$ ineinander transformierbar sind, heißen ,innerhalb der Gruppe $G_{m}$ gleichberechtigt" oder kurz "gleichberechtigt".

Die Elemente der beiden Gruppen $G_{\mu}$ und $G_{\mu}^{\prime}$ sind umkehrbar eindeutig aufeinander bezogen, indem allgemein dem Elemente $T_{\alpha}$ von $G_{\mu}$ das Element $T_{\alpha}^{\prime}=U \cdot T_{\alpha} \cdot U^{-1}$ von $G_{\mu}^{\prime}$ zugewiesen ist. Diese Beziehung ist eine solche, daB, wenn $T_{\beta} \cdot T_{\alpha}=T_{\gamma}$ ist, zufolge (2) stets auch für die zugeordneten Elemente $T_{\alpha}^{\prime}, T_{\beta}^{\prime}$ und $T_{\gamma}^{\prime}$ die Gleichung $T_{\beta}^{\prime} \cdot T_{\alpha}^{\prime}=T_{\gamma}^{\prime}$ gilt. Die Regel, nach der sich je zwei Elemente von $G_{\mu}$ wieder zu einem Elemente dieser Gruppe zusammensetzen, liefert also beim Übergang zur $G_{\mu}^{\prime}$ gerade genau die Regel für die gleichberechtigte Gruppe. Zwei Gruppen gleicher Ordnung, deren Elemente in der hiermit dargelegten Art umkehrbar eindeutig einander zugeordnet sind, heißen allgemein "isomorph". Da die wesentlichen Eigenschaften einer Gruppe aus dem Gesetze hervorgehen, nach dem sich je zwei Elemente zu einem dritten zusammensetzen, so stimmen zwei isomorphe Gruppen in ihren wesentlichen Eigenschaften überein. Wir notieren den Satz: Zwei innerhalb der $G_{m}$ gleichberechtigte Untergruppen sind isomorph.

Sind die Elemente $T_{\alpha}^{\prime}=U \cdot T_{\alpha} \cdot U^{-1}$ der mit $G_{\mu}$ gleichberechtigten $G_{\mu}^{\prime}$, abgesehen von der Reihenfolge, den $T_{\alpha}$ gleich, so ist $G_{\mu}^{\prime}$ wieder die Gruppe $G_{\mu}$. Wir bringen dies durch die Gleichungen:

$$
U \cdot G_{\mu} \cdot U^{-1}=G_{\mu}, \quad U \cdot G_{\mu}=G_{\mu} \cdot U
$$

zum Ausdruck und sagen, die Gruppe $G_{\mu}$ sei mit dem Elemente $U$,vertauschbar". Offenbar ist $G_{\mu}$ mit jedem ihrer eigenen Elemente vertauschbar. Allgemein besteht der Satz: Alle in $G_{m}$ enthaltenen Elemente $U$, mit denen $G_{\mu}$ vertauschbar ist, bilden eine Gruppe $G_{\mu \cdot \tau}$, in $\operatorname{der} G_{\mu}$ als Untergruppe enthalten ist. Aus:

$$
U_{1} \cdot G_{\mu} \cdot U_{1}^{-1}=G_{\mu}, \quad U_{2} \cdot G_{\mu} \cdot U_{9}^{-1}=G_{\mu}
$$

folgt nämlich auf Grund des assoziativen Gesetzes, das man leicht auf die hier vorliegenden symbolischen Produkte überträgt:

$\left(U_{2} \cdot U_{1}\right) \cdot G_{\mu} \cdot\left(U_{2} \cdot U_{1}\right)^{-1}=U_{2} \cdot\left(U_{1} \cdot G_{\mu} \cdot U_{1}^{-1}\right) \cdot U_{2}^{-1}=U_{2} \cdot G_{\mu} \cdot U_{2}^{-1}=G_{\mu}$.

Die Ordnung der Gruppe aller Elemente $U$, mit denen $G_{m}$ vertauschbar ist, muB aber ein Vielfaches $\mu \approx$ ron $\mu$ sein, da $G_{\mu}$ in jener Gruppe als Untergruppe enthalten ist.

Der Index dieser Gruppe $G_{\mu \tau}$ als Untergruppe der $G_{m}$ ist $\frac{t}{\tau}$. Die $G_{\mu \tau}$ liefere als Zerlegung von $G_{m}$ in Nebengruppen:

$$
G_{m}=G_{u \tau}+V_{1} \cdot G_{u \tau}+V_{2} \cdot G_{u \tau}+\cdots+V_{\frac{t}{\tau}-1} \cdot G_{\mu \tau} .
$$


Alle Elemente der einzelnen Nebengruppe $V_{a} \cdot G_{\mu \tau}$ liefern dann, zur Transformation von $G_{\mu}$ verwendet, ein und dieselbe transformierte Gruppe $G_{\mu}^{(a)}=V_{a} \cdot G_{\mu} \cdot V_{a}^{-1}$. Zwei verschiedene Nebengruppen liefern indessen verschiedene transformierte Gruppen. Wäre nämlich:

$$
V_{b} \cdot G_{\mu} \cdot V_{b}^{-1}=V_{a} \cdot G_{\mu} \cdot V_{a}^{-1},
$$

so wäre $G_{\mu}$ mit $V_{a}^{-1} \cdot V_{b}$ vertauschbar, und also würde $V_{a}^{-1} \cdot V_{b}$ ein Element $U$ von $G_{\mu \tau}$ sein, woraus die nicht zutreffende Gleichung $V_{b}=V_{a} \cdot U$ folgen würde. Es ergibt sich also der Satz: Die Untergruppe $G_{\mu}$ ist durch die gesamten Elemente. von $G_{m}$ im ganzen in $\frac{t}{\tau}$ verschiedene gleichberechtigte und also isomorphe Untergruppen transformierbar. Wir nennen sie ein "System von $\frac{t}{\tau}$ gleichberechtigten Untergruppen".

Ist insbesondere $\tau=t, \mathrm{~d}$. h. ist $G_{\mu}$ mit allen Elementen von $G_{m}$ vertauschbar, so ist $G_{\mu}$ nur mit sich selbst gleichberechtigt und heiBt dann eine "ausgezeichnete Untergruppe" von $G_{m}$. Die nur aus $S_{0}=1$ bestehende Untergruppe $G_{1}$ ist eine ausgezeichnete, desgleichen die aus allen Elementen bestehende $G_{m}$. Gibt es auBer diesen beiden keine weiteren ausgezeichneten - Untergruppen in $G_{m}$, so heißt die Gruppe $G_{m}$ "einfach"; andernfalls nennt man sie „zusammengesetzt".

Sind $G_{\lambda}, G_{\mu}, G_{v}, \ldots$ irgendwelche Untergruppen von $G_{m}$, so nennt man das System aller Elemente, deren einzelnes in jeder dieser Gruppen enthalten ist, den "Durchschnitt" der Gruppen $G_{\lambda}, G_{\mu}, G_{v}, \ldots$ und bezeichnet denselben durch $D\left(G_{\lambda}, G_{\mu}, G_{v}, \ldots\right)$. Dieser Durchschnitt $D\left(G_{\lambda}, G_{\mu}, G_{v}, \ldots\right)$ bildet wieder eine Gruppe, die als Untergruppe in jeder der Gruppen $G_{\lambda}, G_{\mu}, G_{v}, \ldots$ enthalten ist. Gehören nämlich die Elemente $S_{a}$ und $S_{b}$ dem Durchschnitt $D$ an, so sind sie in jeder Gruppe $G_{\lambda}, G_{\mu}, G_{v}, \ldots$ enthalten; also ist auch $S_{b} \cdot S_{a}$ in jeder Gruppe und damit im Durchschnitt enthalten. Gehen die Gruppen $G_{\lambda}, G_{\mu}, G_{v}, \ldots$ durch Transformation mit irgendeinem Elemente $U$ von $G_{m}$ in $G_{\lambda}^{\prime}, G_{\mu}^{\prime}$, $G_{v}^{\prime}, \ldots$ über, so geht der Durchschnitt $D\left(G_{\lambda}, G_{\mu}, G_{v}, \ldots\right)$ bei Transformation mit $U$ in denjenigen der Gruppen $G_{\lambda}^{\prime}, G_{\mu}^{\prime}, G_{v}^{\prime}, \ldots$ über. Ein System gleichberechtigter Untergruppen $G_{\mu}, G_{\mu}^{\prime}, G_{\mu}^{\prime \prime}, \ldots$ geht bei einer solchen Transformation, abgesehen von der Anordnung der Gruppen, stets in sich selbst über. Hieraus folgt: Der Durchschnitt $D\left(G_{\mu}, G_{\mu}^{\prime}, G_{\mu}^{\prime \prime}, \ldots\right)$. eines Systems gleichberechtigter Untergruppen ist eine ausgezeichnete Untergruppe von $G_{m}$.

\section{\$ 4. Sätze über ausgezeichnete Untergruppen.}

Ist die Untergruppe $G_{\mu}$ des Index $t$ und der Elemente $T_{0}=1, T_{1}$, $T_{2}, \ldots, T_{\mu-1}$ ausgezeichnet, so gilt für jedes Element $U_{a}$ von $G_{m}$ die Gleichung $G_{\mu} \cdot U_{a}=U_{a} \cdot G_{\mu}$, so daß die beiden Arten (2) und (4) S. $4 \mathrm{ff}$. 
der Zerlegung von $G_{m}$ in Nebengruppen im Falle einer ausgezeichneten $G_{\mu}$ nicht verschieden sind. Nach S. 5 haben wir also jetzt nur ein System von Nebengruppen, dem mit der einzelnen Nebengruppe immer auch die ihr ,inverse“ Nebengruppe angehört.

Wir nehmen nun an, daß das Element $U_{b} \cdot U_{a}$ in der zu $U_{c}$ gehörenden Nebengruppe enthalten und also in der Gestalt $U_{b} \cdot U_{a}=T \cdot U_{c}$ darstellbar ist, wo $T$ eines der Elemente von $G_{\mu}$ ist. Dann werden sich irgend zwei Elemente $T_{\alpha} \cdot U_{a}$ und $T_{\beta} \cdot U_{b}$ der beiden Nebengruppen $G_{\mu} \cdot U_{a}$ und $G_{\mu} \cdot U_{b}$ stets wieder zu einem Elemente der zu $U_{c}$ gehörenden Nebengruppe zusammensetzen, wie mit Rücksicht auf $U_{b} \cdot T_{\alpha}=T_{\gamma} \cdot U_{b}$ aus

$$
\left(T_{\beta} \cdot U_{b}\right) \cdot\left(T_{\alpha} \cdot U_{a}\right)=\left(T_{\beta} \cdot T_{\gamma}\right) \cdot\left(U_{b} \cdot U_{a}\right)=\left(T_{\beta} \cdot T_{\gamma} \cdot T\right) \cdot U_{c}
$$

folgt. Wir bringen diese Tatsache durch die Gleichung zum Ausdruck:

$$
\left(G_{\mu} \cdot U_{b}\right) \cdot\left(G_{\mu} \cdot U_{a}\right)=G_{\mu} \cdot U_{c},
$$

in die wir die den verschiedenen Kombinationen $T_{\alpha}, T_{\beta}$ entsprechenden Gleichungen (1) zusammengefaßt denken.

Die Gleichung (2) veranlaßt uns, die $t$ zu einer ausgezeichneten Untergruppe des Index $t$ gehörenden Nebengruppen $G_{\mu}, G_{\mu} \cdot U_{1}, \ldots, G_{\mu} \cdot U_{t-1}$ selbst wieder als „Elemente" aufzufassen, was nach dem allgemeinen Begriffe eines Elementes (S. 2) statthaft ist. Als solche neuen Elemente bezeichnen wir die Nebengruppen kurz durch $\bar{U}_{0}=G_{\mu}, \bar{U}_{1}=G_{\mu} \cdot U_{1}$, $\bar{U}_{2}=G_{\mu} \cdot U_{2}, \ldots, \bar{U}_{t-1}=G_{\mu} \cdot U_{t-1}$. Dann ist leicht zu zeigen, daß die $\bar{U}_{a}$ selbst wieder eine "Gruppe" der Ordnung $t$ bilden, die wir durch $\bar{G}_{t}$ bezeichnen. Irgend zwei Elemente $\bar{U}_{a}$ und $\bar{U}_{b}$ geben nämlich zufolge (2) bei Zusammensetzung das Element $\bar{U}_{b} \cdot \bar{U}_{a}=\bar{U}_{c}$, das wieder zu den $t$ Elementen $\bar{U}$ gehört. Da ein symbolisches Produkt $\bar{U}_{b} \cdot \bar{U}_{a}$, wie bemerkt, die Bedeutung einer zusammenfassenden Bezeichnung für eine Anzahl symbolischer Produkte von Elementen der $G_{m}$ hat, so überträgt sich das assoziative Gesetz auf die neuen Produkte. Sind endlich $\bar{U}_{b}$ und $\bar{U}_{c}$ irgend zwei verschiedene Elemente, ist also $\bar{U}_{b} \neq \bar{U}_{c}$, so ist auch $U_{b} \neq U_{c}$, und dann gehören auch $U_{a} \cdot U_{b}$ und $U_{a} \cdot U_{c}$ nicht der gleichen Nebengruppe an, da aus $U_{a} \cdot U_{b}=T \cdot U_{d}, U_{a} \cdot U_{c}=T^{\prime} \cdot U_{d}$ sofort auf zwei Gleichungen:

$$
U_{b}=T^{\prime \prime} \cdot\left(U_{a}^{-1} \cdot U_{d}\right), \quad U_{c}=T^{\prime \prime \prime} \cdot\left(U_{a}^{-1} \cdot U_{d}\right)
$$

geschlossen würde, aus denen hervorginge, daß $U_{b}$ und $U_{c}$ entgegen der Annahme in der gleichen Nebengruppe enthalten wären. Aus $\bar{U}_{b} \neq \bar{U}_{c}$ folgt also $\bar{U}_{a} \cdot \bar{U}_{b} \neq \bar{U}_{a} \cdot \bar{U}_{c}$, und ebenso beweist man, daß auch $\bar{U}_{b} \cdot \bar{U}_{a}$ $\neq \bar{U}_{c} \cdot \bar{U}_{a}$ zutrifft. Damit ist der Satz gewonnen: Die eindeutig bestimmten Nebengruppen $\bar{U}_{0}=1, \bar{U}_{1}, \bar{U}_{2}, \ldots, \bar{U}_{t-1}$ einer ausgezeichneten Untergruppe $G_{u}$ des Index $t$ bilden, aufs neue als Elemente aufgefa $\beta t$, eine Gruppe $\bar{G}_{t}$ 
der Ordnung $t=\frac{m}{\mu}$, die man die "Quotientengruppe" von $G_{m}$ und $G_{\mu}$ nennt und durch $G_{m} / G_{\mu}$ bezeichnet.

Die Gruppen $G_{m}$ und $\bar{G}_{t}$ sind 1- $\mu$-deutig aufeinander bezogen, indem dem einzelnen Elemente $S=T \cdot U_{a}$ von $G_{m}$ das Element $\bar{U}_{a}$ von $\bar{G}_{t}$ entspricht, dem einzelnen Elemente $\bar{U}_{a}$ von $\bar{G}_{t}$ aber die $\mu$ Elemente $U_{a}, T_{1} \cdot U_{a}$ $T_{2} \cdot U_{a}, \ldots, T_{\mu-1} \cdot U_{a}$ zugeordnet sind. Dabei entspricht, indem den Elementen $T_{\alpha} \cdot U_{a}$ und $T_{\beta} \cdot U_{b}$ von $G_{m}$ die Elemente $\bar{U}_{a}$ und $\bar{U}_{b}$ von $\bar{G}_{t}$ zugehören, zufolge (1) dem aus $T_{\alpha} \cdot U_{a}$ und $T_{\beta} \cdot U_{b}$ zusammengesetzten Elemente $\left(T_{\beta} \cdot U_{b}\right) \cdot\left(T_{\alpha} \cdot U_{a}\right)$ stets auch wieder das aus $\bar{U}_{a}$ und $\bar{U}_{b}$ zusammengesetzte Element $\bar{U}_{b} \cdot \bar{U}_{a}=\bar{U}_{c}$. Die Beziehung zwischen $G_{m}$ und $\bar{G}_{t}$ ist also eine Verallgemeinerung des oben (S. 7) zwischen Gruppen gleicher Ordnung erklärten "Isomorphismus" (der hier für $u=1$ vorliegen würde); wir nennen die Gruppen $G_{m}$ und $\bar{G}_{t}$ einander $" 1-\mu$-deutig homomorph".

Es mögen nun die Elemente $W_{0}=1, W_{1}, W_{2}, \ldots, W_{\lambda-1}$ von $G_{m}$ eine Untergruppe $G_{\lambda}$ bilden. Diesen Elementen $W$ mögen im ganzen $s$ verschiedene Elemente $\bar{V}_{0}=1, \bar{V}_{1}, \ldots, \bar{V}_{t-1}$ von $\bar{G}_{t}$ entsprechen. Sind den beiden Elementen $\bar{V}_{\alpha}$ und $\bar{V}_{\beta}$ (vielleicht außer anderen) die Elemente $W_{a}$ und $W_{b}$ der $G_{2}$ zugeordnet, so entspricht dem Elemente $W_{b} \cdot W_{a}$ eindeutig das Element $\vec{V}_{\beta} \cdot \bar{V}_{\alpha}$. Da nun $W_{b} \cdot W_{a}$ in $G_{2}$ enthalten ist, so findet sich $\bar{V}_{\beta} \cdot \bar{V}_{\alpha}$ unter den Elementen $\bar{V}_{0}, \bar{V}_{1}, \ldots, \bar{V}_{s-1}$ : Jeder Untergruppe $G_{2}$ von $G_{m}$ entspricht eindeutig eine Untergruppe $\bar{G}_{\text {, von }} \bar{G}_{t}$. Um zu prüfen, ob im Falle einer ausgezeichneten Untergruppe $G_{2}$ vielleicht auch $\bar{G}$, ausgezeichnet ist, bilden wir mit irgendeinem Elemente $\bar{U}$ von $\bar{G}_{t}$ das Element $\bar{U} \cdot \bar{V}_{\alpha} \cdot \bar{U}^{-1}$. Ist $S$ ein $\bar{U}$ entsprechendes Element, so ist $S \cdot W_{a} \cdot S^{-1}$ ein dem Elemente $\bar{U} \cdot \bar{V}_{\alpha} \cdot \bar{U}^{-1}$ zugeordnetes Element. Nun findet sich $S \cdot W_{a} \cdot S^{-1}$ in der ausgezeichneten $G_{\lambda}$, und also ist $\bar{U} \cdot \bar{V}_{\alpha} \cdot \bar{U}^{-1}$ in $\bar{G}_{s}$ enthalten: Einer ausgezeichneten Untergruppe $G_{\lambda}$ von $G_{m}$ entspricht wieder eine ausgezeichnete Untergruppe $\bar{G}_{\text {s }}$ von $\bar{G}_{t}$.

Alle in $G_{\lambda}$ und $G_{\mu}$ gemeinsam enthaltenen Elemente bilden den Durchschnitt $D\left(G_{\lambda}, G_{\mu}\right)$ der beliebigen Untergruppe $G_{2}$ und unserer ausgezeichneten $G_{\mu}$. Ist $G_{\lambda}$ auch an der Nebengruppe $G_{\mu} \cdot U_{a}$ beteiligt, so sei $V_{a}$ ein in $G_{2}$ und $G_{\mu} \cdot U_{a}$ zugleich enthaltenes Element. Wir können die Nebengruppe dann auch in der Gestalt $G_{\mu} \cdot V_{a}$ schreiben, da nach S. 4 zur Bildung der einzelnen Nebengruppe das Element $U_{a}$ durch irgendein in der Nebengruppe enthaltenes Element ersetzt werden kann. Mit $D\left(G_{2}, G_{\mu}\right)$ und $V_{a}$ enthält $G_{2}$ sogleich alle durch das symbolische Produkt $D\left(G_{\lambda}, G_{\mu}\right) \cdot V_{a}$ zu bezeichnenden Elemente, die aus denen von $D\left(G_{\lambda}, G_{\mu}\right)$ und $V_{a}$ zusammengesetzt sind. Hiermit sind aber auch die in $G_{2}$ und $G_{\mu} \cdot \nabla_{a}$ zugleich enthaltenen Elemente erschöpft. Soll nämlich neben $V_{a}$ auch $T \cdot V_{a}$ in $G_{2}$ enthalten sein, so ist auch $\left(T \cdot V_{a}\right) \cdot V_{a}^{-1}=T$ 
in $G_{2}$ und also im Durchschnitt $D\left(G_{\lambda}, G_{\mu}\right)$ enthalten, so daB sich $T \cdot V_{a}$ in der Tat in $D\left(G_{\lambda}, G_{\mu}\right) \cdot V_{a}$ findet. Nun ist aber $G_{\lambda}$ im ganzen an $s$ Nebengruppen von $G_{\mu}$ beteiligt. Bezeichnen wir diese Nebengruppen durch $G_{\mu}, G_{\mu} \cdot V_{1}, \ldots, G_{\mu} \cdot V_{s-1}{ }^{1}$ ), so ergibt sich der Satz: Die Ordnung $s$ der einer beliebigen Untergruppe $G_{\lambda}$ entsprechenden Untergruppe $\bar{G}_{8}$ von $\bar{G}_{t}$ ist gleich dem Index, den die Gruppe $D\left(G_{\lambda}, G_{\mu}\right)$ als Untergruppe der $G_{2}$ besitzt, da sich die $G_{2}$ ihrer Untergruppe $D\left(G_{2}, G_{\mu}\right)$ entsprechend in die $s$ Nebengruppen:

$$
\begin{gathered}
G_{\lambda}=D\left(G_{\lambda}, G_{\mu}\right)+D\left(G_{\lambda}, G_{\mu}\right) V_{1}+D\left(G_{\lambda}, G_{\mu}\right) V_{2}+\cdots \\
\cdots+D\left(G_{\lambda}, G_{\mu}\right) V_{s-1}
\end{gathered}
$$

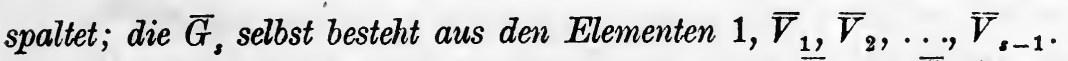

Umgekehrt können der einzelnen Untergruppe $\bar{G}_{\text {, von }} \bar{G}_{t}$, bestehend aus den Elementen $1, \bar{V}_{1}, \bar{V}_{2}, \ldots, \bar{V}_{3-1}$, mehrere Untergruppen $G_{2}$ von $G_{m}$ zugeordnet sein. Unter ihnen findet sich eine eindeutig bestimmte, die alle übrigen in sich enthält, und die wir deshalb als die "größte" der $\bar{G}_{\text {s }}$ entsprechende Untergruppe von $G_{m}$ bezeichnen. Sie hat die Ordnung $\mu s$, enthält $G_{\mu}$ in sich und ist durch die Zerlegung in Nebengruppen charakterisiert:

$$
G_{\mu s}=G_{\mu}+G_{\mu} \cdot V_{1}+G_{\mu} \cdot V_{q}+\cdots G_{\mu} \cdot V_{s-1},
$$

wo $V_{1}, V_{2}, \ldots, V_{s-1}$ irgend $(s-1)$ den $\bar{V}_{1}, \bar{V}_{2}, \ldots, \bar{V}_{s-1}$ entsprechende Elemente sind. DaB alle von den Nebengruppen in (4) rechts gelieferten Elemente wieder eine Gruppe bilden, folgt mit Rücksicht auf die Gruppeneigenschaft von $\bar{G}$, leicht aus einer dem Ansatze (1) entsprechenden

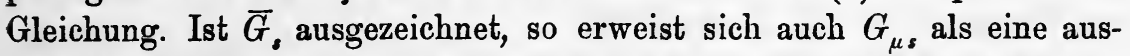
gezeichnete Untergruppe. Ist nämlich $T_{\alpha} \cdot V_{a}$ irgendein Element aus $G_{\mu s}$ und $S$ ein beliebiges Element von $G_{2 n}$, so ist:

$$
S \cdot\left(T_{\alpha} \cdot V_{a}\right) \cdot S^{-1}=\left(S \cdot T_{\alpha} \cdot S^{-1}\right) \cdot\left(S \cdot V_{a} \cdot S^{-1}\right) .
$$

In der ersten Klammer rechts steht, da $G_{\mu}$ ausgezeichnet ist, ein $T_{\beta}$ aus $G_{\mu}$. Entspricht dem $S$ das Element $\bar{U}$ von $\bar{G}_{t}$, so entspricht dem Prodakte $S \cdot V_{a} \cdot S^{-1}$ das Element $\bar{U} \cdot \bar{V}_{a} \cdot \bar{U}^{-1}=\bar{V}_{b}$, das sich in der ausgezeichneten $\bar{G}$, findet. Diesem entsprechen aber die in $G_{\mu} \cdot V_{b}$ zusammengefaßten Elemente, unter denen sich demnach $S \cdot V_{a} \cdot S^{-1}$ findet. Es gilt also:

$$
S \cdot V_{a} \cdot S^{-1}=T_{\gamma} \cdot V_{b}, \quad S \cdot\left(T_{a} \cdot V_{a}\right) \cdot S^{-1}=\left(T_{\beta} \cdot T_{\gamma}\right) \cdot V_{b},
$$

und das in der zweiten Gleichung rechts stehende Element findet sich in $G_{\mu s}$ : Einer beliebigen Untergruppe $\bar{G}_{s}$ von $\bar{G}_{t}$ entspricht als ,größte"

1) Es ist natürlich keineswegs gemeint, daB dies gerade die an erster Stelle unserer ursprünglichen Anordnung (2) S. 4 stehenden Nebengruppen $G_{\mu}, G_{\mu} \cdot U_{1}$, $\ldots, G_{\mu} \cdot U_{s-1}$ sein sollen. 
Untergruppe von $G_{m}$ die durch (4) gegebene $G_{\mu s}$ der Ordnung $\mu s$, die eine ausgezeichnete Untergruppe der $G_{m}$ ist, falls $\vec{G}_{s}$ eine solche von $\bar{G}_{t}$ ist.

\section{$\S$ 5. Kompositionsreihe einer Gruppe $G_{m}$.}

Eine ausgezeichnete Untergruppe $G_{\mu}$ einer Ordnung $\mu<m$ heißt eine, ,größle ausgezcichnete Untergruppe" von $G_{m}$, wenn außer $G_{m}$ und $G_{\mu}$ keine ausgezeichnete Untergruppe existiert, die $G_{\mu}$ enthält und in $G_{m}$ enthalten ist. Es besteht der Satz: $G_{\mu}$ ist stets und nur dann eine größte ausgezeichnete Untergruppe von $G_{m}$, wenn die Quotientengruppe $G_{m} / G_{\mu}=\bar{G}_{t}$ einfach ist. Einer ausgezeichneten Untergruppe $G_{\mu s}$ mit $1<s<t$, die $G_{\mu}$ enthält, gehört nämlich eine ausgezeichnete Untergruppe $\bar{G}_{s}$ von $\bar{G}_{t}$ $\mathrm{zu}$, wie umgekehrt jeder ausgezeichneten $\bar{G}_{s}$ mit $1<s<t$, die in $\bar{G}_{t}$ enthalten ist, als "gröBte“ zugehörige Untergruppe von $G_{m}$ eirie $G_{\mu}$ enthaltende ausgezeichnete $G_{\mu s}$ entspricht.

Es sei weiterhin $G_{\mu}$ eine größte ausgezeichnete Untergruppe und $\bar{G}$ die zugehörige Quotientengruppe $G_{m} / G_{\mu}$. Einer von $G_{\mu}$ verschiedenen ausgezeichneten Untergruppe $G_{\lambda}$ entspricht in $\bar{G}_{t}$ eine ausgezeichnete $\bar{G}_{s t}$ die, da $\bar{G}_{t}$ einfach ist, entweder die $\bar{G}_{1}^{\prime}$ oder die $\bar{G}_{t}$ selbst ist. Im ersten Falle ist $G_{\lambda}$ in $G_{\mu}$ enthalten. Soll also auch $G_{\lambda}$ eine gröBte ausgezeichnete Untergruppe von $G_{m}$ sein, so muB ihr die $\bar{G}_{t}$ entsprechen, die Gleichung (3) S. 11 hat demnach die Gestalt:

$$
G_{\lambda}=D\left(G_{\lambda}, G_{\mu}\right)+D\left(G_{\lambda}, G_{\mu}\right) \cdot V_{1}+\cdots+D\left(G_{\lambda}, G_{\mu}\right) \cdot V_{t-1} \text {, }
$$

d. h. sie ist rechts $t$-gliedrig. Wir wählen hier die $V$ so, daB $V_{a}$ stets der Nebengruppe $G_{\mu} \cdot U_{a}$ angehört.

Nun liefert der Durchschnitt $D\left(G_{\lambda}, G_{\mu}\right)$ zweier ausgezeichneter $G_{\lambda}$, $G_{\mu}$ eine gleichfalls ausgezeichnete Untergruppe, da. die Transformation irgendeines Elementes von $D\left(G_{2}, G_{\mu}\right)$ durch ein beliebiges $S$ stets wieder ein in $G_{2}$ und $G_{\mu}$ und also in $D\left(G_{\lambda}, G_{\mu}\right)$ enthaltenes Element ergibt.. Zur Bildung der Quotientengruppe $G_{t}^{\prime}=G_{2} / D\left(G_{2}, G_{\mu}\right)$ betrachten wir die $t$ in (1) rechts stehenden Nebengruppen aufs neue als Elemente:

$$
\begin{gathered}
D\left(G_{\lambda}, G_{\mu}\right)=\overline{\bar{V}}_{0}=1, D\left(G_{\lambda}, G_{\mu}\right) \cdot V_{1}=\bar{V}_{1}, \ldots, \\
D\left(G_{2}, G_{\mu}\right) \cdot \nabla_{t-1}=\bar{V}_{t-1} .
\end{gathered}
$$

Indem wir die Elemente $\bar{U}_{a}$ und $\bar{V}_{a}$ einander entsprechen lassen, werden die beiden Gruppen $\bar{G}_{t}$ und $\bar{G}_{t}^{\prime}$ einander ,isomorph", da ja aus $\bar{U}_{b}: \bar{U}_{a}=\bar{U}_{\text {o }}$ stets wieder $\bar{V}_{b} \cdot \bar{V}_{a}=\bar{V}_{c}$ folgt. Also ist auch $\bar{G}_{t}^{\prime}$ einfach, und $D\left(G_{\lambda}, G_{\mu}\right)$ erweist sich als "größte" ausgezeichnete Untergruppe von $G_{\lambda}$. Da man diese Betrachtung auch in der Art ausführen kann, daB man $G_{2}$ an Stelle yon $G_{\mu}$ voranstellt und $G_{\mu}$ folgen läßt, so gilt der Satz: Sind $G_{2}$ und $G_{\mu}$ zwei verschiedene größte ausgezeichnete Untergruppen von $G_{m}$, so ist der Durchschnitt $D\left(G_{\lambda}, G_{\mu}\right)$ eine größte ausgezeichnete Untergruppe sowohl 
von $G_{2}$ als von $G_{\mu}$; die Quotientengruppen $G_{m} / G_{\mu}$ und $G_{2} / D\left(G_{2}, G_{\mu}\right)$ sind isomorph, und dasselbe gilt von den Quotientengruppen $G_{m} / G_{2}$ und $G_{\mu} / D\left(G_{\lambda}, G_{\mu}\right)$.

Wir denken jetzt eine Reihe von Untergruppen $G_{m}, G_{\mu_{1}}, G_{\mu_{2}}, G_{\mu_{3}}, \ldots$ in der Art gewählt, daB jede eine gröBte ausgezeichnete Untergruppe in der voraufgehenden ist. Da hierbei $m>\mu_{1}>\mu_{2}>\cdots$ gilt, so gelangt man nach einer endlichen Anzahl $n$ von Schritten zur $G_{1}$ und gewinnt die sich schließende Reihe:

$$
G_{m}, G_{\mu_{1}}, G_{\mu_{2}}, G_{\mu_{3}}, \ldots, G_{\mu_{n}}=G_{1} \text {, }
$$

in der die vorletzte Gruppe einfach ist. Die $n$ zugehörigen Indizes:

$$
\frac{m}{\mu_{1}}=t_{1}, \quad \frac{\mu_{1}}{\mu_{2}}=t_{2}, \quad \frac{\mu_{2}}{\mu_{3}}=t_{3}, \quad \ldots, \quad \frac{\mu_{n-1}}{\mu_{n}}=\mu_{n-1}=t_{n}
$$

iefern als Produkt die Ordnung $m=t_{1} \cdot t_{2} \cdot t_{3} \cdots t_{n}$ der Gesamtgruppe. Die zugehörigen Quotientengruppen bezeichnen wir durch:

$$
G_{m} / G_{\mu_{2}}=\bar{G}_{t_{1}}, \quad G_{\mu_{1}} / G_{\mu_{2}}=\bar{G}_{t_{2}}, \ldots
$$

Die Reihe (3) heißt eine „Reihe der Zusammensetzung“ oder eine „Kompositionsreihe" der Gruppe $G_{m}$, die Reihe (4) heibt die zugehörige "Indexreihe".

Neben (3) liege nun auch in:

$$
G_{m}, G_{\lambda_{1}}, G_{\lambda_{2}}, G_{\lambda_{3}}, \ldots, G_{1}
$$

eine Kompositionsreihe von $G_{m}$ vor mit der zugehörigen Indexreihe:

$$
\frac{m}{\lambda_{1}}=s_{1}, \quad \frac{\lambda_{1}}{\lambda_{2}}=s_{2}, \quad \frac{\lambda_{9}}{\lambda_{3}}=s_{3}, \quad \ldots
$$

und den Quotientengruppen.

$$
G_{m} / G_{\lambda_{1}}=\bar{G}_{s_{1}}, \quad \dot{G}_{\lambda_{1}} / G_{\lambda_{2}}=\bar{G}_{s_{2}}, \quad G_{\lambda_{2}} / G_{\lambda_{3}}=\bar{G}_{s_{3}}, \ldots
$$

Dann gilt folgender Satz: Die Kompositionsreihen (3) und (6) unserer $G_{m}$ haben stets gleiche Gliederanzahl $(n+1)$, die $n$ Indizes (4) sind, abgesehen von der Anordnung, den $n$ Indizes (7) gleich, und es lassen sich die Quotientengruppen (5) und (8) zu Paaren, $\bar{G}_{t_{\alpha}}$ und $\bar{G}_{s_{\beta}}$, so einander zuordnen, $d a \beta \bar{G}_{t_{\alpha}}$ und $\bar{G}_{\delta_{\beta}}$ isomorph sind.

Diesen Satz kann man durch vollständige Induktion beweisen. Ist $m$ eine Primzahl, so gibt es nur die eine Kompositionsreihe $G_{m}, G_{1}$, und dann ist der Satz selbstrerständlich. Wir zerlegen nun bei beliebiger Ordnung $m$ die Zahl $m$ in ihre Primfaktoren und zählen deren Anzahl ab. Wir nehmen den Satz als bereits bewiesen an für alle Ordnungen $m^{\prime}$, deren Primfaktorenanzahl mindestens um eine Einheit geringer ist als die von $m$. Dann läßt sich zeigen, daß der Satz auch noch für $m$ gilt.

Es ist nämlich der Durchschnitt $D\left(G_{\lambda_{1}}, G_{\mu_{1}}\right)=G_{v_{1}}$ eine gröBte ausgezeichnete Untergruppe sowohl in $G_{\mu_{1}}$ als $G_{\lambda_{1}}$. Irgendeine Kompositions- 
reihe $G_{v_{1}}, G_{v_{2}}, \ldots, G_{1}$ von $G_{v_{1}}$ liefert demnach in:

$$
G_{m}, G_{\mu_{1}}, G_{v_{1}}, G_{v_{2}}, \ldots, G_{1} \text { und } G_{m}, G_{\lambda_{1}}, G_{v_{1}}, G_{v_{2}}, \ldots, G_{1}
$$

zwei Kompositionsreihen für $G_{m}$, wobei (nach dem vorausgesandten Satze) die Gruppen $G_{m} / G_{\mu_{1}}$ und $G_{\lambda_{1} / G_{v_{1}}}$ isomorph sind und ebenso die Gruppen $G_{m} / G_{\lambda_{1}}$ und $G_{\mu_{1}} / G_{v_{1}}$. Nun sind:

$$
G_{\mu_{1}}, G_{\mu_{2}}, \ldots, 1 \text { und } G_{\mu_{1}}, G_{v_{1}}, G_{v_{2}}, \ldots, 1
$$

Kompositionsreihen für $G_{\mu_{1}}$. Da $\mu_{1}$ mindestens einen Primfaktor weniger hat als $m$, so haben diese beiden Reiben der Annahme gemäB gleiche Gliederanzahlen, und es gilt für die Systeme der Quotientengruppen $G_{\mu_{1}} / G_{\mu_{2}}, G_{\mu_{2}} / G_{\mu_{2}}, \ldots$ und $G_{\mu_{1}} / G_{v_{1}}, G_{v_{1}} / G_{v_{2}}, \ldots$ die im Satze behauptete Zusammenordnung zu Paaren. Insbesondere findet sich im ersten Systeme eine zu $G_{\mu_{1}} / G_{v_{1}}$ und also zu $G_{m} / G_{\lambda_{1}}$ isomorphe Gruppe, während die übrigen $(n-2)$ Gruppen des Systems $G_{\mu_{1}} / G_{\mu_{2}}, G_{\mu_{2}} / G_{\mu_{3}}, \ldots$ in irgendeiner Anordnung den Quotientengruppen $G_{v_{1}} / G_{v_{2}}, G_{v_{2}} / G_{v_{3}}, \ldots$ als isomorph zugewiesen sind. In derselben Art findet man im Systeme $G_{\lambda_{1}} / G_{\lambda_{2}}, G_{\lambda_{2}} / G_{\lambda_{2}}$, ... eine mit $G_{m} / G_{\mu_{1}}$ isomorphe Gruppe, während jede der übrigen im Systeme $G_{v_{1}} / G_{v_{2}}, G_{v_{2}} / G_{v_{3}}, \ldots$ und damit im Systeme $G_{\mu_{1}} / G_{\mu_{2}}, G_{\mu_{2}} / G_{\mu_{3}}, \ldots$, jedoch unter AusschluB der bereits der Gruppe $G_{m} / G_{\lambda_{1}}$ zugeordneten, ihre isomorphe findet. Damit ist der aufgestellte Satz bewiesen.

\section{§ 6. Sätze über Abelsche Gruppen.}

Eine Gruppe $G_{m}$ sollte als eine kommutative oder Abelsche bezeichnet werden, wenn jedes ihrer Elemente mit jedem anderen vertauschbar ist. Die Elemente einer solchen Gruppe seien $S_{0}=1, S_{1}, S_{2}, \ldots, S_{m-1}$, und die Periode von $S_{a}$ werde durch $\nu_{a}$ bezeichnet, so daB $\nu_{0}=1, v_{1}>1$, $v_{2}>1, \ldots$ gilt.

Man bilde die Produkte:

$$
S_{1}^{h_{2}} \cdot S_{2}^{h_{2}} \cdot S_{2}^{h_{2}} \cdots S_{m-1}^{h_{m-1}}, \quad 0 \leqq h_{a}<\nu_{a},
$$

die sich auf alle $\nu_{1} \cdot \nu_{2} \cdots \nu_{m-1}$ Kombinationen ganzzahliger Exponenten $h$ beziehen, welche den in (1) rechts angegebenen Ungleichungen genügen. Im Systeme der Produkte (1) kommt jedes Element von $G_{m}$ zur Darstellung, nämlich das Einheitselement z. B. dann, wenn alle $h$ gleich 0 gesetzt werden, und das von $S_{0}$ verschiedene Element $S_{a}$, wenn z. B. der Exponent $h_{a}=1$, alle übrigen $h$ aber $=0$ gesetzt werden. Das Einheitselement möge nun im ganzen $e$ Male unter den Produkten (1) auftreten, d. h. es möge im ganzen $e$ verschiedene Kombinationen der Exponenten $h$ geben, für welche das Produkt (1) gleich $S_{0}=1$ wird. Wir belegen diese Kombinationen mit der besonderen Bezeichnung $\eta_{1}, \eta_{2}, \eta_{3}, \ldots, \eta_{m-1}$ und haben dann :

$$
S_{1}^{\eta_{1}} \cdot S_{2}^{\eta_{2}} \cdot S_{3}^{\eta_{3}} \cdots S_{m-1}^{\eta_{m-1}}=1
$$


Ist $S$ ein beliebiges Element von $G_{m}$ mit einer ersten Darstellung:

$$
S=S_{1}^{h_{1}} \cdot S_{2}^{h_{2}} \cdot S_{3}^{h_{3}} \cdots S_{m-1}^{h_{m-1}},
$$

so ergeben sich sofort $e$ verschiedene Darstellungen von $S$ in der Gestalt:

$$
S=S_{1}^{h_{1}+\eta_{1}} \cdot S_{2}^{h_{2}+\eta_{2}} \cdots S_{m-1}^{h_{m-1}+\eta_{m-1}}
$$

aus der Vertauschbarkeit der Elemente, wobei wir jeden Exponenten $\left(h_{a}+\eta_{a}\right) \bmod \nu_{a}$ nötigenfalls auf seinen kleinsten nicht-negativen Rest reduziert denken. Ist andrerseits:

$$
S=S_{1}^{h_{1}^{\prime}} \cdot S_{2}^{h_{2}^{\prime}} \cdot S_{3}^{h_{2}} \cdots S_{m-1}^{h_{m-1}}
$$

irgendeine Darstellung des fraglichen Elementes $S$, so ergibt sich wieder aus der Vertauschbarkeit der Elemente in:

$$
S \cdot S^{-1}=S_{1}^{h_{1}^{\prime}{ }_{1}-h_{1}} \cdot S_{2}^{h_{2}^{\prime}-h_{2}} \cdot S_{3}^{h_{3}^{\prime}-h_{3}} \cdots S_{m-1}^{h^{\prime} m_{m-1}-h_{m-1}}=1
$$

(nötigenfalls nach Reduktion der Exponenten) eine Darstellung (2) des Einheitselementes. Hieraus folgt, daß wir in den $e$ verschiedenen Darstellungen (4) von $S$ bereits alle von den Produkten (1) gelieferten Darstellungen von $S$ vor uns haben: Jedes Element von $G_{m}$ wird vom System der $\nu_{1} \cdot v_{2} \cdots v_{m-1}$ Produkte gerade so oft geliefert wie jedes andere, nämlich e Male, so daß die Gleichung gilt:

$$
\nu_{1} \cdot \nu_{2} \cdot \nu_{3} \cdots v_{n-1}=e \cdot m .
$$

Irgendein Primfaktor $p$ von $m^{1}$ ) geht zufolge (5) in mindestens einer der Zahlen $\nu$, etwa in $\nu_{a}$, auf. Dann ist $S_{a}^{\frac{v_{a}}{p}}$ ein Element der Periode $p$. Ist $p$ irgendein Primfaktor der Ordnung in der Abelschen Gruppe $G_{m}$, so gibt es in $G_{m}$ sicher ein Element $S$ der Periode $p$ und damit eine zyklische Untergruppe $G_{p}$ der Ordnung $p$. Es seien $S_{a}, S_{b}, S_{c}, \ldots$ Elemente aus $G_{m}$ von den Perioden $v_{a}, v_{b}, \nu_{c}, \ldots$, und es sei $v$ das kleinste gemeinschaftliche Vielfache von $v_{a}, v_{b}, v_{c}, \ldots$ Dann gilt für das aus $S_{a}, S_{b}, S_{c}, \ldots$ zusammengesetzte Element $S$ :

$$
S^{0}=\left(S_{a} \cdot S_{b} \cdot S_{c} \ldots\right)^{0}=S_{a}^{\prime} \cdot S_{b}^{0} \cdot S_{c}^{o} \ldots=1 .
$$

Also ist nach S. $5 \mathrm{ff}$. die Periode von $S$ ein Teiler von $v$ : Die Periode des aus den Elementen $S_{a}, S_{b}, S_{c}, \ldots$ zusammengesetzten Elementes $S=S_{a} \cdot S_{b} \cdot S_{c} \ldots$ ist ein Teiler des kleinsten gemeinsamen Vielfachen der Perioden $v_{a}, \nu_{b}, \nu_{c}, \ldots$ der zusammensetzenden Elemente.

Die Ordnung $m$ der Abelschen Gruppe sei als Produkt $k \cdot l$ zweier teilerfremder Zahlen $k, l$ darstellbar. In $G_{m}$ mögen $k^{\prime}$, durch $U_{0}=1$, $U_{1}, U_{2}, \ldots, U_{k^{\prime}-1}$ zu bezeichnende Elemente auftreten, deren Perioden in $k$ aufgehen. Da nach dem eben bewiesenen Satze die Periode von $U_{a} \cdot U_{b}$ gleichfalls in $k$ aufgeht, so gehört $U_{a} \cdot U_{b}$ zu den $k^{\prime}$ Elementen $U$.

1) Falls nichts weiter gesagt ist, gilt $p$ als von 1 verschieden. 
Die $k^{\prime}$ Elemente $U$ bilden hiernach eine Gruppe $G_{k^{\prime}}$, die natürlich wieder eine Abelsche ist. Ebenso gelangen wir zu einer Abelschen Gruppe $G_{z^{\prime}}$ aller $l^{\prime}$ in $G_{m}$ enthaltenen Elemente $V_{0}=1, V_{1}, V_{2}, \ldots, V_{l^{\prime}-1}$, deren Perioden in $l$ aufgehen. Da keine Periode $\nu>1$ in $k$ und $l$ zugleich aufgeht, so haben die Gruppen $G_{k^{\prime}}$ und $G_{l^{\prime}}$ nur das Einheitselement gemein. Die Ordnung $k^{\prime}$ der $G_{k^{\prime}}$ ist teilerfremd gegen $l$, und entsprechend ist $l^{\prime}$ teilerfremd gegen $k$. Hätten nämlich $k^{\prime}$ und $l$ einen Primfaktor $p$ gemein, so gäbe es in $G_{k^{\prime}}$ ein $U$ der Periode $p$, die in $l$ aufgeht und also teilerfremd gegen $k$ ist. Dies würde der Erklärung der Elemente $U$ widersprechen.

Die $k^{\prime} \cdot l^{\prime}$ Produkte $U_{a} \cdot V_{b}$ stellen lauter verschiedene Elemente von $G_{m}$ dar. Soll nämlich $U_{a} \cdot V_{b}=U_{c} \cdot V_{d}$ sein, so folgt:

$$
U_{c}^{-1} \cdot U_{a}=V_{d} \cdot V_{b}^{-1}=1 ;
$$

denn $U_{c}^{-1} \cdot U_{a}$ ist in $G_{k^{\prime}}$ und $V_{d} \cdot V_{b}^{-1}$ in $G_{l}$ enthalten, beide Gruppen haben aber nur das Einheitselement gemein. Aus (6) aber ergibt sich sofort $U_{a}=U_{c}, V_{b}=V_{d}$. Man kann weiter zeigen, daB jedes Element $S$ von $G_{m}$ als ein Produkt $U_{a} \cdot V_{b}$ darstellbar ist. Da nämlich $k$ und $l$ teilerfremd sind, so lassen sich nach I, 281 zwei ganze (positive oder negative) Zahlen $x, \lambda$ angeben, die die Gleichung $k x+l \lambda=1$ befriedigen. Wir setzen dann:

$$
S=S^{k x+l \lambda}=S^{\imath \lambda} \cdot S^{k x}
$$

und folgern aus den beiden Gleichungen:

$$
\left(S^{\imath \imath}\right)^{k}=S^{m \cdot \lambda}=1, \quad\left(S^{k *}\right)^{l}=S^{m \cdot *}=1,
$$

daB $S^{l \lambda}$ ein $U_{a}$ und $S^{k \varkappa}$ ein $V_{b}$ ist. In (7) liegt also die Darstellung $S=U_{a} \cdot V_{b}$ vor.

Die $k^{\prime} \cdot l^{\prime}$ Produkte $U_{a} \cdot V_{b}$ bilden selbst wieder eine Gruppe der Ordnung $k^{\prime} \cdot l^{\prime}$, die wir $G_{k^{\prime} \cdot l^{\prime}}$ nennen und symbolisch als Produkt $G_{k^{\prime}} \cdot G_{l^{\prime}}$ bezeichnen können. Nach der eben beendeten Überlegung ist diese $G_{k^{\prime} \cdot l^{\prime}}$ mit der Gesamtgruppe $G_{m}$ identisch. Es gilt also:

$$
k^{\prime} \cdot l^{\prime}=m=k \cdot l,
$$

und da wir bereits wissen, daB $k^{\prime}$ teilerfremd gegen $l$ und $l^{\prime}$ teilerfremd gegen $k$ ist, so folgt $k^{\prime}=k, l^{\prime}=l$. Gestattet die Ordnung $m$ einer Abelschen Gruppe $G_{m}$ die Zerlegung $m=k \cdot l$ in zwei teilerfremde Falktoren $k, l$, so gibt es in $G_{m}$ genau $k$ eine $G_{k}$ bildende Elemente $U$, deren Perioden 7 teilen, und genau $l$ eine $G_{l}$ bildende Elemente $V$, deren Perioden in $l$ aufgehen; die Gesamtgruppe $G_{m}$ aber ist in der Gestalt $G_{k} \cdot G_{l}$ der Gruppe aller Elemente $U_{a} \cdot V_{b}$ darstellbar.

Da jedes Element einer in $G_{m}$ enthaltenen Untergruppe $G_{\mu}$ mit einem beliebigen Elemente $S$ von $G_{m}$ vertauschbar ist, so ist auch $G_{\mu}$ mit $S$ vertauschbar: Jede Untergruppe $G_{\mu}$ einer Abelschen Gruppe $G_{m}$ ist 
„ausgezeichnet" und übrigens selbst wieder eine Abelsche Gruppe. Zur Aufstellung der Quotientengruppe $G_{m} / G_{\mu}$ führte die Gleichung (2) S. 9. Da im Falle unserer Abelscnen Gruppe aus jener Gleichung wegen der Vertauschbarkeit der Elemente

$$
\left(G_{\mu} \cdot U_{a}\right) \cdot\left(G_{\mu} \cdot U_{b}\right)=G_{\mu} \cdot U_{c}
$$

folgt, so gilt weiter der Satz: Die zu einer (ausgezeichneten) Untergruppe $G_{\mu}$ einer Abelschen Gruppe $G_{m}$ gehörende Quotientengruppe $G_{m} / G_{\mu}=\bar{G}_{t}$ ist gleichfalls eine Abelsche Gruppe.

Ist $p^{\sigma}$ eine höchste in $m$ aufgehende Primzahlpotenz, so setzen wir $m=p^{\sigma} \cdot l$ und finden in $G_{m}$ eine Untergruppe der Ordnung $p^{\sigma}$. Daran schlieBt sich der Satz: In einer Abelschen Gruppe, deren Ordnung die Primzahlpotenz $p^{\sigma}$ ist, gibt es stets eine Untergruppe der Ordnung $p^{\sigma-1}$. Der Beweis wird durch vollständige Induktion geführt. DaB in einer Abelschen $G_{p^{2}}$ stets eine Untergruppe $G_{p}$ nachweisbar ist, steht bereits fest. Wir nehmen an, daß der Satz für die Ordnungen $p^{2}, p^{3}, \ldots, p^{\sigma-1}$ richtig ist, und können dann leicht zeigen, daß er auch für die Ordnung $p^{\sigma}$ gilt. Die Abelsche $G_{p^{\sigma}}$ enthält nämlich eine (ausgezeichnete) $G_{p}$. Die zugehörige Abelsche Gruppe $G_{p^{\sigma}} / G_{p}$ enthält der Annahme nach eine Untergruppe $\bar{G}_{p^{\sigma-2}}$, da jene Quotientengruppe die Ordnung $p^{\sigma-1}$ hat. Nach S. $11 \mathrm{ff}$. entspricht dieser $\bar{G}_{p^{\sigma-2}}$ in der $G_{p^{\sigma}}$ eine Untergruppe der Ordnung $p \cdot p^{\sigma-2}=p^{\sigma-1}$, womit der Beweis des Satzes beendet ist.

Wir halten an der Zerlegung $m=p^{\sigma} \cdot l$ fest und multiplizieren nach S. 16 die eben nachgewiesene $G_{p^{\sigma-1}}$ mit $\operatorname{der} G_{l}$. Es entsteht eine Gruppe der Ordnung $p^{\sigma-1} \cdot l=\frac{m}{p}$. In jeder Abelschen Gruppe der Ordnung $m$, die durch die Primzahl $p$ teilbar ist, gibt es eine Untergruppe der Ordmung $\frac{m}{p}$, die in $\dot{G}_{m}$ ausgezeichnet enthalten ist; die zugehörige Quotientengruppe $\bar{G}_{p}$ ist als Gruppe von Primzahlordnung ,zyklisch“ und „einfach“. Selbstverständlich ist die Untergruppe der Ordnung $\frac{m}{p}$ eine „gröBte“ ausgezeichnete Untergruppe.

Durch wiederbolte Anwendung dieses Ergebnisses folgt der Hauptsatz: Jede Indexreihe einer Abelschen Gruppe $G_{m}$ besteht aus den gesamten Primfaktoren von m, wobei jeder Primfaktor so oft als Reihenglied auftritt, wie er in $m$ enthalten ist; jede Quotientengruppe ist als Gruppe von Primzahlordnung zyklisch.

\section{$\S 7$. Permutationsgruppen.}

Es seien $n$ gleichartige Dinge vorgelegt, die wir numerieren und mit ihren Nummern 1, 2, 3, ., $n$ als Namen belegen. Eine erste Anordnung der Dinge ist durch $1,2,3, \ldots, n$ gegeben, irgendeine der $n$ ! 
Anordnungen sei durch $a_{1}, a_{2}, a_{3}, \ldots, a_{n}$ bezeichnet, so daB die $a_{k}$ die Zahlen $1,2,3, \ldots, n$ in der neuen Anordnung bedeuten. Unter der Operation $S_{a}$ verstehen wir den gleichzeitigen Ersatz des Dinges 1 durch $a_{1}, 2$ durch $a_{2}$ usw., allgemein $k$ durch $a_{k}$. Wir nennen diese Operation $S_{a}$ eine "Permutation" der $n$ Dinge, so da $B$ es den $n$ ! Anordnungen $a_{1}, a_{2}, \ldots, a_{n}$ entsprechend $n$ ! verschiedene Permutationen der $n$ Dinge gibt.

Die Permutation $S_{a}$ kann man symbolisch durch:

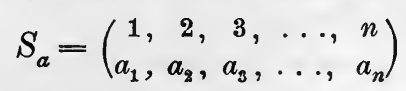

bezeichnen, wobei also dasjenige Ding $a_{k}$, durch welches $k$ ersetzt werden soll, genau unter $k$ steht. Die in der oberen Zeile gewählte Anordnung ist unwesentlich; man kann $S_{a}$ z. B. auch durch:

$$
S_{a}=\left(\begin{array}{ccc}
3, & 5,1, \ldots, n-3 \\
a_{3}, & a_{5}, & a_{1}, \ldots, \\
a_{n-3}
\end{array}\right)
$$

bezeichnen, wenn nur in der ersten Zeile jedes der $n$ Dinge und jedes nur einmal untergebracht ist und unter $k$ allemal $a_{k}$ steht. Wir haben also im ganzen $n$ ! Schreibweisen für die einzelne Permutation zur Hand. Als abgekürzte Bezeichnung für $S_{a}$ benutzen wir $S_{a}=\left(k, a_{k}\right)$.

Die Permutation $S_{0}=(k, k)$, bei der also jedes Ding durch sich selbst ersetzt wird, heißt die "identische Permutation" und wird unten als Element der zu erklärenden Gruppen auch durch 1 bezeichnet, da sie das "Einheitselement" dieser Gruppen liefern wird. Die Permutation $\left(a_{k}, k\right)$, bei der also umgekehrt $a_{1}$ durch $1, a_{2}$ durch 2 usw. ersetzt wird, heiBt zur Permutation $S_{a}$,invers" und wird durch $S_{a}^{-1}$ bezeichnet.

Üben wir auf die $n$ Dinge zuerst die Permutation $S_{a}=\left(k, a_{k}\right)$, sodann die Permutation $S_{b}=\left(k, b_{k}\right)=\left(a_{k}, b_{a_{k}}\right)$ aus, so ist das Ergebnis wiẹder eine Permutation, nämlich offenbar $\left(k, b_{a_{k}}\right)$, die wir symbolisch durch das Produkt $S_{b} \cdot S_{a}$ bezeichnen:

$$
S_{b} \cdot S_{\alpha}=\left(k, b_{a_{k}}\right)=\left(\begin{array}{c}
1,2, \ldots, n \\
b_{a_{1}}, b_{a_{2}}, \ldots, b_{a_{n}}
\end{array}\right)
$$

Man kann auch sofort solche Produkte mit drei oder noch mehr Faktoren bilden, die stets wieder Permutationen darstellen, und findet, daB für diese Produkte das assoziative Gesetz gilt. Ist nämlich $S_{c}=\left(k, c_{k}\right)=\left(b_{a_{k}}, c_{b_{a_{k}}}\right)$ eine dritte Permutation, so gewinnt man als Permutation $S_{c} \cdot\left(S_{b} \cdot S_{a}\right)$ :

$$
\begin{gathered}
S_{c} \cdot\left(S_{b} \cdot S_{a}\right)=\left(k, c_{b_{a_{k}}}\right) . \\
S_{c} \cdot S_{b}=\left(k, c_{b_{k}}\right)=\left(a_{k}, c_{b_{a_{k}}}\right),
\end{gathered}
$$

Andrerseits gilt:

so daß $\left(S_{c} \cdot S_{b}\right) \cdot S_{a}$ zu der schon in (3) gewonnenen Permutation zurückführt. Sind die beiden Permutationen $S_{b}=\left(a_{k}, b_{a_{k}}\right)$ und $S_{c}=\left(a_{k}, c_{a_{k}}\right)$ verschieden, so sind stets auch $S_{b} \cdot S_{a}=\left(k, b_{a_{k}}\right)$ und $S_{c} \cdot S_{a}=\left(k, c_{a_{k}}\right)$ ver- 
schieden, und ebenso erweist sich $S_{a} \cdot S_{b} \neq S_{a} \cdot S_{c}$ als zutreffend. Es besteht hiernach der Satz: Alle $n$ ! Permutationen von $n$ Dingen bilden, als „Elemente" aufgefaßt, eine Gruppe $G_{n}$ ! der Ordnung n!, die wir als eine Permutationsgruppe bezeichnen.

Wir bestimmen weiter, daß die Benennung „Permutationsgruppe $e^{\text {" }}$ auch auf alle in der $G_{n !}$ enthaltenen Untergruppen ïbertragen werden soll. Ihnen gegenüber wird die Gesamtgruppe $G_{n}$ als die „symmetrische Permutationsgruppe" oder kurz die „symmetrische Gruppe" bezeichnet. Die Anzahl $n$ der Dinge, die den Permutationen unterworfen werden, heißt der „Grad" der fraglichen Gruppen.

Außer der symmetrischen Gruppe betrachten wir zunächst nur die "zyklischen" Permutationsgruppen. Die Bestimmung der Periode einer einzelnen Permutation $S$ und damit der Ordnung der aus ihr zu erzeugenden zyklischen Permutationsgruppe geschieht durch folgende Betrachtung. Irgendeines der $n$ Dinge $\alpha_{1}$ möge bei Ausübung von $S$ in $\alpha_{2}$ übergehen, $\alpha_{2}$ aber in $\alpha_{3}, \alpha_{3}$ in $\alpha_{4}$ usw. Die zu $S$ inverse Permutation $S^{-1}$ führt dann $\alpha_{2}$ in $\alpha_{1}$ über, $\alpha_{3}$ in $\alpha_{2}, \alpha_{4}$ in $\alpha_{3}$ usw. Man verfolge nun die Reihe $\alpha_{1}, \alpha_{2}, \alpha_{3}, \ldots$, bis man zu einem Dinge $\alpha_{\tau+1}$ gelangt, das schon einmal aufgetreten ist. Dann sind die $\tau$ Dinge $\alpha_{1}, \alpha_{2}, \alpha_{3}, \ldots, \alpha_{\tau}$ verschieden, und $\alpha_{\tau+1}$ ist notwendig gleich $\alpha_{1}$. Wäre nämlich $\alpha_{\tau+1}=\alpha_{k}$ mit $k>1$, so würden auch die durch $S^{-1}$ aus $\alpha_{\tau+1}$ und $\alpha_{k}$ hervorgehenden Dinge $\alpha_{\tau}$ - und $\alpha_{k-1}$ gleich sein, und also wären die $\alpha_{1}, \alpha_{2}, \alpha_{3}, \ldots, \alpha_{\tau}$ nicht alle voneinander verschieden. Die Dinge $\alpha_{1}, \alpha_{2}, \alpha_{3}, \ldots, \alpha_{\tau}$ werden, wie man sagt, durch $S$ „im Zyklus permutiert“; man nennt auch die Zusammenstellung $\alpha_{1}, \alpha_{2}, \alpha_{3}, \ldots, \alpha_{\tau}$ einen , $\tau$-gliedrigen Zyklus" der Permutation $S$. Ist insbesondere $\tau=n$, so ist $S$ gegeben durch:

$$
S=\left(\begin{array}{l}
\alpha_{1}, \alpha_{2}, \alpha_{3}, \ldots, \alpha_{n} \\
\alpha_{2}, \alpha_{3}, \alpha_{4}, \ldots, \alpha_{1}
\end{array}\right)
$$

Diese Permutation $S$, die bei der in der ersten Zeile stehenden Anordnung jedes Ding durch das folgende, das letzte aber durch das erste ersetzt, heißt insbesondere eine „zyklische Permutation"; offenbar erzeugt sie eine zyklische Permutationsgruppe $G_{n}$ der Ordnung n. Ist indessen $\tau<n$, so erschöpfen die $\alpha_{1}, \alpha_{2}, \ldots, \alpha_{\tau}$ noch nicht alle $n$ Dinge. Es mögen dann die $\tau^{\prime}$ Dinge $\alpha_{1}^{\prime}, \alpha_{2}^{\prime}, \ldots, \alpha_{\tau^{\prime}}^{\prime}$ einen zweiten $\tau^{\prime}$-gliedrigen Zyklus von $S$ bilden, sowie vorkommenden Falles die $\alpha_{1}^{\prime \prime}, \alpha_{2}^{\prime \prime}, \ldots, \alpha_{\tau^{\prime}}^{\prime \prime}$ einen $\boldsymbol{\tau}^{\prime \prime}$-gliedrigen Zyklus usw. Man hat nun:

$$
S=\left(\begin{array}{ll}
\alpha_{1}, \alpha_{2}, \ldots, \alpha_{\tau}, & \alpha_{1}^{\prime}, \alpha_{2}^{\prime}, \ldots, \alpha_{\tau^{\prime}}^{\prime}, \ldots \\
\alpha_{2}, \alpha_{3}, \ldots, \alpha_{1}, & \alpha_{2}^{\prime}, \alpha_{3}^{\prime}, \ldots, \alpha_{1}^{\prime}, \ldots
\end{array}\right)
$$

und findet auf diese Weise $S$ in eine Anzahl von Zyklen aufgelöst. Es ist einleuchtend, daß die Periode $\nu$ von $S$ und damit die Ordnung $\nu$ der aus $S$ zu erzeugenden zyklischen Gruppe das kleinste gemeinschaftliche Vielfache von $\tau, \tau^{\prime}, \tau^{\prime \prime}, \ldots$ ist. 
Die Bedeutung der Permutationsgruppen für die allgemeine Theorie der endlichen Gruppe wird durch folgenden Satz gekennzeichnet: Jede endliche Gruppe $G_{m}$ der Ordnung $m$ ist als Permutationsgruppe, z. B. als eine solche $m^{\text {ten }}$ Grades darstellbar. Man fasse nämlich die $m$ "Elemente" $S_{0}, S_{1}, S_{2}, \ldots, S_{m-1}$ in dieser Reihenfolge als $m$ Dinge, wie wir sie bisher durch $1,2,3, \ldots, m$ bezeichneten, auf. Dem Elemente $S_{a} \operatorname{der} G_{m}$ möge dann die Permutation:

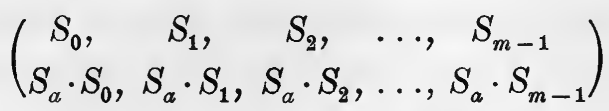

der $m$ "Dinge" $S$ zugeordnet sein. Alle $m$ so zu gewinnenden Permutationen bilden dann die "Einkleidung“ unserer $G_{m}$ als einer Permutationsgruppe $m^{\text {ten }}$ Grades.

\section{§ 8. Transitivität und Primitivität der Permutationsgruppen.}

Es sei jetzt $G_{m}$ eine beliebige unserer Permutationsgruppen $\boldsymbol{n}^{\text {ten }}$ Grades. Zwei Dinge $\alpha_{1}$ und $\alpha_{2}$ heißen „durch $G_{m}$ verbunden", wenn es eine Permutation $S$ in $G_{m}$ gibt, die $\alpha_{1}$ durch $\alpha_{2}$ ersetzt; die gleichfalls in $G_{m}$ enthaltene Permutation $S^{-1}$ ersetzt dann natürlich $\alpha_{2}$ durch $\alpha_{1}$. Sind zwei Dinge durch $G_{m}$ mit einem dritten verbunden, so sind sie auch untereinander durch $G_{m}$ verbunden. Sind demnach $\alpha_{1}, \alpha_{2}, \ldots, \alpha_{\tau}$ die gesamten mit $\alpha_{1}$ durch $G_{m}$ verbundenen Dinge, so ist jedes dieser $\tau$ Dinge mit jedem unter ihnen, aber mit keinem weiteren Dinge durch $G_{m}$ verbunden.

Ist $\tau=n, \mathrm{~d}$. h. ist jedes der $n$ Dinge mit jedem anderen durch $G_{m}$ verbunden, so heißt die Gruppe $G_{m}$,transitiv“. Ist $\tau<n$, so können wir ein zweites System $\alpha_{1}^{\prime}, \alpha_{2}^{\prime}, \ldots, \alpha_{\tau^{\prime}}^{\prime}$ miteinander durch $G_{m}$ verbundener Dinge aufstellen, sowie vorkommenden Falles ein drittes usw. Zwei aus verschiedenen Systemen entnommene Dinge sind dann nicht durch $G_{m}$ verbunden. Die Gruppe $G_{m}$ heißt jetzt ,intransitiv", und die verschiedenen Systeme verbundener Dinge nennt man die "Systeme der Intransitivität" von $G_{m}$. Die aus (4) S. $19 \mathrm{zu}$ erzeugende zyklische $G_{n}$ ist offenbar transitiv; dagegen erzeugt die Permutation (5) S. 19, die in mehr als einen Zyklus verfällt, eine intransitive zyklische $G_{v}$, bei der die verschiedenen Zyklen die Systeme der Intransitivität liefern.

Der Begriff der Transitivität kann in folgender Art weiterentwickelt werden. Die Gruppe $G_{m}$ heißt , $k$-fach transitiv", wenn es bei willkürlicher Auswahl der $k$ Dinge $\alpha_{1}, \alpha_{2}, \ldots, \alpha_{k}$ in $G_{m}$ stets eine Permutation:

$$
\left(\begin{array}{c}
1,2,3, \ldots, k, \ldots \\
\alpha_{1}, \alpha_{2}, \alpha_{3}, \ldots, \alpha_{k}, \ldots
\end{array}\right)
$$

gibt, die also die $k$ Dinge $1,2, \ldots, k$ bzw. in die $k$ willkürlich gewählten Dinge $\alpha_{1}, \alpha_{2}, \ldots, \alpha_{k}$ überführt. Es gibt dann sicher in $G_{m}$ auch eine 
Permutation, die $k$ willkürlich gewählte Dinge $\alpha_{1}, \alpha_{2}, \ldots, \alpha_{k}$ bzw. in $k$ gleichfalls willkürlich gewählte $\beta_{1}, \beta_{2}, \ldots, \beta_{k}$ überführt. Genauer wollen wir die $G_{m}$ immer dann $k$-fach transitiv nennen, wenn sie nicht auch noch $(k+1)$-fach oder $(k+2)$-fach usw. transitiv ist.

Eine weitere Einteilung der transitiven Gruppen in zwei Arten geschieht nach folgendem Grundsatze. Es mögen die $n$ Dinge in eine Anzahl von Systemen, die wir symbolisch durch $A, A^{\prime}, A^{\prime \prime}, \ldots$ bezeichnen

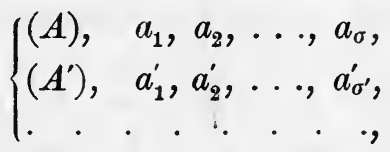

in der Art zerlegbar sein, daß diese Zerlegung gegenüber jeder Permutation von $G_{m}$ invariant ist. Die letzte Aussage soll folgenden Sinn haben. Es sollen, wenn die Anordnung (1) der $n$ Dinge durch eỉne beliebige Permutation von $G_{m}$ in die Anordnung:

(B), $b_{1}, b_{2}, \ldots, b_{\sigma}$,

$\left(B^{\prime}\right), \quad b_{1}^{\prime}, b_{2}^{\prime}, \ldots, b_{\sigma^{\prime}}^{\prime}$,

übergeht, die Systeme $B, B^{\prime}, \ldots$, als ganze betrachtet (d. h. abgesehen von irgendeiner Umordnung der Dinge im einzelnen Systeme), wieder nur die Systeme $A, A^{\prime}, \ldots$ in irgendeiner Anordnung darstellen.

Da $G_{m}$ transitiv sein sollte, so gibt es in $G_{m}$ eine Permutation, die das Anfangsglied $a_{1}^{(i)}$ einer beliebigen unter den Reihen $A^{(i)}$ in $b_{1}$ überführt. Dann geht der Annahme zufolge das System $A^{(i)}$ der $\sigma^{(i)}$ Dinge $a_{1}^{(i)}, a_{2}^{(i)}, \ldots$ in das System $B$ der $\sigma$ Dinge $b_{1}, b_{2}, \ldots, b_{\sigma}$ über. Wir ziehen hieraus die Folgerung: Die Anzahlen $\sigma, \sigma^{\prime}, \sigma^{\prime \prime}, \ldots$ der Dinge in den einzelnen Systemen (1) sind einander gleich $\sigma=\sigma^{\prime}=\sigma^{\prime \prime}=\cdots$, so daß $\sigma$ ein Teiler des Gruppengrades $n$ ist.

Zwei solche Einteilungen der $n$ Dinge können wir für jede $G_{m}$ angeben, nämlich die für $\sigma=n$ und für $\sigma=1$. Existieren keine weiteren Anordnungen (1), so heißt die transitive Gruppe $G_{m}$ "primitiv"; gibt es indessen eine Anordnung (1) mit $1<\sigma<n$, so wird $G_{m}$ "imprimitiv" genannt, die $\frac{n}{\sigma}$ Systeme $A, A^{\prime}, A^{\prime \prime}, \ldots$ werden als „Systeme der Imprimitivität" bezeichnet.

Es liege nun eine imprimitive Gruppe $G_{m}$ vor. Zur Abkürzung schreiben wir $\frac{n}{\sigma}=t$, so daB $t$ ein von 1 und $n$ verschiedener Teiler von $n$ ist. Da $G_{m}$ transitiv ist, so findet sich in $G_{m}$ eine Permutation $T$, die $a_{1}$ in ein beliebiges Ding des ersten Systems $A$ überführt. Diese Permutation transformiert dann das ganze System $A$ in sich, was wir durch $T(A)=A$ andeuten. Man sammle nun alle Permutationen $T_{0}=1$, 
$T_{1}, T_{2}, \ldots, T_{\mu-1}$ von $G_{m}$, welche $A$ in sich überführen. Da mit $T_{\alpha}$ und $T_{\beta}$ auch $T_{\beta} \cdot T_{\alpha}$ das System $A$ in sich transformiert, so bilden die $T_{0}=1, T_{1}, T_{2}, \ldots, T_{\mu-1}$ eine in $G_{m}$ enthaltene Untergruppe $G_{\mu}$, die offenbar intransitiv ist und $A$ zu einem ersten Systeme der Intransitivität hat.

Da die $G_{m}$ transitiv ist, so erhält sie weiter eine Permutation $V_{i}$, die $A$ in ein beliebiges System $A^{(i)}$ überführt, was durch $V_{i}(A)=A^{(i)}$ angedeutet werde. Ist aber $S$ irgendeine Permutation der Gruppe $G_{m}$, für die gleichfalls $S(A)=A^{(i)}$ zutrifft, so folgt $V_{i}^{-1} \cdot S(A)=V_{i}^{-1}\left(A^{(i)}\right)=A$, so daB $V_{i}^{-1} \cdot S=T$ eine Permutation der $G_{\mu}$ ist. Da andrerseits alle Permutationen $V_{i} \cdot T$ das System $A$ in $A^{(i)}$ transformieren, so ergibt sich der Satz: Die Nebengruppe $V_{i} \cdot G_{\mu}$ besteht aus den gesamten Permutationen $\operatorname{der} G_{m}$, die $A$ in $A^{(i)} \ddot{u b e r f u ̈ h r e n, ~ w a s ~ w i r ~ d u r c h ~} V_{i} \cdot G_{\mu}(A)=A^{(i)}$ andenten. Nun führt jede Permutation $S$ von $G_{m}$ das System $A$ in eines der Systeme $A, A^{\prime}, \ldots, A^{(t-1)}$ über. Alle $t$ Nebengruppen $G_{\mu}, V_{1} \cdot G_{\mu}, V_{2} \cdot G_{\mu}, \ldots$, $V_{t-1} \cdot G_{\mu}$ erschöpfen demnach die ganze $G_{m}$ : In der Gleichung:

$$
G_{m}=G_{\mu}+V_{1} \cdot G_{\mu}+V_{2} \cdot G_{\mu}+\cdots+V_{t-1} \cdot G_{\mu}
$$

haben wir die der Untergruppe $G_{\mu}$ entsprechende Zerlegung von $G_{m}$ in Nebengruppen vor uns; es gilt demnach:

$$
\mu \cdot t=\mu \cdot \frac{n}{\sigma}=m, \quad \mu=\frac{\sigma m}{n},
$$

so $d a \beta$ б $\mathrm{m}$ ein Vielfaches von $n$ ist.

Ist $T$ eine beliebige Permutation von $G_{\mu}$, so gilt $V_{i} \cdot T \cdot V_{i}^{-1}\left(A^{(i)}\right)=A^{(i)}$. Andrerseits zeigt man leicht, daß jede Permutation $S$ von $G_{m}$, die $A^{(i)}$ in sich überführt, in die Gestalt $S=V_{i} \cdot T \cdot V_{i}^{-1}$ gesetzt werden kann. Nun haben wir in:

$G_{\mu}, G_{\mu}^{\prime}=V_{1} \cdot G_{\mu} \cdot V_{1}^{-1}, G_{\mu}^{\prime \prime}=V_{2} \cdot G_{\mu} \cdot V_{2}^{-1}, \ldots, G_{\mu}^{(t-1)}=V_{t-1} \cdot G_{\mu} \cdot V_{t-1}^{-1}$

die gesamten mit $G_{\mu}$ innerhalb $G_{m}$ gleichberechtigten Untergruppen, die natürlich keineswegs alle verschieden zu sein brauchen, und die im Falle einer ausgezeichneten $G_{\mu}$ sogar alle gleich sind. Es ergibt sich der Satz: Die $t$ mit $G_{\mu}$ gleichberechtigten Untergruppen $G_{\mu}, G_{\mu}^{\prime}, \ldots, G_{\mu}^{(t-1)}$ sind den $t$ Systemen $A, A^{\prime}, \ldots, A^{(t-1)}$ zugeordnet, indem die einzelne dieser Untergruppen alle Permutationen der $G_{m}$ umfaßt, die das zugehörige System in sich transformieren. Insbesondere ergibt sich die Folgerung: Der Durchschnitt $G_{\lambda}=D\left(G_{\mu}, G_{\mu}^{\prime}, G_{\mu}^{\prime \prime}, \ldots, G_{\mu}^{(t-1)}\right)$ liefert eine in der $G_{m}$ ausgezeichnete intransitive Untergruppe, die aus allen Permutationen besteht, welche jedes System (1) in sich überführen. Es kann hierbei natürlich der Fall vorliegen, daB $G_{2}$ die Untergruppe $G_{1}$ ist.

Umgekehrt gilt folgender Satz: Gibt es in einer transitiven Gruppe $G_{m}$ eine intransitive ausgezeichnete Untergruppe $G_{2}$ einer Ordnung $\lambda>1$, 
so ist $G_{m}$ imprimitiv, und die Systeme der Intransitivität von $G_{\lambda}$ liefern für $G_{m}$ Systeme der Imprimitivität. Die Systeme der Intransitivität von $G_{\lambda}$, für welche wir die Bezeichnungen (1) heranziehen, sind eindeutig bestimmt, und insbesondere steht ibre Anzahl fest. Eine beliebige Permutation $S$ aus $G_{m}$ führe die Systeme $A, A^{\prime}, A^{\prime \prime}, \ldots$ in die oben durch $B, B^{\prime}, B^{\prime \prime}, \ldots$ bezeichneten Systeme über, deren Anzahl also gleich derjenigen $\operatorname{der} A, A^{\prime}, A^{\prime \prime}, \ldots$ ist. Dann gelten die Gleichungen:

$$
G_{\lambda}\left(A^{(i)}\right)=A^{(i)}, \quad S \cdot G_{\lambda}\left(A^{(i)}\right)=S\left(A^{(i)}\right)=B^{(i)}, \quad A^{(i)}=S^{-1}\left(B^{(i)}\right),
$$

aus denen $S \cdot G_{\lambda} \cdot S^{-1}\left(B^{(i)}\right)=B^{(i)}$ hervorgeht. Da nun $G_{\lambda}$ eine ausgezeichnete Untergruppe ist, so folgt $S \cdot G_{\lambda} \cdot S^{-1}=G_{\lambda}$ und also $G_{\lambda}\left(B^{(i)}\right)=B^{(i)}$. Das einzelne $B^{(i)}$ stellt also wieder ein System durch $G_{\lambda}$ verbundener Dinge vor oder mehrere solche Systeme. Die letztere Möglichkeit ist aber ausgeschlossen, da sonst aus den gesamten $B, B^{\prime}, B^{\prime \prime}, \ldots$ Systeme der Intransitivität für $G_{\lambda}$ in einer Anzahl hervorgehen würden, die größer als die Anzahl der $A, A^{\prime}, A^{\prime \prime}, \ldots$ wäre. Also sind die $B, B^{\prime}, B^{\prime \prime} \ldots$ wieder die Systeme der Intransitivität von $G_{2}$ und als solche mit den Systemen $A, A^{\prime}, A^{\prime \prime}, \ldots$, von der Anordnung abgesehen, gleich. Die Zerlegung $A, A^{\prime}, A^{\prime \prime} \ldots$ aller $n$ Dinge besitzt somit gegenüber jeder Permutation von $G_{m}$ den oben bezeichneten Charakter der Invarianz. Da die Anzahl der Systeme $>1$ (die Ordnung $\lambda$ ist $>1$ ) sowie $<n\left(G_{\lambda}\right.$ ist intransitiv) ist, so ist der Satz bewiesen.

Als eine unmittelbare Folge notieren wir noch: Jede in einer primitiven Gruppe $G_{m}$ enthaltene ausgezeichnete Untergruppe ist transitiv.

\section{Algebraische Gleichungen. ${ }^{1}$ )}

\section{§ 1. Symmetrische Funktionen.}

Unter $g\left(z_{1}, z_{2}, \ldots, z_{n}\right)$ verstehen wir eine rationale ganze Funktion der $n$ unabhängigen Veränderlichen $z_{1}, z_{2}, \ldots, z_{n}$, die wir in der Gestalt:

$$
g\left(z_{1}, z_{2}, \ldots, z_{n}\right)=\sum A_{\lambda_{1}, \lambda_{2}}, \ldots, \lambda_{n} z_{1}^{\lambda_{1}} z_{2}^{\lambda_{2}} \ldots z_{n}^{\lambda_{n}}
$$

mit von den $z$ unabhängigen Koeffizienten $A$ geben. Die Gruppe $G_{n 1}$ sei die "symmetrische“ Gruppe aller $n$ ! Permutationen $\operatorname{der} z_{1}, z_{2}, \ldots, z_{n}$. Eine einzelne Permutation $S_{a}=\left(z_{k}, z_{a_{k}}\right)$ der $G_{n 1}$ wird die Funktion (1) entweder in eine neue Funktion überführen oder in sich transformieren. Der letztere Fall charakterisiert sich dadurch, dab $g\left(z_{a_{1}}, z_{a_{2}}, \ldots, z_{a_{n}}\right)$ durch Umrechnung auf die Gestalt $g\left(z_{1}, z_{2}, \ldots, z_{n}\right)$ zurückgebracht werden kann und also mit $g\left(z_{1}, z_{2}, \ldots, z_{n}\right)$ bei unabhängig variablen

1) Neben den S. 1 genannten Werken vgl. man noch E. Landau „Einführung in die elementare und analytische Theorie der algebraischen Zahlen und der Ideale", Teil I (Leipzig 1918). 
$z_{1}, z_{2}, \ldots, z_{n}$ „identisch“. ist. Falls jede der beiden Permutationen $S_{a}$ und $S_{b}$ die Funktion (1) in sich transformieren, so geschieht dasselbe durch $S_{b} \cdot S_{a}$. Die gesamten Permutationen, welche $g\left(z_{1}, z_{2}, \ldots, z_{n}\right)$ in sich transformieren, bilden eine in $\operatorname{der} G_{n}$ enthaltene Untergruppe, die $G_{\mu}$ heiße und deren Index $t=\frac{n !}{\mu}$ ist.

Alle $\mu$. Permutationen der einzeluen zu $G_{\mu}$ gehörenden Nebengruppe $V_{i} \cdot G_{\mu}$ transformieren $g\left(z_{1}, z_{2}, \ldots, z_{n}\right)$ in eine und dieselbe Funktion. Zwei aus verschiedenen Nebengruppen entnommene Permutationen $V_{a}$ und $V_{b}$ ergeben indessen stets verschiedene Funktionen, da andernfalls $V_{b}^{-1} \cdot V_{a}$ die Funktion (1) in sich transformieren würde und also $\operatorname{der} G_{\mu}$ angehören müßte. Die $t$ Funktionen, in welche $g\left(z_{1}, z_{2}, \ldots, z_{n}\right)$ durch die $t$. Permutationen $V_{0}=1, V_{1}, V_{2}, \ldots, V_{t-1}$ übergeführt wird, heißen einander „,konjugiert", und $g\left(z_{1}, z_{2}, \ldots, z_{n}\right)$ wird als eine "t-wertige" Funktion bezeichnet. Gegenüber irgendeiner Permutation der $G_{n !}$ erfahren die $t$ konjugierten Funktionen selbst eine Permutation.

Eine einwertige Funktion, die also durch alle Permutationen der "symmetrischen" Gruppe $G_{n,}$ in sich transformiert wird, heißt eine „symmetrische Funktion". Ein Beispiel einer solchen Funktion ist:

$$
g=z_{1}+z_{2}+z_{3}+\cdots+z_{n} \text {. }
$$

Als Beispiel einer $n$-wertigen Funktion nennen wir $g=z_{1}$. Sie bleibt bei den Permutationen derjenigen $G_{(n-1) !}$ unverändert, welche nur die Argumente $z_{2}, z_{3}, \ldots, z_{n}$ auf alle Arten umstellt; die zugehörigen $n$ konjugierten Funktionen sind $z_{1}, z_{2}, \ldots, z_{n}$. Als Beispiel einer $n$ !-wertigen Funktion nennen wir endlich etwa:

$$
g\left(z_{1}, z_{2}, \ldots, z_{n}\right)=z_{1}+2 z_{2}+3 z_{3}+\cdots+n z_{n}
$$

sie wird nur durch die identische Permutation in sich transformiert.

Die Funktion $z_{1} \cdot z_{2} \cdot z_{3} \cdots z_{k}$ bleibt bei den $k ! \cdot(n-k)$ ! Permutationen unverändert, welche die $z_{1}, z_{2}, \ldots, z_{k}$ und ebenso die $z_{k+1}$, $z_{k+2}, \ldots, z_{n}$ nur unter sich permutieren. Die Wertigkeit $t$ dieser Funktion ist also, da sie durch alle übrigen Permutationen geändert wird:

$$
t=\frac{n !}{k ! \cdot(n-k) !}=\left(\begin{array}{l}
n \\
k
\end{array}\right) \text {. }
$$

Die Summe der $\left(\begin{array}{l}n \\ k\end{array}\right)$ zugehörigen konjugierten Funktionen ist symmetrisch und wird als die $k^{\text {te }}$ symmetrische Grundfunktion $\sigma_{k}$ bezeichnet. Den Zahlen $k=1,2, \ldots, n$ entsprechend gewinnen wir im ganzen n symmetrische Grundfunktionen:

$$
\left\{\begin{array}{l}
\sigma_{1}=z_{1}+z_{2}+z_{3}+\cdots+z_{n}, \\
\sigma_{2}=z_{1} z_{2}+z_{1} z_{3}+\cdots+z_{n-1} z_{n}, \\
\cdot \cdot \cdot \cdot \cdot \cdot \cdot \cdot \cdot . \\
\sigma_{n}=z_{1}: z_{2} \cdot z_{3} \cdots z_{n} .
\end{array}\right.
$$


Bildet man mit irgendeinem nicht-verschwindenden Faktor $a_{0}$ und einer Unbekannten $z$ die Gleichung $n^{\text {ten }}$ Grades:

$$
a_{0}\left(z-z_{1}\right)\left(z-z_{2}\right)\left(z-z_{3}\right) \ldots\left(z-z_{n}\right)=0,
$$

deren $n$ "Wurzeln" $z$ die $z_{1}, z_{2}, z_{3}, \ldots, z_{n}$ sind, und kleidet man diese Gleichung nach Ausmultiplikation der Klammern in die Gestalt:

$$
a_{0} z^{n}+a_{1} z^{n-1}+a_{2} z^{n-2}+\cdots+a_{n}=0,
$$

so ist bekanntlich die Beziehung der $n$ Grundfunktionen (3) zu den Gleichungskoeffizienten gegeben durch:

$$
\sigma_{1}=-\frac{a_{1}}{a_{0}}, \sigma_{2}=\frac{a_{2}}{a_{0}}, \sigma_{3}=-\frac{a_{3}}{a_{0}}, \ldots, \sigma_{n}=(-1)^{n} \frac{a_{n}}{a_{0}} .
$$

Der Hauptsatz der Theorie der symmetrischen Funktionen lautet Jede ganze symmetrische Funktion (1) kann umgerechnet werden in die Gestalt einer rationalen ganzen Funktion:

$$
g\left(z_{1}, z_{2}, \ldots, z_{n}\right)=\sum B_{\mu_{1}, \mu_{2}, \ldots, \mu_{n}} \sigma_{1}^{u_{1}} \cdot \sigma_{2}^{\mu_{2}} \cdots \sigma_{n}^{\mu_{n}}
$$

der $n$ symmetrischen Grundfunktionen (3); dabei sind die Koeffizienten $B$ lineare homogene, mit "ganzzahligen" Koeffizienten versehene Ausdrücke in den ursprünglichien Koeffizienten $A$ der Funktion g, der Grad der in (5) rechtsstehenden Funktion in den $\sigma$ aber ist gleich dem größten im Ausdrucke (1) von $g$ auftretenden Exponenten $\lambda_{.}{ }^{1}$ )

Nennen wir eine Funktion (1) mit ausschließlich ganzzahligen Koeffizienten kurz eine "ganzzahlige" Funktion, so folgt insbesondere der Satz: Eine ganze ganzzahlige symmetrische Funktion (1) läßt sich in eine ganze ganzzahlige Funktion der symmetrischen Grundfunktionen umrechnen, deren Gràd in den $\sigma$ gleich dem höchsten in (1) rechts auftretenden Exponenten $\lambda$ ist.

Die bekanntesten Beispiele liefern die „Potenzsummen “ $\operatorname{der} z_{1}, z_{2}, \ldots, z_{n}$. Die $\nu^{\text {te }}$ Potenzsumme bezeichnen wir durch:

$$
s_{y}=z_{1}^{v}+z_{2}^{v}+z_{3}^{v}+\cdots+z_{n}^{v} .
$$

Die Darstellung der niedersten Potenzsummen als ganzer ganzzahliger Funktionen der $\sigma$ ist:

$$
\left\{\begin{array}{l}
s_{1}=\sigma_{1}, \quad s_{2}=\sigma_{1}^{2}-2 \sigma_{2}, \\
s_{3}=\sigma_{1}^{3}-3 \sigma_{1} \sigma_{2}+3 \sigma_{3} \\
s_{4}=\sigma_{1}^{4}-4 \sigma_{1}^{2} \sigma_{2}+4 \sigma_{1} \sigma_{3}+2 \sigma_{2}^{2}-4 \sigma_{4}, \\
. \text {. . . . . . . . . . . . }
\end{array}\right.
$$

1) Dieser Satz ist sehr bekannt, aber nicht ganz kurz beweisbar. Man findet den Nachweis in allen ausfïhrlicheren Lehrbüchern der Algebra, z. B. bei Weber, a. a. $0 .$, Bd. 1, S. $160 \mathrm{ff}$. 
Wir erinnern ferner noch an das "Differenzenprodukt" der $z$ :

$$
\left(z_{1}-z_{2}\right)\left(z_{1}-z_{3}\right) \cdots\left(z_{1}-z_{n}\right)\left(z_{2}-z_{3}\right) \cdots\left(z_{n-1}-z_{n}\right),
$$

dessen Quadrat eine ganze ganzzahlige symmetrische Funktion der $z$ und also eine ganze ganzzahlige Funktion der $\sigma$ ist. Für die oben aufgestellte algebraische Gleichung $n^{\text {ten }}$ Grades, deren Wurzeln die $z_{1}, z_{2}, \ldots, z_{n}$ sind, ist dieses Quadrat die „Diskriminante", deren Verschwinden das Auftreten einer mindestens zweifachen Wurzel jener Gleichung anzeigt. Das Differenzenprodukt (8) selbst, d. h. die Quadratwurzel der Diskriminante, ist eine zweiwertige Funktion der $z_{1}, z_{2}, \ldots, z_{n}$; sie wird durch alle "geraden" Permutationen in sich transformiert und erleidet gegenüber allen „ungeraden"Permutationen Zeichenwechsel. Die zur Funktion (8) gehörende Untergruppe ist die sogenannte "alternierende" Gruppe aller "geraden" Permutationen, die die Ordnung ${ }_{2}^{1} n$ ! hat und ausgezeichnet ist. ${ }^{1}$ )

\section{\$2. Tschirnhausentransformation.}

Eine erste Anwendung der entwickelten Sätze können wir bei Gelegenheit der nach Tschirnhausen benaunten Transformation einer algebraischen Gleichung machen. Eine Gleichung $n^{\text {ten }}$ Grades sei durch:

$$
z^{n}+a_{1} z^{n-1}+a_{2} z^{n-2}+\cdots+a_{n}=0
$$

gegeben, ihre Wurzeln seien $z_{1}, z_{2}, z_{3}, \ldots, z_{n}$. Es soll nun die Gleichung (1) für $z$ auf eine Gleichung für eine neue Unbekannte $w$ umgerechnet werden, die mit $z$ durch die Beziehung:

$$
w=c_{0}+c_{1} z+c_{2} z^{2}+\cdots+c_{n-1} z^{n-1}
$$

zusammenhängt, unter den $c$ gegebene Konstante verstanden.

$\mathrm{Um}$ diese Transformation zu vollziehen, berechnen wir die $n$ Werte:

$$
w_{k}=c_{0}+c_{1} z_{k}+c_{2} z_{k}^{2}+\cdots+c_{n-1} z_{k}^{n-1}, \quad k=1,2, \ldots, n,
$$

die den $n$ Wurzeln $z_{k}$ der Gleichung (1) entsprechen. Die Gleichung $n^{\text {ten }}$ Grades für $w$ :

$$
\left(w-w_{1}\right)\left(w-w_{2}\right)\left(w-w_{3}\right) \cdots\left(u-w_{n}\right)=0,
$$

welche die $n$ Wurzeln (3) hat, möge entwickelt so lauten:

$$
w^{n}+b_{1} w^{n-1}+b_{2} w^{n-2}+\cdots+b_{n}=0
$$

sie heibt eine "Tschirnhausenresolvente" der Gleichung (1), und die durch (2) gegebene Transformation wird als eine "Tschirnhausentransformation" der Gleichung (1) bezeichnet.

Der einzelne Koeffizient $b_{i}$ ist eine ganze homogene Funktion $i^{\text {ten }}$ Grades der $w_{1}, w_{2}, \ldots, w_{n}$, die zugleich in diesen Größen symmetrisch ist. Tragen wir für die $w_{k}$ die Ausdrücke (3) ein, so wird $b_{i}$ eine ganze

1) S. das Nähere bei Weber, a. a. O. Bd. I, S. ̌37ff. 
symmetrische Funktion der $z_{1}, z_{2}, \ldots, z_{n}$, deren Koefizienten ganze ganzzahlige homogene Ausdrücke $i^{\text {ten }}$ Grades der $c_{0}, c_{1}, \ldots, c_{n-1}$ sind. Da nun die symmetrischen Grundfunktionen $\operatorname{der} z_{1}, z_{2}, \ldots, z_{n}$, von den Vorzeichen abgesehen, einfach die Koeffizienten $a_{1}, a_{2}, \ldots, a_{n}$ der Gleichung (1) sind, so folgt aus dem Hauptsatze von S. 25 das Ergebnis: Die Koeffizienten $b_{i}$ der Tschirnhausenresolvente (4) sind rationale ganze Funktionen der ursprïnglichien Koeffizienten $a_{1}, a_{2}, \ldots, a_{n}$ mit Koeffizienten, die ganze ganzzahlige homogene Ausdrücke $i^{\text {ten }}$ Grades der $c_{0}, c_{1}, c_{2}, \ldots, c_{n-1}$ sind.

\section{$\S 3$. Hilfssatz über ganze Funktionen.}

Es seien $n$ unabhängige Variable $z_{1}, z_{2}, z_{3}, \ldots, z_{n}$ und $m$ rationale ganze Funktionen der $z$ :

$$
g_{1}\left(z_{1}, z_{2}, \ldots, z_{n}\right), \quad g_{2}\left(z_{1}, z_{2}, \ldots, z_{n}\right), \ldots, g_{m}\left(z_{1}, z_{2}, \ldots, z_{n}\right)
$$

gegeben, unter $m$ und $n$ beliebige positive ganze Zahlen verstanden. Wir ordnen jede der Funktionen so, daB in ihr die Glieder, die in ihren variablen Bestandteilen $z_{1}^{v_{1}} \cdot z_{2}^{\gamma_{2}} \cdots z_{n}^{v_{n}}$ übereinstimmen, zusammengefaßt sind. Die einzelne Funktion verschwindet „identisch“, wenn in der so geordneten Gestalt jeder Koeffizient gleich 0 ist. Wir nehmen an, daß keine der Funktionen (1) identisch verschwindet, daß also in jeder mindestens ein Glied mit einem von 0 verschiedenen Koeffizienten auftritt. Dann gilt folgender, bald zur Verwendung kommender Satz: Sind $m$ nicht identisch verschwindende ganze rationale Funktionen von $n$ Variablen vorgelegt, so kann man auf unendlich viele Arten für die $z_{1}, z_{2}, \ldots, z_{n}$ ganze Zahlen eintragen, für welche keine der Funltionen verschwindet und also ihr Produkt von 0 verschieden ist.

Der Beweis kann leicht durch vollständige Induktion geführt werden. Ist zunächst $n=1$, so haben wir $m$ nicht identisch verschwindende ganze Funktionen $g_{k}\left(z_{1}\right)$ einer Variablen. Für die einzelne gibt es nur eine beschränkte Anzahl von Werten $z_{1}$, die $g_{k}\left(z_{1}\right)=0$ befriedigen. Meiden wir also die endlich vielen Werte $z_{1}$, für die mindestens eine der Funktionen $g_{k}\left(z_{1}\right)$ verschwindet, so bleiben in der Tat noch unendlich viele ganze Zahlen $z_{1}$, für die keine der Funktionen verschwindet. Unser Satz ist also für $n=1$ richtig.

Wir nehmen nun an, der Satz gelte auch noch für mehrere Variable, und zwar jedenfalls bis zum Falle von $(n-1)$ Variablen. Dann können wir leicht zeigen, daß er auch noch im nächstfolgenden Falle von $n$ Variablen richtig ist, womit der allgemeine Beweis des Satzes beendet sein wird. Ordnen wir nämlich die Funktionen (1) nach Potenzen der letzten Variablen $z_{n}$, so werden die Koeffizienten dieser Potenzen offenbar ganze Funktionen der $(n-1)$ Variablen $z_{1}, z_{2}, \ldots, z_{n-1}$. Dabei können in der einzelnen Funktion $g_{k}$ diese "Koeffizienten" nicht alle identisch 
verschwinden, da sonst $g_{k}\left(z_{1}, z_{2}, \ldots, z_{n}\right)$ selbst identisch verschwinden würde. Der Annahme gemäB können wir dann auf unendlich viele Arten für die $z_{1}, z_{2}, \ldots, z_{n-1}$ solche ganze Zahlen eintragen, daß alle nicht identisch verschwindenden unter jenen „Koeffizienten" von 0 versschiedene Werte annehmen. Wir haben dann mit einem System nicht identisch verschwindender ganzer Funktionen der einzigen Variablen $z_{n} z u$ tun und können nach dem für $n=1$ bereits bewiesenen Satze auf unendlich viele Arten für $z_{n}$ eine gleichfalls ganze Zahl so eintragen, daß keine dieser Funktionen verschwindet. Damit ist der Beweis unseres Satzes allgemein geführt.

\section{\$4. Funktionen in Zahlkörpern.}

Neben den Begriff der Gruppe tritt in der Theorie der algebraischen Glejchungen als nicht minder wichtig der von Dedekind ${ }^{1}$ ) eingeführte Begriff des „Körpers“. Ein System konstanter Zahlen heißt ein „Zahlkörper" oder kurz ein "Körper" $\left.\Re^{2}\right)$, wenn mit irgendzwei Zahlen $a, b$ des Systems stets auch $(a+b),(a-b), a \cdot b$ und, sofern $b$ von 0 verschieden ist, auch $a: b$ im System enthalten ist. Das Ergebnis irgendwelcher rationaler Rechnungen, angewandt auf Zahlen von $\mathfrak{\kappa}$, ist also, wenn nur die Division durch 0 stets vermieden wird, immer wieder in $\Omega$ enthalten. Die Zahlen von $\Omega$ mögen irgendwelche reelle oder komplexe endliche Konstante sein; der zunächst mögliche Fall, daß $\mathfrak{A}$ nur aus der Zahl 0 besteht, soll übrigens ausgeschlossen sein.

Nach der letzten Bemerkung enthält $\Omega$ sicher eine von 0 verschiedene Zahl $a$, dann aber auch $a: a=1$ und damit sogleich alle rationalen Zahlen. Die gesamten rationalen Zahlen bildẹ offenbar für sich einen Körper, der der ,rationale Körper" genannt wird und durch $\Re$ bezeichnet werden möge. Der rationale Körper $\Re$ ist, wie wir sahen, in jedem Zahlkörper $\mathfrak{\Omega}$ enthalten. Ein weiteres Beispiel eines Körpers liefert das System aller Zahlen $(a+i b)$ nit rationalen $a, b$; wir nennen ferner den Körper aller reellen Zahlen sowie den alle übrigen Körper umfassenden Körper aller reellen und komplexen Zahlen.

Es sei jetzt irgendein Zahlkörper $\Re$ vorgelegt. Eine rationale ganze Funktion einer Variablen $\boldsymbol{z}$ :

$$
f(z)=a_{0} z^{n}+a_{1} z^{n-1}+a_{2} z^{n-2}+\cdots+a_{n}
$$

heiße „eine Funktion im Körper $\Re^{*}$ oder kurz eine „Funktion in $\Re^{\star}$, falls

1) Siehe die Angaben am Anfang des vierten T'eiles der vorliegenden Einleitung sowie über den Begriff des „Funktionenkörpers" die Ausführungen in I, 81.

2) Mit gewissen Eigenschaften ausgestattete Systeme unendlich vieler Zahlen oder Systeme unendlich vieler Funktionen werden weiterhin vielfach auftreten. Zur Bezeichnung solcher Systeme benutzen wir stets die Frakturschrift. 
die Koeffizienten $a_{0}, a_{1}, a_{2}, \ldots, a_{n}$ Zahlen aus $\Omega$ sind. Damit der „Grad“" $n$ in (1) wirklich vorliegt, gelte der Koeffizient $a_{0}$ des höchsten Gliedes stets als von 0 verschieden. Jede von 0 verschiedene Zahl aus $\Omega$ gilt hiernach als eine "Funktion nullten Grades in $\Re^{*}$; die Zahl 0 steht für sich als ,identisch verschwindende Funktion in $\Re^{\prime \prime}$.

Das Produkt zweier Funktionen in $\Omega$ ist offenbar wieder eine Funktion in $\Re$, deren Grad gleich der Summe der Grade der Faktoren ist. Läßt sich andrerseits $f(z)$ als Produkt $f(z)=\chi_{1}(z) \cdot \chi_{2}(z)$ zweier Funktionen in $\Omega$ darstellen, so heiBt jede der Funktionen $\chi_{1}(z), \chi_{2}(z)$ ein "Teiler" der Funktion $f(z)$. Es ist einleuchtend, daß $f(z)$ jede Funktion nullten Grades in $\Omega$ zum Teiler hat, und da $B$ auch jedes Produkt von $f(z)$ und einer Funktion nullten Grades Teiler von $f(z)$ ist.

Es seien $f(z)$ und $g(z)$ zwei festgewählte Funktionen in $\Omega$, deren Grade $n$ und $m>0$ seien. ${ }^{1}$ ) Man bilde alle Ausdrücke:

$$
f(z) \psi(z)+g(z) \varphi(z)
$$

unter $\varphi(z)$ und $\psi(z)$ irgendwelche Funktionen in $\mathfrak{R}$ verstanden. Es entsteht so ein System unendlich vieler Funktionen in $\mathfrak{\Omega}$, das wir durch $\mathfrak{F}$ bezeichnen, und das folgende Eigenschaften besitzt:

1. Die Summe und die Differenz zweier Funktionen aus $\mathfrak{F}$ liefern siets wieder Funktionen aus $\mathfrak{F}$;

2. Das Prodult einer Funktion aus $\mathfrak{F}$ und einer Funktion in $\Re$ ist stets wieder eine Funktion aus $\mathfrak{F}$.

Das System $\mathfrak{F}$ enthält die mit 0 identische Funktion in $\Omega$, die wir z. B. erhalten, wenn wir $\varphi(z)$ mit $f(z)$ und $\psi(z)$ mit $-g(z)$ identisch wählen. Auch die Funktionen $f(z)$ und $g(z)$ selbst kommen in $\mathfrak{F}$ vor. Wir sehen von der mit 0 identischen Funktion ab und ordnen die übrigen Funktionen von $\mathfrak{F}$ nach ihren Graden. Es sei $\nu$ der hierbei auftretende „Minimalgrad“, der dann jedenfalls weder $>m$ noch $>n$ ist. Eine in $\mathfrak{F}$ auftretende Funktion des Minimalgrades $\nu$ sei $\chi(z)$, die wir nötigenfalls nach Division durch den Koeffizienten des höchsten Gliedes in die Gestalt setzen:

$$
\chi(z)=z^{\nu}+c_{1} z^{\nu-1}+c_{2} z^{\nu-2}+\cdots+c_{v} .
$$

Dann gilt der Satz: Die Funktion $\chi(z)$ ist durch $f(z)$ und $g(z)$ eindeutig bestimmt. Ist nämlich $\chi^{\prime}(z)=z^{v}+c_{1}^{\prime} z^{v-1}+\cdots$ irgendeine in $\mathfrak{F}$ enthaltene Funktion des Minimalgrades $\nu$ mit dem höchsten Koeffizienten 1, so ist $\chi^{\prime}(z)-\chi(z)$ eine in $\mathfrak{F}$ enthaltene Funktion eines Grades $<\boldsymbol{\nu}$, die demnach mit 0 identisch ist. Also ist $\chi^{\prime}(z)$ mit $\chi(z)$ identisch.

1) In dem Falle, daß mindestens eine der Funktionen $f(z), g(z)$ dem nullten Grade angehört, gestalten sich die folgenden Entwicklungen elementar. 
Da $\nu \leqq n$ ist, so können wir $f(z)$ durch $\chi(z)$ teilen und ein Ergebnis der Gestalt:

$$
f(z)=q(z) \chi(z)+r(z)
$$

aufstellen, wo $q(z)$, der Quotient, und $r(z)$, der Rest der Division, Funktionen in $\Re$ sind und der Grad von $r(z)$ kleiner als $\nu$ ist. Ziehen wir die Darstellung:

$$
\chi(z)=f(z) \psi(z)+g(z) \varphi(z)
$$

der Funktion $\chi$ als einer solchen des Systems $\mathfrak{F}$ heran, so folgt aus (4) und (5):

$$
r(z)=f(z)(1-q(z) \psi(z))-g(z) q(z) \varphi(z) .
$$

Die Funktion $r(z)$ ist hiernach gleichfalls in $\mathfrak{F}$ enthalten; sie ist also als einem Grade $<v$ angehörig mit 0 identisch, so daß $f(z)$ durch $\chi(z)$ ohne Rest teilbar ist. Da man dieselbe Betrachtung auf $g(z)$ anwenden kann, so ist $\chi(z)$ ein gemeinsamer Teiler von $f(z)$ und $g(z)$. Aus (5) folgt weiter, daß jeder gemeinsame Teiler von $f(z)$ und $g(z)$ ein Teiler ron $\chi(z)$ ist; die Funktion $\chi(z)$ heißt demnach der ,größte gemeinsame Teiler" von $f(z)$ und $g(z)$ : Die durch $f(z)$ und $g(z)$ eindeutig bestimmte Funktion (3) des in $\mathfrak{F}$ auftretenden Minimalgrades $\nu$ ist der größte gemeinschaftliche Teiler von $f(z)$ und $g(z)$.

Ist der Minimalgrad $v=1$ und also $\chi(z)$ mit 1 identisch, so heißen die beiden Funktionen $f(z)$ und $g(z)$,teilerfremd". Es folgt: Sind $f(z)$ und $g(z)$ zwei teilerfremde Funlitionen in $\Re$, so kann man $\varphi(z)$ und $\psi(z)$ als Funktionen in $\Re$ so wählen, daß die Gleichung:

$$
f(z) \psi(z)+g(z) \varphi(z)=1
$$

identisch besteht; umgekehrt folgt aus dem Bestehen einer Gleichung (6), $d a \beta f(z)$ und $g(z)$ teilerfremd sind.

Eine weitere wichtige Folgerung ist: Sind $f(z), g(z)$ und $h(z)$ Funktionen in $\Omega$, von denen die beiden ersten teilerfremd sind, und ist $g(z) \cdot h(z)$ durch $f(z)$ teilbar, so ist $f(z)$ ein Teiler von $h(z)$. Für teilerfremde $f(z)$, $g(z)$ folgt nämlich aus $(6)$ :

$$
h(z)=f(z) \cdot \psi(z) h(z)+g(z) h(z) \cdot \varphi(z),
$$

und da die beiden Glieder rechter Hand den Teiler $f(z)$ haben, so hat auch $h(z)$ diesen Teiler.

Mit $\varphi(z)$ und $\psi(z)$ genügen auch die beiden Funktionen:

$$
\varphi_{1}(z)=\varphi(z)+\omega(z) f(z), \quad \psi_{1}(z)=\psi(z)-\omega(z) g(z)
$$

der identischen Gleichung (6), wobei $\omega(z)$ eine beliebige Funktion in $\Omega$ ist. Man kann über $\omega(z)$ so verfügen, $\operatorname{daB} \varphi_{1}(z)$ einen Grad $<n$ erhält. Aus der identischen Gleichung:

$$
f(z) \psi_{1}(z)=1-g(z) \varphi_{1}(z)
$$


folgt dann, daß $\psi_{1}(z)$ einen Grad $<m$ hat. Ist aber weiter $\varphi_{1}^{\prime}(z)$ und $\dot{\psi}_{1}^{\prime}(z)$ irgendein Funktionenpaar mit Graden $<n$ bzw. $<m$, das die Gleichung (6) befriedigt, so folgt als identische Gleichung:

$$
f(z)\left(\psi_{1}^{\prime}(z)-\psi_{1}(z)\right)=g(z)\left(\varphi_{1}(z)-\varphi_{1}^{\prime}(z)\right) .
$$

Da $f(z)$ und $g(z)$ teilerfremd sind, so muß nach dem letzten Satze $f(z)$ ein Teiler von $\left(\varphi_{1}(z)-\varphi_{1}^{\prime}(z)\right)$ seị, so daß die letztere Funktion, da sie den Grad $n$ von $f(z)$ nicht erreicht, mit 0 identisch ist. Es sind also $\varphi_{1}(z)$ und $\varphi_{1}^{\prime}(z)$ identisch, und ebenso folgert man die Identität von $\psi_{1}(z)$ und $\psi_{1}^{\prime}(z)$. Sind $f(z)$ und $g(z)$ teilerfremd, so gibt es ein und nur ein Paar, die identische Gleichung (6) befriedigender Funktionen $\varphi(z), \psi(z)$, deren Grade $b z w .<n$ und $<m$ sind.

Sind $f(z)$ und $g(z)$ wieder teilerfremd und sind $\varphi(z)$ und $\psi(z)$ zunächst zwei beliebige die Gleichung (6) erfüllende Funktionen, so folgt durch Multiplikation mit irgendeiner Funktion $h(z)$ die Gleichung (7). Schreiben wir in ihr für $h(z) \varphi(z)$ und $h(z) \psi(z)$ gleich selbst wieder $\varphi(z)$ und $\psi(z)$, so folgt:

$$
h(z)=f(z) \psi(z)+g(z) \varphi(z) .
$$

Hieran schließe man die oben mit den Gleichungen (8) begonnene Betrachtung, die jetzt zu folgendem Ergebnisse führt: Sind $f(z)$ und $g(z)$ teilerfremd, so kann man jede Funktion $h(z)$ in $\Omega$ in der Gestalt (9) mit zwei Funktionen $\varphi(z)$ und $\psi(z)$ in $\Omega$ darstellen, und zwar kann man die Funktionen $\varphi(z)$ und $\psi(z)$ in einer und nur einer Art so wählen, daß der Grad von $\varphi(z)$ kleiner als der Grad $n$ von $f(z)$ ist.

Die Funktion $f(z)$ heißt , $i n \Re$ reduzibel", falls sie eine Funktion $\chi(z)$ in $\Omega$ von einem Grade, der $>0$ und $<n$. ist, zum Teiler hat; besitzt sie keinen solchen Teiler, so heißt sie „in $\Re$ irreduzibel". Der Zusatz ,in $\Re^{\prime 6}$ wird hierbei, wenn er sich von selbst versteht, gewöhnlich fortgelassen. Eine reduzibele Funktion $f(z)$ ist in das Produkt $f(z)=\chi(z) \cdot \chi_{1}(z)$ zweier Funktionen in $\Re$ spaltbar, deren Grade zwischen 0 und $n$ liegen. Sie hat nämlich einen Teiler $\chi(z)$, worauf wir vermittelst der Division von $f(z)$ durch $\chi(z)$ einen Quotienten $\chi_{1}(z)$ erhalten, der die Bedingungen des Satzes erfüllt:

Ist $\mathcal{\Omega}$ der Körper aller reellen Zahlen, so ist jede Funktion $f(z)$ eines Grades $n>2$ reduzibel; sie ist nämlich in $\Omega$ in Faktoren ersten oder zweiten Grades spaltbar. Ist $\mathcal{\Omega}$ der Körper aller Zahlen, so ist jede Funktion $f(z)$ eines Grades $n>1$ reduzibel, nämlich in Faktoren ersten Grades zerlegbar. Diese Angaben folgen aus dem Fundamentaltheorem der Algebra.

Es besteht der Satz: Ist von den beiden Funktionen $f(z)$ und $g(z)$ in $\Re$ die erste irreduzibel, so sind $f(z)$ und $g(z)$ entweder teilerfremd oder $g(z)$ hat den Teiler $f(z)$. Sind sie nämlich nicht teilerfremd, so haben sie einen 
gröBten gemeinsamen Teiler $\chi(z)$ eines Grades $>0$, der als Teiler der irreduzibelen Funktion $f(z)$, abgesehen von einem konstanten"Faktor, nur $f(z)$ selbst sein kinn. Einfache Folgerungen des letzten Satzes sind: Zuvei irreduzibele Funktionen $f(z)$ und $g(z)$ sind entweder teilerfremd oder bis auf cinen konstanten Faktor, der eine Zahl aus $\mathfrak{\Omega}$ ist, identisch. Sind sie nämlich nicht teilerfremd, so ist jede Funktion ein Teiler der anderen. Eine irreduzibele Funktion $f(z)$ ist stets teilerfremd zu iher Ableitung $f^{\prime}(z)$. Es kann nämlich $f^{\prime}(z)$ als Funktion $(n-1)^{\text {ten }}$ Grades in $\Omega$ nicht durch $f(z)$ teilbar sein.

Endlich besteht der Satz: Eine reduzibele Funlition $f(z)$ ist nur auf eine Art als Produkt irreduzibeler Funktionen darstellbar, abgesehen davon, daß jede irreduzibele Funktion noch um eine Zahl aus $\Omega$ als Falitor abgeändert werden mag. Haben wir nämlich für $f(z)$ die beiden Zerlegungen:

$$
f_{1}(z) \cdot f_{2}(z) \cdots f_{\mu}(z) \text { und } g_{1}(z) \cdot g_{2}(z) \cdots g_{v}(z)
$$

in irreduzibele Faktoren, so ist $g_{1}(z)$ Teiler des Produktes von $f_{1}(z)$ und $f_{2}(z) \cdot f_{3}(z) \cdots f_{\mu}(z)$. Da $f_{1}(z)$ und $g_{1}(z)$ irreduzibel sind, so sind diese Funktionen entweder (bis auf einen konstanten Faktor) identisch oder teilerfremd. Im letzteren Falle ist $g_{1}(z)$ Teiler von $f_{2}(z) \cdot f_{3}(z) \cdots f_{\mu}(z)$. Indem man alsdann dieselbe Schlußweise für $g_{1}(z)$ und das Produkt $f_{2}(z) \cdot\left(f_{3}(z) \cdots f_{\mu}(z)\right)$ wiederholt und in derselben Weise fortfährt, ergibt sich, daß $g_{1}(z)$ notwendig unter den Faktoren $f_{1}(z), f_{2}(z), \ldots, f_{\mu}(z)$ auftritt. Die Fortsetzung des Beweises ist einleuchtend.

\section{$\$ 5$. Algebraische Zahlen in bezug auf einen Körper $\mathfrak{A}$.}

Durch Nullsetzen einer Funktion $f(z)$ in $\Omega$ entsteht eine "Gleichung im Körper $\Omega^{*}$ oder kurz eine „Gleichung in $\Omega^{*} f(z)=0$, die , in $\Omega$ redu$z i b e l^{\prime}$ oder "irreduzibel" heißt, je nachdem die Funktion $f(z)$ reduzibel

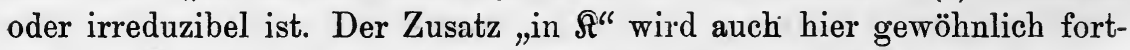
gelassen. Zwei Gleichungen $f(z)=0$ und $g(z)=0$ in $\Re$ haben keine gemeinsame Lösung, falls $f(z)$ und $g(z)$ teilerfremd sind. Haben diese Funktionen aber einen größten gemeinsamen Faktor $\chi(z)$ eines Grades $\boldsymbol{\nu}>0$, so ist jede gemeinsame Wurzel der Gleichungen $f(z)=0$ und $g(z)=0$ eine Wurzel der Gleichung $\chi(z)=0$ und umgekehrt. Diese Angaben folgen leicht aus den Formeln (5)ff. von $\S 4$.

Die Sätze aus dem letzten Teile des vorigen Paragraphen ergeben nun unmittelbar einige wichtige Folgerungen: Eine irreduzibele Gleichung $f(z)=0$ hat nie eine mehrfache Wurzel. Hätte nämlich $f(z)=0$ eine mehrfache Wurzel, so würde diese auch der Gleichung $f^{\prime}(z)=0$ genügen, während doch $f(z)$ and $f^{\prime}(z)$ teilerfremd sind. Ist von den beiden Gleichungen $f(z)=0$ und $g(z)=0$ die erste irreduzibel, so wird die zweite entweder durch keine oder durch alle Wurzeln der ersten befriedigt. Es 
sind nämlich $f(z)$ und $g(z)$ entweder teilerfremd oder $g(z)$ hat den Teiler $f(z)$. Als besonderer Fall ergibt sich hieraus: Zwei irreduzibele Gleichungen in $\Re$ haben entweder keine gemeinsame Wurzel, oder ihre linken Seiten sind, abgesehen von einem konstanten Faktor, identisch.

Wir stellen nun folgende Erklärung auf: Eine Zahl $\theta$ heiBt „alge-

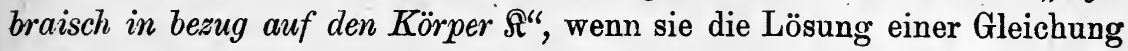
in $\Re$ ist. Ist diese Gleichung reduzibel, so hat ihre linke Seite mindestens einen irreduzibelen Faktor, der für $\theta$ verschwindet. Dieser Faktor sei vom $n^{\text {ten }}$ Grade und liefere für $\theta$ die irreduzibele Gleichung:

$$
f(z)=z^{n}+a_{1} z^{n-1}+a_{2} z^{n-2}+\cdots+a_{n}=0
$$

mit dem Koeffizienten 1 im höchsten Gliede. Mit Rücksicht auf den letzten Satz folgt: Jede in bezug auf $\Omega$ algebraische Zahl $\theta$ genügt einer eindeutig bestimmten irreduzibelen Gleichung (1) in $\Re$. Ist $n=1$, so ist $\theta$ in $\Omega$ enthalten. Ist $n>1$, so bezeichnen wir die $n$ Wurzeln von (1) durch $\theta, \theta^{\prime}, \theta^{\prime \prime}, \ldots, \theta^{(n-1)}$; sie liefern $n$ verschiedene in bezug auf $\Omega$ algebraische Zahlen, die einander ,tconjugiert" genannt werden, und von denen keine in $\Omega$ enthalten ist.

Unter der "Adjunktion" von $\theta$ zum Körper $\Re$ versteht man den $\mathrm{Zu}$ satz von $\theta$ sowie aller durch rationale Rechnungen aus $\theta$ und Zahlen von $\Re$ berechenbarer Zahlen zum Körper $\Re$; natürlich bleibt wieder die Division durch 0 ausgeschlossen. Die Adjunktion führt $\mathrm{zu}$ einem durch $(\mathfrak{\Omega}, \theta)$ zu bezeichnenden Zahlkörper, der $\Re$ in sich enthält. Ist $\theta$ in $\Re$ enthalten, d. h. ist $n=1$, so ist natürlich $\operatorname{der} \operatorname{Körper}(\Re, \theta)$ kein anderer als $\Re$ selbst. Ist indessen $n>1$, so tritt eine Erweiterung von $\Re$ ein; dieser Fall wird uns demnach vornehmlich interessieren.

Jede Zahl $\zeta$ des erweiterten Körpers $(\Omega, \theta)$ ist in der Gestalt:

$$
\xi=\frac{h(\theta)}{g(\theta)}
$$

darstellbar, wo $h(z)$ und $g(z)$ Funktionen in $\mathfrak{K}$ sind, von denen die letztere für $z=\theta$ nicht verschwindet. Da hiernach $f(z)$ und $g(z)$ teilerfremd sind, so ist $h(z)$ nach einem Satze von S. 31 in der Gestalt:

$$
h(z)=f(z) \psi(z)+g(z) \varphi(z)
$$

darstellbar. Dabei sind die Funktionen $\varphi(z)$ und $\psi(z)$ eindeutig bestimmt, wenn wir fordern, dab $\varphi(z)$ einen Grad $<n$ hat und damit die Gestalt besitzt:

$$
\varphi(z)=c_{0}+c_{1} z+c_{2} z^{2}+\cdots+c_{n-1} z^{n-1} .
$$

Tragen wir nun in (3) insbesondere $z=\theta$ ein, so wird $f(\theta)=0$, und wir finden:

$$
\zeta=\frac{h(\theta)}{g(\theta)}=\varphi(\theta) \text {. }
$$


Wir sind damit zu dem Satze gelangt: Jede Zahl $\xi$ des erweiterten Körpers $(\Re, \theta)$ ist auf eine und nur eine Art in der Gestalt:

$$
\zeta=c_{0}+c_{1} \theta+c_{2} \theta^{2}+\cdots+c_{n-1} \theta^{n-1}
$$

darstellbar (unter $c_{0}, c_{1}, \ldots, c_{n-1}$ Zahlen aus $\Omega$ verstanden), und umgekehrt ist jeder Ausdruck (5) eine Zahl von $(\Re, \theta)$. Es bleibt hierbei nur noch zu zeigen, daB die einzelne Zahl $\zeta$ nur auf eine Art in der Gestalt (5) darstellbar ist. Gäbe es nämlich noch eine zweite solche Darstellung $\xi=c_{0}^{\prime}+c_{1}^{\prime} \theta+\cdots$, so wäre:

$$
\left(c_{n-1}^{\prime}-c_{n-1}\right) \theta^{n-1}+\cdots+\left(c_{2}^{\prime}-c_{2}\right) \theta^{2}+\left(c_{1}^{\prime}-c_{1}\right) \theta+\left(c_{0}^{\prime}-c_{0}\right)=0 .
$$

Die Gleichung $\left(c_{n-1}^{\prime}-c_{n-1}\right) z^{n-1}+\cdots+\left(c_{1}^{\prime}-c_{1}\right) z+\left(c_{0}^{\prime}-c_{0}\right)=0$ hat demnach die Wurzel $\theta$, so daB die linksstehende Funktion den Teiler $n^{\text {ten }}$ Grades $f(z)$ hat und also notwendig die identisch verschwindende Funktion ist. Daraus folgt die Behauptung.

Es besteht folgender Satz: Ist $\theta$ algebraisch in bezug auf $\Re$ und $\theta_{1}$ algebraisch in bezug auf $(\Omega, \theta)$, so ist $\theta_{1}$ auch algebraisch in bezug auf $\AA$. Die Zahl $\theta_{1}$ genügt einer Gleichung:

$$
g(z, \theta)=z^{l}+d_{1}(\theta) z^{l-1}+d_{2}(\theta) z^{l-2}+\cdots+d_{l}(\theta)=0,
$$

deren Koeffizienten als Zahlen von $(\Omega, \theta)$ die Gestalt (5) haben. Man bilde das Produkt:

$$
h(z)=g(z, \theta) \cdot g\left(z, \theta^{\prime}\right) \cdot g\left(z, \theta^{\prime \prime}\right) \cdots g\left(z, \theta^{(n-1)}\right),
$$

dessen Faktoren man gewinnt, indem man in $g(z, \theta)$ für $\theta$ der Reihe nach die $n$ konjugierten Zahlen $\theta, \theta^{\prime}, \theta^{\prime \prime}, \ldots, \theta^{(n-1)}$, d. h. die $n$ Wurzeln der irreduzibelen Gleichung (1) einträgt. In Abhängigkeit von $\theta, \theta^{\prime}, \theta^{\prime \prime}, \ldots$, $\theta^{(n-1)}$ ist $h(z)$ eine symmetrische Funktion, deren "Koeffizienten" zufolge der Bauart der $d$ "Funktionen in $\Re^{*}$ sind. Nach dem Hauptsatze von S. 25 läbt sich diese symmetrische Funktion in eine rationale ganze Funktion der Koeffizienten $a_{1}, a_{2}, \ldots, a_{n}$ von (1) umrechnen, wobei die Koeffizienten dieser Funktion der $a$ ganzzahlige lineare homogene Verbindungen der eben genannten Funktionen in $\mathfrak{\Re}$ sind. Also ist $h(z)$ selbst eine Funktion in $\Re$ und $h(z)=0$ eine Gleichung in $\Re$, deren Lösung $\theta_{1}$ ist.

\section{\$ 6. Gleichzeitige Adjunktion mehrerer algebraischer Zahlen.}

Es seien $\theta_{1}$ und $\theta_{2}$ zwei verschiedene in bezug auf $\Omega$ algebraische Zahlen, die den beiden irreduzibelen Gleichungen $f_{1}(z)=0$ und $f_{2}(z)=0$ der Grade $n_{1}$ und $n_{2}$ mit den höchsten Koeffizienten 1 genügen. Da die Koeffizienten von $f_{2}(z)$ in $\Re$ und also in $\left(\Omega, \theta_{1}\right)$ enthalten sind, so ist $\theta_{2}$ auch in bezug auf $\left(\Omega, \theta_{1}\right)$ algebraisch. Ist $\theta_{2}$ in $\left(\Omega, \theta_{1}\right)$ enthalten, so ergibt die Adjunktion von $\theta_{2} \mathrm{zu}\left(\Omega, \theta_{1}\right)$ keine Erweiterung dieses Körpers. Ist aber $\theta_{2}$ nicht in $\left(\Omega, \theta_{1}\right)$ enthalten, so liefert die Adjunktion von $\theta_{2} \mathrm{zu}$ 
$\left(\Re, \theta_{1}\right)$ einen durch $\left(\Re, \theta_{1}, \theta_{\mathbf{2}}\right)$ zu bezeichnenden erweiterten Körper, dessen sämtliche Zahlen durch $\theta_{2}$ und Zahlen $C_{0}, C_{1}, C_{2}, \ldots$ aus $\left(\Re, \theta_{1}\right)$ in der Gestalt:

$$
C_{0}+C_{1} \theta_{2}+C_{2} \theta_{2}^{2}+\cdots+C_{n_{2}-1} \theta_{2}^{n_{2}-1}
$$

darstellbar sind. ${ }^{1}$ ) Trägt man für die $C$ ihre Darstellungen (5) S. 34 ein, so nehmen die Zahlen von $\left(\Omega, \theta_{1}, \theta_{2}\right)$ die Gestalt an:

$$
\sum_{\lambda, \mu} c_{\lambda \mu} \theta_{1}^{\lambda} \theta_{2}^{\mu}, \quad \lambda=0,1, \ldots, n_{1}-1, \quad \mu=0,1, \ldots, n_{2}-1,
$$

wo die $c_{\lambda \mu}$ Zahlen aus $\Re$ sind. Umgekehrt ist jeder Ausdruck (2) eine Zahl aus ( $\Omega ; \theta_{1}, \theta_{2}$ ). Offenbar gelangt man zu demselben Körper, wenn man zu $\Omega$ zunächst $\theta_{2}$ und dann $\theta_{1}$ adjungiert; man kann auch sagen, es entstehe $\left(\Re, \theta_{1}, \theta_{2}\right)$ durch gleichzeitige Adjunktion von $\theta_{1}$ und $\theta_{2}$.

Diese Betrachtung ist leicht zu verallgemeinern. Sind sogleich $\theta_{1}$, $\theta_{2}, \ldots, \theta_{m}$ irgend $m$ verschiedene in bezug auf $\Re$ algebraische Zahlen, die den irreduzibelen Gleichungen $f_{1}(z)=0, f_{2}(z)=0, \ldots, f_{m}(z)=0$ von den Graden $n_{1}, n_{2}, \ldots, n_{m}$ und mit den höchsten Koeffizienten 1 genügen, so entsteht durch gleichzeitige Adjunktion aller $m$ Zahlen ein durch $\left(\boldsymbol{\Omega}, \theta_{1}, \theta_{2}, \ldots, \theta_{m}\right)$ zu bezeichnender Körper, dessen Zahlen $\Theta$ in der Gestalt:

$$
\Theta=\sum_{\lambda, \mu, v, \ldots} c_{\lambda \mu \nu} \ldots \theta_{1}^{\lambda} \theta_{2}^{\mu} \theta_{3}^{\nu} \ldots, \quad \lambda=0,1, \ldots, n_{1}-1, \quad \mu=0,1, \ldots, n_{2}-1, \ldots
$$

mit Koeffizienten $c$ aus $\mathfrak{K}$ darstellbar sind. Zugleich liefert jeder Ausdruck (3) eine Zahl aus $\left(\Omega, \theta_{1}, \theta_{2}, \ldots, \theta_{m}\right)$. Die angenommene Verschiedenheit der $\theta_{1}, \theta_{2}, \ldots, \theta_{m}$ schließt nicht aus, da $\beta$ zwei oder mehrere dieser Größen oder vielleicht sogar alle der gleichen irreduzibelen Gleichung genügen und also konjugiert sind. Es ist ja nur die Verschiedenheit $\operatorname{der} \theta$, aber nicht diejenige der irreduzibelen Gleichungen $f_{1}(z)=0, f_{2}(z)=0$, $\ldots, f_{m}(z)=0$ gefordert.

Es besteht nun der grundlegende Satz: Der durch Adjunktion einer beliebigen Anzahl in bezug auf $\Re$ algebraischer Zahlen $\theta_{1}, \theta_{2}, \ldots, \theta_{m}$ entstehende Körper $\left(\Re, \theta_{1}, \theta_{2}, \ldots, \theta_{m}\right)$ ist auch herstellbar durch Adjunktion einer einzigen in bezug auf $\Omega$ algebraischen Zahl $\eta$, die man in der Gestalt:

$$
\eta=\gamma_{1} \theta_{1}+\gamma_{2} \theta_{2}+\cdots+\gamma_{m} \theta_{m}
$$

mit rationalen ganzen Zahlen $\gamma$ wählen kann.

1) Diese Darstellung ist übrigens nur dann eindeutig für die einzelne Zahl von $\left(\Re, \theta_{1}, \theta_{2}\right)$ bestimmt, wenn $f_{2}(z)$ auch im Körper $\left(\AA, \theta_{1}\right)$ irreduzibel ist. Trifft dies nicht zu, so genügt $\theta_{2}$ einer in $\left(\Re, \theta_{1}\right)$ irreduzibelen Gleichung eines Grades $\dot{n}_{2}^{\prime}<n_{2}$, und wir haben dann für die einzelne Zahl von $\left(\Omega, \theta_{1}, \theta_{2}\right)$ eine Darstellung (1) jedoch nur vom Grade $\left(n_{2}^{\prime}-1\right)$ in $\theta_{2}$, die dann eindentig ist. Durch diesen Umstand wird die folgende SchluBreihe nicht berührt. 
Zum Beweise erklären wir $\eta$ zunächst als lineare homogene Funktion von $m$ unabhängigen Variablen $z_{1}, z_{2}, \ldots, z_{m}$ :

$$
\eta=z_{1} \theta_{1}+z_{2} \theta_{2}+z_{3} \theta_{3}+\cdots+z_{m} \theta_{m} .
$$

Die Zahl $\theta_{1}$ ist eine unter $n_{1}$ voneinander verschiedenen konjugierten Zahlen $\theta_{1}, \theta_{1}^{\prime}, \ldots, \theta_{1}^{\left(n_{1}-1\right)}$. Ebenso haben wir $n_{2}$ roneinander verschiedene konjugierte Zahlen $\theta_{2}, \theta_{2}^{\prime}, \ldots, \theta_{2}^{\left(n_{2}-1\right)}$ usw. Greift man je eine Zahl aus dem einzelnen der $m$ Systeme konjugierter Zahlen heraus, so lassen sich auf diese Weise $N=n_{1} \cdot n_{2} \cdot n_{3} \cdots n_{m}$ Kombinationen bilden, von denen keine zwei in der vorliegenden Anordnung dasselbe Zahlensystem darstellen. Man ersetze nun in (5) die $\theta_{1}, \theta_{2}, \ldots, \theta_{m}$ nach und nach durch alle $N$ Kombinationen und gewinnt auf diese Weise $N$ lineare Funktionen $\eta, \eta^{\prime}, \eta^{\prime \prime}, \ldots, \eta^{(N-1)}$, von denen keine zwei identisch sind. Es stellen demnach die $\frac{1}{2} N(N-1)$ Differenzen:

$$
\eta-\eta^{\prime}, \eta-\eta^{\prime \prime}, \ldots, \eta-\eta^{(N-1)}, \eta^{\prime}-\eta^{\prime \prime}, \ldots, \eta^{(N-2)}-\eta^{(N-1)}
$$

ebenso viele lineare Funktionen der $z_{1}, z_{2}, \ldots, z_{m}$ dar, von denen keine einzige identisch verschwindet.

Diese Funktionen genügen den Voraussetzungen des Satzes von S.27. Nach jenem Satze können wir jetzt den Argumenten $z_{1}, z_{2}, \ldots, z_{m}$ der $\frac{1}{2} N(N-1)$ Funktionen solche ganzzahlige Werte $\gamma_{1}, \gamma_{2}, \ldots, \gamma_{m}$ erteilen, für welche keine der Funktionen (6) verschwindet. Für diese ganzzahligen Argumente $\gamma$ nehmen also die $n$ linearen Funktionen $\eta, \eta^{\prime}, \ldots, \eta^{(N-1)}$ durchgängig voneinander verschiedene Werte an. Wir nennen diese Werte gleich selbst wieder $\eta, \eta^{\prime}, \ldots, \eta^{(N-1)}$; der erste der Werte soll die in (4) in Ansatz gebrachte Zahl sein.

Wir bilden nun mit den $N$ Zahlen $\eta, \eta^{\prime}, \ldots, \eta^{(N-1)}$ und einer Variablen $Z$ die Funktion $N^{\text {ten }}$ Grades:

$$
F(Z)=(Z-\eta)\left(Z-\eta^{\prime}\right)\left(Z-\eta^{\prime \prime}\right) \cdots\left(Z-\eta^{(N-1)}\right),
$$

die entwickelt die Gestalt habe:

$$
F(Z)=Z^{N}+A_{1} Z^{N-1}+A_{2} Z^{N-2}+\cdots+A_{N} .
$$

Die Koeffizienten $A_{1}, A_{2}, \ldots, A_{N}$ sind (abgesehen vom Vorzeichen) die symmetrischen Grundfunktionen der $\eta, \eta^{\prime}, \ldots, \eta^{(N-1)}$. Stellen wir die $\eta, \eta^{\prime}, \ldots, \eta^{(N-1)}$ durch die $\theta$ dar, so werden die $A_{1}, A_{2}, \ldots, A_{N}$ zu ganzen ganzzahligen Funktionen der Lösungen der $m$ Gleichungen $f_{1}(z)=0$, $f_{2}(z)=0, \ldots, f_{m}(z)=0$, und zwar sind sie symmetrisch in den Wurzeln jeder einzelnen dieser Gleichungen. Wir rechnen nun die $A$ zunächst als symmetrische Funktionen der $n_{1}$ Wurzeln von $f_{1}(z)=0$ in rationale ganze Funktionen der Koeffizienten dieser Gleichung um, wobei nach dem Hauptsatze von S. 25 als „Koeffizienten“ ganze ganzzahlige Funktionen der Wurzeln von $f_{2}(z)=0, \ldots, f_{m}(z)=0$ auftreten, die wieder in den Wurzeln jeder dieser Gleichungen symmetrisch sind. Durch Wiederho- 
lung dieses Verfahrens finden wir für die $A$ schlieblich ganze ganzzahlige Ausdrücke in den Koeffizienten aller Gleichungen $f_{1}(z)=0, f_{2}(z)=0$, $\ldots, f_{m}(z)=0$. Es ist also $F(Z)=0$ eine Gleichung in $\Omega$, so da $\beta$ als Lösung dieser Gleichung eine in bezug auf $\AA$ algebraische Zahl ist; zugleich gilt für die Ableitung $F^{\prime}(Z)$ die Ungleichung $F^{\prime}(\eta) \neq 0$, da die Gleichung $F(Z)=0$ nur einfache Wurzeln hat.

Es sei nun irgendeine Zahl $\Theta$ des Körpers $\left(\Re, \theta_{1}, \theta_{2}, \ldots, \theta_{m}\right)$ gewählt. Indem wir in der Darstellung (3) dieser Zahl die $\theta_{1}, \theta_{2}, \ldots, \theta_{m}$ durch alle $N$ Kombinationen der Wurzeln von $f_{1}(z)=0, f_{2}(z)=0, \ldots$, $f_{m}(z)=0$ ersetzen, mögen wir die den $\eta, \eta^{\prime}, \ldots, \eta^{(N-1)}$ zugeordneten Zahlen $\Theta, \Theta^{\prime}, \ldots, \Theta^{(N-1)}$ erbalten. Dann haben wir in:

$$
\frac{F(Z)}{Z-\eta} \cdot \Theta+\frac{F(Z)}{Z-\eta^{\prime}} \cdot \Theta^{\prime}+\cdots+\frac{F(Z)}{Z-\eta^{(N-1)}} \cdot \Theta^{(N-1)}=H(Z)
$$

eine ganze Funktion $(N-1)^{\text {ten }}$ Grades von $Z$ :

$$
H(Z)=B_{1} Z^{N-1}+B_{2} Z^{N-2}+\cdots+B_{N},
$$

und zwar sind die $B_{1}, B_{2}, \ldots, B_{N}$ ganze symmetrische Funktionen der Wurzeln jeder der $m$ Gleichungen $f_{1}(z)=0, \ldots, f_{m}(z)=0$ mit Koeffizienten, die dem Körper $\mathcal{\Omega}$ angehören. Auf Grund des Hauptsatzes von S. 25 folgern wir hieraus wie oben, daß $H(Z)$ eine „Funktion in $\Re^{\text {“ ist. }}$

Für $Z=\eta$ ergibt sich aus der Gleichung (9):

$$
\Theta=\frac{H(\eta)}{F^{\prime}(\eta)},
$$

und da $F^{\prime}(\eta) \neq 0$ ist, so gehört $\Theta$ zufolge $(10)$ dem $\operatorname{Körper~}(\Re, \eta)$ an. $\mathrm{Da}$ andrerseits wegen (4) die Zahl $\eta$ in $\left(\Omega, \theta_{1}, \theta_{2}, \ldots, \theta_{m}\right)$ enthalten ist, so findet sich in diesem Körper überhaupt jede Zahl aus $(\Omega, \eta)$. Jede Zahl des einen der beiden Körper $(\Re, \eta)$ und $\left(\Re, \theta_{1}, \theta_{2}, \ldots, \theta_{m}\right)$ ist demnach im anderen enthalten, so daß beide Körper, insofern sie das gleiche Zahlensystem darstellen, einander gleich zu nennen sind. Der aufgestellte Satz ist damit bewiesen.

Aus dem letzten Satze von $\S 5$ (S. 34) ergibt sich noch eine Erweiterung des eben bewiesenen Satzes. Wir nehmen jetzt die Adjunktionen in der Art hintereinander vor, daB $\theta_{2}$ algebraisch in bezug auf $\left(\Omega, \theta_{1}\right)$ ist, ebenso $\theta_{3}$ in bezug auf $\left(\Omega, \theta_{1}, \theta_{2}\right)$ usw. Dann ist nach dem Schlußsatze von $\S 5$ zunächst $\theta_{2}$ auch in bezug auf $\Omega$ algebraisch; ebenso ist, da wir $\left(\Omega, \theta_{1}, \theta_{2}\right)$ als einen Körper $\left(\Omega, \eta_{1}\right)$ darstellen können, nach jenem Satze auch $\theta_{3}$ algebraisch in bezug auf $\Re$, und man folgert in derselben Weise weiter, daß überhaupt alle $\theta_{1}, \theta_{2}, \ldots, \theta_{m}$ in bezug auf $\Re$ algebraisch sind. Auch der durch diese Art der Adjunktion entstehende Körper $\left(\Omega, \theta_{1}, \theta_{2}, \ldots, \theta_{m}\right)$ ist als ein Körper $(\Re, \eta)$ mittelst einer einzigen Adjunktion herstellbar. 


\section{§ 7. Konjugierte Körper. Primitive und imprimitive Zahlen.}

Die in bezug auf $\Omega$ algebraische Zahl $\theta$ genüge der irreduzibelen Gleichung $n^{\text {ten }}$ Grades (1) S. 33. Dem Grade $n$ dieser Gleichung entsprechend heiBt der durch Adjunktion von $\theta$ zu $\Omega$ entstehende Körper $(\Re, \theta)$ ein algebraischer Körper $n^{\text {ten }}$ Grades in bezug auf $\Re$. Ist $n=1$, so ist $\theta$ in $\Omega$ enthalten, und die beiden $\operatorname{Körper}(\Omega, \theta)$ und $\Omega$ sind gleich, d. h. sie stellen das gleiche Zahlensystem dar, was wir durch $(\Omega, \theta)=\Omega$ zum Ausdruck bringen. Ist $n>1$, so ist $\theta$ nicht in $\Omega$ enthalten, und also enthält $(\Re, \theta)$ den Körper $\Re$, ohne durch ihn erschöpft zu werden. Enthält allgemein ein Körper $\Omega^{\prime}$ einen Körper $\Omega$ in sich, ohne durch ihn erschöpft zu werden, so bringen wir dies Sachverhältnis durch $\mathfrak{\Omega}^{\prime}>\Omega$ oder $\AA<\Omega^{\prime}$ zum Ausdruck. In unserem Falle $n>1$ gilt also $(\Omega, \theta)>\Omega$.

Die $n$ durchweg verschiedenen Lösungen der Gleichung $f(z)=0$ wurden als $n$,konjugierte" in bezug auf $\Re$ algebraische Zahlen bezeichnet (S. 33). Statt der bisherigen Bezeichnung ist es fortan zweckmäBiger, diese $n$ konjugierten Zahlen durch untere Indizes zu unterscheiden und sie durch $\theta_{1}=\theta, \theta_{2}, \theta_{3}, \ldots, \theta_{n}$ zu bezeichnen. Ihnen entsprechen $n$ in bezug auf $\Re$ algebraische Körper $n^{\text {ten }} \operatorname{Grades}\left(\Re, \theta_{1}\right)=(\Re, \theta),\left(\Re, \theta_{2}\right), \ldots$ $\left(\Omega, \theta_{n}\right)$, die $n$,konjugierte" Körper heißen. Die Zahlen dieser $n$ Körper entsprechen einander umkehrbar eindeutig, indem wir einer Zahl:

$$
\zeta=c_{0}+c_{1} \theta+c_{2} \theta^{2}+\cdots+c_{n-1} \theta^{n-1}
$$

von $(\Omega, \theta)$ im Körper $\left(\Re, \theta_{i}\right)$ die Zahl:

$$
\xi_{i}=c_{0}+c_{1} \theta_{i}+c_{2} \theta_{i}^{2}+\cdots+c_{n-1} \theta_{i}^{n-1}
$$

mit den gleichen Koeffizienten $c$ zuordnen. Je $n$ solche Zahlen $\xi_{1}=\xi$, $\xi_{2}, \xi_{3}, \ldots, \xi_{n}$ sollen wieder ,konjugiert" heißen.

Wir setzen nun die Gleichung $n^{\text {ten }}$ Grades an, die ein solches System. von $n$ konjugierten Zahlen zu Wurzeln hat; sie lautet:

$$
g(w)=\left(w-\xi_{1}\right)\left(w-\xi_{2}\right) \cdots\left(w-\xi_{n}\right)=0
$$

oder in ausgerechneter Gestalt:

$$
g(w)=w^{n}+b_{1} w^{n-1}+b_{2} w^{n-2}+\cdots+b_{n}=0 .
$$

Nach dem Hauptsatze von S. 25 sind die $b_{1}, b_{2}, \ldots, b_{n}$ Zahlen in $\Re$, also $g(w)=0$ eine "Gleichung in $\Omega^{*}$. Mit Benutzung der Entwicklungen in $\S 2$, S. 26, finden wir als Ergebnis: Je $n$ konjugierte Zahlen $\xi_{1}=\xi, \xi_{2}, \ldots$, $\xi_{n}$ unserer $n$ Körper sind die Wurzeln einer Gleichung $n^{\text {ten }}$ Grades (4) in $\Re$, die eine Tschirnhausenresolvente der Gleichung $f(z)=0$ ist; umgekehrt liefert jede Tschirnhausentransformation (2), S. 26, mit Koeffizienten c aus $\Omega$ eine Resolvente $g(w)=0$ von $f(z)=0$, deren Wurzeln $n$ konjugierte Zahlen unserer Körper sind. 
Die Zerlegung der Funktion $g(w)$ in ihre in $\Re$ irreduzibelen Faktoren sei:

$$
g(w)=h_{1}(w) \cdot h_{2}(w) \cdots h_{\mu}(w)
$$

der Bestimmtheit halber seien die höchsten Koeffizienten der Faktoren alle gleich 1 genommen. Ein beliebiger dieser Faktoren liefert eine Gleichung $h(w)=0$ in $\Re$, die durch mindestens ein $\xi_{i}$ befriedigt wird. Dann wird das zugehörige $\theta_{i}$ der Gleichung in $\Re$ :

$$
h\left(c_{0}+c_{1} z+c_{2} z^{2}+\cdots+c_{n-1} z^{n-1}\right)=0
$$

genügen. Da $f(z)=0$ irreduzibel ist, so wird nach S. 32 die Gleichung (6) mit $\theta_{i}$ sogleich alle $n$ konjugierten Zahlen $\theta_{1}, \theta_{2}, \ldots, \theta_{n}$ zu Wurzeln haben. Daraus folgt umgekehrt, daß $h(w)=0$ durch alle Zahlen $\xi_{1}, \xi_{2}$, ..., $\xi_{n}$ befriedigt wird. Andere Wurzeln kann die Gleichung $h(w)=0$ nicht besitzen, da sie ein Bestandteil der Gleichung $g(w)=0$ ist. Da $h(w)$ irreduzibel ist, so hat die Gleichung $h(w)=0$ nur einfache Wurzeln. Da diese Überlegung für jeden Faktor $h(w)$ gilt, so ergibt sich: Die linke Seite $g(w)$ der Tschirnhausenresolvente (4) ist die $\mu^{\text {te }}$ Potenz einer irreduzibelen Funktion $h(w)$ vom Grade $\nu$, wo $\mu \cdot \nu=n$ gilt; die $n$ konjugierten Zahlen $\xi_{1}, \xi_{2}, \ldots, \xi_{n}$ sind zu je $\mu$ einander gleich und liefern $\boldsymbol{v}$ verschiedene Zahlen, die die Wurzeln der irreduzibelen Gleichung $h(w)=0$ sind.

Wir wollen nun die beiden Fälle, daß $\mu=1$ oder $\mu>1$ ist, unterscheiden und stellen folgende Erklärung auf: Eine Zahl $\xi$ des Körpers $(\Omega, \theta)$ heißt „primitiv“ oder „imprimitiv“, je nachdem die $n$ konjugierten Zahlen $\xi_{1}=\xi, \xi_{2}, \ldots, \xi_{n}$ alle verschieden sind oder nicht. Dann besteht der Satz: In einem Körper $(\Omega, \theta)$ eines Grades $n>1$ gibt es sicher unendlich viele primitive Zahlen. ${ }^{1}$ ) Verstehen wir nämlich für den Augenblick unter $\xi$ die Funktion:

$$
\zeta=z_{0}+z_{1} \theta+z_{2} \theta^{2}+\cdots+z_{n-1} \theta^{n-1}
$$

der $n$ unabhängigen Variablen $z_{0}, z_{1}, \ldots, z_{n-1}$ und entsprechend unter $\xi_{i}$ die Funktion:

$$
\xi_{i}=z_{0}+z_{1} \theta_{i}+z_{2} \theta_{i}^{2}+\cdots+z_{n-1} \theta_{i}^{n-1},
$$

so sind keine zwei dieser Funktionen $\xi_{1}=\xi, \xi_{2}, \ldots, \xi_{n}$ identisch, da z. B. ihre Koeffizienten von $z_{1}$ durchweg verschieden sind. Von den $\frac{1}{z} n(n-1)$ Funktionen $\left(\xi_{1}-\xi_{2}\right),\left(\xi_{1}-\xi_{3}\right), \ldots,\left(\xi_{1}-\xi_{n}\right),\left(\xi_{2}-\xi_{3}\right), \ldots,\left(\xi_{n-1}-\xi_{n}\right)$ verschwindet also keine identisch. Durch Wiederholung der Überlegung von S. 27 findet man demnach, daB man die $z_{0}, z_{1}, \ldots, z_{n-1}$ auf unendlich viele Arten als rationale ganze Zahlen so wählen kann, daß $n$ konjugierte, durchweg verschiedene Zahlen $\xi_{1}, \xi_{2}, \ldots, \xi_{n}$ unserer $n$ Zahlkörper gewonnen werden.

1) Für $n=1$ würde die Unterscheidung primitiver und imprimitiver Zahlen ibre Bedeutung verlieren. 
Es sei jetzt $\zeta=\xi_{1}$ primitiv und also $g^{\prime}(\xi) \neq 0$. Ferner sei $\eta=\eta_{1}$ irgendeine Zahl aus $(\Re, \theta)$ und $\eta_{1}, \eta_{2}, \ldots, \eta_{n}$ die zugehörigen $n$ konjugierten Zahlen. Wir bilden den Ansatz:

$$
\frac{g(w)}{w-\xi_{1}} \eta_{1}+\frac{g(w)}{w-\xi_{2}} \eta_{2}+\cdots+\frac{g(w)}{w-\xi_{n}} \eta_{n}=H(w)
$$

und erkennen genau wie S. 37 auf Grund des Hauptsatzes von S. 25 in $H(w)$ eine Funktion $(n-1)^{\text {ten }}$ Grades in $\Omega$. Setzen wir $w=\xi_{1}=\xi$ in (7) ein, so folgt:

$$
\eta=\frac{H(\zeta)}{g^{\prime}(\zeta)}
$$

so daß jede Zahl $\eta$ aus $(\Re, \theta)$ im Körper $(\Re, \xi)$ enthalten ist. Da umgekehrt $\xi$ in $(\Omega, \theta)$ enthalten ist, so gilt $(\Omega, \xi)=(\Omega, \theta)$. Durch Adjunktion irgendeiner primitiven Zahl $\xi$ von $(\Omega, \theta)$ zum Körper $\Omega$ ergibt sich stets der gesamte Körper $(\Re, \theta)$ wieder. Ist hingegen $\xi$ imprimitiv, so ist $(\Re, \xi)$ ein Körper eines Grades $v<n$, dessen sämtliche Zahlen irreduzibelen Gleichungen mit Graden $\leqq \nu$ genügen. In diesem Körper kann also keine primitive Zahl von $(\Re, \theta)$ enthalten sein: Für eine imprimitive Zahl $\xi$ von $(\Re, \theta)$ gilt $(\Re, \xi)<(\Re, \theta), d . h .(\Re, \xi)$ ist in $(\Re, \theta)$ enthalten, erschöpft aber den Körper $(\Re, \theta)$ noch nicht.

Der Körper $(\Re, \theta)$ möge selbst „primitiv“ oder „imprimitiv“ heißen, je nachdem seine sämtlichen Zahlen (außer denen, die den Körper $\mathfrak{\Omega}$ bilden) primitiv sind oder neben primitiven auch imprimitive vorkommen. Ist $\zeta$ eine noch nicht in $\Omega$ enthaltene imprimitive Zahl von $(\Re, \theta)$, so genügt der Körper $\Omega^{\prime}=(\Re, \xi)$ der Bedingung $\Omega<\Omega^{\prime}<(\Omega, \theta)$. Dieser Satz ist umkehrbar: Jeder die Bedingung $\Omega<\Omega^{\prime}<(\Omega, \theta)$ erfüllende Körper $\AA^{\prime}$, der also $\Re$ enthält und in $(\Omega, \theta)$ enthalten ist, ohne mit einem dieser Körper gleich zu sein, ist ein Körper $(\Omega, \xi)$, der aus $\AA$ durch $A d$ junktion einer imprimitiven Zahl $\xi$ von $(\Omega, \theta)$ gewinnbar ist. Die Existenz eines solchen Körpers $\Omega^{\prime}$ ist also charakteristisch für einen imprimitiven Körper $(\Omega, \theta)$.

Zum Beweise verstehen wir unter $\xi_{1}$ eine nicht in $\Re$ enthaltene Zahl von $\Re^{\prime}$. Dann gilt $\left(\Re, \xi_{1}\right) \leqq \Omega^{\prime}$, und $\xi_{1}$ ist eine imprimitive Zahl von $(\Re, \theta)$, so daß der Grad $\nu_{1}$ von $\left(\Omega, \xi_{1}\right)$ die Ungleichung $\nu_{1}<n$ befriedigt. Ist $\left(\Re, \xi_{1}\right)=\Re^{\prime}$, so ist der Satz bewiesen. Ist $\left(\Omega, \xi_{1}\right)<\Re^{\prime}$, so sei $\xi_{2}$ eine nicht in $\left(\Re, \xi_{1}\right)$ enthaltene Zahl von $\Omega^{\prime}$. Nach S. 35ff. können wir dann eine gleichfalls in $\Omega^{\prime}$ enthaltene Zahl $\xi_{2}$ so wählen, daß $\left(\Omega, \xi_{1}, \xi_{2}^{\prime}\right)=\left(\Omega, \xi_{2}\right)$ wird. Der Körper $\left(\Omega, \xi_{2}\right)$ befriedigt die Bedingung $\left(\Omega, \xi_{2}\right) \leqq \Omega^{\prime}$, und sein Grad $\nu_{2}$ liegt im Intervall $\nu_{1}<\nu_{2}<n$. Ist auch jetzt noch nicht $\left(\Re, \xi_{2}\right)$ $=\Re^{\prime}$, so können wir in derselben Art einen $\operatorname{Körper}\left(\Re, \xi_{3}\right)$ bilden, für den $\left(\Re, \xi_{3}\right) \leqq \Re^{\prime}$ gilt, und dessen Grad $\nu_{3}$ im Intervalle $\nu_{2}<\nu_{3}<n$ liegt. Dieser Prozeß führt nach endlich vielen Schritten zum Ziele, d. h. zu einem Körper $\left(\Omega, \xi_{i}\right)$, der gleich $\Omega^{\prime}$ ist; denn die Grade $\nu$ sind ganze po- 
sitive Zahlen, von denen jede größer als die vorhergehende ist, und die alle $<n$ sind.

Einige bemerkenswerte Folgerungen sind noch: Ein Körper $(\Re, \theta)$ vom Primzahlgrade $n$ ist stets primitiv. Der Grad $\nu$ der irreduzibelen Gleichung für eine imprimitive Zahl aus $(\Re, \theta)$ ist nämlich ein von $n$ selbst verschiedener Teiler von $n$; im Falle einer Primzahl $n$ haben wir also nur $v=1$. Ein die Bedingung:

$$
\Re<\Omega^{\prime} \leqq(\Omega, \theta)
$$

erfüllender Körper $\mathfrak{\Re}^{\prime}$ ist, falls er vom $n^{\text {ten }}$ Grade ist, notwendig gleich $(\Re, \theta)$. Wie soeben stellen wir nämlich $\Re^{\prime}$ als einen Körper $(\Omega, \xi)$ dar und haben in $\zeta$ wegen des Grades $n$ von $\Omega^{\prime}=(\Re, \xi)$ eine primitive Zahl von $(\Re, \theta)$.

\section{§ 8. Galoissche Körper und Galoissche Resolventen. $\left.{ }^{1}\right)$}

Wie bisher seien $(\Re, \theta)=\left(\Re, \theta_{1}\right),\left(\Omega, \theta_{2}\right), \ldots,\left(\Re, \theta_{n}\right)$ konjugierte in bezug auf $\Re$ algebraische Körper $n^{\text {ten }}$ Grades. Soll $\theta_{i}$ in $(\Re, \theta)$ enthalten sein, so ist hierfür hinreichend und notwendig das Bestehen einer Gleichung:

$$
\theta_{i}=c_{i 0}+c_{i 1} \theta+c_{i 2} \theta^{2}+\cdots+c_{i, n-1} \theta^{n-1},
$$

wo die $c$ hier und weiterhin stets Zahlen aus $\Omega$ sind. Es ist dann $\left(\Re, \theta_{i}\right) \leqq(\Re, \theta)$ und also nach dem Schlußsatze von $\S 7$ genauer $\left(\Re, \theta_{i}\right)$ $=(\Omega, \theta)$, so daß aus (1) umgekehrt auch die Gültigkeit einer entsprechenden Darstellung von $\theta$ in $\theta_{i}$ entspringt.

Hieran schließt sich folgende Erklärung: Der Körper $n^{\text {ten }}$ Grades $(\Re, \theta)$ heißt ein "Galoisscher Körper" oder „Normalkörper", falls er mit seinen sämtlichen konjugierten Körpern gleich ist. Ein notwendiges und hinreichendes Kennzeichen für einen Normalkörper ist also, daB sich alle mit $\theta=\theta_{1}$ konjugierten Zahlen $\theta_{i}$ in der Gestalt (1) durch $\theta$ darstellen lassen. Es wird sich dann in entsprechender Gestalt überhaupt jedes $\theta_{i}$ in jedem $\theta_{k}$ darstellen lassen. Einleuchtend ist der Satz: Ein Galoisscher Körper enthält mit irgendeiner Zahl $\xi$ stets alle mit $\xi$ konjugierten Zahlen in sich.

Ein den Körper $(\Omega, \theta)$ umfassender Galoisscher Körper wird neben $\theta=\theta_{1}$ auch die konjugierten Zahlen $\theta_{2}, \theta_{3}, \ldots, \theta_{n}$ und damit den Körper $\left(\Re, \theta_{1}, \theta_{2}, \ldots, \theta_{n}\right)$ enthalten. Es gilt aber der Satz: Der Körper $\left(\Re, \theta_{1}, \theta_{2}, \ldots, \theta_{n}\right)$ ist selbst ein Galoisscher Körper und ist demnach als

1) Die folgenden Entwicklungen geben die Grundzüge der Galoisschen Gleichungstheorie in der neueren auf den Körperbegriff aufgebauten Gestalt. Galois' Werke sind im Zusammenhang von Liouville im Bd. 11 des Journ. de math. (1846) veröffentlicht, eine deutsche Ausgabe ist von Maser veranstaltet (Berlin, 1889). 
der „kleinste" den gegebenen Körper $(\Omega, \theta)$ enthaltende Galoissche Körper $z u$ bezeichnen. Nach S. 35 können wir nämlich den $\operatorname{Körper}\left(\Re, \theta_{1}, \theta_{2}, \ldots, \theta_{n}\right)$ auch als einen Körper $(\Omega, \eta)$ durch Adjunktion einer einzigen Zahl:

$$
\eta=\gamma_{1} \theta_{1}+\gamma_{2} \theta_{2}+\cdots+\gamma_{n} \theta_{n}
$$

mit rationalen ganzen Koeffizienten $\gamma$ herstellen. Die Zahl $\eta$ genügt einer Gleichung in $\Omega$ vom Grade $N=n^{n}$, deren sämtliche Wurzeln in der Gestalt:

$$
\gamma_{1} \theta_{i}+\gamma_{2} \theta_{k}+\cdots+\gamma_{n} \theta_{l}
$$

enthalten sind. ${ }^{1}$ ) Wir nennen jetzt den irreduzibelen Bestandteil dieser Gleichung, welcher $\eta$ als Wurzel hat, $F(Z)=0$ und bezeichnen den Grad dieser in $\Re$ irreduzibelen Gleichung durch $m$. Da alle Wurzeln dieser Gleichung die Gestalt (3) haben, so sind sie in $\left(\Omega, \theta_{1}, \theta_{2}, \ldots, \theta_{n}\right)$ enthalten, d.h. alle mit $\eta$ konjugierten Zahlen gehören wieder dem Kör$\operatorname{per}(\Omega, \eta)$ an, so daß $(\Re, \eta)=\left(\Re, \theta_{1}, \theta_{2}, \ldots, \theta_{n}\right)$ tatsächlich ein Normalkörper ist.

Es mag noch bemerkt werden, daß sich die eben gewonnenen Ergebnisse von der Voraussetzung der Irreduzibilität der Gleichung $f(z)=0$ frei machen lassen. Den Vorbedingungen der Entwicklung von S. $35 \mathrm{ff}$. entsprechend baben wir nur zu fordern, da $\beta$ die $n$ Wurzeln $\theta_{1}, \theta_{2}, \ldots, \theta_{n}$ der Gleichung $f(z)=0$ durchweg verschieden sind. Wir gewinnen zufolge jener Entwicklungen auch dann in $\left(\Omega, \theta_{1}, \theta_{2}, \ldots, \theta_{n}\right)=(\Omega, \eta)$ einen Galoisschen Körper, nur ist er nicht mehr notwendig der „kleinste“, den Körper $(\Omega, \theta)$ umfassende Galoissche Körper. Doch werden wir weiterhin immer nur beiläufig auf den Fall einer reduzibelen Gleichung $f(z)=0$ eingehen.

Bezeichnen wir jetzt allgemein die Zahlen des Galoisschen Körpers $\left(\Omega, \theta_{1}, \theta_{2}, \ldots, \theta_{n}\right)$ durch $\eta$, so ist jede von ihnen in der Gestalt:

$$
\eta=R\left(\theta_{1}, \theta_{2}, \ldots, \theta_{n}\right)
$$

als rationale Funktion $\operatorname{der} \theta_{1}, \theta_{2}, \ldots, \theta_{n}$ mit Koeffizienten aus $\Re$ darstellbar. Jede primitive Zahl (4) des Galoisschen Körpers genügt einer irreduzibelen Gleichung in $\Omega$ vom Grade $m$ :

$$
F(Z)=0 \text {, }
$$

die man als eine "Galoissche Resolvente" der Gleichung $f(z)=0$ bezeichnet, mag die letztere Gleichung irreduzibel oder reduzibel sein. Betrachtet man den Körper " $(\Re, \eta)$ ohne Beziehung auf $(\Omega, \theta)$ und die Gleichung $f(z)=0$ als einen durch Adjunktion von $\eta$ zu $\Omega$ entstehenden „Normalkörper“, so erscheint es zweckmäBiger, die Gleichung (5) als eine „Nor-

1) Zur Vorbereitung der hier vorliegenden Utberlegung wurde bereits S. 35 darauf aufmerksam gemacht, da $B$ nur die damals $\theta_{1}, \theta_{2}, \ldots, \theta_{m}$ genannten Zahlen verschieden sein sollten, daB dagegen die Gleichungen $f_{1}(z)=0, f_{2}(z)=0$, ..., $f_{m}(z)=0$ (wie es hier zutrifft) alle einander gleich sein dürfen. 
malgleichung" zu bezeichnen. Aus den Entwicklungen am Anfang des Paragraphen ergibt sich der Satz: Eine irreduzibele Gleichung $m^{\text {ten }}$ Grades in $\Omega$ ist dadurch als eine Normalgleichung charakterisiert, daß jede ihrer Lösungen $\eta_{i}$ in einer beliebigen unter ihnen $\eta_{k}$ in der Gestalt darstellbar ist:

$$
\eta_{i}=c_{i 0}^{(k)}+c_{i 1}^{(k)} \eta_{k}+c_{i 2}^{(k)} \eta_{k}^{2}+\cdots+c_{i, m-1}^{(k)} \eta_{k}^{m-1} .
$$

Da alle Zahlen $\eta_{2}, \eta_{3}, \ldots, \eta_{m}$ in $\left(\Omega, \eta_{1}\right)$ enthalten sind und also der $\operatorname{Körper}\left(\Re, \eta_{1}, \eta_{2}, \ldots, \eta_{m}\right)=\left(\Re, \eta_{1}\right)$ ist, so kann man auch sagen, eine Normalgleichung sei dadurch charahterisiert, daß sie ihre eigene Galoissche Resolvente ist.

Die Bedeutung der Galoisschen Resolvente geht aus folgenden Angaben hervor. In der Theorie der algebraischen Gleichungen bezeichnet man, falls irgendein Zahlkörper vorgelegt ist, die Zahlen desselben als „rational bekannte" Größen. Ist eine Gleichung $f(z)=0 \mathrm{zu}$ lösen, so sind mit ihr die Koeffizienten von $f(z)$ gegeben. Als vorgelegt gilt also ein Körper, der jedenfalls alle Koeffizienten von $f(z)$ enthält. Uim für den Augenblick mit einem bestimmten Körper zu tun zu haben, denken wir etwa $\Re$ als Körper aller Zahlen, die sich aus den Gleichungskoeffizienten rational mit rationalen Zahlenkoeffizienten berechnen lassen. Ist die Gleichung $f(z)=0$ irreduzibel, und ist ihr Grad $n>1$, so gehören die Wurzeln der Gleichung noch nicht zu den „rational bekannten" Größen. Aber es gilt der Satz: Hat man eine "einzige" Wurzel $\eta$ einer Galoisschen Resolvente (5) von $f(z)=0$ gewonnen und damit die Erweiterung des Körpers $\Omega$ durch Adjunktion von $\eta$ zum Körper $(\mathfrak{i}, \eta)$ vollzogen, so sind nicht nur alle übrigen Wurzeln der Galoisschen Resolvente, sondern auch alle Wurzeln der Gleichung $f(z)=0$ selbst „rational bekannt".

\section{§ 9. Die Transformationen eines Galoisschen Körpers in sich.}

Wie soeben bedeute $\eta$ eine fest gewählte primitive Zahl des Galoisschen Körpers, die der irreduzibelen Normalgleichung $F(Z)=0$ vom Grade $m$ genügt. Beliebige Zahlen aus $(\Omega, \eta)$ mögen durch $\xi$ bezeichnet werden; jede von ihnen ist eindeutig in der Gestalt darstellbar:

$$
\xi=c_{0}+c_{1} \eta+c_{2} \eta^{2}+\cdots+c_{m-1} \eta^{m-1}
$$

Die mit $\xi$ konjugierten Zahlen unterscheiden wir wieder durch die Bezeichnungen $\xi_{1}=\xi, \xi_{2}, \xi_{3}, \ldots, \xi_{m}$. Ist $\xi$ primitiv, so sind diese $m$ Zahlen alle verschieden; andrenfalls werden sie zu je $\mu$ einander gleich und stellen $\nu$ verschiedene Zahlen $\xi_{1}=\xi, \xi_{2}, \ldots, \xi_{v}$ dar, wo $\mu$ ein von 1 verschiedener Teiler von $m$ und $\mu \cdot \nu=m$ ist. Bezeichnen wir allgemein ein System konjugierter Zahlen durch $\xi_{1}, \xi_{2}, \ldots, \xi_{v}$, so können wir den Fall primitiver Zahlen für $\nu=m$ hier mit einbegreifen.

Im Ausdruck (1) aller Zahlen von $(\Re, \eta)$ ersetzen wir jetzt $\eta$ durch 
irgendeine konjugierte Zahl $\eta_{\alpha}$. Hierbei geht jede Zahl $\zeta$ von $(\Re, \eta)$ in eine bestimmte mit ihr konjugierte Zahl über, und zwar zwei verschiedene Zahlen stets wieder in zwei verschiedene. Ist nämlich $\xi^{\prime}=c_{0}^{\prime}+c_{1}^{\prime} \eta+\cdots$ $+c_{m-1}^{\prime} \eta^{m-1}$ von der in (1) dargestellten Zahl $\xi$ verschieden, so gelten nicht gleichzeitig alle $m$ Gleichungen $c_{0}^{\prime}=c_{0}, c_{1}^{\prime}=c_{1}, \ldots, c_{m-1}^{\prime}=c_{m-1}$. Dann sind aber notwendig auch die beiden Zahlen:

$c_{0}+c_{1} \eta_{\alpha}+c_{2} \eta_{\alpha}^{2}+\cdots+c_{m-1} \eta_{\alpha}^{m-1}, \quad c_{0}^{\prime}+c_{1}^{\prime} \eta_{\alpha}+c_{2}^{\prime} \eta_{\alpha}^{2}+\cdots+c_{m-1}^{\prime} \eta_{\alpha}^{m-1}$ verschieden, da aus ihrer Gleichheit eine nicht identische Gleichung in $\Omega$ für $\eta_{\alpha}$ von einem Grade $<m$ hervorgehen würde, was der Irreduzibilität von $F(Z)=0$ widerspricht. Es folgt: Beim Ersatz von $\eta$ durch irgendeine der $m$ konjugierten Zahlen $\eta_{\alpha}$ erfahren je $v$ konjugierte Zahlen $\xi_{1}, \xi_{2}, \ldots, \xi_{v}$ des Galoisschen Körpers $(\Omega, \eta)$ eine bestimmte Permutation. Wir bezeichnen diese Permutation (damit $S_{0}=1$ die identische Permutation wird) durch $S_{\alpha-1}$ und erhalten (da die mit $\xi$ konjugierten Zahlen alle in $(\Re, \eta)$ enthalten sind), indem wir der Reihe nach $\eta_{\alpha}=\eta_{1}, \eta_{2}, \ldots, \eta_{m}$ setzen, im ganzen $m$ Transformationen des Galoisschen Körpers $(\Re, \eta)$ in sich, die wir gleich selbst wieder $S_{0}, S_{1}, \ldots, S_{m-1}$ nennen, und von denen $S_{0}=1$ die „iden-

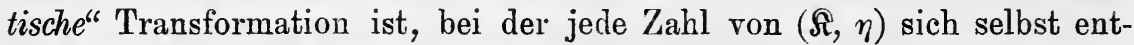
spricht.

Diese Transformationen haben eine wichtige aus der Irreduzibilität von $F(Z)=0$ folgende Eigenschaft. Es seien durch das Symbol $R$ bei der folgenden Überlegung stets rationale Funktionen bezeichnet, deren Koeffizienten in $\Omega$ enthalten sind. Es seien zunächst $R_{1}(z)$ und $R_{2}(z)$ solche Funktionen einer Variablen, und es mögen die Zerlegungen der $R_{1}(z), R_{2}(z)$ in Quotienten je zweier ganzer Funktionen gegeben sein durch:

$$
R_{1}(z)=\frac{h_{1}(z)}{g_{1}(z)}, \quad R_{z}(z)=\frac{h_{2}(z)}{g_{2}(z)} .
$$

Dann besteht der Satz: Haben die Funktionen $R_{1}(z), R_{2}(z)$ für $z=\eta=\eta_{1}$ gleiche endliche Werte, $R_{1}(\eta)=R_{2}(\eta)$, so haben sie für jede der $m$ konjugierten Zahlen $\eta_{\alpha}$ gleiche endliche Werte, $R_{1}\left(\eta_{\alpha}\right)=R_{2}\left(\eta_{\alpha}\right)$. Der Voraussetzung nach gelten nämlich die drei Bedingungen:

$$
g_{1}(\eta) \neq 0, \quad g_{2}(\eta) \neq 0, \quad g_{1}(\eta) h_{2}(\eta)-g_{2}(\eta) h_{1}(\eta)=0
$$

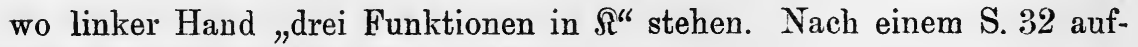
gestellten Satze folgt sofort für jedes $\eta_{\alpha}$ :

$$
g_{1}\left(\eta_{\alpha}\right) \neq 0, \quad g_{2}\left(\eta_{\alpha}\right) \neq 0, \quad g_{1}\left(\eta_{\alpha}\right) h_{2}\left(\eta_{\alpha}\right)-g_{2}\left(\eta_{\alpha}\right) h_{1}\left(\eta_{\alpha}\right)=0,
$$

woraus die Richtigkeit des Satzes hervorgeht.

Es handelt sich hierbei um einen Spezialfall des folgenden grundlegenden Satzes: Besteht zwischen irgendwelchen Zahlen $\xi, \xi^{\prime}, \xi^{\prime \prime}, \ldots$ und $\bar{\zeta}, \bar{\zeta}^{\prime}, \bar{\zeta}^{\prime \prime}, \ldots$ aus $(\Re, \eta)$ eine Gleichung:

$$
R_{1}\left(\xi, \xi^{\prime}, \xi^{\prime \prime}, \ldots\right)=R_{2}\left(\bar{\xi}, \bar{\zeta}^{\prime}, \bar{\zeta}^{\prime \prime}, \ldots\right),
$$


wo rechts und links rationale Ausdrücke endlicher Werte mit Koeffizienten des Körpers $\Omega$ stehen, so sind auch die beiden Zahlen $R_{1}\left(\xi_{\alpha}, \xi_{\alpha}^{\prime}, \ldots\right)$ und $R_{2}\left(\bar{\xi}_{\alpha}, \bar{\zeta}_{\alpha}^{\prime}, \ldots\right)$ endlich und einander gleich:

$$
R_{1}\left(\xi_{\alpha}, \xi_{\alpha}^{\prime}, \xi_{\alpha}^{\prime \prime}, \ldots\right)=R_{2}\left(\bar{\xi}_{\alpha}, \bar{\xi}_{\alpha}^{\prime}, \bar{\xi}_{\alpha}^{\prime \prime}, \ldots\right) \text {, }
$$

wenn wir unter $\xi_{\alpha}, \xi_{\alpha}^{\prime}, \xi_{\alpha}^{\prime \prime}, \ldots$ und $\bar{\xi}_{\alpha}, \bar{\zeta}_{\alpha}^{\prime}, \bar{\zeta}_{\alpha}^{\prime \prime}, \ldots$ die aus den Argumenten in (2) durch die Transformation $S_{\alpha-1}$ hervorgehenden Zahlen verstehen. Der Beweis folgt sofort aus dem voraufgehenden Satze, wenn wir in (2) rechts und links für die Argumente ihre Ausdrücke (1) in $\eta$ eintragen.

Die Transformation $S_{\alpha-1}$ mag nun als Permutation der $\eta$ die folgende sein:

$$
S_{\alpha-1}=\left(\begin{array}{l}
\eta_{1}, \eta_{3}, \ldots, \eta_{m} \\
\eta_{\alpha_{1}}, \eta_{\alpha_{2}}, \ldots, \eta_{\alpha_{m}}
\end{array}\right)
$$

und also $\eta_{k}$ in $\eta_{\alpha_{k}}$ überführen. Dann gilt der Satz: Die Transformation $S_{\alpha-1}$ des Galoisschen Körpers in sich kann auch dadurch vollzogen werden, daß man alle Zahlen dieses Körpers durch $\eta_{k}$ darstellt und in diesen Darstellungen $\eta_{k}$ durch $\eta_{\alpha_{k}}$ ersetzt. Hat nämlich die beliebige Zahl $\xi$ des Körpers in $\eta_{k}$ die Darstellung:

$$
\zeta=c_{0}^{\prime}+c_{1}^{\prime} \eta_{k}+c_{2}^{\prime} \eta_{k}^{2}+\cdots+c_{m-1}^{\prime} \eta_{k}^{m-1},
$$

und geht $\xi$ durch die Transformation $S_{\alpha-1}$ in $\xi_{\alpha}$ über, so folgt aus (5) nach dem soeben bewiesenen Satze die Gleichung:

$$
\zeta_{\alpha}=c_{0}^{\prime}+c_{1}^{\prime} \eta_{\alpha_{k}}+c_{2}^{\prime} \eta_{\alpha_{k}}^{2}+\cdots+c_{m-1}^{\prime} \eta_{\alpha_{k}}^{m-1} .
$$

Daraus geht die Behauptung unmittelbar hervor.

Aus dem letzten Satze folgt die Möglichkeit der Zusammensetzung je zweier Transformationen des Körpers in sich. Üben wir zunächst die Transformation $S_{\alpha-1}$ aus, die $\eta$ in $\eta_{\alpha}$ überführt, und hierauf die Transformation $S_{\beta-1}$, die vermittelst des Ersatzes von $\eta$ durch $\eta_{\beta}$ oder also vermittelst des Ersatzes von $\eta_{\alpha}$ durch $\eta_{\beta_{\alpha}}$ erzielt werden kann, so gelangen wir zu einer durch $S_{\beta-1} \cdot S_{\alpha-1}$ zu bezeichnenden Transformation, die unmittelbar vermöge des Ersatzes von $\eta$ durch $\eta_{\beta_{\alpha} \alpha}$ erzielbar ist, also unserem System der $m$ Transformationen wieder angehört und für die $\eta$ die Permutation liefert:

$$
S_{\beta-1} \cdot S_{\alpha-1}=\left(\begin{array}{l}
\eta_{1}, \eta_{2}, \ldots, \eta_{m} \\
\eta_{\beta \alpha_{1}}, \eta_{\beta \alpha_{2}}, \ldots, \eta_{\beta \alpha_{m}}
\end{array}\right) \text {. }
$$

Das System der m Transformationen $S_{0}, S_{1}, \ldots, S_{m-1}$ erfüllt hiernach die erste Gruppenbedingung von S. 2. Aber auch die beiden anderen Gruppeneigenschaften liegen vor; man zeigt dies wie S. 18, indem man die $S$ als Permutationen der $\eta_{1}, \eta_{2}, \ldots \eta_{m}$ schreibt und beachtet, daB die einzelne Permutation die zugehörige Transformation des ganzen Körpers in sich eindeutig festlegt: 
Die $m$ Transformationen eines Galoisschen Körpers $m^{\text {ten }}$ Grades in sich bilden eine Gruppe $G_{m}$ der $m^{\text {ten }}$ Ordnung, die sich für die $\eta_{1}, \eta_{2}, \ldots, \eta_{m}$ sowie iiberhaupt für jedes System konjugierter primitiver Zahlen als eine Permutationsgruppe $G_{m}$ des $m^{\text {ten }}$ Grades darstellt.

Unter den $m$ Permutationen der $\eta$ gibt es eine und nur eine, die $\eta_{1}$ in die beliebig vorgeschriebene konjugierte Zahl $\eta_{\alpha}$ überführt, nämlich die Permutation $S_{\alpha-1}$. Sie führt dann $\eta_{2}$ in eine bestimmte Zahl $\eta_{\alpha_{2}}$, nicht mehr in eine frei wählbare unter den konjugierten Zahlen über. Nach der S. 20 eingeführten Bezeichnung ist somit die Gruppe $G_{m}$ als Permutationsgruppe $m^{\text {ten }}$ Grades für $\eta_{1}, \eta_{2}, \ldots, \eta_{m}$ oder irgendein System von $m$ primitiven konjugierten Zahlen „einfach transitiv“.

\section{\$ 10. Die Galoissche Gruppe einer Gleichung $f(z)=0$.}

Wir kehren jetzt zur Gleichung $n^{\text {ten }}$ Grades $f(z)=0$ zurück, die wir einstweilen als irreduzibel vorraussetzen, und aus deren Wurzeln wir in der Gestalt $\left(\Omega, \theta_{1}, \theta_{2}, \ldots, \theta_{n}\right)=(\Omega, \eta)$ den Galoisschen Körper herstellten. Ist $\theta=\theta_{1}$ eine primitive Zahl dieses Körpers, so ist $n=m$, und wir haben in $f(z)=0$ eine „Normalgleichung“. Ist $n<m$, so ist $\theta$ imprimitiv und also $n$ ein vom ganzen verschiedener Teiler der Zahl $m$. Im ersten Falle liefert die $G_{m}$, eingekleidet in die Gestalt einer Permutationsgruppe der $n=m$ Wurzeln $\theta_{1}, \theta_{2}, \ldots$ eine einfach transitive Gruppe $G_{m}$ des Grades $m$, wie in $\S 9$ am Schlusse gesagt wurde. Im zweiten Falle zeigen wir zunächst, daß die $m$ Transformationen des Galoisschen Körpers in sich $m$ verschiedene Permutationen $\operatorname{der} \theta_{1}, \theta_{2}, \ldots, \theta_{n}$ liefern. Sollen nämlich $S_{\alpha}$ und $S_{\beta}$ die gleiche Permutation der $\theta$ bewirken, so liefert $S_{\beta}^{-1} \cdot S_{\alpha}$ die identische Permutation der $\theta$ und damit die Transformation, bei der jede Zahl des Körpers $\left(\Re, \theta_{1}, \theta_{2}, \ldots, \theta_{n}\right)$ $=(\Re, \eta)$ sich selbst zugeordnet ist. Also ist $S_{\beta}^{-1} \cdot S_{\alpha}=S_{0}=1$ und $S_{\beta}=S_{\alpha}$.

In jedem Falle (mag $n=m$ oder $n<m$ sein) stellen wir folgende Erklärung auf: Die von den Transformationen des Galoisschen Körpers $\left(\Omega, \theta_{1}, \theta_{2}, \ldots, \theta_{n}\right)=(\Omega, \eta)$ in sich gelieferten $m$ Permutationen der $\theta_{1}, \theta_{2}, \ldots, \theta_{n}$ ergeben eine besondere Einkleidung der Gruppe $G_{m}$ als einer Permutationsgruppe $m^{\text {ter }}$ Ordnung $n^{\text {ten }}$ Grades, die man als die "Galoissche Gruppe" der Gleichung $f(z)=0$ bezeichnet. Sie liefert den im Mittelpunkte der Galoisschen Theorie der Gleichungen stehenden Begriff. Für die Normalgleichung $F(Z)=0$ des vorigen Paragraphen ist die Galoissche Gruppe die daselbst betrachtete einfach transitive Permutationsgruppe $m^{\text {ten }}$ Grades der $\eta_{1}, \eta_{2}, \ldots, \eta_{m}$.

Die Eigenschaften der Galoisschen Gruppe ergeben sich leicht aus den Entwicklungen des vorigen Paragraphen. Wir notieren zunächst, daß die Galoissche Gruppe einer irreduzibelen Gleichung $f(z)=0$ stets 
transitiv ist. Eine beliebig vorgeschriebene Wurzel $\theta_{\alpha}$ gewinnen wir nämlich aus $\theta_{1}$ durch die Transformation $S_{\alpha}$ des Körpers $(\Omega, \eta)$ in sich.

Von grundsätzlicher Bedeutung sind die folgenden Ausführungen: Unter $R\left(\theta_{1}, \theta_{2}, \ldots, \theta_{n}\right)$ verstehen wir wieder einen rationalen Ausdruck mit Koeffizienten aus $\Re$. Hat der Ausdruck $R\left(\theta_{1}, \theta_{2}, \ldots, \theta_{n}\right)$ als Wert eine bereits in $\Omega$ enthaltene Zahl $c$, so sagen wir, es bestehe für die $\theta_{1}, \theta_{2}, \ldots, \theta_{n}$ die ,rationale Gleichung. in $\mathfrak{\Re}^{“}$ :

$$
R\left(\theta_{1}, \theta_{2}, \ldots, \theta_{n}\right)=c .
$$

Da jede Zahl $c$ von $\mathfrak{K}$ bei den Transformationen $S_{\alpha}$ nur sich selbst zugeordnet ist, so folgt nach dem Satze von S. $44 \mathrm{ff}$. aus (1):

$$
R\left(\theta_{\alpha_{1}}, \theta_{\alpha_{2}}, \ldots, \theta_{\alpha_{n}}\right)=c,
$$

wenn $\theta_{k}$ durch $S_{\alpha}$ in $\theta_{\alpha_{k}}$ übergeführt wird. Damit haben wir den Satz: Jede rationale Gleichung in $\Re$, die für die $\theta_{1}, \theta_{2}, \ldots, \theta_{n}$ zutrifft, bleibt richtig, falls man in ihr die $\theta_{1}, \theta_{2}, \ldots, \theta_{n}$ einer beliebigen Permutation der Galoisschen Gruppe unterwirft. Umgekehrt gilt der Satz: Ein rationaler Ausdruck $R\left(\theta_{1}, \theta_{2}, \ldots, \theta_{n}\right)$ mit endlichem Werte, der sich gegenüber den Permutationen der Galoisschen Gruppe $G_{m}$ nicht ändert, stellt eine Zahl aus $\Omega$ dar. Die Zahlen aus $\Re$ sind nämlich die einzigen Zahlen des Körpers $(\Re, \eta)$, die nur sich selbst konjugiert sind. Sehen wir nur erst den Körper $\Re$ als gegeben und also dessen Zahlen als „rational bekannt" an, so können wir auch sagen: Ein rationaler Ausdruck $R\left(\theta_{1}, \theta_{2}, \ldots, \theta_{n}\right)$ der Wurzeln von $f(z)=0$, der sich gegenüber den Permutationen der Galoisschen Gruppe $G_{m}$ nicht ändert, ist „rational bekannt".

Der erste dieser drei Sätze ist in folgender Art umkehrbar: Eine Permutation der $\theta_{1}, \theta_{2}, \ldots, \theta_{n}$, die ,jede" zwischen den $\theta_{1}, \theta_{2}, \ldots, \theta_{n}$ gültige rationale Gleichung in $\AA$ in eine gleichfalls richtige solche Gleichung überführt, gehört der Galoisschen Gruppe $G_{m}$ an, so daß diese $G_{m}$ auch als Gruppe aller Permutationen der Wurzeln von $f(z)=0$ erklärt werden kann, bei denen „alle" für die $\theta_{1}, \theta_{2}, \ldots, \theta_{n}$ bestehenden rationalen Gleichungen (1) wieder in richtige Gleichungen übergehen. Wir können nämlich mittelst rationaler ganzer Zahlen $\gamma$ eine Zahl:

$$
\eta=\gamma_{1} \theta_{1}+\gamma_{2} \theta_{2}+\cdots+\gamma_{n} \theta_{n}
$$

so wählen, daß die $n$ ! Zahlen $\eta_{1}=\eta, \eta_{2}, \eta_{3}, \ldots$, die aus (3) durch alle $n$ ! Permutationen der $\theta_{1}, \theta_{2}, \ldots, \theta_{n}$ hervorgehen, durchweg verschieden sind. Man beweist dies durch Wiederholung einer S. 36 ausgeführten Überlegung. In der Reihe $\eta_{1}, \eta_{2}, \ldots$ mögen die $m$ in $(\AA, \eta)$ mit $\eta=\eta_{1}$ konjugierten Zahlen an erster Stelle stehen; sie gehen aus (3) durch die Permutationen der Galoisschen Gruppe $G_{m}$ hervor, während der Rest $\eta_{m+1}, \eta_{m+2}, \ldots, \eta_{n 1}$ von den nicht in $G_{m}$ enthaltenen $(n !-m)$ Permu- 
tationen herrührt. ${ }^{1}$ ) Ist nan $F^{\prime}(Z)=0$ die zur Zahl (3) gehörende Galoissche Resolvente $m^{\text {ten }}$ Grades, so gilt:

$$
F\left(\eta_{1}\right)=0, \quad F\left(\eta_{2}\right)=0, \ldots, \quad F\left(\eta_{m}\right)=0,
$$

während andrerseits:

$$
F\left(\eta_{m+1}\right) \neq 0, \quad F\left(\eta_{m+2}\right) \neq 0, \ldots, \quad F\left(\eta_{n i}\right) \neq 0
$$

zutrifft. Die Wurzeln von $F(Z)=0$ sind nämlich die $m$ konjugierten Zahlen $\eta_{1}, \eta_{2}, \ldots, \eta_{m}$, von denen die $\eta_{m+1}, \ldots, \eta_{n}$ ! durchweg verschieden sind. Denken wir nun $F(\eta)=0$ durch Einsetzung des Ausdrucks (3) für $\eta$ als eine für die $\theta_{1}, \theta_{2}, \ldots, \theta_{n}$ gültige rationale Gleichung in $\Omega$ geschrieben, und soll eine auf die $\theta$ auszuïbende Permutation diese Gleichung wieder in eine richtige Gleichung überführen, so kann es sich zufolge (4) und (5) nur um eine der $m$ ersten Permutationen handeln, also um eine Permution der $G_{m}$, die $\eta$ in eine der Zahlen $\eta_{1}, \eta_{2}, \ldots, \eta_{m}$ überführt. Damit ist der Satz bewiesen.

Die Übertragung der gewonnenen Ergebnisse auf reduzibele Gleichungen $f(z)=0$ ist leicht. Auch im Falle einer reduzibelen Gleichung $n^{\text {ten }}$ Grades $f(z)=0$ mit $n$ verschiedenen Wurzeln $\theta_{1}, \theta_{2}, \ldots, \theta_{n}$ hatten wir oben (S. 42) einen Galoisschen Körper $\left(\Omega, \theta_{1}, \theta_{2}, \ldots, \theta_{n}\right)=(\Omega, \eta)$ hergestellt. Die $m$ Transformationen dieses Körpers in sich liefern wieder $m$ Permutationen der $\theta_{1}, \theta_{2}, \ldots, \theta_{n}$, die man wie vorhin (S. 46) als durchweg verschieden erkennt. Diese $m$ Permutationen liefern uns die „Galoissche Gruppe" $G_{m}$ der reduzibelen Gleichung $f^{\prime}(z)=0$. Zerfällt $f(z)$ in die irreduzibelen Faktoren $f_{1}(z), f_{2}(z), \ldots, f_{\lambda}(z)$ der Grade $n_{1}, n_{2}, \ldots, n_{\lambda}$, so sind die Wurzeln des einzelnen Faktors nur unter sich konjugiert und werden demnach durch die $G_{m}$ auch nur unter sich permutiert: Die Galoissche Gruppe der reduzibelen Gleichung $f(z)=0$ ist also ,intransitiv“, und die Systeme der $n_{1}, n_{2}, \ldots, n_{\lambda}$ Wurzeln der irreduzibelen Bestandteile von $f(z)=0$ liefern die "Systeme der Intransitivität". Die Gruppenordnung $m$ ist ein Multiplum jedes der Grade $n_{1}, n_{2}, \ldots, n_{2}$, ist aber keineswegs mehr notwendig ein Multiplum von $n$ selbst. Die wesentlichen Eigenschaften der Galoisschen Gruppe, die auf der Irreduzibilität der Galoisschen Resolventen $F(Z)=0$ beruhen, bleiben auch bei reduzibelen Gleichungen $f(z)=0$ bestehen.

Es sei wieder $f(z)=0$ eine irreduzibele Gleichung $x^{\text {ten }}$ Grades mit den Wurzeln $\theta_{1}, \theta_{2}, \ldots, \theta_{n}$. Ist der Körper $(\Re, \theta)=\left(\Re, \theta_{1}\right)$ (und damit natürlich jeder der konjugierten Körper $\left(\Re, \theta_{i}\right)$ im Sinne von $S .40$ imprimitiv, so nennen wir auch die Gleichung $f(z)=0$,imprimitiv“. Es liege dieser Fall vor, und es sei insbesondere $\xi=R(\theta)$ eine imprimitive

1) Wir dürfen $m<n$ ! annehmen, da für $m=n$ ! der zu beweisende Satz selbstverständlich ist. 
Zahl aus $(\Omega, \theta)$. Dann sind die $n$ mit $\xi$ konjugierten Zahlen zu je $\mu$ einander gleich und stellen nur $\nu$ verschiedene Zahlen $\xi_{1}, \xi_{2}, \ldots, \xi_{\nu}$ dar; dabei ist $\mu$ ein von 1 und $n$ verschiedener Teiler der Zahl $n$. Wir ordnen nun die $n$ Zahlen $\theta$ so in $\nu$ Zeilen zu je $\mu$ Zahlen an:

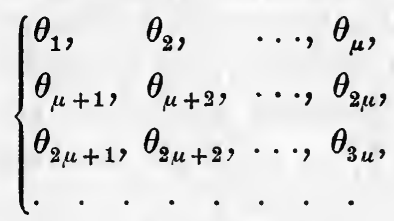

daß die $\theta$ der $i^{\text {ten }}$ Zeile, als Argumente in $R$ eingesetzt, übereinstimmend die Zahl $\xi_{i}$ liefern. Für zwei Argumente $\theta_{\alpha}$ und $\theta_{\beta}$ gilt alsdann:

$$
R\left(\theta_{\alpha}\right)=R\left(\theta_{\beta}\right) \text { oder } R\left(\theta_{\alpha}\right) \neq R\left(\theta_{\beta}\right),
$$

je nachdem die $\theta_{\alpha}, \theta_{\beta}$ in der gleichen Zeile (6) oder in verschiedenen Zeilen stehen. Bei Ausübung einer Permutation der Galoisschen Gruppe $G_{m}$ geht nun aus einer Gleichung (7) stets wieder eine Gleichung, aus einer Ungleichung (7) stets wieder eine Ungleichung hervor. Die Zeilenanordnung (6) der $n$ Zahlen $\theta_{1}, \theta_{2}, \ldots, \theta_{n}$ besitzt demnach gegenüber der Gruppe $G_{m}$ die S. 21 bei der Erklärung der Imprimitivität einer Permutationsgruppe näher dargelegte Invarianz. Es folgt somit: Die Galoissche Gruppe der imprimitiven Gleichung $f(z)=0$ ist eine, ,imprimitive Gruppe", und die Zeilen (6) liefern die „Systeme der Imprimitivität". ${ }^{\text {) }}$ )

\section{\$11. Untergruppen der Galoisschen Gruppe und zugehörige Zahlen.}

Wir gehen auf die ursprüngliche Erklärung der Galoisschen Gruppe $G_{m}$ als Gruppe der $m$ Transformationen des Galoisschen Körpers $(\Re, \eta)$ in sich zurück. Aus diesem Körper greifen wir eine beliebige Zahl $\zeta$ heraus, die in der Gestalt $\xi=R(\eta)$ darstellbar ist, wo man $R(\eta)$ als rationale ganze Funktion $(m-1)^{\text {ten }}$ Grades von $\eta$ mit Koeffizienten aus $\Omega$ schreiben kann. Die mit $\xi$ konjugierten Zahlen sind zu je $\mu$ einander gleich, wo $\mu$ ein Teiler von $m$ ist, zunächst unter Einschluß von $\mu=m$ und $\mu=1$; für $\mu=m$ ist $\xi$ eine schon in $\Omega$ enthaltene Zahl, für $\mu=1$ haben wir in $\zeta$ eine primitive Zahl des Körpers $(\Re, \eta)$.

Unter den Transformationen der $G_{m}$, die nach S. 44 die mit $\xi$ konjugierten Zahlen $\xi_{1}=\xi, \xi_{2}, \ldots, \xi_{\nu}$ untereinander permutieren, kommen im ganzen $\mu$ vor, die die Zahl $\xi$ in sich selbst überführen. Diese $\mu$ Transformationen bilden eine in $\operatorname{der} G_{m}$ enthaltene Untergruppe $G_{\mu}$, deren Index $\frac{m}{\mu}=t$ die bisher durch $\nu$ bezeichnete Anzahl ist. Wir nennen die $G_{\mu}$ „die zur Zahl $\zeta$ gehörende Untergruppe $G_{\mu}{ }^{\prime \prime}$ und umgekehrt $\zeta$,eine zur Untergruppe $G_{\mu}$ gehörende $Z a h l^{“}$. Zu einer Zahl von $\Re$ gehört dann die

1) Weiteres über imprimitive Gleichungen findet man bei Weber, a. a. O., Bd. 1, S. $524 \mathrm{ff}$. 
Gesamtgruppe $G_{m}$, zu einer primitiven Zahl des Körpers $(\Omega, \eta)$ die Untergruppe $G_{1}$. Zerlegen wir die $G_{m}$ entsprechend $\operatorname{der} G_{\mu}$ in die Nebengruppen $G_{\mu}, V_{1} \cdot G_{\mu}, V_{2} \cdot G_{\mu}, \ldots, V_{t-1} \cdot G_{\mu}$, so führen die Transformationen der einzelnen Nebengruppe $V_{i-1} \cdot G_{\mu}$ die Zahl $\zeta$ in eine und dieselbe konjugierte Zahl $\xi_{i}$ über; allen $t$ Nebengruppen entsprechen auf diese Weise die $t(=\nu)$ verschiedenen konjugierten Zahlen $\xi_{1}=\xi, \xi_{2}$, $\xi_{3}, \ldots, \xi_{t}$. Zur Zahl $\xi_{i}$ ihrerseits gehört die mit $G_{\mu}$ gleichberechtigte Untergruppe $V_{i-1} \cdot G_{\mu} \cdot V_{i-1}^{-1}: Z u$ den $t$ verschiedenen mit $\zeta$ konjugierten Zahlen des Körpers $(\Re, \eta)$ gehören die $t$ mit $G_{\mu}$ gleichberechtigten Untergruppen $G_{\mu}, V_{1} \cdot G_{\mu} \cdot V_{1}^{-1}, V_{2} \cdot G_{\mu} \cdot V_{2}^{-1}, \ldots$, die natürlich leineswegs alle verschieden zu sein brauchen.

Es besteht nun folgender grundlegende Satz: Zu jeder Untergruppe $G_{\mu}$ von $G_{m}$ gibt es unendlich viele zugehörige Zahlen im Körper $(\Omega, \eta)$; ist $\zeta$ irgendeine dieser Zahlen, so ist jede andere sowie überhaupt jede Zahl, die zu einer $G_{\mu}$ in sich enthaltenden Untergruppe gehört, im Körper $(\Omega, \xi)$ enthalten und also rational in $\zeta$ mit Koeffizienten aus $\Re$ darstellbar.

Da der Satz für $\mu=1$ und $\mu=m$ selbstverständlich ist, so setzen wir $1<\mu<m$ voraus. Wir bezeichnen die mit $\eta$ konjugierten Zablen so, daß $\eta=\eta_{1}$ durch die Transformationen $\operatorname{der} G_{\mu}$ in die in der ersten Zeile (1) stehenden Zahlen $\eta_{1}, \eta_{2}, \ldots, \eta_{\mu}$ übergeht, während diese ganze Zeile durch die Transformation $V_{i}$ in die Zeile $\eta_{i \mu+1}, \eta_{i \mu+2}, \ldots, \eta_{(i+1) \mu}$ übergeführt wird:

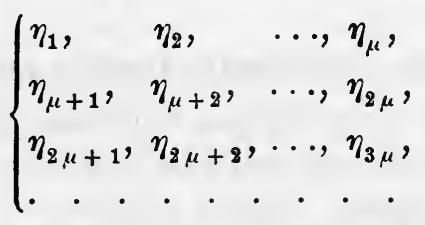

Wir bilden nun die $\mu$ symmetrischen Grundfunktionen $\sigma_{1}^{(i)}, \sigma_{2}^{(i)}, \ldots, \sigma_{\mu}^{(i)}$ der in der Zeile $\eta_{i \mu+1}, \eta_{i \mu+2}, \ldots$ stehenden $\eta$ und gewinnen so $t$ neue Zahlenreihen:

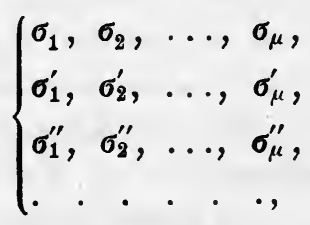

von denen keine zwei einander gleich sind; es würden ja sonst die $\eta$ der beiden entsprechenden Zeilen (1) von der Anordnung abgesehen übereinstimmen. Nach einer wiederholt ausgeübten SchluBweise können wir somit (und zwar auf unendlich viele Arten) $\mu$ rationale ganze Zahlen $\gamma$ so wählen, daB die $t$ Zahlen von $(\Omega, \eta)$ :

$$
\gamma_{1} \sigma_{1}+\gamma_{2} \sigma_{2}+\cdots+\gamma_{\mu} \sigma_{\mu}, \quad \gamma_{1} \sigma_{1}^{\prime}+\gamma_{2} \sigma_{2}^{\prime}+\cdots+\gamma_{\mu} \sigma_{\mu}^{\prime}, \cdots
$$

durchweg voneinander verschieden sind. Die erste dieser Zahlen gehört 
zur $G_{\mu}$, die übrigen gehen aus ihr durch die Transformationen $V_{1}, V_{2}, \ldots$ hervor und sind also die mit der ersten konjugierten Zahlen. Wir haber demnach in der Tat in:

$$
\xi=\gamma_{1} \sigma_{1}+\gamma_{2} \sigma_{2}+\cdots+\gamma_{\mu} \sigma_{\mu}
$$

eine zur Gruppe $G_{\mu}$ gehörende Zahl konstruiert.

Aus den mit $\xi=\xi_{1}$ konjugierten Zahlen bilden wir mittelst einer Variablen $w$ die Funktion $g(w)=\left(w-\xi_{1}\right)\left(w-\xi_{2}\right) \ldots\left(w-\xi_{t}\right)$ vom Grade $t$, die ausgerechnet eine irreduzibele Funktion in $\Re$ ergibt. Es sei alsdann $\xi^{\prime}$ irgendeine Zahl aus $(\Re, \eta)$, die durch die Transformationen $\operatorname{der} G_{\mu}$ in sich selbst übergeführt wird, die also entweder auch zur $G_{\mu}$ oder zu einer $G_{\mu}$ umfassenden Untergruppe gehört. Durch $1, V_{1}, V_{2}, \ldots$, $V_{t-1}$ werde $\zeta^{\prime}=\xi_{1}^{\prime}$ in die Zahlen $\xi_{1}^{\prime}, \xi_{2}^{\prime}, \ldots, \zeta_{t}^{\prime}$ übergeführt, die nicht alle voneinander verschieden zu sein brauchen. Es ist nun, wie man durch Division von $g(w)$ durch $\left(w-\xi_{1}\right)$ feststellt:

$$
\frac{g(w)}{w-\zeta} \cdot \zeta^{\prime}=\frac{g(w)}{w-\zeta_{1}} \cdot \xi_{1}^{\prime}
$$

eine Funktion $(t-1)^{\text {ten }}$ Grades von $v$, deren Koeffizienten Zahlen aus $(\Omega, \eta)$ sind, die alle durch die Transformationen $\operatorname{der} G_{\mu}$ in sich übergeführt werden. Durch $1, V_{1}, V_{2}, \ldots, V_{t-1}$ geht die Funktion (5) in die $t$,konjugierten Funktionen“ über:

$$
\frac{g(w)}{w-\xi_{1}} \cdot \xi_{1}^{\prime}, \quad \frac{g(w)}{w-\xi_{2}} \cdot \xi_{2}^{\prime}, \ldots, \quad \frac{g(w)}{w-\xi_{t}} \cdot \xi_{t}^{\prime},
$$

die sich bei Ausübung irgendwelcher Transformationen der $G_{m}$ untereinander permutieren. Die Summe der Funktionen (6) bleibt demnach bei allen Transformationen $\operatorname{der} G_{m}$ unverändert und erweist sich also als eine „Funktion in $\Re^{\leftrightarrow:}$

$$
\frac{g(w)}{w-\zeta_{1}} \cdot \zeta_{1}^{\prime}+\frac{g(w)}{w-\xi_{2}} \cdot \xi_{2}^{\prime}+\cdots+\frac{g(w)}{w-\zeta_{t}} \cdot \zeta_{t}=h(w) .
$$

Wie üblich gewinnen wir hieraus für $w=\xi_{1}=\xi$ :

$$
\xi^{\prime}=\frac{h(\zeta)}{g^{\prime}(\zeta)}
$$

so daß $\xi^{\prime}$ tatsächlich dem Körper $(\Re, \xi)$ angehört.

Kehren wir zu unserer ursprünglichen Gleichung $n^{\text {ten }}$ Grades $f(z)=0$ zurück, so ist der betrachtete Galoissche Körper in der Gestalt $\left(\Omega, \theta_{1}, \theta_{2}, \ldots, \theta_{n}\right)$ zu erklären, und die $G_{m}$ wird die als Galoissche Gruppe der Gleichung $f(z)=0$ erklärte Permutationsgruppe. Man nennt in der Theorie der Gleichungen eine Zahl aus $\left(\Omega, \theta_{1}, \theta_{2}, \ldots, \theta_{n}\right)$, d. h. eine Zahl, die durch einen rationalen Ausdruck:

$$
\xi=R\left(\theta_{1}, \theta_{2}, \ldots, \theta_{n}\right)
$$

mit Koeffizienten aus $\Re$ gegeben ist, eine ,natürliche Irrationalität" der 
Gleichung $f(z)=0$. Demgegenüber heißt eine beim AuflösungsprozeB der Gleichung etwa zu benutzende Zahl, die dem Körper $\left(\Omega, \theta_{1}, \theta_{2}, \ldots \theta_{n}\right)$ nicht angehört, eine ,akzessorische Irrationalität" der Gleichung $f(z)=0$. Wir übertragen die Bezeichung der "Zugehörigkeit" von den Transformationsgruppen $G_{\hat{p}}$ und Zahlen $\xi$ auf die Untergruppen der Galoisschen Gruppe und die natürlichen Irrationalitäten der Gleichung. Den eben bewiesenen Satz können wir dann auch in folgende Gestalt kleiden: $Z u$ jeder Untergruppe $G_{\mu}$ der Galoisschen Gruppe $G_{m}$ unserer Gleichung $f(z)=0$ gibt es unendlich viele zugehörige natürliche Irrationalitäten; in einer unter ihnen ist jede andere, sowie jede natürliche Irrationalität, die zu einer die $G_{\mu}$ umfassenden Untergruppe gehört, rational mit Koeffizienten aus $\Re$ darstellbar.

\section{§ 12. Die rationalen Resolventen einer Gleichung $f(z)=0$.}

Irgendeine Zahl $\xi=R\left(\theta_{1}, \theta_{2}, \ldots, \theta_{n}\right)$ des Galoisschen Körpers $\left(\Omega, \theta_{1}, \theta_{2}, \ldots, \theta_{n}\right)$ genügt einer irreduzibelen Gleichung in $\Omega$ :

$$
g(w)=0
$$

des Grades $t$, die wir als eine „rationale Resolvente" der Gleichung $f(z)=0$ bezeichnen. Die $t$ Wurzeln der Resolvente $\xi_{1}=\xi, \xi_{2}, \ldots, \xi_{t}$ sind Zahlen des genannten Galoisschen Körpers, die zu den gleichberechtigten Untergruppen $G_{\mu}, V_{1} \cdot G_{\mu} \cdot V_{1}^{-1}=G_{\mu}^{\prime}, \ldots, V_{t-1} \cdot G_{\mu} \cdot V_{t-1}^{-1}=G_{\mu}^{(t-1)}$ der Ordnung $\mu$ gehören mögen. Das Produkt der Gruppenordnung $\mu$ und des Grades $t$ der Resolvente ist gleich $m$. Die im Extremfalle $t=m, \mu=1$ für primitive Zahlen $\zeta$ des Galoisschen Körpers eintretenden Resolventen sind natürlich die Galoisschen Resolventen der gegebenen Gleichung $f(z)=0$.

Die $t$ gleichberechtigten Untergruppen $G_{\mu}, G_{\mu}^{\prime}, \ldots, G_{\mu}^{(t-1)}$ brauchen keineswegs alle voneinander verschieden zu sein, können sogar alle einander gleich sein, nämlich wenn $G_{\mu}$ eine ausgezeichnete Untergruppe ist. Jedenfalls ist der Durchschnitt:

$$
G_{l}=D\left(G_{\mu}, G_{\mu}^{\prime}, G_{\mu}^{\prime \prime}, \ldots, G_{\mu}^{(t-1)}\right)
$$

eine ausgezeichnete Untergruppe, deren Ordnung wir $l$ nennen. Diese $G_{l}$ setzt sich zusammen aus allen Transformationen der Gesamtgruppe $G_{m}$, die jede einzelne der $t$ Zahlen $\xi_{1}, \xi_{2}, \ldots, \xi_{t}$ in sich überführen. Den Index der Untergruppe $G_{l}$ bezeichnen wir durch $\frac{m}{l}=\bar{m}$, die Zerlegung $\operatorname{der} G_{m}$ in Nebengruppen entsprechend $\operatorname{der} G_{l}$ sei durch:

$$
G_{m}=G_{l}+U_{1} \cdot G_{l}+U_{2} \cdot G_{l}+\cdots+U_{\bar{m}-1} \cdot G_{l}
$$

gegeben. Alle Transformationen der einzelnen Nebengruppe $U_{i} \cdot G_{l}$ ergeben die gleiche, etwa durch $T_{i}$ zu bezeichnende Permutation der 
$\xi_{1}, \xi_{2}, \ldots, \xi_{t}$, wobei $T_{0}=1$ die identische Permutation ist. Es ergibt sich der Satz: Gegenüber allen $m$ Transformationen der $G_{m}$ erfahren die $t$ Wurzeln $\xi_{1}, \xi_{2}, \ldots, \xi_{t}$ der rationalen Resolvente (1) im ganzen $\bar{m}$ Permutationen $T_{0}=1, T_{1}, \ldots, T_{\bar{m}_{-1}}$, die eine mit der "Quotientengruppe" $G_{m} / G_{l}$ isomorphe und also wie diese auf die $G_{m}$ 1-l-deutig homomorph bezogene Gruppe $G_{\bar{m}}$ bilden. Wir bezeichnen diese Permutationsgrappe $G_{\bar{m}}$ gleich selbst als Quotientengruppe $G_{m} / G_{l}$ von $G_{m}$ und $G_{l}$.

Ist $l=1$ und also $\bar{m}=m$, so erhalten wir in der Permutationsgruppe $G_{\bar{m}}$ eine neue Darstellung unserer $G_{m}$ und erkennen in dieser Permutationsgruppe einfach die "Galoissche Gruppe" der Resolvente (1).

Besonders wichtig aber ist nun der Fall, daß $l>1$ ist. Wir bilden wie oben bei der Gleichung $f(z)=0$ jetzt für die Resolvente (1) den Galoisschen Körper:

$$
\bar{\Re}=\left(\Re, \xi_{1}, \xi_{2}, \ldots, \xi_{t}\right),
$$

den wir mittelst irgendeiner zur $G_{l}$ gehörenden Zahl $\bar{\eta}$ auch in der Gestalt $\bar{\Omega}=(\Re, \bar{\eta})$ darstellen können. Diese Zahl $\bar{\eta}$ genügt dann einer in $\Re$ ir reduzibelen "Normalgleichung“:

$$
\bar{F}(W)=0
$$

vom Grade $\bar{m}=\frac{m}{l}$, welche eine „Galoissche Resolvente" der Gleichung (1) ist; die Gruppe $G_{\bar{m}}$ der Transformationen des Körpers $\bar{\AA}$ in sich liefert in der Gestalt der Permutationsgruppe $G_{m} / G_{l}$ der $T_{0}=T_{1}, T_{2}, \ldots, T_{\bar{m}_{-1}}$ die „Galoissche Gruppe" der Gleichung (1).

Nicht minder wichtig sind die Folgerungen für die ursprüngliche Galoissche Resolvente $F(Z)=0$. Die Gruppe $G_{l}$ ist als Permutationsgruppe der $m$ Wurzeln von $F(Z)=0$ intransitiv und besitzt $\bar{m}$ Systeme der Intransitivität zu je $l$ Wurzeln. Ein beliebiges dieser Systeme sei $\eta_{1}, \eta_{2}, \ldots, \eta_{l}$. Die symmetrischen Grundfunktionen dieser $l$ Zahlen $\eta$ gehören zur $G_{l}$ und sind also im Körper $\bar{\AA}=(\Re, \bar{\eta}$ ) enthalten (S. 50 ). Die $\eta_{1}, \eta_{2}, \ldots, \eta_{l}$ sind also die Wurzeln einer Gleichung $H(Z)=0$ in $\bar{\AA}$ vom Grade $l$. Wir können leicht zeigen, daß diese Gleichung in $\bar{\Omega}$ irreduzibel ist. Sollte sie es nicht sein, so bezeichnen wir mit $h(Z)=0$ den irreduzibelen Bestandteil, dem $\eta_{1}$ genügt. Ersetzen wir die in den Koeffizienten von $h(Z)$ auftretende Zahl $\bar{\eta}$ durch ihren rationalen ganzen Ausdruck in $\left.\eta_{1}{ }^{1}\right)$, so entsteht aus $h\left(\eta_{1}\right)=0$ eine für $\eta_{1}$ gültige ,Gleichung in $\overline{\Omega^{\prime}}$. Diese Gleichung bleibt demnach bei allen $m$ Transformationen der $G_{m}$ richtig, und also insbesondere bei allen $l$ Transformationen $\operatorname{der} G_{l}$. Die letzteren aber lassen $\bar{\eta}$ unverändert und führen $\eta_{1}$ in $\eta_{1}, \eta_{2}, \ldots, \eta$ über. Man beachte noch, daß die Transformationen der einzelnen zu $G_{l}$

1) $\eta_{1}$ ist eine primitive Zahl des Körpers $(\Re, \eta)$. 
gehörenden Nebengruppe die Zahl $\bar{\eta}$ in eine ihrer konjugierten Zahlen überführt, das System $\eta_{1}, \eta_{2}, \ldots, \eta_{l}$ aber in eines der $\bar{m}$ Systeme der Intransitivität von $G_{l}$. Wir haben damit den Satz gewonnen: In dem nach Adjunktion von $\bar{\eta} z u \Omega$ entstehenden Körper $\bar{\Omega}=(\Omega, \bar{\eta})$ ist die ursprüngliche Galoissche Resolvente $F(Z)=0$ reduzibel und zerfällt in $\bar{m}$ irreduzibele Gleichungen in $\bar{\AA}$ vom Grade $l$, deren einzelne $H(Z)=0$ je eines der $\bar{m}$ genannten Systeme der Intransitivität zu Wurzeln hat; aus einer Gleichung $H(Z)=0$ gehen die übrigen hervor, wenn man die in den Koeffizienten von $H(Z)$ auftretende Zahl $\bar{\eta}$ durch ihre konjugierten Zahlen ersetzt.

Bilden wir für die einzelne in $\bar{\Omega}$ irreduzibele Gleichung $l^{\text {ten }}$ Grades $H(Z)=0$ den Galoisschen Körper $\left(\bar{\Omega}, \eta_{1}, \eta_{2}, \ldots, \eta_{l}\right)$, so ist dieser natürlich unser bisheriger Körper $(\boldsymbol{\Omega}, \eta)$ und kann auch als Körper $\left(\bar{\Re}, \eta_{1}\right)$ geschrieben werden. Nach S. $44 \mathrm{ff}$. werden die $l$ Transformationen dieses Körpers in sich dadurch gewonnen, daß wir $\eta_{1}$ der Reihe nach durch $\eta_{1}, \eta_{2}, \ldots, \eta_{l}$ ersetzen. Wir gelangen dabei zu den $l$ Permutationen der $\eta_{1}, \eta_{2}, \ldots, \eta_{l}$, die von $\operatorname{der} G_{l}$ für dieses System der Intransitivität geliefert werden. In dieser Gestalt einer Permutationsgruppe $l^{\text {ten }}$ Grades ist dann die $G_{l}$ wieder einfach transitiv: Die einzelne in $\bar{\AA}$ irreduzibele Gleichung $l^{\text {ten }}$ Grades $H(Z)=0$ ist eine "Normalgleichung", deren Galoissche Gruppe die als Permutationsgruppe $l^{\text {ten }}$ Grades der $\eta_{1}, \eta_{2}, \ldots, \eta_{l}$ geschriebene Gruppe $G_{5}$ ist.

Wegen der grundlegenden Wichtigkeit fassen wir die gewonnenen Ergebnisse nochmals zusammen: Ist $G_{l}$ eine ausgezeichnete Untergruppe der ursprünglichen Galoisschen Gruppe $G_{m}$, so genügt eine zur $G_{l}$ gehörende natürliche Irrationalität $\bar{\eta}$ von $f^{\prime}(z)=0$ einer in $\Re$ irreduzibelen Gleichung (5) des Grades $\bar{m}=\frac{m}{l}$, deren Galoissche Gruppe die Quotientengruppe $G_{m} / G_{l}$ ist; nach Adjunktion von $\bar{\eta} z u \Omega$ wird die ursprüngliche Galoissche Resolvente $F(Z)=0$ im Körper $\bar{\Re}=(\Re, \bar{\eta})$ reduzibel und liefert nach Zerlegung $\bar{m}$ in $\bar{\AA}$ irreduzibele, innerhalb $(\boldsymbol{\Omega}, \eta)$,konjugierte" Gleichungen $l^{\text {ten }}$ Grades $H(Z)=0$, die wieder "Normalgleichungen" sind, und für deren einzelne $G_{l}$ die Galoissche Gruppe ist.

\section{\$ 13. Auflösung einer algebraischen Gleichung $f^{\prime}(z)=0$.}

Am Schlusse von $\S 8$, S. 43, wurde die Bedeutung der Galoisschen Resolvente $F(Z)=0$ für die algebraische Gleichung $n^{\text {ten }}$ Grades $f(z)=0$ dargelegt. Wir nehmen diese Gleichung in einem vorgelegten Körper $\Omega$ als irreduzibel an. ${ }^{1}$ ) Das Problem, die Gleichung vollständig zu lösen, d. h. alle Wurzeln anzugeben oder doch auf Grund „rationaler" Rechnungen

1) Andernfalls würden wir mit dem einzelnen irreduzibelen Bestandteile von $f(z)=0$ arbeiten. 
finden zu können, erfordert alsdann die.Gewinnung des Körpers $(\Omega, \eta)$, in dem in der Tat alle Wurzeln $\theta_{1}, \theta_{2}, \ldots, \theta_{n}$ von $f(z)=0$,rational bekannt" sind.

Die in $\$ 12$ aufgestellten Sätze im Verein mit den Entwicklungen von S. 12 ff. über die „Kompositionsreihe“ einer Gruppe $G_{m}$ zeigen, wie die Lösung dieses Problems zu vollziehen ist: Wir gewinnen durch Auflösung einer Kette ron „Normalgleichungen" je mit „einfachen" Galoisschen Gruppen die Erweiterung von $\Omega$ zum Körper. $(\Omega, \eta)$, in dem $f(z)=0$, wie auch die Galoissche Resolvente $F(Z)=0$, in lauter "lineare" Gleichungen reduzibel sind.

Die Galoissche Gruppe $G_{m}$ unserer Gleichung besitze nämlich als eine Kompositionsreihe die Gruppen:

$$
G_{m}, G_{m_{1}}, G_{m_{2}}, \ldots, G_{1}
$$

und als zugehörige Indexreihe:

$$
\frac{m}{m_{1}}=t_{1}, \quad \frac{m_{1}}{m_{2}}=t_{3}, \quad \frac{m_{2}}{m_{3}}=t_{3}, \ldots,
$$

wobei das Produkt der Indizes $t_{1} \cdot t_{2} \cdot t_{3} \cdots=m$ ist. Die zugehörigen Quotientengruppen:

$$
G_{m} / G_{m_{1}}=G_{t_{1}}, \quad G_{m_{1}} / G_{m_{2}}=G_{t_{2}}, \ldots
$$

sind einfach, d.h. keine der Gruppen $G_{t}$ enthält (von $G_{t}$ und $G_{1}$ abgesehen) eine ausgezeichnete Untergruppe. Da die Berechnung einer Wurzel $\eta$ von $F(Z)=0$ unser Ziel ist, so gehen wir nach dem Schlußsatze von $\S 12$ so vor: Wir nehmen für die daselbst $G_{l}$ genannte Gruppe die ,gröBte" ausgezeichnete Untergruppe $G_{m_{1}}$. Die zugehörige Gleichung (5) S. 53 möge jetzt durch $f_{1}(w)=0$ bezeichnet werden; sie hat den Grad $t_{1}$ und ist eine "Normalgleichung“ mit „einfacher" Galoisscher Gruppe $G_{t_{1}}$. Es genügt, eine beliebige Wurzel $\eta_{1}$ dieser Normalgleichung zu berechnen, dereu Adjunktion zu $\Omega$ den Körper $\Omega_{1}=\left(\Omega, \eta_{1}\right)$ liefere. In $\Omega_{1}$ ist nun $F(Z)=0$ reduzibel, und zwar in $t_{1}$ irreduzibele Gleichungen $m_{1}^{\text {ten }}$ Grades zerfällbar. Eine beliebige unter ihnen sei $F_{1}(Z)=0$; sie ist wieder eine Normalgleichung und hat $G_{m_{1}}$ zur Galoisschen Gruppe. Nun wiederholen wir den gleichen ProzeB, indem wir in $G_{m_{1}}$ die gröBte ausgezeichnete Untergruppe $G_{m_{2}}$ aufgreifen usw. Nach einer endlichen Anzahl solcher Schritte gelangen wir bis zur $G_{1}$ und damit zur Kenntnis der gesuchten Wurzel $\eta$ : Die Lösung der Gleichung $f(z)=0$ wird geleistet, indem man für die endliche Kette von Normalgleichungen:

$$
f_{1}(w)=0, f_{2}(w)=0, \quad f_{3}(w)=0, \ldots
$$

mit den die Bedingung $t_{1} \cdot t_{2} \cdot t_{3} \cdots=m$ erfüllenden Graden $t_{1}, t_{2}, t_{3}, \ldots$ je eine Wurzel $\eta_{1}, \eta_{2}, \ldots$ berechnet $\left.{ }^{1}\right)$; die einzelne Normalgleichung $f_{v}(w)=0$

1) Beiläufig bemerken wir, daB die Berechnung einer Wurzel der einzelnen 
ist irreduzibel im Körper $\AA_{v-1}=\left(\Re, \eta_{1}, \eta_{2}, \ldots, \eta_{v-1}\right)$ und hat die einfache Galoissche Gruppe $G_{t_{\nu}}$. Hat man die letzte Gruppe $G_{1}$ der Reihe (1) erreicht, so liegt für die gesuchte Wurzel $\eta$ eine "lineare" Gleichung vor, d. $h . \eta$ ist bekannt. Der Satz von S. 13 über die Kompositionsreihen einer Gruppe $G_{m}$ aber liefert uns noch das Ergebnis: Irgendeine andere Kompositionsreihe der Galoisschen Gruppe $G_{m}$ unserer Gleichung $f(z)=0$ liefert eine Kette von Normalgleichungen, die in der Anzahl sowie (abgesehen von der Reihenfolge) in den Graden und den Galoisschen Gruppen mit den Gleichungen (4) übereinstimmen. Dabei gelten zwei isomorphe Gruppen als gleich. Hiermit sind die im Mittelpunkte der Galoisschen Gleichungstheorie stehenden Sätze gewonnen. ${ }^{1}$ )

\section{\$ 14. Beispiel der Kreisteilungsgleichungen. ${ }^{2}$ )}

Ein einfaches Beispiel zur Erläuterung der Galoisschen Theorie liefern die Kreisteilungsgleichungen. Diejenige für den $n^{\text {ten }}$ Teilungsgrad lautet zunächst $z^{n}=1$, ihre Lösungen sind die $n$ Wurzeln $n^{\text {ton }}$ Grades der Einheit:

$$
\varepsilon_{1}=e^{\frac{2 i \pi}{n}}, \quad \varepsilon_{2}=e^{\frac{4 i \pi}{n}}, \quad \varepsilon_{3}=e^{\frac{6 i \pi}{n}}, \ldots, \varepsilon_{n-1}=e^{\frac{2(n-1) i \pi}{n}}, \quad \varepsilon_{n}=1,
$$

die als die aufeinanderfolgenden Potenzen $\varepsilon, \varepsilon^{2}, \ldots, \varepsilon^{n-1}, \varepsilon^{n}=1$ der ersten unter ihnen $\varepsilon=\varepsilon_{1}$ darstellbar sind. Zugrunde zu legen ist der rationale Körper $\Re$. Der bei der Auflösung der Gleichung zu erreichende Galoissche Körper $\left(\Re, \varepsilon_{1}, \varepsilon_{2}, \ldots, \varepsilon_{n}\right)$ ist auch bereits als Körper $(\Re, \varepsilon)$ darstellbar und heißt der "Kreisteilungskörper" für den $n^{\text {ten }}$ Teilungsgrad.

Unter den $n$ Wurzeln $\varepsilon_{k}=\varepsilon^{k}$ sind primitive Zahlen von $(\Re, \varepsilon)$ alle und nur die, bei denen $k$ teilerfremd gegen $n$ ist. Ist die Zerlegung von $n$ in Primfaktoren durch $n=p_{1}^{\gamma_{1}} \cdot p_{2}^{\gamma_{2}} \ldots$ gegeben, so hat man bekanntlich:

$$
\varphi(n)=n\left(1-\frac{1}{p_{1}}\right)\left(1-\frac{1}{p_{2}}\right) \cdots
$$

mod $n$ inkongruente, gegen $n$ teilerfremde Zahlen $k$, so daß im ganzen $\varphi(n)$ unter den Einheitswurzeln (1) primitive Zahlen von $(\Re, \varepsilon)$ sind.

Hilfsgleichung $f_{\nu}(w)=0$ auch ersetzt werden kann durch die vollständige Auflösung irgendeiner rationalen Resolvente, die die Normalgleichung $f_{\nu}(w)=0$ zur Galoisschen Resolvente hat.

1) Die im Lösungsprozeb der Gleichung vollzogenen Adjunktionen beziehen sich ausschließlich auf „natürliche Irrationalitäten" von $f(z)=0$. Die Heranziehung irgendwelcher „akzessorischer Irrationalitäten“ vermag den Prozeß nicht zu vereinfachen, insofern auch dann unter den zu lösenden Hilfsgleichungen sich immer wieder solche der Gruppen $G_{t_{1}}, G_{t_{2}}, \ldots$ einstellen. Man vgl. hierüber Weber, a. a. O., Bd. 1, S. 555 ff. sowie Loewy, a. a. O., S. 303.

2) Wegen genauerer Begründung der in den nächsten vier Paragraphen gegegebenen Ausführungen ist wieder auf Weber, a. a. 0., Bd. 1, S. $452 \mathrm{ff}$. und 564 ff. zu verweisen. 
Sie heißen die „primitiven $n^{\text {ten }}$ Einheitswurzeln" und genügen einer in $\Re$ irreduzibelen Gleichung $\varphi^{\text {ten }}$ Grades:

$$
z^{\varphi}+a_{1} z^{\varphi-1}+a_{2} z^{\varphi-2}+\cdots+a_{\varphi}=0,
$$

welche die ,irreduzibele Kreisteilungsgleichung" für den $n^{\text {ten }}$ Teilungsgrad heibt. ${ }^{1}$ )

Da jede der $\varphi(n)$ Lösungen von (3) rational in einer unter ihnen $\varepsilon=\varepsilon_{1}$ darstellbar ist, so haben wir in der irreduzibelen Gleichung (3) nach S. $41 \mathrm{ff}$. eine "Normalgleichung“" und in $(\Re, \varepsilon)$ einen „Galoisschen Körper" des Grades $\varphi(n)$. Die Transformationen $S$ und $S^{\prime}$ des Galoisschen Körpers $(\Re, \varepsilon)$ in sich mögen $\varepsilon$ in $\varepsilon^{\alpha}$ bzw. $\varepsilon^{\Im}$ überführen. Dann wird sowohl durch $S \cdot S^{\prime}$ als auch durch $S^{\prime} \cdot S$ die Wurzel $\varepsilon^{k}$ in $\varepsilon^{\alpha \beta \cdot k}$ übergeführt. Es gilt demgemäß $S \cdot S^{\prime}=S^{\prime} \cdot S$, so daß wir den Satz gewinnen: Die irreduzibele Kreisteilungsgleichung (3) ist eine "Normalgleichung", deren Galoissche Gruppe $G_{\varphi(n)}$ eine „,kommutative" oder „Abelsche Gruppe" ist.

Nach dem Hauptsatze von S. 17 über die Kompositions- und Indexreihen Abelscher Gruppen haben wir nun die Gruppenordnung $\varphi(n)$ in ibre Primfaktoren zu zerlegen:

$$
\varphi(n)=q_{1} \cdot q_{2} \cdot q_{3} \ldots,
$$

wobei jede mehrfach vorkommende Primzahl $q$ entsprechend oft hintereinander als Faktor zu setzen ist. Die Reihe der Primzahlen $q_{1}, q_{2}, q_{3}, \ldots$ liefert dann eine Indexreihe der Gruppe $G_{\varphi(n)}$. Die Lösung der irreduzibelen Kreisteilungsgleichung (3) für den $n^{\text {ten }}$ Teilungsgrad erfordert demnach die Lösung einer Kette von normalen Hilfsgleichungen, deren Grade die Primzahlen $q_{1}, q_{2}, q_{3}, \ldots$ sind, und deren Galoissche Gruppen als Gruppen der Primzahlordnungen $q_{1}, q_{2}, q_{3}, \ldots$ durchweg zyklisch sind. Auf Gleichungen mit zyklischen Gruppen kommen wir im nächsten Paragraphen zurück. Vorerst seien noch folgende Bemerkungen über die Kreisteilungsgleichungen angeschlossen.

Bekanntlich ist die wirkliche Durchführung der Lösung der Kreisteilungsgleichnngen bereits lange vor Galois durch GauB entwickelt. Ist $n=n_{1} \cdot n_{2}$ das Produkt zweier teilerfremder Zahlen $n_{1}, n_{2}$, so gilt:

$$
\left(\Re, e^{\frac{2 i \pi}{n}}\right)=\left(\Re, e^{\frac{2 i \pi}{n_{1}}}, e^{\frac{2 i \pi}{n_{2}}}\right)
$$

d. h. wir erhalten den Kreisteilungskörper für den $n^{\text {ten }}$ Teilungsgrad durch

$$
2 i \pi \quad 2 i \pi
$$

gleichzeitige Adjunktion von $e^{\frac{2 n_{1}}{n_{1}}}$ und $e^{\frac{2 \pi}{n_{2}}}$. Es bestehen nämlich erstens die Gleichungen:

$$
e^{\frac{2 i \pi}{n_{1}}}=e^{n_{2} \cdot \frac{2 i \pi}{n}}, \quad e^{\frac{2 i \pi}{n_{2}}}=e^{n_{2} \cdot \frac{2 i \pi}{n}},
$$

1) Über die Beweise der Irreduzibilität der Gleichung (3) im rationalen Körper sehe man auch die geschichtlichen Angaben bei Loewy, a. a. O., S. 314. 
sowie zweitens, wenn $\alpha$ und $\beta$ zwei die Bedingung $\alpha n_{3}-\beta n_{1}=1$ erfüllende rationale ganze Zahlen sind, die Darstellung:

$$
e^{\frac{2 i \pi}{n}}=e^{\alpha \cdot \frac{2 i \pi}{n_{1}}} \cdot e^{-\beta \cdot \frac{2 i \pi}{n_{2}}},
$$

woraus die Gleichung (5) hervorgeht. Die Auflösung der Gleichung (3) für den Teilungsgrad $n$ kann demnach in der Weise vollzogen werden, daß man gesondert voneinander die irreduzibelen Kreisteilungsgleichungen für die Teilungsgrade $n_{1}$ und $n_{2}$ löst. Die wirkliche Durchführung der Auflösung darf man demnach auf den Fall beschränken, da $B$ der Teilungs$\operatorname{grad} n$ eine Primzahlpotenz $n=p^{v}$ ist. Hier gilt:

$$
\varphi\left(p^{v}\right)=p^{v-1}(p-1)=q_{1} \cdot q_{2} \cdots p^{\nu-1},
$$

wo $q_{1} \cdot q_{2} \cdots$ die Primfaktorenzerlegung von $(p-1)$ ist. Wir haben also Normalgleichungen der Grade $q_{1}, q_{2}, \ldots$ und $(\nu-1)$ Normalgleichungen $p^{\text {ten }}$ Grades zu lösen. Die Gleichungen der Grade $q_{1}, q_{2}, \ldots$ dienen zur Berechnung der Einheitswurzeln $p^{\text {ten }}$ Grades. Die weiteren $(\nu-1)$ Gleichung $p^{\text {ten }}$ Grades sind dann einfach die $(\nu-1)$ „reinen“ Gleichungen:

$$
z^{p}=e^{\frac{2 i \pi}{p}}, \quad z^{p}=e^{\frac{2 i \pi}{p^{2}}}, \ldots, z^{p}=e^{\frac{2 i \pi}{p^{\nu-1}}}
$$

wobei mittelst der Lösung jeder Gleichung die rechte Seite der nächsten Gleichung gewonnen wird. Hier handelt es sich also um Gleichungen, die durch "Wurzelziehungen" (Einteilungen von Winkeln in $p$ gleiche Teile) lösbar sind. G a u B sagt (in Art.336 der „Disquisitiones arithmeticae“) von diesen Gleichungen, dab sie sich in keiner Weise auf Gleichungen niederen Grades zurückführen lassen. ${ }^{1}$ )

Demgegenüber bezieht sich der Hauptinhalt der Gaußschen Theorie auf die Lösung der irreduzibelen Kreisteilungsgleichung:

$$
z^{p-1}+z^{p-2}+\cdots+z+1=0
$$

für die Primzahl $n=p$. Der erste Teil der Theorie führt zu der Kette der Gleichungen, welche in der späteren allgemeinen Galoisschen Theorie den Hilfsgleichungen (4) S. 55 entsprechen. Daran reiht sich sodann die wirkliche Auflösung der Hilfsgleichungen, die auf „reine“ Gleichungen zurückgeführt werden und demnach wieder durch „Wurzelziehungen“ lösbar sind.

\section{\$ 15. Zyklische Gleichungen.}

Nicht nur für die Kreisteilungsgleichungen, sondern auch für die gleich zu besprechende allgemeinere Klasse der „Abelschen Gleichungeu“

1) Wir kommen auf Gleichungen dieser Art im nächsten Paragraphen zurück. 
sind die Gleichungen mit zyklischer Gruppe von grundlegender Bedeutung. Wir stellen folgende Erklärung auf: Eine in einem Körper $\Re$ ivreduzibele Gleichung $n^{\text {ien }}$ Grades $f(z)=0$, deren Galoissche Gruppe zyklisch ist, soll selbst eine ,zyklische" Gleichung genannt werden. Die Galoissche Gruppe soll sich also aus einer einzigen Permutation $S$ erzeugen lassen. Zerlegen wir diese Permutation $S$ nach S. 19 in ihre Zyklen, so kann nur ein Zyklus vorliegen, der alle $n$ Wurzeln der Gleichung verbindet, da andernfalls die Gruppe intransitiv sein würde. Wir ordnen die Wurzeln, die wir übrigens hier zweckmäBig durch $\theta_{0}, \theta_{1}, \theta_{2}, \ldots, \theta_{n-1}$ bezeichnen, in der Art an, daB:

$$
S=\left(\begin{array}{l}
\theta_{0}, \theta_{1}, \theta_{2}, \ldots, \theta_{n-1} \\
\theta_{1}, \theta_{2}, \theta_{3}, \ldots, \theta_{0}
\end{array}\right)
$$

wird. Wir erhalten damit den Satz: Die Galoissche Gruppe einer irreduzibelen zyklischen Gleichung $n^{\text {ten }}$ Grades ist eine Gruppe $G_{n}$ von der $n^{\text {ten }}$ Ordnung, so daß jede zyklische Gleichung $f(z)=0$ eine Normalgleichung ist.

Für den vorliegenden $\mathrm{Z}$ weck ist es ausreichend, den Grad $n$ der zyklischen Gleichung gleich einer Primzahl $p$ zu nehmen, wodurch die Schlußweise wesentlich erleichtert wird. Wir bemerken dann zunächst, da $\beta$ die primitive $p^{\text {te }}$ Einheitswurzel $\varepsilon=e^{\frac{2 i \pi}{p}}$ entweder in $\mathcal{\AA}$ enthalten ist oder eine alizessorische Irrationalität von $f(z)=0$ darstellt. Im Galoisschen Körper $(\Omega, \theta)$ vom Primzahlgrade $p$ sind nämlich, abgesehen von den Zahlen des Körpers $\Omega$, nur primitive Zahlen enthalten (vgl. S. 41), die als solche irreduzibelen Gleichungen $p^{\text {ten }}$ Grades genügen; $\varepsilon$ aber genügt einer Gleichung $(p-1)^{\text {ten }}$ Grades in $\Omega$. Ist $\varepsilon$ nicht in $\Omega$ enthalten, so bleibt die Gleichung $f(z)=0$ auch im Körper $(\Omega, \varepsilon)$ irreduzilel. Jede Zerlegung $f(z)=\varphi(z) \cdot \psi(z)$ mit zwei Faktoren $\varphi(z), \psi(z)$ von Graden $<n$ und den höchsten Koeffizienten 1 liefert Funktionen $\varphi(z), \psi(z)$, deren Koeffizienten im Körper $p^{\text {ten }}$ Grades $(\Omega, \theta)$, aber noch nicht alle in $\Omega$ enthalten sind. Jeder solche noch nicht in $\Re$ enthaltene Koeffizient kann aber als „primitive" Zahl des Körpers $(\Omega, \theta)$ nicht in Körper $(p-1)^{\text {ten }}$ Grades $(\Re, \varepsilon)$ enthalten sein.

Entsprechend unseren ursprünglichen Festsetzungen hat der Körper $\Re$ nur den Bedingungen zu genügen, daß die Koeffizienten von $f(z)=0$ in ibm enthalten sind. Diese Forderung befriedigt mit $\Omega$ auch der Körper $(\Re, \varepsilon)$. Sollte demnach die Einheitswurzel $\varepsilon$ nicht ohnehin schon in $\Re$ auftreten, so wollen wir sie adjungieren und fortan den gleich selbst wieder durch $\Re$ zu bezeichnenden Körper $(\Re, \varepsilon)$ der Untersuchung zugrunde legen. Auf dieser Grundlage können wir die Lösung der zyklischen Gleichung in folgender Art durchführen. Wir bilden, unter $\alpha$ eine der Zahlen $0,1,2, \ldots, p-1$ verstanden, den Ausdruck:

$$
\xi_{\alpha}=\theta_{0}+\varepsilon^{-\alpha} \theta_{1}+\varepsilon^{-2 \alpha} \theta_{2}+\cdots+\varepsilon^{-(p-1) \alpha} \theta_{p-1},
$$


der eine "Lagrangesche Resolvente" ${ }^{\text {1) }}$ heißt und (nach Adjunktion von $\varepsilon$ ) zu den natürlichen Irrationalitäten der Gleichung $f(z)=0$ gehört. Bei Ausübung der erzeugenden Permutation (1) der Galoisschen Gruppe $G_{p}$ wird $\xi_{\alpha}$ übergeführt in $\varepsilon^{\alpha} \xi_{\alpha}$. Durch wiederholte Ausübung von $S$ findet man als die $p$ mit $\xi_{\alpha}$ konjugierten Zahlen:

$$
\xi_{\alpha}, \varepsilon^{\alpha} \xi_{\alpha}, \varepsilon^{2 \alpha} \xi_{\alpha}, \varepsilon^{3 \alpha} \xi_{\alpha}, \ldots ; \varepsilon^{(p-1) \alpha} \xi_{\alpha} .
$$

Die $p$ Zahlen $1, \varepsilon^{\mu}, \varepsilon^{2 \mu}, \ldots, \varepsilon^{(p-1) \mu}$ sind für $\mu=0$ alle gleich 1 , für eine der Zahlen $\mu=1,2,3, \ldots, p-1$ stellen sie die $p$ Wurzeln $p^{\text {ten }}$ Grades der Einheit in irgendeiner Reihenfolge dar und geben dann die Summe 0. Die für $\alpha=0,1,2, \ldots, p-1$ zu bildenden Gleichungen (2) lassen sich daraufhin in folgender Art nach $\theta_{0}, \theta_{1}, \ldots, \theta_{p-1}$ auflösen:

$$
\theta_{\beta}=\frac{1}{p} \sum_{\alpha=0}^{p-1} \varepsilon^{\alpha \beta} \xi_{\alpha}, \quad \beta=0,1,2, \ldots, p-1 .
$$

Die Zahl $\xi_{0}$ ist bereits in $\Re$ enthalten. Würde dasselbe von allen Zahlen $\zeta$ gelten, so würden zufolge (4) auch die $\theta_{\beta}$ in $\mathfrak{R}$ enthalten sein, während doch keine einzige Wurzel der irreduzibelen Gleichung $f(z)=0$ eines Grades $p>1$ in $\Re$ vorkommt. Also gibt es unter den Zahlen (2) mindestens eine primitive und eben deshalb vón 0 verschiedene Zahl des Körpers $(\Re, \theta)$. Die $p^{\text {te }}$ Potenz jeder Zahl $\xi_{\alpha}$ ist zufolge (3) nur mit sich selbst konjugiert und stellt dieserhalb eine Zahl $c_{\alpha}$ aus $\Omega$ dar: Jede der $p$ Zahlen $\xi_{\alpha}$ genïgt einer, ,reinen" Gleichung in $\Omega$ :

$$
w^{p}=c_{\alpha}
$$

vom Grade p, und es findet sich unter diesen Gleichungen mindestens eine, die in $\Re$ irreduzibel ist, und für die $c_{\alpha} \neq 0$ ist.

Nennen wir eine beliebige unter den Wurzeln dieser reinen Gleichung $\xi=\sqrt[p]{c_{\alpha}}$, so gilt $(\Re, \theta)=(\Re, \zeta)$, und es ist jede Wurzel $\theta_{\beta}$ unserer zyklischen Gleichung in der Gestalt:

$$
\theta_{\beta}=c_{\beta, 0}+c_{\beta, 1} \xi+c_{\beta, 2} \xi^{2}+\cdots+c_{\beta, p-1} \xi^{p-1}
$$

mit Koeffizienten $c$ aus $\Omega$ darstellbar. Wir haben also den Satz gewonnen: Eine zyklische Gleichung des Primzahlgrades $p$ ist nötigenfalls nach Adjunktion der Einheitswurzel $p^{\text {ten }}$ Grades $\varepsilon$ mittelst einer einzigen Wurzelziehung $p^{\text {ten }}$ Grades lösbar.

Dieser Satz begründet die am Schlusse des vorigen Paragraphen über die Kreisteilungsgleichungen gemachten Angaben näher. Die zur Berechnung der Einheitswurzeln $p^{\text {ten }}$ Grades zu lösenden Hilfsgleichungen sind zyklische Gleichungen der Grade $q_{1}, q_{2}, \ldots$ Jede dieser Gleichungen

1) Hier bedeutet also der Ausdruck „Resolvente“" nicht eine Gleichung für eine natürliche Irrationalität, sondern eine besonders gewählte Irrationalität dieser Art selbst. 
ist durch eine einzige Wurzel $q^{\text {ten }}$ Grades lösbar, wobei freilich der vorstehenden Theorie zufolge eine $q^{\text {to }}$ Einheitswurzel zu adjungieren ist. Schon hieraus folgt ohne näheres Eingehen auf die Gaußsche Theorie durch den Schluß der vollständigen Induktion, da $\beta$ die Kreisteilungsgleichungen und mit ihnen jedenfalls auch alle zyklischen Gleichungen von Primzahlgraden allein durch Wurzelziehungen (und rationale Rechnungen) lösbar sind.

\section{\$ 16. Abelsche Gleichungen.}

Eine in einem Körper $\Re$ irreduzibele Gleichung $n^{\text {ten }}$ Grades, deren Wurzeln wir etwa wieder $\theta=\theta_{0}, \theta_{1}, \theta_{2}, \ldots, \theta_{n-1}$ nennen, heißt eine "Abelsche Gleichung", wenn ihre Galoissche Gruppe eine kommutative oder Abelsche ist. Da die Gruppe transitiv ist, so können wir $n$ Permutationen $S_{0}=1, S_{1}, \ldots, S_{n-1}$ aus ihr entnehmen, von denen $S_{k}$ die Wurzel $\theta_{0}$ in $\theta_{k}$ überführt. Diese $n$ Permutationen bilden bereits die ganze Galoissche Gruppe der Gleichung. Es sei nämlich $G_{\mu}$ die Untergruppe aller Permutationen, die $\theta_{0}$ in sich überführen. Dann bilden diejenigen Permutationen, die eine beliebige Wurzel $\theta_{k}$ in sich überführen, die mit $G_{\mu}$ gleichberechtigte Untergruppe $S_{k} \cdot G_{\mu} \cdot S_{k}^{-1}$. Da aber jede Untergruppe einer Abelschen Gruppe ausgezeichnet ist, so ist $S_{k} \cdot G_{\mu} \cdot S_{k}^{-1}=G_{\mu}$, d. h. die Permutationen der $G_{\mu}$ führen jede Wurzel der Gleichung in sich über, so daß $G_{\mu}=G_{1}$ nur aus der identischen Permutation $S_{0}=1$ besteht. Führt neben $S_{k}$ auch $S_{k}^{\prime}$ die Wurzel $\theta_{0}$ in $\theta_{k}$ über, so transformiert $S_{k}^{-1} \cdot S_{k}^{\prime}$ die Wurzel $\theta_{0}$ in sich, so daß $S_{k}^{-1} \cdot S_{k}^{\prime}=1$ und also $S_{k}^{\prime}=S_{k}$ gilt. Es gibt also eine und nur eine Permutation in der Gruppe, die $\theta_{0}$ in eine beliebig vorgeschriebene Wurzel $\theta_{k}$ überführt. Damit ist der Satz bewiesen: Die Galoissche Gruppe einer Abelschen Gleichung $n^{\text {ten }}$ Grades ist eine einfach transitive Gruppe $G_{n}$ der Ordnung $n$; eine Abelsche Gleichung ist also stets eine Normalgleichung, und der zugehörige Galoissche Körper ist der durch $(\Omega, \theta)$ gegebene Körper $n^{\text {ten }}$ Grades.

Jede Wurzel $\theta_{k}$ der Abelschen Gleichung ist in einer ersten unter ibnen $\theta$ in der Gestalt:

$$
\theta_{k}=c_{k, 0}+c_{k, 1} \theta+c_{k, 2} \theta^{2}+\cdots+c_{k, n-1} \theta^{n-1}
$$

mit Koeffizienten $c$ aus $\Re$ darstellbar. Die in (1) rechts stehende Funktion, die durch $R_{k}$ bezeichnet werden möge, liefert die Wurzel $\theta_{k}$, in welche $\theta$ bei der Permutation $S_{k}$ übergeht. Üben wir zuerst $S_{k}$ und dann $S_{l}$ aus, so gehe $\theta$ in $\theta_{m}=R_{k}\left(R_{l}(\theta)\right)$ über. Da nun $S_{k} \cdot S_{l}=S_{l} \cdot S_{k}$ ist, so folgt: Je zwei der bei unserer Abelschen Gleichung auftretenden rationalen ganzen Funktionen $R$ befriedigen die Bedingung:

$$
R_{k}\left(R_{l}(\theta)\right)=R_{l}\left(R_{k}(\theta)\right) .
$$

Bei der Zusammensetzung zweier Funktionen $R$ hat man die zunächst 
auftretenden Potenzen von $\theta$ mit Exponenten $\geq n$ mittelst der Gleichung $f(\theta)=0$ auf solche mit Exponenten $<n$ zu reduzieren. In (2) rechts und links erscheinen dann nach Ausführung dieser Reduktionen identische Funktionen $(n-1)^{\text {ten }}$ Grades von $\theta$.

Umgekehrt gilt der Satz: Eine in $\mathfrak{S}$ irreduzibele Gleichung $n^{\text {ten }}$ Grades $f(z)=0$ ist eine Abelsche Gleichung, wenn jede ihrer Wurzeln $\theta_{k}$ in einer ersten unter ihnen $\theta$ rational in der Gestalt (1) darstellbar ist, und wenn diese rationalen Funktionen $R$ die Bedingung (2) erfüllen. Der Galoissche Körper $\left(\Re, \theta, \theta_{1}, \ldots, \theta_{n-1}\right)$ ist dann nämlich als Körper $(\Re, \theta)$ darstellbar und also vom Grade $n$, so daß $f(z)=0$ eine Normalgleichung ist. Die Galoissche Gruppe $G_{n}$ der Ordnung $n$ ist aber zufolge (2) kommutativ.

Nach S. 17 wird die Indexreihe der kommutativen Gruppe $G_{n}$ von den die Zahl $n$ zusammensetzenden Primfaktoren $t_{1}, t_{2}, t_{3}, \ldots$ geliefert. Die Gruppen $G_{t_{1}}, G_{t_{2}}, \ldots$ der Hilfsgleichungen sind demnach als Gruppen von Primzahlordnung durchweg zyklisch, so daß die Hilfsgleichungen selbst ohne Ausnahme zyklische Gleichungen von Primzahlgraden sind, deren Auflösung in $\S 15$ behandelt ist. Eine Gleichung, deren vollständige Auflösung ausschließlich durch rationale Rechnungen und Wurzelziehungen, also durch „Operationen der Algebra“ vollzogen werden kann, wird als eine „algebraisch lösbare Gleichung" bezeichnet. Nach S. $60 \mathrm{ff}$. sind die zyklischen Gleichungen von Primzahlgraden solche Gleichungen. Die vorstehende Betrachtung hat uns also zu dem Satze geführt: Die Abelschen Gleichungen, zu denen insbesondere die Kreisteilungsgleichungen und alle zyklischen Gleichungen gehören, sind algebraisch lösbar.

\section{§ 17. Algebraisch lösbare Gleichungen.}

Um die Gesamtheit aller algebraisch lösbaren Gleichungen zu überblicken, gehen wir noch etwas weiter, als es in $\$ 15$ geschah, auf die algebraische Bedeutung der Ausziehung einer Wurzel $n^{\text {ten }}$ Grades ein. Ist die Primfaktorenzerlegung von $n$ durch $n=p_{1} \cdot p_{2} \cdot p_{3} \cdots$ gegeben, so kommt das Ausziehen einer Wurzel $n^{\text {ten }}$ Grades auf eine Kette von Wurzelziehungen der Primzahlgrade $p_{1}, p_{2}, p_{3}, \ldots$ hinaus. Für die einzelne dieser Wurzelziehungen, d. h. für die Lösung einer „reinen“ Gleichung:

$$
z^{p}=c
$$

des Primzahlgrades $p$ gilt nun der folgende Satz: Die Gleichung (1) ist in einem die Zahl $c$ enthaltenden Körper $\mathfrak{\Omega}$ entweder irreduzibel oder $c$ ist die $p^{\text {te }}$ Potenz einer Zahl $c_{0}$ aus $\Omega$.

Soll es nämlich eine Zerlegung:

$$
z^{p}-c=\varphi(z) \cdot \psi(z)
$$

von $\left(z^{p}-c\right)$ in zwei Funktionen $\varphi(z)$ und $\psi(z)$ in $\mathfrak{\kappa}$ von niederem als 
$p^{\text {ten }}$ Grade geben, so sei $m$ der Grad von $\varphi(z)$. Die $m$ Wurzeln von $\varphi(z)=0$ gehören zu denen der Gleichung (1) und haben also alle die Gestalt $\varepsilon^{\lambda} \sqrt[p]{c}$, wo $\sqrt[p]{c}$ eine unter ihnen ist, $\varepsilon$ eine primitive $p^{\text {te }}$ Einheitswurzel bedeutet und $\lambda$ ganzzahlig ist. Das Produkt der $m$ Wurzeln der Gleichung $\varphi(z)=0$ ist als Absolutglied der Funktion $\varphi(z)$ eine in $\Re$ enthaltene Zahl $a$. Wir haben also:

$$
\varepsilon^{u}(\sqrt[p]{c})^{m}=a,
$$

wo auch $\mu$ eine ganze Zahl ist, und finden durch Erheben der letzten Gleichung zur $p^{\text {ten }}$ Potenz:

$$
c^{n n}=a^{p} .
$$

Da nun $m$ teilerfremd gegen $p$ ist, so kann man zwei ganze Zahlen $\alpha$ und $\beta$ angeben, die die Gleichung $\alpha m+\beta p=1$ befriedigen. Mit Benutzung von (3) ergibt sich hieraus:

$$
c=c^{\alpha m} \cdot c^{\beta p}=a^{\alpha p} \cdot c^{\beta p}=\left(a^{\alpha} \cdot c^{\beta}\right)^{p},
$$

so daß $c$ in der Tat als $p^{\text {te }}$ Potenz einer Zahl aus $\mathfrak{K}$ dargestellt ist.

Ist nun die Gleichung (1) reduzibel und also $c=c_{0}^{p}$, so sind die Wurzeln dieser Gleichung $c_{0}, \varepsilon c_{0}, \varepsilon^{2} c_{0}, \ldots, \varepsilon^{p-1} c_{0}$, so daß die Auflösung der Gleichung einfach auf die der Kreisteilungsgleichung für den $p^{\text {ten }} T e i-$ lungsgrad zurïckkommt.

Im Falle der Irreduzibilität sei $\theta=\sqrt[p]{c}$ eine beliebige unter den Wurzeln der Gleichung (1), die dann noch nicht in $\Re$ enthalten ist. Da sich die gesamten Wurzeln dann in der Gestalt:

$$
\theta_{0}=\theta, \theta_{1}=\varepsilon \theta, \theta_{2}=\varepsilon^{2} \theta, \ldots, \theta_{p-1}=\varepsilon^{p-1} \theta
$$

ansetzen lassen, so ist $\varepsilon$ sicher im Galoisschen Körper ( $\left(\theta, \theta, \theta_{1}, \ldots\right.$, $\left.\theta_{p-1}\right)$ enthalten. Sollte $\varepsilon$ noch nicht in $\Omega$ enthalten sein, so wird doch gleichwohl die Gleichung (1) auch noch im Körper $(\Re, \varepsilon)$ irreduzibel sein. Wäre sie nämlich reduzibel, so fänden wir wie oben, daB $c$ die $p^{\text {te }}$ Potenz einer Zahl aus $(\Omega, \varepsilon)$ sein müBte, und also wären alle Wurzeln der in $\Omega$ irreduzibelen Gleichung $p^{\text {ten }}$ Grades $(1)$ im $\operatorname{Körper}(\Omega, \varepsilon)$ vom $(p-1)^{\text {ten }}$ Grade enthalten, was unmöglich ist.

Wir adjungieren nun $\varepsilon$ nötigenfalls und bezeichnen den Körper $(\Omega, \varepsilon)$ gleich selbst wieder durch $\Omega$. Aus (4) erkennen wir in der Gleichung (1) etzt nach den Sätzen von S. 61 ff. sofort eine „Abelsche Gleichung", und zwar ist sie offenbar "zyklisch“, so daß die Wurzelziehung $p^{\text {ten }}$ Grades jetzt auf die Lösung einer zyklischen Gleichung des Primzahlgrades $p$ hinauskommt.

Da auch die Kreisteilungsgleichungen ihrerseits auf zyklische Gleichungen von Primzahlgraden zurückgeführt werden, so bedeutet überhaupt jede Radizierung im Sinne der Gleichungstheorie die Lösung einer Kette zyklischer Gleichungen von Primzahlgraden. Hiernach können wir sofort beantworten, wann irgendeine in einem Körper $\Re$ irreduzibele Glei- 
chung $f(z)=0$ algebraisch lösbar ist. Die bei der fortgesetzten Reduktion der Galoisschen Gruppe $G_{m}$ bis zur $G_{1}$ hin nach und nach zu lösenden Hilfsgleichungen haben lauter einfache Galoissche Gruppen. Sollen diese Gleichungen alle algebraisch lösbar sein, so müssen sie ohne Ausnahme zyklische Gruppen von Primzahlordnung zu Galoisschen Gruppen haben. Ist dieses aber der Fall, so sind sie auch sicher algebraisch lösbar. Da jede Gruppe von Primzahlordnung zyklisch ist, so ergibt sich der Satz: Eine in einem Körper $\Re$ irredusibele Gleichung ist stets und nur dann algebraisch lösbar, wenn die Indexreihe der zugehörigen Galoisschen Gruppe aus lauter Primzahlen besteht.

\section{Algebraische Funktionen.}

\section{§1. Funktionen und Gleichungen in Funktionenkörpern.}

Die Entwicklungen des zweiten Teiles übertragen wir jetzt auf solche Gleichungen mit variablen Koeffizienten, wie sie in der Theorie der algebraischen Funktionen (vgl. I, 76 ff.) auftreten. Wesentliche Änderungen sind dabei, daß erstens an Stelle von Zahlkörpern hier „Funktionenkörper" treten, deren Begriff bereits in I, 81 aufgestellt wurde, und daß zweitens an die Stelle der Gleichheit zweier Zahlen jetzt die Identität zweier Funktionen tritt. Im übrigen gestalten sich die Entwicklungen durchaus nach dem Vorbilde derjenigen im Teile II, so daß es der Kürze halber vielfach gestattet sein wird, auf die obigen Darlegungen Bezug zu nehmen.

Die Funktionenkörper, mit denen wir zu arbeiten haben, sind in folgender Art zu erklären. Wir legen zunächst einen bestimmten Zahlkörper $\Omega$ zugrunde, verstehen unter $x$ eine komplexe Variable und unter $R_{1}(x), R_{2}(x), \ldots$ rationale Funktionen von $x$ mit Koeffizienten aus $\Re$. Weiter sei $y$ eine algebraische Funktion von $x$, gegeben durch eine im. Sinne von I, 79 irreduzibele Gleichung $l^{\text {ten }}$ Grades in $y$ :

$$
y^{l}+R_{1}(x) y^{l-1}+R_{2}(x) y^{l-2}+\cdots+R_{l}(x)=0 .
$$

Dieser algebraischen Funktion $y$ gehört eine gewisse zusammenhängende $l$-blättrige Riemannsche Fläche $F_{l}$ über der $x$-Ebene zu. Die gesamten algebraischen Funktionen dieser Fläche sind nach I, 80 rational in $x$ und $y$ darstellbar, und umgekehrt ist jede rationale Funktion von $x$ und $y$ mit nicht identisch verschwindendem Nenner eine algebraische Funktion der Fläche. Wir greifen unter diesen Funktionen diejenigen heraus, welche als rationale Ausdrücke $R(x, y)$ in $x$ und $y$ mit Koeffizienten aus $\Re$ darstellbar sind. ${ }^{1}$ ) Es ist einleuchtend, daß die herausgegriffenen Funk-

1) Unter (5) S. 68 wird eine eindeutig bestimmte Normaldarstellung für die einzelne dieser Funktionen $R(x, y)$ angegeben werden. 
tionen $R(x, y)$ in ihrer Gesamtheit einen „Funktionenkörper" bilden; wir bezeichnen diesen Körper durch $\Re_{x}$ und legen ihn den nachfolgenden Entwicklungen zugrunde. Ist insbesondere $l=1$, so handelt es sich einfach um den Körper $\Re_{x}$ aller rationalen Funktionen von $x$ mit Koeffizienten aus $\Re$. Dieser letztere Körper, der durch Adjunktion der Variablen $x$ zum Körper $\Re$ entsteht, kann entsprechend auch durch $(\Omega, x)$ bezeichnet werden.

Es seien nun $a_{0}(x), a_{1}(x), \ldots, a_{n}(x)$ oder kurz $a_{0}, a_{1}, \ldots, a_{n}$ irgend $(n+1)$ Funktionen aus $\Omega_{x}$, von denen die erste nicht identisch verschwinden soll. Vermittelst einer komplexen Variablen $z$ bilden wir dann den Ausdruck:

$$
f(z)=a_{0} z^{n}+a_{1} z^{n-1}+a_{2} z^{n-2}+\cdots+a_{n},
$$

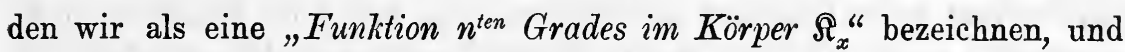
aus dem wir durch Nullsetzen eine "Gleichung $n^{\text {ten }}$ Grades in $\mathfrak{R}_{x}^{\text {" }}$ gewinnen. Die Funktionen nullten Grades in $\Omega_{x}$ sind dann einfach die den Körper $\Omega_{x}$ bildenden Funktionen, abgesehen von der identisch verschwindenden Funktion, die wieder für sich steht. Für funktionentheoretische Schlüsse ist die Tatsache wichtig, daß jede Lösung einer Gleichung $f(z)=0$ in $\AA_{x}$ eine algebraische Funtion der ursprïnglichen Variablen $x$ liefert. Durch Elimination von $y$ aus $f(z)=0$ und der Gleichung (1) erhalten wir nämlich eine algebraische Relation zwischen $z$ und $x$.

Es lassen sich nun zunächst die Entwicklungen von S. $29 \mathrm{ff}$. auf die hier vorliegenden Funktionen $f(z)$ übertragen. Kann $f(z)$ als das Produkt $\chi_{1}(z) \cdot \chi_{2}(z)$ zweier Funktionen $\chi_{1}(z), \chi_{2}(z)$ in $\Omega_{x}$ dargestellt werden, so heißt jede dieser beiden Funktionen ein "Teiler" von $f^{\prime}(z)$. Einleuchtend ist dann wieder, da $B$ jede nicht identisch verschwindende Funktion aus $\Re_{x}$ (d. h. jede Funktion nullten Grades in $\Re_{x}$ ) ein Teiler von $f(z)$ ist und ebenso jedes Produkt von $f(z)$ mit einer solchen Funktion aus $\Omega_{x}$.

Aus zwei fest gegebenen Funktionen $f(z)$ und $g(z)$ in $\Omega_{x}$, deren Grade $n$ und $m>0$ seien, bilden wir mittelst beliebiger Funktionen $\varphi(z)$, $\psi(z)$ in $\mathfrak{\Omega}_{x}$ die Gesamtheit $\mathfrak{F}$ aller Funktionen:

$$
f(z) \psi(z)+g(z) \varphi(z) .
$$

Aus der Körpereigenschaft der Koeffizienten unserer Funktionen geht dann wieder hervor, daß das System $\mathfrak{F}$ die S. 29 unter 1. und 2. genannten Eigenschaften besitzt, wobei $\Omega_{x}$ an Stelle von $\Omega$ tritt. Hieran schließt sich die Überlegung von S. $29 \mathrm{ff}$., die zum "größten gemeinsamen Teiler" der beiden Funktionen $f(z)$ und $g(z)$ :

$$
\chi(z)=z^{\nu}+c_{1} z^{\nu-1}+c_{2} z^{\nu-2}+\cdots+c_{v}
$$

hinführt. Dieser größte gemeinsame Teiler ist eine durch $f(z)$ und $g(z)$ eindeutig bestimmte Funktion in $\Re_{x}$, deren Grad $\nu \geqq 0$ ist und jedenfalls 
66 Einleitung, Teil III: Arithmetische Theorie der algebraischen Funktionen

keinen der Grade $n, m$ übertrifft. Die Funktion $\chi(z)$ gehört dem Systeme $\mathfrak{F}$ an, ist also darstellbar in der Gestalt:

$$
\chi(z)=f(z) \psi(z)+g(z) \varphi(z) .
$$

Ist der Grad $\nu=0$ und also $\chi(z)$ mit 1 identisch, so heißen die Funktionen $f(z), g(z)$,teilerfremd“: Für zwei teilerfremde $f(z), g(z)$ kann. man zwei Funktionen $\varphi(z), \psi(z)$ in $K_{x}$ so bestimmen, daß die Gleichung:

$$
f(z) \psi(z)+g(z) \varphi(z)=1
$$

identisch besteht. Auch umgekehrt ist unmittelbar einleuchtend, daß die Funktionen $f(z)$ und $g(z)$ durch die Existenz einer identischen Gleichung (6) als teilerfremd charaliterisiert sind. Hätten nämlich $f(z)$ und $g(z)$ einen gemeinsamen Teiler eines Grades $\nu>0$, so würde in (6) links das Produkt einer Funktion des Grades $\nu>0$ mit einer Funktion in $\boldsymbol{\Omega}_{x}$ stehen, die wegen (6) nicht mit 0 identisch sein kann. Also würde in (6) links eine Funktion mindestens vom Grade $\nu>0$ stehen, die nicht mit der Funktion nullten Grades 1 identisch ist.

Hieran schließen sich genau wieder die Überlegungen von S. $30 \mathrm{ff}$. Wir dürfen sogleich folgende Sätze notieren: Sind $f(z), g(z)$ und $h(z)$ Funktionen in $\Omega_{x}$, von denen die beiden ersten teilerfremd sind, und ist $g(z) \cdot h(z)$ durch $f(z)$ teilbar, so ișt $f(z)$ ein Teiler von $h(z)$. Auf die Gleichung (6) bezieht sich der Satz: Sind $f(z)$ und $g(z)$ teilerfremd, so. gibt es ein und nur ein Paar, die Gleichung (6) identisch befriedigender Funktionen $\varphi(z), \psi(z)$, deren Grade bzw. $<n$ und $<m$ sind. Für eine beliebige Funktion $h(z)$ in $\Omega_{x}$ gilt endlich der Satz: Sind $f(z)$ und $g(z)$, teilerfremd, so kann man jede Funktion $h(z)$ in $\Omega_{x}$ in der Gestalt:

$$
h(z)=f(z) \psi(z)+g(z) \varphi(z)
$$

mit zwei Funktionen $\varphi(z)$ und $\psi(z)$ in $\mathfrak{\Omega}_{x}$ darstellen, und zwar leann man die Funktionen $\varphi(z)$ und $\psi(z)$ in einer und nur einer Art so wählen, daß der Grad von $\varphi(z)$ kleiner als der Grad $n$ von $f(z)$ ist.

Die Funktion $f(z)$ heißt ,in $\Omega_{x}$ reduzibel", falls sie eine Funktion $\chi(z)$ in $\Omega_{x}$ eines Grades, der $>0$ und $<n$ ist, zum Teiler hat; existiert ein solcher Teiler nicht, so heißt $f(z)$, in $\Re_{x}$ irreduzibel". $\left.{ }^{1}\right)$ Hieran schlie Ben sich wie S. $31 \mathrm{ff}$. die folgenden Sätze:

Ist von den beiden Funktionen $f(z)$ und $g(z)$ in $\mathfrak{\Re}_{x}$ die erste irreduzibel, so sind $f(z)$ und $g(z)$ entweder teilerfremd oder $g(z)$. hat $f(z)$ zum. Teiler.

Zwei irreduzibele Funktionen $f(z)$ und $g(z)$ sind entweder teilerfremd oder bis auf einen Faktor, der eine Funktion aus $\Omega_{x}$ ist, identisch.

1) Der Znsatz „,in $\mathfrak{N}_{x}$ “ wird wieder, falls er selbstverständlich ist, gewöhnlich fortgelassen. 
Reduzibilität und Irreduzibilität. Algebraische Funktionen in bezug auf $\Re_{x} 67$

Eine irreduzibele Funktion $f(z)$ ist stets teilerfremd zur Funktion $f^{\prime}(z)$, die man durch partielle Differcntiation nach $z$ aus ihr erzielt.

Jede Funktion $f(z)$ in $\Omega_{x}$ ist nur auf eine Art als Produkt irreduzibeler Funktionen darstellbar, abgesehen davon, daß jede irreduzibele Funktion noch um eine nicht identisch verschwindende Funktion aus $\Omega_{x}$ als Faktor geändert werden kann.

\section{\$2. Algebraische Funktionen in bezug auf einen Körper $\boldsymbol{A}_{\boldsymbol{x}}$.}

Die Gleichung $n^{\text {ten }}$ Grades $f(z)=0$ in $\Re_{x}$, die wir durch Nullsetzen einer Funktion $n^{\text {ten }}$ Grades in $\Omega_{x}$ gewinnen, heißt "in $\Omega_{x}$ reduzibel" oder "irreduzibel", je nachdem $f(z)$ reduzibel oder irreduzibel ist. Eine Lösung oder Wurzel $z=\theta(x)$ der Gleichung $f(z)=0$ ist nach S. 65 eine ,algebraische Funktion" von $x$; genauer ist sie als eine mehrdeutige Funktion der Stelle der Riemannschen Fläche $\mathbf{F}_{\mathbf{l}}$ zu denken und werde als eine ,in bezug auf den Körper $\mathfrak{R}_{x}$ algebraische Funktion" bezeichnet. Ist $f(z)=0$ reduzibel, so muB für $z=\theta(x)$ mindestens einer der irreduzibelen Faktoren von $f(z)$ identisch verschwinden, so daß jede in bezug auf $\Omega_{x}$ algebraische Funktion sicher mindestens einer irreduzibelen Gleichung in $\mathfrak{\Omega}_{x}$ genügt.

Es gilt nun zunächst der Satz: Haben die beiden Gleichungen $f(z)=0$ und $g(z)=0$, von denen die erste irreduzibel ist, eine gemeinsame Wurzel $z=\theta(x)$, so ist $f(z)$ ein Teiler von $g(z)$. Wäre dies nämlich nicht der Fall, so müBten, da $f(z)$ irreduzibel ist, $f(z)$ und $g(z)$ teilerfremd sein Also gäbe es zwei Funktionen $\varphi(z)$ und $\psi(z)$ in $\AA_{x}$, die mit $f(z)$ und $g(z)$ die Relation (6) S. 56 identisch befriedigen. Wählen wir aber irgendeine Stelle $x_{0}$ auf $\mathrm{F}_{l}$, für welche alle Koeftizienten unserer Funktionen endliche Werte haben, so ist sicher:

$$
f\left(\theta\left(x_{0}\right)\right) \psi\left(\theta\left(x_{0}\right)\right)+g\left(\theta\left(x_{0}\right)\right) \varphi\left(\theta\left(x_{0}\right)\right)=0,
$$

da $\varphi\left(\theta\left(x_{0}\right)\right), \psi\left(\theta\left(x_{0}\right)\right)$ nicht unendlich sind und $f\left(\theta\left(x_{0}\right)\right), g\left(\theta\left(x_{0}\right)\right)$ verschwinden. Dies widerspricht aber der identischen Relation (6) S. 66, so $\mathrm{dab}$ der Satz richtig ist.

Als einfache Folgerungen ergeben sich die Sätze: Zuei irreduzibele Gleichungen in $\Re_{x}$ haben entueder keine gemeinsame Wurzel oder ihre linken Seiten sind, abgesehen ron einem Fahtor, der eine Funktion in $\mathfrak{\kappa}_{x}$ ist, identisch. Eine in bezug auf den Körper $\AA_{x}$ algebraische Funktion $z=\theta(x)$ genügt im wesentlichen nur einer einzigen irreduzibelen Gleichung in $\Re_{x}$. Der Ausdruck „im wesentlichen" bezieht sich darauf, daB die linke Seite der Gleichung noch mit einer Funktion aus $\Omega_{x}$ als Faktor versehen werden kann. Weiter besteht der Satz: Ist $z=\theta(x)$ eine Wurzel der irreduzibelen Gleichung $f(z)=0$, so kann $f^{\prime}(\theta(x))$ nicht identisch verschwinden.

Ist $n$ der Grad der irreduzibelen Gleichung für $z=\theta(x)$, so nennen 
68 Einleitung, Teil III: Arithmetische Theorie der algebraischen Funktionen wir $\theta(x)$ eine in bezug auf $\Re_{x}$ algebraische Funktion ,vom $n^{\text {ten }}$ Grade“. Für $n=1$ haben wir in $\theta(x)$ natürlich eine Funktion aus $\Omega_{x}$. Ist $n>1$, so gehört $\theta(x)$ dem Körper $\Omega_{x}$ nicht an, so daß wir durch Adjunktion von $\theta=\theta(x)$ zu $\Re_{x}$ einen durch $\left(\Omega_{x}, \theta\right)$ zu bezeichnenden erweiterten Funktionenkörper erhalten. Dieser Körper besteht aus allen in der Gestalt:

$$
\xi(x)=\frac{h(\theta(x))}{g(\theta(x))} \quad \text { oder kurz } \quad \xi=\frac{h(\theta)}{g(\theta)}
$$

darstellbaren Funktionen, wo $g(x)$ und $h(x)$ Funktionen in $\Re_{x}$ sind, von denen die erste für $\theta(x)$ nicht identisch verschwinden und also $f(z)$ nicht als Faktor haben darf. Da hiernach $f(z)$ und $g(z)$ teilerfremd sind, so können wir nach (7) S. 66 die im Zähler von $\zeta$ stehende Funktion $h(z)$ in der Gestalt:

$$
h(z)=f(z) \psi(z)+g(z) \varphi(z)
$$

darstellen, wo $\varphi(z)$ und $\psi(z)$ Funktionen in $\Re_{x}$ sind und der Grad von $\varphi(z)$ kleiner als $n$ ist. Schreiben wir demnach:

$$
\varphi(z)=c_{0}+c_{1} z+c_{2} z^{2}+\cdots+c_{n-1} z^{n-1},
$$

so ergibt sich durch Eintragen von $\theta(x)$ für $z$ in (2) und (3) der Satz: Jede Funktion $\xi=\xi(x)$ des erweiterten Körpers $\left(\Omega_{x}, \theta\right)$ ist in der Gestalt:

$$
\xi=c_{0}+c_{1} \theta+c_{2} \theta^{2}+\cdots+c_{n-1} \theta^{n-1}
$$

darstellbar, wo die c Funltionen aus $\mathfrak{l}_{x}$ sind; zugleich ist diese Darstellung für die einzelne Funktion $\zeta(x)$ eindeutig bestimmt. Der letzte Teil des Satzes folgt genau wie S. 34 aus der Irreduzibilität von $f(z)$.

Man kann diesen Satz auch auf die Gleichung,(1) S. 64 und damit auf die Darstellung der algebraischen Funktionen $R(x, y)$ des Körpers $\mathfrak{\Re}_{x}$ anwenden. Jene Gleichung ist irreduzibel im Körper $(\Re, x)$ der rationalen Funktionen von $x$ mit Koeffizienten aus $\Re^{1}$ ) Für die Funktionen $R(x, y)$ des Körpers $\mathfrak{R}_{x}$ ergibt sich demnach je eine eindeutig bestimmte Darstellung:

$$
R(x, y)=c_{0}^{\prime}+c_{1}^{\prime} y+c_{2}^{\prime} y^{2}+\cdots+c_{l-1}^{\prime} y^{l-1},
$$

wo die $c^{\prime}$ dem Körper $(\Omega, x)$ angehören und also rationale Funktionen von $x$ mit Koeffizienten aus $\Omega$ sind.

\section{§ 3. Gleichzeitige Adjunktion mehrerer algebraischer Funktionen.}

Von grundsätzlicher Bedeutung ist auch hier die Tatsache, daß man die gleichzeitige Adjunktion einer beliebigen Anzahl algebraischer Funktionen $\theta_{1}(x), \theta_{2}(x), \ldots, \theta_{m}(x)$ zu $\Omega_{x}$ durch die Adjunktion einer einzigen Funktion $\eta(x)$ ersetzen kann. Der Beweis dieses Satzes kann wieder durch die

1) Sie ist nach I, 79 sogar irreduzibel im Körper aller rationalen Funktionen von $x$ mit beliebigen konstanten Koeffizienten. 
Darstellung der Funktionen von $\left(\Omega_{x}, \theta\right)$. Adjunktion mehrerer Funktionen

Überlegungen von S. $35 \mathrm{ff}$. geführt werden, nur sind ein paar Änderungen dadurch bedingt, daß wir hier mit Funktionen und nicht mit Zahlen zu tun haben.

Wir gehen schrittweise vor, bezeichnen zunächst die Funktion $\theta_{1}(x)$ kurz durch $\theta(x)$ und stellen nach (4) und (5) $\S 2$ die Funktionen $\xi$ des Körpers $\left(\Omega_{x}, \theta\right)$ in der Gestalt:

$$
\zeta=\sum_{\lambda, v} c_{\lambda v} y^{\lambda} \theta^{v}, \quad \lambda=0,1, \ldots, l-1, \quad \nu=0,1, \ldots, n-1
$$

dar, wo die $c_{\lambda v}$ Funktionen des soeben mit $(\Omega, x)$ bezeichneten Körpers der rationalen Funktionen von $x$ mit Koeffizienten aus $\Omega$ sind. Die Funktion $\theta(x)$ war zunächst eine mehrdeutige Funktion auf der Riemannschen Fläche $F_{l}$. Sie ist aber auch direkt in bezug auf $(\Omega, x)$ algebraisch und möge als solche der in $(\Omega, x)$ irreduzibelen Gleichung:

$$
\theta^{m}+R_{1}^{\prime}(x) \theta^{m-1}+R_{2}^{\prime}(x) \theta^{m-2}+\cdots+R_{m}^{\prime}(x)=0
$$

genügen, die wir der Gleichung (1) S. 64 für $y$ anreihen.

Wir setzen nun:

$$
y_{1}(x)=\alpha y(x)+\beta \theta(x) \text { oder kurz } y_{1}=\alpha y+\beta \theta,
$$

wo $\alpha, \beta$ als rationale ganze Zahlen in folgender Art bestimmt werden sollen: Da keine zwei Lösungen $y(x), y^{\prime}(x), \ldots, y^{(l-1)}(x)$ der Gleichung (1) S. 64 identisch sind und ebenfalls keine zwei Lösungen $\theta(x), \theta^{\prime}(x)$, $\ldots, \theta^{(n-1)}(x)$ von (2), so können wir ein Argument $x_{0}$ so wählen, daß die $l$ Zahlen $y\left(x_{0}\right), y\left(x_{0}\right), \ldots$ durchweg verschieden sind und ebenso die $m$ Zahlen $\theta\left(x_{0}\right), \theta^{\prime}\left(x_{0}\right), \ldots$ Wir bilden die $l \cdot m$ Kombinationen:

$$
\alpha y^{(\lambda)}(x)+\beta \theta^{(\mu)}(x), \quad \lambda=0,1, \ldots, l-1, \quad \mu=0,1, \ldots, m-1
$$

und erhalten auf diese Weise $l \cdot m$ Funktionen, die wir in irgendeiner Reihenfolge $y_{1}, y_{1}^{\prime}, y_{1}^{\prime \prime}, \ldots, y_{1}^{(l m-1)}$ nennen. Wegen der Verschiedenheit der Zahlen $y^{(\lambda)}\left(x_{0}\right)$ und derjenigen der $\theta^{(u)}\left(x_{0}\right)$ können wir nach einer oben wiederholt ausgeübten Überlegung die $\alpha, \beta$ als rationale ganze Zahlen so bestimmen, daß die $l \cdot m$ Zahlen $y_{1}\left(x_{0}\right), y_{1}^{\prime}\left(x_{0}\right), \ldots, y_{1}^{(l m-1)}\left(x_{0}\right)$ durchweg verschieden sind. Daraus folgt dann, daß von den $l m$ Funktionen (4) keine zwei identisch sein können.

Hieran schließt sich nun die Wiederholung der Überlegung, die wir oben (S. 36) mit der Gleichung (7) begonnen hatten. Wir finden, daß die $l m$ Funktionen $y_{1}, y_{1}^{\prime}, \ldots, y_{1}^{(l m-1)}$ die Wurzeln einer Gleichung $F(z)=0$ im Körper $(\Omega, x)$ vom Grade $l m$ sind. Diese Gleichung braucht in $(\Re, x)$ nicht irreduzibel zu sein; aber es verschwindet wegen der Verschiedenheit der $y_{1}, y_{1}^{\prime}, \ldots$ sicher $F^{\prime}\left(y_{1}\right)$ nicht identisch, was für die weitere Überlegung ausreichend ist. Durch Übertragung der an (9) S. 37 angeschlossenen Überlegung finden wir, daß der Körper $\left(\Omega_{x}, \theta\right)=(\Re, x, y, \theta)$ auch als Körper $\left(\Re, x, y_{1}\right)$, der durch $\Re_{x}^{(1)}$ bezeichnet werde, durch Ad- 
70 Einleitung, Teil III: Arithmetische Theorie der algebraischen Funktionen

junktion der einzigen Funktion $y_{1}$ zu $(\Omega, x)$ an der Stelle der beiden $y, \theta$ gewinnbar ist.

Wir setzen jetzt genauer $\theta_{1}(x)$ für die.Funktion $\theta(x)$ ein und halten an der Bezeichnung $\left(\Omega_{x}, \theta_{1}\right)=\Re_{x}^{(1)}$ fest. Wesentlich ist, daß der so erhaltene Körper $\Omega_{x}^{(1)}$ wieder ein Körper derselben Art wie $\Omega_{x}$ ist. Die Funktionen des Körpers $\left(\Re_{x}^{(1)}, \theta_{2}\right)=\left(\Re_{x}, \theta_{1}, \theta_{2}\right)$ lassen sich demnach in der Gestalt:

$$
\sum_{\nu=0}^{n_{2}-1} c_{\nu}^{(1)} \theta_{\Sigma}^{v}=\sum_{\lambda, \mu} c_{\lambda, \mu} \theta_{1}^{\lambda} \theta_{2}^{u}, \quad \lambda=0,1, \ldots, n_{1}-1, \quad \mu=0,1, \ldots, n_{2}-1
$$

darstellen, wo die $c_{v}^{(\mathfrak{l})}$ Funktionen aus $\mathfrak{\Re}_{x}^{(1)}$ und die $c_{\lambda \mu}$ solche aus $\boldsymbol{\Omega}_{x}$ sind, und $n_{1}, n_{2}$ die Grade von $\theta_{1}(x), \theta_{2}(x)$ bedeuten. Die Darstellungen (4) sind freilich nicht mehr notwendig eindeutig bestimmt ${ }^{1}$ ); doch wird dadurch die weitere Schlußweise nicht beeinflußt. Der Körper $\left(\boldsymbol{\Omega}_{x}^{(1)}, \theta_{2}\right)$ kann dann wieder als ein Körper $\mathfrak{\Omega}_{x}^{(2)}$ von der Art des Körpers $\boldsymbol{\Omega}_{x}$ durch Adjunktion einer einzigen Funktion $y_{2}$ zu $(\Omega, x)$ gewonnen werden.

Um das Ergebnis möglichst dem Satze von S. 35 anzupassen, entnehmen wir aus der vorstehenden Betrachtung die Tatsache, daß die bei den weiteren Adjunktionen eintretenden Körper $\left(\Omega_{x}, \theta_{1}, \theta_{2}, \theta_{3}\right), \ldots$ stets wieder als Körper $\Re_{x}^{(3)}, \ldots$ von der Art des Körpers $\AA_{x}$ bei der Fortsetzung der Betrachtung zugrunde gelegt werden können. Wir gelangen so für die Funktionen des Körpers $\left(\Omega_{x}, \theta_{1}, \theta_{2}, \ldots, \theta_{m}\right)$ zu Darstellungen der Gestalt:

(6) $\sum_{\lambda, \mu, v, \ldots} c_{\lambda \mu \nu} \ldots \theta_{1}^{\lambda} \theta_{2}^{u} \theta_{3}^{v} \ldots, \quad \lambda=0,1, \ldots, n_{1}-1, \quad \mu=0,1, \ldots, n_{2}-1, \ldots$,

die für die einzelnen Funktionen natürlich wieder nicht notwendig eindeutig bestimmt sind. Die Zahlen $n_{1}, n_{2}, \ldots$ sind die Grade der irreduzibelen Gleichungen $f_{1}(z)=0, f_{2}(z)=0, \ldots$, denen die Funktionen $\theta_{1}(x)$, $\theta_{\mathbf{2}}(x), \ldots$ genügen. Diese Gleichungen brauchen natürlich, wenn wir auch die $m$ Funktionen $\theta_{1}(x), \theta_{2}(x), \ldots$ als durchweg verschieden voraussetzen, keineswegs alle voneinander verschieden zu sein.

Die Überlegungen von S. 36 ff. zum Beweise des am Anfang des Paragraphen aufgestellten Satzes wiederholen sich nun genau wie dort, wobei nur der Schluß auf die Verschiedenheit der $N=n_{1} \cdot n_{2} \ldots n_{m}$ Funktionen $\eta(x), \eta^{\prime}(x), \ldots, \eta^{(N-1)}(x)$ einer funktionentheoretischen Betrachtung bedarf, bei der man von einer geeigneten speziellen Stelle der Riemannschen Fläche $\mathbf{F}_{l}$ auszugehen hat. Wir gelangen zu dem Ergebnis: Der durch Adjunktion von $m$ in bezug auf $\Re_{x}$ algebraischen Funktionen $\theta_{1}(x)$, $\theta_{2}(x), \ldots, \theta_{m}(x) z u \Re_{x} z u$ gewinnende Funktionenkërper $\left(\Omega_{x}, \theta_{1}, \theta_{2}, \ldots, \theta_{m}\right)$

1) Die für $\theta_{2}$ vorgelegte in $\mathfrak{N}_{x}^{\prime}$ irreduzibele Gleichung des Grades $n_{2}$ kann nämlich im Körper $\mathfrak{\Re}_{x}^{(1)}$ reduzibel sein. 
kann auch durch Adjunktion einer einzigen in bezug auf $\Omega_{x}$ algebraischen Funktion $\eta(x)$, die in der Gestalt:

$$
\eta(x)=\gamma_{1} \theta_{1}(x)+\gamma_{2} \theta_{2}(x)+\cdots+\gamma_{m} \theta_{m}(x)
$$

mittelst rationaler ganzer Zahlen $\gamma$ darstellbar ist, zu $\AA_{x}$ geuronnen werden.

\section{§ 4. Konjugierte Körper. Primitive und imprimitive Funktionen.}

In den folgenden vier Paragraphen übertragen wir die Entwicklungen von S. $38 \mathrm{ff}$. auf unsere jetzt vorliegenden Gleichungen mit variablen Koeffizienten. Die Beweise gestalten sich wie oben: sie dürfen demnach zumeist übergangen werden.

Es sei wieder $f(z)=0$ eine irreduzibele Gleichung in $\boldsymbol{\Omega}_{x}$ vom $n^{\text {ten }}$ Grade, deren $n$ Lösungen wir jetzt durch $\theta(x)=\theta_{1}(x), \theta_{2}(x), \ldots, \theta_{n}(x)$ bezeichnen. Die $\theta=\theta_{1}, \theta_{2}, \ldots, \theta_{n}$ heißen $n$,konjugierte" in bezug auf $\Omega_{x}$ algebraische Funktionen, und entsprechend werden die $\boldsymbol{n}$ Funktionenkörper $\left(\Re_{x}, \theta\right)=\left(\Re_{x}, \theta_{1}\right),\left(\Re_{x}, \theta_{z}\right), \ldots,\left(\Omega_{x}, \theta_{n}\right)$ als $n$, ,konjugierte ${ }^{\prime \prime}$ in bezug auf $\Re_{x}$ algebraische Körper $n^{\text {ten }}$ Grades bezeichnet. Irgendeine Funktion $\zeta=\zeta(x)$ des Körpers $\left(\AA_{x}, \theta\right)$ ist nach (4) S. 68 in der Gestalt:

$$
\xi=c_{0}+c_{1} \theta+c_{2} \theta^{2}+\cdots+c_{n-1} \theta^{n-1}
$$

darstellbar, unter $c_{0}, c_{1}, \ldots, c_{n-1}$ Funktionen aus $\mathfrak{\Re}_{x}$ verstanden. Ersetzen wir in (1) rechts $\theta=\theta_{1}$ durch $\theta_{2}, \theta_{3}, \ldots, \theta_{n}$, so erhalten wir die mit $\zeta=\xi_{1}(x)$ "konjugierten Funktionen" $\xi_{2}(x), \xi_{3}(x), \ldots, \xi_{n}(x)$. Wie S. 38 beweist man, daB $\xi(x)=\xi_{1}(x), \xi_{2}(x), \ldots, \xi_{n}(x)$ die Wurzeln einer Gleichung $n^{\text {ten }}$ Grades in $\Re_{x}$ :

$$
g(w)=w^{n}+b_{1} w^{n-1}+b_{2} u^{n-2}+\cdots+b_{n}=0
$$

sind, die aus der Gleichung $f(z)=0$ als „Tschirnhausenresolvente" mittelst der Transformation gewonnen wird:

$$
w=c_{0}+c_{1} z+c_{2} z^{2}+\cdots+c_{n-1} z^{n-1} .
$$

Die Frage der Reduzibilität der Gleichung (2) führt zu der Überlegung von S. 39 zurück. Wir haben den Satz: Die linke Seite $g(w)$ der Tschirnhausenresolvente (2) ist die $\mu^{\text {te }}$ Potenz einer irreduzibelen Funktion $\boldsymbol{\nu}^{\text {ten }}$ Grades in $\mathfrak{\Omega}_{x}$, wobei $\boldsymbol{\mu} \cdot \boldsymbol{\nu}=\boldsymbol{n}$ zutrifft; die $n$ konjugierten Funktionen $\xi_{1}, \xi_{2}, \ldots, \xi_{n}$ sind $z u$ je $\mu$ einander gleich und liefern $\nu$ verschiedene Funktionen. Ist $\mu=1$, so ist die Gleichung (2) irreduzibel, und die $n$ konjugierten Funktionen $\xi_{1}, \xi_{2}, \ldots, \xi_{n}$, die in diesem Falle durchweg verschieden sind, heißen ,primitive" Funktionen des Körpers $\left(\Omega_{x}, \theta\right)$. Für $m>1$ werden die $\xi$,imprimitive" Funktionen des fraglichen Körpers genannt.

Es gilt der Satz: In jedem in bezug auf $\mathfrak{\Omega}_{x}$ algebraischen Körper $n^{\text {ten }}$ Grades $\left(\Re_{x}, \theta\right)$ gibt es unendlich viele primitive Funktionen. Man kann 
Einleitung, Teil III: Arithmetische Theorie der algebraischen Funktionen

nämlich bereits auf unendlich viele Arten ein System rationaler ganzer Zahlen $\gamma_{0}, \gamma_{1}, \gamma_{2}, \ldots, \gamma_{n-1}$ so wählen, daB die $n$ mit

$$
\xi=\gamma_{0}+\gamma_{1} \theta+\gamma_{2} \theta^{2}+\cdots+\gamma_{n-1} \theta^{n-1}
$$

konjugierten Funktionen durchweg verschieden sind. Hierzu ist hinreichend, daB die $n$ mit (4) konjugierten Funktionen an irgendeiner speziellen Stelle $x_{0}$ der Riemannschen Fläche $F_{l}$ durchweg verschiedene numerische Werte annehmen. Dies aber ist durch unendlich viele Auswahlen von Zahlen $\gamma$ erreichbar, wenn nur, was keine Schwierigkeit hat, die Stelle $x_{0}$ etwa so gewählt ist, daß die Zahlen $\theta_{1}\left(x_{0}\right), \theta_{2}\left(x_{0}\right), \ldots, \theta_{n}\left(x_{0}\right)$ durchweg verschieden sind.

Genau wie S. 40 gestaltet sich der Beweis des Satzes: Durch $A d-$ junktion irgendeiner „primitiven“ Funltion $\xi$ von $\left(\Omega_{x}, \theta\right)$ zum Körper $\mathfrak{\Omega}_{x}$ ergibt sich stets der gesamte Körper $\left(\Omega_{x}, \theta\right)$ wieder, während für eine „imprimitive" Funktion $\xi$ der Körper $\left(\Re_{x}, \xi\right)$ den Körper $\left(\Re_{x}, \theta\right)$ noch nicht erschöpft.

\section{\$. Galoissche Körper und Galoissche Resolventen.}

Entsprechend den Entwicklungen von S. 41 haben wir nun folgende Erklärung aufzustellen: Der Funktionenkörper $\left(\Re_{x}, \theta\right)$ heißt ein „Galoisscher" Körper oder „Normalliörper", falls er mit seinen sämtlichen konjugierten Körpern gleich ist. Notwendig und hinreichend hierfür ist, daB jede der Funktionen $\theta_{2}(x), \theta_{3}(x), \ldots, \theta_{n}(x)$ im Körper $\left(\Omega_{x}, \theta_{1}\right)=\left(\Re_{x}, \theta\right)$ enthalten ist, d. h. daB $(n-1)$ Gleichungen gelten:

$$
\theta_{k}=c_{k 0}+c_{k 1} \theta+c_{k 2} \theta^{2}+\cdots+c_{k, n-1} \theta^{n-1}, \quad k=2,3, \ldots, n,
$$

wo die $c$ Funktionen aus $\Omega_{x}$ sind. Es ist dann in entsprechender Weise jede Funktion $\theta_{k}(x)$ in jeder $\theta_{i}(x)$ darstellbar. Einleuchtend ist, daB ein Normalkörper mit jeder seiner Funktionen $\xi(x)$ alle ihr konjugierten Funktionen $\xi_{1}(x)=\xi(x), \xi_{2}(x), \ldots, \xi_{n}(x)$ enthält.

Wie oben besteht auch hier der Satz: Ist $\left(\mathfrak{K}_{x}, \theta\right)$ ein beliebiger in bezug auf $\AA_{x}$ algebraischer Körper $n^{\text {ten }}$ Grades, und sind $\theta_{1}(x)=\theta(x)$, $\theta_{2}(x), \ldots, \theta_{n}(x)$ die mit $\theta(x)$ konjugierten Funktionen, so ist der Körper $\left(\Omega_{x}, \theta_{1}, \theta_{2}, \ldots, \theta_{n}\right)$ der, kleinste" Galoissche oder Normalkörper, $\operatorname{der}\left(\Omega_{x}, \theta\right)$ in sich enthält. Der Beweis wird durch Wiederholung der Betrachtung von S. 42 geführt.

Ist $\left(\AA_{x}, \theta_{1}, \theta_{2}, \ldots, \theta_{n}\right)$ in bezug auf $\Omega_{x}$ ein algebraischer Körper $m^{\text {ten }}$ Grades, so genügt irgendeine primitive Funktion $\eta(x)$ dieses Körpers einer irreduzibelen Gleichung $m^{\text {ten }}$ Grades in $\AA_{x}$ :

$$
F(Z)=0,
$$

die wir als eine „Galoissche Resolvente" $\operatorname{der}$ Gleichung $f(z)=0$ bezeichnen oder auch, für sich betrachtet, eine „Normalgleichung" nennen. Eine Nor- 
malgleichung ist durch die Eigenschaft charakterisiert, daß sie in $\Re_{x}$ irreduzibel ist, und daß jede ihrer Lösungen $\eta_{i}(x)$ in einer beliebigen unter ihnen $\eta_{k}(x)$ in der Gestalt:

$$
\eta_{i}=c_{i 0}^{(k)}+c_{i 1}^{(k)} \eta_{k}+c_{i 2}^{(k)} \eta_{k}^{2}+\cdots+c_{i, n-1}^{(k)} \eta_{k}^{n-1}
$$

mit Funktionen $c$ aus $\mathfrak{K}_{x}$ darstellbar ist. Eine Normalgleichung ist stets selbst eine ihrer Galoisschen Resolventen.

\section{\$ 6. Galoissche Gruppe einer Gleichung $f(\approx)=0$.}

Wie eben bedeute $\eta(x)$ eine bestimmt gewählte primitive Funktion des Galoisschen Körpers $\left(\Omega_{x}, \theta_{1}, \theta_{2}, \ldots, \theta_{n}\right)$, die der irreduzibelen Gleichung (2) $\$ 5$ genüge. Wir gewinnen dann alle Funktionen des Körpers $\left(\Omega_{x}, \theta_{1}, \theta_{2}, \ldots, \theta_{n}\right)=\left(\Omega_{x}, \eta\right)$ in der Gestalt:

$$
\xi=c_{0}+c_{1} \eta+c_{2} \eta^{2}+\cdots+c_{m-1} \eta^{m-1}
$$

und zwar jede Funktion nur einmal, wenn wir hier für die $c_{0}, c_{1}, \ldots, c_{m-1}$ alle möglichen Systeme von Funktionen aus $\Re_{x}$ eintragen. Statt $\eta$ können wir aber auch jede mit $\eta(x)$ konjugierte Funktion $\eta_{k}(x)$ benutzen, d. h. wir gewinnen auch durch den Ansatz:

$$
\xi_{k}=c_{0}+c_{1} \eta_{k}+c_{2} \eta_{k}^{2}+\cdots+c_{m-1} \eta_{k}^{m-1}
$$

jede Funktion von $\left(\Omega_{x}, \eta\right)$ und jede nur einmal.

Ersetzen wir nun $\eta$ durch $\eta_{k}$, so geht entsprechend $\zeta$ in $\xi_{k}$ über, und wir erhalten einen umkehrbar eindeutigen Ersatz jeder Funktion aus $\left(\Omega_{x}, \eta\right)$ durch eine bestimmte mitihr konjugierte Funktion aus $\left(\Omega_{x}, \eta\right)$. Wir nennen diesen Ersatz wie oben eine „Transformation des Galoisschen Körpers in sich". Nehmen wir der Reihe nach $\eta_{k}$ gleich $\eta_{1}, \eta_{2}, \ldots, \eta_{m}$, so erhalten wir $m$ verschiedene Transformationen des Galoisschen Körpers in sich, die wir symbolisch durch $S_{0}=1, S_{1}, S_{2}, \ldots, S_{m-1}$ bezeichnen; sie haben für jedes System von konjugierten Funktionen $m$ Permutationen zur Folge, die wir gleichfalls durch die Symbole $S_{k}$ bezeichnen.

Für das System dieser $m$ Transformationen gelten nun wieder alle Ausführungen von S. $44 \mathrm{ff}$. mit denjenigen Abänderungen der Begründungen, welche auf dem Umstande beruhen, daß wir hier mit Funktionenkörpern zu tun haben. Es besteht insbesondere der Satz: Wenn die $m$ Permutationen auch nicht für jedes System konjugierter imprimitiver Funktionen von $\left(\Omega_{x}, \eta\right)$ durchgängig verschieden sind, so sind sie doch sicher durchgängig verschieden für die $n$ Lösungen $\theta_{1}(x), \theta_{2}(x), \ldots, \theta_{n}(x)$ der ursprünglich vorgelegten irreduzibelen Gleichung $f(z)=0$.

Die Gruppeneigenschaft der $m$ Transformationen folgt wie S. 45: Die $m$ Transformationen $S_{0}=1, S_{1}, S_{2}, \ldots, S_{m-1}$ des Galoisschen Körpers $\left(\Omega_{x}, \eta\right)$ in sich bilden eine endliche Gruppe $G_{m}$ der Ordnung $m$, die in ihrer Gestalt als Permutationsgruppe der $m$ konjugierten Funktionen $\eta$ die 
74 Einleitung, Teil III: Arithmetische Theorie der algebraischen Funktionen „Galoissche Gruppe" der Normalgleichung $F(Z)=0$ heißt, in ihrer Gestalt als Permutationsgruppe der $\theta$ aber "die Galoissche Gruppe" der Gleichung $f(z)=0$. Die Galoissche Gruppe $G_{m}$ der irreduzibelen Gleichung $f(z)=0$ ist transitiv. Die Galoissche Gruppe einer Normalgleichung $m^{\text {ten }}$ Grades ist eine $G_{m}$ der Ordnung $m$ und des Grades $m$, die einfach transitiv ist.

Die Eigenschaften der $G_{m}$, die auf der Irreduzibilität der Gleichungen beruhen, gestalten sich gleichfalls wie oben. Es seien:

$$
R_{1}(z)=\frac{h_{1}(z)}{g_{1}(z)}, \quad R_{2}(z)=\frac{h_{2}(z)}{g_{2}(z)}
$$

Quotienten von „Funktionen in $\Re_{x}$ “, deren Nenner nicht durch $F(z)$ teilbar seien. Dann sind $g_{1}\left(\eta_{k}(x)\right)$ und $g_{2}\left(\eta_{k}(x)\right)$ für jedes $\eta_{k}(x)$ Funktionen aus $\left(\Omega_{x}, \eta\right)$, die nicht identisch verschwinden. Damit die beiden in $\left(\Omega_{x}, \eta\right)$ enthaltenen Funktionen $R_{1}(\eta(x))$ und $R_{2}(\eta(x))$ identisch sind, ist hinreichend und notwendig, daß die Gleichung:

$$
h_{1}(z) g_{2}(z)-h_{2}(z) g_{1}(z)=0
$$

die Lösung $z=\eta(x)$ hat. Nach S. 67 hat sie dann aber jede Funktion $\eta_{k}(x)$ als Lösung, so daß die Gleichung $R_{1}\left(\eta_{k}(x)\right)=R_{2}\left(\eta_{k}(x)\right)$ für jedes $\eta_{k}(x)$ identisch besteht, sobald sie für eine der $m$ Funktionen $\eta(x)$ zutrifft.

Die Übertragung dieses Satzes auf die Galoissche Gruppe der Gleichung $f(z)=0$ ergibt wie oben (S. 47) den Satz: Jede rationale „Gleichung in $\AA_{x}$ ", die für die Funktionen $\theta_{1}(x), \theta_{2}(x), \ldots, \theta_{n}(x)$ identisch besteht, bleibt eine identisch gültige Gleichung, falls man die $\theta_{1}, \theta_{2}, \ldots, \theta_{n}$ irgendeiner Permutation der Galoisschen Gruppe unterwirft. Berücksichtigt man noch, daß eine Funktion des Körpers $\left(\Re_{x}, \theta_{1}, \theta_{2}, \ldots, \theta_{n}\right)$ durch die Permutation der $G_{m}$ in ihre "konjugierten" Funktionen übergeführt wird, so folgt der Satz: Ein rationaler Ausdruck $R\left(\theta_{1}, \theta_{2}, \ldots, \theta_{n}\right)$ mit Koeffizienten aus $\mathfrak{\Omega}_{x}$, der bei allen Permutationen der $G_{m}$ mit sich selbst als Funktion auf der Fläche $\mathbf{F}_{l}$ identisch bleibt, ist eine Funktion aus $\mathfrak{\Omega}_{x}$.

Als Umkehrung des vorletzten Satzes haben wir nooh den folgenden Satz zu nennen: Eine Permutation der $\theta_{1}, \theta_{2}, \ldots, \theta_{n}$, die ,jede" zwischen diesen Funktionen identisch gültige "Gleichung in $\mathfrak{\Omega}_{x}$ " wieder in eine ebensolche Gleichung überführt, gehört der Galoisschen Gruppe $G_{m}$ an; diese $G_{m}$ kann demnach als Gruppe aller Permutationen der $\theta$ erklärt werden, bei denen "alle" zwischen den $\theta_{1}(x), \theta_{2}(x), \ldots, \theta_{n}(x)$ identisch bestehenden Gleichungen in $\AA_{x}$ wieder in solche übergehen. Man führt den Beweis genau wie S. $47 \mathrm{ff}$. mit einer besonders gewählten Funktion $\eta(x)$ der Gestalt (3) S. 47. Die Auswahl der ganzzahligen Koeffizienten $\gamma$ kann wieder so getroffen werden, dab von den $n$ ! Funktionen, die bei allen $n$ ! Permutationen der $\theta$ aus $\eta$ hervorgehen, keine zwei identisch sind. 


\section{$\S$ \%. Auflösung einer algebraischen Gleichung $f(z)=0$.}

Es sei $G_{\mu}$ irgendeine Untergruppe der Galoisschen Gruppe $G_{m}$ unserer irreduzibelen Gleichung $f(z)=0$. Eine Funktion $\xi(x)$ des Galoisschen Körpers $\left(\Omega_{x}, \theta_{1}, \theta_{2}, \ldots, \theta_{n}\right)$, die bei den Permutationen der $G_{\mu}$ und nur bei diesen in sich übergeführt wird, nennen wir ,eine zur $G_{\mu}$ gehörende Funktion" und sagen auch umgekehrt, die Gruppe $G_{\mu}$ gehöre $z u \xi(x)$. Es besteht der Satz: $Z u$ irgendeiner Untergruppe $G_{\mu}$ von $G_{m}$ gibt es unendlich viele zugehörige Funktionen; ist $\xi(x)$ eine unter ihnen, so ist jede Funktion des Galoisschen Körpers, die durch die Permutationen der $G_{\mu}$ in sich übergeführt wird, im Körper $\left(\Omega_{x}, \xi\right)$ enthalten. Der Beweis überträgt sich von S. 50 ff. ohne weiteres, wenn nur überall an Stelle des Wortes "Zahl" das Wort „Funktion" gesetzt wird und die "Identität" der Funktionen an Stelle der "Gleichheit" der Zahlen tritt.

Irgendeine Funktion $\xi(x)$ des Galoisschen Körpers nennen wir auch hier eine ,natürliche Irrationalität" der Gleichung $f(z)=0$. Die Gleichung in $\Omega_{x}$, der $\xi$ genügt, heißt wie oben eine „rationale Resolvente" der gegebenen Gleichung $f(z)=0$. Die Entwicklungen von S. $52 \mathrm{ff}$. übertragen sich auf die vorliegenden Verhältnisse mit den eben genannten formalen Abänderungen, wobei insbesondere alle gruppentheoretischen Überlegungen unberührt bleiben. Als Hauptsatz gilt der folgende: Ist $G_{l}$ eine ausgezeichnete Untergruppe der Galoisschen Gruppe $G_{m}$, so genüg! eine zur $G_{l}$ gehörende Funktion $\bar{\eta}$ einer in $\Re_{x}$ irreduzibelen Gleichung des Grades $\bar{m}=\frac{m}{l}$, deren Galoissche Gruppe die Quotientengruppe $G_{m} / G_{l}$ ist; nach Adjunktion von $\bar{\eta} z u \Re_{x}$ wird die Galoissche Resolvente $F(Z)=0$ im Körper $\bar{\Omega}_{x}=\left(\AA_{x}, \bar{\eta}\right)$ reduzibel und liefert nach Zerlegung $\bar{m}$ in $\bar{\Omega}_{x}$ irreduzibele Gleichungen $l^{\text {ten }}$ Grades, die wieder Normalgleichungen sind, und für deren einzelne $G_{l}$ die Galoissche Gruppe ist.

Der Prozeß der "vollständigen Auflösung" der Gleichung $f(z)=0$ oder, was auf dasselbe hinausläuft, die Berechnung „einer" Lösung der Galoisschen Resolvente $F(Z)=0$ gestaltet sich nun genau so wie für die Gleichungen des vorigen Teiles. Wir haben eine Kette von Hilfsgleichungen, die "Normalgleichungen" mit „einfacher Galoisscher Gruppe" sind, zu lösen und erreichen nach der einzelnen Lösung eine Erniedrigung der Ordnung der jeweils vorliegenden Galoisschen Gruppe unter. entsprechender Zerfällung der bis dahin erreichten Galoisschen Resolvente. Insbesondere sind wieder die Kennzeichen für die „algebraische Lösbarkeit" der Gleichung $f(z)=0$ nach der oben (S. 64) angegebenen Regel aus der Struktur der Galoisschen Gruppe $G_{m}$ zu entnehmen. 


\section{§ 8. Monodromiegruppe einer Gleichung $f(\approx)=0$.}

Die bei der Lösung einer Gleichung $f(z)=0$ nach und nach zu adjungierenden natürlichen Irrationalitäten sind Funktionen des Körpers $\left(\Omega_{x}, \theta_{1}, \theta_{2}, \ldots, \theta_{n}\right)$. Es ist nicht ausgeschlossen, daß hierbei auch Funktionen auftreten, die bereits in $x$ und der ursprünglich vorgelegten algebraischen Funktion $y$ von $x$ rational sind. Solche zu adjungierende Funktionen werden dann freilich nicht schon im Körper $\AA_{x}$ enthalten sein, d. h. im rationalen Ausdruck $R(x, y)$ dieser Funktionen müssen unter den Koeffizienten „Zahlen" auftreten, die noch nicht im Zahlkörper $\Re$ enthalten sind. Wir nennen diese Zahlen „numerische Irrationalitüten", die für die Gleichung $f(z)=0$,natürlich“ sind. Indem wir sie zu $\boldsymbol{\Omega}$ und $\boldsymbol{\Omega}_{\boldsymbol{x}}$ adjungieren, erhalten wir einen Zahlkörper $\mathfrak{\Re}^{\prime}$ und einen Funktionenkörper $\AA_{x}^{\prime}$, welchem letzteren die zu adjungierenden Funktionen $R(x, y)$ angehören.

Es entsteht nun die Frage, wie weit wir durch Adjunktion ,numerischer" Irrationalitäten die Auflösung der Gleichung $f(z)=0$ zu treiben vermögen. Hierauf antwortet die folgende Betrachtung:

Um die Lösungen $\eta_{1}(x), \eta_{2}(x), \ldots, \eta_{m}(x)$ der Galoisschen Resolvente $F(Z)=0$ eindeutig zu erklären, wählen wir auf der Riemannschen Fläche $F_{3}$ eine Stelle $x_{0}$, in deren Umgebung keine dieser Funktionen ver-

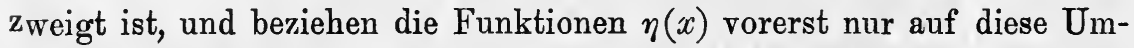
gebung. Setzen wir jetzt von $x_{0}$ aus eine dieser Funktionen $\eta(x)$ längs eines auf die $F_{\text {l }}$ geschlossenen Weges fort, so erbalten wir nach Rückkehr zum Ausgangspunkte $x_{0}$ der $F_{2}$ wieder eine Lösung von $F(Z)=0$, also eine der $m$ Funktionen $\eta(x)$. Auch liefern zwei verschiedene Funktionen $\eta(x)$ bei dieser Fortsetzung über einen geschlossenen Weg der Fläche am Schlusse notwendig wieder zwei verschiedene $\eta(x)$. Einem geschlossenen Wege auf der $\mathbf{F}_{\mathbf{l}}$ gehört demnach eine bestimmte Permutation $T$ der $m$ Lösungen der Galoisschen Resolvente $F(Z)=0 z u$.

Es sei irgendeine zwischen den $\eta_{1}(x), \eta_{2}(x), \ldots, \eta_{m}(x)$ identisch bestehende rationale Gleichung:

$$
R\left(\eta_{1}(x), \eta_{2}(x), \ldots, \eta_{m}(x)\right)=0
$$

vorgelegt, deren Koeffizienten rational in $x$ und $y$ mit irgendwelchen numerischen Konstanten (keineswegs nur mit Zahlen aus $\mathfrak{K}$ ) aufgebaut sind. Bei analytischer.Fortsetzung über die Fläche $F_{z}$ hin bleibt die Gleichung (1) gültig; sie geht demnach wieder in eine identisch bestehende Gleichung über, falls wir die $\eta$ der Permutation $T$ unterwerfen. Da dies insbesondere auch für „jede" Relation (1) gilt, deren Koeffizienten Funktionen aus $\mathfrak{\Omega}_{x}$ sind, so folgt nach S. 74: Alle durch geschlossene Umläufe auf der Fläche $\mathbf{F}_{l}$ herstellbaren Permutationen $T$ der $\eta_{1}, \eta_{2}, \ldots, \eta_{m}$ sind in der Galoisschen Gruppe $G_{m}$ enthalten. 
Zwei geschlossene Umläufe, die wir hintereinander ausüben, lassen sich zu einem dritten Umlaufe zusammensetzen. Also folgt der Satz: Die gesamten durch geschlossene Umläufe herstellbaren Permutationen $T_{0}, T_{1}, T_{2}$, $\ldots, T_{\mu-1}$ bilden eine Gruppe $G_{\mu}$, die in der Galoisschen Gruppe $G_{m}$ enthalten ist und als "Monodromiegruppe" der Gleichung $F(Z)=0$ bezeichnet wird. In ihrer Gestalt als Permutationsgruppe $n^{\text {ten }}$ Grades $\operatorname{der} \theta_{1}, \theta_{2}, \ldots$, $\theta_{n}$ nennen wir die $G_{\mu}$ die „Monodromiegruppe" der Gleichung $f(z)=0$. Es ist einleuchtend, daß wir diese Gestalt der $G_{\mu}$ unmittelbar gewinnen, wenn wir alle Permutationen $T_{0}=1, T_{1}, T_{2}, \ldots, T_{\mu-1}$ der Funktionen $\theta_{1}(x), \theta_{2}(x), \ldots, \theta_{n}(x)$ bei geschlossenen Umläufen auf der $F_{l}$ sammeln.

Es ist möglich, daß die Monodromiegruppe $G_{\mu}$ mit der Gesamtgruppe $G_{m}$ gleich ist. Liegt dieser Fall nicht vor, so gilt folgende Überlegung: Die Monodromiegruppe $G_{\mu}$ ist als Permutationsgruppe der $\eta$ für $\mu<m$ intransitiv. Da das einzelne $\eta$ durch die $\mu$ Permutationen $\operatorname{der} G_{\mu}$ in $\mu$ verschiedene $\eta$ übergeführt wird, so erhalten wir $t=\frac{m}{\mu}$ Systeme der Intransitivität, die wir durch:

$$
\eta_{k \mu+1}, \eta_{k \mu+2}, \ldots, \eta_{k \mu+\mu}, \quad k=0,1,2, \ldots, t-1
$$

bezeichnen. Die $\eta$ jedes dieser $t$ Systeme werden bei den Umläufen auf $\operatorname{der} F_{l}$ nur unter sich permutiert. Die symmetrischen Grundfunktionen der $\eta$ des einzelnen Systemes (2) sind demnach algebraische Funktionen $\operatorname{der} F_{l}$ und als solche rational in $x$ und $y$. Es folgt der Satz: Die $\eta(x)$ eines jeden der $t$ Systeme (2) sind die Lösungen einer Gleichung $\mu^{\text {ten }}$ Grades:

$$
H_{k}(Z)=0, \quad k=0,1,2, \ldots, t-1,
$$

deren Koeffizienten rationale Funktionen von $x$ und $y$ sind. Da $F(Z)=0$ im Körper $\mathfrak{\Omega}_{x}$ irreduzibel ist, so treten in diesen rationalen Funktionen von $x$ und y Zahlenkoeffizienten auf, die als ,"numerische Irrationalitäten “ fïr die Gleichung $f(z)=0$,natürlich" sind. Es besteht weiter der Satz: Jede der $t$ Gleichungen (3) ist in dem Sinne irreduzibel, daß $H_{k}(Z)$ nicht in Faktoren von niederem als $\mu^{\text {ten }}$ Grade zerfällbar ist, die gleichfalls in $x$ und y rationale Koeffizienten hätten. Jeder solche Faktor würde nämlich, wie die an (1) angeschlossene Überlegung zeigt, durch alle $\eta$ seines Systems (2) befriedigt.

Diese Ergebnisse legen die Bedeutung der Monodromiegruppe $G_{\mu}$ dar: Durch Adjunktion „,numerischer Irrationalitäten" ist die Zerfällung der Galoisschen Resolvente in die $t$ Gleichungen (3) erreichbar; jede weitere Zerfällung erfordert die Adjunktion von „Funktionen“. Durch Adjunktion jener numerischen Irrationalitäten werde der Körper $\Omega$ auf $\AA^{\prime}$ und entsprechend $\Omega_{x}$ auf $\Omega_{x}^{\prime}$ erweitert. Jede der $t$ Gleichungen (3) ist eine „Gleichung in $\Omega_{x}^{\prime \prime \prime}$, die in diesem Körper, sowie überhaupt in jedem Körper $\mathfrak{\Omega}_{x}^{\prime \prime}$, der durch weitere Adjunltionen von "Zahlen" herstellbar ist, irreduzibel ist; 
dabei ist die Gleichung (3) wieder eine "Normalgleichung", deren Galoissche Gruppe die aus den $\mu$ Lösungen der Gleichung aufgebaute $G_{\mu}$ ist.

Über die Berechnung der numerischen Irrationalitäten kann hier nur erst folgendes gesagt werden: Entsprechend dem ersten Satze von $\S 7$ haben wir zunächst eine zur $G_{\mu}$ gehörende „Funktion" $\xi(x)$ zu bilden, die einer Gleichung $t^{\text {ten }}$ Grades in $\Omega_{x}$ genügt:

$$
\xi^{t}+c_{1} \xi^{t-1}+c_{2} \zeta^{t-2}+\cdots+c_{t-1}=0 .
$$

Im Körper $\left(\Omega_{x}, \zeta\right)$ sind dann die sämtlichen Koeffizienten der $t$ Gleichungen (3) enthalten. Die Gleichung (4) ist durch eine "rationale Funktion" von $x$ und $y$ lösbar; nichtrationale Operationen bei der Auflösung von (4) beziehen sich also nur auf die Berechnung von „Zablen“. Indessen muB es späteren besonderen Fällen vorbehalten bleiben, an Stelle der Gleichung (4) für eine „Funktion“ eine solche für eine "Zahl“ zu setzen.

\section{Algebraische Zahlen. ${ }^{1}$ )}

\section{\$1. Algebraische und ganze algebraische Zahlen.}

An die Entwicklungen von S. 32 ff. schließen wir für den einfachsten Fall, daß der damalige Körper $\Re$ der rationale Zahlkörper $\Re$ ist, folgende Erklärung an: Unter einer „algebraischen Zahl" schlechthin versteht man eine Zahl, die in bezug auf den rationalen Körper $\Re$ algebraisch ist. Eine algebraische Zahl $\theta$ genügt also einer Gleichung:

$$
f(z)=z^{n}+a_{1} z^{n-1}+a_{2} z^{n-2}+\cdots+a_{n}=0
$$

mit rationalen Zahlenkoeffizienten $a$, und jede Wurzel einer solchen Gleichung, mag sie reduzibel oder irreduzibel in $\Re$ sein, ist eine algebraische Zahl. Da wir die Irreduzibilität der Gleichung (1) einstweilen nicht fordern, so lassen sich für eine einzelne Zahl $\theta$ unendlich viele Gleichungen angeben, deren Wurzel sie ist.

Insbesondere gilt die Erklärung: Eine algebraische Zahl heißt speziell eine "ganze algebraische Zahl" $\eta$, wenn sich mindestens eine in $\Re$ reduzibele oder irreduzibele Gleichung (1) mit rationalen "ganzen" Koeffizienten a angeben läßt, deren Wurzel $\eta$ ist.

Es besteht der Satz: Sind $\eta$ und $\eta^{\prime}$ ganze algebraische Zahlen, so

1) Es kommen in diesem Teile einige späterhin unentbehrliche Hauptsätze der „Idealtheorie" Dedekind's zur Behandlung; s. das Supplement XI zu Dirichlet's "Vorlesungen über Zahlentheorie", $3^{\text {to }}$ Aufl. (Braunschweig, 1879), S. $434 \mathrm{ff}$., $4^{\text {to }}$ Aufl. (Braunschweig, 1894), S. 434 ff. S. auch den ersten Teil des S. 23 genannten Werkes von Land au. Beim Hauptsatze der Idealtheorie (S. 97) folgt die vorliegende Darstellung der besonders kurzen von A. Hurwitz herrührenden Beweismethode; s. dessen Note „Über die Theorie der Ideale“, Göttinger Nachrichten von 1894. 
sind auch ihre Summe $\left(\eta+\eta^{\prime}\right)$, ihre Differenz $\left(\eta-\eta^{\prime}\right)$ und ihr Produkt. $\eta \cdot \eta^{\prime}$ ganze algebraische Zahlen. Für $\eta$ bestehe die Gleichung (1) mit den Wurzeln $\eta_{1}=\eta, \eta_{2}, \ldots, \eta_{n}$, für $\eta^{\prime}$ eine entsprechende Gleichung des Grades $n^{\prime}$ mit den Wurzeln $\eta_{1}^{\prime}=\eta^{\prime}, \eta_{2}^{\prime}, \ldots, \eta_{n^{\prime}}^{\prime}$. Man bilde die $n \cdot n^{\prime}$ Summen $\left(\eta_{i}+\eta_{k}^{\prime}\right)$ je einer Wurzel der ersten und einer der zweiten Gleichung. Die symmetrischen Grundfunktionen dieser $n \cdot n^{\prime}$ Summen sind nach dem Hauptsatze der Theorie der symmetrischen Funktionen (S. 25) ganze ganzzahlige Funktionen der Koeffizienten $a_{1}, a_{2}, \ldots$ und $a_{1}^{\prime}, a_{2}^{\prime}, \ldots$ jener beiden Gleichungen, sind also selbst rationale ganze Zahlen. Also genügt $\left(\eta+\eta^{\prime}\right)$ einer Gleichung:

$$
z^{n n^{\prime}}+b_{1} z^{n \cdot n^{\prime}-1}+\cdots+b_{n n^{\prime}}=0
$$

mit rationalen ganzen $b$ und ist somit eine ganze algebraische Zahl. Da offenbar mit $\eta^{\prime}$ auch $-\eta^{\prime}$ eine ganze algebraische Zahl ist, so gilt unser Satz auch für die Differenz $\left(\eta-\eta^{\prime}\right)$. Endlich wird für das Produkt $\eta \cdot \eta^{\prime}$ der Beweis gerade so wie für die Summe $\left(\eta+\eta^{\prime}\right)$ geführt. Durch wiederholte Bildung von Summen, Differenzen und Produkten folgt: Jede rationale ganze, ganzzahlige Funktion von ganzen algebraischen Zahlen ist wieder eine ganze algebraische Zahl.

Der Hauptsatz über symmetrische Funktionen gestattet uns auch noch folgenden Satz zu beweisen: Jede Wurzel $\eta$ einer Gleichung (1), deren Koeffizienten $a_{1}, a_{2}, \ldots, a_{n}$ ganze algebraische Zahlen sind, ist selbst eine ganze algebraische Zahl. Der einzelne Koeffizient $a_{k}$ genügt jetzt selbst einer Gleichung:

$$
y^{m_{k}}+b_{k 1} y^{m_{k}-1}+b_{k 2} y^{m_{k}-2}+\cdots+b_{k, m_{k}}=0
$$

mit rationalen ganzen $b$, deren sämtliche Wurzeln $a_{k}, a_{k}^{\prime}, \ldots, a_{k}^{\left(m_{k}-1\right)}$ seien. Wir bilden die $m_{1} \cdot m_{2} \cdots m_{n}$ Funktionen:

$$
z^{n}+a_{1}^{\left(i_{1}\right)} z^{n-1}+a_{2}^{\left(i_{2}\right)} z^{n-2}+\cdots+a_{n}^{\left(i_{n}\right)}
$$

für alle $m_{1} \cdot m_{2} \cdots m_{n}$ Kombinationen der Zahlen $a_{k}^{(i)}$. Die erste dieser Funktionen ist die linke Seite der für $\eta$ vorgelegten Gleichung. Das Produkt aller dieser Funktionen gibt, gleich 0 gesetzt, eine Gleichung für $\eta$, deren Koeffizienten ganze ganzzahlige Funktionen der $b$ und also selbst rationale ganze Zahlen sind. Also ist $\eta$ eine ganze algebraische Zahl.

Irgendeine algebraische Zahl $\theta$ genüge der Gleichung (1), deren Koeffizienten $a_{1}, a_{2}, \ldots, a_{n}$ rationale Brüche sind. Ihr Hauptnenner sei die rationale ganze positive $\mathrm{Zahl} a$, so $\mathrm{da} B a \cdot a_{1}, a \cdot a_{2}, \ldots, a \cdot a_{n}$ rationale ganze Zahlen sind. Das Produkt $a \cdot \theta=\eta$ genügt der Gleichung:

$$
\eta^{n}+a a_{1} \eta^{n-1}+a^{2} a_{2} \eta^{n-2}+\cdots+a^{n} a_{n}=0,
$$

deren Koeffizienten durchweg rationale ganze Zahlen sind. Es folgt hieraus der Satz: Jede algebraische Zahl $\theta$ liefert durch Multiplikation mit 
einer geeignet gewählten rationalen ganzen positiven Zahl a als Produkt $a \theta=\eta$ eine ,ganze" algebraische Zahl.

Unter einer "ganzen Zahl“ verstehen wir im vorliegenden Teile stets eine ganze „algebraische“ Zahl. Die ganzen „rationalen“ Zahlen, die zu den ganzen algebraischen Zahlen gehören, mögen immer durch den $\mathrm{Zu}$ satz „rational" gekennzeichnet werden.

\section{$\$$ 2. Ein algebraischer Hilfssatz.}

Ist eine ganze algebraische Zahl $\eta$ als Produkt $\eta=\eta^{\prime} \cdot \eta^{\prime \prime}$ zweier ganzer algebraischer Zahlen $\eta^{\prime}, \eta^{\prime \prime}$ darstellbar, so heiBt jeder Faktor, z. B. $\eta^{\prime}$, ein "Teiler" von $\eta$ oder $\eta$ heißt durch $\eta^{\prime}$ "teilbar" oder man sagt, $\eta^{\prime}$,gehe in $\eta$ auf". Die Gesetze der Teilbarkeit der ganzen algebraischen Zahlen werden den wichtigsten Gegenstand unserer Untersuchungen ausmachen. Um den hierbei auftretenden Hauptsatz besonders kurz beweisen zu können, soll zunächst ein Hilfssatz aufgestellt werden.

Mit irgendwelchen ganzen (algebraischen) Zahlen $a_{0}, a_{1}, \ldots, a_{\mu}$ und $b_{0}, b_{1}, \ldots, b_{v}$, von denen $a_{0}$ und $b_{0}$ von 0 verschieden seien, bilde man die Funktionen:

$$
\varphi(z)=a_{0} z^{\mu}+a_{1} z^{\mu-1}+\cdots+a_{\mu}, \quad \psi(z)=b_{0} z^{\nu}+b_{1} z^{\nu-1}+\cdots+b_{v},
$$
deren Produkt:

$$
\varphi(z) \cdot \psi(z)=f(z)=c_{0} z^{n}+c_{1} z^{n-1}+c_{2} z^{n-2}+\cdots+c_{n}
$$

vom Grade $n=\mu+\nu$ ist und wieder ganzzahlige Koeffizienten hat. Dann gilt der Satz: Sind alle Zahlen $c_{0}, c_{1}, \ldots, c_{n}$ durch die ganze Zahl $\eta$ teilbar, so hat auch jedes der $(\mu+1)(\nu+1)$ ganzzahligen Produkte $a_{i} \cdot b_{k}$, $z u$ bilden für $i=0,1, \ldots, \mu$ und $k=0,1, \ldots, v$, den Teiler $\eta$.

Einen sehr kurzen Beweis dieses Satzes hat Hurwitz ${ }^{1}$ ) geliefert. Es wird zunächst bewiesen, daß die $(\nu+1)$ Produkte $a_{0} b_{0}, a_{0} b_{1}, a_{0} b_{2}$, $\ldots, a_{0} b_{v}$ durch $\eta$ teilbar sind. Für $a_{0} b_{0}=c_{0}$ folgt dies bereits aus der Voraussetzung; für die übrigen Produkte gilt folgende Betrachtung: Die Gleichung:

$$
a_{0} \psi(z)=c_{0} z^{\nu}+a_{0} b_{1} z^{\nu-1}+a_{0} b_{2} z^{\nu-2}+\cdots+a_{0} b_{v}=0
$$

hat $\nu$ Wurzeln $z_{1}, z_{2}, \ldots, z_{v}$, die zu den $n$ Wurzeln von $f(z)=0$ gehören. Der Quotient:

$$
(-1)^{k} \frac{a_{0} b_{k}}{c_{0}}=\sigma_{k}\left(z_{1}, z_{2}, \ldots, z_{v}\right)
$$

iefert die $k^{\text {te }}$ symmetrische Grundfunktion der $z_{1}, z_{2}, \ldots, z_{v}$. Als Funktion der $n$ Wurzeln $z_{1}, z_{2}, \ldots, z_{n}$ der Gleichung $f(z)=0$ ist eine beliebige der $\nu$ Funktionen $\sigma_{k}$, die wir kurz $\sigma$ nennen, noch nicht symmetrisch, bleibt vielmehr nur erst bei denjenigen $\nu !(n-\nu)$ ! Permutationen unverändert, welche die $z_{1}, z_{2}, \ldots, z_{v}$ unter sich vertauschen und ebenso die

1) In der S. 78 genannten Note. 
$z_{v+1}, z_{v+2}, \ldots, z_{n}$. Somit ist $\sigma$ im Sinne von S. 24 eine $t$-wertige ganze ganzzahlige Funktion der Wurzeln von $f(z)=0$, wo

$$
t=\frac{n !}{\nu !(n-v) !}=\left(\begin{array}{l}
n \\
\nu
\end{array}\right)
$$

ist. Als $t$-wertige Funktion genügt $\sigma$ einer Gleichung $t^{\text {ten }}$ Grades:

$$
\sigma^{t}+d_{1} \sigma^{t-1}+d_{2} \sigma^{t-2}+\cdots+d_{t}=0,
$$

wo die $-d_{1}, d_{2},-d_{3}, d_{4}, \ldots$ die symmetrischen Grundfunktionen der $t$ verschiedenen Ausdrücke sind, die aus $\sigma\left(z_{1}, z_{2}, \ldots, z_{v}\right)$ bei allen $n$ ! Permutationen der $z_{1}, z_{2}, \ldots, z_{n}$ hervorgehen. Nach dem Hauptsatze von S. 25 sind die $d$ als ganze ganzzahlige symmetrische Funktionen der $z_{1}$, $z_{2}, \ldots, z_{n}$ rationale ganze ganzzahlige Funktionen der symmetrischen Grundfunktionen der $z_{1}, z_{2}, \ldots, z_{n}$ und damit der $\frac{c_{1}}{c_{0}}, \frac{c_{2}}{c_{0}}, \ldots, \frac{c_{n}}{c_{0}}$, und zwar ist der Grad von $d_{1}$ als Funktion $\operatorname{der} \frac{c_{1}}{c_{0}}, \frac{c_{2}}{c_{0}}, \ldots, \frac{c_{n}}{c_{0}}$ gleich 1, der von $d_{2}$ gleich 2, der von $d_{3}$ gleich 3 usw.

Man multipliziere die Gleichung (4) mit $c_{0}^{t}$ und schreibe $c_{0} \sigma=\tau$ oder ausführlich:

$$
c_{0} \sigma_{k}=\tau_{k}=(-1)^{k} a_{0} b_{k} \text {. }
$$

Die Produkte $c_{0} d_{1}=e_{1}, c_{0}^{2} d_{2}=e_{2}, \ldots, c_{0}^{t} d_{t}=e_{t}$ sind alsdann ganze homogene Funktionen ersten, zweiten, $\ldots, t^{\text {ten }}$ Grades von $c_{0}, c_{1}, \ldots, c_{n}$, die als solche der Voraussetzung zufolge bzw. durch $\eta, \eta^{2}, \ldots, \eta^{t}$ teilbar sind. Die mit $c_{0}^{t}$ multiplizierte Gleichung (4) aber hat die Gestalt:

$$
\tau^{t}+e_{1} \tau^{t-1}+e_{2} \tau^{t-2}+\cdots+e_{t}=0
$$

und liefert, durch $\eta^{t}$ geteilt:

$$
\left(\frac{\tau}{\eta}\right)^{t}+\frac{e_{1}}{\eta}\left(\frac{\tau}{\eta}\right)^{t-1}+\frac{e_{2}}{\eta^{2}}\left(\frac{\tau}{\eta}\right)^{t-2}+\cdots+\frac{e_{t}}{\eta^{t}}=0 .
$$

Somit befriedigt $\frac{\tau}{\eta}$ eine Gleichung mit ganzzahligen Koeffizienten und ist demnach zufolge des vorletzten Satzes in $\S 1$ selbst eine ganze Zahl. Also sind in der Tat alle $(v+1)$ Produkte $a_{0} b_{0}, a_{0} b_{1}, a_{0} b_{2}, \ldots, a_{0} b_{v}$ durch $\eta$ teilbar.

Nachdem bewiesen ist, daß alle Produkte $a_{0} b_{0}, a_{0} b_{1}, \ldots, a_{0} b_{v}$ durch $\eta$ teilbar sind, stellen wir weiter fest, daB auch das Produkt von:

$$
\varphi(z)-a_{0} z^{\mu}=a_{1} z^{\mu-1}+a_{2} z^{\mu-2}+\cdots+a_{\mu}
$$

und $\psi(z)$, nämlich die Funktion:

$$
\left(\varphi(z)-a_{0} z^{\mu}\right) \cdot \psi(z)=f(z)-z^{\mu}\left(a_{0} b_{0} z^{\nu}+a_{0} b_{1} z^{\nu-1}+\cdots+a_{0} b_{v}\right)
$$

lauter durch $\eta$ teilbare Koeffizienten hat. Ist $a_{1} \neq 0$, so finden wir durch Wiederholung der vorstehenden Überlegung, $\mathrm{da} B$ auch alle Produkte $a_{1} b_{0}, a_{1} b_{1}, a_{1} b_{2}, \ldots, a_{1} b_{v}$ durch $\eta$ teilbar sind. Im Falle $a_{1}=0$ ist dies selbstrerständlich. In gleicher Weise fortfahrend erkennen wir die Richtigkeit des aufgestellten Satzes. 


\section{\$ 3. Folgerungen betreffs rationaler ganzer Zahlen.}

Es gilt der Satz: Eine ganze algebraische Zahl $\eta$, die dem rationalen Körper $\Re$ angehört, ist eine rationale "ganze" Zahl. Als eine in $\Re$ enthaltene Zahl kann $\eta=\frac{q}{r}$ gesetzt werden, wo $q$ und $r$ zwei teilerfremde rationale ganze Zahlen sind. Als ganze (algebraische) Zahl genügt $\eta$ einer Gleichung (1) S. 78 mit rationalen ganzen $a_{1}, 4 \ldots$ Also folgt, wenn wir $\eta=\frac{q}{r}$ für $z$ in jene Gleichung eintragen und mit $r^{n}$ multiplizieren:

$$
q^{n}=-r\left(a_{1} q^{n-1}+a_{2} q^{n-2} r+a_{3} q^{n-3} r^{2}+\cdots+a_{n} r^{n-1}\right) .
$$

Hiernach ist $q^{n}$ und also $q$ durch jeden Primfaktor von $r$ teilbar. Da aber $q$ und $r$ teilerfremd sind, so hat $r$ keinen Primfaktor, der gröBer als 1 wäre, d. h. es ist $r=1$ und also $\eta$ rational und ganz.

Wir ziehen nun einige Folgerungen aus dem Satze des $\S 2$ für den Fall, daB die Koeffizienten $a_{0}, a_{1}, \ldots, a_{\mu}$ und $b_{0}, b_{1}, \ldots, b_{v}$ der Funktionen $\varphi(z)$ und $\psi(z)$ rationale ganze Zahlen sind. Eine ganze Funktion mit rationalen ganzen Koeffizienten soll "ursprünglich" heißen, wenn diese Koeffizienten keine rationale ganze Zahl, die $>1$ ist, als Teiler gemeinsam haben. Dann besteht der Satz: Das Produkt zweier ursprünglicher Funktionen $\varphi(z)$ und $\psi(z)$ ist stets wieder eine ursprüngliche Funktion $f(z)$. Wäre dies nicht der Fall, so gäbe es mindestens eine Primzahl $p>1$, die in allen Koeffizienten $c$ von $f(z)$ und also in allen $(\mu+1)(\nu+1)$ Produkten $a_{i} b_{k}$ aufgeht. Da $\varphi(z)$ ursprünglich ist, so gibt es mindestens eine Zahl $a_{i}$, die nicht durch $p$ teilbar ist, und ebenso können wir ein gegen $p$ primes $b_{k}$ angeben. Also ist $a_{i} b_{k}$ nicht durch $p$ teilbar, so daB die Annahme einer nicht ursprünglichen Funktion $f(z)$ unhaltbar ist.

Es gelte ferner die Annahme, daB die Funktion:

$$
f(z)=z^{n}+c_{1} z^{n-1}+c_{2} z^{n-2}+\cdots+c_{n}
$$

mit rationalen „ganzen“ Koeffizienten $c$ im Körper $\Re$ reduzibel sei und in das Produkt der beiden Funktionen:

$$
\varphi(z)=z^{\mu}+a_{1} z^{\mu-1}+\cdots+a_{\mu}, \quad \psi(z)=z^{\nu}+b_{1} z^{\nu-1}+\cdots+b^{\nu}
$$

mit, ,rationalen" Koeffizienten $a, b$ zerfalle. Die rationalen Brüche $a$ mögen den Hauptnenner $a_{0}$ haben, so daß $a_{0}, a_{1}^{\prime}=a_{0} a_{1}, a_{2}^{\prime}=a_{0} a_{2}, \ldots, a_{\mu}^{\prime}=a_{0} a_{\mu}$ rationale ganze Zahlen ohne einen allen gemeinsamen Teiler $>1$ sind; ebenso mag $b_{0}$ der Hauptnenner der rationalen Brüche $b$ sein, so dab auch $b_{0}, b_{1}^{\prime}=b_{0} b_{1}, \ldots, b_{v}^{\prime}=b_{0} b_{v}$ rationale ganze Zahlen ohne einen allen gemeinsamen Teiler sind. Es stehen also auf der linken Seite der Gleichung:

$$
\left(a_{0} z^{\mu}+a_{1}^{\prime} z^{\mu-1}+\cdots+a_{\mu}^{\prime}\right)\left(b_{0} z^{\nu}+b_{1}^{\prime} z^{\nu-1}+\cdots+b_{\nu}^{\prime}\right)=a_{0} b_{0} f(z)
$$

zwei ursprüngliche Funktionen, und also ist nach dem eben bewiesenen 
Satze auch $a_{0} b_{0} f(z)$ ursprünglich. Hieraus ergibt sich $a_{0} b_{0}=1, a_{0}=1$, $b_{0}=1$, so daB der Satz gilt: Ist die Funktion (1) mit rationalen ganzen Koeffizienten $c$ im rationalen Körper $\Re$ reduzibel und zwar zerfällbar in das Produkt der beiden Funktionen (2) mit rationalen Koeffizienten $a, b$, so sind diese Koeffizienten $a, b$ notwendig „ganze" rationale Zahlen.

\section{§ 4. Algebraische Zahlkörper.}

Aus den Sätzen von S. $33 \mathrm{ff}$. entnimmt man unmittelbar die folgenden Ergebnisse: Eine algebraische $\mathrm{Zahl} \theta$ genügt einer eindeutig bestimmten, im rationalen Körper $\Re$ irreduzibelen ${ }^{1}$ ) Gleichung:

$$
f(z)=z^{n}+a_{1} z^{n-1}+a_{2} z^{n-2}+\cdots+a_{n}=0
$$

mit rationalen Koeffizienten, deren sämtliche Wurzeln $\theta_{1}=\theta, \theta_{2}, \ldots, \theta_{n}$ verschieden sind und $n$,konjugierte" algebraische Zahlen heißen. Die Adjunktion von $\theta$ zu $\Re$ liefert einen in bezug auf $\Re$ algebraischen Körper $\Omega=(\Re, \theta)$, der weiterhin kurz als ein „algebraischer Körper $n^{\text {ten }}$ Grades“ bezeichnet wird. Die $n$ Körper $\left(\Re, \theta_{1}\right)=(\Re, \theta),\left(\Re, \theta_{2}\right), \ldots,\left(\Re, \theta_{n}\right)$ heißen „,konjugiert" und sollen kurz $\Omega_{1}=\Omega, \Omega_{2}, \ldots, \Omega_{n}$ genannt werden. Diese Körper brauchen nicht alle voneinander verschieden $\mathrm{zu}$ sein. Sind sie insbesondere alle einander gleich, so heißt $\mathfrak{A}$ ein "Galoisscher Körper" oder „Normalkörper".

Jede Zahl $\zeta$ des Körpers $\Omega$ ist auf eine und nur eine Art in der Gestalt:

$$
\xi=c_{0}+c_{1} \theta+c_{2} \theta^{2}+\cdots+c_{n-1} \theta^{n-1}
$$

mit rationalen $c$ darstellbar; sie genügt der durch die Tschirnhausentransformation: $w=c_{0}+c_{1} z+c_{2} z^{2}+\cdots+c_{n-1} z^{n-1}$

aus (1) hervorgehenden Gleichung $n^{\text {ten }}$ Grades und ist deshalb wieder eine algebraische Zahl. Umgekehrt ist jede mit rationalen $c$ dargestellte Zahl (2) in $\Omega$ enthalten. Die $n$ mit $\zeta$,konjugierten" Zahlen:

$$
\xi_{i}=c_{0}+c_{1} \theta_{i}+c_{2} \theta_{i}^{2}+\cdots+c_{n-1} \theta_{i}^{n-1}, \quad i=1,2, \ldots, n
$$

sind $\mathrm{zu}$ je $\mu$ einander gleich und stellen $\nu$ verschiedene Zahlen dar, wobei $\mu \cdot \nu=n$ ist. Gilt $\mu=1$, so heißt $\zeta$ eine "primitive" Zahl des Körpers $\Omega$, für $\mu>1$ wird sie ,imprimitiv" genannt.

Hieran schließen sich einige weitere Entwicklungen über die Darstellung von Zahlen aus $\Re$. Irgend $n$ Zahlen $\xi, \zeta^{\prime}, \ldots, \xi^{(n-1)}$ aus $\Omega$ heißen "linear-abhängig", falls ein System nicht durchgängig verschwindender rationaler Zahlen $b, b^{\prime}, \ldots, b^{(n-1)}$ angebbar ist, für das:

$$
b \xi+b^{\prime} \xi^{\prime}+\cdots+b^{(n-1)} \xi^{(n-1)}=0
$$

gilt; existiert ein solches Zahlensystem $b$ nicht, so heißen die $\xi, \zeta^{\prime}, \ldots, \zeta^{(n-1)}$

1) Der Zusatz „im rationalen Körper $\Re^{\prime \prime}$ bleibt gewöhnlich fort, da sich die Irreduzibilität hier stets auf $\Re$ bezieht. 
„linear-unabhängig“. Zufolge der Irreduzibilität von (1) sind jedenfalls die Zahlen $1, \theta, \theta^{2}, \ldots, \theta^{n-1}$ linear-unabhängig. Für die $n$ Zahlen $\xi^{(0)}=\xi, \xi^{\prime}, \ldots$ $\xi^{(n-1)}$ mögen als Darstellungen (2) gelten:

$$
\xi^{(k)}=c_{0}^{(k)}+c_{1}^{(k)} \theta+c_{2}^{(k)} \theta^{9}+\cdots+c_{n-1}^{(k)} \theta^{n-1}, \quad k=0,1, \ldots, n-1 .
$$

Damit die Zahlen $\xi, \xi^{\prime}, \ldots, \xi^{(n-1)}$ linear-abhängig sind, ist dann hinreichend und notwendig, daß $n$ nicht durchgängig verschwindende rationale Zahlen $b$ existieren, die den Gleichungen:

$$
b c_{i}+b^{\prime} c_{i}^{\prime}+\cdots+b^{(n-1)} c_{i}^{(n-1)}=0, \quad i=0,1, \ldots,(n-1)
$$

genügen. Nach bekannten Sätzen der Determinantentheorie folgt: Irgend $n$ durch (5) gegebene Zahlen $\xi, \xi, \ldots, \xi^{(n-1)}$ des Körpers $\Re$ sind linearunabhängig oder nicht, je nachdem die Determinante $\left|c_{i .}^{(k)}\right| \operatorname{der} n^{2}$ Koeffizienten $c$ in (5) von 0 verschieden ist oder verschwindet.

Es seien $\xi_{1}^{(k)}=\xi^{(k)}, \xi_{2}^{(k)}, \ldots, \xi_{n}^{(k)}$ die mit $\xi^{(k)}$ konjugierten Zahlen. Dann gilt folgende Erklärung: Das Quadrat der $n$-reihigen Determinante:

$$
\left|\begin{array}{l}
\xi_{1}, \xi_{1}^{\prime}, \ldots, \xi_{1}^{(n-1)} \\
\xi_{2}, \xi_{2}^{\prime}, \ldots, \xi_{2}^{(n-1)} \\
\cdot \cdot \\
\xi_{n}, \xi_{n}^{\prime}, \ldots, \xi_{n}^{(n-1)}
\end{array}\right|=\left|\xi_{i}^{(k)}\right|
$$

heißt die "Diskriminante" $\operatorname{der} n$ Zahlen $\xi, \xi^{\prime}, \ldots, \xi^{(n-1)}$ und wird durch $D\left(\xi, \xi^{\prime}, \ldots, \xi^{(n-1)}\right)$ bezeichnet. Insbesondere ist die Diskriminante $D\left(1, \theta, \ldots, \theta^{n-1}\right)$ der $n$ Zahlen $1, \theta, \ldots, \theta^{n-1}$ zugleich die Diskriminante der irreduzibelen Gleichung (1) in $\mathfrak{R}$ (S. 26); diese Diskriminante ist eine von 0 verschiedene rationale Zahl. Schreibt man alle mit (5) konjugierten Gleichungen auf, so ergibt das Multiplikationsgesetz der Determinanten:

$$
D\left(\xi, \xi^{\prime}, \ldots, \xi^{(n-1)}\right)=\left|c_{i}^{(k)}\right|^{2} \cdot D\left(1, \theta, \theta^{2}, \ldots, \theta^{n-1}\right) .
$$

Hieraus folgt mit Rücksicht auf den letzten Satz: Die Diskriminante $D\left(\xi, \xi^{\prime}, \ldots, \xi^{(n-1)}\right)$ der $n$ Zahlen $\xi, \xi^{\prime}, \ldots, \xi^{(n-1)}$ verschwindet oder hat einen von 0 verschiedenen rationalen Zahlwert, der mit $D\left(1, \theta, \theta^{2}, \ldots, \theta^{n-1}\right)$ im Vorzeichen übereinstimmt, je nachdem die $\xi, \xi^{\prime}, \ldots, \xi^{(n-1)}$ linear-abhängig sind oder nicht.

Ist $\left|c_{i}^{(k)}\right| \neq 0$, so lassen sich die $n$ Gleichungen (5) nach $1, \theta, \ldots, \theta^{n-1}$ lösen. Es ergeben sich so Darstellungen der $1, \theta, \theta^{2}, \ldots, \theta^{n-1}$ und damit Darstellung aller Zahlen von $\Omega$ in der Gestalt:

$$
c \xi+c^{\prime} \xi^{\prime}+c^{\prime \prime} \xi^{\prime \prime}+\cdots+c^{(n-1)} \xi^{(n-1)}
$$

durch die $n$ linear-unabhängigen Zahlen $\xi, \xi^{\prime}, \ldots, \xi^{(n-1)}$ mittels rationaler $c, c^{\prime}, \ldots$ Auch diesø Darstellung ist für die einzelne Zahl von $\Omega$ eindeutig bestimmt, wie aus der linearen Unabhängigkeit der $\xi, \xi^{\prime}, \ldots$ folgt. Umgekehrt liefert natürlich jeder mit rationalen $c$ gebildete Ausdruck (8) eine Zahl aus $\Omega$. 
Es mögen sich noch folgende Erklärungen hier anschließen: Sind $\xi_{1}=\xi, \xi_{2}, \ldots, \xi_{n}$ die $n$ mit $\xi$ konjugierten Zahlen, so versteht man unter der „Spur" $S(\xi)$ von $\xi$ die Summe und unter der „Norm“ $N(\xi)$ von $\xi$ das Produkt jener konjugierten Zahlen:

$$
S(\xi)=\xi_{1}+\xi_{2}+\cdots+\xi_{n}, \quad N(\xi)=\xi_{1} \cdot \xi_{2} \cdots \xi_{n} .
$$

$S(\xi)$ und $N(\xi)$ sind rationale Zahlen, die an bekannten Stellen als Koeffizienten in der Gleichung $n^{\text {ten }}$ Grades für $\xi$ auftreten. Konjugierte Zahlen haben natürlich gleiche Spuren sowie auch gleiche Normen. Bildet man das Quadrat der Determinante (6) nach dem Multiplikationsgesetze der Determinanten, so gelangt man zu folgender Darstellung der Diskriminante der Zahlen $\xi, \zeta^{\prime}, \ldots, \xi^{(n-1)}$ durch Spuren:

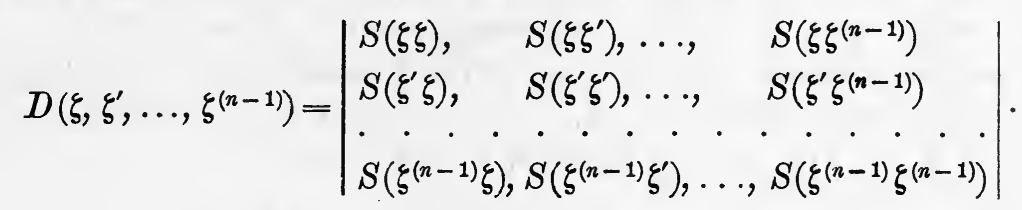

\section{§. Die ganzen Zahlen des Körpers $\mathfrak{R}$.}

Die in $\Re=(\Re, \theta)$ enthaltenen ganzen Zahlen sollen allgemein $\eta$ genannt werden, das System aller dieser ganzen Zahlen werde e genannt. Es gilt der Satz: Sind $\eta$ und $\eta^{\prime}$ irgend zwei Zahlen aus e, so sind auch ihre Summe $\left(\eta+\eta^{\prime}\right)$, ihre Differenz $\left(\eta-\eta^{\prime}\right)$ und ihr Produkt $\left(\eta \cdot \eta^{\prime}\right)$ in e enthalten. Es sind nämlich $\left(\eta \pm \eta^{\prime}\right)$ und $\eta \cdot \eta^{\prime}$ nach S. 79 wieder ganze Zahlen, und andrerseits gehören $\left(\eta \pm \eta^{\prime}\right)$ und $\eta \cdot \eta^{\prime}$ dem Körper $\Omega$ an und sind demnach im System e enthalten. Allgemein gilt der Satz: Jede rationale ganze Funktion von Zahlen aus e mit rationalen ganzen Koeffizienten ist wicder eine Zahl aus e. Für die Spuren und Normalen bestehen die Regeln:

$$
S\left(\eta \pm \eta^{\prime}\right)=S(\eta)^{-} \pm S\left(\eta^{\prime}\right), \quad N\left(\eta \cdot \eta^{\prime}\right)=N(\eta) \cdot N\left(\eta^{\prime}\right)
$$

Sind nämlich zu $\eta$ und $\eta^{\prime}$ im Körper $\Omega_{i}$ die Zahlen $\eta_{i}$ und $\eta_{i}^{\prime}$ konjugiert, so ist $\mathrm{zu}\left(\eta \pm \eta^{\prime}\right)$ die Zahl $\left(\eta_{i} \pm \eta_{i}^{\prime}\right)$ und zu $\eta \cdot \eta^{\prime}$ die Zahl $\eta_{i} \cdot \eta_{i}^{\prime}$ konjugiert, woraus die Regeln (1) leicht folgen.

Eine Zahl aus $e$, deren Norm gleich \pm 1 ist, heiBt eine „Einheit" des Körpers $\mathcal{R}$ oder des Systems $e$ und möge speziell durch $\varepsilon$ bezeichnet werden. Genügt $\varepsilon$ der irreduzibelen Gleichung:

$$
z^{v}+a_{1} z^{\nu-1}+\cdots+a_{v-1} z \pm 1=0
$$

mit rationalen ganzen Koeffizienten, so genügt die gleichfalls in $\mathfrak{N}$ enthaltene Zahl $\varepsilon^{-1}$ der Gleichung:

$$
z^{v} \pm\left(a_{v-1} z^{\nu-1}+\cdots+a_{1} z+1\right)=0,
$$

stellt also gleichfalls eine ganze Zahl dar. Ist andrerseits mit der von 0 
verschiedenen Zahl $\eta$ aus e auch $\eta^{-1}$ eine ganze Zahl und also wieder in e enthalten, so genügt $\eta$ einer irreduzibelen Gleichung mit dem Absolutgliede \pm 1 . Es gilt also der Satz: Eine von 0 verschiedene Zahl $\eta$ aus $\mathrm{e}$ ist stets und nur dann eine Einheit $\varepsilon$, wenn auch $\eta^{-1}$ eine ganze Zahl ist. Jede mit einer Einheit konjugierte Zahl ist natürlich wieder eine Einheit ihres Körpers. Ist $\eta$ eine beliebige Zahl aus $e$ und $\varepsilon$ eine Einheit, so heißt $\varepsilon \cdot \eta$ eine mit $\eta$ „assoziierte Zahl“. Zwei assoziierte Zahlen haben, abgesehen vom Vorzeichen, gleiche Normen.

Nach S. 80 liefert jede Zahl $\xi$ aus $\Re$, mit einer geeignet gewählten von 0 verschiedenen rationalen ganzen Zahl $a$ multipliziert, eine ganze Zahl $\eta=a \cdot \xi$. Es mögen auf diese Weise aus den $n$ Zahlen $\xi_{1}, \xi_{2}, \ldots, \xi_{n}{ }^{1}$ ) von $\Re$ die $n$ Zahlen $\eta_{1}=a_{1} \xi_{1}, \eta_{2}=a_{2} \xi_{2}, \ldots, \eta_{n}=a_{n} \xi_{n}$ von $e$ gewonnen werden. Sind die $\xi$ linear-unabhängig, so gilt dasselbe offenbar von den $n$ ganzen Zahlen $\eta_{1}, \eta_{2}, \ldots, \eta_{n}$. Wir können somit stets $n$ linear-unabhängige ganze Zahlen $\eta_{1}, \eta_{2}, \ldots, \eta_{n}$ zugrunde legen, in denen jede Zahl $\xi$ von $\Omega$ auf eine und nur eine Art in der Gestalt:

$$
\xi=c_{1} \eta_{1}+c_{2} \eta_{2}+\cdots+c_{n} \eta_{n}
$$

mittels rationaler Koeffizienten $c$ darstellbar ist (vgl. S. 84).

Die Diskriminante $D\left(\eta_{1}, \eta_{2}, \ldots, \eta_{n}\right)$ irgendeines Systems linearunabhängiger ganzer Zahlen $\eta_{1}, \eta_{2}, \ldots, \eta_{n}$ aus $\mathrm{e}$ ist nach $\mathrm{S} .84$ eine ganze Zahl, sowie nach dem an (7) S. 84 angeschlossenen Satze eine rationale, von 0 verschiedene und mit $D\left(1, \theta, \ldots, \theta^{n-1}\right)$ im Vorzeichen übereinstimmende Zahl. Also folgt: Die Diskriminanten aller Systeme linear-unabhängiger Zahlen $\eta_{1}, \eta_{2}, \ldots, \eta_{n}$ aus e sind von 0 verschiedene, rationale ganze Zahlen, die alle das gleiche Vorzeichen haben.

Unter allen von 0 verschiedenen rationalen ganzen Zahlen, die als Diskriminanten bei den Systemen linear-unabhängiger Zahlen $\eta_{1}, \eta_{2}, \ldots, \eta_{n}$ aus $\mathfrak{e}$ auftreten, gibt es eine absolut kleinste. Diese absolut kleinste Zahl $D$ heibt die „Grundzahl" oder „Disliriminante" des Körpers $\Re$; ein System, dessen Diskriminante jenen Minimalwert hat, wird eine "Basis" des Zahlsystems e genannt. Fis besteht der Satz: Bilden die Zahlen $\eta_{1}, \eta_{2}, \ldots, \eta_{n}$ eine Basis von e, so ist nicht nur jede Zahl:

$$
\eta=e_{1} \eta_{1}+e_{2} \eta_{2}+\cdots+e_{n} \eta_{n}
$$

mit rationalen ganzen $e$ in e enthalten, sondern umgekehrt ist auch "jede" Zahl aus e auf eine und nur eine Art in der Gestalt (4) mittels rationaler "ganzer" $e$ darstellbar. Zu beweisen ist hier nur noch, daB keine ganze

1) Bisher bezeichneten wir mit $\xi_{1}, \zeta_{2}, \ldots, \xi_{n}$ ein System von $n$ konjugierten Zahlen. Da solche Systeme weiterhin nur noch selten zu betrachten sind, so benutzen wir die bequeme Schreibweise der unteren Indizes zur Unterscheidung irgendwelcher Zahlen aus $\Re$. 
Zahl in der Gestalt:

$$
\eta=c_{1} \eta_{1}+c_{2} \eta_{2}+\cdots+c_{n} \eta_{n}
$$

mittels rationaler, aber nicht durchweg ganzer $c$ darstellbar ist. Sollte aber eine ganze Zahl $\eta$ mit solcher Darstellung vorkommen, so stellen wir die $c$ als Quotienten kleinster ganzer Zahlen dar und nennen ihren Hauptnenner $h$. Dann sind:

$$
h c_{1}=e_{1}, \quad h c_{2}=e_{2}, \quad h c_{n}=e_{n}
$$

$\boldsymbol{n}$ rationale ganze Zahlen, deren größter, allen gemeinsamer Teiler prim gegen $h$ ist. Irgendein Primfaktor $p>1$ von $h$ geht demnach nicht in allen diesen $e$ auf und möge etwa teilerfremd gegen $e_{1}$ sein. Schreiben wir $h=a p$, so ist auch:

$$
a \eta=\frac{e_{1} \eta_{1}+e_{2} \eta_{2}+\cdots+e_{n} \eta_{n}}{p}
$$

eine ganze Zahl. Da $e_{1}$ und $p$ teilerfremd sind, so kann man eine rationale ganze Zahl $b$ entsprechend der Kongruenz $b e_{1} \equiv 1(\bmod p)$ wählen und hat dann auch in:

$$
\eta_{1}^{\prime}=a b \eta-\frac{b e_{1}-1}{p} \eta_{1}=\frac{1}{p} \eta_{1}+a b\left(c_{2} \eta_{2}+\cdots+c_{n} \eta_{n}\right)
$$

eine ganze Zahl. Für die Diskriminante des Systems $\eta_{1}^{\prime}, \eta_{2}, \ldots, \eta_{n}$ ergibt sich nun leicht:

$$
D\left(\eta_{1}^{\prime}, \eta_{2}, \ldots, \eta_{n}\right)=\frac{1}{p^{2}} D\left(\eta_{1}, \eta_{2}, \ldots, \eta_{n}\right),
$$

so daß wir in $D\left(\eta_{1}, \eta_{2}, \ldots, \eta_{n}\right)$ noch nicht die minimale Diskriminante erreicht haben würden. Damit ist der Satz aber bewiesen.

Irgendein System von $n$ Zahlen $\eta_{1}^{\prime}, \eta_{2}^{\prime}, \ldots, \eta_{n}^{\prime}$ aus $e$ besitze in der Basis $\eta_{1}, \eta_{2}, \ldots, \eta_{n}$ die Darstellung:

$$
\eta_{i}^{\prime}=e_{i 1} \eta_{1}+e_{i 2} \eta_{2}+\cdots+e_{i n} \eta_{n}, \quad i=1,2, \ldots, n .
$$

Wie S. 84 folgt aus dem Multiplikationsgesetze der Determinanten:

$$
D\left(\eta_{1}^{\prime}, \eta_{2}^{\prime}, \ldots, \eta_{n}^{\prime}\right)=\left|e_{i k}\right|^{2} \cdot D\left(\eta_{1}, \eta_{2}, \ldots, \eta_{n}\right) .
$$

Hieraus folgt der Satz: Die Zahlen $\eta_{1}^{\prime}, \eta_{2}^{\prime}, \ldots, \eta_{n}^{\prime}$ bilden stets und nur dann gleichfalls eine Basis von e, wenn die Determinante der $n^{2}$ ganzzahligen Koeffizienten in (5) gleich \pm 1 ist. Weiter liest man aus (6) das Ergebnis ab: Ist die rationale ganze Zahl $D\left(\eta_{1}^{\prime}, \eta_{2}^{\prime}, \ldots, \eta_{n}^{\prime}\right)$ durch kein Quadrat (außer 1) teilbar, so bilden die $\eta_{1}^{\prime}, \eta_{2}^{\prime}, \ldots, \eta_{n}^{\prime}$ eine Basis, und $D\left(\eta_{1}^{\prime}, \ldots, \eta_{n}^{\prime}\right)$ ist die Grundzahl des Körpers.

\section{$\S$ 6. Teilbarkeit der Zahlen $\boldsymbol{\eta}$ im Systeme e.}

Eine Zahl $\eta$ des Systems e heißt durch die gleichfalls in e enthaltene Zahl $\eta^{\prime}$,teilbar" oder $\eta^{\prime}$ ist ein "Teiler" von $\eta$ oder "geht in $\eta$ auf", falls es eine Zahl $\eta^{\prime \prime}$ in e gibt, die mit $\eta$ und $\eta^{\prime}$ die Gleichung $\eta=\eta^{\prime} \cdot \eta^{\prime \prime}$ be- 
friedigt. Natürlich ist dann auch $\eta^{\prime \prime}$, ein Teiler von $\eta$. Es ist einleuchtend, daß $\eta$ durch jede Einheit $\varepsilon$ des Körpers $\Re$ und durch jede mit $\eta$ assoziierte Zahl $\varepsilon \eta$ teilbar ist.

Im rationalen Körper sind die Gesetze der Teilbarkeit sehr einfach. Die einzigen Einheiten von $\Re$ sind +1 und -1 . Eine von 0 und \pm 1 verschiedene rationale ganze Zahl $p$, die als Teiler nur die vier Zahlen \pm 1 und $\pm p$ hat, heißt eine "Primzahl". Es besteht der Satz: Jede von 0 verschiedene rationale ganze Zahl $a$ ist als Produkt einer der Einheiten \pm 1 und einer Anzahl positiver Primzahlen darstellbar, und zwar sind diese Primzahlen (natürlich abgesehen von ihrer Reihenfolge) durch a eindeutig bestimmt. Der Satz von der eindeutigen Bestimm theit dieser „Primfaktorenzerlegung" ist die Grundlage vieler arithmetischer Überlegungen und gehört zu den wichtigsten Grundsätzen der Zahlentheorie.

Bei der Ausdehnung der Gesetze der Teilbarkeit auf die ganzen Zahlen $\eta$ eines algebraischen Körpers $\Omega$ stellte sich nun die Tatsache ein, $d a \beta$ der eben für den rationalen Körper $\mathfrak{R}$ ausgesprochene Satz keinezwegs allgemein auf algebraische Körper $\mathfrak{\Omega}$ verallgemeinert werden konnte, indem bereits Körper zweiten Grades nachweisbar waren, in denen er nicht mehr gilt.

Als Beispiel betrachten wir den durch die irreduzibele Gleichung $z^{2}+5=0$ gegebenen Körper zweiten Grades $\Re=(\Re, i \sqrt{5})$. Derselbe ist ein Normalkörper, dem $i \sqrt{5}$ als ganze Zahl angehört und dessen sämtliche Zahlen in der Gestalt $\left(c_{0}+c_{1} i \sqrt{5}\right)$ mit rationalen $c$ darstellbar sind. Da:

$$
D(1, i \sqrt{5})=\left|\begin{array}{l}
1,+i \sqrt{5} \\
1,-i \sqrt{5}
\end{array}\right|^{2}=-20
$$

nur den quadratischen Teiler 4 hat, so sind sicher alle ganzen Zahlen von $\Re$ mittelst rationaler ganzer $e$ in der Gestalt $\frac{1}{2}\left(e_{0}+e_{1} i \sqrt{5}\right)$ darstellbar. Nun ist aber:

$$
N\left(\frac{e_{0}+e_{1} i \sqrt{5}}{2}\right)=\frac{e_{0}^{2}+5 e_{1}^{2}}{4}
$$

nur dann eine ganze Zahl, wenn $e_{0}$ und $e_{1}$ gerade Zahlen sind. Also bilden die beiden Zahlen $1, i \sqrt{5}$ eine Basis von $e$, und die Grundzahl von $\Re$ ist -20 . Aus $N\left(e_{0}+e_{1} i \sqrt{5}\right)=e_{0}^{2}+5 e_{1}^{2}$ liest man sofort weiter ab, daB \pm 1 die einzigen Einheiten von $\Omega$ sind.

Die Zahl 21 ist nun in das Produkt 3.7 spaltbar. Keiner der Faktoren 3 und 7 ist in e weiter zerlegbar. Wäre nämlich z. B. die Zahl 3 als Produkt $\eta_{1} \cdot \eta_{2}$ zweier von \pm 1 verschiedener ganzer Zahlen von $\mathbb{R}$ darstellbar, so wäre nach (1) S. 85 :

$$
N\left(\eta_{1}\right) \cdot N\left(\eta_{2}\right)=N(3)=9
$$

und also würde, da $N\left(\eta_{1}\right)$ und $N\left(\eta_{2}\right)$ rational, ganz und von \pm 1 verschieden sind, $N\left(\eta_{1}\right)=N\left(\eta_{2}\right)= \pm 3 \mathrm{zu}$ treffen. Ist also $\eta_{1}=e_{0}+e_{1} i \sqrt{5}$, 
so würde $e_{0}^{2}+\check{5} e_{1}^{2}= \pm 3$ folgen, eine Gleichung, die durch rationale ganze $e_{0}, e_{1}$ nicht zu befriedigen ist. Die Zahl 3 ist also im vorliegenden Systeme $e$ unzerlegbar, und man zeigt in derselben Art, daB auch 7 unzerlegbar ist. Neben der Zerlegung $21=3 \cdot 7$ der Zahl 21 besteht aber noch eine zweite Zerlegung:

$$
21=(1+2 i \sqrt{5})(1-2 i \sqrt{5})
$$

von 21 in das Produkt zweier in $\Re$ enthaltener ganzer Zahlen. Diese Faktoren sind gleichfalls unzerlegbar. Wäre nämlich etwa $1+2 i \sqrt{5}$ $=\eta_{1} \cdot \eta_{2}$, wo wieder $\eta_{1}, \eta_{2}$ keine Einheiten sind, so würde:

$$
N\left(\eta_{1}\right) \cdot N\left(\eta_{3}\right)=N(1+2 i \sqrt{5})=21
$$

folgen, und also wäre für einen der beiden Faktoren $N(\eta)= \pm 3$, was wir bereits als unmöglich erkannten. Die Zahl 21 ist hiernach in zwei wesentlich verschiedenen Arten als Produkt unzerlegbarer ganzer Zahlen unseres Körpers $(\Re, i \sqrt{5})$ darstellbar.

Es ist ein naheliegender Gedanke, durch eine Erweiterung des Gebietes e unserer ganzen Zahlen den Satz von der „Eindeutigkeit“ der Zerlegung jeder ganzen Zahl des erweiterten Gebietes in unzerlegbare Faktoren zu retten. In unserem Falle müßte etwa eine Zerlegung von 21 in vier unzerlegbare Faktoren $\mathfrak{p}_{1}, \mathfrak{p}_{2}, \mathfrak{p}_{3}, \mathfrak{p}_{4}$ stattfinden, und es müBte:

(1) $3=\mathfrak{p}_{1} \cdot \mathfrak{p}_{2}, \quad 7=\mathfrak{p}_{3} \cdot \mathfrak{p}_{4} \quad$ und $\quad 1+2 i \sqrt{5}=\mathfrak{p}_{1} \cdot \mathfrak{p}_{3}, \quad 1-2 i \sqrt{5}=\mathfrak{p}_{2} \cdot \mathfrak{p}_{4}$ gelten. Die Durchführung dieses Gedankens in einer für alle algebraischen Körper $\Omega$ gültigen Gestalt ist von R. Dedekind in seiner "Idealtheorie" geleistet. Die wichtigsten Grundlagen dieser Theorie sind nun zu entwickeln.

\section{§ 7. Begriff und Darstellung eines Ideals.}

Der Grundgedanke der Dedekindschen Theorie ist der, daß die Begriffe und Gesetze der Teilbarkeit nicht auf einzelne Zahlen angewandt werden, sondern auf gewisse Systeme unendlich vieler ganzer Zahlen aus e, die Dedekind „Ideale“ nennt. Es mögen zumächst die elementaren Vorstellungen und Überlegungen, die die Teilbarkeit der rationalen ganzen Zahlen betreffen, in die Sprache der Idealtheorie übersetzt werden. Es sei demnach zunächst $\mathfrak{e}$ das System aller ganzen Zahlen des rationalen Körpers $\Re$.

Ist $\alpha$ eine von 0 verschiedene Zahl aus e, und durchläuft $\eta$ alle Zahlen von $e$, so heißt das System $\eta \alpha$ aller durch $\alpha$ teilbaren Zahlen von $e$ ein in e enthaltenes „Ideal". Als Bezeichnung für ein solches Ideal benutzen wir wieder die Frakturschrift $\mathfrak{a}$ und schreiben auch, wenn wir ausdrücken wollen, dab $\mathfrak{a}$ aus allen Vielfachen von $\alpha$ besteht, $\mathfrak{a}=[\alpha]$, so daß auch $[\alpha]$ ein Symbol für das System der unendlich vielen Zahlen $\eta \alpha$ ist. Die charakteristischen Eigenschaften eines solchen Ideals sind die folgenden: 
1. Die Summe und die Differenz zweier Zahlen aus a sind wieder in $\mathfrak{a}$ enthalten.

2. Das Produkt irgendeiner Zahl $\eta$ aus e und einer Zahl aus a ist wieder in a enthalten.

3. Das Ideal a soll nicht nur aus der einzigen Zahl 0 bestehen. ${ }^{1}$ )

Es ist leicht zu zeigen, daß diese drei Eigenschaften im System $e$ der rationalen ganzen Zahlen stets ein Zahlsystem $\eta \alpha$ obiger Art festlegen. Versteht man also jetzt unter einem Ideale $\mathfrak{a}$ in jenem Systeme $e$ irgendein in $e$ enthaltenes Zahlsystem, das die drei genannten Eigenschaften hat, so ist $\mathrm{zu}$ beweisen, $\mathrm{da} \mathfrak{a} \mathfrak{a}$ stets aus den gesamten durch eine nicht-verschwindende ganze Zahl $\alpha$ teilbaren Zahlen von e besteht. Ist nämlich $\alpha$ eine absolut kleinste von 0 verschiedene Zahl in a, die zufolge 3. existiert, so enthält $\mathfrak{a}$ sicher alle Zahlen $\eta \alpha$ wegen der Eigenschaft 2. Weitere Zahlen können aber in a nicht auftreten, da sonst zufolge 1 . sofort eine absolut zwischen 0 und $|\alpha|$ gelegene Zahl in $\mathfrak{a}$ nachweisbar wäre. Man beachte übrigens gleich noch, daß die Gesamtheit $e$ aller rationalen ganzen Zahlen offenbar selbst ein Ideal darstellt, das wir auch durch [1] bezeichnen können.

Da zu jeder Zahl $\alpha$ aus $e$ eindeutig ein Ideal $\mathfrak{a}=[\alpha]$ gehört und ungekehrt zu jedem Ideal a eindeutig ein Paar ,assoziierter" Zahlen $\pm \alpha$ aus $e$ (die sich in Rücksicht auf Teilbarkeit im wesentlichen gleich verhalten), so erscheint es möglich, die Zahlen durch ihre zugehörigen Ideale zu ersetzen und die Regeln der Teilbarkeit in die Sprache der Ideale zu übertragen.

Als ,Produkt" zweier Ideale $\mathfrak{a}=[\alpha]$ und $\mathfrak{b}=[\beta]$ bezeichnen wir das Ideal $\mathfrak{c}=\mathfrak{a} \cdot \mathfrak{b}=[\alpha \cdot \beta]$. Dann ist offenbar $\mathfrak{b} \cdot \mathfrak{a}=\mathfrak{a} \cdot \mathfrak{b}$, und wir finden, wenn eines der Ideale $\mathfrak{a}, \mathfrak{b}$, etwa $\mathfrak{b}=\mathfrak{e}=[1]$ ist, $\mathfrak{a} \cdot \mathfrak{e}=\mathfrak{e} \cdot \mathfrak{a}=\mathfrak{a}$, so da $\mathfrak{b}$ $\mathfrak{e}$ als "Einheitsideal" bezeichnet werden kann. Ist $\mathfrak{c}=\mathfrak{a} \cdot \mathfrak{b}$, so heißt jedes der Ideale $\mathfrak{a}, \mathfrak{b}$ ein "Teiler" von $\mathfrak{c}$, oder man sagt, $\mathfrak{c}$ sei durch $\mathfrak{a}$ und $\mathfrak{b}$ „teilbar", oder $\mathfrak{a}$ und $\mathfrak{b}$,gehen in $\mathfrak{c}$ auf"; umgekehrt ist $\mathfrak{c}$ ein "Vielfaches" von $\mathfrak{a}$ und auch von $\mathfrak{b}$. Das Ideal $\mathfrak{a}$ ist stets und nur dann ein Teiler von $\mathfrak{c}$, wenn jede Zahl von $\mathfrak{c}$ in $\mathfrak{a}$ enthalten ist. ${ }^{2}$ ) Setzen wir nämlich $\mathfrak{c}=[\gamma]$, so ist $\gamma$ in $\mathfrak{a}$ enthalten, also $\gamma=\beta \cdot \alpha$, wo $\beta$ eine Zahl aus $e$ ist.

Sind $\alpha$ und $\beta$ zwei von 0 verschiedene Zahlen aus $e$, so bilden alle Zahlen $\left(\eta_{1} \alpha+\eta_{2} \beta\right)$ mit irgendwelchen Paaren $\eta_{1}, \eta_{2}$ aus e wieder ein Ideal. Wir bezeichnen dieses Ideal durch $\delta=[\alpha, \beta]$. Offenbar sind sowohl die Zahlen von $\mathfrak{a}$ als auch die von $\mathfrak{b}$ in $\mathfrak{b}$ enthalten, so da $\mathfrak{b}$ ein gemeinsamer Teiler von $\mathfrak{a}$ und $\mathfrak{b}$ ist. Da jeder gemeinsame Teiler von $\mathfrak{a}$ und $\mathfrak{b}$ die

1) Das aus der Zahl 0 allein bestehende "System" würde die Eigenschaften 1. und 2. besitzen. Durch die Forderung 3. ist dieses "System" ausgeschlossen.

2) Der "Teiler" a ist hier also ein „umfassenderes" Zahlsystem als das „Vielfache $c \mathfrak{c}$, sofern nicht etwa $\mathfrak{c}=\mathfrak{a} \cdot \mathrm{e}$ vorliegen sollte. 
Zahlen $\eta_{1} \alpha$ und $\eta_{2} \beta$ und also die von $\delta$ in sich enthält, so heißt $\delta=[\alpha, \beta]$ der "größte gemeinsame Teiler" von $\mathfrak{a}$ und $\mathfrak{b}$. Dies ist mit dem elementaren Begriffe des größten gemeinsamen Teilers zweier rationaler ganzer Zahlen in Übereinstimmung. Ist nämlich $\delta=[\alpha, \beta]=[\delta]$, wo $\delta$ positiv gewählt sein mag, so ist $\delta$ die kleinste positive, mit rationalen ganzen $\eta_{1}, \eta_{2}$ in der Gestalt $\delta=\eta_{1} \alpha+\eta_{2} \beta$ darstellbare Zahl. Diese ist aber in der Tat der größte gemeinsame Teiler von $\alpha$ und $\beta$ (vgl. S. 30).

Wir kehren nun zu einem beliebigen Körper $n^{\text {ten }}$ Grades $\Omega$ zurück, dessen ganze Zahlen das System e bilden. Irgendein System von Zahlen aus $\mathfrak{e}$ bildet ein „Ideal“ $\mathfrak{a}$ des Körpers $\Re$, wenn das System die drei oben genannten Eigenschaften 1., 2. und 3. besitzt. Wir stellen im Anschluß an dieșe Erklärung sogleich fest: Zwei Ideale $\mathfrak{a}$ und $\mathfrak{b}$ von $\mathfrak{\Re}$ heißen einander gleich, $\mathfrak{a}=\mathfrak{b}$, wenn jede Zahl des einen Ideals auch im anderen enthalten ist. Auf Grund des folgenden Satzes kann man Ideale des Körpers $\Re$ herstellen: Sind $\alpha_{1}, \alpha_{2}, \ldots, \alpha_{\lambda}$ irgendwelche $\lambda$ festgewählte, nicht durchweg verschwindende Zahlen aus e, so liefern die gesamten Zahlen:

$$
\eta_{1} \alpha_{1}+\eta_{2} \alpha_{2}+\cdots+\eta_{\lambda} \alpha_{\lambda}
$$

zu bilden für alle möglichen Systeme von $\lambda$ Zahlen $\eta_{1}, \eta_{2}, \ldots, \eta_{\lambda}$ aus $\mathrm{e}$, ein Ideal $\mathfrak{a}$, das wir durch:

$$
\mathfrak{a}=\left[\alpha_{1}, \alpha_{2}, \ldots, \alpha_{2}\right]
$$

bezeichnen. Man zeigt nämlich am Systeme (1) sofort die drei charakteristischen Eigenschaften eines Ideals. Hierbei ist übrigens keineswegs behauptet, daB die einzelne Zahl aus a nur auf eine Weise in der Gestalt (1) darstellbar sei.

Auch umgekehrt gilt der Satz: Jedes Ideal a von $\Re$ ist mittelst einer „endlichen" Anzahl seiner Zahlen $\alpha_{1}, \alpha_{2}, \ldots, \alpha_{\lambda}$ in der Gestalt $\left[\alpha_{1}, \alpha_{2}, \ldots, \alpha_{2}\right]$ als System aller Zahlen (1) darstellbar. $\mathrm{Zu}$ einer weiterhin besonders wichtigen Art einer solchen Darstellung irgendeines vorgelegten Ideals $a$ führt folgende Überlegung: Es gibt sicher in a Systeme von $n$ linearunabhängigen Zahlen $\alpha_{1}, \alpha_{2}, \ldots, \alpha_{n}$. Ist nämlich $\alpha$ eine von 0 verschiedene Zahl aus $a$, und bilden $\eta_{1}, \eta_{2}, \ldots, \eta_{n}$ eine Basis von $e$, so bilden z. B. die $n$ Zahlen $\alpha_{1}=\eta_{1} \alpha, \alpha_{2}=\eta_{2} \alpha, \ldots, \alpha_{n}=\eta_{n} \alpha$ ein System linear-unabhängiger Zahlen von $\mathfrak{a}$. Für jedes solche System ist $D\left(\alpha_{1}, \alpha_{2}, \ldots, \alpha_{n}\right)$ eine von 0 verschiedene rationale ganze Zahl. Wie S. 86 nennen wir ein System linear-unabhängiger Zahlen $\alpha_{1}, \alpha_{2}, \ldots, \alpha_{n}$ aus a eine "Basis" des Ideals $\mathfrak{a}$, falls die von 0 verschiedene rationale ganze Zahl $D\left(\alpha_{1}, \alpha_{2}, \ldots, \alpha_{n}\right)$, absolut genommen, einen möglichst kleinen Wert hat. Dann gilt der Satz: Jede Zahl:

$$
\alpha=e_{1} \alpha_{1}+e_{2} \alpha_{2}+\cdots+e_{n} \alpha_{n}
$$

mit rationalen ganzen $e$ ist in a enthalten, und jede Zahl $\alpha$ von $\mathfrak{a}$ ist auf eine und nur eine Art in der Gestalt (3) mittelst rationaler ganzer Zahlen 
$e$ durch die Basis $\alpha_{1}, \alpha_{2}, \ldots, \alpha_{n}$ darstellbar. Der Beweis wird durch Wiederholung der Überlegung von S. 87 geführt, wobei an die Stelle der damaligen Zahlen $\eta_{1}, \eta_{2}, \ldots, \eta_{n}$ die Zahlen $\alpha_{1}, \alpha_{2}, \ldots, \alpha_{n}$ treten und das System $e$ durch $\mathfrak{a}$ zu ersetzen ist. Hiernach ist $\mathfrak{a}$ sicher z. B. in den $n$ Zahlen einer Basis $\alpha_{1}, \alpha_{2}, \ldots, \alpha_{n}$ als Ideal $\left[\alpha_{1}, \alpha_{2}, \ldots, \alpha_{n}\right]$ darstellbar, womit der letzte Satz bewiesen ist.

Während später der Gebrauch einer Basis von a wichtig wird, ist es einstweilen zweckmäBiger, die Anzahl $\lambda$ der Zahlen $\alpha$ in (2) unbestimmt zu lassen und als "Koeffizienten" nicht nur rationale ganze Zahlen, sondern wie in (1) beliebige ganze Zahlen $\eta$ aus e zuzulassen. Sollen alle Zahlen eines zweiten Ideals $\mathfrak{b}=\left[\beta_{1}, \beta_{2}, \ldots, \beta_{\mu}\right]$ in $\mathfrak{a}$ enthalten sein, so ist hierfür das Bestehen der $\mu$ Gleichungen:

$$
\beta_{i}=\eta_{i 1} \alpha_{1}+\eta_{i 2} \alpha_{2}+\cdots+\eta_{i \lambda} \alpha_{\lambda}, \quad i=1,2, \ldots, \mu
$$

erforderlich und hinreichend, wo natürlich die $\eta$ Zahlen aus e sind. Bestehen außerdem $\lambda$ Gleichungen:

$$
\alpha_{k}=\eta_{k 1}^{\prime} \beta_{1}+\eta_{k 2}^{\prime} \beta_{2}+\cdots+\eta_{k \mu}^{\prime} \beta_{\mu}, \quad k=1,2, \ldots, \lambda
$$

wieder mit Zahlen $\eta^{\prime}$ aus $\mathfrak{e}$, so sind auch alle Zahlen von $\mathfrak{a}$ in $\mathfrak{b}$ enthalten, d. h. wir haben $\mathfrak{a}=\mathfrak{b}$.

Ein Ideal $\mathfrak{a}=[\alpha]$, das also aus allen durch die von 0 verschiedene ganze Zahl $\alpha$ teilbaren Zahlen aus e besteht, heißt ein "Hauptideal". Sollen die beiden Hauptideale $\mathfrak{a}=[\alpha]$ und $\mathfrak{b}=[\beta]$ einander gleich sein, so müssen zwei Gleichungen $\beta=\eta \alpha$ und $\alpha=\eta^{\prime} \beta$ mit Zahlen $\eta$ und $\eta^{\prime}$ aus $e$ gelten. Aus ihnen folgt $\eta \cdot \eta^{\prime}=1$, so daB die Zahlen $\eta$ und $\eta^{\prime}$ Einheiten sein müssen, $\eta=\varepsilon$ und $\eta^{\prime}=\varepsilon^{\prime}=\varepsilon^{-1}$. Aus $\beta=\varepsilon \alpha$ folgt auch sofort $\alpha=\varepsilon^{\prime} \beta$, so daß der Satz gilt: Die beiden Hauptideale $\mathfrak{a}=[\alpha]$ und $\mathfrak{b}=[\beta]$ sind stets und nur dann einander gleich, wenn $\alpha$ und $\beta$ assoziierte Zahlen sind. Insbesondere folgt: Jedes aus einer Einheit $\varepsilon$ hergestellte Hauptideal $[\varepsilon]$ ist gleich e.

Die „Hauptideale" sind es, die den Zahlen von e, genauer den Systemen assoziierter Zahlen von e zugeordnet sind. Soll der Begriff des Ideals allgemein die S. $88 \mathrm{ff}$. besprochene Schwierigkeit heben, so müBte die zu vollziehende „Erweiterung“ des Gebietes $e$ der ganzen Zahlen von $\Omega$ darin bestehen, daß wir, nachdem die Systeme der assoziierten Zahlen durch ihre Hauptideale ersetzt sind, die gesamten übrigen Ideale von $\Omega$, sofern solche vorhanden sind, als selbständige Elemente, gewissermaßen als neue ganze Zahlen, den bisherigen hinzuzufügen. In dem so erweiterten Gebiete müBten dann die Gesetze der Teilbarkeit wieder denselben einfachen Charakter annehmen wie im rationalen Körper. Daß dies in der Tat der Fall ist, wird der in §9, S. 97 aufzustellende Hauptsatz der Idealtheorie zeigen. 


\section{§ 8. Multiplikation der Ideale.}

Die Multiplikation zweier Ideale ist so zu erklären, daB insbesondere die Multiplikation zweier Hauptideale auf diejenige zweier ganzer Zahlen hinausläuft. Dies leistet folgende Festsetzung: Das Produkt $\mathfrak{c}=\mathfrak{a} \cdot \mathfrak{b}$ der beiden Ideale $\mathfrak{a}=\left[\alpha_{1}, \alpha_{2}, \ldots, \alpha_{2}\right]$ und $\mathfrak{b}=\left[\beta_{1}, \beta_{2}, \ldots, \beta_{\mu}\right]$ ist das Ideal:

$$
c=\left[\alpha_{1} \beta_{1}, \alpha_{1} \beta_{2}, \ldots, \alpha_{1} \beta_{\mu}, \alpha_{2} \beta_{1}, \ldots, \alpha_{\lambda} \beta_{\mu}\right] .
$$

Dem Produkte gehört jede Zahl an, die durch Multiplikation einer Zahl $\alpha$ aus $\mathfrak{a}$ und einer Zahl $\beta$ aus $\mathfrak{b}$ entsteht, und damit auch jede Summe solcher Produkte:

$$
\alpha \beta+\alpha^{\prime} \beta^{\prime}+\alpha^{\prime \prime} \beta^{\prime \prime}+\cdots+\alpha^{(v)} \beta^{(v)} .
$$

Umgekehrt ist jede Zahl des Ideals (1) in der Gestalt (2) darstellbar z. B. als eine Summe:

$$
\alpha_{1} \beta^{\prime}+\alpha_{2} \beta^{\prime \prime}+\cdots+\alpha_{\lambda} \beta^{(\lambda)}
$$

wo $\alpha_{1}, \alpha_{2}, \ldots, \alpha_{2}$ die zur Darstellung des Ideals $\mathfrak{a}=\left[\alpha_{1}, \alpha_{2}, \ldots, \alpha_{2}\right]$ ausgewählten Zahlen sind und $\beta^{\prime}, \beta^{\prime \prime}, \ldots, \beta^{(\lambda)}$ Zahlen des Ideals $\mathfrak{b}$ bedeuten. Man kann das Produkt $\mathfrak{a} \cdot \mathfrak{b}$ geradezu als das ein Ideal bildende System aller Zahlen (2) erklären. Hieraus erkennt man, $d a \beta$ das Produkt $\mathfrak{a} \cdot \mathfrak{b}$ durch die Faltoren $\mathfrak{a}$ und $\mathfrak{b}$ eindeutig bestimmt ist, $\mathfrak{d} . \mathrm{h}$. da $\mathfrak{a} \cdot \mathfrak{b}$ nicht etwa abhängig ist von der besonderen Auswahl der beiden Zahlensysteme $\alpha_{1}, \alpha_{2}, \ldots, \alpha_{\lambda}$ und $\beta_{1}, \beta_{2}, \ldots, \beta_{\mu}$, die der Erklärung (1) zugrunde liegen.

Aus (1) folgt der Satz: Für die Multiplikation der Ideale gelten die Gesetze $\mathfrak{a} \cdot \mathfrak{b}=\mathfrak{b} \cdot \mathfrak{a}$ und $(\mathfrak{a} \cdot \mathfrak{b}) \cdot \mathfrak{c}=\mathfrak{a} \cdot(\mathfrak{b} \cdot \mathfrak{c})$. Für das assoziative Gesetz wolle man sich das Produkt dreier Ideale entsprechend dem Ansatze (1) anschreiben. Die Gesetze bestehen für die Ideale dann einfach deshalb, weil sie für die Zahlen gelten.

Von grundsätzlicher Bedeutung ist nun der folgende Satz: Ist $\mathfrak{a}=\left[\alpha_{0}, \alpha_{1}, \alpha_{2}, \ldots, \alpha_{\mu}\right]$ ein beliebiges Ideal des Körpers $\Re$, so kann man ein zweites Ideal $\mathfrak{b}$ von $\mathfrak{\Omega}$ so angeben, da $\beta$ das Produkt von $\mathfrak{a}$ und $\mathfrak{b}$ ein Hauptideal $\mathfrak{a} \cdot \mathfrak{b}=[a]$ mit rationaler ganzer positiver Zahl a wird. Um dies zu zeigen, bilden wir mit einer Variablen $z$ die ganze Funktion $\mu^{\text {ten }}$ Grades:

$$
\varphi(z)=\alpha_{0} z^{\mu}+\alpha_{1} z^{\mu-1}+\cdots+\alpha_{\mu}
$$

und stellen die $(n-1)$ mit $\varphi(z)$,konjugierten“ Funktionen her:

$$
\left\{\begin{array}{l}
\alpha_{0}^{\prime} z^{\mu}+\alpha_{1}^{\prime} z^{\mu-1}+\cdots+\alpha_{\mu}^{\prime}, \\
\alpha_{0}^{\prime \prime} z^{\mu}+\alpha_{1}^{\prime \prime} z^{\mu-1}+\cdots+\alpha_{\mu}^{\prime \prime}, \\
. .+. . . .
\end{array},\right.
$$

unter $\alpha_{k}, \alpha_{k}^{\prime}, \alpha_{k}^{\prime \prime}, \ldots, \alpha_{k}^{(n-1)}$ die $n$ mit $\alpha_{k}$ konjugierten Zahlen verstanden. Das Produkt der $(n-1)$ Funktionen (4) bezeichnen wir durch:

$$
\psi(z)=\beta_{0} z^{\nu}+\beta_{1} z^{\nu-1}+\cdots+\beta_{v}
$$


der Grad $\nu$ von $\psi(z)$ ist $\mu(n-1)$, die Koeffizienten $\beta$ sind ganze Zahlen. Durch Multiplikation der Funktionen $\varphi(z)$ und $\psi(z)$ entstehe die Funktion $\mu n^{\text {ten }}$ Grades:

$$
\varphi(z) \psi(z)=f(z)=\gamma_{0} z^{\mu n}+\gamma_{1} z^{\mu n-1}+\cdots+\gamma_{\mu n} .
$$

Da $f(z)$ das Produkt der $n$ konjugierten Funktionen (3) und (4) ist, so sind die $\gamma_{0}, \gamma_{1}, \ldots, \gamma_{\mu n}$ rational $\left.^{1}\right)$ und stellen also nach einem Satze von S. 82 rationale ganze Zahlen dar. Da ferner $\psi(z)$ der Quotient von $f(z)$ und $\varphi(z)$ ist, so gehören die Koeffizienten $\beta$ dem Körper $\Omega$ an und sind als ganze Zahlen in e enthalten. Aus dem Hilfssatze von $\S 2$, S. 80 folgt nun leicht, daß das Ideal $\mathfrak{b}=\left[\beta_{0}, \beta_{1}, \beta_{2}, \ldots, \beta_{v}\right]$ dem zu beweisenden Satze genügt. Haben nämlich die $\gamma_{0}, \gamma_{1}, \ldots, \gamma_{u n}$ die rationale ganze positive Zahl $a$ als größten gemeinsamen Teiler, so ist nach jenem Hilfssatze $a$ ein Teiler jedes Produktes $\alpha_{i} \beta_{k}$. Demnach ist jede Zahl des Ideals $\mathfrak{c}=\mathfrak{a} \cdot \mathfrak{b}$ durch $a$ teilbar und also in der Gestalt $\eta a$ darstellbar. Andrerseits gehören dem Ideale $\mathfrak{c}=\mathfrak{a} \cdot \mathfrak{b}$ alle Zahlen $\dot{\gamma}_{0}, \gamma_{1}, \ldots, \gamma_{\mu n}$ und damit die Zahl:

$$
e_{0} \gamma_{0}+e_{1} \gamma_{1}+e_{2} \gamma_{2}+\cdots+e_{\mu n} \gamma_{\mu n}
$$

mit irgendwelchen rationalen ganzen $e$ an. Man kann aber die $e$ so wählen, daB die Zahl (7) gleich dem gröBten gemeinsamen Teiler $a$ aller $\gamma$ wird. $\left.{ }^{2}\right)$ Hiernach ist auch $a$ in $c$ enthalten. Es ist also nicht nur jede Zahl von $c$ in der Gestalt $\eta a$ darstellbar, sondern jedes Produkt $\eta a$ mit beliebigem Faktor $\eta$ aus $e$ ist in $\mathfrak{c}$ enthalten, $d$. $h$. es gilt $\mathfrak{c}=\mathfrak{a} \cdot \mathfrak{b}=[a]$ womit unser Satz bewiesen ist.

Aus dem eben bewiesenen Satze kann man leicht auf den folgenden schließen: Sind die Produkte $\mathfrak{a} \cdot \mathfrak{a}^{\prime}$ und $\mathfrak{a} \cdot \mathfrak{a}^{\prime \prime}$ eines Ideals $\mathfrak{a}$ mit den beiden Idealen $\mathfrak{a}^{\prime}=\left[\alpha_{1}^{\prime}, \alpha_{2}^{\prime}, \ldots, \alpha_{\lambda}^{\prime}\right]$ und $\mathfrak{a}^{\prime \prime}=\left[\alpha_{1}^{\prime \prime}, \alpha_{9}^{\prime \prime}, \ldots, \alpha_{\mu}^{\prime \prime}\right]$ einander gleich, so sind auch $\mathfrak{a}^{\prime}$ und $\mathfrak{a}^{\prime \prime}$ gleich, $d$. $h$. aus $\mathfrak{a} \cdot \mathfrak{a}^{\prime}=\mathfrak{a} \cdot \mathfrak{a}^{\prime \prime}$ folgt $\mathfrak{a}^{\prime}=\mathfrak{a}^{\prime \prime}$. Ist nämlich $\mathfrak{b}$ ein Ideal, das in $\mathfrak{b} \cdot \mathfrak{a}$ ein Hauptideal $[a]$ mit rationaler ganzer positiver Zahl $a$ liefert, so folgt durch Multiplikation von $\mathfrak{a} \cdot \mathfrak{a}^{\prime}=\mathfrak{a} \cdot \mathfrak{a}^{\prime \prime}$ mit $\mathfrak{b}$ zufolge der Gültigkeit des assoziativen Gesetzes $[a] \cdot \mathfrak{a}^{\prime}=[a] \cdot \mathfrak{a}^{\prime \prime}$. Diese Gleichung besagt, daß jede Zahl $\left(\eta_{1}^{\prime} a \alpha_{1}^{\prime}+\eta_{2}^{\prime} a \alpha_{2}^{\prime}+\cdots+\eta_{2}^{\prime} a \alpha_{2}^{\prime}\right)$ des Ideals $[a] \cdot \mathfrak{a}^{\prime}$ auch als eine $\mathrm{Zahl}\left(\eta_{1}^{\prime \prime} a \alpha_{1}^{\prime \prime}+\eta_{2}^{\prime \prime} a \alpha_{2}^{\prime \prime}+\cdots+\eta_{\mu}^{\prime \prime} a \alpha_{\mu}^{\prime \prime}\right)$ von $[a] \cdot \mathfrak{a}^{\prime \prime}$

1) Um den Hauptsatz über symmetrische Funktionen bequem anwenden zu können, stelle man die $\alpha_{0}, \alpha_{1}, \ldots$ als Zahlen von $\mathfrak{f}$ in der Gestalt (2) S. 83 dar und ordne geradezu die Funktion $\varphi(z)$ nach Potenzen von $\theta$ an. Für die Funktionen (4) ergeben sich dann die entsprechenden Ausdrücke in den mit $\theta$ konjugierten Zahlen $\theta^{\prime}, \theta^{\prime \prime}, \ldots$

2) Daß man bei zwei rationalen ganzen Zahlen $\gamma_{0}, \gamma_{1}$ die gleichfalls rationalen ganzen Zahlen $e_{0}, e_{1}$ so wählen kann, daB $\left(e_{0} \gamma_{0}+e_{1} \gamma_{1}\right)$ der gröBte gemeinsame Teiler von $\gamma_{0}$ und $\gamma_{1}$ wird, ist ein bekannter Elementarsatz der Zahlentheorie (S. $90 \mathrm{ff}$.). Hieraus folgt die Behauptung des Textes leicht durch vollständige Induktion. 
darstellbar sei und umgekehrt. Teilen wir durch $a$, so folgt, daB jede Zahl $\left(\eta_{1}^{\prime} \alpha_{1}^{\prime}+\eta_{2}^{\prime} \alpha_{2}^{\prime}+\cdots+\eta_{2}^{\prime} \alpha_{\lambda}^{\prime}\right)$ auch als eine Zahl $\left(\eta_{1}^{\prime \prime} \alpha_{1}^{\prime \prime}+\eta_{2}^{\prime \prime} \alpha_{2}^{\prime \prime}+\cdots+\eta_{\mu}^{\prime \prime} \alpha_{\mu}^{\prime \prime}\right)$ darstellbar ist und umgekehrt. Dies aber heiBt, dab $\mathfrak{a}^{\prime}=\mathfrak{a}^{\prime \prime}$ ist.

\section{\$ 9. Faktorenzerlegung eines Ideals.}

Um einen bei der Faktorenzerlegung eines Ideals zu benutzenden Hilfssatz zu beweisen, schicken wir folgende Erklärung voraus: Zwei Zahlen $\eta$ und $\eta^{\prime}$ aus e, deren Differenz durch die ganze Zahl $\alpha$ aus e teilbar ist, sollen modulo $\alpha$ kongruent heißen:

$$
\eta^{\prime} \equiv \eta \quad(\bmod \alpha)
$$

Alle $\bmod \alpha$ kongruenten Zahlen von e fassen wir in eine "Zahllklasse" zusammen. Es gilt der Satz: Ist a rational, ganz und positiv, so gibt es $\bmod a$ im ganzen $a^{n}$ inkongruente Zahlkilassen in e. Stellen wir nämlich die Zahlen $\eta$ von $e$ in einer Basis $\eta_{1}, \eta_{2}, \ldots, \eta_{n}$ dar:

$$
\eta=e_{1} \eta_{1}+e_{2} \eta_{2}+\cdots+e_{n} \eta_{n}
$$

so ist die Zahl (1) mit der Zahl $\eta^{\prime}=e_{1}^{\prime} \eta_{1}+e_{2}^{\prime} \eta_{2}+\cdots+e_{n}^{\prime} \eta_{n}$ stets und nur dann $\bmod a$ kongruent, wenn $e_{k} \equiv e_{k}^{\prime}(\bmod a)$ für $k=1,2, \ldots, n$ gilt. Man erhält demuach ein volles System $\bmod a$ inkongruenter Zahlen $\eta$, wenn man die $n$ Koeffizienten $e_{k}$ in (1) auf alle $a^{n}$ Arten entsprechend den Bedingungen $0 \leqq e_{k}<a$ auswählt.

Hieraus ergibt sich der unten anzuwendende Satz: Es gibt in $\Omega$ nur eine beschränkte Anzahl von Idealen, die eine vorgeschriebene rationale ganze positive Zahl a enthalten. Ein solches Ideal enthält nämlich mit einer Zahl $\eta$ sogleich die ganze Klasse der $\bmod a \operatorname{mit} \eta$ kongruenten Zahlen, setzt sich also aus einer gewissen Anzahl der $a^{n} \bmod a$ inkongruenten Zahlklassen zusammen. Aus einer endlichen Anzahl $a^{n}$ von Klassen können wir aber nur eine endliche Anzahl von Kombinationen herstellen, woraus der Satz hervorgeht.

Es gelte nun folgende Erklärung: Ein Ideal $\mathfrak{c}$ hat ein Ideal $\mathfrak{a}$ als "Teiler" oder ist „,durch a teilbar" oder $\mathfrak{a}$,geht in $\mathfrak{c}$ auf", falls es ein Ideal $\mathfrak{a}^{\prime}$ gibt, das mit $\mathfrak{a}$ multipliziert $\mathfrak{c}$ liefert, $\mathfrak{a} \cdot \mathfrak{a}^{\prime}=\mathfrak{c}$. Aus $\mathfrak{a} \cdot \mathfrak{e}=\mathfrak{a}$ folgt, daß jedes Ideal $\mathfrak{a}$ sowohl das Einheitsideal $\mathfrak{e}$ als sich selbst zum Teiler hat. Sollte es Ideale $\mathfrak{a}$ in $\mathfrak{\Omega}$ geben, die außer $\mathfrak{e}$ und $\mathfrak{a}$ keinen Teiler haben, so werden wir solche Ideale als „Primideale" bezeichnen. An die Erklärung des Teilers schliebt sich der Satz: Ist a ein Teiler von $\mathfrak{c}$, so gibt es auch nur ein Ideal $\mathfrak{a}^{\prime}$, das mit $\mathfrak{a}$ multipliziert $\mathfrak{c}$ als Produkt liefert. Wäre nämlich neben $\mathfrak{a} \cdot \mathfrak{a}^{\prime}=\mathfrak{c}$ auch $\mathfrak{a} \cdot \mathfrak{a}^{\prime \prime}=\mathfrak{c}$, so wäre $\mathfrak{a} \cdot \mathfrak{a}^{\prime}=\mathfrak{a} \cdot \mathfrak{a}^{\prime \prime}$ und also nach dem letzten Satze des vorigen Paragraphen $\mathfrak{a}^{\prime}=\mathfrak{a}^{\prime \prime}$.

In den nächstfolgenden Sätzen wird man Verallgemeinerungen der S. $90 \mathrm{ff}$. für den rationalen Körper aufgestellten Sätze erkennen. Das Ideal 
$\mathfrak{a}$ ist stets und nur dann ein Teiler von $\mathfrak{c}$, wenn jede Zahl von $\mathfrak{c}$ in $\mathfrak{a}$ enthalten ist. Daß jede $\mathrm{Zahl}$ von $\mathfrak{c}=\mathfrak{a} \cdot \mathfrak{a}^{\prime}$ in $\mathfrak{a}$ enthalten ist, folgt aus der Erklärung (1) S. 93 des Produktes zweier Ideale. Ist andrerseits c irgendein Ideal, dessen sämtliche Zahlen in $\mathfrak{a}$ enthalten sind, und ist $\mathfrak{b}$ ein Ideal, das mit a multipliziert wie oben als Produkt ein Hauptideal $\mathfrak{a} \cdot \mathfrak{b}=[a]$ liefert, so ist auch jede Zahl des Ideals $\mathfrak{c} \cdot \mathfrak{b}=\mathfrak{b} \cdot \mathfrak{c}$ durch $a$ teilbar. Es gibt demnach für $\mathfrak{b} \cdot \mathfrak{c}$ eine Darstellung:

$$
\mathfrak{b} \cdot \mathfrak{c}=\left[a \alpha_{1}^{\prime}, a \alpha_{2}^{\prime}, \ldots, a \alpha_{v}^{\prime}\right]
$$

wo die $\alpha_{1}^{\prime}, \alpha_{2}^{\prime}, \ldots, \alpha_{v}^{\prime}$ ganze Zahlen sind. Setzen wir $\left[\alpha_{1}^{\prime}, \alpha_{2}^{\prime}, \ldots, \alpha_{v}^{\prime}\right]=\mathfrak{a}^{\prime}$, so wird $\mathfrak{b} \cdot \mathfrak{c}=[a] \cdot \mathfrak{a}^{\prime}=\mathfrak{b} \cdot \mathfrak{a} \cdot \mathfrak{a}^{\prime}$, woraus $\mathfrak{c}=\mathfrak{a} \cdot \mathfrak{a}^{\prime}$ und also der zu beweisende Satz folgt.

Ein Ideal, das in jedem der beiden Ideale $\mathfrak{a}$ und $\mathfrak{a}^{\prime}$ aufgeht, und das jeden gemeinsamen Teiler von $\mathfrak{a}$ und $\mathfrak{a}^{\prime}$ selbst zum Teiler hat, heißt ein "größter gemeinsamer Teiler" von a und $\mathfrak{a}^{\prime}$. Es gilt der Satz: Je zwei Ideale $\mathfrak{a}$ und $\mathfrak{a}^{\prime}$ des Körpers $\mathfrak{\Omega}$ haben einen eindeutig bestimmten größten gemeinsamen Teiler $\mathrm{b} ;$ ist $\mathfrak{a}=\left[\alpha_{1}, \alpha_{2}, \ldots, \alpha_{2}\right]$ und $\mathfrak{a}^{\prime}=\left[\alpha_{1}^{\prime}, \alpha_{2}^{\prime}, \ldots, \alpha_{\mu}^{\prime}\right]$, so ist dieser Teiler D:

$$
\delta=\left[\alpha_{1}, \alpha_{2}, \ldots, \alpha_{\lambda}, \alpha_{1}^{\prime}, \alpha_{2}^{\prime}, \ldots, \alpha_{\mu}^{\prime}\right] .
$$

Jede Zahl von $\mathfrak{a}$ ist nämlich in diesem $\delta$ enthalten, ebenso jede von $\mathfrak{a}^{\prime}$; also ist $\mathfrak{b}$ ein gemeinsamer Teiler von $\mathfrak{a}$ und $\mathfrak{a}^{\prime}$. Ist aber $\mathfrak{b}^{\prime}$ irgendein gemeinsamer Teiler von $\mathfrak{a}$ und $\mathfrak{a}^{\prime}$, so sind alle Zahlen $\alpha_{1}, \alpha_{2}, \ldots, \alpha_{2}, \alpha_{1}^{\prime}, \alpha_{2}^{\prime}$, $\ldots, \alpha_{\mu}^{\prime}$ in $\delta^{\prime}$ enthalten. Also sind auch alle Zahlen von $\delta$ in $\delta^{\prime}$ enthalten, d. h. es ist $\delta^{\prime}$ Teiler von $\delta$. Hieran schlieBt sich die Erklärung: Ist der größte gemeinsame Teiler von $\mathfrak{a}$ und $\mathfrak{a}^{\prime}$ das Einheitsideal $\mathfrak{e}$, so heißen $\mathfrak{a}$ und $\mathfrak{a}^{\prime}$, ,teilerfremd".

Aus den bisherigen Sätzen ergeben sich einige einfache Folgerungen: Gilt für zuei Ideale $\mathfrak{a}$ und $\mathfrak{a}^{\prime}$ die Gleichung $\mathfrak{a} \cdot \mathfrak{a}^{\prime}=\mathfrak{e}$, so ist $\mathfrak{a}=\mathfrak{a}^{\prime}=e$. Es ist nämlich jede Zahl von $\mathfrak{e}$ sowohl im Teiler $\mathfrak{a}$ als im Teiler $\mathfrak{a}^{\prime}$ enthalten. Irgendein vorgelegtes Ideal $\mathfrak{a}$ hat nur endlich viele Teiler. Ist wie oben $\mathfrak{a} \cdot \mathfrak{b}=[a]$, so ist jede Zahl von $[a]$, also auch $a$ selbst in $\mathfrak{a}$ und damit auch in jedem Teiler von $\mathfrak{a}$ enthalten. Es gibt aber nach S. 95 nur endlich viele Ideale, die die Zahl $a$ enthalten. Ist $\mathfrak{a} \cdot \mathfrak{a}^{\prime}=\mathfrak{c}$, und ist $\mathfrak{a}^{\prime}$ nicht das Einheitsideal $\mathfrak{e}$, so hat $\mathfrak{a}$ weniger Teiler als $\mathfrak{c}$. Jeder Teiler von $\mathfrak{a}$ ist nämlich ein Teiler von $c$. Aber nicht jeder Teiler von $\mathfrak{c}$ ist ein solcher von a. Es geht z. B. $\mathfrak{c}$ selbst wohl in $\mathfrak{c}$, aber nicht in $\mathfrak{a}$ auf. Wäre nämlich $\mathfrak{a}=\mathfrak{c} \cdot \mathfrak{a}^{\prime \prime}$, so würde durch Multiplikation mit $\mathfrak{a}^{\prime}$ :

$$
\mathfrak{c} \mathfrak{a}^{\prime \prime} \cdot \mathfrak{a}^{\prime}=\mathfrak{a} \cdot \mathfrak{a}^{\prime}=\mathfrak{c}=\mathfrak{c} \cdot \mathfrak{e}
$$

und also $\mathfrak{a}^{\prime} \cdot \mathfrak{a}^{\prime \prime}=\mathfrak{e}, \mathfrak{a}^{\prime}=\mathfrak{e}$ entgegen der Annahme folgen.

Das Ideal $e$ ist nur durch sich selbst teilbar und hat demnach den Charakter eines Primideals. Indes verstehen wir weiterhin unter einem „Prim- 
ideal" ein von $e$ verschiedenes Ideal $\mathfrak{p}$, welches nur $\mathfrak{e}$ und $\mathfrak{p}$ selbst zu Teilern hat. Es gilt dann der Satz: Jedes von e verschiedene Ideal a ist durch mindestens ein Primideal teilbar. Es hat nämlich a sicher mindestens einen von $e$ verschiedenen Teiler, nämlich $\mathfrak{a}$ selbst, und andrerseits auch nur eine endliche Anzahl solcher Teiler. Unter diesen von $e$ verschiedenen Teilern des Ideals $\mathfrak{a}$ können wir einen aussuchen, der seinerseits nicht mehr Teiler hat als irgendein anderer von ihnen. Jeder Teiler hat nämlich selbst nicht mehr Teiler als $\mathfrak{a}$; unter den Teileranzahlen gibt es also eine kleinste. Den ausgesuchten Teiler nennen wir $\mathfrak{b}$ und erkennen leicht, daß er ein Primideal darstellt. Gäbe es nämlich eine Zerlegung $b=\mathfrak{c} \cdot \mathfrak{c}^{\prime}$, wo keines der Ideale $\mathfrak{c}$ gleich $\mathfrak{e}$ wäre, so wären auch $\mathfrak{c}$ und $\mathfrak{c}^{\prime}$ Teiler von $\mathfrak{a}$, die nach dem letzten Satze entgegen der Annahme über $\mathfrak{b}$ weniger Teiler als $\mathfrak{b}$ hätten.

Jetzt ist auch der folgende Satz beweisbar: Geht ein Primideal $\mathfrak{p}$ in dem Produkte $\mathfrak{a}=\mathfrak{a}^{\prime} \cdot \mathfrak{a}^{\prime \prime}$ der beiden Ideale $\mathfrak{a}^{\prime}$ und $\mathfrak{a}^{\prime \prime}$ auf, so ist mindestens einer der Faktoren $\mathfrak{a}^{\prime}, \mathfrak{a}^{\prime \prime}$ durch $\mathfrak{p}$ teilbar. Wir nehmen an, daß $\mathfrak{p}$ nicht in $\mathfrak{a}^{\prime \prime}$ aufgeht, und haben zu zeigen, dab $\mathfrak{p}$ Teiler von $\mathfrak{a}^{\prime}$ ist. Der Annahme entsprechend sind $\mathfrak{a}^{\prime \prime}=\left[\alpha_{1}^{\prime \prime}, \alpha_{2}^{\prime \prime}, \ldots, \alpha_{2}^{\prime \prime}\right]$ und $\mathfrak{p}=\left[\pi_{1}, \pi_{2}, \ldots, \pi_{\mu}\right]$ teilerfremd:

$$
\left[\alpha_{1}^{\prime \prime}, \alpha_{2}^{\prime \prime}, \ldots, \alpha_{2}^{\prime \prime}, \pi_{1}, \pi_{2}, \ldots, \pi_{\mu}\right]=\mathrm{e} .
$$

Da die Zahl 1 in e enthalten ist, so gibt es eine Darstellung:

$$
1=\eta_{1}^{\prime \prime} \alpha_{1}^{\prime \prime}+\eta_{2}^{\prime \prime} \alpha_{2}^{\prime \prime}+\cdots+\eta_{\lambda}^{\prime \prime} \alpha_{\lambda}^{\prime \prime}+\eta_{1} \pi_{1}+\cdots+\eta_{u} \pi_{\mu}
$$

dieser Zahl. Ist nun $\alpha^{\prime}$ eine beliebige Zahl aus $\mathfrak{a}^{\prime}$, so folgt durch Multiplikation der letzten Gleichung mit $\alpha^{\prime}$ :

$$
\alpha^{\prime}=\alpha^{\prime}\left(\eta_{1}^{\prime \prime} \alpha_{1}^{\prime \prime}+\eta_{2}^{\prime \prime} \alpha_{2}^{\prime \prime}+\cdots+\eta_{\lambda}^{\prime \prime} \alpha_{\lambda}^{\prime \prime}\right)+\alpha^{\prime} \eta_{1} \pi_{1}+\cdots+\alpha^{\prime} \eta_{\mu} \pi_{\mu} .
$$

Das erste Glied rechts, als Produkt einer Zahl $\alpha^{\prime}$ aus $\mathfrak{a}^{\prime}$ und einer Zahl $\left(\eta_{1}^{\prime \prime} \alpha_{1}^{\prime \prime}+\cdots\right)$ aus $\mathfrak{a}^{\prime \prime}$, ist in $\mathfrak{a}^{\prime} \cdot \mathfrak{a}^{\prime \prime}=\mathfrak{a}$ und also auch im Teiler $\mathfrak{p}$ von $\mathfrak{a}$ enthalten. Da auch die Zahl $\left(\alpha^{\prime} \eta_{1} \pi_{1}+\cdots\right)$ in $\mathfrak{p}$ enthalten ist, so gilt dasselbe von $\alpha^{\prime}$, also von jeder beliebigen Zahl aus $\mathfrak{a}^{\prime}$. Also ist $\mathfrak{p}$ Teiler von $\mathfrak{a}^{\prime}$.

In allen diesen Sätzen erkennt man die Übertragungen bekannter Elementarsätze über die Teilbarkeit der rationalen ganzen Zahlen auf die Ideale des Körpers $\Re$. Der Hauptsatz der Idealtheorie ist endlich der folgende: Jedes von e verschiedene Ideal a von $\Omega$ ist als Produkt von Primidealen darstellbar:

$$
\mathfrak{a}=\mathfrak{p}_{1} \cdot \mathfrak{p}_{2} \cdot \mathfrak{p}_{3} \cdots \mathfrak{p}_{l}
$$

und zwar im wesentlichen, d. h. abgesehen von der Anordnung der Faktoren nur auf eine einzige Art, wobei wir ïbrigens in (3) rechts etwa gleiche Primideale auch in Primidealpotenzen zusammenfassen lönnen. Ist a nicht selbst Primideal, in welchem Falle die Gleichung (3) für $l=1$ gilt, so sei $\mathfrak{p}_{1}$ ein erstes in $\mathfrak{a}$ aufgehendes Primideal, das nach dem vorletzten Satze existiert. Wir setzen $\mathfrak{a}=\mathfrak{p}_{1} \cdot \mathfrak{a}^{\prime}$ und haben in $\mathfrak{a}^{\prime}$ ein Ideal, das weniger 
Teiler hat als $\mathfrak{a}$. Ist $\mathfrak{a}^{\prime}$ noch nicht Primideal, so setzen wir $\mathfrak{a}^{\prime}=\mathfrak{p}_{\mathbf{2}} \cdot \mathfrak{a}^{\prime \prime}$, wo die Teileranzahl von $\mathfrak{a}^{\prime \prime}$ wieder geringer ist als die von $\mathfrak{a}^{\prime}$. Da die Teileranzahl von $\mathfrak{a}$ endlich war, so kommt dieser Prozeß zum Abschluß und liefert eine Darstellung (3) von a. Gibt es noch eine zweite solche Darstellung:

$$
\mathfrak{a}=\mathfrak{p}_{1}^{\prime} \cdot \mathfrak{p}_{2}^{\prime} \cdot \mathfrak{p}_{3}^{\prime} \cdots \mathfrak{p}_{m}^{\prime},
$$

sô gelten folgende Schlüsse. Es sei etwa $l \leqq m$. Das Ideal $\mathfrak{p}_{1}$ geht im Produkte $\mathfrak{p}_{1}^{\prime} \cdot\left(\mathfrak{p}_{2}^{\prime} \cdot \mathfrak{p}_{3}^{\prime} \cdots \mathfrak{p}_{m}^{\prime}\right)$ auf; also ist entweder $\mathfrak{p}_{1}=\mathfrak{p}_{1}^{\prime}$, oder $\mathfrak{p}_{1}$ geht in $\mathfrak{p}_{2}^{\prime} \cdot \mathfrak{p}_{3}^{\prime} \cdots \mathfrak{p}_{m}^{\prime}$ auf. Durch Fortsetzung dieses Verfahrens findet man, daB $p_{1}$ unter den in (4) rechts stehenden Idealen auftritt. Wir können demnach, nötigenfalls nach Umordnung der Ideale in (4) rechts, $\mathfrak{p}_{1}^{\prime}=\mathfrak{p}_{1}$ setzen. Dann folgt nach einem Satze von S. 94:

$$
\mathfrak{p}_{2} \cdot \mathfrak{p}_{3} \cdots \mathfrak{p}_{l}=\mathfrak{p}_{2}^{\prime} \cdot \mathfrak{p}_{3}^{\prime} \cdots \mathfrak{p}_{m}^{\prime} .
$$

Auf dieselbe Art ergibt sich $\mathfrak{p}_{2}^{\prime}=\mathfrak{p}_{2}, \mathfrak{p}_{3}^{\prime}=\mathfrak{p}_{3}, \ldots, \mathfrak{p}_{l-1}^{\prime}=\mathfrak{p}_{l-1}$, worauf die Gleichung übrig bleibt:

$$
\mathfrak{p}_{l}=\mathfrak{p}_{l}^{\prime} \cdot \mathfrak{p}_{l+1}^{\prime} \cdots \mathfrak{p}_{m}^{\prime} \text {. }
$$

Diese Gleichung lehrt, daß $l=m$ sein $m u B$, und daß $\mathfrak{p}_{l}^{\prime}=\mathfrak{p}_{l}$ zutrifft. Damit ist der Satz bewiesen.

In dem S. 88 ff. betrachteten quadratischen Körper der Grundzahl - 20 konnten wir das Hauptideal [21] als Produkt sowohl von [3] und [7] als auch als Produkt von $[1+2 i \sqrt{5}]$ und $[1-2 i \sqrt{5}]$ darstellen, was den Zerlegungen $21=3 \cdot 7$ und $21=(1+2 i \sqrt{5})(1-2 i \sqrt{5})$ entspricht. Die Hauptideale [3], [7], [1 $\pm 2 i \sqrt{5}]$ sind nun noch keine Primideale. Die Zerlegung von [21] in Primideale enthält vier Faktoren, nämlich:

$$
\begin{array}{ll}
\mathfrak{p}_{1}=[3,1+2 i \sqrt{5}], & \mathfrak{p}_{2}=[3,1-2 i \sqrt{5}], \\
\mathfrak{p}_{3}=[7,1+2 i \sqrt{5}], & \mathfrak{p}_{4}=[7,1-2 i \sqrt{5}],
\end{array}
$$

in denen man auf Grund späterer Sätze leicht Primideale erkennt, und die übrigens die größten gemeinschaftlichen Teiler von [3] und $[1+2 i \sqrt{5}]$, von [3] und $[1-2 i \sqrt{5}]$ usw. sind. Bildet man nach (1) S. 93 das Produkt $\mathfrak{p}_{1} \cdot \mathfrak{p}_{2}$, so ist:

$$
\mathfrak{p}_{1} \cdot \mathfrak{p}_{2}=[9,3+6 i \cdot \sqrt{5}, 3-6 i \cdot \sqrt{5}, 21] .
$$

Das Ideal $\mathfrak{p}_{1} \cdot \mathfrak{p}_{2}$ enthält auch die Zahl $21-2 \cdot 9=3$ und also alle Zahlen von [3]. Da andrerseits alle vier Zahlen in (5) rechts in [3] enthalten sind, so sind auch alle Zahlen von $\mathfrak{p}_{1} \cdot \mathfrak{p}_{2}$ in [3] enthalten, d. h. man hat die erste. der vier Gleichungen:

$\mathfrak{p}_{1} \cdot \mathfrak{p}_{2}=[3], \mathfrak{p}_{1} \cdot \mathfrak{p}_{3}=[1+2 i \sqrt{5}], \mathfrak{p}_{3} \cdot \mathfrak{p}_{4}=[7], \mathfrak{p}_{2} \cdot \mathfrak{p}_{4}=[1-2 i \sqrt{5}]$, deren drei übrige entsprechend zu zeigen sind. Aus der eindeutig bestimmten Zerlegung $[21]=\mathfrak{p}_{1} \cdot \mathfrak{p}_{2} \cdot \mathfrak{p}_{3} \cdot \mathfrak{p}_{4}$ kommen wir demnach bei den beiden Anordnungen: 


$$
\begin{aligned}
& {[21]=\left(\mathfrak{p}_{1} \cdot \mathfrak{p}_{2}\right) \cdot\left(\mathfrak{p}_{3} \cdot \mathfrak{p}_{4}\right)=[3] \cdot[7],} \\
& {[21]=\left(\mathfrak{p}_{1} \cdot \mathfrak{p}_{3}\right) \cdot\left(\mathfrak{p}_{2} \cdot \mathfrak{p}_{4}\right)=[1+2 i \sqrt{5}] \cdot[1-2 i \sqrt{5}]}
\end{aligned}
$$

zu den beiden Zerlegungen von [21] in Produkte von Hauptidealen zurück. Die Erweiterung auf alle Ideale des vorliegenden Körpers $\Omega$ stellt die Eindeutigkeit der Primidealzerlegung wieder her.

\section{$\S 10$. Die Basen eines Ideals a.}

Eine „Basis" eines Ideals a wurde von irgendeinem Systeme linearunabhängiger Zahlen $\alpha_{1}, \alpha_{2}, \ldots, \alpha_{n}$ aus $\mathfrak{a}$ gebildet, deren Diskriminante $D\left(\alpha_{1}, \alpha_{2}, \ldots, \alpha_{n}\right)$ die absolut kleinste von 0 verschiedene Zahl ist, die als Diskriminante von $n$ Zahlen aus a auftritt (S. 91). In der Basis $\alpha_{1}, \alpha_{2}, \ldots, \alpha_{n}$ ist jede Zahl $\alpha$ von $\mathfrak{a}$ auf eine und nur eine Art in der Gestalt:

$$
\alpha=e_{1} \alpha_{1}+e_{2} \alpha_{2}+\cdots+e_{n} \alpha_{n}
$$

mit rationalen ganzen $e$ darstellbar, und umgekehrt ist jeder Ausdruck (1) eine Zahl von $\mathfrak{a}$. Dieser Satz ist für eine Basis charakteristisch, d. h. ein System linear-unabhängiger Zahlen $\alpha_{1}^{\prime}, \alpha_{2}^{\prime}, \ldots, \alpha_{n}^{\prime}$ von a, in dem jede Zahl des Ideals in der Gestalt $e_{1}^{\prime} \alpha_{1}^{\prime}+e_{2}^{\prime} \alpha_{2}^{\prime}+\cdots+e_{n}^{\prime} \alpha_{n}^{\prime}$ mittels rationaler ganzer $e^{\prime}$ darstellbar ist, liefert immer eine Basis von a. Es sind nämlich sowohl die $\alpha_{1}^{\prime}, \alpha_{2}^{\prime}, \ldots, \alpha_{n}^{\prime}$ in den $\alpha_{1}, \alpha_{2}, \ldots, \alpha_{n}$ linear homogen mit rationalen ganzen Koeffizienten darstellbar wie auch umgekehrt die $\alpha_{1}, \alpha_{2}, \ldots, \alpha_{n}$ in den $\alpha_{1}^{\prime}, \alpha_{2}^{\prime}, \ldots, \alpha_{n}^{\prime}$. Hieraus folgt (bei Benutzung des Multiplikationsgesetzes der Determinanten), daB jede der beiden rationalen ganzen Zahlen $D\left(\alpha_{1}, \alpha_{2}, \ldots, \alpha_{n}\right)$ und $D\left(\alpha_{1}^{\prime}, \alpha_{2}^{\prime}, \ldots, \alpha_{n}^{\prime}\right)$ in der anderen aufgeht, so daB sie als Zahlen von gleichem Vorzeichen einander gleich sind. Also ist auch $\alpha_{1}^{\prime}, \alpha_{2}^{\prime}, \ldots, \alpha_{n}^{\prime}$ eine Basis.

Die Darstellungen der eben betrachteten Zahlen $\alpha_{1}^{\prime}, \alpha_{2}^{\prime}, \ldots, \alpha_{n}^{\prime}$ in der ersten Basis seien:

$$
\alpha_{i}^{\prime}=e_{i 1} \alpha_{1}+e_{i 2} \alpha_{2}+\cdots+e_{i n} \alpha_{n}, \quad i=1,2, \ldots, n .
$$

Aus der Gleichheit der beiden Diskriminanten folgt dann der Satz: In einer ersten Basis $\alpha_{1}, \alpha_{2}, \ldots, \alpha_{n}$ von a ist jede andere Basis $\alpha_{1}^{\prime}, \alpha_{2}^{\prime}, \ldots, \alpha_{n}^{\prime}$ dieses Ideals in der Gestalt (2) mit rationalen ganzen Koeffizienten $e_{i k}$ der Determinante \pm 1 darstellbar, und jedes so gewonnene Zahlsystem $\alpha_{1}^{\prime}, \alpha_{2}^{\prime}, \ldots, \alpha_{n}^{\prime}$ ist auch offenbar wieder eine Basis von a.

Es seien nun die Zahlen $\alpha_{1}, \alpha_{2}, \ldots, \alpha_{n}$ einer Basis von $\mathfrak{a}$ in einer Basis $\eta_{1}, \eta_{2}, \ldots, \eta_{n}$ von $e$ in der Gestalt:

$$
\alpha_{i}=h_{i 1} \eta_{1}+h_{i 2} \eta_{2}+\cdots+h_{i n} \eta_{n}, \quad i=1,2, \ldots, n
$$

mit rationalen ganzen $h$ dargestellt. Dann gilt nach (7) S. 84:

$$
D\left(\alpha_{1}, \dot{\alpha}_{2}, \ldots, \alpha_{n}\right)=\left|h_{i k}\right|^{2} D\left(\eta_{1}, \eta_{2}, \ldots, \eta_{n}\right),
$$


wo $\left|h_{i k}\right|$ die $n$-reihige Determinante der Koeffizienten $h$ ist. Der absolute Wert dieser Determinante hat für das Ideal a eine wichtige Bedeutung, welche im nächsten Paragraphen zur Besprechung gelangt. Zur Vorbereitung wählen wir hier zunächst eine besonders gebaute Basis für a aus. Es besteht nämlich folgender Satz: Man kann eine Basis von a stets durch $n$ Zahlen der Gestalt:

$$
\left\{\begin{array}{l}
\alpha_{1}=h_{11} \eta_{1} \\
\alpha_{2}=h_{21} \eta_{1}+h_{22} \eta_{2} \\
\alpha_{3}=h_{31} \eta_{1}+h_{32} \eta_{2}+h_{33} \eta_{3} \\
\cdot \cdot \cdot \cdot \cdot \cdot \cdot \cdot \cdot \cdot \cdot \cdot \cdot \cdot \cdot \cdot \cdot h_{n n} \eta_{n}
\end{array}\right.
$$

mit positiven $h_{11}, h_{22}, \ldots, h_{n n}$ aufbauen.

Um dies einzusehen, betrachten wir alle in a enthaltenen Zahlen:

$$
\alpha=e_{1} \eta_{1}+e_{2} \eta_{2}+\cdots+e_{v} \eta_{v}
$$

mit $e_{v} \neq 0$, wo $v$ eine der Zahlen $1,2, \ldots, n$ ist. Solche Zahlen kommen sicher für jedes $\nu$ in $\mathfrak{a}$ vor; denn es gibt in a von 0 verschiedene rationale ganze Zahlen $e_{v}$, also z. B. auch Zahlen $e_{v} \eta_{v}$. Die Gesamtheit der in a enthaltenen Zahlen (6) heiße $\mathfrak{A}_{v}$. Da mit $\alpha$ auch $-\alpha$ in $\mathfrak{a}$ enthalten ist, so kommen auch Zahlen (6) mit $e_{v}>0$ in $\mathfrak{A}_{v}$ vor. Die kleinste positive Zahl $e_{v}$, die vorkommt, heiße $h_{v v}$, eine Zahl aus $\mathfrak{A}_{v}$, bei der sie auftritt, sei:

$$
\alpha_{v}=h_{v_{1}} \eta_{1}+h_{v 2} \eta_{2}+\cdots+h_{v} \eta_{v} .
$$

Wir können nun zunächst leicht zeigen, daß alle in $\mathfrak{A}_{v}$ auftretenden $e_{v}$ Vielfache von $h_{v v}$ sind. Gäbe es nämlich eine Zahl (6), bei der $e_{v}$ nicht durch $h_{v v}$ teilbar ist, so könnten wir von ihr durch wiederholten Zusatz oder Abzug von $\alpha_{v}$ zu einer Zahl (6) mit $0<e_{v}<h_{v v}$ gelangen, d. h. $h_{v v}$ wäre der Annahme entgegen nicht die kleinste positive Zahl $e_{v}$. Ist nun für irgendeine Zahl $\alpha$ aus $\mathfrak{A}_{v}$ der letzte Koeffizient $e_{v}=e_{v}^{\prime} \cdot h_{v v}$, wo also $e_{v}^{\prime}$ rational und ganz ist, so ist offenbar $\left(\alpha-e_{v}^{\prime} \alpha_{v}\right)$ eine Zahl, die bereits einem der Systeme $\mathfrak{A}_{v-1}, \mathfrak{A}_{v-2}, \ldots$ angehört. Nun bilden alle Systeme $\mathfrak{A}_{n}, \mathfrak{A}_{n-1}, \ldots, \mathfrak{A}_{2}, \mathfrak{A}_{1}$ zusammen mit der Zahl 0 das ganze Ideal $\mathfrak{a}$. Durch wiederholte Anwendung der eben durchgeführten Überlegung finden wir, daß jede Zahl $\alpha$ aus $a$ in der Gestalt:

$$
\alpha=e_{1}^{\prime} \alpha_{1}+e_{2}^{\prime} \alpha_{2}+\cdots+e_{n}^{\prime} \alpha_{n}
$$

darstellbar ist. Dabei bildet die Zahl 0 keine Ausnahme, da sie in (8) für $e_{1}^{\prime}=0, e_{2}^{\prime}=0, \ldots, e_{n}^{\prime}=0$ gewonnen wird. Nehmen wir noch hinzu, daß offenbar:

$$
D\left(\alpha_{1}, \alpha_{2}, \ldots, \alpha_{n}\right)=\left(h_{11} \cdot h_{22} \cdots h_{n n}\right)^{2} \cdot D\left(\eta_{1}, \eta_{2}, \cdots, \eta_{v}\right) \neq 0
$$

gilt, so erkennen wir in $\alpha_{1}, \alpha_{2}, \ldots, \alpha_{n}$ eine Basis von $\mathfrak{a}$. 


\section{11. Norm eines Ideals.}

Wir gehen jetzt auf die Gleichung (4) $\S 10$ und die in ihr als Spezialfall enthaltene Gleichung (9) daselbst zurück und stellen folgende Erklärung auf: Unabhängig von der ausgewählten Basis $\alpha_{1}, \alpha_{2}, \ldots, \alpha_{n}$ des Ideals a ist die positiv genommene Wurzel:

$$
\sqrt{\frac{D\left(\alpha_{1}, \alpha_{2}, \ldots, \alpha_{n}\right)}{D\left(\eta_{1}, \eta_{2}, \ldots, \eta_{n}\right)}}
$$

eine eindeutig bestimmte, dem Ideal a eigentïmliche rationale ganze positiveZahl, die die „Norm" des Ideals a genannt und durch $N(\mathfrak{a})$ bezeichnet wird. Die Norm $N(\mathfrak{a})$ ist also der absolute Betrag der Determinante der $n^{2}$ Koeffizienten $h_{i k}$ in (3) $\S 10$; insbesondere folgt für die in (5) $\S 10$ vorliegenden Zahlen $h$ :

$$
N(\mathfrak{a})=h_{11} \cdot h_{22} \cdot h_{33} \cdots h_{n n} .
$$

Für ein Hauptideal $[\alpha]$ gilt der Satz: Die Norm $N([\alpha])$ eines Hauptideals $[\alpha]$ ist gleich dem absoluten Betrage der Norm $N(\alpha)$ der ganzen Zahl $\alpha$. Für $[\alpha]$ haben wir nämlich eine Basis in $\alpha_{1}=\alpha \eta_{1}, \alpha_{2}=\alpha \eta_{2}, \ldots$, $\alpha_{n}=\alpha \eta_{n}$. Sind $\alpha, \alpha^{\prime}, \alpha^{\prime \prime}, \ldots, \alpha^{(n-1)}$ die mit $\alpha$ konjugierten Zahlen, so liefert die zur Berechnung von $D\left(\alpha_{1}, \alpha_{2}, \ldots, \alpha_{n}\right)$ anzusetzende $n$-reihige Determinante :

$$
D\left(\alpha_{1}, \alpha_{2}, \ldots, \alpha_{n}\right)=\left(\alpha \cdot \alpha^{\prime} \ldots \alpha^{(n-1)}\right)^{2} D\left(\eta_{1}, \eta_{2}, \ldots, \eta_{n}\right),
$$

so daB sich aus (1) und der Erklärung der Norm (S. 85) der Satz ergibt.

Um die Bedeutung der Zahl $N(\mathfrak{a})$ darzulegen, erweitern wir den Begriff der Kongruenz zweier Zahlen in folgender Art: Zwei Zahlen $\beta, \gamma$ aus e sollen mod a kongruent heißen:

$$
\beta \equiv \gamma \quad \text { oder } \quad \gamma \equiv \beta \quad(\bmod \mathfrak{a}),
$$

wenn $(\beta-\gamma)$ und damit auch $(\gamma-\beta)$ in a enthalten ist. Für ein Hauptideal $\mathfrak{a}=[\alpha]$ ist die Kongruenz (3) gleichbedeutend mit $\beta \equiv \gamma(\bmod \alpha)$. Die Elementarregeln für das Rechnen mit Kongruenzen in bezug auf Zahlen als Moduln übertragen sich auf die Kongruenzen (3). Ist $\beta \equiv \gamma$ $(\bmod \mathfrak{a})$ und $\gamma \equiv \delta(\bmod \mathfrak{a})$, so ist auch $\beta \equiv \delta(\bmod \mathfrak{a}) ; \operatorname{denn} \operatorname{mit}(\beta-\gamma)$ und $(\gamma-\delta)$ ist auch $(\beta-\gamma)+(\gamma-\delta)=\beta-\delta$ in $\mathfrak{a}$ enthalten. Besteht neben (3) die Kongruenz $\beta^{\prime} \equiv \gamma^{\prime}(\bmod \mathfrak{a})$, so gilt auch $\left(\beta \pm \beta^{\prime}\right) \equiv\left(\gamma \pm \gamma^{\prime}\right)$ $(\bmod \mathfrak{a})$. Ebenso folgt aus $(3)$ sofort $\eta \beta \equiv \eta \gamma(\bmod \mathfrak{a})$, da mit $(\beta-\gamma)$ auch $\eta(\beta-\gamma)$ in $\mathfrak{a}$ enthalten ist; etwas allgemeiner folgt aus (3) und $\beta^{\prime} \equiv \gamma^{\prime}$ $(\bmod \mathfrak{a})$ auch $\beta \beta^{\prime} \equiv \gamma \gamma^{\prime}(\bmod \mathfrak{a})$.

Alle mod a kongruenten Zahlen von e vereinigen wir in eine "Zahlklasse" modulo a. Es besteht dann der Satz: Das System e der ganzen Zahlen von $\Re$ zerfällt $\bmod \mathfrak{a}$ in $N(\mathfrak{a})$ Klassen, als deren „Repräsentanten" 
wir die $N(\mathfrak{a})=h_{11} \cdot h_{2 \mathfrak{2}} \cdots h_{n n}$ Zahlen:

$$
e_{1} \eta_{1}+e_{2} \eta_{2}+\cdots+e_{n} \eta_{n}, \quad 0 \leqq e_{1}<h_{11}, 0 \leqq e_{2}<h_{22}, \cdots, 0 \leqq e_{n}<h_{n n}
$$

wählen können, unter $h_{11}, h_{22}, \ldots, h_{n n}$ die $n$ in (5) S. 100 auftretenden Zahlen verstanden. Erstlich sind nämlich die $N(\mathfrak{a})$ Zahlen (4) durchweg inkongruent mod a. Die Differenz zweier verschiedenen unter ihnen hat nämlich die Gestalt:

$$
d_{1} \eta_{1}+d_{2} \eta_{2}+\cdots+d_{n} \eta_{n}
$$

mit rationalen ganzen, nicht durchweg verschwindenden $d$, die die Bedingungen $0 \leqq\left|d_{i}\right|<h_{i i}$ erfüllen. Es kann demnach die sicher von 0 verschiedene Zahl (5) keinem der oben (S. 100) durch $\mathfrak{A}_{n}, \mathfrak{A}_{n-1}, \ldots, \mathfrak{A}_{1}$ bezeichneten Zahlsysteme angehören und ist demnach auch nicht in $\mathfrak{a}$ enthalten. Andrerseits kann, wenn wir für a die Basis (5) (S. 100) benutzen, jede Zahl $\eta$ aus e auf eine Zahl des Systems (4) reduziert werden, indem wir von $\eta$ nach und nach die Produkte $e_{n}^{\prime} \alpha_{n}, e_{n-1}^{\prime} \alpha_{n-1}, \ldots, e_{1}^{\prime} \alpha_{1}$ mit geeignet gewählten rationalen ganzen $e^{\prime}$ abziehen. Also ist jede Zahl aus $\mathfrak{e}$ mit einer Zahl (4) $\bmod \mathfrak{a}$ kongruent.

Ein Repräsentantensystem der $N(\mathfrak{a})$ verschiedenen Zahlklassen mod $\mathfrak{a}$ sei von den Zahlen $\varrho_{1}, \varrho_{2}, \ldots, \varrho_{N}$ geliefert. Dann sind auch die $N$ Zahlen $\varrho_{1}+1, \varrho_{2}+1, \ldots, \varrho_{N}+1$, die alle in enthalten sind, mod $\mathfrak{a}$ durchweg inkongruent, bilden also gleichfalls ein Repräsentantensystem mod a. Hieraus folgt:

$$
\left(\varrho_{1}+1\right)+\left(\varrho_{2}+1\right)+\cdots+\left(\varrho_{N}+1\right) \equiv \varrho_{1}+\varrho_{2}+\cdots+\varrho_{N} \quad(\bmod \mathfrak{a})
$$

und damit $N(\mathfrak{a}) \equiv 0(\bmod \mathfrak{a}):$ Im Ideal $\mathfrak{a}$ ist die rütionale ganze positive Zahl $N(\mathfrak{a})$ enthalten. Da nach S. 95 eine gegebene rationale ganze Zahl nur in endlich vielen Idealen auftritt, so folgt weiter der Satz: Es gibt nur endlich viele Ideale mit gegebenem Werte der Norm.

Ferner besteht der wichtige Satz: Ist a ein beliebiges Ideal und $\mathfrak{p}$ ein Primideal des Körpers $\mathfrak{\Omega}$, so gilt für $N(\mathfrak{a} \cdot \mathfrak{p})$ die Regel:

$$
N(\mathfrak{a}: \mathfrak{p})=N(\mathfrak{a}) \cdot N(\mathfrak{p}) .
$$

Der etwas umständliche Beweis läßt sich so gliedern:

Alle Zahlen von $\mathfrak{a} \cdot \mathfrak{p}$ sind in $\mathfrak{a}$ enthalten, aber nicht umgekehrt alle Zahlen von $\mathfrak{a}$ in $\mathfrak{a} \cdot \mathfrak{p}$, da sonst $\mathfrak{p}=\mathfrak{e}$ wäre. Wir können demnach aus $\mathfrak{a}$ eine Zahl $\alpha$ entnehmen, die nicht in $\mathfrak{a} \cdot \mathfrak{p}$ enthalten ist und deshalb auch sicher von 0 verschieden ist. Da $a$ ein Teiler von $[\alpha]$ ist, so gibt es ein Ideal $\mathfrak{b}$, das die Gleichung $\mathfrak{a} \cdot \mathfrak{b}=[\alpha]$ befriedigt. Dieses Ideal $\mathfrak{b}$ ist sicher zu $\mathfrak{p}$ teilerfremd. Es müßte nämlich andernfalls durch $\mathfrak{p}$ teilbar sein; dann aber wäre $\mathfrak{a} \cdot \mathfrak{b}=[\alpha]$ durch $\mathfrak{a} \cdot \mathfrak{p}$ teilbar, und also wäre $\alpha$ entgegen der getroffenen Auswahl dieser Zahl in $\mathfrak{a} \cdot \mathfrak{p}$ enthalten.

Zur Abkürzung schreiben wir $N(\mathfrak{a})=l$ und $N(\mathfrak{p})=m$ und verstehen 
unter $\varrho_{1}, \varrho_{2}, \ldots, \varrho_{l}$ und $\pi_{1}, \pi_{2}, \ldots, \pi_{m}$ Repräsentantensysteme $\bmod a$ und mod p. Wir können dann zeigen, daß die $l \cdot m$ Zahlen:

$$
\varrho_{\lambda}+\alpha \pi_{\mu}, \quad \lambda=1,2, \ldots, l, \quad \mu=1,2, \ldots, m
$$

ein Repräsentantensystem $\bmod \mathfrak{a} \cdot \mathfrak{p}$ bilden, womit die Regel (6) bewiesen sein würde.

Erstlich können keine zwei verschiedene Zahlen (7) $\bmod \mathfrak{a} \cdot \mathfrak{p}$ kongruent sein. Aus $\varrho_{\lambda^{\prime}}+\alpha \pi_{\mu^{\prime}} \equiv \varrho_{\lambda}+\alpha \pi_{\mu}(\bmod \mathfrak{a} \mathfrak{p})$ folgt nämlich, da jede Zahl von $\mathfrak{a} \cdot \mathfrak{p}$ auch in $\mathfrak{a}$ enthalten ist, die Kongruenz $\varrho_{\lambda^{\prime}}+\alpha \pi_{\mu^{\prime}} \equiv \varrho_{\lambda}+\alpha \pi_{\mu}$ $(\bmod \mathfrak{a})$ und also, da $\alpha$ in $\mathfrak{a}$ enthalten ist, $\varrho_{\lambda^{\prime}} \equiv \varrho_{\lambda}(\bmod \mathfrak{a})$. Somit ist, $\varrho_{\lambda^{\prime}}=\varrho_{\lambda}$ und also $\alpha \pi_{\mu^{\prime}} \equiv \alpha \pi_{\mu}(\bmod \mathfrak{a} \cdot \mathfrak{p})$, d. h. es ist $\alpha\left(\pi_{\mu^{\prime}}-\pi_{\mu}\right)$ in $\mathfrak{a} \cdot \mathfrak{p}$ enthalten. Es ist demnach $\mathfrak{a} \cdot \mathfrak{p}$ ein Teiler des Hauptideals:

$$
\left[\alpha\left(\pi_{\mu^{\prime}}-\pi_{\mu}\right)\right]=[\alpha] \cdot\left[\pi_{\mu^{\prime}}-\pi_{\mu}\right]=\mathfrak{a} \mathfrak{b} \cdot\left[\pi_{\mu^{\prime}}-\pi_{\mu}\right],
$$

und also geht $\mathfrak{p}$ im Ideal $\mathfrak{b} \cdot\left[\pi_{\mu^{\prime}}-\pi_{\mu}\right]$ auf. Da aber $\mathfrak{p}$ kein Teiler von $\mathfrak{b}$ ist, so ist $\left[\pi_{\mu^{\prime}}-\pi_{\mu}\right]$ durch $\mathfrak{p}$ teilbar, woraus $\pi_{\mu^{\prime}} \equiv \pi_{\mu}(\bmod \mathfrak{p})$ und also $\pi_{\mu^{\prime}}=\pi_{\mu}$ folgt. Also sind die $l \cdot m$ Zahlen (7) mod $\mathfrak{a} \cdot \mathfrak{p}$ durchweg inkongruent.

Wir haben endlich zu zeigen, daß jede Zahl $\eta$ aus $e \bmod \mathfrak{a} \cdot \mathfrak{p}$ mit einer Zahl (7) kongruent ist. Gehört $\eta \bmod \mathfrak{a}$ in die durch $\varrho_{\lambda}$ repräsentierte Klasse, so gilt $\eta=\varrho_{\lambda}+\alpha^{\prime}$, unter $\alpha^{\prime}$ eine 'Zahl aus $\mathfrak{a}$ verstanden. Da $\mathfrak{b}$ und $\mathfrak{p}$ teilerfremd sind, so ist $\mathfrak{a}$ der größte gemeinsame Teiler von $\mathfrak{a} \cdot \mathfrak{b}=[\alpha]$ und $\mathfrak{a} \cdot \mathfrak{p}$. Also läßt sich nach S. 96 die in $\mathfrak{a}$ enthaltene Zahl $\alpha^{\prime}$ als Summe $\alpha^{\prime}=\eta^{\prime} \alpha+\alpha^{\prime \prime}$ einer Zahl $\eta^{\prime} \alpha$ aus $[\alpha]$ und einer Zahl $\alpha^{\prime \prime}$ aus $\mathfrak{a} \cdot \mathfrak{p}$ darstellen. Gehört $\eta^{\prime} \bmod \mathfrak{p}$ zur Klasse des Repräsentanten $\pi_{\mu}$, so ist $\eta^{\prime}=\pi_{\mu}+\pi$, wo $\pi$ in $\mathfrak{p}$ enthalten ist. Es folgt also:

$$
\alpha^{\prime}=\alpha \pi_{\mu}+\alpha \pi+\alpha^{\prime \prime}, \quad \eta=\varrho_{\lambda}+\alpha \pi_{\mu}+\left(\alpha \pi+\alpha^{\prime \prime}\right) .
$$

Da nun $\alpha$ in $\mathfrak{a}$ und $\pi$ in $\mathfrak{p}$ enthalten ist, so ist $\alpha \pi$ in $\mathfrak{a} \cdot \mathfrak{p}$ enthalten; und da dasselbe von $\alpha^{\prime \prime}$ gilt, so folgt $\eta \equiv \varrho_{\lambda}+\alpha \pi_{\mu}(\bmod \mathfrak{a} \mathfrak{p})$, womit unsere Behauptung eingelöst und also der Satz (6) bewiesen ist.

Die Regel (6) ist als Spezialfall im folgenden Satze enthalten: Die Norm des Produktes $\mathfrak{a} \cdot \mathfrak{b}$ zweier Ideale $\mathfrak{a}, \mathfrak{b}$ ist gleich dem Produkte der Normen der Faktoren:

$$
N(\mathfrak{a} \cdot \mathfrak{b})=N(\mathfrak{a}) \cdot N(\mathfrak{b})
$$

Für $\mathfrak{b}=\mathfrak{e}$ ist der Satz (8) selbstrerständlich, da $\mathfrak{a} \cdot \mathfrak{e}=\mathfrak{a}$ und $N(\mathfrak{e})=1$ gilt. Für ein Primideal $\mathfrak{b}=\mathfrak{p}$ ist (8) soeben bewiesen. Der allgemeine Beweis kann durch vollständige Induktion geführt werden. Der Satz sei richtig, falls $\mathfrak{b}$ in weniger als $\nu$ Primideale zerfällt. Dann zeigt man leicht, $\mathrm{da} B$ er auch noch gilt, wenn $\mathfrak{b}$ ein Produkt von $\nu$ Primidealen ist. Man setze nämlich $\mathfrak{b}=\mathfrak{p} \cdot \mathfrak{c}$, w.o $\mathfrak{c}$ ein Produkt von $(\nu-1)$ Primidealen ist, und 
beweist leicht folgende Kette von Gleichungen:

$$
\begin{gathered}
N(\mathfrak{a} \cdot \mathfrak{b})=N(\mathfrak{a} \cdot(\mathfrak{p} \cdot \mathfrak{c}))=N((\mathfrak{a} \cdot \mathfrak{p}) \cdot \mathfrak{c})=N(\mathfrak{a} \cdot \mathfrak{p}) \cdot N(\mathfrak{c}) \\
=N(\mathfrak{a}) \cdot N(\mathfrak{c}) \cdot N(\mathfrak{p})=N(\mathfrak{a}) \cdot N(\mathfrak{p} \mathfrak{c})=N(\mathfrak{a}) \cdot N(\mathfrak{b})
\end{gathered}
$$

\section{\$ 12. Äquivalenz der Ideale.}

Der Erklärung der Äquivalenz zweier Ideale schicken wir folgenden Hilfssatz voraus: Liefern die beiden Ideale $\mathfrak{a}_{1}$ und $a_{2}$, mit einem und demselben Ideale $\mathfrak{b}$ multipliziert, als Produkte zwei Hauptideale:

$$
\mathfrak{a}_{1} \cdot \mathfrak{b}=\left[\alpha_{1}\right], \quad \mathfrak{a}_{2} \cdot \mathfrak{b}=\left[\alpha_{2}\right],
$$

und ist auch das Produkt von $\mathfrak{a}_{1}$ mit dem Ideale $\mathfrak{c}$ ein Hauptideal $\mathfrak{a}_{1} \cdot \mathfrak{c}=\left[\alpha_{1}^{\prime}\right]$, so ist stets auch das Prodult $\mathfrak{a}_{2} \cdot \mathfrak{c}$ ein Hauptideal. Es gilt nämlich:

$$
\left[\alpha_{1}^{\prime} \cdot \alpha_{2}\right]=\left[\alpha_{1}^{\prime}\right] \cdot\left[\alpha_{2}\right]=\left(\mathfrak{a}_{1} \cdot \mathfrak{c}\right) \cdot\left(\mathfrak{a}_{2} \cdot \mathfrak{b}\right)=\left(\mathfrak{a}_{1} \cdot \mathfrak{b}\right) \cdot\left(\mathfrak{a}_{2} \cdot \mathfrak{c}\right)
$$

und also, wenn wir $\mathfrak{a}_{1} \cdot \mathfrak{b}=\left[\alpha_{1}\right]$ setzen:

$$
\left(\mathfrak{a}_{2} \cdot \mathfrak{c}\right) \cdot\left[\alpha_{1}\right]=\left[\alpha_{1}^{\prime} \cdot \alpha_{2}\right] \text {. }
$$

Alle Zahlen des rechts stehenden Ideals, also auch $\alpha_{1}^{\prime} \cdot \alpha_{2}$, haben somit den Teiler $\alpha_{1}$, so dab wir schreiben können:

$$
\left(\mathfrak{a}_{2} \cdot \mathfrak{c}\right) \cdot\left[\alpha_{1}\right]=\left[\frac{\alpha_{1}^{\prime} \cdot \alpha_{2}}{\alpha_{1}} \cdot \alpha_{1}\right]=\left[\frac{\alpha_{1}^{\prime} \cdot \alpha_{2}}{\alpha_{1}}\right] \cdot\left[\alpha_{1}\right]
$$

Das Ideal $\mathfrak{a}_{2} \cdot \mathfrak{c}$ ist also gleich $\left[\frac{\alpha_{1}^{\prime} \cdot \alpha_{2}}{\alpha_{1}}\right], d$. h. es ist ein Hauptideal.

Hieran schließt sich folgende Erklärung: Zwei Ideale $\mathfrak{a}_{1}$ und $\mathfrak{a}_{2}$ aus $\Re$ heißen einander äquivalent, wenn es ein Ideal $\mathfrak{b}$ in $\mathfrak{\Omega}$ gibt, das, mit $\mathfrak{a}_{1}$ und $\mathfrak{a}_{2}$ multipliziert, in den Produkten $\mathfrak{a}_{1} \cdot \mathfrak{b}$ und $\mathfrak{a}_{2} \cdot \mathfrak{b}$ Hauptideale liefert. Als Zeichen der Äquivalenz möge dienen $\mathfrak{a}_{1} \sim \mathfrak{a}_{2}$. Der vorausgeschickte Hilfssatz ergibt die Folgerung: Gilt $\mathfrak{a}_{1} \sim \mathfrak{a}_{2}$ und $\mathfrak{a}_{1} \sim \mathfrak{a}_{3}$, so gilt auch $\mathfrak{a}_{2} \sim \mathfrak{a}_{3}, d . h$. sind zwei Ideale einem dritten äquivalent, so sind sie auch untereinander äquivalent. Sind nämlich $\mathfrak{a}_{1} \cdot \mathfrak{b}$ und $\mathfrak{a}_{2} \cdot \mathfrak{b}$ Hauptideale, und gilt dasselbe vou $\mathfrak{a}_{1} \cdot \mathfrak{c}$ und $\mathfrak{a}_{3} \cdot \mathfrak{c}$, so ist nach dem Hilfssatze auch $\mathfrak{a}_{2} \cdot \mathfrak{c}$ ein Hauptideal, so daß $\mathfrak{a}_{2} \sim a_{3}$ gilt.

Aus dem für zwei äquivalente Ideale $\mathfrak{a}_{1}$ und $\mathfrak{a}_{2}$ charakteristischen Gleichungen (1) folgt:

$$
\mathfrak{a}_{1} \cdot\left[\alpha_{2}\right]=\mathfrak{a}_{2} \cdot\left[\alpha_{1}\right] .
$$

Besteht andrerseits für zwei Ideale $\mathfrak{a}_{1}, \mathfrak{a}_{2}$ und zwei Zahlen $\alpha_{1}$ und $\alpha_{2}$ aus $\mathfrak{e}$ die Gleichung (2), so folgt durch Multiplikation mit einem Ideale $\mathfrak{b}$ :

$$
\mathfrak{a}_{1} \cdot \mathfrak{b} \cdot\left[\alpha_{2}\right]=\mathfrak{a}_{2} \cdot \mathfrak{b} \cdot\left[\alpha_{1}\right] .
$$

Nun können wir nach S. 93 das Ideal $\mathfrak{b}$ so wählen, daß $\mathfrak{a}_{1} \cdot \mathfrak{b}=[\alpha]$ ein Hauptideal wird. Dann gilt $\mathfrak{a}_{2} \cdot \mathfrak{b} \cdot\left[\alpha_{1}\right]=\left[\alpha \cdot \alpha_{2}\right]$, woraus wir wie im Anfang des Paragraphen folgern, dab auch $\mathfrak{a}_{2} \cdot \mathfrak{b}$ ein Hauptideal ist. Somit 
kann man auch sagen: Zwei Ideale $\mathfrak{a}_{1}$ und $\mathfrak{a}_{2}$ sind stets und nur dann äquivalent, wenn es zwei von 0 verschiedene Zahlen $\alpha_{1}, \alpha_{2}$ in e gibt, die mit $\mathfrak{a}_{1}$ und $\mathfrak{a}_{2}$ die Gleichung (2) erfüllen. Wir notieren als unmittelbare Folgerung noch den Satz: Gilt $\mathfrak{a}_{1} \sim \mathfrak{a}_{2}$, so gewinnt man die Zahlen von $\mathfrak{a}_{2}$ aus denen von $\mathfrak{a}_{1}$, indem man die letzteren sämtlich mit einer gewissen Zahl $\frac{\alpha_{2}}{\alpha_{1}}$ aus $\Re$ multipliziert.

\section{$\$$ 13. Die Idealklassen des Körpers $\Re$.}

Alle mit einem einzelnen Ideale $\mathfrak{a}$ äquivalenten Ideale vereinigen wir in eine „Klasse" von Idealen oder „Idealklasse“, die wir durch $\mathfrak{A}$ bezeichnen. Dann sind je zwei Ideale der Klasse $\mathfrak{A}$ miteinander äquivalent, und irgend zwei Ideale verschiedener Klassen nennen wir ,inäquivalent“. Jedes Ideal $\mathfrak{a}$ von $\Omega$ gehört einer bestimmten Klasse an, da $\mathfrak{a}$, mit einem geeigneten Ideale $\mathfrak{b}$ multipliziert, ein Hauptideal liefert. Als „Repräsentanten" der einzelnen Klasse können wir ein beliebiges Ideal derselben wählen. Es gilt der Satz: Die gesamten Hauptideale von $\AA$ bilden eine Klasse für sich, die die „Hauptklasse" heißt und durch \& bezeichnet werden mag. Jedes Hauptideal $\mathfrak{a}$ ergibt nämlich, mit $\mathfrak{e}$ multipliziert, ein Hauptideal, nämlich $\mathfrak{a}$ selbst, und soll umgekehrt $\mathfrak{a} \cdot \mathfrak{e}$ ein Hauptideal sein, so muß $\mathfrak{a}$ selbst ein solches sein.

Es besteht der grundlegende Satz: Die gesamten Ideale des Körpers $\Re$ ordnen sich in ,endlich viele" Idealklassen an.

Dem Beweise müssen wir einen Hilfssatz vorausschicken, zu dem die folgende Betrachtung hinführt: Es sei $\eta_{1}, \eta_{2}, \ldots, \eta_{n}$ eine Basis von $e$, und es seien $\eta_{i}, \eta_{i}^{\prime}, \eta_{i}^{\prime \prime}, \ldots, \eta_{i}^{(n-1)}$ die mit $\eta_{i}$ konjugierten Zahlen, $\eta_{i}^{(k)}$ eine beliebige unter ihnen. Die Summe der $n$ absoluten Beträge $\left|\eta_{1}^{(k)}\right|,\left|\eta_{2}^{(k)}\right|, \ldots$, $\left|\eta_{n}^{(k)}\right|$ hat einen reellen positiven Wert, ebenso das Produkt aller $n$ Summen:

$$
\prod_{k=0}^{n-1}\left(\left|\eta_{1}^{(k)}\right|+\left|\eta_{2}^{(k)}\right|+\cdots+\left|\eta_{n}^{(k)}\right|\right)=M
$$

Dieser reelle positive Wert $M$ ist mit der Auswahl der Basis fest bestimmt. Jede Zahl $\eta$ von $e$ ist nun in der Gestalt:

$$
\eta=e_{1} \eta_{1}+e_{2} \eta_{2}+\cdots+e_{n} \eta_{n}
$$

mittels rationaler ganzer $e$ darstellbar. Wir lassen jetzt nur noch diejenigen Zahlen (2) zu, bei denen die absoluten Beträge $\left|e_{i}\right|$ eine rationale ganze positive Zahl $g$, deren Auswahl wir vorbehalten, nicht übersteigen, $\left|e_{i}\right| \leqq g$. Dann gilt, falls $\eta, \eta^{\prime}, \eta^{\prime \prime}, \ldots, \eta^{(n-1)}$ die mit $\eta$ konjugierten Zahlen sind:

$$
\left|\eta^{(k)}\right| \leqq g\left(\left|\eta_{1}^{(k)}\right|+\left|\eta_{2}^{(k)}\right|+\cdots+\left|\eta_{n}^{(k)}\right|\right) \text {. }
$$

Mit Rücksicht auf (1) folgt hieraus:

$$
|N(\eta)| \leqq M g^{n}
$$


Es sei jetzt $\mathfrak{b}$ irgendein Ideal von $\mathfrak{\Re}$. Da $N(\mathfrak{b})$ eine rationale ganze positive Zahl ist, so können wir die ganze Zahl $g$ so wählen, daB:

$$
g^{n} \leqq N(\mathfrak{b})<(g+1)^{n}
$$

zutrifft. Wir bilden nun die $(g+1)^{n}$ verschiedenen ganzen Zahlen (2), die allen Kombinationen rationaler ganzer Zahlen $e$ mit den Bedingungen $0 \leqq e_{i} \leqq g$ entsprechen. Da es $\bmod \mathfrak{b}$ nur $N(\mathfrak{b})$ inkongruente ganze Zahlen in $\mathfrak{e}$ gibt, so sind unter jenen $(g+1)^{n}$ Zahlen $\eta$ wegen (4) mindestens zwei mod $\mathfrak{b}$ kongruente Zahlen nachweisbar, deren Differenz $\beta$ eine von 0 verschiedene Zahl (2) mit $\left|e_{i}\right| \leqq g$ ist, die sicher in $\mathfrak{b}$ enthalten ist. Also ist zufolge (3) der absolute Betrag $|N(\beta)| \leq M g^{n}$, und da $g^{n} \leqq N(\mathfrak{b})$ ist, so folgt der Satz: In jedem Ideal $\mathfrak{b}$ von $\Re$ ist eine von 0 verschiedene Zahl $\beta$ nachweisbar, für deren Norm die Ungleichung gilt:

$$
N(\beta) \mid \leqq M \cdot N(\mathfrak{b})
$$

Es sei nun $\mathfrak{A}$ irgendeine Idealklasse des Körpers $\mathfrak{R}$ und $\mathfrak{b}$ ein Ideal, das, mit den Idealen $\mathfrak{a}$ von $\mathfrak{A}$ multipliziert, Hauptideale $\mathfrak{a} \cdot \mathfrak{b}$ liefert. Wir wählen aus $\mathfrak{b}$ eine der Bedingung (5) entsprechende Zahl $\beta$ und können dann, da das Hauptideal $[\beta]$ den Teiler $\mathfrak{b}$ hat, $[\beta]=\mathfrak{a} \cdot \mathfrak{b}$ setzen, wo $\mathfrak{a}$ der Klasse $\mathfrak{A}$ angehört. Nach S. $101 \mathrm{ff}$. folgt:

$$
N(\beta) \mid=N(\mathfrak{a}) \cdot N(\mathfrak{b}) \leq M \cdot N(\mathfrak{b}),
$$

und also gilt $N(\mathfrak{a}) \leqq M$, so daß in jeder Klasse $\mathfrak{X}$ ein Ideal a nachweisbar ist, dessen Norm nicht größer als der reelle positive Wert $M$ ist. Insbesondere können wir als Repräsentanten der Klasse $\mathfrak{A}$ ein solches Ideal a wählen.

Nun gibt es nur endlich viele rationale ganze positive Zahlen, die $\leq M$ sind. $Z u$ jeder dieser Zahlen, als Norm $N(\mathfrak{a})$ aufgefaßt, gibt es nach S. 102 nur endlich viele Ideale $\mathfrak{a}$. Wir können also für jede Klasse $\mathfrak{A}$ des Körpers $\Re$ einen Repräsentanten $\mathfrak{a}$ aus einer begrenzten Anzahl von Idealen wählen. Also haben wir in der Tat auch nur eine begrenzte Anzahl von Idealklassen in $\Omega$.

Aus dem Satze am Anfang des vorigen Paragraphen folgt weiter: Ist $\mathfrak{a} \cdot \mathfrak{b}$ ein Hauptideal, so ergibt irgendein Ideal $\mathfrak{a}^{\prime}$ der Klasse $\mathfrak{A}$ von $\mathfrak{a}$ mit irgendeinem Ideale $\mathfrak{b}^{\prime}$ der Klasse $\mathfrak{B}$ von $\mathfrak{b}$ als Produkt $\mathfrak{a}^{\prime} \cdot \mathfrak{b}^{\prime}$ wieder ein Hauptideal. Man kann demnach von einer Multiplikation der beiden Klassen $\mathfrak{A}$ und $\mathfrak{B}$ sprechen und den vorstehenden Satz durch die Gleichung $\mathfrak{A} \cdot \mathfrak{B}=\mathfrak{E}$ oder auch $\mathfrak{B} \cdot \mathfrak{A}=\mathfrak{E}$ zum Ausdruck bringen. Jede der beiden Klassen $\mathfrak{A}, \mathfrak{B}$ heißt zur andern „invers"; auch mag $\mathfrak{B}$ durch $\mathfrak{A}^{-1}$ und entsprechend $\mathfrak{A}$ durch $\mathfrak{B}^{-1}$ bezeichnet werden.

Gilt $\mathfrak{a} \sim \mathfrak{a}^{\prime}$, und ist $\mathfrak{c}$ irgendein Ideal, so gilt auch $\mathfrak{a} \cdot \mathfrak{c} \sim \mathfrak{a}^{\prime} \cdot \mathfrak{c}$. Sind nämlich $\mathfrak{a} \cdot \mathfrak{b}$ und $\mathfrak{a}^{\prime} \cdot \mathfrak{b}$ Hauptideale, und gehört das Ideal $\mathfrak{b}$ der zur 
Klasse $\mathfrak{C}$ von $\mathfrak{c}$ inversen Klasse an, so sind die beiden Ideale:

$$
(\mathfrak{a} \cdot \mathfrak{c}) \cdot(\mathfrak{b} \cdot \mathfrak{d})=(\mathfrak{a} \cdot \mathfrak{b}) \cdot(\mathfrak{c} \cdot \mathfrak{b}), \quad\left(\mathfrak{a}^{\prime} \cdot \mathfrak{c}\right) \cdot(\mathfrak{b} \cdot \mathfrak{d})=\left(\mathfrak{a}^{\prime} \cdot \mathfrak{b}\right) \cdot(\mathfrak{c} \cdot \mathfrak{d})
$$

Hauptideale, da rechts beide Male Produkte von Hauptidealen stehen. Also ist in der Tat $\mathfrak{a} \cdot \mathfrak{c} \sim \mathfrak{a}^{\prime} \cdot \mathfrak{c}$. Weiter folgt durch zweimalige Anwendung dieses Satzes: Gilt $\mathfrak{a} \sim \mathfrak{a}^{\prime}$ und $\mathfrak{b} \sim \mathfrak{b}^{\prime}$, so gilt auch $\mathfrak{a} \cdot \mathfrak{b} \sim \mathfrak{a}^{\prime} \cdot \mathfrak{b}^{\prime}$.

Hieraus ergibt sich die Möglichkeit, allgemein die Idealklassen miteinander zu multiplizieren: Sind $\mathfrak{A}$ und $\mathfrak{B}$ irgendzwei Ideallilassen, so ergeben zwei Ideale $\mathfrak{a}$ und $\mathfrak{b}$ aus $\mathfrak{A}$ und $\mathfrak{B}$ als Produkt $\mathfrak{a} \cdot \mathfrak{b}=\mathfrak{c}$ stets ein Ideal einer durch $\mathfrak{A}$ und $\mathfrak{B}$ bestimmten dritten Klasse $\mathfrak{C}$, die wir symbolisch als Produkt $\mathfrak{A} \cdot \mathfrak{B}=\mathfrak{C}$ von $\mathfrak{A}$ und $\mathfrak{B}$ schreiben. Für Produkte dieser Art gelten das kommutative und das assoziative Gesetz, $\mathfrak{A} \cdot \mathfrak{B}=\mathfrak{B} \cdot \mathfrak{A}$ und $(\mathfrak{A} \cdot \mathfrak{B}) \cdot \mathfrak{C}=\mathfrak{A} \cdot(\mathfrak{B} \cdot \mathfrak{C})$, da diese Gesetze für die Multiplikation der der Ideale gelten. Produkte gleicher Faktoren können wir natürlich als Potenzen schreiben.

Wir bezeichnen jetzt mit $h$ die endliche Anzahl aller Idealklassen des Körpers $\mathfrak{\Re}$, diese Klassen selbst aber mit $\mathfrak{A}_{0}, \mathfrak{A}_{1}, \mathfrak{A}_{2}, \ldots, \mathfrak{A}_{h-1}$, wobei $\mathfrak{A}_{0}$ die oben mit $\mathfrak{F}$ bezeichnete "Hauptklasse" sei. Dann können wir die $\mathfrak{A}_{0}, \mathfrak{A}_{1}, \ldots, \mathfrak{A}_{h-1}$ im Sinne von S. 2 als die Elemente einer Gruppe $G_{h}$ auffassen, wobei das Gesetz der Zusammensetzung zweier Elemente $\mathfrak{A}_{i}, \mathfrak{A}_{k}$ zu einem dritten $\mathfrak{A}_{\boldsymbol{l}}$ durch die Multiplikation der Idealklassen gegeben ist, $\mathfrak{A}_{i} \cdot \mathfrak{A}_{k}=\mathfrak{A}_{l}$. Man erkennt sofort, daß dieses Gesetz den drei Gruppenbedingungen von S. 2 gehorcht, und. zwar der zweiten Bedingung, da das assoziative Gesetz für die Produkte der Idealklassen gilt, der dritten Bedingung, wie man leicht aus dem Umstande folgert, daß mit der einzelnen Klasse $\mathfrak{A}_{i}$ immer auch die inverse Klasse $\mathfrak{A}_{i}^{-1}$ dem Systeme $\mathfrak{A}_{0}, \mathfrak{A}_{1}, \ldots, \mathfrak{A}_{h-1}$ angehört. Da überdies $\mathfrak{A}_{i} \cdot \mathfrak{A}_{k}=\mathfrak{A}_{k} \cdot \mathfrak{A}_{i}$ gilt, so haben wir den Satz: Die $h$ Idealklassen des Körpers $\Re$ bilden gegenüber Multiplikation eine kommutative oder Abelsche Gruppe $G_{h}$ der Ordnung $h$, in der die Hauptklasse $\mathfrak{A}_{0}=$ \& das "Einheitselement" darstellt.

Es sei $\boldsymbol{\nu}$ die „Periode“ irgendeines Elementes $\mathfrak{A}$ der Gruppe $G_{h}$, d.h. es sei $\boldsymbol{\nu}$ die kleinste rationale ganze positive Zahl, für die $\mathfrak{A}^{v}=\mathfrak{A}_{0}=\mathbb{E}$ ist. Dann bilden die Elemente $\mathfrak{A}, \mathfrak{A}^{2}, \ldots, \mathfrak{A}^{v-1}, \mathfrak{A}^{v}=\mathfrak{A}_{0}$ eine zyklische Untergruppe $G_{v} \operatorname{der} G_{h}$, und also ist $\nu$ ein Teiler von $h$. Insbesondere gilt stets $\mathfrak{A}^{h}=\mathfrak{A}_{0}$; wir können auch sagen: Die $h^{\text {to }}$ Potenz jedes Ideals a aus $\mathfrak{\Omega}$ ist ein Hauptideal.

\section{$\$ 14$. Zerfällung der rationalen Primzahlen in Primideale.}

In jedem Ideale $\mathfrak{a}$ gibt es von 0 verschiedene rationale ganze positive Zahlen, z. B. die Zahl $N(\mathfrak{a})$. Ist $a$ die kleinste rationale ganze positive Zahl von $a$, so sind $0, \pm a, \pm 2 a, \pm 3 a, \ldots$ alle in $a$ auftretenden rationalen ganzen Zahlen. Gäbe es nämlich in $\mathfrak{a}$ noch eine weitere ratio- 
nale ganze Zahl, so würde auch noch eine zwischen 0 und $a$ liegende Zahl auftreten. Da $N(\mathfrak{a})$ in $\mathfrak{a}$ enthalten ist, so folgt: Die Zahl $N(\mathfrak{a})$ ist durch die kleinste in a vorkommende rationale ganze positive Zahl a teilbar. Da ferner $\mathfrak{a}$ im Hauptideal $[a]$ aufgeht, so kann man $[a]$ als Produkt $[a]=\mathfrak{a} \cdot \mathfrak{b}$ darstellen, woraus:

$$
N([a])=a^{n}=N(\mathfrak{a}) \cdot N(\mathfrak{b})
$$

folgt: Die Norm $N(\mathfrak{a})$ ist eine in $a^{n}$ aufgehende rationale ganze positive Zahl, unter a die kleinste rationale ganze positive Zahl von a verstanden.

Ist $\mathfrak{a}$ ein Primideal $\mathfrak{p}$, so ist die Zahl $a$ notwendig eine rationale Primzahl $p$. Man beachte zunächst, daß $a=1$ nur für das Ideal e zutrifft; denn in diesem Falle ist $N(\mathfrak{a})=1$, also $\mathfrak{a}=\mathfrak{e}$. Wäre nun für $\mathfrak{a}=\mathfrak{p}$ die Zahl $a=a_{1} \cdot a_{2}$, wo $a_{1}$ und $a_{2}$ zwei rationale ganze Zahlen $>1$ sind, so würde $\mathfrak{p}$ in $[a]=\left[a_{1}\right] \cdot\left[a_{2}\right]$ und also mindestens in einem Faktor $\left[a_{1}\right]$ oder $\left[a_{2}\right]$ aufgehen, so daß $a$ nicht die kleinste rationale ganze positive Zahl von $\mathfrak{p}$ wäre. $\mathrm{Da} N(\mathfrak{p})$ in $p^{n}$ aufgeht und übrigens $N(\mathfrak{p})>1$ ist (sonst wäre $\mathfrak{p}=\mathrm{e}$ ), so folgt: Die kleinste rationale ganze positive Zahl, die in einem Primideale $\mathfrak{p}$ auftritt, ist eine rationale Primzahl $p$; für die Norm eines Primideals $\mathfrak{p}$ gilt:

$$
N(\mathfrak{p})=p^{\lambda}
$$

wo der Exponent $\lambda$ eine Zahl der Reihe 1, 2, .., n ist und als der „Grad" des Primideals $p$ bezeichnet wird.

Die Primfaktorenzerlegung des Hauptideals $[p]$ sei:

$$
[p]=\mathfrak{p}_{1} \cdot \mathfrak{p}_{2} \cdots \mathfrak{p}_{l} \text {. }
$$

Nach (8) S. 103 folgt hieraus:

$$
N([p])=p^{n}=N\left(\mathfrak{p}_{1}\right) \cdot N\left(\mathfrak{p}_{2}\right) \cdots N\left(\mathfrak{p}_{l}\right) .
$$

Es ist demnach jeder der rechts stehenden Faktoren eine Potenz von $p$ (mit einem Exponenten $>0$ ), so dab der Satz gilt: Ist $p$ eine rationale Primzahl, so zerfällt das Hauptideal $[p]$ in $l$ Primideale $\mathfrak{p}_{1}, \mathfrak{p}_{2}, \ldots, \mathfrak{p}_{l}$, wo $l$ eine der Zahlen 1, 2, .., $n$ ist; jedes dieser Primideale hat $p$ als kleinste rationale ganze positive Zahl, und die Summe ihrer Grade $\lambda_{1}, \lambda_{2}$, $\ldots, \lambda_{l}$ ist gleich $n$.

Die tieferen Entwicklungen, die sich an die Zerlegung (2) des Hauptideals $[p]$ anschließen, gehören zu den schwierigsten und interessantesten Teilen der Dedekindschen Idealtheorie. $\left.{ }^{1}\right)$ Ein ziemlich leicht beweisbarer Satz, den wir nicht entbehren können, ist der folgende: Geht p nicht in der Grundzahl $D$ des Körpers $\mathfrak{R}$ auf, so sind die Primideale $\mathfrak{p}_{1}, \mathfrak{p}_{2}, \ldots$, $\mathfrak{p}_{l}$, in welche $[p]$ zerfällt, alle voneinander verschieden.

1) Vgl. Dedekind, „Über die Diskriminanten endlicher Körper", Göttinger Abhandl., Bd. 19 (1882). 
Dem Beweise dieses Satzes schicken wir zwei Hilfssätze voraus: Sind $\beta_{1}, \beta_{2}, \ldots, \beta_{n}$ Zahlen aus $\mathfrak{e}$, und ist:

$$
\alpha=b_{1} \beta_{1}+b_{2} \beta_{2}+\cdots+b_{n} \beta_{n},
$$

wo die $b$ rationale Zahlen mit dem Hauptnenner $h$ sind, eine ,ganze" Zahl, so ist die Diskriminante $D\left(\beta_{1}, \beta_{2}, \ldots, \beta_{n}\right)$ der $\beta$ durch das Quadrat $h^{2}$ des Hauptnenners $h$ teilbar. Sind die $\beta$ linear-abhängig, so ist ihre Diskriminante 0 , und dann ist der Satz richtig. Sind die $\beta$ linear-unabhängig, so mögen sie in einer Basis $\eta_{1}, \eta_{2}, \ldots, \eta_{n}$ die Darstellungen besitzen:

$$
\beta_{i}=e_{i 1} \eta_{1}+e_{i 2} \eta_{2}+\cdots+e_{i n} \eta_{n}, \quad i=1,2, \ldots, n .
$$

Die Determinante $d=\left|e_{i k}\right|$ der $n^{2}$ ganzen Zahlen $e_{i k}$ ist jetzt von 0 verschieden, und die $\eta_{1}, \eta_{2}, \ldots, \eta_{n}$ sowie damit jede Zahl $\eta$ von e stellen sich durch die $\beta$ je auf eine und nur eine Art in der Gestalt dar:

$$
\eta=c_{1} \beta_{1}+c_{2} \beta_{2}+\cdots+c_{n} \beta_{n},
$$

wo die rationalen $c$, insoweit sie Brüche sind, einen in $d$ aufgehenden Hauptnenner haben. Da:

$$
D\left(\beta_{1}, \beta_{2}, \ldots, \beta_{n}\right)=d^{2} \cdot D
$$

ist, wo rechts die Grundzahl $D$ von $\Omega$, also eine rationale ganze Zahl steht, so ist $D\left(\beta_{1}, \beta_{2}, \ldots, \beta_{n}\right)$ durch $d^{2}$ teilbar. Nun ist das in (4) dargestellte $\alpha$ eine Zahl aus $\mathrm{e}$; der Hauptnenner $h$ der $b_{1}, b_{2}, \ldots, b_{n}$ geht also in $d$ und das Quadrat $h^{2}$ demnach in $d^{2}$ und damit in der Diskriminante $D\left(\beta_{1}, \beta_{2}, \ldots, \beta_{n}\right)$ auf. Damit ist der erste Hilfssatz bewiesen.

Zwei ganze algebraische Zahlen (mögen sie in $\Re$ enthalten sein oder nicht) sollen $\bmod p$ kongruent heißen, falls ihre durch die rationale Primzahl $p$ geteilte Differenz eine ganze algebraische Zahl liefert. Da die Binomialkoeffizienten $\left(\begin{array}{l}p \\ 1\end{array}\right),\left(\begin{array}{l}p \\ 2\end{array}\right), \ldots, .\left(\begin{array}{c}p \\ p-1\end{array}\right)$ durch $p$ teilbar sind, so gilt für irgendzwei ganze algebraische 'Zahlen $\alpha, \alpha^{\prime}$ :

$$
\left(\alpha+\alpha^{\prime}\right)^{p} \equiv \alpha^{p}+\alpha^{p} \quad(\bmod p),
$$

woraus man leicht für irgendeine Anzahl ganzer Zahlen $\alpha, \alpha^{\prime}, \ldots, \alpha^{(v-1)}$ folgert:

$$
\left(\alpha+\alpha^{\prime}+\cdots+\alpha^{(v-1)}\right)^{p} \equiv \alpha^{p}+\alpha^{p}+\cdots+\left(\alpha^{(v-1)}\right)^{p} \quad(\bmod p) .
$$

Ist $\alpha$ eine Zahl aus $\mathrm{e}$, und sind $\alpha, \alpha^{\prime}, \ldots, \alpha^{(n-1)}$ die mit $\alpha$ konjugierten Zahlen, so folgt aus (6) für die Spur $S(\alpha)=\alpha+\alpha^{\prime}+\cdots+\alpha^{(n-1)}$ :

$$
(S(\alpha))^{p} \equiv \alpha^{p}+\alpha^{p}+\cdots+\left(\alpha^{(n-1)}\right)^{p}=S\left(\alpha^{p}\right) \quad(\bmod p) .
$$

Nach dem Fermatschen Satze gilt aber für die ganze rationale Zahl $S(\alpha)$ die Kongruenz $(S(\alpha))^{p} \equiv S(\alpha)(\bmod p)$. Wir finden demnach als für jede Zahl $\alpha$ aus e gültig:

$$
S(\alpha) \equiv S\left(\alpha^{p}\right) \quad(\bmod p)
$$

Sind $\alpha_{1}, \alpha_{2}, \ldots, \alpha_{n}$ irgend $n$ Zahlen aus $\mathrm{e}$, so können wir ihre Dis- 
kriminante $D\left(\alpha_{1}, \alpha_{2}, \ldots, \dot{\alpha}_{n}\right)$ nach der Regel (10) S. 85 durch die Spuren der Produkte der $\alpha$ zu zweien darstellen. Bilden wir entsprechend die Diskriminante $D\left(\alpha_{1}^{p}, \alpha_{2}^{p}, \ldots, \alpha_{n}^{p}\right)$, so folgt aus der Kongruenz (7) als zweiter Hilfssatz: Für irgend $n$ Zahlen $\alpha_{1}, \alpha_{2}, \ldots, \alpha_{n}$ aus e gilt, unter $p$ eine rationale Primzahl verstanden, die Kongruenz:

$$
D\left(\alpha_{1}, \alpha_{2}, \ldots, \alpha_{n}\right) \equiv D\left(\alpha_{1}^{p}, \alpha_{2}^{p}, \ldots, \alpha_{n}^{p}\right) \quad(\bmod p) .
$$

Zum Beweise des Satzes über die Primideale $\mathfrak{p}_{1}, \mathfrak{p}_{2}, \ldots, \mathfrak{p}_{l}$ von $[p]$ nehmen wir an, $[p]$ sei durch mindestens ein Primidealquadrat $\mathfrak{p}^{2}$ teilbar, und haben dann zu zeigen, daß $p$ in der Grundzahl $D$ des Körpers $\Re$ aufgeht. Wir schreiben $[p]=\mathfrak{a} \cdot \mathfrak{p}^{2}$ und haben in $\mathfrak{a} \cdot \mathfrak{p}$ ein nicht durch $[p]$ teilbares Ideal. Folglich gibt es in $\mathfrak{a} \cdot \mathfrak{p}$ eine nicht durch $p$ teilbare Zahl $\eta$. Da aber $(\mathfrak{a} \cdot \mathfrak{p})^{2}=\mathfrak{a} \cdot[p]$ durch $[p]$ teilbar ist, so ist $\eta^{2}$ und also auch $\eta^{p}$ durch $p$ teilbar.

Ist die Darstellung von $\eta$ in der Basis von $\mathrm{e}$ :

$$
\eta=e_{1} \eta_{1}+e_{2} \eta_{2}+\cdots+e_{n} \eta_{n},
$$

so sind die $e$ rationale ganze Zahlen, die nicht alle durch $p$ teilbar sind. Durch Erheben zur $p^{\text {ten }}$ Potenz folgt mit Rücksicht auf (6) und den Fermatschen Satz $e_{k}^{p} \equiv e_{k}(\bmod p)$ :

$$
\eta^{p} \equiv e_{1} \eta_{1}^{p}+e_{2} \eta_{2}^{p}+\cdots+e_{n} \eta_{n}^{p} \quad(\bmod p) .
$$

$\mathrm{Da} \eta^{p}$ durch $p$ teilbar ist, so gilt:

$$
e_{1} \eta_{1}^{p}+e_{2} \eta_{2}^{p}+\cdots+e_{n} \eta_{n}^{p} \equiv 0 \quad(\bmod p)
$$

so daß wir in: $\quad \alpha=\frac{e_{1}}{p} \eta_{1}^{p}+\frac{e_{2}}{p} \eta_{2}^{p}+\cdots+\frac{e_{n}}{p} \eta_{n}^{p}$

eine „ganze“ Zahl gewonnen haben, während rechts mindestens ein Bruch des Nenners $p$ als Koeffizient auftritt. Nach dem ersten Hilfssatze ist also $D\left(\eta_{1}^{p}, \eta_{2}^{p}, \ldots, \eta_{n}^{\nu}\right)$ durch $p^{2}$ teilbar, worauf die Kongruenz (8) lehrt, daß $D\left(\eta_{1}, \eta_{2}, \ldots, \eta_{n}\right)$, d. h. die Grundzahl $D$ des Körpers $\Omega$ durch $p$ teilbar ist.

Hiermit ist bewiesen, $\mathrm{daB}$ in der Zerlegung (2) von $[p]$ lauter verschiedene Primideale auftreten, falls die rationale Primzahl $p$ nicht in der Grundzahl $D$ von $\Omega$ aufgeht. Über die Zerlegung der in $D$ aufgehenden rationalen Primzahlen, der sogenannten "kritischen" Primzahlen des Körpers $\Omega$, sei auf die S. 108 genannte Arbeit von Dedekind verwiesen.

\section{§ 15. Sätze über Galoissche Zahlkörper.}

Unter $\Omega$ verstehen wir den bisher betrachteten Körper $n^{\text {ten }}$ Grades und unter $\mathfrak{\Omega}^{\prime}$ einen seiner konjugierten Körper. Einer ganzen Zahl $\eta$ aus $\Re$ entspricht als konjugiert wieder eine ganze Zahl $\eta^{\prime}$ aus $\AA^{\prime}$, so dab dem "Ideale" $e$ von $\mathbb{R}$ das Ideal $e^{\prime}$ von $\mathbb{R}^{\prime}$ zugeordnet ist. Allgemeiner ent- 
spricht einem Ideale $\mathfrak{a}$ von $\mathfrak{\Omega}$ stets wieder ein Ideal $\mathfrak{a}^{\prime}$ von $\mathfrak{\Omega}^{\prime}$. Sind nämlich $\alpha_{1}$ und $\alpha_{2}$ zwei Zahlen aus $\mathfrak{a}$, die die gleichfalls in $\mathfrak{a}$ enthaltene Summe $\alpha_{1}+\alpha_{2}=\alpha_{3}$ liefern, so besteht nach S. $71 \mathrm{ff}$. auch zwischen den konjugierten Zahlen die Gleichung $\alpha_{1}^{\prime}+\alpha_{2}^{\prime}=\alpha_{3}^{\prime}$, und ebenso überträgt sich die Gleichung $\eta \cdot \alpha_{1}=\alpha_{4}$ auf die für $\mathfrak{a}^{\prime}$ gültige Gleichung $\eta^{\prime} \cdot \alpha_{1}^{\prime}=\alpha_{4}^{\prime}$ zwischen den konjugierten Zahlen. Sind die Zahlen eines Ideals $\mathfrak{b}$ in $\mathfrak{a}$ enthalten, so sind auch die Zahlen des zu $\mathfrak{b}$ konjugierten Ideals $\mathfrak{b}^{\prime}$ in $\mathfrak{a}^{\prime}$ enthalten. Man folgert hieraus leicht, daß sich die Ideale von $\mathfrak{\Omega}^{\prime}$ in bezug auf Teilbarkeit genau so verhalten, wie die ihnen konjugierten Ideale von $\Re$. Insbesondere entspricht einem Primideale $p$ von $\Omega$ stets wieder ein Primideal $\mathfrak{p}^{\prime}$ von $\mathfrak{\Re}^{\prime}$. Zwei nach einem Ideale kongruente ganze Zahlen des einen Körpers liefern als konjugiert zwei Zahlen, die bezüglich des konjugierten Ideals kongruent sind, so daB sich die Klasseneinteilung aller Zahlen von $e$ bezüglich eines Ideals $\mathfrak{a}$ auf eine Klasseneinteilung aller Zahlen von $e^{\prime} \bmod \mathfrak{a}^{\prime}$ überträgt. Hieraus folgt der Satz: Konjugierte Ideale $\mathfrak{a}$ und $\mathfrak{a}^{\prime}$ der beiden Körper $\mathfrak{\Omega}$ und $\mathfrak{\Omega}^{\prime}$ haben stets gleiche Normen, $d . h$. es gilt $N(\mathfrak{a})=N\left(\mathfrak{a}^{\prime}\right)$.

Es sei jetzt $\Re$ insbesondere ein „Galoisscher Körper“, der mit seinen sämtlichen konjugierten Körpern gleich ist. Nach S. $44 \mathrm{ff}$. gestattet der Galoissche Körper $\Omega$ vom $n^{\text {ten }}$ Grade $n$ Transformationen $S_{0}, S_{1}, \ldots, S_{n-1}$ in sich, die eine Gruppe, $G_{n}$ bildeu, und bei denen je $n$ konjugierte Zahlen untereinander permutiert werden. Jetzt sind die mit einem einzelnen Ideale $\mathfrak{a}$ konjugierten Ideale $\mathfrak{a}, \mathfrak{a}^{\prime}, \mathfrak{a}^{\prime \prime}, \ldots, \mathfrak{a}^{(n-1)}$ alle in $\mathfrak{\Omega}$ enthalten. Eine erste Folgerung knüpfen wir an die nun vorliegende Möglichkeit, diese $n$ Ideale nach S. 93 miteinander zu multiplizieren. Hierbei ergibt sich der Satz: In einem Galoisschen Körper $\Re$ liefert die gemeinsame Norm $N(\mathfrak{a})$ von $n$ konjugierten Idealen $\mathfrak{a}, \mathfrak{a}^{\prime}, \ldots, \mathfrak{a}^{(n-1)}$ ein Hauptideal $[N(\mathfrak{a})]$, das gleich dem Produkte der $n$ konjugierten Ideale ist:

$$
\mathfrak{a} \cdot \mathfrak{a}^{\prime} \cdot \mathfrak{a}^{\prime \prime} \cdots \mathfrak{a}^{(n-1)}=[N(\mathfrak{a})]
$$

Dieser Satz ist für Hauptideale $\mathfrak{a}=[\alpha], \mathfrak{a}^{\prime}=\left[\alpha^{\prime}\right], \ldots, \mathfrak{a}^{(n-1)}=\left[\alpha^{(n-1)}\right]$ einleuchtend; denn nach der Erklärung der Multiplikation der Ideale ist:

$$
[\alpha] \cdot\left[\alpha^{\prime}\right] \cdots\left[\alpha^{(n-1)}\right]=\left[\alpha \cdot \alpha^{\prime} \cdots \alpha^{(n-1)}\right]=[N(\mathfrak{a})] .
$$

Für ein beliebiges Ideal $\mathfrak{a}$ gilt nach S. 107 die Gleichung $\mathfrak{a}^{h}=[\alpha]$, wo $h$ die Anzahl der Idealklassen ist und $\alpha$ eine Zahl aus $e$ bedeutet. Da die Gleichung (1) für Hauptideale schon bewiesen ist, so folgt:

$$
\left(\mathfrak{a} \cdot \mathfrak{a}^{\prime} \cdots \mathfrak{a}^{(n-1)}\right)^{h}=\mathfrak{a}^{h} \cdot \mathfrak{a}^{h} \cdots\left(\mathfrak{a}^{(n-1)}\right)^{h}=\left[N\left(\mathfrak{a}^{h}\right)\right]=\left[N(\mathfrak{a})^{h}\right]=[N(\mathfrak{a})]^{h} .
$$

Sind aber die $h^{\text {ten }}$ Potenzen zweier Ideale gleich, so sind diese selbst gleich, wie aus der eindeutigen Zerlegung der Ideale in Primideale folgt. Also gilt die Gleichung (1) allgemein.

Die $n$ konjugierten Ideale $\mathfrak{a}, \mathfrak{a}^{\prime}, \ldots, \mathfrak{a}^{(n-1)}$ brauchen keineswegs alle 
voneinander verschieden zu sein. Sind im ganzen $\mu$ unter jenen Idealen mit $\mathfrak{a}$ gleich, so gibt es $\mu$ unter den $n$ Transformationen $S_{0}, S_{1}, \ldots, S_{n-1}$ $\operatorname{der} G_{n}$, die $\mathfrak{a}$ in sich überführen. Diese Transformationen bilden für sich eine Untergruppe $G_{\mu}$ der $G_{n}$ von der Ordnung $\mu$, die wir als die ,zum Ideal a gehörige Untergruppe" bezeichnen. Den $\nu=\frac{n}{\mu}$ Nebengruppen entsprechend bilden dann die $\mathfrak{a}, \mathfrak{a}^{\prime}, \ldots, \mathfrak{a}^{(n-1)}$ im ganzen $\nu$ verschiedene Systeme von je $\mu$ einander gleichen Idealen. Sind alle $n$ Ideale $\mathfrak{a}, \mathfrak{a}^{\prime}, \ldots$, $\mathfrak{a}^{(n-1)}$ verschieden, so gilt $\mu=1$; ist $\mathfrak{a}=[a]$ ein Hauptideal mit rationaler ganzer Zahl $a$, so gilt $\mu=n$.

Die in (2) S. 108 angesetzte Zerlegung eines Hauptideals [ $p]$ mit rationaler Primzahl $p$ läßt sich in einem Galoisschen Körper in folgender Art weiter entwickeln: Ist $\mathfrak{p}_{1}$ ein in $[p]$ aufgehendes Primideal $\lambda^{\text {ten }}$ Grades, so war $N\left(\mathfrak{p}_{1}\right)=p^{\lambda}$, und $\lambda$ war eine der Zahlen $1,2, \ldots, n$. Zu $\mathfrak{p}_{1}$ gehört eine Untergruppe $G_{\mu} \operatorname{der} G_{n}$ vom Index $\nu=\frac{n}{\mu}$. Wir haben dann $\nu$ verschiedene mit $\mathfrak{p}_{1}$ konjugierte Primideale $\mathfrak{p}_{1}, \mathfrak{p}_{2}, \ldots, \mathfrak{p}_{v}$, und der Satz (1) ergibt die Gleichung:

$$
\left(\mathfrak{p}_{1} \cdot \mathfrak{p}_{2} \cdots \mathfrak{p}_{v}\right)^{\mu}=[p]^{\lambda}
$$

Ist demnach $\mathfrak{p}_{1}^{*}$ die höchste in $[p]$ aufgehende Potenz von $\mathfrak{p}_{1}$, so ist $x \lambda=\mu$ und also $x \lambda \nu=n$. Zugleich ergibt sich: Das Hauptideal $[p]$ mit einer rationalen Primzahl $p$ zerfällt im Galoisschen Körper $\Omega$ in das Produkt:

$$
[p]=\mathfrak{p}_{1}^{*} \cdot \mathfrak{p}_{2}^{*} \cdots \mathfrak{p}_{v}^{*}
$$

von $\nu$ konjugierten Primidealpotenzen gleicher Exponenten $\varkappa$. Dabei ist die Anzahl $\nu$ ein Teiler von $n$, der Exponent $\varkappa$ ein Teiler von $\frac{n}{v}$; es ist ferner $\lambda=\frac{n}{x \nu}$ der gemeinsame Grad der Primideale $\mathfrak{p}_{1}, \mathfrak{p}_{2}, \ldots, \mathfrak{p}_{v}$, und endlich ist $\mu=x \lambda$ die Ordnung der zum einzelnen Primideale gehörenden Gruppe. Dies gilt, mag $p$ eine kritische Primzahl des Körpers sein oder nicht. Für nichtkritische Primzahlen $p$ ist nach S. $108 \mathrm{ff}$. stets $x=1$. Hier also gilt der Satz: Ist die rationale Primzahl $p$ kein Teiler der Grundzahl $D$ des Körpers, so zerfällt $[p]$ in das Produkt von v verschiedenen Primidealen:

$$
[p]=\mathfrak{p}_{1} \cdot \mathfrak{p}_{\mathbf{2}} \cdots \mathfrak{p}_{v}
$$

des Grades $\mu=\frac{n}{\nu}$, der mit der Ordnung der zum einzelnen Primideale gehörenden Gruppe $G_{\mu}$ gleich ist.

\section{\$ 16. Beispiel der quadratischen Körper.}

Ein Körper zweiten Grades oder quadratischer Körper $\Re$ wird nach S. 83 durch eine Gleichung zweiten Grades mit rationalen ganzen Koeffizienten $a_{0} z^{2}+a_{1} z+a_{2}=0$ bestimmt, die im rationalen Körper irredu- 
zibel ist, d. h. deren Diskriminante $\left(a_{1}^{2}-4 a_{0} a_{\mathrm{q}}\right)$ nicht das Quadrat einer rationalen ganzen Zahl ist. Sondern wir das gröBte in dieser Diskriminante als Teiler enthaltene Quadrat einer rationalen ganzen Zahl ab, so bleibe die rationale ganze Zahl $d$ übrig, die dann durch kein Quadrat (außer 1) teiỉbar ist. Der Körper $\Omega$ setzt sich zusammen aus allen Zahlen:

$$
\xi=c_{0}+c_{1} \sqrt{d}
$$

unter $c_{0}$ und $c_{1}$ irgendwelche rationale Zahlen verstanden. Da mit $\xi$ stets auch die konjugierte Zahl $\xi^{\prime}=c_{0}-c_{1} \sqrt{d}$ in $\AA$ enthalten ist, so ist jeder quadratische Körper ein Galoisscher Körper.

Es ist zunächst festzustellen, welche unter deu Zahlen (1) ganz sind. Ist $\xi=c_{0}+c_{1} \sqrt{d}$ und also auch $\xi^{\prime}=c_{0}-c_{1} \sqrt{d}$ ganz, so gilt dasselbe von $\xi+\xi^{\prime}=2 c_{0}$ und $\left(\xi-\xi^{\prime}\right)^{2}=\left(2 c_{1}\right)^{2} d$. Es sind also $2 c_{0}=e_{0}$ und, da $d$ durch kein Quadrat teilbar ist, auch $2 c_{1}=e_{1}$ rationale ganze Zahlen, so daß die ganzen Zahlen von $\Re$ jedenfalls in der Gestalt:

$$
\xi=\frac{e_{0}+e_{1} \sqrt{d}}{2}
$$

mit rationalen ganzen $e$ enthalten sind. Da die Zahl (2) der Gleichung

$$
\xi^{2}-e_{0} \xi+\frac{e_{0}^{2}-d e_{1}^{2}}{4}=0
$$

genügt, so ist dafür, daß sie ganzzahlig ist, die Bedingung:

$$
e_{0}^{2} \equiv d e_{1}^{2} \quad(\bmod 4)
$$

hinreichend und notwendig. Nun ist $d$ modulo 4 mit einer der Zahlen 1, 2, 3 kongruent. Für $d \equiv 2$ oder $3(\bmod 4)$ folgt $e_{0} \equiv e_{1} \equiv 0(\bmod 2)$ aus (3), womit diese Kongruenz erfüllt ist; für $d \equiv 1(\bmod 4)$ ist bereits das Bestehen der Kongruenz $e_{0} \equiv e_{1}(\bmod 2)$ hinreichend. Indem wir im letzten Falle der Zahl (2) die Gestalt verleihen:

$$
\zeta=\frac{e_{0}-e_{1}}{2}+e_{1} \frac{1+\sqrt{d}}{2},
$$

finden wir für $d \equiv 1(\bmod 4)$ in $1, \frac{1+\sqrt{d}}{2}$ eine Basis für das System e aller ganzen Zahlen von $\Omega$, für $d \equiv 2$ oder $3(\bmod 4)$ aber in $1, \sqrt{d}$. Die Grundzahl $D$ von $\mathfrak{R}$ ist entsprechend gleich $d$ bzw. $4 d$. Für die Basis können wir in den beiden eben unterschiedenen Fällen einen gemeinsamen Ausdruck in der Grundzahl $D$ finden. Setzen wir:

$$
\frac{D+\sqrt{D}}{2}=\theta \text {, }
$$

so gilt der Satz: Die Grundzahl des quadratischen Körpers $\Re$ ist $D=d$, falls $d \equiv 1(\bmod 4)$ ist, und $D=4 d$, falls $d \equiv 2$ oder $3(\bmod 4)$ gilt; eine Basis für das System e der ganzen Zahlen von $\Re$ ist in allen Fällen durch $1, \theta$ gegeben, unter $\theta$ die ganze Zahl (4) verstanden. 
Ist $p$ eine rationale Primzahl, so ist das Hauptideal $[p]$ im Körper $\Re$ entweder ein Primideal zweiten Grades oder das Produkt zweier Primideale ersten Grades, die konjugiert sind und nur dann einander gleich sein können, wenn $p$ in $D$ aufgeht. Wir nehmen den Fall $[p]=\mathfrak{p} \cdot \mathfrak{p}^{\prime}$ an, ohne die Möglichkeit $\mathfrak{p}=\mathfrak{p}^{\prime}$ auszuschlieBen. Da $\mathfrak{p}$ ein Primideal ersten Grades ist, so gilt $N(\mathfrak{p})=p$, so daB es mod $\mathfrak{p}$ im ganzen $p$ inkongruente Zahlklassen in $e$ gibt. Als Repräsentanten dieser $p$ Klassen können wir die Zahlen $0,1,2, \ldots, p-1$ wählen, da $p$ die kleinste rationale ganze positive Zahl in $p$ ist und also die Zahlen $0,1, \ldots, p-1 \bmod \mathfrak{p}$ durchweg inkongruent sind. Die in (4) erklärte ganze Zahl $\theta$ gehöre in die durch die rationale ganze Zahl $b$ repräsentierte Klasse $\bmod \mathfrak{p}$, so daB $\theta-b \equiv 0(\bmod \mathfrak{p})$ gilt. Setzen wir zur Abkürzung $\theta-b=\eta$ und $D-2 b=a$, so gilt:

$$
\eta=\frac{a+\sqrt{D}}{2} \equiv 0 \quad(\bmod \mathfrak{p}) .
$$

Die zu $\eta$ konjugierte Zahl $\eta^{\prime}$ ist demnach im konjugierten Ideale $\mathfrak{p}^{\prime}$ enthalten:

$$
\eta^{\prime}=\frac{a-\sqrt{D}}{2} \equiv 0 \quad\left(\bmod \mathfrak{p}^{\prime}\right),
$$

und also gehört das Produkt $\eta \cdot \eta^{\prime}$ dem Hauptideale $\mathfrak{p} \cdot \mathfrak{p}^{\prime}=[p]$ an:

$$
\eta \cdot \eta^{\prime}=\frac{a^{2}-D}{4} \equiv 0 \quad(\bmod p), \quad D \equiv a^{2} \quad(\bmod 4 p) .
$$

Die letzte Kongruenz liefert den Satz: Ist das Hauptideal $[p]$ mit rationaler Primzahl $p$ in das Produkt $\mathfrak{p} \cdot \mathfrak{p}^{\prime}$ zweier Primideale ersten Grades spaltbar, so ist die Grundzahl $D$ des Körpers quadratischer Rest von $4 p$.

Dieser Satz ist umkehrbar. Ist nämlich $D$ quadratischer Rest von $4 p$, so gibt es eine die zweite Kongruenz (5) befriedigende rationale ganze Zahl $a$. Da diese Zahl $a$ auch $a \equiv D(\bmod 2)$ befriedigt, so sind:

$$
\frac{a \pm \sqrt{D}}{2}=\frac{a \mp D}{2} \pm \theta
$$

zwei ganze Zahlen, deren Produkt zufolge (5) durch $p$ teilbar ist. Da aber keine dieser beiden Zahlen einzeln durch $p$ teilbar ist ${ }^{1}$ ), so kann $[p]$ kein Primideal sein. Es besteht also der Satz: Ist $D$ quadratischer Rest von $4 p$, so zerfällt das Hauptideal $[p]$ in das Produkt zweier Primideale $\mathfrak{p}, \mathfrak{p}^{\prime}$ ersten Grades.

Wir haben endlich noch festzustellen, ob im Falle einer in $D$ aufgehenden rationalen Primzahl $p$ etwa $\mathfrak{p}^{\prime}=\mathfrak{p}$ zutrifft. Die Grundzahl $D$ ist zufolge ihrer Erklärung aus $d$ entweder $\equiv 0$ oder $\equiv 1(\bmod 4)$. Ist $p$ eine ungerade Primzahl, so kann die zweite Kongruenz (5) durch $a=0$

1) Aus dem Umstande, daß 1, $\theta$ eine Basis von $e$ ist, folgt leicht, daB die durch $p$ teilbaren Zahlen $\left(e_{0}+e_{1} \theta\right)$ von $e$ durch $p$ teilbare $e_{0}, e_{1}$ haben. 
oder $a=p$ befriedigt werden, je nachdem $D \equiv 0$ oder $\equiv 1(\bmod 4)$ ist. Ist aber $p=2$ und also $D$ (als durch $p$ teilbar) $\equiv 0(\bmod 4)$, so genügt der zweiten Kongruenz (5) die Zahl $a=0$ oder $a=2$, je nachdem $D \equiv 0$ oder $\equiv 4(\bmod 8)$ gilt. Geht $p$ in der Grundzahl $D$ auf, so ist $D$ quadratischer Rest von $4 p$, und die zweite Kongruenz (5) wird mittelst einer durch $p$ teilbaren Zahl a befriedigt. Wir zerlegen nun $[p]$ in das Produkt $\mathfrak{p} \cdot \mathfrak{p}^{\prime}$ der beiden Primideale $\mathfrak{p}$ und $\mathfrak{p}^{\prime}$ und beachten, daß das Produkt der beiden ganzen Zahlen $\eta, \eta^{\prime}=\frac{a \pm \sqrt{D}}{2}$ durch $p$ teilbar ist. Es geht also $\mathfrak{p} \cdot \mathfrak{p}^{\prime}$ in $[\eta] \cdot\left[\eta^{\prime}\right]$ auf, so daB $\mathfrak{p}$ in einem der Faktoren $[\eta],\left[\eta^{\prime}\right]$, etwa in $[\eta]$, aufgeht. Dann aber geht $\mathfrak{p}^{\prime}$ in $\left[\eta^{\prime}\right]$ auf, d. h. $\eta^{\prime}$ ist in $\mathfrak{p}^{\prime}$ enthalten. Da auch $a$ (als durch $p$ teilbar) in $\mathfrak{p}^{\prime}$ enthalten ist, so findet sich in $\mathfrak{p}^{\prime}$ auch $\eta=a-\eta^{\prime}$, so dab $\eta$ in $\mathfrak{p}$ und in $\mathfrak{p}^{\prime}$ enthalten ist. Wären nun $\mathfrak{p}$ und $\mathfrak{p}^{\prime}$ verschieden, so würde $[\eta]$, als durch $\mathfrak{p}$ und $\mathfrak{p}^{\prime}$ teilbar, auch durch $\mathfrak{p} \cdot \mathfrak{p}^{\prime}=[p]$ teilbar sein, d. h. $\eta$ hätte den Teiler $p$, was indessen nicht der Fall ist. $\left.{ }^{1}\right)$ Also sind $\mathfrak{p}$ und $\mathfrak{p}^{\prime}$ einander gleich.

Unter Zusammenfassung der Ergebnisse haben wir folgenden Satz: Ist $p$ eine rationale Primzahl, so ist das Hauptideal $[p]$ das Quadrat eines Primideals ersten Grades, falls $p$ in $D$ aufgeht; dagegen ist $[p]$ für eine nicht-kritische rationale Primzahl $p$ das Produkt zweier verschiedener konjugierter Primideale ersten Grades oder ein Primideal zweiten Grades, je nachdem die Grundzahl $D$ quadratischer Rest oder Nichtrest von $4 p$ ist.

Auf Grund dieses Satzes bestätigen sich die Angaben von S. $98 \mathrm{ff}$. über die Primidealzerlegung von [3] und [7] in dem damals betrachteten quadratischen Körper $\Re$ der Grundzahl $D=-20$.

\section{$\$ 17$. Gegen ein Ideal $\mathfrak{a}$ teilerfremde Zahlklassen.}

Der soeben für quadratische Körper ausgesprochene Satz ist bei einer Untersuchung zu verwenden, die sich zunächst auf einen beliebigen Körper $\Re$ vom $n^{\text {ten }}$ Grade bezieht. Von einer ganzen Zahl $\eta$ dieses Körpers sagt man, sie habe mit einem Ideal $\mathfrak{a}$ desselben den größten gemeinsamen Teiler $D$, wenn $[\eta]$ und $\mathfrak{a}$ das Ideal $b$ als größten gemeinsamen Teiler haben. Ist $\mathfrak{b}=\mathfrak{e}$, so heißt $\eta$ zu a teilerfremd. Gilt $\eta^{\prime} \equiv \eta(\bmod \mathfrak{a})$, so haben auch $\eta^{\prime}$ und a den größten gemeinsamen Teiler $\delta$. Da nämlich $\eta$ sowie alle Zahlen von $\mathfrak{a}$ in $\delta$ enthalten sind, und da andrerseits $\left(\eta^{\prime}-\eta\right)$ dem Ideal $\mathfrak{a}$ und also auch $\mathfrak{b}$ angehört, so ist auch $\eta^{\prime}=\eta+\left(\eta^{\prime}-\eta\right)$ in $\delta$ enthalten, so daB $\delta$ ein Teiler von $\left[\eta^{\prime}\right]$ ist. Nennen wir nun $\delta^{\prime}$ den größten gemeinsamen Teiler von $\eta^{\prime}$ und $\mathfrak{a}$, so ist $\mathfrak{b}$ Teiler von $\mathfrak{b}^{\prime}$. Kehren wir diese Betrachtung um, indem wir an $\eta^{\prime}$ und $\delta^{\prime}$ statt an $\eta$ und $\delta$ anknüpfen, so findet sich in derselben Weise, daß $\delta^{\prime}$ Teiler von $\downarrow$ ist. Also

1) S. die vorige Note. 
ist $\mathfrak{b}^{\prime}=\mathfrak{b}:$ Alle Zahlen der einzelnen der $N(\mathfrak{a}) \bmod \mathfrak{a}$ inkongruenten Zahlklassen von e haben mit a einen und denselben größten gemeinsamen Teiler.

Es soll nun festgestellt werden, wie viele unter den $N(\mathfrak{a}) \bmod \mathfrak{a}$ inkongruenten Zahlklassen teilerfremd gegen $\mathfrak{a}$ sind. Die Anzahl dieser Klassen bezeichnen wir durch das Symbol $\psi(\mathfrak{a})$ im Anschluß an das bekannte Symbol $\varphi(m)$ der rationalen Zahlentheorie, das für eine rationale ganze positive Zahl $m$ die Anzahl der $\bmod m$ inkongruenten und zu $m$ teilerfremden Zahlklassen darstellt.

Das Symbol $\psi(\mathfrak{a})$ hat die folgende Eigenschaft: Sind $\mathfrak{a}$ und $\mathfrak{b}$ teilerfremde Ideale, so gilt $\psi(\mathfrak{a} \cdot \mathfrak{b})=\psi(\mathfrak{a}) \cdot \psi(\mathfrak{b})$. Da nämlich der größte gemeinsame Teiler von $\mathfrak{a}$ und $\mathfrak{b}$ gleich $\mathfrak{e}$ ist, so gibt es (vgl. S. 96) eine Zahl $\alpha$ in $\mathfrak{a}$ und eine Zahl $\beta$ in $\mathfrak{b}$, deren Summe $\alpha+\beta=1$ ist. Hieraus folgt $\alpha \equiv 1(\bmod \mathfrak{b})$ und $\beta \equiv 1(\bmod \mathfrak{a})$; die in $\mathfrak{a}$ enthaltene Zahl $\alpha$ ist also teilerfremd gegen $\mathfrak{b}$, und die in $\mathfrak{b}$ enthaltene Zahl $\beta$ ist teilerfremd gegen a. Es mögen nun die Zahlen $r_{1}, r_{2}, \ldots$ ein Repräsentantensystem der $N(\mathfrak{a}) \bmod \mathfrak{a}$ inkongruenten Klassen bilden und die $s_{1}, s_{2}, \ldots$ ein solches für die $N(\mathfrak{b}) \bmod \mathfrak{b}$ inkongruenten Klassen. Wir haben dann in:

$$
t_{i, k}=r_{i} \beta+s_{k} \alpha
$$

ein System von $N(\mathfrak{a}) \cdot N(\mathfrak{b})=N(\mathfrak{a} \cdot \mathfrak{b})$ Zahlen, das ein Repräsentantensystem der $N(\mathfrak{a} \cdot \mathfrak{b}) \bmod \mathfrak{a} \cdot \mathfrak{b}$ inkongruenten Klassen bildet. Es ist nämlich leicht zu zeigen, daß keine zwei verschiedenen Zahlen (1) $\bmod \mathfrak{a} \cdot \mathfrak{b}$ kongruent sind. Soll aber:

$$
r^{\prime} \beta+s^{\prime} \alpha \equiv r \beta+s \alpha \quad(\bmod \mathfrak{a} \cdot \mathfrak{b})
$$

gelten, so folgt, da $\alpha \equiv 0(\bmod \mathfrak{a})$ ist:

$$
\left(\boldsymbol{r}^{\prime}-r\right) \boldsymbol{\beta} \equiv 0 \quad(\bmod \mathfrak{a}) .
$$

Da nun $\beta$ teilerfremd gegen $\mathfrak{a}$ ist, so ist $\left[r^{\prime}-r\right]$ teilbar durch $\mathfrak{a}$, woraus $r^{\prime} \equiv r(\bmod a)$ und also $r^{\prime}=r$ folgt. In derselben Weise folgt $s^{\prime}=s$, so daß die Behauptung über die Zahlen (1) zutrifft. Soll jetzt die Zahl (1) teilerfremd zu $\mathfrak{a} \cdot \mathfrak{b}$ sein, so ist hierzu notwendig und hinreichend, daB $\boldsymbol{r}_{\boldsymbol{i}}$ teilerfremd zu $\mathfrak{a}$ und $s_{k}$ teilerfremd zu $\mathfrak{b}$ ist. Da nämlich $\alpha \equiv 0(\bmod \mathfrak{a})$ gilt und $\beta$ teilerfremd $\mathrm{zu} \mathfrak{a}$ ist, so ist der gröBte gemeinsame Teiler von $r_{i} \beta$ und also von $t_{i k}$ und dem Ideale $\mathfrak{a}$ derjenige von $r_{i}$ und $\mathfrak{a}$. Wir erhalten also alle gegen $\mathfrak{a} \cdot \mathfrak{b}$ teilerfremden Repräsentanten (1), wenn wir $r_{i}$ auf die $\psi(\mathfrak{a})$ gegen $\mathfrak{a}$ teilerfremden Repräsentanten $r$ und $s_{k}$ auf die $\psi(\mathfrak{b})$ gegen $\mathfrak{b}$ teilerfremden Repräsentanten $s$ beschränken. Damit ist die Regel $\psi(\mathfrak{a} \cdot \mathfrak{b})=\psi(\mathfrak{a}) \cdot \psi(\mathfrak{b})$ bewiesen.

Die Primidealzerlegung von $\mathfrak{a}$ sei (unter Zusammenfassung gleicher Faktoren zu Potenzen):

$$
\mathfrak{a}=\mathfrak{p}_{1}^{v_{1}} \cdot \mathfrak{p}_{2}^{v_{2}} \cdot \mathfrak{p}_{3}^{v_{3}} \cdots
$$


Aus der eben bewiesenen Regel folgt dann:

$$
\psi(\mathfrak{a})=\psi\left(\mathfrak{p}_{1}^{v_{2}}\right) \cdot \psi\left(\mathfrak{p}_{2}^{v_{2}}\right) \cdot \psi\left(\mathfrak{p}_{3}^{v_{2}}\right) \cdots
$$

so daB nur noch die Anzahl $\psi\left(p^{v}\right)$ für eine Primidealpotenz $p^{v}$ zu bestimmen ist. $\mathrm{Zu}$ diesem $\mathrm{Z}$ wecke zerlegen wir $\mathfrak{e}$ in die $N(\mathfrak{p})$ mod $\mathfrak{p}$ inkongruenten Klassen, die wir durch die Zahlen $\varrho_{1}, \varrho_{2}, \ldots$ repräsentieren. Wir zerlegen sodann erneut alle durch $\mathfrak{p}$ teilbaren Zahlen ${ }^{1}$ ), die eine der eben abgetrennten Klassen bilden, bezüglich des Moduls $\mathfrak{p}^{v}$ in Klassen, deren Anzahl wir durch das Symbol $(\mathfrak{p}, \nu)$ bezeichnen, und die wir durch $\sigma_{1}$, $\sigma_{2}, \ldots$ repräsentieren. Bildet man nun die $(p, \nu) \cdot N(p)$ Summen $\left(\varrho_{i}+\sigma_{k}\right)$, so zeigt sich, da $B$ keine zwei verschiedene von diesen Summen mod $\mathfrak{p}^{v}$ kongruent sind, daß aber jede Zahl $\eta$ aus $e$ mit einer der Summen mod $\mathfrak{p}^{\nu}$ kongruent ist, woraus sich die Regel ergibt:

$$
N\left(\mathfrak{p}^{\nu}\right)=(\mathfrak{p}, \boldsymbol{v}) \cdot N(\mathfrak{p}) .
$$

Aus $\varrho^{\prime}+\sigma^{\prime} \equiv \varrho+\sigma\left(\bmod \mathfrak{p}^{2}\right)$ folgt nämlich $\varrho^{\prime} \equiv \varrho(\bmod \mathfrak{p})$ und damit $\rho^{\prime}=\rho$ sowie $\sigma^{\prime} \equiv \sigma\left(\bmod p^{2}\right)$. Diese letzte Kongruenz ergibt dann sofort auch $\sigma^{\prime}=\sigma$. Andrerseits ist irgendeine ganze Zahl $\eta \bmod \mathfrak{p}$ mit einem bestimmten $\varrho$ kongruent, und weiter ist die durch $\mathfrak{p}$ teilbare Zahl $(\eta-\varrho)$ $\bmod \mathfrak{p}^{v}$ mit einem bestimmten $\sigma$ kongruent, woraus $\eta \equiv \varrho+\sigma\left(\bmod \mathfrak{p}^{2}\right)$ folgt. Damit ist die Regel (5) sichergestellt.

Da nun $N\left(\mathfrak{p}^{v}\right)=(N(\mathfrak{p}))^{v}$ ist, so folgt $(\mathfrak{p}, v)=(N(\mathfrak{p}))^{v-1}$. Alle $N\left(\mathfrak{p}^{v}\right)$ $\bmod \mathfrak{p}^{v}$ inkongruenten Klassen setzen sich nun aus den $\psi\left(\mathfrak{p}^{v}\right)$ gegen $\mathfrak{p}^{v}$ teilerfremden und den $(\mathfrak{p}, \boldsymbol{v})$ Klassen der durch $\mathfrak{p}$ teilbaren Zahlen zusammen. Also ist:

$$
\begin{aligned}
& N\left(\mathfrak{p}^{v}\right)=\psi\left(\mathfrak{p}^{v}\right)+(\mathfrak{p}, \boldsymbol{v})=\psi\left(\mathfrak{p}^{\nu}\right)+N\left(\mathfrak{p}^{v-1}\right), \\
& \psi\left(\mathfrak{p}^{v}\right)=N\left(\mathfrak{p}^{v}\right)-N\left(\mathfrak{p}^{v-1}\right)=N\left(\mathfrak{p}^{v}\right)\left(1-\frac{1}{N(\mathfrak{p})}\right) .
\end{aligned}
$$

Bei Rückgang auf die Gleichung (4) ergibt sich damit der Satz: Die Anzahl $\psi(\mathfrak{a})$ der mod $\mathfrak{a}$ inkongruenten und gegen a teilerfremden Zahlklassen von e ist gegeben durch:

$$
\psi(\mathfrak{a})=N(\mathfrak{a}) \prod_{i} T\left(1-\frac{1}{N\left(\mathfrak{p}_{i}\right)}\right),
$$

wo sich das Produkt auf alle unterschiedenen in a aufgehenden Primideale bezieht.

Die Formel (6) soll jetzt für einen quadratischen Körper $\Re$ und ein in $\Omega$ enthaltenes Hauptideal $\mathfrak{a}=[a]$ mit rationaler ganzer positiver Zahl $a$ spezialisiert werden. Die rationalen Primfaktoren von $a$, die etwa kritische Primzahlen des Körpers $\Omega$ sind und also in der Grundzahl $D$ des

1) Es darf als selbstverständlich gelten, daB eine Zahl $\eta$ durch ein Ideal teilbar heißt, wenn $[\eta]$ durch dieses Ideal teilbar ist, d. h. also wenn $\eta$ im Ideal enthalten ist. 
Körpers aufgehen, sollen durch $p_{0}$ bezeichnet werden; die übrigen rationalen Primfaktoren von $a$ mögen $p_{1}$ oder $p_{2}$ heißen, je nachdem $D$ quadratischer Rest oder Nichtrest von $4 p$ ist. Zur Unterscheidung dieser drei Fälle bedient man sich einer Verallgemeinerung des Legendre-Jacobischen Zeichens. Man versteht für eine rationale Primzahl $p$ unter dem Symbole $(D, p)$ die Zshlen $0,+1$ oder -1 , je nachdem $p$ eine Zahl $p_{0}$ $p_{1}$ oder $p_{2}$ ist:

$$
\left(D, p_{0}\right)=0, \quad\left(D, p_{1}\right)=+1, \quad\left(D, p_{2}\right)=-1 .
$$

Nach S. 115 ist nun im quadratischen Körper $\Re$ das Hauptideal [ $\left.p_{0}\right]$ das Quadrat eines Primideals $\mathfrak{p}_{0}$ ersten Grades, während ein Hauptideal $\left[p_{1}\right]$ das Produkt zweier verschiedenen konjugierten Primideale $\mathfrak{p}_{1}, \mathfrak{p}_{1}^{\prime}$ ersten Grades ist und ein Hauptideal $\left[p_{2}\right]$ selbst ein Primideal zweiten Grades darstellt. Für die Normen gelten also die Regeln:

$$
N\left(\mathfrak{p}_{0}\right)=p_{0}, \quad N\left(\mathfrak{p}_{1}\right)=N\left(\mathfrak{p}_{1}^{\prime}\right)=p_{1}, \quad N\left(\left[p_{2}\right]\right)=p_{2}^{2} .
$$

Da $N([a])=a^{2}$ ist, so nimmt die Regel (6) hier die Gestalt an:

$$
\psi([a])=a^{2} \prod\left(1-\frac{1}{p_{0}}\right) \cdot \prod\left(1-\frac{1}{p_{1}}\right)^{2} \cdot \prod\left(1-\frac{1}{p_{2}^{2}}\right),
$$

wo sich die Produkte auf die verschiedenen rationalen Primzahlen der einzelnen der drei Arten, die in $a$ enthalten sind, beziehen.

Die Gleichung (8) kann mittelst des schon oben erwähnten Zeichens $\varphi(a)$ aus der rationalen Zahlentheorie vereinfacht werden. Bekanntlich ist:

$$
\varphi(a)=a \prod\left(1-\frac{1}{p}\right)
$$

wo sich das Produkt auf alle verschiedenen in $a$ aufgehenden rationalen Primzahlen bezieht. Da wir die Gleichung (9) auch:

$$
\varphi(a)=a \prod\left(1-\frac{1}{p_{0}}\right) \cdot \prod\left(1-\frac{1}{p_{1}}\right) \cdot \prod\left(1-\frac{1}{p_{2}}\right)
$$

schreiben können, so kann die Gleichung (8) in die Gestalt:

$$
\psi([a])=\varphi(a) \cdot a \cdot \prod\left(1-\frac{1}{p_{1}}\right) \cdot \prod\left(1+\frac{1}{p_{2}}\right)
$$

gesetzt werden. Ziehen wir also das in (7) erklärte Zeichen heran, so ergibt sich der Satz: Ist a eine rationale ganze positive Zahl, so sind von den $N([a])=a^{2} \bmod [a]$ inkongruenten Zahlklassen, in die das System e der ganzen Zahlen des quadratischen Körpers $\Re$ zerfällt, im ganzen:

$$
\psi([a])=\varphi(a) \cdot a \prod\left(1-\frac{(D, p)}{p}\right)
$$

Klassen gegen a teilerfremd, wo sich das Produkt auf alle verschiedenen, in a aufgehenden rationalen Primzahlen bezieht. 


\section{§18. Satz über die zu einem gegebenen Ideale teilerfremden Ideale.}

Es seien $\mathfrak{a}$ und $\mathfrak{c}$ irgend zwei Ideale des Körpers $\Re$. Alle in $\mathfrak{a}$ und $c$ zugleich enthaltenen Zahlen, zu denen jedenfalls alle Zahlen des Ideals $\mathfrak{a} \cdot \mathfrak{c}$ gehören, bilden offenbar wieder ein Ideal $\mathfrak{m}$, das als das ,kleinste gemeinschaftliche Vielfache" oder "Multiplum" von a und c bezeichnet wird. Die Benennung rechtfertigt sich dadurch, daß $m$ als Teiler in jedem gemeinschaftlichen Vielfachen von $\mathfrak{a}$ und $\mathfrak{c}$ enthalten ist. Sind für $\mathfrak{a}$ und $\mathfrak{c}$ die Primidealzerlegungen bekannt, so kann man diejenige von $\mathfrak{m}$ genau in derselben Art herstellen, wie man für zwei in ibre Primfaktoren zerlegte rationale ganze Zahlen $a$ und $c$ die Primfaktorenzerlegung ihres kleinsten gemeinsamen Multiplums $m$ gewinnt.

Der Begriff des kleinsten gemeinschaftlichen Vielfachen zweier Ideale $a$ und $c$ kommt beim Beweise des folgenden Satzes zur Geltung ${ }^{1}$ ): Ist ein Ideal a durch keines der endlich vielen Ideale $\mathfrak{c}_{1}, \mathfrak{c}_{2}, \ldots, \mathfrak{c}_{v}$ teilbar, so ist in a eine Zahl $\alpha$ nachweisbar, die in keinem der $v$ Ideale $\mathfrak{c}$ enthalten ist. $^{2}$ ) Ist $\nu=1$, so ist der Satz richtig, da $c$ ein Teiler von a wäre, falls alle Zahlen von $\mathfrak{a}$ in $\mathfrak{c}$ enthalten wären. Der allgemeine Beweis des Satzes kann weiter durch vollständige Induktion geführt werden. Wir nehmen an, der Satz sei richtig, falls die Anzahl der Ideale $c$ kleiner als $\nu$ ist, und beweisen, daB er dann auch noch für die Anzahl $\nu$ gilt.

Das kleinste gemeinschaftliche Vielfache von $\mathfrak{a}$ und $\mathfrak{c}_{i}$ sei $\mathfrak{m}_{i}=\mathfrak{a} \cdot \mathfrak{b}_{i}$, wo $\mathfrak{b}_{i}$ ein bestimmtes von $e$ verschiedenes Ideal ist. ${ }^{3}$ ) Ist die Zahl $\alpha$ von $\mathfrak{a}$ nicht in $\mathfrak{m}_{\mathfrak{i}}$ enthalten, so ist sie auch nicht in $\mathfrak{c}_{i}$ enthalten, da $\mathfrak{m}_{i}$ aus allen $\mathfrak{a}$ und $\mathfrak{c}_{i}$ gemeinsamen Zahlen besteht. Es genügt demnach in $\mathfrak{a}$ eine Zahl $\alpha$ nachzuweisen, die in keinem der Ideale $\mathfrak{m}_{1}, \mathfrak{m}_{2}, \ldots, \mathfrak{m}_{v}$ enthalten ist. Es mögen sich nun erstlich unter den Idealen $\mathfrak{b}_{1}, \mathfrak{b}_{2}, \ldots, \mathfrak{b}_{v}$ zwei finden, die nicht teilerfremd sind. Wir stellen sie an den Schluß der Reihe und nennen $\mathfrak{b}_{v-1}^{\prime}$ den größten gemeinsamen Teiler von $\mathfrak{b}_{v-1}$ und $\mathfrak{b}_{v}$, der dann also von $e$ verschieden sein wird. In $\mathfrak{m}_{1}=\mathfrak{a} \cdot \mathfrak{b}_{1}, \ldots, \mathfrak{m}_{v-2}=\mathfrak{a} \cdot \mathfrak{b}_{v-2}, \mathfrak{m}_{v-1}^{\prime}=\mathfrak{a} \mathfrak{b}_{v-1}^{\prime}$ haben wir dann $(\nu-1)$ Ideale, von denen keines in $\mathfrak{a}$ aufgeht. Der Annahme zufolge gibt es demnach in $\mathfrak{a}$ eine Zahl $\alpha$, die in keinem der Ideale $\mathfrak{m}_{1}, \mathfrak{m}_{2}, \ldots, \mathfrak{m}_{v-2}, \mathfrak{m}_{v-1}^{\prime}$ enthalten ist. Da aber $\mathfrak{m}_{v-1}^{\prime}$ sowohl $\mathfrak{m}_{v-1}$ als $\mathfrak{m}_{v}$ teilt, so ist $\alpha$ auch nicht in $\mathfrak{m}_{v-1}$ und $\mathfrak{m}_{v}$ enthalten, so daB in diesem Falle unser Satz bewiesen ist.

Es braucht jetzt nur noch die Möglichkeit betrachtet zu werden, daB je zwei unter den $\nu$ Idealen $\mathfrak{b}_{1}, \mathfrak{b}_{2}, \ldots, \mathfrak{b}_{v}$ teilerfremd sind. Dann ist

1) S. hierzu Dedekind in Dirichlets "Vorlesungen über Zahlentheorie“" (4. Aufl.), S. $558 \mathrm{ff}$.

2) Ist $\mathfrak{a}=[\alpha]$ ein Hauptideal, so ist der Satz einleuchtend, da in diesem Falle $\alpha$ der Voraussetzung nach in keinem der Ideale $c$ enthalten ist.

3) Wäre $\mathfrak{b}_{i}=\mathrm{e}$, so wäre $\mathfrak{a}\left(=\mathfrak{m}_{i}\right)$ durch $\mathfrak{c}_{i}$ teilbar, entgegen der Voraussetzung. 
unser Satz aber leicht direkt beweisbar. Ist nämlich $\mathfrak{b}_{i}^{\prime}$ das Produkt der Ideale $\mathfrak{b}$, abgesehen von $\mathfrak{b}_{i}$, so ist $\mathfrak{b}_{i}^{\prime}$ nicht durch das von $e$ verschiedene Ideal $\mathfrak{b}_{i}$ teilbar, und also ist auch $\mathfrak{a} \cdot \mathfrak{b}_{i}^{\prime}$ nicht teilbar durch $\mathfrak{a} \cdot \mathfrak{b}_{i}$. Es gibt demnach in $\mathfrak{a} \cdot \mathfrak{b}_{i}^{\prime}$ eine Zahl $\alpha_{i}$, die nicht in $\mathfrak{a} \cdot \mathfrak{b}_{i}$ enthalten ist. Die $\nu$ so zu erklärenden Zahlen $\alpha_{1}, \alpha_{2}, \ldots, \alpha_{v}$ sind aber alle in $a$ enthalten, und dasselbe gilt also von ibrer Summe:

$$
\alpha=\alpha_{1}+\alpha_{2}+\cdots+\alpha_{v} .
$$

Für diese Zahl $\alpha$ von $\mathfrak{a}$ aber finden wir $\alpha \equiv \alpha_{i}\left(\bmod \mathfrak{a} \cdot \mathfrak{b}_{i}\right)$, da alle $\alpha_{k}$ mit $k \neq i$ zufolge der Erklärung von $\mathfrak{b}_{k}^{\prime}$ in dem Ideale $\mathfrak{a} \cdot \mathfrak{b}_{i}$ enthalten sind. $\left.{ }^{1}\right)$ Die Zahl $\alpha_{i}$ ist indessen nicht in $\mathfrak{a} \cdot \mathfrak{b}_{i}$ enthalten, und also gehört auch $\alpha$ dem Ideale $\mathfrak{m}_{i}=\mathfrak{a} \cdot \mathfrak{b}_{i}$ nicht an. Hiermit ist unser Satz vollständig bewiesen.

Aus dem aufgestellten Satze ziehen wir nun eine Folgerung, die später grundsätzliche Bedeutung erlangt. Zunächst ist folgender Satz zu nennen: Sind $\mathfrak{a}$ und $\mathfrak{b}$ irgend zwei Ideale des Körpers $\mathfrak{R}$, so kann man eine von 0 verschiedene Zahl $\alpha$ aus $\mathfrak{a}$ so auswählen, daß die beiden Ideale $\mathfrak{a} \cdot \mathfrak{b}$ und $[\alpha]$ das Ideal $\mathfrak{a}$ selbst als größten gemë̈nschaftlichen Teiler haben. Wir verstehen nämlich unter $\mathfrak{c}_{1}, \mathfrak{c}_{2}, \ldots, \mathfrak{c}_{v}$ alle endlich vielen Teiler von $\mathfrak{a} \cdot \mathfrak{b}$, die zugleich $\mathfrak{a}$ als Teiler enthalten, aber von $\mathfrak{a}$ selbst verschieden sind. Dann geht keines der Ideale $\mathfrak{c}$ in $\mathfrak{a}$ auf, und wir können also aus $\mathfrak{a}$ eine Zahl $\alpha$ wählen, die in keinem der Ideale $c$ enthalten ist. Der gröBte gemeinschaftliche Teiler von $\mathfrak{a} \cdot \mathfrak{b}$ und $[\alpha]$ hat das Ideal $\mathfrak{a}$, das sowohl in $\mathfrak{a} \cdot \mathfrak{b}$ als in $[\alpha]$ aufgeht, zum Teiler und geht seinerseits in $\mathfrak{a} \cdot \mathfrak{b}$ auf. Dieser größte gemeinschaftliche Teiler $m u B$ also entweder eines der Ideale $c$ sein oder stellt das Ideal $\mathfrak{a}$ vor. Hiervon bleibt aber nur die zweite Möglichkeit, da $\alpha$ in keinem der Ideale $c$ enthalten ist. Der ausgesprochene Satz ist also richtig.

Durch Vermittlung dieses Satzes gelangen wir nun zu dem schon genannten, für später wichtigen Ergebnisse. Das Hauptideal $[\alpha]$ als durch $\mathfrak{a}$ teilbar, kann als Produkt $[\alpha]=\mathfrak{a} \cdot \mathfrak{a}^{\prime}$ dargestellt werden. Aus der Tatsache, da $\mathfrak{B} \mathfrak{a} \cdot \mathfrak{a}^{\prime}$ und $\mathfrak{a} \cdot \mathfrak{b}$ den gröBten gemeinschaftlichen Teiler $\mathfrak{a}$ haben, folgt, da $\mathfrak{a} \mathfrak{a}^{\prime}$ und $\mathfrak{b}$ teilerfremd sind. Man kann also aus dem Ideale $\mathfrak{a}$ ein Hauptideal stets durch Multiplikation mit einem solchen Ideale $\mathfrak{a}^{\prime}$ herstellen, das teilerfremd gegen ein beliebiges Ideal $\mathfrak{b}$ ist. In der zur Idealklasse von $\mathfrak{a}$ inversen Klasse gibt es demnach stets ein Ideal $\mathfrak{a}^{\prime}$, das teilerfremd $\mathrm{zu} \mathfrak{b}$ ist. Bei der freien Wahl der Ideale $\mathfrak{a}$ und $\mathfrak{b}$ sind wir damit zu dem aufzustellenden Hauptsatze gelangt: In jeder Idealklasse des Körpers $\boldsymbol{\Omega}$ gibt es Ideale, die zu einem beliebig vorgeschriebenen Ideale $\mathfrak{b}$ des Körpers teilerfremd sind.

1) $\alpha_{k}$ ist in $\mathfrak{a} \cdot \mathfrak{b}_{k}^{\prime}$ und also im Teiler $\mathfrak{a} \cdot \mathfrak{b}_{i}$ von $\mathfrak{a} \cdot \mathfrak{b}_{k}^{\prime}$ enthalten. 


\section{Quadratische Körper und Formen negativer Diskriminante. $\left.{ }^{1}\right)$}

\section{\$1. Zweige und Zweigideale im quadratischen Körper $\Re$.}

In einer nahen Beziehung zur Theorie der elliptischen Funktionen stehen die quadratischen Körper negativer Grundzahl oder Diskriminante D. $\left.{ }^{2}\right)$ Über diese Körper $\AA$ sind demnach noch etwas weitergehende Untersuchungen anzustellen. Nach S. 113 ist die Diskriminante $\mathbf{D}=d$ oder $=4 d$, je nachdem die von quadratischen Teilern freie, rationale ganze negative Zahl $d \equiv 1(\bmod 4)$ ist oder einer der Zahlen 2, $3 \bmod 4$ kongruent ist. Das System $\mathfrak{e}$ der ganzen Zahlen von $\Omega$ stellen wir hinfort in der Basis 1, $\Theta$ dar, wo:

$$
\Theta=\frac{-1+\sqrt{D}}{2}=\frac{-1+i \sqrt{|D|}}{2} \text { oder } \theta=\frac{\sqrt{D}}{2}=\frac{i \sqrt{|D|}}{2}
$$

ist, je nachdem $\mathbf{D}=d$ oder $=4 d$ gilt. Offenbar gilt der Satz: Die Diskriminante D ist durch kein ungerades Quadrat teilbar; es ist entweder $\mathbf{D} \equiv 1$ oder $\equiv 0(\bmod 4)$, und in letzterem Falle gilt genauer $\mathbf{D} \equiv 8$ oder $\equiv 12(\bmod 16)$.

Für eine Einheit $\varepsilon=e_{0}+e_{1} \Theta$ von $\mathfrak{\Omega}$ gilt, wenn $\varepsilon^{\prime}$ und $\Theta^{\prime}$ die zu $\varepsilon$ und $\Theta$ konjugierten Zahlen sind:

$$
\varepsilon \cdot \varepsilon^{\prime}=\left(e_{0}+e_{1} \Theta\right)\left(e_{0}+e_{1} \Theta^{\prime}\right)=e_{0}^{2}+e_{1}^{2} \Theta \Theta^{\prime}+e_{0} e_{1}\left(\Theta+\Theta^{\prime}\right)=1 \text {. }
$$

Durch Eintragung der Ausdrücke (1) von $\Theta$ und der entsprechenden Ausdrücke von $\Theta^{\prime}$ zeigt man leicht den Satz: Der Körper der Diskriminante $\mathbf{D}=-3$ hat die sechs Einheiten $\varepsilon= \pm 1, \pm \varrho, \pm \rho^{2}$, unter $\rho$ die dritte Einheitswurzel $\varrho=\frac{-1+i \sqrt{3}}{2}$ verstanden, derjenige der Diskriminante $\mathbf{D}=-4$ hat die vier Einheiten $\varepsilon= \pm 1, \pm i$, alle übrigen haben nur die beiden Einheiten $\varepsilon= \pm 1$.

1) Den folgenden Entwicklungen liegt vornehmlich die Schrift Dedekinds "Über die Anzahl der Idealklassen in den verschiedenen Ordnungen eines endlichen Körpers" (Braunschweig 1877) zugrunde. Die Theorie der quadratischen Formen im geometrischen Gewande and die Beziehungen dieser Theorie zu den elliptischen Funktionen behandelte Klein in seinen (autographierten) Vorlesungen "Ausgewählte Kapitel der Zahlentheorie I und II" (Göttingen 1896 und 1897).

2) Wir bevorzugen fortan die Benennung „Diskriminante“ für D, da diese in der Theorie der quadratischen Formen die übliche ist. Übrigens werden die Diskriminanten D der Körper $\Re$ weiterhin durch Fettdruck hervorgehoben, damit wir die Bèzeichnung $\boldsymbol{D}$ in anderem Sinne verwenden können. 
Wir sondern jetzt aus dem Systeme $e$ aller ganzen Zahlen $\left(e_{0}+e_{1} \Theta\right)$ von $\Omega$ das System $e_{n}$ aller Zahlen aus, bei denen $e_{1}$ durch eine vorgeschriebene rationale ganze positive Zahl $n$ teilbar ist. Dieses Zahlsystem $\mathrm{e}_{n}$, bestehend aus allen in der Gestalt $\left(e_{0}+e_{1} n \Theta\right)$ wieder mit rationalen ganzen $e$ darstellbaren Zahlen von $\mathfrak{R}$, bezeichnen wir als einen "Zweig" von e und nennen $n$ den "Grad" des Zweiges, sprechen auch kurz vom „n $n^{\text {ten }}$ Zueige" $\mathrm{e}_{n}$ des Systems e.

Es gilt der Satz: Die Summe, die Differenz und das Produkt zweier Zahlen des Zweiges $e_{n}$ liefern stets wieder Zahlen dieses Zweiges. ${ }^{1}$ )

Für $n=1$ erhalten wir als ,ersten Zweig" das System e selbst; dieses System, dem alle übrigen Zweige angehören, soll demnach auch als "Stamm" bezeichnet werden.

Die negative Zahl $D=n^{2} \mathrm{D}$ heiße die "Zueigdiskriminante", D selbst wird demgegenüber "Stammdisliriminante“ genannt." ) Ordnen wir die Stammdiskriminanten den Zweigdiskriminanten (nämlich für $n=1$ ) unter, so läßt sich jede rationale ganze negative $Z a h l D$, die $\equiv 1$ oder $0(\bmod 4)$ ist, als eine Zweigdiskriminante auffassen. Ist nämlich erstens $D \equiv 1$ (mod 4$)$, so verstehe man unter $n^{2}$ das größte in $D$ aufgehende (ungerade) Quadrat und setze $D=n^{2} \mathrm{D}=n^{2} d$. Dann ist $d$ eine von quadratischen Teilern freie Zahl, die $\equiv 1(\bmod 4)$ ist. Gilt aber $D \equiv 0(\bmod 4)$, so sei $n_{0}^{2}$ das größte in $D$ aufgehende ungerade Quadrat und $4^{v}$ die größte in $D$ aufgehende Potenz von 4 . Dann ist $4^{-v} \cdot n_{0}^{-2} \cdot D$ ganzzahlig und $\equiv 1,2$ oder $3(\bmod 4)$. Im ersten Falle setzen wir:

$$
n=2^{v} \cdot n_{0}, \quad D=n^{2} \mathrm{D}=n^{2} \cdot d,
$$

wo auch $d \equiv 1(\bmod 4)$ und von quadratischen Teilern frei ist. Im zweiten und dritten Falle schreiben wir:

$$
n=2^{v-1} \cdot n_{0}, \quad D=n^{2} \mathrm{~B}=n^{2} \cdot 4 d,
$$

wo $d$ wieder quadratfrei und $\equiv 2$ oder $\equiv 3(\bmod 4)$ ist.

Eine „Basis“ für $e_{n}$ haben wir in den Zahlen $1, n \Theta$, insofern $e_{n}$ gerade von allen Zahlen $\left(e_{0}+e_{1} \cdot n \Theta\right)$ mit irgendwelchen ganzzahligen $e$ gebildet wird. Besseren Anschluß an die oben für e gebrauchte Basis gewinnen wir, wenn wir statt $n \Theta$ die auf folgende Art zu erklärende Zahl $\theta$ heranziehen:

1) Dedekind bezeichnet ein Zahlsystem dieser Art als eine "Ordnung“ und nennt $n$ den „Führer" der Ordnung; Hilbert bedient sich der Bezeichnnng "Ring“" oder „Zahlring“.

2) Der Name „Stammdiskriminante" ist von W eber eingeführt, der Bezeichnung „Zweigdiskriminante" bedient sich $\mathrm{Klein}$ in seinen oben genannten Vorlesungen. 


$$
\left\{\begin{array}{ll}
\theta=n \Theta=\frac{\sqrt{D}}{2} & \text { für } D \equiv 0, \mathrm{D} \equiv 0 \\
\theta=\frac{n}{2}+n \Theta=\frac{\sqrt{D}}{2} & \text { für } D \equiv 0, \mathbf{D} \equiv 1 \\
\theta=\frac{n-1}{2}+n \Theta=\frac{-1+\sqrt{D}}{2} & \text { für } D \equiv 1, \mathbf{D} \equiv 1
\end{array}\right\}(\bmod 4) .
$$

Wir haben dann in allen Fällen (auch unter Einschluß von $n=1$ ) den Satz: Eine Basis von $\mathrm{e}_{n}$ wird durch die Zahlen 1, $\theta$ geliefert, wo:

$$
\theta=\frac{-1+\sqrt{D}}{2}=\frac{-1+i \sqrt{|D|}}{2} \text { oder } \theta=\frac{\sqrt{D}}{2}=i \frac{\sqrt{|D|}}{2}
$$

ist, je nachdem $D \equiv 1$ oder $\equiv 0(\bmod 4)$ gilt.

Die folgenden Entwicklungen beziehen sich auf Ideale $\mathfrak{a}$, die teilerfremd gegen das zum Grade $n$ des Zweiges $e_{n}$ gehörende Hauptideal [ $n$ ] sind. Alle Zahlen, die zugleich in $\mathfrak{a}$ und $e_{n}$ enthalten sind, bilden ein durch $\mathfrak{a}_{n}$ zu bezeichnendes System, das wir als den " $n^{\text {ten }}$ Zweig des Ideals

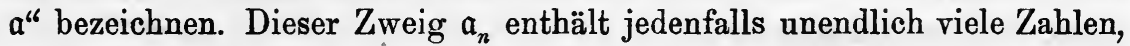
z. B. alle Zahlen des kleinsten gemeinschaftlichen Vielfachen von $\mathfrak{a}$ und [n]. Zwei Zahlen des $n^{\text {ten }}$ Zweiges $\mathfrak{a}_{n}$ geben, addiert oder subtrahiert, stets wieder Zahlen von $\mathfrak{a}_{n}$; denn die Summe und die Differenz sind wieder sowohl in $\mathfrak{a}$ als in $\mathfrak{e}_{n}$ enthalten. Ferner ist das Produkt einer beliebigen Zahl aus $e_{n}$ und einer solchen aus $\mathfrak{a}_{n}$ stets wieder in $\mathfrak{a}_{n}$ enthalten, da dieses Produkt eben auch wieder in $\mathfrak{a}$ und $e_{n}$ zugleich enthalten ist.

Eine dritte Eigenschaft von $\mathfrak{a}_{n}$ folgt aus dem Umstande, dab $\mathfrak{a}$ teilerfremd gegen $[n]$ ist. Der größte gemeinsame Teiler von $\mathfrak{a}$ und $[n]$ ist also e, so daB sich nach S. 96 jede Zahl von e als Summe einer Zahl $\alpha$ aus $\mathfrak{a}$ und einer Zahl $n \eta$ aus [n] darstellen läßt. Ist insbesondere für irgendeine Zahl $\eta_{n}$ aus $e_{n}$ diese Darstellung $\eta_{n}=\alpha+n \eta$, so gehört $\alpha=\eta_{n}-n \eta$ offenbar dem Zweige $e_{n}$ und also auch dem Zweige $\mathfrak{a}_{n}$ des Ideals $\mathfrak{a}$ an und mag demnach genauer durch $\alpha_{n}$ bezeichnet werden. Die Gleichung $\eta_{n}=\alpha_{n}+n \eta$ zeigt, daß jede Zahl des Zweiges $e_{n}$ als Summé einer Zahl aus $\mathfrak{a}_{n}$ und einer solchen aus $[n]$ darstellbar ist, eine Tatsache, die wir kurz durch die Aussage kennzeichnen, $\mathfrak{a}_{n}$ sei „teilerfremd“" $z u[n]$.

Als "Produkt" $\mathfrak{e} \cdot \mathfrak{a}_{n}$ von $\mathfrak{e}$ und $\mathfrak{a}_{n}$ erklären wir das System aller Zahlen, die als Produkte einer Zahl von $\mathfrak{e}$ und einer von $\mathfrak{a}_{n}$ oder als Summen solcher Produkte darstellbar sind (vgl. die Erklärung des Produktes zweier Ideale S. 93). Dann gilt der Satz: Das Produkt $\mathfrak{e} \cdot \mathfrak{a}_{n}$ von e und dem $n^{\text {ten }} Z$ Wweige des gegen $[n]$ teilerfremden Ideals a stellt wieder dieses Ideal a selbst dar. Zunächst überzeuge man sich, daß das Produkt $e \cdot \mathfrak{a}_{n}$ die drei Grundeigenschaften eines Ideals hat (S. 90). Alle Zahlen des Ideals $\mathfrak{e} \cdot \mathfrak{a}_{n}$ sind in $\mathfrak{a}$ enthalten, so $\operatorname{daB} \mathfrak{a}$ ein Teiler von $\mathfrak{e} \cdot \mathfrak{a}_{n}$ ist. Da ferner $a_{n}$ teilerfremd gegen $[n]$ ist, so besitzt die Zahl 1 , die in $e_{n}$ ent- 
halten ist, eine Darstellung:

$$
1=\alpha_{n}+n \eta \text {. }
$$

Ist jetzt $\alpha$ eine beliebige Zahl aus $\mathfrak{a}$, so folgt aus (4) die Gleichung $\alpha=\alpha \alpha_{n}+n \eta \alpha$. Das erste Glied dieser Summe $\alpha \cdot \alpha_{n}$ gehört dem Ideale $\mathfrak{e} \cdot \mathfrak{a}_{n}$ an. Die Zahl $n \eta \alpha$ ist in $\mathfrak{e}_{n}$ sowie als Produkt $(n \eta) \cdot \alpha$ in $\mathfrak{a}$, also in $\mathfrak{a}_{n}$ und damit auch in $\mathfrak{e} \cdot \mathfrak{a}_{n}$ enthalten. Demnach ist auch die Summe $\alpha$ der Zahlen $\alpha \cdot \alpha_{n}$ und $n \eta \alpha$ und also jede Zahl $\alpha$ von $\mathfrak{a}$ im Ideal $\mathfrak{e} \cdot \mathfrak{a}_{n}$ enthalten. Somit ist $\mathfrak{e} \cdot \mathfrak{a}_{n}$ ein Teiler von $\mathfrak{a}$. Da hiernach jedes der Ideale $\mathfrak{a}$ und $\mathfrak{e} \cdot \mathfrak{a}_{n}$ ein Teiler des anderen ist, so gilt in der Tat $\mathfrak{e} \cdot \mathfrak{a}_{n}=\mathfrak{a}$.

Die Zweige $\mathfrak{a}_{n}$ der zu $[n]$ teilerfremden Ideale spielen im Zweige $\mathfrak{e}_{n}$ offenbar dieselbe Rolle wie die Ideale a selbst im Stamme e. Wir bezeichnen die $\mathfrak{a}_{n}$ dieserhalb auch kurz als „Zweigideale“, genauer als „die zum Grade $n$ gehörenden Zweigideale" und nennen ihnen gegenüber die $\mathfrak{a}$ selbst „Stammideale“. Es ist sogar möglich, die Zweigideale unabhängig von den Stammidealen in folgender Art zu erklären: Ein System $\mathfrak{a}_{n}$ von Zahlen des $n^{\text {ten }}$ Zweiges $\mathfrak{e}_{n}$ soll ein "Zweigideal" heißen, wern es folgende Eigenschaften besitzt:

1. Die Summen und die Differenzen zweier Zahlen von $\mathfrak{a}_{n}$ sind wieder in $\mathfrak{a}_{n}$ enthalten.

2. Das Prodult einer Zahl von $\mathfrak{e}_{n}$ und einer von $\mathfrak{a}_{n}$ ist wieder in $\mathfrak{a}_{n}$ enthalten.

3. Das System $\mathfrak{a}_{n}$ ist gegen [n] teilerfremd, d. h. jede Zahl von $\mathfrak{e}_{n}$ ist als Summe einer Zahl aus $\mathfrak{a}_{n}$ und einer solchen aus $[n]$ darstellbar.

Die Eigenschaft 3. hat natürlich nur für $n>1$ Bedeutung. Sie ersetzt zugleich die bei einem Stammideale $\mathfrak{a}$ geforderte Bedingung 3 (S. 90), daß a nicht aus der Zahl 0 allein bestehen solle. Da nämlich für $n>1$ das Hauptideal $[n]$ den Zweig $\mathfrak{e}_{n}$ nicht erschöpft, kommen sicher in $\mathfrak{a}_{n}$ von 0 verschiedene Zahlen vor.

$\mathrm{Da} \mathfrak{B}$ die $n^{\text {ten }}$ Zweige $\mathfrak{a}_{n}$ der gegen $[n]$ teilerfremden Stammideale $\mathfrak{a}$ die drei Eigenschaften 1 . bis 3 . besitzen, wurde bereits gezeigt. DaB diese $n^{\text {ten }}$ Zweige aber auch alle den Bedingungen 1. bis 3. genügenden Zweigideale erschöpfen, geht aus folgendem Satze hervor: Ist $\mathfrak{a}_{n}$ ein der independenten Erklärung entsprechendes Zweigideal, so ist das wie oben zu erklärende Produkt $\mathfrak{e} \cdot \mathfrak{a}_{n}=\mathfrak{a}$ ein gegen [ $n$ ] teilerfremdes Stammideal, und $\mathfrak{a}_{n}$ ist der $n^{\text {te }} Z$ weig von $\mathfrak{a}$. Zunächst ist wieder einleuchtend, daß das Zahlsystem $\mathfrak{e} \cdot \mathfrak{a}_{n}$, das sich zusammensetzt aus allen Produkten einer Zahl aus $e$ und einer solchen aus $a_{n}$, sowie aus allen Summen solcher Produkte, ein Stammideal $\mathfrak{a}=\mathfrak{e} \cdot \mathfrak{a}_{n}$ bildet. Zufolge der Eigenschaft 3. von $\mathfrak{a}_{\mathfrak{n}}$ gibt es eine Darstellung (4) der Zahl 1, unter $\alpha_{n}$ eine Zabl des Zweigideals $\mathfrak{a}_{n}$ verstanden. Ist $\eta^{\prime}$ eine beliebige Zahl aus e, so folgt aus (4):

$$
\eta^{\prime}=\eta^{\prime} \alpha_{n}+n \eta \eta^{\prime},
$$


so daB jede Zahl $\eta^{\prime}$ als Summe einer in $e \cdot \mathfrak{a}_{n}=\mathfrak{a}$ enthaltenen Zahl $\eta^{\prime} \alpha_{n}$ und einer Zahl $n \eta \eta^{\prime}$ des Hauptideals [ $\left.n\right]$ darstellbar ist. Also ist das Stammideal $\mathfrak{a}=\mathfrak{e} \cdot \mathfrak{a}_{n}$ teilerfremd zu $[n]$. Ist endlich $\mathfrak{a}_{n}^{\prime}$ der $n^{\text {te }} Z_{\text {weig }}$ von $\mathfrak{a}=\mathfrak{e} \cdot \mathfrak{a}_{n}$, so haben wir noch die Gleichheit von $\mathfrak{a}_{n}^{\prime}$ mit $\mathfrak{a}_{n}$ zu zeigen. Da alle Zahlen von $\mathfrak{a}_{n}$ zugleich in $\mathfrak{a}=\mathfrak{e} \cdot \mathfrak{a}_{n}$ und $\mathfrak{e}_{n}$ enthalten sind, so gehören sie auch alle dem $Z$ weige $\mathfrak{a}_{n}^{\prime}$ von $\mathfrak{a}$ an. Ist andrerseits $\alpha_{n}^{\prime}$ eine beliebige Zahl des Zweiges $\mathfrak{a}_{n}^{\prime}$, so gibt es wegen der Eigenschaft 3. des Zweigideals $\mathfrak{a}_{n}$ eine Darstellung:

$$
\alpha_{n}^{\prime}=\alpha_{n}+n \eta,
$$

wo $\alpha_{n}$ dem Zweigideal $\mathfrak{a}_{n}$ angehört. Die Zahl $n \eta=\alpha_{n}^{\prime}-\alpha_{n}$, als Differenz zweier in $\mathfrak{a}$ enthaltenen Zahlen, findet sich gleichfalls in $\mathfrak{a}$, so daß das Ideal $[n \eta]=[n] \cdot[\eta]$ durch die beiden teilerfremden Ideale $[n]$ und $\mathfrak{a}$, also auch durch $[n] \cdot \mathfrak{a}$ teilbar ist. Nun gewinnt man die Zahlen von $[n] \cdot \mathfrak{a}=[n] \cdot\left(\mathfrak{e} \cdot \mathfrak{a}_{\mathfrak{n}}\right)$, indem man die Zahlen von $\mathfrak{a}_{n}$ mit denen von $[n] \cdot e=[n]$ multipliziert und alle Summen solcher Produkte hinzufügt. Alle so erhaltenen Zahlen sind, da die Zahlen von [n] in $\mathrm{e}_{n}$ enthalten sind, zufolge der Eigenschaften 2. und 1. des Zweigideales $\mathfrak{a}_{n}$ in diesem enthalten. Dies gilt also auch von der Zahl $n \eta$ selbst, und mit $\alpha_{n}$ und $n \eta$ gehört zufolge (5) auch $\alpha_{n}^{\prime}$ dem Zweigideale $\mathfrak{a}_{n}$ an. Alle Zahlen des Zweiges $\mathfrak{a}_{n}^{\prime}$ sind demnach im Zweigideale $\mathfrak{a}_{n}$ enthalten, so daß die Gleichheit von $\mathfrak{a}_{n}^{\prime}$ und $\mathfrak{a}_{n}$ jetzt feststeht. Wir haben somit den Satz gewonnen: Die gegen $[n]$ teilerfremden Stammideale a sind den Ziweigidealen $\mathfrak{a}_{n}$ des $n^{\text {ten }}$ Zweiges $\mathrm{e}_{n}$ umliehrbar eindeutig zugeordnet, indem einerseits $\mathfrak{a}_{n}$ der $n^{\text {te }}$ Zweig von $\mathfrak{a}$ ist und andrerseits $\mathfrak{a}$ das Produlit $\mathfrak{e} \cdot \mathfrak{a}_{n}$ darstellt.

\section{§ 2. Zahlstrahlen im quadratischen Körper.}

Um die Äquivalenz der Zweigideale behandeln zu können, ist eine längere Zwischenentwicklung einzuschalten. Zwei Zahlen $\eta$ und $\eta^{\prime}$ aus e sollen "teilerfremd" heißen, wenn die Hauptideale $[\eta]$ und $\left[\eta^{\prime}\right]$ teilerfremd sind. Wir greifen nun erstens aus e alle Zahlen heraus, die teilerfremd gegen den Grad $n$ unseres Zweiges $e_{n}$ sind. Den ausgewählten Zahlen fügen wir sodann noch alle diejenigen gebrochenen Zahlen von $\Re$ hinzu, deren einzelne als Quotient zweier gegen $n$ teilerfremden ganzen Zahlen von $\Re$ darstellbar ist. Das so gebildete Zahlsystem hat die Eigenschaft, daß sowohl das Produkt wie auch der Quotient zweier seiner Zahlen stets wieder dem System angehört. Ein mit dieser Eigenschaft ausgestattetes Zahlsystem heißt ein „Zahlstrahl“ oder kurz ein „Strahl“1); der besondere, soeben ausgewählte Strahl werde durch $\mathcal{S}^{(n)}$ oder, wenn der obere Index entbehrlich ist, durch $\subseteq$ bezeichnet.

1) Weber gebraucht statt Strahl die Bezeichnung "Gruppe". 
Zweitens greifen wir aus dem $n^{\text {ten }} Z_{w e i g e ~} e_{n}$ alle gegen $n$ teilerfremden Zahlen heraus und fügen ihnen wieder alle gebrochenen Zahlen hinzu, die als Quotienten zweier herausgegriffener Zahlen darstellbar sind. Das so zu gewinnende in $\mathbb{S}$ enthaltene Zahlsystem stellt wieder einen Strahl dar, der durch $\mathfrak{j}^{(n)}$ oder kurz durch $\{$ bezeichnet werden soll.

Wenn nun auch die Strahlen $\subseteq$ und $\{$ neben ganzen auch gebrochene Zahlen enthalten, so können wir diese Strahlen doch mod [n] oder, was auf dasselbe hinausläuft, mod $n$ in "Zahlklassen" zerlegen, nämlich $\subseteq$ in $\psi([n])$ und $\{$ in $\varphi(n)$ Klassen, unter $\psi([n])$ und $\varphi(n)$ die in $(10)$ und (9) S. 118 erklärten Anzahlen verstanden. Wir führen dies zunächst im einfacheren Falle des Strahles $\{$ aus; die Übertragung auf $\subseteq$ wird hernach leicht sein.

Für die ganzen Zahlen $\left(e_{0}+e_{1} n \Theta\right)$ von $\mathfrak{j}$ ist die Einteilung einleuchtend. Die Zahl $\left(e_{0}+e_{1} n \Theta\right)$ ist $\bmod n$ mit $e_{0}$ kongruent, und da $e_{0}$ teilerfremd gegen $n$ ist, so haben wir für die ganzen Zahlen von ¡ insgesamt $\varphi(n)$ Klassen, die wir repräsentieren durch die $\varphi(n)$ rationalen ganzen Zahlen $r_{1}=1, r_{2}, \ldots, r_{\varphi}=n-1$ zwischen 0 und $n$, die teilerfremd zu $n$ sind. Eine gebrochene Zahl $\mu$ von $\left\{\right.$ habe $\mu=\frac{\alpha}{\beta}$ als eine erste Darstellung als Quotient zweier ganzer Zahlen $\alpha, \beta$ von $\left\{\right.$. Es sei $\alpha \equiv r_{i}$, $\beta \equiv r_{k}(\bmod n)$. Dann gibt es, da $r_{k}$ teilerfremd gegen $n$ ist, einen und nur eipen Repräsentanten $r_{l}$, der die Kongruenz $r_{k} \cdot r_{l} \equiv 1(\bmod n)$ erfüllt und im AnschluB an diese Kongruenz auch durch $r_{k}^{-1}$ bezeichnet sein mag. Ist $r_{i} \cdot r_{l}=r_{i} \cdot r_{k}^{-1} \equiv r_{m}(\bmod n)$, so fügen wir die Zahl $\mu$ der durch $r_{m}$ vertretenen Klasse bei, was durch:

$$
\mu=\frac{\alpha}{\beta} \equiv r_{i} \cdot r_{k}^{-1} \equiv r_{m} \quad(\bmod n)
$$

zum Ausdruck komme. Zu zeigen ist noch, daB die Klasse, der $\mu$ zuerteilt ist, von der besonderen Darstellung $\mu=\frac{\alpha}{\beta}$ unabhängig ist. Eine zweite Darstellung von $\mu$ sei $\mu=\frac{\alpha^{\prime}}{\beta^{\prime}}$, und es gelte $\alpha^{\prime} \equiv r_{i}^{\prime}, \beta^{\prime} \equiv r_{k}^{\prime}(\bmod n)$, so daB von dieser Darstellung aus die Zahl $\mu$ in die Klasse von $r_{i}^{\prime} \cdot r_{k}^{\prime-1}$ gehören würde. Aus $\alpha \beta^{\prime}=\alpha^{\prime} \beta$ folgt dann $r_{i} r_{k}^{\prime} \equiv r_{i}^{\prime} r_{k}(\bmod n)$, und also ergibt sich weiter durch Multiplikation mit $r_{k}^{-1} \cdot r_{k}^{\prime-1}$ die Kongruenz $r_{i} r_{k}^{-1} \equiv r_{i}^{\prime} r_{k}^{\prime-1}(\bmod n)$. Durch unsere Maßregel ist also auch jede gebrochene Zahl von $\uparrow$ einer und nur einer unter den $\varphi(n)$ Zahlklassen zuerteilt.

Es besteht der Satz: Zwei Kongruenzen $\mu \equiv r, \mu^{\prime} \equiv r^{\prime}(\bmod n)$ für Zahlen $\mu, \mu^{\prime}$ aus ; können miteinander multipliziert oder durcheinander dividiert werden, ohne ein unrichtiges Ergebnis zu liefern. Die Division durch $r$ läuft dabei natürlich auf die Multiplikation mit $r^{-1}$ hinaus. Der Satz ist zunächst einleuchtend für die Multiplikation ganzzahliger $\mu, \mu^{\prime}$ (S. 101). Dann gilt er aber auch für die Multiplikation, in dem Falle, daß eine der 
beiden Zahlen $\mu, \mu^{\prime}$ oder beide gebrochen sind. Ist nämlich $\mu=\frac{\alpha}{\beta}$, $\boldsymbol{\mu}^{\prime}=\frac{\alpha^{\prime}}{\beta^{\prime}}$, und gehören die $\alpha, \beta, \alpha^{\prime}, \beta^{\prime}$ in die Zahlklassen von $r_{i}, r_{k}, r_{i}^{\prime}, r_{k}^{\prime}$, so ist: $\quad \alpha \cdot \alpha^{\prime} \equiv r_{i} \cdot r_{i}^{\prime}, \quad \beta \cdot \beta^{\prime} \equiv r_{k} \cdot r_{k}^{\prime} \quad(\bmod n)$,

so daB $\mu \mu^{\prime}$ in die Zahlklasse von $\left(r_{i} r_{i}^{\prime}\right) \cdot\left(r_{k} r_{k}^{\prime}\right)^{-1}$ hineingehört. Nun ist aber $\left(r_{k} r_{k}^{\prime}\right)^{-1} \equiv r_{k}^{-1} \cdot r_{k}^{\prime-1}$, woraus:

$$
\left(r_{i} r_{i}^{\prime}\right) \cdot\left(r_{k} r_{k}^{\prime}\right)^{-1} \equiv r_{i} r_{i}^{\prime} \cdot r_{k}^{-1} r_{k}^{\prime-1} \equiv\left(r_{i} r_{k}^{-1}\right) \cdot\left(r_{i}^{\prime} r_{k}^{\prime-1}\right) \equiv r r^{\prime}
$$

folgt. Also gehört $\mu \cdot \mu^{\prime}$ in die Zahlklasse von $r r^{\prime}$, d. h. aus $\mu \equiv r, \mu^{\prime} \equiv r^{\prime}$ $(\bmod n)$ folgt $\mu \mu^{\prime} \equiv r r^{\prime}(\bmod n)$. In ähnlicher Art zeigt man auch die Erlaubnis der Division zweier Kongruenzen $\mu \equiv r, \mu^{\prime} \equiv r^{\prime}(\bmod n)$ dureheinander. Den bewiesenen Satz verallgemeinert man leicht noch dahin, daß irgend zwei Kongruenzen $\mu \equiv \mu^{\prime \prime}, \mu^{\prime}=\mu^{\prime \prime \prime}(\bmod n)$ bei Multiplikation und Division wieder richtige Kongruenzen liefern.

Das System aller in die durch $r_{i}$ repräsentierte Klasse gehörenden Zahlen von $\left\{\right.$ werde durch $\mathfrak{G}_{i}^{(n)}$ oder kurz durch $\mathfrak{g}_{i}$ bezeichnet. Die Tatsache, daB alle $\varphi(n)$ Systeme $\Xi_{1}, \Xi_{2}, \ldots, \xi_{\varphi}$ den Strahl $\lceil$ gerade erschöpfen, bringen wir durch die symbolische Gleichung:

$$
\mathfrak{i}=\vec{g}_{1}+\mathfrak{g}_{2}+\cdots+\mathfrak{g}_{\varphi}
$$

zum Ausdruck. Das System $\Xi_{1}$ bildet wieder einen „Strahl“, da für zwei Zahlen aus $\mathfrak{i}$, die $\equiv 1(\bmod n)$ sind, sowohl das Produkt als der Quotient wieder $\equiv 1(\bmod n)$ sind. Nach dem soeben bewiesenen Satze gehört das Produkt von $r_{i}$ und einer Zahl aus $\mathfrak{g}_{1}$ in das System $\mathfrak{g}_{i}$, und umgekehrt ist der Quotient einer Zahl aus $\mathfrak{\Xi}_{i}$ und der Zahl $r_{i}$ stets in $\Xi_{1}$ enthalten. Wir erhalten also gerade das ganze System $\tilde{\xi}_{i}$, indem wir $r_{i}$ mit allen Zahlen von $\mathfrak{g}_{1}$ multiplizieren, was durch die Gleichung $\mathfrak{g}_{i}=r_{i} \cdot \mathfrak{g}_{1}$ zum Ausdruck komme. Die Zusammensetzung des Strahles \{ aus seinen $\varphi(n)$ Zahlklassen können wir demnach an Stelle von (2) auch so schreiben:

$$
\mathfrak{i}=\Xi_{1}+r_{2} \cdot \Xi_{1}+r_{3} \cdot \Xi_{1}+\cdots+r_{\varphi} \cdot \Xi_{1} \text {. }
$$

Es wiederholen sich entsprechende Betrachtungen für die Zerlegung des Strahles $\mathfrak{S}$ in Zahlklassen mod $n$. Da die ganzen Zahlen von $\mathfrak{\subseteq}$ alle gegen [n] teilerfremden Zahlen von e sind, so verteilen sie sich auf $\psi([n])$ Klassen (S. 117). Wir repräsentieren diese Klassen durch $\psi$ geeignet gewählte Zahlen $\varrho_{1}=1, \varrho_{2}, \varrho_{3}, \ldots, \varrho_{\psi}$ und dürfen die ersten $\varphi$ unter ihnen mit den obigen $r_{1}, r_{2}, \ldots, r_{\varphi}$ identisch nehmen. Für irgendein $\varrho_{k}$ gibt es dann stets ein und nur ein die Kongruenz $\varrho_{k} \cdot \varrho_{l} \equiv 1(\bmod n)$ befriedigendes $\varrho_{l}$, das demnach auch durch $\varrho_{k}^{-1}$ bezeichnet werden mag. ${ }^{1}$ )

1) Der Beweis folgt (wie in der rationalen Zahlentheorie) aus dem Umstande, $\mathrm{daB}$ die $\psi$ Produkte $\varrho_{k}, \varrho_{k} \varrho_{2}, \varrho_{k} \varrho_{3}, \ldots, \varrho_{k} \varrho_{\psi}$ durchweg $\bmod n$ inkongruent und teilerfremd zu $n$ sind, so daB eines dieser Produkte mit $\rho_{1}=1$ kongruent sein muB. 
Die gebrochenen Zahlen von $\subseteq$ verteilen wir daraufhin genau nach den oben beim Strahle \{ befolgten Grundsätzen auf die $\psi([n])$ Klassen. Auch die Fortsetzung der Betrachtung gestaltet sich Wort für Wort wie oben: Jede Zahl $\lambda$ von $\subseteq$ gehört in eine und nur eine der $\psi([n]) \bmod n$ inkongruenten Klassen; zwei Kongruenzen $\lambda \equiv \lambda^{\prime \prime}, \lambda^{\prime} \equiv \lambda^{\prime \prime \prime}(\bmod n)$ zwischen Zahlen von $\mathfrak{S}$ dürfen miteinander multipliziert oder durcheinander dividiert werden, ohne ein unrichtiges Ergebnis zu liefern.

Eine beliebige Zahl der durch $\varrho_{1}=1$ repräsentierten Klasse von $\subseteq$ sei $\lambda=\frac{\gamma}{\delta}$. Mit der ganzen Zahl $\delta$ ist auch die ihr konjugierte Zahl $\delta^{\prime}$ teilerfremd gegen $[n]$, da ein $\left[\delta^{\prime}\right]$ und $[n]$ gemeinsamer Primidealfaktor $\mathfrak{p}^{\prime}$ im konjugierten Ideale $\mathfrak{p}$ einen gemeinsamen Faktor von $[\delta]$ und $[n]$ liefern würde. Schreiben wir $\lambda=\frac{\gamma \delta^{\prime}}{\delta \delta^{\prime}}$, so ist der Nenner $\delta \delta^{\prime}$ als rationale ganze Zahl in $\mathrm{e}_{n}$ enthalten und als teilerfremd gegen $n$ einer Zahl $r_{i}$ $\bmod n$ kongruent. Aus $\lambda \equiv 1, \delta \delta^{\prime} \equiv r_{i}(\bmod n)$ folgt weiter:

$$
\gamma \delta^{\prime}=\lambda \cdot \delta \delta^{\prime} \equiv r_{i} \quad(\bmod n),
$$

so daß auch $\gamma \delta^{\prime}$ in $e_{n}$ und also $\lambda$ in $\{$ enthalten ist. Als mit $1 \bmod n$ kongruent gehört dann $\lambda$ in das Teilsystem $\xi_{1}$. Umgekehrt ist jede Zahl von $\Xi_{1}$ auch in $\subseteq$ enthalten und gehört daselbst zu der durch $\varrho_{1}=1$ repräsentierten Klasse. Also führt die durch $\varrho_{1}=1$ repräsentierte Klasse von 5 einfach zum Systeme $\Xi_{1}$ zurïck. Wie oben zeigt man dann weiter leicht, daß man das System aller mit $\varrho_{i}$ kongruenten Zahlen von $\mathcal{S}$ gerade genau erschöpft, indem man das symbolisch durch $\varrho_{i} \cdot \Xi_{1}$ zu bezeichnende System aller Produkte von $\varrho_{i}$ und den Zahlen von $\Xi_{1}$ bildet. Es ergibt sich der Satz: Der Strahl $\subseteq$ setzt sich aus den $\psi([n])$ Systemen $\mathfrak{\Xi}_{1}$, $\varrho_{2} \cdot \mathfrak{s}_{1}, \varrho_{3} \cdot \Xi_{1}, \ldots, \varrho_{\psi} \cdot \mathfrak{g}_{1}$ zusammen und kann demnach wieder symbolisch in der Gestalt einer Summe geschrieben werden:

$$
\widetilde{S}=\mathfrak{s}_{1}+\varrho_{2} \cdot \mathfrak{g}_{1}+\varrho_{3} \cdot \mathfrak{s}_{1}+\cdots+\varrho_{\psi} \cdot \mathfrak{s}_{1} \text {. }
$$

Da $\varrho_{2}=r_{2}, \varrho_{3}=r_{3}, \ldots, \varrho_{\varphi}=r_{\varphi}$ sein sollte, so liefern die ersten $\varphi$ Glieder in (4) rechts zufolge (3) als Summe ₹. Wir schreiben nun $\varrho_{\varphi+1}=\sigma_{2}$ und haben in $\sigma_{2}, \sigma_{2} \cdot \varrho_{3}, \sigma_{2} \cdot \varrho_{3}, \ldots, \sigma_{2} \cdot \varrho_{\varphi}$ weitere $\varphi$ Zahlen von $\subseteq$, die untereinander und gegen $\varrho_{1}=1, \varrho_{2}, \ldots, \varrho_{\varphi}$ durchweg inkongruent sind. Wir können demnach die Zahlen $\sigma_{2}, \sigma_{2} \cdot \varrho_{2}, \ldots, \sigma_{2} \cdot \varrho_{\varphi}$ als weitere $\varphi$ Repräsentanten $\varrho_{\varphi+1}, \varrho_{\varphi+2}, \ldots, \varrho_{2 \varphi}$ benutzen; die zugehörigen Glieder der Summe (4) fassen sich zur Teilsumme:

$$
\varrho_{\varphi+1} \cdot \mathfrak{g}_{1}+\cdots+\varrho_{2 \varphi} \cdot \mathfrak{B}_{1}=\sigma_{2}\left(\mathfrak{g}_{1}+\varrho_{2} \cdot \mathfrak{B}_{1}+\cdots+\varrho_{\varphi} \cdot \mathfrak{g}_{1}\right)=\sigma_{2} \cdot\{
$$

zusammen. Wir setzen weiter $\varrho_{2 \varphi+1}=\sigma_{3}$ und wiederholen dieselbe SchluBweise, bis alle Glieder auf der rechten Seite von (4) erschöpft sind, was nach $\psi([n]): \varphi(n)=\chi(n)$ Schritten erreicht sein wird. Da übrigens $\psi([n]) \bmod n$ inkongruente, gegen $n$ teilerfremde ganze Zahlen in $e$ vor- 
kommen, so steht nichts im Wege, die Repräsentanten $\varrho$ durchweg ganzzahlig zu wählen; auch die $\chi(n)$ Zahlen $\sigma_{1}=1, \sigma_{2}, \ldots, \sigma_{\chi}$ werden dann ganz. Wir gelangen zu dem Satze: Die gesamten Zahlen des Strahles $\mathfrak{S}$ lassen sich auf $\chi(n)$ Zahlsysteme verteilen, entsprcchend der symbolischen Darstellung von $\mathbb{S}$ als Summe:

$$
\widetilde{S}=\mathfrak{i}+\sigma_{2} \cdot \mathfrak{i}+\sigma_{3} \cdot \mathfrak{j}+\cdots+\sigma_{\%} \cdot \mathfrak{j},
$$

wo $\chi(n)$ die durch $\chi(n)=\psi([n]): \varphi(n)$ erklärte Anzahl ist, die sich im Anschlu $\beta$ an (10) S. 118 in der Gestalt darstellen läßt:

$$
\chi(n)=n \prod\left(1-\frac{(\mathbf{D}, p)}{p}\right)
$$

$\left\lceil\right.$ bedeutet den oben näher erklärten zum $n^{\text {ten }}$ Zweige gehörenden Strahl, und $\sigma_{1}=1, \sigma_{2}, \ldots, \sigma_{\%}$ sind gewisse $\chi(n)$ inkongruente, geeignet gevö̈hlte ,ganze" Zahlen aus $\mathfrak{S}$.

\section{Zerlegung der Idealklassen von $\Re$ in Zweigklassen.}

Nach S. $104 \mathrm{ff}$. sind zwei Ideale $\mathfrak{a}$ und $\mathfrak{b}$ von $\Re$ dann und nur dann äquivalent, wenn es eine Zahl $\lambda$ in $\Re$ von der Art gibt, daß die Zahlen des Ideals $\mathfrak{a}$, mit $\lambda$ multipliziert, gerade genau alle Zahlen von $\mathfrak{b}$ ergeben. Wir bringen diese Tatsache durch die Gleichung $\mathfrak{b}=\lambda \cdot \mathfrak{a}$ zum Ausdruck. Alle mit einem gegebenen Ideale äquivalenten Ideale bildeten eine „Idealklasse", und es gab in $\Re$ nur eine endliche Anzahl solcher Klassen. Nach S. 120 gibt es in jeder Idealklasse gegen $[n]$ teilerfremde Ideale.

Es besteht der Satz: Sind $\mathfrak{a}$ und $\mathfrak{b}=\lambda \cdot \mathfrak{a}$ zwei äquivalente Ideale, so ist die Zahl $\lambda$ bis auf einen Faktor, der eine Einheit $\varepsilon$ von $\mathcal{\Omega}$ ist, eindeutig bestimmt. Gilt nämlich neben $\mathfrak{b}=\lambda \cdot \mathfrak{a}$ auch $\mathfrak{b}=\lambda^{\prime} \cdot \mathfrak{a}$, und setzen wir $\lambda=\frac{\alpha}{\beta}$ und $\lambda^{\prime}=\frac{\alpha^{\prime}}{\beta^{\prime}}$, so müssen wegen der Gleichheit der Zahlsysteme $\lambda \cdot \mathfrak{a}$ und $\lambda^{\prime} \cdot \mathfrak{a}$ auch die beiden Systeme:

$$
\beta \beta^{\prime} \cdot \lambda \cdot \mathfrak{a}=\alpha \beta^{\prime} \cdot \mathfrak{a} \text { und } \beta \beta^{\prime} \cdot \lambda^{\prime} \cdot \mathfrak{a}=\alpha^{\prime} \beta \cdot \mathfrak{a}
$$

gleich sein. Also folgt die Gleichheit der Ideale $\left[\alpha \beta^{\prime}\right] \cdot \mathfrak{a}$ und $\left[\alpha^{\prime} \beta\right] \cdot \mathfrak{a}$ und damit auch diejenige von $\left[\alpha \beta^{\prime}\right]$ und $\left[\alpha^{\prime} \beta\right]$. Die Zahlen $\alpha \beta^{\prime}$ und $\alpha^{\prime} \beta$ sind demnach assoziiert, woraus der aufgestellte Satz folgt.

Wir stellen weiter den Satz auf: Ist eines der äquivalenten Ideale $\mathfrak{a}$ und $\mathfrak{b}=\lambda \cdot \mathfrak{a}$ teilerfremd gegen [n], und gehört $\lambda$ dem Strahle $\subseteq$ an, so ist auch das andere Ideal teilerfremd gegen $[n]$. Wir setzen $\lambda=\frac{\alpha}{\beta}$, wo $\alpha$ und $\beta$ ganze Zahlen des Körpers $\Omega$ bedeuten, die gegen $n$ teilerfremde Normen $N(\alpha)$ und $N(\beta)$ haben. Aus $\mathfrak{b}=\lambda \cdot \mathfrak{a}$ folgt $\mathfrak{b} \cdot[\beta]=\mathfrak{a} \cdot[\alpha]$ und also:

$$
N(\mathfrak{b}) \cdot N(\beta)=N(\mathfrak{a}) \cdot N(\alpha) .
$$


Ist nun eines dẹ Ideale $\mathfrak{a}, \mathfrak{b}$, etwa $\mathfrak{a}$, teilerfremd gegen $[n]$ und damit $N(\mathfrak{a})$ teilerfremd gegen $n^{1}$ ), so folgt aus (1), dab $N(\mathfrak{b})$ teilerfremd gegen $n$ und also $\mathfrak{b}$ teilerfremd gegen $[n]$ ist. Der aufgestellte Satz ist also richtig. Hieran schließt sich der Satz: Sind die beiden äquivalenten Ideale a und $\mathfrak{b}=\lambda \cdot \mathfrak{a}$ teilerfremd gegen $[n]$, so gehört $\lambda$ dem Strahle $\mathfrak{S}$ an. Wir können nämlich aus der zur Klasse von $\mathfrak{a}$ und $\mathfrak{b}$ inversen Idealklasse ein gegen $[n]$ teilerfremdes Ideal $\mathfrak{c}$ entnehmen und finden dann in $\mathfrak{b} \cdot \mathfrak{c}=\left[\alpha^{\prime}\right]$ und $\mathfrak{a} \cdot \mathfrak{c}=\left[\beta^{\prime}\right]$ zwei Hauptideale, die gegen $[n]$ teilerfremd sind und also gegen $n$ teilerfremde Zahlen $\alpha^{\prime}, \beta^{\prime}$ liefern. Aus $\mathfrak{b}=\lambda \cdot \mathfrak{a}$ oder der damit gleichbedeutenden Gleichung $[\beta] \cdot \mathfrak{b}=[\alpha] \cdot \mathfrak{a}$ folgt durch Multiplikation mit c:

$$
[\beta] \cdot \mathfrak{b} \mathfrak{c}=[\alpha] \cdot \mathfrak{a} c, \quad[\beta] \cdot\left[\alpha^{\prime}\right]=[\alpha] \cdot\left[\beta^{\prime}\right], \quad\left[\beta \alpha^{\prime}\right]=\left[\alpha \beta^{\prime}\right] .
$$

Also sind $\beta \alpha^{\prime}$ und $\alpha \beta^{\prime}$ assoziiert, und wir finden:

$$
\alpha \beta^{\prime}=\varepsilon \cdot \alpha^{\prime} \beta, \quad \lambda=\frac{\alpha}{\beta}=\frac{\varepsilon \alpha^{\prime}}{\beta^{\prime}},
$$

unter $\varepsilon$ eine Einheit verstanden. Zufolge der letzten Darstellung von $\lambda$ gehört diese Zahl dem Strahle S an.

Es sei jetzt $n>1$. Die Zahlen $\lambda$ des Strahles $\subseteq$ waren entsprechend der Gleichung (5) S. 129 in $\chi(n)$ Systeme $\left\{, \sigma_{2} \cdot\left\{, \ldots, \sigma_{\chi} \cdot\{\right.\right.$ verteilt, wo ¡ der zum $n^{\text {ten }}$ Zweige $e_{n}$ gehörende Strahl $\mathfrak{j}^{(n)}$ war und $1=\sigma_{1}, \sigma_{2}, \ldots$, $\sigma_{\chi}$ gewisse $\chi(n)$ ganze Zahlen von $\subseteq$ bedeuten. Mit einer Zahl $\lambda$ gehört $-\lambda$ stets dem gleichen Systeme $\sigma_{k} \cdot j$ an, da der Strahl $j$ mit einer einzelnen Zahl immer auch ihre entgegengesetzte enthält. Nach den Angaben von S. 121 über die Einheiten von $\AA$ ergibt sich: Für $|\mathbf{D}|>4$ sind je zwei assoziierte Zahlen $\pm \lambda^{2}$ ) immer in dem gleichen Systeme $\sigma_{k} \cdot \mathfrak{i}$ enthalien. Ist $|\mathbf{D}|=4$, so gehören die beiden assoziierten Zahlen $\lambda$ und $\lambda^{\prime}=i \lambda$ stets verschiedenen Systemen $\sigma_{k} \cdot\{$ an, da sonst ihr Quotient $\lambda^{\prime}: \lambda=i$ in $\{$ enthalten wäre, was jedoch wegen $n>1$ nicht der Fall ist. Ebenso gehören für $|\mathbf{D}|=3$ die drei assoziierten Zahlen $\lambda$, $\varrho \lambda, \varrho^{2} \lambda$ stets drei verschịedenen Systemen $\sigma_{k} \cdot\left\{\right.$ an. Ist für $|\mathbf{D}|=4$ die zu $\sigma_{k}$ assoziierte Zahl $i \sigma_{k}$ in $\sigma_{l} \cdot\left\lceil\right.$ enthalten, so können wir, wenn nicht bereits $\sigma_{l}=i \sigma_{k}$ sein sollte, $\sigma_{l}$ durch $i \sigma_{k}$ ersetzen $^{3}$ ) und finden, daß $\sigma_{i} \cdot \tilde{j}$ einfach aus allen Zahlen besteht, die aus denen von $\sigma_{k} \cdot\{$ durch Multiplikation mit $i$ entstehen. Die beiden Systeme $\sigma_{k} \cdot\left\{\right.$ und $\sigma_{i} \cdot\{$ mögen dann selbst „assoziiert" heißen. Durch Übertragung der Betrachtung auf $|\mathbf{D}|=3$ folgt der Satz:

1) Hätten $N(\mathfrak{a})$ und $n$ eine rationale Primzahl $p$ als gemeinsamen Faktor, so würde mindestens ein in $[p]$ aufgehendes Primideal $\mathfrak{a}$ und $[n]$ zugleich teilen.

2) Wir übertragen den Begriff der „assoziierten" Zahlen in sofort verständlicher Art auch auf die gebrochenen Zahlen von $\Re$.

3) Ist $\sigma_{l}^{\prime}$ irgendeine Zahl des Systems $\sigma_{l} \cdot\left\{\right.$, so ist offenbar das System $\sigma_{l}^{\prime}$. mit $\sigma_{l} \cdot j$ identisch. 
Für $|\mathbf{D}|>4$ sind unter den $\chi(n)$ Systemen $\sigma_{k} \cdot\{$ keine zwei assoziiert, für $|\mathbf{D}|=4$ sind sie zu je zweicn assoziiert und bilden $\frac{1}{2} \chi(n)$ nicht-assoziierte Systempaare, fiir $|\mathbf{D}|=3$ sind sie zu je dreien assoziiert und bilden $\frac{1}{3} \chi(n)$ nicht-assoziierte Systemtripel.

Wir stellen nun folgende Erklärung auf: Zwei gegen $n$ teilerfremde, der gleichen Klasse angehörende Ideale $\mathfrak{a}$ und $\mathfrak{b}$ sollen ,im Zweige $\mathfrak{e}_{n}$ äquivalent" heißen, wenn sich eine Gleichung $\mathfrak{b}=\boldsymbol{\mu} \cdot \mathfrak{a}$ angeben läßt, in der $\mu$ dem Strahle $\{$ angehört; läßt sich eine solche Gleichung nicht angeben, so heißen $\mathfrak{a}$ und $\mathfrak{b}$, ,in $\mathfrak{e}_{n}$ inäquivalent". Man bilde nun zunächst aus einem ersten gegen $[n]$ teilerfremden Ideale $\mathfrak{a}$ die $n$ äquivalenten, gleichfalls gegen $n$ teilerfremden Ideale $\mathfrak{a}, \sigma_{2} \cdot \mathfrak{a}, \sigma_{3} \cdot \mathfrak{a}, \ldots, \sigma_{\chi} \cdot \mathfrak{a}$. Nach dem Satze über die Bestimmtheit der Faktoren $\lambda$ in den Gleichungen $\mathfrak{b}=\lambda \cdot \mathfrak{a}$ zwischen je zwei äquivalenten Idealen und nach den Angaben über die Verteilung aller Zahlen $\lambda$ von $\subseteq$ auf die Systeme $\sigma_{k} \cdot\{$ ergibt sich der Satz: Die $\chi(n)$ Ideale $\mathfrak{a}, \sigma_{2} \cdot \mathfrak{a}, \sigma_{3} \cdot \mathfrak{a}, \ldots, \sigma_{\varkappa} \cdot \mathfrak{a}$ sind für $|\mathbf{D}|>4$ in $\mathrm{e}_{n}$ inäquivalent, für $|\mathbf{D}|=4$ werden sie zu Paaren in $\mathrm{e}_{n}$ äquivalent und stellen $\frac{1}{2} \chi(n)$ in $\mathrm{e}_{n}$ inäquivalente Idealpaare dar, für $|\mathbf{D}|=3$ werden sie $z u$ dreien in $\mathrm{e}_{n}$ äquivalent und liefern $\frac{1}{3} \chi(n)$ in $\mathrm{e}_{n}$ inäquivalente Idealtripel.

Um die beiden Ausnahmefälle nicht stets besonders nennen zu müssen, verabreden wir, daß die $Z a h l \tau=1, \frac{1}{2}$ oder $\frac{1}{3}$ sein soll, je nachdem $|\mathbf{D}|>4$, $|\mathbf{D}|=4$ oder $=3$ ist. Auch ordnen wir in den Ausnahmefällen die $\mathfrak{a}$, $\sigma_{2} \cdot \mathfrak{a}, \ldots, \sigma_{\chi} \cdot \mathfrak{a}$ so an, dab $\tau \chi(n)$ durchweg in $e_{n}$ inäquivalente Ideale $\mathfrak{a}$, $\sigma_{2} \cdot \mathfrak{a}, \ldots, \sigma_{\tau_{\ell}} \cdot \mathfrak{a}$ voranstehen. Einleuchtend ist, dab irgend zwei gegen $[n]$ teilerfremde Ideale $\mathfrak{b}=\lambda \cdot \mathfrak{a}$ und $\mathfrak{b}^{\prime}=\lambda^{\prime} \cdot \mathfrak{a}$ stets und nur dann in $\mathfrak{e}_{n}$ äquivalent sind, wenn $\lambda$ und $\lambda^{\prime}$ dem gleichen Systeme $\sigma_{k} \cdot j$ oder assoziierten Systemen angehören. Alle einander in $\mathfrak{e}_{n}$ äquivalenten Ideale fügen wir in eine "Zweighlasse" zusammen und bezeichnen ihnen gegenüber die ursprünglichen Klassen auch als "Stammklassen". Wir haben dann den folgenden Satz: Die gesamten gegen $[n]$ teilerfremden Ideale einer vorgelegten Stammklasse verteilen sich auf $\tau \chi(n)$ "Zweigklassen", die wir durch $\mathfrak{a}, \sigma_{2} \cdot \mathfrak{a}, \ldots, \sigma_{\tau \chi} \cdot \mathfrak{a}$ repräsentieren lönnen; zwei Ideale aus verschiedenen Zweigklassen sind stets in $\mathrm{e}_{n}$ inäquivalent.

\section{\$4. Multiplikation und Äquivalenz der Zweigideale.}

Die Erklärung der Multiplikation zweier Zweigideale des $n^{\text {ten }}$ '/weiges $\mathrm{e}_{n}$ schliebt sich an diejenige der Stammideale an. Unter dem Produkte $\mathfrak{a}_{n} \cdot \mathfrak{b}_{n}$ zweier Zweigideale $\mathfrak{a}_{n}$ ind $\mathfrak{b}_{n}$ verstehen wir das System aller Zahlen, die entweder Produlte je einer Zahl $\alpha_{n}$ aus $\mathfrak{a}_{n}$ und einer Zahl $\boldsymbol{\beta}_{n}$ aus $\mathfrak{b}_{n}$ sind oder als irgendwelche Summen solcher Produkte gewonnen werden. Am Produkte $\mathfrak{a}_{n} \cdot \mathfrak{b}_{n}$ erkennt man leicht wieder die drei S. 124 genannten Grundeigenschaften eines Zweigideals. Jedenfalls enthält das System $\mathfrak{a}_{n} \cdot \mathfrak{b}_{n}$ nur Zahlen des Zweiges $\mathfrak{e}_{n}$. Betreffs der Eigenschaft 1 beachte 
man nur wegen der Differenzen, daß ein Zweigideal mit einer einzelnen Zahl immer auch deren entgegengesetzte Zahl enthält. Die Eigenschaft 2 kann man für $\mathfrak{a}_{n} \cdot \mathfrak{b}_{n}$ leicht aus dem Umstande ableiten, daß diese Eigenschaft für $\mathfrak{a}_{n}$ zutrifft. Um die Eigenschaft 3 am Systeme $\mathfrak{a}_{n} \cdot \mathfrak{b}_{n}$ nachzuweisen, verstehen wir unter $\eta_{n}$ eine beliebige Zahl des Zweiges $\mathfrak{e}_{n}$. Da $\mathfrak{a}_{n}$ und $\mathfrak{b}_{n}$ die Eigenschaft 3 besitzen, so gibt es zwei Darstellungen:

$$
\eta_{n}=\alpha_{n}+n \eta, \quad 1=\beta_{n}+n \eta^{\prime}
$$

der in $\mathfrak{e}_{n}$ enthaltenen Zahlen $\eta_{n}$ und 1. Es folgt:

$$
\eta_{n}=\alpha_{n} \beta_{n}+n \eta^{\prime \prime}, \quad \text { wo } \eta^{\prime \prime}=\alpha_{n} \eta^{\prime}+\beta_{n} \eta+n \eta \eta^{\prime}
$$

ist, so daß jede Zahl von $\mathfrak{e}_{n}$ als Summe einer Zahl aus $\mathfrak{a}_{n} \cdot \mathfrak{b}_{n}$ und einer solchen aus [n] darstellbar ist. Das Produkt zweier Zweigideale $\mathfrak{a}_{n}, \mathfrak{b}_{n}$ ist wieder ein Zweigideal $\mathfrak{c}_{n}=\mathfrak{a}_{n} \cdot \mathfrak{b}_{n}$. Daß für unsere Produkte das kommutative und das assoziative Gesetz gelten, folgt wieder leicht aus dem Umstande, dab diese Gesetze für die Produkte der Zahlen zutreffen.

Die Multiplikation der Zweigideale steht zu derjenigen der Stammideale in enger Beziehung: Sind $\mathfrak{a}=\mathfrak{e} \cdot \mathfrak{a}_{n}$ und $\mathfrak{b}=\mathfrak{e} \cdot \mathfrak{b}_{n}$ die zu den Zweigidealen $\mathfrak{a}_{n}$ und $\mathfrak{b}_{n}$ gehörenden Stammideale, so gehört zum Produlite $\mathfrak{c}_{n}=\mathfrak{a}_{n} \cdot \mathfrak{b}_{n}$ von $\mathfrak{a}_{n}$ und $\mathfrak{b}_{n}$ als Stammideal $\mathfrak{e} \cdot \mathfrak{c}_{n}$ das Prodult $\mathfrak{c}=\mathfrak{a} \cdot \mathfrak{b}$ der beiden Stammideale $\mathfrak{a}$ und $\mathfrak{b}$. Das Ideal $\mathfrak{a}$ besteht nämlich aus allen Produkten $\eta \cdot \alpha_{n}$ und allen Summen solcher Produkte, und ebenso besteht $\mathfrak{b}$ aus allen Produkten $\eta \cdot \boldsymbol{\beta}_{n}$ und deren Summen. Da nun $e \cdot e$ wieder gleich $e$ ist, so setzt sich das Ideal $\mathfrak{c}=\mathfrak{a} \cdot \mathfrak{b}$ aus allen Prodúkten $\eta \cdot \alpha_{n} \beta_{n}$ und allen Summen solcher Produkte zusammen. Zu genau demselben Zahlsystem aber gelangt man, wenn man vom Zweigideal $\mathfrak{c}_{n}=\mathfrak{a}_{n} \cdot \mathfrak{b}_{n}$ durch Multiplikation mit $\mathfrak{e}$ zum zugehörigen Stammideal $e \cdot \mathfrak{c}_{n}$ übergeht. Die Umkehrung des letzten Satzes lautet: Sind $\mathfrak{a}$ und $\mathfrak{b}$ gegen [n] teilerfremde Stammideale, denen die Zweigideale $\mathfrak{a}_{n}$ und $\mathfrak{b}_{n}$ zugehören, so entspricht dem offenbar gleichfalls gegen $[n]$ teilerfremden Stammideale $\mathfrak{c}=\mathfrak{a} \cdot \mathfrak{b}$ als Zweigideal das Produkt $\mathfrak{a}_{n} \cdot \mathfrak{b}_{n}$ von $\mathfrak{a}_{n}$ und $\mathfrak{b}_{n}$. Nach S. 125 entsprechen die $\mathrm{Zweigideale}$ und die gegen $[n]$ teilerfremden Stammideale einander umkehrbar eindeutig. Zufolge des letzten Satzes entspricht aber dem Zweigideal $\mathfrak{a}_{n} \cdot \mathfrak{b}_{n}$ das Stammideal $\mathfrak{c}=\mathfrak{a} \cdot \mathfrak{b}$; also ist umgekehrt das $z \mathfrak{u} \mathfrak{c}=\mathfrak{a} \cdot \mathfrak{b}$ gehörige Zweigideal $\mathfrak{c}_{n}=\mathfrak{a}_{n} \cdot \mathfrak{b}_{n}$.

Der Äquivalenz der Zweigideale legen wir folgende Erklärung zugrunde: Zwei Zweigideale $\mathfrak{a}_{n}$ und $\mathfrak{b}_{n}$ heißen stets und nur dann „äquivalent", wenn es eine Zahl $\mu$ des Strahles $\left\{\right.$ gibt, die der Gleichung $\mathfrak{b}_{n}=\mu \cdot \mathfrak{a}_{n}$ genïgt, was besagt, daß die Zahlen von $\mathfrak{b}_{n}$ einfach die mit $\mu$ multiplizierten Zahlen von $\mathfrak{a}_{n}$ sind. Wir können dann folgenden Satz beweisen: Zwei Zweigideale $\mathfrak{a}_{n}$ und $\mathfrak{b}_{n}$ sind stets und nur dann äquivalent, wenn die beiden zugehörigen Stammideale $\mathfrak{a}$ und $\mathfrak{b}$ „im Zwveige $\mathfrak{e}_{n}$ äquivalent" sind. Trifft 
nämlich die letzte Bedingung zu, so gibt es eine Zahl $\mu=\frac{a}{\beta}$ in $\mathfrak{j}$, die die Gleichung $\mathfrak{b}=\mu \cdot \mathfrak{a}$ erfüllt. Die Zahl $\beta$ ist teilerfremd gegen $n$ und in $e_{n}$ enthalten. Dasselbe gilt von der zu $\beta$ konjugierten Zahl $\beta^{\prime}$, so daß $\beta \cdot \beta^{\prime}$ eine gegen $n$ teilerfremde rationale ganze Zahl ist. Nun ist jede Zahl des Systems:

$$
\boldsymbol{\mu} \cdot \mathfrak{a}_{n}=\frac{\alpha}{\beta} \cdot \mathfrak{a}_{n}=\frac{\alpha \beta^{\prime}}{\beta \beta^{\prime}} \cdot \mathfrak{a}_{n}
$$

in $\mathfrak{b}$ enthalten und also ganz. Da jede dieser ganzen Zahlen bei Multiplikation mit der gegen $n$ teilerfremden rationalen ganzen Zahl $\boldsymbol{\beta} \cdot \boldsymbol{\beta}^{\prime}$ als Produkt eine Zahl des Systems $\alpha \beta^{\prime} \cdot \mathfrak{a}_{n}$ und also des Zweiges $\mathfrak{e}_{n}$ liefert, so gehört sie bereits selbst dem Zweige $e_{n}$ an. ${ }^{1}$ ) Da außerdem alle Zahlen von $\mu \cdot \mathfrak{a}_{n}$ in $\mathfrak{b}$ enthalten sind, so finden sie sich alle im $Z$ weige $\mathfrak{b}_{n}$ von $\mathfrak{b}$. In derselben Art zeigt man, daß jede Zahl von $\mu^{-1} \cdot \mathfrak{b}_{n}$ in $\mathfrak{a}_{n}$ enthalten ist. Sind also die beiden Stammideale $\mathfrak{a}$ und $\mathfrak{b}$ in $e_{n}$ äquivalent, so gilt sicher $\mathfrak{b}_{n}=\boldsymbol{\mu} \cdot \mathfrak{a}_{n}, \mathrm{~d}$. h. die beiden Zweigideale $\mathfrak{a}_{n}$ und $\mathfrak{b}_{n}$ sind äquivalent. Umgekehrt folgt aus $\mathfrak{b}_{n}=\mu \cdot \mathfrak{a}_{n}$ sehr leicht $\left(\mathfrak{e} \cdot \mathfrak{b}_{n}\right)=\mu \cdot\left(\mathfrak{e} \cdot \mathfrak{a}_{n}\right)$, womit der aufgestellte Satz im rollen Umfange bewiesen ist.

Auch für die Äquivalenz der Zweigideale $\mathfrak{a}_{n}$ und $\mathfrak{b}_{n}$ benutzen wir das oben (S. 104) eingeführte Zeichen $\mathfrak{a}_{n} \sim \mathfrak{b}_{n}$. Da sich die Zahlen des Strahles $j$ bei Multiplikation reproduzieren, so sind auch hier wieder zwei $\mathrm{Zweigideale,} \mathrm{die} \mathrm{einem} \mathrm{dritten} \mathrm{äquivalent} \mathrm{sind,} \mathrm{miteinander} \mathrm{äqui-}$ valent. Alle mit einem gegebenen $\mathfrak{a}_{n}$ äquivalenten $\mathrm{Z}$ weigideale fügen wir zu einer „Ideallilasse“ $\mathfrak{A}_{n}$ zusammen, wobei dann je zwei Zweigideale der Klasse $\mathfrak{A}_{n}$ äquivalent sind. Die mit dem $Z$ weigideale $\mathfrak{e}_{n}$ äquivalenten Zweigideale bilden die "Haupthlasse" and mögen wieder „Hauptideale" genannt werden. ${ }^{2}$ )

Es ist nun leicht, die Betrachtung so zu ergänzen, daß Übereinstimmung mit den bei den Stammidealen aufgestellten Sätzen vorliegt. Zunächst haben wir den Satz: Ist $\mathfrak{a}_{n} \cdot \mathfrak{b}_{n}=\mathfrak{c}_{n}$, und gilt $\mathfrak{a}_{n}^{\prime} \sim \mathfrak{a}_{n}, \mathfrak{b}_{n}^{\prime} \sim \mathfrak{b}_{n}$, so ist auch $\mathfrak{c}_{n}^{\prime}=\mathfrak{a}_{n}^{\prime} \cdot \mathfrak{b}_{n}^{\prime} \sim \mathfrak{c}_{n}$. Es gibt nämlich zwei Zahlen $\mu$ und $\mu^{\prime}$ in $\mathfrak{\{}$, die die Gleichungen $\mathfrak{a}_{n}^{\prime}=\mu \cdot \mathfrak{a}_{n}, \mathfrak{b}_{n}^{\prime}=\mu^{\prime} \cdot \mathfrak{b}_{n}$ befriedigen. Die Zahlen des Produktes $\mathfrak{c}_{n}^{\prime}=\mathfrak{a}_{n}^{\prime} \cdot \mathfrak{b}_{n}^{\prime}$ gehen dann aus denen des Produktes $\mathfrak{a}_{n} \cdot \mathfrak{b}_{n}$ einfach durch Multiplikation mit $\mu \cdot \mu^{\prime}$ hervor. Da diese Zahl in $\{$ enthalten ist, so gilt $\mathfrak{c}_{n}^{\prime} \sim \mathfrak{c}_{n}$. Nennen wir $\mathfrak{A}_{n}$ und $\mathfrak{B}_{n}$ die zu $\mathfrak{a}_{n}$ und $\mathfrak{b}_{n}$ gehörenden Idealklassen, so gibt irgendein Ideal aus $\mathfrak{A}_{n}$, mit irgendeinem aus $\mathfrak{B}_{n}$ multipliziert, als Produkt stets ein Ideal einer bestimmten dritten Klasse

1) Soll nämlich eine $\mathrm{Zahl}\left(e_{0}+e_{1} n \Theta\right)$ von $\mathfrak{e}_{n}$, durch die gegen $n$ teilerfremde, rationale ganze Zahl $\beta \beta^{\prime}$ geteilt, eine ganze Zahl als Quotienten liefern, so müssen $e_{0}$ und $e_{1}$ durch $\beta \beta^{\prime}$ teilbar sein.

2) Wenn wir bei den letzten Penennungen den Zusatz „Zweig“ der Kürze halber vermeiden, so wird daraus kaum eine Zweideutigkeit entstehen, da aus dem 7usammenhange stets die genaue Bedeutung der Benennung zu entnehmen ist. 
$\mathfrak{C}_{n}$, die wir das "Produkt" der beiden Klassen $\mathfrak{A}_{n}$ und $\mathfrak{B}_{n}$ nennen und symbolisch durch $\mathfrak{C}_{n}=\mathfrak{A}_{n} \cdot \mathfrak{B}_{n}$ bezeichnen.

Ist $\mathfrak{a}_{n}$ irgendein Zweigideal und $\mathfrak{a}$ das zugehörige Stammideal, so gibt es ein gegen $[n]$ teilerfremdes Stammideal $\mathfrak{b}^{\prime}$, das in $\mathfrak{a} \cdot \mathfrak{b}^{\prime}=[\gamma]$ ein Hauptideal des Stammes e liefert. Die ganze Zahl $\gamma$ ist teilerfremd gegen $n$, gehört also in eine der $\psi([n])$ oben (S. $127 \mathrm{ff}$.) betrachteten Zahlklassen mod $n$ hinein. Wir können nach den dortigen Darlegungen eine gleichfalls gegen $n$ teilerfremde Zahl $\mu_{0}$ so wählen, $\mathrm{daB} \mu=\mu_{0} \gamma$ in die Zahlklasse $\mathfrak{g}_{1}$ und damit zum Strahle $\{$ gehört. Indem wir die Zahlen des Ideals $\mathfrak{a} \cdot \mathfrak{b}^{\prime}=[\gamma]$ mit $\mu_{0}$ multiplizieren und $\mu_{0} \cdot \mathfrak{b}^{\prime}=\mathfrak{b}$ setzen, ist auch $\mathfrak{b}$ ein gegen $[n]$ teilerfremdes Ideal, und wir finden $\mathfrak{a} \cdot \mathfrak{b}=[u]$. Der $n^{\text {te }} \mathrm{Zweig}$ des Hauptideals $[\mu]$ ist nun einfach das Hauptideal $\mu \cdot \mathfrak{e}_{n}$ des Zweiges $\mathfrak{e}_{n}$. $\mathrm{DaB}$ alle Zahlen von $\mu \cdot e_{n}$ im $n^{\text {ten }} \mathrm{Zweige}$ von $[\mu]$ vorkommen, ist nämlich einleuchtend. Soll andrerseits die Zahl $\mu \cdot \eta$ von $[\mu]$ in $e_{n}$ enthalten sein, so ist auch $\left(\mu \cdot \mu^{\prime}\right) \cdot \eta$ in $e_{n}$ enthalten, unter $\mu^{\prime}$ die zu $\mu$ konjugierte Zahl verstanden. Dann gehört aber, da $\mu \cdot \mu^{\prime}$ rational, ganz und gegen $n$ teilerfremd ist, auch schon $\eta$ dem Zweige $\mathfrak{e}_{n}$ an. Aus $\mathfrak{a} \cdot \mathfrak{b}=[\mu]$ folgt nun durch Übergang zu den Zweigidealen $\mathfrak{a}_{n} \cdot \mathfrak{b}_{n}=\mu \cdot \mathfrak{e}_{n}$ und also, da $\mu$ in $\left\{\right.$ enthalten ist, der Satz: Fïr jedes Zweigideal $\mathfrak{a}_{n}$ gibt es sicher ein Ideal $\mathfrak{b}_{n}$ von der Art, daß das Produkt $\mathfrak{a}_{n} \cdot \mathfrak{b}_{n}$ ein Hauptideal des $n^{\text {ten }}$ Zweiges ist. Natürlich gibt dann jedes Ideal der Klasse $\mathfrak{A}_{n}$ von $\mathfrak{a}_{n}$, mit irgendeinem Ideale der Klasse $\mathfrak{B}_{n}$ von $\mathfrak{b}_{n}$ multipliziert, als Produkt ein Hauptideal des Zweiges $\mathfrak{e}_{n}$. Wir nennen die Klassen $\mathfrak{U}_{n}$ und $\mathfrak{B}_{n}$ einander „invers" und bezeichnen im Anschluß daran auch wohl $\mathfrak{B}_{n}$ durch $\mathfrak{A}_{n}^{-1}$ und $\mathfrak{A}_{n} \operatorname{durch} \mathfrak{B}_{n}^{-1}$.

Die durch $h(|D|)=h\left(n^{2}|\mathbf{D}|\right)$ zu bezeichnende „Anzahl der Idealklassen" des Zweiges $e_{n}$ wird durch den S. 132 sogleich an die Erklärung äquivalenter $\mathfrak{a}_{n}, \mathfrak{b}_{n}$ angeschlossenen Satz auf die Anzahl $h(|\mathbf{D}|)$ der Idealklassen im Stamme e zurückgeführt. Aus der Zerlegung der einzelnen Klasse der Stammideale in je $\chi(n)$ "Zweigklassen" ergibt sich unmittel-

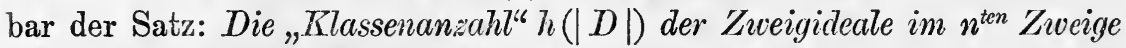
$\mathbf{e}_{n}$ des quadratischen Körpers $\Re$ der Diskriminante $\mathbf{D}$ ist gegeben durch:

$$
h(|D|)=h(|\mathbf{D}|) \cdot \tau n \prod\left(1-\frac{(\mathbf{D}, p)}{p}\right)
$$

wo $h(|\mathbf{D}|)^{\circ}$ die endliche Anzahl der Idealklassen im Stamme $\mathfrak{c}$ ist und die iibrigen Bestandtcile der rechten Seite von (1) die oben (S. 118 und 131) dargelegte Bedeutung haben.

Endlich bleibt uns noch übrig, die gruppentheoretische Auffassung der Multiplikation der Idealklassen auf den Zweig $\boldsymbol{e}_{n}$ zu übertragen, wobei die $h$ Klassen $\mathfrak{H}_{0}, \mathfrak{A}_{1}, \ldots, \mathfrak{A}_{l_{h-1}}$ die „Elemente“ der Gruppe bilden und unter ihnen insbesondere die "Hauptklasse" $\mathfrak{A}_{0}$ "das Einheitselement" liefert. Die Überlegungen schließen sich genau an S. 107 an; wir notieren 
sogleich den Satz: Die $h$ Idealllassen $\mathfrak{A}_{0}, \mathfrak{A}_{1}, \ldots, \mathfrak{A}_{h-1}$ des $n^{\text {ten }}$ Zweiges vom Körper $\Re$ bilden gegeniiber Multiplikation eine kommutative oder Abelsche Gruppe $G_{h}$ der Ordnung $h$, in der das Einheitsclement von der Hauptklasse $\mathfrak{A}_{0}$ geliefert wird.

\section{§. Basen der Ideale und ebene Punktgitter.}

Die Überlegung, welche uns oben (S. 86 ff.) zur Existenz einer „Basis“ für das Zahlsystem $\mathfrak{e}$ hinführte, war auch auf jedes Ideal $\mathfrak{a}$ anwendbar (S.91 ff.) und ist ohne Änderung auch bei jedem Zweigideal $\mathfrak{a}_{n}$ durchführbar. Wir schließen jetzt, wenn wir von einem „Ideale $\mathfrak{a}_{n}$ " sprechen, den Fall $n=1$ mit ein, so daß $\mathfrak{a}_{n}$ ein Zweigideal oder ein Stammideal bedeuten mag. Um den schon erwähnten Zusammenhang der quadratischen Körper $\Re$ negativer Diskriminanten mit der Theorie der elliptischen Funktionen gleich äußerlich hervortreten zu lassen, bezeichnen wir die Zahlen einer Basis von $\mathfrak{a}_{n}$ durch $\omega_{1}, \omega_{2}$; die zu $\omega_{1}, \omega_{2}$ konjugierten Zahlen, die zugleich ihre „konjugiert komplexen“ Zahlen sind, mögen $\bar{\omega}_{1}, \bar{\omega}_{2}$ heißen.

Als ein erstes aus den allgemeinen Sätzen über die Basen folgendes Ergebnis haben wir anzumerken: Irgendeine Basis $\omega_{1}^{\prime}, \omega_{2}^{\prime}$ des Ideals $\mathfrak{a}_{n}$ ist durch eine erste Basis $\omega_{1}, \omega_{2}$ in der Gestalt:

$$
\omega_{1}^{\prime}=\alpha \omega_{1}+\beta \omega_{2}, \quad \omega_{3}^{\prime}=\gamma \omega_{1}+\delta \omega_{2}, \quad \alpha \delta-\beta \gamma= \pm 1
$$

darstellbar, wo $\alpha, \beta, \gamma, \delta$ vier rationale ganze Zahlen der Determinante +1 oder -1 sind. Da die $\omega_{1}, \omega_{2}$ linear-unabhängig sind, so ist der Quotient $\omega=\frac{\omega_{1}}{\omega_{2}}$ eine nicht-rationale Zahl von $\Re$, die wegen der negativen Diskriminante D komplex ist. Wir können nötigenfalls durch Zeichenwechsel einer der beiden Zahlen $\omega_{1}, \omega_{2}$, der für den Gebrauch dieser Zahlen als einer Basis von $\mathfrak{a}_{n}$ ohne Folge ist, erreichen, daß der Quotient $\omega$ eine komplexe Zahl mit positivem imaginären Bestandteile wird. Wir wollen in diesem Falle $\omega_{1}, \omega_{2}$ eine ,positive ${ }^{\prime \prime}$ Basis und $\omega=\frac{\omega_{1}}{\omega_{2}}$ einen „positiven“ Basisquotienten nennen. Dann gilt insbesondere der Satz: Irgendeine positive Basis $\omega_{1}^{\prime}, \omega_{2}^{\prime}$ von $\mathfrak{a}_{n}$ ist durch eine erste unter ihnen $\omega_{1}$, $\omega_{2}$ in der Gestalt darstellbar:

$$
\omega_{1}^{\prime}=\alpha \omega_{1}+\beta \omega_{2}, \quad \omega_{2}^{\prime}=\gamma \omega_{1}+\delta \omega_{2}, \quad \alpha \delta-\beta \gamma=1,
$$

wo $\alpha, \beta, \gamma, \delta$ vier rationale ganze Zahlen der Determinante 1 sind; andrerseits bildet jedes durch eine Substitution (2) aus einer ersten positiven Basis $\omega_{1}, \omega_{2}$ gewinnbare Zahlenpaar $\omega_{1}^{\prime}$, $\omega_{2}^{\prime}$ wieder eine positive Basis von $\mathfrak{a}_{n}$.

Wir sind hiermit zu der Lehre von den linearen Transformationen der Perioden $\omega_{1}, \omega_{2}$, die in I, $182 \mathrm{ff}$. entwickelt wurde, zurückgeführt. Unter Aufnahme der gruppentheoretischen Sprechweise können wir dem letzten Satze die Gestalt geben: Aus einer ersten positiven Basis $\omega_{1}, \omega_{2}$ 
von $a_{n}$ gewinnen wir gerade genau die gesamten positiven Basen dieses Ideals, indem wir auf $\omega_{1}, \omega_{2}$ die gesamten Substitutionen der "homogenen Modulgruppe" $\Gamma^{(\omega)}$ ausüben (s. I, 283), und in entsprechender Art ergeben sich aus einem ersten positiven Basisquotienten w alle solche Quotienten für $\mathfrak{a}_{n}$ durch Ausübung der ,nicht-homogenen Modulgruppe" $\Gamma^{(\omega)}$ auf $\omega$.

Die geometrischen Hilfsmittel aus Bd. I mögen nun auch hier herangezogen werden. Wir kleiden die gesamten Zahlen von $\mathfrak{a}_{n}$ in die Gestalt:

$$
u=m_{1} \omega_{1}+m_{2} \omega_{2},
$$

wo $m_{1}, m_{2}$ alle Paare rationaler ganzer Zahlen durchlaufen sollen. Indem wir in der „u-Ebene“ alle Bildpunkte dieser Zahlen markieren, erhalten wir ein ebenes „Punktgitter" von der Art, wie uns solche Gitter in I, $174 \mathrm{ff}$. von den Eckpunkten der Parallelogrammnetze der $u$-Ebene geliefert wurden. ${ }^{1}$ ) Das vorliegende Gitter (3) gewinnen wir vom Parallelogrammnetze der Perioden $\omega_{1}, \omega_{2}$. Doch können wir nach der Lehre von der linearen Transformation der Perioden das gleiche Gitter (3) noch durch unendlich viele weitere Parallelogrammnetze ausschneiden, wie sie eben durch die Substitutionen der $\Gamma^{(\omega)}$ aus dem ersten Netze hervorgehen. Zur geometrischen Deutung der Werte $\omega$ der Basisquotienten ziehen wir die „w-Halbebene" heran, in der wir das „Dreiecksnetz" der Modulgruppe $\Gamma^{(\omega)}$ gezeichnet denken. Die gesamten positiven Basisquotienten $\omega$ unseres Ideals $a_{n}$ stellen dann ein System bezüglich der $\Gamma^{(\omega)}$ äquivalenter Punkte $\omega$ im Dreiecksnetze dar.

Für das zu $\mathfrak{a}_{n}$ konjugierte Ideal $\overline{\mathfrak{a}}_{n}$ hat man das Zahlenpaar $-\overline{\boldsymbol{\omega}}_{1}, \overline{\boldsymbol{\omega}}_{2}$ als eine positive Basis, wenn das Paar $\omega_{1}, \omega_{2}$ eine solche von $\mathfrak{a}_{n}$ darstellt. ${ }^{2}$ ) Das zu $\overline{\mathfrak{a}}_{n}$ gehörende Gitter geht aus dem von $\mathfrak{a}_{n}$ durch Spiegelung an der reellen $u$-Achse hervor. Der zu $-\bar{\omega}_{1}, \bar{\omega}_{2}$ gehörende Quotient - $\bar{\omega}$ liefert den bezüglich der imaginären $\omega$-Achse mit $\omega$ symmetrisch gelegenen Punkt: Die gesamten positiven Basisquotienten von $\bar{a}_{n}$ liefern ein Punktsystem der $\omega$-Halbebene, das mit dem zu $\mathfrak{a}_{n}$ gehörenden Punktsystem besüglich der durch Spiegelungen erweiterten Modulgruppe $\Gamma^{(\omega)}$ äquivalent ist. Insbesondere können diese beiden Punktsysteme dadurch identisch werden, dab sie auf Symmetriekreise des Dreiecksnetzes der $\omega$-Halbebene rücken. Wir kommen unten auf diesen Fall zurück.

Ein mit $\mathfrak{a}_{n}$ "äquivalentes" Ideal $\mathfrak{a}_{n}^{\prime}$ ist nach S. 132 symbolisch als Produkt $\mathfrak{a}_{n}^{\prime}=\mu \cdot \mathfrak{a}_{n}$ darstellbar, wo $\mu$ eine Zahl des Strahles $\mathfrak{j}$ ist. Es ist einleuchtend, dab wir aus einer Basis $\omega_{1}, \omega_{2}$ von $\mathfrak{a}_{n}$ in:

$$
\omega_{1}^{\prime}=\mu \omega_{1}, \quad \omega_{2}^{\prime}=\mu \omega_{2}
$$

1) Die Vorstellung des Punktgitters ist das wichtigste geometrische Hilfsmittel, mit dem Klein in seinen S. 121 genannten Vorlesungen arbeitet.

2) Wie in Bd. I verstehen wir unter $\bar{\omega}_{1}, \bar{\omega}_{2}, \bar{\omega}$ die zu $\omega_{1}, \omega_{2}$, $\omega$ konjugiert komplexen Zahlen. 
eine Basis für $\mathfrak{a}_{n}^{\prime}$ gewinnen. Das zu $\mathfrak{a}_{n}^{\prime}$ gehörende Punktgitter wird demnach aus dem Gitter (3) durch die Transformation $u^{\prime}=\mu u$ erhalten. Wir haben den Satz: Äquivalente Ideale $\mathfrak{a}_{n}$ und $\mathfrak{a}_{n}^{\prime}$ haben "ähnliche" Punktgitter, und ihnen kommt ein und dasselbe System beziiglich der $\Gamma^{(\omega)}$ äquivalenter Punkte der w-Halbebene zu, so daß dicses Punltsystem als ein Attribut der Ideallilasse anzusehen ist.

Die Perioden $\omega_{1}, \omega_{2}$ sind in der Theorie der elliptischen Funktionen abgesehen davon, daß ihr Quotient nicht reell sein darf, frei wählbar, und sie werden in der Theorie der Modulfunktionen als variabel betrachtet. Demgegenüber sind die als Basen der Ideale $\mathfrak{a}_{n}$ auftretenden $\omega_{1}, \omega_{2}$ in der Art beschränkt, daß sie gewisse Paare ganzer Zahlen eines quadratischen Körpers $\mathcal{A}$ negativer Diskriminante $D$ sind, woraus dann folgt, daB auch die Basisquotienten $\omega$ diesem Körper angehören. In der Theorie der elliptischen Funktionen heißen diese $\omega_{1}, \omega_{2}$, ,singuläre Periodenpaare und die $\omega$,singuläre Pcriodenquotienten"; ihre Bedeutung für die Theorie der elliptischen Funktionen wird unten ausführlich darzulegen sein.

\section{§ 6. Notizen über quadratische Formen negativer Diskriminante.}

Um die Beziehung zwischen den Idealen $\mathfrak{a}_{n}$ und den ganzzahligen binären quadratischen Formen negativer Diskriminante entwickeln zu können, sind zunächst einige Notizen über diese Formen vorauszuschicken. ${ }^{1}$ ) Unter einer "ganzzahligen binären quadratischen Form" verstehen wir einen Ausdruck der Gestalt:

$$
a x^{2}+b x y+c y^{2}
$$

dessen Koeffizienten $a, b, c$ rationale ganze Zahlen sind, während $x, y$ willkürliche Gröben bedeuten. Je nach Umständen gelten die $x, y$ als komplexe Variable oder sind auf willkürliche rationale ganze Zahlen eingeschränkt. Als Bezeichnung für eine Form (1) benutzen wir, wenn es nicht erforderlich ist, die Variablen $x, y$ besonders hervorzuheben, das Symbol $(a, b, c)$.

Die größte rationale ganze positive Zahl $t$, die in $a, b, c$ zugleich aufgeht, heißt der „Teiler" der Form $(n, b, c)$. Ist $t=1$, so heißt die Form ,ursprünglich". Für $t>1$ setzen wir $a=t a_{0}, b=t b_{0}, c=t c_{0}$ und können jetzt $(a, b, c)$ aus der ursprünglichen Form $\left(a_{0}, b_{0}, c_{0}\right)$ durch Multiplikation mit $t$ ableiten. Für $t>1$ heißt demnach $(a, b, c)$ eine „abgeleitete" Form. Der Ausdruck $\left(b^{2}-4 a c\right)$ wird die „Disliriminante" der Form $(a, b, c)$ genannt und durch $D$ bezeichnet. Die Diskriminante ist offenbar durch das Quadrat $t^{2}$ des Teilers $t$ teilbar. Ist die Diskriminante negativ, so sind $a$ und $c$ von 0 verschieden und haben gleiches

1) Vgl. Dirichlet-Dedekind, „Vorlesungen über Zahlentheorie“, 4. Aufl., S. $128 \mathrm{ff}$. und "Modulfunktionen" Bd. I, S. $243 \mathrm{ff}$. 
Vorzeichen. Je nachdem dieses Vorzeichen positiv oder negativ ist, heißt $(a, b, c)$ selbst eine „positive" oder eine "negative Form". Da eine negative Form einfach durch Zeichenwechsel ihrer drei Koeffizienten in eine positive übergeführt wird, so wird bei den folgenden Untersuchungen der alleinige Gebrauch positiver Formen keine wesentliche Beschränkung bedeuten.

Führt nuan in die Form (1) an Stelle der $x, y$ neue Variable $x^{\prime}, y^{\prime}$ mittels einer "ganzzahligen linearen Substitution der Determinante 1“:

$$
x^{\prime}=\alpha x+\beta y, \quad y^{\prime}=\gamma x+\delta y, \quad \alpha \delta-\beta \gamma=1
$$

ein, so erhält man aus $(a, b, c)$ die Form:

$$
\left(a^{\prime}, b^{\prime}, c^{\prime}\right)=a^{\prime} x^{\prime 2}+b^{\prime} x^{\prime} y^{\prime}+c^{\prime} y^{2},
$$

deren ganzzahlige Koeffizienten sich aus den $a, b, c$ und den Substitutionskoeffizienten $\alpha, \beta, \gamma, \delta$ nach der Regel berechnen:

$$
\left\{\begin{array}{l}
a^{\prime}=\delta^{2} a-\gamma \delta b+\gamma^{2} c \\
b^{\prime}=-2 \beta \delta a+(\alpha \delta+\beta \gamma) b-2 \alpha \gamma c, \\
c^{\prime}=\beta^{2} a-\alpha \beta b+\alpha^{2} c .
\end{array}\right.
$$

Umgekehrt wird die Form $\left(a^{\prime}, b^{\prime}, c^{\prime}\right)$ durch die zu (2) „inverse“ Substitution mit den Koeffizienten $\delta,-\beta,-\gamma, \alpha$ wieder in $(a, b, c)$ übergeführt, wobei sich die Gleichungen (4) invertieren zu:

$$
\left\{\begin{array}{l}
a=\alpha^{2} a^{\prime}+\alpha \gamma b^{\prime}+\gamma^{2} c^{\prime}, \\
b=2 \alpha \beta a^{\prime}+(\alpha \delta+\beta \gamma) b^{\prime}+2 \gamma \delta c^{\prime}, \\
c=\beta^{2} a^{\prime}+\beta \delta b^{\prime}+\delta^{2} c^{\prime} .
\end{array}\right.
$$

In (2) haben wir die Substitutionen der homogenen Modulgruppe $\Gamma^{(\omega)}$ wiedergewonnen, abgesehen davon, daß die Bezeichnungen $\omega_{1}, \omega_{2}$ der der Variablen hier durch $x, y$ ersetzt sind. Indem wir die in I benutzte abgekürzte Bezeichnung $\left(\begin{array}{l}\alpha, \beta \\ \gamma, \delta\end{array}\right)$ für die Substitution (2) wieder heranziehen, können wir sagen, die Form $(a, b, c)$ gehe durch die Substitution $\left(\begin{array}{l}\alpha, \beta \\ \gamma, \delta\end{array}\right)$ in $\left(a^{\prime}, b^{\prime}, c^{\prime}\right)$ über und umgekehrt $\left(a^{\prime}, b^{\prime}, c^{\prime}\right)$ durch $\left(\begin{array}{l}\delta,-\beta \\ -\gamma, \alpha\end{array}\right)$ wieder in $(a, b, c)$.

Jede durch eine Substitution (2) aus $(a, b, c)$ entstehende Form $\left(a^{\prime}, b^{\prime}, c^{\prime}\right)$ heißt mit $(a, b, c)$, $\ddot{a} q u i v a l e n t "$. Aus der Gruppeneigenschaft der Substitutionen (2) folgt dann, daß umgekehrt auch $(a, b, c)$ mit $\left(a^{\prime}, b^{\prime}, c^{\prime}\right)$ äquivalent ist, und $\mathrm{da} a$ zwei Formen, die mit einer dritten äquivalent sind, stets auch miteinander äquivalent sind. Vereinigen wir daher alle mit einer gegebenen Form $(a, b, c)$ äquivalenten Formen in eine "Klasse von Formen" oder "Formllasse" $\mathfrak{F}$, so sind je zwei Formen der Klasse $\mathfrak{F}$ äquivalent. Aus (4) ergibt sich $b^{\prime 2}-4 a^{\prime} c^{\prime}$ 
$=b^{2}-4 a c$, äquivalente Formen haben demnach gleiche Diskriminanten D. Aus (4) und (5) folgt ferner, daß äquivalente Formen stets gleiche Teiler $t$ haben. Fndlich folgert man aus (4) leicht:

$$
4 a a^{\prime}=(2 \delta a-\gamma b)^{2}-D \gamma^{2},
$$

so daB für $D<0$ stets $a a^{\prime}>0$ zutrifft: Zwei äquivalente Formen negativer Diskriminante sind demnach immer zugleich positiv baw. negativ. Die Diskriminante $D$ und der Teiler $t$ sind demnach Attribute der Formklasse $\mathfrak{F}$, auch gehören für $D<0$ der Klasse entweder nur positive oder nur negative Formen an.

Die Hauptaufgabe ist nun für uns, bei gegebener Diskriminante $D$ die gesamten hier eintretenden Formklassen $\mathfrak{F}$ festzustellen und insbesondere ihre Anzahl abzuzählen. Wir behandeln diese Aufgabe nur für den weiterhin allein in Betracht kommenden Fall $D<0$ und setzen die Formen als positiv voraus. Zur Durchführung der Aufgabe setzen wir den Quotienten der Variablen $\frac{x}{y}=\omega$ und führen eine geometrische Deutung der Form $(a, b, c)$ durch denjenigen Punkt der positiven $\omega$-Halbebene ein, für welchen die Form $(a, b, c)$ verschwindet. Dieser „Nullpunkt" der Form berechnet sich zu:

$$
\omega=\frac{-b+\sqrt{D}}{2 a}=\frac{-b+i \sqrt{|D|}}{2 a},
$$

und es ergibt sich aus (5) durch eine einfache Rechnung im Falle der $\ddot{A}$ quivalenz der beiden Formen $(a, b, c)$ und $\left(a^{\prime}, b^{\prime}, c^{\prime}\right)$ für ihre Nullpunkte die Gleichung:

$$
\omega^{\prime}=\frac{\alpha \omega+\beta}{\gamma \omega+\delta},
$$

so daß die Nullpunkte $\omega$ und $\omega^{\prime}$ der beiden äquivalenten Formen stets bezüglich der nicht-homogenen Modulgruppe äquivalent sind. Besteht andrerseits zwischen den beiden Nullpunkten $\omega$ und $\omega^{\prime}$ zweier positiver Formen $(a, b, c)$ und $\left(a^{\prime}, b^{\prime}, c^{\prime}\right)$ mit gleicher Diskriminante $D<0$ eine Relation (7), so folgt:

$\frac{-b^{\prime}+i \sqrt{|D|}}{2 a^{\prime}}=\frac{(2 \beta a-\alpha b)+\alpha i \sqrt{|D|}}{(2 \delta a-\gamma b)+\gamma i \sqrt{|D|}}=\frac{(2 \beta \delta a-(\alpha \delta+\beta \gamma) b+2 \alpha \gamma c)+i \sqrt{|D|}}{2\left(\delta^{2} a-\gamma \delta b+\gamma^{2} c\right)}$

woraus man leicht auf die Gleichungen (4) zurückschließt. Die beiden positiven Formen $(a, b, c)$ und $\left(a^{\prime}, b^{\prime}, c^{\prime}\right)$ gleicher negativer Diskriminante $D$ sind also auch stets äquivalent, wenn ihre Nullpunkte $\omega, \omega^{\prime}$ bezüglich $\operatorname{der} \Gamma^{(\omega)}$ äquivalent sind.

Die in I, $185 \mathrm{ff}$. entwickelte Reduktionstheorie der Periodenquotienten setzt uns nun unmittelbar in den Stand, die gestellte Aufgabe der Aufzählung aller Formklassen $\mathfrak{F}$ gegebener negativer Diskriminante $D$ 
zu lösen. Der Nullpunkt einer gegebenen Form ist mit einem und nur einem Punkte $\omega$ des durch Fig. 43 in I, 179 gegebenen Diskontinuitätsbereiches der Gruppe $\Gamma^{(\omega)}$ äquivalent. Ein diesem Bereiche angehörender Punkt $\omega$ lieferte uns einen „reduzierten" Periodenquotienten. Die für einen solchen Punkt $\omega$ charakteristischen Bedingungen sind:

$$
-1 \leqq \omega+\bar{\omega}<+1, \quad \omega \bar{\omega}>1
$$

oder (für ein $\omega$ auf dem unteren Rande des Diskontinuitätsbereiches):

$$
-1 \leqq \omega+\bar{\omega} \leqq 0, \quad \omega \bar{\omega}=1,
$$

wo $\bar{\omega}$ wie bisher der zu $\omega$ konjugiert komplexe Wert ist. Die zu einem solchen „reduzierten" $\omega$ gehörende Form $(a, b, c)$ möge selbst als eine „reduzierte Form" bezeichnet werden. Aus (8) und (9) ergibt sich dann als Kennzeichen einer reduzierten positiven Form negativer Diskriminante $D$ :

$$
-a<b \leqq a<c \text { bzw. } 0 \leqq b \leqq a=c .
$$

Nach den voraufgehenden Überlegungen und Rechnungen ist nun in jeder unserer Formklassen eine und nur eine reduzierte Form enthalten. Für eine solche Form ergibt sich aus (10) und der Erklärung von $D$ :

$$
4 b^{2} \leqq 4 a c, \quad 3 b^{2} \leqq|D|, \quad 4 a c=b^{2}+|D| .
$$

Bei gegebenem $D$ sind hiernach nur endlich viele $b$ zulässig und beim einzelnen $b$ zufolge der letzten Bedingung (11) nur endlich viele Kombinationen $a, c$. Indem wir daher die einzelne Formklasse $\mathfrak{F}$ durch ihre reduzierte Form „repräsentieren“, haben wir durch Aufzählung aller reduzierten Formen auf Grund der zweiten und dritten Bedingung (11) unsere Aufgabe der Feststellung aller Formklassen einer gegebenen Diskriminante $D$ gelöst. Wir haben insbesondere den Satz gefunden: Bei gegebener negativer Diskriminante $D$ gibt es nur eine endliche Anzahl zugehöriger Klassen $\mathfrak{F}$ positiver Formen.

Es mögen sich weiter folgende Notizen anschließen: Zwei Formen $(a, b, c)$ und $(a,-b, c)$, die sich nur im Vorzeichen des zweiten Koeffizienten unterscheiden, heißen ,entgegengesetzt". Die-Nullpunkte entgegengesetzter Formen liegen symmetrisch zur imaginären $\omega$-Achse. Eine Form, deren Nullpunkt auf einem Symmetriekreise des Dreiecksnetzes der $\omega$-Halbebene liegt, heiBt, zwveiseitig" oder ,ambig“. Äquivalente Formen sind immer zugleich zweiseitig, so daß die Benennung ,zweiseitig“ oder „ambig“ auf die Formklasse $\mathfrak{F}$ übertragen werden kann. Aus der Struktur des Dreiecksnetzes der $\omega$-Halbebene geht der Satz hervor: Eine Form ist stets und nur dann mit ihrer entgegengesetzten Form äquivalent, wenn sie zweiseitig ist. Für die reduzierten Formen, die, falls sie zweiseitig sind, ihre Nullpunkte auf der imaginären $\omega$-Achse oder auf dem Rande 
Endlichkeit der Klassenanzahl. Ambige Formen, Hauptformen usw.

des Diskontinuitätsbereiches der $\Gamma^{(\omega)}$ haben, ist dies unmittelbar einleuchtend. Die gesamten Formklassen $\mathfrak{F}$ negativer Diskriminante. $D$ setzen sich also aus den ambigen Klassen und den Paaren entgegengesetzter Klassen zusammen.

Die Diskriminante $D$ ist mod 4 entweder mit 0 oder mit 1 kongruent. Je nachdem der erste oder zweite dieser Fälle vorliegt, haben wir in:

$$
\left(1,0,-\frac{1}{4} D\right) \text { bzw. }\left(1,1, \frac{1}{4}(1-D)\right)
$$

eine reduzierte Form der Diskriminante $D$, die offenbar ambig ist, da ihr Nullpunkt:

$$
\omega=\frac{i \sqrt{|D|}}{2} \quad \text { bzw. } \quad \omega=\frac{-1+i \sqrt{|D|}}{2}
$$

auf der imaginären $\boldsymbol{\omega}$. Achse oder auf dem linken Rande des Diskontinuitätsbereiches der $\Gamma^{(\omega)}$ liegt. Man bezeichnet die Form (12) als die "Hauptform" und ihre Klasse als die „Hauptklasse" der Diskriminante $D$.

Endlich haben wir mit Rücksicht auf die weitere Entwicklung noch folgenden Satz zu nennen: In jeder Klasse ursprïnglicher Formen der Diskriminante $D$ ist eine Form $(a, b, c)$ nachweisbar, die einen gegen die beliebig vorgeschriebene rationale ganze positive Zahl $n$ teilerfremden ersten Koeffizienten a hat. Zum Beweise entnebmen wir der Klasse eine beliebige Form $\left(a^{\prime}, b^{\prime}, c^{\prime}\right)$ und verstehen unter $p_{1}, p_{2}, \ldots, p_{v}$ die verschiedenen Primfaktoren von $n$. Wir setzen sodann:

$$
\alpha=p_{1}^{\lambda_{1}} \cdot p_{2}^{\lambda_{2}} \cdots p_{v}^{\lambda_{y}}, \quad \gamma=p_{1}^{\mu_{1}} \cdot p_{2}^{\mu_{2}} \cdots p_{v}^{\mu_{\nu}}
$$

mit folgender Bedeutung der Exponenten:

$$
\left\{\begin{array}{lllll}
\lambda_{k}=0, & \mu_{k}=1 & \text { für } & a^{\prime} \neq 0 & \\
\lambda_{k}=1, & \mu_{k}=0 & \text { für } & a^{\prime} \equiv 0, & c^{\prime} \neq 0 \\
\lambda_{k}=0, & \mu_{k}=0 & \text { für } & a^{\prime} \equiv 0, & c^{\prime} \equiv 0
\end{array}\right\}\left(\bmod p_{k}\right),
$$

woraus hervorgeht, daß $\alpha$ und $\gamma$ teilerfremd sind. Von den drei Fällen (14) liegt für jeden Primteiler $p_{k}$ von $n$ einer und nur einer vor. Für $\alpha$ und $\gamma$ bestimmen wir irgend zwei, die Gleichung $\alpha \delta-\beta \gamma=1$ befriedigende rationale ganze Zahlen $\beta, \delta$ und berechnen nach (5) die mit $\left(a^{\prime}, b^{\prime}, c^{\prime}\right)$ äquivalente Form $(a, b, c)$. Man zeigt dann in der Tat leicht, daß $a$ durch kein $p_{k}$ teilbar ist, wobei für den dritten Fall (14) in Betracht kommt, dab $a^{\prime}, b^{\prime}, c^{\prime}$ nicht zugleich durch $p_{k}$ teilbar sind.

\section{$\S 7$. Beziehung zwischen den Zweigidealen $\mathfrak{a}_{n}$ und den quadratischen Formen.}

Es sei $\mathfrak{a}_{n}$ irgendeines unserer Zweigideale und $\mathfrak{a}$ das zugehörige Stammideal; der Fall $n=1$, wo $\mathfrak{a}_{n}=\mathfrak{a}$ ist, sei mit eingeschlossen. Eine positive Basis von $\mathfrak{a}_{n}$ möge durch $\omega_{1}, \omega_{9}$ gebildet werden. Um in (3) 
S. 136 die $m_{1}, m_{2}$ als „willkürliche" rationale ganze Zahlen zu kennzeichnen, schreiben wir $m_{1}=-y, m_{2}=x$, so daß die Zahlen des Ideals $\mathfrak{a}_{n}$ durch $\left(x \omega_{2}-y \omega_{1}\right)$ gegeben sind. Die Norm der einzelnen Zahl von $\mathfrak{a}_{n}$ ist dann:

$$
N\left(x \omega_{2}-y \omega_{1}\right)=\omega_{2} \bar{\omega}_{2} x^{2}-\left(\omega_{1} \bar{\omega}_{2}+\omega_{2} \bar{\omega}_{1}\right) x y+\omega_{1} \bar{\omega}_{1} y^{2},
$$

wo $\bar{\omega}_{1}, \bar{\omega}_{2}$ die zu $\omega_{1}, \omega_{2}$ konjugierten Zahlen sind. Die in (1) rechts auftretenden rationalen ganzen Zahlen $\omega_{2} \bar{\omega}_{2},-\left(\omega_{1} \bar{\omega}_{2}+\omega_{2} \bar{\omega}_{1}\right), \omega_{1} \bar{\omega}_{1}$ gehören dem Produkte $\mathfrak{a} \cdot \overline{\mathfrak{a}}$ des Stammideals $\mathfrak{a}$ mit seinem konjugierten Ideale $\overline{\mathfrak{a}}$ an. Da dieses Produkt das Hauptideal $[N(\mathfrak{a})]$ ist (s. (1) S. 111), so sind die drei Zahlen $\omega_{2} \bar{\omega}_{2}, \ldots$ durch die rationale ganze positive Zahl $N(\mathfrak{a})$ teilbar. Schreiben wir also:

$$
\omega_{2} \bar{\omega}_{2}=N(\mathfrak{a}) \cdot a, \quad-\omega_{1} \bar{\omega}_{2}-\omega_{2} \bar{\omega}_{1}=N(\mathfrak{a}) \cdot b, \quad \omega_{1} \bar{\omega}_{1}=N(\mathfrak{a}) \cdot c,
$$

so sind $a, b, c$ drei rationale ganze Zahlen, von denen die erste und dritte positiv sind. Die Gleichung (1) schreibt sich in die Gestalt um:

$$
N\left(x \omega_{2}-y \omega_{1}\right)=N(\mathfrak{a})\left(a x^{2}+b x y+c y^{2}\right),
$$

wo wir rechts in der Klammer eine ganzzahlige binäre quadratische Form gewonnen haben. Wir bezeichnen die Diskriminante $\left(b^{2}-4 a c\right)$ dieser Form $(a, b, c)$ vorläufig durch $D^{\prime}$, um durch $D$ wie früher die Diskriminante des Zweiges $e_{n}$ zu bezeichnen.

Zum Zwecke einer ersten Angabe über $D^{\prime}$ stellen wir die $\omega_{1}, \omega_{2}$ durch die in (3) S. 123 gegebene Basis $1, \theta$ von $e_{n}$ in der Gestalt:

$$
\omega_{1}=e_{0}^{(1)}+e_{1}^{(1)} \theta, \quad \omega_{2}=e_{0}^{(2)}+e_{1}^{(2)} \theta
$$

dar und erhalten aus (2) leicht:

$$
N(\mathfrak{a})^{2} \cdot D^{\prime}=N(\mathfrak{a})^{2}\left(b^{2}-4 a c\right)=\left(\omega_{1} \bar{\omega}_{2}-\omega_{2} \bar{\omega}_{1}\right)^{2}=\left(e_{0}^{(1)} e_{1}^{(2)}-e_{1}^{(1)} e_{0}^{(2)}\right)^{2} \cdot D,
$$

wo $D=n^{2} \cdot \mathbb{D}$ die Diskriminante von $\mathfrak{e}_{n}$ ist. Da $D<0$ ist und $a>0$, $c>0$ schon festgestellt wurde, so handelt es sich in (3) rechts um eine. positive Form $(a, b, c)$ negativer Diskriminante $D^{\prime}$.

Der Übergang von $\omega_{1}, \omega_{2}$ zu irgendeiner positiven Basis $\omega_{1}^{\prime}, \omega_{2}^{\prime}$ von $\mathfrak{a}_{n}$ wird durch die Substitution (2) S. 135 vermittelt, die für die konjugiert komplexen Werte $\bar{\omega}_{1}, \bar{\omega}_{2}$ die gleiche Substitution nach sich zieht. Bei Zugrundelegung der Basis $\omega_{1}^{\prime}$, $\omega_{2}^{\prime}$ führt die Regel (2) zur Form $\left(a^{\prime}, b^{\prime}, c^{\prime}\right)$, deren Koeffizienten sich nach (4) S. 138 aus $a, b, c$ und den Substitutionskoeffizienten berechnen. Also besteht der Satz: Den unendlich vielen positiven Basen des Zweigideals $\mathfrak{a}_{n}$ gehören die unendlich vielen Formen der zu $(a, b, c)$ gehörenden Formklasse $\mathfrak{F}$ umkehrbar eindeutig zu, so daß dem Zweigideale $\mathfrak{a}_{n}$ die Formklasse zugeordnet erscheint.

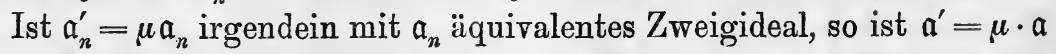
das zu $\dot{a}_{n}^{\prime}$ gehörende Stammideal. Auf Grund der Regel (7) S. 103 zeigt 
man (unter Spaltung von $\mu$ in den Quotienten zweier ganzer Zahlen) leicht die Regel $N\left(\mathfrak{a}^{\prime}\right)=\mu \bar{\mu} \cdot N(\mathfrak{a})$, wo $\bar{\mu}$ die zu $\mu$ konjugierte Zahl ist. Eine erste Basis von $\mathfrak{a}_{n}^{\prime}$ haben wir in $\omega_{1}^{\prime}=\mu \omega_{1}, \omega_{2}^{\prime}=\mu \omega_{2}$, unter $\omega_{1}, \omega_{2}$ eine solche von $\mathfrak{a}_{n}$ verstanden. Für die zu $\omega_{1}^{\prime}, \omega_{2}^{\prime}$ gehörende quadratische Form $\left(a^{\prime}, b^{\prime}, c^{\prime}\right)$ folgt aus (2):

$$
a^{\prime} N\left(\mathfrak{a}^{\prime}\right)=a^{\prime} \cdot \mu \bar{\mu} N(\mathfrak{a})=\omega_{2}^{\prime} \bar{\omega}_{2}^{\prime}=\mu \bar{\mu} \cdot \omega_{2} \bar{\omega}_{2}, \ldots
$$

so daß der Vergleich mit (2) zu $a^{\prime}=a, b^{\prime}=b, c^{\prime}=c$ führt: Den gesamten Idealen der $z u \mathfrak{a}_{n}$ gehörenden Klasse $\mathfrak{A}$ ist hiernach ein und dieselbe Formklasse $\mathfrak{F}$ zugeordnet, so daß der Idealklasse $\mathfrak{A}$ die Formlilasse $\mathfrak{F}$ eindeutig zugehört.

Es ist nun die Diskriminante $D^{\prime}$ und der Teiler $t$ der Formklasse $\mathfrak{F}$ festzustellen. Nach den gewonnenen Ergebnissen dürfen wir zu diesem Zwecke der Idealklasse $\mathfrak{A}$ ein beliebiges Zweigideal $\mathfrak{a}_{n}$ entnehmen und dem gewählten Ideale $\mathfrak{a}_{n}$ irgendeine seiner positiven Basen zugrunde legen. $\mathrm{Zu}$ zweckmäßigen Auswahlen führt die folgende Überlegung:

Die größte rationale ganze positive Zahl, durch die alle Zahlen des Stammideals a teilbar sind, heiße der "größte rationale Divisor" oder "Teiler" von $\mathfrak{a}$ und werde durch $d$ bezeichnet. Ist. $d=1$, so heiße das Ideal a „ursprünglich", für $d>1$ werde a ,abgeleitet" genannt. Aus den Grundeigenschaften eines Ideals folgt, daß die Zahlen von $\mathfrak{a}$, durch $d$ geteilt, wieder die Zahlen eines Ideals $\mathfrak{a}^{(0)}$ bilden, das dann ursprünglich ist. Da $\mathfrak{a}=[d] \cdot \mathfrak{a}^{(0)}$ teilerfremd $\mathrm{zu}[n]$ ist, so ist $d$ teilerfremd zu $n$ und also im Strahle $\left\{\right.$ enthalten. Die beiden Stammideale $\mathfrak{a}$ und $\mathfrak{a}^{(0)}$ sind also ,im Zweige $\mathfrak{e}_{n}$ äquivalent", und somit sind die zugehörigen Zweigideale $\mathfrak{a}_{n}$ und $\mathfrak{a}_{n}^{(0)}$ schlechthin äquivalent. Es ist hiernach statthaft, für die Untersuchung der Diskriminante $D^{\prime}$ und des Teilers $t$ von $(a, b, c)$ ein Ideal $\mathfrak{a}_{n}$ der Idealklasse $\mathfrak{A}$ zu entnehmen, dessen zugehöriges Stammideal $\mathfrak{a}$ ursprünglich ist.

Es besteht nun der folgende auch für sich bemerkenswerte Satz: Ist $\mathfrak{a}$ ein ursprüngliches Ideal, so ist $N(\mathfrak{a})$ die kleinste rationale ganze positive in a auftretende $Z a h l_{.}{ }^{1}$ ) Bezeichnen wir nämlich mit a die kleinste rationale ganze positive $\mathrm{Zahl}^{2}$ ) von $\mathfrak{a}$, so sind alle rationalen ganzen Zahlen von $\mathfrak{a}$ Vielfache dieser Zahl $a$. Insbesondere ergibt sich für die Zahl $N(\mathfrak{a})$ von $\mathfrak{a}$ eine Darstellung $N(\mathfrak{a})=a \cdot c$, wo $c$ rational und ganz ist. Hieraus folgt:

$$
\mathfrak{a} \cdot \overline{\mathfrak{a}}=[N(\mathfrak{a})]=[a] \cdot[c] .
$$

1) Übrigens erweitert man diesen Satz leicht $\mathrm{zu}$ dem folgenden noch etwas allgemeineren Satze: Die kleinste in einem Ideale $\mathfrak{a}$ auftretende rationale ganze positive Zahl ist $d^{-1} \cdot N(\mathfrak{a})$, unter $d$ den größten rationalen Divisor von $\mathfrak{a}$ verstanden.

2) Die Wahl der Bezeichnung $a$ ist deshalb zweckmäBig, weil diese Zahl hernach der erste Koeffizient einer quadratischen Form wird. 
Da $\mathfrak{a}$ ein Teiler von $[a]$ ist, so können wir $[a]=\mathfrak{a} \cdot \mathfrak{b}$ setzen und folgern hieraus, da $[a]$ sich selbst konjugiert ist $[a]=\overline{\mathfrak{a}} \cdot \overline{\mathfrak{b}}$, unter $\overline{\mathfrak{b}}$ das zu $\mathfrak{b}$ konjugierte Ideal verstanden. Tragen wir $\overline{\mathfrak{a}} \cdot \overrightarrow{\mathfrak{b}}$ für $[a]$ in (5) ein und heben die entstehende Gleichung durch $\overline{\mathfrak{a}}$, so erhalten wir $\mathfrak{a}=[c] \cdot \overline{\mathfrak{b}}$. Also geht $c$ im größten rationalen Teiler 1 von $\mathfrak{a}$ auf, so daß $c=1$ und damit $a=N(\mathfrak{a})$ folgt; wie zu beweisen war.

Es zerfallen nun die Zahlen von $\mathfrak{e} \bmod \mathfrak{a}$ in $N(\mathfrak{a})=a$ Zahlklassen, die wir durch die offenbar inkongruenten Zahlen $0,1,2, \ldots, a-1$ repräsentieren können. Die zweite in der Basis des Zweiges $e_{n}$ auftretende Zahl $\theta$, die durch:

$$
\theta=\frac{-1+i \sqrt{|D|}}{2} \text { oder } \theta=\frac{i \sqrt{|D|}}{2}
$$

gegeben ist, je nachdem $D \equiv 1$ oder $\equiv 0(\bmod 4)$ gilt, möge in die durch $m$ repräsentierte Klasse gehören:

$$
\theta \equiv m \quad(\bmod \mathfrak{a}),
$$

wo also $m$ eine bestimmte der Zahlen $0,1,2, \ldots, a-1$ ist. Setzen wir $b=2 m+1$ oder $b=2 m$, je nachdem $D \equiv 1$ oder $\equiv 0(\bmod 4)$ gilt, so haben wir in $b$ eine der Kongruenz $b \equiv D(\bmod 2)$ genügende rationale ganze Zahl, und es folgt:

$$
\theta-m=\frac{-b+i \sqrt{|D|}}{2} \equiv 0 \quad(\bmod \mathfrak{a})
$$

so daß die Zahl $\frac{-b+i \sqrt{|D|}}{2}$ dem Ideale a angehört.

Statt $1, \theta$ können wir nun auch $1, \theta-m$ als Basis für $\mathfrak{e}_{n}$ benutzen. Die Zahlen von $e_{n}$ sind also in der Gestalt:

$$
e_{0}+e_{1} \frac{-b+i \sqrt{|D|}}{2}
$$

mittelst rationaler ganzer $e$ darstellbar. Soll die Zahl (9) von $\mathbf{e}_{n}$ auch in $\mathfrak{a}$ enthalten sein und also eine Zahl des Zweigideals $\mathfrak{a}_{n}$ liefern, so muß wegen (8) die Zahl $e_{0}$ in a vorkommen, also ein Vielfaches von $a$ darstellen. Eine Basis des Zweigideals $a_{n}$ erhalten wir somit in den beiden Zahlen:

$$
\omega_{1}=\frac{-b+i \sqrt{|D|}}{2}, \quad \omega_{2}=a .
$$

Mit der Zahl $\omega_{1}$ ist auch $N\left(\omega_{1}\right)$ in $\mathfrak{a}$ enthalten und stellt als rational und ganz ein Multiplum $a c$ von $a$ dar; es gilt also:

$$
N\left(\frac{-b+i \sqrt{|D|}}{2}\right)=\frac{b^{2}-D}{4}=a c
$$

wo auch $c$ rational, ganz und positiv ist. $\mathrm{Da} N(\mathfrak{a})=a$ ist, so berechnet sich aus (2) als die zur Basis (10) gehörende quadratische Form $(a, b, c)$. 
Zur Idealklasse $\mathfrak{A}$ gehört demnach eine Formklasse $\mathfrak{F}$, deren Diskriminante gleich der Diskriminante $D$ des Zweiges $\mathrm{e}_{n}$ ist.

Wir können endlich zeigen, daß die drei Zahlen $a, b, c$ keinen gemeinsamen Teiler $t>1$ besitzen. Da nämlich $\mathfrak{a}$ und also $\overline{\mathfrak{a}}$ teilerfremd gegen $[n]$ sind, so sind auch $a \cdot \overline{\mathfrak{a}}=[a]$ und $[n]$ teilerfremd, so dab $a$ und $n$ teilerfremde rationale ganze Zahlen sind. Hätten nun $a, b, c$ den ungeraden Primfaktor $p$ gemein, so würde $p^{2}$ in $b^{2}-4 a c=D=n^{2} \mathrm{D}$ aufgehen. Es wäre also, da D durch kein ungerades Quadrat teilbar ist, $p$ auch in $n$ enthalten, was aber der Tatsache teilerfremder $a, n$ widersprechen würde. Wären aber $a, b, c$ zugleich durch 2 teilbar, so wäre $n$ als teilerfremd zu $a$ ungerade. Da $D$ und also D durch 4 teilbar wäre, so würde nach $\mathrm{S} .121$ entweder $\mathbf{D} \equiv 8$ oder $\mathbf{D} \equiv 12(\bmod 16)$ gelten, woraus wegen $n^{2} \equiv 1(\bmod 4)$ auch $D \equiv 8$ bzw. $\equiv 12(\bmod 16)$ folgen würde. Dies steht im Widerspruche dazu, daB bei drei geraden Zahlen $a, b, c$ notwendig:

$$
D=b^{2}-4 a c \equiv b^{2} \equiv 0 \text { oder } \equiv 4 \quad(\bmod 16)
$$

zutrifft. Also ist auch der gemeinsame Teiler 2 von $a, b, c$ ausgeschlossen.

Unter Zusammenfassung aller Ergebnisse haben wir folgenden für $n=1$ und $n>1$ geltenden Satz: Jeder Idealklasse $\mathfrak{A}$ im $n^{\text {ten }}$ Zweige $\mathfrak{e}_{n}$ des Körpers $\Re$ der negativen Diskriminante $\mathbb{D}$ ist eindeutig cine bestimmte Klasse $\mathfrak{F}$ ussprünglicher positiver quadratischer Formen der Zweigdiskriminante $D=n^{2} \mathbf{D}$ zugeordnet, und swar werden von irgendeinem Ideale $\mathfrak{a}_{n}$ der Klasse $\mathfrak{A}$ aus die gesamten Formen $\left(a x^{2}+b x y+c y^{2}\right)$ von $\mathfrak{F}$ als die durch $N(\mathfrak{a})=N\left(\mathfrak{e} \cdot \mathfrak{a}_{n}\right)$ geteilten Normen der Zahlen $\left(x \omega_{3}-y \omega_{1}\right)$ von $\mathfrak{a}_{n}$ erhalten, indem man nach und nach alle positiven Basen $\omega_{1}, \omega_{2}$ zur Darstellung dieser Zahlen heransieht.

Der gewonnene Satz ist umkehrbar. Man kann zunächst beweisen, daß man eine beliebige Klasse $\mathfrak{F}$ ursprünglicher positiver Formen der Zweigdiskriminante $D$ von einer geeigneten Idealklasse $\mathfrak{A}$ des $n^{\text {ten }} Z$ weiges aus gewinnt. Da wir soeben alle Formen der Klasse $\mathfrak{F}$ den verschiedenen Basen $\omega_{1}, \omega_{2}$ von $\mathfrak{a}_{n}$ entsprechend fanden, so dürfen wir beim Versuch der Umkehrung der Entwicklung der beliebig vorgelegten Klasse $\mathfrak{F}$ irgendeine Form $(a, b, c)$ entuehmen. Wir wählen, entsprechend dem Schlußsatze von 66 , S. 141, eine Form $(a, b, c)$ mit einem gegen $n$ teilerfremden $a$. Wir erklären sodann zwei Zahlen $\omega_{1}, \omega_{2}$ wie in (10). Beide Zahlen $\omega_{1}, \omega_{2}$ sind ganzzahlig, und zwar $\omega_{1}$ deshalb, weil $b \equiv D(\bmod 2)$ gilt; zugleich gehören beide Zahlen dem Zweige $\mathfrak{e}_{n}$ an. Wir bilden nun das System aller Zahlen:

$$
x \omega_{2}-y \omega_{1}=a x+\frac{b-i V|D|}{2} y,
$$

wo $x, y$ alle Paare rationaler ganzer Zahlen durchlaufen, und können 
leicht zeigen, daß diese offenbar auch durchweg in $\mathfrak{e}_{n}$ enthaltenen Zahlen ein Zweigideal $\mathfrak{a}_{n}$ liefern. Das Zutreffen der Eigenschaft 1 eines Zweigideals (S. 124) ist einleuchtend. Zur Prüfung der Eigenschaft 2 können wir $1, \omega_{1}$ an Stelle von $1, \theta$ als Basis von $e_{n}$ benutzen. Es genügt dann zu zeigen, daß $\omega_{1}^{2}$ und $\omega_{1} \omega_{2}$ im Systeme (12) enthalten sind, weil damit das Produkt irgendeiner Zahl $\eta_{n}$ von $e_{n}$ mit einer beliebigen Zahl (12) wieder diesem Systeme angehört. Nun erweist sich $\omega_{1} \omega_{2}=a \omega_{1}$ unmittelbar als dem Systeme (12) angehörig; dasselbe folgt aber für $\omega_{1}^{2}$ aus der Gleichung:

$$
\omega_{1}^{2}=-a c-b \frac{-b+i \sqrt{|D|}}{2}=-b \omega_{1}-c \omega_{2} .
$$

Die Eigenschaft 3 der Zweigideale fordert, daß jede Zahl $\eta_{n}$ von $\mathfrak{e}_{n}$ als Summe:

$$
\eta_{n}=\left(x \omega_{2}-y \omega_{1}\right)+n \eta
$$

einer Zahl des Systems (12) und einer solchen des Hauptideals [ $n]$ darstellbar ist. Es genügt, diese Darstellbarkeit für die beiden Basiszahlen $\eta_{n}=1$ und $\eta_{n}=\omega_{1}$ zu zeigen. Für $\eta_{n}=\omega_{1}$ haben wir in (13) zu setzen $x=0, y=-1, \eta=0$; für $\eta_{n}=1$ bestimme man $x$ aus der wegen teilerfremder $a, n$ lösbaren Kongruenz $a x \equiv 1(\bmod n)$, setze $y=0$ und findet für $\eta$ eine rationale ganze Zahl. Die Zahlen (13) bilden also tatsächlich ein Zweigideal $\mathfrak{a}_{n}$.

Der Basis $\omega_{1}, \omega_{2}$ dieses Zweigideals entspricht nun auf Grund von (2) eine ursprüngliche quadratische Form, deren Koeffizienten die von ihrem größten gemeinschaftlichen Teiler befreiten rationalen ganzen Zahlen $\omega_{2} \bar{\omega}_{2},-\omega_{1} \bar{\omega}_{2}-\omega_{2} \bar{\omega}_{1}, \omega_{1} \bar{\omega}_{1}$ sind. Durch Eintragen der Werte (10) für $\omega_{1}, \omega_{2}$ findet man als Form $(a, b, c)$ wieder und als jenen gröBten gemeinsamen Teiler, der nach (2) zugleich die Norm des Stammideals $\mathfrak{a}=\mathfrak{e} \cdot \mathfrak{a}_{n}$ ist, die Zahl $a$. Also wird in der Tat die vorgelegte Klasse $\mathfrak{F}$ von einer unserer Idealklassen $\mathfrak{U}$ geliefert.

Wir können endlich zeigen, daß $\mathfrak{F}$ auch nur von einer Idealklasse $\mathfrak{A}$ geliefert wird. Ist nämlich $\omega_{1}^{\prime}, \omega_{2}^{\prime}$ die Basis irgendeines Zweigideals, die nach (2) die zuletzt betrachtete Form $(a, b, c)$ ergibt, so gilt für die durch die Form eindeutig bestimmten Basisquotienten:

$$
\omega_{1}^{\prime}: \omega_{2}^{\prime}=\omega_{1}: \omega_{2}=\frac{-b+i \sqrt{|D|}}{2}: a \text {. }
$$

Wir schreiben demnach $\omega_{1}^{\prime}=\mu \omega_{1}, \omega_{2}^{\prime}=\mu \omega_{2}$ mit einem Proportionalitätsfaktor $\mu$, für den wir die Darstellung haben:

$$
\mu=\frac{\omega_{1}^{\prime}}{\omega_{1}}=\frac{\omega_{2}^{\prime}}{\omega_{2}}=\frac{\omega_{2}^{\prime}}{a}
$$

Die im letzten Nenner stehende Zahl $a$ ist teilerfremd gegen $n$ und in $\mathrm{e}_{n}$ enthalten. Auch der Zähler $\omega_{2}^{\prime}$ ist in $\mathfrak{e}_{n}$ enthalten. Wir nehmen nun an, 
$\mathrm{da} B\left[\omega_{2}^{\prime}\right]$ und $[n]$ mindestens ein Primideal $\mathfrak{p}$ als Faktor gemein haben. Aus $[a] \cdot\left[\omega_{1}^{\prime}\right]=\left[\omega_{2}^{\prime}\right] \cdot\left[\omega_{1}\right]$ würde dann wegen teilerfremder $a, n$ folgen $\mathrm{da} B$ auch $\left[\omega_{1}^{\prime}\right]$ den Faktor $\mathfrak{p}$ hat. Mit $\omega_{1}^{\prime}$ und $\omega_{2}^{\prime}$ ist aber auch jede Zahl, von $\mathfrak{a}^{\prime}=\mathfrak{e} \cdot \mathfrak{a}_{n}^{\prime}$. in $\mathfrak{p}$ enthalten, $\mathfrak{d}$. h. $\mathfrak{a}^{\prime}$ hätte selbst den Faktor $\mathfrak{p}$ mit $[n]$ gemein, während doch das zu einem Zweigideale $\mathfrak{a}_{n}^{\prime}$ gehörende Stammideal $\mathfrak{a}^{\prime}$ teilerfremd gegen $[n]$ ist. Also ist auch $\omega_{2}^{\prime}$ teilerfremd gegen $n$, so dab wir in $\mu$ eine Zahl des Strahles $j$ und damit in $\mathfrak{a}_{n}^{\prime}=\mu \mathfrak{a}_{n}$ ein mit $\mathfrak{a}_{n}$ äquivalentes Ideal erkenmen. Jede Formklasse $\mathfrak{F}$ wird also nur von einer Idealklasse geliefert.

Wir haben so den Satz gewonnen: Die Idealklassen $\mathfrak{A}$ des $n^{\text {ten }}$ Zweiges $\mathfrak{e}_{n}$ vom quadratischen Körper $\AA$ der negativen Diskriminante Klassen $\mathfrak{F}$ ursprünglicher positiver quadratischer Formen der negativen Zweigdiskriminante $D=n^{2} \mathbb{B}$ entsprechen in der oben näher erörterten Weise einander umkehrbar eindeutig. Insbesondere geht hieraus hervor, daß die Anzahl der Klassen ursprüngiicher positiver Formen der negativen Diskriminante $D$ gleich der Anzahl $h(|D|)$ der Idealklassen des $n^{\text {ten }}$ Zweiges ist. Für die Anzahlen $h(|D|)$ und $h(|\mathbf{D}|)$ der Formklassen der beiden Diskriminanten $D=n^{2}$ und D bleibt demnach die in (1) S. 134 aufgestellte Relation gültig.

Die „Hauptklasse“ $\mathfrak{A}_{0}$ wurde nach S. 133 von den mit $\mathfrak{e}_{n}$ äquivalenten Idealen geliefert. Benutzen wir wieder die Basis $1, \theta$ für $e_{n}$, so finden wir als zugehörige Form sofort $\left(1,1, \frac{1-D}{4}\right)$ bzw. $\left(1,0, \frac{-D}{4}\right)$, je nachdem $D \equiv 1$ oder $\equiv 0(\bmod 4)$ ist. Wir gelangen also zur Hauptform, so daß der Hauptllasse $\mathfrak{A}_{0}$ der Ideale $\mathfrak{a}_{n}$ die Hauptklasse $\mathfrak{F}_{0}$ der Formen zugeordnet ist.

Eine später zur Verwendung kommende Folgerung aus der Gleichung (1) S. 134 möge hier gleich noch angeschlossen werden. Ist $D \equiv 1(\bmod 4)$, so ist auch $D \equiv 1(\bmod 4)$, und $n$ ist ungerade. Wir wollen in diesem Falle eine Beziehung zwischen den Klassenanzahlen $h(|D|)$ und $h\left(\left|D^{\prime}\right|\right)$ $=h(4|D|)$ der Diskriminanten $D$ und $D^{\prime}=4 D$ aufstellen.

Ist erstlich $D=-3$ und also Stammdiskriminante, so folgt aus (1) S. 134:

$$
h(12)=h(3) \cdot \frac{1}{3} \cdot 2\left(1-\frac{(-3,2)}{2}\right),
$$

wo $(-3,2)$ das S. 118 erklärte Symbol ist. Da die Kongruenz $x^{2} \equiv-3$ (mod 8) keine rationale ganze lösung $x$ hat, so ist $(-3,2)=-1$, und wir finden $h(12)=h(3)$. Ist $D$ eine von -3 verschiedene Stammdiskriminante, so gilt nach (1) S. 134:

$$
h(4|D|)=h(|D|) \cdot 2\left(1-\frac{(D, 2)}{2}\right) .
$$

Ist hingegen $D$ eine Zweigdiskriminante, $\mathrm{D}=n^{-2} \cdot D$ aber die zugehörige Stammdiskriminante, so gelten die beiden Gleichungen: 


$$
\begin{gathered}
h(|D|)=h(|\mathbf{D}|) \cdot \tau \cdot n \prod^{(n)}\left(1-\frac{(\mathbf{D}, p)}{p}\right), \\
h(4|D|)=h(|\mathbf{D}|) \cdot \tau \cdot 2 n \prod^{(2 n)}\left(1-\frac{(\mathbf{D}, p)}{p}\right) .
\end{gathered}
$$

Da $n$ ungerade ist, so hat das Produkt auf der rechten Seite der letzten Gleichung neben den im Produkte der ersten Gleichung auftretenden Faktoren noch den zu $p=2$ gehörenden weiteren Faktor. Die Division der letzten Gleichung durch die vorletzte führt also zur Relation (14) zurück. Die Kongruenz $x^{2} \equiv D(\bmod 8)$ ist nun aber in rationalen ganzen Zahlen $x$ lösbar oder nicht lösbar, je nachdem $D \equiv 1$ oder $\equiv 5(\bmod 8)$ gilt. Im ersten Falle ist also $(D, 2)=+1$, im zweiten $(D, 2)=-1$. So ergibt sich aus $(14)$ der Satz: Ist $D=-3$ oder $\equiv 1(\bmod 8)$, so sind die Klassenanzahlen $h(4|D|)$ und $h(|D|)$ einander gleich; ist $D \equiv 5(\bmod 8)$, jedoch nicht gleich -3 , so ist $h(4|D|)$ das Dreifache der Klassenanzahl $h(|D|)$.

\section{Komposition der quadratischen Formen.}

Die Multiplikation der Ideale $\mathfrak{a}_{n}$ im $\boldsymbol{n}^{\text {ten }} /$ weige $\mathfrak{e}_{n}$ des quadratischen Körpers $\mathfrak{\Re}$ führte uns S. 134 ff. zur "MLultiplikation der Ideallilassen“. Die Grundlage dieser Entwicklung war der Satz, daB das Produkt eines beliebigen Ideals der Klasse $\mathfrak{A}$ und eines beliebigen der Klasse $\mathfrak{I}^{\prime}$ ein Ideal einer durch $\mathfrak{A}$ und $\mathfrak{U}^{\prime}$ eindeutig bestimmten dritten Klasse $\mathfrak{I}^{\prime \prime}$ liefert, die wir dann selbst als Produkt $\mathfrak{A} \cdot \mathfrak{\mathfrak { Y } ^ { \prime }}$ der beiden gegebenen Klassen $\mathfrak{A}$ und $\mathfrak{H}^{\prime}$ bezeichneten. Gegenüber dieser Multiplikation bildeten die $\vec{h}$ Idealklassen $\mathfrak{A}_{0}, \mathfrak{A}_{1}, \ldots, \mathfrak{A}_{h-1}$ von $\mathfrak{e}_{n}$ eine Abelsche Gruppe $G_{h}$ der Ordnung $h$, in der das Einheitselement von der Hauptklasse $\mathfrak{A}_{0}$ geliefert wird.

Die Multiplikation der Idealklassen $\mathfrak{A}_{0}, \mathfrak{A}_{1}, \ldots, \mathfrak{A}_{h-1}$ des $n^{\text {ten }}$ Zweiges $\mathfrak{e}_{n}$ ergibt nun, auf die den $\mathfrak{A}$ eindeutig zugeordneten Klassen ursprünglicher positiver quadratischer Formen der Zweigdiskriminante $D$ übertragen, die Lehre von der "Komposition" dieser Formklassen $\mathfrak{F}_{0}, \mathfrak{F}_{1}, \ldots$, $\mathfrak{F}_{h-1}$, die von $G a u \beta$ begründet ist ${ }^{1}$ ), und die sich in etwas kürzerer Gestalt im Supplement $X$ der "Vorlesungen über Zahlentheorie" von DirichletDedekind ${ }^{2}$ ) dargestellt findet. Entsprechen den drei Idealklassen $\mathfrak{A}, \mathfrak{I}^{\prime}$ und $\mathfrak{U}^{\prime \prime}=\mathfrak{A} \cdot \mathfrak{U}^{\prime}$ die Formklassen $\mathfrak{F}, \mathfrak{F}^{\prime}$ und $\mathfrak{F}^{\prime \prime}$, so sagt man, die Klasse $\mathfrak{F}^{\prime \prime}$ entstehe aus $\mathfrak{F}$ und $\mathfrak{F}^{\prime}$ durch „Komposition" und bezeichnet $\mathfrak{F}^{\prime \prime}$ wieder symbolisch durch das Produkt $\mathfrak{F} \cdot F^{\prime}$, wobei entsprechend den Produkten der Idealklassen das kommutative Gesetz $\mathfrak{F} \cdot \mathfrak{F}^{\prime}=\mathfrak{F}^{\prime} \cdot \mathfrak{F}$ gilt. Alle $h$ Formklassen $\mathfrak{F}_{0}, \mathfrak{F}_{1}, \ldots, \mathfrak{F}_{h-1}$ bilden dann gegenüber der Komposition

1) In Art. 234 der "Disquisitiones arithmeticae".

2) S. $387 \mathrm{ff}$. der 4. Auflage. 
die Elemente einer Alslschen Gruppe $G_{h}$ der Ordnung $h$, in der das Einheitselement von der Hauptklasse $\mathfrak{F}_{0}$ geliefert wird.

Die Komposition der Klassen wird von Gau $\beta$ auf diejenige der Formen gegründet, die „Komposition der Formen" aber wird direkt, und zwar mit einem erheblichen Rechnungsaufwande durchgeführt. Der einfachste Ansatz zur Behandlung der Komposition der Formen wird von der Multiplikation der Ideale $\mathfrak{a}_{n}$ geliefert. Der Komposition zweier Formen $(a, b, c)$ und $\left(a^{\prime}, b^{\prime}, c^{\prime}\right)$ entsprechend haben wir hier nicht nur zwei ihnen zugehörige Ideale $\mathfrak{a}_{n}$ und $\mathfrak{a}_{n}^{\prime}$ zu wählen, sondern auch für diese Ideale zwei besondere Basen $\omega_{1}, \omega_{2}$ und $\omega_{1}^{\prime}, \omega_{2}^{\prime}$ zugrunde zu legen. Die Produkte der Zahlen $\left(x \omega_{2}-y \omega_{1}\right)$ und $\left(x^{\prime} \omega_{3}^{\prime}-y^{\prime} \omega_{1}^{\prime}\right)$ von $\mathfrak{a}_{n}$ und $\mathfrak{a}_{n}^{\prime}$ sind dann im Produkte $\mathfrak{a}_{n}^{\prime \prime}=\mathfrak{a}_{n} \cdot \mathfrak{a}_{n}^{\prime}$ enthalten. Wenn wir also für $\mathfrak{a}_{n}^{\prime \prime}$ irgendeine Basis $\omega_{1}^{\prime \prime}, \omega_{2}^{\prime \prime}$ auswählen, so gibt es für vier beliebige rationale ganze Zahlen $x, y, x^{\prime}, y^{\prime}$ stets zwei bestimmte rationale ganze Zahlen $x^{\prime \prime}, y^{\prime \prime}$, die der Gleichung genügen:

$$
\left(x \omega_{2}-y \omega_{1}\right)\left(x^{\prime} \omega_{2}^{\prime}-y^{\prime} \omega_{1}^{\prime}\right)=x^{\prime \prime} \omega_{2}^{\prime \prime}-y^{\prime \prime} \omega_{1}^{\prime \prime} .
$$

Aus dieser Grundgleichung lassen sich alle Formeln der Kompositionstheorie entwickeln. Wir folgern zunächst für die $\mathrm{zu} \omega_{1}, \omega_{2}, \ldots$ konjugierten Zahlen $\bar{\omega}_{1}, \bar{\omega}_{2}, \ldots$ :

$$
\left(x \bar{\omega}_{2}-y \bar{\omega}_{1}\right)\left(x^{\prime} \bar{\omega}_{2}^{\prime}-y^{\prime} \bar{\omega}_{1}^{\prime}\right)=x^{\prime \prime} \bar{\omega}_{2}^{\prime \prime}-y^{\prime \prime} \bar{\omega}_{1}^{\prime \prime} .
$$

Nach einem S. 132 aufgestellten Satze gehört zum Produkte $\mathfrak{a}_{n} \cdot \mathfrak{a}_{n}^{\prime}$ als Stammideal das Produkt $\mathfrak{a} \cdot \mathfrak{a}^{\prime}$ der zu $\mathfrak{a}_{n}$ und $\mathfrak{a}_{n}^{\prime}$ gehörenden Stammideale. Da nun $N\left(\mathfrak{a} \cdot \mathfrak{a}^{\prime}\right)=N(\mathfrak{a}) \cdot N\left(\mathfrak{a}^{\prime}\right)$ gilt, so ergibt sich durch Multiplikation der Gleichungen (1) und (2) auf Grund von (3), S. 142:

(3) $\left(a x^{2}+b x y+c y^{2}\right) \cdot\left(a^{\prime} x^{\prime 2}+b^{\prime} x^{\prime} y^{\prime}+c^{\prime} y^{\prime 2}\right)=a^{\prime \prime} x^{\prime \prime 2}+b^{\prime \prime} x^{\prime \prime} y^{\prime \prime}+c^{\prime \prime} y^{\prime \prime 2}$, wo $\left(a^{\prime \prime}, b^{\prime \prime}, c^{\prime \prime}\right)$ die zur Basis $\omega_{1}^{\prime \prime}$, $\omega_{2}^{\prime \prime}$ gehörende Form ist. Lösen wir die Gleichungen (1) und (2) nach $x^{\prime \prime}$ und $y^{\prime \prime}$ auf ${ }^{1}$ ), so berechnen sich die $x^{\prime \prime}$, $y^{\prime \prime}$ aus den $x, y$ und $x^{\prime}, y^{\prime}$ in Gestalt einer sogenannten "bilinearen Substitution":

$$
\left\{\begin{array}{l}
x^{\prime \prime}=\alpha x x^{\prime}+\alpha^{\prime} x y^{\prime}+\alpha^{\prime \prime} x^{\prime} y+\alpha^{\prime \prime \prime} y y^{\prime} \\
y^{\prime \prime}=\beta x x^{\prime}+\beta^{\prime} x y^{\prime}+\beta^{\prime \prime} x^{\prime} y+\beta^{\prime \prime \prime} y y^{\prime} .
\end{array}\right.
$$

Durch diese bilineare Substitution wird die in (3) rechts stehende, aus $(a, b, c)$ und $\left(a^{\prime}, b^{\prime}, c^{\prime}\right)$,homponierte" Form $\left(a^{\prime \prime}, b^{\prime \prime}, c^{\prime \prime}\right)$ in das in (3) lintis stehende Prodult der, ,komponierenden" Formen zerlegt.

Durch Auswahl besonders zweckmäBiger Formen aus zwei vorgelegten Klassen $\mathfrak{F}$ und $\mathfrak{F}^{\prime}$ kann man die vorstehenden Gleichungen in sehr einfache Gestalten kleiden und dadurch Regeln zur Bestimmung der komponierten Klasse $\mathfrak{F} \cdot \mathfrak{F}^{\prime}$ gewinnen. Wir entnehmen z. B. den Klassen $\mathfrak{F}$,

1) Die Determinante des Gleichungssystems (1), (2) ist als Diskriminante $D\left(\omega_{1}^{\prime \prime}, \omega_{2}^{\prime \prime}\right)$ der beiden linear-unabhängigen Zahlen $\omega_{1}^{\prime \prime}, \omega_{2}^{\prime \prime}$ von 0 rerschieden. 
$\mathfrak{F}^{\prime}$ zunächst zwei Formen $\left(a, b_{1}, c_{1}\right)$ und $\left(a^{\prime}, b_{1}^{\prime}, c_{1}^{\prime}\right)$, in denen $a$ teilerfremd gegen $n$. und $a^{\prime}$ teilerfremd gegen $a n$ ist, was nach dem Schlubsatze von $\S 6$, S. 141 keine Schwierigkeiten hat. Wir üben sodann auf diese beiden Formen zwei Substitutionen der $\Gamma^{(\omega)}$ mit $\alpha=\delta=1, \gamma=0$ aus, wobei nach (4) S. 138 die ersten Koeffizienten der Formen unverändert bleiben, während sich als mittlere Koeffizienten $\left(b_{1}-2 \beta a\right)$ und $\left(b_{1}^{\prime}-2 \beta^{\prime} a^{\prime}\right)$ einstellen. Da $a$ und $a^{\prime}$ teilerfremd sind und $b_{1}^{\prime} \equiv b_{1}(\bmod 2)$ wegen der gleichen Diskriminanten der Formen gilt, so kann man die beiden rationalen ganzen Zahlen $\beta$ und $\beta^{\prime}$ so wählen, daß:

$$
\beta a-\beta^{\prime} a^{\prime}=\frac{1}{2}\left(b_{1}-b_{1}^{\prime}\right) \text { und also } b_{1}+2 \beta a=b_{1}^{\prime}-2 \beta^{\prime} a^{\prime}
$$

zutrifft. Wir gelangen zu zwei Formen $(a, b, c)$ und $\left(a^{\prime}, b, c^{\prime}\right)$ mit gleichen mittleren Koeffizienten und folgern aus der Gleichheit der Diskriminanten die Gleichung $a c=a^{\prime} c^{\prime}$. Da nun $a$ und $a^{\prime}$ teilerfremd sind, so geht $a$ in $c^{\prime}$ auf. Wir setzen also $c^{\prime}=a c_{0}$ und finden $c=a^{\prime} c_{0}$ : Aus zwei beliebig vorgelegten Klassen $\mathfrak{F}, \mathfrak{F}^{\prime}$ kann man zwei Formen $\left(a, b, a^{\prime} c_{0}\right)$ und $\left(a^{\prime}, b, a c_{0}\right)$ wählen, in denen $a$ und $a^{\prime}$ gegeneinander und gegen $n$ teilerfremd sind, während $c_{0}$ rational und ganz ist.

Als zugehörige Ideale benutzen wir nun die der Zahlen:

$$
a x+\frac{b-i \sqrt{|D|}}{2} y, \quad a^{\prime} x^{\prime}+\frac{b-i \sqrt{|D|}}{2} y^{\prime} .
$$

Die Gleichung (1) nimmt dann die Gestalt an:

$$
\left(x x^{\prime}-c_{0} y y^{\prime}\right) a a^{\prime}+\left(a x y^{\prime}+a^{\prime} x^{\prime} y+b y y^{\prime}\right) \frac{b-i \sqrt{|D|}}{2}=x^{\prime \prime} \omega_{2}^{\prime \prime}-y^{\prime \prime} \omega_{1}^{\prime \prime} .
$$

Die Zahl $a \cdot a^{\prime}$ ist im Ideal $\mathfrak{a}_{n}^{\prime \prime}=\mathfrak{a}_{n} \cdot \mathfrak{a}_{n}^{\prime}$ enthalten. Aber auch $\frac{-b+i \sqrt{|D|}}{2}$ findet sich in $\mathfrak{a}_{n}^{\prime \prime} ;$ denn sowohl $a \cdot \frac{-b+i \sqrt{|D|}}{2}$ als auch $a^{\prime} \cdot \frac{-b+i \sqrt{|D|}}{2}$ kommen in $\mathfrak{a}_{n}^{\prime \prime}$ vor, und $a, a^{\prime}$ sind teilerfremd. Aus (6) geht demnach hervor, daß man:

$$
\omega_{1}^{\prime \prime}=\frac{-b+i \sqrt{|D|}}{2}, \quad \omega_{2}^{\prime \prime}=a a^{\prime}
$$

als Basis von $\mathfrak{a}_{n}^{\prime \prime}$ benutzen kann, worauf die zugehörige bilineare Substitution (4) die Gestalt annimmt:

$$
x^{\prime \prime}=x x^{\prime}-c_{0} y y^{\prime}, \quad y^{\prime \prime}=a x y^{\prime}+a^{\prime} x^{\prime} y+b y y^{\prime} .
$$

Wir haben also den Satz gewonnen: Repräsentieren wir die beiden gegebenen Klassen $\mathfrak{F}$ und $\mathfrak{F}^{\prime}$ durch die Formen $\left(a, b, a^{\prime} c_{0}\right)$ und $\left(a^{\prime}, b, a c_{0}\right)$, so ist die komponierte Klasse $\mathfrak{F} \cdot \mathfrak{F}^{\prime}$ diejenige der Form $\left(a a^{\prime}, b, c_{0}\right)$. Dies ist in der Tat die der Basis (7) entsprechende Form.

Die zu zwei ,entgegengesetzten“ Formen $(a, b, c)$ und $(a,-b, c)$ gehörenden Klassen nannten wir bereits S. 141 selbst „entgegengesetzt". Diese Klassen sind stets und nur dann einander gleich, wenn sie ambig 
sind. Wir nehmen $a$ teilerfremd gegen $n$ an und haben in den beiden zugehörigen Idealen:

$$
a x+\frac{b-i \sqrt{|D|}}{2} y, \quad a x^{\prime}-\frac{b+i \sqrt{|D|}}{2} y^{\prime}
$$

zwei „,konjugierte“ Ideale $\mathfrak{a}_{n}, \mathfrak{a}_{n}^{\prime}$. Es läßt sich nun leicht zeigen, daß die beiden zugehörigen Idealklassen $\mathfrak{A}, \mathfrak{A}^{\prime}$ im Sinne von S. 134 einander invers sind und also in $\mathfrak{A} \cdot \mathfrak{A}^{\prime}=\mathfrak{A}_{0}$ die Hauptklasse liefern. Wir stellen nämlich in $\mathfrak{a}_{n} \cdot \mathfrak{a}_{n}^{\prime}$ zunächst die drei Zahlen fest:

$a^{2}, \quad a \frac{b+i \sqrt{|D|}}{2}+a \frac{b-i \sqrt{|D|}}{2}=a b, \quad \frac{b-i \sqrt{|D|}}{2} \cdot \frac{b+i \sqrt{|D|}}{2}=a c$, damit aber auch (weil $a, b, c$ keinen Teiler $t>1$ gemein haben) die Zahl $a$. Mit $a$ und $a \frac{-b+i \sqrt{|D|}}{2}$ enthält $\mathfrak{a}_{n} \cdot \mathfrak{a}_{n}^{\prime}$ alle Zahlen:

$$
a\left(x+\frac{b-i \sqrt{|D|}}{2} y\right) \text {. }
$$

Andrerseits sind, wie man leicht feststellt, alle Produkte zweier Zahlen (9) im Systeme (10) enthalten. Also ist, da $1, \frac{-b+i \sqrt{|D|}}{2}$ eine Basis von $\mathfrak{e}_{n}$ ist, $\mathfrak{a}_{n} \cdot \mathfrak{a}_{n}^{\prime}=a \cdot \mathfrak{e}_{n}$, so daß $\mathfrak{a}_{n} \cdot \mathfrak{a}_{n}^{\prime}$ tatsächlich mit $\mathfrak{e}_{n}$ äquivalent ist. Wir kleiden das Ergebnis in die Gestalt: Zwei entgegengesetzte Formklassen $\mathfrak{F}, \mathfrak{F}^{\prime}$ geben, miteinander komponiert, stets die Hauptklasse $\mathfrak{F}_{0}$, so daß $\mathfrak{F}^{\prime}$ auch durch $\mathfrak{F}^{-1}$ bezeichnet werden mag. Da mit $\mathfrak{F}$ die Klasse $\mathfrak{F}^{\prime}$, die der Gleichung $\mathfrak{F} \cdot \mathfrak{F}^{\prime}=\mathfrak{F}_{0}$ genügt, eindeutig bestimmt ist, und da nur die ambigen Klassen sich selbst entgegengesetzt sind, so folgt insbesondere: Nur die ambigen Formklassen geben, mit sich selbst komponiert, die Hauptklasse $\mathfrak{F}_{0}$.

\section{\$ 9. Einteilung der Formklassen in Geschlechter.}

Von einer rationalen ganzen Zahl $m$ sagt man, sie sei durch die quadratische Form $(a, b, c)$,darstellbar“, wenn es zwei rationale ganze Zahlen $x, y$ gibt, für welche $\left(a x^{2}+b x y+c y^{2}\right)$ gleich $m$ wird:

$$
m=a x^{2}+b x y+c y^{2} \text {. }
$$

Nach (4) S. 138 folgt, daß z. B. alle Zablen, die als erste Koeffizienten der mit $(a, b, c)$ äquivalenten Formen auftreten, durch $(a, b, c)$ darstellbar sind. Wir können demnach zufolge eines S. 141 aufgestellten Satzes durch $(a, b, c)$ darstellbare Zahlen $m$ angeben, die zu irgendeiner vorgeschriebenen Zahl $n$ teilerfremd sind. Es gibt sogar unendlich viele gegen eine vorgeschriebene Zahl $n$ teilerfremde, durch $(a, b, c)$ darstellbare Zahlen. Ist nämlich $m$ eine erste, so können wir auch eine gegen $n m$ teilerfremde, durch $(a, b, c)$ darstellbare Zahl $m^{\prime}$ angeben, sodann eine gegen $\mathrm{nmm}^{\prime}$ teilerfremde usw. 
Durch Ausübung einer Substitution (2) S. 138 auf (1) folgt leicht der Satz: Ist die Zahl $m$ durch $(a, b, c)$ darstellbar, so ist diese Zahl auch durch jede mit $(a, b, c)$ äquivalente Form darstellbar. Wir dürfen uns demnach der Ausdrucksweise bedienen, die Zahl m sei „durch eine Formklasse $\mathfrak{F}$ darstellbar". Weiter ergibt sich aus (1) sofort:

$$
m=a x^{2}+(-b) x(-y)+c(-y)^{2} .
$$

Da nun $(a,-b, c)$ der zu $\mathfrak{F}$ entgegengesetzten Klasse $\mathfrak{F}^{-1}$ angehört, so besteht der Satz: Ist eine Zahl $m$ durch eine der beiden entgegengesetzten Klassen $\mathfrak{F}, \mathfrak{F}^{-1}$ darstellbar, so ist sie stets auch durch die andere Klasse darstellbar. Aus (3) S. 149 geht endlich noch folgender Satz herror: Ist $m$ durch die Klasse $\mathfrak{F}$ und $m^{\prime}$ durch $\mathfrak{F}^{\prime}$ darstellbar, so ist das Produkt $m \cdot m^{\prime}$ durch die aus der Komposition der Klassen $\mathfrak{F}$ und $\mathfrak{F}^{\prime}$ zu gewinnende Klasse $\mathfrak{F} \cdot \mathfrak{F}^{\prime}$ darstellbar.

Es seien nun $m$ und $m^{\prime}$ irgend zwei durch $\mathfrak{F}$ darstellbare Zahlen. Dann ist $m^{\prime}$ auch durch $\mathfrak{F}^{-1}$ und also $m \cdot m^{\prime}$ durch die Hauptklasse $\mathfrak{F} \cdot \mathfrak{F}^{-1}=\mathfrak{F}_{0}$ darstellbar. Benutzen wir die Hauptform $\left(1,0,-\frac{D}{4}\right)$ bzw. $\left(1,1, \frac{1-D}{4}\right)$ selbst, so folgt:

$$
m m^{\prime}=x^{2}-\frac{D}{4} y^{2} \quad \text { oder } \quad 4 m m^{\prime}=(2 x+y)^{2}-D y^{2},
$$

je nachdem $D \equiv 0$ oder $\equiv 1(\bmod 4)$ ist.

Wir verstehen weiter unter $p$ irgendeine in $D$ aufgehende ungerade Primzahl und unter $m, m^{\prime}$ irgend zwei gegen $p$ teilerfremde ganze Zahlen, die durch $\mathfrak{F}$ darstellbar sind. Aus (2) folgt dann:

$$
m m^{\prime} \equiv x^{2} \quad \text { bzw. } \quad 4 m m^{\prime} \equiv(2 x+y)^{2} \quad(\bmod p),
$$

so daß $m \cdot m^{\prime}$ quadratischer Rest von $p$ ist. Also besteht der Satz: Alle gegen den ungeraden Primfaktor $p$ von $D$ teilerfremden, durch $\mathfrak{F}$ darstellbaren Zahlen $m, m^{\prime}, m^{\prime \prime}$, ... haben gleichen quadratischen Charakter in bezug auf $p, d . h$. sie sind entweder durchweg quadratische Reste oder durchweg quadratische Nichtreste von $p$, so daß wir mit Hilfe des Legendreschen Zeichens schreiben können:

$$
\left(\begin{array}{c}
m \\
p
\end{array}\right)=\left(\begin{array}{c}
m^{\prime} \\
p
\end{array}\right)=\left(\begin{array}{c}
m^{\prime \prime} \\
p
\end{array}\right)=\cdots
$$

Der gemeinsame Wert +1 oder -1 dieser Legendreschen Zeichen, der durch die in $D$ enthaltene ungerade Primzahl $p$ und die Formklasse $\mathfrak{F}$ bestimmt ist, heißt ein "Charakter" der Klasse $\mathfrak{F}$ und möge durch $\chi$ bezeichnet sein. Jede Klasse $\mathfrak{F}$ hat demnach zunächst so viele Charaktere $\chi$, als verschiedene ungerade Primzahlen $p$ in der Diskriminante $D$ enthalten sind. Eine wesentliche Eigenschaft dieser Charaktere kommt durch folgenden Satz zum Ausdruck: Sind $\chi$ und $\chi^{\prime}$ die zum ungeraden Primfaktor $p$ von $D$ gehörenden Charalktere der Klassen $\mathfrak{F}$ und 
$\mathfrak{F}^{\prime}$, so ist der zu p gehörende Charakter der durch Komposition von $\mathfrak{F}$ und $\mathfrak{F}^{\prime}$ entstehenden Klasse $\mathfrak{F} \cdot \mathfrak{F}^{\prime}$ das Produkt $\chi \cdot \chi^{\prime}$ der Charaktere $\chi$ und $\chi^{\prime}$. Sind nämlich $m$ und $m^{\prime}$ zwei gegen $p$ teilerfremde durch $\mathfrak{F}$ bzw. $\mathfrak{F}^{\prime}$ darstellbare Zahlen, so ist $m \cdot m^{\prime}$ durch $\mathfrak{F} \cdot \mathfrak{F}^{\prime}$ darstellbar und teilerfremd gegen $p$. Dann aber gilt:

$$
\left(\begin{array}{c}
m \cdot m^{\prime} \\
p
\end{array}\right)=\left(\begin{array}{c}
m \\
p
\end{array}\right) \cdot\left(\begin{array}{c}
m^{\prime} \\
p
\end{array}\right)=\chi \cdot \chi^{\prime} .
$$

Ist $D$ gerade, aber nicht $\equiv 4(\bmod 16)$, so können wir für jede Klasse $\mathfrak{F}$ noch einen, in einem gewissen Falle sogar noch zwei weitere Charaktere erklären, die wieder die bisherigen Eigenschaften der $\chi$ besitzen. Ist nämlich erstens $\frac{1}{4} D \equiv 3(\bmod 4)$, hat also $D$ die Gestalt $D=16 k+12$, unter $k$ hier und weiterhin rationale ganze Zablen verstanden, so folgt aus (2) für irgend zwei ungerade durch $\mathfrak{F}$ darstellbare Zahlen $m, m^{\prime}$ :

$$
m m^{\prime} \equiv x^{2}+y^{2} \quad(\bmod 4) .
$$

Da hier eine der Zahlen $x, y$ gerade, die andere ungerade sein muß, so folgt $m \cdot m^{\prime} \equiv 1, m^{\prime} \equiv m(\bmod 4)$, so daß für alle ungeraden, durch $\mathfrak{F}$ darstellbaren Zahlen $m, m^{\prime}, m^{\prime \prime}, \ldots$ die Gleichungen gelten:

$$
(-1)^{\frac{m-1}{2}}=(-1)^{\frac{m^{\prime}-1}{2}}=(-1)^{\frac{m^{\prime \prime}-1}{2}}=\cdots \text {. }
$$

Ist zweitens $\frac{1}{4} D \equiv 2(\bmod 8)$, also $D=32 k+8$, so folgt aus $(2)$ für irgend zwei ungerade, durch $\mathfrak{F}$ darstellbare Zahlen $m, m^{\prime}$ :

$$
m m^{\prime} \equiv x^{2}-2 y^{2} \quad(\bmod 8) \text {. }
$$

Also ist $x$ ungerade, und es folgt $m m^{\prime} \equiv \pm 1, m^{\prime} \equiv \pm m(\bmod 8)$. In beiden Fällen folgt $\frac{1}{8}\left(m^{2}-1\right) \equiv \frac{1}{8}\left(m^{\prime 2}-1\right)(\bmod 2)$, so daß sich jetzt für alle ungeraden, durch $\mathfrak{F}$ darstellbaren Zahlen:

$$
(-1)^{\frac{m^{2}-1}{8}}=(-1)^{\frac{n^{\prime 2}-1}{8}}=(-1)^{\frac{m^{\prime \prime 2}-1}{8}}=\cdots
$$

ergibt. Ist drittens $\frac{1}{4} D \equiv 4(\bmod 8)$, also $D=32 \%+16$, so schlieBt man wieder auf $m^{\prime} \equiv m(\bmod 4)$ und damit auf das Bestehen der Gleichungen (4). Haben wir viertens $\frac{1}{4} D \equiv 6(\bmod 8)$, also $D=32 k+24$, so folgt aus (2):

$$
m m^{\prime} \equiv x^{2}+2 y^{2} \quad(\bmod 8) \text {. }
$$

Da $x$ ungerade sein muß, so schließen wir auf $m \cdot m^{\prime} \equiv 1$ oder $m \cdot m^{\prime} \equiv 3$ $(\bmod 8)$, also auf $m^{\prime} \equiv m$ bzw. $m^{\prime} \equiv 3 m(\bmod 8)$. In beiden Fällen gelangt man leicht zu den Gleichungen:

$$
(-1)^{\frac{m-1}{2}+\frac{m^{2}-1}{8}}=(-1)^{\frac{m^{\prime}-1}{2}+\frac{m^{\prime 2}-1}{8}}=\cdots \text {. }
$$

Ist endlich $\frac{1}{4} D \equiv 0(\bmod 8)$, also $D=32 k$, so folgt in der bisherigen Weise $m m^{\prime} \equiv 1(\bmod 8)$ und also $m^{\prime} \equiv m(\bmod 8)$. Jetzt bestehen also die Gleichungen (4) und (5) zugleich.

Wir führen nun, falls $D=16 k+12$ oder $=32 k+16$ ist, den 
Wert $(-1)^{\frac{m-1}{2}}$ als neuen Charakter $\chi$ von $\mathfrak{F}$ ein. Ebenso führen wir für $D=32 k+8$ den Charakter $\chi=(-1)^{\frac{m^{2}-1}{8}}$ und für $D=32 z+24$ den Charakter $\chi=(-1)^{\frac{m-1}{2}+\frac{m^{2}-1}{8}}$ ein, sowie endlich für $D=32 k$ die beiden Charaktere $(-1)^{\frac{m-1}{2}}$ and $(-1)^{\frac{m^{2}-1}{8}}$. Auch diese Charaktere haben stets einen der Werte +1 oder -1 . Da man aber für irgend zwei ungerade ' $Z$ ahlen $m, m^{\prime}$ leicht die Gleichungen zeigt:

$$
\begin{aligned}
& (-1)^{\frac{m-1}{2}} \cdot(-1)^{\frac{m^{\prime}-1}{2}}=(-1)^{\frac{m m^{\prime}-1}{2}}, \\
& (-1)^{\frac{m^{2}-1}{8}} \cdot(-1)^{\frac{m^{\prime 2}-1}{8}}=(-1)^{\frac{\left(m m^{\prime}\right)^{2}-1}{8}},
\end{aligned}
$$

so besteht auch für jede Art der neu erklärten Charaktere der Satz: Der Charakter einer komponierten Klasse $\mathfrak{F} \cdot \mathfrak{F}^{\prime}$ ist das Produlit der Charaktere der komponierenden Klassen.

Die Anzahl $\boldsymbol{\nu}$ der Charaktere der einzelnen Klasse $\mathfrak{F}$ ist gleich der Anzahl der verschiedenen Primfaktoren von $D$, falls $D=4 k+1$ oder $=16 k+12$ oder mod 32 mit einer der Zahlen 8, 16, 24 kongruent ist; für $D=16 z+4$ ist diese Anzahl $\nu$ um eine Einheit geringer als die Anzahl der verschiedenen Primfaktoren von $D$, für $D=32 \%$ um eine Einheit größer. Wir bezeichnen die Charaktere der einzelnen Klasse $\mathfrak{F}$ in einer bestimmten Anordnung jetzt genauer durch $\chi_{1}, \chi_{2}, \ldots, \chi_{v}$ und nennen diese Zusammenstellung aller $\nu$ Charaktere den "Totalcharakter" der Klasse $\mathfrak{F}$. Dann gilt der Satz: Der Totalcharakter $\chi_{1}^{\prime \prime}, \chi_{2}^{\prime \prime}, \ldots, \chi_{v}^{\prime \prime}$ der durch Komposition von $\mathfrak{F}$ und $\mathfrak{F}^{\prime}$ entstehenden Klasse $\mathfrak{F}^{\prime \prime}=\mathfrak{F} \cdot \mathfrak{F}^{\prime}$ berechnet sich aus den Totalcharalteren der komponierenden Klassen $\chi_{1}, \chi_{2}$, $\ldots, \chi_{v}$ und $\chi_{1}^{\prime}, \chi_{2}^{\prime}, \ldots, \chi_{v}^{\prime}$ nach dem Gesetze:

$$
\chi_{1}^{\prime \prime}=\chi_{1} \cdot \chi_{1}^{\prime}, \quad \chi_{2}^{\prime \prime}=\chi_{2} \cdot \chi_{2}^{\prime}, \ldots, \quad \chi_{v}^{\prime \prime}=\chi_{v} \cdot \chi_{v}^{\prime}
$$

Da jeder einzelne Charakter einen der beiden Werte \pm 1 hat, so sind im ganzen $2^{v}$ verschiedene Totalcharaktere kombinatorisch möglich. Wie viele von diesen $2^{v}$ verschiedenen Kombinationen als Totalcharaktere unserer Formklassen wirklich auftreten, muß hier unentschieden bleiben. Da zwei entgegengesetzte Klassen $\mathfrak{F}$ und $\mathfrak{F}^{-1}$ gleiche Totalcharaktere haben, so ist nach der Regel (7) einleuchtend, daß die Hauptklasse $\mathfrak{F}_{0}$ den Totalcharakter $\chi_{1}=1, \chi_{2}=1, \ldots, \chi_{v}=1$ hat.

Wir vereinigen nun alle Klassen, welche im Totalcharakter übereinstimmen, zu einem "Geschlechte" von Formklassen und nennen insbesondere dasjenige Geschlecht, dem die Hauptklasse angehört, das „Hauptgeschlecht". Die Anzahl der Klassen des Hauptgeschlechtes heiße $\mu$, die Klassen selbst seien $\mathfrak{F}_{0}, \mathfrak{F}_{1}, \ldots, \mathfrak{F}_{\mu-1}$. Dann besteht zufolge der Regel (7) der Satz: In der Abelschen Gruppe $G_{h}$ aller $h(|D|)$ Klassen 
der Diskriminante $D$ bilden die $\mu$ Klassen $\mathfrak{F}_{0}, \mathfrak{F}_{1}, \ldots, \mathfrak{F}_{\mu-1}$ des Hauptgeschlechtes eine ausgezeichnete ${ }^{1}$ ) Untergruppe $G_{\mu}$ der Ordnung $\mu$, so daß $\mu$ ein Teiler von $h$ ist. Komponiert man irgendeine Klasse $\mathfrak{F}$ mit den $\mu$ Klassen $\mathfrak{F}_{0}, \mathfrak{F}_{1}, \ldots, \mathfrak{F}_{\mu-1}$ des Hauptgeschlechtes, so haben alle Klassen $\mathfrak{F} \cdot \mathfrak{F}_{0}, \mathfrak{F} \cdot \mathfrak{F}_{1}, \ldots, \mathfrak{F} \cdot \mathfrak{F}_{\mu-1}$ gleiche Totalcharaktere, und zugleich erschöpfen diese $\mu$ Klassen das ganze zu diesem Totalcharakter gehörende Geschlecht. Den $\frac{h}{\mu} \operatorname{zur} G_{\mu}$ gehörenden Nebengruppen (vgl. S. 4) entsprechen also ebenso viele Geschlechter von Formklassen, und hiermit werden zugleich alle Geschlechter erschöpft: Alle Geschlechter der Formklassen der Diskriminante $D$ enthalten gleich viele Klassen; die Klassenanzahl h, geteilt durch die Anzahl $\mu$ der Klassen im einzelnen Geschlechte, ergibt uns somit als Quotienten die Anzahl der Geschlechter und damit die der wirklich auftretenden Totalcharaktere.

Die $2^{v}$ kombinatorisch möglichen Totalcharaktere bilden gegenüber Multiplikation (entsprechend der Gleichung (7)) eine Abelsche Gruppe $G_{2^{v}}$ der Ordnung $2^{v}$. Die bei der Diskriminante $D$ wirklich auftretenden Totalcharaktere bilden für sich eine Gruppe, da mit zwei solchen Totalcharakteren stets auch ihr Produkt auftritt. Die Ordnung jeder Untergruppe der $G_{2}$ ist ein Teiler von $2^{v}$, also wieder eine Potenz $2^{\lambda}$ von 2. Für die Klassenanzahl $h(|D|)$ und damit für die Ordnung der Gruppe $G_{h}$ folgt hieraus $h=2^{\lambda} \cdot \mu$, wo $\mu$ die Anzahl der Klassen im einzelnen Geschlechte ist. Die Anwendung des S. 17 (am Schlusse von §6) aufgestellten Satzes ergibt somit das Resultat: Die Abelsche Gruppe $G_{h}$ besitzt als Indexreihe die in $\mu$ aufgehenden Primzahlen, vereint mit $\lambda$ Indizes, die alle gleich 2 sind.

1) Alle Untergruppen einer Abelschen Gruppe sind ausgezeichnet. 
Erster Abschnitt.

\section{Die Additions-, Multiplikations- und Divisionssätze der elliptischen Funktionen.}

Die in diesem Abschnitte zur Sprache kommenden Entwicklungen über die Additions-, Multiplikations- und Divisionssätze der elliptischen Funktionen stammen in ihren Grundlagen aus der älteren Geschichte der elliptischen Funktionen. Die Additionstheoreme wurden bereits gegen Mitte des vorletzten Jahrhunderts von Euler entdeck $\ell^{1}$ ) und gehören zu den in der älteren und neueren Geschichte der elliptischen Funktionen am häufigsten behandelten Gegenständen. Für die Ausbildung der Multiplikations- und Divisionssätze sind vornehmlich die Schöpfungen Abels grundlegend gewesen. ${ }^{2}$ ) Insbesondere ist von $\mathrm{Abel}$ der algebraische Charakter der Teilungsgleichungen erkannt, und die hier gewonnenen Sätze sind für seine allgemeinen Untersuchungen über die Auflösung algebraischer Gleichungen vorbildlich gewesen.

Die nachfolgende Darstellung schließt sich durchweg an die in Bd. I des vorliegenden Werkes entwickelte Gestalt der Theorie der elliptischen Funktionen an. Die Theoreme sind also überall zunächst für die Funktionen erster Stufe dargelegt; bei der zweiten Stufe aber werden sie in derjenigen Gestalt entwickelt, wie sie bei Jacobi vorliegen.

\section{Erstes Kapitel. \\ Die Additionssätze der elliptischen Funktionen.}

Eine vorläufige Betrachtung der Additionssätze der Funktionen erster Stufe wurde schon in I, 202 ff. durchgeführt. Es sei insbesondere an die daselbst S. 204 entwickelte Auffassung erinnert, nach welcher die Addi-

1) Vgl. A.Enneper, „Elliptische Funktionen, Theorie und Geschichte“, 2. Aufl., bearb. von F. Müller (Halle 1890), S. 182 ff. Dieses Werk wird weiterhin kurz dureh die Namen der Autoren zitiert.

2) S. die „Recherches sur les fonctions elliptiques“, Art. 9 ff., Journ. für Math., Bd. 2 (1827) oder "Werke", Bd.1, S. $279 \mathrm{ff}$. 
tionsformeln für $\wp$ und $\wp^{\prime}$ :

$$
\begin{aligned}
& \wp(u+v)=R_{1}\left(\wp(u), \wp^{\prime}(u), \wp(v), \wp^{\prime}(v)\right) \\
& \wp^{\prime}(u+v)=R_{2}\left(\wp(u), \wp^{\prime}(u), \wp(v), \wp^{\prime}(v)\right)
\end{aligned}
$$

eine algebraische Darstellung der kontinuierlichen Gruppe zweifach unendlich vieler Transformationen der Riemannschen Fläche $F_{2}$ in sich lieferten. Funktionentheoretisch ist die Existenz zweier Gleichungen der angegebenen Gestalt für $\wp(u+v)$ und $\wp^{\prime}(u+v)$ leicht einzusehen. Es ist z. B. $\wp(u+v)$ als Funktion von $u$ doppeltperiodisch mit den Perioden $\omega_{1}, \omega_{2}$ nnd lä6t sich also nach den Sätzen von I, 198 als rationale Funktion von $\wp(u), \wp^{\prime}(u)$ darstellen. Weierstra $B$ hat in seinen Vorlesungen $\left.{ }^{1}\right)$ die Existenz eines Additionstheorems sogar an die Spitze der ganzen Entwicklung gestellt und teilt den Satz mit, daß die Existenz eines solchen Theorems eine charakteristische Eigenschaft der elliptischen Funktionen und ihrer Ausartungen sei. Als Eingang in die Entwicklung der Additionssätze wählen wir eine gleichfalls von Weierstraß aufgestellte dreigliedrige Sigmarelation ${ }^{2}$ ), aus welcher im wesentlichen nur noch durch das Mittel analytischer Umformungen die Hauptformeln der Additionssätze gewonnen werden sollen.

\section{$\$ 1$. Additionstheoreme der elliptischen Funktionen erster Stufe.}

Sind $u_{1}, u_{2}, u_{3}$ drei beliebig gewählte Werte von $u$, so stellen die drei Produkte:

$$
\sigma\left(u+u_{1}\right) \sigma\left(u-u_{1}\right), \sigma\left(u+u_{2}\right) \sigma\left(u-u_{2}\right), \sigma\left(u+u_{3}\right) \sigma\left(u-u_{3}\right)
$$

drei gleichändrige ganze elliptische Funktionen dritter Art zweiter Ordnung dar, zwischen denen nach I, 227 eine lineare Gleichung:

$$
\begin{gathered}
A_{1} \sigma\left(u+u_{1}\right) \sigma\left(u-u_{1}\right)+A_{2} \sigma\left(u+u_{2}\right) \sigma\left(u-u_{2}\right) \\
+A_{3} \sigma\left(u+u_{3}\right) \sigma\left(u-u_{3}\right)=0
\end{gathered}
$$

mit nicht durchweg verschwindenden, von $u$ unabhängigen Koeffizienten $A$ identisch besteht. Zur Bestimmung $\operatorname{der} A$ setzt man in (1) nacheinander $u=u_{1}, u_{2}, u_{3}$ ein und findet:

$$
\begin{aligned}
A_{2} \sigma\left(u_{1}+u_{2}\right) \sigma\left(u_{1}-u_{2}\right)+A_{3} \sigma\left(u_{1}+u_{3}\right) \sigma\left(u_{1}-u_{3}\right) & =0, \\
A_{1} \sigma\left(u_{2}+u_{1}\right) \sigma\left(u_{9}-u_{1}\right) & +A_{3} \sigma\left(u_{2}+u_{3}\right) \sigma\left(u_{2}-u_{3}\right)=0 \\
A_{1} \sigma\left(u_{3}+u_{1}\right) \sigma\left(u_{3}-u_{1}\right)+A_{2} \sigma\left(u_{3}+u_{2}\right) \sigma\left(u_{3}-u_{2}\right) & =0 .
\end{aligned}
$$

1) Vgl. H. A. Schwarz, „Formeln und Lehrsätze zum Gebrauche der elliptischen Funktionen", nach Vorlesungen und Aufzeichuungen des Herrn K. Weierstraß (Göttingen $1881 \mathrm{ff}$.) S. $1 \mathrm{ff}$.

2) S. die eben genannte Schrift S. 47 . 
Aus diesen Gleichungen berechnen sich die Verhältnisse der $A$ wie folgt: $A_{1}: A_{2}: A_{3}=\sigma\left(u_{2}+u_{3}\right) \sigma\left(u_{2}-u_{3}\right): \sigma\left(u_{3}+u_{1}\right) \sigma\left(u_{3}-u_{1}\right): \sigma\left(u_{1}+u_{2}\right) \sigma\left(u_{1}-u_{2}\right)$. Durch Eintragen dieser Werte in (1) findet man die dreigliedrige Weierstraßsche Sigmarelation.

$$
\begin{aligned}
& \sigma\left(u+u_{1}\right) \sigma\left(u-u_{1}\right) \sigma\left(u_{2}+u_{3}\right) \sigma\left(u_{2}-u_{3}\right) \\
+ & \sigma\left(u+u_{2}\right) \sigma\left(u-u_{2}\right) \sigma\left(u_{3}+u_{1}\right) \sigma\left(u_{3}-u_{1}\right) \\
+ & \sigma\left(u+u_{3}\right) \sigma\left(u-u_{3}\right) \sigma\left(u_{1}+u_{2}\right) \sigma\left(u_{1}-u_{2}\right)=0,
\end{aligned}
$$

die man als die gemeinsame Quelle der weiteren Additionsformeln benutzen kann.

Bevor dies ausgeführt wird, schließen wir an (2) noch folgende, unten zur Benutzung kommende Betrachtung an. Für die zwölf Argumente der in(2)auftretenden Sigmafunktionen benutzen wirfolgende Abkürzungen:

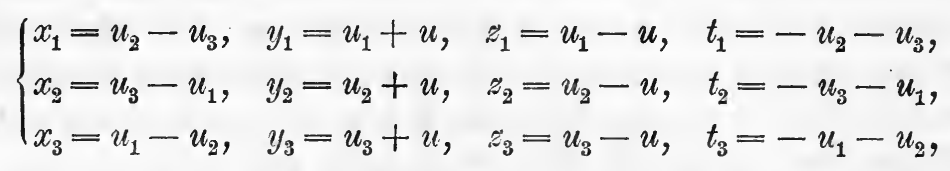

mit deren Hilfe sich die Relation (2) in die Gestalt kleidet:

$$
\begin{gathered}
\sigma\left(x_{1}\right) \sigma\left(y_{1}\right) \sigma\left(z_{1}\right) \sigma\left(t_{1}\right)+\sigma\left(x_{2}\right) \sigma\left(y_{2}\right) \sigma\left(z_{2}\right) \sigma\left(t_{2}\right) \\
+\sigma\left(x_{3}\right) \sigma\left(y_{3}\right) \sigma\left(z_{3}\right) \sigma\left(t_{3}\right)=0 .
\end{gathered}
$$

Man kann hierbei an Stelle der vier unabhängigen Variablen $u, u_{1}, u_{2}, u_{3}$ auch die $x, y, z, t$ irgendeiner der drei Reihen (3) als solche benutzen. Nehmen wir z. B. die erste Reihe (3), so ergeben sich für die $u$ die Darstellungen:

(5) $u=\frac{1}{2}\left(y_{1}-z_{1}\right), u_{1}=\frac{1}{2}\left(y_{1}+z_{1}\right), u_{2}=\frac{1}{2}\left(x_{1}-t_{1}\right), u_{3}=\frac{1}{2}\left(-x_{1}-t_{1}\right)$.

Durch Vermittlung dieser Formeln findet man die $x, y, z, t$ der zweiten und dritten Reihe (3) in denen der ersten durch die beiden quaternären linearen Substitutionen der Determinante 1 dargestellt:

$$
\begin{aligned}
& \left\{\begin{array}{l}
x_{2}=-\frac{1}{2} x_{1}-\frac{1}{2} y_{1}-\frac{1}{2} z_{1}-\frac{1}{2} t_{1}, \\
y_{2}=+\frac{1}{2} x_{1}+\frac{1}{2} y_{1}-\frac{1}{2} z_{1}-\frac{1}{2} t_{1}, \\
z_{2}=+\frac{1}{2} x_{1}-\frac{1}{2} y_{1}+\frac{1}{2} z_{1}-\frac{1}{2} t_{1}, \\
t_{2}=+\frac{1}{2} x_{1}-\frac{1}{2} y_{1}-\frac{1}{2} z_{1}+\frac{1}{2} t_{1},
\end{array}\right. \\
& \left\{\begin{array}{l}
x_{3}=-\frac{1}{2} x_{1}+\frac{1}{2} y_{1}+\frac{1}{2} z_{1}+\frac{1}{2} t_{1}, \\
y_{3}=-\frac{1}{2} x_{1}+\frac{1}{2} y_{1}-\frac{1}{2} z_{1}-\frac{1}{2} t_{1}, \\
z_{3}=-\frac{1}{2} x_{1}-\frac{1}{2} y_{1}+\frac{1}{2} z_{1}-\frac{1}{2} t_{1}, \\
t_{3}=-\frac{1}{2} x_{1}-\frac{1}{2} y_{1}-\frac{1}{2} z_{1}+\frac{1}{2} t_{1} .
\end{array}\right.
\end{aligned}
$$

Die erste dieser Substitutionen, die wir symbolisch durch $S$ bezeichnen, 
liefert, zweimal ausgeübt, die Substitution (7), die demnach durch $S^{2}$ zu bezeichnen ist. Dreimalige Wiederholung von $S$ liefert aber die identische Substitution, so daß die Substitutionen (6) und (7) im Verein mit der identischen Substitution eine zyklische Gruppe $G_{3}$ der Ordnung 3 bilden. Bilden die $x_{1}^{\prime}, y_{1}^{\prime}, z_{1}^{\prime}, t_{1}^{\prime}$ ein zweites Variablensystem, das gleichfalls den Substitutionen $S$ und $S^{2}$ unterworfen werden soll, so erweist sich der bilineare Ausdruck $\left(x_{1} x_{1}^{\prime}+y_{1} y_{1}^{\prime}+z_{1} z_{1}^{\prime}+t_{1} t_{1}^{\prime}\right)$ als invariant gegenüber $S$ und $S^{2}$, d. h. es besteht die Relation:

$$
\begin{gathered}
x_{1} x_{1}^{\prime}+y_{1} y_{1}^{\prime}+z_{1} z_{1}^{\prime}+t_{1} t_{1}^{\prime}=x_{2} x_{2}^{\prime}+y_{2} y_{3}^{\prime}+z_{2} z_{2}^{\prime}+t_{2} t_{2}^{\prime} \\
=x_{3} x_{3}^{\prime}+y_{3} y_{3}^{\prime}+z_{3} z_{3}^{\prime}+t_{3} t_{3}^{\prime} .
\end{gathered}
$$

Dieserhalb werden $S$ und $S^{2}$ als „orthogonale" Substitutionen bezeichnet. ${ }^{1}$ ) Unter Zusammenfassung können wir den Satz notieren: Sind $x_{1}, y_{1}, z_{1}, t_{1}$ vier unabhängige Variable, die durch die orthogonalen Substitutionen $S$ und $S^{2}$ der zyklischen $G_{3}$ in die Variablensysteme (6) und (7) übergehen, so besteht für diese drei Variablensysteme $x_{i}, y_{i}, z_{i}, t_{i}$ die Sigmarelation (4) identisch.

In die Relation (2) werde jetzt eingesetzt $u_{2}=0, u_{3}=v$, wodurch sie die Gestalt annimmt:

$$
\begin{gathered}
-\sigma\left(u+u_{1}\right) \sigma\left(u-u_{1}\right) \sigma(v)^{2}+\sigma\left(v+u_{1}\right) \sigma\left(v-u_{1}\right) \sigma(u)^{2} \\
+\sigma(u+v) \sigma(u-v) \sigma\left(u_{1}\right)^{2}=0 .
\end{gathered}
$$

Verstehen wir unter $\pm u_{1}$ die beiden Nullpunkte der $\wp$-Funktion, so können wir $\sigma\left(u+u_{1}\right) \sigma\left(u-u_{1}\right)$ durch das Produkt $-\wp(u) \sigma(u)^{2} \sigma\left(u_{1}\right)^{2}$ ersetzen (vgl. (11) in I, 216) und $\sigma\left(v+u_{1}\right) \sigma\left(v-u_{1}\right)$ entsprechend ausdrücken:

$$
\begin{gathered}
\wp(u) \sigma(u)^{2} \sigma\left(u_{1}\right)^{2} \sigma(v)^{2}-\wp(v) \sigma(v)^{2} \sigma\left(u_{1}\right)^{2} \sigma(u)^{2} \\
+\sigma(u+v) \sigma(u-v) \sigma\left(u_{1}\right)^{2}=0 .
\end{gathered}
$$

Hieraus folgt die schon in I, 217 unter (14) gewounene Gleichung:

$$
\frac{\sigma(u+v) \sigma(u-v)}{\sigma(u)^{2} \sigma(v)^{2}}=-\wp(u)+\wp(v) .
$$

Man logarithmiere diese Gleichung und differenziere nach $u$ bzw. $v$; bei Benutzung von (6) in I, 209 ergibt sich:

$$
\begin{aligned}
& \xi(u+v)+\xi(u-v)-2 \zeta(u)=+\frac{\wp^{\prime}(u)}{\rho(u)-\wp(v)}, \\
& \xi(u+v)-\xi(u-v)-2 \xi(v)=-\frac{\wp^{\prime}(v)}{\wp(u)-\xi(v)} .
\end{aligned}
$$

Die Addition dieser Gleichungen ergibt das sogenaunte „Additionstheorem des Normalintegrals zweiter Gattung $\zeta(u)^{\prime}$, das auch bereits in I, 202 unter

1) In der analytischen Geometrie des Raumes liefern die Drehungen eines rechtwinkligen Achsenkreazes um den Nullpunkt ternäre orthogonale Substitutionen der Koordinaten. 
(2) gewonnen wurde:

$$
\zeta(u+v)=\xi(u)+\xi(v)+\frac{1}{2} \frac{\wp^{\prime}(u)-\wp^{\prime}(v)}{\wp(u)-\xi(v)} .
$$

Durch die in I, 203 ausgeführte Rechnung findet man aus (9) die a. a. 0. unter ( 5 ) angegebene Gleichung:

$$
\wp(u+v)=\frac{1}{4}\left(\frac{\wp^{\prime}(u)-\wp^{\prime}(v)}{\wp(u)-\wp(v)}\right)^{2}-\wp(u)-\wp^{\prime}(v) .
$$

Hier hat man eine erste Gestalt des "Additionstheorems der §o-Funktion" vor sich; das Theorem bringt zum Ausdruck, daß die $\wp$-Funltion, gebildet für die Summe $(u+v)$, sich rational durch die Funktionen $\wp$, $\wp^{\prime}$ der einzelnen Summanden $u, v$ darstellt, und zeigt zugleich die Gestalt dieser Darstellung. Aus (9) und (10) folgt:

$$
\wp(u+v)+\wp(u)+\wp(v)=(\xi(u+v)-\zeta(u)-\xi(v))^{2} .
$$

Durch Differentiation nach $u$ bzw. $v$ und Addition der entstehenden Gleichungen folgt bei nochmaliger Benutzung von (9):

$$
2 \wp^{\prime}(u+v)+\wp^{\prime}(u)+\wp^{\prime}(v)=\frac{\wp^{\prime}(u)-\wp^{\prime}(v)}{\wp(u)-\wp(v)}(\wp(u)+\wp(v)-2 \wp(u+v)),
$$

eine Gleichung, der man die übersichtliche Gestalt geben kann:

$$
\left|\begin{array}{rrr}
\wp(u+v), & -\wp^{\prime}(u+v), & 1 \\
\wp(u), & \wp^{\prime}(u), & 1 \\
\wp(v), & \wp^{\prime}(v), & 1
\end{array}\right|=0 .
$$

Ersetzt man $\wp(u+v)$ durch den Ausdruck (10), so hat man eine erste Gestalt des Additionstheorems der $\wp^{\prime}$-Funktion gewonnen.

Mit Hilfe der zwischen $\wp$ und $\wp^{\prime}$ bestehenden Gleichung kann man die vorstehenden Additionsformeln in verschiedene andere Gestalten kleiden. Da sich $\wp^{\prime 2}$ rational in $\wp$ ausdrückt, so kann man z. B. Gestalten der Gleichungen erzielen, die in $\wp^{\prime}(u)$ und $\wp^{\prime}(v)$ linear sind. Für die $\wp^{-}$-Funktion gelangt man so zu der in I, 203 unter (4) angegebenen Gleichung:

$$
\begin{gathered}
2 \wp(u+v)(\wp(u)-\wp(v))^{2} \\
=\left(2 \wp(u) \wp(v)(\wp(u)+\wp(v))-{ }_{2}^{1} g_{2}(\wp(u)+\wp(v))-g_{3}\right)-\wp^{\prime}(u) \wp^{\prime}(v) .
\end{gathered}
$$

Als entsprechende Gestalt des Additionstheorems der $\xi^{\prime}$-Funktion findet man nach einer nicht ganz kurzen Rechnung:

$$
\begin{gathered}
2 \wp^{\prime}(u+v)(\wp(u)-\wp(v))^{3} \\
=\left(2 \wp(u)^{2}(\wp(v)+3 \wp(u))-\frac{1}{2} g_{3}(3 \wp(u)+\wp(v))-2 g_{3}\right) \wp^{\prime}(v) \\
-\left(2 \wp(v)^{2}(\wp(v)+3 \wp(u))-\frac{1}{2} g_{2}(3 \wp(v)+\wp(u))-2 g_{3}\right) \wp^{\prime}(u) .
\end{gathered}
$$

Eine weitere bemerkenswerte Gestalt der Additionsformeln erzielt man auf folgendem Wege: Man multipliziert (13) mit $2(\wp(u)-\wp(v))$ und er- 
setzt im Produkte rechts die dritten Potenzen von $\rho$ auf Grund der Regel:

$$
4 \wp^{3}=\wp^{\prime 2}+g_{2} \wp+g_{3} .
$$

Dabei erscheint ein Aggregat, das in jedem der beiden Größenpaare $\wp(u), \wp^{\prime}(u)$ und $\wp(v), \wp^{\prime}(v)$ rational und ganz rom zweiten Grade ist. Einer entsprechenden Umformung unterziehe man die rechte Seite der mit 2 multiplizierten Gleichung (14). Endlich folgere man aus (15):

$$
4(\wp(u)-\wp(v))^{3}=\wp^{\prime}(u)^{2}-\wp^{\prime}(v)^{2}-\left(12 \wp(u) \wp(v)-g_{2}\right)(\wp(u)-\wp(v)) .
$$

Als zusammenfassender Ausdruck der Additionsformeln für $\wp$ und $\wp^{\prime}$ entsteht so:

$$
\wp(u+v): \wp^{\prime}(u+v): 1=
$$

$\left\{\wp^{\prime}(u)^{2} \wp(v)-\wp(u) \wp^{\prime}(v)^{2}-\left(2 \wp^{\prime}(u) \wp^{\prime}(v)+g_{2}(\wp(u)+\wp(v))+3 g_{3}\right)(\wp(u)-\wp(v))\right\}$ $:\left\{\left(\wp^{\prime}(u) \wp^{\prime}(v)+3 g_{3}\right)\left(\wp^{\prime}(u)-\wp^{\prime}(v)\right)+2\left(6 \wp(u) \wp(v)-g_{2}\right)\left(\wp(u) \wp^{\prime}(v)-\wp^{\prime}(u) \wp(v)\right)\right.$

$$
\left.+g_{\mathrm{a}}\left(\wp(u) \wp^{\prime}(u)-\wp(v) \wp^{\prime}(v)\right)\right\}
$$

$:\left\{\wp^{\prime}(u)^{2}-\wp^{\prime}(v)^{2}-\left(12 \wp(u) \wp(v)-g_{2}\right)(\wp(u)-\wp(v)\}\right.$.

Hier stehen rechts in jedem Gliede der Proportion rationale ganze Funktionen zweiten Grades in jedem der beiden Größenpaare $\wp(u), \wp^{\prime}(u)$ und $\wp(v), \wp^{\prime}(v)$.

Man kann das Additionstheorem der $\wp$-Funktion auch dadurch zum Ausdruck bringen, daB nach demselben zwischen den drei Funktionen $\wp(u+v), \wp(u), \wp(v)$ eine algebraische Beziehung bestehen muß. Diese Beziehung nimmt symmetrische Gestalt an, wenn wir $-(u+v)=w$ und also $\wp(u+v)=\wp(w)$ setzen. Die fragliche Beziehung gewinnt man leicht aus (13) durch Auflösung nach $\wp^{\prime}(u) \wp^{\prime}(v)$, Quadrieren und Ersatz von $\wp^{\prime}(u)^{2}$ und $\wp^{\prime}(v)^{2}$ durch ihre Ausdrücke in $\wp(u)$ und $\wp(v)$ : Für irgend drei Argumente $u, v, w$ der Summe $u+v+w=0$ gilt folgende Relation zweiten Grades in jeder der Funktionen $\wp(u), \wp(v), \wp(w)$ :

$$
\begin{aligned}
(\wp(v) \wp(w) & \left.+\wp(w) \wp(u)+\wp(u) \wp(v)+\frac{1}{4} g_{2}\right)^{2} \\
& -\left(4 \wp(u) \wp(v) \wp(u v)-g_{3}\right)(\wp(u)+\wp(v)+\wp(w))=0 .
\end{aligned}
$$

\section{\$2. Invariante algebraische Gestalten der Additionsformeln.}

Verlegt man die Betrachtung aus der $u$-Ebene auf die Riemannsche Fläche $F_{2}$, so nehmen die Additionsformeln des $\$ 1$ algebraische Gestalten an, die sich an die Rechnungen in I, $146 \mathrm{ff}$. anschließen. Wir setzen $f(z)=4 z^{3}-g_{2} z-g_{3}$ und haben den einzelnen Punkt der $\mathbf{F}_{2}$ durch ein zusammengehöriges Wertepaar $z, \sqrt{f(z)}$ festzulegen. Den beiden Argumenten $u$ und $v$ mögen die Stellen $x, \sqrt{f(x)}$ und $y, \sqrt{f(y)}$ der $\mathbf{F}_{2}$ entsprechen, dem Argumente $(u+v)$ aber die Stelle $z, \sqrt{f(z)}$. Die Bedeutung der Additionsformeln ist dann die, daß zwei willkürlich gewählte Stellen $x, \sqrt{f(x)}$ 
und $y, \sqrt{f(y)}$ stets eine dritte Stelle $z, \sqrt{f(z)}$ eindeutig bestimmen, die sich auf Grund von (13) und (14) S. 160 algebraisch aus den gegebenen Stellen berechnen läßt.

Es hat nun Klein ${ }^{1}$ ) gezeigt, daß man die zu gewinnenden neuen Formeln für die Additionstheoreme (13) und (14) S. 160 in sehr eineinfache invariante Gestalten setzen kann. Wir spalten $z$ (und natürlich entsprechend die Werte $x$ und $y$ von $z$ ) in den Quotienten $z_{1}: z_{2}$ zweier homogenen Variablen und ziehen neben $f(z)$ die "Verzweigungsform":

$$
f_{z}=4 z_{1}^{3} z_{2}-g_{2} z_{1} z_{2}^{3}-g_{3} z_{2}^{4}
$$

heran. Die Gleichung (13) S. 160 nimmt dann, in algebraische Gestalt umgeschrieben, nach Multiplikation mit $x_{2}^{2} y_{2}^{2}$ die Form an:

$$
2(x, y)^{2} z=\left(2 x_{1} y_{1}-\frac{1}{2} g_{2} x_{2} y_{2}\right)\left(x_{1} y_{2}+x_{2} y_{1}\right)-g_{3} x_{2}^{2} y_{2}^{2}-\sqrt{f_{x}} \cdot \sqrt{f_{y}},
$$

wo das Symbol $(x, y)$ wie in I, 146 eine Abkürzung für $\left(x_{1} y_{2}-x_{2} y_{1}\right)$ ist. Entsprechend kleidet sich die Gleichung (14) S. 160 nach Multiplikation mit $x_{2}^{3} y_{2}^{3}$ in die Gestalt:

$$
\begin{aligned}
(x, y)^{3} \sqrt{f(z)} & =\left(x_{1}^{2}\left(x_{1} y_{2}+3 x_{2} y_{1}\right)-\frac{1}{4} g_{2} x_{2}^{2}\left(3 x_{1} y_{2}+x_{2} y_{1}\right)-g_{3} x_{2}^{3} y_{2}\right) \sqrt{f_{y}} \\
& -\left(y_{1}^{2}\left(y_{1} x_{2}+3 y_{2} x_{1}\right)-\frac{1}{4} g_{2} y_{2}^{2}\left(3 y_{1} x_{2}+y_{2} x_{1}\right)-g_{3} y_{2}^{3} x_{2}\right) \sqrt{f_{x}} .
\end{aligned}
$$

Es sei nun $g_{x, y}$ die durch 4 geteilte erste Polare der Verzweigungsform $f_{x}$ und $h_{x, y}$ die durch 12 geteilte zweite Polare:

$$
\left\{\begin{aligned}
4 g_{x, y} & =\frac{\partial f_{x}}{\partial x_{1}} y_{1}+\frac{\partial f_{x}}{\partial x_{2}} y_{8}, \\
12 h_{x, y} & =\frac{\partial^{2} f_{x}}{\partial x_{1}^{2}} y_{1}^{2}+2 \frac{\partial^{2} f_{x}}{\partial x_{1} \partial x_{2}} y_{1} y_{2}+\frac{\partial^{2} f_{x}}{\partial x_{g}^{2}} y_{2}^{2}
\end{aligned}\right.
$$

Aus (1) berechnet man:

$$
\begin{aligned}
& g_{x, y}=\left(3 x_{1}^{2} x_{2}-\frac{1}{4} g_{2} x_{2}^{3}\right) y_{1}+\left(x_{1}^{3}-\frac{3}{4} g_{2} x_{1} x_{2}^{2}-g_{3} x_{2}^{3}\right) y_{2}, \\
& h_{x, y}=2 x_{1} x_{2} y_{1}^{2}+\left(2 x_{1}^{2}-\frac{1}{2} g_{2} x_{2}^{2}\right) y_{1} y_{2}-\left(\frac{1}{2} g_{2} x_{1} x_{2}+g_{3} x_{2}^{2}\right) y_{2}^{2} .
\end{aligned}
$$

Der Vergleich mit den vorstehenden Gleichungen ergibt als invariante. Schreibweise der Additionsformeln der Funktionen $\wp$ und $\wp^{\prime}$ :

$$
\left\{\begin{array}{c}
z=\frac{h_{x, y}-\sqrt{f_{x}} \sqrt{f_{y}}}{2(x, y)^{2}}, \\
\sqrt{f(z)}=\frac{g_{x, y} \sqrt{f_{y}}-g_{y, x} \sqrt{f_{x}}}{(x, y)^{3}} .
\end{array}\right.
$$

Diese Gleichungen erscheinen als algebraische Ausdrucksformen der transzendenten, zwischen den Stellen $x, y, z$ bestehenden Gleichung:

$$
\int_{\infty}^{x} \frac{d t}{\sqrt{f(t)}}+\int_{\infty}^{y} \frac{d t}{\sqrt{f(t)}}=\int_{\infty}^{z} \frac{d t}{\sqrt{f(t)}} .
$$

1) „Über hyperelliptische Sigmafunktionen“, Math. Ann. Bd. 27 (1886), S. 455 ff. 
In algebraischer Gestalt tritt das Additionstheorem bei Euler auf. ${ }^{1}$ ) Das Hauptinteresse wendet"sich dann aber in den ältesten Untersuchungen unseres Gegenstandes sogleich der Annahme zu, daB der Wert $z$ festgehalten wird. Dadurch werden die Stellen $x$ und $y$ in Abhängigkeit voneinander gesetzt, und zwar drückt sich diese Abhängigkeit zunächst in Gestalt der Differentialgleichung:

$$
\frac{d x}{\sqrt{f(x)}}+\frac{d y}{\sqrt{f(y)}}=0
$$

aus, der man auch die homogene Gestalt verleihen kann:

$$
\frac{(x, d x)}{\sqrt{f_{x}}}+\frac{(y, d y)}{\sqrt{f_{y}}}=0 .
$$

Indem man etwa in der ersten Gleichung (3) für $z$ den konstanten Wert $C$ einträgt, ergibt sich als algebraische Beziehung zwischen den Stellen $x, \sqrt{f(x)}$ und $y, \sqrt{f(y)}$ :

$$
h_{x, y}-2 C(x, y)^{2}-\sqrt{f_{x}} \sqrt{f_{y}}=0 .
$$

In dieser Gleichung hat unser Additionssatz das allgemeine Integral der Differentialgleichung erster Ordnung (5), und zwar als algebraische Beziehung zwischen $x$ und $y$ ergeben. Auf dieses Ergebnis wurde ursprünglich das Hauptgewicht gelegt. ${ }^{2}$ )

Sowohl die Differentialgleichung (6) als auch ihr Integral (7) sind invariant gebaut. Geht man demnach durch lineare Transformation zu einer beliebigen Verzweigungsform:

$$
f_{z}=a_{0} z_{1}^{4}+4 a_{1} z_{1}^{3} z_{2}+6 a_{2} z_{1}^{2} z_{2}^{2}+4 a_{3} z_{1} z_{2}^{3}+a_{4} z_{2}^{4},
$$

so behalten für diese sowohl die Differentialgleichung (6) als auch ihr Integral (7) unverändert ihre Gestalt bei. Übrigens gestattet die Gleichung (7) noch eine Vereinfachung. Man berechnet zunächst aus (8):

$h_{x, y}=x_{2}^{2} y_{2}^{2}\left(a_{0} x^{2} y^{2}+2 a_{1} x y(x+y)+a_{2}\left(x^{2}+4 x y+y^{2}\right)+2 a_{3}(x+y)+a_{4}\right)$ und gestaltet den Klammerausdruck unter Aufnahme der beiden Funktionen $f(x)$ und $f(y)$ leicht so um:

$$
\begin{aligned}
h_{x, y}=x_{2}^{2} y_{2}^{2}\left(\frac{1}{3} f(x)+\frac{1}{2} f(y)\right. & -\frac{1}{2} a_{0}\left(x^{2}-y^{2}\right)^{2} \\
& \left.-2 a_{1}\left(x^{2}-y^{2}\right)(x-y)-2 a_{2}(x-y)^{2}\right) .
\end{aligned}
$$

Durch Eintragung dieses Ausdrucks in (7) entwickelt man als Gestalt des allgemeinen Integrales der Gleichung (5):

$$
\left(\frac{\sqrt{f(x)}-\sqrt{f(y)}}{x-y}\right)^{2}=a_{0}(x+y)^{2}+4 a_{1}(x+y)+C^{\prime},
$$

wo $C^{\prime}=4 a_{2}+4 C$ als neue Konstante eingeführt ist. Dieses Integral ist bereits von Euler entdeckt.

1) S. die geachichtlichen Angaben bei "Enneper-Müller", S. $184 \mathrm{ff}$.

2) S. "Enneper-Müller", S. $185 \mathrm{ff}$. 
I, 1. Die Additionssätze der elliptischen Funktionen

In eine sehr einfache invariante Gestalt, die bisher nicht bemerkt zu sein scheint, kann man die Additionsformeln (16) S. 161 durch Aufnahme ternärer Variablen auf Grund der Proportion:

$$
x_{1}: x_{2}: x_{3}=\wp(u): \wp^{\prime}(u): 1
$$

kleiden. Zwischen den drei $x_{k}$ besteht dann eine algebraische Beziehung, die man durch Nullsetzen der ternären kubischen Form:

$$
F_{x}=4 x_{1}^{3}-g_{2} x_{1} x_{3}^{2}-x_{2}^{2} x_{3}-g_{3} x_{3}^{3}
$$

erhält. Man setze $u+v_{\star}+w=0$, wie S. 161, und bezeichne die den Argumenten $u, v, w$ zugehörigen ternären Variablen durch $x_{k}, y_{x}, z_{k}$. Die Proportion (16) nimmt dann die Gestalt an:

$$
z_{1}: z_{2}: z_{3}=
$$

$$
\begin{gathered}
\left\{x_{2}^{2} y_{1} y_{3}-x_{1} x_{3} y_{2}^{2}-\left(2 x_{2} y_{2}+g_{2}\left(x_{1} y_{3}+x_{3} y_{1}\right)+3 g_{3} x_{3} y_{3}\right)\left(x_{1} y_{3}-x_{3} y_{1}\right)\right\} \\
\left\{-\left(x_{2} y_{2}+3 g_{3} x_{3} y_{3}\right)\left(x_{2} y_{3}-x_{3} y_{2}\right)-2\left(6 x_{1} y_{1}-g_{2} x_{3} y_{3}\right)\left(x_{1} y_{2}-x_{2} y_{1}\right)\right. \\
\left.+g_{2}\left(x_{1} x_{2} y_{3}^{2}-x_{3}^{2} y_{1} y_{2}\right)\right\} \\
\left\{x_{2}^{2} y_{3}^{2}-x_{3}^{2} y_{2}^{2}-\left(12 x_{1} y_{1}-g_{2} x_{3} y_{3}\right)\left(x_{1} y_{3}-x_{3} y_{1}\right)\right\} .
\end{gathered}
$$

Bezeichnet $G_{x, y}$ die erste Polare der Form (11):

so gilt explizite:

$$
G_{x, y}=\frac{\partial F_{x}}{\partial x_{1}} y_{1}+\frac{\partial F_{x}}{\partial x_{2}} y_{2}+\frac{\partial F_{x}}{\partial x_{3}} y_{3}
$$

$$
G_{x, y}=12 x_{1}^{2} y_{1}-g_{2} x_{3}^{2} y_{1}-2 x_{2} x_{3} y_{2}-2 g_{2} x_{1} x_{3} y_{3}-x_{2}^{2} y_{3}-3 g_{3} x_{3}^{2} y_{3} .
$$

Die Proportion (12) läßt sich nun mit dieser Polare einfach so schreiben:

$$
z_{1}: z_{3}: z_{3}=\left(x_{1} G_{y, x}-y_{1} G_{x, y}\right):\left(x_{2} G_{y, x}-y_{2} G_{x, y}\right):\left(x_{3} G_{y, x}-y_{3} G_{x, y}\right) \text {. }
$$

Da die homogenen Variablen nur als Verhältnisgrößen für die Riemannsche Fläche in Betracht kommen, so kann man die $z_{k}$ auch direkt den drei in der letzten Proportion rechts stehenden Gliedern gleich setzen: Bei Gebrauch der durch (10) eingeführten ternären Variablen kann man die Additionsformeln in die einfachen Ausdrücke:

$$
z_{k}=x_{k} G_{y, x}-y_{k} G_{x, y},
$$

$k=1,2,3$

zusammenziehen, wo $G_{x, y}$ die erste Polare der ternären kubischen Form (11) ist.

\section{§ 3. Übergang zu den Additionsformeln der Jacobischen Funktionen.}

Bei Ausübung einer linearen Transformation auf die homogenen Variablen verhalten sich die rechten Seiten der Gleichungen (3) S. 162 invariant. Übt man die unimodulare Substitution $z_{1}=z_{1}^{\prime}+e_{x} z_{2}^{\prime}, z_{2}=z_{2}^{\prime}$ aus, so geht die Verzweigungsform $f_{z}=4 z_{2}\left(z_{1}-e_{1} z_{2}\right)\left(z_{1}-e_{2} z_{2}\right)\left(z_{1}-e_{3} z_{2}\right)$ bei Fortlassung der oberen Indizes an den neuen Variablen über in:

$$
f_{z}=4 z_{1} z_{2}\left(z_{1}-\left(e_{2}-e_{\varkappa}\right) z_{2}\right)\left(z_{1}-\left(e_{\mu}-e_{\chi_{2}}\right) z_{2}\right) \text {, }
$$


wo $x, \lambda, \mu$ die Indizes 1, 2, 3 in irgendeiner Anordnung sein sollen. Die erste Gleichung (3) S. 162 ergibt somit in den neuen Variablen und also für die Form (1):

$$
z=\frac{h_{x, y}-2 e_{x}(x, y)^{2}-\sqrt{f_{x}} \sqrt{f_{y}}}{2(x, y)^{2}}
$$

Berechnet man nun für die Form (1) die zweite Polare $12 h_{x, y}$, so ergibt sich:

$$
h_{x, y}-2 e_{x}(x, y)^{2}=2 x_{1} x_{2} y_{1}^{2}
$$

$+2\left(x_{1}^{2}+6 e_{\chi} x_{1} x_{2}+\left(e_{\lambda}-e_{\varkappa}\right)\left(e_{\mu}-e_{\chi}\right) x_{2}^{2} y_{1} y_{2}+2\left(e_{\lambda}-e_{\varkappa}\right)\left(e_{\mu}-e_{\varkappa}\right) x_{1} x_{2} y_{2}^{2}\right)$, wofür man nach Multiplikation mit $2 x_{1} x_{2} y_{1} y_{2}$ schreiben kann:

$$
2 x_{1} x_{2} y_{1} y_{2}\left(h_{x, y}-2 e_{x}(x, y)^{2}\right)=x_{1}^{2} x_{2}^{2} f_{y}+y_{1}^{2} y_{2}^{2} f_{x} \text {. }
$$

Die Gleichung (2) gestattet demnach die Schreibweise:

$$
z=\frac{\left(x_{1} x_{2} \sqrt{f_{y}}-y_{1} y_{2} \sqrt{f_{x}}\right)^{2}}{4 x_{1} x_{2} y_{1} y_{2}(x, y)^{2}}
$$

Nach Ausziehen der Quadratwurzel und Übergang zur nichthomogenen Schreibweise folgt:

$\sqrt{z}= \pm \frac{\sqrt{x} \sqrt{\left(y-\left(e_{\lambda}-e_{x}\right)\right)\left(y-\left(e_{\mu}-e_{x}\right)\right)}-\sqrt{y} \sqrt{\left(x-\left(e_{\lambda}-e_{x}\right)\right)\left(x-\left(e_{\mu}-e_{x}\right)\right)}}{x-y}$

Diese Gleichung schreibt sich bei Einführung der in I, $383 \mathrm{ff}$. erklärten doppeltperiodischen Funktionen zweiter Stufe $\psi_{*}(u), \varphi_{*}(u)$ so:

$$
\psi_{*}(u+v)= \pm \frac{\psi_{x}(u) \varphi_{x}(v)-\psi_{x}(v) \varphi_{*}(u)}{\psi_{*}(u)^{2}-\psi_{*}(v)^{2}} .
$$

Für $\lim u=0$ folgt, da $\lim _{u=0} \psi_{\varkappa}(u)=\infty$ ist:

$$
\psi_{x}(v)=\mp \psi_{x}(v) \cdot \lim _{u=0}\left(\frac{\varphi_{x}(u)}{\psi_{x}(u)^{2}}\right) \text {. }
$$

Nach (4) und (9) in I, 384ff. ist aber der rechts stehende limes gleich 1, so daß das untere Zeichen gilt: Die Additionstheoreme für die drei Funktionen zweiter Stufe $\psi_{\psi^{\prime}}(u)=\sqrt{\wp(u)-e_{*}}$ lauten also:

$$
\psi_{\varkappa}(u+v)=\frac{\varphi_{\varkappa}(u) \psi_{\varkappa}(v)-\psi_{\psi}(u) \varphi_{\varkappa}(v)}{\psi_{\varkappa}(u)^{2}-\psi_{\varkappa}(v)^{2}},
$$

wo $\varphi_{x}(u)$ nach I, 385 erklärt werden kann durch:

$$
\varphi_{\chi}(u)=\psi_{\lambda}(u) \psi_{\mu}(u)=\sqrt{\left(\psi_{\varkappa}(u)^{2}-\left(e_{\lambda}-e_{\varkappa}\right)\right)\left(\psi_{\varkappa}(u)^{2}-\left(e_{\mu}-e_{\varkappa}\right)\right)} .
$$

Von (3) aus kann man unmittelbar zu den Additionssätzen der Jacobischen Funktionen gelangen. Nach I, 389 gilt:

$$
\left\{\begin{array}{l}
\psi_{1}\left(\frac{u}{\sqrt{e_{2}-e_{1}}}\right)=\frac{\sqrt{e_{2}-e_{1}}}{\operatorname{sn} u} \\
\psi_{2}\left(\frac{u}{\sqrt{e_{2}-e_{1}}}\right)=\frac{\operatorname{cn} u \sqrt{e_{2}-e_{1}}}{\operatorname{sn} u} \\
\psi_{3}\left(\frac{u}{\sqrt{e_{2}-e_{1}}}\right)=\frac{\mathrm{d} \mathrm{n} u \sqrt{e_{2}-e_{1}}}{\operatorname{sn} u}
\end{array}\right.
$$


so dab man mit Rücksicht auf (4) weiter findet:

$$
\left\{\begin{array}{l}
\varphi_{1}\left(\frac{u}{\sqrt{e_{2}}}\right)=\frac{\left(e_{2}-e_{1}\right) \operatorname{cn} u \operatorname{dn} u}{\operatorname{sn} u^{2}} \\
\varphi_{2}\left(\frac{u}{\sqrt{e_{2}-e_{1}}}\right)=\frac{\left(e_{2}-e_{1}\right) \operatorname{dn} u}{\operatorname{sn} u^{2}}, \\
\varphi_{3}\left(\frac{u}{\sqrt{e_{2}-e_{1}}}\right)=\frac{\left(e_{2}-e_{1}\right) \operatorname{cn} u}{\operatorname{sn} u^{2}} .
\end{array}\right.
$$

Die Gleichungen (3) führen damit zu dem Ergebnis: Die Additionsformeln der Jacobischen Funktionen sn, cn, dn sind:

$$
\left\{\begin{array}{l}
\operatorname{sn}(u+v)=\frac{\operatorname{sn} u^{2}-\operatorname{sn} v^{2}}{\operatorname{sn} u \operatorname{cn} v \operatorname{dn} v-\operatorname{sn} v \operatorname{cn} u \operatorname{dn} u}, \\
\operatorname{cn}(u+v)=-\frac{\operatorname{dn} u \operatorname{sn} v \operatorname{cn} v-\operatorname{dn} v \operatorname{sn} u \operatorname{cn} u}{\operatorname{sn} u \operatorname{cn} v \operatorname{dn} v-\operatorname{sn} v \operatorname{cn} u \operatorname{dn} u} \\
\operatorname{dn}(u+v)=-\frac{\operatorname{cn} u \operatorname{dn} v \operatorname{sn} v-\operatorname{cn} v \operatorname{dn} u \operatorname{sn} u}{\operatorname{sn} u \operatorname{cn} v \operatorname{dn} v-\operatorname{sn} v \operatorname{cn} u \operatorname{dn} u}
\end{array}\right.
$$

Bei Aufstellung der zweiten und dritten Gleichung hat man von den bekannten Relationen Gebrauch zu machen:

$$
\mathrm{cn}^{2}=1-\mathrm{sn}^{2}, \quad \mathrm{dn}^{2}=1-k^{2} \mathrm{sn}^{2} .
$$

Gewöhnlich benutzt man eine andere Gestalt der Additionsformeln der Funktionen sn, cn, dn, die aus (7) durch eine einfache Umrechnung hervorgeht. Mit Hilfe von (8) beweist man leicht die Gleichung

$(\operatorname{sn} u \operatorname{cn} v \operatorname{dn} v-\operatorname{sn} v \operatorname{cn} u \operatorname{dn} u)(\operatorname{sn} u \operatorname{cn} v \operatorname{dn} v+\operatorname{sn} v \operatorname{cn} u \operatorname{dn} u)$

$$
=\left(\operatorname{sn} u^{2}-\operatorname{sn} v^{2}\right)\left(1-k^{2} \operatorname{sn} u^{2} \operatorname{sn} v^{2}\right) \text {. }
$$

Erweitert man nun die Gleichungen (7) rechts mit dem Ausdrucke ( $\operatorname{sn} u$ cn $v \operatorname{dn} v+\operatorname{sn} v$ cn $u \operatorname{dn} u$ ), so lassen sich auch die Zähler mittelst der Relationen (8) derart umformen, daß sich aus allen drei Brüchen die Faktoren ( $\left.\operatorname{sn} u^{2}-\operatorname{sn} v^{2}\right)$ fortheben lassen. Als neue Ausdrücke für die Additionsformeln der Jacobischen Funktionen sn, cn, dn gewinnt man so:

$$
\left\{\begin{array}{l}
\operatorname{sn}(u+v)=\frac{\operatorname{sn} u \operatorname{cn} v \operatorname{dn} v+\operatorname{sn} v \operatorname{cn} u \operatorname{dn} u}{1-k^{2} \operatorname{sn} u^{2} \operatorname{sn} v^{2}}, \\
\operatorname{cn}(u+v)=\frac{\operatorname{cn} u \operatorname{cn} v-\operatorname{sn} u \operatorname{sn} v \operatorname{dn} u \operatorname{dn} v}{1-k^{2} \operatorname{sn} u^{2} \operatorname{sn} v^{2}}, \\
\operatorname{dn}(u+v)=\frac{\operatorname{dn} u \operatorname{dn} v-k^{2} \operatorname{sn} u \operatorname{sn} v \operatorname{cn} u \operatorname{cn} v}{1-k^{2} \sin u^{2} \operatorname{sn} v^{2}} .
\end{array}\right.
$$

Für die in I, 472 unter (7) angegebene Ausartung der elliptischen Funktionen, die im Falle $k^{2}=0$ eintritt, gelangen wir hier zu den Additionssätzen der trigonometrischen Funktionen sin und cos zurück.

\section{\$ 4. Einführung einer Abelschen Gruppe $G_{256}$.}

Nach I, 384 führen die Änderungen des Argumentes $u$ der ursprünglichen Sigmafunktion um Periodenhälften zu den drei Sigmafunktionen 
$\sigma_{i}\left(u \mid \omega_{1}, \omega_{2}\right)$ der zweiten Stufe. Um allgemein das Verhalten der dreigliedrigen Relation (4) S. 158 bei solchen Änderungen festzustellen, erklären wir zunächst für die Argumente $x_{1}, y_{1}, z_{1}, t_{1}$ im ersten Gliede unserer Relation (4) S. 158 die symbolisch durch $V$ zu bezeichnende Substitution:

$$
\left\{\begin{array}{l}
x_{1}^{\prime}=x_{1}+\alpha_{1}^{\prime} \frac{\omega_{1}}{2}+\alpha_{1}^{\prime \prime} \frac{\omega_{2}}{2}, \\
y_{1}^{\prime}=y_{1}+\beta_{1}^{\prime} \frac{\omega_{1}}{2}+\beta_{1}^{\prime \prime} \frac{\omega_{2}}{2}, \\
z_{1}^{\prime}=z_{1}+\gamma_{1}^{\prime} \frac{\omega_{1}}{2}+\gamma_{1}^{\prime \prime} \frac{\omega_{2}}{2}, \\
t_{1}^{\prime}=t_{1}+\delta_{1}^{\prime} \frac{\omega_{1}}{2}+\delta_{1}^{\prime \prime} \frac{\omega_{2}}{2},
\end{array}\right.
$$

und schreiben für diese Substitution auch abkürzend:

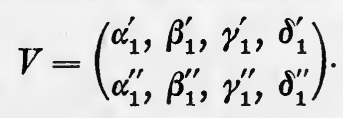

Hierbei sollen die $\alpha_{1}^{\prime}, \alpha_{1}^{\prime \prime}, \ldots, \delta_{1}^{\prime \prime}$ irgendwelche acht ganze Zahlen sein, die nur der einen Bedingung zu unterliegen haben, daß die vermöge der in (6) und (7) S. 158 gegebenen Transformationen $S$ und $S^{2}$ auf die Argumentreihen $x_{2}, y_{2}, \ldots$ und $x_{3}, y_{3}, \ldots$ umgerechnete Substitution $V$ wieder Substitutionen $S \cdot V \cdot S^{-1}$ und $S^{2} \cdot V \cdot S^{-2}$ der Gestalten:

$$
\left\{\begin{array}{l}
x_{i}^{\prime}=x_{i}+\alpha_{i}^{\prime} \frac{\omega_{1}}{2}+\alpha_{i}^{\prime \prime} \frac{\omega_{2}}{2}, \\
y_{i}^{\prime}=y_{i}+\beta_{i}^{\prime} \frac{\omega_{1}}{2}+\beta_{i}^{\prime \prime} \frac{\omega_{2}}{2}, \\
z_{i}^{\prime}=z_{i}+\gamma_{i}^{\prime} \frac{\omega_{1}}{2}+\gamma_{i}^{\prime \prime} \frac{\omega_{2}}{2}, \\
t_{i}^{\prime}=t_{i}+\delta_{i}^{\prime} \frac{\omega_{1}}{2}+\delta_{i}^{\prime \prime} \frac{\omega_{2}}{2}
\end{array}\right.
$$

mit ganzzahligen Koeffizienten $\alpha_{2}^{\prime}, \alpha_{2}^{\prime \prime}, \ldots, \delta_{3}^{\prime \prime}$ liefern. Nun berechnen sich aber die vier Koeffizienten $\alpha_{i}^{\prime}, \beta_{i}^{\prime}, \gamma_{i}^{\prime}, \delta_{i}^{\prime}$ aus den $\alpha_{1}^{\prime}, \beta_{1}^{\prime}, \gamma_{1}^{\prime}, \delta_{1}^{\prime}$ einfach durch die Substitution $S$ bzw. $S^{2}$, und ebenso erhält man die $\alpha_{i}^{\prime \prime}, \beta_{i}^{\prime \prime}, \gamma_{i}^{\prime \prime}, \delta_{i}^{\prime \prime}$ aus den $\alpha_{1}^{\prime \prime}, \beta_{1}^{\prime \prime}, \gamma_{1}^{\prime \prime}, \delta_{1}^{\prime \prime}$. Für die Ganzzahligkeit der $\alpha_{2}^{\prime}, \alpha_{2}^{\prime \prime}, \ldots, \delta_{3}^{\prime \prime}$ ist demnach das Bestehen der beiden Kongruenzen:

$$
\alpha_{1}^{\prime}+\beta_{1}^{\prime}+\gamma_{1}^{\prime}+\delta_{1}^{\prime} \equiv 0, \quad \alpha_{1}^{\prime \prime}+\beta_{1}^{\prime \prime}+\gamma_{1}^{\prime \prime}+\delta_{1}^{\prime \prime} \equiv 0 \quad(\bmod .2)
$$

notwendig und hinreichend.

Alle Substitutionen $V$ mit ganzzahligen, die Kongruenzen (4) erfüllenden Koeffizienten bilden nun eine kommutative oder Abelsche Gruppe $\Gamma$. Der Abelsche Charakter dieser Gruppe ist eine Folge des Umstandes, daB sich bei Kombination zweier Substitutionen $V$ entsprechende Koeffizienten addieren.

Soll die G-Relation (4) S. 158 durch ein einzelnes $V$ in sich transformiert werden, so müssen sowohl die acht Koeffizienten von $V$, wie die 
sechzehn von $S \cdot V \cdot S^{-1}$ und $S^{2} \cdot V \cdot S^{-2}$ durchweg gerade Zahlen sein. Aus den auf unsere Koeffizientensysteme umgeschriebenen Substitutionen (6) und (7) S. 158 geht hervor, daß für die Geradzahligkeit der Koeffizienten von $V, S \cdot V \cdot S^{-1}, S^{2} \cdot V \cdot S^{-2}$ die Kongruenzen:

$$
\left\{\begin{array}{lll}
\alpha_{1}^{\prime} \equiv \beta_{1}^{\prime} \equiv \gamma_{1}^{\prime} \equiv \delta_{1}^{\prime} \equiv 0, & \alpha_{1}^{\prime \prime} \equiv \beta_{1}^{\prime \prime} \equiv \gamma_{1}^{\prime \prime} \equiv \delta_{1}^{\prime \prime} \equiv 0 \quad(\bmod 2), \\
\alpha_{1}^{\prime}+\beta_{1}^{\prime}+\gamma_{1}^{\prime}+\delta_{1}^{\prime} \equiv 0, & \alpha_{1}^{\prime \prime}+\beta_{1}^{\prime \prime}+\gamma_{1}^{\prime \prime}+\delta_{1}^{\prime \prime} \equiv 0 \quad(\bmod 4),
\end{array}\right.
$$

notwendig und hinreichend sind. Alle Substitutionen $V$, die die Kongruenzen (5) befriedigen, bilden eine in $\Gamma$ enthaltene ausgezeichnete Untergruppe. $\left.{ }^{1}\right)$

Die gefundene Untergruppe nennen wir $\Gamma^{\prime}$ und zerlegen nach dem bei endlichen Gruppen angewandten Grundsatze die Gesamtgruppe $\Gamma$ in eine symbolische Summe von „Nebengruppen" (vgl. S. 4):

$$
\Gamma=\Gamma^{\prime}+\Gamma^{\prime} \cdot U_{1}+\Gamma^{\prime} \cdot U_{2}+\cdots,
$$

wo die $U$ geeignet gewählte Substitutionen $V$ sind. Aus dem additiven Gesetze, das für die Kombination unserer Substitutionen gilt, ist dann folgendes einleuchtend: In irgend zwei Substitutionen der einzelnen Nebengruppe sind je zwei entsprechende Koeffizienten mod 2 und je zwei entsprechende Koeffizientensummen $(\alpha+\beta+\gamma+\delta) \bmod 4$ kongruent; für irgend zwei Substitutionen aus verschiedenen Nebengruppen bestehen diese Kongruenzen nicht zugleich. Die Anzahl der Nebengruppen bestimmt man demnach durch folgende Abzählung: Auf Grund von (4) ist von den vier Zahlen $\alpha^{\prime}, \beta^{\prime}, \gamma^{\prime}, \delta^{\prime}$ eine mod 2 durch die übrigen bestimmt, und dasselbe gilt von einer der Zahlen $\alpha^{\prime \prime}, \beta^{\prime \prime}, \gamma^{\prime \prime}, \delta^{\prime \prime}$. Man hat also $\left(2^{3}\right)^{2}=64$ inkongruente Klassen von Substitutionen mod 2. Jede dieser 64 Klassen zerlegt sich wieder in 4 Unterklassen, je nachdem von den Zahlen $\left(\alpha^{\prime}+\beta^{\prime}+\gamma^{\prime}+\delta^{\prime}\right)$ und $\left(\alpha^{\prime \prime}+\beta^{\prime \prime}+\gamma^{\prime \prime}+\delta^{\prime \prime}\right)$, die zufolge (4) gerade sind, beide durch 4 teilbar sind oder nur die erste oder nur die zweite oder endlich keine von beiden. Indem wir den Begriff des „Index" einer Untergruppe aufnehmen, (vgl. S. 4), hat sich ergeben: Die durch die Kongruenzen (5) erliärte ausgezeichnete Untergruppe der Gruppe $\Gamma$ hat den Index 256 und möge demnach $\Gamma_{256}$ genannt werden.

Nach den S. 9 ff. für ausgezeichnete Untergruppen entwickelten Sätzen fassen wir nun die 256 Nebengruppen (6) selbst als Elemente einer Gruppe und bezeichnen diese Elemente gleich wieder durch $U_{0}=1, U_{1}, U_{2}, \ldots, U_{255}$. Die ausgezeichnete Untergruppe $\Gamma_{256}$ liefert auf diese Weise eine Abelsche Gruppe $G_{256}$ der endlichen Ordnung 256.

Von den Untergruppen dieser $G_{256}$ kommt diejenige zur Benutzung, deren Substitutionen den Kongruenzen:

$$
\alpha_{1}^{\prime} \equiv \beta_{1}^{\prime} \equiv \gamma_{1}^{\prime} \equiv \delta_{1}^{\prime}, \quad \alpha_{1}^{\prime \prime} \equiv \beta_{1}^{\prime \prime} \equiv \gamma_{1}^{\prime \prime} \equiv \delta_{1}^{\prime \prime} \quad(\bmod 2)
$$

1) Es ist selbstverständlich, $d_{a} B$ auch Abelsche Gruppen der Ordnung $\infty$ nur ausgezeichnete Untergruppen enthalten. 
genügen. Unter den 64 mod 2 inkongruenten Klassen von Substitutionen befriedigen vier diese Kongruenzen; man hat nämlich die beiden Fälle $\alpha_{1}^{\prime} \equiv \beta_{1}^{\prime} \equiv \cdots \equiv 0$ oder $\equiv 1(\bmod 2)$ mit den beiden Fällen $\alpha_{1}^{\prime \prime} \equiv \beta_{1}^{\prime \prime} \cdots \equiv 0$ oder $\equiv 1(\bmod 2)$ zu kombinieren. Jede der vier Klassen zerfällt aber, wie wir sahen, in vier Unterklassen, so daB im ganzen 16 unter den 256 bezüglich der $\Gamma_{256}$ inäquivalenten Klassen von Substitutionen die Kongruenzen (7) befriedigen. Die den Bedingungen (7) genïgenden Substitutionen der $G_{256}$ bilden eine ausgezcichnete Untergruppe $G_{16}$ der Ordnung 16 und des Index 16. Dieser $G_{16}$ entspricht innerhalb der Gruppe $\Gamma$ eine ausgezeichnete Untergruppe $\Gamma_{16}$ des Index 16.

Bevor wir etwas näher auf die $G_{16}$ eingehen, stellen wir die Wirkung der Transformation unserer Gruppen $\Gamma, \Gamma_{16}, \Gamma_{256}$ durch die Substitution $S$ fest. DaB die Substitution $S \cdot V \cdot S^{-1}$ wieder ganzzahlige Koeffizienten $\alpha_{2}^{\prime}, \beta_{2}^{\prime}, \ldots, \delta_{2}^{\prime \prime}$ hat, wurde bereits oben (S. 167) ausgesprochen. Da sich jede der beiden Reihen $\alpha_{2}, \beta_{2}, \gamma_{2}, \delta_{2}$ aus der entsprechenden Reihe $\alpha_{1}, \beta_{1}, \gamma_{1}, \delta_{1}$ durch die Substitution $S$ selbst berechnet, so ist für beide Reihen:

$$
\alpha_{2}+\beta_{2}+\gamma_{2}+\delta_{2}=\alpha_{1}-\beta_{1}-\gamma_{1}-\delta_{1},
$$

wie aus (6) S. 158 folgt. Also erfüllt auch $S \cdot V \cdot S^{-1}$ wieder die Kongruenzen (4), so daB $S \cdot \Gamma \cdot S^{-1}$ wieder die Gruppe $\Gamma$ ist (natürlich für die Variablen $x_{2}, y_{2}, z_{2}, t_{2}$ geschrieben). Da die Substitution $S$ orthogonal ist, so gilt nach S. 159 für jede der beiden Reihen $\alpha_{2}^{\prime}, \ldots$ und $\alpha_{2}^{\prime \prime} \ldots$ :

$$
\alpha_{2}^{2}+\beta_{2}^{2}+\gamma_{2}^{2}+\delta_{2}^{2}=\alpha_{1}^{2}+\beta_{1}^{2}+\gamma_{1}^{2}+\delta_{1}^{2} .
$$

Bestehen also insbesondere die Kongruenzen (7), so folgt:

$$
\alpha_{2}^{2}+\beta_{2}^{2}+\gamma_{2}^{2}+\delta_{2}^{2} \equiv 0 \quad(\bmod 4) \text {. }
$$

Da das Quadrat einer ganzen Zahl mod 4 mit 0 oder 1 kongruent ist, je nachdem die Zahl gerade oder ungerade ist, so folgt aus der vorstehenden Kongruenz, da $B$ die Zahlen $\alpha_{2}, \beta_{2}, \gamma_{2}, \delta_{2}$ der einzelnen der beiden Reihen mod 2 einander kongruent sind. Somit ist $S \cdot \Gamma_{16} \cdot S^{-1}$ wieder die Gruppe $\Gamma_{16}$ selbst. Gelten endlich die Kongruenzen (5), so erweist sich (wegen der ersten Gleichung (6) S. 158) jede der Zahlen $\alpha_{2}^{\prime}, \alpha_{2}^{\prime \prime}$ als gerade, und also sind nach der eben beendeten Überlegung alle acht Zahlen $\alpha_{2}, \beta_{2}, \gamma_{2}, \delta_{2}$ beider Reihen gerade. Dann aber folgt aus (8) und (5) weiter:

$$
\alpha_{2}+\beta_{2}+\gamma_{2}+\delta_{2} \equiv \alpha_{1}+\beta_{1}+\gamma_{1}+\delta_{1} \equiv 0 \cdot(\bmod 4)
$$

so daß auch $S \cdot V \cdot S^{-1}$ die Kongruenzen (5) befriedigt. Es hat sich also gezeigt, daß jede der drei Gruppen $\Gamma, \Gamma_{16}$ und $\Gamma_{256}$ durch $S$ und also auch durch $S^{2}$ in sich transformiert wird.

Wir bauen nun zunächst die $G_{16}$ in folgender Weise auf: Ein einzelnes Zahlquadrupel $(\alpha, \beta, \gamma, \delta)$ geht durch die Substitutionen $1, S, S^{2}$ 
in drei zusammengehörige Quadrupel über. Für $(\alpha, \beta, \gamma, \delta)=(0,0,0,0)$ sind diese drei Quadrupel einander gleich. Setzen wir weiter $(\alpha, \beta, \gamma, \delta)$ $=(2,0,0,0)$, so erhalten wir die drei Quadrupel:

$$
(2,0,0,0),(-1,1,1,1),(-1,-1,-1,-1) \text {. }
$$

Wir fügen zu diesen drei Quadrupeln noch $(0,0,0,0)$ hinzu und kombinieren die vier Quadrupel zu den sechzehn Paaren $\left(\begin{array}{c}\alpha^{\prime}, \beta^{\prime}, \gamma^{\prime}, \delta^{\prime} \\ \alpha^{\prime \prime}, \beta^{\prime \prime}, \gamma^{\prime \prime}, \delta^{\prime \prime}\end{array}\right)$. Diese sechzehn Paare können wir als die Substitutionen $\operatorname{der} G_{16}$ verwerten. Unter ihnen haben wir zunächst die identische Substitution $\left(\begin{array}{l}0,0,0,0 \\ 0,0,0,0\end{array}\right)$. Die 15 übrigen ordnen sich in fünf Systeme zu je dreien, wobei die Substitutionen des einzelnen Tripels durch $S$ zyklisch permutiert werden. Bei dreien unter diesen Tripeln, neun Substitutionen liefernd, besteht jedesmal eine der Substitutionen nur aus geraden Zahlen; ein Beispiel ist

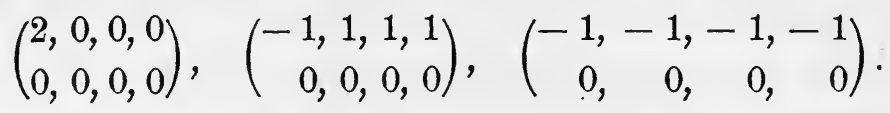

Bei den beiden übrigen Tripeln, die noch sechs Substitutionen ergeben, ist keine der Substitutionen aus durchweg geraden Zahlen zusammengesetzt; ein Beispiel hierfür ist das Tripel:

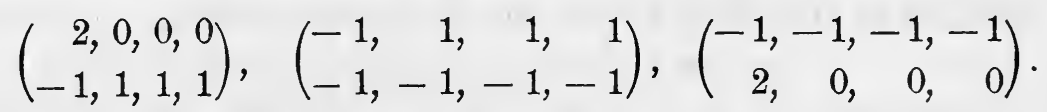

Man zerlege nun die $G_{256}$ entsprechend ihrer ausgezeichneten Untergruppe $G_{16}$ in die sechzehn Nebengruppen:

$$
G_{256}=G_{16}+G_{16} \cdot T_{1}+G_{16} \cdot T_{2}+\cdots+G_{16} \cdot T_{15} .
$$

Es sind dann endlich auch noch die sechzehn hierbei $\mathrm{zu}$ benutzenden Substitutionen $T_{0}=1, T_{1}, T_{2}, \ldots, T_{15}$ zweckmäßig zu wählen. Es gehören aber zwei Substitutionen

$$
\left(\begin{array}{l}
\alpha^{\prime}, \beta^{\prime}, \gamma^{\prime}, \delta^{\prime} \\
\alpha^{\prime \prime}, \beta^{\prime \prime}, \gamma^{\prime \prime}, \delta^{\prime \prime}
\end{array}\right), \quad\left(\begin{array}{l}
\overline{\alpha^{\prime}}, \overline{\beta^{\prime}}, \bar{\gamma}, \overline{\delta^{\prime}} \\
\bar{\alpha}^{\prime \prime}, \overline{\beta^{\prime \prime}}, \bar{\gamma}^{\prime \prime}, \overline{\delta^{\prime \prime}}
\end{array}\right)
$$

stets und nur dann der gleichen Nebengruppe $G_{16} \cdot T_{k}$ an, wenn die Kongruenzen gelten:

$$
\left.\begin{array}{l}
\bar{\alpha}^{\prime}-\alpha^{\prime} \equiv \overline{\beta^{\prime}}-\beta^{\prime} \equiv \bar{\gamma}^{\prime}-\gamma^{\prime} \equiv \overline{\delta^{\prime}}-\delta^{\prime} \\
\bar{\alpha}^{\prime \prime}-\alpha^{\prime \prime} \equiv \overline{\beta^{\prime \prime}}-\beta^{\prime \prime} \equiv \gamma^{\prime \prime}-\bar{\gamma}^{\prime \prime} \equiv \overline{\delta^{\prime \prime}}-\delta^{\prime \prime}
\end{array}\right\}
$$

Neben $T_{0}=1$ treffen wir nun für die weiter folgenden neun Substitutionen $T_{1}, T_{2}, \ldots, T_{9}$ die Wahlen: 
Die ausgezeichnete Untergruppe $G_{16}$ in der $G_{256}$

(13)

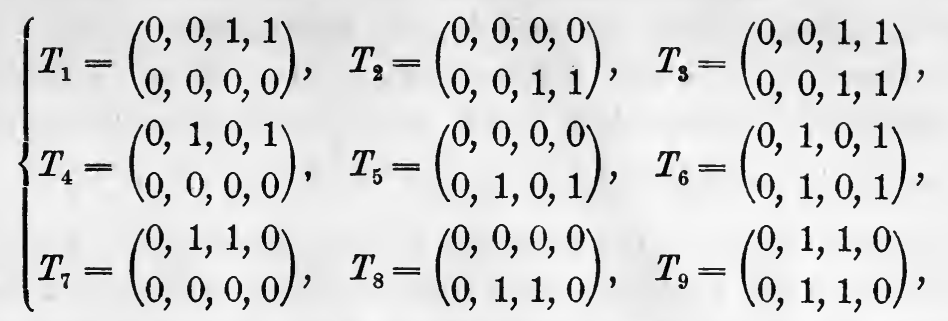

während wir den Rest der sechs Substitutionen $T_{10}, \ldots$ so bestimmen:

$$
\left\{\begin{array}{l}
T_{10}=\left(\begin{array}{l}
0,0,1,1 \\
0,1,0,1
\end{array}\right), \quad T_{11}=\left(\begin{array}{l}
0,1,0,1 \\
0,0,1,1
\end{array}\right), \quad T_{12}=\left(\begin{array}{l}
0,1,1,0 \\
0,0,1,1
\end{array}\right), \\
T_{13}=\left(\begin{array}{l}
0,0,1,1 \\
0,1,1,0
\end{array}\right), \quad T_{14}=\left(\begin{array}{l}
0,1,0,1 \\
0,1,1,0
\end{array}\right), \quad T_{15}=\left(\begin{array}{l}
0,1,1,0 \\
0,1,0,1
\end{array}\right) .
\end{array}\right.
$$

Man wolle mittelst der elementaren Durchrechnung feststellen, daB keine zwei der sechzehn Substitutionen $T_{0}, T_{1}, \ldots, T_{15}$ der gleichen Nebengruppe (12) angehören. Damit ist dann bewiesen, daB man diese Substitutionen zum Zwecke der Zerlegung (12) in der Tat gebrauchen kann.

\section{§ 5. Die 256 dreigliedrigen Sigmarelationen. ${ }^{1}$ )}

Mittelst der Relation (8) in I, 209 stellt man die Wirkung einer Substitution $V$ der Untergruppe $\Gamma_{256}$ auf die Relation (4) S. 158 fest. Das erste Glied der Relation erfährt die Substitution $V$, während auf die Argumente $x_{2}, \ldots$ und $x_{3}, \ldots$ der beiden anderen Glieder die Substitutionen $S \cdot V \cdot S^{-1}$ und $S^{2} \cdot V \cdot S^{-2}$ auszuüben sind. Alle Sigmafunktionen gehen bis auf Exponentialfaktoren in sich über. Der bei der ersten Funktion $\sigma\left(x_{1}\right)$ auftretende Faktor aber ist:

$$
e^{\pi i\left(\frac{\alpha_{1}^{\prime} \alpha_{1}^{\prime \prime}}{4}+\frac{\alpha_{1}^{\prime}+\alpha_{1}^{\prime \prime}}{2}\right)+\frac{\alpha_{1}^{\prime} \eta_{1}+\alpha_{1}^{\prime \prime} \eta_{2}}{2}\left(x_{1}+\frac{\alpha_{1}^{\prime} \omega_{1}+\alpha_{1}^{\prime \prime} \omega_{2}}{4}\right),}
$$

und man findet, daB das erste Glied unserer Sigmarelation in sich, multipliziert mit der Exponentialfunktion von:

$$
\begin{aligned}
& \frac{\pi i}{4}\left(\alpha_{1}^{\prime} \alpha_{1}^{\prime \prime}+\beta_{1}^{\prime} \beta_{1}^{\prime \prime}+\gamma_{1}^{\prime} \gamma_{1}^{\prime \prime}+\delta_{1}^{\prime} \delta_{1}^{\prime \prime}\right)+\frac{1}{2}\left(\left(\alpha_{1}^{\prime} \eta_{1}+\alpha_{1}^{\prime \prime} \eta_{2}\right) x_{1}+\cdots+\left(\delta_{1}^{\prime} \eta_{1}+\delta_{1}^{\prime \prime} \eta_{2}\right) t_{1}\right) \\
& +\frac{1}{8}\left(\left(\alpha_{1}^{\prime} \eta_{1}+\alpha_{1}^{\prime \prime} \eta_{2}\right)\left(\alpha_{1}^{\prime} \omega_{1}+\alpha_{1}^{\prime \prime} \omega_{2}\right)+\cdots+\left(\delta_{1}^{\prime} \eta_{1}+\delta_{1}^{\prime \prime} \eta_{2}\right)\left(\delta_{1}^{\prime} \omega_{1}+\delta_{1}^{\prime \prime} \omega_{2}\right)\right),
\end{aligned}
$$

übergeht. Die beiden anderen Glieder verhalten sich entsprechend; die bei ihnen auftretenden Exponentialfaktoren gehen aus dem eben angegebenen

1) Über das Auftreten solcher Relationen in Gestalt von Thetarelationen und über zahlreiche sie betreffende Untersuchungen vgl. man die sachlichen und geschichtlichen Darlegungen bei „Enneper-Müller", S. $135 \mathrm{ff}$. In erschöpfender Weise behandelt den Gegenstand E. Study in der Arbeit "Sphärische Trigonometrie, orthogonale Substitutionen und elliptische Funktionen", Leipz. Abhandl. Bd.20 (1893). 
hervor, indem man die Indizes 1 bei den Koeffizienten $\alpha, \beta, \ldots$ und den Variablen $x, y, \ldots$ durch 2 bzw. 3 ersetzt. Nun ist es die Wirkung der ,orthogonalen" Substitution $S$, daB bei diesem Ersatze die Ausdrücke:

$$
\alpha_{1} \alpha_{1}^{\prime \prime}+\beta_{1}^{\prime} \beta_{1}^{\prime \prime}+\gamma_{1}^{\prime} \gamma_{1}^{\prime \prime}+\delta_{1}^{\prime} \delta_{1}^{\prime \prime}, \quad \alpha_{1}^{\prime} x_{1}+\cdots+\delta_{1}^{\prime} t_{1}, \ldots, \alpha_{1}^{\prime \prime 2}+\cdots+\delta_{1}^{\prime \prime 2}
$$

unverändert bleiben. Die Faktoren der drei Glieder sind demnach einander gleich und können aus der transformierten Gleichung fortgehoben werden: Die dreigliedrige Sigmarelation (4) S. 158 wird durch die Substitutionen $V$ der Untergruppe $\Gamma_{256}$ in sich transformiert und nimmt demnach gegenüber den Substitutionen $V$ der Gesamtgruppe höchstens 256 verschiedene Gestalten an.

Die Gewinnung dieser Relationen ist nur noch eine etwas umständliche Rechenarbeit, die hier nicht in allen Einzelheiten dargestellt werden kann. Man wird zunächst die sechzehn Substitutionen der $G_{16}$ zur Ausübung bringen und die entstehenden sechzehn Relationen sodann vermittelst der sechzehn Substitutionen $T_{0}, T_{1}, \ldots, T_{15}$ umformen. Es ist zweckmäBig, neben $\omega_{1}, \omega_{2}$ auch noch als dritte Periode $\omega_{3}=-\omega_{1}-\omega_{2}$ einzuführen und $\eta_{3}$ als entsprechende Periode des Integrals zweiter Gattung zu benutzen. Bei den Rechnungen ist wiederholt die Legendresche Relation (6) aus I, $160 \mathrm{zu}$ benutzen. Im übrigen gründen sich die Rechnungen auf das Verhalten der ursprünglichen $\sigma-F u n k t i o n$ und der drei Funktionen $\sigma_{1}(u), \sigma_{2}(u), \sigma_{3}(u)$ zweiter Stufe bei Änderung der Argumente um Perioden oder um Periodenhälften. Wir notieren zunächst aus I,384:

$$
\left\{\begin{array}{l}
\sigma\left(u \pm \omega_{x}\right)=-e^{ \pm \eta_{x} u+\frac{1}{2} \eta_{x} \omega_{x}} \sigma(u), \\
\sigma_{x}\left(u \pm \omega_{x}\right)=-e^{ \pm \eta_{x} u+\frac{1}{2} \eta_{x} \omega_{x}} \sigma_{x}(u), \\
\sigma_{x}\left(u \pm \omega_{\lambda}\right)=+e^{ \pm \eta_{\lambda} u+\frac{1}{2} \eta_{\lambda} \omega_{\lambda}} \sigma_{x}(u),
\end{array}\right.
$$

wo $x, \lambda$ zwei verschiedene der drei Indizes $1,2,3$ sind. Weiter folgt aus der Erklärung der $\sigma_{\%}(u)$ in I, 384 und den übrigen daselbst entwickelten Formeln:

$$
\left\{\begin{array}{l}
\sigma\left(u \pm \frac{\omega_{x}}{2}\right)= \pm e^{ \pm \frac{1}{2} \eta_{x} u} \sigma\left(\frac{\omega_{x}}{2}\right) \sigma_{x}(u), \\
\sigma_{x}\left(u \pm \frac{\omega_{x}}{2}\right)=\mp e^{ \pm \frac{1}{2} \eta_{x} u+\frac{1}{4} \eta_{x} \omega_{x}} \cdot \frac{1}{\sigma\left(\frac{\omega_{x}}{2}\right)} \cdot \sigma(u), \\
\sigma_{x}\left(u \pm \frac{\omega_{\lambda}}{2}\right)=-e^{ \pm \frac{1}{2} \eta_{\lambda} u-\frac{1}{4} \eta_{x} \omega_{\lambda}} \cdot \frac{\sigma\left(\frac{\omega_{\mu}}{2}\right)}{\sigma\left(\frac{\omega_{x}}{2}\right)} \cdot \sigma_{\mu}(u),
\end{array}\right.
$$

wo $x, \lambda, \mu$ die Indizes $1,2,3$ in irgendeiner Anordnung sind. Die Werte der Sigmafunktion für die Periodenhälften können nach I, $416 \mathrm{ff}$. durch 
die daselbst unter (12) eindeutig erklärten vierten Wurzeln aus den Differenzen der $e_{1}, e_{2}, e_{3}$ so dargestellt werden:

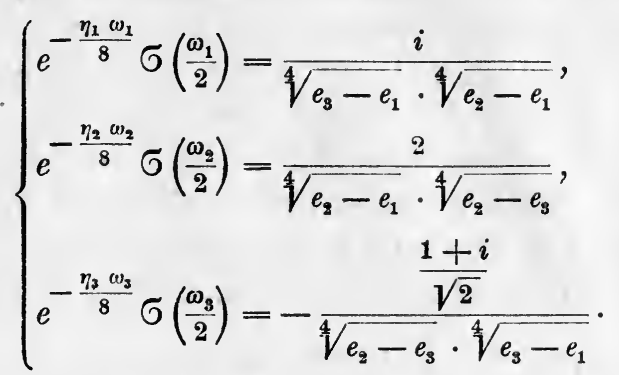

Die 15 von der identischen Substitution verschiedenen Substitutionen $\operatorname{der} G_{16}$ zerlegten wir in zwei Systeme zu neun bzw. sechs Substitutionen. Um ein Beispiel für die Wirkung der ersten neun Substitutionen auszuführen, so üben wir auf die drei Glieder der Relation (4) S. 158 gleichzeitig die drei Substitutionen (10) S. 170 aus. Im ersten Gliede bleiben die ursprünglichen $\sigma$-Funktionen bestehen, im zweiten und dritten Gliede findet sich überall die Funktion $\sigma_{1}$ ein. Entsprechend ist es überhaupt der Charakter der neun sich ergebenden neuen Relationen, da $B$ immer in einem der drei Glieder die ursprünglichen $\sigma$ verbleiben, während übrigens entweder nur $\sigma_{1}$ oder nur $\sigma_{2}$ oder endlich nur $\sigma_{3}$ auftritt. Als ein Beispiel für die sechs noch fehlenden Substitutionen der $G_{16}$ üben wir auf die drei liederG der Relation (4) S. 158 gleichzeitig die drei Substitutionen (11) S. 170 aus. Dabei treten im ersten Gliede nur Faktoren $\sigma_{2}$, im zweiten nur $\sigma_{3}$ und im dritten nur $\sigma_{1}$ auf. Entsprechend erscheinen wieder die übrigen fünf Relationen gebaut.

Etwaige gemeinsame Exponentialfaktoren in den drei Gliedern einer transformierten Relation wird man fortheben. Auch hat man sich bei den Umrechnungen neben der Legendreschen Relation zur Vereinfachung der drei Gleichungen (3) zu bedienen. Die Rechnungen führen zu einem sehr übersichtlichen Ergebnisse, falls man sich folgender Abkürzungen bedient:

$$
\left\{\begin{array}{l}
\xi^{(i)}=\sigma\left(x_{i}\right) \sigma\left(y_{i}\right) \sigma\left(z_{i}\right) \sigma\left(t_{i}\right), \\
\xi_{x}^{(i)}=-\frac{\sigma\left(x_{i}\right) \sigma_{x}\left(y_{i}\right) \sigma_{x}\left(z_{i}\right) \sigma_{x}\left(t_{i}\right)}{\left(e_{x}-e_{\lambda}\right)\left(e_{x}-e_{\mu}\right)} .
\end{array}\right.
$$

Die der $G_{16}$ entsprechenden sechzehn dreigliedrigen Sigmarelationen setzen sich dann zusammen erstens aus der ursprïnglichen Relation:

$$
\xi^{(1)}+\xi^{(2)}+\xi^{(3)}=0,
$$

sodann den neun Relationen:

$$
\xi^{(1)}-\xi_{x}^{(2)}+\xi_{x}^{(3)}=0, \quad \xi_{x}^{(1)}+\xi^{(2)}-\xi_{x}^{(3)}=0, \quad-\xi_{x}^{(1)}+\xi_{x}^{(2)}+\xi^{(3)}=0,
$$

wo $x=1,2,3$ zu nehmen ist, drittens aus den sechs Relationen:

$$
\xi^{(1)}+\xi_{\lambda}^{(2)}+\xi_{\mu}^{(3)}=0,
$$


wo $x, \lambda, \mu$ die sechs Anordnungen der drei Indizes 1, 2, 3 zu durchlaufen haben.

Auf die gewonnenen sechzehn Relationen sind nun weiter die neun Transformationen (13) S. 171 sowie die sechs Transformationen (14) S. 171 auszuüben. Aus der Bauart dieser Substitutionen ersieht man dann wieder leicht, welche Sigmafunktionen in den transformierten Relationen auftreten. Die Ergebnisse kleiden sich nach längeren Zwischenrechnungen wieder in eine sehr übersichtliche Gestalt. Wir erklären im. AnschluB an (4) drei Systeme von je vier Größen $\eta$ durch:

$$
\left\{\begin{array}{l}
\eta^{(i)}=+\sigma\left(x_{i}\right) \sigma\left(y_{i}\right) \sigma_{k}\left(z_{i}\right) \sigma_{k}\left(t_{i}\right), \\
\left.\eta_{1}^{(i)}=-\sigma_{k}\left(x_{i}\right) \sigma_{k}\left(y_{i}\right) \sigma\left(z_{i}\right) \sigma_{(}\right) \\
\eta_{2}^{(i)}=-\frac{\sigma_{k+1}\left(x_{i}\right) \sigma_{k+1}\left(y_{i}\right) \sigma_{k+2}\left(z_{i}\right) \sigma_{k+2}\left(t_{i}\right)}{e_{k+1}-e_{k+2}} \\
\eta_{3}^{(i)}=+\frac{\sigma_{k+2}\left(x_{i}\right) \sigma_{k+2}\left(y_{i}\right) \sigma_{k+1}\left(z_{i}\right) \sigma_{k+1}\left(t_{i}\right)}{e_{k+1}-e_{k+2}}
\end{array}\right.
$$

wo $k$ die Indizes 1, 2, 3 durchläuft und die unteren Indizes bei den $\sigma$ und $e$ nötigenfalls mod 3 zu reduzieren sind. Sechs weitere Systeme von. je vier Größen $\eta$ erklären wir durch:

und durch:

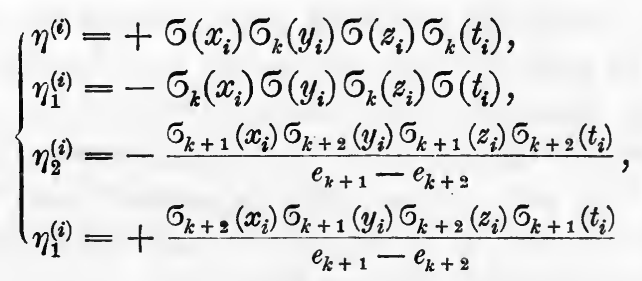

$$
\left\{\begin{array}{l}
\left.\eta^{(i)}=+\sigma\left(x_{i}\right) \sigma_{k}\left(y_{i}\right) \sigma_{k}\left(z_{i}\right) \sigma_{(}\right) \\
\eta_{1}^{(i)}=-\sigma_{k}\left(x_{i}\right) \sigma\left(y_{i}\right) \sigma\left(z_{i}\right) \sigma_{k}\left(t_{i}\right), \\
\eta_{2}^{(i)}=-\frac{\sigma_{k+1}\left(x_{i}\right) \sigma_{k+2}\left(y_{i}\right) \sigma_{k+2}\left(z_{i}\right) \sigma_{k+1}\left(t_{i}\right)}{e_{k+1}-e_{k+2}} \\
\eta_{3}^{(i)}=+\frac{\sigma_{k+2}\left(x_{i}\right) \sigma_{k+1}\left(y_{i}\right) \sigma_{k+1}\left(z_{i}\right) \sigma_{k+2}\left(t_{i}\right)}{e_{k+1}-e_{k+2}}
\end{array}\right.
$$

An die 16 Relationen (5) ff. reihen sich dann den neun Substitutionen (13) S. 171 entsprechend die weiteren $9 \cdot 16$ Relationen:

$$
\begin{aligned}
& \eta^{(1)}-\eta_{\%}^{(2)}+\eta_{x}^{(3)}=0, \eta_{x}^{(1)}+\eta^{(2)}-\eta_{x}^{(3)}=0,-\eta_{\varkappa}^{(1)}+\eta_{\varkappa}^{(2)}+\eta^{(3)}=0, \\
& \eta_{x}^{(1)}+\eta_{\lambda}^{(2)}+\eta_{\mu}^{(3)}=0
\end{aligned}
$$

die sich auf die neun erklärten Größensysteme $\eta, \eta_{1}, \eta_{2}, \eta_{3}$ beziehen.

Endlich sind noch die sechs Substitutionen (14) S. 171 auf die sechzehn Relationen (5)ff. auszuüben. Das Charakteristische ist nun, daß wir zu dreigliedrigen Relationen geführt werden, bei denen im einzelnen Gliede 
als Faktoren die ursprüngliche Sigmafunktion und die drei Funktionen zweiter Stufe zugleich auftreten. Wir erklären sechs Systeme zu je vier Größen $\xi$ durch:

$$
\left\{\begin{array}{l}
\xi^{(i)}=\sigma\left(x_{i}\right) \sigma_{k}\left(y_{i}\right) \sigma_{l}\left(z_{i}\right) \sigma_{m}\left(t_{i}\right), \\
\xi_{1}^{(i)}=\sigma_{k}\left(x_{i}\right) \sigma_{\left(y_{i}\right)} \sigma_{m}\left(z_{i}\right) \sigma_{l}\left(t_{i}\right), \\
\xi_{2}^{(i)}=\sigma_{l}\left(x_{i}\right) \sigma_{m}\left(y_{i}\right) \sigma_{\left(z_{i}\right)} \sigma_{k}\left(t_{i}\right), \\
\xi_{3}^{(i)}=\sigma_{m}\left(x_{i}\right) \sigma_{l}\left(y_{i}\right) \sigma_{k}\left(z_{i}\right) \sigma_{\left(t_{i}\right),}
\end{array}\right.
$$

wo auch die Indizes $k, l, m$ alle sechs Anordnungen von 1, 2, 3 durchlaufen sollen. Die noch fehlenden $6 \cdot 16$ Relationen haben dann wieder die Gestalt:

$$
\begin{gathered}
\xi^{(1)}+\xi^{(2)}+\xi^{(3)}=0, \\
\xi^{(1)}-\xi_{*}^{(2)}+\xi_{x}^{(3)}=0, \xi_{*}^{(1)}+\xi^{(2)}-\xi_{x}^{(3)}=0,-\xi_{x}^{(1)}+\xi_{\varkappa}^{(2)}+\xi^{(3)}=0, \\
\xi_{x}^{(1)}+\xi_{\lambda}^{(2)}+\xi_{\mu}^{(3)}=0 .
\end{gathered}
$$

Jede der 256 Relationen kann auch unabhängig von den übrigen durch eine funktionentheoretische Überlegung gewonnen werden, die uns oben zur ursprünglichen Relation (4) S. 158 führte. Übrigens sind die Relationen natürlich in mannigfachster Art voneinander abhängig. Sehr einfach gestaltet sich diese Abhängigkeit für je sechzehn zusammengehörige Relationen, wie im Falle der Relationen (5), (6) und (7) ausgeführt werden möge. Den ursprünglichen vier unabhängigen Variablen $u, u_{1}, u_{2}, u_{3}$ entsprechend wählen wir etwa die vier Größen $\xi^{(1)}, \xi_{1}^{(1)}, \xi_{2}^{(1)}, \xi_{3}^{(1)}$ als unabhängig. Die Auflösung der Relationen (5) ff. nach den $\xi^{(2)}, \xi_{1}^{(2)}, \ldots$ ergibt:

$$
\left\{\begin{array}{l}
\xi^{(2)}=-\frac{1}{2} \xi^{(1)}-\frac{1}{2} \xi_{1}^{(1)}-\frac{1}{2} \xi_{2}^{(1)}-\frac{1}{2} \xi_{3}^{(1)} \\
\xi_{1}^{(2)}=+\frac{1}{2} \xi^{(1)}+\frac{1}{2} \xi_{1}^{(1)}-\frac{1}{2} \xi_{2}^{(1)}-\frac{1}{2} \xi_{3}^{(1)} \\
\xi_{2}^{(2)}=+\frac{1}{3} \xi^{(1)}-\frac{1}{2} \xi_{1}^{(1)}+\frac{1}{2} \xi_{2}^{(1)}-\frac{1}{2} \xi_{3}^{(1)} \\
\xi_{3}^{(2)}=+\frac{1}{2} \xi^{(1)}-\frac{1}{2} \xi_{1}^{(1)}-\frac{1}{2} \xi_{2}^{(1)}+\frac{1}{2} \xi_{3}^{(1)}
\end{array}\right.
$$

während wir für die $\xi^{(3)}, \xi_{1}^{(3)}, \ldots$ zu den Ausdrücken gelangen:

$$
\left\{\begin{array}{l}
\xi^{(3)}=-\frac{1}{2} \xi^{(1)}+\frac{1}{2} \xi_{1}^{(1)}+\frac{1}{2} \xi_{2}^{(1)}+\frac{1}{2} \xi_{3}^{(1)}, \\
\xi_{1}^{(3)}=-\frac{1}{2} \xi^{(1)}+\frac{1}{2} \xi_{1}^{(1)}-\frac{1}{2} \xi_{2}^{(1)}-\frac{1}{2} \xi_{3}^{(1)} \\
\xi_{2}^{(3)}=-\frac{1}{2} \xi^{(1)}-\frac{1}{2} \xi_{1}^{(1)}+\frac{1}{2} \xi_{2}^{(1)}-\frac{1}{2} \xi_{3}^{(1)} \\
\xi_{3}^{(3)}=-\frac{1}{2} \xi^{(1)}-\frac{1}{2} \xi_{1}^{(1)}-\frac{1}{2} \xi_{2}^{(1)}+\frac{1}{2} \xi_{3}^{(1)} .
\end{array}\right.
$$

Aus diesen acht unabhängigen Relationen, die uns übrigens, wie man sieht, zu unseren beiden orthogonalen Substitutionen $S$ und $S^{2}$ zurückgeführt haben, sind dann die sechzehn Relationen (5) ff. einfache Folgen.

\section{§ 6. Die Additionstheoreme der Jacobischen Funktionen.}

Nach I, $416 \mathrm{ff}$. ist die Beziehung zwischen den Sigmafunktionen und den vier Jacobischen $\vartheta$-Funktionen die folgende: 


$$
\begin{aligned}
& \sigma(u)=\sqrt{\frac{\overline{2 \pi}}{\omega_{2}}} \frac{1}{\sqrt[8]{\Delta}} e^{\frac{\eta_{2} u^{2}}{2 \omega_{2}}} \vartheta_{1}(v), \\
& \sigma_{1}(u)=\sqrt{\frac{\pi}{\omega_{2}}} \frac{1}{\sqrt[4]{e_{2}-e_{3}}} e^{\frac{\eta_{2} u^{2}}{2 \omega_{2}}} \vartheta_{0}(v), \\
& \sigma_{2}(u)=\sqrt{\frac{\pi}{\omega_{2}}} \frac{1}{\sqrt[4]{e_{3}-e_{1}}} e^{\frac{\eta_{2} u^{2}}{2 \omega_{2}}} \vartheta_{2}(v), \\
& \sigma_{3}(u)=\sqrt{\frac{\pi}{\omega_{2}}} \frac{1}{\sqrt[4]{e_{2}-e_{1}}} e^{\frac{\eta_{2} u^{2}}{2 \omega_{2}}} \vartheta_{3}(v),
\end{aligned}
$$

wo $u=v \omega_{2}$ gilt und die rechts auftretenden Wurzeln durch die Relation verbunden sind:

$$
\sqrt{\frac{\omega_{2}}{\pi}} \sqrt[4]{e_{2}-e_{3}} \cdot \sqrt[4]{e_{3}-e_{1}} \cdot \sqrt[4]{e_{2}-e_{1}}=\sqrt{\frac{\omega_{2}}{2 \pi}} \sqrt[8]{\Delta}
$$

Rechnet man nun die einzelne dreigliedrige Sigmarelation auf ihren Ausdruck in den $\vartheta$-Funktionen um, so erweisen sich die in den drei Gliedern auftretenden Exponentialfaktoren auf Grund der Eigenschaft der $S$ und $S^{2}$ als "orthogonaler" Substitutionen als gleich. Diese Faktoren und ebenso die von den Wurzeln $\sqrt{\frac{\pi}{\omega_{2}}}$ herrührenden gemeinsamen Faktoren der drei Glieder können demnach fortgehoben werden. Die Rechnungen führen zu folgendem Ergebnis: In den 256 für die $\xi, \eta, \xi$ aufgestellten Relationen liann man diesen Größen auch folgende Bedeutungen unterlegen:

$$
\begin{aligned}
& \xi^{(i)}=+\vartheta_{1}\left(x_{i}\right) \vartheta_{1}\left(y_{i}\right) \vartheta_{1}\left(z_{i}\right) \vartheta_{1}\left(t_{i}\right), \\
& \xi_{1}^{(i)}=-\vartheta_{0}\left(x_{i}\right) \vartheta_{0}\left(y_{i}\right) \vartheta_{0}\left(z_{i}\right) \vartheta_{0}\left(t_{i}\right), \\
& \xi_{2}^{(i)}=-\vartheta_{2}\left(x_{i}\right) \vartheta_{2}\left(y_{i}\right) \quad \vartheta_{2}\left(z_{i}\right) \vartheta_{2}\left(t_{i}\right), \\
& \xi_{3}^{(i)}=+\vartheta_{3}\left(x_{i}\right) \vartheta_{3}\left(y_{i}\right) \vartheta_{3}\left(z_{i}\right) \vartheta_{3}\left(t_{i}\right), \\
& \eta^{(i)}=+\vartheta_{1}\left(x_{i}\right) \vartheta_{1}\left(y_{i}\right) \vartheta_{k}\left(z_{i}\right) \vartheta_{k}\left(t_{i}\right), \\
& \eta_{1}^{(i)}=-\vartheta_{k}\left(x_{i}\right) \vartheta_{k}\left(y_{i}\right) \vartheta_{1}\left(z_{i}\right) \vartheta_{1}\left(t_{i}\right), \\
& \eta_{2}^{(i)}=-\vartheta_{l}\left(x_{i}\right) \vartheta_{l}\left(y_{i}\right) \vartheta_{m}\left(z_{i}\right) \vartheta_{m}\left(t_{i}\right), \\
& \eta_{3}^{(i)}=+\vartheta_{m}\left(x_{i}\right) \vartheta_{m}\left(y_{i}\right) \vartheta_{l}\left(z_{i}\right) \vartheta_{l}\left(t_{i}\right), \\
& \xi^{(i)}=\vartheta_{1}\left(x_{i}\right) \vartheta_{k}\left(y_{i}\right) \vartheta_{l}\left(z_{i}\right) \vartheta_{m}\left(t_{i}\right), \\
& \xi_{1}^{(i)}=\vartheta_{k}\left(x_{i}\right) \vartheta_{1}\left(y_{i}\right) \vartheta_{m}\left(z_{i}\right) \vartheta_{i}\left(t_{i}\right), \\
& \xi_{2}^{(i)}=\vartheta_{l}\left(x_{i}\right) \vartheta_{m}\left(y_{i}\right) \vartheta_{1}\left(z_{i}\right) \vartheta_{k}\left(t_{i}\right), \\
& \xi_{3}^{(i)}=\vartheta_{m}\left(x_{i}\right) \vartheta_{l}\left(y_{i}\right) \vartheta_{k}\left(z_{i}\right) \vartheta_{1}\left(t_{i}\right) .
\end{aligned}
$$

Hierbei ist noch folgendes zu bemerken: In den vier Formeln für die $\eta$ hat man die Indizes $k, l, m$ zuerst gleich $0,2,3$, sodann gleich $2,3,0$, endlich gleich 3, 0,2 zu nehmen. Neben jedes der drei so zu gewinnenden Systeme der $\eta$ treten dann noch zwei weitere, die man aus ihnen einfach durch zyklische Permutation der Argumente $y, z, t$ ableiten kann. 
Auf diese Weise entstehen, wie es sein muB, die neun Systeme der $\eta$. In den Formeln für die $\xi$ hat man für $k, l, m$ der Reihe nach die sechs Anordnungen der Indizes $0,2,3$ einzutragen, womit wir die sechs Systeme der $\xi$ gewinnen. Die drei Systeme der $x_{i}, y_{i}, z_{i}, t_{i}$ hängen wieder durch die Relationen (6) und (7) S. 158 zusammen.

Der Ansatz der $\vartheta$-Relationen führt uns in das Gebiet der Untersuchungen, welche Jacobi in seiner Vorlesung „Theorie der elliptischen Funktionen aus den Eigenschaften der Thetareihen abgeleitet" ${ }^{\text {(1) }}$ zur Grundlage gewählt hat. Analytische Entwicklungen über diese Relationen sind seither vielfach wiederholt und weitergeführt..2) Die Möglichkeit, jede dieser Relationen auch einzeln auf Grund eines funktionentheoretischen Schlusses (mittelst des Hermiteschen Satzes von I, 227) zu gewinnen, wurde bereits erwähnt. Im übrigen erscheinen die Relationen in ihrer Anzahl 256 deshalb in einer gewissen abgeschlossenen Vollständigkeit, weil man in ihnen eben alle Relationen besitzt, die man aus einer unter ihnen vermittelst der Änderung der Argumente um Periodenhälften abzuleiten imstande ist.

$\mathrm{Zu}$ den Additionssätzen der Jacobischen Funktionen gelangt man nun, indem man für die Argumente $x_{1}, y_{1}, z_{1}, t_{1}$ insbesondere

$$
x_{1}=y_{1}=u, \quad z_{1}=t_{1}=v
$$

einträgt, was nach (6) und (7) S. 128 für $x_{2}, y_{2}, \ldots, t_{3}$ die Werte:

$$
x_{2}=-u-v, y_{2}=u-v, z_{2}=t_{2}=0, x_{3}=-y_{3}=v, z_{3}=t_{3}=-u
$$

nach sich zieht. Die 256 Relationen werden dann z. T. identisch erfüllt, z. T. werden sie miteinander identisch.

Zunächst gewinnen die zwölf Größen $\xi$ folgende Bedeutungen:

$$
\begin{array}{ll}
\xi^{(1)}=-\xi^{(3)}=+\vartheta_{1}(u)^{2} \vartheta_{1}(v)^{2}, & \xi^{(2)}=0, \\
\xi_{1}^{(1)}=+\xi_{1}^{(3)}=-\vartheta_{0}(u)^{2} \vartheta_{0}(v)^{2}, & \xi_{1}^{(2)}=-\vartheta_{0}^{2} \vartheta_{0}(u+v) \vartheta_{0}(u-v), \\
\xi_{2}^{(1)}=+\xi_{2}^{(3)}=-\vartheta_{2}(u)^{2} \vartheta_{2}(v)^{2}, & \xi_{2}^{(2)}=-\vartheta_{2}^{2} \vartheta_{2}(u+v) \vartheta_{2}(u-v), \\
\xi_{3}^{(1)}=+\xi_{3}^{(3)}=+\vartheta_{3}(u)^{2} \vartheta_{3}(v)^{2}, & \xi_{3}^{(2)}=+\vartheta_{3}^{2} \vartheta_{3}(u+v) \vartheta_{3}(u-v) .
\end{array}
$$

Bei Eintragung in die sechzehn Relationen (5), (6) und (7) S. 173 werden vier von ihnen identisch erfüllt, während die zwölf übrigen zu Paaren identisch werden und folgende sechs verschiedene $\vartheta$-Relationen liefern:

$$
\left\{\begin{aligned}
\vartheta_{0}^{2} \vartheta_{0}(u+v) \vartheta_{0}(u-v) & =\vartheta_{0}(u)^{2} \vartheta_{0}(v)^{2}-\vartheta_{1}(u)^{2} \vartheta_{1}(v)^{2} \\
& =\vartheta_{3}(u)^{2} \vartheta_{3}(v)^{2}-\vartheta_{2}(u)^{2} \vartheta_{2}(v)^{2} \\
\vartheta_{2}^{2} \vartheta_{2}(u+v) \vartheta_{2}(u-v) & =\vartheta_{2}(u)^{2} \vartheta_{2}(v)^{2}-\vartheta_{1}(u)^{2} \vartheta_{1}(v)^{2} \\
& =\vartheta_{3}(u)^{2} \vartheta_{3}(v)^{2}-\vartheta_{0}(u)^{2} \vartheta_{0}(v)^{2} \\
\vartheta_{3}^{2} \vartheta_{3}(u+v) \vartheta_{3}(u-v) & =\vartheta_{3}(u)^{2} \vartheta_{3}(v)^{2}+\vartheta_{1}(u)^{2} \vartheta_{1}(v)^{2} \\
& =\vartheta_{0}(u)^{2} \vartheta_{0}(v)^{2}+\vartheta_{2}(u)^{2} \vartheta_{2}(v)^{2}
\end{aligned}\right.
$$

1) Ausgearbeitet von C. W. Borchardt; s. Jacobis Werke, Bd. I, S. $503 \mathrm{ff}$.

2) S. die Note. S. 171. 
Man stelle entsprechend die für $x_{1}=y_{1}=u, z_{1}=t_{1}=v$ eintretenden besonderen Gestalten der Größen $\eta$ fest und wird durch Eintragung in die Gleichungen (11) ff. S. 174 finden, daB die 9.16 Relationen der $\eta$ im ganzen 24 verschiedene $\boldsymbol{\vartheta}$-Relationen ergeben. Es sind dies erstens die drei Systeme $\mathrm{zu}$ je sechs Relationen:

$$
\begin{aligned}
& \left\{\begin{aligned}
\vartheta_{0}^{2} \vartheta_{1}(u+v) \vartheta_{1}(u-v) & =\vartheta_{1}(u)^{2} \vartheta_{0}(v)^{2}-\vartheta_{0}(u)^{2} \vartheta_{1}(v)^{2} \\
& =\vartheta_{3}(u)^{2} \vartheta_{2}(v)^{2}-\vartheta_{2}(u)^{2} \vartheta_{3}(v)^{2} \\
\vartheta_{2}^{2} \vartheta_{3}(u+v) \vartheta_{3}(u-v) & =\vartheta_{1}(u)^{2} \vartheta_{0}(v)^{2}+\vartheta_{2}(u)^{2} \vartheta_{3}(v)^{2} \\
& =\vartheta_{0}(u)^{2} \vartheta_{1}(v)^{2}+\vartheta_{3}(u)^{2} \vartheta_{2}(v)^{2} \\
\vartheta_{3}^{2} \vartheta_{2}(u+v) \vartheta_{2}(u-v) & =\vartheta_{3}(u)^{2} \vartheta_{2}(v)^{2}-\vartheta_{1}(u)^{2} \vartheta_{0}(v)^{2} \\
& =\vartheta_{2}(u)^{2} \vartheta_{3}(v)^{2}-\vartheta_{0}(u)^{2} \vartheta_{1}(v)^{2} \\
& =\vartheta_{0}(u)^{2} \vartheta_{3}(v)^{2}-\vartheta_{3}(u)^{2} \vartheta_{0}(v)^{2} \\
& =\vartheta_{2}(u)^{2} \vartheta_{1}(v)^{2}+\vartheta_{0}(u)^{2} \vartheta_{3}(v)^{2} \\
\vartheta_{3}^{2} \vartheta_{0}(u+v) \vartheta_{0}(u-v) & =\vartheta_{1}(u)^{2} \vartheta_{2}(v)^{2}+\vartheta_{3}(u)^{2} \vartheta_{0}(v)^{2} \\
& =\vartheta_{3}(u)^{2} \vartheta_{0}(v)^{2}-\vartheta_{9}(u)^{2} \vartheta_{1}(v)^{2} \\
\vartheta_{0}^{2} \vartheta_{3}(u+v) \vartheta_{3}(u-v) & =\vartheta_{1}(u)^{2} \vartheta_{3}(u)^{2}-\vartheta_{1}(u)^{2} \vartheta_{2}(v)^{2} \vartheta_{3}(v)^{2}-\vartheta_{3}(u)^{2} \vartheta_{1}(v)^{2} \\
& =\vartheta_{0}(u)^{2} \vartheta_{2}(v)^{2}-\vartheta_{2}(u)^{2} \vartheta_{0}(v)^{2} \\
\vartheta_{3}^{2} \vartheta_{1}(u+v) \vartheta_{1}(u-v) & =\vartheta_{1}(u)^{2} \vartheta_{3}(v)^{2}+\vartheta_{2}(u)^{2} \vartheta_{0}(v)^{2} \\
\vartheta_{2}^{2} \vartheta_{0}(u+v) \vartheta_{0}(u-v) & =\vartheta_{3}(u)^{2} \vartheta_{1}(v)^{2}+\vartheta_{0}(u)^{2} \vartheta_{2}(v)^{2} \\
\vartheta_{0}^{2} \vartheta_{2}(u+v) \vartheta_{2}(u-v) & =\vartheta_{0}(u)^{2} \vartheta_{2}(v)^{2}-\vartheta_{1}(u)^{2} \vartheta_{3}(v)^{2} \\
& =\vartheta_{2}(u)^{2} \vartheta_{0}(v)^{2}-\vartheta_{3}(u)^{2} \vartheta_{1}(v)^{2}
\end{aligned}\right.
\end{aligned}
$$

an die sich weiter die drei Systeme zu je zwei Relationen anreihen:

$$
\left\{\begin{aligned}
\vartheta_{2} \vartheta_{3} \vartheta_{2}(u+v) \vartheta_{3}(u-v) & =\vartheta_{2}(u) \vartheta_{3}(u) \vartheta_{2}(v) \vartheta_{3}(v) \\
& -\vartheta_{1}(u) \vartheta_{0}(u) \vartheta_{1}(v) \vartheta_{0}(v), \\
\vartheta_{3} \vartheta_{2} \vartheta_{3}(u+v) \dot{\vartheta}_{2}(u-v) & =\vartheta_{2}(u) \vartheta_{3}(u) \vartheta_{2}(v) \vartheta_{3}(v) \\
& +\vartheta_{1}(u) \vartheta_{0}(u) \vartheta_{1}(v) \vartheta_{0}(v),
\end{aligned}\right.
$$$$
\left\{\begin{aligned}
\vartheta_{3} \vartheta_{0} \vartheta_{3}(u+v) \vartheta_{0}(u-v) & =\vartheta_{3}(u) \vartheta_{0}(u) \vartheta_{3}(v) \vartheta_{0}(v) \\
& -\vartheta_{1}(u) \vartheta_{2}(u) \vartheta_{1}(v) \vartheta_{2}(v), \\
\vartheta_{0} \vartheta_{3} \vartheta_{0}(u+v) \vartheta_{3}(u-v) & =\vartheta_{3}(u) \vartheta_{0}(u) \vartheta_{3}(v) \vartheta_{0}(v) \\
& +\vartheta_{1}(u) \vartheta_{2}(u) \vartheta_{1}(v) \vartheta_{2}(v),
\end{aligned}\right.
$$

$$
\left\{\begin{aligned}
\vartheta_{2} \vartheta_{0} \vartheta_{2}(u+v) \vartheta_{0}(u-v) & =\vartheta_{2}(u) \vartheta_{0}(u) \vartheta_{2}(v) \vartheta_{0}(v) \\
& -\vartheta_{1}(u) \vartheta_{3}(u) \vartheta_{1}(v) \vartheta_{3}(v) \\
\vartheta_{0} \vartheta_{2} \vartheta_{0}(u+v) \vartheta_{2}(u-v) & =\vartheta_{2}(u) \vartheta_{0}(u) \vartheta_{2}(v) \vartheta_{0}(v) \\
& +\vartheta_{1}(u) \vartheta_{3}(u) \vartheta_{1}(v) \vartheta_{3}(v)
\end{aligned}\right.
$$


Endlich bleiben noch die sechs Systeme zu je vier Größen $\xi$ übrig. Ihre 6.16 Relationen ergeben für $x_{1}=y_{1}=u, z_{1}=t_{1}=v$ nur noch sechs verschiedene $\vartheta$-Relationen, die wir in drei Systeme zu je zweien anordnen:

$$
\begin{aligned}
& \left\{\begin{aligned}
\vartheta_{2} \vartheta_{3} \vartheta_{1}(u+v) \vartheta_{0}(u-v) & =\vartheta_{1}(u) \vartheta_{0}(u) \vartheta_{2}(v) \vartheta_{3}(v) \\
& +\vartheta_{2}(u) \vartheta_{3}(u) \vartheta_{1}(v) \vartheta_{0}(v), \\
\vartheta_{2} \vartheta_{3} \vartheta_{0}(u+v) \vartheta_{1}(u-v) & =\vartheta_{1}(u) \vartheta_{0}(u) \vartheta_{2}(v) \vartheta_{3}(v) \\
& -\vartheta_{2}(u) \vartheta_{3}(u) \vartheta_{1}(v) \vartheta_{0}(v),
\end{aligned}\right. \\
& \left\{\begin{aligned}
\vartheta_{0} \vartheta_{3} \vartheta_{1}(u+v) \vartheta_{2}(u-v) & \vartheta_{1}(u) \vartheta_{2}(u) \vartheta_{0}(v) \vartheta_{3}(v) \\
& +\vartheta_{0}(u) \vartheta_{3}(u) \vartheta_{1}(v) \vartheta_{2}(v), \\
\vartheta_{0} \vartheta_{3} \vartheta_{2}(u+v) \vartheta_{1}(u-v) & =\vartheta_{1}(u) \vartheta_{2}(u) \vartheta_{0}(v) \vartheta_{3}(v) \\
& -\vartheta_{0}(u) \vartheta_{3}(u) \vartheta_{1}(v) \vartheta_{2}(v),
\end{aligned}\right. \\
& \left\{\begin{aligned}
\vartheta_{0} \vartheta_{2} \vartheta_{3}(u+v) \vartheta_{3}(u-v) & \vartheta_{1}(u) \vartheta_{3}(u) \vartheta_{0}(v) \vartheta_{2}(v) \\
& +\vartheta_{0}(u) \vartheta_{2}(u) \vartheta_{1}(v) \vartheta_{3}(v), \\
\vartheta_{0} \vartheta_{2} \vartheta_{3}(u+v) \vartheta_{1}(u-v) & =\vartheta_{1}(u) \vartheta_{3}(u) \vartheta_{0}(v) \vartheta_{2}(v) \\
& -\vartheta_{0}(u) \vartheta_{2}(u) \vartheta_{1}(v) \vartheta_{3}(v)
\end{aligned}\right.
\end{aligned}
$$

Nach I, 419 ff. gelten für die Jacobischen Funktionen sn, en, dn die Darstellungen:

$$
\begin{aligned}
& \vartheta_{1}(u)=\sqrt{k} \operatorname{sn}(2 K u) \vartheta_{0}(u), \\
& \vartheta_{2}(u)=\sqrt{\frac{k}{k^{\prime}}} \operatorname{cn}(2 K u) \vartheta_{0}(u), \\
& \vartheta_{3}(u)=\frac{1}{\sqrt{k^{\prime}}} \operatorname{dn}(2 K u) \vartheta_{0}(u),
\end{aligned}
$$

während die $\vartheta$-Nullwerte mit den Integralmoduln durch die Beziehungen verknüpft sind:

$$
\vartheta_{0}=\vartheta_{3} \cdot \sqrt{k^{\prime}}, \quad \vartheta_{2}=\vartheta_{3} \cdot \sqrt{k} .
$$

Bildet man nun den Quotienten irgend zweier von den $36 \vartheta$-Relationen, so lassen sich die Quotienten der $\vartheta$-Funktionen durch die Funktionen sn, cn, dn und die Quotienten der $\vartheta$-Nullwerte durch die Integralmoduln ausdrücken. Schreibt man für die Argumente $2 K u$ und $2 K v$ sogleich wieder $u$ und $v$, so gelangt man zu einer Relation, die die Funktionen $\operatorname{sn}(u \pm v), \operatorname{cn}(u \pm v), \operatorname{dn}(u \pm v), \operatorname{sn} u, \operatorname{sn} v, \operatorname{cn} u, \ldots, \operatorname{dn} v$ aneinander bindet, und deren Koeffizienten aus den Integralmoduln aufgebaut sind. Um für die Funktionen sn, en, dn die Additionsformeln im engeren Sinne zu erhalten, d. h. die rationalen Ausdrücke für $\operatorname{sn}(u+v), \operatorname{cn}(u+v)$, dn $(u+v)$, kann man in vier Arten vorgehen. Man muB sich aus den Gleichungen (1) ff. die vier Formeln aussuchen, in denen die Funktionen $\vartheta_{1}(u+v), \vartheta_{2}(u+v), \vartheta_{3}(u+v), \vartheta_{0}(u+v)$ entweder alle vier mit dem gleichen Faktor $\vartheta_{0}(u-v)$ oder mit $\vartheta_{1}(u-v)$ oder mit $\vartheta_{2}(u-v)$ oder endlich mit $\vartheta_{3}(u-v)$ multipliziert erscheinen, und aus ihnen die Quotienten bilden. Greifen wir z. B. die erste Formel (8) und ebenso die ersten 
Formeln (7), (6) und (1) heraus, so ergibt sich nach den nötigen Vereinfachungen die Proportion:

$$
\begin{aligned}
\operatorname{sn}(u+v): \operatorname{cn}(u+v): \operatorname{dn}(u+v): 1 & =(\operatorname{sn} u \operatorname{cn} v \operatorname{dn} v+\operatorname{sn} v \operatorname{cn} u \operatorname{dn} u) \\
& :(\operatorname{cn} u \operatorname{cn} v-\operatorname{sn} u \operatorname{dn} u \operatorname{sn} v \operatorname{dn} v) \\
& :\left(\operatorname{dn} u \operatorname{dn} v-k^{2} \operatorname{sn} u \operatorname{cn} u \operatorname{sn} v \operatorname{cn} v\right) \\
& :\left(1-k^{2} \operatorname{sn} u^{2} \operatorname{sn} v^{2}\right) .
\end{aligned}
$$

Damit sind wir zu den bekannten Formeln (9) S. 166 zurückgelangt.

Gewöhnlich treten bei den fraglichen Quotientenbildungen linker Hand mehrere Funktionen, miteinander multipliziert oder durcheinander geteilt, auf. Besonders einfach sind die drei Gleichungen:

$$
\begin{aligned}
\operatorname{sn}(u+v) \operatorname{sn}(u-v) & =\frac{\operatorname{sn} u^{2}-\operatorname{sn} v^{2}}{1-k^{2} \operatorname{sn} u^{2} \operatorname{sn} v^{2}}, \\
\operatorname{cn}(u+v) \operatorname{cn}(u-v) & =\frac{\operatorname{cn} u^{2} \operatorname{cn}^{2}-k^{\prime 2} \operatorname{sn} u^{2} \operatorname{sn} v^{2}}{1-k^{2} \operatorname{sn} u^{2} \operatorname{sn} v^{2}}, \\
\operatorname{dn}(u+v) \operatorname{dn}(u-v) & =\frac{\operatorname{dn} u^{2} \operatorname{dn} v^{2}+k^{2} k^{\prime} \operatorname{sn} u^{2} \operatorname{sn} v^{2}}{1-k^{2} \operatorname{sn} u^{2} \operatorname{sn} v^{2}},
\end{aligned}
$$

die man leicht aus der ersten Formel (2) und den Formeln (1) herstellt.

\section{§ 7. Additionssätze für mehrgliedrige Argumentsummen. ${ }^{1}$ )}

Setzt man in den beiden Additionsformeln für $\wp(u+v)$ und $\wp^{\prime}(u+v)$ an Stelle von $v$ die Summe $(v+w)$ und wendet auf $\wp(v+w)$ und $\wp^{\prime}(v+w)$ jeneFormeln nochmals an, so gelangt man zu Darstellungen von $\wp(u+v+w)$ und $\wp^{\prime}(u+v+w)$ durch die Funktionen der einzelnen Argumente $u, v, w$. Man kann offenbar in der gleichen Art fortfahren und erkennt die Möglichkeit, die Funktionen $\wp$ und $\wp^{\prime}$ für $n$-gliedrige Argumentsummen $\left(u_{1}+u_{2}+\cdots+u_{n}\right)$ rational in den Funktionen der einzelnen Summenglieder darzustellen. Die Gewinnung dieser „Additionssätze für mehrgliedrige Argumentsummen" auf induktivem Wege ist freilich schon bei den niedersten Werten $n$ recht umständlich. Dagegen gelingt die direkte Behandlung sogar des allgemeinen Falles einer n-gliedrigen Summe nach einer von Weierstra $B^{2}$ ) herrührenden Methode mittels einer Determinante auf folgende Art:

Mit irgendwelchen $(n+1)$ Argumenten $u, u_{1}, u_{2}, \ldots, u_{n}$ bilden wir die $(n+1)$-reihige Determinante:

1) Man vergleiche hierzu die geschichtlichen und literarischen Notizen im Artikel „Elliptische Funktionen" in Bd. II, 2 der "Enzyklopädie“, S. 299 ff. Hinweise auf diesen Artikel sollen weiterhin durch „Enzyklopädie“ unter Angabe der Seitenzahl gemacht werden.

2) Vgl. Schwarz, „Formeln und Lehrsätze zum Gebrauche der elliptischen Funktionen", S. 16. 
Methode von WeierstraB zur Verallgemeinerung der Additionsformeln 181

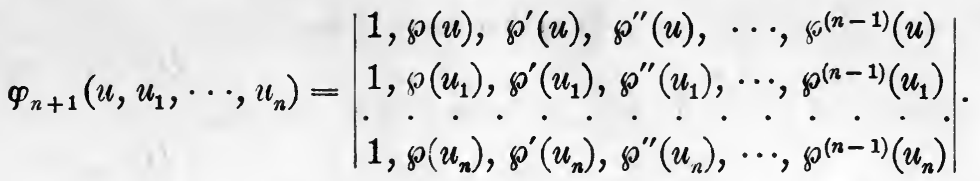

An Stelle der Ableitungen $\wp^{\prime \prime}, \wp^{\prime \prime \prime}, \ldots$ kann man auch Potenzen von $\wp$ und $\wp^{\prime}$ treten lassen. Nach I, 206 ist nämlich die Potenz $\wp(u)^{k+1}$ als $(2 k+2)$-wertige doppeltperiodische Funktion mit einem einzigen Pole $(2 k+2)^{\text {ter }}$ Ordnung bei $u=0$ und insbesondere als gerade Funktion in der Gestalt:

$\wp(u)^{k+1}=\frac{1}{(2 k+1) !} \wp^{(2 k)}(u)+a_{1} \wp^{(2 k-2)}(u)+a_{2} \wp^{(2 k-4)}(u)+\cdots+a_{k} \wp(u)+a_{k+1}$

darstellbar. Der Koeffizient des ersten Gliedes rechts ist aus den Potenzreihen bestimmt, die übrigen Koeffizienten können unbekannt bleiben. Durch Differentiation nach $u$ folgt weiter:

$$
\wp(u)^{k} \wp^{\prime}(u)=\frac{2}{(2 k+2) !} \wp^{(2 k+1)}(u)+a_{1} \wp^{(2 k-1)}(u)+\cdots+a_{k} \wp^{\prime}(u) .
$$

Mit Benutzung bekannter Determinantensätze kann man auf Grund dieser beiden Formeln die Determinante (1) umrechnen, und zwar findet man im Falle eines ungeraden $n$ :

$$
\varphi_{n+1}=\frac{2 ! \cdot 3 ! \cdot 4 ! \cdots n !}{2^{\frac{n-1}{2}}}\left|\begin{array}{l}
1, \wp(u), \wp^{\prime}(u), \wp(u)^{2}, \wp(u) \wp^{\prime}(u), \cdots, \wp(u)^{\frac{n+1}{2}} \\
1, \wp\left(u_{1}\right), \wp^{\prime}\left(u_{1}\right), \wp\left(u_{1}\right)^{2}, \wp\left(u_{1}\right) \wp^{\prime}\left(u_{1}\right), \cdots, \wp\left(u_{1}\right)^{\frac{n+1}{2}} \\
1, \wp\left(u_{n}\right), \wp^{\prime}\left(u_{n}\right), \wp\left(u_{n}\right)^{2}, \wp\left(u_{n}\right) \wp^{\prime}\left(u_{n}\right), \cdots, \wp\left(u_{n}\right)^{\frac{n+1}{2}}
\end{array}\right|,
$$

woran sich für eine gerade Zahl $n$ die Darstellung anschlieBt:

$$
\varphi_{n+1}=\frac{2 ! \cdot 3 ! \cdot 4 ! \cdots n !}{2^{\frac{n}{2}}}\left|\begin{array}{l}
1, \wp(u), \wp^{\prime}(u), \wp(u)^{2}, \cdots, \wp(u)^{\frac{n-2}{2}} \wp^{\prime}(u) \\
1, \wp\left(u_{1}\right), \wp^{\prime}\left(u_{1}\right), \wp\left(u_{1}\right)^{2}, \cdots, \wp\left(u_{1}\right)^{\frac{n-2}{2}} \wp^{\prime}\left(u_{1}\right) \\
\cdot \bullet \cdot \bullet\left(u_{n}\right), \wp^{\prime}\left(u_{n}\right), \wp\left(u_{n}\right)^{2}, \cdots, \wp\left(u_{n}\right)^{\frac{n-2}{2}} \wp^{\prime}\left(u_{n}\right)
\end{array}\right| .
$$

Der bequemen Bezeichnung halber schreiben wir $u_{0}$ statt $u$ und folgern aus (8) S. 159 für $n=1$ als Darstellung der Determinante $\varphi_{2}$ durch die Sigmafunktion:

$$
\varphi_{2}\left(u_{0}, u_{1}\right)=-\sigma\left(u_{0}+u_{1}\right) \frac{\sigma\left(u_{1}-u_{0}\right)}{\sigma\left(u_{0}\right)^{2} \sigma\left(u_{1}\right)^{2}} .
$$

Diese Gleichung liefert den niedersten Fall des folgenden Satzes: Die $(n+1)$-reihige Determinante $\varphi_{n+1}\left(u_{0}, u_{1}, \ldots, u_{n}\right)$ ist durch die Sigmafunktion in folgender Art darstellbar: 


$$
\begin{gathered}
\varphi_{n+1}\left(u_{0}, u_{1}, \cdots, u_{n}\right) \\
=(-1)^{n} 1 ! \cdot 2 ! \cdot 3 ! \cdot 4 ! \cdots n ! \sigma\left(u_{0}+u_{1}+\cdots+u_{n}\right) \frac{\prod_{x>2} \sigma\left(u_{x}-u_{\lambda}\right)}{\prod_{x=0}^{n} \sigma\left(u_{x}\right)^{n+1}},
\end{gathered}
$$

wo sich das Produkt im Zähler der rechten Seite auf alle Kombinationen $x, \lambda$ der Indizes $0,1,2, \ldots, n$ zu zweien mit $x>\lambda$ bezieht.

Der allgemeine Beweis der Formel (5) wird durch vollständige Induktion geführt. Wir nehmen an, daß für $\varphi_{n}\left(u_{1}, u_{2}, \ldots, u_{n}\right)$ die der Gleichung (5) entsprechende Darstellung gilt, und wählen vorerst die $u_{1}, u_{2}, \ldots, u_{n}$ so, daß $\varphi_{n}\left(u_{1}, u_{2}, \ldots, u_{n}\right)$ einen endlichen, nicht verschwindenden Wert hat; es soll also keines der Argumente $u_{1}, u_{2}, \ldots, u_{n}$, auch nicht ihre Summe einen Gitterpunkt des Parallelogrammnetzes der $u$-Ebene liefern, und keine zwei der Argumente $u_{1}, u_{2}, \ldots, u_{n}$ sollen bezüglich der Gruppe $\Gamma^{(u)}$ äquivalent sein. In Abhängigkeit von $u$ ist jetzt die Determinante (1) eine $(n+1)$-wertige doppeltperiodische Funktion, deren $(n+1)$ Pole bei $u=0$ zusammenfallen. Als Anfangsglied der Entwicklung dieser Funktion nach Potenzen von $u$ folgt aus (1):

(6) $\varphi_{n+1}\left(u, u_{1}, u_{2}, \cdots, u_{n}\right)=-n ! \cdot \varphi_{n}\left(u_{1}, u_{2}, \cdots, u_{n}\right) \cdot u^{-n-1}+\cdots$

Von den Nullpunkten unserer Funktion liegen $n$ zufolge (1) bei $u=u_{1}, u_{2}$ $\cdot \cdot, u_{n}$. Nach dem Abelschen Theoreme liegt somit der letzte Nullpunkt bei $u=-u_{1}-u_{2}-\cdots-u_{n}$. Als Darstellung (6) in I, 214 für unsere Funktion von $u$ erhalten wir hiernach:

$$
\begin{gathered}
\varphi_{n+1}\left(u, u_{1}, u_{2}, \cdots, u_{n}\right) \\
=C \frac{\sigma\left(u-u_{1}\right) \sigma\left(u-u_{2}\right) \cdots \sigma\left(u-u_{n}\right) \sigma\left(u+u_{1}+u_{2}+\cdots+u_{n}\right)}{\sigma(u)^{n+1}},
\end{gathered}
$$

wo $C$ eine von $u$ unabhängige Größe ist. Als Anfangsglied der Potenzreihe von $\varphi_{n+1}$ ergibt sich aus (7):

$$
\begin{gathered}
\varphi_{n+1}\left(u, u_{1}, u_{2}, \cdots, u_{n}\right) \\
=(-1)^{n} C \sigma\left(u_{1}+u_{2}+\cdots+u_{n}\right) \cdot \prod_{x=1}^{n} \sigma\left(u_{x}\right) \cdot u^{-n-1}+\cdots
\end{gathered}
$$

Der Vergleich mit (6) liefert für $C$ den Ausdruck:

$$
C=\frac{(-1)^{n-1} n ! \varphi_{n}\left(u_{1}, u_{2}, \cdots, u_{n}\right)}{\sigma\left(u_{1}+u_{2}+\cdots+u_{n}\right) \prod_{x=1}^{n} \sigma\left(u_{x}\right)} .
$$

Indem man diesen Ausdruck für $C$ in (7) einträgt, $\varphi_{n}\left(u_{1}, u_{2}, . ., u_{n}\right)$ durch seinen als gültig vorausgesetzten Ausdruck in der Sigmafunktion ersetzt und $u_{0}$ an Stelle von $u$ schreibt, ergibt sich die zu beweisende Formel (5).

Um nun die Additionssätze für $n$-gliedrige Argumentsummen zu gewinnen, hat man die beiden ersten Glieder der eben bereits mehrfach be- 
trachteten Potenzreihe heranzuziehen. Von (1) aus gelangt man zum zweiten Reihengliede, indem man die zum Elemente $\wp^{(n-2)}(u)$ der ersten Zeile gehörende Unterdeterminante einführt. Wir bezeichnen dieselbe durch:

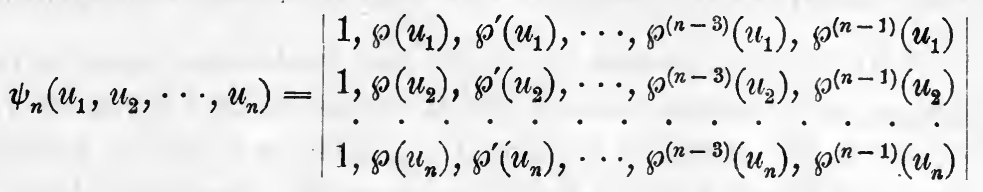

und können sie im AnschluB an (2) bei ungeradem $n$ auch so schreiben:

$$
\psi_{n}=\frac{2 ! \cdot 3 ! \cdot 4 ! \cdots n !}{2^{\frac{n-3}{2}}(n-1) !}\left|\begin{array}{l}
1, \wp\left(u_{1}\right), \wp^{\prime}\left(u_{1}\right), \wp\left(u_{1}\right)^{2}, \cdots, \wp\left(u_{1}\right)^{\frac{n-1}{2}}, \wp\left(u_{1}\right)^{\frac{n+1}{2}} \\
1, \wp\left(u_{2}\right), \wp^{\prime}\left(u_{2}\right), \wp\left(u_{2}\right)^{2}, \cdots, \wp\left(u_{2}\right)^{\frac{n-1}{2}}, \wp\left(u_{2}\right)^{\frac{n+1}{2}} \\
1, \wp\left(u_{n}\right), \wp^{\prime}\left(u_{n}\right), \wp\left(u_{n}\right)^{2}, \cdots, \wp\left(u_{n}\right)^{\frac{n-1}{2}}, \wp\left(u_{n}\right)^{\frac{n+1}{2}}
\end{array}\right|,
$$

woran sich im Falle eines geraden $n$, der Formel (3) entsprechend, reiht:

$$
\text { (10) } \psi_{n}=\frac{2 ! \cdot 3 ! \cdot 4 ! \cdots n !}{2^{\frac{n}{2}}(n-1) !}\left|\begin{array}{l}
1, \wp\left(u_{1}\right), \wp^{\prime}\left(u_{1}\right), \cdots, \wp\left(u_{1}\right)^{\frac{n-4}{2}} \wp^{\prime}\left(u_{1}\right), \wp\left(u_{1}\right)^{\frac{n-2}{2}} \wp^{\prime}\left(u_{1}\right) \\
1, \wp\left(u_{2}\right), \wp^{\prime}\left(u_{2}\right), \cdots, \wp\left(u_{2}\right)^{\frac{n-4}{2}} \wp^{\prime}\left(u_{2}\right), \wp\left(u_{2}\right)^{\frac{n-2}{2}} \wp^{\prime}\left(u_{2}\right) \\
1, \wp\left(u_{n}\right), \wp^{\prime}\left(u_{n}\right), \cdots, \wp\left(u_{n}\right)^{\frac{n-4}{2}} \wp^{\prime}\left(u_{n}\right), \wp\left(u_{n}\right)^{\frac{n-2}{2}} \wp^{\prime}\left(u_{n}\right)
\end{array}\right| .
$$

Auf Grund von (1) erhalten wir damit für die beiden Anfangsglieder der Potenzreihe von $\varphi_{n+1}$ als Funktion von $u$ :

$\varphi_{n+1}=-n ! \varphi_{n}\left(u_{1}, u_{2}, \cdots, u_{n}\right) u^{-n-1}-(n-1) ! \psi_{n}\left(u_{1}, u_{2}, \cdots, u_{n}\right) u^{-n}+\cdots$

Bei Zugrundelegung des Ausdrucks (5) dieser Funktion aber führt die Entwicklung des zweiten Reihengliedes auf Grund der Relation (6) in I, 209 auf das Normalintegral zweiter Gattung. Man gelangt zu folgender Gestalt der beiden ersten Glieder:

$$
\begin{gathered}
\varphi_{n+1}=-n ! \varphi_{n}\left(u_{1}, u_{2}, \cdots, u_{n}\right) u^{-n-1}\left\{1+\left(\zeta\left(u_{1}+u_{2}+\cdots+u_{n}\right)\right.\right. \\
\left.\left.-\zeta\left(u_{1}\right)-\cdots-\xi\left(u_{n}\right)\right) u+\cdots\right\} .
\end{gathered}
$$

Der Vergleich der zweiten Glieder führt zum Additionstheorem des Normalintegrals zweiter Gattung für eine n-gliedrige Argumentsumme:

$$
\begin{gathered}
\zeta\left(u_{1}+u_{2}+\cdots+u_{n}\right)=\xi\left(u_{1}\right)+\xi\left(u_{2}\right)+\cdots+\xi\left(u_{n}\right) \\
+\frac{1}{n} \frac{\psi_{n}\left(u_{1}, u_{2}, \ldots, u_{n}\right)}{\varphi_{n}\left(u_{1}, u_{2}, \ldots, u_{n}\right)} .
\end{gathered}
$$

Wir haben hier die Verallgemeinerung der Gleichung (9) S. 160 vor uns. Wie damals, so können wir auch hier durch Differentiationen zu entsprechenden Sätzen für die Funktionen $\wp$ und $\wp^{\prime}$ gelangen; doch sind die entstehenden Formeln für uns ohne weitergehende Bedeutung. 


\section{Zweites Kapitel.}

\section{Die Multiplikationssätze der elliptischen Funktionen.}

Die Multiplikationssätze der elliptischen Funktionen betreffen die Beziehungen, die zwischen einer Funktion mit $n$-fachem Argumente $n u$ und der gleichen, für einfaches Argument $u$ gebildeten Funktion bestehen; $n$ ist dabei als ganze positive Zahl vorausgesetzt. Man kann diese Sätze aus den Additionsformeln für n-gliedrige Argumentsummen durch Gleichsetzung aller $n$ Summanden entwickeln; doch führt eine direkte Behand. lung leichter zum Ziele. Literarische Notizen über die Multiplikationssätze findet man in „Enzyklopädie“, S. 302 ff.; die ältere Theorie betreffend vgl. man auch „Enneper-Müller“, S. $368 \mathrm{ff}$.

\section{§ 1. Multiplikationssätze der Funktionen erster Stufe.}

Die Funktion $\wp(n u)$, gebildet für irgendeine positive ganze Zahl $n$, hat als Funktion mit den Perioden $\omega_{1}, \omega_{2}$ aufgefaßt, im Periodenparallelogramm $n^{2}$ Pole zweiter Ordnung in den $n^{2}$ Punkten:

$$
u=\frac{\lambda \omega_{1}+\mu \omega_{2}}{n},
$$

2., $\mu=0,1,2, \cdots, n-1$

und stellt demnach eine Funktion der Wertigkeit $2 n^{2}$ dar. Da sie überdies eine gerade Funktion ist, so ist sie bereits in $\wp(u)$ allein rational darstellbar, und zwar vom Grade $n^{2}$. Wir nennen diese rationale Funktion $R(\wp(u))$ und finden durch Differentiation nach $u$ eine entsprechende Darstellung für $\wp^{\prime}(n u)$ :

$$
\wp(n u)=R(\wp(u)), \quad \wp^{\prime}(n u)=\frac{1}{n} R^{\prime}(\wp(u)) \cdot \wp^{\prime}(u)
$$

Um die Funktion $R(\wp)$ zugänglicher zu machen, führt Weierstra $\beta^{1}$ ) folgenden, symbolisch durch $\psi^{(n)}(u)$ zu bezeichnenden б-Quotienten ein:

$$
\psi^{(n)}(u)=\frac{\sigma(n u)}{(\sigma(u))^{n \cdot n}} .
$$

Aus (7) und (8) in I, 209 folgt, daB diese Funktion die Perioden $\omega_{1}, \omega_{2}$ hat; dabei besitzt sie die Wertigkeit $\left(n^{2}-1\right)$, indem sie im Nullpunkte einen Pol der Ordnung $\left(n^{2}-1\right)$ und in den $\left(n^{2}-1\right)$ weiteren Punkten (1) je einen einfachen Nullpunkt hat. Es besteht mun die Gleichung:

$$
\wp(n u)=\wp(u)-\frac{\psi^{(n-1)}(u) \psi^{(n+1)}(u)}{\psi^{(n)}(u)^{2}},
$$

so daß, wenn man die Funktionen $\psi^{(n)}(u)$ in $\wp(u)$ und $\wp^{\prime}(u)$ dargestellt hat, damit die Darstellung von $\wp(n u)$ in der Gestalt (2) zugleich gewonnen

1) S. Schwarz, „Formeln und Lehrsätze zum Gebrauche der elliptischen Funktionen" S. 18. 
ist. Die Gleichung (4) ist nämlich eine einfache Folge der Gleichung (8) S. 159, wenn man in ihr $v=n u$ einträgt. An Stelle von (4) könnte man auch die Gleichung:

$$
\wp(n u)=\wp(u)-\frac{1}{n^{2}} \frac{d^{2} \log \psi^{(n)}(u)}{d u^{2}}
$$

treten lassen, die aus (3) mit Rücksicht auf die zweite Gleichung (1) in I, 212 folgt.

Die Ausdrücke der ersten Funktionen $\psi^{(1)}(u), \psi^{(2)}(u), \ldots$ in $\wp(u)$ und $\wp^{\prime}(u)$ sind:

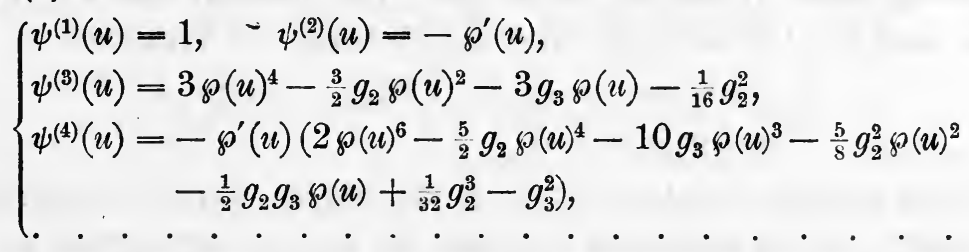

Die zweite Gleichung folgt aus der Übereinstimmung der Nullpunkte und Pole von $\wp^{\prime}(u)$ und $\psi^{(2)}(u)$ mit Rücksicht auf die Anfangskoeffizienten der beiderseitigen Potenzreihen. Zum Beweise der dritten Gleichung folgern wir aus (4) für $n=2$ mit Rücksicht auf die beiden ersten Gleichung (6):

$$
\psi^{(3)}(u)=(\wp(u)-\wp(2 u)) \rho^{\prime}(u)^{2}
$$

und entnehmen den Ausdruck von $\rho(2 u)$ aus (10) S. 160 für $\lim v=u$ :

$$
\wp(2 u)=\frac{1}{4}\left(\frac{\wp^{\prime \prime}(u)}{\wp^{\prime}(u)}\right)^{2}-2 \wp(u)=\frac{1}{4}\left(\frac{12 \wp(u)^{2}-g_{2}}{2 \wp^{\prime}(u)}\right)^{2}-2 \wp(u) .
$$

Zur Entwicklung des Ausdrucks von $\psi^{(4)}(u)$ kann man an:

$$
\psi^{(4)}(u)=\frac{\sigma(4 u)}{\sigma(u)^{16}}=\frac{\sigma(4 u)}{\sigma(2 u)^{4}} \cdot\left(\frac{\sigma(2 u)}{\sigma(u)^{4}}\right)^{4}=-\wp^{\prime}(2 u) \cdot \wp^{\prime}(u)^{4}
$$

anknüpfen und hat aus (7) den Ausdruck von $\wp^{\prime}(2 u)$ in $\wp(u)$ und $\wp^{\prime}(u)$ $\mathrm{zu}$ berechnen. ${ }^{1}$ )

Zur Berechnung der Ausdrücke von $\psi^{(5)}(u), \psi^{(6)}(u), \ldots$ in $\wp(u)$ und $\wp^{\prime}(u)$ kann man sich zweier Rekursionsformeln bedienen. Setzt man in der G-Relation (4) S. 158 für die $x_{1}, y_{1}, z_{1}, t_{1}$ die Werte $x_{1}=(2 n+1) u$, $y_{1}=z_{1}=t_{1}=u$ ein, so ergibt sich:

$\sigma((2 n+1) u) \sigma(u)^{3}-\sigma((n+2) u) \sigma(n u)^{3}+\sigma((n-1) u) \sigma((n+1) u)^{3}=0$. Trägt man zweitens $x_{1}=2 n u, y_{1}=2 u, z_{1}=t_{1}=u$ ein, so folgt:

$$
\begin{aligned}
\sigma(2 n u) \sigma(2 u) \sigma(u)^{2} & -\sigma(n u) \sigma((n+2) u) \sigma((n-1) u)^{2} \\
& +\sigma(n u) \sigma((n-2) u) \sigma((n+1) u)^{2}=0 .
\end{aligned}
$$

In die Funktionen $\psi$ umgeschrieben lauten diese Relationen:

$$
\left\{\begin{array}{l}
\psi^{(2 n+1)}(u)=\psi^{(n+2)}(u) \psi^{(n)}(u)^{3}-\psi^{(n-1)}(u) \psi^{(n+1)}(u)^{3} \\
\psi^{(2 n)}(u) \psi^{(2)}(u)=\psi^{(n)}(u)\left(\psi^{(n+2)}(u) \psi^{(n-1)}(u)^{2}-\psi^{(n-2)}(u) \psi^{(n+1)}(u)^{2}\right)
\end{array}\right.
$$

1) Ein anderer Weg zur Berechnung von $\psi^{(3)}(u)$ and $\psi^{(4)}(u)$ wird sogleich angegeben werden. 
Im Anschluß an (6) kann man mit Hilfe dieser Formeln in der Tat $\psi^{(5)}(u)$, $\psi^{(6)}(u), \ldots$ berechnen.

Diese Rekursionsrechnungen gestalten sich aber alsbald sehr umständlich. Demgegenüber kann man wieder durch Einführung einer Determinante einen Ausdruck für $\psi^{(n)}(u)$ sogar bei beliebigem $n$ angeben. In I, 450 ist unter (1) die von Klein eingeführte Funktion $\sigma_{\lambda, \mu}\left(u \mid \omega_{1}, \omega_{2}\right)$ erklärt $^{1}$ ), wo $\lambda, \mu$ die unter (1) genannten Kombinationen ganzer Zahlen durchlaufen sollen mit AusschluB der Kombination $\lambda=0, \mu=0$, die zur ursprünglichen $\sigma$-Funktion zurückführt. Der Quotient von $\sigma_{\lambda, \mu}(u)$ und $\sigma(u)$ zeigt bei Vermehrung von $u$ um Perioden das Verhalten:

$$
\frac{\sigma_{\lambda, \mu}\left(u+m_{1} \omega_{1}+m_{2} \omega_{2}\right)}{\sigma\left(u+m_{1} \omega_{1}+m_{2} \omega_{2}\right)}=e^{\frac{2 i \pi}{n}\left(m_{1} \mu-m_{2} \lambda\right)} \frac{\sigma_{\lambda, \mu}(u)}{\sigma(u)},
$$

wie man aus der Gleichung (2) in I, 451 folgert. Die $n^{\text {to }}$ Potenz dieses Quotienten hat demnach die Perioden $\omega_{1}, \omega_{2}$, und zwar stellt sie eine $n$-wertige doppeltperiodische Funktion dar, deren $n$ Pole im Gitterpunkte $u=0$ zusammenfallen, während die $n$ Nullpunkte an der Stelle (1) gleichfalls zusammenliegen. Nach I, 206 stellen wir nun diese Funktion in der Gestalt:

$$
\left(\frac{\sigma_{\lambda, \mu}(u)}{\sigma_{(u)}}\right)^{n}=a_{0}+a_{1} \wp(u)+a_{2} \wp^{\prime}(u)+\cdots+a_{n-1} \wp^{(n-2)}(u)
$$

dar, wo der letzte Koeffizient $a_{n-1}$ sicher von 0 verschieden ist. Da aber an der Stelle (1) ein Nullpunkt $n^{\text {ter }}$ Ordnung unserer Funktion liegt ${ }^{2}$ ), so verschwinden ebenda auch noch ihre $(n-1)$ ersten Ableitungen; d. h. für die Stelle $(1)$ sind die $(n-1)$ in den Ableitungen von $\wp(u)$ linearen homogenen Gleichungen erfüllt:

$$
\begin{aligned}
& a_{1} \wp^{\prime}(u)+a_{2} \wp^{\prime \prime}(u)+\cdots+a_{n-1} \wp^{(n-1)}(u)=0, \\
& a_{1} \wp^{\prime \prime}(u)+a_{2} \wp^{\prime \prime \prime}(u)+\cdots+a_{n-1} \wp^{(n)}(u)=0 \text {, } \\
& a_{1} \wp^{(n-1)}(u)+a_{2} \wp^{(n)}(u)+\cdots+a_{n-1} \wp^{(2 n-3)}(u)=0 .
\end{aligned}
$$

Mit Rücksicht auf $a_{n-1} \neq 0$ folgt hieraus aber weiter das Verschwinden $\operatorname{der}(n-1)$-reihigen Determinante

an jeder Stelle (1).

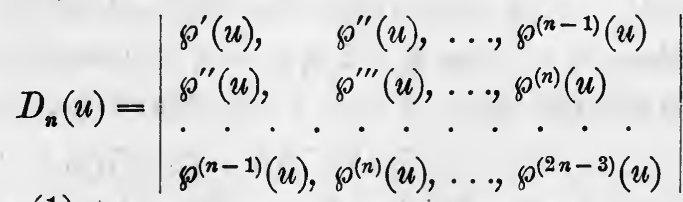

1) An Stelle der in I, 450 im AnschluB an ältere Arbeiten über $\vartheta$-Funktionen benutzten Bezeichnung $\sigma_{g, h}(u)$ wird fortan die in der Theorie der Modulfunktionen übliche Bezeichnung $\sigma_{\lambda, \mu}(u)$ gebraucht.

2) Es gilt hier immer die Kombination $\lambda=0, \mu=0$ als ausgeschlossen. 
Wir haben damit die schon erwähnte Determinante gewonnen, die von F.Brioschi ${ }^{1}$ ) und L. Kiepert ${ }^{2}$ ) zur Aufstellung der Multiplikationssätze der elliptischen Funktionen herangezogen ist. Tragen wir für $\wp^{\prime}(u)$, $\wp^{\prime \prime}(u), \ldots$ die Anfangsglieder der Reihenentwicklungen nach Potenzen von $u$ ein, so gewinnt man als Anfangsglied der Reihe von $D_{n}(u)$ selbst:

$$
D_{n}(u)=(-1)^{n-1} C_{n} \cdot u^{-\left(n^{2}-1\right)}+\cdots,
$$

wo $C_{n}$ die folgende, sogleich weiter zu berechnende Determinante ist:

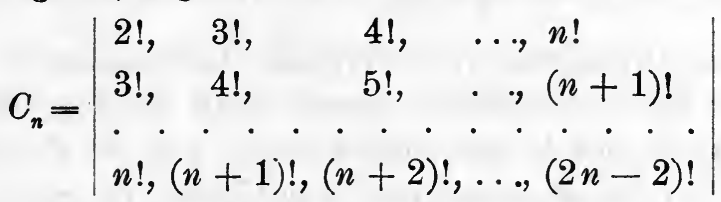

Hiernach ist $D_{n}(u)$ eine $\left(n^{2}-1\right)$-wertige doppeltperiodische Funktion, die mit $\psi^{(n)}(u)$ in bezug auf Pole und Nullpunkte genau übereinstimmt und also mit $\psi^{(n)}(u)$ bis auf einen konstanten Faktor identisch ist.

Um diesen Faktor zu bestimmen, haben wir zunächst $C_{n}$ zu berechnen. Wir sondern aus den Zeilen der Determinante (12) bzw. die Faktoren 2 !, 3!, ..., $n$ ! ab, hierauf aus den Spalten die Faktoren 1, 1!, 2!, ..., $(n-2)$ ! und gewinnen auf diese Weise:

$$
\begin{aligned}
& C_{n}=n(2 ! \cdot 3 ! \cdot 4 ! \cdots(n-1) !)^{2} \cdot C_{n}^{\prime},
\end{aligned}
$$

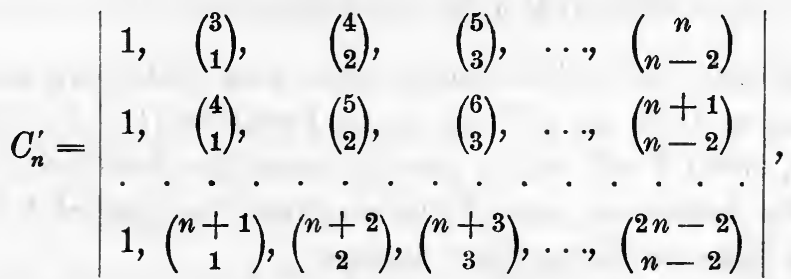

wo $\left(\begin{array}{l}n \\ k\end{array}\right)$ der $k^{\text {te }}$ Binomialkoeffizient der $n^{\text {ten }}$ Potenz ist. Die Determinante $C_{n}^{\prime}$ erweist sich als von $n$ unabhängig und hat demnach den Wert $C_{3}^{\prime}=1$. Zieht man nämlich jede Spalte, mit der vorletzten beginnend, von der folgenden ab, und verfährt man darauf mit den Zeilen genau so, so kürzt sich bei Benutzung einer bekannten Regel der Binomialkoeffizienten $C_{n}^{\prime} \mathrm{zu}$ :

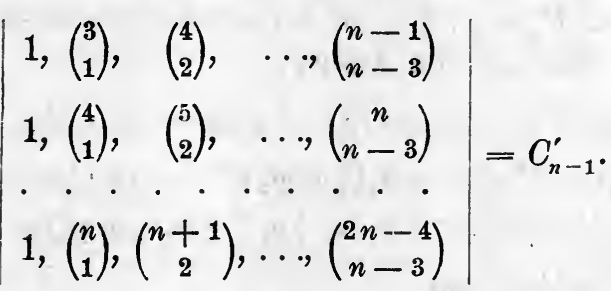

1) "Sur quelques formules pour la multiplication des fonctions elliptiques", Compt. Rend. Bd. 59 (1864) S. 999.

2) „Wirkliche Ausführung der ganzzahligen Multiplikation der elliptischen Funktionen", Journ. f. Math. Bd. 76 (1875), S. 21. 
Hiernach haben wir an Stelle von (11) genauer:

$$
D_{n}(u)=(-1)^{n-1} n(2 ! \cdot 3 ! \cdot 4 ! \cdots(n-1) !)^{2} u^{-\left(n^{2}-1\right)}+\cdots
$$

als Anfangsglied der Reihe von $D_{n}(u)$, und der Vergleich mit dem Anfangsgliede der Reihe für $\psi^{(n)}(u)$ ergibt den Satz: Die Funktion $\psi^{(n)}(u)$ besitzt für beliebiges $n$ in den Ableitungen von $\wp(u)$ die Darstellung:

$$
\psi^{(n)}(u)=\frac{(-1)^{n-1}}{(2 ! \cdot 3 ! \cdot 4 ! \cdots(n-1) !)^{2}} D_{n}(u)
$$

wo $D_{n}(u)$ die in (10) gegebene $(n-1)$-reihige Determinante ist. Der „Multiplikationssatz" für die $\wp$-Funktion nimmt damit die abgeschlossene Gestalt an, daß $\wp(n u)$ sich in dem Ausdrucke (4) oder (5) darstellt, wo die Funktionen $\psi^{(n)}(u)$ für alle $n$ sich nach dem Gesetze (14) und (10) aus den Ableitungen $\wp^{\prime}(u), \wp^{\prime \prime}(u), \ldots$ berechnen.

Um schlieBlich bis zur Gleichung (2) vorzudringen, ist hiernach in der Hauptsache weiter nichts mehr nötig als die Ableitungen der $\wp$-Funktion von $\wp^{\prime \prime}(u)$ ab in $\wp(u)$ und $\wp^{\prime}(u)$ darzustellen (vgl. I, 207). Für die Ableitungen $\wp^{\prime \prime}(u), \wp^{\prime \prime \prime}(u), \wp^{(4)}(u), \wp^{(5)}(u)$ finden wir bei Fortlassung der Argumente $u^{1}$ ):

$$
\left\{\begin{array}{l}
\wp^{\prime \prime}=3 ! \wp^{2}-\frac{1}{2} g_{2}, \wp^{\prime \prime \prime}=2 \cdot 3 ! \wp \wp^{\prime}, \\
\wp^{(4)}=5 ! \wp^{3}-2 \cdot 3^{2} g_{2} \wp-2^{2} \cdot 3 g_{3}, \\
\wp^{(5)}=\left(3 \cdot 5 ! \wp^{2}-2 \cdot 3^{2} g_{2}\right) \wp^{\prime} .
\end{array}\right.
$$

Bei Fortführung dieser Rechnung erkennt man leicht, daB sich jede Ableitung gerader Ordnung $\wp^{(2 k)}$ als ganze Funktion $(k+1)^{\text {ten }}$ Grades von $\wp$ darstellt, deren Koeffizienten ganze ganzzahlige Ausdrücke in $\frac{1}{2} g_{2}$ und $g_{3}$ sind. Wir bezeichnen diese Funktion durch das Symbol $P_{k}\left(\wp, \frac{1}{2} g_{2}, g_{3}\right)$ und haben dann die allgemeinen Ansätze:

$$
\wp^{(2 k)}=P_{k}\left(\wp, \frac{1}{2} g_{2}, g_{3}\right), \quad \wp^{(2 k+1)}=P_{k}^{\prime}\left(\wp, \frac{1}{2} g_{2}, g_{3}\right) \cdot \wp^{\prime},
$$

wo $P_{k}^{\prime}$ die Ableitung von $P_{k}$ nach $\wp$ ist. Der Koeffizient des höchsten Gliedes von $P_{k}$ bestimmt sich aus den Anfangsgliedern der Potenzreihen nach $u$ zu $(2 k+1)$ !. Da übrigens $\gamma^{(2 k)}$ eine homogene Funktion der Dimension - $(2 k+2)$ in $u, \omega_{1}, \omega_{2}$ ist, so kann man den rationalen ganzen Ausdruck von $P_{k}$ in $\wp, \frac{1}{2} g_{2}, g_{3}$ bis auf die numerischen Koeffizienten sofort angeben. Man hat den Ansatz:

$$
\begin{aligned}
\wp^{(2 k)} & =P_{k}\left(\wp, \frac{1}{2} g_{2}, g_{3}\right)=(2 k+1) ! \wp^{k+1}+a_{1}\left(\frac{1}{2} g_{2}\right) \wp^{k-1}+a_{2} g_{3} \wp^{k-2} \\
& +a_{3}\left(\frac{1}{2} g_{2}\right)^{2} \wp^{k-3}+a_{4}\left(\frac{1}{2} g_{2}\right) g_{3} \wp^{k-4}+\left(a_{5}\left(\frac{1}{2} g_{2}\right)^{3}+a_{5}^{\prime} g_{3}^{2}\right) \wp^{k-5} \\
& +a_{6}\left(\frac{1}{2} g_{2}\right)^{2} g_{3} \wp^{k-6}+\left(a_{7}\left(\frac{1}{2} g_{2}\right)^{4}+a_{7}^{\prime}\left(\frac{1}{2} g_{2}\right) g_{3}^{2}\right) \wp^{k-7}+\cdots
\end{aligned}
$$

wo die $a$ ganze Zahlen sind.

1) Mit Rücksicht auf die sogleich auszuführende Rekursionsrechnung ist es zweckmäBig, die numerischen Koeffizienten in den nachfolgenden Gleichungen (abgesehen vom ersten) in ihre Primfaktoren zu zerlegen. 
Differenziert man die zweite Gleichung (16) nochmals nach $u$, so ergibt sich als „Rekursionsformel“ für die Berechnung der Funktionen $P_{k}$ : (18)

$$
P_{k+1}=\left(6 \wp^{2}-\frac{1}{2} g_{2}\right) P_{k}^{\prime}+\left(4 \wp^{3}-g_{2} \wp-g_{3}\right) P_{k}^{\prime \prime} \text {. }
$$

Mittels dieser Gleichung findet man im AnschluB an (15) für die nächstfolgenden Ableitungen $\wp^{(6)}, \ldots$ gerader Ordnungen:

$$
\left\{\begin{array}{c}
\wp^{(6)}=7 ! \wp^{4}-2^{4} \cdot 3^{2} \cdot 7 g_{2} \wp^{2}-2^{4} \cdot 3^{2} \cdot 5 g_{3} \wp+3^{2} g_{2}^{2}, \\
\wp^{(8)}=9 ! \wp^{5}-2^{5} \cdot 3^{4} \cdot 5 \cdot 7 g_{2} \wp^{3}-2^{4} \cdot 3^{2} \cdot 5 \cdot 13 g_{3} \wp^{2}+2^{4} \cdot 3^{3} \cdot 7 g_{2}^{2} \wp \\
+2^{3} \cdot 3^{3} \cdot 11 g_{2} g_{3}, \\
\wp^{(10)}=11 ! \wp^{6}-2^{7} \cdot 3^{5} \cdot 5 \cdot 7 \cdot 11 g_{2} \wp^{4}-2^{6} \cdot 3^{2} \cdot 5^{2} \cdot 11 \cdot 47 g_{3} \wp^{3} \\
+2^{4} \cdot 3^{4} \cdot 7^{2} \cdot 11 g_{2}^{2} \wp^{2}+2^{4} \cdot 3^{3} \cdot 5^{2} \cdot 53 g_{2} g_{3} \wp \\
-2^{3} \cdot 3^{3} \cdot 7 g_{2}^{3}+2^{5} \cdot 3^{2} \cdot 5 \cdot 13 g_{3}^{2} .
\end{array}\right.
$$

Zufolge (3) ist $\psi^{(n)}(u)$ und damit $D_{n}(u)$ eine gerade oder ungerade Funktion von $u$, je nachdem $n$ ungerade oder gerade ist. Somit läBt sich $D_{n}(u)$ für ungerades $n$ als ganze ganzzahlige Funktion von $\wp(u), \frac{1}{2} g, g_{3}$ darstellen, für gerades $n$ aber als Produkt einer solchen Funktion mit $\wp^{\prime}(u)$. Der Grad dieser Funktion in $\wp(u)$, die Dimension in $u, \omega_{1}, \omega_{2}$ und der Koeffizient der höchsten Potenz von $\wp(u)$ werden aus dem oben angegebenen Anfangskoeffizienten der Entwicklung von $D_{n}(u)$ nach Potenzen von $u$ bestimmt. Wir haben allgemein für ungerades $n$ den Ansatz:

$$
\begin{aligned}
D_{n}(u) & =n(2 ! \cdot 3 ! \cdot 4 ! \cdots(n-1) !)^{2} \wp^{\frac{1}{2}\left(n^{2}-1\right)}+b_{1}\left(\frac{1}{2} g_{2}\right) \wp^{\frac{1}{2}\left(n^{2}-5\right)} \\
& +b_{2} g_{3} \wp^{\frac{1}{2}\left(n^{2}-7\right)}+b_{3}\left(\frac{1}{2} g_{2}\right)^{2} \wp^{\frac{1}{2}\left(n^{2}-9\right)}+b_{4}\left(\frac{1}{2} g_{2}\right) g_{3} \wp^{\frac{1}{2}\left(n^{2}-11\right)} \\
& +\left(b_{5}\left(\frac{1}{2} g_{2}\right)^{3}+b_{5}^{\prime} g_{3}^{2}\right) \wp^{\frac{1}{2}\left(n^{2}-13\right)}+\cdots
\end{aligned}
$$

während sich für gerades $n$ der Ansatz ergibt:

$$
\begin{aligned}
D_{n}(u) & =\wp^{\prime} \cdot\left\{\frac{1}{2} n(2 ! \cdot 3 ! \cdot 4 ! \cdots(n-1) !)^{2} \wp^{\frac{1}{2}\left(n^{2}-4\right)}+c_{1}\left(\frac{1}{2} g_{2}\right) \wp^{\frac{1}{2}\left(n^{2}-8\right)}\right. \\
& +c_{2} g_{3} \wp^{\frac{1}{2}\left(n^{2}-10\right)}+c_{3}\left(\frac{1}{2} g_{2}\right)^{2} \wp^{\frac{1}{2}\left(n^{2}-12\right)}+c_{4}\left(\frac{1}{2} g_{2}\right) g_{3} \wp^{\frac{1}{2}\left(n^{2}-14\right)} \\
& \left.+\left(c_{5}\left(\frac{1}{2} g_{2}\right)^{3}+c_{5}^{\prime} g_{3}^{2}\right) \wp^{\frac{1}{2}\left(n^{2}-16\right)}+\cdots\right\},
\end{aligned}
$$

wo die $b$ und $c$ ganze Zahlen sind. Wir reihen an die Gleichungen (6) wenigstens noch die fertigen Ausdrücke von $\psi^{(5)}$ und $\psi^{(6)}$ an:

$$
\begin{aligned}
\psi^{(5)}(u) & =\frac{D_{5}(u)}{(2 ! \cdot 3 ! \cdot 4 !)^{2}}=5 \wp^{12}-\frac{31}{2} g_{2} \wp^{10}-5 \cdot 19 g_{3} \wp^{9}-\frac{3 \cdot 5 \cdot 7}{2^{4}} g_{2}^{2} \wp^{8} \\
& +3 \cdot 5 g_{2} g_{3} \wp^{7}+\left(\frac{3 \cdot 5^{2}}{2^{4}} g_{2}^{3}-3 \cdot 5 g_{3}^{2}\right) \wp^{6}+\frac{3 \cdot 29}{2^{3}} g_{2}^{2} g_{3} \wp^{5} \\
& -\left(\frac{5^{3}}{2^{8}} g_{2}^{4}-2 \cdot 3 \cdot 5 g_{2} g_{3}^{2}\right) \wp^{4}-\left(\frac{5}{2^{4}} g_{2}^{3} g_{3}-5^{2} g_{3}^{3}\right) \wp^{3} \\
& +\left(\frac{5^{2}}{2^{9}} g_{2}^{5}-\frac{3 \cdot 5}{2^{3}} g_{2}^{2} g_{3}^{2}\right) \wp^{2}+\left(\frac{5^{2}}{2^{8}} g_{2}^{4} g_{3}-\frac{5}{2} g_{2} g_{3}^{3}\right) \wp \\
& -\left(\frac{1}{2^{12}} g_{2}^{6}-\frac{1}{2^{5}} g_{2}^{3} g_{3}^{2}+g_{3}^{4}\right) .
\end{aligned}
$$


Für $\psi^{(6)}$ findet sich aus der zweiten Rekursionsformel (8) der Ausdruck:

$$
\psi^{(6)}=\psi^{(2)} \cdot \psi^{(3)}\left(\psi^{(5)}-\left(\frac{\psi^{(4)}}{\wp^{\prime}}\right)^{2}\right),
$$

so dab $\psi^{(6)}$ die Funktionen $\psi^{(2)}$ und $\psi^{(3)}$ als Faktoren enthält. Für den dritten Faktor findet man:

$$
\begin{aligned}
\frac{\psi^{(6)}(u)}{\psi^{(2)}(u) \psi^{(3)}(u)} & =\wp^{12}-\frac{11}{2} g_{2} \wp^{10}-5 \cdot 11 g_{3} \wp^{9}-\frac{3 \cdot 5 \cdot 11}{2^{4}} g_{2}^{2} \wp^{8} \\
& -3 \cdot 11 g_{2} g_{3} \wp^{7}+\left(\frac{23}{2^{4}} g_{2}^{3}-3 \cdot 37 g_{31}^{2}\right) \rho^{6}-\frac{3 \cdot 11}{2^{3}} g_{2}^{2} g_{3} \wp^{5} \\
& -\left(\frac{5 \cdot 37}{2^{8}} g_{2}^{4}-3 \cdot 5 g_{2} g_{3}^{2}\right) \wp^{4}-\left(\frac{5}{2^{4}} g_{2}^{3} g_{3}-5 g_{3}^{3}\right) \wp^{3} \\
& +\left(\frac{3^{2} \cdot 5}{2^{9}} g_{2}^{5}-\frac{3^{3}}{2^{3}} g_{2}^{2} g_{3}^{2}\right) \wp^{2}+\left(\frac{3 \cdot 11}{2^{8}} g_{3}^{4} g_{3}-\frac{7}{2} g_{2} g_{3}^{3}\right) \wp \\
& -\left(\frac{5}{2^{12}} g_{2}^{6}-\frac{3}{2^{5}} g_{2}^{3} g_{3}^{2}+2 g_{3}^{4}\right)
\end{aligned}
$$

Wie man sieht, werden die Ausdrücke der $\psi^{(n)}(u)$ in $\wp(u)$ und $\wp^{\prime}(u)$ bei wachsendem $n$ schnell sehr umständlich. Wertvoll für später sind übrigens namentlich die allgemeinen Ansätze (20) und (21), an die wir wieder anzuknüpfen haben werden.

\section{§ 2. Partielle Differentialgleichung der Funktionen $\psi^{(n)}$.}

Es gibt noch ein paar andere Methoden zur Berechnung von $\wp(n u)$ bzw. von $\psi^{(n)}(u)$, die, wenn sie auch nicht mehr leisten als die Methoden von $\S 1$, immerhin der Erwähnung wert sind. Eine erste solche Methode beruht auf einer partiellen Differentialgleichung, der $\psi^{(n)}$ als Funktion von $\wp, g_{2}$ und $g_{3}$ genügt.

Nach I, 322 befriedigt die б-Funktion die Differentialgleichung:

$$
\frac{\partial^{2} \log \sigma(u)}{\partial u^{2}}+\left(\frac{\partial \log \sigma(u)}{\partial u}\right)^{2}+2 D_{\eta}(\log \sigma(u))+\frac{1}{12} g_{2} u^{2}=0 \text {. }
$$

Setzt man $n u$ statt $u$ ein und multipliziert mit $n^{2}$, so folgt:

$$
\frac{\partial^{2} \log \sigma(n u)}{\partial u^{2}}+\left(\frac{\partial \log \sigma(n u)}{\partial u}\right)^{2}+2 n^{2} D_{\eta}(\log \sigma(n u))+\frac{n^{4}}{12} g_{2} u^{2}=0 .
$$

Von dieser Gleichung ziehe man die mit $n^{4}$ multiplizierte erste Gleichung ab, führe die kurz $\psi$ zu nennende Funktion $\psi^{(n)}$, sowie nach (1) in I, 212 die Funktionen $\zeta(u)$ und $\wp(u)$ ein. Das Ergebnis kleidet sich bei Fortlassung der Argumente $u$ in die Gestalt:

$$
\frac{\partial^{2} \log \psi}{\partial u^{2}}+\left(\frac{\partial \log \psi}{\partial u}\right)^{2}+2 n^{2} \xi \frac{\partial \log \psi}{\partial u}+2 n^{2} D_{\eta}(\log \psi)+n^{2}\left(n^{2}-1\right) \wp=0
$$

wofür wir unter Zusammenfassung der beiden ersten Glieder nach ..Multiplikation mit $\psi$ schreiben können:

$$
\frac{\partial^{2} \psi}{\partial u^{2}}+2 n^{2} \zeta \frac{\partial \psi}{\partial u}+2 n^{2} D_{\eta}(\psi)+n^{2}\left(n^{2}-1\right) \wp \psi=0 .
$$


In dieser Gleichung gelten $u, g_{2}, g_{3}$ als die unabhängigen Variablen. Führt man $\wp^{\circ}$ an Stelle von $u$ ein, so ist für die Differentiationen nach $g_{2}$ und $g_{3}$ zu berücksichtigen, daß $\wp$ die Argumente $u, g_{2}$ und $g_{3}$ enthält. Die Ableitungen von $\psi$ nach $g_{2}$ und $g_{3}$ sind also:

$$
\frac{\partial \psi}{\partial \xi} \frac{\partial \wp}{\partial g_{s}}+\frac{\partial \psi}{\partial g_{2}}, \quad \frac{\partial \psi}{\partial \wp} \frac{\partial \wp}{\partial g_{s}}+\frac{\partial \psi}{\partial g_{s}},
$$

und als algebraische Gestalt des Differentialausdrucks $D_{\eta}(\psi)$ tritt an Stelle von (9) in I, 317:

$$
D_{\eta}(\psi)=\frac{\partial \psi}{\partial \dot{\xi}} D_{\eta}(\wp)-6 g_{3} \frac{\partial \psi}{\partial g_{2}}-\frac{1}{3} g_{2}^{2} \frac{\partial \psi}{\partial g_{3}} .
$$

Andrerseits gilt, da $u$ in $\wp$ allein (und nicht in $g_{2}$ und $g_{3}$ ) enthalten ist:

$$
\frac{\partial \psi}{\partial u}=\frac{\partial \psi}{\partial \wp^{\prime}} \wp^{\prime}, \quad \frac{\partial^{2} \psi}{\partial u^{2}}=\frac{\partial^{2} \psi}{\partial \rho^{2}} \gamma^{\prime 2}+\frac{\partial \psi}{\partial \wp^{\prime}} \wp^{\prime \prime} .
$$

Die Gleichung (1) gewinnt damit die Gestalt:

$$
\begin{gathered}
\frac{\partial^{2} \psi}{\partial \wp^{2}} \wp^{\prime 2}+\frac{\partial \psi}{\partial \rho^{\prime \prime}}+2 n^{2} \frac{\partial \psi}{\partial \rho^{\prime}}\left(\xi \rho^{\prime}+D_{\eta}(\xi)\right)-12 n^{2} g_{3} \frac{\partial \psi}{\partial g_{2}}-\frac{2}{3} n^{2} g_{2}^{2} \frac{\partial \psi}{\partial g_{3}} \\
+n^{2}\left(n^{2}-1\right) \wp \psi=0 .
\end{gathered}
$$

Der im dritten Gliede auftretende Klammerausdruck ist nach (7) in I, 322 gleich $\left(-2 \wp^{2}+\frac{1}{3} g_{2}\right)$. Ersetzen wir noch $\wp^{\prime 2}$ und $\wp^{\prime \prime}$ durch ihre Ausdrücke in $\varsigma^{\circ}$, so ergibt sich: Die Funltion $\psi^{(n)}$ genügt als solche von $\wp$, $g_{2}$ und $g_{3}$ der partiellen Differentialgleichung zweiter Ordnung:

$$
\begin{gathered}
\left(4 \wp^{3}-g_{2} \varsigma-g_{3}\right) \frac{\partial^{2} \psi}{\partial \wp^{2}}-\left(\left(4 n^{2}-6\right) \wp^{2}-\left(\frac{2}{3} n^{2}-\frac{1}{2}\right) g_{2}\right) \frac{\partial \psi}{\partial \wp} \\
-12 n^{2} g_{3} \frac{\partial \psi}{\partial g_{2}}-\frac{2}{3} n^{2} g_{2}^{2} \frac{\partial \psi}{\partial g_{3}}+n^{2}\left(n^{2}-1\right) \wp \psi=0 .
\end{gathered}
$$

Man kann diese Differentialgleichung zur Berechnung von $\psi^{(n)}$ benutzen, was freilich schon bei $n=5$ einen erheblichen Aufwand von Rechnung erfordert. Um die Methode für $n=3$ durchzuführen, haben wir dem Ansatze (20) entsprechend:

$$
\psi=3 \wp_{\rho^{4}}+a g_{2} \wp^{2}+b g_{3} \wp+c g_{2}^{2}
$$

mit numerischen Konstanten $a, b, c$ in die Differentialgleichung:

$$
\begin{gathered}
\left(4 \wp^{3}-g_{2} \wp-g_{3}\right) \frac{\partial^{2} \psi}{\partial \wp^{2}}-\left(30 \rho^{2}-\frac{11}{2} g_{2}\right) \frac{\partial \psi}{\partial \wp}-108 g_{3} \frac{\partial \psi}{\partial g_{2}}-6 g_{2}^{2} \frac{\partial \psi}{\partial g_{3}} \\
+72 \wp \psi=0
\end{gathered}
$$

einzutragen und müssen dadurch eine in $\wp, g_{2}, g_{3}$ identisch bestehende Gleichung gewinnen. Die entstehende Gleichung muB also z. B. auch gelten, wenn wir $g_{3}=0$ setzen; auf diese Weise erhalten wir:

$$
\begin{aligned}
\left(4 \wp^{3}-g_{2} \wp\right)\left(36 \wp^{2}\right. & \left.+2 a g_{2}\right)-\left(30 \wp^{2}-\frac{11}{2} g_{3}\right)\left(12 \wp^{3}+2 a g_{2} \wp\right)-6 b g_{2}^{2}{ }^{2} \\
& +72 \wp\left(3 \wp^{4}+a g_{2} \rho^{2}+c g_{2}^{2}\right)=0 .
\end{aligned}
$$


Ordnet man nach Potenzen von $\wp$, so muß jeder dabei auftretende Koeffizient der einzelnen Potenz für sich verschwinden, was:

$$
2 a+3=0, \quad 3 a-2 b+24 c=0
$$

liefert. Nimmt man ferner $\wp=0$, so gelangt man auf entsprechendem Wege zur Gleichung:

$$
4 a-11 b+432 c=0 .
$$

Die Auflösung der Gleichungen (3) und (4) liefert $a=-\frac{3}{2}, b=-3$, $c=-\frac{1}{16}$ und führt zu dem in (6) S. 185 angegebenen Ausdrucke von $\psi^{(3)}$ zurück.

\section{§ 3. Berechnung von $\wp(\mathrm{nu})$ durch ein Kettenbruchverfahren.}

Nur beiläufig gehen wir endlich auf eine Methode ein, den Ausdruck von $\wp(n u)$ in $\wp(u)$ mittelst eines Kettenbruchverfahrens zu gewinnen. Diese Methode führt in ihren Ergebnissen freilich nur zu bereits Bekanntem zurück; sie wird hier erwähnt wegen der bedeutenden Rolle, die sie in der Literatur des vorigen Jahrhunderts spielt. Einem Zusammenhange zwischen Integralen mit der Quadratwurzel einer ganzen Funktion und der Kettenbruchentwicklung dieser Quadratwurzel ist schon $\mathrm{Abel}^{1}$ ) nachgegangen. Wenig später stellte $\mathrm{Jacobi}^{2}$ ) ohne Beweis die Formeln auf, die im Falle der Quadratwurzel einer ganzen Funktion vierten Grades zwischen der Kettenbruchentwicklung und den Multiplikationssätzen der zugehörigen elliptischen Funktionen bestehen. In einer an die allgemeineren Fragen Abels anknüpfenden Arbeit von C. W. Borchardt ${ }^{3}$ ) sind sodann die Jacobischen Resultate neu behandelt und bewiesen. Späterhin sind endlich G. Frobenius und L. Stickelberger ${ }^{4}$ ) in einer ausführlichen Arbeit auf diese Gegenstände zurückgekommen und hàben die Multiplikationsformeln der $\wp$-Funktion bis zur Gewinnung der obigen Determinantenformeln hingeführt.

Um die Kettenbruchentwicklung zunächst auf transzendenter Grundlage zu gewinnen, bilden wir die Funktion:

$$
\Phi_{n}(u)=\frac{1}{2} \frac{\wp^{\prime}(u)-\wp^{\prime}\left(n u_{0}\right)}{\wp(u)-\wp\left(n u_{0}\right)}-\frac{1}{2} \frac{\wp^{\prime}\left(u_{0}\right)-\wp^{\prime}\left(n u_{0}\right)}{\wp\left(u_{0}\right)-\wp\left(n u_{0}\right)}
$$

mit einer ganzen Zahl $n>1$. Die Stelle $u_{0}$ denken wir vorerst fest ge-

1) „Sur l'intégration de la formule differentielle $\frac{\rho d x}{\sqrt{R}}, R$ et $\varrho$ étant des fonctions entières", Journ. f. Math., Bd. 1 (1826).

2) „Note sur une nouvelle application de l'analyse des fonctions elliptiques à l'algèbre", Journ. f. Math., Bd. 7 (1831).

3) „Application des transcendantes abéliennes à la théorie des fractions continues“, Journ. f. Math., Bd. 48 (1852).

4) "Über Addition und Multiplikation der elliptischen Funktionen", Journ. f. Math., Bd. 88 (1879). 
wählt, und zwar so, daß $\wp\left(n u_{0}\right), \wp^{\prime}\left(n u_{0}\right),\left(\wp\left(u_{0}\right)-\wp\left(n u_{0}\right)\right)$ endliche und von 0 verschiedene Werte sind. Es ist dann jedenfalls $n u_{0}$ keine ganzzahlige Kombination von Periodenhälften, woraus hervorgeht, daß auch $\wp\left(u_{0}\right), \wp^{\prime}\left(u_{0}\right)$ endlich sind, und daß die beiden Stellen $\pm n u_{0}$ bezüglich der Gruppe $\Gamma^{(u)}$ nicht äquivalent sind.

Die Funktion (1) hat im Periodenparallelogramm zwei Pole und ist also zweiwertig. Der eine Pol liegt bei $u=0$, wo das Anfangsglied der Potenzreihe durch $\Phi_{n}(u)=-u^{-1}+\cdots$ gegeben ist. Ein weiterer Pol kann nur in einem der beiden getrennt liegenden Nullpunkte erster Ordnung $\pm n u_{0}$ des Nenners $\left(\wp(u)-\wp\left(n u_{0}\right)\right)$ auftreten. Da aber an der Stelle $u=n u_{0}$ auch der Zähler $\left(\wp^{\prime}(u)-\wp^{\prime}\left(n u_{0}\right)\right)$ verschwindet, so bleibt als weiterer Pol nur noch $u=-n u_{0}$ übrig. Von den beiden Nullpunkten der Funktion (1) liegt einer bei $u=u_{0}$ und also der andere zufolge des Abelschen Theorems (5) in I, 213 bei $u=-(n+1) u_{0}$. Man kann daraufhin sofort die Darstellung (6) in I, 214 der Funktion (1) durch die б-Funktion ansetzen, wobei der von $u$ unabhängige Faktor aus dem schon genannten Anfangsgliede $-u^{-1}$ von $\Phi_{n}(u)$ gewonnen wird. Man findet:

$$
\Phi_{n}(u)=\frac{\sigma\left(u-u_{0}\right) \sigma\left(u+(n+1) u_{0}\right) \sigma\left(n u_{0}\right)}{\sigma(u) \sigma\left(u+n u_{0}\right) \sigma\left(u_{0}\right) \sigma\left((n+1) u_{0}\right)},
$$

eine Gleichung, in der fortan $u_{0}$ als unabhängige Variable gelten darf.

Für $n>2$ findet man weiter mit Benutzung von (14) in I, 217:

$$
\frac{\wp(u)-\wp\left(u_{0}\right)}{\Phi_{n-1}(u)}=-\frac{\sigma\left(u+u_{0}\right) \sigma\left(u+(n-1) u_{0}\right) \sigma\left(n u_{0}\right)}{\sigma(u) \sigma\left(u+n u_{0}\right) \sigma\left(u_{0}\right) \sigma\left((n-1) u_{0}\right)} .
$$

Der links stehende Quotient ist also gleichfalls eine zweiwertige doppeltperiodische Funktion, die mit $\Phi_{n}(u)$ die Pole gemein hat und wie $\Phi_{n}(u)$ das Anfangsglied $-u^{-1}$ der Potenzreihe nach $u$ hat. Die Differenz der Funktionen (3) und (2) hat demnach eine unter 2 herabsinkende Wertigkeit und stellt also eine von $u$ unabhängige Größe dar, die als Funktion von $u_{0}$ durch $\varphi_{n}\left(u_{0}\right)$ bezeichnet werden soll:

$$
\frac{\wp(u)-\wp\left(u_{0}\right)}{\Phi_{n-1}(u)}-\Phi_{n}(u)=\varphi_{n}\left(u_{0}\right)
$$

Zur Berechnung von $\varphi_{n}\left(u_{0}\right)$ tragen wir $u=-u_{0}$ ein und finden aus (1):

$$
\varphi_{n}\left(u_{0}\right)=-\Phi_{n}\left(-u_{0}\right)=\frac{\wp^{\prime}\left(u_{0}\right)}{\wp\left(u_{0}\right)-\wp\left(n u_{0}\right)} .
$$

Für $n=1$ ist die den Funktionen (1) entsprechende zweiwertige Funktion durch:

$$
\Phi_{1}(u)=\frac{1}{2} \frac{\wp^{\prime}(u)-\wp^{\prime}\left(u_{0}\right)}{\wp(u)-\wp\left(u_{0}\right)}-\frac{1}{2} \frac{\wp^{\prime \prime}\left(u_{0}\right)}{\wp^{\prime}\left(u_{0}\right)}
$$

gegeben. Sie hat ihre beiden Pole bei $u=0$ und $u=-u_{0}$, während 
ihre Nullpunkte bei $u=u_{0}$ und $u=-2 u_{0}$ liegen. Erklären wir $\varphi_{1}\left(u_{0}\right)$ durch:

$$
\varphi_{1}\left(u_{0}\right)=\frac{1}{2} \frac{\wp^{\prime \prime}\left(u_{0}\right)}{\wp^{\prime}\left(u_{0}\right)}
$$

so reiht sich an (4) für $n=1$ die Gleichung an:

$$
\frac{1}{2} \frac{\wp^{\prime}(u)-\wp^{\prime}\left(u_{0}\right)}{\wp(u)-\wp\left(u_{0}\right)}-\Phi_{1}(u)=\varphi_{1}\left(u_{0}\right) \text {. }
$$

Im übrigen gilt auch für die Funktion $\Phi_{1}(u)$ die allgemein unter (2) gewonnene Darstellung durch die Sigmafunktion. Hieraus folgt wie oben, daB die Gleichung (4) auch für $n=2$ bestehen bleibt, falls wir $\varphi_{2}\left(u_{0}\right)$ durch die allgemeine Vorschrift (5) erklären.

Schreiben wir die entwickelte Formelkette in der Gestalt:

$$
\begin{gathered}
\frac{1}{2} \frac{\wp^{\prime}(u)-\wp^{\prime}\left(u_{0}\right)}{\wp(u)-\wp\left(u_{0}\right)}=\varphi_{1}\left(u_{0}\right)+\Phi_{1}(u), \\
\frac{\wp(u)-\wp\left(u_{0}\right)}{\Phi_{1}(u)}=\varphi_{2}\left(u_{0}\right)+\Phi_{2}(u), \\
\frac{\wp(u)-\wp\left(u_{0}\right)}{\Phi_{2}(u)}=\varphi_{3}\left(u_{0}\right)+\Phi_{3}(u), \\
\cdot \cdot \cdot \cdot \cdot \cdot \cdot \cdot \cdot \cdot \cdot \cdot \cdot \\
\frac{\wp(u)-\wp\left(u_{0}\right)}{\Phi_{n-1}(u)}=\varphi_{n}\left(u_{0}\right)+\Phi_{n}(u),
\end{gathered}
$$

unter $n$ irgendeine ganze Zahl $>1$ verstanden, so sind die ersten Glieder der rechten Seiten stets als die Grenzen der links stehenden Ausdrücke für $u=u_{0}$ eindeutig bestimmt, womit dann zugleich auch die in den zweiten Gliedern rechts stehenden $\Phi_{v}(u)$ als für $u=u_{0}$ verschwindende Funktionen bestimmt sind. Die Elimination von $\Phi_{1}, \Phi_{2}, \ldots, \Phi_{n-1}$ ergibt aber für die in der ersten Gleichung links stehende Funktion die Entwicklung in einen $n$-gliedrigen Kettenbruch:

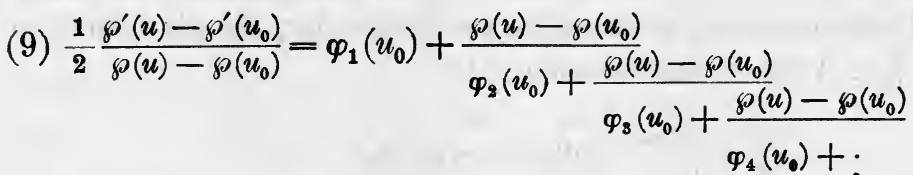

$$
\begin{aligned}
& \vdots+\frac{\wp(u)-\wp\left(u_{0}\right)}{\varphi_{n}\left(u_{0}\right)+\Phi_{n}(u)} .
\end{aligned}
$$

- Diese Kettenbruchentwicklung ist nun auch in algebraischer Gestalt. durchführbar, nämlich auf Grund der Regel, nach der man eine Potenzreihe in einen Kettenbruch umrechnet. Wir setzen zunächst die Formel (9) in algebraische Gestalt, indem wir:

$$
\wp(u)=x, \quad \wp\left(u_{0}\right)=x_{0}, \quad \wp^{\prime}(u)=\sqrt{f(x)}, \quad \wp^{\prime}\left(u_{0}\right)=\sqrt{f\left(x_{0}\right)}
$$

schreiben, unter $f(x)$ die ganze Funktion $\left(4 x^{3}-g_{2} x-g_{3}\right)$ verstanden: 
Die Kettenbruchentwicklung in transzendenter und in algebraischer Gestalt

$$
\begin{aligned}
& \frac{1}{2} \frac{\sqrt{f(x)}-\sqrt{f\left(x_{0}\right)}}{x-x_{0}}=\varphi_{1}+\frac{x-x_{0}}{\varphi_{2}+\underline{x-x_{0}}} \\
& \varphi_{3}+\frac{x-x_{0}}{\varphi_{4}+} \text {. } \\
& \therefore+\frac{x-x_{0}}{\varphi_{n}+\Phi_{n}} .
\end{aligned}
$$

Um die Taylorsche Reihe der links stehenden Funktion:

$$
\begin{gathered}
\frac{1}{2} \frac{\sqrt{f(x)}-\sqrt{f\left(x_{0}\right)}}{x-x_{0}}=a_{0}+a_{1}\left(x-x_{0}\right)+a_{2}\left(x-x_{0}\right)^{2}+\cdots, \\
2 a_{n-1}=\frac{1}{n !} \frac{d^{n} \sqrt{f\left(x_{0}\right)}}{d x^{n}}
\end{gathered}
$$

in den Kettenbruch (10) umzuwandeln, hat man wiederholt von der Gleichung:

$$
\begin{gathered}
\left(c_{0}+c_{1} z+c_{2} z^{2}+c_{3} z^{3}+\cdots\right)^{-1}=\frac{1}{c_{0}}-\frac{c_{1}}{c_{0}^{2}} z+\left(\frac{c_{1}^{2}}{c_{0}^{3}}-\frac{c_{2}}{c_{0}^{2}}\right) z^{2} \\
-\left(\frac{c_{1}^{3}}{c_{0}^{4}}-\frac{2 c_{1} c_{2}}{c_{0}^{3}}+\frac{c_{3}}{c_{0}^{2}}\right) z^{3}+\cdots
\end{gathered}
$$

Gebrauch zu machen, mittelst deren man den reziproken Wert einer Potenzreihe wieder in eine solche Reihe umwandelt. Die Anfangsglieder des Kettenbruchs (10) in der neuen Gestalt sind gegeben durch:

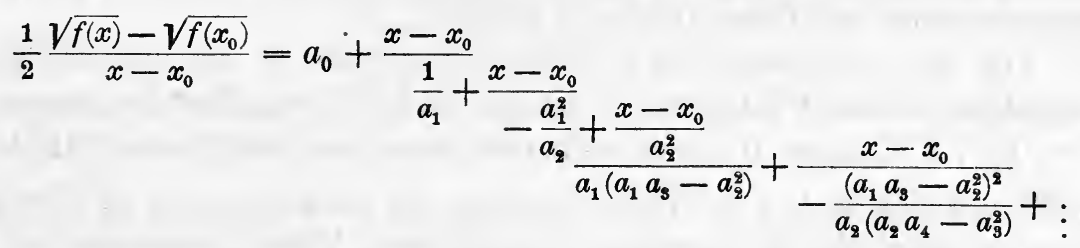

Der Vergleich mit (10) lehrt die folgenden Darstellungen der $\varphi_{1}, \varphi_{2}, \ldots$ :

$$
\begin{gathered}
\varphi_{1}=a_{0}, \quad \varphi_{2}=\frac{1}{a_{1}}, \quad \varphi_{3}=-\frac{a_{1}^{2}}{a_{2}}, \quad \varphi_{4}=\frac{a_{2}^{2}}{a_{1}\left(a_{1} a_{3}-a_{2}^{2}\right)}, \\
\varphi_{5}=-\frac{\left(a_{1} a_{3}-a_{2}^{2}\right)^{2}}{a_{2}\left(a_{2} a_{4}-a_{3}^{2}\right)}, \cdots,
\end{gathered}
$$

wo die $a_{0}, a_{1}, a_{2}, \ldots$ durch die zweite Gleichung (11) gegeben sind.

Bei Rückgang zu den transzendenten Funktionen wollen wir den Index an $u_{0}$ fortlassen und haben dann erstlich:

$$
\varphi_{1}=\frac{1}{2} \frac{\wp^{\prime \prime}(u)}{\wp^{\prime}(u)}, \quad \varphi_{n}=\frac{\wp^{\prime}(u)}{\wp(u)-\wp(n u)}, \quad n>1,
$$

während andrerseits aus der zweiten Gleichung (11):

$$
a_{0}=\frac{1}{2} \frac{d \wp^{\prime}(u)}{d \wp(u)}=\frac{1}{2} \frac{\wp^{\prime \prime}(u)}{\wp^{\prime}(u)}, \quad a_{n}=\frac{1}{n+1} \frac{d a_{n-1}}{d \wp}=\frac{1}{n+1} \frac{d a_{n-1}}{d u} \cdot \frac{1}{\wp^{\prime}(u)}
$$

als Regel für die Berechnung der $a$ folgt. In den ersten Fällen haben wir bei Fortlassung der Argumente $u$ die Darstellungen: 


$$
a_{0}=\frac{\wp^{\prime \prime}}{2 \wp^{\prime}}, \quad a_{1}=\frac{\wp^{\prime} \wp^{\prime \prime \prime}-\wp^{\prime \prime 2}}{4 \wp^{\prime 3}}, \quad a_{2}=\frac{\wp^{\prime 2} \wp^{(4)}-4 \gamma^{\prime} \wp^{\prime \prime} \wp^{\prime \prime \prime}+3 \wp^{\prime \prime 3}}{12 \wp^{\prime 5}}, \ldots
$$

Man prüfe etwa den Fall $n=3$, wo die Gleichung:

$$
\wp(3 u)=\wp(u)-\frac{\wp^{\prime}(u)}{\varphi_{3}(u)}=\wp(u)+\frac{a_{9} \wp^{\prime}(u)}{a_{1}^{2}}
$$

nach Eintragung der Ausdrücke von $\wp^{\prime \prime}, \wp^{\prime \prime \prime}, \wp^{(4)}$ in $\wp$ und $\wp^{\prime}$ auf die von früher bekannte Darstellung von $\wp(3 u)$ in $\wp(u)$ und $\wp^{\prime}(u)$ zurückführt. Im übrigen sei wegen Durchbildung der Methode nochmals auf die letzte der S. 192 genannten Abhandlungen verwiesen.

\section{\$ 4. Ansatz der Multiplikationsformeln für sn, cn und dn.}

Die Multiplikationsformeln für die früher allein betrachteten Funktionen zweiter Stufe sind von $\mathrm{Abel}^{1}$ ) aufgestellt. Jacobi bezieht sich in den „Fundamenten“ (am Ende des Artikels 28) auf Abel und übergeht daher die Einzelheiten der Multiplikationsformeln. Er kommt jedoch alsbald auf den Gegenstand zurück ${ }^{2}$ ) und erkennt die Existenz einer partiellen Differentialgleichung, welche zur Berechnung der Multiplikationsformeln dienen kann. Es handelt sich um die der Gleichung des $\$ 2$ entsprechende Differentialgleichung für die Funktionen zweiter Stufe; wir kommen unten auf diese Gleichung zurück.

Die drei Funktionen $\operatorname{sn} w, \mathrm{cn} w, \operatorname{dn} w$ gehören je als zweiwertige doppeltperiodische Funktionen zu den drei in I, 378 eingeführten Gruppen $\Gamma_{2_{1}}^{(w)}, \Gamma_{2_{2}}^{(w)}, \Gamma_{2_{3}}^{(w)}$, deren Diskontinuitätsbereiche durch die Figuren 75 ff. in I, 393 gegeben sind. Die Werteverteilung der Funktionen ist in diesen Figuren durch die in Klammern eingetragenen Werte veranschaulicht. Ist nun $n$ irgendeine positive ganze Zahl, so gehören auch die drei Funktionen sn $(n w)$, en $(n w), \mathrm{dn}(n w)$ bzw. zu den drei genannten Gruppen. Es hat nämlich z. B. die Funktion sn $(n w)$ die Perioden $\frac{4 K}{n}$ und $\frac{2 i K^{\prime}}{n}$, und zwar gehört sie als zweiwertige Funktion zu dem diesen beiden Perioden entsprechenden Parallelogramme. Man mache sich deutlich, daß sich $n^{2}$ Parallelogramme dieser letzteren Art zu dem in Fig. 75 a. a. 0. gegebenen Diskontinuitätsbereiche der $\Gamma_{2_{1}}^{(w)}$ zusammenordnen lassen. Indem man die Betrachtung auch auf die beiden anderen Fälle überträgt, erkennt man in sn $(n w), \operatorname{cn}(n w)$, dn $(n w)$ Funktionen der Wertigkeit $2 n^{2}$ der fraglichen Gruppen. Auf Grund des Satzes in I, 394 (unten) und der entsprechenden Sätze für die $\Gamma_{2_{2}}^{(w)}, \Gamma_{2_{3}}^{(w)}$ sind nun die sn $(n w)$, cn $(n w)$,

1) Im zweiten Paragraphen der „Recherches sur les fonctions elliptiques“, Journ. f. Math., Bd. 2 (1828).

2) "Suite des notices sur les fonctions elliptiques", Journ. f. Math., Bd. 3 1828) oder Jacobis Werke, Bd. 1, S. 264, Abs. III. 
Ansätze für $\operatorname{sn}(n w), \operatorname{cn}(n w), \operatorname{dn}(n w)$ als Funktionen von sn $w, \operatorname{cn} w, \operatorname{dn} w 197$ $\operatorname{dn}(n w)$ rational in sn $w$, cn $w, d n w$ darstellbar. Die Ansätze für diese Darstellungen gewinnt man durch einfache funktionentheoretische Überlegung, wie wenigstens im Falle der Funktion sn ( $n w)$ etwas näher ausgeführt werden soll.

Ist erstlich $n$ ungerade, so bleibt die ungerade Funktion sn ( $n w)$ bei der Substitution $w^{\prime}=2 K-w$ unverändert und ist demnach eine rationale Funktion von sn $w$ allein, und zwar wegen der Wertigkeit eine solche vom Grade $n^{2}$. Bei $w=0$ haben $\operatorname{sn}(n w)$ und sn $w$ einen Nullpunkt erster Ordnung und bei $w=i K^{\prime}$ einen Pol erster Ordnung gemein. Hieraus schließt man, daß der Zähler der fraglichen rationalen Funktion den Faktor sn $w$ hat, und dab der Grad des Nenners um eine Einheit kleiner als der Grad des Zählers ist. Hiernach ist sn $(n w)$ darstellbar als Produkt von sn $w$ und einer rationalen Funktion des Grades $\left(n^{2}-1\right)$. Diese Funktion kann nur gerade Potenzen von sn $w$ enthalten, da $\operatorname{sn}(n w): \operatorname{sn} w$ eine gerade Funktion von $w$ ist. Übrigens nimmt die fragliche rationale Funktion von $(\operatorname{sn} w)^{2}$ für sn $w=0$ den Wert $n$ an, da man aus (10) in I, 399 sofort $\lim (\operatorname{sn}(n w): \operatorname{sn} w)=n$ feststellt.

$w=0$

Etwas umständlicher gestaltet sich die Überlegung im Falle einer geraden Zahl $n$, einfacher hingegen wieder für cn $(n w)$ und $d n(n w)$. Wir fassen sogleich die Ergebnisse zusammen und schreiben hierbei zur Abkürzung:

$$
(\operatorname{sn} w)^{2}=z \text {. }
$$

Im Falle einer ungeraden Zahl $n$ gelten für die Multiplikationsformeln der Jacobischen Funktionen sn, cn, dn die Ansätze:

(1) $\operatorname{sn}(n w)=\frac{\operatorname{sn} w \cdot G_{1}^{(n)}(z)}{G_{0}^{(n)}(z)}, \operatorname{cn}(n w)=\frac{\operatorname{cn} w \cdot G_{2}^{(n)}(z)}{G_{0}^{(n)}(z)}, \operatorname{dn}(n w)=\frac{\operatorname{dn} w \cdot G_{3}^{(n)}(z)}{G_{0}^{(n)}(z)}$, wo die $G$ rationale ganze Funktionen folgender Gestalten sind:

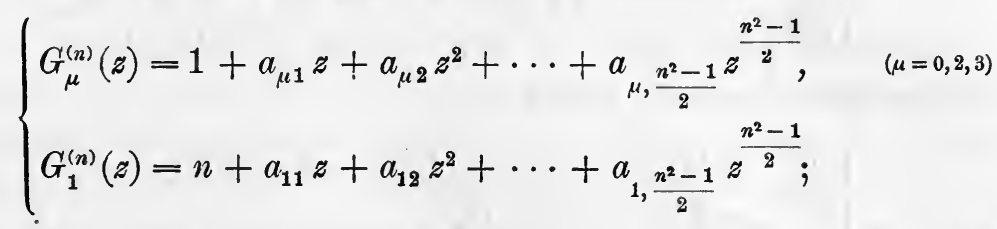

für eine gerade Zahl $n$ schließen sich hieran die Ansätze:

(3) $\operatorname{sn}(n w)=\frac{\operatorname{sn} w \operatorname{cn} w \operatorname{dn} w \cdot G_{1}^{(n)}(z)}{G_{0}^{(n)}(z)}, \operatorname{cn}(n w)=\frac{G_{2}^{(n)}(z)}{G_{0}^{(n)}(z)}, \operatorname{dn}(n w)=\frac{G_{3}^{(n)}(z)}{G_{0}^{(n)}(z)}$,

wo die rationalen ganzen Funktionen $G$ folgende Gestalten haben:

$$
\left\{\begin{array}{l}
G_{\mu}^{(n)}(z)=1+a_{\mu 1} z+a_{\mu 2} z^{2}+\cdots+a_{\mu, \frac{n^{2}}{2} z^{\frac{n^{2}}{2}},} \\
G_{1}^{(n)}(z)=n+a_{11} z+a_{12} z^{2}+\cdots+a_{1, \frac{n^{2}-4}{2}} z^{\frac{n^{2}-4}{2}} .
\end{array} \quad(\mu=0,2,3)\right.
$$


Zwischen den vier Funktionen eines und desselben $n$ bestehen zwei identische Gleichungen, die sich aus den beiden in I, 389 gewonnenen Relationen zwischen sn, en und dn ergeben. Diese Gleichungen lauten im Falle eines ungeraden $n$ :

$$
(1-z) G_{2}(z)^{2}=G_{0}(z)^{2}-z G_{1}(z)^{2},\left(1-k^{2} z\right) G_{3}(z)^{2}=G_{0}(z)^{2}-k^{2} z G_{1}(z)^{2}
$$

während sie bei geradem $n$ die Gestalt haben:

$$
\left\{\begin{array}{l}
G_{2}(z)^{2}=G_{0}(z)^{2}-z(1-z)\left(1-k^{2} z\right) G_{1}(z)^{2} \\
G_{3}(z)^{2}=G_{0}(z)^{2}-k^{2} z(1-z)\left(1-k^{2} z\right) G_{1}(z)^{2}
\end{array}\right.
$$

$k^{2}$ ist der Legendre-Jacóbische Integralmodul (vgl. I, 367).

Das Ziel der weiteren Entwicklung ist nun die genauere Berechnung der ganzen Funktionen $G$. Zunächst bietet sich ein rekurrentes Verfahren dar. Setzen wir in den drei Additionsformeln (9), S. 166 für $u$ und $v$ übereinstimmend $n w$ ein, so folgt:

$$
\left\{\begin{array}{l}
\operatorname{sn} 2 n w=\frac{2 \operatorname{sn} n w \cdot \operatorname{cn} n w \cdot \operatorname{dn} n w}{1-k^{2} \operatorname{sn} n w^{4}}, \\
\operatorname{cn} 2 n w=\frac{\operatorname{cn} n w^{2}-\operatorname{sn} n w^{2} \operatorname{dn} n w^{2}}{1-k^{2} \operatorname{sn} n w^{4}}, \\
\operatorname{dn} 2 n w=\frac{\operatorname{dn} n w^{2}-k^{2} \operatorname{sn} w^{2} \operatorname{cn} n w^{2}}{1-k^{2} \operatorname{sn} n w^{4}} .
\end{array}\right.
$$

Hieraus berechnen sich, wenn wir der Kürze halber die Argumente $z$ der Funktionen $G$ fortlassen, mit Rücksicht auf die Anfangskoeffizienten der $G$ bei ungeradem $n$ die Formeln:

$$
\left\{\begin{array}{l}
G_{0}^{(2 n)}=G_{0}^{(n)_{4}}-k^{2} z^{2} G_{1}^{(n)_{4}}, \\
G_{1}^{(2 n)}=2 G_{0}^{(n)} G_{1}^{(n)} G_{2}^{(n)} G_{3}^{(n)} \\
G_{2}^{(2 n)}=(1-z) G_{0}^{(n)_{2}} G_{2}^{(n)_{2}}-\left(z-k^{2} z^{2}\right) G_{1}^{(n)_{2}} G_{3}^{(n)_{2}}, \\
G_{3}^{(2 n)}=\left(1-k^{2} z\right) G_{0}^{(n)_{2}} G_{3}^{(n)_{2}}-\left(k^{2} z-k^{2} z^{2}\right) G_{1}^{(n)_{2}} G_{2}^{(n)_{2}},
\end{array}\right.
$$

bei geradem $n$ aber die Beziehungen:

$$
\left\{\begin{array}{l}
G_{0}^{(2 n)}=G_{0}^{(n)_{4}}-k^{2} z^{2}(1-z)^{2}\left(1-k^{2} z\right)^{2} G_{1}^{(n)_{4}}, \\
G_{1}^{(2 n)}=2 G_{0}^{(n)} G_{1}^{(n)} G_{2}^{(n)} G_{3}^{(n)}, \\
G_{2}^{(2 n)}=G_{0}^{(n)_{2}} G_{2}^{(n)}-\left(z-\left(1+k^{2}\right) z^{2}+k^{2} z^{3}\right) G_{1}^{(n)_{2}} G_{3}^{(n)_{2}}, \\
G_{3}^{(2 n)}=G_{0}^{(n)_{2}} G_{3}^{(n)_{2}}-\left(k^{2} z-\left(k^{2}+k^{4}\right) z^{2}+k^{4} z^{3}\right) G_{1}^{(n)_{2}} G_{2}^{(n)_{2}} .
\end{array}\right.
$$

Setzt man andrerseits $u=(n+1) w, v=n w$ in die Additionsformeln ein, so gelangt man entsprechend zu den drei in jedem Falle $n$ gültigen Formeln:

$$
\left\{\begin{array}{l}
G_{0}^{(2 n+1)}=G_{0}^{(n)_{2}} G_{0}^{(n+1)_{2}}-\left(k^{2} z^{2}-\left(k^{2}+k^{4}\right) z^{3}+k^{4} z^{4}\right) G_{1}^{(n)_{2}} G_{1}^{(n+1)_{2}} \\
G_{2}^{(2 n+1)}=G_{0}^{(n)} G_{2}^{(n)} G_{0}^{(n+1)} G_{2}^{(n+1)}-\left(z-k^{2} z^{2}\right) G_{1}^{(n)} G_{3}^{(n)} G_{1}^{(n+1)} G_{3}^{(n+1)} \\
G_{3}^{(2 n+1)}=G_{0}^{(n)} G_{3}{ }^{n)} G_{0}^{(n+1)} G_{3}^{(n+1)}-\left(k^{2} z-k^{2} z^{2}\right) G_{1}^{(n)} G_{2}^{(n)} G_{1}^{(n+1)} G_{2}^{(n+1)}
\end{array}\right.
$$


während sich $G_{1}^{(2 n+1)}$ bei ungeradem $n$ durch die Formel:

$$
\begin{aligned}
G_{1}^{(2 n+1)} & =G_{0}^{(n)} G_{1}^{(n)} G_{2}^{(n+1)} G_{3}^{(n+1)} \\
& +\left(1-\left(1+k^{2}\right) z+k^{2} z^{2}\right) G_{2}^{(n)} G_{3}^{(n)} G_{0}^{(n+1)} G_{1}^{(n+1)}
\end{aligned}
$$

bei geradem $n$ aber durch:

$$
\begin{aligned}
G_{1}^{(2 n+1)} & =G_{2}^{(n)} G_{3}^{(n)} G_{0}^{(n+1)} G_{1}^{(n+1)} \\
& +\left(1-\left(1+k^{2}\right) z+k^{2} z^{2}\right) G_{0}^{(n)} G_{1}^{(n)} G_{2}^{(n+1)} G_{3}^{(n+11)}
\end{aligned}
$$

berechnet.

Da alle vier Funktionen $G^{(1)}$ mit 1 identisch sind, so ergibt sich aus den Formeln (8):

$$
G_{0}^{(2)}=1-k^{2} z^{2}, G_{1}^{(2)}=2, G_{2}^{(2)}=1-2 z+k^{2} z^{2}, G_{3}^{(2)}=1-2 k^{2} z+k^{2} z^{2}
$$

sowie weiter mittelst der Formeln (10) und (11):

$$
\left\{\begin{array}{l}
G_{0}^{(3)}=1-6 k^{2} z^{2}+4 k^{2}\left(1+k^{2}\right) z^{3}-3 k^{4} z^{4} \\
G_{1}^{(3)}=3-4\left(1+k^{2}\right) z+6 k^{2} z^{2}-k^{4} z^{4} \\
G_{2}^{(3)}=1-4 z+6 k^{2} z^{2}-4 k^{4} z^{3}+k^{4} z^{4} \\
G_{3}^{(3)}=1-4 k^{2} z+6 k^{2} z^{2}-4 k^{2} z^{3}+k^{4} z^{4}
\end{array}\right.
$$

Mit Rücksicht auf die Gestalt der Rekursionsformeln gewinnen wir aus diesen Angaben den allgemeinen Satz, $d a \beta$ die Koeffizienten a der ganzen Funktionen $G$ durchweg rationale ganze ganzzahlige Funktionen von $k^{2}$ sind. Einige weitere allgemeine Gesetze über unsere Funktionen $G$ erhalten wir durch Heranziehung bisher noch nicht benutzter Hilfsmittel.

\section{Weitere Beziehungen zwischen den Funktionen $G(z)$.}

Da weiterhin nur noch die Funktionen $G_{0}^{(n)}, G_{1}^{(n)}, \ldots$ eines und desselben $n$ zur Sprache kommen, so wird der obere Index $n$ fortgelassen. Dagegen wird ausführlicher $G_{0}\left(z, k^{2}\right), G_{1}\left(z, k^{2}\right), \ldots$ geschrieben, um die Abhängigkeit der Funktionen von $k^{2}$ hervorzuheben. Es ist nun zunächst möglich, die Koeffizienten der höchsten Potenzen in den Ausdrücken (2) und (4) S. 197 der Funktionen $G$ allgemein zu bestimmen. Diese Koeffizienten $a_{0, \frac{n^{2}-1}{2}}, a_{1, \frac{n^{2}-1}{2}}, \ldots$ der vier Ausdrücke (2) S. 197, die sich auf ungerades $n$ beziehen, sind bzw.:

$$
(-1)^{\frac{n-1}{2}} n k^{\frac{n^{2}-1}{2}}, \quad(-1)^{\frac{n-1}{2}} k^{\frac{n^{2}-1}{2}}, k^{\frac{n^{2}-1}{2}}, k^{\frac{n^{2}-1}{8}}
$$

im Falle eines geraden $n$ hat man für $a_{0, \frac{n^{2}}{2}}, a_{1, \frac{n^{2}-4}{2}}, \ldots$ bzw.:

$$
(-1)^{\frac{n}{2}} k^{\frac{n^{2}}{2}}, \quad(-1)^{\frac{n-2}{2}} n k^{\frac{n^{2}-4}{2}}, \quad k^{\frac{n^{2}}{2}}, \quad k^{\frac{n^{2}}{2}} \text {. }
$$


Für $n=1,2$ und 3 geht die Richtigkeit dieser Angaben aus den am Schlusse von $\S 4$ zusammengestellten Ausdrücken der zugehörigen Funktionen $G$ hervor. Die Rekursionsformeln (8)ff. S. 198 zeigen dann mittelst des Schlusses der vollständigen Induktion die allgemeine Gültigkeit. Schreiben wir auch noch bei den Koeffizienten $a$ das Argument $k^{2}$ hinzu, so gilt hiernach als Ansatz bei einem ungeraden $n$ :

$$
\left\{\begin{array}{l}
G_{0}\left(z, k^{2}\right)=1+a_{01}\left(k^{2}\right) z+a_{02}\left(k^{2}\right) z^{2}+\cdots+(-1)^{\frac{n-1}{2}} n k^{\frac{n^{2}-1}{2}} z^{\frac{n^{2}-1}{2}} \\
G_{1}\left(z, k^{2}\right)=n+a_{11}\left(k^{2}\right) z+a_{12}\left(k^{2}\right) z^{2}+\cdots+(-1)^{\frac{n-1}{2}} k^{\frac{n^{2}-1}{2}} z^{\frac{n^{2}-1}{2}} \\
G_{\mu}\left(z, k^{2}\right)=1+a_{\mu 1}\left(k^{2}\right) z+a_{\mu 2}\left(k^{2}\right) z^{2}+\cdots+k^{\frac{n^{2}-1}{2}} z^{\frac{n^{2}-1}{2}}, \quad(\mu=2,8)
\end{array}\right.
$$

und bei einem geraden $n$ :

$$
\left\{\begin{array}{l}
G_{0}\left(z, k^{2}\right)=1+a_{01}\left(k^{2}\right) z+a_{02}\left(k^{2}\right) z^{2}+\cdots+(-1)^{\frac{n}{2}} k^{\frac{n^{2}}{2}} z^{\frac{n^{2}}{2}}, \\
G_{1}\left(z, k^{2}\right)=n+a_{11}\left(k^{2}\right) z+a_{12}\left(k^{2}\right) z^{2}+\cdots+(-1)^{\frac{n-2}{2}} n k^{\frac{n^{2}-4}{2}} z^{\frac{n^{2}-4}{2}}, \\
G_{\mu}\left(z, k^{2}\right)=1+a_{\mu 1}\left(k^{2}\right) z+a_{\mu 2}\left(k^{2}\right) z^{2}+\cdots+k^{\frac{n^{2}}{2}} z^{\frac{n^{2}}{2}},
\end{array}\right.
$$

Man gelangt nun zur Kenntnis einer Anzahl von Relationen zwischen den vier Funktionen $G$, wenn man das Argument $w$ der Funktionen sn, cn, dn um Periodenhälften ändert, und wenn man diese Funktionen der linearen Transformation unterwirft (vgl. I,475). Das Verhalten von sn $w, \ldots$, sn $(n w), \ldots$ bei Vermehrung von $w$ um $i K^{\prime}$ geht aus der Tabelle in I, 395 und den Formeln (13) in I, 390 hervor; der Integralmodul bleibt unverändert. Die für ungerades $n$ gültigen Gleichungen (1) S. 197 gehen demnach bei dieser Änderung von $w$ in die folgenden über:

$$
\begin{aligned}
& \frac{1}{\operatorname{sn}(n w)}=\frac{1}{\operatorname{sn} w} \cdot \frac{G_{1}\left(\frac{1}{k^{2} z}, k^{2}\right)}{G_{0}\left(\frac{1}{k^{2} z}, k^{2}\right)}, \\
& \frac{\operatorname{dn}(n w)}{\operatorname{sn}(n w)}=(-1)^{\frac{n-1}{2}} \frac{\operatorname{dn} w}{\operatorname{sn} w} \cdot \frac{G_{2}\left(\frac{1}{k^{2} z}, k^{2}\right)}{G_{0}\left(\frac{1}{k^{2} z}, k^{2}\right)}, \\
& \frac{\operatorname{cn}(n w)}{\operatorname{sn}(n w)}=(-1)^{\frac{n-1}{2}} \frac{\operatorname{cn} w}{\operatorname{sn} w} \cdot \frac{G_{3}\left(\frac{1}{k^{2} z}, k^{2}\right)}{G_{0}\left(\frac{1}{k^{2} z}, k^{2}\right)} .
\end{aligned}
$$

Der Vergleich mit den ursprünglichen Gleichungen lehrt die bei ungeradem $n$ bestehenden Beziehungen: 
(3)

$$
\left\{\begin{array}{l}
G_{1}\left(z, k^{2}\right)=(-1)^{\frac{n-1}{2}}(k z)^{\frac{n^{2}-1}{2}} G_{0}\left(\frac{1}{k^{2} z}, k^{2}\right), \\
G_{3}\left(z, k^{2}\right)=(k z)^{\frac{n^{2}-1}{2}} G_{2}\left(\frac{1}{k^{2} z}, k^{2}\right) .
\end{array}\right.
$$

Bei geradem $n$ gewinnt man auf entsprechendem Wege die Regeln:

$$
\left\{\begin{array}{l}
G_{0}\left(z, k^{2}\right)=(-1)^{\frac{n}{2}}(k z)^{\frac{n^{2}}{2}} G_{0}\left(\frac{1}{k^{2} z}, k^{2}\right), \\
G_{1}\left(z, k^{2}\right)=(-1)^{\frac{n-2}{2}}(k z)^{\frac{n^{2}-4}{2}} G_{1}\left(\frac{1}{k^{2} z}, k^{2}\right), \\
G_{\mu}\left(z, k^{2}\right)=(k z)^{\frac{n^{2}}{2}} G_{\mu}\left(\frac{1}{k^{2} z}, k^{2}\right) .
\end{array}\right.
$$

Man übe zweitens die in I, 475 mit - STS bezeichnete lineare Transformation aus. Das Verhalten der Funktionen und des Integralmoduls ist a. a. 0 . angegeben; insbesondere ist $z$ durch $k^{2} z$ zu ersetzen und $k^{2}$ durch $\frac{1}{k^{2}}$. Man findet, daß sowohl bei ungeradem als geradem $n$ die Regeln gelten:

$$
\left\{\begin{array}{l}
G_{\mu}\left(z, k^{2}\right)=G_{\mu}\left(k^{2} z, \frac{1}{k^{2}}\right), \\
G_{2}\left(z, k^{2}\right)=G_{3}\left(k^{2} z, \frac{1}{k^{2}}\right), \\
G_{3}\left(z, k^{2}\right)=G_{2}\left(k^{2} z, \frac{1}{k^{2}}\right) .
\end{array}\right.
$$

$(\mu=0,1)$

Bei Anwendung der linearen Transformation $T$ geht $k^{2}$ zufolge I, 475 in $\left(1-k^{2}\right)$ über und $z$ in $\frac{z}{z-1}$. Hier findet man bei ungeradem $n$ die Gesetze:

$$
\left\{\begin{array}{l}
G_{0}\left(z, k^{2}\right)=(z-1)^{\frac{n^{2}-1}{2}} G_{2}\left(\frac{z}{z-1}, 1-k^{2}\right), \\
G_{2}\left(z, k^{2}\right)=(z-1)^{\frac{n^{2}-1}{2}} G_{0}\left(\frac{z}{z-1}, 1-k^{2}\right), \\
G_{\mu}\left(z, k^{2}\right)=(z-1)^{\frac{n^{2}-1}{2}} G_{\mu}\left(\frac{z}{z-1}, 1-k^{2}\right), \quad(\mu=1,3\rangle
\end{array}\right.
$$

während sich bei geradem $n$ die Regeln anschließen:

$$
\left\{\begin{array}{l}
G_{0}\left(z, k^{2}\right)=(z-1)^{\frac{n^{2}}{2}} G_{2}\left(\frac{z}{z-1}, 1-k^{2}\right), \\
G_{1}\left(z, k^{2}\right)=(z-1)^{\frac{n^{2}-4}{2}} G_{1}\left(\frac{z}{z-1}, 1-k^{2}\right), \\
G_{2}\left(z, k^{2}\right)=(z-1)^{\frac{n^{2}}{2}} G_{0}\left(\frac{z}{z-1}, 1-k^{2}\right), \\
G_{3}\left(z, k^{2}\right)=(z-1)^{\frac{n^{2}}{2}} G_{3}\left(\frac{z}{z-1}, 1-k^{2}\right) .
\end{array}\right.
$$


$\mathrm{Zu}$ neuen Regeln würde auch noch die Vermehrung von $w$ um $K$ führen; doch sind diese etwas umständlicher und kommen weiterhin nicht zur Verwendung.

Die gewonnenen Formeln zeigen, $d a \beta$ bei ungeradem $n$ mit $G_{0}$ die drei anderen Funktionen als bekannt gelten können, da man, falls $G_{0}$ bekannt ist, $G_{2}$ aus der zweiten Formel (6) und sodann $G_{1}$ und $G_{3}$ aus den Formeln (3) gewinnt. Bei geradem n dürfen mit $G_{0}$ und $G_{1}$ auch $G_{2}$ und $G_{3}$ als bekannt gelten, da man $G_{2}$ mittelst der dritten Formel (7) aus $G_{0}$ und sodann $G_{3}$ aus $G_{2}$ durch die dritte Formel (5) bestimmt. Übrigens ist $G_{1}^{(n)}$, falls die Funktionen $G_{\mu}^{\left(\frac{n}{2}\right)}$ schon bekannt sind, aus der zweiten Rekursionsformel (9) S. 198 unmittelbar abzulesen, so daß sich unsere Aufgabe im wesentlichen auf die Berechnung von $G_{0}^{(n)}$ beschränkt.

Besonders wertvoll sind die Regeln (5), die wir für die Koeffizienten $a$ in die Gestalten kleiden:

$$
\left\{\begin{array}{l}
a_{\mu v}\left(\frac{1}{k^{2}}\right) \cdot k^{2 v}=a_{\mu v}\left(k^{2}\right), \\
a_{2 v}\left(\frac{1}{k^{2}}\right) \cdot k^{2 v}=a_{3 v}\left(k^{2}\right), \quad a_{3 v}\left(\frac{1}{k^{2}}\right) k^{2 v}=a_{2 v}\left(k^{2}\right) .
\end{array}\right.
$$

Der Schlußsatz von $\S 4$ kann demnach so ergänzt werden: Der einzelne Koeffizient $a_{\mu \nu}$ ist eine ganze ganzzahlige Funktion höchstens $\boldsymbol{\nu}^{\text {ten }}$ Grades von $k^{2}$.

Ehe wir weitere Folgerungen aus den Relationen (3)ff. ziehen, stellen wir allgemein die Werte der Koeffizienten $a$ für den Fall $k^{2}=0$ fest. In diesem Falle sind die Funktionen $G_{0}$ und $G_{3}$ für $n=1,2$ und 3 nach $\S 4$ mit 1 identisch. Die Rekursionsformeln (8) ff. S. 198 zeigen alsdann durch den SchluB der vollständigen Induktion, daß für jedes $n$ die Funktionen $G_{0}(z, 0)$ und $G_{3}(z, 0)$ mit 1 identisch sind. Es folgt hieraus: Die Koeffizienten $a_{0 v}\left(k^{2}\right)$ und $a_{3 v}\left(k^{2}\right)$ in den Ansätzen (1) und (2) verschwinden mit $k^{2}$, haben also als ganze Funktionen höchstens $v^{\text {ten }}$ Grades von $k^{2}$ die Absolutglieder 0. Die Regeln (8) zeigen daraufhin weiter: Die Grade von $a_{0 v}\left(k^{2}\right)$ und $a_{2 v}\left(k^{2}\right)$ in $k^{2}$ sind höchstens $(\nu-1)$, so daß insbesondere $a_{01}\left(k^{2}\right)$ mit 0 identisch ist.

Auch die Funktionen $G_{1}(z, 0)$ und $G_{2}(z, 0)$ können allgemein angegeben werden, da bei verschwindendem Integralmodul die Funktionen $\operatorname{sn} w$ und en $w$ nach I, 472 in die trigonometrischen Funktionen $\sin w$ und $\cos w$ übergehen und also unsere Multiplikationsformeln einfach auf die Elementarformeln zurückkommen, in denen $\sin w$ und $\cos w$ nach Potenzen von $\sin w$ entwickelt sind. Wir haben also bei ungeradem $n$ die Darstellungen: 
(9)

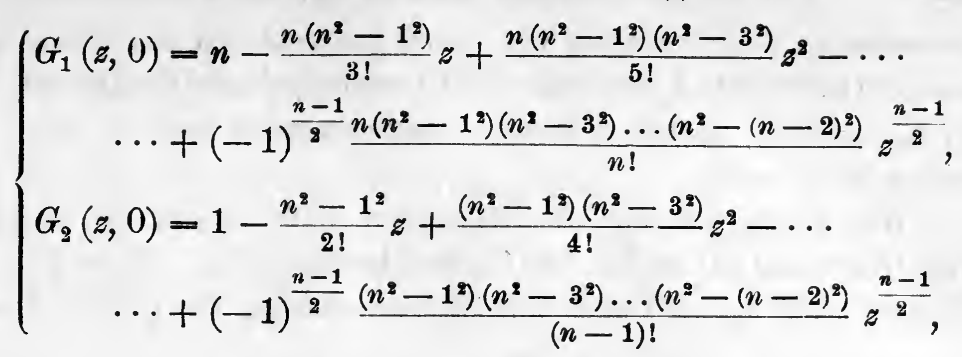

während bei geradem $n$ sich die Gleichungen anschließen:

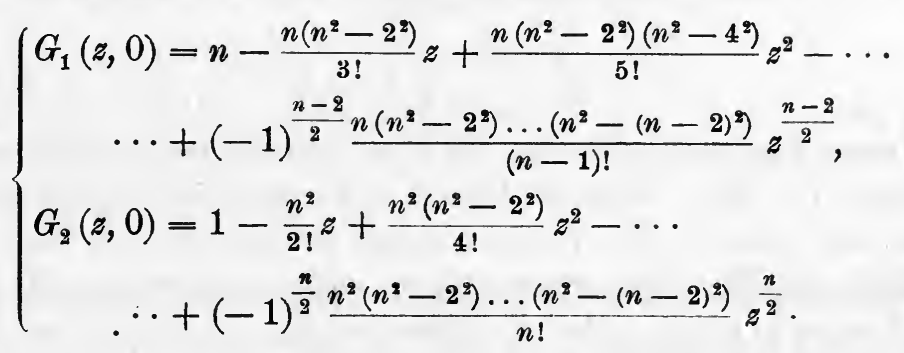

Die hier bei den Potenzen $z, z^{2}, z^{3}$ auftretenden Koeffizienten sind die $A b$ solutglieder der rationalen ganzen Ausdrücke der $a_{\mu 1}\left(k^{2}\right), a_{\mu 2}\left(k^{2}\right), a_{\mu 3}\left(k^{2}\right), \ldots$ in $k^{2}$ für $\mu=1$ bzw. 2 ; diese Absolutglieder verschwinden bei ungeradem $n$ von $a_{\mu, \frac{n+1}{2}}$ an, bei geradem $n$ von $a_{1, \frac{n}{2}}$ und $a_{2, \frac{n+2}{2}}$ an.

Was die übrigen Koeffizienten in den Ausdrücken der $a_{\mu \nu}$ als Funktionen von $k^{2}$ angeht, so folgt aus der ersten Gleichung (8), daß sowohl bei $a_{0 v}$ als bei $a_{1 v}$ die Koeffizienten, die gleich weit vom Anfang und Ende des Ausdrucks abstehen, einander gleich sind. In dem für uns wichtigsten Falle der $a_{0 v}$ haben wir somit den Ansatz:

$$
a_{0 v}=\alpha_{1} k^{2}+\alpha_{2} k^{4}+\cdots+\alpha_{2} k^{2 v-4}+\alpha_{1} k^{2 v-2}
$$

mit ganzen Zahlen $\alpha$, wofür wir unter Zusammenziehung entsprechender Glieder auch schreiben können:

$$
k^{-v} \cdot a_{0 v}=\alpha_{1}\left(k^{v-2}+\frac{1}{k^{\nu-2}}\right)+\alpha_{2}\left(k^{v-4}+\frac{1}{k^{\nu-4}}\right)+\cdots
$$

Führt man die Größe:

$$
x=k+\frac{1}{k}
$$

ein, so kann man die in (12) auftretenden Klammerausdrücke in folgender Art durch die Potenzen von $x$ darstellen:

(14) $k^{2}+\frac{1}{k^{2}}=x^{2}-2, k^{3}+\frac{1}{k^{3}}=x^{3}-3 x, k^{4}+\frac{1}{k^{4}}=x^{4}-4 x^{2}+2, \ldots$

Die rechte Seite von (12) wird dann eine rationale ganze Funktion:

$$
k^{-v} \cdot a_{0 v}=\alpha_{1}^{\prime} x^{v-2}+\alpha_{2}^{\prime} x^{v-4}+\alpha_{3}^{\prime} x^{v-6}+\cdots
$$


höchstens $(\nu-2)^{\text {ten }}$ Grades von $\varkappa$ mit ganzzahligen Koeffizienten $\alpha^{\prime}$. Ist aber in (12) rechts $\alpha_{i}$ der erste nicht verschwindende Koeffizient, während $\alpha_{1}, \alpha_{2}, \ldots, \alpha_{i-1}$ gleich 0 sind, so verschwinden auch $\alpha_{1}^{\prime}, \alpha_{2}^{\prime}, \ldots, \alpha_{i-1}^{\prime}$, und es ist $\alpha_{i}^{\prime}=\alpha_{i}$.

Wir gehen nun auf die Relationen (3)ff. zurück und tragen in die erste Gleichung (3) rechts für $G_{0}$ den Ansatz (1) ein. Es ergibt sich mit Rücksicht auf $a_{01}=0$ nach kurzer Umrechnung für $G_{1}$ der Ausdruck:

$$
\begin{aligned}
& G_{1}\left(z, k^{2}\right)=n+(-1)^{\frac{n-1}{2}}\left(k^{-\frac{n^{2}-5}{2}} a_{0, \frac{n^{2}-3}{2}} z+k^{-\frac{n^{2}-9}{2}} a_{0, \frac{n^{2}-5}{2} z^{2}}+\cdots\right. \\
& \left.\cdots+k^{\frac{n^{2}-13}{2}} a_{03} z^{\frac{n^{2}-7}{2}}+k^{\frac{n^{2}-9}{2}} a_{02} z^{\frac{n^{2}-5}{2}}+(k z)^{\frac{n^{2}-1}{2}}\right) \text {. }
\end{aligned}
$$

Dieser Ausdruck geht aber für $k^{2}=0$ in die rechte Seite der ersten Gleichung (9) über. Hieraus können wir einen Schluß ziehen auf die Gestalt des ersten in (11) rechts wirklich auftretenden Gliedes. Um ein abschließendes Ergebnis aussprechen zu können, setzen wir entsprechend dem Ansatze (1) den ersten Koeffizienten $a_{00}=1$ und gehen übrigens sogleich auf (15) zurück: Im Falle eines ungeraden $n$ haben wir für die höchsten Koeffizienten von $G_{0}\left(z, k^{2}\right)$ die Darstellungen:

$$
\left\{\begin{array}{l}
7^{-\frac{n^{2}-1}{2}} a_{0, \frac{n^{2}-1}{2}}=(-1)^{\frac{n-1}{2}} n \\
k^{-\frac{n^{2}-3}{2}} a_{0, \frac{n^{2}-3}{2}}=-(-1)^{\frac{n-1}{2} \frac{n\left(n^{2}-1^{2}\right)}{3 !} x} \\
k^{-\frac{n^{2}-5}{2}} a_{0, \frac{n^{2}-5}{2}}=+(-1)^{\frac{n-1}{2}} \frac{n\left(n^{2}-1^{2}\right)\left(n^{2}-3^{2}\right)}{5 !} x^{2}+a_{n^{2}-5}, 1 \\
k^{-\frac{n^{2}-7}{2}} a_{0, \frac{n^{2}-7}{2}}=-(-1)^{\frac{n-1}{2}} \frac{n\left(n^{2}-1^{2}\right)\left(n^{2}-3^{2}\right)\left(n^{2}-5^{2}\right)}{7 !} x^{3}+\frac{\alpha^{2}-7}{2}, 1
\end{array},\right.
$$

während sich für die Anfangskoeffizienten aus (15) ergibt:

$$
\begin{cases}a_{00}=1, \quad a_{01}=0, & k^{-2} a_{02}=\alpha_{2,1}, \\ k^{-3} a_{03}=\alpha_{31} x, & k^{-4} a_{04}=\alpha_{41} x^{2}+\alpha_{42}, \ldots,\end{cases}
$$

wo die $\alpha$ durchweg ganze Zahlen sind.

Im Falle eines geraden $n$ könnte man im Anschlub an die erste Gleichung (6) die Entwicklung der Koeffizienten $a_{0 v}$ ebensoweit fördern wie soeben bei ungeradem $n$. Doch wollen wir hier nur erst die erste Gleichung (4) anwenden, die, wie man leicht feststellt, Beziehungen zwischen je zwei vom Anfang und Ende des Ausdrucks (2) für $G_{0}$ gleich weit abstehenden Koeffizienten $a$ ergibt. Wir gewinnen den Satz: Im Falle eines geraden $n$ gelten für die Koeffizienten von $G_{0}\left(z, k^{2}\right)$ die Darstellungen: 
(18)

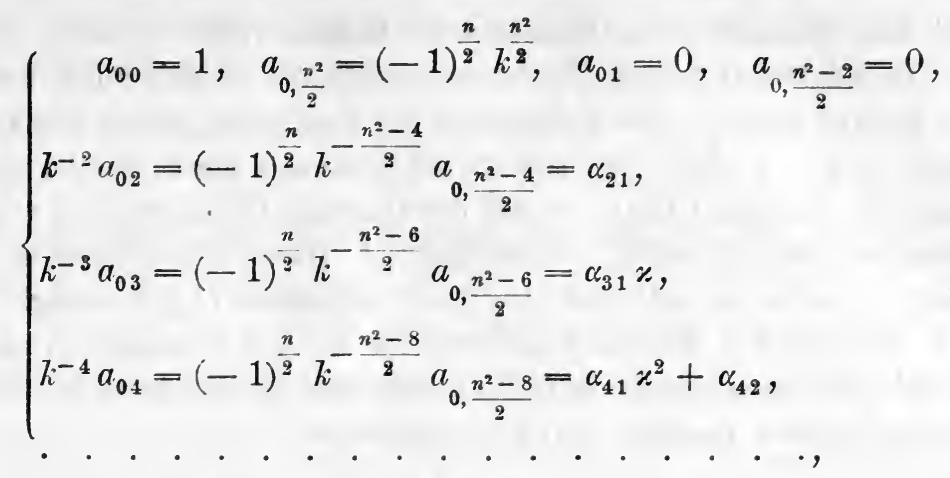

wo die $\alpha$ wieder durchueg ganze Zahlen sind. Für die Bestimmung dieser ganzen Zahlen kann die S. 196 erwähnte von Jacobi aufgefundene Differentialgleichung benutzt werden.

\section{\$ 6. Differentialgleichungen zur Berechnung der Funktionen $G(z)$.}

Aus (1) S. 172 folgert man mit Hilfe der Legendreschen Relation, daB die Funktion $\sigma_{1}(u)$ folgender Gleichung bei Vermehrung von $u$ um Periodenmultipla genügt:

$$
\sigma_{1}\left(u+m_{1} \omega_{1}+m_{2} \omega_{2}\right)=(-1)^{m_{1} m_{2}+m_{1}} e^{\left(m_{1} \eta_{1}+m_{2} \eta_{2}\right)\left(u+\frac{m_{1} \omega_{1}+m_{2} \omega_{2}}{2}\right)} \sigma_{1}(u) .
$$

Hieraus geht hervor, daB der Quotient:

$$
\frac{\sigma_{1}(n u)}{\left(\sigma_{1}(u)\right)^{n \cdot n}}
$$

die Perioden $\omega_{1}, \omega_{2}$ hat. Der Nenner dieses Quotienten hat im Periodenparallelogramm einen einzigen Nullpunkt der Ordnung $n^{2}$ an der Stelle $\frac{\omega_{1}}{2}$. Der Zähler hat $n^{2}$ Nullpunkte erster Ordnung im Periodenparallelogramm an den Stellen:

$$
u=\frac{\omega_{1}}{2 n}+\frac{\alpha \omega_{1}+\beta \omega_{2}}{n}=\frac{(2 \alpha+1) \omega_{1}+2 \beta \omega_{2}}{2 n}, \alpha, \beta=0,1, \ldots,(n-1),
$$

von denen im Falle eines ungeraden $n$ eine, im Falle eines geraden $\boldsymbol{n}$ keine mit dem Nullpunkte des Nenners zusammenfällt. Der Quotient (1) hat demnach die Wertigkeit $\left(n^{2}-1\right)$ bzw. $n^{2}$ und ist übrigens als gerade Funktion von $u$ rational in $\wp(u)$ darstellbar.

Auf Grund der vierten Gleichung (1) in I, 401 schreiben wir die Funktion (1) in der Weierstraßschen Funktion $\mathrm{Al}(w)$ so:

$$
\frac{\sigma_{1}(n u)}{\left(\sigma_{1}(u)\right)^{n \cdot n}}=\frac{\operatorname{Al}(n w)}{(\operatorname{Al}(w))^{n \cdot n}}
$$

als solche werden wir sie dann statt in $\wp(u)$ rational in $z=(\operatorname{sn} w)^{2}$ darstellen. Nun fällt der Pol der Ordnung $\left(n^{2}-1\right)$ bzw. $n^{2}$ unseres Quotienten mit dem Pole zweiter Ordnung von $z$ zusammen, so daB der Quo- 
tient eine rationale ganze Funktion des Grades $\frac{1}{2}\left(n^{2}-1\right)$ bzw. $\frac{1}{2} n^{2}$ von $z$ ist. Die Zählernullpunkte (2) führen uns genau zu den Polen der Funktion sn $(n \omega)$ zurück. Die Nullpunkte der fraglichen ganzen Funktion des Grades $\frac{1}{2}\left(n^{2}-1\right)$ bzw. $\frac{1}{2} n^{2}$ von $z$ sind demnach genau wieder die Nullpunkte der Funktion $G_{0}(z)$, so daB der Quotient (3), abgesehen von einem konstanten Faktor, mit $G_{0}(z)$ identisch ist. Dieser Faktor aber ist einfach gleich 1 , da für $w=0$ und also $z=0$ einerseits $G_{0}(z)$ zufolge (1) und (2) S. 200 gleich 1 wird und andererseits $\mathrm{Al}(0)=1$ zufolge (7) in I, 403 zutrifft: Die ganze Funktion $G_{0}^{(n)}(z)$ läßt sich als'Funktion von $w$ in der Weierstraßschen Funktion $\mathrm{Al}(w)$ so darstellen:

$$
G_{0}^{(n)}(z)=\frac{\operatorname{Al}(n w)}{(\mathrm{Al}(w))^{n \cdot n}} .
$$

Entsprechende Darstellungen der Funktionen $G_{1}^{(n)}(z), G_{2}^{(n)}(z), G_{3}^{(n)}(z)$ in allen vier Funktionen $\mathrm{Al}(w)$ wird man mit Benutzung der eben aufgestellten Gleichung (4) aus (1) und (3) S. 197 auf Grund von (2) in I, 401 leicht ableiten.

Wie aus der Gleichung (3) S. 184, der unsere jetzige Gleichung (4) offenbar genau entspricht, in § 2, S. $190 \mathrm{ff}$., eine partielle Differentialgleichung für $\psi^{(n)}$ abgeleitet wurde, so können wir aus (4) eine ebensolche Gleichung für die fortan kurz durch $G$ zu bezeichnende Funktion $G_{0}^{(n)}\left(z, k^{2}\right)$ ableiten. Der Differentialgleichung (5) in I, 470 von $\mathrm{Al}$ als Funktion von $w$ und $k^{2}$ kann man die Gestalt:

$$
\begin{gathered}
\frac{\partial^{2} \log \mathrm{Al}(w)}{\partial w^{2}}+\left(\frac{\partial \log \mathrm{Al}(w)}{\partial w}\right)^{2}+2 k^{2} w \frac{\partial \log \mathrm{Al}(w)}{\partial w} \\
+4 k^{2}\left(1-k^{2}\right) \frac{\partial \log \mathrm{Al}(w)}{\partial\left(k^{2}\right)}+k^{2} w^{2}=0
\end{gathered}
$$

geben. Man setze $n w$ an Stelle von $w$, multipliziere mit $n^{2}$ und ziehe vom Ergebnis die mit $n^{4}$ multiplizierte Gleichung (5) ab. Die entstehende Gleichung nimmt bei Einführung von $G$ auf Grund von (4) und bei Benutzung der dritten Gleichung (6) in I, 402 die Gestalt an:

$$
\begin{gathered}
\frac{\partial^{2} \log G}{\partial w^{2}}+\left(\frac{\partial \log G}{\partial w}\right)^{2}+2 n^{2} \frac{\partial \log G}{\partial w}\left(\frac{\partial \log \mathrm{Al}(w)}{\partial w}+k^{2} w\right) \\
+4 n^{2} k^{2}\left(1-k^{2}\right) \frac{\partial \log G}{\partial\left(k^{2}\right)}+n^{2}\left(n^{2}-1\right) k^{2} z=0 .
\end{gathered}
$$

Hierfür kann man nach Zusatz des Faktors $G$ auch schreiben:

$$
\begin{gathered}
\frac{\partial^{2} G}{\partial w^{2}}+2 n^{2} \frac{\partial G}{\partial w}\left(\frac{\partial \log \mathrm{Al}(w)}{\partial w}+k^{2} w\right) \\
+4 n^{2} k^{2}\left(1-k^{2}\right) \frac{\partial G}{\partial\left(k^{2}\right)}+n^{2}\left(n^{2}-1\right) k^{2} z G=0 .
\end{gathered}
$$

Führt man an Stelle von $w$ und $k^{2}$ als neue unabhängige Variable $z$ und $k^{2}$ ein, so ist zu beachten, $\mathrm{daB} w$ nur in $z$ enthalten ist, während $k^{2}$ 
nicht nur als zweite unabhängige Variable auftritt, sondern auBerdem noch in $z$ als zweites Argument vorkommt. Wir haben also einerseits:

$$
\frac{\partial G}{\partial w}=\frac{\partial G}{\partial z} \frac{\partial z}{\partial w}, \quad \frac{\partial^{2} G}{\partial w^{2}}=\frac{\partial^{2} G}{\partial z^{2}}\left(\frac{\partial z}{\partial w}\right)^{2}+\frac{\partial G}{\partial z} \frac{\partial^{*} z}{\partial w^{2}},
$$

während andererseits die in (6) gemeinte partielle Ableitung von $G$ nach $k^{2}$ durch:

$$
\frac{\partial G}{\partial z} \frac{\partial z}{\partial\left(k^{2}\right)}+\frac{\partial G}{\partial\left(k^{2}\right)}
$$

zu ersetzen ist. Die Gleichung (6) nimmt so die Gestalt an:

$$
\text { (7) } \begin{aligned}
\frac{\partial^{2} G}{\partial z^{2}}\left(\frac{\partial z}{\partial w}\right)^{2} & +\frac{\partial G}{\partial z}\left(\frac{\partial^{2} z}{\partial w^{2}}+2 n^{2}\left(\frac{\partial \log \mathrm{Al}(w)}{\partial w}+k^{2} w\right) \frac{\partial z}{\partial w}+4 n^{2} k^{2}\left(1-k^{2}\right) \frac{\partial z}{\partial\left(k^{2}\right)}\right) \\
& +4 n^{2} k^{2}\left(1-k^{2}\right) \frac{\partial G}{\partial\left(k^{2}\right)}+n^{2}\left(n^{2}-1\right) k^{2} z G=0 .
\end{aligned}
$$

Die mit den Ableitungen von $G$ nach $z$ multiplizierten Faktoren sind nun noch auf $z$ und $k^{2}$ umzurechnen. Zunächst leitet man aus (1) und (2) in I, 396 leicht die Gleichungen ab:

$$
\left\{\begin{array}{l}
\left(\frac{\partial z}{\partial w}\right)^{2}=4 z(1-z)\left(1-k^{2} z\right) \\
\frac{\partial^{2} z}{\partial w^{2}}=2\left(1-2\left(1+k^{2}\right) z+3 k^{2} z^{2}\right)
\end{array}\right.
$$

Die zweite Ableitung von $z$ nach $w$ können wir auch so entwickeln:

$$
\frac{\partial^{2} z}{\partial w^{2}}=\frac{\partial^{2}\left(\operatorname{sn} w^{2}\right)}{\partial w^{2}}=2 \operatorname{sn} w \frac{\partial^{2} \operatorname{sn} w}{\partial w^{2}}+\frac{1}{2(\operatorname{sn} w)^{2}}\left(\frac{\partial(\operatorname{sn} w)^{2}}{\partial w}\right)^{2}
$$

und folgern mit Rücksicht von (8) die sogleich zu benutzende Gleichung:

$$
\operatorname{sn} w \frac{\partial^{2} \operatorname{sn} w}{\partial w^{2}}=-\left(1+k^{2}\right) z+2 k^{2} z^{2} \text {. }
$$

Der Rest der Zwischenrechnung ist etwas umständlicher. Subtrahiert man die durch $\mathrm{Al}(w)$ und $\mathrm{Al}_{1}(w)$ geteilten beiden ersten Differentialgleichungen (5) in I, 470 voneinander, so ergibt sich bei Einführung der Logarithmen der Funktionen $\mathrm{Al}$ :

$$
\begin{gathered}
\frac{\partial^{2} \log \mathrm{Al}_{1}(w)}{\partial w^{2}}-\frac{\partial^{2} \log \mathrm{Al}(w)}{\partial w^{2}}+\left(\frac{\partial \log \mathrm{Al}_{1}(w)}{\partial w}\right)^{2}-\left(\frac{\partial \log \mathrm{Al}(w)}{\partial w}\right)^{2}+2 k^{2} w\left(\frac{\partial \log \mathrm{Al}_{1}(w)}{\partial w}\right. \\
\left.-\frac{\partial \log \mathrm{Al}(w)}{\partial w}\right)+4 k^{2}\left(1-k^{2}\right)\left(\frac{\partial \log \mathrm{Al}_{1}(w)}{\partial\left(k^{2}\right)}-\frac{\partial \log \mathrm{Al}(w)}{\partial\left(k^{2}\right)}\right)+1-k^{2}=0
\end{gathered}
$$

Setzt man für $\mathrm{Al}_{1}(w)$ das dieser Funktion gleiche Produkt sn $w \cdot \operatorname{Al}(w)$, so folgt nach Multiplikation der entstehenden Gleichung mit $2 n^{2}(\operatorname{sn} w)^{2}$ $=2 n^{2} z$

$$
\begin{gathered}
2 n^{2} \operatorname{sn} w \frac{\partial^{2} \operatorname{sn} w}{\partial w^{2}}+2 n^{2}\left(\frac{\partial \log \mathrm{Al}(w)}{\partial w}+k^{2} w\right) \frac{\partial z}{\partial w}+4 n^{2} k^{2}\left(1-k^{2}\right) \frac{\partial z}{\partial\left(k^{2}\right)} \\
+2 n^{2}\left(1-k^{2}\right) z=0 .
\end{gathered}
$$


Mit Benutzung von (9) finden wir somit:

$$
2 n^{2}\left(\frac{\partial \log \mathrm{Al}(w)}{\partial w}+k^{2} w\right) \frac{\partial z}{\partial w}+4 n^{2} k^{2}\left(1-k^{2}\right) \frac{\partial z}{\partial\left(k^{2}\right)}=4 n^{2} k^{2} z(1-z) .
$$

Auf Grund dieser Gleichung sowie der Gleichungen (8) und (9) lassen sich die Koeffizienten der Gleichung (7) durchweg auf $z$ und $k^{2}$ umrechnen: Die Funktion $G=G_{0}^{(n)}\left(z, k^{2}\right)$ befriedigt die partielle Differentialgleichung zweiter Ordnung:

$$
4 z(1-z)\left(1-k^{2} z\right) \frac{\partial^{2} G}{\partial z^{2}}
$$

$$
\begin{gathered}
+2\left(1+2\left(-1+\left(n^{2}-1\right) k^{2}\right) z-\left(2 n^{2}-3\right) k^{2} z^{2}\right) \frac{\partial G}{\partial z}+4 n^{2} k^{2}\left(1-k^{2}\right) \frac{\partial G}{\partial\left(k^{2}\right)} \\
+n^{2}\left(n^{2}-1\right) k^{2} z G=0 .
\end{gathered}
$$

In diese Gleichung tragen wir den Ansatz:

$$
G=a_{0}+a_{1} z+a_{2} z^{2}+\cdots
$$

ein, ordnen nach Potenzen von $z$ und setzen den Koeffizienten der einzelnen Potenz $z^{v}$ gleich 0 . Es ergibt sich:

$$
\begin{gathered}
(2 v+1)(2 \nu+2) a_{v+1}+4 n^{2} k^{2}\left(1-k^{2}\right) \frac{d a_{v}}{d\left(k^{2}\right)}+4 v\left(\left(n^{2}-v\right) k^{2}-v\right) a_{v} \\
+\left(n^{2}-2 v+1\right)\left(n^{2}-2 v+2\right) k^{2} a_{v-1}=0 .
\end{gathered}
$$

Da die Produkte $k^{-v} \cdot a_{v}$ rationale ganze Funktionen der in (13) S. 203 eingeführten Größe $x$ sind, so ist es zweckmäßig, an Stelle der $a_{v}$ diese Produkte zu berechnen und an Stelle von $k^{2}$ die Größe $x$ als unabhängige Variable einzuführen. Wir gelangen so zu dem abschließenden Ergebnis, das die von J a co bi entdeckte Methode der Berechnung von $G_{0}^{(n)}$ begründet: Je drei aufeinander folgende Produkte $k^{-v-1} \cdot a_{v+1}, k^{-v} a_{v}, k^{-v+1} a_{v-1}$ sind durch die folgende Relation aneinander gebunden:

$$
(2 \nu+1)(2 \nu+2)\left(k^{-v-1} \cdot a_{v+1}\right)+2 n^{2}\left(4-x^{2}\right) \frac{d\left(k^{-v} a_{\nu}\right)}{d x}
$$

$+2 v\left(n^{2}-2 v\right) x \cdot\left(k^{-v} \cdot a_{v}\right)+\left(n^{2}-2 v+1\right)\left(n^{2}-2 v+2\right)\left(k^{-v+1} \cdot a_{v-1}\right)=0$.

Mittelst dieser Gleichung kann man die Ergebnisse des vorigen Paragraphen bestätigen und wesentlich ergänzen. Da aus (16) und (17) S. 204 in jedem Falle $n$ sowohl die beiden niedrigsten als auch die beiden höchsten Koeffizienten bekannt sind, so ist die Berechnung der übrigen auf rekurrentem Wege sowohl in aufsteigender als in absteigender Linie möglich. So findet man bei $n=4$ unter zweckmäBiger Zusammenfassung der Glieder:

$$
\begin{gathered}
G_{0}^{(4)}\left(z, k^{2}\right)=\left(1+(k z)^{8}\right)-20\left((k z)^{2}+(k z)^{6}\right) \\
+32 \varkappa\left((k z)^{3}+(k z)^{5}\right)-2\left(8 x^{2}+13\right)(k z)^{4} .
\end{gathered}
$$


Für $n=5$ reiht sich hieran:

(13)

$$
\begin{aligned}
& G_{0}^{(5)}\left(z, k^{2}\right)=5-20 x(k z)+2\left(8 x^{2}+31\right)(k z)^{2}-80 x(k z)^{3}-105(k z)^{4} \\
& +360 x(k z)^{5}-60\left(4 x^{2}+5\right)(k z)^{6}+16\left(4 x^{3}+23 x\right)(k z)^{7} \\
& -5\left(32 x^{2}+25\right)(k z)^{8}+140 x(k z)^{9}-50(k z)^{10}+(k z)^{12}
\end{aligned}
$$

Endlich ergibt sich für $n=6$ wieder unter Zusammenfassung je zweier Glieder:

$$
\begin{aligned}
& G_{0}^{(6)}\left(z, k^{2}\right)=\left(1-(k z)^{18}\right)-105\left((k z)^{2}-(k z)^{16}\right)+448 x\left((k z)^{3}-(k z)^{15}\right) \\
& -12\left(72 x^{2}+37\right)\left((k z)^{4}-(k z)^{14}\right)+768\left(x^{3}+3 x\right)\left((k z)^{5}-(k z)^{13}\right) \\
& -4\left(64 x^{4}+840 x^{2}+621\right)\left((k z)^{6}-(k z)^{12}\right) \\
& +192\left(8 x^{3}+33 x\right)\left((k z)^{7}-(k z)^{11}\right)-126\left(32 x^{2}+15\right)\left((k z)^{8}-(k z)^{10}\right) .
\end{aligned}
$$

Das Glied mit $z^{9}$ fällt aus. Durch die erste Relation (4) S. 201 zeigt man allgemein, daB der Koeffizient $a_{0, \frac{n^{2}}{4}}$ stets verschwindet, falls $n$ das Doppelte einer ungeraden Zahl ist.

\section{Drittes Kapitel.}

\section{Die Divisionssätze der elliptischen Funktionen.}

Die Divisionssätze der elliptischen Funktionen sind von Abel entdeckt und zum ersten Male in den „Recherches sur les fonctions elliptiques “ $^{1}$ ) Art. 14 ff. dargestellt. Die Erkenntnis Abels, daß die allgemeinen Teilungsgleichungen durch Wurzelziehungen lösbar sind, dürfte für seine allgemeinen Untersuchungen über algebraisch lösbare Gleichungen von wesentlichem Einfluß gewesen sein. Bei dem Interesse, das die Teilungsgleichungen als Beispiele für die Galoissche Gleichungstheorie darbieten, kommen die Divisionssätze ausführlicher in denjenigen Darstellungen zur Geltung, bei denen algebraische Fragen im Vordergrunde stehen. So widmet C. Jordan in seinem bekannten Werke ${ }^{2}$ ) ein besonderes Kapitel den Gruppen der Teilungsgleichungen, und zwar sowohl der hier zunächst zu behandelnden "allgemeinen" Teilungsgleichungen, wie der im nächsten Kapitel zur Sprache kommenden „speziellen“ Teilungsgleichungen. Besonders ausführlich behandelt $\mathrm{H}$. Weber die algebraische Seite der Divisionssätze in seinem Buche „Elliptische Funktionen und algebraische Zahlen“.3) Über die wirkliche Durchfïhrung des Lösungsprozesses der allgemeinen Teilungsgleichung vgl. man L. Kiepert, „Auflösung der Transformationsgleichung und Division der elliptischen Funktionen“. ${ }^{4}$ )

1) Journ. f. Math. Bd. 2 (1828).

2) "Traité des substitutions et des équations algébriques" (Paris, 1870).

3) Braunschweig, 1891. Eine zweite Auflage ist 1908 als dritter Band des S. 1 genannten "Lehrbuches des Algebra“ erschienen.

4) Journ. f. Math., Bd. 76 (1873), S. 34. 


\section{§ 1. Die allgemeine Teilungsgleichung der $\wp$-Funktion.}

Während das Ziel der Multiplikation bei den Funktionen $\wp(u)$ und $\wp^{\prime}(u)$ die Berechnung von $\wp(n u)$ und $\wp^{\prime}(n u)$ aus $\wp(u)$ und $\wp^{\prime}(u)$ ist, unter $n$ irgendeine positive ganze Zahl verstanden, ist das Problem der Division, bei gegebenen und durch die bekannte Relation verknüptten Funktionswerten $\wp(u), \wp^{\prime}(u)$ die zugehörigen Werte $\wp\left(\frac{u}{n}\right), \wp^{\prime}\left(\frac{u}{n}\right) z u$ berechnen. Die ganze Zahl $n$ heißt der "Grad" der Division oder Teilung. Wird in den Gleichungen (2) S. 184 an Stelle von $u$ der Wert $\frac{u}{n}$ gesetzt, so folgt:

$$
R\left(\wp\left(\frac{u}{n}\right)\right)-\wp(u)=0, \quad \wp^{\prime}\left(\frac{u}{n}\right)=\frac{n \wp^{\prime}(u)}{R^{\prime}\left(\wp\left(\frac{u}{n}\right)\right)} .
$$

Die erste dieser Gleichungen läBt sich auf Grund von (4) S. 184 nach Multiplikation mit dem Nenner $\left(\psi^{(n)}\right)^{2}$ der Funktion $R$ in die Gestalt setzen:

$$
\wp\left(\frac{u}{n}\right)\left(\psi^{(n)}\left(\frac{u}{n}\right)\right)^{2}-\psi^{(n-1)}\left(\frac{u}{n}\right) \psi^{(n+1)}\left(\frac{u}{n}\right)-\wp(u)\left(\psi^{(n)}\left(\frac{u}{n}\right)\right)^{2}=0 .
$$

Die Darstellung der Funktionen $\psi^{(n)}(u)$ in $\wp(u), \wp^{\prime}(u)$ geht aus (14) S. 188 und (20) ff. S. 189 hervor. Man hat bei ungeradem $n$ :

$$
\psi^{(n)}(u)=n \wp(u)^{\frac{1}{2}\left(n^{2}-1\right)}+\beta_{1}\left(\frac{1}{4} g_{2}\right) \wp(u)^{\frac{1}{2}\left(n^{2}-5\right)}+\beta_{2} g_{3} \wp(u)^{\frac{1}{2}\left(n^{2}-7\right)}+\cdots
$$

woran sich für ein gerades $n$ die Gleichung schließt:

$$
\begin{aligned}
\psi^{(n)}(u)=-\wp^{\prime}(u)\left(\frac{1}{2} n \wp(u)^{\frac{1}{2}\left(n^{2}-4\right)}+\gamma_{1}\left(\frac{1}{4} g_{2}\right) \wp(u)^{\frac{1}{2}\left(n^{2}-8\right)}\right. \\
\left.+\gamma_{2} g_{3} \wp(u)^{\frac{1}{2}\left(n^{2}-10\right)}+\cdots\right)
\end{aligned}
$$

Die $\beta$ und $\gamma$ erweisen sich zunächst als rationale Zahlen; sie sind aber sogar ganze Zahlen, wie auf Grund der Rekursionsformeln (8) S. 185 aus den auf die niedersten $n$ bezogenen Gleichungen (6) S. 185 hervorgeht. Benutzt man diese Ausdrücke der $\psi^{(n)}$ zur weiteren Entwicklung der Gleichung (2), so ergibt die Zwischenrechnủng, daß der Koeffizient der höchsten Potenz der unbekannten Funktion $\wp\left(\frac{u}{n}\right)$ sowohl bei geradem als bei ungeradem $n$ in einfachster Weise gleich 1 wird. Die Koeffizienten der Gleichung sind rationale ganze, ganzzahlige Funktionen von $\frac{1}{4} g_{2}, g_{3}, \wp(u)$, und sie sind speziell in $\wp(u)$ linear; die Dimension der Gleichung in $u, \omega_{1}, \omega_{2}$ ist $-2 n^{2}$. Indem wir die Funktion $\wp\left(\frac{u}{n}\right)$ als „Unbekannte" der Gleichung 'durch $Z$ bezeichnen, können wir die Gleichung (2) in die entwickelte Gestalt kleiden: 
Ansatz der allgemeinen Teilungsgleichung und des Teilungsproblems 211

$$
\begin{aligned}
& Z^{n^{2}}-n^{2} \wp(u) Z^{n^{2}-1}+a_{1}\left(\frac{1}{4} g_{2}\right) Z^{n^{2}-2}+\left(a_{2} g_{3}+a_{2}^{\prime}\left(\frac{1}{4} g_{2}\right) \wp(u)\right) Z^{n^{2}-3} \\
& +\left(a_{3}\left(\frac{1}{4} g_{2}\right)^{2}+a_{3}^{\prime} g_{3} \wp(u)\right) Z^{n^{2}-4}+\cdots=0,
\end{aligned}
$$

wo die $a_{1}, a_{2}, a_{2}^{\prime}, a_{3}, \ldots$ ganze Zahlen sind.

Die Gleichung (3) soll die „allgemeine Teilungsgleichung ${ }^{1}$ ) der §-Funktion für den $n^{\text {ten }}$ Teilungsgrad" heißen. Das Teilungsproblem darf als erledigt gelten, wenn die Gleichung (3) nach $Z$ aufgelöst ist. Kennt man nämlich $\wp\left(\frac{u}{u}\right)$, so ist $\wp^{\prime}\left(\frac{u}{n}\right)$ auf Grund der zweiten Gleichung (1) rational berechenbar.

Die Funktionen $\wp\left(\frac{u}{n}\right), \wp^{\prime}\left(\frac{u}{n}\right)$ haben die Perioden $n \omega_{1}, n \omega_{2}$. Der einzelne Punkt des zugehörigen Periodenparallologramms der Ecken $0, n \omega_{2}$, $n \omega_{1}+n \omega_{2}, n \omega_{1}$ ist durch das hier auftretende Wertepaar $\wp\left(\frac{u}{n}\right), \wp^{\prime}\left(\frac{u}{n}\right)$ eindeutig angebbar. Das fragliche Parallelogramm läbt sich nun aus $n^{2}$ Parallelogrammen der ursprünglichen, auf $\omega_{1}, \omega_{2}$ bezogenen Teilung aufbauen. Die Angabe eines Paares zusammengehöriger Werte $\wp(u), \wp^{\prime}(u)$ legt aber in den $n^{2}$ kleinen Parallelogrammen ein System von $n^{2}$ homologen, bezüglich der Gruppe $\Gamma^{(u)}$ äquivalenten Punkten fest. Ist $u$ der im ersten Parallelogramm der Ecken 0, $\omega_{2}, \omega_{1}+\omega_{2}, \omega_{1}$ gelegene Punkt dieses Systems, so sind alle $n^{2}$ Punkte durch

$$
u+\lambda \omega_{1}+\mu \omega_{2},
$$$$
\lambda, \mu=0,1,2, \ldots, n-1
$$

gegeben, wo $\lambda, \mu$ alle $n^{2}$ Kombinationen der $n$ ganzen Zahlen $0,1,2, \ldots$, $n-1$ durchlaufen. Abkürzend verstehen wir unter $\wp_{\lambda \mu}(u), \wp_{\lambda \mu}^{\prime}(u)$ die beiden Funktionen:

$$
\wp_{\lambda_{\mu}}(u)=\wp\left(u+\frac{\lambda \omega_{1}+\mu \omega_{2}}{n}\right), \quad \wp_{\lambda_{\mu}}^{\prime}(u)=\wp^{\prime}\left(u+\frac{\lambda \omega_{1}+\mu \omega_{2}}{n}\right) .
$$

Unser Teilungsproblem läuft dann darauf hinaus, bei Festlegung eines Systems von $n^{2}$ Stellen (4) im großen Parallelogramm durch Angabe eines Paares zusammengehöriger Werte $\wp(u), \wp^{\prime}(u)$ die in jenen $n^{2}$ Stellen auftretenden Wertepaare $\wp_{\lambda \mu}\left(\frac{u}{n}\right), \wp_{\lambda \mu}^{\prime}\left(\frac{u}{n}\right) z u$ berechnen.

Im AnschluB hieran kann man das Teilungsproblem auch noch rein algebraisch aussprechen. Das einzelne Parallelogramm der ursprünglichen Teilung wird durch die Funktion $z=\wp(u)$ auf die in Bd. I mit $\mathrm{F}_{2}$ bezeichnete zweiblättrige Riemannsche Fläche des Gesehlechtes $p=1$ abgebildet, deren einzelner Punkt durch zwei zusammengehörige Werte $z$, $w=\sqrt{4 z^{3}-g_{2} z-g_{3}}$ angebbar ist. Entsprechend gibt die Abbildung des Parallellogramms der Perioden $n \omega_{1}, n \omega_{2}$ durch $z=\wp(u)$ eine Riemann-

1) Im Gegensatze zur „speziellen Teilungsgleichung ", die unten betrachtet

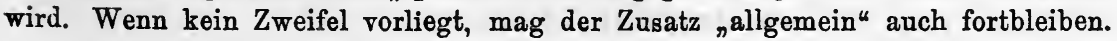


sche Fläche $\mathbf{F}_{2 n^{2}}$ des Geschlechtes $p=1$, die mit $2 n^{2}$ Blättern über der $z$-Ebene lagert. Ein Funktionssystem dieser $F_{2 n^{2}}$ haben wir dann in $Z=\wp\left(\frac{u}{n}\right), W=\sqrt{4 Z^{3}-g_{2} Z-g_{3}}$. Nun kann die $\mathbf{F}_{2 n^{2}}$ offenbar auch durch eine $n^{2}$-fache Überlagerung der $F_{2}$ gewonnen werden. Unser Problem läuft dann darauf hinaus, für $n^{2}$ in den Exemplaren der $\mathbf{F}_{2}$ homologe Stellen der $\mathbf{F}_{2 n^{2}}$, wie sie durch Angabe eines Wertsystems $z$, w festgelegt sind, die $n^{2}$ zugehörigen Wertsysteme $Z, W$ zu berechnen.

Indem wir die in der Einleitung entwickelte Galoissche Gleichungstheorie auf die Teilungsgleichung (3) anwenden, gewinnt die Untersuchung neben dem algebraisch-funktionentheoretischen Interesse noch einen wesentlichen arithmetischen Charakter, der durch den Bau der Koeffizienten der Gleichung (3) begründet ist. Als gegeben gilt mit der Gleichung (3) der weiterhin mit $\Omega$ zu bezeichnende Körper der rationalen Funktionen von $\wp(u), \wp^{\prime}(u)$, deren Koeffizienten rationale Funktionen von $g_{2}, g_{3}$ mit rationalen Zahlenkoeffizienten sind. Dabei liegt hier insofern noch eine Besonderheit vor, daß für uns nur solche Ausdrïcke des Körpers $\Omega$ in Betracht kommen, die in $u, \omega_{1}, \omega_{2}$ homogen sind. Sehen wir die Perioden $\omega_{1}, \omega_{2}$ als veränderlich an, so bilden übrigens die von $u$ unabhängigen Größen von $\Omega$ ihrerseits auch noch einen Funktionenkörper, da sie rationale Funktionen der Modulformen $g_{2}\left(\omega_{1}, \omega_{2}\right), g_{3}\left(\omega_{1}, \omega_{2}\right)$ mit rationalen Zahlenkoeffizienten sind. Dieser Umstand wird für die Entwicklungen des nächsten Kapitels grundlegend.

Als erster Satz der Teilungstheorie ist zu nennen: Die allgemeine Teilungsgleichung (3) ist im Körper $\Re$ irreduzibel und bleibt auch dann noch irreduzibel, wenn man zu $\Re$ irgendwelche "von u unabhängige" Irrationalitäten adjungiert. Ist nämlich nach irgendwelchen Adjunktionen dieser Art $F\left(Z, \wp(u), \wp^{\prime}(u)\right)=0$ der irreduzible Bestandteil von (3), dem die erste Lösung $\wp\left(\frac{u}{n}\right)$ genügt, so besteht die Gleichung:

$$
F\left(\wp\left(\frac{u}{n}\right), \wp(u), \wp^{\prime}(u)\right)=0
$$

in $u$ identisch. Die Gleichung (6) bleibt also richtig, falls man $u$ um das Periodenmultiplum $\lambda \omega_{1}+\mu \omega_{2}$ zunehmen läßt. Hierbei gehen $\wp(u)$ und $\wp^{\prime}(u)$ in sich über. Die Gleichung $F\left(Z, \wp(u), \wp^{\prime}(u)\right)=0$ wird also durch alle $n^{2}$ Lösungen $Z=\wp_{\lambda \mu}\left(\frac{u}{n}\right)$ des Teilungsproblems befriedigt und ist demnach die Teilungsgleichung (3) selbst, die damit als irreduzibel erkannt wird.

$\mathrm{Zu}$ den Funktionen (5) gehören für die Kombination $\lambda=0, \mu=0$ auch die ursprünglichen $\wp(u), \wp^{\prime}(u)$. Setzt man in den übrigen $\left(n^{2}-1\right)$ Paaren (5) das erste Argument $u=0$, so gelangt man zu den abgekürzt durch $\wp_{\lambda \mu}, \wp_{\lambda \mu}^{\prime}$ zu bezeichnenden Ausdrücken: 


$$
\wp_{\lambda \mu}=\wp\left(\frac{\lambda \omega_{1}+\mu \omega_{2}}{n}\right), \quad \wp_{\lambda \mu}^{\prime}=\wp^{\prime}\left(\frac{\lambda \omega_{1}+\mu \omega_{2}}{n}\right),
$$

die homogene Funktionen der Dimension $(-2)$ bzw. ( -3$)$ der Perioden $\omega_{1}, \omega_{2}$ allein sind und als die zum $n^{\text {ten }}$ Teilungsgrade gehörenden ,Teil-

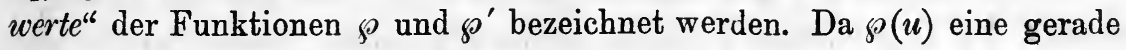
und $\wp^{\prime}(u)$ eine ungerade Funktion ist, so gilt:

$$
\wp_{n-\lambda, n-\mu}=\wp_{\lambda, \mu}, \quad \wp_{n-\lambda, n-\mu}^{\prime}=-\wp_{\lambda, \mu}^{\prime} .
$$

Man folgert hieraus leicht die Sätze: Bei ungeradem $n$ gibt es nur $\frac{1}{2}\left(n^{2}-1\right)$ verschiedene Teilwerte $\wp_{\lambda \mu}$, und die $\left(n^{2}-1\right)$ Teilwerte $\wp_{\lambda \mu}^{\prime}$ sind zu je zweien einander entgegengesetzt. Bei geradem $n$ hat man erstlich $\frac{1}{2}\left(n^{2}+2\right)$ Teilwerte $\wp_{\lambda \mu}$, unter denen sich die drei schon beim Grade 2 auftretenden Teilwerte:

$$
\wp_{\frac{n}{2}, 0}=\wp\left(\frac{\omega_{1}}{2}\right)=e_{1}, \wp_{0, \frac{n}{2}}=\wp\left(\frac{\omega_{2}}{2}\right)=e_{2}, \quad \wp_{\frac{n}{2}, \frac{n}{2}}=\wp_{(}\left(\frac{\omega_{1}+\omega_{2}}{2}\right)=e_{3}
$$

finden (vgl.(13) in I, 217); von den $\wp_{\lambda, \mu}^{\prime}$ verschwinden die den drei Teilwerten (9) entsprechenden identisch, während die $\left(n^{2}-4\right)$ übrigen zu je zweien entgegengesetzt sind.

Nach den Additionssätzen sind übrigens $\wp_{\lambda_{\mu}}, \wp_{\lambda_{\mu}}^{\prime}$ rational mit rationalen Zahlenkoeffizienten in $g_{2}, g_{3}, \wp_{\lambda, 0}, \wp_{0 \mu}, \wp_{\lambda 0}^{\prime}, \wp_{0 \mu}^{\prime}$ darstellbar. Ferner sind nach den Multiplikationssätzen $\wp_{\lambda_{0}}$ und $\wp_{0 \mu}$. wieder rational mit rationalen Zahlenkoeffizienten in $g_{2}, g_{3}$ und $\wp_{10}$ bzw. $\wp_{01}$ darstellbar, und für $\wp_{\lambda 0}^{\prime}, \wp_{0 \mu}^{\prime}$ hat man entsprechende Darstellungen unter Hinzunahme von $\wp_{10}^{\prime}$ bzw. $\wp_{01}^{\prime}$. Jedenfalls sind also die gesamten Teilwerte des $n^{\text {ten }}$ Teilungsgrades rational mit rationalen Zahlenkoeffizienten in $g_{2}, g_{3}, \wp_{10}, \wp_{01}$, $\wp_{10}^{\prime}, \wp_{01}^{\prime}$ darstellbar, wobei übrigens die beiden $\wp_{10}^{\prime}, \wp_{01}^{\prime}$ aus $g_{2}, g_{3}$ und $\wp_{10}$ bzw. $\wp_{01}$ mittels zweier Quadratwurzeln berechenbar sind.

Es besteht nun der wichtige Satz: Die Teilwerte des $n^{\text {ten }}$ Teilungsgrades gehören zu den natürlichen Irrationalitäten der allgemeinen Teilungsgleichung dieses Grades. Der zur Teilungsgleichung gehörende Galoissche Körper entsteht nämlich aus $\Omega$ durch Adjunktion aller $n^{2}$ Lösungen $\wp_{\lambda \mu}\left(\frac{u}{n}\right)$ der Teilungsgleichung. Diesem Galoisschen Körper gehören wegen der Gleichung:

$$
\wp_{\lambda \mu}^{\prime}\left(\frac{u}{n}\right)=\frac{n \wp^{\prime}(u)}{R^{\prime}\left(\wp_{\lambda \mu}\left(\frac{u}{n}\right)\right)}
$$

dann auch die $n^{2}$ Funktionen $\wp_{\lambda \mu}^{\prime}\left(\frac{u}{n}\right)$ an. Nun folgt aber aus dem Additionstheoreme (10) S. 160:

$\wp_{\lambda \mu}=\wp\left(\left(\frac{u}{n}+\frac{\lambda \omega_{1}+\mu \omega_{2}}{n}\right)-\frac{u}{n}\right)=\frac{1}{4}\left(\frac{\wp_{\lambda \mu}^{\prime}\left(\frac{u}{n}\right)+\wp^{\prime}\left(\frac{u}{n}\right)}{\wp_{\lambda \mu}\left(\frac{u}{n}\right)-\wp\left(\frac{u}{n}\right)}\right)^{2}-\wp_{\lambda \mu}\left(\frac{u}{n}\right)-\wp\left(\frac{u}{n}\right)$, 
woraus unmittelbar hervorgeht, daß $\wp_{\lambda \mu}$ dem fraglichen Galoisschen Körper angehört. In derselben Weise erkennt man auch die Zugehörigkeit der $\wp_{\lambda \mu}^{\prime}$ zu diesem Körper.

Wir wenden uns nun zunächst zu der Frage, wie sich der Lösungsproze $B$ der allgemeinen Teilungsgleichung gestaltet, wenn bereits alle natürlichen Irrationalitäten, die von $u$ unabhängig sind, also insbesondere die Teilwerte $\wp_{\lambda_{\mu}}, \wp_{\lambda_{\mu}}^{\prime}$ adjungiert sind. Den alsdann zugrunde liegenden Körper $\left(\Omega, \wp_{i \mu}, \wp_{\lambda_{\mu}}^{\prime}, \ldots\right)$ nennen wir kurz $\Omega^{\prime}$. Entsprechend der allgemeinen Theorie (S. 76 ff.) haben wir zur Beantwortung dieser Frage zunächst die „Monodromiegruppe“ der allgemeinen Teilungsgleichung aufzustellen.

\section{\$2. Die Monodromiegruppe der allgemeinen Teilungsgleichung.}

Nach S. 77 wird die Monodromiegruppe der Teilungsgleichung (3) S. 211 von allen Permutationen der $n^{2}$ Lösungen $Z=\wp_{\lambda \mu}\left(\frac{u}{n}\right)$ geliefert, die bei den Umläufen von $z$ auf der Riemannschen Fläche $F_{2}$ oder (was auf dasselbe hinauskommt) bei den Substitutionen:

$$
u^{\prime}=u+m_{1} \omega_{1}+m_{2} \omega_{2}
$$

der Gruppe $\Gamma^{(u)}$ auftreten. Bei der einzelnen Substitution (1) aber geht $\wp_{\lambda \mu}\left(\frac{u}{n}\right)$ einfach in $\wp_{\lambda^{\prime} \mu^{\prime}}\left(\frac{u}{n}\right)$ über, wo $\lambda^{\prime}, \mu^{\prime}$ aus:

$$
\lambda^{\prime} \equiv \lambda+m_{1}, \quad \mu^{\prime} \equiv \mu+m_{2} \quad(\bmod . n)
$$

$\mathrm{zu}$ berechnen ist. Nennen wir die Substitution $u^{\prime}=u+m_{1}^{\prime} \omega_{1}+m_{2}^{\prime} \omega_{2}$ mit der Substitution (1) kongruent $\bmod n$, falls $m_{1}^{\prime} \equiv m_{1}, m_{2}^{\prime} \equiv m_{2}(\bmod . n)$ zutrifft, so ist einleuchtend, daB alle $\bmod n$ miteinander kongruenten Substitutionen ein und dieselbe Permutation der $n^{2}$ Wurzeln der Teilungsgleichung bewirken. Insbesondere ergeben die Substitutionen der in I, 375 mit $\Gamma_{n^{2}}^{(u)}$ bezeichneten „Hauptkongruenzgruppe $n^{\text {ter }}$ Stufe“ innerhalb $\operatorname{der} \Gamma^{(u)}$ die identische Permutation der $\wp_{\lambda \mu}\left(\frac{u}{n}\right)$.

Andererseits liefern $n^{2} \bmod n$ inkongruente Substitutionen (1) ebenso viele verschiedene Permutationen der $n^{2}$ Wurzeln der Teilungsgleichung; denn es wird irgendeine erste Lösung $\wp_{\lambda \mu}\left(\frac{u}{n}\right)$, wie aus (2) leicht folgt, durch jene $n^{2}$ Substitutionen in alle $n^{2}$ verschiedenen Lösungen unserer Gleichung übergefübrt. Je zwei der $n^{2}$ Permutationen sind zufolge des Gesetzes (2) „kommutativ". Nach der allgemeinen Theorie haben wir also folgenden grundlegenden Satz: Die Monodromiegruppe der allgemeinen Teilungsgleichung (3) S. 211 ist eine ,tommutative", einfach transitive Gruppe $G_{n^{2}}$ der Ordnung $n^{2}$; die Teilungsgleichung selbst ist demnach im Körper $\boldsymbol{\Omega}=\left(\Omega, \wp_{\lambda_{\mu}}, \wp_{\lambda \mu}^{\prime}, \ldots\right)$ eine Abelsche Gleichung und als solche algebraisch 
lösbar. Das Kennzeichen einer Abelschen Gleichung, daB jede ihrer Wúrzeln in jeder anderen rational? darstellbar ist, und dab je zwei hierbei auftretende rationale Funktionen kommutativ sind, muB also an unserer Teilungsgleichung erfüllt sein. Die rationale Darstellbarkeit ist aus dem Additionstheorem unmittelbar ersichtlich; so gilt z. B. als Darstellung von $\wp_{\lambda \mu}\left(\frac{u}{n}\right)$ in $\rho\left(\frac{u}{n}\right)$ :

$$
\left.\wp_{\lambda \mu}\left(\frac{u}{n}\right)=\frac{1}{4}\left(\frac{\wp^{\prime}\left(\frac{u}{n}\right)-\wp^{\prime} \lambda_{\mu}}{\wp\left(\frac{u}{n}\right)-\wp_{\lambda \mu}}\right)^{2}-\wp^{2}\left(\frac{u}{n}\right)-\wp_{\lambda \mu}{ }^{1}\right) .
$$

Um die algebraische Lösung der Teilungsgleichung wirklich durchzuführen, haben wir auf die Struktur der Gruppe $G_{n^{2}}$ und die in ihr enthaltenen Untergruppen einzugehen. Ehe dies geschieht, sollen noch einige bemerkenswerte Darstellungsformen $\operatorname{der} G_{n^{2}}$ erwähnt werden.

Sieht man die $\bmod n$ kongruenten Substitutionen (1) als nicht verschieden an, so reduziert sich die $\Gamma^{(u)}$ auf eine endliche Gruppe $G_{n^{2}}$, deren Substitutionen man zweckmäßig symbolisch durch die Kongruenzen:

$$
u^{\prime} \equiv u+m_{1} \omega_{1}+m_{2} \omega_{2} \quad(\bmod n)
$$

an Stelle der Gleichungen (1) darstellt. Die ganzen Zahlen $m_{1}, m_{2}$ wird man dabei auch nach Kombination zweier Substitutionen stets auf ihre kleinsten, nicht-negativen Reste $\bmod n$ reduzieren. Diese $G_{n^{2}}$ ist der Monodromiegruppe der Teilungsgleichung isomorph und darf als eine Darstellungsform jener Gruppe benutzt werden. Für die Untersuchung der Untergruppen jener Gruppe ist diese Darstellung besonders geeignet.

Nach I, 190 liefert jede Substitution (1) eine eindeutige Transformation der Riemannschen Fläche $\boldsymbol{F}_{\mathbf{2} n^{2}}$ in sich, die wir oben vom Parallelogramm der Perioden $n \omega_{1}, n \omega_{2}$ aus herstellten. Dabei ergeben zwei $\bmod n$ kongruente Substitutionen (1) ein und dieselbe Transformation der Fläche in sich, während die $n^{2} \bmod n$ inkongruenten Substitutionen ebenso viele verschiedene Transformationen liefern. Diese $n^{2}$ Transformationen der $\mathbf{F}_{2 n^{2}}$ in sich bilden dann ihrerseits wieder eine Gruppe $G_{n^{2}}$, in der wir eine neue Ausdrucksform unserer Monodromiegruppe finden. Das Additionstheorem gestattet eine algebraische Darstellung der Gruppe zu gewinnen, und zwar ist hier die Gestalt (16) S. 161 der Additionsformeln besonders geeignet. Setzt man in dieser Proportion $\frac{u}{n}$ statt $u$ und $\frac{\lambda \omega_{1}+\mu \omega_{2}}{n}$ statt $v$ ein, so erhält man bei Benutzung der algebraischen

1) Man beachte, daB $\wp^{\prime}\left(\frac{u}{n}\right)$ zufolge der zweiten Gleichung (1) S. 210 rational in $\wp\left(\frac{u}{n}\right)$ und Größen des Korpers $\Re$ darstellbar ist. 
Schreibweise:

$$
\wp\left(\frac{u}{n}\right)=Z, \quad \wp^{\prime}\left(\frac{u}{n}\right)=W, \quad \wp_{\lambda \mu}\left(\frac{u}{n}\right)=Z^{\prime}, \quad \wp_{\lambda \mu}^{\prime}\left(\frac{u}{n}\right)=W^{\prime}
$$

als der Substitution $u^{\prime} \equiv u+\lambda \omega_{1}+\mu \omega_{2}$ entsprechend die Transformation:

$$
\begin{aligned}
Z^{\prime}: W^{\prime}: 1 & =\left(W^{2} \wp_{\lambda \mu}-Z \wp_{\lambda \mu}^{\prime 2}-\left(2 W \wp_{\lambda \mu}^{\prime}+g_{2}\left(Z+\wp_{\lambda \mu}\right)+3 g_{3}\right)\left(Z-\wp_{\lambda \mu}\right)\right) \\
: & \left(\left(W \wp_{\lambda \mu}^{\prime}+3 g_{3}\right)\left(W-\wp_{\lambda \mu}^{\prime}\right)+2\left(6 Z \wp_{\lambda \mu}-g_{2}\right)\left(Z \wp_{\lambda \mu}^{\prime}-W \wp_{\lambda \mu}\right)\right. \\
& \left.+g_{2}\left(Z W-\wp_{\lambda \mu} \wp_{\lambda \mu}^{\prime}\right)\right) \\
& :\left(W^{2}-\wp_{\lambda \mu}^{\prime 2}-\left(12 Z \wp_{\lambda \mu}-g_{2}\right)\left(Z-\wp_{\lambda \mu}\right)\right) .
\end{aligned}
$$

Die $G_{n^{2}}$ erscheint hier eingekleidet in eine Gruppe eindeutig umkehrbarer quadratischer Transformationen der $Z, W$.

Eine Erniedrigung des Grades dieser Transformationen tritt in den beiden niedersten Fällen $n=2$ und 3 ein. Im Falle $n=2$ ist $\wp_{\lambda \mu}$ eine der drei Größen $e_{1}, e_{2}, e_{3}$, etwa $e_{i}$, während $\wp_{\lambda \mu}^{\prime}=0$ ist. Ersetzt man auf der rechten Seite von (5) die $g_{2}, g_{3}$ durch ihre Ausdrücke in $e_{1}, e_{2}, e_{3}$ und $W^{2}$ durch $4\left(Z-e_{1}\right)\left(Z-e_{2}\right)\left(Z-e_{3}\right)$, so läßt sich nach den nötigen Zwischenrechnungen aus den drei Gliedern der Proportion der gemeinsame Faktor $4\left(Z-e_{i}\right)$ herausheben, und es verbleibt die Proportion:

$$
Z^{\prime}: W^{\prime}: 1=\left(Z-e_{i}\right)\left(e_{i} Z+e_{i}^{2}+e_{k} e_{l}\right):-\left(2 e_{i}^{2}+e_{k} e_{l}\right) W:\left(Z-e_{i}\right)^{2}
$$

Die drei von der Identität verschiedenen Transformationen der $G_{4}$ werden demnach in $Z$ allein linear und führen uns zu den Substitutionen (2) in I, 133 zurück. In der Tat ist ja die vorliegende Gruppe für diejenige zweiblättrige Riemannsche Fläche $F_{2}^{\prime}$ des Geschlechtes 1 , welche durch Abbildung der $F_{8}$ vermittelst der Funktion $Z$ gewonnen wird, einfach die Vierergruppe der Transformationen der zugehörigen Verzweigungsform in sich (vgl. I, $133 \mathrm{ff}$.).

Im Falle $n=3$ handelt es sich bei der Herabminderung des Grades der Transformationen (5) um einen bei den Anwendungen der elliptischen. Funktionen auf die Theorie der Kurven dritten Grades bekannten Satz, der übrigens in der von Klein entwickelten „Theorie der elliptischen Normalkurven " verallgemeinert ist. ${ }^{1}$ )

Statt übrigens die Transformationen (5) einer direkten algebraischen Umformung zu unterwerfen, kann man sich zum Beweise des Satzes, daB für $n=3$ die neun Transformationen der Fläche $\mathbf{F}_{18}$ in sich in $Z$ und $W$ linear werden, der folgenden funktionentheoretischen Überlegung bedienen:

Die beiden Funktionen $\wp_{\lambda_{\mu}}(u), \wp_{\lambda_{\mu}}^{\prime}(u)$ sind zwei- bzw. dreiwertig und haben einen Pol zweiter bzw. dritter Ordnung an der Stelle $-\frac{\lambda \omega_{1}+\mu \omega_{2}}{3}$.

1) Vgl. Klein; „Über die elliptischen Normalkurven der $n^{\text {ten } O r d n u n g ~ u n d ~}$

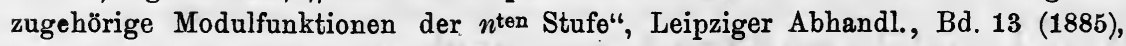
sowie die Darstellung in "Modulfunktionen", Bd. 2, S. $237 \mathrm{ff}$., insbesondere S. 248. 
Nach dem Abelschen Theorem (vgl.I,214 ff.)gibt es eine dreiwertige doppeltperiodische Funktion $\psi(u)$, die an jener Stelle einen Nullpunkt dritter Ordnung und bei $u=0$ einen Pol dieser Ordnung hat. Diese Funktion $\psi(u)$ läBt sich nach der Regel (4) in I, 206 so darstellen:

$$
\psi(u)=c_{0}+c_{1} \wp(u)+c_{2} \wp^{\prime}(u),
$$

wo die $c$ von $u$ unabhängige Größen sind und $c_{2}$ von 0 verschieden ist. Übrigens ist $\psi(u)$ durch Pol und Nullpunkt nur erst bis auf einen von $u$ unabhängigen, beliebig wählbaren Faktor bestimmt. Wir verfügen über diesen Faktor so, daß $c_{2}=-\gamma_{\lambda, \mu}^{\prime}$ wird. Die Werte $c_{0}$ und $c_{1}$ ergeben sich dann aus dem Umstande, daß wegen des Nullpunktes dritter Ordnung an der. Stelle $-\frac{\lambda \omega_{1}+\mu \omega_{2}}{3}$ sowohl $\psi(u)$ als auch die Ableitung:

$$
\psi^{\prime}(u)=c_{1} \wp^{\prime}(u)+c_{2} \wp^{\prime \prime}(u)=c_{1} \wp^{\prime}(u)+c_{2}\left(6 \wp(u)^{2}-\frac{1}{2} g_{2}\right)
$$

verschwindet. Hierans ergeben sich, wenn wir noch $c_{2}=-\wp_{\lambda \mu}^{\prime}$ eintragen, die beiden Gleichungen:

$$
\begin{gathered}
c_{0}+c_{1} \wp_{\lambda \mu}=-\wp_{\lambda \mu}^{\prime 2}=-4 \wp_{\lambda \mu}^{3}+g_{2} \wp_{\lambda \mu}+g_{3}, \\
c_{1}+6 \rho_{\lambda \mu}^{2}-\frac{1}{2} g_{2}=0,
\end{gathered}
$$

aus denen sich $c_{1}$ und $c_{2}$ sofort bestimmen. Man findet:

$$
\psi(u)=\left(2 \wp_{\lambda \mu}^{3}+\frac{1}{2} g_{2} \wp_{\lambda \mu}+g_{3}\right)-\left(6 \wp_{\lambda u}^{2}-\frac{1}{2} g_{2}\right) \wp(u)-\wp_{2 \mu}^{\prime} \wp^{\prime}(u) .
$$

Die beiden Produkte $\psi(u) \cdot \wp_{\lambda \mu}(u)$ und $\psi(u) \cdot \wp_{\lambda \mu}^{\prime}(u)$ sind nun als dreiwertige doppeltperiodische Funktionen mit Polen dritter Ordnung bei $u=0$ gleichfalls in den Gestalten:

$$
\left\{\begin{array}{l}
\psi(u) \wp_{2 \mu}(u)=a_{0}+a_{1} \wp(u)+a_{2} \wp^{\prime}(u), \\
\psi(u) \wp_{\lambda \mu}^{\prime}(u)=b_{0}+b_{1} \wp(u)+b_{2} \wp^{\prime}(u)
\end{array}\right.
$$

darstellbar. Die Koeffizienten $a_{0}, a_{1}, \ldots, b_{2}$ lassen sich aber leicht mittelst der Reihenentwicklungen bestimmen. Man hat erstlich wegen der rechten Seiten der Ansätze (8):

$$
\left\{\begin{array}{l}
\psi(u) \wp_{\lambda \mu}(u)=-2 a_{2} u^{-3}+a_{1} u^{-2}+a_{0}+\cdots, \\
\psi(u) \wp_{\lambda \mu}^{\prime}(u)=-2 b_{2} u^{-3}+b_{1} u^{-2}+b_{0}+\cdots
\end{array}\right.
$$

Auf der anderen Seite folgt aus (7):

$$
\psi(u)=2 \wp_{\lambda \mu}^{\prime} u^{-3}-\left(6 \delta_{\lambda \mu}^{2}-\frac{1}{2} g_{2}\right) u^{-2}+\left(2 \wp_{\lambda \mu}^{3}+\frac{1}{2} g_{2} \wp_{\lambda \mu}+g_{3}\right)+\cdots,
$$

und man findet bei Benutzung der Regeln (15) S. 188:

$$
\begin{aligned}
& \wp_{\lambda \mu}(u)=\wp_{\lambda \mu}+\wp_{\lambda \mu}^{\prime} u+\frac{1}{2}\left(6 \wp_{\lambda \mu}^{2}-\frac{1}{2} g_{2}\right) u^{2}+2 \wp_{\lambda \mu} \wp_{\lambda \mu}^{\prime} u^{3}+\cdots, \\
& \wp_{\lambda \mu}^{\prime}(u)=\wp_{\lambda \mu}^{\prime}+\left(6 \delta_{\lambda \mu}^{2}-\frac{1}{2} g_{2}\right) u+6 \wp_{\lambda \mu} \wp_{\lambda \mu}^{\prime} u^{2}+ \\
& \quad+\left(20 \wp_{\lambda \mu}^{3}-3 g_{2} \wp_{\lambda \mu}-2 g_{3}\right) u^{3}+\cdots
\end{aligned}
$$

Durch Multiplikation dieser Reihen mit der für $\psi(u)$ gewinnt man die fertige 
Gestalt der Anfangsglieder der Reihen für $\psi(u) \wp_{\lambda \mu}(u)$ und $\psi(u) \wp_{\lambda \mu}^{\prime}(u)$, so dab der Vergleich mit den Ansätzen (8) die Bedeutung der Koeffizienten $a_{0}, a_{1}, \ldots, b_{2}$ aufklärt.

Man schreibe endlich in den Gleichungen (7) und (8) noch $\frac{u}{n}$ an Stelle von $u$ und führe übrigens wie oben (S. 216) die algebraischen Bezeichnungen $Z, W, Z^{\prime}, W^{\prime}$ ein. Als Ergebnis findet man: Im Falle $n=3$ stellen sich die. 9 Transformationen der Riemannschen Fläche $\mathbf{F}_{18}$ in sich mittelst der $Z, W$ durch folgende lineare Substitutionen dar:

$$
\begin{gathered}
Z^{\prime}: W^{\prime}: 1=\left(\left(2 \wp_{\lambda \mu}^{3}-\frac{3}{2} g_{2} \wp_{\lambda \mu}-2 g_{3}\right) Z-\wp_{\lambda \mu} \wp_{\lambda \mu}^{\prime} W\right. \\
\left.-\left(\frac{1}{2} g_{2} \wp_{\lambda \mu}^{2}+3 g_{3} \wp_{\lambda \mu}+\frac{1}{8} g_{2}^{2}\right)\right) \\
:\left(\left(6 \wp_{\lambda \mu}^{2}-\frac{1}{2} g_{2}\right) \wp_{\lambda \mu}^{\prime} Z-\wp_{\lambda_{\mu}}^{\prime 2} W+\left(6 \wp_{\lambda \mu}^{3}-\frac{5}{2} g_{2} \wp_{\lambda \mu}-3 g_{3}\right) \wp_{\lambda \mu}^{\prime}\right) \\
:\left(-\left(6 \wp_{\lambda \mu}^{2}-\frac{1}{2} g_{2}\right) Z-\wp_{\lambda \mu}^{\prime} W+\left(2 \wp_{\lambda \mu}^{3}+\frac{1}{2} g_{2} \wp_{\lambda_{\mu}}+g_{3}\right)\right) .
\end{gathered}
$$

\section{§ 3. Zyklische Untergruppen der $G_{n^{2}}$ und Kongruenzgruppen $n^{\text {ter }}$ Stufe.}

Für die Substitutionen der Gruppe $G_{n^{2}}$ gebrauchen wir fortan die Schreibweise:

$$
u^{\prime} \equiv u+l \omega_{1}+m \omega_{2} \quad(\bmod n) ;
$$

wir bezeichnen diese Substitutionen durch $S_{0}=1, S_{1}, S_{2}, \ldots, S_{n^{2}-1}$ und deuten die einzelne unter ihnen auch durch das Symbol $(l, m)$ an. Es soll nun zunächst die Entwicklung, welche in I, 377 für $n=2$ ausgeführt wurde, auf beliebiges $n$ übertragen werden. Zu diesem Zwecke stellen wir erstlich die „Perioden" der Substitutionen $S$ und damit die „zyklischen Untergruppen" der $G_{n^{2}}$ fest.

Die $\nu^{\text {te }}$ Potenz von $S=(l, m)$ ist einfach $S^{v}=(\nu l, \nu m)$. Soll $S^{v}=1$ sein, so müssen die beiden Zahlen $\nu l, \nu m$ zugleich durch $n$ teilbar sein. Ist $t$ der größte gemeinsame Teiler von $l$ und $m$, so gibt es zwei ganze Zahlen $a, b$, die die Gleichung $a l+b m=t$ befriedigen. Soll demnach $S^{v}=1$ sein, so ist hierzu notwendig und offenbar auch ausreichend, daß:

$$
a \cdot \nu l+b \cdot v m=\nu t
$$

durch $n$ teilbar ist. Die kleinste, dieser Bedingung genügende positive Zahl $\nu$ ist $\frac{n}{\tau}$, wo $\tau$ der gröBte gemeinsame Teiler von $t$ und $n$ ist. Im Falle $\tau=1$ nennen wir das Zahlenpaar $l, m$ teilerfremd gegen $n$. Die sogleich näher zu bestimmende Anzahl inkongruenter und gegen $n$ teilerfremder Zahlenpaare mod $n$ werde durch das Symbol $\chi(n)$ bezeichnet. Wir merken vorläufig den Satz an: Die Periode der einzelnen Substitution $S$ ist stets ein Teiler von $n$; insbesondere hat man $\chi(n)$ Substitutionen der Periode $n$. 
Zur Bestimmung der Anzahl $\chi(n)$ nehmen wir an, daB $n$ das Produkt $n_{1} \cdot n_{2}$ zweier teilerfremder ganzer Zahlen $n_{1}, n_{2}$ sei. Für das einzelne Paar $l, m$ gelte:

$$
l \equiv l_{i}, \quad m \equiv m_{i} \quad\left(\bmod n_{i}\right),
$$

$(i=1,2)$

wo die $l_{i}, m_{i}$ Zahlen der Reihe $0,1,2, \ldots, n_{i}-1$ sind. Das einzelne Paar $l, m$ reduziert sich also nach den Moduln $n_{i}$ auf zwei bestimmte Restpaare $l_{i}, m_{i}$. Auch umgekehrt gelangen wir durch Kombination der $n_{1}^{2}$ Restpaare $l_{1}, m_{1}$ mit den $n_{2}^{2}$ Restpaaren $l_{2}, m_{2}$ zu den gesamten $n_{1}^{2} \cdot n_{2}^{2}=n^{2}$ Restpaaren $\bmod n$ zurück. Die einzelne Zahlklasse $\bmod n$ ergibt sich nämlich entsprechend durch Kombination der $n_{1}$ Zahlklassen $\bmod n_{1}$ mit den $n_{2}$ Klassen $\bmod n_{2}$. Aus den Gleichungen:

$$
l=l_{i}+\alpha_{i} n_{i}, \quad m=m_{i}+\beta_{i} n_{i},
$$

in denen die $\alpha, \beta$ ganze Zahlen sind, ist einleuchtend, daß der größte gemeinsame Teiler $\tau_{i}$ von $l_{i}, m_{i}, n_{i}$ auch als Teiler in $l, m$ und $n$ enthalten ist. Haben andrerseits $l, m$ und $n$ einen Primteiler $p$ gemein, so ist $p$ in $n_{i}$, d. h. in einer der Zahlen $n_{1}, n_{2}$, als Faktor enthalten und geht zufolge (1) auch in $l_{i}$ und $m_{i}$ auf. Die sämtlichen gegen $n$ teilerfremden Paare $l, m$ erhalten wir also gerade genau, wenn wir die $\chi\left(n_{1}\right)$ gegen $n_{1}$ teilerfremden Paare $l_{1}, m_{1}$ mit den $\chi\left(n_{2}\right)$ gegen $n_{2}$ teilerfremden Paaren kombinieren. Es gilt demnach für die Anzahl $\chi$ das Gesetz $\chi\left(n_{1} \cdot n_{2}\right)=\chi\left(n_{1}\right) \cdot \chi\left(n_{2}\right)$, falls $n_{1}$ und $n_{2}$ teilerfremd sind.

Hiernach braucht man $\chi(n)$ nur noch in dem Falle zu berechnen, daß $n$ eine Primzahlpotenz $p^{v}$ ist. Unter den $p^{v}$ inkongruenten Zahlen $0,1,2, \ldots, p^{v}-1$ sind aber $p^{v-1}$ durch $p$ teilbar und $\left(p^{v}-p^{v-1}\right)$ teilerfremd gegen $p$. Wird $l$ gleich einem dieser $\left(p^{v}-p^{v-1}\right)$ teilerfremden Reste gesetzt, so liefert jedes $m$ ein gegen $p^{v}$ teilerfremdes Paar $l, m$, was $p^{v}\left(p^{v}-p^{v-1}\right)$ inkongruente Paare ergibt. Setzt man aber $l$ gleich einem der $p^{\nu-1}$ durch $p$ teilbaren Reste, so sind für $m$ nur noch die $\left(p^{\nu}-p^{v-1}\right)$ gegen $p$ teilerfremden Reste zugänglich, was noch $p^{\nu-1}\left(p^{\nu}-p^{\nu-1}\right)$ Paare liefert. Hieraus folgt:

$$
\chi\left(p^{v}\right)=p^{v}\left(p^{v}-p^{v-1}\right)+p^{v-1}\left(p^{v}-p^{v-1}\right)=p^{2 v}\left(1-\frac{1}{p^{2}}\right)
$$

und damit der allgemeine Satz: Ist die Primfaktorenzerlegung der Zahl n durch $n=p_{1}^{v_{1}} \cdot p_{2}^{v_{2}} \cdot p_{3}^{v_{2}} \ldots$ gegeben, so ist die Anzahl $\chi(n)$ der gegen $n$ teilerfremden Paare $l, m$ und damit die Anzahl der in $G_{n^{2}}$ enthaltenen Substitutionen der Periode $n$ :

$$
\chi(n)=n^{2}\left(1-\frac{1}{p_{1}^{2}}\right)\left(1-\frac{1}{p_{2}^{2}}\right)\left(1-\frac{1}{p_{3}^{2}}\right) \cdots
$$

Jede dieser $\chi(n)$ Substitutionen $S$ der Periode $n$ erzeugt eine zyklische Untergruppe $G_{n}$ der Ordnung $n$. Soll unter den $n$ Substitutionen 
$S^{v}$ dieser $G_{n}$ die einzelne $S^{v}$ wieder die Periode $n$ haben, so ist hierzu notwendig und hinreichend, daß $\nu$ teilerfremd gegen $n$ ist. Da unter den Resten $\bmod n$ im ganzen:

$$
\varphi(n)=n\left(1-\frac{1}{p_{1}}\right)\left(1-\frac{1}{p_{2}}\right)\left(1-\frac{1}{p_{3}}\right) \cdots
$$

teilerfremd gegen $n$ sind, so enthält die $G_{n}$ insgesamt $\varphi(n)$ Substitutionen der Periode $n$ und kann aus jeder dieser Substitutionen erzeugt werden. Erklären wir neben $\chi(n)$ und $\varphi(n)$ eine dritte von $n$ abhängige Anzahl $\psi(n)$ durch:

$$
\psi(n)=n\left(1+\frac{1}{p_{1}}\right)\left(1+\frac{1}{p_{2}}\right)\left(1+\frac{1}{p_{3}}\right) \cdots,
$$

so gilt $\chi(n)=\varphi(n) \cdot \psi(n)$, und wir gewinnen den Satz: Als zyklische Untergruppen höchster Ordnung treten in der $G_{n^{2}}$ im ganzen $\psi(n)$ Untergruppen $G_{n}$ der Ordnung $n$ auf. Da $G_{n^{2}}$ eine Abelsche Gruppe ist, so ist jede dieser $G_{n}$ eine ,ausgezeichnete" Untergruppe der $G_{n^{2}}$.

Innerhalb der Gruppe $\Gamma^{(u)}$ der Substitutionen $u^{\prime}=u+l \omega_{1}+m \omega_{2}$ bilden nun alle Substitutionen, die mit den Substitutionen einer vorgelegten zyklischen $G_{n} \bmod n$ kongruent sind, eine durch $\Gamma_{n}^{(u)}$ zu bezeichnende Untergruppe, welche die in I, 375 erklärte Hauptkongruenzgruppe $\Gamma_{n^{2}}^{(u)}$ der $n^{\text {ten }}$ Stufe in sich enthält. Wir nennen auch $\Gamma_{n}^{(u)}$ eine „Kongruenzgruppe $n^{\text {ter }}$ Stufe $e^{\text {" }}$ und haben unseren $\psi(n)$ zyklischen $G_{n}$ entsprechend $\psi(n)$ innerhalb der Gesamtgruppe $\Gamma^{(u)}$ ausgezeichnete Kongruenzgruppen $n^{\text {ter }}$ Stufe $\Gamma_{n}^{(u)}$. Daß sie ausgezeichnet sind, folgt in der Tat unmittelbar wieder aus dem kommutativen Charakter der $\Gamma^{(u)}$.

Wie im Falle $n=2$ (vgl. I, $379 \mathrm{ff}$.) gestalten sich die Verhältnisse aber wieder anders innerhalb der ternären Gruppe $\Gamma^{(u, \omega)}$, in welcher die $\Gamma^{(u)}$ als ternäre Untergruppe der Substitutionen:

$$
u^{\prime}=u+l \omega_{1}+m \omega_{2}, \quad \omega_{1}^{\prime}=\omega_{1}, \quad \omega_{2}^{\prime}=\omega_{3}
$$

und die Modulgruppe $\Gamma^{(\omega)}$ als ternäre Untergruppe der Substitutionen:

$$
u^{\prime}=u, \quad \omega_{1}^{\prime}=\alpha \omega_{1}+\beta \omega_{2}, \quad \omega_{2}^{\prime}=\gamma \omega_{1}+\delta \omega_{2}
$$

enthalten ist. Transformiert man nämlich die kurz durch $S=(l, m)$ zu bezeichnende Substitution (5) mittelst der unter (6) gegebenen Substitution $V$ der $\Gamma^{(\omega)}$, so wird, wie schon in I, 379 festgestellt ist, die transformierte Subsitution:

$$
S^{\prime}=V \cdot S \cdot V^{-1}=\left(l^{\prime}, m^{\prime}\right)=(l \delta-m \gamma,-l \beta+m \alpha) .
$$

Gilt nun zunächst $l \equiv m \equiv 0(\bmod n)$, so ist freilich auch $l^{\prime} \equiv m^{\prime} \equiv 0$ $(\bmod n)$, woraus man folgert, daß die Hauptkongruenzgruppe $\Gamma_{n^{2}}^{(u)}$ auch in der ternären Gruppe $\Gamma^{(u, \omega)}$ ausgezeichnet ist. Demgegenüber wird z. B. die Substitution $S=(0,1)$ in $S^{\prime}=(-\gamma, \alpha)$ transformiert. Nun kommen in der $\Gamma^{(\omega)}$ als Zahlen $-\gamma, \alpha$ alle Paare teilerfremder ganzer Zahlen vor. 
Geben $-\gamma, \alpha$ als kleinste nichtnegative Reste $\bmod n$ die Zahlen $l_{0}, m_{0}$, so haben wir in $l_{0}, m_{0}$ sicher ein gegen $n$ teilerfremdes Zahlenpaar, da ein gemeinsamer Teiler $\tau$ von $l_{0}, m_{0}$ und $n$ auch $\gamma$ und $\alpha$ zugleich teilen würde. Andrerseits kann man zu irgendeinem gegen $n$ teilerfremden Paare $l_{0}, m_{0}$ stets zwei $\bmod n$ mit $l_{0}$ bzw, $m_{0}$ kongruente Zahlen $-\gamma, \alpha$ angeben, die zueinander teilerfremd sind. $\left.{ }^{1}\right)$ Die Substitution $S=(0,1)$ ist also durch Transformation mit einem geeignet gewählten $V$ in ein $S^{\prime}$ überführbar, welches mit einer "beliebigen“" unserer obigen Substitutionen $(l, m)$ der Periode $n$ kongruent ist. Hieraus ergibt sich insbesondere: Die $\psi(n)$ innerhalb der $\Gamma^{(u)}$ ausgezeichneten Kongruenzgruppen $\Gamma_{n}^{(u)}$ werden innerhalb der ternären Gruppe $\Gamma^{(u, \omega)}$ miteinander ,gleichberechtigt", d.h. ineinander transformierbar. Es gelten also für beliebiges $n$ dieselben Sätze, die in I, $378 \mathrm{ff}$. für $n=3$ gewonnen wurden.

Die Ergebnisse kann man auch in geometrische Gestalt kleiden. Als Diskontinuitätsbereich der Hauptkongruenzgruppe $\Gamma_{n^{2}}^{(u)}$ kann man das Parallelogramm der Ecken 0, $n \omega_{2}, n \omega_{1}+n \omega_{2}, n \omega_{1}$ benutzen, das aus $n^{2}$ "quadratisch" angeordneten Parallelogrammen des ursprünglichen Netzes zusammensetzbar ist. .Übt man jetzt alle linearen Transformationen der $\Gamma^{(\omega)}$. aus (die offenbar auch für die beiden Perioden $n \omega_{1}, n \omega_{2}$ die gesamten „linearen Transformationen" (vgl.I,184) liefern), so nimmt das große Parallelogramm unendlich viele verschiedene Gestalten an, die aber alle nur wechselnde Formen des Diskontinuitätsbereiches einer und derselben Gruppe, nämlich der ausgezeichneten $\Gamma_{n^{2}}^{(u)}$ sind. Für eine einzelne der $\psi(n)$ Kongruenzgruppen $\Gamma_{n}^{(u)}$, etwa die durch $l \equiv 0(\bmod n)$ erklärte, ist ein Diskontinuitätsbereich als Parallelogramm der Ecken $0, \omega_{2}, n \omega_{1}+\omega_{2}$, $n \omega_{1}$ wählbar, das aus $n$,linear" aneinander gereihten Parallelogrammen des ursprünglichen Netzes besteht. Wendet man jetzt auf dieses Parallelogramm der Ecken $0, \omega_{2}, n \omega_{1}+\omega_{2}, n \omega_{1}$ die gesamten linearen Transformationen der $\Gamma^{(\omega)}$ an, so sind die entstehenden unendlich vielen Parallelogramme nicht mehr Diskontinuitätsbereiche einer und derselben Gruppe; aber sie liefern, als Diskontinuitätsbereiche aufgefaßt, doch nur endlich viele verschiedene Gruppen, nümlich eben unsere $\psi(n)$ gleichberechtigten Kongruenzgruppen $\Gamma_{n}^{(u)}$.

1) Man nehme etwa $\gamma=-l_{0}$ und bezeichne mit $\gamma_{0}$ das Produkt aller Primfaktoren von $\gamma$, die nicht zugleich in $n$ aufgehen. Dann ist eine ganze Zahl $a$ entsprechend der Kongruenz $a n \equiv 1-m_{0}\left(\bmod \gamma_{0}\right)$ angebbar, da der Faktor $n$ der linken Seite zum Modul $\gamma_{0}$ teilerfremd ist. Setzt man nun $\alpha=m_{0}+a n$, so ist $\alpha \equiv 1\left(\bmod \gamma_{0}\right)$ und also teilerfremd gegen $\gamma_{0}$. Hätte also $\alpha$ mit $\gamma=-l_{0}$ mindestens einen Primteiler $\boldsymbol{p}$ gemein, so würde es sich um einen zugleich in $n$ aufgehenden Teiler handeln, der zufolge $m_{0}=\alpha-a n$ auch $m_{0}$ teilen würde. Dies widerspricht aber dem Umstande, daB das Paar $l_{0}, m_{0}$ teilerfremd gegen $n$ ist. In $-\gamma, \alpha$ haben wir also teilerfremde Zahlen, die $\bmod n$. mit $l_{0}$ bzw. $m_{0}$ kongruent sind. 


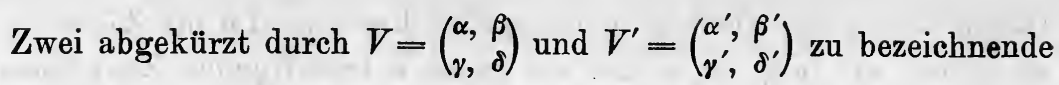
Substitutionen der $\Gamma^{(\omega)}$ heißen $\bmod n$ kongruent, wenn die vier Kongrnenzen:

$$
\alpha^{\prime} \equiv \alpha, \quad \beta^{\prime} \equiv \beta, \quad \gamma^{\prime} \equiv \gamma, \quad \delta^{\prime} \equiv \delta \quad(\bmod n)
$$

bestehen. Wir kennzeichnen ihr Zutreffen kurz durch. $V^{\prime} \equiv V(\bmod n)$. Gilt $V^{\prime} \equiv V(\bmod n)$, so ergibt $V^{\prime}$, mit der zu $V$ inversen Substitution $V^{-1}$ kombiniert, in $V^{\prime} \cdot V^{-1}$ eine mit der identischen Substitution $V_{0}=\left(\begin{array}{ll}1 & 0 \\ 0, & 1\end{array}\right)$ oder kurz $V_{0}=1$ kongruente Substitution. Ist zweitens eine der beiden Substitutionen $V, V^{\prime}$ mit der identischen Substitution kongruent, so ist $V^{\prime} \cdot V$ mit der anderen kongruent. Diese Angaben folgen sofort aus der Regel, nach der sich die Koeffizienten einer aus zwei Substitutionen zusammengesetzten Substitution berechnen (s. Gleichung (2) in I, 127). Es folgt, daß alle mit der identischen Substitution $V_{0}=1 \bmod n$ kongruenten Substitutionen der $\Gamma^{(\omega)}$ eine Untergruppe bilden, die nach I,375 die „Hauptkongruenzgruppe $n^{\text {ter }}$ Stufe $e^{\text {"6 }}$ innerhalb der $\Gamma^{(\omega)}$ heiBt. $\left.{ }^{1}\right)$ Ist $V \equiv 1(\bmod n)$ und $V^{\prime}$ beliebig, so ist $V^{\prime} \cdot V \equiv V^{\prime}$ und also $V^{\prime} \cdot V \cdot V^{\prime-1} \equiv 1$ (mod. $n$ ). Die Hauptkongruenzgruppe $n^{\text {ter }}$ Stufe ist also eine ausgezeichnete Untergruppe der $\Gamma^{(\omega)}$.

Bildet man für diese Untergruppe nach dem Schema (2) oder (4) S. $4 \mathrm{ff}$. die Nebengruppen, so erscheinen in der einzelnen Nebengruppe alle Substitutionen der $\Gamma^{(\omega)}$ vereint, die mit einer unter ihnen $\bmod n$ kongruent sind. Der Index der Hauptkongruenzgruppe $\boldsymbol{n}^{\text {ter }}$ Stufe ist demnach gleich der Anzahl mod $n$ inkongruenter Substitutionen in der $\Gamma^{(\omega)}$. Da aber jede Substitution $V$ mit ihren vier Koeffizienten eine Lösung der Kongruenz:

$$
\alpha \delta-\beta \gamma \equiv 1 \quad(\bmod n)
$$

in ganzen Zahlen $\alpha, \beta, \gamma, \delta$ ergibt, so ist die Anzahl der mod $n$ inkongruenten Substitutionen sicher nicht größer als die Anzahl inkongruenter Lösungen der Kongruenz (9). Diese Anzahl stellen wir leicht fest. Zunächst muß $\alpha, \gamma$ wegen (9) ein gegen $n$ teilerfremdes Restpaar sein, und wir haben $\chi(n)$ derartige Paare. Ist beim einzelnen solchen Paare $\alpha$ teilerfremd gegen $n$, so ist $\beta$ unbeschränkt, und für jedes $\beta$ ist ein zugehöriges $\delta$ aus (9) eindeutig bestimmt, was für dieses' Paar $\alpha, \gamma$ im ganzen $n$ inkongruente Lösungen von (9) ergibt. Hat aber $\alpha$ mit $n$ den größten Teiler $\tau$ gemein, so ist $\gamma$ teilerfremd gegen $\tau$, und die aus (9) folgende Kongruenz $\beta \gamma \equiv-1(\bmod \tau)$ hat eine und nur eine Lösung $\beta_{0}$. Wir finden

1) Es ist dies die in „Modulfunktionen“ Bd. 1, S. 387 ff. ausführlich untersuchte Gruppe. 
aus ihr $\frac{n}{\tau} \bmod n$ inkongruente Zahlen:

$$
\beta \equiv \beta_{0}, \quad \beta_{0}+\tau, \quad \beta_{0}+2 \tau, \ldots, \quad \beta_{0}+\left(\frac{n}{\tau}-1\right) \tau(\bmod n)
$$

als zugehörig. Für jede einzelne dieser Zahlen $\left(\beta_{0}+\nu \tau\right)$ ist weiter die Kongruenz:

$$
\frac{\alpha}{\tau} \delta \equiv \frac{\beta_{0} \gamma+1}{\tau}+\gamma \nu \quad\left(\bmod \frac{n}{\tau}\right)
$$

nach $\delta \mathrm{zu}$ lösen. Sie hat $\bmod n \tau^{-1}$,eine“ Lösung $\delta_{0}$, aus der $\tau \bmod n$ inkongruente Zahlen:

$$
\delta \equiv \delta_{0}, \quad \delta_{0}+\frac{n}{\tau}, \quad \delta_{0}+2 \frac{n}{\tau}, \ldots, \delta_{0}+(\tau-1) \frac{n}{\tau}
$$

als brauchbar hervorgehen. Wir haben also auch jetzt für das einzelne Paar $\alpha, \gamma$ im ganzen $\frac{n}{\tau} \cdot \tau=n$ Lösungen der Kongruenz (9), so daß wir als Anzahl inkongruenter Lösungen von (9) finden:

$$
n \chi(n)=n^{3}\left(1-\frac{1}{p_{1}^{2}}\right)\left(1-\frac{1}{p_{2}^{2}}\right)\left(1-\frac{1}{p_{3}^{2}}\right) \cdots .
$$

Es geht nun schon aus der Fußnote von S. 221 hervor, daB alle $\chi(n)$ inkongruenten Paare $\alpha, \gamma$ in der $\Gamma^{(\omega)}$ wirklich auftreten. Zum einzelnen Paare teilerfremder Zahlen $\alpha, \gamma$ gebören aber nach (1) in I, 292 die einfach unendlich vielen Substitutionen:

$$
V=\left(\begin{array}{l}
\alpha, \beta+\nu \alpha \\
\gamma, \delta+v \gamma
\end{array}\right) . \quad(v=\cdots,-2,-1,0,1,2, \cdots)
$$

Wir erkennen sofort, daß unter ihnen genau $n$ inkongruente Substitutionen enthalten sind, die wir etwa für $v=0,1,2, \ldots, n-1$ gewinnen. Also haben wir für alle $n \chi(n)$ inkongruenten Lösungen von (9) zugehörige Substitutionen $V$, woraus hervorgeht, daß der Index. der Hauptkongruenzgruppe $n^{\text {ter }}$ Stufe innerhalb der $\Gamma^{(\omega)}$ durch die in (10) dargestellte Anzahl $n \chi(n)$ gegeben ist. Die fragliche Untergruppe möge dementsprechend durch $\Gamma_{n \chi(n)}^{(\omega)}$ oder kurz durch $\Gamma_{n \chi}^{(\omega)}$ bezeichnet werden.

Sehen wir je zwei mod $n$ kongruente Substitutionen $V$ als nicht verschieden an, so reduziert sich die Gruppe $\Gamma^{(\omega)}$ hiernach auf eine Gruppe $G_{n \chi(n)}$ der endlichen Ordnung $n \chi(n)$, deren Substitutionen wir zweckmäßig durch:

$$
\omega_{1}^{\prime} \equiv \alpha \omega_{1}+\beta \omega_{2}, \quad \omega_{2}^{\prime} \equiv \gamma \omega_{1}+\delta \omega_{2} \quad(\bmod n)
$$

bezeichnen. Die Kongruenzzeichen beziehen sich natürlich auf die ganzzahligen Koeffizienten $\alpha, \beta, \gamma, \delta$, die nur $\bmod n \mathrm{zu}$ unterscheiden sind. Das Gesetz der Kombination zweier Substitutionen ist selbstverständlich das bisherige; nur tritt an Stelle der "Gleichung“ (2) in I, 127 hier eine entsprechend gebauté „Kongruenz“. Zur deutlicheren Unterscheidung der 
Gruppe $G_{n \chi(n)}$ von den oben betrachteten Gruppen $G_{n^{2}}$ und $G_{n}$ der Substitutionen $S$ schreiben wir genauer $G_{n \chi(n)}^{(\omega)}$ sowie $G_{n^{2}}^{(u)}$ und $G_{n}^{(u)}$.

Reduzieren wir endlich die ternäre Gruppe $\Gamma^{(u, \omega)} \bmod n$, so gelangen wir zu einer Gruppe $G_{n^{3} \chi(n)}^{(u, \omega)}$ der Ordnung $n^{3} \chi(n)$, in der (nach der oben bei den Gruppen $\Gamma^{(u, \omega)}, \Gamma^{(u)}, \Gamma^{(\omega)}$ dargestellten Auffassung) sowohl die $G_{n^{2}}^{(u)}$ (und damit auch ihre zyklischen $G_{n}^{(u)}$ ) als auch die $G_{n \chi(n)}^{(\omega)}$ als Untergruppen enthalten sind. Da die Regel (7), nur als Kongruenz mod $n$ geschrieben, gültig bleibt, so folgert man aus den oben aufgestellten Sätzen betreffs der zyklischen $G_{n}^{(u)}$ die Angaben: Die $\psi(n)$ zyklischien Untergruppen $G_{n}^{(u)}$ sind zwar in der $G_{n^{2}}^{(u)}$ ausgezeichnet, dagegen werden sie in $\operatorname{der} G_{n^{3} \chi(n)}^{(u, \omega)}$ gleichberechtigt, ja innerhalb dieser letateren Gruppe sind sogar alle $\chi(n)$ Substitutionen $S$ der Periode $n$ gleichberechtigt.

Zwei unter den $\psi(n)$ zyklischen Untergruppen $G_{n}^{(u)}$ mögen zu einander „komplementär" heißen, wenn sie außer der identischen Substitution keine Substitution gemein haben. Es soll festgestellt werden, wie viele unter den $\psi(n)$ zyklischen Gruppen mit einer vorgelegten unter ihnen komplementär sind. Da alle $G_{n}^{(u)}$ innerhalb $\operatorname{der} G_{n^{3} \chi}^{(u, \omega)}$ ineinander transformierbar sind, so ist die gesuchte Anzahl ein und dieselbe, welche $G_{n}^{(u)}$ wir auch vorlegen mögen. Wir wählen etwa die aus $S=(0,1)$ zu erzeugende $G_{n}^{(u)}$ und schreiben $T=(l, m)$ als erzeugende Substitution einer komplementären $G_{n}^{(u)}$. Dann darf keine der $(n-1)$ Substitutioneu $T^{v}=(\nu l, \nu m)$ mit $\nu=1,2, \ldots, n-1$ der aus $S$ zu erzeugenden $G_{n}^{(u)}$ angehören, d. h. keine der Zahlen $l, 2 l, \ldots,(n-1) l$ darf durch $n$ teilbar sein, was einfach darauf hinaủsläuft, daB $l$ einer der $\varphi(n)$ gegen $n$ teilerfremden Reste $\bmod n$ ist. Da $m$ unbeschränkt bleibt, so haben wir $n \varphi(n)$ brauchbare $T$ und gewinnen den Satz: Mit der einzelnen der $\psi(n)$ Gruppen $G_{n}^{(u)}$ sind immer $n$ dieser zyklischen Gruppen komplementär.

Die Bedeutung des Begriffs der komplementären $G_{n}^{(u)}$ geht aus folgendem Satze hervor: Die aus zwei Substitutionen $S$ und $T$ der Periode $n$ hergestellten $n^{2}$ Substitutionen: $S^{v} \cdot T^{\nu^{\prime}}$,

$v, v^{\prime}=0,1,2, \ldots, n-1$

sind stets und nur dann alle von einander verschieden und erschöpfen also die $G_{n^{2}}^{(u)}$, wenn die aus $S$ und $T$ zu erzeugenden $G_{n}^{(u)}$ komplementär sind. Ist $G_{n}^{(u)}$ die aus $S \mathrm{zu}$ erzeugende Untergruppe, so können wir die Zerlegung der Gesamtgruppe $G_{n^{2}}^{(u)}$ in die zugehörigen $n$ Nebengruppen so schreiben:

$$
G_{n^{2}}^{(u)}=G_{n}^{(u)}+G_{n}^{(u)} \cdot T+G_{n}^{(u)} \cdot T^{2}+\cdots+G_{n}^{(u)} \cdot T^{n-1} .
$$

$Z u$ der in der $G_{n^{2}}^{(u)}$ ausgezeichneten $G_{n}^{(u)}$ gehört demgemäß als entsprechende "Quotientengruppe" $G_{n^{2}}^{(u)} / G_{n}^{(u)}$ eine mit der Gruppe der Substitutio- 
nen $1, T, T^{2}, \ldots, T^{n-1}$ isomorphe Gruppe, d.h. wieder eine zyklische $G_{n}$. Wir folgern hieraus für die Lösung der allgemeinen Teilungsgleichung das Ergebnis: Die im Körper $\boldsymbol{\Omega}^{\prime}=\left(\Omega, \wp_{2 \mu}, \wp_{2 \mu}^{\prime} \ldots\right)$ Abelsche Teilungsgleichung des Grades $n^{2}$ ist mittelst zweier zyklischer Gleichungen $n^{\text {ten }}$ Grades lösbar.

\section{$\S 4$. Elliptische Funktionen $n^{\text {ter }}$ Stufe.}

Nach I, 376 ist eine elliptische Funktion $n^{\text {ter }}$ Stufe neben anderen Eigenschaften dadurch charakterisiert, dab sie homogen in $u, \omega_{1}, \omega_{2}$ ist und gegenüber den Substitutionen der ternären Hauptkongruenzgruppe $n^{\text {ter }}$ Stufe unverändert bleibt. Zu diesen Funktionen gehören insbesondere die Lösungen des Teilungsproblems $\wp_{\lambda \mu}\left(\frac{u}{n}\right), \delta_{\lambda \mu}^{\prime}\left(\frac{u}{n}\right)$. Schreiben wir nämlich z. B. die erster dieser Funktionen ausführlich:

$$
\wp_{\lambda \mu}\left(\frac{u}{n}\right)=\wp\left(\frac{u}{n}+\frac{\lambda \omega_{1}+\mu \omega_{2}}{n} \mid \omega_{1}, \omega_{2}\right),
$$

so erweist sie sich gegenüber einer Substitution $u^{\prime}=u+m_{1} \omega_{1}+m_{2} \omega_{2}$ mit $m_{\mathbf{1}} \equiv 0, m_{\mathbf{2}} \equiv 0(\bmod n)$ unmittelbar als invariant. Die Ausübung einer Substitution $\omega_{1}^{\prime}=\alpha \omega_{1}+\beta \omega_{2}, \omega_{2}^{\prime}=\gamma \omega_{1}+\delta \omega_{2}$ auf das zweite und dritte Argument in (1) läßt diese Funktion gleichfalls unverändert. Für das erste Argument finden wir:

$$
\frac{\lambda \omega_{1}^{\prime}+\mu \omega_{2}^{\prime}}{n}=\frac{\lambda \omega_{1}+\mu \omega_{2}}{n}+\left(\lambda \frac{\alpha-1}{n}+\mu \frac{\gamma}{n}\right) \omega_{1}+\left(\lambda \frac{\beta}{n}+\mu \frac{\delta-1}{n}\right) \omega_{2},
$$

so daß für $\alpha \equiv \delta \equiv 1, \beta \equiv \gamma \equiv 0(\bmod n)$ die Unveränderlichkeit der Funktion (1) feststeht.

Es gibt nun noch einfachere elliptische Funktionen $n$ ter Stufe, die in doppelter Hinsicht wichtig sind. Sie werden uns einmal eine einfache Lösung des Teilungsproblems vermitteln, andrerseits stellt ihre Theorie eine planmäBige Erweiterung der in I, $382 \mathrm{ff}$. entworfenen Theorie der elliptischen Funktionen zweiter Stufe auf eine beliebige Stufe $n$ dar.

Wir bilden entsprechend dem Ansatze (1) in I, 450 für die vorliegende Stufe $n$ die $n^{2}$-Funktionen

$$
\sigma_{\lambda \mu}\left(u \mid \omega_{1}, \omega_{2}\right)
$$$$
\lambda, \mu=0,1,2 \ldots, n-1,
$$

von denen $\sigma_{0,0}\left(u \mid \omega_{1}, \omega_{2}\right)$ mit der ursprünglichen $\sigma$-Funktion identisch ist. Etwas kürzer schreiben wir $\sigma_{\lambda \mu}(u)$ statt (2) und verstehen unter $\sigma_{\lambda \mu}$ den Wert dieser Funktion für $u=0$; dabei ist $\sigma_{0,0}$ mit 0 identisch, während die übrigen $\sigma_{\lambda \mu}$ nicht identisch verschwindende Funktionen der $\omega_{1}, \omega_{2}$ sind. Die Verallgemeinerung der drei unter (4) in I, 384 erklärten Funktionen zweiter Stufe $\psi_{k}\left(u \mid \omega_{1}, \omega_{2}\right)$ sehen wir nun in den $\left(n^{2}-1\right)$ Funktionen: 


$$
\Psi_{\lambda \mu}\left(u \mid \omega_{1}, \omega_{2}\right)=\frac{\sigma_{\lambda \mu}\left(u \mid \omega_{1}, \omega_{2}\right)}{\sigma_{\lambda \mu} \cdot \sigma\left(u \mid \omega_{1}, \omega_{1}\right)}
$$

wo nur die Kombination $\lambda=0, \mu=0$ auszuschließen ist. Nach I, 451 zeigt diese Funktion bei Vermehrung von $u$ um Perioden das Verhalten:

$$
\Psi_{\lambda \mu}\left(u+l \omega_{1}+m \omega_{2}\right)=\varepsilon^{3 \mu-m \imath} \Psi_{\lambda_{\mu}}(u),
$$

unter $\varepsilon$ die Einheitswurzel $e^{\frac{2 i \pi}{n}}$ verstanden; sie bleibt sicher unverändert, wenn $l \equiv 0, m \equiv 0(\bmod n)$ gilt. Nach $(5)$ in I, 451 bleibt die Funktion $\Psi_{\lambda_{\mu}}$ gleichfalls unverändert, wenn $\operatorname{man}$ auf die $\omega_{1}, \omega_{2}$ eine $\bmod n \operatorname{mit}$ 1 kongruente Substitution $V$ ausübt. Wir haben hiernach in (3) im ganzen $\left(n^{2}-1\right)$ verschiedene Funktionen $n^{\text {ter }}$ Stufe gewonnen, die offenbar in $u$, $\omega_{1}, \omega_{2}$ homogen von der Dimension -1 sind. Die Funktion $\Psi_{{ }_{\mu}}(u)$ hat einen Pol erster Ordnung in jedem Gitterpunkte des ursprünglichen Parallelogrammnetzes. Speziell bei $u=0$ gilt die Entwicklung:

$$
\Psi_{2 \mu}(u)=\frac{1}{u}+\frac{\sigma_{\lambda \mu}^{\prime}}{\sigma_{\lambda \mu}}+\frac{\sigma_{\lambda \mu}^{\prime \prime}}{2 \sigma_{\lambda \mu}} u+\ldots
$$

wo neben den $\sigma_{\lambda \mu}$ auch noch die „Nullwerte“ der Ableitungen $\sigma_{\lambda_{\mu}}^{\prime}(u)$, $\sigma_{\lambda_{\mu}}^{\prime \prime}(u), \ldots$ in bezug auf $u$ auftreten. Vornehmlich wichtig sind die $\sigma_{\lambda \mu}$ und $\sigma_{\lambda \mu}^{\prime}$; wir bezeichnen sie als „die $\sigma-b z w$. $\sigma^{\prime}$-Teilwerte“ des $n$ ten Teilungsgrades. Nullpunkte erster Ordnung hat $\Psi_{\lambda \mu}(u)$ an allen mit $\frac{\lambda \omega_{1}+\mu \omega_{2}}{n}$ bezüglich der $\Gamma^{(u)}$ äquivalenten Stellen. Sonstige Pole oder Nullpunkte treten aber nicht auf. Ändern wir in der Erklärung (1) in I, 450 der Funktion $\sigma_{\lambda \mu}(u)$ die $\lambda, \mu$ um Vielfache von $n$, so zeigt die Funktion das in (4) daselbst notierte Verhalten. Bei der Bauart der rechten Seite von (3) geht hieraus hervor, daß $\Psi_{\lambda \mu}(u)$ unverändert bleibt, wenn wir $\lambda$ und $\mu$ um Vielfache von $n$ ändern. Dieser Umstand ist gelegentlich für die Schreibweise unserer Gleichungen wichtig.

Haben $\lambda, \mu, n$ einen Teiler $t>1$ gemein, so tritt $\Psi_{\lambda, u}(u)$ bereits bei der Stufe $\frac{n}{t}$ auf. Demgegenüber nennen wir die $\chi(n)$ Funktionen $\Psi_{\lambda_{\mu}}(u)$, welche zu den $\chi(n)$ gegen $n$ teilerfremden Paaren $\lambda, \mu$ gehören, die „eigentlich zur Stufe n gehörigen" Funktionen $\Psi_{\lambda_{\mu}}(u)$. Soll die eigentlich zur Stufe $n$ gehörende Funktion $\Psi_{\lambda_{\mu}}(u)$ bei der Substitution $S=(l, m)$ $\operatorname{der} \Gamma^{(u)}$ unverändert bleiben, so ist hierzu die Kongruenz:

$$
l \mu-m \lambda \equiv 0 \quad(\bmod n)
$$

hinreichend und notwendig. Da $\lambda, \mu$ ein gegen $n$ teilerfremdes Paar sind, so kann man zwei ganze Zahlen $a, b$ angeben, die die Kongruenz $a \mu+b \lambda \equiv 1(\bmod n)$ befriedigen (vgl. S. $222 \mathrm{ff}$.). Durch Multiplikation dieser Kongruenz mit $l \mathrm{bzw}$. $m$ findet man bei Benutzung von (6):

$$
l \equiv(a m+b l) \lambda, \quad m \equiv(a m+b l) \mu \quad(\bmod n) .
$$


Die elliptischen Funktionen $n^{\text {tex }}$ Stufe $\Psi_{\lambda \mu}\left(u \mid \omega_{1}, \omega_{2}\right)$

Setzt man also zur Abkürzung $a m+b l=\nu$, so gelten die Kongruenzen $l \equiv \nu \lambda, \quad m \equiv \nu \mu(\bmod n)$. Umgekehrt folgt aus diesen Kongruenzen bei beliebigem $v$ auch wieder (6). Hieraus ergibt sich der Satz: Die eigentlich zur Stufe n gehörende Funktion $\Psi_{\lambda_{\mu}}(u)$ bleibt bei den Substitutionen derjenigen Kongruenzgruppe $n^{\text {ter }}$ Stufe $\Gamma_{n}^{u_{1}}$ unverändert, die sich mod $n$ auf die aus $S=(\lambda, \mu)$ zu erzeugende zyklische $G_{n}^{(u)}$ reduziert; zur gleichen $\Gamma_{n}^{(u)}$ gehören alle $\varphi(n)$ Funktionen $\Psi_{\lambda v, \mu v}(u)$, wo jetzt $\nu$ die $\varphi(n)$ inkongruenten und gegen $n$ teilerfremden Zahlen durchläuft.

Unter den $\psi(n)$ innerhalb der $\Gamma^{(n, \omega)}$ gleichberechtigten Gruppen $\Gamma_{n}^{(u)}$ bevorzugen wir zunächst die aus den beiden Substitutionen $u^{\prime}=u+n \omega_{1}, u^{\prime}=u+\omega_{2}$ erzeugbare. Als Diskontinuitätsbereich dieser Gruppe kann man das Parallelogramm der Ecken $0, \omega_{2}, n \omega_{1}+\omega_{2}, n \omega_{1}$ benutzen, das sich aus $n$ Parallelogrammen des ursprünglichen Netzes aufbaut. In $\Psi_{01}(u), \Psi_{02}(u), \ldots, \Psi_{0, n-1}(u)$ haben wir $(n-1)$ Funktionen, die bei den Substitutionen der ausgewählten $\Gamma_{n}^{(u)}$ unverändert bleiben, und deren einzelne bei Ausübung von $u^{\prime}=u+\omega_{1}$ das Verhalten zeigt $\Psi_{0 u}\left(u+\omega_{1}\right)=\varepsilon^{\mu} \Psi_{0 \mu}(u)$. Hieraus geht hervor, daß die $(n-1)$ Produkte:

$$
\Psi_{01}(u)^{v} \cdot \Psi_{0, n-v}(u),
$$$$
r=1, \mathbf{2}, \ldots, n-1
$$

die Perioden $\omega_{1}, \omega_{2}$ haben. Das einzelne Produkt $(7)$ stellt eine $(\nu+1)$ wertige Funktion mit einem Pole $(\nu+1)^{\text {ter }}$ Ordnung bei $u=0$ und $(\nu+1)$ Nullpunkten im Parallelogramm, von denen $\nu$ an der Stelle $\frac{\omega_{2}}{n}$ zusammenfallen. Das Anfangsglied der Reihe nach Potenzen ron $u$ ist zufolge (5) gleich $u^{-(v+1)}$.

Die Funktion (7) soll nun auf Grund der Regel (4) in I, 206 dargestellt werden. Wir benutzen dabei sogleich die Lage des $\boldsymbol{v}$-fachen Nullpunktes und verstehen unter $\wp_{\lambda \mu}^{\prime \prime}, \wp_{\lambda \mu}^{\prime \prime \prime}, \ldots$ die entsprechend den Gleichungen (7) S. 213 zu erklärenden "Teilwerte“ der Funktionen $\wp^{\prime \prime}(u)$, $\wp^{\prime \prime \prime}(u), \ldots$ Mit Rücksicht auf die Anfangsglieder der Reihen nach Potenzen von $u$ hat man den Ansatz:

$$
\begin{aligned}
& \Psi_{01}(u)^{v} \cdot \Psi_{0, n-v}(u)=a_{v 1}\left(\wp(u)-\wp_{01}\right)+a_{v 2}\left(\wp^{\prime}(u)-\wp_{01}^{\prime}\right)+\cdots \\
& \cdots+a_{v, v-1}\left(\wp^{(v-2)}(u)-\wp_{01}^{(v-2)}\right)+\frac{(-1)^{v-1}}{\nu !}\left(\wp^{(v-1)}(u)-\wp_{01}^{(v-1)}\right) .
\end{aligned}
$$

Zur Bestimmung der noch unbekannten Koeffizienten benutzen wir die Tatsache, daß (wegen des Nullpunktes $\dot{\nu}^{\text {ter }}$ Ordnung) auch noch die $(\nu-1)$ ersten Ableitungen der Funktion (8) an der Stelle $\frac{\omega_{2}}{n}$ verschwinden. Es gelten also die $(v-1)$ Gleichungen: 


$$
\left\{\begin{array}{l}
a_{v 1} \wp_{01}^{\prime}+a_{v 2} \wp_{01}^{\prime \prime}+\ldots+a_{v, v-1} \wp_{01}^{(v-1)}=\frac{(-1)^{v}}{v !} \wp_{01}^{(v)}, \\
a_{v 1} \wp_{01}^{\prime \prime}+a_{v 2} \wp_{01}^{\prime \prime \prime}+\ldots+a_{v, v-1} \wp_{01}^{(v)}=\frac{(-1)^{v}}{v !} \wp_{01}^{(v+1)} \\
\ldots \ldots \ldots \ldots \ldots \ldots \ldots \ldots \ldots \\
a_{v 1} \wp_{01}^{(v-1)}+a_{v 2} \wp_{01}^{(v)}+\ldots \ldots+a_{v, v-1} \wp_{01}^{(2 v-3)}=\frac{(-1)^{v}}{\nu !} \wp_{01}^{(2 v-2)}
\end{array}\right.
$$

Da für $v=1$ in (8) noch kein unbekannter Koeffizient auftritt, so haben wir dieses Gleichungssystem nur auf die $(n-2)$ Zahlen $\boldsymbol{\nu}=2,3, \ldots$, $(n-1) \mathrm{zu}$ beziehen. Als Determinante dieses Systems haben wir nach den Gleichungen (10) und (14) S. 186 ff.:

$$
D_{v}\left(\frac{\omega_{q}}{n}\right)=(-1)^{\nu-1}(2 ! \cdot 3 ! \cdot 4 ! \cdots(\nu-1) !)^{2} \psi^{(v)}\left(\frac{\omega_{2}}{n}\right),
$$

wo die Funktion $\psi^{(v)}(u)$ nach S. 189 bei ungeradem $\nu$ durch:

$$
\psi^{(v)}(u)=v \wp(u)^{\frac{1}{2}\left(v^{2}-1\right)}+\beta_{1}\left(\frac{1}{4} g_{2}\right) \wp(u)^{\frac{1}{2}\left(v^{2}-5\right)}+\beta_{2} g_{3} \wp(u)^{\frac{1}{2} \cdot\left(v^{2}-\tau\right)}+\cdots
$$

bei geradem $\nu$ aber durch:

$$
\begin{gathered}
\psi^{(v)}(u)=-\delta^{\prime}(u)\left(\frac{1}{2} \nu \wp(u)^{\frac{1}{2}\left(v^{2}-4\right)}+\gamma_{1}\left(\frac{1}{4} g_{2}\right) \wp(u)^{\frac{1}{2}\left(v^{2}-8\right)}\right. \\
\left.+\gamma_{2} g_{3} \wp(u)^{\frac{1}{2}\left(v^{2}-10\right)}+\cdots\right)
\end{gathered}
$$

gegeben ist und die $\beta$ und $\gamma$ ganze Zahlen sind. Diese in (3) S. 184 eingeführte und oben bereits ausführlich betrachtete Funktion hat die Wertigkeit $\left(\boldsymbol{\nu}^{2}-1\right)$, und ihre durchweg einfachen Nullpunkte liegen in den " $\nu^{\text {ten }}$ Teilpunkten" des Parallelogramms, d. h. an den Stellen, die sich als ganzzahlige Multipla von $\frac{\omega_{1}}{\nu}, \frac{\omega_{2}}{\nu}$ darstellen, unter Ausschlub des Nullpunktes $u=0$, in dem $\psi^{(\nu)}(u)$ unendlich wird. Mit $\psi_{\lambda \mu}^{(\eta)}$ bezeichnen wir die entsprechend (7) S. 213 zu erklärenden „Teilwerte" von $\psi^{(v)}(u)$ für den $n^{\text {ten }}$ Teilungsgrad. Da $\nu<n$ ist, so ist der in (10) rechts auftretende Tfilwert $\psi_{01}^{(2)}$ sicher von 0 verschieden, so daß das System (9) nach den $a$ auflösbar ist. Für die mit dem Faktor $\psi_{01}^{(\eta)}$ versehenen $a$ finden sich rationale ganze Ausdrücke der Teilwerte $\varsigma_{01}^{\prime}, \wp_{01}^{\prime \prime}, \ldots$ mit rationalen Zahlenkoeffizienten.

In dem daraufhin für das Produkt:

$$
\psi_{01}^{(v)} \cdot \Psi_{01}(u)^{\nu} \cdot \Psi_{0, n-v}(u)
$$

sich ergebenden Ausdrucke sollen endlich alle höheren Ableitungen $\wp^{\prime \prime}(u), \wp^{\prime \prime \prime}(u), \ldots, \wp^{(v-1)}(u)$ auf Grund der Gleichungen (16) S. 188 durch $\wp(u), \wp^{\prime}(u), g_{z}, g_{3}$ ausgedrückt werden, und ebenso mögen die Teilwerte $\wp_{01}^{\prime \prime}, \wp_{01}^{\prime \prime \prime}, \ldots$ durch die aus jenen Gleichungen für sie entspringenden Ausdrücke in $\wp_{01}, \wp_{01}^{\prime}, g_{2}, g_{3}$ ersetzt werden. In der neuen Gestalt des 
Produktes (13) kommt dann $\wp^{\prime}(u)$ sogleich nur in der ersten Potenz vor. Aber auch von $\wp_{01}^{\prime}$ können wir etwa zunächst auftretende höhere Potenzen mittelst der Relation $\wp_{01}^{\prime 2}=4 \wp_{01}^{3}-g_{2} \wp_{01}-g_{3}$ entfernen. Aus der in (8) auftretenden höchsten Ableitung $\rho_{(\nu}^{(v-1)}(u)$ folgern wir auf Grund der Gleichungen (16) ff. S. 188, daß bei ungeradem $v$ im neuen Ausdruck des Produktes (13) das Glied höchsten Grades die Potenz $\wp(u)^{\frac{v+1}{2}}$, das nächste Glied aber das Produkt $\wp^{\prime}(u) \cdot \wp(u)^{\frac{\nu-3}{2}}$ enthält. Bei geradem $\nu$ tritt im höchsten Gliede das Produkt $\wp^{\prime}(u) \cdot \wp(u)^{\frac{y-2}{2}}$ auf, im nächsten aber die Potenz $\wp(u)^{\frac{v}{2}}$. Die Koeffizienten sind nunmehr rationale ganze Funktionen von $\wp_{01}, \wp_{01}^{\prime}, g_{3}, g_{3}$ mit rationalen numerischen Koeffizienten, wobei wie bemerkt $\wp_{01}^{\prime}$ nur in erster Potenz auftritt. Die nähere Bauart der Koeffizienten geht aus dem Umstande hervor, daB das Produkt (13) in $u, \omega_{1}, \omega_{2}$ homogen von der Dimension $-\nu(\nu+1)$ ist. Da diese Zahl gerade ist, so wird in jedem Gliede, das $\wp^{\prime}(u)$ enthält, zugleich der Faktor $\wp_{01}^{\prime}$ auftreten. Fïr das Produkt (13) haben wir bei ungeradem $v$ die Darstellung:

$$
\begin{aligned}
& \psi_{01}^{(v)} \cdot \Psi_{01}(u)^{\nu} \Psi_{0, n-v}(u)=A_{0}^{(v)}+A_{1}^{(v)} \wp(u)+A_{2}^{(v)} \wp(u)^{2}+\cdots \\
+ & A_{\frac{v+1}{2}}^{(v)} \wp(u)^{\frac{v+1}{2}}+\wp_{01}^{\prime} \wp^{\prime}(u)\left(B_{0}^{(v)}+B_{1}^{(v)} \wp(u)+\cdots+\frac{\left.B_{\frac{v-3}{2}}^{(v)} \wp(u)^{\frac{v-3}{2}}\right),}{}\right.
\end{aligned}
$$

während sich für gerades $\nu$ ergibt:

$$
\begin{gathered}
\psi_{01}^{(v)} \cdot \Psi_{01}(u)^{\nu} \Psi_{0, n-\nu}(u)=A_{0}^{(v)}+A_{1}^{(\nu)} \wp(u)+A_{2}^{(v)} \wp(u)^{2}+\cdots \\
+A_{\frac{v}{2}}^{(\nu)} \wp(u)^{\frac{v}{2}}+\wp_{01}^{\prime} \wp^{\prime}(u)\left(B_{0}^{(\nu)}+B_{1}^{(v)} \wp(u)+\cdots+B_{\frac{\nu-2}{2}}^{(v)} \wp(u)^{\frac{\nu-2}{2}}\right) ;
\end{gathered}
$$

die Koeffizienten haben die Gestalten:

$$
\left\{\begin{array}{l}
A_{k}^{(v)}=\alpha_{k 1}^{(v)} \wp_{01}^{\frac{1}{2} v(v+1)-k}+\alpha_{k 2}^{(v)} g_{2} \wp_{01}^{\frac{1}{2} v(v+1)-k-2}+\alpha_{k 3}^{(v)} g_{3} \wp_{01}^{\frac{1}{2} v(v+1)-k-3}+\cdots \\
B_{k}^{(\nu)}=\beta_{k 1}^{(\nu)} \wp_{01}^{\frac{1}{2} v(v+1)-k-3}+\beta_{k 2}^{(v)} g_{2} \wp_{01}^{\frac{1}{2} v(v+1)-k-5}+\beta_{k 3}^{(v)} g_{3} \wp_{01}^{\frac{1}{2} v(v+1)-k-6}+\cdots
\end{array}\right.
$$

wo die $\alpha, \beta$ rationale Zahlen sind. Übrigens bestimmen sich die Koeffizienten der höchsten Glieder leicht aus den Anfangskoeffizienten der Reihenentwicklungen; man findet, je nachdem $\nu$ ungerade oder gerade ist:

$$
A_{\frac{v+1}{2}}^{(v)}=\psi_{01}^{(v)} \quad \text { bzw. } \quad B_{\frac{v-2}{2}}^{(v)}=-\frac{\psi_{01}^{(\nu)}}{2 \wp_{01}^{\prime}} .
$$

In den beiden niedersten Fällen $\nu=1$ und $\nu=2$, von denen der erste 
direkt aus (8) hervorgeht, findet man:

$$
\begin{gathered}
\Psi_{01}(u) \Psi_{0, n-1}(u)=\wp(u)-\wp_{01}, \\
\Psi_{01}(u)^{2} \Psi_{0, n-2}(u)=-\frac{4 \wp_{01}^{3}+g_{2} \wp_{01}+2 g_{3}}{4 \gamma_{01}^{\prime}}+\frac{12 \wp_{01}^{2}-g_{2}}{4 \wp_{01}^{\prime}} \wp(u)-\frac{1}{2} \wp^{\prime}(u) .
\end{gathered}
$$

Im nächsten Falle $\nu=3$ gilt:

$$
\Psi_{01}(u)^{3} \Psi_{0, n-3}(u)=\frac{A_{0}^{(3)}+A_{1}^{(3)} \wp(u)+B_{0}^{(3)} \wp_{01}^{\prime} \wp^{\prime}(u)}{3 \wp_{01}^{4}-\frac{3}{2} g_{2} \wp_{01}^{2}-3 g_{3} \wp_{01}-\frac{1}{16} g_{5}^{9}}+\wp(u)^{2}
$$

mit folgender Bedeutung der $A_{0}^{(3)}, A_{1}^{(3)}, B_{0}^{(3)}$ :

$$
\begin{aligned}
& A_{0}^{(3)}=-\wp_{01}^{6}-\frac{3}{2} g_{2} \wp_{01}^{4}-4 g_{3} \wp_{01}^{3}+\frac{3}{16} g_{2}^{2} \wp_{01}^{2}+{ }_{4}^{3} g_{2} g_{3} \wp_{01}+\frac{1}{2} g_{3}^{2}, \\
& A_{1}^{(3)}=+6 \wp_{01}^{5}-g_{2} \wp_{01}^{3}+3 g_{3} \wp_{01}^{2}+\frac{3}{8} g_{2}^{2} \wp_{01}^{2}+\frac{1}{4} g_{2} g_{3}, \\
& B_{0}^{(3)}=-2 \wp_{01}^{3}+\frac{1}{2} g_{2} \wp_{01}+\frac{1}{2} g_{3} .
\end{aligned}
$$

Auf die vorstehenden Rechnungen gründet sich die Methode, die elliptischen Funktionen $n^{\text {ter }}$ Stufe $\Psi_{2, \mu}(u)$ aus denjenigen der ersten Stufe zu berechnen. Wir haben in (14) bzw. (15) für $\Psi_{01}(u)^{v} \cdot \Psi_{0, n-v}(u)$ einen durch $R_{v}\left(\wp(u), \wp^{\prime}(u), \wp_{01}, \delta_{01}^{\prime}\right)$ zu bezeichnenden Ausdruck gewonnen, der $\operatorname{dem}$ Körper $\left(\Re, \wp_{01}, \wp_{01}^{\prime}\right)$ angehört, unter $\Omega$ den S. $212 \mathrm{im}$ AnschluB an die Koeffizienten der allgemeinen Teilungsgleichung erklärten Körper verstanden. Wir haben dann die $(n-1)$ Gleichungen:

$$
\Psi_{01}(u)^{v} \Psi_{0, n-v}(u)=R_{v}\left(\wp(u), \wp^{\prime}(u), \wp_{01}, \wp_{01}^{\prime}\right), \quad v=1 \text { \&, ,., ,n-1, }
$$

in deren letzter linker Hand die $n^{\text {to }}$ Potenz von $\Psi_{01}(u)$ steht. Die Auflösung nach den $\Psi_{0_{\mu}}(u)$ ist geleistet durch:

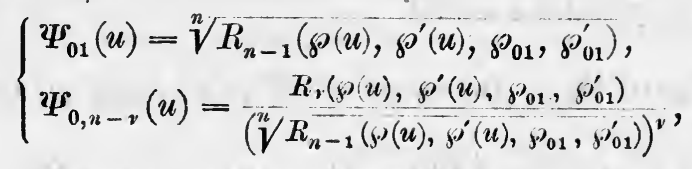

so daß zur Gewinnung der $(n-1)$ Funlitionen $\Psi_{0 \mu}(u)$ zum Körper $\left(\Re, \wp_{01}, \wp_{01}^{\prime}\right)$ nur die eine in der Gleichung (19) enthaltene $n^{\text {tc }}$ Wurzel zu adjungieren ist.

Da die übrigen Gruppen $\Gamma_{n}^{(u)}$ innerhalb der $\Gamma^{(u,(v)}$ mit der eben betrachteten Gruppe gleichberechtigt sind, so gelangen wir durch Ausübung geeigneter Substitutionen der $\Gamma^{(w)}$ auf (19) zu den entsprechend zu den übrigen Gruppen gehörenden Gleichungen. Bei diesen Substitutionen bleiben die Größen des Körpers $\Re$ invariant. Üben wir insbesondere die Substitution $\omega_{1}^{\prime}=-\omega_{2}, \omega_{2}^{\prime}=\omega_{1}$ aus, so folgt auf Grund der Regel (3) in I, 451 das Gleichungensystem:

$$
\left\{\begin{array}{l}
\Psi_{10}(u)=\sqrt[n]{R_{n-1}\left(\wp(u), \wp^{\prime}(u), \wp_{10}, \wp_{10}^{\prime}\right)}, \\
\Psi_{n-v, 0}^{\circ}(u)=\frac{R_{v}\left(\wp(u), \wp^{\prime}(u), \wp_{10}, \wp_{01}^{\prime}\right)}{\left(\sqrt[n]{R_{n-1}\left(\wp^{\prime}(u), \wp^{\prime}(u), \wp_{10}, \gamma_{10}^{\prime}\right)}\right)} .
\end{array}\right.
$$

Die hier auftretenden Funktionen $R_{v}$ gehören dem Körper $\left(\boldsymbol{\Re}, \wp_{10}, \wp_{10}^{\prime}\right)$ an. 
Nach S. 213 sind im Körper $\left(\Omega, \wp_{10}, \wp_{10}^{\prime}, \wp_{01}, \wp_{01}^{\prime}\right)$ bereits alle $\wp^{\circ-}$ und $\wp^{\prime}$-Teilwerte des $n^{\text {ten }}$ Teilungsgrades enthalten, so daB dieser Körper, wie schon S. $214 \mathrm{ff}$. geschah, durch $\left(\Re, \wp_{\lambda_{\mu}}, \wp_{\lambda_{\mu}}^{\prime}\right)$ bezeichnet werden mag. Es besteht nun der wichtige Satz: Nach Adjunktion der „beiden" in den ersten Gleichungen (19) und (20) gegebenen Wurzeln $n^{\text {ten }}$ Grades zum Körper ( $\left(, \wp_{i, \mu}, \wp_{\lambda \mu}^{\prime}\right)$ sind bereits ,alle $\left(n^{2}-1\right)$ eigentlich oder uneigentlich zur $n^{\text {ten }}$ Stufe gehörenden Funktionen $\Psi_{\lambda \mu}(u)$,rational bekannt". $\left.{ }^{1}\right)$ Ist nämlich $\Psi_{n-\lambda, n-\mu}(u)$ irgendeine unserer Funktionen mit zwei von 0 verschiedenen Indizes $n-\lambda, n-\mu$, so erweist sich:

$$
\Psi_{n-\lambda, n-\mu}(u) \cdot \Psi_{\lambda_{0}}(u) \Psi_{0 \mu}(u)
$$

auf Grund der Regel (4) S. 226 als eine Funktion der Perioden $\omega_{1}, \omega_{3}$, die übrigens dreiwertig ist und bei $u=0$ ihren Pol dritter Ordnung mit dem Anfangsgliede $u^{-3}$ der Reihenentwicklung hat. Nach (4) in I, 206 gestattet diese Funktion eine Darstellung:

$$
\Psi_{n-\lambda, n-\mu}(u) \cdot \Psi_{\lambda 0}(u) \Psi_{0 \mu}(u)=C_{0}+C_{1} \wp(u)-\frac{1}{2} \wp^{\prime}(u),
$$

in $\operatorname{der} C_{0}$ und $C_{1}$ von $u$ unalbhängig sind. Zur Bestimmung dieser Konstanten dient der Umstand, daß die dargestellte Funktion die Nullpunkte $\frac{\lambda \omega_{1}}{n}$ und $\frac{\mu \omega_{2}}{n}$ hat:

$$
C_{0}+C_{1} \wp_{\lambda 0}=\frac{1}{2} \wp_{\lambda 0}^{\prime}, \quad C_{0}+C_{1}^{\gamma} \wp_{0 \mu}=\frac{1}{8} \wp_{0 \mu}^{\prime} .
$$

Die Differenz $\left(\wp_{\lambda_{0}}-\wp_{0 \mu}\right)$ verschwindet nicht, da die zweiwertige Funktion $\left(\wp(u)-\varsigma_{0 \mu}\right)$ ihre beiden Nullpunkte im Periodenparallelogramm bei $\frac{\mu \omega_{2}}{n}$ und $\frac{(n-\mu) \omega_{2}}{\cdots n}$ hat und also nicht bei $\frac{\lambda \omega_{1}}{n}$. Nach Berechnung $\operatorname{der} C_{0}$, $C_{1}$ findet man

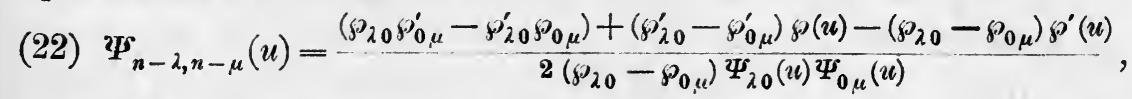

woraus der zu beweisende Satz hervorgeht.

\section{$\$ 5$. Lösung der allgemeinen Teilungsgleichung.}

Um die Auflösung der Teilungsgleichung nicht zu unterbrechen, soll eine Betrachtung über das Absolutglied der Reihenentwicklung (5) S. 226 vorausgesandt werden. Für die $n^{\text {te }}$ Potenz von $\Psi_{01}(u)$ ergibt sich mit Rïcksicht auf (19) S. 230:

$$
u^{-n}+\frac{n \sigma_{01}^{\prime}}{\sigma_{01}} u^{-n+1}+\cdots=R_{n-1}\left(\wp(u), \wp^{\prime}(u), \wp_{01}, \wp_{01}^{\prime}\right) .
$$

1) An Stelle der beiden zu den Wurzeln (19) und (20) gehörenden Kongruenzgruppen $\Gamma_{n}^{(i)}$ kann man irgend zwei dieser Gruppen, die komplementären $G_{n}^{(u)}$ entsprechen, zugrunde legen. 
Entwickelt man auch die rechte Seite nach Potenzen von $u$, so findet man als Koeffizienten von $u^{-n+1}$ einen rationalen Ausdruck in $g_{2}, g_{3}$, $\varsigma_{01}, \varsigma_{01}^{\prime}$ mit rationalen Zahlenkoeffizienten. In einer solchen Gestalt läßt sich also $\frac{\sigma_{01}^{\prime}}{\sigma_{01}}$ darstellen.

Die wirkliche Aufstellung des fraglichen Ausdrucks, und zwar sogleich für einen beliebigen Quotienten $\frac{\sigma_{2 \mu}^{\prime}}{\sigma_{2 \mu}}$, führt man indessen zweckmäßiger auf folgendem Wege aus: Aus der Erklärung von $\sigma_{\lambda \mu}(u)$ folgt unter Heranziehung des Integrals zweiter Gattung:

$$
\frac{\sigma_{\lambda \mu}^{\prime}(u)}{\sigma_{\lambda \mu}(u)}=\frac{\lambda \eta_{1}+\mu \eta_{2}}{n}+\xi\left(u-\frac{\lambda \omega_{1}+\mu \omega_{2}}{n}\right) \text {. }
$$

Für $u=0$ ergibt sich, wenn wir die , $\xi$-Teilwerte" $\xi_{2 \mu}$ wie üblich erklären:

$$
\frac{\sigma_{2 \mu}^{\prime}}{\sigma_{\lambda \mu}}=\frac{\lambda \eta_{1}+\mu \eta_{2}}{n}-\xi_{2, \mu^{\circ}}
$$

Durch Differentiation des Logarithmus der zur nächst niederen Zahl $(n-1)$ gehörenden Funktion:

$$
\psi^{(n-1)}(u)=\frac{\sigma((n-1) u)}{(\sigma(u))^{(n-1)(n-1)}}
$$

in bezug auf $u$ findet man:

$$
\frac{1}{n-1} \cdot \frac{d \log \psi^{(n-1)}(u)}{d u}=\xi((n-1) u)-(n-1) \xi(u) .
$$

Trägt man $u=\frac{\lambda \omega_{1}+\mu \omega_{2}}{n}$ ein, so möge das aus der linksstehenden'Ableitung folgende Ergebnis wieder durch Anhängung des Indexpaares $\lambda, \mu$ gekennzeichnet werden:

$\frac{1}{n-1}\left(\frac{d \log \psi^{(n-1)}(u)}{d u}\right)_{\lambda \mu}=\xi\left(-\frac{\lambda \omega_{1}+\mu \omega_{2}}{n}+\lambda \omega_{1}+\mu \omega_{2}\right)-(n-1) \xi_{\lambda \mu}$.

Das erste Glied der rechten Seite ist nach (5) in I, 196 gleich $\lambda \eta_{1}+\mu \eta_{2}-\xi_{\lambda \mu}$; die gesamte rechte Seite ist also gleich dem $n$-fachen Werte der rechten Seite von (1). So ergibt sich der Satz: Der Quotient (1) ist eine Modulform $n^{\text {ter }}$ Stufe $(-1)^{\text {ter }}$ Dimension, die sich aus der Funktion $\psi^{(n-1)}(u)$ nach der Regel:

$$
\frac{\sigma_{\lambda \mu}^{\prime}}{\sigma_{\lambda \mu}}=\frac{1}{n(n-1) \psi_{\lambda \mu}^{(n-1)}}\left(\frac{d \psi^{(n-1)}(u)}{d u}\right)_{\lambda \mu}
$$

berechnen läßt und also eine rationale Funktion von $g_{2}, g_{3}, \wp_{\lambda_{\mu}}, \wp_{\lambda_{\mu}}^{\prime}$ mit rationalen Zahlenkoeffizienten ist. Für $n=2$ verschwindet der Quotient (2); in den nächsten Fällen $n=3,4,5$ findet man: 
$6 \psi_{\lambda \mu}^{(2)} \frac{\sigma_{\lambda \mu}^{\prime}}{\sigma_{\lambda \mu}}=-6 \wp_{\lambda \mu}^{2}+\frac{1}{2} g_{2}$

$12 \psi_{2 \mu}^{(3)} \frac{\sigma_{\lambda \mu}^{\prime}}{\sigma_{\lambda \mu}}=3 \wp_{\lambda \mu}^{\prime}\left(4 \wp_{\lambda \mu}^{3}-g_{2} \wp_{\lambda \mu}-g_{3}\right)$,

$20 \psi_{\lambda \mu}^{(4)} \frac{\sigma_{\lambda \mu}^{\prime}}{\sigma_{\lambda, \mu}}=-60 \wp_{\lambda \mu}^{8}+68 g_{2} \wp_{\lambda \mu}^{6}+192 g_{3} \delta_{\lambda, \mu}^{5}-{ }_{2}^{5} g_{2}^{2} \wp_{\lambda \mu}^{4}-30 g_{2} g_{3} \wp_{\lambda, \mu}^{3}$

$$
-\left(\frac{23}{64} g_{2}^{3}+24 g_{3}^{2}\right) \wp_{\lambda_{\mu}}^{2}-2 g_{2}^{2} g_{3} \delta_{\lambda_{\mu}}+\left(\frac{1}{64} g_{2}^{4}-g_{2} g_{3}^{2}\right) \text {, }
$$

wo die Ausdrücke der $\psi_{\lambda \mu}$ in $g_{2}, g_{3}, \wp_{\lambda \mu} ; \wp_{\lambda \mu}^{\prime}$ aus (6) S. 185 abzulesen sind.

Um nun die Auflösung der Teilungsgleichung (3) S. 211 zu vollziehen, adjungieren wir zu dem S. 212 erklärten Körper $\Omega$ die Teilwerte $\wp_{\lambda \mu}, \wp_{\lambda \mu}^{\prime}$ des $n^{\text {ten }}$ Grades. Es wird sich im nächsten Kapitel zeigen, daß dem so zu gewinnenden Körper $\Omega^{\prime}=\left(\Re, \wp_{\lambda \mu}, \wp_{\lambda_{\mu}}^{\prime}\right)$ die $n^{\text {to }}$ Wurzel der Einheit $\varepsilon$ angehört, die demnach fortan als „rational bekannt" gelten darf.

Für die Summe der $n^{2}$ Lösungen der Teilungsgleichung lesen wir aus dieser Gleichung selbst die Darstellung ab:

$$
\sum_{\lambda, \mu} \wp_{\lambda, \mu}\left(\frac{u}{n}\right)=\sum_{\lambda, \mu} \wp_{\lambda \mu}\left(\frac{u}{n}+\frac{\lambda \omega_{1}+\mu \omega_{2}}{n}\right)=n^{2} \wp(u) .
$$

Weiter bilden wir, unter $l$ und $\lambda$ zwei Zahlen der Reihe $0,1,2, \ldots, n-1$ verstanden, die Summe:

$$
\sum_{\mu=0}^{n-1} \varepsilon^{-l \mu} \rho_{\lambda \mu}\left(\frac{u}{n}\right)=\sum_{\mu=0}^{n-1} \varepsilon^{-l \mu} \wp\left(\frac{u}{n}+\frac{\lambda \omega_{1}+\mu \omega_{2}}{n}\right) .
$$

Bei Zunahme von $u$ um $\omega_{2}$ geht diese Summe in sich selbst, multipliziert mit $\varepsilon^{b}$, über. Das Produkt der Summe und der Funktion $\Psi_{l m}(u)$ hat demnach (vgl. (4) S. 226) die Periode $\omega_{2}$; dabei ist $m$ aus der Reihe 0, 1, 2,.., $n-1$ willkürlich wählbar, nur unter Vermeidung der Kombination $l=0$, $m=0$. Man multipliziere dieses Produkt mit $\varepsilon^{m \lambda}$ und bilde die Summe aller Produkte für $\lambda=0,1, \ldots, n-1$. Die entstehende Funktion hat dann, wie man mit Hilfe von (4) S. 226 leicht feststellt, die Perioden $\omega_{1}, \omega_{2}$.

Von dieser Summe:

$$
\Psi_{l m}(u) \sum_{\lambda=0}^{n-1}\left(\varepsilon^{m \lambda} \sum_{\mu=0}^{n-1} \varepsilon^{-l \mu} \wp\left(\frac{u}{n}+\frac{\lambda \omega_{1}+\mu \omega_{2}}{n}\right)\right)
$$

bestimme man nun die beiden ersten Glieder der Entwicklung nach Potenzen von $u$. Für den Faktor $\Psi_{\imath m}(u)$ ist die Gleichung (5) S. 226 heranzuziehen. Bei der Doppelsumme rühren Glieder mit negativen Exponenten von $u$ nur von der Kombination $\lambda=0, \mu=0$ her, und zwar nur das eine Glied $n^{2} u^{-2}$. Man hat somit: 


$$
\Psi_{l m}(u) \sum_{\lambda, \mu} \varepsilon^{m \lambda_{2}-l_{l \mu} \gamma_{\lambda \mu}}\left(\frac{u}{n}\right)=n^{2} u^{-3}+\frac{n^{2} \sigma_{l m}^{\prime}}{\sigma_{l m}} u^{-2}+\cdots
$$

als die gesuchten Anfangsglieder der Reihenentwicklung.

Der Ausdruck (4) stellt nun eine dreiwertige Funktion der Perioden $\omega_{1}, \omega_{2}$ dar, deren drei Pole bei $u=0$ zusammenfallen, während einer der Nullpunkte von demjenigen der Funktion $\Psi_{l m}(u)$ geliefert wird. Sie ist demnach in der Gestalt:

$$
A\left(\wp(u)-\wp_{\imath m}\right)+B\left(\wp^{\prime}(u)-\wp^{\prime}{ }^{\prime}\right)
$$

darstellbar. Die Koeffizienten $A, B$ bestimmen sich vermittelst der beiden ersten in (5) angegebenen Reihengliedern. Für irgendeine der $\left(n^{2}-1\right)$ von $l=0, m=0$ verschiedenen Kombinationen $l, m$ gilt:

$$
\sum_{\lambda, \mu} \varepsilon^{m \lambda-l_{l \mu} \rho_{\eta_{\mu} \mu}}\left(\frac{u}{n}\right)=\frac{n^{z}}{\Psi_{l m}(u)}\left(\frac{\sigma_{l m}^{\prime}}{\sigma_{l m}^{\prime}}\left(\rho(u)-\rho_{l m}\right)-\frac{1}{2}\left(\rho^{\prime}(u)-\rho_{l m}^{\prime}\right)\right) .
$$

Man multipliziere nun, unter $\lambda_{0}, \mu_{0}$ irgendeine der $n^{2}$ Zahlkombinationen verstanden, die einzelne Gleichung (6) mit $\varepsilon^{l \mu_{0}-m \nu_{0}}$ und addiere alle $\left(n^{2}-1\right)$ Gleichungen zur Gleichung (3). In der Summe tritt linker Hand bei der einzelnen Funktion $\rho_{\lambda \mu}\left(\frac{u}{n}\right)$ der Faktor:

$$
\sum_{l, m} \varepsilon^{m\left(\lambda-\lambda_{0}\right)-l\left(\mu-\mu_{0}\right)}=\sum_{m} \varepsilon^{m\left(\lambda-\lambda_{0}\right)} \cdot \sum_{l} \varepsilon^{-l\left(\mu-\mu_{0}\right)}
$$

auf. Falls nicht $\lambda=\lambda_{0}, \mu=\mu_{0}$ zutrifft, ist mindestens eine der Summen rechter Hand gleich 0 ; nur wenn $\lambda=\lambda_{0}, \mu=\mu_{0}$ ist, sind beide rechts stehenden Summen von 0 verschieden und gleich $n$. Es ergibt sich somit unter Fortlassung der Indizes 0 bei $\lambda_{0}$ und $\mu_{0}$ :

$$
\rho_{\lambda, u}\left(\frac{u}{n}\right)=\wp^{\prime}(u)+\sum_{l, m}^{\prime} \varepsilon^{l, u-m \lambda} \cdot \frac{1}{\Psi_{l m}(u)}\left(\sigma_{l m}^{\prime}\left(\wp(u)-\rho_{l m}^{\prime}\right)-\frac{1}{2}\left(\rho^{\prime}(u)-\rho_{l m}^{\prime}\right)\right),
$$

wo durch den oberen Index am Summenzeichen angedeutet sein soll, daß die Kombination $l=0, m=0$ auszulassen ist. Diese Gleichung gibt die Auflösung der allgemeinen Teilungsgleichung; die sämtlichen Wurzeln sind rational bekannt, sobald zum Körper $\boldsymbol{\Omega}^{\prime}=\left(\boldsymbol{\Omega}, \wp_{\lambda_{\mu}}, \rho_{\lambda_{\mu}}^{\prime}\right)$ die beiden Wurzeln $n^{\text {ten }}$ Grades adjungiert sind, welche nach S.230ff. zur Berechnung der Funktionen $\Psi_{l m}(u)$ dienen. Das Ergebnis ist in Übereinstimmung mit der allgemeinen Theorie der zyklischen Gleichungen (S. $59 \mathrm{ff}$.) und dem S. 225 ausgesprochenen Satze, daB die allgemeine Teilungsgleichung nach Adjunktion der Teilwerte durch zwei zyklische Gleichungen $n^{\text {ten }}$ Grades lösbar sei.

\section{\$ 6. Divisionssätze der elliptischen Funktionen zweiter Stufe.}

Im Gebiete der elliptischen Funktionen zweiter Stufe führt das Problem der Division nicht mehr zu wesentlich neuen Sätzen; es handelt sich viel- 
mehr nur um eine formale Umgestaltung der bisherigen, auf die erste Stufe bezüglichen Sätze. Als gegeben hat man den Körper $\mathfrak{R}$ anzusehen, der durch Adjunktion des Integralmoduls $k^{2}$ und der drei Funktionen $\operatorname{sn} w$, en $w, \mathrm{dn} w$ aus dem rationalen Körper $\Re$ entsteht. $\mathrm{Zu}$ berechnen sind die Funktionen sn $\frac{w}{n}$, en $\frac{w}{n}, \mathrm{dn} \frac{w}{n}$, und zwar durch Auflösung der ,allgemeinen Teilungsgleichungen“, die aus den Multiplikationsformeln (1) und (3) S. 197 durch den Ersatz von $w$ durch $\frac{w}{n}$ hervorgehen.

Zur Erleichterung der Entwicklung kann man von dem Umstande Gebrauch machen, daB bei zusammengesetztem Grade $n=n_{1} \cdot n_{2}$ unser Problem zerlegt werden kann in die beiden auf $n_{1}$ und $n_{8}$ einzeln bezogenen Probleme. Man kann demnach so vorgehen, daß man zunächst die Teilung zweiten Grades behandelt, deren wiederholte Ausübung die Grade $n=2^{v}$ erledigt. Es verbleibt dann nur noch die Besprechung der Teilung ungeraden Grades.

Aus den drei Gleichungen ( 7 ) S. 198 folgt, falls man in ihnen $w$ durch $\frac{w}{2 n}$ ersetzt:

$$
\left\{\begin{aligned}
\operatorname{sn} w & =\frac{2 \operatorname{sn} \frac{w}{2} \operatorname{cn} \frac{w}{2} \operatorname{dn} \frac{w}{2}}{1-k^{2} \operatorname{sn} \frac{w^{4}}{2}}, \\
\operatorname{cn} w & =\frac{\operatorname{cn} \frac{w^{2}}{2}-\operatorname{sv} \frac{w^{2}}{2} \operatorname{dn} \frac{w^{2}}{2}}{1-k^{2} \operatorname{sn} \frac{w^{4}}{2}}, \\
\operatorname{dn} w & =\frac{\operatorname{dn} \frac{w^{2}}{2}-k^{2} \operatorname{sn} \frac{w^{2}}{2} \operatorname{cn} \frac{w^{2}}{2}}{1-k^{2} \operatorname{sn} \frac{w^{4}}{2}} .
\end{aligned}\right.
$$

Das Teilungsproblem zweiten Grades fordert nun die Berechnung von $\operatorname{sn} \frac{w}{2}$, en $\frac{w}{2}, d n \frac{w}{2}$ aus diesen drei Gleichungen, d. h. bei gegebenen $\operatorname{sn} w$, en $w, \operatorname{dn} w$. Die Lösung dieses Problems erfordert an irrationalen Operationen das Ausziehen zweier Quadratwurzeln. Mittelst der beiden zwischen den Funktionen sn, cn, dn bestehenden quadratischen Relationen (vgl. I, 389) folgert man aus (1):

$$
\begin{gathered}
1+\operatorname{cn} w=\frac{2 \operatorname{cn} \frac{w^{2}}{2}}{1-k^{2} \operatorname{sn} \frac{w^{4}}{2}}, \quad 1+\operatorname{dn} w=\frac{2 \operatorname{dn} \frac{w^{2}}{2}}{1-k^{2} \operatorname{sn} \frac{x^{4}}{2}}, \\
\operatorname{cn~} w+\operatorname{dn} w=\frac{2 \operatorname{cn} \frac{w^{2}}{2} \operatorname{dn} \frac{w^{2}}{2}}{1-k^{2} \operatorname{sn} \frac{w^{4}}{2}} .
\end{gathered}
$$


Als Auflösungen dieser Gleichungen nach en $\frac{w}{2}$ und dn $\frac{w}{2}$ ergeben sich:

$$
\operatorname{cn} \frac{w}{2}= \pm \sqrt{\frac{\operatorname{cn} w+\operatorname{dn} w}{1+\operatorname{dn} w}}, \quad \operatorname{dn} \frac{w}{2}= \pm \sqrt{\frac{\operatorname{cn} w+\operatorname{dn} w}{1+\operatorname{cn} w}}
$$

während sich sn $\frac{w}{2}$ mit Hilfe der ersten Gleichung (1) in en $\frac{w}{2}$ und $\mathrm{dn} \frac{w}{2}$ eindeutig berechnet:

$$
\operatorname{sn} \frac{w}{2}=\frac{\operatorname{sn} w}{1+\operatorname{cn} w} \cdot \frac{\operatorname{cn} \frac{w}{2}}{\operatorname{dn} \frac{w}{2}}=\frac{\operatorname{sn} w}{1+\operatorname{dn} w} \cdot \frac{\operatorname{dn} \frac{w}{2}}{\operatorname{cn} \frac{w}{2}} .
$$

Aus (1) ergeben sich zugleich die Formeln, mittelst deren wir die Divisionsgleichungen erster Stufe auf diejenigen der zweiten Stufe umzurechnen haben. Die drei Gleichungen (10) in I, 389 nehmen bei Ersatz von $u$ und $w$ durch $\frac{u}{2}$ und $\frac{w}{2}$ die Gestalten an:

$\operatorname{sn} \frac{w}{2}=\frac{\sqrt{e_{2}-e_{1}}}{\sqrt{\wp\left(\frac{u}{2}\right)-e_{1}}}, \quad \operatorname{cn} \frac{w}{2}=\frac{\sqrt{\wp\left(\frac{u}{2}\right)-e_{2}}}{\sqrt{\wp\left(\frac{u}{2}\right)-e_{1}}}, \quad \operatorname{dn} \frac{w}{2}=\frac{\sqrt{\wp\left(\frac{u}{2}\right)-e_{s}}}{\sqrt{\wp\left(\frac{u}{2}\right)-e_{1}}}$.

Durch Eintragen dieser Ausdrücke in die drei Gleichungen (1) ergibt sich:

$$
\left\{\begin{array}{l}
\operatorname{sn} w=\frac{\sqrt{e_{2}-e_{1}} \wp^{\prime}\left(\frac{u}{2}\right)}{\left(\wp\left(\frac{u}{2}\right)-e_{1}\right)^{2}-k^{2}\left(e_{2}-e_{1}\right)^{2}}, \\
\operatorname{cn} w=\frac{\left(\wp\left(\frac{u}{2}\right)-e_{2}\right)^{2}-\left(2 e_{2}^{2}+e_{3} e_{1}\right)}{\left(\wp\left(\frac{u}{2}\right)-e_{1}\right)^{2}-k^{2}\left(e_{2}-e_{1}\right)^{2}} \\
\operatorname{dn} w=\frac{\left(\wp\left(\frac{u}{2}\right)-e_{3}\right)^{2}-\left(2 e_{3}^{2}-e_{1} e_{2}\right)}{\left(\wp\left(\frac{u}{2}\right)-e_{1}\right)^{2}-k^{2}\left(e_{2}-e_{1}\right)^{2}}
\end{array}\right.
$$

wo bei der Umrechnung von den beiden bekannten Gleichungen:

$$
k^{2}=\frac{e_{3}-e_{1}}{e_{2}-e_{1}}, \quad e_{1}+e_{2}+e_{3}=0
$$

Gebrauch zu machen ist. Es erscheint nun zweckmäBig, an Stelle von $\wp(u)$ und $\wp^{\prime}(u)$ weiterhin die beiden Funktionen nullter Dimension einzuführen:

$$
p(u)=\frac{\wp(u)}{e_{2}-e_{1}}, \quad p^{\prime}(u)=\frac{\wp^{\prime}(u)}{\left(\sqrt{e_{2}-e_{1}}\right)^{3}} .
$$

In den auf diese Funktionen umgerechneten Gleichungen (4) stellen sich die Koeffizienten rational in $k^{2}$ allein dar: 


$$
\left\{\begin{array}{l}
\operatorname{sn} w=\frac{9 p^{\prime}\left(\frac{u}{2}\right)}{\left(3 p\left(\frac{u}{2}\right)+1+k^{2}\right)^{2}-9 k^{2}}, \\
\operatorname{cn} w=\frac{\left(3 p\left(\frac{u}{2}\right)+k^{2}-2\right)^{2}-9\left(1-k^{2}\right)}{\left(3 p\left(\frac{u}{2}\right)+1+k^{2}\right)^{2}-9 k^{2}}, \\
\text { dn } w=\frac{\left(3 p\left(\frac{u}{2}\right)+1-2 k^{2}\right)^{2}+9 k^{2}\left(1-k^{2}\right)}{\left(3 p\left(\frac{u}{2}\right)+1+k^{2}\right)^{2}-9 k^{2}}
\end{array} .\right.
$$

Man kann diese Gleichungen in folgender Art deuten: In $p\left(\frac{u}{2}\right)$, $p^{\prime}\left(\frac{u}{2}\right)$ hat man ein Funktionssystem der Hauptkongruenzgruppe zweiter Stufe $\Gamma_{4}^{(u)}$. Zu dieser Gruppe gehören auch die Funktionen sn $w$, en $w$, dn $w$, so daB sie sich rational in $p\left(\frac{u}{2}\right), p^{\prime}\left(\frac{u}{2}\right)$ darstellen lassen, was eben durch die Formeln (6) geschehen ist. Es muB sich aber auch umgekehrt in $\operatorname{sn} w, \operatorname{cn} w, \mathrm{dn} w$ jede weitere Funktion jener Gruppe rational darstellen lassen. Um z. B. $p\left(\frac{u}{2}\right)$ und $p^{\prime}\left(\frac{u}{2}\right)$ in sn $w$, en $w$, dn $w$ darzustellen, entnehmen wir aus (6):

$$
\begin{aligned}
& \frac{3 p\left(\frac{u}{2}\right)+1+k^{2}}{3 p^{\prime}\left(\frac{u}{2}\right)}=\frac{\mathrm{dn} w-\operatorname{cn} w}{2\left(1-k^{2}\right) \operatorname{cn} w}, \\
& \frac{3 p\left(\frac{u}{2}\right)+k^{2}-2}{3 p^{\prime}\left(\frac{u}{2}\right)}=\frac{1-\operatorname{dn} w}{2 k^{2} \operatorname{sn} w}, \\
& \frac{3 p\left(\frac{u}{2}\right)+1-2 k^{2}}{3 p^{\prime}\left(\frac{u}{2}\right)}=\frac{1-\operatorname{cn} w}{2 \operatorname{sn} w} .
\end{aligned}
$$

Durch Addition dieser drei Gleichungen, sowie zweitens durch Subtraktion der zweiten von der dritten gelangt man leicht zu folgenden Ausdrücken der $p\left(\frac{u}{2}\right), p^{\prime}\left(\frac{u}{2}\right)$ in $\operatorname{sn} w$, en $w, \operatorname{dn} w$ :

$$
\left\{\begin{array}{l}
p\left(\frac{u}{2}\right)=\frac{1-k^{4}+k^{2}\left(k^{2}-2\right) \operatorname{cn} w+\left(2 k^{2}-1\right) \operatorname{dn} w}{-3+3 k^{2}-3 k^{2} \operatorname{cn} w+3 \operatorname{dn} w} \\
p^{\prime}\left(\frac{u}{2}\right)=\frac{2 k^{2}\left(1-k^{2}\right) \operatorname{sn} w}{-1+k^{2}-k^{2} \operatorname{cn} w+\operatorname{dn} w} .
\end{array}\right.
$$

Für einen ungeraden 'Teilungsgrad $n$ bezeichnen wir als zugehörige ,Teilwerte" der Funktionen sn, cn, dn die Größen: 


$$
\left\{\begin{array}{l}
\mathrm{sn}_{\lambda \mu}=\mathrm{sn} \frac{4 \lambda i K^{\prime}+4 \mu K}{n}, \\
\mathrm{cn}_{\lambda \mu}=\mathrm{cn} \frac{4 \lambda i K^{\prime}+4 \mu K}{n}, \\
\mathrm{dn}_{\lambda \mu}=\mathrm{dn} \frac{4 \lambda i K^{\prime}+4 \mu K}{n} .
\end{array}\right.
$$

Nach (5) in I, 388 entspricht dem rechts stehenden Argumente der Wert $u=\frac{2 \lambda \omega_{1}+2 \mu \omega_{2}}{n}$ und also $\frac{u}{2}=\frac{\lambda \omega_{1}+\mu \omega_{2}}{n}$. Für die Funktionen (5) erklären wir die Teilwerte natürlich durch:

$$
p_{\lambda \mu}=\frac{\wp_{\lambda \mu}}{e_{2}-e_{1}}, \quad p_{\lambda \mu}^{\prime}=\frac{\rho_{\lambda \mu}^{\prime}}{\left(\sqrt{e_{2}-e_{1}}\right)^{3}} .
$$

Aus (6) ergibt sich nun:

$$
\begin{aligned}
& \mathrm{su}_{\lambda \mu}: \mathrm{cn}_{\lambda_{\mu}}: \mathrm{dn}_{\lambda \mu}: 1=9 p_{\lambda_{\mu}}^{\prime}:\left(\left(3 p_{\lambda_{\mu}}+k^{2}-2\right)^{2}-9\left(1-k^{2}\right)\right) \\
& \quad:\left(\left(3 p_{\lambda \mu}+1-2 k^{2}\right)^{2}+9 k^{2}\left(1-k^{2}\right)\right):\left(\left(3 p_{\lambda \mu}+1+k^{2}\right)^{2}-9 k^{2}\right),
\end{aligned}
$$

und andrerseits folgt aus (7):

$$
\begin{aligned}
p_{\lambda \mu}: p_{\lambda \mu}^{\prime}: 1= & \left(1-k^{4}+k^{2}\left(k^{2}-2\right) \mathrm{cn}_{\lambda \mu}+\left(2 k^{2}-1\right) \mathrm{dn}_{\lambda \mu}\right) \\
& : 6 k^{2}\left(1-k^{2}\right) \mathrm{sn}_{\lambda \mu}: 3\left(-1+k^{2}-k^{2} \mathrm{cn}_{\lambda \mu}+\mathrm{dn}_{\lambda \mu}\right) .
\end{aligned}
$$

Es gilt also der Satz: Die für ein ungerades $n$ erklärten T'eilwerte $\mathrm{sn}_{\lambda \mu}, \mathrm{cn}_{\lambda \mu}, \mathrm{dn}_{\lambda_{\mu}}$ sind unkehrbar rational in den zum gleichen $n$ gehörenden Teilwerten $p_{\lambda_{\mu}}, p_{\lambda_{\mu}}^{\prime}$ darstellbar, wobei die Koeffizienten rationale Ausdrücke in $k^{2}$ mit rationalen Zahlenkoeffizienten sind.

Um nun bei ungeradem $n$ die Divisionssätze für die zweite Stufe zu erhalten, tragen wir erstlich in die Gleichungen von S. $211 \mathrm{ff} . \frac{2}{2}^{u}$ statt $u$ ein und schreiben zur Abkürzung $\frac{u}{2}=v$. Wir beachten sodann, daB jene Gleichungen in den $u, \omega_{1}, \omega_{2}$ homogen sind und demnach durch Division mit einer geeigneten Potenz der Wurzel $\sqrt{e_{2}-e_{1}}$, die nach I, 473 ein Modulform $4^{\text {ter }}$ Stufe der Dimension -1 ist, in Gleichungen der Dimension 0 umgewandelt werden können. Indem wir diese Umwandlung an den einzelnen in den Gleichungen verbundenen Größen vornehmen, haben wir an Stelle von $\wp(v)$ und $\wp^{\prime}(v)$ mit $p(v)$ und $p^{\prime}(v)$ zu tun, an Stelle $\operatorname{der} g_{2}$ und $g_{3}$ aber mit den rationalen Funktionen von $k^{2}$ :

$$
\frac{g_{2}}{\left(e_{2}-e_{1}\right)^{2}}=\frac{4}{3}\left(1-k^{2}+k^{4}\right), \quad \frac{g_{3}}{\left(e_{2}-e_{1}\right)^{3}}=\frac{4}{27}\left(2-3 k^{2}-3 k^{4}+2 k^{6}\right),
$$

die man leicht aus den Darstellungen der $g_{2}, g_{3}$ in den $e_{1}, e_{2}, e_{3}$ ableitet. An die Stelle des Körpers, der durch Adjunktion von $g_{2}, g_{3}, p(u), p^{\prime}(u)$ zum rationalen Körper $\Re$ entsteht, tritt bei Ersatz von $u$ durch $v$ und Übergang zur Dimension 0 der folgende Körper: $\Re=\left(\Re, k^{2}, p(v), p^{\prime}(v)\right)$. 
Der Hauptsatz der Teilungstheorie war, daß die Lösung der auf die Dimension 0 umgerechneten Teilungsgleichung (3) S. 211 nach Adjunktion $\operatorname{der} p_{\lambda_{\mu}}, p_{\lambda \mu}^{\prime}$ an irrationalen Operationen nur das Ausziehen zweier Wurzeln $n^{\text {ten }}$ Grades erfordert. Nun kann $\Re$ zufolge $(6)$ und $(7)$ auch durch $\left(\Re, k^{2}, \operatorname{sn} w\right.$, en $\left.w, \operatorname{dn} w\right)$ erklärt werden, und ebenso ist $\left(\Re, p_{\lambda_{\mu}}, p_{\lambda_{\mu}}^{\prime}\right)$ zufolge (10) und (11) mit dem Körper $\left(\Re, \mathrm{sn}_{\lambda \mu}, \mathrm{cn}_{\lambda, \mu}, \mathrm{dn}_{\lambda, \mu}\right)$ gleich. Endlich aber sind die Lösungen des Teilungsproblems $p_{\lambda \mu}\left(\frac{v}{n}\right), p_{\lambda \mu}^{\prime}\left(\frac{v}{n}\right)$ einerseits und $\operatorname{sn}_{\lambda \mu}\left(\frac{w}{n}\right), \mathrm{cn}_{\lambda \mu}\left(\frac{w}{n}\right), \mathrm{dn}_{\lambda \mu}\left(\frac{w}{n}\right)$, gegeben durch:

$$
\operatorname{sn}_{\lambda \mu}\left(\frac{w}{n}\right)=\operatorname{sn}\left(\frac{w}{n}+\frac{4 \lambda i K^{\prime}+4 \mu K}{n}\right), \cdots,
$$

andrerseits gegenseitig rational ineinander mit Koeffizienten des Körpers $\left(\Re, k^{2}\right)$ darstellbar (Gleichungen (6) und (7)). Also ist in der Tat mit der algebraischen Lösung der Teilungsgleichung (3) S. 211 die Lösung des Teilungsproblems für die zweite Stufe sogleich mitgegeben.

Es erübrigt, noch einige Angaben über die äußere Gestalt des Teilungsproblems zweiter Stufe hinzuzufügen. Da $n$ als ungerade gilt, so haben wir an die Gleichungen (1) S. 197 anzuknüpfen, in denen $\frac{w}{n}$ an Stelle von $w$ einzusetzen ist. Aus der zweiten und dritten der genannten Gleichungen folgt:

$$
\operatorname{cn}_{\lambda \mu}\left(\frac{w}{n}\right)=\frac{\operatorname{cn} w \cdot G_{0}^{(n)}\left(\operatorname{sn}_{\lambda \mu}\left(\frac{w}{n}\right)^{2}\right)}{G_{:}^{(n)}\left(\operatorname{sn}_{\lambda \mu}\left(\frac{w}{n}\right)^{2}\right)}, \operatorname{dn}_{\lambda \mu}\left(\frac{w}{u}\right)=\frac{\operatorname{dn} w \cdot G_{0}^{(n)}\left(\operatorname{sn}_{\lambda \mu}\left(\frac{w}{n}\right)^{2}\right)}{G_{3}^{(n)}\left(\operatorname{si}_{i \mu}\left(\frac{w}{n}\right)^{2}\right)},
$$

während aus der ersten Gleichung, in der wir sn $v=\sqrt{z}=Z$ eintragen, sich als ,allgemeine Teilungsgleichung der sn-Funlition fïr den ungeraden Teilungsgrad $n^{\text {" }}$ ergibt:

$$
Z \cdot G_{1}^{(n)}\left(Z^{2}\right)-\operatorname{sn} w \cdot G_{0}^{(n)}\left(Z^{2}\right)=0
$$

oder explizite unter Angabe der höchsten und niedersten Glieder:

$$
\cdots-(-1)^{\frac{n-1}{2}} \frac{n\left(n^{2}-1\right)}{3 !}\left(1+k^{2}\right) Z^{3}+(-1)^{\frac{n-1}{2}} n Z-(-1)^{\frac{n-1}{2}} \operatorname{sn} w=0 \text {. }
$$

Diese Gleichung, deren Glieder alternierend den Faktor sn $w$ haben und übrigens ganze ganzzahlige Funktionen von $k^{2}$ zu Koeffizienten besitzen, tritt also für die zweite Stufe bei ungeradem $n$ an die Stelle der Gleichung (3) S. 211.

Als beiläufiges Ergebnis ziehen wir noch aus (13) für $w=0$ die Folgerung: 


$$
\mathrm{cn}_{\lambda \mu}=\frac{G_{0}^{(n)}\left(\mathrm{sn}_{\lambda \mu}^{2}\right)}{G_{2}^{(n)}\left(\operatorname{si}_{\lambda \mu}^{2}\right)}, \quad \mathrm{dn}_{\lambda \mu}=\frac{G_{0}^{(n)}\left(\mathrm{sn}_{\lambda \mu}^{2}\right)}{G_{3}^{(n)}\left(\mathrm{sn}_{\lambda \mu}^{2}\right)} .
$$

Bei ungeradem $n$ sind also die Teilwerte $\mathrm{cn}_{\lambda_{\mu}}, \mathrm{dn}_{\lambda_{\mu}}$ rational in $\mathrm{sn}_{\lambda_{\mu}}^{2}$ darstellbar mit Koeffizienten des Körpers $\left(\Re, k^{2}\right)$.

\section{\$ 7. Die Abelschen Relationen.}

Für die algebraischen Überlegungen des nächsten Kapitels sind gewisse Relationen zwischen den Teilwerten des $n^{\text {ten }}$ Teilungsgrades und den $n^{\text {ten }}$ Wurzeln der Einheit von Wert, die wir zweckmäßig schon hier aufstellen, da sich die Methode ihrer Aufstellung an die voraufgehenden Entwicklungen anschließt. Die fraglichen Relationen betreffen ein beliebiges ungerades $n$ und sind nach Abel benannt, weil sie von ihm für die Funktionen zweiter Stufe in Nr. 6 der Einleitung zum „Précis d'une théorie des fonctions elliptiques" ${ }^{\text {(1) }}$ angegeben sind. Bewiesen und für die algebraische Berechnung der Teilwerte verwertet wurden die Abelschen Relationen durch Sylow ${ }^{2}$ ) sowie später durch Kronecker ${ }^{3}$ ), dem die Untersuchungen Sylows zunächst unbekannt geblieben waren. Für die erste Stufe sind die Abelschen Relationen im Jahre 1884 durch Klein in einer Vorlesung aufgestellt. ${ }^{4}$ )

Man kann die Abelschen Relationen zunächst für die Funktionen erster Stufe auf folgendem Wege gewinnen. Der Quotient:

$$
\frac{\wp(u)-e_{2}}{\wp^{\prime}(u)}=\varphi_{2}(u)
$$

stellt eine zweiwertige Funktion der Perioden $\omega_{1}, \omega_{2}$ dar, deren Nullpunkte bei $u=0$ und $\frac{\omega_{2}}{2}$, und deren Pole bei $u=\frac{\omega_{1}}{2}$ und $\frac{\omega_{1}+\omega_{2}}{2}$ liegen. Die Funktion $\varphi_{2}\left(u+\frac{\omega_{2}}{2}\right)$ hat dieselben Nullpunkte und Pole, ist also bis auf einen von $u$ unabhängigen Faktor gleich $\varphi_{2}(u)$. Dieser Faktor kann nur gleich +1 oder -1 sein, da $\varphi_{2}(u)$ bei zweimaliger Ausübung der Substitution $u^{\prime}=u+\frac{\omega_{1}}{2}$ unverändert bleibt. Der Faktor +1 ist nicht brauchbar, da $\varphi_{2}(u)$ sonst eine einwertige Funktion der Perioden $\omega_{1}$, $\frac{\omega_{2}}{2}$ wäre; es gilt also:

$$
\varphi_{2}\left(u+\omega_{1}\right)=\varphi_{2}(u), \quad \varphi_{2}\left(u+\frac{\omega_{2}}{2}\right)=-\varphi_{2}(u) .
$$

1) Journ. f. Math., Bd. 4 (1829).

2) In den Schriften der Gesellsch. d. Wiss. zu Christiania von 1864 und 71.

3) „Über die algebraischen Gleichungen, von denen die Teilung der elliptischen Funktionen abhängt", Berliner Berichte von 1875.

4) Mit Erweiterungen veröffentlicht durch Engel in den Berichten der Gesellsch. d. Wiss. zu Leipzig von 1884. 
Für beliebiges ungerades $n$ bilde man die Summe:

$$
\Phi_{2}(u)=\sum_{\mu=0}^{n-1} \varepsilon^{2 \lambda, "} \varphi_{2}\left(u+\frac{u \omega_{2}}{n}\right),
$$

wo $\varepsilon$ in der bisherigen Bedeutung als $n^{\text {te }}$ Wurzel der Einheit gebraucht ist und $\lambda$ der Zahlenreihe $0,1,2, \ldots, n-1$ angehört. Wächst $u$ um $\frac{\omega_{2}}{2 n}$, so kann man mit Rücksicht auf die zweite Gleichung (2) schreiben:

$$
\varphi_{2}\left(u+\frac{(2 \mu+1) \omega_{2}}{2 n}\right)=-\varphi_{2}\left(u+\frac{\left(\mu+\frac{1}{2}(n+1)\right) \omega_{2}}{n}\right)=-\varphi_{2}\left(u+\frac{\mu^{\prime} \omega_{2}}{n}\right),
$$

wo zur Abkürzung $\mu^{\prime}=\mu+\frac{1}{2}(n+1)$ gesetzt ist. Da

$$
\varepsilon^{2 \lambda \mu}=\varepsilon^{2 \lambda\left(\mu^{\prime}-\frac{1}{2}(n+1)\right)}=\varepsilon^{-\lambda} \cdot \varepsilon^{2 \lambda \mu^{\prime}}
$$

ist, so folgt aus (3):

$$
\Phi_{2}\left(u+\frac{\omega_{2}}{2 n}\right)=-\varepsilon^{-\lambda} \sum_{\mu^{\prime}} \varepsilon^{2 \lambda u^{\prime}} \varphi_{2}\left(u+\frac{\mu^{\prime} \omega_{2}}{n}\right),
$$

wo $\mu^{\prime}$ beginnend mit $\frac{1}{2}(n+1)$ ein volles Restsystem mod $n$ durchläuft. Aber bei Änderung von $\mu^{\prime}$ um ein Vielfaches von $n$ bleibt das einzelne Glied dieser Summe unverändert, so daß die in der letzten Gleichung rechts stehende Summe wieder gleich $\Phi_{2}(u)$ ist. Die Funktion $\Phi_{2}(u)$ genügt den Bedingungen:

$$
\Phi_{2}\left(u+\omega_{1}\right)=\Phi_{2}(u), \quad \Phi_{2}\left(u+\frac{\omega_{2}}{2 n}\right)=-\varepsilon^{-\imath} \Phi_{2}(u),
$$

stellt also nach I, 217 eine elliptische Funktion zweiter Art der Perioden $\omega_{1}^{\prime}=\omega_{1}, \omega_{2}^{\prime}=\frac{\omega_{2}}{2 n}$ und des Faktorensystems $\lambda_{1}=1, \lambda_{2}=-\varepsilon^{-\lambda}$ dar.

Diese Funktion hat nun im zugehörigen Parallelogramme der Perioden $\omega_{1}^{\prime}, \omega_{2}^{\prime}$ nur einen einzigen Pol, nämlich bei $u=w=\frac{\omega_{1}^{\prime}}{2}$. Sie hat demnach daselbst auch nur einen Nullpunkt $v$, für den aus (8) in I, 220 die Gleichung folgt:

$$
v-w=v-\frac{\omega_{1}^{\prime}}{2}=m_{1} \omega_{1}^{\prime}+m_{2} \omega_{2}^{\prime}-\frac{\omega_{1}^{\prime}}{2 i \pi} \log \left(-\varepsilon^{-\lambda}\right) .
$$

Sehen wir vom Periodenmultiplum $\left(m_{1} \omega_{1}^{\prime}+m_{2} \omega_{2}^{\prime}\right)$ ab und schreiben:

$$
\omega_{1}^{\prime}=\omega_{1}, \quad \log \left(-\varepsilon^{-\lambda}\right)=\pi i-\lambda \log \varepsilon=\pi i-\lambda \frac{2 i \pi}{n},
$$

so ergibt sich als Lage des einzigen Nullpunktes von $\Phi_{2}(\imath)$ im Parallelogramme der Perioden $\omega_{1}^{\prime}=\omega_{1}, \omega_{2}^{\prime}$ sofort $v=\frac{\lambda \omega_{1}}{n}$. Unter Rückgang auf die Gestalt (3) von $\Phi_{2}(u)$ und die Bedeutung (1) von $\varphi_{3}(u)$ ergibt sich bei Einführung der Teilwerte: Fïr irgendein $\lambda$ der Zahlenreihe $0,1,2, \ldots$, $n-1$ gilt: 


$$
\sum_{\mu=0}^{n-1} \varepsilon^{22 . \mu} \frac{\wp_{\lambda \mu}-e_{2}}{\wp_{\lambda \mu}^{\prime}}=0
$$

dagegen ist für zwei „verschiedene" $\lambda^{\prime}, \lambda$ stets:

$$
\sum_{\mu=0}^{n-1} \varepsilon^{2 \lambda^{\prime} \mu} \frac{\rho^{3} \lambda_{\mu}-e_{2}}{\gamma^{\prime} \lambda_{\mu}} \neq 0
$$

Eine entsprechende Entwicklung kann man an den Quotienten:

$$
\frac{\wp(u)-e_{1}}{\wp^{\prime}(u)}=\varphi_{1}(u)
$$

knüpfen, der den Bedingungen:

$$
\varphi_{1}\left(u+\frac{\omega_{1}}{2}\right)=-\varphi_{1}(u), \quad \varphi_{1}\left(u+\omega_{2}\right)=\varphi_{1}(u)
$$

genügt und in der Summe:

$$
\Phi_{1}(u)=\sum_{\mu=0}^{n-1} \varepsilon^{2 \lambda \mu} \varphi_{1}\left(u+\frac{\mu \omega_{2}}{n}\right)
$$

eine elliptische Funktion zweiter Art der Perioden $\frac{\omega_{1}}{2}, \frac{\omega_{2}}{n}$ und des Faktorensystems $\lambda_{1}=-1, \lambda_{2}=\varepsilon^{-2 \lambda}$ ergibt. Durch Fortsetzung der Betrachtung in obiger Art findet man den Satz: Für irgendein $\lambda$ der Zahlenreihe $0,1,2, \ldots, n-1$ gilt:

$$
\sum_{\mu=0}^{n-1} \varepsilon^{2 \lambda \mu} \frac{\rho_{\lambda \mu}-e_{1}}{\xi_{\lambda, \mu}^{\prime}}=0
$$

während für zwei „,verschiedene" $\lambda, \lambda^{\prime}$ stets die Ungleichung besteht:

$$
\sum_{\mu=0}^{n-1} \varepsilon^{2 \lambda^{\prime} \mu} \frac{\jmath^{3} \lambda_{\mu}-e_{1}}{\wp_{\lambda \mu}^{\prime}} \neq 0 \text {. }
$$

Durch Kombination der beiden Gleichungen (5) und (7) gelangt man zu den beiden, mit ihnen gleichwertigen Gleichungen:

$$
\sum_{\mu=0}^{n-1} \varepsilon^{2 \lambda, \mu} \frac{1}{\rho_{\lambda, \mu}^{\prime}}=0, \quad \sum_{\mu=0}^{n-1} \varepsilon^{2 \lambda \mu} \frac{\rho^{2} \lambda \mu}{\rho_{\lambda, \mu}^{\prime}}=0 .
$$

In ihnen haben wir die für ungerades $n$ und für jede Zahl $\lambda$ der Reihe $0,1,2, \ldots, n-1$ bestehenden "Abelschen Relationen" der $\wp$ - und $\wp^{\prime}-T e i l-$ werte erreicht. Aus den beiden Ungleichungen (6) und (8) können wir freilich nur noch die folgende Aussage herleiten: Sollen für zwei Zahlen $\lambda$ und $\lambda^{\prime}$ der Reihe $0,1,2, \ldots, n-1$ die Gleichungen: 


$$
\sum_{n=0}^{n-1} \varepsilon^{2 \lambda^{\prime} \mu} \frac{1}{\jmath^{\prime} \lambda_{\mu \mu}^{\prime}}=0, \quad \sum_{\mu=0}^{n-1} \varepsilon^{2 \lambda^{\prime} \mu} \frac{\wp^{\prime} \lambda_{\mu}}{\wp_{\lambda_{\mu}}^{\prime}}=0
$$

gleichzeitig gelten, so ist notwendig $\lambda^{\prime}=\lambda$.

Durch Ausübung von Substitutionen der homogenen Gruppe $\Gamma^{\left({ }^{\prime \prime \prime}\right)}$ kann man aus (9) andere Relationen dieser Art ableiten. So findet man z. B. mittelst der Substitution $\omega_{1}^{\prime}=\omega_{2}, \omega_{2}^{\prime}=-\omega_{1}$ aus (9):

$$
\sum_{\lambda=0}^{n-1} \varepsilon^{-2 \lambda_{\mu}} \frac{1}{\rho_{\lambda_{\mu}^{\prime}}^{\prime}}=0, \quad \sum_{\lambda=0}^{n-1} \varepsilon^{-2 \lambda \mu} \frac{\rho_{\lambda \mu}}{\rho^{\prime} \lambda_{\mu \mu}}=0 .
$$

Die in der älteren Literatur vorliegenden Abelschen Relationen betreffen die sn-Funktion. Wir gelangen zu ihnen, indem wir die an (1) angeschlossene Überlegung auf den reziproken Wert der in I, 384 erklärten Funktion $\psi_{1}(u)$ anwenden. Aus ihr bilden wir die Summe:

$$
\Psi_{1}(u)=\sum_{u=0}^{n-1} \frac{\varepsilon^{4 \lambda \mu}}{\psi_{1}\left(u+\frac{2 \mu \omega_{2}}{n}\right)},
$$

in welcher wir unter Benutzung der Regel (7) in I, 384 leicht eine elliptische Funktion zweiter Art der Perioden $\omega_{1}, \frac{\omega_{2}}{n}$ und des Multiplikatorensystems $1,-\varepsilon^{-2 \lambda}$ erkennen. Im zugehörigen Parallelogramme liegt ein Pol erster Ordnung bei $\frac{\omega_{1}}{2}$; den einen im Parallogramme auftretenden Nullpunkt findet man daraufhin bei $u=\frac{2 \lambda \omega_{1}}{n}$ gelegen. Den Übergang zur sn-Funktion vermitteln die Formeln (5) und (8) in I, 388ff. Wir notieren sogleich als Beispiel einer Abelschen Relation älterer Gestalt:

$$
\sum_{\mu=0}^{n-1} \varepsilon^{4 \lambda \mu} \operatorname{sn}_{\lambda \mu}=0
$$

\section{Viertes Kapitel.}

\section{Die Teilwerte der elliptischen Funktionen.}

Der Satz, daß die allgemeine Teilungsgleichung eine Abelsche Gleichung ist, setzt die Adjunktion und damit die Kenntnis der Teilwerte $\wp_{\lambda \mu}, \wp_{\lambda \mu}^{\prime}$ voraus. Diese genügen ihrerseits Gleichungen, die man als „spezielle Teilungsgleichungen" bezeichnet und deren Theorie zu den interessantesten Gegenständen unserer Darstellung gehört. Die Galoissche Gruppe der zum Grade $n$ gehörenden speziellen Teilungsgleichung hat 
C. Jordan in seinem bekannten Gruppenwerke ${ }^{1}$ ) betrachtet, ohne zu endgültigen Ergebnissen zu gelangen. Solche werden jedoch in den schon S. 240 genannten Untersuchungen von Sylow und Kronecker erreicht. In funktionentheoretischer Hinsicht hat namentlich Kiepert ${ }^{2}$ ) die Teilwerte der Funktionen erster Stufe untersucht und ihre Beziehung zur Transformationstheorie verfolgt.

\section{$\$ 1$. Die Teilwerte $\wp_{\lambda \mu}, \wp_{\lambda_{\mu}}^{\prime}$ und die speziellen Teilungsglèichungen.}

Beim Teilungsgrade $n$ haben wir nach der S. 213 vollzogenen Abzählung für ungerades $n$ im ganzen $\frac{1}{2}\left(n^{2}-1\right)$ verschiedene Teilwerte $\wp_{\lambda_{\mu}}$ und $\left(n^{2}-1\right)$ paarweise entgegengesetzte Teilwerte $\wp_{\lambda_{\mu}}^{\prime}$. Bei geradem $n$ reihten sich $\frac{1}{2}\left(n^{2}+2\right)$ verschiedene $\wp_{\lambda_{\mu}}$ an, unter ihnen die drei schon bei $n=2$ auftretenden "Teilwerte" $e_{1}, e_{2}, e_{3}$, sowie $\left(n^{2}-4\right)$ paarweise entgegengesetzte $\wp_{\lambda \mu}^{\prime}$.

Für die Teilwerte $\wp_{\lambda \mu}$ versehen wir uns zu späterem Gebrauche sogleich mit einer analytischen Darstellung, die wir aus der Gleichung (14) in I, 270 durch Eintragen des Wertes $u=\frac{\lambda \omega_{1}+\mu \omega_{2}}{n}$ entnehmen. Dabei möge für den Quotienten $\frac{\eta_{9}}{\omega_{3}}$ die Darstellung (15) in I, 271 eingeführt werden; auch ersetze man die trigonometrischen Funktionen durch ihre Ausdrücke in der Exponentialfunktion. Eine einfache Zwischenrechnung ergibt:

$$
\begin{gathered}
\delta_{\lambda_{\mu}=}=\left(\frac{2 \pi}{\omega_{2}}\right)^{2}\left\{-\frac{1}{12}+2 \sum_{m=1}^{\infty} \frac{m q^{2 m}}{1-q^{2 m}}-\varepsilon^{\mu} q^{\frac{2 \lambda}{n}}\left(\sum_{m=0}^{\infty} \varepsilon^{\mu m} q^{\frac{22 m}{n}}\right)^{2}\right. \\
\left.-\sum_{m=1}^{\infty} \frac{m q^{2 m}}{1-q^{2 m}}\left(\varepsilon^{\mu m} q^{\frac{22 m}{n}}+\varepsilon^{-\mu m} q^{-\frac{2 \lambda m}{n}}\right)\right\}
\end{gathered}
$$

wo wie bisher $\varepsilon=e^{\frac{2 i \pi}{n}}$ ist. Wesentlich an dieser Darstellung ist, daß erstlich $\mu$ nur in den Exponenten der Potenzen von $\varepsilon$ auftritt, und daß zweitens, abgesehen vom Absolutgliede $-\frac{1}{12}$ in den Koeffizienten der Potenzen von $q$ neben $\varepsilon$ nur rationale ganze Zahlen vorkommen. Bei Umordnung nach ansteigenden Potenzen von $q^{\frac{2}{4}}$ erhalten wir somit eine Darstellung:

$$
\wp_{\lambda \mu}=\left(\frac{2 \pi}{\omega_{2}}\right)^{2}\left\{-\frac{1}{12}+A_{\lambda 1}\left(\varepsilon^{\mu}\right) q^{\frac{2}{n}}+A_{\lambda 2}\left(\varepsilon^{\mu}\right) q^{\frac{4}{n}}+A_{\lambda 3}\left(\varepsilon^{\mu}\right) q^{\frac{6}{n}}+\cdots\right\},
$$

wo sich die Koeffizienten $A_{\lambda, k}\left(\varepsilon^{\mu}\right)$ in der Gestalt:

1) Traité des substitutions et des équations algébriques" (Paris 1870) S. $93 \mathrm{ff}$.

2) „Über Teilung und Transformation der elliptischen Funktionen", Math. Ann. Bd. 20 (1885). 


$$
A_{\lambda k}\left(\varepsilon^{\mu}\right)=a_{\lambda k}^{(0)}+a_{\lambda k}^{(1)} \varepsilon^{\mu}+a_{\lambda k}^{(2)} \varepsilon^{2 \mu}+\cdots+a_{\lambda k}^{(n-1)} \varepsilon^{(n-1) \mu}
$$

mit rationalen ganzen Zahlen $a_{\lambda k}^{(i)}$ darstellen und also ganze algebraische Zahlen des zum Teilungsgrade $n$ gehörenden „Kreisteilungskörpers" sind.

Nach S. 225 gehen die Funktionen $\wp_{\lambda_{\mu}}\left(\frac{u}{n}\right), \wp_{\lambda \mu}^{\prime}\left(\frac{u}{n}\right)$ bei Ausübung einer Substitution der Hauptkongruenzgruppe $n^{\text {ter }}$ Stufe $\Gamma_{n \chi(n)}^{(\omega)}$ in sich selbst über. Setzen wir $u=0$, so folgt: Die zum $n^{\text {ten }}$ Teilungsgrade gehörenden Teilwerte $\wp_{\lambda \mu}, \wp_{i, \mu}^{\prime}$ 'sind invariant gegenüber den Substitutionen der Hauptkongruenzgruppe $n^{\text {ter }}$ Stufe $\Gamma_{n \chi(n)}^{(\omega)}$ und heißen dieserhalb „Modulformen $n^{\text {ter }}$ Stufe".

Die in (3) S. 184 eingeführte $\left(n^{2}-1\right)$-wertige Funktion $\psi^{(n)}(u)$ hatte gerade die $\left(n^{2}-1\right)$ Teilpunkte $\frac{\lambda \omega_{1}+\mu \omega_{2}}{n}$ des Periodenparallelogramms zu Nullpunkten. Setzt man also in den Ausdruck von $\psi^{(n)}(u)$ als rationale ganze Fúnktion von $\wp(u)$ an Stelle von $\wp(u)$ eine Unbekannte $z$ ein, so gelangt man zu einer Gleichung für die Teilwerte $\wp_{\lambda, \mu}$. Aus den S. 189 für $\psi^{(n)}(u)$ angegebene Ausdrücken folgt somit der Satz: Bei ungeradem $n$ sind die $\frac{1}{2}\left(n^{2}-1\right)$ verschiedenen $\wp_{\lambda_{\mu}}$ die Wurzeln einer Gleichung:

$$
n z^{\frac{1}{2}\left(n^{2}-1\right)}+\beta_{1}\left(\frac{1}{4} g_{2}\right) z^{\frac{1}{2}\left(n^{2}-5\right)}+\beta_{2} g_{3} z^{\frac{1}{2}\left(n^{2}-7\right)}+\cdots=0
$$

während bei geradem $n$ die $\frac{1}{2}\left(n^{2}-4\right)$ von den $e_{1}, e_{2}, e_{3}$ verschiedenen $\wp_{\lambda \mu}$ der Gleichung:

$$
\frac{1}{2} n z^{\frac{1}{2}\left(n^{2}-4\right)}+\gamma_{1}\left(\frac{1}{4} g_{2}\right) z^{\frac{1}{2}\left(n^{2}-8\right)}+\gamma_{2} g_{3} z^{\frac{1}{2}\left(n^{2}-10\right)}+\cdots=0
$$

genügen ${ }^{1}$ ); die $\beta$ und $\gamma$ sind dabei rationale ganze Zahlen. Bis $n=6$ sind diese Gleichungen in den Entwicklungen von S. $185 \mathrm{ff}$. explizite enthalten. Entsprechende Gleichungen für die $\gamma^{\prime} \lambda_{\mu}^{\prime}$ kann man durch Elimination von $z$ aus:

$$
4 z^{3}-g_{2} z-\left(z^{\prime 2}+g_{3}\right)=0
$$

und (4) bzw. (5) gewinnen. Da jedoch die Berechnung von $\wp_{\lambda \mu}^{\prime}$ aus $\wp_{\lambda \mu}$ nur noch das Ausziehen einer Quadratwurzel erfordert, so beschäftigen wir uns zunächst vornehmlich mit den $\wp_{\lambda \mu}$ und den Gleichungen (4) und (5).

Als „eigentlich“ zum Teilungsgrade $n$ oder zur Stufe $n$ gehörig bezeichnen wir diejenigen Teilwerte, die nicht bereits bei einer niederen Stufe auftreten. Haben die drei Zahlen $\lambda, \mu, n$ den größten gemeinsamen Teiler $t$, so gehören die $\wp_{\lambda \mu}, \wp_{\lambda \mu}^{\prime}$ eigentlich zur Stufe $\frac{n}{t} \cdot$ Nach S. 218

1) Der Fall $n=2$, bei dem die drei $\wp$-Teilwerte durch $e_{1}, e_{2}, e_{3}$ geliefert werden, während noch keine (von 0 verschiedene) $\wp_{\lambda, \mu}^{\prime}$ auftreten, gelte als ausgeschlossen. 
gibt es $\chi(n)$ gegen $n$ teilerfremde $\bmod n$ inkongruente Zahlenpaare, wo $\chi(n)$ die in (2) S. 219 berechnete Anzahl ist. Diese Zahlenpaare liefern die eigentlich zur $n^{\text {ten }}$ Stufe gehörenden Teilwerte. Da mit $\lambda, \mu$ auch $n-\lambda, n-\mu$ ein gegen $n$ teilerfremdes Paar ist, so folgt für $n>2$ : Es gibt $\frac{1}{2} \chi(n)$ eigentlich zur Stufe $n$ geliörige Teilwerte $\delta_{\lambda_{\mu}}$ und $\chi(n)$ paarweise nur im Vorzeichen verschiedene eigentlich zur Stufe $n$ gehörige $\wp_{\lambda \mu}^{\prime}$. Bei $n=2$ hat man $\chi(2)=3$ Teilwerte $\jmath_{\nu_{\mu},}$, während die $\wp_{\lambda_{\mu}}^{\prime}$ identisch verschwinden.

Üben wir eine Substitution $V=\left(\begin{array}{c}\alpha, \beta \\ \beta, \delta\end{array}\right)$ der homogenen $\Gamma^{(\omega)}$ aus, so geht $\wp_{\lambda \mu}$ in $\delta_{\lambda^{\prime}, \mu^{\prime}}=\wp_{\alpha \lambda+\gamma \mu, \beta \lambda+\delta \mu}$ über, und eine entsprechende Aussage gilt für $\wp_{\lambda \mu^{\prime}}^{\prime}$ Da die Indizes mod $n$ beliebig reduzierbar sind, so gilt der Satz: Bei Ausübung einer Substitution $V=\left(\begin{array}{l}\alpha, \beta \\ \gamma, \delta\end{array}\right)$ der homogenen $\Gamma^{(\omega)}$ er fahren die Indizes $\lambda, \mu$ eine durch die Kongruenzen:

$$
\lambda^{\prime} \equiv \alpha \lambda+\gamma \mu, \quad \mu^{\prime} \equiv \beta \lambda+\delta \mu \quad(\bmod n)
$$

darstellbare Transformation. Bei den $\wp_{\lambda_{\mu}}$ ist für $n>2$ neben Reduktionen $\bmod n$ nötigenfalls auch noch ein gleichzeitiger Zeichenvechsel der Indizes vorzunehmen.

Bei diesen Träsformationen werden nun die gegen $n$ teilerfremden Zahlenpaare stets nur unter sich permutiert. Auch können wir etwa aus dem Paare $\lambda \equiv 1, \mu \equiv 0$ mittels einer geeigneten Transformation (7) jedes beliebige gegen $n$ teilerfremde Paar herstellen, da nach $\mathrm{S}_{:} 220 \mathrm{ff}$. für jedes solche Paar $\lambda^{\prime}, \mu^{\prime}$ in der $\Gamma^{(\omega)}$ Substitutionen $\left(\begin{array}{l}\alpha, \beta \\ \gamma, \delta\end{array}\right)$ mit $\alpha \equiv \lambda^{\prime}, \beta \equiv \mu^{\prime}$ aufauftreten. Mit Rücksicht auf die Gruppeneigenschaft der $\Gamma^{(\omega)}$ können wir also den Satz aussprechen: Die ${ }_{2}^{1} \chi(n)$ eigentlich zur $n^{\text {ten }}$ Stufe gehörenden Teilwerte $\varsigma_{\lambda_{u}}$ werden bei Ausïbung irgendeiner Substitution der homogenen $\Gamma^{(\omega)}$ stets mur untereinander permutiert; auch können wir jeden dieser Teilwerte durch eine geeignete Substitution in jeden vorgeschriebenen unter ihnen transformieren. Die ${ }_{2}^{1} \chi(n)$ Teilwerte $\wp_{\lambda_{\mu}}$ werden in diesem Sinne als ,gleichberechtigt" bezeichnet. Übrigens kann man das gewonnene Ergebnis kurz auch so ausdrücken: Gegenüber den Substitutionen der Gruppe $\Gamma^{(\omega)}$ erfahren die $\frac{1}{2} \chi(n)$ eigentlich zur $n^{\text {ten }}$ Stufe gehörenden Teilwerte $\varsigma_{\lambda_{u}}$ die Permutationen einer "transitiven" Gruppe, deren nähere Gesetzmäßigkeit durch die Kongruenzen (7) nötigenfalls mit gleichzeitigem Zeichenuechsel von $\lambda^{\prime}, \mu^{\prime}$ festgelegt ist. Entsprechende Sätze gelten natïrlich für die $\chi(n)$ Teilwerte $\wp_{2, \mu}^{\prime}$.

Auf Grund dieser Ergebnisse können wir die Frage der Reduzibilität der Gleichung (4) bzw. (5) im Körper $\Re^{\prime}=\left(\Re, g_{2}, g_{3}\right)$ behandeln. Bilden wir die symmetrischen Grundfunktionen der $\frac{1}{2} \chi(n)$ eigentlich zur Stufe $n$ gehörenden $\wp_{\lambda_{\mu}}$, so gelangen wir zu Ausdrücken, die gegen- 
über allen Substitutionen der $\Gamma^{(*)}$ invariant sind. Als ganze Funktionen der $\wp_{\lambda \mu}$ werden sie sich zufolge (2) in Potenzreihen nach $q^{2}$ entwickeln lassen, die für $|q|<1$ konvergent sind, und die ausschlieblich "positive“ Exponenten der Potenzen von $q^{2}$ aufweisen. Auf Grund der Sätze in I, $305 \mathrm{ff}$. erkennen wir in jenen symmetrischen Grundfunktionen ,ganze Modulformen erster Stufe“, die als solche nach dem Gesetze (8) in I, 309 durch $g_{2}$ und $g_{3}$ darstellbar sind. Ziehen wir noch die Dimension in den $\omega_{1}, \omega_{2}$ heran, so folgt, daß die $\frac{1}{2} \chi(n)$ Teilwerte $\rho_{1 .,}$ die Wurzeln einer Gleichung:

$$
z^{\frac{1}{2} \chi(n)}+\alpha_{1} g_{2} z^{\frac{1}{2} \chi(n)-2}+\alpha_{2} g_{3} z^{\frac{1}{2} \chi(n)-3}+\alpha_{3} g_{2}^{2} z^{\frac{1}{2} \chi(n)-4}+\cdots=0
$$

sind, in der die $\alpha_{1}, \alpha_{2}, \ldots$ numerische, $d$. h. von den $\omega_{1}, \omega_{2}$ unabhängige Konstante sind.

Von diesen Konstanten kann man durch den Schluß der vollständigen Induktion zeigen, daB sie rationale Zahlen sind. Die Wurzeln der Gleichung (4) bzw. (5) sind nämlich die eigentlich zur Stufe $n$ gehörenden $\wp_{\lambda_{u}}$, sowie außerdem alle eigentlich zu den Stufen $\frac{n}{t}$ gehörenden Teilwerte, wo $t$ alle Teiler $>1$ von $n$ durchläuft. Nur ist natürlich $t=n$ ausgeschlossen, und außerdem ist aus der Gleichung ( 5 ) bereits der zur zweiten Stufe gehörende Bestandteil $\left(4 z^{3}-g_{2} z-g_{3}\right)$ entfernt. Man kann aber durch „rationale“ Divisionen aus (4) bzw. (5) alle Wurzeln entfernen, die zu Stufen $\frac{n}{t}<n$ gehören. Gilt das Gesetz der rationalen numerischen Koeffizienten für alle Stufen $<n$, so ist hiernach einleuchtend, daB es auch für $n$ gilt. Nun ist dieses Gesetz jedenfalls für alle Primzahlen $n$ richtig, da für eine ungerade Primzabl $n$ die Gleichung (8) einfach die durch $n$ geteilte Gleichung (4) ist. Unsere Behauptung ist damit allgemein bewiesen.

Denkt man in (8) für $z$ eine einzelne Wurzel $\zeta_{\eta_{\mu}}$ eingetragen, so ergibt sich eine in $\omega_{1}, \omega_{2}$ identisch bestehende Gleichung. Die Gleichung bleibt demnach richtig bei allen solchen Veränderungen der $\omega_{1}, \omega_{2}$, bei denen der Periodenquotient $\omega$ in seiner positiven Halbebene verbleibt. Durch Änderungen dieser Art kann man nun von einem ersten Wertepaare $\omega_{1}, \omega_{2}$ zu jedem bezüglich der $\Gamma^{(\omega)}$ äquivalenten Paare gelangen. Hieraus folgt mit Rücksicht auf die Gleichberechtigung der $-\frac{1}{2} \chi(n)$ Teilwerte $\wp_{\lambda_{\mu}}$ die Irreduzibilität der Gleichung (8) selbst nach Adjunktion irgendwelcher „numerischer“ Irrationalitäten. Genügt nämlich etwa $z=\wp_{10}$ einer „irreduzibelen" Gleichung $F\left(z, g_{2}, g_{3}\right)=0$ in einem Körper, der ans $\left(\Re, g_{2}, g_{3}\right)$ durch Adjunktion irgendwelcher numerischer Irrationalitäten entsteht, so zeigt sich durch Wiederholung der vorstehenden Betrachtung, daB jene Gleichung durch alle $\frac{1}{2} \chi(n)$ Teilwerte erfüllt wird. Sie enthält also 
die Gleichung (8) und ist demnach als irreduzibel bis auf einen von $z$ unabhängigen Faktor mit (8) identisch.

Wir fassen die Ergebnisse in folgenden Satz zusammen: Die $\frac{1}{2} \chi(\boldsymbol{n})$ eigentlich zur $n^{\text {ten }}$ Stufe gehörenden Teilwerte $\jmath_{2, \mu}$ sind für $n>2$ die Wurzeln der als "spezielle Teilungsgleichung" für den $n^{\text {ten }}$ Teilungsgrad bezeichneten Gleichung (8), deren Koeffizienten dem Körper $\mathfrak{\Re}=\left(\Re, g_{2}, g_{3}\right)$ angehören, und die in diesem Körper irreduzibel ist, auch irreduzibel bleiben würde, falls noch irgendwelche "numerische" Irrationalitäten adjungiert würden. Im Falle $n=2$ bleibt dieser Satz mit der Abänderung bestehen, daß der Grad der Gleichung nicht $\frac{1}{2} \chi(2)$, sondern $\chi(2)=3$ ist.

Übrigens findet man durch Elimination von $z$ aus (8) und der Gleichung:

$$
4 z^{3}-g_{2} z-\left(z^{2}+g_{3}\right)=0,
$$

daß die $\chi(n)$ eigentlich zur Stufe $n$ gehörenden Teilwerte $\wp_{\lambda \mu}^{\prime}$ einer Gleichung $\chi(n)^{\text {ten }}$ Grades:

$$
z^{\prime} \chi(n)+\beta_{1} g_{3} z^{\prime} \chi(n)-2+\left(\beta_{2} g_{2}{ }^{3}+\beta_{3} g_{3}{ }^{2}\right) z^{\prime} \chi(n)-4+\cdots=0
$$

genügen, welche die soeben über die spezielle Teilungsgleichung (3) ausgesagten Eigenschaften gleichfalls besitzt.

Der Schluß auf die Rationalität der numerischen Koeffizienten der speziellen Teilungsgleichung kann auch noch in anderer Art vollzogen werden. Ersetzt man den zweiten Index $\mu$ der $\rho_{\lambda \mu}$ durch $x \mu$, unter $\varkappa$ einen der $\varphi(n)$ gegen $n$ teilerfremden Reste $\bmod n$ verstanden, so permutieren sich die $\frac{1}{2} \chi(n)$ Teilwerte $\delta_{\lambda_{\mu}}$ untereinander. Die symmetrischen Grundfunktionen der $\wp_{\lambda \mu}$ bleiben demnach bei jenem Ersatze unverändert. Die aus (2) zu entnehmende Reihenentwicklung:

$$
\sigma_{v}\left(\rho_{2, \mu}\right)=\left(\frac{2 \pi}{\omega_{2}}\right)^{2 v}\left(a_{0}+a_{1} q^{2}+a_{2} q^{4}+\cdots\right)
$$

für die $\nu^{\text {te }}$ symmetrische Grundfunktion hat also als Koeffizienten nur noch Zahlen des zum Teilungsgrade $n$ gehörenden Kreisteilungskörpers, die beim Ersatze von $\varepsilon$ durch irgendeine der $\varphi(n)$ primitiven Einheitswurzeln $n^{\text {ten }}$ Grades unverändert bleiben, d. h. die Koeffizienten $a$ in (10) sind rationale Zahlen. Als ganze Modulform der ersten Stufe bezeichnen wir $\sigma_{v}\left(\wp_{\lambda \mu}\right)$ durch $G_{v}\left(\omega_{1}, \omega_{2}\right)$. Nach (8) in I, 309 läBt sich eine solche Form in der Gestalt:

$$
G_{v}\left(\omega_{1}, \omega_{2}\right)=\sum_{l, m} C_{l m} g_{2:}^{l} g_{3}^{m}
$$

mittelst numerischer Koeffizienten $C_{l m}$ durch $g_{2}, g_{3}$ darstellen. Diese Darstellung von $G_{v}\left(\omega_{1}, \omega_{2}\right)$ ist einzig, da sonst zwischen $g_{2}$ und $g_{3}$ eine identische Relation bestände.

Eine nicht identisch verschwindende ganze Modulform erster Stufe der Dimension $-2 \nu$ hat nach I, 309 im Diskontinuitätsbereiche der $\Gamma^{\left(\omega^{*}\right.}$ 
Nullpunkte in der Gesamtordnung $\frac{v}{6}$. Verschwinden demnach in der Potenzreihe einer solchen Form die Koeffizienten bis zu einem Gliede mit einem Exponenten von $q^{2}$, der $>\frac{\nu}{6}$ ist, so liegt bereits in der Spitze $\omega=i \infty$ des Diskontuinitätsbereiches ein Nullpunkt von einer Ordnung $>\frac{v}{6}$, so daß die Form dann notwendig mit 0 identisch ist. Dieserhalb sind zwei ganze Modulformen erster Stufe der Dimension $-2 v$, deren Potenzreihen in den Anfangsgliedern, und zwar bis zu einem Exponenten $>\frac{v}{6}$ von $q^{2}$, übereinstimmen, notwendig miteinander identisch.

Tragen wir nun in den Ausdruck (11) irgendeiner Form $G_{v}\left(\omega_{1}, \omega_{2}\right)$ für $g_{2}, g_{3}$ die Potenzreihen (3) in I, 274 ein, so ergibt sich bei Umordnung nach ansteigenden Potenzen von $q^{2}$ :

$$
G_{v}\left(\omega_{1}, \omega_{2}\right)=\left(\frac{2 \pi}{\omega_{2}}\right)^{2 v}\left(b_{0}+b_{1} q^{2}+b_{2} q^{4}+\cdots\right),
$$

wo die $b_{k}$ lineare homogene Funktionen der $C_{l m}$ :

$$
b_{k}=\sum_{l, m} \alpha_{l m}^{(k)} C_{l m}
$$

mit „rationalen“ Koeffizienten $\alpha$ sind. Soll jetzt die Form (11) mit $\sigma_{\nu}\left(\wp_{\lambda_{\mu}}\right)$ identisch sein, so ist hierzu nach den vorausgeschickten Überlegungen notwendig und hinreichend, daß die Reihe (12) von $G_{v}\left(\omega_{1}, \omega_{3}\right)$ mit der Reihe (10) in einer gewissen Anzahl von Anfangskoeffizienten übereinstimmt, daB also die $C_{l m}$ einer gewissen Anzahl linearer Gleichungen:

$$
\sum_{l, m} \alpha_{l m}^{(0)} C_{l m}=a_{0}, \quad \sum_{l, m} \alpha_{l m}^{(1)} C_{l m}=a_{1}, \ldots
$$

mit durchweg rationalen Koeffizienten genügen. Nun gibt es sicher ein System endlicher Zahlen $C_{l m}$, die diese Gleichungen befriedigen, da $\sigma_{v}\left(\wp_{\lambda \mu}\right)$ als ganze Modulform erster Stufe in der Gestalt (11) darstellbar ist. Da aber jedes IJösungssystem $C_{l m}$ der Gleichungen (14) eine Darstellung (11) liefern würde und diese Darstellung für $\sigma_{v}\left(\wp_{\lambda_{\mu}}\right)$, wie wir wissen einzig ist, so gibt es auch nur ein Lösungssystem $C_{l m}$ der Gleichungen (14). Die linearen Gleichungen (14) sind also zur eindeutigen Berechnung der endlichen Größen $C_{l m}$ geeignet, so daß sich die $C_{l m}$ als Quotienten von Determinanten der a und $a$ und damit als rationale Zahlen aus (14) bestimmen. Diese Schlußweise ist hier gleich ausführlich dargelegt, da wir sie noch öfter zu verwenden haben werden.

\section{$\S 2$. Kongruenzgruppen $\boldsymbol{n}^{\text {ter }}$ Stufe in der Modulgruppe $\boldsymbol{\Gamma}$.}

Da sich die nächsten Überlegungen nur auf die Gruppen $\Gamma^{(\omega)}$ und $G_{n \chi(n)}^{(\omega)}$ beziehen, so wird der obere Index $\omega$ der Kürze halber fortgelassen. 
Es sei jetzt in der endlichen Gruppe $G_{n_{\chi}(n)}$, auf die sich die Gruppe $\Gamma$ mod $n$ reduziert, irgendeine Untergruppe $G_{t}$ der Ordnung $t$ und des Index $\mu=\frac{n \chi(n)}{t}$ vorgelegt. Die zugehörige Zerlegung der Gesamtgruppe in Nebengruppen sei etwa in der Gestalt gegeben:

$$
G_{n /(n)}=G_{t}+G_{t} \cdot U_{1}+G_{t} \cdot U_{2}+\cdots+G_{t} \cdot U_{\mu-1},
$$

wo $U_{1}, U_{2}, \ldots, U_{\mu-1}$ zweckmäßig gewählte Substitutionen $\operatorname{der} G_{n \chi(n)}$ oder der Gruppe $I$ sind. Nun können wir die $G_{n \chi(n)}$ auch im Anschluß an die Zerlegung:

$$
\Gamma=\Gamma_{n \%}+\Gamma_{n \%} \cdot V_{1}+\Gamma_{n \%} \cdot V_{2}+\cdots+\Gamma_{n \gamma} \cdot V_{n \chi-1}
$$

der Gruppe $\Gamma$ in die der Hauptkongruenzgruppe $n^{\text {ter }}$ Stufe zugehörigen Nebengruppen erklären. Da die $\Gamma_{n \%(n)}$ ausgezeichnet ist, so liefern die $n \chi(n)$ Nebengruppen, selbst wieder als Elemente gefaßt (vgl. S. 9), eine endliche Gruppe der Ordnung $n \chi(n)$, nämlich unsere $G_{n_{\chi}(n)}$. Die Substitutionen derjenigen $t$ Nebengruppen (2), die die Elemente der vorgenannten $G_{t}$ sind, bilden nun für sich eine Untergruppe von $\Gamma$, und zwar eine solche des Index $\mu$ die dieserhalb $\Gamma_{\mu}$ heiße; der Zerlegung (1) entspricht nämlich die Zerlegung:

$$
\Gamma=\Gamma_{\mu}+\Gamma_{\mu} \cdot U_{1}+\Gamma_{\mu} \cdot U_{2}+\cdots+\Gamma_{\mu} \cdot U_{n-1}
$$

der Gesamtgruppe $\Gamma$ in die entsprechenden Nebengruppen. Alle so zu gewinnenden Gruppen $\Gamma_{\|}$bezeichnen wir als "Kongruenzgruppen $n^{\text {ter }}$

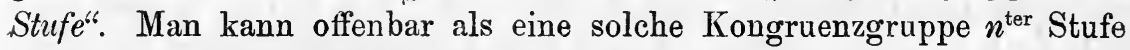
auch jede Gruppe erklären, die die Hauptkongruenzgruppe dieser Stufe $\Gamma_{n \chi(n)}$ als Untergruppe in sich enthält; denn jede solche Gruppe wird sich aus einer bestimmten Anzahl von Nebengruppen (2) zusammensetzen und liefert demnach ihrerseits eine bestimmte $G_{t}$ innerhalb der $G_{n \ell(n)}$. Einem Systeme gleichberechtigter Untergruppen $G_{t} \operatorname{des}$ Index $\mu$ entspricht ein System ebenso vieler gleichberechtigter Kongruenzgruppen $\Gamma_{\mu}$ des Index $\mu$; eine ausgezeichnete $G_{t}$ ergibt eine ausgezeichnete $\Gamma_{\mu}$, speziell die $G_{1}$ liefert die Hauptkongruenzgruppe $\Gamma_{n} \%(n)$.

Das Problem, alle Kongruenzgruppen $n^{\text {ter }}$ Stufe anzugeben, kommt also auf die Aufgabe zurück, die Gruppe $G_{n \%(n)}$ in ihre gesamten Untergruppen zu zerlegen. Diese Aufgabe ist, wie beiläufig erwähnt sei, im wesentlichen als gelöst anzusehen. Ist $n$ das Produkt $n_{1} \cdot n_{2}$ zweier teilerfremder Zahlen, so ist, wie in „Modulfunktionen“, Bd. 1, S. $402 \mathrm{ff}$. gezeigt wird, die vollständige Zerlegung der $G_{n \chi(n)}$ auf die Zerlegungen der beiden Gruppen $G_{n_{1} \chi\left(n_{1}\right)}$ und $G_{n_{2} \chi_{\left(n_{2}\right)}}$ zurückführbar. Man hat demnach weiter nur noch Primzahlpotenzen $n$ zu behandeln. Nun hat J. Gierster zunächst für den Fall einer Primzahl $n^{1}$ ) und sodann für den Fall einer

1) „Die Untergruppen der Galoisschen Gruppe der Modulargleichung für den Fall eines primzahligen Transformationsgrades", Math. Ann.. Bd.18 (1881). 
beliebigen Potenz einer ungeraden Primzahl ${ }^{1}$ ) die vollständige Zerlegung der $G_{n \chi(n)}$ wirklich durchführen können. Rückständig sind demnach nur die Potenzen der Primzahl 2, von denen allein die drei niedersten Fälle 2, 4, 8 erschöpfend behandelt sind.

Für die Theorie der speziellen Teilungsgleichung ist folgender Satz grundlegend: Ist $n=p^{v}$ eine Potenz der Primzahl $p$ mit einem Exponenten $v \geqq 2$, so bilden alle Substitutionen $\operatorname{der} G_{n \chi(n)}$, die mod $p^{\nu-1}$ mit der identischen Substitution 1 kongruent sind, eine Abelsche Gruppe $G_{p^{3}}$ der Ordnung $p^{3}$, die in der $G_{n y(n)}$ ausgezeichnet enthalten ist, und die, abgesehen von der identischen Substitution nur aus Substitutionen der Periode p besteht. Die fraglichen Substitutionen haben nämlich die Gestalt:

$$
\left.V \equiv\left(\begin{array}{l}
1+a p^{\nu-1}, b p^{\nu-1} \\
c p^{\nu-1}, 1-a p^{\nu-1}
\end{array}\right) \quad\left(\bmod p^{\nu}\right)^{2}\right)
$$

mit beliebigen ganzen Zahlen $a, b, c$. Man erhält in der Tat $p^{3}$ Substitutionen, wenn man $a, b$, c, unabhängig voneinander Restsysteme mod $p$ durchlaufen läBt. Zwei Substitutionen $V$ und $V^{\prime}$ dieser Art kombinieren sich nach dem Gesetze:

$$
V \cdot V^{\prime} \equiv\left(\begin{array}{cc}
1+\left(a+a^{\prime}\right) p^{\nu-1}, & \left(b+b^{\prime}\right) p^{\nu-1} \\
\left(c+c^{\prime}\right) p^{\nu-1}, & 1-\left(a+a^{\prime}\right) p^{\nu-1}
\end{array}\right) \quad\left(\bmod p^{\nu}\right) .
$$

Hieraus sind die Angaben des Satzes abgesehen von der Behauptung, daß die $G_{p^{3}}$ ausgezeichnet ist, leicht abzulesen. Die $G_{p^{3}}$ aber ist ausgezeichnet, weil sie der ausgezeichneten Kongruenzgruppe $\Gamma_{p^{v-1}} \chi_{\left(p^{p-1}\right)}$ der Stufe $p^{\nu-1}$ entspricht. ${ }^{3}$ )

Bei beliebigem $n$ kommen für die speziellen Teilungsgleichungen gewisse zyklische Untergruppen $G_{n}$ der Ordnung $n$ in Betracht. Unter $S$ verstehen wir wie in I, 298 die Substitution $\left(\begin{array}{l}1,1 \\ 0,1\end{array}\right)$; da $S^{n} \equiv 1(\bmod n)$ gilt, so erweist sich $S$ in $\operatorname{der} G_{n \chi(n)}$ als eine Substitution der Periode $n$ und erzeugt eine zyklische Untergruppe der Ordnung $n$, bestehend aus den Substitutionen $S^{0}=1, S, S^{2}, \ldots, S^{n-1}$, die offenbar alle voneinander verschieden sind. Die Anzahl der mit dieser $G_{n}$ gleichberechtigten Gruppen bestimmt man durch folgende Überlegung: Soll die $G_{n}$ durch die Substitution $V \equiv\left(\begin{array}{l}\alpha, \beta \\ \gamma, \delta\end{array}\right)$ in sich transformiert werden, so muB $V^{-1} \cdot S \cdot V \equiv S^{v}$

1) „Über die Galoissche Gruppe der Modulargleichung, wenn der Transformationsgrad die Potenz einer Primzahl $>2$ ist", Math. Ann., Bd. 26 (1885). Übrigens betreffen die Giersterschen Untersuchungen die weiterhin noch zu betrachtenden nichthomogenen Gruppen $G_{\frac{1}{2} n \chi(n)}$.

2) Wie in (11) S. 223 bezieht sich das Kongruenzzeichen natürlich auf die Koeffizienten der Substitution.

3) Nach der Begriffserklärung der Kongruenzgruppen $n^{\text {tor }}$ Stufe gehören zu ihnen auch alle Kongruenzgruppen der Stufen, die Teiler von $n$ sind. 
sein. Die Kongruenz lautet ausführlich:

$$
V^{-1} \cdot S \cdot V \equiv\left(\begin{array}{cc}
1+\gamma \delta, & \delta^{2} \\
-\gamma^{2} & , 1-\gamma \delta
\end{array}\right) \equiv\left(\begin{array}{l}
1, \nu \\
0,1
\end{array}\right) \quad(\bmod n)
$$

und führt also zu den beiden Bedingungen $\gamma \delta \equiv 0, \gamma^{2} \equiv 0(\bmod n)$. Multipliziert man diese beiden Kongruenzen mit $\alpha$ und $-\beta$, so liefert ihre Addition $\gamma \equiv 0(\bmod n)$, womit sie beide erfüllt sind. Die Zahl $\alpha$ kann jeden der $\varphi(n)$ gegen $n$ teilerfremden Reste $\bmod n$ bedeuten, $\beta$ bleibt beliebig wählbar, und für $\delta$ gilt die Kongruenz $\delta \equiv \alpha^{-1}(\bmod n)$. Alle so gewonnenen Substitutionen:

$$
V \equiv\left(\begin{array}{l}
\alpha, \beta \\
0, \alpha^{-1}
\end{array}\right)(\bmod n)
$$

bilden eine Untergruppe $G_{n \varphi(n)}$ der Ordnung $n \varphi(n)$ und also des Index $\left.\psi(n)^{1}\right)$, die als eine "metazyklische" Gruppe bezeichnet werden soll und in der die aus $S$ erzeugte zyklische $G_{n}$ als ausgezeichnete Untergruppe enthalten ist. Im niedersten Falle $n=2$ ist iibrigens die $G_{n \varphi(n)}$ mit der $G_{n}$ selbst gleich.

Wir zerlegen nun die Gesamtgruppe $G_{n \chi(n)}$ in die $\psi(n)$ der $G_{n \varphi(n)}$ entsprechenden Nebengruppen:

$$
G_{n \chi(n)}=G_{n \varphi(n)}+G_{n \varphi(n)} \cdot U_{1}+G_{n \varphi(n)} \cdot U_{2}+\cdots+G_{n \varphi(n)} \cdot U_{\psi(n)-1} .
$$

Die Substitutionen der einzelnen Nebengruppe transformieren $G_{n}$ in eine und dieselbe mit $G_{n}$ gleichberechtigte Gruppe. Man hat also höchstens $\psi(n)$ mit der $G_{n}$ gleichberechtigte Gruppen:

$G_{n}=U_{0}^{-1} \cdot G_{n} \cdot U_{0}, \quad G_{n}^{\prime}=U_{1}^{-1} \cdot G_{n} \cdot U_{1}, \ldots, \quad G_{n}^{(\psi-1)}=U_{\psi-1}^{-1} \cdot G_{n} \cdot U_{\psi-1}$, unter $U_{0}$ die identische Substitution verstanden. Diese $\psi(n)$ Gruppen sind aber durchweg verschieden. Wäre nämlich $U_{k}^{-1} \cdot G_{n} \cdot U_{k}=U_{i}^{-1} \cdot G_{n} \cdot U$ bei verschiedenen $U_{i}, U_{k}$, so würde $U_{k} \cdot U_{i}^{-1}$ die $G_{n}$ in sich transformieren also in $G_{n \varphi(n)}$ enthalten sein. Dies ist aber nicht der Fall, da $U_{k}$ sonst der Nebengruppe $G_{n} \cdot U_{i}$ angehören würde. In der $G_{n \chi(n)}$ sind demnach $\psi(n)$ gleichberechtigte zylilische Untergruppen $G_{n}$ der Ordnung $n$ enthalten, von denen eine die aus $S$ zu erzeugende $G_{n}$ ist, und deren einzelne in einer metazyklischen Gruppe $G_{n \varphi(n)}$ als ausgezeichnete Untergruppe enthalten ist. Die symbolisch durch -1 zu bezeichnende Substitution $\left(\begin{array}{l}-1,0 \\ 0,-1\end{array}\right)$ ist im Falle $n=2$, aber auch nur in diesem Falle mit der identischen Substitution kongruent. Wir nehmen $n>2$ und stellen fest, daB die Substitution -1 mit jeder Substitution der $G_{n \chi(n)}$ vertauschbar ist. Eine Untergruppe $G_{\mu}$, die die Substitution -1 nicht enthält, wird demnach

1) $\psi(n)$ bedeutet die in (4) S. 220 erklärte, der Gleichung $\varphi(n) \cdot \psi(n)=\chi(n)$ genügende Anzahl. 
durch Zusatz dieser Substitution zu einer $G_{2 \mu}$ der Ordnung $2 \mu$ erweitert, in der die $G_{\mu}$ ausgezeichnet enthalten ist. So wird die aus der Substitution $S$ zu erzeugende $G_{n}$ durch Zusatz von -1 zu einer $G_{2 n}$ erweitert, die iibrigens in der $G_{n \varphi(n)}$ der Substitutionen (6) enthalten ist. Soll $V \equiv\left(\begin{array}{l}\alpha, \beta \\ \gamma, \delta\end{array}\right)$ diese $G_{2 n}$ in sich transformieren, so ist, da -1 durch $V$ in sich transformiert wird, hierzu notwendig und hinreichend, daB $V^{-1} \cdot S \cdot V$ in $\operatorname{der} G_{2 n}$ enthalten ist:

$$
V^{-1} \cdot S \cdot V \equiv\left(\begin{array}{c}
1+\gamma \delta, \delta^{2} \\
-\gamma^{2}, 1-\gamma \delta
\end{array}\right) \equiv\left(\begin{array}{c} 
\pm 1, \nu \\
0, \pm 1
\end{array}\right) \quad(\bmod n) .
$$

Gilt das obere Zeichen, so gehört $V$ der $G_{n \varphi(n)}$ an. Das untere Zeichen kann aber, wie man durch Addition des ersten und vierten Koeffizienten von $V^{-1} \cdot S \cdot V$ findet, nur für $n=4$ gelten. In diesem Falle kommen neben den acht Substitutionen (6) noch die acht inkongruenten Substitutionen mit $\gamma \equiv 2(\bmod 4)$ für $V$ zur Geltung, so daß hier die $G_{2 n}$ in einer $G_{2 n \varphi(n)}$ ausgezeichnet enthalten ist. Wie oben findet man den Satz: Für $n=3$ und $n>4$ hat man $\psi(n)$ gleichberechtigte $G_{2 n}$, deren einzelne eine zyklische $G_{n}$ als ausgezeichnete Untergruppe enthält, aus der sie durch Zusatz von -1 entsteht; für $n=4$ hat man $\frac{1}{2} \psi(4)=3$ solche $G_{3}$, deren einzelne zwei zyklische $G_{4}$ als ausgezeichnet enthält.

Den Untergruppen $G_{n}$ und $G_{2 n}$ entsprechen ebenso viele gleichberechtigte Kongruenzgruppen $n^{\text {ter }}$ Stufe $\Gamma_{\chi(n)}$ und $\Gamma_{\frac{1}{2} \chi_{(n)}}$ der Indizes $\chi(n)$ bzw. $\frac{1}{2} \chi(n)$. Zu diesen Gruppen gehören nun gerade die Teilwerte $\wp_{\lambda, \mu}^{\prime}$ und $\wp_{\lambda_{\mu}}$. Die $\wp_{0, u}$ und $\wp_{0 \mu}^{\prime}$ bleiben unverändert bei der Substitution $S$, die $\wp_{0 \mu}$ auch gegenüber der Substitution -1 . Umgekehrt folgt aus der Regel (7) S. 246, daB eine Substitution, die $\wp_{0 \mu}$ in sich überführt, der $\Gamma_{\frac{1}{2} \chi(n)}$ angehört, und daB eine Substitution, die $\wp_{0_{\mu}}^{\prime}$ in sich transformiert, zur $\Gamma_{\chi(n)}$ gehört $\left.^{1}\right)$; gemeint sind hierbei natürlich diejenigen Gruppen, welche zu den aus $S$ und -1 bzw. aus $S$ zu erzeugenden $G_{2_{n}}$ und $G_{n}$ gehören. Nun gehen alle eigentlich zur Stufe $n$ gehörenden Teilwerte $\wp_{\lambda \mu}, \wp_{\lambda \mu}^{\prime}$ aus den $\wp_{01}, \wp_{01}^{\prime}$ durch Transformationen der $\Gamma^{(\omega)}$ hervor; die $\wp_{\lambda_{\mu}}$ (und ebenso die $\wp_{\Lambda_{\mu}}^{\prime}$ ) heißen dieserhalb ,gleichberechtigt" (vgl. S. 7). Hieraus ergibt sich der Satz: Dic $\frac{1}{2} \chi(n)=\frac{1}{2} \varphi(n) \psi(n)$ Teilwerte $\wp_{\lambda_{\iota}}$ gehören in Systemen zu je $\frac{1}{2} \varphi(n)$ den $\psi(n)$ gleichberechtigten Kongruenzgruppen $\Gamma_{\frac{1}{2}}{ }^{\prime}{ }^{(n)}$ an, indem sie bei den Substitutionen der betreffenden $\Gamma_{\frac{1}{2} \chi_{(n)}}$ und nur bei

1) Bei den eigentlich zur Stufe $n$ gehörenden $\wp_{0 \mu}, \varsigma_{0 \mu}^{\prime}$, um die es sich hier allein handelt, ist $\mu$ teilerfremd gegen $n$. 
ihnen unverändert bleiben ${ }^{1}$ ); ebenso gehören die $\chi(n)$ Teilwerte $\wp_{\lambda \mu}^{\prime} z u$ je $\varphi(n)$ den $\psi(n)$ gleichberechtigten Kongruenzgruppen $\Gamma_{\chi(n)}$ an.

Ein paar weitere später zur Benutzung kommende Ausführungen über Kongruenzgruppen mögen sich gleich hier anschließen. Die Kongruenz zweiten Grades $\mu^{2} \equiv 1(\bmod n)$ ist bekänntlich stets lösbar. $\left.{ }^{2}\right)$ Die Anzahl б ihrer $\bmod n$ inkongruenten Lösungen ist gleich 1 für $n=2$; weiter ist $\sigma=2$, wenn $n$ die Potenz einer ungeraden Primzahl oder das Doppelte einer solchen Potenz ist oder $n=4$ gilt; in allen übrigen Fällen ist $\sigma$ durch 4 teilbar. Nennen wir die Lösungen jener Kongruenz $\mu_{1}, \mu_{2}, \ldots$, $\mu_{\sigma}$, so bilden die $\sigma$ Substitutionen $\left(\begin{array}{c}\mu_{1}, 0 \\ 0, \mu_{1}\end{array}\right),\left(\begin{array}{c}\mu_{2}, 0 \\ 0, \mu_{2}\end{array}\right), \ldots,\left(\begin{array}{c}\mu_{\sigma}, 0 \\ 0, \mu_{\sigma}\end{array}\right)$ eine ausgezeichnete $G_{\sigma}$ in der $G_{n \chi(n)}$; denn $\left(\begin{array}{l}\mu, 0 \\ 0, \mu\end{array}\right)$ ist mit jeder Substitution der $G_{n \chi(n)}$ vertauschbar. In den Fällen mit $\sigma=2$ besteht die $G_{\sigma}$ aus den beiden Substitutionen 1 und -1 ; in den Fällen mit $\sigma \geqq 4$ bilden diese beiden Substitutionen eine in $d e r G_{n_{\chi(n)}}$ ausgezeichnet enthaltene Untergruppe $G_{2} \operatorname{der} G_{\sigma}$.

Zur $G_{2}$ gehört eine "Quotientengruppe“ $G_{n \chi(n)} / G_{2}=G_{\frac{1}{2} n_{\gamma(n)}}$, auf welche sich die $G_{n_{\chi}(n)}$ reduziert, wenn wir je zwei Substitutionen $\left(\begin{array}{l}\alpha, \beta \\ \gamma, \delta\end{array}\right)$ und $\left(\begin{array}{l}-\alpha,-\beta \\ -\gamma,-\delta\end{array}\right)$ als nicht verschieden ansehen. Dies ist der Standpunkt der nicht-homogenen Modulgruppe $\Gamma^{(\omega)}$, wie in I, $280 \mathrm{ff}$. näher erörtert ist. Man kann auch sagen, daß sich die nicht-homogene Modulgruppe $\Gamma$ mod $n$ auf eine Gruppe der endlichen Ordnung $\frac{1}{2} n \chi(n)$ reduziert, die mit der obigen $G_{\frac{1}{2} n \chi(n)}$ isomorph ist. Die Zerlegung dieser $G_{\frac{1}{2} n \chi(n)}$ in ihre Untergruppen liefert dann wie oben die gesamten Kongruenzgruppen $n^{\text {tpr }}$ Stufe in der nicht-homogenen Modulgruppe. Insbesondere entspricht der Untergruppe $G_{1}$ die "Hauptkongruenzgruppe $n^{\text {ter }}$ Stufe" $\Gamma_{\frac{1}{2} n \chi(n)}$ des Index $\frac{1}{2} n \chi(n)$. Die zyklischen Gruppen $G_{n}$ erfahren keine Reduktion der Ordnung bei Fortgang zu den nicht-homogenen Substitutionen. Dagegen reduzieren sich die metazyklischen Gruppen auf Untergruppen $G_{\frac{1}{2} n \varphi(n)}$ der Ordnung $\frac{1}{2} n \varphi(n)$, da in der einzelnen homogenen $G_{n \varphi(n)}$ die Substitution -1 enthalten ist und also ihre Substitutionen paarweise durch Zeichenwechsel der vier Koeffizienten ineinander übergehen. Da die einzelne $G_{n}$ nach wie vor durch die Substitutionen der zugehörigen $G_{\frac{1}{2} n \varphi(n)}$ und durch

1) Nur für $n=4$ gehören die $\frac{1}{2} \chi(n)=6$ Teilwerte $\wp_{\lambda \mu}$ zu je $\varphi(n)=2$ den $\frac{1}{2} \psi(n)=3$ Gruppen $\Gamma_{\frac{1}{2} \chi(n)}=\Gamma_{6}$ an.

2) S. etwa Dirichlet-Dedekind, „Vorles. über Zahlentheorie", S. 88 der vierten Auflage. 
keine anderen in sich transformiert wird, so haben wir in der $G_{\frac{1}{3} n_{\chi}(n)}$ wieder $\psi(n)$ gleichberechtigte zyklische $G_{n}$ der Ordnung n, von denen eine die aus $S$ zu erzeugende Gruppe ist.

Allgemein entspricht der ausgezeichneten $G_{\sigma}$ eine Quotientengruppe $G_{n \chi(n)} / G_{\sigma}=G_{\frac{n}{\sigma} \chi(n)}$ der Ordnung $\stackrel{n}{\sigma}_{-}^{n} \chi(n)$, während ihr andrerseits eine ausgezeichnete Kongruenzgruppe $\Gamma_{\sigma_{\sigma}} \chi(n)$ des Index $\frac{n}{\sigma} \chi(n)$ in der nichthomogenen Modulgruppe zugehört. Diese Gruppen kommen in der Theorie der Modulargleichungen zur Verwendung.

\section{\$ 3. Die Galoisschen Resolventen der speziellen Teilungsgleichungen.}

Die in den Teilen II und III der Einleitung entwickelte Galoissche Gleichungstheorie soll jetzt auf die spezielle Teilungsgleichung (8) S. 247 in Anwendung gebracht werden. Falls man diese Gleichung den S. 65 eingeführten Gleichungen genau anpassen will, so hat man etwa an Stelle der $\wp_{\lambda \mu}$ die Größen nullter Dimension:

$$
\frac{g_{2} g_{3} \wp_{\lambda_{u, u}}}{\Delta}
$$

benutzen. Sie genügen einer Gleichung, die aus der Gleichung (8) S. 247 durch die Substitution:

$$
z=\frac{\Delta}{g_{2} g_{3}} z_{0}
$$

hervorgeht. Diese „auf die nullte Dimension reduzierte“ spezielle Teilungsgleichung hat, wie man mit Hilfe von (16) in I, 124 zeigt, die Gestalt:

$$
z_{0}^{\frac{1}{2} \chi(n)}+\alpha_{1}^{\prime} J(J-1) z_{0}^{\frac{1}{2} \chi(n)-2}+\alpha_{2}^{\prime} J(J-1)^{2} z_{0}^{\frac{1}{2} \chi(n)-3}+\cdots=0,
$$

wo der Koeffizient von $z_{0}^{\frac{1}{2} \chi(n)-k}$ eine ganze Funktion $k^{\text {ten }}$ Grades von $J$ mit rationalen Zahlenkoeffizienten ist. Den Koeffizienten dieser Gleichung entsprechend hat man den Körper $\Re_{J}$ der rationalen Funktionen von $J$ mit rationalen Zahlenkoeffizienten als gegeben anzusehen, der dann an Stelle des S. $65 \mathrm{ff}$. durch $\Re_{x}$ bezeichneten Körpers tritt. Nach dem Satze von S. 248 ist die Gleichung (2) in diesem Körper $\Re_{J}$ irreduzibel und würde auch nach Adjunktion irgendwelcher ,numerischer" Irrationalitäten irreduzibel bleiben. Zur Erleichterung der SchluBweise werden wir in der Tat gelegentlich von der Gleichung (2) Gebrauch machen. Doch legen wir zunächst die Gleichung (8) S. 247 direkt zugrunde; vom Körper $\Re=\left(\Re, g_{2}, g_{3}\right)$ kommen dann natürlich nur Größen, die in $\omega_{1}, \omega_{2}$ homogen sind, d. h. „Modulformen“, zur Benutzung. 
Wir bilden nun durch Adjunktion der $\frac{1}{2} \chi(n)$ Teilwerte $\wp_{\lambda_{\mu}}$ den zur Gleichung (8) S. 247 gehörenden Galoisschen Körper:

$$
\left(\Re, \ldots, \wp_{\lambda \mu}, \ldots\right)=\left(\Re, g_{2}, g_{3}, \ldots, \wp_{\lambda \mu}, \ldots\right) .
$$

Nach S. 70 kann dieser Körper auch durch Adjunktion einer einzigen Größe hergestellt werden, die man mit Hilfe zweckmäßig gewählter rationaler ganzer Zahlen $c$ aus den $\rho_{\lambda_{\mu}}$ in der Gestalt:

$$
p_{1}\left(\omega_{1}, \omega_{2}\right)=\sum_{\lambda, \mu} c_{\lambda \mu} \wp_{\lambda \mu}
$$

bilden kann. Diese Größe ist eine. Modulform $n^{\text {ter }}$ Stufe der Dimension -2 ; die Bedeutung des Index 1 wird unten erklärt werden.

Die $\wp_{\lambda \mu}$ als Größen des Körpers (3) sind in $p_{1}$ rational mit Koeffizienten des Körpers $\Omega$ darstellbar:

$$
\wp_{\lambda \mu}=R_{\lambda \mu}\left(p_{1}\right) \text {. }
$$

Hieraus folgt leicht, daß zur Modulform $p_{1}\left(\omega_{1}, \omega_{2}\right)$ diejenige ausgezeichnete Kongruenzgruppe $n^{\text {ter }}$ Stufe $\Gamma_{\frac{1}{2} n \chi(n)}$ gehört, deren Substitutionen $\bmod n$ mit 1 oder -1 kongruent sind, d. h. daß diese und nur diese Substitutionen der $\Gamma^{(\omega)}$ die Form $p_{1}\left(\omega_{1}, \omega_{2}\right)$ in sich transformieren. DaB nämlich $p_{1}$ gegenüber diesen Substitutionen invariant ist, geht aus (4) hervor. Jede Substitution, die $p_{1}$ in sich transformiert, wird aber auch zufolge (5) z. B. die beiden Formen $\wp_{01}$ und $\wp_{10}$ zugleich in sich überführen, ist also zugleich mit einer Substitution $\left(\begin{array}{c} \pm 1, \beta \\ 0, \pm 1\end{array}\right)$, als auch einer Substitution $\left(\begin{array}{l} \pm 1,0 \\ \gamma, \pm 1\end{array}\right) \bmod n$ kongruent, d. h. sie ist $\equiv \pm 1$.

Durch die Substitutionen der $\Gamma^{(\omega)}$ wird hiernach $p_{1}\left(\omega_{1}, \omega_{2}\right)$ im ganzen in $\frac{1}{2} n \chi(n)$ verschiedene Modulformen transformiert, deren symmetrische Grundfunktionen ganze Modulformen erster.Stufe sind. Zur Darstellung dieser Modulformen als rationaler ganzer Funktionen von $g_{2}, g_{3}$ bedienen wir uns der Methode von S. $248 \mathrm{ff}$. Wir entnehmen zunächst aus (2) S. 244 eine Potenzreihe für $p_{1}$ :

(6) $p_{1}\left(\omega_{1}, \omega_{2}\right)=\left(\frac{2 \pi}{\omega_{2}}\right)^{2}\left(B_{0}+B_{1}(\varepsilon) q^{\frac{2}{n}}+B_{2}(\varepsilon) q^{\frac{4}{n}}+B_{3}(\varepsilon) q^{\frac{6}{n}}+\cdots\right)$,

wo $B_{0}$ eine rationale Zahl des Nenners 12 ist und die $B_{1}(\varepsilon), B_{2}(\varepsilon), \ldots$ Zahlen (und zwar ganze Zahlen) des zum $n^{\text {ten }}$ Teilungsgrade gehörenden Kreisteilungskörpers $(\Re, \varepsilon)$ sind, der ein Körper des Grades $\varphi(n)$ ist. Eine entsprechende Darstellung gestattet jede der $\frac{1}{2} n \chi(n)$ mit $p_{1}$ gleichberechtigten Formen, und also wird auch die $\nu^{\text {te }}$ symmetrische Grundfunktion dieser Formen, abgesehen vom Faktor $\left(\frac{2 \pi}{\omega_{2}}\right)^{2 v}$, als Entwicklungskoeffizienten der Potenzreihe Zahlen des Kreisteilungskörpers haben. Die 
Methode von S. 248 zeigt darauf hin, daß auch die numerischen Koeffizienten in den Darstellungen der fraglichen symmetrischen Grundfunktionen durch $g_{8}, g_{3}$ Zahlen von $(\Re, \varepsilon)$ sind. Man gelangt auf diese Weise zu dem Satze: Die $\frac{1}{2} n \chi(n)$ mit $p_{1}$ gleichberechtigten Formen sind die Lösungen einer Gleichung:

(7) $Z^{\frac{1}{2} n \chi(n)}+\beta_{11} g_{2} Z^{\frac{1}{2} n \chi(n)-2}+\beta_{12} g_{3} Z^{\frac{1}{2} n \chi(n)-3}+\beta_{13} g_{2}^{2} Z^{\frac{1}{2} n \chi(n)-4}+\cdots=0$,

deren Koeffizienten dem Körper $\left(\Re, g_{2}, g_{3}, \varepsilon\right)$ angehören; die Gleichung ist in diesem Körper irreduzibel und würde auch bei Adjunktion sonstiger „numerischer" Irrationalitäten irreduzibel bleiben. Den letzten Teil des Satzes beweist man genau so wie S. 247 die Irreduzibilität der speziellen Teilungsgleichung.

Ersetzt man in der Potenzreihe (2) S. 244 von $\wp_{\lambda_{\mu}}$ die Einheitswurzel $\varepsilon$ durch irgendeine der $\varphi(n)$ primitiven Einheitswurzeln $\varepsilon^{*}$, so gelangt man zur Reihenentwicklung für $\wp_{\lambda, x \mu}$. Wir erklären nun im Anschluß an (4) die $\varphi(n)$ Modulformen $p_{x}\left(\omega_{1}, \omega_{2}\right)$ durch:

$$
p_{x}\left(\omega_{1}, \omega_{2}\right)=\sum_{\lambda, \mu} c_{\lambda, \mu} \wp_{\lambda, x, \mu},
$$

wo $x$ die $\varphi(n)$ gegen $n$ teilerfremden Reste $\bmod n$ durchläuft. Da die $c_{\lambda_{\mu}}$ rational sind, so folgt aus (6) als Reihenentwicklung von $p_{\%}\left(\omega_{1}, \omega_{2}\right)$ :

(9) $p_{\varkappa}\left(\omega_{1}, \omega_{2}\right)=\left(\frac{2 \pi}{\omega_{2}}\right)^{2}\left(B_{0}+B_{1}\left(\varepsilon^{\varkappa}\right) q^{\frac{2}{n}}+B_{2}\left(\dot{\varepsilon}^{\varkappa}\right) q^{\frac{4}{n}}+B_{3}\left(\varepsilon^{\varkappa}\right) q^{\frac{6}{n}}+\cdots\right)$.

Die Gleichung $(\tilde{5})$ besteht in $\omega_{1}, \omega_{2}$ oder, wenn man die rechte und linke Seite nach Potenzen von $q$ entwickelt denkt, in $\omega_{9}$ und $q$ identisch, so daB die Koeffizienten gleich hoher Potenzen rechts und links gleich sind. Diese Koeffizienten sind Zahlen aus $\left.(\Re, \varepsilon)^{1}\right)$; je zwei einander gleiche Koeffizienten rechts und links bleiben demnach gleich, falls man $\varepsilon$ durch irgendeine primitive Wurzel $\varepsilon^{*}$ ersetzt. Da die Koeffizienten von $R_{\lambda \mu}$ dem Körper $\left(\Re, g_{2}, g_{3}\right)$ angehören, also bei jenem Ersatz unverändert bleiben, so bleibt die Gleichung (5) richtig, falls man $p_{1}$ durch $p_{x}$ und $\wp_{\lambda_{\mu}}$ durch $\wp_{\lambda \times u}$ ersetzt. Wir haben also $\varphi(n)$ Systeme zu je $\frac{1}{2} n \chi(n)$ Gleichungen:

$$
\rho_{\lambda, x \mu}=R_{\lambda, \mu}\left(p_{\nu}\right) \text {. }
$$

Für das einzelne $x$ und die $\frac{1}{2} \chi(n)$ Paare $\lambda, \mu$ haben wir auch in $\wp_{\lambda, x \mu}$ alle eigentlich zur $n^{\text {ten }}$ Stufe gehörenden $\wp$-Teilwerte. Die $\frac{1}{2} \chi(n)$ auf dieses $x$ bezogenen Gleichungen (10) zeigen demnach, daß nicht nur $p_{1}$, sondern jede der $\varphi(n)$ Größen $p_{\%}$ benutzt werden kann, um durch ihre Adjunktion den Galoisschen Körper (3) zu gewinnen. Indem wir die zur

1) Natürlich wieder abgesehen vom gemeinsamen Faktor $\left(\frac{2 \pi}{\omega_{2}}\right)^{2}$. 
Gleichung (7) führende Überlegung auf $p_{. x}$ anwenden, ergibt sich, daB diese Form und die mit ihr gleichberechtigten die Wurzeln einer Gleichung sind:

$$
\begin{gathered}
Z^{\frac{1}{2} n \chi(n)}+\beta_{\chi 1} g_{2} Z^{\frac{1}{2} n \chi(n)-2}+\beta_{\chi 2} g_{3} Z^{\frac{1}{2} n \chi(n)-3}+\beta_{\varkappa 3} g_{2}^{2} Z^{\frac{1}{2^{n}} \chi \chi(n)-4} \\
+\cdots=0
\end{gathered}
$$

welche alle oben von der Gleichung (7) ausgesagten Eigenschaften besitzt. Nun wird die Gleichung (7) für $Z=p_{1}$ nach Eintragung der Reihenentwicklungen wieder zu einer in $\omega_{2}$ und $q$ identisch bestehenden. Sie bleibt demnach auch gültig, wenn wir in ihr überall, d. h. auch in den Koeffizienten $\beta_{11}, \beta_{12}, \ldots$ die Einheitswurzel $\varepsilon$ durch $\varepsilon^{*}$ ersetzen. Es genügt demnach $p_{*}$ einer Gleichung des Grades $\frac{1}{2} n \chi(n)$, die aus (7) hervorgeht, indem man in den Koeffizienten $\beta_{11}, \beta_{12}, \ldots$ die Einheitswurzel $\varepsilon$ durch $\varepsilon^{*}$ ersetzt. Die so zu gewinnende Gleichung muß aber direkt die Gleichung (11) sein, wie sich aus der Irreduzibilität dieser Gleichung ergibt. Die $\frac{1}{2} n \chi(n)$ mit $p_{\varkappa}$ gleichbercchtigten Formen genügen der im Körper $\left(\Re, g_{2}, g_{3}, \varepsilon\right)$ irreduzibelen Gleichung (11), in der die $\beta_{x 1}, \beta_{x_{2}}, \beta_{* 3}, \ldots$ diejenigen Zahlen des Kreisteilungskörpers ( $\Re, \varepsilon$ ) sind, die aus den numerischen Kocffizienten $\beta_{11}, \beta_{12}, \beta_{13}, \ldots$ der Gleichung (7) durch den Ersatz von $\varepsilon$ durch $\varepsilon^{*}$ hervorgehen.

Es ist nun zu entscheiden, ob die $\varphi(n)$ Gleichungen (11) alle verschieden sind oder nicht. Zu diesem Zwecke wird folgende Hilfsbetrachtung vorausgeschickt, bei der wir den niedersten Fall $n=2$ als elementar ausschließen. Wir üben auf das Formentripel $\rho_{01}, \wp_{11}, \rho_{21}^{\prime}$ zunächst die $\frac{1}{2} n \chi(n)$ bezüglich der $\Gamma_{\frac{1}{2} n \chi(n)}$ zu unterscheidenden Substitutionen aus und erzeugen auf diese Art die $\frac{1}{2} n \chi(n)$ verschiedenen ,gleichberechtigten Tripel":

$$
\delta_{\gamma \delta}, \quad \rho_{\alpha+\gamma, \beta+\delta}, \quad \rho_{2 \alpha+\gamma, 2 \beta+\delta} .
$$

Ersetzen wir andrerseits in den Potenzreihen des ersten Tripels die Einbeitswurzel $\varepsilon$ durch die $\varphi(n)$ primitiven Einheitswurzeln $\varepsilon^{x}$, so erhalten wir die $\varphi(n)$ Tripel:

$$
\wp_{0 x}, \quad \wp_{1 x}, \quad \wp_{2 x},
$$

die wir als $\varphi(n)$ „konjugierte“ Tripel bezeichnen wollen. Abgesehen davon, daB $\wp_{01}, \wp_{11}, \wp_{21}$ sowohl unter (12) als unter (13) auftritt, kommt keines der gleichberechtigten Tripel (12) zugleich unter den konjugierten Tripeln vor. Aus dem gleichzeitigen Bestehen der drei Gleichungen:

$$
\wp_{\gamma \delta}=\wp_{0 \varkappa}, \quad \wp_{\alpha+\gamma, \beta+\delta}=\wp_{1 \psi}, \quad \wp_{2 \alpha+\gamma, 2 \beta+\delta}=\wp_{2 \varkappa}
$$

folgt nämlich zunächst $\gamma \equiv 0$ auf Grund der ersten Gleichung, sowie dann weiter $\alpha \equiv \pm 1$ auf Grund der zweiten. Da ein gleichzeitiger Zei- 
chenwechsel von $\alpha, \beta, \gamma, \delta$ vorgenommen werden kann, so soll $\alpha \equiv 1$. und damit $\delta \equiv 1$ (wegen $\gamma \equiv 0$ ) gesetzt werden. Die zweite und dritte Gleichung (14) lauten nun $\wp_{1, \beta+1}=\wp_{1, z}, \wp_{2,2} \beta+1=\wp_{2, x}$ und führen auf $x \equiv \beta+1 \equiv 2 \beta+1$ und damit auf $\beta \equiv 0, * \equiv 1$, womit die behauptete Verschiedenheit der Tripel (12) und (13) bewiesen ist.

Man bilde nun alle Tripel von Differenzen:

$$
\wp_{\gamma \delta}-\wp_{0 \varkappa}, \quad \wp_{\alpha+\gamma, \beta+\delta}-\wp_{1 \gamma}, \quad \wp_{2 \alpha+\gamma, 2 \beta+\delta}-\wp_{2 \varkappa},
$$

indem man nur daron absieht, das sowohl unter (12) als (13) anftretende Tripel $\wp_{01}, \wp_{11}, \wp_{21}$ mit sich selbst zur Subtraktion zu bringen. Man hat dann eine begrenzte Anzahl von Tripeln (15), von denen nach dem eben bewiesenen Satze keines aus drei mit 0 identischen Differenzen besteht. Wie S. 36 und S. 69 kann man hieraus den SchluB ziehen, daß man drei rationale ganze Zahlen $a, b, c$ so wäblen kann, dab von allen, den Tripeln (15) entsprechenden Formen:

$$
a\left(\wp_{\gamma \delta}-\wp_{0 \alpha}\right)+b\left(\wp_{\alpha+\gamma, \beta+\delta}-\wp_{1 k}\right)+c\left(\wp_{2 \alpha+\gamma, 2 \beta+\delta}-\wp_{2 \alpha}\right)
$$

keine einzige identisch rerschwindet. Demnach ist von allen $\frac{1}{2} n \chi(n)$ mit $\left(a \wp_{01}+b \wp_{11}+c \wp_{21}\right)$,gleichberechtigten" Formen keine einzige mit einer der "konjugierten“ Formen $\left(a \wp_{0 x}+b_{\wp_{1 x}}+c \varphi_{\varphi_{2,}}\right)$ mit $x \neq 1(\bmod n)$ identisch.

Nun ist aber $\left(a_{\wp_{01}}+b_{\wp_{11}}+c \wp_{21}\right)$, als im Galoissehen Körper (3) enthalten, in der Gestalt:

$$
a_{\wp_{01}}+b_{\wp_{11}}+c_{\wp_{21}}=R\left(p_{1}\right)
$$

mit Koeffizienten des Körpers $\left(\Re, g_{2}, g_{3}\right)$ darstellbar. Hieraus schließt man wie oben für die konjugierten Formen auf Darstellungen:

$$
a \wp_{0 x}+b \wp_{1 x}+c \varsigma_{2 x}=R\left(p_{x}\right) \text {. }
$$

Wäre nun die $\varkappa^{\text {te }}$ Gleichung (11) mit $x \neq 1$ identisch mit der Gleichung (7), so würde $p_{\eta}$, zu den mit $p_{1}$,gleichberechtigten" Formen gehören und also die Form (17) unter den mit (16) gleichberechtigten Formen auftreten. Dies aber trifft, wie wir wissen, nicht zu. Es ist also keine der Gleichungen (11) mit $k$ 丰 1 identisch mit der Gleichung (7). Da es uns nun unbenommen bleibt, irgendeine der $\varphi(n)$ Formen $p_{k}$ als Form $p_{1}$ an die Spitze der Entwicklung zu stellen, so ist die wichtige Tatsache erkannt, daß die $\varphi(n)$ Gleichungen (11) durchweg voneinander verschieden sind.

Zur Erleichterung der nächsten Überlegung passen wir die vorliegenden Voraussetzungen dadurch etwas besser an die allgemeinen Sätze der Einleitung über die Galoissche Gleichungstheorie an, daB wir mit den in (1) gegebenen Teilwerten nullter Dimension arbeiten. Die entsprechend an Stelle der $p_{x}$ tretenden GröBen $\frac{g_{2} g_{s} p_{x}}{\Delta}$ genügen $\varphi(n)$ „konjugierten“ 
Gleichungen:

$$
Z_{0}^{\frac{1}{2} n \chi(n)}+\beta_{x 1}^{\prime} J(J-1) Z_{0}^{\frac{1}{2} n \chi(n)-3}+\beta_{x 2}^{\prime} J(J-1)^{2} Z_{0}^{\frac{1}{2} n \chi(n)-3}+\cdots=0
$$

mit Koeffizienten des Körpers $\left(\Re_{J}, \varepsilon\right)$, der durch Adjunktion von $\varepsilon$ zu $\Re_{J}$ entsteht und einen in bezug auf $\Re_{J}$ algebraischen Körper $\varphi(n)^{\text {ten }}$ Grades darstellt. Wir nennen die Koeffizienten der Gleichung (18) kurz $A_{* 1}, A_{\varkappa 2}, A_{\varkappa 3}, \ldots$ und stellen fest, daB die Differenzen:

$$
A_{x_{1}}-A_{\varkappa^{\prime} 1}, A_{x_{2}}-A_{\varkappa^{\prime} 2}, \ldots
$$

für irgend zwei verschiedene Indizes $x, \varkappa^{\prime}$ ein System nicht durchweg identisch verschwindender Funktionen von $\left(\Re_{J}, \varepsilon\right)$ darstellen, da die $\varphi(n)$ Gleichungen durchweg verschieden sind. Man kann demnach ein System rationaler ganzer Zahlen $\alpha_{1}, \alpha_{2}$, . . so wählen, daß

$$
\alpha_{1}\left(A_{x_{1}}-A_{x^{\prime} 1}\right)+\alpha_{2}\left(A_{x_{2}}-A_{x^{\prime} 2}\right)+\cdots
$$

für keine Kombination zweier verschiedener $\varkappa, \varkappa^{\prime}$ identisch verschwindet Hieraus aber folgt, daB:

$$
\theta=\alpha_{1} A_{\gamma_{1}}+\alpha_{2} A_{\chi_{2}}+\alpha_{3} A_{\varkappa_{3}}+\cdots
$$

eine „primitive“ Funktion des Körpers $\left(\Re_{J}, \varepsilon\right)$ ist, und daß demnach dieser Körper auch durch Adjunktion von $\theta$ zu $\Re_{J}$ gewonnen werden kann. Nun gehören die $A_{\varkappa 1}, A_{x 2}, \ldots$ als Koeffizienten der Gleichung (18) dem Galoisschen Körper (3) an. Im gleichen Körper ist also, da die $\alpha_{1}, \alpha_{2}, \ldots$ rationale ganze Zahlen sind, auch $\theta$ und damit der $\operatorname{Körper}\left(\Re_{J}, \theta\right)=\left(\Re_{J}, \varepsilon\right)$ enthalten. Hiermit ist die grundlegende Tatsache bewiesen, $d a \beta$ die Einheitswurzel $n^{\text {ten }}$ Grades $\varepsilon$ eine natürliche Irrationalität der speziellen Teilungsgleichung des $n^{\text {ten }}$ Teilungsgrades ist (vgl. oben S. 233).

Die Frage nach der Galoisschen Resolvente der speziellen Teilungsgleichung ist jetzt unmittelbar zu beantworten. Durch Multiplikation der $\varphi(n)$ Gleichungen (11) entsteht eine im Körper ( $\left.\Re, g_{2}, g_{3}\right)$ irreduzibele Gleichung des Grades $\frac{1}{2} n \chi(n) \varphi(n)$, welche die Galoissche Resolvente der speziellen Teilungsgleichung, bezogen auf den durch die Koeffizienten dieser Gleichung gegebenen Körper $\Re=\left(\Re, g_{2}, g_{3}\right)$ ist. Weiter aber ergibt sich sofort: Nach Adjunktion der natürlichen Irrationalität \& wird die Galoissche Resolvente im Körper $(\Re, \varepsilon)=\left(\Re, g_{2}, g_{3}, \varepsilon\right)$ reduzibel und zerfällt in die $\varphi(n)$ nunmehr irreduzibelen Gleichungen (11), die auch bei Adjunktion irgendwelcher sonstiger numerischer Irrationalitäten irreduzibel bleiben.

Die „Galoissche Gruppe“ der speziellen Teilungsgleichung ist hiernach eine $G_{\frac{1}{2} n \chi(n) \varphi(n)}$ der Ordnung $\frac{1}{2} n \chi(n) \varphi(n)$, die eine Gruppe $G_{1^{\prime}, n_{\chi(n)}}$ der Ordnung $\frac{1}{2} n \chi(n)$, die "Monodromiegruppe“ unserer Gleichung, als ausgezeichnete Untergruppe besitzt. Als zugehörige „Quo- 
tientengruppe“:

$$
G_{\frac{1}{2} n \%(n) \varphi(n)} / G_{\frac{1}{2} n \chi(n)}=G_{\varphi(n)}
$$

aber ergibt sich die Gruppe der Kreisteilungsgleichung des $n^{\text {ten }}$ Teilungsgrades. Bei Benutzung der Gleichung (2) würde sich die Monodromiegruppe auf die Umläufe von $J$ in seiner Ebene beziehen (S. 76). Nach den Darlegungen in I, 299 ff. erzielen wir diese Umläufe durch Ausübung der Substitutionen der Modulgruppe auf $\omega$ bzw. $\omega_{1}, \omega_{2}$. Als Permutationsgruppe der Wurzeln $\wp_{\lambda \mu}$ der speziellen Teilungsgleichung ist demnach die Monodromiegruppe durch die $\frac{1}{2} n \chi(n)$ inkongruenten Substitutionen (7) S. 246 gegeben, vobei zwei Substitutionen, die durch gleichzeitigen Zeichenwechsel der vier Koeffizienten ineinander übergehen, als nicht verschieden gelten. Natürlich ist dies so zu verstehen, daB man sich die $\frac{1}{2} \chi(n)$ Wurzeln unserer Gleichung in eine Reihe geschrieben denkt und dann nacheinander die $\frac{1}{2} n \chi(n)$ Substitutionen (7) S. 246 auf die Indizes $\lambda, \mu$ ausübt, wodurch die Permutationen der $\wp_{\lambda \mu}$ hergestellt werden. Übrigens können wir nach S. 246 den Satz aussprechen, daß die Monodromiegruppe der speziellen Teilungsgleichung isomorph mit der $G_{\frac{1}{2} n \chi_{(n)}}$ ist, auf die sich. die nicht-homogene Modulgruppe $\bmod n$ reduziert.

Um zur Galoisschen Gruppe zu gelangen, haben wir $\varepsilon$ der Reibe nach durch alle $\varphi(n)$ primitiven Einheitswurzeln $n^{\text {ten }}$ Grades zu ersetzen. Dieser Ersatz bewirkt auf die Indizes $\lambda_{,}, \mu$ der Teilwerte $\rho_{\lambda \mu}$ die Substitution:

$$
\lambda^{\prime} \equiv \lambda, \quad \mu^{\prime} \equiv \varkappa \mu \quad(\bmod n),
$$

wo $x$ alle $\varphi(n)$ inkongruenten gegen $n$ teilerfremden Reste $\bmod n z u$ durchlaufen hat. Kombinieren wir die Substitutionen (19) mit den $\frac{1}{2} n \chi(n)$ Substitutionen (7) S. 246, so gelangen wir zur Galoisschen $G_{\frac{1}{2} n \chi(n) \varphi(n)}$ : Die Galoissche Gruppe $G_{\frac{1}{2} \eta_{\chi(n) \varphi(n)}}$ der speziellen Teilungsgleichung wird als Permutationsgruppe der $\rho_{\lambda_{\mu}}$ durch die $\frac{1}{2} n \chi(n) \varphi(n)$ inkongruenten Substitutionen:

$$
\lambda^{\prime} \equiv \alpha \lambda+\gamma \mu, \quad \mu^{\prime} \equiv \beta \lambda+\delta \mu \quad(\bmod n)
$$

erzielt, deren Determinanten $(\alpha \delta-\beta \gamma)$ alle gegen $n$ teilerfremden inkongruenten Reste $x$ sind, und bei denen zwei durch gleichzeitigen Zeichenwechsel der Koeffizienten ineinander überführbare Substitutionen als nicht verschieden gelte?.

\section{\$4. Lösung der speziellen Teilungsgleichung.}

Es ist jetzt möglich, über den ProzeB der Berechnung der Teilwerte $\wp_{2 \mu}$ durch Auflösung der speziellen Teilungsgleichung endgültige Angaben zu machen. Ist $n=n_{1} \cdot n_{2}$ das Produkt zweier teilerfremder Zahlen $n_{1}, n_{2}$, so kann zunächst die Berechnung der Teilwerte des Grades $n$ auf 
die der Grade $n_{1}, n_{2}$ zurückgeführt werden. Wir verstehen unter $\lambda_{1}, \lambda_{2}$ zwei ganze Zahlen, die die Gleichung $\lambda_{1} n_{2}+\lambda_{2} n_{1}=1$ befriedigen, setzen:

$$
\wp\left(\frac{\omega_{i}}{n_{1} n_{2}}\right)=\wp\left(\frac{\lambda_{1} \omega_{i}}{n_{1}}+\frac{\lambda_{2} \omega_{i}}{n_{2}}\right), \quad \wp^{\prime}\left(\frac{\omega_{i}}{n_{1} n_{2}}\right)=\wp^{\prime}\left(\frac{\lambda_{1} \omega_{i}}{n_{1}}+\frac{\lambda_{2} \omega_{i}}{n_{2}}\right), \quad i=1,2
$$

und entwickeln die rechten Seiten dieser Gleichungen nach dem Additionstheorem. Aus den Teilwerten $\wp\left(\frac{\lambda_{1} \omega_{i}}{n_{1}}\right), \wp\left(\frac{\lambda_{2} \omega_{i}}{n_{2}}\right)$ und den durch Quadratwurzeln zu berechnenden zugehörigen Teilwerten der $\wp^{\prime}$-Funktion berechnen wir uns also die Teilwerte (1) und aus ihnen weiter auf Grund der Additionssätze die übrigen Teilwerte des Grades $n=n_{1} \cdot n_{2}$. Auf diese Weise wird die Berechnung der Teilwerte irgendwelcher Grade mit Hilfe von Quadratwurzeln und rationalen Rechnungen auf die Berechnung der Teilwerte solcher Graden zurückgeführt, die Primzahlpotenzen $=p^{v}$ sind

In der zu $n=p^{v}$ gehörenden Monodromiegruppe haben wir nun für $\nu>1$ oben (S. 251) eine ausgezeichnete Untergruppe gefunden, deren zugehörige Quotientengruppe eine Abelsche Gruppe $G_{p^{3}}$ der Ordnung $p^{8}$ ist. Dem entspricht folgende einfache algebraische Tatsache: Nach dem Satze von S. 234 berechnet man aus $\wp\left(\frac{\omega_{1}}{p^{\nu-1}}\right), \wp^{\prime}\left(\frac{\omega_{1}}{p^{\nu-1}}\right)$ durch Lösung der „allgemeinen" Teilungsgleichung für den $p^{\text {ten }}$ Teilungsgrad die Teilwerte $\wp\left(\frac{\omega_{1}}{p^{v}}\right), \wp^{\prime}\left(\frac{\omega_{1}}{p^{v}}\right)$, was nach Adjunktion der $p^{\text {ten }}$ Einheitswurzel $\varepsilon$ an irrationalen Operationen das Ausziehen zweier Wurzeln $p^{\text {ten }}$ Grades erfordert. Mittelst zweier weiteren Wurzeln $p^{\text {ten }}$ Grades bestimmt man entsprechend $\wp\left(\frac{\omega_{2}}{p^{v}}\right), \wp^{\prime}\left(\frac{\omega_{2}}{p^{v}}\right)$, womit dann alle weiteren Teilwerte des Teilungsgrades $p^{v}$ rational bekannt sind. Eine der vier Wurzeln $p^{\text {ten }}$ Grades muß freilich überflüssig sein. Jedenfalls aber besteht der Satz, daß, falls die Teilwerte der primzahligen Teilungsgrade $p$ bekannt sind, die Teilwerte aller weiteren Grade allein durch rationale Rechnungen und Wurzelziehungen berechenbar sind.

Wir haben demnach unsere Aufmerksamkeit allein noch auf die Primzahlgrade $n=p$ zu richten und betrachten nunmehr nach Adjunktion der $p^{\text {ten }}$ Einheitswurzel $\varepsilon$ die Monodromiegruppe $G_{\frac{1}{2} p\left(p^{2}-1\right)}$ der speziellen Teilungsgleichung, welche wir nach S. 246 in der Gestalt der $\bmod n$ reduzierten nicht-homogenen Modulgruppe $\Gamma$ vorlegen. Es besteht nun der grundlegende Satz: Für alle Primzahlen $p>3$ ist die Gruppe $G_{\frac{1}{2} p\left(p^{2}-1\right)}$ „einfach", d.h. sie besitzt (außer der $G_{1}$ und $\left.\operatorname{der} G_{\frac{1}{9} p\left(p^{2}-1\right)}\right)$ keine ausgezeichnete Untergruppe.

Zum Beweise dieses Satzes erinnern wir daran, daß nach I, 297-die

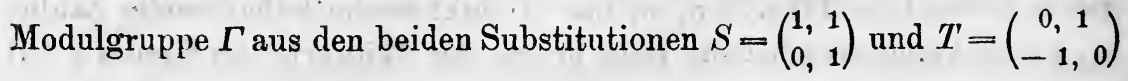


erzeugbar ist. Entsprechend wird die $G_{\frac{1}{2} p\left(p^{2}-1\right.}$ aus den Substitutionen $S \equiv\left(\begin{array}{l}1,1 \\ 0,1\end{array}\right)$ und $T \equiv\left(\begin{array}{c}0,1 \\ -1,0\end{array}\right)$ erzeugbar sein. Wir können nun beweisen, daB für $p>3$ eine ausgezeichnete Untergruppe $\operatorname{der} G_{\frac{1}{2} p\left(p^{2}-1\right)}$, die nicht nur aus der Substitution 1 besteht, notwendig die Substitutionen $S$ und $T$ besitzt und also die $G_{\frac{1}{2} p\left(p^{2}-1\right)}$ sein $m u B$, womit der Satz ersicbtlich sein würde.

Eine ausgezeichnete Untergruppe muß mit einer ihrer Substitutionen $V \equiv\left(\begin{array}{l}\alpha, \beta \\ \gamma, \delta\end{array}\right)$ alle mit $V$ gleichberechtigten, $d$. h. durch Transformation aus $V$ hervorgehenden Substitutionen enthalten. Transformieren wir aber die vorgelegte Substitution $V$ durch die Substitution:

$$
S^{b} \equiv\left(\begin{array}{l}
1, b \\
0,1
\end{array}\right), \quad T \equiv\left(\begin{array}{c}
0,1 \\
-1,0
\end{array}\right), \quad U \equiv\left(\begin{array}{c}
a, 0 \\
0, a^{-1}
\end{array}\right) \quad(\bmod p)
$$

unter $a$ und $b$ irgendwelche Zahlen der Reihe $1,2, \ldots, p-1$ verstanden, so gewinnen wir:

$$
\left\{\begin{array}{l}
S^{-b} \cdot V \cdot S^{b} \equiv\left(\begin{array}{c}
\alpha-b \gamma, \beta+b(\alpha-\delta)-b^{2} \gamma \\
\gamma, \delta+b \gamma
\end{array}\right), \\
T^{-1} \cdot V \cdot T \equiv\left(\begin{array}{c}
\delta,-\gamma \\
-\beta, \alpha
\end{array}\right), \quad U^{-1} \cdot V \cdot U=\left(\begin{array}{c}
\alpha, a^{-2} \beta \\
a^{2} \gamma, \delta
\end{array}\right) .
\end{array}\right.
$$

Enthält nun die von der $G_{1}$ verschiedene ausgezeichnete Untergruppe $G$ eine Substitution $V \equiv\left(\begin{array}{l}1, \beta \\ 0,1\end{array}\right)$ mit $\beta \neq 0$, so enthält sie auch $S$ und damit auch:

$$
S \cdot\left(T^{-1} \cdot S \cdot T\right) \cdot S \equiv\left(\begin{array}{c}
0,1 \\
-1,0
\end{array}\right) \equiv T
$$

sie ist also notwendig die $G_{\frac{1}{9} p\left(p^{2}-1\right)}$. Enthält $G$ zweitens eine Substitution $V \equiv\left(\begin{array}{c}\alpha, \beta \\ 0, \alpha^{-1}\end{array}\right)$ mit $\alpha \neq \pm 1$, so ist $\left(\alpha-\alpha^{-1}\right)$ teilerfremd gegen $p$. Zufolge der ersten Kongruenz (2) haben wir nun:

$$
S^{-b} \cdot V \cdot S^{b} \equiv\left(\begin{array}{l}
\alpha, \beta+b\left(\alpha-\alpha^{-1}\right) \\
0, \alpha^{-1}
\end{array}\right) \equiv\left(\begin{array}{c}
\alpha, \nu \\
0, \alpha^{-1}
\end{array}\right) \equiv V_{v},
$$

wo $\nu$ durch zweckmäBige Auswahl von $b$ mit jeder Zahl $0,1,2, \ldots, p-1$ kongruent werden kann. Mit $V_{0}$ und $V_{\alpha}$ ist auch:

$$
V_{0}^{-1} \cdot V_{\alpha} \equiv\left(\begin{array}{l}
1,1 \\
1,0
\end{array}\right) \equiv S
$$

in $G$ enthalten, so daB wieder $G=G_{\frac{1}{2} p\left(p^{2}-1\right)}$ ist. Eine von $\operatorname{der} G_{1}$ und $\operatorname{der} G_{\frac{1}{2} p\left(p^{2}-1\right)}$ verschiedene ausgezeichnete $G$ hat demnach, abgesehen von der identischen Substitution, keine Substitution mit $\gamma \equiv 0$ und also wegen der zweiten. Kongruenz (2) auch keine mit $\beta \equiv 0$. Ist demnach $V \equiv\left(\begin{array}{l}\alpha, \beta \\ \gamma, \delta\end{array}\right) \neq 1$ irgendeine Substitution von $G$, so ist $\gamma \neq 0(\bmod p)$, so daB es eine Zahl $b \equiv \alpha \gamma^{-1}(\bmod p)$ gibt. Für dieses $b$ hat $V^{\prime} \equiv S^{-b} \cdot V \cdot S^{\prime \prime}$ 
einen durch $p$ teilbaren ersten Koeffizienten, so dab es in $G$ sicher eine Substitution $V^{\prime}=\left(\begin{array}{c}0, \beta^{\prime} \\ -\beta^{\prime}-1, \delta^{\prime}\end{array}\right)$ gibt und demnach auch die Substitution:

$$
V^{\prime} \cdot\left(T^{-1} \cdot V^{\prime} \cdot T^{\prime-1}\right) \equiv\left(\begin{array}{c}
0, \beta^{\prime} \\
-\beta^{\prime-1}, \delta^{\prime}
\end{array}\right) \cdot\left(\begin{array}{l}
\delta^{\prime}, \beta^{\prime-1} \\
-\beta^{\prime}, 0
\end{array}\right) \equiv\left(\begin{array}{l}
\beta^{\prime 2}, \\
\delta^{\prime}\left(\beta^{\prime}+\beta^{\prime-1}\right), \beta^{\prime-2}
\end{array}\right) .
$$

Da sie einen durch $p$ teilbaren zweiten Koeffizienten hat, so ist sie $\equiv 1$, d. h. wir haben $\beta^{\prime} \equiv \pm 1, \delta^{\prime} \equiv 0$. In $G$ kommt stets die Substitution $T$ vor; zugleich ist diese Substitution, wenn $G$ nicht die $G_{\frac{1}{2} p\left(p^{2}-1\right)}$ sein soll, die einzige in $G$ auftretende Substitution mit $\alpha \equiv 0$. Nun kommt aber in $G$ zufolge der dritten Kongruenz (2) mit $T$ auch $\left(\begin{array}{c}0, a^{-2} \\ -a^{2}, 0\end{array}\right)$ vor. Man kann aber $a$, wenn $p>5$ ist, sicher so wählen, daB diese Substitution von $T$ verschieden ist. Dieser Widerspruch zeigt, daß für $p>5$ keine von $G_{1}$ und $G_{\frac{1}{2} p\left(p^{2}-1\right)}$ verschiedene ansgezeichnete Untergruppe rorkommt. Bei $p=5$ gelangen wir zu dem gleichen Resultate, indem wir bemerken, daB hier $S^{-2} \cdot T \cdot S^{2}$, ohne $\equiv 1$ zu sein, ein durch 5 teilbares $\beta$ hat.

Für $p>3$ besteht hiernach die „Indexreihe“ (vgl. S. 13) unserer Gruppe $G_{\frac{1}{2} p\left(p^{2}-1\right)}$ nur aus dem einzigen Gliede $\frac{1}{2} p\left(p^{2}-1\right)$; und da diese Zahl keine Primzahl ist, so ist nach dem Theorem von $\mathrm{S} .75$ die spezielle Teilungsgleichung des $p^{\text {ten }}$ Teilungsgrades für $p>3$ nicht algebraisch lösbar. Für $p=2$ und $p=3$ gehören die Teilungsgleichungen den Graden 3 und 4 an: In den beiden niedersten Fällen $p=2$ und $p=3$ ist die Berechnung der Teilwerte allein durch Wurzelziehungen durchführbar.

Bei dieser Sachlage war es nun ein besonders wichtiges Ziel der von Klein geschaffenen Theorie der elliptischen Modulfunktionen, die eigenartigen algebraischen Probleme, welche den Monodromiegruppen für $p=5,7$, $11, \ldots$ entsprechend als "Galoissche Probleme" der Grade 60, 168, 660, ..., allgemein des Grades $\frac{1}{2} p\left(p^{2}-1\right)$, auftreten, näher zu erforschen. Bei $p=5$ gelangt man zur "Ikosaedertheorie“. ${ }^{1}$ ) Im Falle $p=7$ entwarf Klein seine besonders schöne Theorie der zugrunde liegenden Gruppe $G_{168}$ durch direkte algebraische Methoden ohne Zuhilfenahne von Reihenentwicklungen der elliptischen Funktionen ${ }^{2}$ ), und auch im Falle $p=11$ gelang ihm die Durchführung einer entsprechenden Theorie. ${ }^{3}$ ) Für die

1) „Vorlesungen über das Ikosaeder und die Anflösung der Gleichungen vom fünften Grade" (Leipzig 1884); s. auch „Über die Transformation der elliptischen Funktionen und die Auflösung der Bleichungen fünften Grades", Math. Ann., Bd. 14 (1878).

2) "Über Transformation siebenter Ordnung der elliptischen Funktionen", Math. Ann., Bd. 14 (1878).

3) „Über Transformation elfter Ordnung der elliptischen Funktionen“, Math. Ann., Bd. 15 (1879). 
höheren Fälle bediente sich Klein indessen der analytischen Hilfsmittel der elliptischen Funktionen.

Die Behandlung dieser Galoisschen Probleme ist in dem Werke „Modulfunktionen“, Bd. 1 und 2 mit großer Ausführlichkeit gegeben. Demgegenüber nimmt die vorliegende Entwicklung die Wendung, daB sie sich den der älteren Theorie entstammenden algebraischen Gesichtspunkten enger anschlieBt. Es handelt sich dabei nicht um die Galoisschen Resolventen, sondern um die „Resolventen niedersten Grades“ der speziellen Teilungsgleichungen. Es sind dies wenigstens im allgemeinen die Modular- und Multiplikatorgleichungen oder, wie wir sagen werden, die "speziellen Transformationsgleichungen", die bei der Transformation höheren Grades der elliptischen Funktionen auftreten. Auch in diesem Gebiete haben übrigens; wie unten näher darzulegen sein wird, die Kleinschen Methoden mannigfach bahnbrechend gewirkt. Ehe wir indessen allgemein auf die Transformationstheorie der elliptischen Funktionen eingehen, sind noch ein paar Ausführungen über die Teilwerte der Jacobischen Funktionen sn, cn, dn nachzutragen.

\section{$\S 5$. Die Teilwerte der Funktionen sn, en und $\mathrm{dn}$.}

Die S. 240 genannten Untersuchungen von Sylow und Kronecker beziehen sich auf die Teilwerte der sn-Funktion und betreffen übrigens nur ungerade Teilungsgrade $n$. Die Aufgabe der Berechnung der Quadrate $\mathrm{sn}_{\lambda \mu}^{2}$ unterscheidet sich vom Probleme der Berechnung der $\wp_{\lambda_{2 \mu}}$ nur dadurch, daß noch die Adjunktion der Modulform zweiter S'tufe $\left(e_{\mathrm{a}}-e_{1}\right)$ zu vollziehen ist. Diese Adjunktion hat zunächst die Bedeutung, daß an Stelle von $\left(\Re, g_{2}, g_{3}\right)$ der $\operatorname{Körper}\left(\Re, k^{2}\right)$ tritt, sowie daß andrerseits die Teilwerte $\wp_{\lambda \mu}, \wp_{\lambda \mu}^{\prime}$ durch die in (9) S. 238 erklärten Teilwerte nullter Dimension $p_{2 \mu}, p_{2 \mu}^{\prime}$ zu ersetzen sind. Dann aber gelten nach (10) und (11) S. 238 die Gleichungen:

$$
\left\{\begin{aligned}
\mathrm{sn}_{\lambda, \mu}^{2} & =\frac{12\left(27 p_{\lambda \mu}^{3}-9\left(1-k^{2}+k^{4}\right) p_{\lambda \mu}-2+3 k^{2}+3 k^{4}-2 k^{6}\right)}{\left(\left(3 p_{\lambda \mu}+1+k^{2}\right)^{2}-9 k^{2}\right)^{2}} \\
p_{\lambda \mu} & =\frac{1-k^{2}+k^{2}\left(k^{2}-2\right) \mathrm{cn}_{\lambda \mu}+\left(2 k^{2}-1\right) \mathrm{dn}}{3\left(-1+k^{2}-k^{2} \mathrm{cn}_{\lambda \mu}+\mathrm{dn}_{\lambda \mu}\right)}
\end{aligned}\right.
$$

während sich die $\mathrm{cn}_{2 \mu}, \mathrm{dn}_{2 \mu}$ auf Grund von (16) S. 240 rational in $\mathrm{sn}_{2 \mu}^{2}$ ausdrücken. Die Gleichwertigkeit der beiden genannten Teilungsprobleme geht aus diesen Gleichungen hervor.

Gleichwohl ist es zweckmäBig, auf das Problem der Berechnung der $\mathrm{sn}_{2 \mu}$ noch etwas näher einzugehen, um die früher hierbei benutzten $\mathrm{Me}$ thoden und Überlegungen zu kennzeichnen. Setzt man, unter $n$ nach wie vor eine beliebige ungerade Zahl verstanden, $w=0$ in (14) S. 239 ein, so ergibt sich $G_{1}^{(n)}\left(Z^{2}\right)=0$ als Gleichung $\left(n^{2}-1\right)^{\text {ten }}$ Grades für $Z=\operatorname{sn}_{\lambda \text {. }}$ 
oder als Gleichung des Grades $\frac{1}{2}\left(n^{2}-1\right)$ für $Z^{2}=s_{\lambda \mu}^{2}$. Diese Gleichung ist nur im Falle eines primzahligen $n$ im Körper $\left(\Re, k^{2}\right)$ irreduzibel. Bei zusammengesetztem $n$ stellt man indessen wie S. 247 durch einen DivisionsprozeB die irreduzible, „spezielle Teilungsgleichung" des Grades $\frac{1}{2} \chi(n)$ für $Z^{2}=\operatorname{sn}_{\lambda \mu}^{2}$ dar, deren Lösungen die „eigentlich" zum Teilungsgrade $n$ gehörenden Teilwerte der sn-Funktion sind.

Die Hauptaufgabe ist nun die Bestimmung der Gruppe dieser speziellen Teilungsgleichung, wobei man so verfahren kann: Nach (1) S. 197 gilt die Regel:

$$
\mathrm{sn}_{z \lambda, \varkappa \mu}=\frac{\operatorname{sn}_{\lambda \mu} G_{1}^{(*)}\left(\sin _{\lambda, \mu}^{2}\right)}{G_{0}^{(\alpha)}\left(\sin _{\lambda, \mu}^{2}\right)}
$$

für jedes positive ganzzahlige $\varkappa$, wobei rechts eine rationale Funktion von $\mathrm{sn}_{2, \mu}$ mit Koeffizienten des Körpers $\left(\Re, k^{2}\right)$ steht. Insbesondere folgt hieraus:

$$
\mathrm{sn}_{\varkappa 0}=\frac{\mathrm{sn}_{10} G_{1}^{(*)}\left(\mathrm{sn}_{10}^{2}\right)}{G_{0}^{(*)}\left(\mathrm{sn}_{10}^{2}\right)}, \quad \mathrm{sn}_{0 \varkappa}=\frac{\mathrm{sn}_{01} G_{1}^{(*)}\left(\mathrm{sn}_{01}^{2}\right)}{G_{0}^{(*)}\left(\mathrm{sn}_{01}^{2}\right)} .
$$

Eine einzelne vorgelegte Permutation der Galoisschen Gruppe unserer Gleichung möge nun $\mathrm{sn}_{10}$ und $\mathrm{sn}_{01}$ in $\mathrm{sn}_{\alpha \beta}$ bzw. $\mathrm{sn}_{\gamma \delta}$ überführen, wo $\alpha, \beta$ und $\gamma, \delta$ gewisse, im Sinne von S. 218 gegen $n$ teilerfremde Zahlenpaare sind. Nach den Sätzen von S. 74 über die Galoissche Gruppe einer Gleichung gehen die Relationen (3) bei der fraglichen Permutation wieder in richtige Relationen über. Nimmt man demnach noch auf die Relation (2) Rücksicht, so zeigt sich, daß bei der vorgelegten Permutation unserer Gruppe $\mathrm{sn}_{y_{0}}$ in $\mathrm{sn}_{x \alpha, x \beta}$ und $\mathrm{sn}_{0 x}$ in $\mathrm{sn}_{x \gamma, x \delta}$ übergeht. Weiter folgt aus dem Additionstheorem mit Rücksicht auf die Formeln (16) S. 240 eine Darstellung von $\mathbf{s n}_{\lambda+\lambda^{\prime}, \mu+\mu^{\prime}}$ in der Gestalt:

$$
\mathrm{sn}_{\lambda+\lambda^{\prime}, \mu+\mu^{\prime}}=R\left(\mathrm{sn}_{\lambda, \mu}, \mathrm{sn}_{\lambda^{\prime} \mu^{\prime}}\right)
$$

als rationale Funktion von $\mathrm{sn}_{\lambda \mu}$ und $\mathrm{sn}_{\lambda^{\prime} \mu^{\prime}}$ mit Koeffizienten aus $\left(\mathfrak{R}, k^{2}\right)$. Als Spezialfall von (4) notieren wir:

$$
\mathrm{sn}_{\lambda \mu}=R\left(\mathrm{sn}_{\lambda_{0}}, \mathrm{sn}_{0 \mu}\right) \text {. }
$$

Durch die fragliche Permutation geht nun $\mathrm{sn}_{20}$ in $\mathrm{sn}_{\alpha \lambda, \beta \lambda}$ und $\mathrm{sn}_{0 \mu}$ in $\mathrm{sn}_{\gamma \mu, \delta \mu}$ über. Da nun auch die Relation (5) in eine richtige Relation übergeführt wird, so liest man aus (4) $\mathrm{ab}, \mathrm{daB}^{\mathrm{si}} \mathrm{sn}_{2 \mu}$ in $\mathrm{sn}_{\alpha \lambda+\gamma \mu, \beta \lambda+\delta \mu}$ übergeht. $\mathrm{Da}$ die Indizes beliebig $\bmod n$ reduziert werden können, so finden wir, daß die einzelne Permutation der Galoisschen Gruppe $\mathrm{sn}_{2, \mu}$ in $\mathrm{sn}_{\lambda^{\prime} \mu^{\prime}} \ddot{u}$ berführt, wo:

$$
\lambda^{\prime} \equiv \alpha \lambda+\gamma \mu, \quad \mu^{\prime} \equiv \beta \lambda+\delta \mu \quad(\bmod n)
$$

gilt und $\alpha, \beta, \gamma, \delta$ vier ganze Zahlen sind, von denen jedenfalls $\alpha, \beta$ und $\gamma, \delta$ gegen $n$ teilerfremde Paare bilden.

Hier dürfen nur solche Zahlquadrupel $\alpha, \beta, \gamma, \delta$ auftreten, welche die $\chi(n)$ gegen $n$ teilerfremden Zahlenpaare $\lambda, \mu$ wieder in diese $\chi(n)$ 
Paare $\lambda^{\prime}, \mu^{\prime}$ überführen. Hieraus kann man den SchluB ziehen, daB $(\alpha \delta-\beta \gamma)$ teilerfremd gegen $n$ sein muß. Hätte nämlich $(\alpha \delta-\beta \gamma)$ mit $n$ mindestens einen Primfaktor $p$ gemein, so beachte man, daB sich die $\chi(n)$ Paare $\lambda, \mu \bmod p$ auf die $\chi(p)$ gegen $p$ teilerfremden und $\bmod p$ inkongruenten Paare reduzieren. Auch diese $\chi(p)$ Paare müssen sich demnach bei den auf den Modul $p$ bezogenen Kongruenzen (6) permutieren. Setzen wir aber in (6) das gegen $p$ teilerfremde Paar $\lambda \equiv-\gamma, \mu \equiv \alpha$ ein $^{1}$ ), so entsteht wegen $\alpha \delta-\beta \gamma \equiv 0(\bmod p)$ das nicht gegen $p$ teilerfremde Paar $\lambda^{\prime} \equiv 0, \mu^{\prime} \equiv 0$. Also ist notwendig $(\alpha \delta-\beta \gamma)$ teilerfremd gegen $n$.

Die gesamten Substitutionen (6), in denen $\alpha \delta-\beta \gamma \equiv \varkappa$ die $\varphi(n)$ gegen $n$ teilerfremden Zahlen durchläuft, bilden nun, wie wir wissen, eine Gruppe $G_{n \chi(n) \varphi(n)}$, in der die Substitutionen mit $\alpha \delta-\beta \gamma \equiv 1(\bmod n)$ eine ausgezeichnete Untergruppe $G_{n \chi(n)}$ der Ordnung $n \chi(n)$ bilden. In der $G_{n \chi(n) \varphi(n)}$ ist die Galoissche Gruppe der speziellen Teilungsgleichung der sn-Funktion sicher enthalten. Bestimmen wir nun zunächst die Monodromiegruppe für die $\mathrm{sn}_{\lambda \mu}$ als algebraische Funktionen ron $h^{2}$, so sind, wenn wir die Umläufe von $k^{2}$ in seiner Ebene durch Periodensubstitutionen ersetzen, nach I, 475 die homogenen Substitutionen der Hauptkongruenzgruppe zweiter Stufe $\Gamma_{6}$ anzuwenden. Bei Ausübung der Substitution $T^{2}=-1$ wird der einzelne Teilwert $\operatorname{sn}_{\lambda \mu}$ durch $\mathrm{sn}_{n-\lambda, n-\mu}=-\mathrm{sn}_{\lambda \mu}$ ersetzt. Für ungerades $n$ ist die Anzahl der $\bmod n$ inkongruenten Substitutionen der homogenen Gruppe $\Gamma_{6}$ gleich $n_{\chi}(n)$. Man kann nämlich zu jeder Substitution $\left(\begin{array}{l}\alpha, \beta \\ \gamma, \delta\end{array}\right)$, indem man die Koeffizienten nötigenfalls um $n$ ändert, sofort eine ihr $\bmod n$ kongruente Substitution:

$$
\omega_{1}^{\prime} \equiv \alpha^{\prime} \omega_{1}+\beta^{\prime} \omega_{2}, \quad \omega_{2}^{\prime} \equiv \gamma^{\prime} \omega_{1}+\delta^{\prime} \omega_{2}, \quad \alpha^{\prime} \delta^{\prime}-\beta^{\prime} \gamma^{\prime} \equiv 1 \quad(\bmod 2 n)
$$

angeben, die mod 2 mit 1 kongruent ist. $\mathrm{Zu}$ dieser Substitution gibt es dann aber mod $2 n$ kongruente Substitutionen in der homogenen $\Gamma^{(\omega)}$ (vgl. S. 221), so dab umgekehrt innerhalb der homogenen $\Gamma_{6}$ alle $n \chi(n)$ inkongruenten Substitutionen $\bmod n$ vertreten sind. Diese Substitutionen liefern dann in der Tat die oben schon genannte $G_{n \chi(n)}$ mit $\alpha \delta-\beta \gamma \equiv 1$ $(\bmod n)$ als Monodromiegruppe der speziellen Teilungsgleichung.

Was nun die auf den Körper $\left(\Re, k^{2}\right)$ bezogene Galoissche Gruppe der speziellen Teilungsgleichung betrifft, so besteht sie nach S. 74 aus allen Permutationen der $\mathrm{sn}_{2 \mu}$, bei denen jede zwischen den Teilwerten bestehende rationale Gleichung mit Koeffizienten aus $\left(\Re, k^{2}\right)$ wieder in eine richtige Gleichung übergeht. Tragen wir aber in irgendeine solche Gleichung für die $\mathrm{sn}_{\lambda \mu}$ und für $k^{2}$ die Reihen nach Potenzen von $q$ ein und ordnen die linke Seite der Gleichung selbst nach Potenzen von $q$, so

1) $\alpha$ und $\gamma$ können nicht zugleich durch $p$ teilbar sein, weil sonst $\lambda^{\prime} \equiv 0$ mod $p$ ) infolge (6) für jedes Paar $\lambda, \mu$ zutreffen würde. 
muB jeder Koeffizient, der übrigens eine Zahl des Körpers $(\Re, \varepsilon)$ ist, für sich verschwinden. Hieraus kann man mit Rücksicht auf die Irreduzibilität der Kreisteilungsgleichung den Schluß ziehen, daß die gedachte rationale Gleichung $z$ wischen den $\mathbf{s n}_{\lambda \mu}$ richtig bleibt, falls $\mathbf{s n}_{\lambda \mu}$ durch $\mathrm{sn}_{\lambda, x \mu}$ ersetzt wird, unter $*$ eine beliebige der $\varphi(n)$ gegen $n$ teilerfremden, $\bmod n$ inkongruenten Zahlen verstanden. Die Galoissche Gruppe der Teilungsgleichung ist also wirklich die $G_{n \chi(n) \varphi(n)}$.

Die Reduktion der Galoisschen Gruppe auf die Monodromiegruppe kann man nun auch mit Benutzung der "Abelschen Relationen" in folgender Art vollziehen. Ist $\varepsilon$ adjungiert und damit der Körper auf $\left(\Re, k^{2}, \varepsilon\right)$ erweitert, so ist die Abelsche Relation (12) S. 243 eine zwischen den $\mathbf{s n}_{2 \mu}$ bestehende Relation mit rational bekannten Koeffizienten. Eine Permutation der $\mathrm{sn}_{\lambda \mu}$, bei welcher $\mathrm{sn}_{\lambda \mu}$ in $\mathrm{sn}_{\lambda, x_{\mu}}$ mit $x \neq 1(\bmod n)$ übergeht, kann jetzt der Gruppe der Teilungsgleichung nicht mehr angehören. Aus den Betrachtungen von S. 243 folgt nämlich:

$$
\left.\sum_{\mu=1}^{n-1} \varepsilon^{4 \lambda \mu} \mathrm{sn}_{2, x \mu} \neq 0 \text { für } x \neq 1(\bmod n) \cdot{ }^{1}\right)
$$

Die Abelsche Relation (12) S. 243 würde also durch eine jener Permutationen mit $x \neq 1(\bmod n)$ nicht wieder in eine richtige Gleichung übergeführt, so daß jene Permutationen nach Adjunktion von $\varepsilon$ nicht mehr der Gruppe unserer Gleichung angehören. Nach Adjunktion von $\varepsilon$ kommt also die Gruppe der Gleichung in der Tat auf die $G_{n \chi(n)}$ zurück.

1) Man wolle sich in dieser Formel wie auch in der Abelschen Relation (12) S. 243 die nicht eigentlich zum Teilungsgrade $n$ gehörenden $\mathrm{sn}_{2 \mu}$ durch die Wurzeln unserer jrreduziblen Teilungsgleichung nach den Multiplikationssätzen ausgedrückt denken. 


\section{Zweiter Abschnitt.}

\section{Die Transformationstheorie der elliptischen Funktionen.}

Als Ziel der weiteren Entwicklung wurde S. 265 die Untersuchung der Resolventen niedersten Grades der speziellen Teilungsgleichung bezeichnet. Wir erweitern diese Aufgabe in der Art, daB wir auch für die allgemeine Teilungsgleichung nach bemerkenswerten Resolventen niederen Grades suchen. In der Tat werden uns solche Resolventen von den Gleichungen der Transformationstheorie, den „allgemeinen“ und den „speziellen Transformationsgleichungen" geliefert. Es handelt sich hierbei um eine außerordentlich vielseitig entwickelte Theorie, deren Anfänge in das vorletzte Jahrhundert zurückreichen, deren wichtigste Grundsätze von Abel und Jacobi aufgestellt wurden, die aber erst im letzten Drittel des vorigen Jahrhunderts durch die Ausbildung der Theorie der elliptischen Modulfunktionen ihre jetzt vorliegende Gestalt gewonnen hat.

\section{Erstes Kapitel.}

\section{Die Transformation $n^{\text {ten }}$ Grades und die allgemeinen Transformationsgleichungen.}

Die Grundaufgabe der Transformationstheorie tritt bereits bei Euler und Lagrange ${ }^{1}$ ), und zwar bei Behandlung gewisser mechanischer Aufgaben, auf. Es handelt sich in der Sprechweise der elliptischen Funktionen um die Frage, ob ein elliptisches Differential, das $z$ als Variable hat, in ein anderes elliptisches Differential mit $z^{\prime}$ als Variable dadurch transformiert werden kann, daß man zwischen $z$ und $z^{\prime}$ eine algebraische Relation vorschreibt. Es ist dies die algebraische Fassung des Transformationsproblems, während sich die transzendente Gestalt, wie sogleich in $\S 1$ ausgeführt wird, an den Begriff der doppeltperiodischen Funktion anschlieBt. Diese transzendente Gestalt bahnt in einfachster Weise die

1) Vgl. „Enzyklopädie“ S. 186. 
270 II, 1. Transformation $n^{\text {ten }}$ Grades $u$. allgemeine Transformationsgleichungen allgemeine Lösung des Problems an. In den grundlegenden Untersuchungen von Abel und Jacobi kommen natürlich beide Seiten des Problems zur Geltung, und zwar die algebraische vornehmlich im „Précis d'une théorie des fonctions elliptiques"1) und im ersten Teile der „Fundamenta nova“" ${ }^{\text {(2) }}$, die transzendente in den „Recherches sur les fonctions elliptiques" (vgl. die erste Note S. 209) und den späteren Entwicklungen der "Fundamenta nova“. In neuerer Zeit pflegt man das Transformationsproblem in transzendenter Gestalt anzusetzen und die algebraische Fassung nur mehr beiläufig zu erwähnen. ${ }^{3}$ ) In dieser Art soll auch hier verfahren werden.

\section{$\S 1$ Aufstellung des Transformationsproblems und Ansatz zur Lösung.}

Es seien $\varphi\left(u \mid \omega_{1}, \omega_{2}\right)$ und $\psi\left(u^{\prime} \mid \omega_{1}^{\prime}, \omega_{2}^{\prime}\right)$ zwei elliptische Funktionen mit fest gegebenen Perioden. Das Grundproblem der allgemeinen Transformation ist dann in transzendenter Gestalt: Unter welchen Umständen hat die Annahme einer linearen Relation $u^{\prime}=m u+\mu$ mit konstanten $m, \mu$ eine "algebraische" Relation $F(\varphi, \psi)=0$ zwischen den elliptischen Funktionen $\varphi$ und $\psi$ zur Folge?

Da $\varphi\left(u \mid \omega_{1}, \omega_{2}\right)$ und $\wp\left(u \mid \omega_{1}, \omega_{2}\right)$ algebraisch zusammenhängen und ebenso $\psi\left(u^{\prime} \mid \omega_{1}^{\prime}, \omega_{2}^{\prime}\right)$ und $\wp\left(u^{\prime} \mid \omega_{1}^{\prime}, \omega_{2}^{\prime}\right)$, so können wir unsere Frage ohne Beschränkung der Allgemeinheit gleich auf $\wp\left(u \mid \omega_{1}, \omega_{2}\right)$ und $\wp\left(u^{\prime} \mid \omega_{1}^{\prime}, \omega_{2}^{\prime}\right)$ an Stelle der Funktionen $\varphi\left(u \mid \omega_{1}, \omega_{2}\right)$ und $\psi\left(u^{\prime} \mid \omega_{1}^{\prime}, \omega_{2}^{\prime}\right)$ beziehen. Weiter hängt $\wp\left(m u+\mu \mid \omega_{1}^{\prime}, \omega_{2}^{\prime}\right)$ mit $\wp\left(m u \mid \omega_{1}^{\prime}, \omega_{2}^{\prime}\right)$ algebraisch zusammen (auf Grund des Additionstheorems), und es gilt, da die $\wp-$-Funktion homogen von der Dimension -2 in ihren drei Argumenten ist:

$$
\wp\left(m u \mid \omega_{1}^{\prime}, \omega_{2}^{\prime}\right)=m^{-2} \cdot \wp\left(u \mid \frac{\omega_{1}^{\prime}}{m}, \frac{\omega_{2}^{\prime}}{m}\right) .
$$

Setzt man demnach für $\frac{\omega_{1}^{\prime}}{m}, \frac{\omega_{2}^{\prime}}{m}$ sogleich wieder $\omega_{1}^{\prime}, \omega_{2}^{\prime}$, so hat das aufgestellte Problem ohne Beschränkung der Allgemeinheit folgende Gestalt angenommen: Unter welchen Umständen, $d . h$. für welche Periodenpaare $\omega_{1}, \omega_{2}$ und $\omega_{1}^{\prime}, \omega_{2}^{\prime}$ besteht zwischen $\wp\left(u \mid \omega_{1}, \omega_{2}\right)$ und $\wp\left(u \mid \omega_{1}^{\prime}, \omega_{2}^{\prime}\right)$ eine algebraisclee Relation:

$$
F\left(\wp\left(u \mid \omega_{1}, \omega_{2}\right), \wp\left(u \mid \omega_{1}^{\prime}, \omega_{2}^{\prime}\right)\right)=0
$$

mit Koeffizienten, die natürlich von u unabhängig sein sollen?

Schreiben wir für die beiden in der Relation (1) verbundenen $\wp$-Funk-

1) Journ. f. Math., Bd. 4 (1829) oder Abels Werke, nene Augg. Bd. 1, S. 518.

2) Königsberg 1829 oder Jacobis Werke, Bd. 1, S. $49 \mathrm{ff}$.

3) Man vgl. z. B. WeierstraB, „Vorlesungen über die Theorie der elliptitischen Funktionen", Werke, Bd. 5, S. 276. 
Das Transformationsproblem in transzendenter und in algebraischer Gestalt 271 tionen $z$ und $z^{\prime}$, und bezeichnen wir zur Einführung der algebraischen Schreibweise des Differentials $d u$ die $g_{2}\left(\omega_{1}^{\prime}, \omega_{2}^{\prime}\right)$ und $g_{3}\left(\omega_{1}^{\prime}, \omega_{2}^{\prime}\right)$ kurz durch $g_{2}^{\prime}, g_{3}^{\prime}$, so können wir unser Problem in folgende algebraische Gestalt umkleiden: Unter welchen Umständen kann man allgcmein die Differentialgleichung:

$$
\frac{d z}{\sqrt{4 z^{3}}-g_{2} z-g_{3}}=\frac{d z^{\prime}}{\sqrt{4 z^{\prime 3}-g_{9}^{\prime} z^{\prime}-g_{3}^{\prime}}}
$$

durch eine algebraische Relation $F\left(z, z^{\prime}\right)=0$ integrieven, oder wie kann das in (2) links stehende Differential mittelst einer algebraischen Funktion $z^{\prime}$ von $z$ in ein glcichgebautes Differential transformiert werden?

Die Lösung des Problems wird durch folgende Betrachtung angebahnt: Die in I, 229 eingeführte Gruppe $\Gamma^{(u)}$ für die Perioden $\omega_{1}, \omega_{2}$ werde kurz $\Gamma$ genannt; die entsprechende Gruppe für die Perioden $\omega_{1}^{\prime}, \omega_{2}^{\prime}$ der zweiten $\wp$-Funktion heiße $\Gamma^{\prime}$. Da die Gleichung (1) in $u$ identisch bestehen soll, so bleibt sie bei Ausübung der Substitutionen von $\Gamma$ richtig; und da bei ihnen $\wp\left(u \mid \omega_{1}, \omega_{2}\right)$ unverändert bleibt, so genügen bei gegebenem $\wp\left(u \mid \omega_{1}, \omega_{2}\right)$ alle Funktionen:

$$
\wp\left(u+m_{1} \omega_{1}+m_{2} \omega_{2} \mid \omega_{1}^{\prime}, \omega_{2}^{\prime}\right)
$$

der Gleichung (1), wo $m_{1}, m_{2}$ alle Paare ganzer Zahlen durchlaufen. Nun liegt aber in (1) eine algebraische Gleichung für $s\left(u \mid \omega_{1}^{\prime}, \omega_{z}^{\prime}\right)$ vor; man hat also in (3) nur endlich viele, etwa $n$, verschiedene Funktionen vor sich. Die gesamten Substitutionen der Gruppe $\Gamma$, die auch $\wp\left(u \mid \omega_{1}^{\prime}, \omega_{2}^{\prime}\right)$ in sich transformieren, bilden dann eine Untergruppe des Index $n$, die durch $\Gamma_{n}$ bezeichnet werden mag. Die zugehörige Zerlegung der Gruppe $\Gamma$ in Nebengruppen sei:

$$
\Gamma=\Gamma_{n}+\Gamma_{n} \cdot S^{(1)}+\Gamma_{n} \cdot S^{(2)}+\cdots+\Gamma_{n} \cdot S^{(n-1)},
$$

wo $S^{(0)}=1, S^{(1)}, S^{(2)}, \ldots$, $S^{(n-1)}$ gewisse $n$ zweckmäBig gewählte Substitutionen von $\Gamma$ sind, die die $n$ verschiedenen Funktionen (3) liefern.

Übertragen wir den Begriff des "Durchschnittes" endlicher Gruppen (S. 8) auf Gruppen unendlicher Ordnung, so ist $\Gamma_{n}$ als "Durchschnitt" $D\left(\Gamma, \Gamma^{\prime}\right)$ der Gruppen $\Gamma$ und $\Gamma^{\prime}$ zu bezeichnen. Da die Gleichung (1) auch in $\wp\left(u \mid \omega_{1}, \omega_{2}\right)$ algebraisch ist, so ist $D\left(\Gamma, \Gamma^{\prime}\right)$ auch in $\Gamma^{\prime}$ als Untergruppe eines endlichen Index $n^{\prime}$ enthalten und werde als solche $\Gamma_{n^{\prime}}^{\prime}$ genannt. Zwei Gruppen $\Gamma$ und $\Gamma^{\prime}$ heißen nun ,kommensurabel", wenn der Durchschnitt $D\left(\Gamma, \Gamma^{\prime}\right)$ in jeder derselben eine Untergruppe von endlichem Index ist. Eine algebraische Relation (1) kann zwischen $\wp\left(u \mid \omega_{1}, \omega_{2}\right)$ und $\wp\left(u \mid \omega_{1}^{\prime}, \omega_{z}^{\prime}\right)$ jedenfalls nur dann bestehen, wenn die Gruppen $\Gamma$ und $\Gamma^{\prime}$ "7iommensurabel" sind. Aus den folgenden Betrachtungen wird hervorgehen, daß umgekelırt die Kommensurabilität der beiden Gruppen $\Gamma$ und $\Gamma^{\prime}$ auch hinreichend für das Bestehen einer algebraischen Relation (1) ist. 
Um einen Diskontinuitätsbereich der Untergruppe $\Gamma_{n}$ zu gewinnen, gehen wir auf das zur Gruppe $\Gamma$ gehörende Parallelogrammnetz zurück und benennen, wie Fig. 47 in I, 233 darlegt, die einzelnen Parallelogramme nach den zugehörigen Substitutionen. Die beiden erzeugenden Substitutionen der Gruppe $u^{\prime}=u+\omega_{1}$ und $u^{\prime}=u+\omega_{2}$ nennen wir wieder $S_{1}$ und $S_{2}$. Da nur $n$ bezüglich der $\Gamma_{n}$ inäquivalente Parallelogramme existieren, so sind unter den Parallelogrammen $S_{0}=1, S_{2}, S_{2}^{2}, \ldots, S_{2}^{n}$ sicher mindestens zwei äquivalente $S_{2}^{v}$ und $S_{2}^{v+D}$ vorhanden, wo $D$ eine positive ganze, möglichst klein gewählte Zahl sei. Die letztere Angabe soll bedeuten, daß unter den Parallelogrammen $S_{2}^{\nu}, S_{2}^{v+1}, \ldots, S_{2}^{\nu+D-1}$ noch nicht zwei bezüglich der $\Gamma_{n}$ äquivalente auftreten. Aus der Äquivalenz zweier Parallelogramme $S$ und $S^{\circ}$ folgt nun auch diejenige der Parallelogramme $S_{2}^{-v} \cdot S$ und $S_{2}^{-v} \cdot S^{\prime}$. Es sind demnach die Parallelogramme $S_{0}=1$ und $S_{2}^{D}$ bezüglich $\Gamma_{n}$ äquivalent, die $D$ Parallelogramme $1, S_{2}, S_{2}^{2}, \ldots, S_{2}^{D-1}$ aber durchweg inäquivalent; die Substitution $u^{\prime}=\imath+D \omega_{2}$ ist daraufhin in der $\Gamma_{n}$ enthalten.

Weiter reihen wir an das Parallelogramm 1 die Parallelogramme $S_{1}$, $S_{1}^{2}, S_{1}^{3}, \ldots$ an, bis wir zu einem $S_{1}^{A}$ kommen, das mit einem der voraufgehenden $1, S_{1}, S_{1}^{2}, \ldots, S_{1}^{A-1}$ oder mit einem der schon an 1 angereihten Parallelogramme $S_{2}, S_{2}^{2}, \ldots, S_{2}^{D-1}$ äquivalent ist. Dies tritt natürlich wieder spätestens für $A=n$ ein. Dahei kann $S_{1}^{A}$ mit keinem der Parallelogramme $S_{1}, S_{1}^{2}, \ldots, S_{1}^{A-1}$ äquivalent sein, da sonst schon $S_{1}^{A-1}$ mit einem der Reihe $1, S_{1}, \ldots, S_{1}^{A-2}$ äquivalent wäre. Also ist $S_{1}^{A}$ mit einem der Parallelogramme $1, S_{2}, S_{2}^{2}, \ldots, S_{2}^{D-1}$ äquivalent, etwa mit $S_{2}^{D-B}$, wo $B$ eine der Zahlen $1,2, \ldots, D$ ist.

Man reihe nun die $A \cdot D$ Parallelogramme:

$$
S_{1}^{\mu} \cdot S_{2}^{\prime \prime}, \quad \quad \mu=0,1, \ldots, A-1 ; v=0,1, \ldots, D-1
$$

zu einem größeren Parallelogramme der Ecken $0, D \omega_{2}, A \omega_{1}+D \omega_{2}, A \omega_{1}$ zusammen. Von den $A \cdot D$ Parallelogrammen (5) können keine zwei verschiedene bezüglich der $\Gamma_{n}$ äquivalent sein. Soll nämlich $S_{1}^{u^{\prime}} \cdot S_{2}^{v^{\prime}}$ mit $S_{1}^{\mu} \cdot S_{2}^{\prime}$ äquivalent sein, wo wir $\mu^{\prime} \geqq \mu$ voraussetzen dürfen, so folgt daraus die Äquivalenz von $S_{1}^{\mu^{\prime}-\mu}$ mit $S_{2}^{v-v^{\prime}}$ oder auch mit $S_{2}^{\nu-v^{\prime}+D}$, welches Parallelogramm wir für $v-v^{\prime}<0$ bevorzugen. Aus der Reihe $S_{1}^{0}=1, S_{1}$, $S_{1}^{2}, \ldots, S_{1}^{A-1}$ ist aber, wie wir wissen, nur das Parallelogramm 1 mit einem solchen der Reihe $1, S_{2}, S_{2}^{2}, \ldots, S_{2}^{D-1}$ äquivalent, und zwar ist es auch nur mit sich selbst äquivalent. Also gilt $\mu^{\prime}=\mu, \nu^{\prime}=\nu$, womit die Behauptung bewiesen ist.

Von der die Punkte 0 und $D \omega_{2}$ verbindenden Seite des großen Parallelogramms wird nun, wenn $B<D$ ist, das durch 0 und $(D-B) \omega_{2}$ begrenzte Stück durch die in $\Gamma_{n}$ enthaltene Substitution:

$$
u^{\prime}=u+A \omega_{1}+B \omega_{2}
$$


in das äquiralente, von $\left(A \omega_{1}+B \omega_{2}\right)$ und $\left(A \omega_{1}+D \omega_{2}\right)$ begrenzte Stück der Gegenseite transformiert. Der von $(D-B) \omega_{2}$ und $D \omega_{2}$ begrenzte Rest aber wird durch $u^{\prime}=u+A \omega_{1}+(B-D) \omega_{2}$ in das noch freie, durch die Punkte $A \omega_{1}$ und $\left(A \omega_{1}+B \omega_{2}\right)$ begrenzte Stück der Gegenseite übergeführt. Die letzte Substitution kann man aus den beiden durch $S_{1}^{\prime}$ und $S_{2}^{\prime}$ zu bezeichnenden Substitutionen:

$$
u^{\prime}=u+A \omega_{1}+B \omega_{2}, \quad u^{\prime}=u+D \omega_{2}
$$

herstellen. Um jene entbehren zu können, schneiden wir vom großen Parallelogramm das Dreieck der Ecken 0, $A \omega_{1}+B \omega_{2}, A \omega_{1}$ ab und ersetzen es durch das äquivalente Dreieck der Ecken $D \omega_{2}, A \omega_{1}+(B+D) \omega_{2}$,

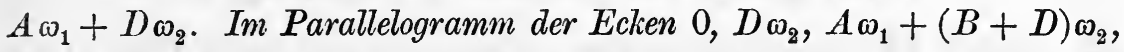
$A \omega_{1}+B \omega_{2}$ haben wir nun einen Diskontinuitätsbereich der Untergruppe $\Gamma_{n}$ in besonders sinfacher Gestalt gewonnen; die Gegenseiten dieses Parallelogramms sind durch die Substitutionen (6), die ein System von erzeugenden Substitutionen der $\Gamma_{n}$ bilden, aufeinander bezogen. Dieser Satz bezieht sich zwar auch auf den Fall $B=D$. Da jedoch in diesem Falle die Seite zwischen 0 und $I \omega_{2}$ des ursprünglichen großen Parallelogramms durch die Substitution $\omega=u+A \omega_{1}$ ungeteilt in die äquivalente Gegenseite übergeht, so behalten wir dieses Parallelogramm unmittelbar als Diskontinuitätsbereich der $\Gamma_{n}$ bei. Es läuft dies darauf hinaus, $d a \beta$ die ganze Zahl $B$ in (6) auf die Werte $0,1,2, \ldots, D-1$ beschränkt ist.

Da nun die $A \cdot D$ Substitutionen (5) ein System bezüglich der $\Gamma_{n}$ inäquivalenter Substitutionen bilden müssen, so ist notwendig $A \cdot D=n$. Nehmen wir andrerseits eine beliebige Zerlegung der Zahl $n$ in zwei positive ganzzahlige Faktoren $n=A \cdot D$ und verstehen unter $B$ eine beliebige der Zahlen $0,1,2, \ldots, D-1$, so bilden die beiden mit diesen Zahlen angesetzten Substitutionen (6) die Erzeugenden einer Untergruppe $\Gamma_{n}$ des Index $n$, deren Diskontinuitätsbereich das Parallelogramm der Ecken $0, D \omega_{2}, A \omega_{1}+(B+D) \omega_{2}, A \omega_{1}+B \omega_{2}$ ist. Zwei verschiedenen Zahlentripeln $A, B, D$ und $A^{\prime}, B^{\prime}, D^{\prime}$ dieser Art entsprechen dabei auch sicher zwei verschiedene Gruppen $\Gamma_{n}$ und $\Gamma_{n}^{\prime}$. Sollten nämlich diese beiden Gruppen gleich sein, so muB erstlich jede der Zahlen $D, D^{\prime}$ ein Vielfaches der anderen sein, woraus $D^{\prime}=D$ und also $A^{\prime}=A$ folgt. Da alsdann weiter in $\Gamma_{n}=\Gamma_{n}^{\prime}$ die Substitution $u^{\prime}=u+\left(B^{\prime}-B\right) \omega_{2}$ enthalten sein muB, so folgt auch noch $B^{\prime}=B$. Damit ist der Satz bewiesen: Die Anzahl verschiedener Untergruppen des Index $n$ in $\Gamma$ ist gleich der durch $\Phi(n) z u$ bezeichnenden Teilersumme der Zahl $n$. Man gewinnt nämlich alle brauchbaren Paare (6) von Erzeugenden, wenn man für $D$ der Reihe nach alle Teiler von $n$ wählt, $A=\frac{n}{D}$ setzt und für $B$ nacheinander $0,1,2, \ldots, D-1$ einträgt.

Die gewonnenen Ergebnisse setzen uns in den Stand, einen Ansatz 
274 II, 1. Transformation $n^{\text {ten }}$ Grades $u$. allgemeine Transformationsgleichungen zur Lösung des Transformationsproblems zu entwickeln. Als Untergruppe $\Gamma_{n}$ des Index $n$ von $\Gamma$ ist der Durchschnitt $D\left(\Gamma, \Gamma^{\prime}\right)$ eine unserer eben gefundenen Gruppen, deren $\wp$-Funktion $\wp\left(u \mid A \omega_{1}+B \omega_{2}, D \omega_{2}\right)$ wir zur Vermittlung zwischen $\wp\left(u \mid \omega_{1}, \omega_{2}\right)$ und $\wp\left(u \mid \omega_{1}^{\prime}, \omega_{2}^{\prime}\right)$ einführen. Da $\wp\left(u \mid \omega_{1}, \omega_{2}\right)$ und $\wp\left(u \mid \omega_{1}^{\prime}, \omega_{2}^{\prime}\right)$ bei den Substitutionen der $\Gamma_{n}$ unverändert bleiben und übrigens gerade Funktionen sind, so gilt der Satz: Für die beiden Funktionen $\wp\left(u \mid \omega_{1}, \omega_{z}\right)$ und $\wp\left(u \mid \omega_{1}^{\prime}, \omega_{2}^{\prime}\right)$ mit kommensurabelen Gruppen $\Gamma, \Gamma^{\prime}$ gelten Darstellungen:

$$
\left\{\begin{array}{l}
\wp\left(u \mid \omega_{1}, \omega_{2}\right)=R\left(\wp\left(u \mid A \omega_{1}+B \omega_{2}, D \omega_{2}\right)\right), \\
\wp\left(u \mid \omega_{1}^{\prime}, \omega_{2}^{\prime}\right)=R^{\prime}\left(\wp\left(u \mid A \omega_{1}+B \omega_{2}, D \omega_{2}\right)\right)
\end{array}\right.
$$

als rationale Funktionen des gleichen Argumentes $\wp\left(u \mid A \omega_{1}+B \omega_{2}, D \omega_{2}\right)$, durch dessen Elimination aus den Gleichungen (7) eine algebraische Relation (1) zwischen $\wp\left(u \mid \omega_{1}, \omega_{2}\right)$ und $\wp\left(u \mid \omega_{1}^{\prime}, \omega_{2}^{\prime}\right)$ gewonnen wird. Die Kommensurabilität der Gruppen ist demnach, wie schon oben behauptet wurde, auch hinreichend für die Existenz einer Relation (1).

\section{§ 2. Die Repräsentanten der Transformationen $\boldsymbol{n}^{\text {ten }}$ Grades.}

Eine Untergruppe $I_{n}$ des Index $n$ in der Gruppe $\Gamma$ hat als Diskontinuitätsbereich ein Parallelogramm, dessen Ecken 0, $\omega_{2}^{\prime}, \omega_{1}^{\prime}+\omega_{2}^{\prime}, \omega_{1}^{\prime}$ Gitterpunkte des ursprünglichen Netzes sind, und dessen Inhalt $n$ mal so groß wie der Inhalt des Parallelogramms der Ecken $0, \omega_{2}, \omega_{1}+\omega_{2}, \omega_{1}$ ist. Wir nehmen die Bezeichnungen $\omega_{1}^{\prime}, \omega_{2}^{\prime}$ auf die Ecken so verteilt an, daB der Quotient $\omega^{\prime}=\omega_{1}^{\prime}: \omega_{2}^{\prime}$ positiven imaginären Bestandteil hat. Umgekehrt liefert jedes solche Parallelogramm den Diskontinuitätsbereich einer Untergruppe $\Gamma_{n}$ des Index $n$ in $\Gamma$, die aus den beiden Substitutionen:

$$
u^{\prime}=S_{i}^{\prime}(u)=u+\omega_{i}^{\prime}
$$

erzeugbar ist. Die $\omega_{1}^{\prime}, \omega_{2}^{\prime}$ stellen sich als Gitterpunkte des ursprünglichen Netzes in der Gestalt:

$$
\omega_{1}^{\prime}=a \omega_{1}+b \omega_{2}, \quad \omega_{2}^{\prime}=c \omega_{1}+d \omega_{2}
$$

mittelst ganzer Zahlen $a, b, c, d$ dar. Da der Quotient $\omega^{\prime}=\omega_{1}^{\prime}: \omega_{2}^{\prime}$ positiven imaginären Bestandteil hat, so ist die Determinante $(a d-b c)$ positiv, und da der Inhalt des Parallelogramms der Ecken $0, \omega_{2}^{\prime}, \omega_{1}^{\prime}+\omega_{2}^{\prime}, \omega_{1}^{\prime}$ gleich dem $n$-fachen Inhalte des ursprünglichen Parallelogramms ist, so gilt insbesondere:

$$
a d-b c=n .
$$

Man kann demnach sagen: Berechnet man die $\omega_{1}^{\prime}, \omega_{2}^{\prime}$ aus den $\omega_{1}, \omega_{2}$ nach (2) mittelst irgendwelcher ganzer Zahlen $a, b, c, d$ der Determinante n, so erzeugen die in (1) gegebenen Substitutionen $S_{1}^{\prime}, S_{2}^{\prime}$ eine Untergruppe des 
Index $n$ in $\Gamma$; zugleich gelangt man auf diese Weise zu allen Untergruppen $\Gamma_{n}$ von $\Gamma$.

Man sagt nun, in (2) liege eine "Transformation $n^{\text {ten }}$ Grades" der Perioden $\omega_{1}, \omega_{2}$ vor, falls $a, b, c, d$ vier ganze Zahlen der Determinante $n$ sind. Diese Erklärung schließt den Begriff der „linearen Transformation“ oder "Transformation ersten Grades" der Perioden, wie er in I, 184 gegeben wurde, ein und verallgemeinert ihn. Symbolisch möge eine Transformation (2) durch $T$ bezeichnet werden. Als Ziel der Transformationstheorie für die Funktionen erster Stufe sehen wir die Berechnung der ,transformierten Funktionen" $\wp\left(u \mid \omega_{1}^{\prime}, \omega_{2}^{\prime}\right), \wp^{\prime}\left(u \mid \omega_{1}^{\prime}, \omega_{2}^{\prime}\right)$ oder:

$$
\wp\left(u \mid a \omega_{1}+b \omega_{2}, c \omega_{1}+d \omega_{2}\right), \quad \wp^{\prime}\left(u \mid a \omega_{1}+b \omega_{2}, c \omega_{1}+d \omega_{2}\right)
$$

und der ,transformierten Invarianten"

$$
g_{2}\left(a \omega_{1}+b \omega_{2}, c \omega_{1}+d \omega_{2}\right), \quad g_{3}\left(a \omega_{1}+b \omega_{2}, c \omega_{1}+d \omega_{2}\right), \quad J\left(\frac{a \omega+b}{c \omega+d}\right)
$$

aus den ursprïnglichen Funktionen und Invarianten an. Das Transformationsproblem des vorigen Paragraphen ist dadurch ein wenig abgeändert. Die ursprüngliche Funktion $\wp\left(u \mid \omega_{1}, \omega_{2}\right)$ ist rational in der transformierten $\wp\left(u \mid \omega_{1}^{\prime}, \omega_{2}^{\prime}\right)$ darstellbar:

$$
\wp\left(u \mid \omega_{1}, \omega_{2}\right)=R\left(\wp\left(u \mid a \omega_{1}+b \omega_{2}, c \omega_{1}+d \omega_{2}\right)\right) .
$$

In (7) S. 274 liegen zwei Relationen dieser Art vor, die das gleiche Argument der rationalen Funktionen haben. Erst durch Elimination dieses Argumentes ergibt sich eine algebraische Relation der Art (1) S. 270. Indem wir uns auf die einzelne Gleichung (4) beschränken, betrachten wir also nur algebraische Relationen (1) S. 270, in denen die eine der beteiligten $\wp$-Funktionen linear enthalten ist. Demgegenüber haben wir unser Problem in anderer Hinsicht wesentlich erweitert, insofern wir die Aufgabe gestellt haben, die transformierten Funktionen aus den ursprüng lichen zu berechnen.

Während man nun für den einzelnen Grad $n$ unendlich viele, abgekürzt durch $T=\left(\begin{array}{l}a, b \\ c, d\end{array}\right)$ zu bezeichnende Transformationen hat, gibt es nach S. 273 doch nur endlich viele, nämlich $\Phi(n)$ verschiedene Untergruppen $\Gamma_{n}$, unter $\Phi(n)$ die Teilersumme der Zahl $n$ verstanden. In der Tat gelangen wir immer wieder zu derselben Gruppe $\Gamma_{n}$, wenn wir an Stelle der $\omega_{1}^{\prime}, \omega_{2}^{\prime}$ zwei aus ihnen durch irgendeine "lineare“ Transformation entstehende Perioden treten lassen. Alle zu derselben Gruppe $\Gamma_{n}$ und also zu den gleichen transformierten Funktionen und Invarianten führenden Transformationen fassen wir in eine "Klasse von Transformationen $n^{\text {ten }}$ Grades" zusammen. Sie entstehen aus einer ersten unter ihnen $T$ in der Gestalt: 
II, 1. Transformation $n^{t_{\mathrm{t} n}}$ Grades $u$. allgemeine 'Transformationsgleichungen

$$
V \cdot T=\left(\begin{array}{l}
\alpha, \beta \\
\gamma, \delta
\end{array}\right) \cdot\left(\begin{array}{l}
a, b \\
c, d
\end{array}\right)=\left(\begin{array}{l}
\alpha a+\beta c, \alpha b+\beta d \\
\gamma a+\delta c, \gamma b+\delta d
\end{array}\right)
$$

wo $V$ die Substitutionen der homogenen Modulgruppe $\Gamma^{(\omega)}$ durchläuft.

Nach S. 273 muß in jeder Klasse eine und nur eine Transformation der Gestalt:

$$
T=\left(\begin{array}{c}
A, B \\
0, D
\end{array}\right), \quad A \cdot D=n, \quad 0 \leqq B<D
$$

enthalten sein, wo $A, B, D$ positive ganze Zahlen sind, die den unter (6) angegebenen Bedingungen genügen. Dies ist arithmetisch leicht zu bestätigen. Bei gegebener Transformation $\left(\begin{array}{l}a, b \\ c, d\end{array}\right)$ kann man in der Tat die Substitution $V$ in einer und nur einer Art so bestimmen, daß in (5) rechts eine Transformation (6) vorliegt. Ist nämlich $A$ der positiv genommene, größte gemeinsame 'Teiler von $a$ und $c$, so hat man zu setzen:

$$
\gamma=-\frac{c}{A}, \quad \delta=+\frac{a}{A}, \quad \gamma b+\delta d=-\frac{c}{A} b+\frac{a}{A} \bar{d}=\frac{n}{A}=D
$$

und gewinnt, da $A$ in $n$ aufgeht, auch in $D$ eine positive ganze Zahl. Da $\gamma$ und $\delta$ teilerfremde ganze Zahlen sind, so gibt es einfach unendlich viele Substitutionen $V$ in $\Gamma^{(\omega)}$ mit diesen $\gamma$ und $\delta$ als dritten und vierten Koeffizienten. Hat eine erste von ihnen die beiden ersten Koeffizienten $\alpha_{0}, \beta_{0}$, so gewinnt man alle, indem man:

$$
\alpha=\alpha_{0}+\nu \gamma, \quad \beta=\beta_{0}+\nu \delta, \quad(v=\cdots,-2,-1,0,1,2, \cdots)
$$

setzt und $\nu$, wie angedeutet, alle ganzen Zahlen durchlaufen läßt. Daraus findet man weiter:

$$
\alpha a+\beta c=A(\alpha \delta-\beta \gamma)=A, \quad \alpha b+\beta d=\left(\alpha_{0} b+\beta_{0} d\right)+\nu D=B
$$

und kann über $v$ in einer und nur einer Art so verfügen, daß die letzte Bedingung (6) erfüllt ist. Wir wollen nun die eine in der Klasse enthaltene Transformation (6) zum „Repräsentanten“ der Klasse wählen und haben dann folgenden Satz: Beim Grade $n$ hat man im ganzen $\boldsymbol{\Phi}(\boldsymbol{n})$, durch die "Repräsentanten" (6) gelieferte Klassen von Transformationen und entsprechend $\Phi(n)$ verschiedene Systeme transformierter Funktionen und Invarianten.

Ist $t$ der größte gemeinsame Teiler der vier Koeffizienten $a, b, c, d$ der Transformation $T$, so haben auch die vier Koeffizienten jeder weiteren Transformation $V \cdot T$ der gleichen Klasse $t$ als gröBten gemeinsamen Teiler, so daß der Teiler $t$ als ein Attribut der Klasse anzusehen ist. Ist $t=1$, so sagt man, es liege "eigentliche" Transformation $n^{\text {ten }}$ Grades vor. Ist $t>1$, wo alsdann $n$ den ,quadratischen" Teiler $t^{2}$ hat, so schreiben wir $a=t \cdot a_{0}, b=t \cdot b_{0}, c=t \cdot c_{0}, d=t \cdot d_{0}$ und haben: 


$$
\begin{aligned}
\wp\left(u \mid a \omega_{1}+b \omega_{2}, c \omega_{1}+d \omega_{2}\right) & =t^{-2} \cdot \wp\left(\frac{u}{t} \mid a_{0} \omega_{1}+b_{0} \omega_{2}, c_{0} \omega_{1}+d_{0} \omega_{2}\right), \cdots, \\
g_{2}\left(a \omega_{1}+b \omega_{2}, c \omega_{1}+d \omega_{2}\right) & =t^{-4} \cdot g_{2}\left(a_{0} \omega_{1}+b_{0} \omega_{2}, c_{0} \omega_{1}+d_{0} \omega_{2}\right), \ldots, \\
J\left(\frac{a \omega+b}{c \omega+d}\right) & =J\left(\frac{a_{0} \omega+b_{0}}{c_{0} \omega+d_{0}}\right) .
\end{aligned}
$$

Sehen wir von den Faktoren $t^{-2}, \ldots a b$, so liegt bei den Funktionen "eigentliche" Transformation des Grades $\frac{n}{t^{2}}$ in Verbindung mit der Division des Argumentes $u$ durch $t$ vor, bei den Invarianten aber „eigentliche" Transformation des Grades $\frac{n}{t^{2}}$. Sieht man auch über die Division des Argumentes $u$ bei den Funktionen hinweg, so kann man den Satz aussprechen, daß sich die $\Phi(n)$ Fälle der Transformation $n^{\text {ten }}$ Grades zusammensetzen aus den „eigentlich" $h^{\prime \prime}$ allen Graden $\frac{n}{t^{2}}$ gehörenden Transformationen, wo $t^{2}$ die quadratischen Teiler von $n$ unter Einschlu $\beta$ von $t^{2}=1$ durchläuft.

Zur Bestimmung der Anzahl der Klassen aller eigentlich zum Grade $n$ gehörenden Transformationen stellen wir zunächst fest, daß jede Untergruppe $\Gamma_{n}$ des Index $n$ eine Kongruenzgruppe $n^{\text {ter }}$ Stufe ist. Benutzen wir nämlich die beiden in (6) S. 273 gegebenen Substitutionen $S_{1}^{\prime}, S_{2}^{\prime}$ als erzeugende Substitutionen der $\Gamma_{n}$, so sind mit ihnen auch $S_{1}^{\prime}{ }^{D} \cdot S_{2}^{\prime}-B$ und $S_{2}^{\prime}{ }^{4}$ oder explizite die Substitutionen $u^{\prime}=u+n \omega_{1}, u^{\prime}=u+n \omega_{2}$ in $\Gamma_{n}$ enthalten. Aus diesen beiden Substitutionen aber läßt sich die Hauptkongruenzgruppe $\Gamma_{n^{2}}$ der $n^{\text {ten }}$ Stufe erzeugen. Da der Flächeninhalt des Diskontinuitätsbereiches der $\Gamma_{n^{2}} n$-mal so groß wie der des Diskontinuitätsbereiches der $\Gamma_{n}$ ist, so ist die $\Gamma_{n^{2}}$ eine Untergruppe des Index $n$ in $\operatorname{der} \Gamma_{n}$, und die letztere Gruppe $\Gamma_{n}$ reduziert sich $\bmod n$ auf eine Untergruppe $G_{n}$ der Ordnung $n$ in $\operatorname{der} G_{n^{2}}$ aller $\bmod n$ inkongruenten Substitutionen.

Wir sind auf diese Weise zu den Entwicklungen von S. $218 \mathrm{ff}$. über Kongruenzgruppen $n^{\text {ter }}$ Stufe in der Gruppe $\Gamma^{(u)}$ zurückgeführt. Dabei stellen sich die Substitutionen der $G_{n}$ in der Gestalt:

$$
u^{\prime} \equiv u+l A \omega_{1}+(l B+m D) \omega_{2}(\bmod n)
$$

dar, unter $l$ und $m$ ganze Zahlen verstanden. Hieran schließt sich der Satz: Die $G_{n}$ ist stets und nur dann eine zyklische Gruppe, wenn eigentliche Transformation $n^{\text {ten }}$ Grades vorliegt. Haben nämlich $A, B, D$ den gemeinsamen Teiler $t>1$, so ist sicher die $\left(\frac{n}{t}\right)^{\text {te }}$ Potenz der Substitution (7) mit der identischen Substitution mod $n$ kongruent, so daB in diesem Falle die $G_{n}$ keine Substitution der Periode $n$ enthält und also nicht zyklisch sein kann. Haben indessen $A, B, D$ keinen Teiler $t>1$ 
278 II, 1. Transformation $n^{\text {ten }}$ Grades u. allgemeine Transformationsgleichungen gemein, so weist man in $\operatorname{der} G_{n}$ leicht eine Substitution:

$$
u^{\prime} \equiv u+A \omega_{1}+(B+m D) \omega_{2}(\bmod n)
$$

der Periode $n$ nach. Man zerlege nämlich $A$ in das Produkt $A_{1} \cdot A_{2}$, wo in $A_{1}$ alle Primfaktoren von $A$ zusammengefaßt sind, die auch in $D$ aufgehen, und wo also $A_{2}$ teilerfremd gegen $D$ ist. Hieraus folgt, daß man $m$ als ganze Zahl entsprechend der Kongruenz:

$$
B_{a x}+m D \equiv 1 \quad\left(\bmod A_{2}\right)
$$

bestimmen kaun. Dann ist $(B+m D)$ teilerfremd gegen $A_{2}$. Da aber eine in $A_{1}$ aufgehende Primzahl auch in $D$ aufgeht und deshalb $B$ sicher nicht teilt, so ist $(B+m D)$ auch teilerfremd gegen $A_{1}$ und also auch gegen $A$. Die beiden ganzen Zahlen $A$ und $(B+m D)$ bilden also im Sinne von S. 218 ein gegen $n$ teilerfremdes Paar, so daB nach den dortigen Sätzen die Sabstitution (8) die Periode $n$ hat. Die $G_{n}$ ist also jetzt zyklisch und gehört zu den $\psi(n)$ schon S. 220 betrachteten Gruppen $G_{n}$.

Umgekehrt lieferte jede der $\psi(n)$ Gruppen $G_{n}$ eine Kongruenzuntergruppe $\Gamma_{n}$, die als Untergruppe des Index $n$ zu unseren bei der Transformation $n^{\text {ten }}$ Grades eintretenden $\Gamma_{n}$ gehört, und die, als einer zyklischen $G_{n}$ entsprechend, eigentliche Transformation $n^{\text {ten }}$ Grades liefern muß. Mit Rücksicht auf die Entwicklungen von S. $220 \mathrm{ff}$. ist also der folgende Satz festgestellt: Die eigentlich zum Grade n gehörenden Transformationen bilden $\psi(n)$ Klassen; die entsprechenden Gruppen $\Gamma_{n}$ sind in der der $\Gamma^{(u)}$ ausgezcichnet, aber in der ternären Gruppe $\Gamma^{(u, \omega)}$ miteinander gleichberechtigt.

Zum Grade $\frac{n}{t^{2}}$ gehören hiernach $\psi\left(\frac{n}{t^{2}}\right)$ Transformationsklassen im eigentlichen Sinne. Ist $n$ rein quadratisch, so tritt auch der Repräsentant $A=D=\sqrt{n}, B=0$ auf, der die lineare Transformation liefert. Unter dem bisher noch nicht erklärten Symbole $\psi(1)$ hat man demnach den Wert 1 zu verstehen. Aus den berechneten Anzahlen der Transformationsklassen ergibt sich daraufhin die Regel: Die Teilersumme $\Phi(n)$ stellt sich in den ganzen Zahlen $\psi$ durch die Gleichung:

$$
\Phi(n)=\sum_{t} \psi\left(\frac{n}{t^{2}}\right)
$$

dar, wo sich die Summe auf alle quadratischen Teiler $t^{2}$ von $n$ bezieht, unter Einschluß von $t^{2}=1$.

\section{\$. Die allgemeine Transformationsgleichung der $\wp$-Funktion.}

Unter den $\psi(n)$ eigentlich zum Grade $n$ gehörenden wesentlich verschiedenen Transformationen nennen wir die durch $A=n, B=0, D=1$ gegebene die „erste Haupttransformation", während die Zahlen $A=1$ 
$B=0, D=n$ die ,zweite Hauptiransformation" liefern mögen. Die Diskontinuitätsbereiche der zugehörigen Gruppen sind leicht herstellbar. Für die erste Gruppe $\Gamma_{n}$ hat man $n$ Parallelogramme des ursprünglichen Netzes zu einem größeren Parallelogramme der Ecken $0, \omega_{2}, n \omega_{1}+\omega_{2}, n \omega_{1}$ zusammenzuordnen. Die beiden Haupttransformationen sind natürlich keineswegs vor den übrigen Transformationen ausgezeichnet. Indem man statt der $\omega_{1}, \omega_{2}$ in geeigneter Weise linear transformierte Perioden einführt, wird man jede beliebige Transformation in den neuen Perioden z. B. als erste Haupttransformation schreiben können. Umgekehrt geht aus den z. B. bei der ersten Haupttransformation eintretenden Größen:

$$
\wp\left(u \mid n \omega_{1}, \omega_{2}\right), \ldots, g_{2}\left(n \omega_{1}, \omega_{2}\right), \ldots, J(n \omega)
$$

das System der Größen irgendeiner anderen Klasse von Transformationen einfach durch eine geeignete "lineare" Transformation der Perioden in der Gestalt:

$$
\wp\left(u \mid n \alpha \omega_{1}+n \beta \omega_{2}, \quad \gamma \omega_{1}+\delta \omega_{2}\right), \ldots
$$

hervor, entsprechend der Gleichberechtigung der $\psi(n)$ Gruppen $\Gamma_{n}$ innerhalb $\Gamma^{(u, \omega)}$. Bei dieser Sachlage darf man sich auf die Betrachtung der Haupttransformationen beschränken und könnte unter ihnen sogar eine, z. B. die erste, bevorzugen.

Im Diskontinuitätsbereiche der zur ersten Haupttransformation gehörenden Gruppe $\Gamma_{n}$ ist $\left.\wp\left(u \mid \omega_{1} ; \omega_{2}\right)=\wp(u)^{1}\right)$ eine $2 n$-wertige gerade Funktion, die dieserhalb eine rationale Funktion $n^{\text {ten }}$ Grades:

$$
\wp(u)=R\left(\wp\left(u \mid n \omega_{1}, \omega_{2}\right)\right)
$$

von $\wp\left(u \mid n \omega_{1}, \omega_{2}\right)$ ist. Als Gleichung $n^{\text {ten }}$ Grades für die transformierte $\wp$-Funktion aufgefaßt, bezeichnen wir diese Gleichung als die ,allgemeine Transformationsgleichung" der $\wp$-Funktion für die erste Haupttransformation $n^{\text {ten }}$ Grades. Sie hat die $n$ Lösungen:

$$
\wp\left(u+\lambda \omega_{1}: n \omega_{1}, \omega_{2}\right),
$$$$
(\lambda=0,1,2, \ldots, n-1)
$$

und ist irreduzibel in jedem Körper, der aus $\left(\Re, \wp(u), \wp^{\prime}(u), g_{9}, g_{3}\right)$ durch Adjunktion irgendwelcher von $u$ unabhängiger Größen entsteht.

Die Theorie dieser allgemeinen Transformationsgleichung ist nun bereits in den Entwicklungen von S. $225 \mathrm{ff}$. über die allgemeine Teilungsgleichung vollständig enthalten. Die beiden zu den Haupttransformationen gehörenden Gruppen $\Gamma_{n}$ sind im Sinne von S. 224 ,komplementär"; auch die beiden Haupttransformationen mögen deshalb „komplemenẗ̈r" heißen. Die aufeinanderfolgende Ausïbung der beiden Haupttransformationen fuihrt zur. Division. Üben wir nämlich auf $\wp\left(u \mid n \omega_{1}, \omega_{2}\right)$ die zweite Haupttransformation aus, so gelangen wir zu:

1) Sind Perioden der Funktionen $\wp, \wp^{\prime}, \sigma, \ldots$ nicht besonders angegeben, so sind immer $\omega_{1}, \omega_{2}$ als solche gedacht. 
II, 1. Transformation $n^{\text {ten }}$ Grades $\mathrm{u}$. allgemeine Transformationsgleichungen

$$
\wp\left(u \mid n \omega_{1}, n \omega_{2}\right)=n^{-2} \cdot \wp\left(\frac{u}{n} \mid \omega_{1}, \omega_{2}\right) \text {. }
$$

Hatten wir nun oben den Satz gefunden, daß die allgemeine Teilungsgleichung nach Adjunktion der Teilwerte durch zwei zyklische Gleichungen $n^{\text {ten }}$ Grades lösbar ist, so werden wir jetzt leicht erkennen, $d a \beta$ die bei den aufeinander folgenden Haupttransformationen eintretenden beiden Transformationsgleichungen $n^{\text {ten }}$ Grades unmittelbar als jene beiden zyklischen Resolventen der allgemeinen Teilungsgleichung angesehen werden können.

Man erinnere sich, daß unter den in (3) S. 226 eingeführten $\Psi_{\lambda_{\mu}}(u)$ die $(n-1)$ Funktionen $\Psi_{0_{\mu}}(u)$ der zur ersten Haupttransformation gehörenden Gruppe $\Gamma_{n}$ angehören. Die Berechnung dieser Funktionen $\Psi_{0 u}(u)$ aus $\wp(u), \wp^{\prime}(u)$ geschieht aber auf Grund von (19) S. 230 nach Adjunktion der Teilwerte mittelst einer einzigen Wurzel $u^{\text {ten }}$ Grades. Es besteht nun einfach der Satz, daß nach Adjunktion dieser $n^{\text {ten }}$ Wurzel und also der $\Psi_{0_{\mu}}(u)$ die Wurzeln (3) der allgemeinen Transformationsgleichung (2) rational bekannt sind.

Man bilde nämlich mittelst der Einheitswurzel $\varepsilon=e^{\frac{2 i \pi}{n}}$ aus den Lösungen (3) der Transformationsgleichung die Summe:

$$
\sum_{\lambda=0}^{n-1} \varepsilon^{\lambda \mu} \wp\left(u+\lambda \omega_{1} \mid n \omega_{1}, \omega_{2}\right)
$$

unter $\mu$ eine Zahl der Reihe $0,1,2, \ldots,(n-1)$ verstanden. Die Summe (4) bleibt bei Vermehrung von $u$ um $\omega_{2}$ unverändert, bei Vermehrung von $u$ um $\omega_{1}$ geht sie in sich selbst, multipliziert mit $\varepsilon^{-\mu}$, über. Für $\mu=0$ liegt also eine Funktion der Perioden $\omega_{1}, \omega_{2}$ vor, die im ursprünglichen Parallelogramm einen einzigen, bei $u=0$ gelegenen Pol zweiter Ordnung mit den Anfangsgliedern der Reihenentwicklung:

$$
\frac{1}{u^{2}}+\sum_{\lambda=1}^{n-1} \wp\left(\lambda \omega_{1} \mid n \omega_{1}, \omega_{2}\right)+\cdots
$$

hat. In diesem Falle ist also die Summe (4), abgesehen vom Absolutgliede der eben angegebenen Reihe, mit der Funktion $\wp(u)$ identisch:

$$
\sum_{\lambda=0}^{n-1} \wp\left(u+\lambda \omega_{1} \mid n \omega_{1}, \omega_{2}\right)=\wp(u)+\sum_{\lambda=1}^{n-1} \wp\left(\lambda \omega_{1} \mid n \omega_{1}, \omega_{2}\right) .
$$

Die rechts stehende Summe gestattet Darstellung durch die Teilwerte der $\wp$-Funktion. Man hat nämlich in $\wp\left(n u \mid n \omega_{1}, \omega_{2}\right)$ eine Funktion der Perioden $\omega_{1}, \omega_{2}$ mit $n$ Polen zweiter Ordnung an den Stellen $u=0, \frac{\omega_{2}}{n}$, $\frac{2 \omega_{2}}{n}, \cdots, \frac{(n-1) \omega_{2}}{n}$ des Periodenparallelogramms. An der einzelnen Stelle 
$\frac{n \omega_{2}}{n}$ wird $n^{2} \wp\left(n u \mid n \omega_{1}, \omega_{2}\right)=\wp\left(u \mid \omega_{1}, \frac{\omega_{2}}{n}\right)$. genau so unendlich, wie die unter (5) S. 211 eingeführte Funktion $\wp_{0,-x}(u)=\wp_{0, n-*}(u)$. Demnach ist die Differenz:

$$
n^{2} \wp\left(n \dot{u} \mid n \omega_{1}, \omega_{2}\right)-\sum_{\mu=0}^{n-1} \wp_{0 \mu}(u)
$$

eine von $\imath$ unabhängige Größe, für die man aus dem Absolutgliede der Reihe nach Potenzen von $u$ den Ausdruck $-\sum_{\mu=1}^{n-1} \wp_{0 \mu}$ abliest:

$$
n^{2} \wp\left(n u \mid n \omega_{1}, \omega_{2}\right)=\wp(u)+\sum_{\mu=1}^{n-1}\left(\wp_{0 \mu}(u)-\wp_{0 \mu}\right) .
$$

Setzt man $u=\frac{\lambda \omega_{1}}{n}$, so folgt:

$$
\wp\left(\lambda \omega_{1} \mid n \omega_{1}, \omega_{2}\right)=\frac{1}{n^{2}}\left(\sum_{\mu=0}^{n-1} \wp_{\lambda \mu}-\sum_{\mu=1}^{n-1} \wp_{0}\right) .
$$

Bildet man diese Gleichung für $\lambda=1,2, \ldots,(n-1)$ und addiert alle $(n-1)$ so entstehenden Gleichungen, so folgt bei zweckmäßiger Zusammenfassung der rechts stehenden Glieder:

$$
\sum_{\lambda=1}^{n-1} \wp\left(\lambda \omega_{1} \mid n \omega_{1}, \omega_{2}\right)=\frac{1}{n^{2}} \sum_{\lambda, \mu}^{\prime} \wp_{\lambda \mu}-\frac{1}{n} \sum_{\mu=1}^{n-1} \wp_{0 \mu},
$$

wo sich die erste Summe rechts auf alle inkongruenten Zahlenpaare mod $n$ unter Auslassung der Kombination $\lambda=0, \mu=0$ bezieht. Nach (8) S. 247 ist diese Summe gleich 0 , da für jeden Teiler von $n$ die im eigentlichen Sinne zugehörigen Teilwerte verschwindende Summe liefern. Es besteht also die Gleichung:

$$
\sum_{\lambda=1}^{n-1} \wp\left(\lambda \omega_{1} \mid n \omega_{1}, \omega_{2}\right)=-\frac{1}{n} \sum_{\mu=1}^{n-1} \wp_{0 \mu},
$$

und die Relation (5) schreibt sich um in:

$$
\sum_{\lambda=0}^{n-1} \wp\left(u+\lambda \omega_{1} \mid n \omega_{1}, \omega_{2}\right)=\wp(u)-\frac{1}{n} \sum_{\mu=1}^{n-1} \wp_{0 \mu} .
$$

Bedeutet $\mu$ in der Summe (4) eine der Zahlen $1,2, \ldots,(n-1)$, so hat das Produkt dieser Summe und der Funktion $\Psi_{0_{\mu}}(u)$ zufolge (4) S. 226 die Perioden $\omega_{1}$ und $\omega_{2}$; und zwar stellt dieses Produkt eine dreiwertige Funktion mit einem Pole dritter Ordnung im Punkte $\imath=0$ dar. Es gilt also für $\mu \neq 0$ der Ansatz:

$$
\Psi_{0 \mu}(u) \cdot \sum_{\lambda=0}^{n-1} \varepsilon^{\lambda \mu} \wp\left(u+\lambda \omega_{1} \mid n \omega_{1}, \omega_{2}\right)=\dot{A}\left(\wp(u)-\wp_{0 \mu}\right)+B\left(\wp^{\prime}(u)-\wp_{0 \mu}^{\prime}\right),
$$


wobei sogleich noch benutzt ist, daB das Produkt im Nullpunkte $u=\frac{\mu \omega_{2}}{n}$ von $\Psi_{0 \mu}(u)$ verschwinden muB. Zur Bestimmung der von $u$ unabhängigen Faktoren benutzen wir die Anfangsglieder der Potenzreihe nach $u$ für das Produkt, die nach (5) S. 226 lauten:

$$
\frac{1}{u^{3}}+\frac{\sigma_{0 \mu}^{\prime}}{\sigma_{0, u}} \cdot \frac{1}{u^{2}}+\cdots
$$

Es ergibt sich für die Summe (4) die Darstellung:

$$
\sum_{\lambda=0}^{n-1} \varepsilon^{\lambda \mu} \wp\left(u+\lambda \omega_{1} \mid n \omega_{1}, \omega_{2}\right)=\frac{\frac{\sigma_{0 \mu}^{\prime}}{\sigma_{0 \mu}}\left(\wp(u)-\wp_{0 \mu}\right)-\frac{1}{2}\left(\wp^{\prime}(u)-\wp_{0 \mu}^{\prime}\right)}{\Psi_{0 \mu}(u)} .
$$

Der Faktor von $\wp(u)$ im Zähler rechter Hand gehört, wie S. 232 festgestellt wurde, dem Körper $\left(\Re, g_{2}, g_{3}, \wp_{0 \mu}, \wp_{0 \mu}^{\prime}\right)$ an.

Durch Auflösung der Gleichungen (7) und (8) gewinnt man nun für die einzelne Wurzel der Transformationsgleichung die Darstellung

$$
\begin{gathered}
\wp\left(u+\lambda \omega_{1} \mid n \omega_{1}, \omega_{2}\right) \\
=\frac{1}{n}\left(\wp(u)-\frac{1}{n} \sum_{\mu=1}^{n-1} \wp_{0 \mu}+\sum_{\mu=1}^{n-1} \varepsilon^{-\lambda \mu \frac{\sigma_{0 \mu}}{\sigma_{0 \mu}}\left(\wp(u)-\wp_{0 \mu}\right)-\frac{1}{2}\left(\wp^{\prime}(u)-\wp_{0 \mu}^{\prime}\right)} \frac{\Psi_{0 \mu}(u)}{2}\right) .
\end{gathered}
$$

Es sind also die Lösungen der allgemeinen Transformationsgleichung (2) nach Adjunktion der in der ersten Gleichung (19) S. 230 stehenden $n^{\text {ton }}$ Wurzel zum Körper $\left(\Re, \wp(u), \wp^{\prime}(u), g_{2}, g_{3}, \wp_{\lambda \mu}, \wp_{2 \mu}^{\prime}\right)$ rational bekannt. Da sich umgekehrt die Funktion $\Psi_{0 \mu}(u)$ zufolge:

$$
\Psi_{0 \mu}(u)=\frac{\frac{\sigma_{0_{\mu}^{\prime}}^{\prime}}{\sigma_{0 \mu}}\left(\wp(u)-\wp_{0 \mu}\right)-\frac{1}{2}\left(\wp^{\prime}(u)-\wp_{0 \mu}^{\prime}\right)}{\sum_{\lambda=0}^{n-1} \varepsilon^{\lambda \mu} \wp\left(u+\lambda \omega_{1} \mid n \omega_{1}, \omega_{2}\right)}
$$

als im „Galoisschen Körper" der Transformationsgleichung (2) enthalten erweist, so ist unsere obige Behauptung über den algebraischen Charakter dieser Gleichung bewiesen.

Die entwickelte Gestalt der Transformationsgleichung (2) kann man aus (5) ableiten. Indem man zur Vereinfachung der Schreibweise vorerst $\frac{\omega_{1}}{n}$ statt $\omega_{1}$ einträgt, kleidet sich die Gleichung (5) in die Gestalt:

$$
\wp\left(u \mid \frac{\omega_{1}}{n}, \omega_{2}\right)=\wp(u)+\sum_{i=1}^{n-1}\left(\wp_{\lambda 0}(u)-\wp_{\lambda 0}\right) .
$$

Im Falle eines ungeraden $n$ schreiben wir hierfür: 


$$
\wp\left(u \mid \frac{\omega_{1}}{n}, \omega_{2}\right)=\wp(u)+\sum_{\lambda=1}^{\frac{n-1}{2}}\left(\wp\left(u+\frac{\lambda \omega_{1}}{n}\right)+\wp\left(u-\frac{\lambda \omega_{1}}{n}\right)-2 \wp_{\lambda 0}\right) .
$$

Ist hingegen der Transformationsgrad $n$ eine gerade Zahl, so ergibt sich entsprechend:

$$
\begin{aligned}
& \wp\left(u \mid \frac{\omega_{1}}{n}, \omega_{2}\right)=\wp(u)+\left(\wp\left(u+\frac{\omega_{1}}{2}\right)-e_{1}\right) \\
& +\sum_{\lambda=1}^{\frac{n-2}{2}}\left(\wp\left(u+\frac{\lambda \omega_{1}}{n}\right)+\wp\left(u-\frac{\lambda \omega_{1}}{n}\right)-\wp_{\lambda 0}\right) .
\end{aligned}
$$

Nun folgt z. B. aus der Gleichung (6) S. 216, falls man $Z$ und $Z^{\prime}$ wieder als $\wp$-Funktion schreibt und $2 u$ an Stelle von $u$ einsetzt:

$$
\wp\left(u+\frac{\omega_{1}}{2}\right)-e_{1}=\frac{2 e_{1}^{2}+e_{2} e_{3}}{\wp(u)-e_{1}},
$$

während das Additionstheorem (4) in I, 203 zur Umformung der einzelnen Glieder der Summen die Gleichung liefert:

$$
\wp(u+v)+\wp(u-v)=\frac{\left(2 \wp(u) \wp(v)-\frac{1}{2} g_{2}\right)(\wp \rho(u)+\wp(v))-g_{3}}{(\wp(u)-\wp(v))^{2}} .
$$

Man findet für ungerades $n$ :

$$
\wp\left(u \mid \frac{\omega_{1}}{n}, \omega_{2}\right)=\wp(u)+\sum_{\lambda=1}^{\frac{n-1}{2}} \frac{\left(12 \wp_{\lambda 0}^{9}-g_{2}\right) \wp(u)-4 \wp_{\lambda 0}^{3}-g_{2} \wp_{\lambda 0}-2 g_{3}}{2\left(\wp(u)-\wp_{\lambda 0}\right)^{2}},
$$

sowie für gerades $n$ :

$$
\begin{aligned}
& \wp\left(u \mid \frac{\omega_{1}}{n}, \omega_{2}\right)=\wp(u)+\frac{2 e_{1}^{2}+e_{2} e_{3}}{\wp(u)-e_{1}} \\
& +\sum_{i=1}^{\frac{n-2}{2}} \frac{\left(12 \wp_{\lambda 0}^{2}-g_{2}\right) \wp(u)-4 \wp_{\lambda 0}^{3}-g_{2} \rho_{20}-2 g_{3}}{2\left(\wp(u)-\wp_{\lambda 0}\right)^{2}} \text {. }
\end{aligned}
$$

Mittelst des Ersatzes von $\omega_{1}$ durch $n \omega_{1}$ erhält man aus (11) bzw. (12) die Transformationsgleichung (2) selbst. Die Koeffizienten sind dann zwar noch nicht (wie wir nach dem Ausdrucke (9) der Lösungen erwarten sollten) in den $g_{2}, g_{3}$ und den ursprünglichen Teilwerten ausgedrückt, sondern stellen sich in denjenigen Größen dar, die aus $g_{2}, g_{3}$ und den $\wp_{20}$ durch die erste Haupttransformation hervorgehen. ${ }^{1}$ )

1) Im Falle eines geraden $n$ ist $e_{1}$ der zu $\lambda=\frac{n}{2}$ gehörende Teilwert $\wp_{\lambda 0}$, während $e_{9} e_{3}=\frac{g_{3}}{4 e_{1}}$ ist. 


\section{$\$$ 4. Transformation $n^{\text {ten }}$ Grades der Sigmafunktion.}

Die eben bei der $\wp$-Funktion befolgte Art, die Transformationsgleichung anzusetzen, bestand darin, daß zunächst $\wp\left(u \mid \frac{\omega_{1}}{n}, \omega_{2}\right)$ als rationale Funktion von $\wp\left(u \mid \omega_{1}, \omega_{2}\right)$ zu Darstellung kam. Beim Übergange vom ursprünglichen $\wp\left(u \mid \omega_{1}, \omega_{2}\right)$ zu $\wp\left(u \mid \frac{\omega_{1}}{n}, \omega_{2}\right)$ handelt es sich um die zur ersten Haupttransformation inverse Operation. Diese Art, die Transformation $n^{\text {ten }}$ Grades anzufassen, ist besonders geeignet bei der G-Funktion und den $\vartheta$-Funktionen, weil hier zur Entwicklung der aufzustellenden Relationen die Sätze aus I, 217 ff. über ganze elliptische Funktionen dritter Art Verwendung finden können.

Um dies zunächst bei der G-Funktion auszuführen, stellen wir fest, daß $\sigma\left(u \mid \frac{\omega_{1}}{n}, \omega_{2}\right)$ eine ganze elliptische Funktion dritter Art ist, die bei Vermehrung von $u$ um $\omega_{1}$ und $\omega_{2}$ bzw. die Faktoren annimmt:

$$
\begin{aligned}
& e^{2 i \pi\left(\mu_{1} u+v_{1}\right)}=e^{n \eta_{1}{ }^{\prime} u+\frac{1}{2} n \eta_{1}{ }^{\prime}\left(\omega_{1}+n \pi i\right.}, \\
& e^{2 i \pi\left(\mu_{2} u+v_{2}\right)}=e^{\eta_{2}{ }^{\prime} u+\frac{1}{2} \eta_{2}{ }^{\prime} \omega_{2}+\pi i} .
\end{aligned}
$$

Hier bedeuten $\eta_{1}^{\prime}, \eta_{2}^{\prime}$ die zu den "transformierten Perioden" $\omega_{1}^{\prime}=\frac{\omega_{1}}{n}$, $\omega_{2}^{\prime}=\omega_{2}$ gehörenden Perioden des Integrals zweiter Gattung:

$$
\eta_{i}^{\prime}=\eta_{i}\left(\omega_{1}^{\prime}, \omega_{2}^{\prime}\right)=\eta_{i}\left(\frac{\omega_{1}}{n}, \omega_{2}\right),
$$

und die Bezeichnungen $\mu, \nu$ sind im Sinne von I, $217 \mathrm{ff}$. gebraucht. Diese Größen $\mu, \nu$ stellen sich in den Perioden wie folgt dar:

$$
\begin{cases}\mu_{1}=\frac{n \eta_{1}^{\prime}}{2 i \pi}, & \nu_{1}=\frac{n \eta_{1}^{\prime} \omega_{1}}{4 i \pi}+\frac{n}{2}, \\ \mu_{2}=\frac{\eta_{2}^{\prime}}{2 i \pi}, & \nu_{2}=\frac{\eta_{2}^{\prime} \omega_{2}}{4 i \pi}+\frac{1}{2} .\end{cases}
$$

Die Nullpunkte der Funktion $\sigma\left(u \mid \frac{\omega_{1}}{n}, \omega_{2}\right)$ im Periodenparallelogramm sind nun bei $u=0, \frac{\omega_{1}}{n}, \frac{2 \omega_{1}}{n}, \cdots, \frac{(n-1) \omega_{1}}{n}$ gelegen, so dab nach (13) in I, 221 anzusetzen ist:

$$
\sigma\left(u \mid \frac{\omega_{1}}{n}, \omega_{2}\right)=C \cdot e^{A u^{2}+B u} \prod_{\lambda=0}^{n-1} \sigma\left(u-\frac{\lambda \omega_{1}}{n}\right),
$$

wo $A, B, C$ von $u$ unabhängig sind. Zur Berechnung von $A$ dient die Gleichung (10) in I, 220. Man findet, indem man $A$ als Funktion der Perioden durch $G_{1}\left(\omega_{1}, \omega_{2}\right)$ oder kurz durch $G_{1}$ bezeichnet:

$$
A=G_{1}\left(\omega_{1}, \omega_{2}\right)=-\frac{1}{2}\left(\eta_{1} \mu_{2}-\eta_{2} \mu_{1}\right)=\frac{1}{4 i \pi}\left(n \eta_{2} \eta_{1}^{\prime}-\eta_{1} \eta_{2}^{\prime}\right)
$$


wofür man auch schreiben kann:

$$
A=G_{1}=\frac{1}{4 i \pi \omega_{1}^{\prime} \omega_{2}^{\prime}}\left(\omega_{1} \eta_{2} \cdot \omega_{2}^{\prime} \eta_{1}^{\prime}-\omega_{2} \eta_{1} \cdot \omega_{1}^{\prime} \eta_{2}^{\prime}\right) \text {. }
$$

Setzt man nun (auf Grund der Legendreschen Relation) erstlich:

$$
\omega_{1} \eta_{2}=2 i \pi+\omega_{2} \eta_{1}, \quad \omega_{1}^{\prime} \eta_{2}^{\prime}=2 i \pi+\omega_{2}^{\prime} \eta_{1}^{\prime}
$$

und sodann zweitens:

$$
\omega_{2}^{\prime} \eta_{1}^{\prime}=-2 i \pi+\omega_{1}^{\prime} \eta_{2}^{\prime}, \quad \omega_{2} \eta_{1}=-2 i \pi+\omega_{1} \eta_{2}
$$

in die rechte Seite von (4) ein, so gelangt man nach kurzer Zwischenrechnung zu den beiden folgenden Ausdrücken für $A=G_{1}$ :

$$
A=G_{1}\left(\omega_{1}, \omega_{2}\right)=\frac{\eta_{1}^{\prime}}{2 \omega_{1}^{\prime}}-\frac{n \eta_{1}}{2 \omega_{1}}=\frac{\eta_{2}^{\prime}}{2 \omega_{2}^{\prime}}-\frac{n \eta_{2}}{2 \omega_{2}}
$$

Eine dritte Darstellung von $A=G_{1}$ durch $\wp$-Teilwerte folgt unten; übrigens haben wir in $G_{1}\left(\omega_{1}, \omega_{2}\right)$ eine wichtige Modulform $n^{\text {ter }}$ Stufe der Dimension -2 gewonnen, die später noch wiederholt $\mathrm{zu}$ betrachten sein wird.

In die Gleichung (14) in I, 221 ist entsprechend den Nullpunkten der Funktion (3) und den Werten (2) der $\mu, \nu$ einzutragen:

$$
\begin{gathered}
v_{1}+v_{2}+\cdots+v_{n}=\frac{n-1}{2} \omega_{1}, \\
-\left(\omega_{1} v_{2}-\omega_{2} \nu_{1}\right)-\frac{1}{2} \omega_{1} \omega_{2}\left(\mu_{1}-\mu_{2}\right)=\frac{n \omega_{2}}{2}-\frac{\omega_{1}}{2}
\end{gathered}
$$

und übrigens natürlich $m=n$. Es ergibt sich $m_{1}=0, m_{2}=0$. Da man übrigens leicht das Zutreffen der Gleichung:

$$
-\left(\eta_{1} \nu_{2}-\eta_{2} \nu_{1}\right)-\frac{1}{2}\left(\mu_{1} \omega_{1} \eta_{2}-\mu_{2} \omega_{2} \eta_{1}\right)=\frac{n \eta_{2}}{2}-\frac{\eta_{1}}{2}
$$

zeigt, so bestimmt $\operatorname{sich} B=\frac{n-1}{2} \eta_{1}$. Auf Grund der in (1) I, 450 gegebenen Erklärung der Funktionen $\sigma_{i \mu}(u)$ kaun man den Exponentialfaktor $e^{B u}$ mit dem Produkte in (3) rechts zum Produkte der $n$ Funktionen $\sigma_{\lambda 0}(u)$ zusammenfassen. Der Faktor $C$ bestimmt sich endlich aus den Anfangsgliedern der Reihen nach Potenzen von $u$. Man gelangt zu dem Ergebnis: Die durch die zur ersten Haupttransformation inverse Operation umgeformte $\sigma-F u n k t i o n$ stellt sich in den Funktionen $\sigma_{\lambda_{0}}(u)$ und ihren Nullwerten wie folgt dar:

$$
\sigma\left(u \mid \frac{\omega_{1}}{n}, \omega_{2}\right)=e^{\left(i_{1} u^{2}\right.} \sigma(u) \prod_{\lambda=1}^{n-1} \sigma_{\lambda 0}(u) .
$$

Durch Logarithmierung und zwoimalige Differentiation folgt aus (6)

$$
\wp\left(u \mid \frac{\omega_{1}}{n}, \omega_{2}\right)=-2 G_{1}+\wp(u)+\sum_{\lambda=1}^{n-1} \wp_{\lambda, 0}(u) .
$$


286 II, 1. Transformation $n^{\text {ten }}$ Grades $u$. allgemeine Transformationggleichungen Nach S. 282 aber gilt:

$$
\wp\left(u \mid \frac{\omega_{1}}{n}, \omega_{2}\right)=-\sum_{\lambda=1}^{n-1} \wp_{\lambda 0}+\wp(u)+\sum_{\lambda=1}^{n-1} \wp_{\lambda 0}(u) .
$$

Der Vergleich mit der voraufgehenden Gleichung liefert demnach noch den Satz: Die Größe $G_{1}\left(\omega_{1}, \omega_{2}\right)$ stellt sich in den $\wp$-Teilwerten des $n^{\text {ten }}$ Teilungsgrades so dar:

$$
G_{1}\left(\omega_{1}, \omega_{2}\right)=\frac{1}{2} \sum_{\lambda=1}^{n-1} \wp_{2,0} .
$$

\section{$\$$ 5. Transformation zweiten Grades der Thetafunktionen.}

Bei den Funktionen der älteren Theorie, die zu den Stufen 2, 4,.. gehören, bringt das Transformationsproblem in seiner in $\S 1$ entwickelten allgemeinen Fassung sachlich nichts Neues, da die Funktionen der zweiten Stufe mit denen der ersten algebraisch zusammenhängen und also eine algebraische Beziehung zwischen Funktionen erster Stufe eine ebensolche zwischen den zugehörigen Funktionen der Stufen 2, 4,... zur Folge hat. Formal ist zu bemerken, daß die Argumente $v$ und $w$ der $\vartheta$ Funktionen und der Funktionen sn, cn, dn mit $u$ durch die Gleichungen zusammenhängen:

$$
v=\frac{u}{\omega_{2}}, \quad w=u \sqrt{e_{2}-e_{1}},
$$

wo der Faktor von $u$ in der letzten Gleichung nach I, 473 eine Modulform vierter Stufe ist. Geht man demnach von $\omega_{1}, \omega_{2}$ zu den transformierten Perioden (2) S. 274, während $u$ unverändert bleibt, so ändern sich gleichwohl die $v$ und $w$, indem sie übergehen in:

$$
v^{\prime}=\frac{v}{c \omega+d}, \quad w^{\prime}=M w,
$$

wo der Faktor $M$ von $w$ der Quotient:

$$
M=\frac{V e_{2}-e_{1}\left(a \omega_{1}+b \omega_{2}, c \omega_{1}+d \omega_{2}\right)}{\sqrt{e_{2}-e_{1}}\left(\omega_{1}, \omega_{2}\right)}
$$

des transformierten Wertes der Modulform $\sqrt{e_{1}-e_{2}}$ und des ursprünglichen Wertes ist. $M$ ist der bei der Transformation eintretende „Multiplikator" des Integrals erster Gattung $w$, von dem Jacobi zeigt, dab er einer gewissen algebraischen Gleichung genügt. Wir kommen späterhin auf diese "Multiplikatorgleichung" für die Transformation $n^{\text {ten }}$ Grades zurück.

Die erste Haupttransformation $n^{\text {ten }}$ Grades läßt sich, wenn $n$ eine zusammengesetzte Zahl $n_{1} \cdot n_{\mathrm{g}}$ ist, ersetzen durch die Aufeinanderfolge der beiden entsprechenden Transformationen der Grade $n_{1}, n_{2}$. Es steht 
uns also frei, aus dem Grade $n$ zunächst die höchste in dieser Zahl enthaltene Potenz $2^{v}$ von 2 auszusondern und die Transformation des Grades 2v durch $\boldsymbol{\nu}$-malige Ausübung der Transformation zweiten Grades zu ersetzen. Wir behandeln demnach, wie es für die Funktionen zweiter Stufe auch schon bei der Teilung zweckmäbig erschien, die Transformation zweiten Grades für sich und schließen dann die Transformation ungeraden Graden an.

Bei den $\vartheta$-Funktionen folgen wir zunächst der Entwicklung, die in $\S 4$ auf die Funktion $\sigma(u)$ angewendet wurde, d. h. wir üben auf die vier Funktionen $\vartheta_{v}(v, q)$ die zu den Transformationen inversen Operationen aus und versuchen, die entstehenden Größen in den ursprünglichen $\vartheta$ Funktionen auszudrücken. Die fraglichen Ausdrücke werden gewonnen auf Grund der Sätze in I, 420 ff. über Thetafunktionen erster und höherer Ordnung mit beliebigen Charakteristiken.

Im Falle des zweiten Grades bedeutet die zur zweiten Haupttransformation inverse Operation den Übergang von $\omega_{2} \mathrm{zu} \frac{\omega_{2}}{2}$ bei unveränderten $u, \omega_{1}$. Infolge (1) handelt es sich hier also um den Übergang von $v, q \mathrm{zu} 2 v, q^{2}$. Dieser Übergang läßt sich aber auch so auffassen, da $\beta$ er die Kombination der ersten Haupttransformation und der Multiplikation des Argumentes $u$ mit 2 ist. Die Hinzunahme der Multiplikation hat zur Folge, da $B$ die umgeformten Funktionen wieder die Perioden der ursprünglichen erhalten und dadurch in den letzteren ausdrückbar werden. Dieser Auffassung folgend pflegt man als erste Haupttransformation zweiten Grades den Übergang von $v, q$ zu $2 v, q^{2}$ bezeichnen, während bei der zweiten Haupttransformation und der noch fehlenden dritten Transformation $q$ durch $q^{\frac{1}{2}}$ bzw. $i q^{\frac{1}{2}}$ ersetzt wird, beide Male bei unverändertem $v$. Es gilt nun also die drei Systeme zu je vier Funktionen:

$$
\boldsymbol{\vartheta}_{v}\left(2 v, q^{2}\right), \quad \vartheta_{v}\left(v, q^{\frac{1}{2}}\right), \quad \vartheta_{v}\left(v, i q^{\frac{1}{2}}\right),
$$$$
v=0,1,2,3
$$

in den ursprünglichen $\vartheta$ auszudrücken.

Aus den Formeln (19) in I, 419 folgt bei Ersatz von $v$ und $\omega$ durch $2 v$ und $2 \omega$ :

$$
\vartheta_{v}\left(2(v+\omega), q^{2}\right)= \pm q^{-2} e^{-4 \pi 0 i} \boldsymbol{\vartheta}_{v}\left(2 v, q^{2}\right),
$$

wo rechts für $\nu=0$ und 1 das negative, für $v=2$ und 3 das positive Zeichen gilt. Da ferner jede $\vartheta$-Funktion bei Vermehrung des Argumentes $v$ um 2 unverändert bleibt, bestehen die Gleichungen:

$$
\vartheta_{v}\left(2(v+1), q^{2}\right)=\vartheta_{v}\left(2 v, q^{2}\right) .
$$

Im Sinne der in I, 428 aufgestellten Erklärung sind hiernach die vier Funktionen $\vartheta_{v}\left(2 v, q^{2}\right)$,allgemeine $e^{\text {" }}$ Thetafuntionen zweiter Ordnung $\theta_{g h}^{(2)}(v)$ der vier Charakteristiken $(0,1),(0,1),(0,0),(0,0) . \mathrm{Zu}$ einem ähnlichen 
288 II, 1. Transformation $n^{\text {ten }}$ Grades $u$. allgemeine Transformationsgleichungen Resultate gelangt man bei dem zweiten und dritten Quadrupel (4). Man knüpfe, um das Verhalten bei Vermehrung von $v$ um $\omega$ festzustellen bzw. an:

$$
\begin{aligned}
\vartheta_{v}\left(v+2 \omega, e^{\pi i \omega}\right) & =q^{-4} e^{-4 \pi v i} \vartheta_{v}\left(v, e^{\pi i \omega}\right), \\
\vartheta_{v}\left(v+2 \omega-1, e^{\pi i \omega}\right) & = \pm q^{-4} e^{-4 \pi i} \vartheta_{v}\left(v, e^{\pi i \omega}\right),
\end{aligned}
$$

ersetze $\omega$ durch $\frac{\omega}{2}$ bzw. $\frac{\omega+1}{2}$ und gelangt durch Fortsetzung der Überlegung zu dem Ergebnis, daß auch die acht noch fehlenden Funktionen (4) ,allgemeine" Thetafunktionen zweiter Ordnung $\theta_{g h}^{(2)}(v)$ sind. Die Charakteristiken des zweiten Quadrupels (4) sind aber der Reihe $\nu=0,1,2,3$ nach $(0,0),(1,0),(1,0),(0,0)$ und die des dritten $(0,0),(1,1),(1,1),(0,0)$.

Nach I, 429 ff. sind somit die zwölf transformierten Funktionen (4) durch die Quadrate und zweigliedrigen Produkte die vier ursprünglichen $\vartheta$-Funktionen darstellbar, deren Verteilung auf die vier Charakteristiken $(g, h)$ in I, 429 unten angegeben ist: Bei der Charakteristik $(0,0)$ darf man irgend zwei der vier Quadrate $\vartheta_{v}(v)^{2}$ für die Darstellung zugrunde legen. $\left.{ }^{1}\right)$ Bei den anderen drei Charakteristiken $(g, h)$ aber beachte man, da $B$ immer ein Produkt $\vartheta_{\mu}(v) \vartheta_{v}(v)$ eine gerade, das andere eine ungerade Funktion darstellt. Unter den zwölf Funktionen (4) sind übrigens dic vier mit $\nu=1$ ungerade, alle übrigen aber gerade.

Dem Ansatze der gesuchten Darstellungen schicken wir noch ein paar Relationen zwischen $\vartheta$-Nullwerten voraus. Die Nullwerte der drei geraden $\vartheta$-Funktionen sind nach (18) in I, 418 durch die Produkte darstellbar:

$$
\left\{\begin{array}{l}
\vartheta_{0}=\prod_{m=1}^{\infty}\left(1-q^{2 m}\right) \prod_{m=1}^{\infty}\left(1-q^{2 m-1}\right)^{2} \\
\vartheta_{2}=2 q^{\frac{1}{4}} \prod_{m=1}^{\infty}\left(1-q^{2 m}\right) \prod_{m=1}^{\infty}\left(1+q^{2 m}\right)^{2} \\
\vartheta_{3}=\prod_{m=1}^{\infty}\left(1-q^{2 m}\right) \prod_{m=1}^{\infty}\left(1+q^{2 m-1}\right)^{2} .
\end{array}\right.
$$

Durch Multiplikation der ersten und dritten Gleichung folgt:

$$
\vartheta_{0} \vartheta_{3}=\prod_{m=1}^{\infty}\left(1-q^{2 m}\right)^{2} \prod_{m=1}^{\infty}\left(1-q^{4 m-2}\right)^{2}=\prod_{m=1}^{\infty}\left(1-q^{4 m}\right)^{2} \prod_{m=1}^{\infty}\left(1-q^{4 m-2}\right)^{4},
$$

wo sich rechts die Produktentwicklung von $\vartheta_{0}\left(q^{2}\right)^{2}$ eingefunden hat. Entsprechend gewinnt man:

1) Bei einer ohne zweites Argument geschriebenen Funktion $\vartheta_{v}(v)$ gilt stets $q$ als zweites Argument. Ebenso ist bei einem ohne Argument geschriebenen Nullwerte $\boldsymbol{\vartheta}_{\boldsymbol{v}}$ als Argument $q$ zu denken. 


$$
\begin{aligned}
\vartheta_{2} \vartheta_{3} & =2 q^{\frac{1}{4}} \prod_{m=1}^{\infty}\left(1-q^{2 m}\right)^{2} \prod_{m=1}^{\infty}\left(1+q^{m}\right)^{2} \\
& =2 q^{\frac{1}{4}} \prod_{m=1}^{\infty}\left(1-q^{m}\right)^{2} \prod_{m=1}^{\infty}\left(1+q^{m}\right)^{4}
\end{aligned}
$$

wo rechts die Produktentwicklung von $\frac{1}{2} \vartheta_{2}\left(q^{\frac{1}{2}}\right)^{2}$ steht. Auf diese Weise erhält man die Relationen:

$$
\boldsymbol{\vartheta}_{0} \boldsymbol{\vartheta}_{3}=\vartheta_{0}\left(q^{2}\right)^{2}, \quad 2 \vartheta_{2} \vartheta_{3}=\vartheta_{2}\left(q^{\frac{1}{2}}\right)^{2}, \quad \vartheta_{2}^{2}=2 \vartheta_{2}\left(q^{2}\right) \vartheta_{3}\left(q^{2}\right)
$$

deren dritte eine unmittelbare Folge der zweiten ist.

Man bilde nun nach den Regeln von I, $429 \mathrm{ff}$., die sich auf den Hermiteschen Satz gründen, zunächst die Ansätze:

$$
\boldsymbol{\vartheta}_{0}\left(2 v, q^{2}\right)=a \vartheta_{0}(v) \boldsymbol{\vartheta}_{3}(v), \quad \boldsymbol{\vartheta}_{3}\left(2 v, q^{2}\right)=b \vartheta_{3}(v)^{2}+c \vartheta_{0}(v)^{2},
$$

wo die $a, b, c$ von $v$ unabhängig sind. Vermehrt man in der zweiten Gleichung $v$ um $\frac{1}{2}$, so bleibt $\vartheta_{3}\left(2 v, q^{2}\right)$ nach I, 419 unverändert, während sich $\vartheta_{3}(v)$ und $\vartheta_{0}(v)$ austauschen; es gilt also $c=b$. Vermehrt man $v$ um $\frac{\omega}{2}$, so folgt aus (7) nach I, 419 bei Fortlassung der rechts und links übereinstimmend auftretenden Faktoren:

$$
\vartheta_{1}\left(2 v, q^{2}\right)=a \vartheta_{1}(v) \vartheta_{2}(v), \quad \vartheta_{2}\left(2 v, q^{2}\right)=b\left(\vartheta_{2}(v)^{2}-\vartheta_{1}(v)^{2}\right)
$$

Jetzt setze man $v=0$ in die erste Gleichung (7) und die zweite Gleichung (8) ein und findet so:

$$
\vartheta_{0}\left(q^{2}\right)=a \vartheta_{0} \vartheta_{3}, \quad \vartheta_{2}\left(q^{2}\right)=b \vartheta_{2}^{2} .
$$

Mit Benutzung von (6) ergibt sich somit:

$$
a=\frac{\boldsymbol{\vartheta}_{0}\left(q^{2}\right)}{\boldsymbol{\vartheta}_{0} \boldsymbol{\vartheta}_{3}}=\frac{1}{\boldsymbol{\vartheta}_{0}\left(q^{2}\right)}, \quad b=\frac{\boldsymbol{\vartheta}_{2}\left(q^{2}\right)}{\boldsymbol{\vartheta}_{2}^{2}}=\frac{1}{2 \boldsymbol{\vartheta}_{3}\left(q^{2}\right)} .
$$

Für die mittelst der ersten Haupttransformation umgeformten $\vartheta$-Funktionen gelten also folgende Darstellungen in den ursprünglichen $\vartheta(v)$ :

$$
\left\{\begin{array}{c}
\vartheta_{0}\left(q^{2}\right) \vartheta_{0}\left(2 v, q^{2}\right)=\vartheta_{0}(v) \vartheta_{3}(v), \quad \vartheta_{0}\left(q^{2}\right) \vartheta_{1}\left(2 v, q^{2}\right)=\vartheta_{1}(v) \vartheta_{2}(v) \\
2 \vartheta_{3}\left(q^{2}\right) \vartheta_{2}\left(2 v, q^{2}\right)=\vartheta_{2}(v)^{2}-\vartheta_{1}(v)^{2} \\
2 \vartheta_{3}\left(q^{2}\right) \vartheta_{3}\left(2 v, q^{2}\right)=\vartheta_{3}(v)^{2}+\vartheta_{0}(v)^{2}
\end{array}\right.
$$

Von den Funktionen $\vartheta_{v}\left(v, q^{\frac{1}{2}}\right)$ haben die beiden ersten die Charakteristiken $(0,0)$ und $(1,0)$, so daB die Ansätze gelten:

$$
\vartheta_{0}\left(v, q^{\frac{1}{2}}\right)=a \vartheta_{0}(v)^{2}+b \vartheta_{1}(v)^{2}, \quad \vartheta_{1}\left(v, q^{\frac{1}{2}}\right)=c \vartheta_{1}(v) \vartheta_{0}(v)
$$

Vermehrt man in der ersten Gleichung $v$ um $\frac{\omega}{2}$, so kommt für die linke 
290 II, 1. Transformation $n^{\text {ten }}$ Grades u. allgemeine Transformationsgleichungen Seite die auf $\frac{\omega}{2}$ statt $\omega$, umgeformte erste Gleichung (19) in I, 419, für die beiden rechts stehenden Funktionen aber die Tabelle daselbst zur Verwendung. Man findet nach:Fortlassung überflüssiger Faktoren:

$$
\vartheta_{0}\left(v, q^{\frac{1}{2}}\right)=a \vartheta_{1}(v)^{2}+b \vartheta_{0}(v)^{2}
$$

so daB $a=b$ gilt. Bei Vermehrung von $v$ um $\frac{1}{2}$ folgt aus (10):

$$
\vartheta_{3}\left(v, q^{\frac{1}{2}}\right)=a\left(\boldsymbol{\vartheta}_{3}(v)^{2}+\vartheta_{2}(v)^{2}\right), \quad \vartheta_{2}\left(v, q^{\frac{1}{2}}\right)=c \vartheta_{2}(v) \vartheta_{3}(v) .
$$

Durch Eintragen von $v=0$ in die erste Gleichung (10) und die zweite Gleichung (11) ergibt sich wieder mit Benutzung von (6):

$$
a=\frac{\vartheta_{0}\left(q^{\frac{1}{2}}\right)}{\vartheta_{0}^{2}}=\frac{1}{\vartheta_{3}\left(q^{\frac{1}{2}}\right)}, \quad c=\frac{\vartheta_{2}\left(q^{\frac{1}{2}}\right)}{\vartheta_{2} \vartheta_{3}}=\frac{2}{\vartheta_{2}\left(q^{\frac{1}{2}}\right)} .
$$

Für die durch die zweite Haupttransformation umgeformten $\vartheta$-Funktionen hat man also folgende Darstellungen in den ursprünglichen $\vartheta(v)$ :

$$
\begin{cases}\vartheta_{3}\left(q^{\frac{1}{2}}\right) \vartheta_{0}\left(v, q^{\frac{1}{2}}\right)=\vartheta_{0}(v)^{2}+\vartheta_{1}(v)^{2}, & \vartheta_{2}\left(q^{\frac{1}{2}}\right) \vartheta_{1}\left(v, q^{\frac{1}{2}}\right)=2 \vartheta_{1}(v) \vartheta_{0}(v) \\ \vartheta_{2}\left(q^{\frac{1}{2}}\right) \vartheta_{2}\left(v, q^{\frac{1}{2}}\right)=2 \vartheta_{2}(v) \vartheta_{3}(v), & \vartheta_{3}\left(q^{\frac{1}{2}}\right) \vartheta_{3}\left(v, q^{\frac{1}{2}}\right)=\vartheta_{3}(v)^{2}+\vartheta_{2}(v)^{2}\end{cases}
$$

An Stelle der zweiten und dritten Gleichung kann man auch schreiben:

$$
\frac{\vartheta_{1}\left(v, q^{\frac{1}{2}}\right)}{\vartheta_{2}\left(q^{\frac{1}{2}}\right)}=\frac{\vartheta_{1}(v) \vartheta_{0}(v)}{\boldsymbol{\vartheta}_{2} \vartheta_{3}}, \quad \frac{\vartheta_{2}\left(v, q^{\frac{1}{2}}\right)}{\vartheta_{2}\left(q^{\frac{1}{2}}\right)}=\frac{\vartheta_{2}(v) \vartheta_{3}(v)}{\boldsymbol{\vartheta}_{2} \vartheta_{3}} .
$$

Endlich trage man in die erste und vierte Gleichung (12) und in die Gleichungen (13) statt $\omega$ noch $\omega+1$ ein und bediene sich bei der Umrechnung der Reihenentwicklungen (17) und (20) in I, $418 \mathrm{ff}$. Man wird leicht zu folgendem Ergebnis gelangen: Die durch die noch fehlende Transformation zweiten Grades umgeformten $\vartheta$-Funktionen stellen sich in den ursprünglichen $\vartheta(v)$ so dar:

$$
\left\{\begin{array}{cc}
\vartheta_{3}\left(i q^{\frac{1}{2}}\right) \vartheta_{0}\left(v, i q^{\frac{1}{2}}\right)= & \vartheta_{3}(v)^{2}+i \vartheta_{1}(v)^{2} \\
\frac{\vartheta_{1}\left(v, i q^{\frac{1}{2}}\right)}{\vartheta_{2}\left(i q^{\frac{1}{2}}\right)}=\frac{\vartheta_{1}(v) \vartheta_{3}(v)}{\vartheta_{0} \vartheta_{2}}, & \frac{\vartheta_{2}\left(v, i q^{\frac{1}{2}}\right)}{\vartheta_{2}\left(i q^{\frac{1}{2}}\right)}=\frac{\vartheta_{0}(v) \vartheta_{2}(v)}{\vartheta_{0} \vartheta_{2}} \\
\vartheta_{3}\left(i q^{\frac{1}{2}}\right) \vartheta_{3}\left(v, i q^{\frac{1}{2}}\right) & =\vartheta_{0}(v)^{2}+i \vartheta_{2}(v)^{2}
\end{array}\right.
$$

\section{§ 6. Transformation zweiten Grades der Funktionen sn, en und dn.}

Auf die Berechnung der transformierten Werte der von den Perioden allein abhängenden Modulfunktionen gehen wir erst in den späteren Kapiteln ein. Um indessen über die Transformation zweiten Grades der 
Funktionen sn, cn und dn abschließende Angaben machen zu können, sind schon hier einige vorläufige Notizen über die Transformation desselben Grades der Integralmoduln und ihrer Wurzeln zu machen.

Nach I, 419 stellen sich die vierten Wurzeln aus dem Integralmodul $k^{2}$ und dem komplementären Modul $k^{2}=1-k^{2}$ in den $\vartheta$-Nullwerten so dar:

$$
\sqrt{k}=\frac{\vartheta_{9}}{\vartheta_{3}}, \quad \sqrt{k^{\prime}}=\frac{\vartheta_{0}}{\boldsymbol{\vartheta}_{3}}
$$

und sie sind hierdurch als eindeutige Funktionen von $\omega$ erklärt. Setzt man im Quotienten der dritten und vierten Gleichung (9) S. 289 für $v$ den Wert 0 ein, so folgt bei Übergang zu den Integralmoduln auf Grund von (1):

$$
\left.\frac{\vartheta_{2}\left(q^{2}\right)}{\vartheta_{3}\left(q^{2}\right)}=\sqrt{k}(2 \omega)=\frac{\vartheta_{2}^{2}}{\vartheta_{3}^{2}+\vartheta_{0}^{2}}=\frac{k}{1+k^{\prime}} \cdot 1\right)
$$

Ebenso findet man aus der ersten und vierten Gleichung (9) S. 289:

$$
\left(\frac{\vartheta_{0}\left(q^{2}\right)}{\vartheta_{3}\left(q^{2}\right)}\right)^{2}=k^{\prime}(2 \omega)=\frac{2 \vartheta_{0} \vartheta_{3}}{\vartheta_{3}^{2}+\vartheta_{0}^{2}}=\frac{2 \sqrt{k^{\prime}}}{1+k^{\prime}} .
$$

Es gilt also der Satz: Die durch die erste Haupttransformation zweiten Grades umgeformten Integralmoduln stellen sich in den ursprünglichen Moduln wie folgt dar:

$$
\sqrt{\bar{k}}(2 \omega)=\frac{k}{1+k^{\prime}}, \quad k(2 \omega)=\frac{1-k^{\prime}}{1+k^{\prime}}, \quad k^{\prime}(2 \omega)=\frac{2 \sqrt{\bar{k}^{\prime}}}{1+k^{\prime}} .
$$

Um entsprechende Gleichungen für die zweite Haupttransformation aufzustellen, kann man entweder an die Gleichungen (12) und (13) S. 290 anzuknüpfen oder in den eben aufgestellten Gleichungen $\frac{\omega}{2}$ an Stelle von $\omega$ eintragen und nach $k\left(\frac{\omega}{2}\right)$ und $\sqrt{k^{\prime}}\left(\frac{\omega}{2}\right)$ lösen. Die mittelst der zweiten Haupttransformation zweiten Grades umgeformten Integralmoduln stehen zu den ursprünglichen Moduln in der Beziehung:

$$
k\left(\frac{\omega}{2}\right)=\frac{2 \sqrt{k}}{1+k}, \cdot \sqrt{k^{\prime}}\left(\frac{\omega}{2}\right)=\frac{k^{\prime}}{1+k}, \quad k^{\prime}\left(\frac{\omega}{2}\right)=\frac{1-k}{1+k} .
$$

Die dritte Transformation zweiten Grades betrachten wir hier nicht besonders.

Die Wirkung der Transformationen zweiten Grades auf die Funktionen sn, cn und dn liest man nun aus den Formeln (9)ff. S. 289 leicht ab. Wir nehmen in diese Funktionen neben $w$ die Quadratwurzel des Integralmoduls $k^{2}$ als zweites Argument auf ${ }^{2}$ ) und fassen die Formeln (28) in

1) Ist bei den Modulfunktionen $k, k^{\prime}, \sqrt{k}, \sqrt{k^{\prime}}$ ein Argument nicht angegeben, so ist stets $\omega$ als solches hinzuzudenken.

2) So oft ein solches Argument nicht angegeben ist, gilt der ursprüngliche Wert $k$ als zweites Argument. 
292 II, 1. 'Transformation $n^{\text {ten }}$ Grades u. allgemeine Transformationsgleichungen I, 420 durch die Proportion zusammen:

$$
\left\{\begin{array}{l}
\operatorname{sn}(w, k): \operatorname{cn}(w, k): \operatorname{dn}(w, k): 1 \\
=\boldsymbol{\vartheta}_{3}^{\mathbf{2}} \boldsymbol{\vartheta}_{1}(v): \boldsymbol{\vartheta}_{0} \boldsymbol{\vartheta}_{3} \boldsymbol{\vartheta}_{\mathbf{2}}(v): \boldsymbol{\vartheta}_{0} \boldsymbol{\vartheta}_{2} \boldsymbol{\vartheta}_{3}(v): \boldsymbol{\vartheta}_{2} \boldsymbol{\vartheta}_{3} \boldsymbol{\vartheta}_{0}(v) .
\end{array}\right.
$$

Die Wirkung der ersten Haupttransformation (vereint mit der Multiplikation) auf $v$ und $\omega$ ist nun $v^{\prime}=2 v, \omega^{\prime}=2 \omega$. Für das Argument $w$, das sich nach I, 388 und (21) in I, 419 so darstellt:

$$
w=u \sqrt{e_{2}-e_{1}}=v \omega_{2} \sqrt{e_{2}-e_{1}}=\pi v \vartheta_{3}^{2},
$$

ergibt sich also die Transformation:

$$
w^{\prime}=2 \pi v \vartheta_{3}\left(q^{2}\right)^{2}=2 w\left(\frac{\vartheta_{3}\left(q^{2}\right)}{\vartheta_{3}}\right)^{2}=\left(1+k^{\prime}\right) w,
$$

wie man mit Hilfe der für $v=0$ gebildeten letzten Gleichung (9) S. 289 und der zweiten Gleichung (1) feststellt. Die Wirkung der ersten Haupttransformation auf $k$ aber ist unter (2) angegeben. Die Proportion (4) ist demgemäß umzuformen in:

$$
\begin{aligned}
\operatorname{sn}\left(\left(1+k^{\prime}\right) w, \frac{1-k^{\prime}}{1+k^{\prime}}\right) & : \operatorname{cn}\left(\left(1+k^{\prime}\right) w, \frac{1-k^{\prime}}{1+k^{\prime}}\right): \operatorname{dn}\left(\left(1+k^{\prime}\right) w, \frac{1-k^{\prime}}{1+k^{\prime}}\right): 1 \\
=\vartheta_{3}\left(q^{2}\right)^{2} \vartheta_{1}\left(2 v, q^{2}\right) & : \vartheta_{0}\left(q^{2}\right) \vartheta_{3}\left(q^{2}\right) \vartheta_{2}\left(2 v, q^{2}\right): \vartheta_{0}\left(q^{2}\right) \vartheta_{\mathbf{2}}\left(q^{2}\right) \vartheta_{3}\left(2 v, q^{2}\right) \\
& : \vartheta_{2}\left(q^{2}\right) \vartheta_{3}\left(q^{2}\right) \vartheta_{0}\left(2 v, q^{2}\right) .
\end{aligned}
$$

Man drücke nun die rechts stehenden transformierten $\vartheta$-Funktionen nach (9) S. 289 durch die ursprünglichen aus, führe auf Grund von (1) und (4) die Moduln und Funktionen sn, cn, dn ein und ersetze noch die transformierten Moduln nach (2) durch die ursprünglichen. Die rechte Seite der letzten Proportion nimmt dabei die Gestalt an:

$$
\left(1+k^{\prime}\right) \operatorname{sn} w \operatorname{cn} w:\left(\operatorname{cn} w^{2}-k^{\prime} \operatorname{sn} w^{2}\right): \frac{1}{1+k^{\prime}}\left(k^{\prime}+\operatorname{dn} w^{2}\right): \operatorname{dn} w .
$$

Im zweiten und dritten Gliede schreibe man noch $\left(1-\mathrm{sn}^{2}\right)$ für $\mathrm{cn}^{2}$ und $\left(1-k^{2} \mathrm{sn}^{2}\right)$ für $\mathrm{dn}^{2}$. Unter Spaltung der Proportion in drei Gleichungen findet sich der Satz: Die mittelst der ersten Haupttransformation umgeformten Funktionen sn, en und dn stellen sich in den ursprünglichen Funktionen so dar:

$$
\left\{\begin{array}{l}
\operatorname{sn}\left(\left(1+k^{\prime}\right) w, \frac{1-k^{\prime}}{1+k^{\prime}}\right)=\left(1+k^{\prime}\right) \frac{\operatorname{sn} w \operatorname{cn} w}{\operatorname{dn} w} \\
\operatorname{cn}\left(\left(1+k^{\prime}\right) w, \frac{1-k^{\prime}}{1+k^{\prime}}\right)=\frac{1-\left(1+k^{\prime}\right) \operatorname{sn} w^{2}}{\operatorname{dn} w}, \\
\operatorname{dn}\left(\left(1+k^{\prime}\right) w, \frac{1-k^{\prime}}{1+k^{\prime}}\right)=\frac{1-\left(1-k^{\prime}\right) \operatorname{sn} w^{2}}{\operatorname{dn} w}
\end{array}\right.
$$

Diese Formeln liefern die sogenannte Landensche Transformation. ${ }^{1}$ )

1) Vgl. die Landensche Abhandlung „An investigation of a general theorem for finding the length of any arc of any conic hyperbel etc.", Philosoph. Transact., Bd. 65 (1775) oder "Math. Mem." (London 1780) Bd. 1, S. 33. 
Die anderen beiden Transformationen zweiten Grades gestatten eine ganz entsprechende Behandlung. Wir notieren nur noch das Ergebnis: Die durch die zweite Haupttransformation umgeformten Funktionen sn, en und dn stellen sich in den ursprünglichen Funktionen und Moduln so dar:

$$
\left\{\begin{array}{l}
\operatorname{sn}\left((1+k) w, \frac{2 \sqrt{k}}{1+k}\right)=\frac{(1+k) \operatorname{sn} w}{1+k \operatorname{sn} w^{2}}, \\
\operatorname{cn}\left((1+k) w, \frac{2 \sqrt{k}}{1+k}\right)=\frac{\operatorname{cn} w \operatorname{dn} w}{1+k \operatorname{sn} w^{2}}, \\
\operatorname{dn}\left((1+k) w, \frac{2 \sqrt{k}}{1+k}\right)=\frac{1-k \operatorname{sn} w^{2}}{1+k \operatorname{sn} w^{2}}
\end{array}\right.
$$

Diese Gleichungen bilden die sogenannte Gaußsche Transformation. ${ }^{1}$ )

\section{$\S 7$. Transformation ungeraden Grades der Funktionen zweiter Stufe.}

Es sei endlich der Transformationsgrad $n$ eine beliebige ungerade Zahl. Beschränken wir uns auf die beiden Haupttransformationen, so haben wir entsprechend der bei $n=2$ befolgten Methode zunächst die transformierten Thetafunktionen:

$$
\boldsymbol{\vartheta}_{v}\left(n v, q^{n}\right), \quad \boldsymbol{\vartheta}_{v}\left(v, q^{\frac{1}{n}}\right),
$$$$
v=0,1,2,3
$$

in den ursprünglichen darzustellen. Die Ansätze zur Lösung dieser Aufgabe gewinnt man wie im Falle $n=2$.

Man folgert erstlich aus den Gleichungen (19) in I, 419:

$$
\begin{aligned}
\vartheta_{v}\left(n v+n \omega, q^{n}\right) & = \pm q^{-n} e^{-2 n \pi v i} \vartheta_{v}\left(n v, q^{n}\right) \\
\vartheta_{v}\left(n v+n, q^{n}\right) & = \pm \vartheta_{v}\left(n v, q^{n}\right),
\end{aligned}
$$

wo die rechts gültigen Vorzeichen aus den eben genannten Gleichungen zu entnehmen sind. Aus diesen Vorzeichen geht hervor, daB die $\boldsymbol{\vartheta}_{\boldsymbol{v}}\left(n \boldsymbol{v}, q^{\boldsymbol{n}}\right)$ als Thetafunktionen $n^{\text {ter }}$ Ordnung die Charakteristiken $(0,1),(1,1),(1,0)$, $(0,0)$ besitzen.

Zur Behandlung des zweiten Quadrupels (1) knüpft man an die aus (19) in I, 419 für beliebiges ungerades $n$ leicht ableitbare Regel:

$$
\boldsymbol{\vartheta}_{\boldsymbol{v}}(v+n \omega, q)= \pm q^{-n^{2}} e^{-2 n \pi v i} \boldsymbol{\vartheta}_{\boldsymbol{v}}(v, q),
$$

wo wieder dieselben Vorzeichen gelten wie in den genannten Formeln. Schreibt man $\frac{\omega}{n}$ statt $\omega$, so folgt:

$$
\vartheta_{v}\left(v+\omega, q^{\frac{1}{n}}\right)= \pm q^{-n} e^{-2 n \pi v v} \vartheta_{v}\left(v, q^{\frac{1}{n}}\right)
$$

1) Vgl. Gau B' Abhandlung „Determinatio attractionis, quam in punctum quodvis positionis datae exerceret planeta etc.", Göttinger Abhandl., Bd. 4 (1818) oder ,Werke", Bd. 3, S. 331. 
294 II, 1. Transformation $n^{\text {ton }}$ Grades u. allgemeine Transformationsgleichungen während sich andrerseits unmittelbar:

$$
\vartheta_{v}\left(v+1, q^{\frac{1}{n}}\right)= \pm \vartheta_{v}\left(v, q^{\frac{1}{n}}\right)
$$

ergibt. Man findet, daB auch das zweite Quadrupel (1) Thetafunktionen $n^{\text {ter }}$ Ordnung der Charakteristiken $(0,1),(1,1),(1,0),(0,0)$ darstellt.

Die Ansätze für die Darstellung der beiden Systeme transformierter Thetafunktionen in den ursprünglichen $\vartheta(v)$ werden durch die Regeln von I, $430 \mathrm{ff}$. geliefert. Für die vier in Frage kommenden Charakteristiken sind daselbst je $n$ linear-unabhängige Ausdrücke in den Funktionen $\vartheta_{v}(v, q)$ gebildet, in denen die einzelnen transformierten Funktionen jeweils linear und homogen darstellbar sind. Für die beiden Funktionen $\vartheta_{0}\left(n v, q^{n}\right)$ und $\vartheta_{0}\left(v, q^{\frac{1}{n}}\right)$ kommen nur die geraden Ausdrücke der Charakteristik $(0,1)$ in Betracht. Dies sind die $\frac{n+1}{2}$ Produkte:

$$
\vartheta_{0}(v)^{n}, \quad \vartheta_{0}(v)^{n-2} \vartheta_{1}(v)^{2}, \quad \vartheta_{0}(v)^{n-4} \vartheta_{1}(v)^{4}, \ldots, \vartheta_{0}(v) \vartheta_{1}(v)^{n-1},
$$

welche in der Zusammenstellung von I, 430 (unten) an erster Stelle genannt sind. Man hat also die beiden Ansätze:

$$
\left\{\begin{array}{l}
\vartheta_{0}\left(n v, q^{n}\right)=a_{0} \vartheta_{0}(v)^{n}+a_{1} \vartheta_{0}(v)^{n-2} \vartheta_{1}(v)^{2}+\cdots+\frac{a_{n-1}}{2} \vartheta_{0}(v) \vartheta_{1}(v)^{n-1} \\
\vartheta_{0}\left(v, q^{\frac{1}{n}}\right)=b_{0} \vartheta_{0}(v)^{n}+b_{1} \vartheta_{0}(v)^{n-2} \vartheta_{1}(v)^{2}+\cdots+\frac{b_{\frac{n-1}{2}}}{2} \vartheta_{0}(v) \vartheta_{1}(v)^{n-1}
\end{array}\right.
$$

wo die Koeffizienten $a, b$ von $v$ unabhängig sind.

Statt für die übrigen drei transformierten Funktionen jedes Quadrupels (1) ähnliche Ansätze auf demselben Wege aufzustellen, kann man diese Ansätze auch durch Umrechnung aus (2) gewinnen. Zur $\vartheta_{1}$-Funktion gelangt man von $\vartheta_{0}$ aus durch Vermehrung von $v$ um $\frac{\omega}{2}$. Erstlich ergibt sich aus der Tabelle in I, 419 leicht:

$$
\vartheta_{0}\left(n v+\frac{n \omega}{2}, q^{n}\right)=i q^{-\frac{n}{4}} e^{-n \pi v i} \vartheta_{1}\left(n v, q^{n}\right)
$$

womit man die erste Gleichung (2) umzurechnen hat. Für die zweite Gleichung (2) knüpfe man an die für jede ganze Zahl $m$ gültige Gleichung:

$$
\vartheta_{0}(v+m \omega, q)=(-1)^{m} q^{-m^{2}} e^{-2 m \pi v i} \vartheta_{0}(v, q) .
$$

Man setze hier $m=\frac{1}{2}(n-1)$ und vermehre $v$ um $\frac{\omega}{2}$, wodurch man erhält:

$$
\vartheta_{0}\left(v+\frac{n \omega}{2}, q\right)=(-1)^{\frac{n-1}{2}} i q^{-\frac{n^{2}}{4}} e^{-n \pi v i} \vartheta_{1}(v, q)
$$


und weiter, wenn man $\frac{\omega}{n}$ statt $\omega$ einträgt:

$$
\vartheta_{0}\left(v+\frac{\omega}{2}, q^{\frac{1}{n}}\right)=(-1)^{\frac{n-1}{2}} i q^{-\frac{n}{4}} e^{-n \pi v i} \vartheta_{1}\left(v, q^{\frac{1}{n}}\right)
$$

Die Wirkung der Vermehrung von $v$ um $\frac{\omega}{2}$ auf die rechten Seiten der Gleichungen (2) ist direkt aus der Tabelle in I, 419 feststellbar. Man erhält aus (2) die weiteren Ansätze:

$$
\left\{\begin{array}{r}
(-1)^{\frac{n-1}{2}} \vartheta_{1}\left(n v, q^{n}\right)=a_{0} \vartheta_{1}(v)^{n}+a_{1} \vartheta_{1}(v)^{n-2} \vartheta_{0}(v)^{2}+\cdots \\
\cdots+a_{\frac{n-1}{2}} \vartheta_{1}(v) \vartheta_{0}(v)^{n-1} \\
\vartheta_{1}\left(v, q^{\frac{1}{n}}\right)=b_{0} \vartheta_{1}(v)^{n}+b_{1} \vartheta_{1}(v)^{n-2} \vartheta_{0}(v)^{2}+\cdots \\
\cdots+\frac{b_{n-1}}{2} \vartheta_{1}(v) \vartheta_{0}(v)^{n-1}
\end{array}\right.
$$

wo die $a$ und $b$ dieselbe Bedeutung haben wie in (2). Durch Vermehrung von $v$ um $\frac{1}{2}$ gelangt man von (2) und (3) aus zu entsprechenden Ansätzen für die transformierten Funktionen $\boldsymbol{\vartheta}_{3}$ und $\boldsymbol{\vartheta}_{2}$.

Von den Koeffizienten ergeben sich $a_{0}$ und $b_{0}$ aus (2), indem man $v=0$ einträgt:

$$
a_{0}=\frac{\vartheta_{0}\left(q^{n}\right)}{\vartheta_{0}^{n}}, \quad b_{0}=\frac{\vartheta_{0}\left(q^{\frac{1}{n}}\right)}{\vartheta_{0}^{n}} .
$$

Ebenso kann man aus (3) für $\lim v=0$ unter Berücksichtigung des aus I, $418 \mathrm{ff}$. sich ergebenden Anfangsgliedes:

$$
\boldsymbol{\vartheta}_{1}(v, q)=\boldsymbol{\vartheta}_{1}^{\prime} \cdot v+\cdots=\pi \boldsymbol{\vartheta}_{0} \boldsymbol{\vartheta}_{2} \boldsymbol{\vartheta}_{3} v+\cdots
$$

der Reihe von $\vartheta_{1}(v)$ nach Potenzen von $v$ die Bedeutung der beiden letzten Koeffizienten entnehmen. Die Rechnung führt auf:

$$
\left\{\begin{array}{l}
a_{\frac{n-1}{2}}=(-1)^{\frac{n-1}{2}} n \frac{\vartheta_{0}\left(q^{n}\right)}{\vartheta_{0}^{n}} \frac{\vartheta_{0}\left(q^{n}\right) \vartheta_{3}\left(q^{n}\right)}{\vartheta_{2} \vartheta_{3}} \\
b_{\frac{n-1}{2}}=\frac{\vartheta_{0}\left(q^{-\frac{1}{n}}\right) \frac{\vartheta_{2}\left(q^{\frac{1}{n}}\right)}{\vartheta_{0}^{n}}\left(q_{\vartheta_{2}} \frac{1}{n}\right)}{\vartheta_{3}} .
\end{array}\right.
$$

Auch die übrigen Koeffizienten $a, b$ sind rational mit rationalen Zahlenkoeffizienten durch die ursprünglichen und transformierten $\vartheta$-Nullwerte darstellbar. Man findet nämlich z.B. zum Zwecke der Berechnung der $a$ bei Division der ersten Gleichung (3) durch die erste Gleichung (2):

(6) $(-1)^{\frac{n-1}{2}} \sqrt{l} \operatorname{sn}\left(w^{\prime}, l\right)=\frac{a_{0}(\sqrt{k})^{n} \operatorname{sn} w^{n}+a_{1}(\sqrt{k})^{n-2} \operatorname{sn} w^{n-2}+\cdots+a_{\frac{n-1}{2}} \sqrt{k} \operatorname{sn} w}{n-1}$

$$
a_{0}+a_{1} k \operatorname{sn} w^{2}+a_{2} k^{2} \operatorname{sn} w^{4}+\cdots+a_{\frac{n-1}{2}} k^{2} \operatorname{sn} w^{n-}
$$


296 II, 1. Transformation $n^{\text {ten }}$ Grades $u$. allgemeine Transformationsgleichungen wo das Argument $w^{\prime}$ und der transformierte Integralmodul $l$ gegeben sind durch:

$$
w^{\prime}=n w \frac{\vartheta_{3}\left(q^{n}\right)^{2}}{\vartheta_{3}^{2}}, \quad \sqrt{l}=\frac{\vartheta_{2}\left(q^{n}\right)}{\vartheta_{3}\left(q^{n}\right)}
$$

Multipliziert man in (6) mit dem Nenner nach links herauf und entwickelt rechts und links nach Potenzen von $w$, so gelangt man durch Gleichsetzung der Koeffizienten gleicher Potenzen rechts und links zu Gleichungen, aus denen man mit Benutzung der schon bekannten Werte, $a_{0}, \frac{a_{n-1}}{2}$ die übrigen Koeffizienten bestimmen kann.

Arbeitet man bei diesen Rechnungen an Stelle der $\vartheta_{1}$-Funktion mit den $\boldsymbol{\vartheta}_{2}$ - und $\boldsymbol{\vartheta}_{3}$-Funktionen, so erscheint die Funktion sn $w$ durch en $w$ bzw. dn $w$ ersetzt. Man gelangt so zu zwei neuen Arten die Koeffizienten darzustellen und erhält durch Gleichsetzung der Ausdrücke, die man fürein und denselben Koeffizienten findet, Relationen zwischen den ursprïnglichen und den transformierten $\vartheta$-Nullwerten. ${ }^{1}$ ) Auf die algebraische Natur dieser $\vartheta$-Relationen wird am Schlusse des vorliegenden Bandes eingegangen.

Eine andere Art die Koeffizienten $a, b$ zu bestimmen benutzt die Nullpunkte der transformierten $\vartheta$-Funktionen. So verschwindet z. B. die Funktion $\vartheta_{1}\left(n v, q^{n}\right)$ für $v=\frac{1}{n}, \frac{2}{n}, \frac{3}{n}, \cdots, \frac{n-1}{n}$. Aus (3) entnimmt man demnach ein System homogener linearer Gleichungen:

$$
a_{0} \vartheta_{1}\left(\frac{\mu}{n}\right)^{n}+a_{1} \vartheta_{1}\left(\frac{\mu}{n}\right)^{n-2} \vartheta_{0}\left(\frac{\mu}{n}\right)^{2}+\cdots+\frac{a_{n-1}}{2} \vartheta_{1}\left(\frac{\mu}{n}\right) \vartheta_{0}\left(\frac{\mu}{n}\right)^{n-1}=0
$$

wo $\mu$ die Werte $1,2, \ldots, n-1$ durchläuft. Nachdem $a_{0}$ aus (4) bereits bekannt ist, könnte man versuchen, diese Gleichungen zur Berechnung der übrigen $a$ zu verwenden. Dieser Weg führt alsdann zu Relar tionen, in denen neben den ursprünglichen und den transformierten $\boldsymbol{\vartheta}$-Nullwerten auch „Teilwerte ${ }^{*}$ der $\boldsymbol{\vartheta}$-Funktionen auftreten.

Die beschriebenen Rechnungen sind übrigens nur für ganz niedereTransformationsgrade durchführbar. Das Hauptinteresse der Transformationstheorie hat sich denn auch wesentlich von den ,allgemeinen Transformationsgleichungen", zu denen z. B. auch die Gleichung (6) gehört, abgewandt und den in algebraischer und arithmetischer Hinsicht weit. interessanteren "speziellen Transformationsgleichungen" zugekehrt, denen die transformierten Modulfunktionen und Modulformen genügen. Zu diesen Gleichungen gehören auch die unten noch ausführlich zu betrachtenden Jacobischen Modular- und Multiplikatorgleichungen.

1) Relationen dieser Art haben sich in groBer Zahl im Nachlasse von GauB gefunden; vgl. die Fragmente IV und V in Ga u B' "Werken“, Bd. 3 S. $461 \mathrm{ff}$. In neuerer Zeit hat sich mit diesen Thetarelationen insbesondere M. Krause beschäftigt; siehe dessen "Theorie der doppeltperiodischen Funktionen einer veränderlichen GröBe (Leipzig 1895), Bd. 1 S. $177 \mathrm{ff}$. 


\section{Zweites Kapitel.}

\section{Systeme ganzer elliptischer Funktionen dritter Art $n^{\text {ter }}$ Stufe.}

Wendet man die Transformation $n^{\text {ten }}$ Grades auf die von den Perioden $\omega_{1}, \omega_{2}$ allein abhängenden Modulfunktionen und Modulformen an, so entstehen Größen, die mit den ursprünglichen Funktionen bzw. Formen wieder algebraisch zusammenhängen. Diese algebraischen Relationen nennen wir „spezielle Transformationsgleichungen“. Sie entsprechen den speziellen Teilungsgleichungen und sind, wie schon gelegentlich bemerkt wurde, im allgemeinen, d.h. von einigen besonderen Transformationsgraden abgesehen, die Resolventen niedersten Grades der speziellen Teilungsgleichungen. Wegen der beziehungsreichen und ausgedehnten Theorie dieser speziellen Transformationsgleichungen erscheint es zweckmäBig, die Betrachtung zunächst auf die Transformation der Funktionen erster Stufe $J(\omega), g_{2}\left(\omega_{1}, \omega_{2}\right), g_{3}\left(\omega_{1}, \omega_{2}\right)$ und $\Delta\left(\omega_{1}, \omega_{2}\right)$ einzuschränken; nur sollen zugleich auch die Wurzeln der Diskriminante $\Delta$, soweit sie eindeutige Modulformen liefern, zugelassen werden.

Bei den algebraischen Einzelausführungen über diese speziellen Transformationsgleichungen bedürfen wir noch der Kenntnis gewisser Systeme elliptischer Modulformen $n^{\text {ter }}$ Stufe, deren man sich bei allen tiefer greifenden Untersuchungen über Transformation der Funktionen erster Stufe mit Vorteil bedient. Es werden jene Systeme von Modulformen geliefert von gewissen Systemen ganzer elliptischer Funktionen $n^{\text {ter }}$ Stufe dritter Art, deren Theorie namentlich in gruppentheoretischer Hinsicht auch an sich bemerkenswert ist. Die Kenntnis dieser Funktionssysteme verdankt man der Abhandlung von Klein „Über die elliptischen Normalkurven der $n^{\text {ten }}$ Ordnung und zugehörige Modulfunktionen $n^{\text {ter }}$ Stufe" ${ }^{\text {"1 }}$ ) sowie derjenigen von A. Hurwitz „Über endliche Gruppen linearer Substitutionen, die in der Theorie der elliptischen Transzendenten auftreten“. ${ }^{2}$ ) Eine ausführliche Besprechung der fraglichen Funktionssysteme findet man in „Modulfunktionen“ Bd. 2, S. 236 ff. Die Darstellung des vorliegenden Kapitels beschränkt sich auf das für die weitere Entwicklung unbedingt Notwendige.

\section{$\S 1$. Teilwerte und Wurzeln der Diskriminante $\Delta$.}

Um die späteren Entwicklungen nicht zu unterbrechen, werden zunächst einige Beziehungen zwischen den Teilwerten und den Wurzeln der

1) Abhandl. der Sächs. Gesellsch. d. Wissensch. zu Leipzig, Bd. 18 (1885).

2) Math. Ann., Bd. 27 (1885). 
298 II, 2. Systeme ganzer elliptischer Funktionen dritter Art $n^{\text {ter }}$ Stufe Diskriminante $\Delta$ aufgestellt. Aus (9) in I, 268 ergibt sich die Produktentwicklung:

$$
\sigma_{0 \mu}^{(n)}=-\frac{\omega_{2}}{\pi} \sin \frac{\mu \pi}{n} \prod_{\nu=1}^{\infty} \frac{\left(1-\varepsilon^{\mu} q^{2 v}\right)\left(1-\varepsilon^{-\mu} q^{2 v}\right)}{\left(1-q^{2 v}\right)^{2}}
$$

wo der Teilungsgrad $n$ der Deutlichkeit halber als oberer Index an der Bezeichnung $\sigma$ angebracht ist. Man nehme $n$ ungerade, setze der Reihe nach $\mu=1,2,3, \ldots, \frac{n-1}{2}$ und bilde das Produkt der entstehenden Gleichungen (1), wobei sich das Produkt der Sinus zu $2^{-\frac{n-1}{2}} \sqrt{n} \mathrm{zu}-$ sammenfaßt:

$$
\prod_{\mu=1}^{\frac{n-1}{2}} \sigma_{0 \mu}^{(n)}=(-1)^{\frac{n-1}{2}}\left(\frac{\omega_{2}}{2 \pi}\right)^{\frac{n-1}{2}} \sqrt{n} \prod_{\nu=1}^{\infty} \frac{1-q^{2 n v}}{\left(1-q^{2 v}\right)^{n}} .
$$

Schreiben wir die transformierten Perioden in der Gestalt:

$$
\omega_{1}^{\prime}=\omega_{1}, \quad \omega_{2}^{\prime}=\frac{\omega_{2}}{n}
$$

und nennen $\Delta^{\prime}$ die zugehörige Diskriminante $\Delta\left(\omega_{1}^{\prime}, \omega_{2}^{\prime}\right)$, so ergibt sich aus der Produktentwicklung (6) in I, 313 der Diskriminante:

$$
\sqrt[24]{\frac{\Delta^{\prime}}{\Delta^{n}}}=\sqrt{n}\left(\frac{\omega_{2}}{2 \pi}\right)^{\frac{n-1}{2}} \prod_{\nu=1}^{\infty} \frac{1-q^{2 n v}}{\left(1-q^{2 v)^{n}}\right.} .
$$

Für jedes ungerade $n$ besteht also zwischen der Diskriminante und den б-Teilwerten die Beziehung:

$$
\sqrt[24]{\frac{\Delta^{\prime}}{\Delta^{n}}}=(-1)^{\frac{n-1}{2}} \prod_{\mu=1}^{\frac{n-1}{2}} \sigma_{0 \mu}^{(n)}
$$

Die $\wp^{\prime}$-Funktion läßt sich durch die $\sigma$-Funktion in der Gestalt:

$$
\wp^{\prime}(u)=-\frac{\sigma(2 u)}{\sigma(u)^{4}}
$$

darstellen. Für den Teilwert $\wp_{0 \mu}^{\prime}$ folgt hieraus:

$$
\wp_{0 \mu}^{\prime}=-\frac{\sigma\left(\frac{2 \mu \omega_{2}}{n}\right)}{\sigma\left(\frac{\mu \omega_{q}}{n}\right)^{4}}=\frac{\sigma_{0,2 \mu}^{(n)}}{\left(\sigma_{0 \mu}^{(n)}\right)^{4}} .
$$

Man setze $\mu=1,2, \ldots, \frac{n-1}{2}$ und bilde das Produkt:

$$
\prod_{\mu=1}^{\frac{n-1}{2}} \wp_{0 \mu}^{\prime}=\prod_{\mu=1}^{\frac{n-1}{2}} \frac{\sigma_{0,2 \mu}^{(n)}}{\left(\sigma_{0 \mu}^{(n)}\right)^{4}}
$$


Beziehungen zwischen der transformierten Diskriminante und den Teilwerten 299 Aus den Formeln (4) und (6) in I, 451 leitet man nun leicht die Regel $\sigma_{0, n-\mu}^{(n)}=\sigma_{0 \mu}^{(n)}$ ab. Die letzten Faktoren im Zähler des eben angegebenen Produktes $\sigma_{0, n-1} ; \sigma_{0, n-3}, \ldots$ kann man demnach durch $\sigma_{01}, \sigma_{03}, \ldots$ ersetzen und findet so:

$$
\prod_{\mu=1}^{\frac{n-1}{2}} \wp_{0 \mu}^{\prime}=\left(\prod_{\mu=1}^{\frac{n-1}{2}} \frac{1}{\sigma_{0 \mu}^{(n)}}\right)^{3}
$$

Aus (3) folgt also der weitere Satz: Für jedes ungerade $n$ besteht zwischen der Diskriminante und den $\wp^{\prime}$-Teilwerten die Beziehung:

$$
\sqrt[8]{\frac{\Delta^{\prime}}{\Delta^{n}}}=\frac{(-1)^{\frac{n-1}{2}}}{\prod_{\mu=1}^{\frac{n-1}{2}} \wp_{0 \mu}^{\prime}}
$$

Endlich leitet man aus der Gleichung (14) in I, 217 leicht:

$$
\wp_{0,2 \mu}-\wp_{0 \mu}=-\frac{\sigma_{0,3 \mu}^{(n)}}{\sigma_{0 \mu}^{(n)} \cdot\left(\sigma_{0,2 \mu}^{(n)}\right)^{2}}
$$

ab. Es sei nun die ungerade Zahl $n$ teilerfremd gegen 3. Lassen wir $\mu$ wieder die Zahlen $1,2,3, \ldots, \frac{n-1}{2}$ durchlaufen, so treten im Zähler die zweiten Indizes auf:

$$
3,6,9, \cdots, \quad \frac{3 n-9}{2}, \quad \frac{3 n-3}{2} .
$$

Reduziert man diese Indizes $\bmod n$ auf ihre absolut kleinsten Reste, d.h. auf Zahlen der Reihe $-\frac{n-1}{2},-\frac{n-3}{2}, \cdots,+\frac{n-1}{2}$, so werden keine zwei gleich oder entgegengesetzt, wie leicht festgestellt wird. Der einzelne б-Teilwert erfährt hierbei höchstens einen Zeichenwechsel. Lassen wir ein sich hier einstellendes, jedoch weiterhin nicht zur Benutzung kommendes Vorzeichen der Kürze halber unbestimmt, so ergibt sich aus (3): Für jeden ungeraden gegen 3 teilerfremden Grad $n$ besteht zwischen der Diskriminante $\Delta$ und den $\wp$-Teilwerten die Beziehung:

$$
\sqrt[12]{\frac{\Delta^{\prime}}{\Delta^{n}}}=\frac{: \pm 1}{\prod_{\mu=1}^{\frac{n-1}{2}}\left(\wp_{0,2 \mu}-\wp_{0 \mu}\right)}
$$

Für ein gerades $n$ bilden wir aus (1) das Produkt:

$$
\prod_{\mu=1}^{n-1} \sigma_{0 \mu}^{(n)}=\sigma_{01}^{(2)} \prod_{\mu=1}^{\frac{n-2}{2}}\left(\sigma_{0 \mu}^{(n)}\right)^{2}=-\left(\frac{\omega_{2}}{\pi}\right)^{n-1} \prod_{\mu=1}^{n-1} \sin \frac{\mu \pi}{n} \prod_{\nu=1}^{\infty} \frac{\left(1-q^{2 n v}\right)^{2}}{\left(1-q^{2 v}\right)^{2 n}}
$$


Das rechts stehende unendliche Produkt ist wieder in einfacher Weise durch die Diskriminante darstellbar: Für jedes gerade $\boldsymbol{n}$ besteht zwischen der Diskriminante und den б-Teilwerten die Beziehung:

$$
\sqrt[12]{\frac{\Delta^{\prime}}{\Delta^{n}}}=-\sigma_{01}^{(2)}\left(\prod_{\mu=1}^{\frac{n-2}{2}} \sigma_{0 \mu}^{(n)}\right)^{2}
$$

Hieraus folgt weiter:

$$
\sqrt[12]{\Delta^{\prime} \Delta}=-(\sqrt[12]{\Delta})^{n}\left(\sqrt[12]{\Delta} \sigma_{01}^{(2)}\right)\left(\prod_{\mu=1}^{\frac{n-2}{3}} \sigma_{0 \mu}^{(n)}\right)^{2}
$$

Nun ergibt sich aus einer in I, 452 aufgestellten Gleichung:

$$
\sqrt[12]{\Delta} \sigma_{01}^{(2)}=-2 q^{\frac{1}{6}} \prod_{\nu=1}^{\infty}\left(1+q^{2 v}\right)^{2}
$$

woraus man durch Vergleich mit der ersten Gleichung (14) in I, 456 die Folgerung zieht:

$$
\sqrt[12]{\Delta} \sigma_{01}^{(2)}=-e^{\frac{5 \pi i}{4}}\left(\sigma_{01}^{(4)} \sigma_{21}^{(4)} \sqrt[6]{\Delta}\right)^{2}
$$

Trägt man diesen Ausdruck für die zweite Klammer auf der rechten Seite von (7) ein, so zeigt sich, daB diese rechte Seite das Quadrat einer eindeutigen Modulform darstellt. Man kann also nochmals die Quadratwurzel ziehen ${ }^{1}$ ) und findet: Für gerades $n$ besteht zwischen der Diskriminante und den $\sigma-T e i l w e r t e n$ die Beziehung:

$$
\sqrt[24]{\Delta^{\prime} \Delta}= \pm e^{\frac{5 \pi i}{8}}(\sqrt[12]{\Delta})^{\frac{n}{2}}\left(\sigma_{01}^{(4} \sigma_{21}^{(4)} \sqrt[6]{\Delta}\right) \prod_{\mu=1}^{\frac{n-8}{2}} \sigma_{0 \mu}^{(n)}
$$

Die Bestimmung des rechts zutreffenden Vorzeichens möge der Kürze halber wieder übergangen werden.

Die transformierte Diskriminante $\Delta^{\prime}=\Delta\left(\omega_{1}, \frac{\omega_{2}}{n}\right)$ wird durch die Substitutionen der durch $\gamma \equiv 0(\bmod n)$ erklärten Kongruenzgruppe $\Gamma_{\psi(n)}$ der $n^{\text {ten }}$ Stufe in sich übergeführt. Bei diesen Substitutionen bleibt also sowohl die Form (3) als die Form (8) bis auf eine multiplikative $24^{\text {ste }}$ Einheitswurzel unverändert. Die bei der einzelnen dieser Formen wirklich auftretenden Einheitswurzeln reproduzieren sich ihrerseits gegenüber Multiplikation irgend zweier unter ihnen; es handelt sich also um die $t$ Einheitswurzeln eines Grades $t$, der ein bestimmter Teiler von 24 ist.

Zerlegen wir nun die Substitutionen der $\Gamma_{\psi(n)}$ in $t$ Klassen, indem wir in die einzelne Klasse alle Substitutionen hineintun, die die Form (3) bzw. (8) mit dem gleichen Faktor versehen, so ist leicht einzusehen,

1) Ohne das Gebiet der eindeutigen Modulformen zu verlassen (vgl. I, 450). 
Beziehungen zwischen der transformierten Diskriminante und den Teilwerten 301 daB wir die $t$ Nebengruppen einer in der $\Gamma_{\psi(n)}$ ausgezeichneten Untergruppe $\Gamma_{t \psi(n)}$ vor uns haben, deren zugehörige Quotientengruppe eine zyklische $G_{t}$ ist. Diese ausgezeichnete $\Gamma_{t \psi(n)}$ besteht dann aus allen Substitutionen, bei denen die fragliche Modulform unverändert blent.

In dem weiterhin zu bevorzugenden Falle eines ungeraden $n$ müssen wir die Gruppe $\Gamma_{t \psi(n)}$ noch näher bestimmen. Wir betrachten zunächst die Modulform (5) und stellen fest, daB $\left(\wp_{0,2 \mu}-\wp_{0 \mu}\right)$ durch eine Substitution $\left(\begin{array}{l}\alpha, \beta \\ \gamma, \delta\end{array}\right)$ mit $\gamma \equiv 0(\bmod n)$ in $\left(\wp_{0,2 \mu \delta}-\wp_{0, \mu \delta}\right)$ übergeführt wird. Nun ist $\delta$ teilerfremd gegen $\gamma$ und also gegen $n$. Die $\frac{n-1}{2}$ Zahlen $\delta, 2 \delta, 3 \delta, \ldots, \frac{n-1}{2} \delta$ darf man ohne Änderung der $\wp$-Teilwerte $\bmod n$ auf ihre absolut kleinsten Reste reduzieren. Von diesen Resten sind dän, eben weil $\delta$ teilerfremd gegen $n$ ist, keine zwei gleich oder entgegengesetzt. Da unsere $\wp$-Teilwerte aber auch bei Zeichenwechsel der Indizes unverändert bleiben, so bleibt das in (5) rechts stehende Produkt überhaupt unverändert: Im Falle eines ungeraden gegen 3 teilerfremden $n$ ist die in (5) dargestellte zwölfte Wurzel des Diskriminantenquotienten eine unmittelbar zur $\Gamma_{\psi(n)}$ gehörende Modulform $n^{\text {ter Stufe. }}$

Etwas umständlicher ist die Feststellung des Verhaltens, welches das in (4) rechts auftretende Produkt:

$$
\wp_{01}^{\prime} \wp_{02}^{\prime} \wp_{03}^{\prime} \cdots \wp_{0, \frac{n-1}{2}}^{\prime}
$$

gegenüber einer Substitution mit $\gamma \equiv 0(\bmod n)$ annimmt. Da

$$
\wp_{0,-\mu}^{\prime}=-\wp_{0 \mu}^{\prime}
$$

gilt, so bleibt das Produkt unverändert oder es erleidet Zeichenwechsel, je nachdem bei Reduktion der $\frac{n-1}{2}$ Zahlen $\delta, 2 \delta, 3 \delta, \ldots, \frac{n-1}{2} \delta$ auf ihre absolut kleinsten Reste $\bmod n$ die Anzahl der hierbei auftretenden negativen Reste gerade oder ungerade ist.

Um diese Anzahl und damit das fragliche Vorzeichen festzustellen, bemerken wir, dab nur für eine Primzahl $n$ alle $\frac{n-1}{2}$ Faktoren (9) „eigentlich" zum $n^{\text {ton }}$ Teilungsgrade gehören. Ist $n$ zusammengesetzt, so gehören nur $\frac{1}{2} \varphi(n)$ Faktoren (9) eigentlich zum Grade $n$, nämlich diejenigen $\wp_{0 \mu}^{\prime}$, bei denen $\mu$ teilerfremd gegen $n$ ist. Ist aber $\tau$ irgendein Teiler von $n$, so treten in (9) auch die zum Teilungsgrade $\tau$ gehörenden Teilwerte $\wp_{01}^{\prime}, \wp_{02}^{\prime}, \ldots$, $\wp_{0, \frac{\tau-1}{2}}^{\prime}$ auf, und zwar $\frac{1}{2} \varphi(\tau)$ unter ihnen als ,eigentlich" zum Grade $\tau$ gehörig. Wir erschöpfen aber das ganze Produkt (9) gerade genau, indem wir $\tau$ alle Teiler von $n$ durchlaufen lassen; unter EinschluB von $n$ und 
302 II, 2. Systeme ganzer elliptischer Funktionen dritter Art $n^{\text {tor }}$ Stufe

Ausschluß von 1, und für jedes $\tau$ die eigentlich zu diesem Grade gehörenden $\frac{1}{2} \varphi(\tau)$ Teilwerte $\wp_{01}^{\prime}, \wp_{02}^{\prime}, \ldots, \wp_{0, \frac{\tau-1}{2}}^{\prime}$ zulassen.

Bei einer Substitution mit $\gamma \equiv 0(\bmod n)$ geht nun jedes dieser Produkte zu je $\frac{1}{2} \varphi(\tau)$ Faktoren in sich über oder erleidet nur einen Zeichenwechsel. Das von uns gesuchte Vorzeichen aber ist einfach das Produkt aller den einzelnen $\tau$ entsprechenden Vorzeichen. Um beim einzelnen $\tau$ dieses Vorzeichen zu bestimmen, reduzieren wir die $\frac{1}{2} \varphi(\tau)$ Produkte $\delta \mu$ mit gegen $\tau$ teilerfremden Zahlen $\mu$ der Reihe $1,2, \ldots, \frac{\tau-1}{2} \bmod \tau$ auf ihre absolut kleinsten Reste und sehen nach, ob unter diesen Resten eine gerade oder ungerade Anzahl negativer vorkommt. Da nach Zeichenwechsel der negativen Reste wieder die $\frac{1}{2} \varphi(\tau)$ anfänglichen Zahlen $\mu$ vorliegen, so gilt für das Produkt der $\frac{1}{2} \varphi(\tau)$ Zahlen $\delta \mu$ die Kongruenz:

$$
\delta^{\frac{1}{2} \varphi(\tau)} \Pi \mu \equiv \pm \Pi \mu,(\bmod \tau),
$$

wo rechts das obere oder untere Zeichen gilt, je nachdem die Anzahl der negativen unter jenen absolut kleinsten Resten gerade oder ungerade ist. Damit haben wir aber das Vorzeichen erreicht, welches wir für das auf $\tau$ bezogene Teilprodukt der $\wp^{\prime}$-Teilwerte suchen. Indem wir noch durch die gegen $\tau$ teilerfremde Zahl $\Pi \mu$ heben, findet sich für das Vorzeichen:

$$
\delta^{\frac{1}{2} \varphi(\tau)} \equiv \pm 1 \quad(\bmod \tau) .
$$

Um diese Kongruenz zu verwerten, stellen wir den Satz auf: Ist die ungerade Zahl $m$ das Produkt $m=m_{1} \cdot m_{2}$ zweier von 1 verschiedener teilerfremder Zahlen $m_{1}, m_{2}$, so ist stets:

$$
\delta^{\frac{1}{2} \varphi(m)} \equiv 1 \quad(\bmod m),
$$

falls $\delta$ teilerfremd gegen $m$ ist. Nach dem verallgemeinerten Fermatschen Satze $\left.^{1}\right)$ gilt nämlich: $\quad \delta^{\varphi\left(m_{1}\right)} \equiv 1 .\left(\bmod m_{1}\right)$.

Da aber $\varphi(m)=\varphi\left(m_{1}\right) \cdot \varphi\left(m_{2}\right)$ gilt und $\varphi\left(m_{2}\right)$ eine gerade Zahl ist, so folgt:

$$
\delta^{\frac{1}{2} \varphi(m)}=\left(\delta^{\varphi\left(m_{1}\right)}\right)^{\frac{1}{2} \varphi\left(m_{2}\right)} \doteq 1 \quad\left(\bmod m_{1}\right) .
$$

$\mathrm{Da}$ die gleiche Kongruenz auch für den Modul $m_{2}$ gewonnen wird, so ist der Satz (11) damit bewiesen.

Für die Potenz $p^{v}$ einer ungeraden Primzahl $p$ gilt $\varphi\left(p^{\nu}\right)=p^{\nu-1} \varphi(p)$, so dab man findet:

$$
\delta^{\frac{1}{2} \varphi\left(p^{\nu}\right)}=\left(\delta^{\frac{1}{2} \varphi(p)}\right) p^{p^{\nu}-1} \equiv \pm 1 \quad\left(\bmod p^{\nu}\right) .
$$

1) Vgl. Dirichlet-Dedekind, „Vorles. über Zahlentheorie", 4. Aufl., S. 38. 
Bestimmung eines Vorzeichens durch das Symbol $\left(\frac{\delta}{n}\right)$

Das hier gesuchte Vorzeichen ist dasselbe, wie dasjenige der Kongruenz:

$$
\left.\left(\delta^{\frac{1}{2} \varphi(p)}\right)\right)^{p^{\nu-1}} \equiv \pm 1 \quad(\bmod p),
$$

wofür wir, da die in der großen Klammer stehende Zahl $\bmod p \operatorname{mit} \pm 1$ kongruent ist und $p^{v-1}$ eine ungerade Zahl bedeutet:

$$
\delta^{\frac{1}{2} \varphi(p)}=\delta^{\frac{p-1}{2}} \equiv \pm 1 \quad(\bmod p)
$$

schreiben können. Das hier gesuchte Vorzeichen ist also einfach das Legendresche Zeichen $\left(\frac{\delta}{p}\right)$ aus der Theorie der quadratischen Reste. $\left.{ }^{1}\right)$

Zufolge (11) brauchen wir von den Teilprodukten des Produktes (9) nur die zuzulassen, welche sich auf Teiler $\tau$ der Gestalt $p^{v}$ beziehen, wo dann jedesmal das gesuchte Vorzeichen $\left(\frac{\delta}{p}\right)$ ist. Ist aber $p^{v}$ eine höchste in $n$, aufgehende Primzahl, so haben wir $\tau$ der Reihe nach gleich $p^{v}$, $p^{\nu-1}, \ldots, p^{2}, p$ zu setzen und also die eben genannte Regel $\nu$ Male anzuwenden. Hieraus ergibt sich das Vorzeichen des Produktes (9) als Produkt der Faktoren $\left(\frac{\delta}{p}\right)^{v}$ bezogen auf alle Primteiler von $n$. Damit aber sind wir zum Legendre-Jacobischen Zeichen ${ }^{2}$ ) geführt und gewinnen den Satz: Die für ein ungerades $n$ in (4) dargestellte achte Wurzel des Diskriminantenquotienten wird durch Ausübung einer Substitution mit $\gamma \equiv 0(\bmod n)$ in:

$$
\left(\frac{\delta}{n}\right) \sqrt[8]{\frac{\Delta^{\prime}}{\Delta^{n}}}
$$

transformiert, wo $\left(\frac{\delta}{n}\right)$ das Legendre-Jacobische. Zeichen ist.

Da man die $24^{\text {ste }}$ Wurzel (3) als Quotienten der beiden Formen (4) und (5) darstellen kann, so folgt aus den über diese letzteren Formen aufgestellten Sätzen: Für die ungeraden, gegen 3 teilerfremden $n$ nimmt die in (3) dargestellte 24te Wurzel des Diskriminantenquotienten bei Ausübung einer Substitution mit $\gamma \equiv 0$ gleichfalls den Faktor $\left(\frac{\delta}{n}\right)$ an. Weitere Sätze über diesen Faktor folgen später.

\section{$\S 2$. Einführung der ganzen elliptischen Funktionen dritter Art $n^{\text {ter }}$ Ordnung $\bar{X}_{\lambda}\left(u \mid \omega_{1}, \omega_{2}\right)$.}

Unter $\omega_{1}^{\prime}, \omega_{2}^{\prime}$ verstehen wir auch weiterhin die transformierten Perioden (2) S. 289 und bezeichnen die zugehörigen Perioden des Normalintegrals zweiter Gattung durch:

$$
\eta_{i}^{\prime}=\eta_{i}\left(\omega_{1}, \frac{\omega_{2}}{n}\right)=\eta_{i}\left(\omega_{1}^{\prime}, \omega_{2}^{\prime}\right)
$$

1) Vgl. Dirichlet-Dedekind, a. a. O., S. 104.

2) Vgl. Dirichlet-Dedekind, a. a. O., S. 78. 
304 II, 2. Systeme ganzer elliptischer Funktionen dritter Art $n^{\text {ter }}$ Stufe

Die entsprechend der Gleichung (5) S. 285 gebildete Größe:

$$
G_{1}\left(\omega_{1}, \omega_{2}\right)=\frac{\eta_{1}^{\prime}}{2 \omega_{1}^{\prime}}-\frac{n \eta_{1}}{2 \omega_{1}}=\frac{\eta_{2}^{\prime}}{2 \omega_{2}^{\prime}}-\frac{n \eta_{2}}{2 \omega_{2}}
$$

ist eine Modulform $n^{\text {ter }}$ Stufe, die durch die $\wp$-Teilwerte in der Gestalt:

$$
G_{1}\left(\omega_{1}, \omega_{2}\right)=\frac{1}{2} \sum_{\mu=1}^{n-1} \wp_{0 \mu}
$$

darstellbar ist. Diese Gleichungen gehen aus (5) und (7) S. $285 \mathrm{ff}$. einfach durch die mit $T$ bezeichnete Substitution $\left(\begin{array}{c}0,1 \\ 1,0\end{array}\right)$ der homogenen Modulgruppe $\Gamma^{(\omega)}$ hervor, wobei in Betracht zu ziehen ist, daB die Perioden des Integrals zweiter Gattung sich zu den $\omega_{1}, \omega_{2}$ "kogredient" substituieren.

Ist nun zunächst $n$ eine beliebige ungerade Zahl, so bilden wir für die transformierten Perioden die Funktion $\sigma_{\lambda, 0}^{(n)}\left(u \mid \omega_{1}^{\prime}, \omega_{2}^{\prime}\right)$ und behaupten, daB sie den Charakter einer Modulform $n^{\text {ter }}$ Stufe hat, d.h. dab sie bei Ausübung einer Substitution $\left(\begin{array}{l}\alpha, \beta \\ \gamma, \delta\end{array}\right)$ der Hauptkongruenzgruppe $n^{\text {ter }}$ Stufe unverändert bleibt. Für die transformierten Perioden rechnet sich die Substitution auf $\left(\begin{array}{c}\alpha, n \beta \\ n^{-1} \gamma, \delta\end{array}\right)$ um, bei der die fragliche G-Funktion nach (3) in I, 451 übergeht in:

$$
\sigma_{\alpha \lambda, n \beta \lambda}^{(n)}\left(u \mid \omega_{1}^{\prime}, \omega_{2}^{\prime}\right)=\sigma_{\lambda+\frac{\alpha-1}{n} \lambda \cdot n, \beta \lambda \cdot n}^{(n)}\left(u \mid \omega_{1}^{\prime}, \omega_{2}^{\prime}\right) .
$$

Die rechts stehende Funktion ist aber nach (4) in I, 451 wieder mit $\sigma_{\lambda, 0}^{(n)}\left(u \mid \omega_{1}^{\prime}, \omega_{2}^{\prime}\right)$ identisch. Man hat bei der erforderlichen Rechnung nur zu berücksichtigen, daß $n$ ungerade sein sollte, und da $B$ bei geradem $\beta$ auch $(\alpha-1)$ gerade sein muB. Wir fügen noch einen mit $G_{1}$ aufgebauten Exponentialfaktor hinzu und haben auch in:

$$
e^{-G_{1} u^{2}} \sigma_{\lambda, 0}^{(n)}\left(u \mid \omega_{1}^{\prime}, \omega_{2}^{\prime}\right)=e^{-G_{1} u^{2}} \sigma_{\lambda, 0}^{(n)}\left(u \mid \omega_{1}, \frac{\omega_{z}}{n}\right)
$$

einen Ausdruck, der den Charakter einer Modulform $n^{\text {ter }}$ Stufe hat.

Im Falle eines geraden $n$ reihen wir an (3) den Ausdruck an:

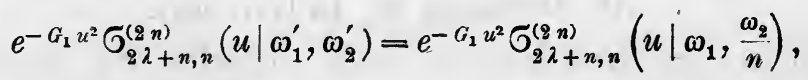

wo $G_{1}$ in der bisherigen Bedeutung gebraucht ist. Als Funktion der Perioden $\omega_{1}, \omega_{2}$ hat auch dieser Ausdruck die Eigenschaft der Invarianz gegenüber den Substitutionen einer gewissen Kongruenzgruppe. Jedoch ist es nicht erforderlich, die Stufe genauer festzustellen.

Die Ausdrücke (3) und (4) gewinnen nun, wie der Erfolg zeigen wird, gruppentheoretisch ein besonders einfaches Verhalten, wenn wir sie 
noch mit $\sqrt[8]{\Delta^{\prime} \Delta}$ multiplizieren, $\Delta^{\prime}$ im Sinne von $\S 1$ gebraucht. Nach (4) S. 299 ist bei ungeradem $n$ :

$$
\sqrt[8]{\frac{\Delta^{\prime}}{\Delta^{n}}}=\sqrt[8]{\Delta^{\prime} \Delta}(\sqrt[4]{\Delta})^{-\frac{n+1}{2}}
$$

unmittelbar eine Modulform $n^{\text {ter }}$ Stufe. Wir wollen demnach bei ungeradem $n$ genauer den Faktor $\sqrt[8]{\Delta^{\prime} \Delta}(\sqrt[4]{\Delta})^{n_{0}}$ hinzuzusetzen, wo $n_{0}$ die kleinste, nicht-negative, der Kongruenz:

$$
n_{0} \equiv-\frac{n+1}{2}(\bmod 4)
$$

genügende ganze Zahl ist. In dem für uns wichtigeren Falle eines ungeraden $n$ haben wir dann auch nach Zusatz des genannten Faktors Ausdrücke mit dem Charakter der Modulformen $n^{\text {ter }}$ Stufe vor uns. $\left.{ }^{1}\right)$ Wir fügen unseren Ausdrücken außerdem noch numerische Faktoren hinzu, die den Zweck haben, die durchzuführenden Rechnungen zu vereinfachen. Als abkürzende Bezeichnung benutzen wir $X_{\lambda}\left(u \mid \omega_{1}, \omega_{2}\right)$, und zwar setzen wir für ungerades $n$ :

$$
X_{\lambda}\left(u \mid \omega_{1}, \omega_{2}\right)=\frac{(-1)^{\lambda}}{\sqrt{n}} \sqrt[8]{\Delta^{\prime} \Delta}(\sqrt[4]{\Delta})^{n_{0}} e^{-G_{1} u^{2}} \sigma_{\lambda, 0}^{(n)}\left(u \mid \omega_{1}, \frac{\omega_{2}}{n}\right),
$$

während für gerades $n$ die Erklärung gelten soll:

$$
X_{\lambda}\left(u \mid \omega_{1}, \omega_{2}\right)=e^{-\frac{\pi i \lambda}{2 n}+\frac{3 \pi i}{4}} \frac{1}{\sqrt{n}} \sqrt[8]{\Delta^{\prime} \Delta} e^{-G_{1} u^{2}} \sigma_{2 \lambda+n, n}^{(2 n)}\left(u \mid \omega_{1}, \frac{\omega_{2}}{n}\right) .
$$

Wächst $\lambda$ um $n$, so erfährt zufolge (4) in I, 451 der 6-Faktor in (7) Zeichenwechsel, während der in (8) den Faktor $i$ annimmt. Es ist die Folge der aufgenommenen numerischen Faktoren, daß die Gesamtausdrücke in (7) und (8) rechts bei Vermehrung von $\lambda$ um $n$ in sich selbst übergehen:

$$
X_{\lambda+n}\left(u \mid \omega_{1}, \omega_{2}\right)=X_{\lambda}\left(u \mid \omega_{1}, \omega_{2}\right) .
$$

Wir haben deshalb in jedem Falle im ganzen höchstens $n$ verschiedene Funktionen $X_{0}, X_{1}, X_{2}, \ldots, X_{n-1}$.

Man setze für $G_{1}$ den zweiten Ausdruck (1) ein und rechne die б-Funktionen in (7) und (8) auf die $\vartheta$-Funktionen, gebildet für die transformierten Perioden, um. Im Falle eines ungeraden $n$ gelangt man zur $\vartheta_{1}$-Funktion auf Grund von (9) in I, 416; bei geradem $n$ führt die Tabelle in I, 419 zur $\vartheta_{3}$-Funktion. Die Rechnung, deren Einzelheiten wir übergehen, ergibt:

1) In der S. 297 genannten Abhandlung von Klein wird die Form (5) unmittelbar als Faktor benutzt. Indem wir negative Potenzen der Diskriminante grundsätzlich meiden, werden die später za erklärenden Modulformen $n^{\text {ter Stufe }}$ unmittelbar ganze Modulformen sein. 
306 II, 2. Systeme ganzer elliptischer Funktionen dritter Art $n^{\text {ter }}$ Stufe

(10) $X_{\lambda}=(-1)^{\lambda} \sqrt{\frac{2 \pi}{\omega_{2}}} \sqrt[8]{\Delta}(\sqrt[4]{\Delta})^{n_{0}} e^{\frac{n \eta_{2}}{2 \omega_{2}} u^{2}} e^{-\frac{2 i \pi u}{\omega_{2}} \lambda} q^{\frac{\lambda^{2}}{n}} \vartheta_{1}\left(\frac{n u-\lambda \omega_{1}}{\omega_{2}}, q^{n}\right)$,

bezogen auf ungerades $n$, während sich bei geradem $n$ anreiht:

$$
X_{\lambda}=\sqrt{\frac{2 \pi}{\omega_{2}}} \sqrt[8]{\Delta} e^{\frac{n \eta_{2}}{2 \omega_{2}} u^{2}} e^{-\frac{2 i \pi u}{\omega_{2}} \lambda} q^{\frac{\lambda^{2}}{n}} \vartheta_{3}\left(\frac{n u-\lambda \omega_{1}}{\omega_{z}}, q^{n}\right) .
$$

Setzt man schließlich die Thetareihen (16) in I, 418 ein, so ergeben sich Reibendarstellungen der Funktionen $X_{\lambda}\left(u \mid \omega_{1}, \omega_{2}\right)$, nämlich im Falle eines ungeraden $n$ :

$$
\begin{gathered}
X_{\lambda}\left(u \mid \omega_{1}, \omega_{2}\right)=\frac{(-1)^{\lambda}}{i} \sqrt{\frac{2 \pi}{\omega_{2}}} \sqrt[8]{\Delta}(\sqrt[4]{\Delta})^{n_{0}} \\
\cdot e^{\frac{n \eta_{2}}{2 \omega_{2}} u^{2}} \sum_{v=-\infty}^{+\infty}(-1)^{v} q^{\frac{((2 v+1) n-2 \lambda)^{2}}{4 n}} e^{\frac{\pi i u}{\omega_{2}}((2 v+1) n-2 \lambda)}
\end{gathered}
$$

während sich für gerades $n$ einfindet:

$$
X_{\lambda}\left(u \mid \omega_{1}, \omega_{2}\right)=\sqrt{\frac{2 \pi}{\omega_{2}}} \sqrt[8]{\Delta} e^{\frac{n \eta_{2}}{2 \omega_{2}}} u^{2} \sum_{\nu=-\infty}^{+\infty} q^{\frac{(\nu n-\lambda)^{2}}{n}} e^{\frac{2 \pi i u}{\omega_{2}}(\nu n-\lambda)} .
$$

Im Parallelogramme der transformierten Perioden ist $X_{\lambda}$ polfrei und hat nur einen Nullpunkt erster Ordnung, der bei

$$
u=\frac{\lambda \omega_{1}^{\prime}}{n} \quad \text { bzw. } \quad u=\frac{\lambda \omega_{1}^{\prime}}{n}+\frac{1}{2} \omega_{1}^{\prime}+\frac{1}{2} \omega_{2}^{\prime}
$$

liegt, je nachdem $n$ ungerade oder gerade ist. Zur Feststellung des Verhaltens der $X_{\lambda}$ bei der Vermehrung von $u$ um die Perioden $\omega_{1}=\omega_{1}^{\prime}$ und $\omega_{2}=n \omega_{2}^{\prime}$ ziehe man die Gleichung (2) in I, 451 heran und benutze die Darstellungen (7) und (8), in denen sich die G-Funktionen auf die Perioden $\omega_{1}^{\prime}$, $\omega_{2}^{\prime}$ beziehen. Die Rechnung liefert das einfache Verhalten:

$$
X_{\lambda}\left(u+\omega_{i}\right)=(-1)^{n} e^{n \eta_{i}\left(u+\frac{1}{2} \omega_{i}\right)} X_{\lambda}(u),
$$

$i=1,2$.

Wir notieren unter. Zusammenfassung der Ergebnisse den Satz: Die $X_{\lambda}\left(u \mid \omega_{1}, \omega_{2}\right)$ stellen für jedes $n$ ein System von $n$ gleichändrigen ganzen

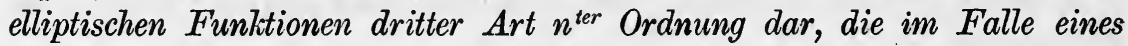
ungeraden $n$ den Charakter von Modulformen $n^{\text {ter }}$ Stufe haben; ${ }^{1}$ ) die $n$ einfachen Nullpunkte im Parallelogramme der Perioden $\omega_{1}, \omega_{2}$ liegen fïr ungerades $n$ bei:

$$
u=\frac{\lambda \omega_{1}+\mu \omega_{2}}{n}
$$

$\mu=0,1,2, \ldots, n-1$

im Falle eines geraden $n$ aber bei:

$$
u=\frac{(2 \lambda+n) \omega_{1}+(2 \mu+1) \omega_{2}}{2 n},
$$

$\mu=0,1,2, \ldots, n-1$.

1) Nach „Modulfunktionen", Bd. 2, S. 289 liegt bei geradem $n$ die Stufe $2 n$ vor, falls $n$ durch 4 teilbar ist, die Stufe $4 n$ dagegen, falls $n$ das Doppelto einer ungeraden Zahl ist. 
Ändert man $u$ um $\frac{\omega_{1}}{n}$ oder $\frac{\omega_{2}}{n}$, so zeigen die $X_{\lambda}$ ein einfaches Verhalten, das man z. B. aus den Formeln (10) und (11) mit Benutzung der Grundformeln der $\vartheta$-Funktionen von I, 419 und der Legendreschen Relation leicht feststellen kann. Die Rechnung, deren Einzelheiten wir wieder übergehen, führt zu dem Satze: Die Funktionen $X_{\lambda}(u)$ erfahren bei Vermehrung von u um $\frac{\omega_{1}}{n}$ und $\frac{\omega_{2}}{n}$ die folgenden Transformationen:

$$
\left\{\begin{array}{l}
X_{\lambda}\left(u+\frac{\omega_{1}}{n}\right)=(-1)^{n} e^{\eta_{1}\left(u+\frac{\omega_{1}}{2 n}\right)} X_{\lambda-1}(u), \\
X_{\lambda}\left(u+\frac{\omega_{2}}{n}\right)=(-1)^{n} \varepsilon^{-\lambda} e^{\eta_{2}\left(u+\frac{\omega_{2}}{2 n}\right)} X_{\lambda}(u),
\end{array}\right.
$$

wo $\varepsilon=e^{\frac{2 i \pi}{n}} i s t$.

Mit Hilfe dieser Regeln kann man beweisen, daß die $n$ Funktionen $X_{\lambda}(u)$ des einzelnen Systems linear-unabhängig sind. Sollte nämlich eine Relation:

$$
c_{0} X_{0}(u)+c_{1} X_{1}(u)+\cdots+c_{n-1} X_{n-1}(u)=0
$$

mit Koeffizienten, die von $u$ unabhängig sind, identisch bestehen, so fände man durch $\mu$-malige Ausübung der ersten Transformation (15) nach Fortlassung eines gemeinsamen Faktors aller Glieder:

$$
c_{\mu} X_{0}(u)+c_{\mu+1} X_{1}(u)+\cdots+c_{\mu-1} X_{n-1}(u)=0 \text {. }
$$

Man übe weiter $\nu$ Male die zweite Transformation (15) aus und lasse jedesmal den auftretenden gemeinsamen Faktor aller Glieder $(-1)^{n} e^{\eta_{2}\left(u+\frac{\omega_{2}}{2 n}\right)}$ fort; auf diese Weise würde man weiter finden:

$c_{\mu} X_{0}(u)+c_{\mu+1} \varepsilon^{-v} X_{1}(u)+c_{\mu+2} \varepsilon^{-2 v} X_{2}(u)+\cdots+c_{\mu-1} \varepsilon^{-(n-1) \nu} X_{n-1}(u)=0$.

Setzt man hier der Reihe nach $\nu=0,1,2, \ldots, n-1$ und addiert alle Gleichungen, so folgt $n c_{\mu} X_{0}(u)=0$ als identische Gleichung, aus der notwendig $c_{\mu}=0$ folgt. Damit aber ist die Behauptung bewiesen.

Die Wirkung eines Zeichenwechsels von $u$ auf $X_{\lambda}$ bestimmt man leicht aus (12) und (13). Man führe zugleich einen neuen Summationsbuchstaben $\nu^{\prime}$ ein, indem man $\nu=-\nu^{\prime}$ oder $\nu=1-\nu^{\prime}$ setzt, je nachdem $n$ ungerade oder gerade ist. Beide Male wird man dann zur Reihe von $X_{n-\lambda}$ geführt. Bei ungeradem $n$ ist noch der Faktor $(-1)^{\lambda}$ zu berücksichtigen, der Zeichenwechsel erfährt, wenn $\lambda$ durch $(n-\lambda)$ ersetzt wird. Bei Zeichenwechsel von u zeigen die Funktionen $X_{\lambda}$ das Verhalten:

$$
X_{\lambda}\left(-u \mid \omega_{1}, \omega_{2}\right)=(-1)^{n} X_{n-\lambda}\left(u \mid \omega_{1}, \omega_{2}\right) \text {. }
$$

In den drei Argumenten $u, \omega_{1}, \omega_{2}$ sind unsere Funktionen homogen, und zwar von der Dimension $-3 n_{0}-2$ bzw. -2 , je nachdem $n$ ungerade 
308 II, 2. Systeme ganzer elliptischer Funktionen dritter Art $\boldsymbol{n}^{\text {ter }}$ Stufe oder gerade ist. Bei gleichzeitigem Zeichenvechsel aller drei Argumente gelten die Regeln:

(17) $X_{\lambda}\left(-u \mid-\omega_{1},-\omega_{2}\right)=(-1)^{\frac{n+1}{2}} X_{\lambda}\left(u \mid \omega_{1}, \omega_{1}\right)$ bzw. $=X_{\lambda}\left(u \mid \omega_{1}, \omega_{2}\right)$, je nachdem $n$ ungerade oder gerade ist. Aus (16) und (17) ergibt sich endlich noch als Folgerung: Bei gleichzeitigem Zeichenwechsel von $\omega_{1}$, $\omega_{2}^{\prime}$ transformieren sich die $X_{2}$ entsprechend den Gleichungen:

$$
X_{\lambda}\left(u \mid-\omega_{1},-\omega_{2}\right)=(-1)^{\frac{n-1}{2}} X_{n-\lambda}\left(u \mid \omega_{1}, \omega_{2}\right) \text { bzw. }=X_{n-\lambda}\left(u \mid \omega_{1}, \omega_{2}\right),
$$

je nachdem $n$ ungerade oder gerade ist. Für $\lambda=0$ hat man die Indizes $n$ auf den rechten Seiten von (16) und (18) durch $0 \mathrm{zu}$ ersetzen.

\section{§ 3. Lineare Transformation der Funktionen $X_{\lambda}\left(u \mid \omega_{1}, \omega_{2}\right)$.}

Ändert man den Summationsbuchstaben $\nu$ in (12) und (13) S. 306 um eine Einheit, so ändert sich der Exponent von $q$ beide Male um eine gerade Zahl. Hieraus folgt, daß alle Glieder der einzelnen Reihe bei Ausübung der Substitution $S=\left(\begin{array}{l}1,1 \\ 0,1\end{array}\right)$ der homogenen Gruppe $\Gamma^{(v)}$ das gleiche Verhalten zeigen. Berücksichtigt man auch die vor den Summen stehenden Faktoren, so zeigt sich, daß $X_{\lambda}$ sich gegenüber $S$ verhält wie:

$$
q^{\frac{1}{4}+\frac{n_{0}}{2}+\frac{(n-2 \lambda)^{2}}{4 n}}=q^{-\frac{\lambda(n-\lambda)}{n}+\frac{1}{2}\left(\frac{n+1}{2}+n_{0}\right)} \text { bzw. } q^{\frac{1}{4}+\frac{\lambda^{2}}{n}} \text {. }
$$

Mit Rücksicht auf die Kongruenz (6) S. 30 ๖̃ findet man den Satz: Bei Ausübung der Substitution $S=\left(\begin{array}{l}1,1 \\ 0,1\end{array}\right)$ geht $X_{\lambda}$ bis auf einen Faltor in sich selbst über:

$$
\begin{cases}X_{\lambda}\left(u \mid \omega_{1}+\omega_{2}, \omega_{2}\right)=\varepsilon^{-\frac{\lambda(n-\lambda)}{2}} X_{\lambda}\left(u \mid \omega_{1}, \omega_{2}\right), & n \equiv 1(\bmod 2) \\ X_{\lambda}\left(u \mid \omega_{1}+\omega_{2}, \omega_{2}\right)=\varepsilon^{\frac{n}{8}+\frac{\lambda^{2}}{2}} X_{\lambda}\left(u \mid \omega_{1}, \omega_{1}\right), & n \equiv 0(\bmod 2)\end{cases}
$$

Wendet man auf die für $i=1$ genommene Gleichung (14) S. 306 die Substitution $T=\left(\begin{array}{c}0,1 \\ -1,0\end{array}\right)$ an, so folgt, da die $\eta_{i}$ mit den $\omega_{i}$ kogredient sind:

$$
X_{\lambda}\left(u+\omega_{2} \mid \omega_{2},-\omega_{1}\right)=(-1)^{n} e^{n \eta_{2}\left(u+\frac{\omega_{2}}{2}\right)} X_{\lambda}\left(u \mid \omega_{2},-\omega_{1}\right) .
$$

Ebenso folgt aus der für $i=2$ genommenen Gleichung (14) S. 306:

$$
X_{\lambda}\left(u-\omega_{1} \mid \omega_{2},-\omega_{1}\right)=(-1)^{n} e^{-n \eta_{1}\left(u-\frac{\omega_{1}}{2}\right)} X_{\lambda}\left(u \mid \omega_{2},-\omega_{1}\right),
$$

woraus man nach Vermehrung von $u$ um $\omega_{1}$ entnimmt: 


$$
X_{\lambda}\left(u+\omega_{1} \mid \omega_{2},-\omega_{1}\right)=(-1)^{n} e^{n \eta_{1}\left(u+\frac{\omega_{1}}{2}\right)} X_{\lambda}\left(u \mid \omega_{2},-\omega_{1}\right) \text {. }
$$

Demnach sind die $X_{\lambda}\left(u \mid \omega_{2},-\omega_{1}\right)$ mit den ursprünglichen $X_{\lambda}\left(u \mid \omega_{1}, \omega_{2}\right)$ gleichändrige ganze elliptische Funktionen dritter Art und als solche nach I, 227 durch die $n$ linear-unabhängigen Funktionen $X_{\lambda}\left(u \mid \omega_{1}, \omega_{2}\right)$ in der Gestalt:

$$
X_{2}\left(u, \omega_{2},-\omega_{1}\right)=\sum_{x=0}^{n-1} c_{\lambda \varkappa} X_{\varkappa}\left(u \mid \omega_{1}, \omega_{2}\right)
$$

mit von $u$ unabhängigen, eindeutig bestimmten Koeffizienten $c$ darstellbar.

Zur Bestimmung dieser Koeffizienten nehmen wir im Ansatze (2) zunächst $\lambda=0$ und vermehren $u$ um $\frac{\omega_{1}}{n}$. Links kommt die zur zweiten Transformation (15) S. 307 inverse Transformation zur Benutzung, da - $\omega_{1}$ die zweite Periode von $X_{0}\left(u \mid \omega_{2},-\omega_{1}\right)$ ist; rechts ist die erste Regel (15) S. 307 anzuwenden. Nach Forthebung überflüssiger Faktoren folgt:

$$
X_{0}\left(u \mid \omega_{2},-\omega_{1}\right)=\sum_{x=0}^{n-1} c_{0, x} X_{\psi-1}\left(u \mid \omega_{1}, \omega_{2}\right),
$$

so da $B$ aus der eindeutigen Bestimmtheit der Koeffizienten $c_{0, x}=c_{0, x-1}$. folgt. Also sind für $\lambda=0$ alle Koeffizienten $c$ des Ansatzes (2) einander gleich :

$$
X_{0}\left(u \mid \omega_{2},-\omega_{1}\right)=c \sum_{x=0}^{n-1} X_{\varkappa}\left(u \mid \omega_{1}, \omega_{2}\right) .
$$

Vermindert man hier $u$ um $\frac{\omega_{2}}{n}$, so sind links und rechts die zu (15) S. 307 inversen Transformationen auszuüben. Es folgt:

$$
X_{1}\left(u \mid \omega_{2},-\omega_{1}\right)=c \sum_{x=0}^{n-1} \varepsilon^{*} X_{*}\left(u \mid \omega_{1}, \omega_{2}\right),
$$

sowie bei wiederholter Ausübung der gleichen Operation allgemein:

$$
X_{\lambda}\left(u, \omega_{2},-\omega_{1}\right)=c \sum_{x=0}^{n-1} \varepsilon^{\kappa \lambda} X_{\nu_{n}}\left(u \mid \omega_{1}, \omega_{2}\right) .
$$

$\mathrm{Um} e$ erstlich für ungerades $n$ zu bestimmen, differenzieren wir die Gleichung (3) nach $u$ und setzen hierauf $u=0$. Ist $X_{\lambda}^{\prime}(u)$ die Ableitung nach $u$, so folgt:

$$
X_{0}^{\prime}\left(0 \mid \omega_{2},-\omega_{1}\right)=c \sum_{\lambda=0}^{n-1} X_{\lambda}^{\prime}\left(0 \mid \omega_{1}, \omega_{2}\right)
$$


310 II, 2. Systeme ganzer elliptischer Funktionen dritter Art $n^{\text {ter }}$ Stufe

Nun folgt aus (10) S. 306:

$$
X_{0}^{\prime}\left(0 \mid \omega_{1}, \omega_{2}\right)=\frac{n}{\omega_{2}} \sqrt{\frac{2 \pi}{\omega_{2}}} \sqrt[8]{\Delta}(\sqrt[4]{\Delta})^{n_{0}} \vartheta_{1}^{\prime}\left(q^{n}\right),
$$

sowie hieraus weiter mit Benutzung von (25) in I, 420:

$$
X_{0}^{\prime}\left(0 \mid \omega_{1}, \omega_{2}\right)=\frac{2 n \pi}{\omega_{2}^{3}}(\sqrt[4]{\Delta})^{n_{0}} \vartheta_{1}^{\prime}(q) \vartheta_{1}^{\prime}\left(q^{n}\right)
$$

Bei Ausübung von $T=\left(\begin{array}{c}0,1 \\ -1,0\end{array}\right)$ nimmt $\sqrt[4]{\Delta}$ nach (10) in I, 454 den Faktor $-i$ an, während aus der ersten Gleichung (4) in I, 482 für den Nullwert der $\vartheta_{1}^{\prime}$-Funktion die Regel folgt ${ }^{1}$ ):

$$
\vartheta_{1}^{\prime}\left(e^{\left.-\frac{\pi i}{\omega}\right)}=e^{-\frac{\pi i}{4}} \omega \sqrt{-\omega} \vartheta_{1}^{\prime}\left(e^{\pi i \omega}\right)\right. \text {. }
$$

Setzt man in (8) statt $\omega$ den Wert $\frac{\omega}{n}$ ein, so folgt:

$$
\vartheta_{1}^{\prime}\left(e^{-\frac{n \pi i}{\omega}}\right)=e^{-\frac{\pi i}{4} \frac{\omega \sqrt{-\omega}}{n \sqrt{n}}} \vartheta_{1}^{\prime}\left(e^{\frac{\pi i \omega}{n}}\right) .
$$

Hiernach berechnet sich als die Wirkung der Substitution $T$ auf die Gleichung (7):

$$
X_{0}^{\prime}\left(0 \mid \omega_{2},-\omega_{1}\right)=\frac{(-i)^{n_{0}+1}}{\sqrt{n}} \frac{2 \pi}{\omega_{2}^{s}}(\sqrt[4]{\Delta})^{n_{0}} \vartheta_{1}^{\prime}(q) \vartheta_{1}^{\prime}\left(q^{\frac{1}{n}}\right)
$$

wo die Wurzel $\sqrt{n}$ positiv zu nehmen ist. $\left.{ }^{2}\right)$ Für die in (5) rechts stehende Summe folgt aus (12) S. 306:

$$
\begin{aligned}
\sum_{\lambda=0}^{n-1} X_{\lambda}^{\prime}\left(0 \mid \omega_{1}, \omega_{2}\right)= & \frac{\pi}{\omega_{2}} \sqrt{\frac{2 \pi}{\omega_{2}}} \sqrt[8]{\Delta}(\sqrt[4]{\Delta})^{n_{0}} \\
& \sum_{\lambda=0}^{\cdot n-1} \sum_{\nu=-\infty}^{+\infty}(-1)^{\lambda+v}((2 \nu+1) n-2 \lambda) q^{\frac{((2 v+1) n-2 \lambda)^{2}}{4 n}} .
\end{aligned}
$$

Nun durchläuft bei Ausführung beider Summen $((2 \nu+1) n-2 \lambda)$ gerade genau alle positiven und negativen ungeraden Zahlen. $W$ ir schreiben also $((2 \nu+1) n-2 \lambda)=2 \nu^{\prime}+1$ unter Einführung eines neuen Summationsbuchstaben $\nu^{\prime}$ und folgern hieraus:

$$
\lambda+\nu \equiv \nu n-\lambda=\nu^{\prime}-\frac{n-1}{2} \equiv \nu^{\prime}+\frac{n-1}{2}(\bmod 2) .
$$

1) Statt der hier gebrauchten Bezeichnung $\vartheta(q)=\boldsymbol{\vartheta}\left(e^{\pi i \omega}\right)$ für die $\vartheta$-Nullwerte ist in I, a. a. 0 . einfacher $\vartheta(\omega)$ geschrieben. teile haben.

2) Nach I, 481 müssen die Werte $\sqrt{-\omega}, \sqrt{-\frac{\omega}{n}}$ positive reelle Bestand- 
Die letzte Gleichung nimmt damit die Gestalt an:

$$
\begin{gathered}
\sum_{\lambda=0}^{n-1} X_{\lambda}^{\prime}\left(0 \mid \omega_{1}, \omega_{2}\right)= \\
=\frac{\pi}{\omega_{2}} \sqrt{\frac{2 \pi}{\omega_{2}}} \sqrt[8]{\Delta}(\sqrt[4]{\Delta})^{n_{0}}(-1)^{\frac{n-1}{2}} \sum_{v^{\prime}=-\infty}^{+\infty}(-1)^{v^{\prime}}\left(2 \nu^{\prime}+1\right) q^{\frac{\left(2 v^{\prime}+1\right)^{2}}{4 n}} .
\end{gathered}
$$

Die mit $\pi$ multiplizierte Summe liefert nach (24) in I, 419 den $\vartheta$-Nullwert $\vartheta_{1}^{\prime}\left(q^{\frac{1}{n}}\right)$. Ersetzen wir wie oben $\sqrt[8]{\Delta}$ durch den Ausdruck in $\vartheta_{1}^{\prime}(q)$ entsprechend der Gleichung (25) in I, 420, so ergibt sich:

$$
\sum_{\lambda=0}^{n-1} X_{\lambda}^{\prime}\left(0 \mid \omega_{1}, \omega_{2}\right)=(-1)^{\frac{n-1}{2}} \frac{2 \pi}{\omega_{2}^{3}}(\sqrt[4]{\Delta})^{n_{0}} \vartheta_{1}^{\prime}(q) \vartheta_{1}^{\prime}\left(q^{\frac{1}{n}}\right)
$$

Trägt man nun die Ausdrücke (9) und (10) in (5) ein, so folgt nach Fortheben gemeinsamer Faktoren beider Seiten der Gleichung bei Benutzung der Kongruenz (6) S. 305 für $n_{0}$ :

$$
\frac{(-i)^{-\frac{n-1}{2}}}{\sqrt{n}}=c \cdot(-1)^{\frac{n-1}{2}}
$$

$$
\frac{1}{c}=i^{\frac{n-1}{2}} \sqrt{n}
$$

mit positiv genommener Wurzel $\sqrt{n}$.

Im Falle eines geraden $u$ setze man in (3) unmittelbar $u=0$ ein und findet:

$$
X_{0}\left(0 \mid \omega_{2},-\omega_{1}\right)=c \sum_{\lambda=0}^{n-1} X_{\lambda}\left(0 \mid \omega_{1}, \omega_{2}\right)
$$

Nun ergibt sich aus (11) S. 306:

$$
X_{0}\left(0 \mid \omega_{1}, \omega_{2}\right)=\sqrt{\frac{2 \pi}{\omega_{2}}} \sqrt[8]{\Delta} \vartheta_{3}\left(q^{n}\right)=\frac{2 \pi}{\omega_{2}^{2}} \vartheta_{1}^{\prime}(q) \vartheta_{3}\left(q^{n}\right)
$$

Die Wirkung der Substitution $T$ findet man aus der obigen Formel (8) und der vierten Regel (4) in I, 482:

$$
X_{0}\left(0 \mid \omega_{2},-\omega_{1}\right)=-\frac{2 \pi}{\omega_{2}^{2}} \cdot \frac{1}{\sqrt{n}} \vartheta_{1}^{\prime}(q) \vartheta_{3}\left(q^{\frac{1}{n}}\right)
$$

mit positiv genommener Wurzel $\sqrt{n}$. Andrerseits findet man aus (13) S.306:

$$
\sum_{\lambda=0}^{n-1} X_{\lambda}\left(0 \mid \omega_{1}, \omega_{2}\right)=\sqrt{\frac{2 \pi}{\omega_{2}}} \sqrt[8]{\Delta} \sum_{\lambda=0}^{n-1} \sum_{\nu=-\infty}^{+\infty} \frac{\frac{(v n-\lambda)^{2}}{n}}{q^{2}}=\sqrt{\frac{2 \pi}{\omega_{2}}} \sqrt[8]{\Delta} \sum_{v^{\prime}=-\infty}^{+\infty} q^{\frac{v^{\prime 2}}{n}}
$$

Nach (16) in I, 418 ist die letzte Reihe gleich $\vartheta_{3}\left(q^{\frac{1}{n}}\right)$, so daB man findet:

$$
\sum_{\lambda=0}^{n-1} X_{\lambda}\left(0 \mid \omega_{1}, \omega_{2}\right)=\frac{2 \pi}{\omega_{2}^{2}} \vartheta_{1}^{\prime}(q) \vartheta_{3}\left(q^{\frac{1}{n}}\right)
$$


312 II, 2. Systeme ganzer elliptischer Funktionen dritter Art $n^{\text {ter }}$ Stufe

Trägt man die in (13) und (14) berechneten Ausdrücke in (12) ein, so folgt nach Forthebung überflüssiger Faktoren:

$$
\frac{1}{c}=-\sqrt{n} \text {. }
$$

In beiden Fällen ist $c$ nicht nur von $u$, sondern auch von den Perioden $\omega_{1}, \omega_{2}$ unabhängig; es ist dies eine wichtige Folge der aus der Diskriminante aufgebauten Faktoren, die oben in die Erklärung der Funktionen $X_{\lambda}$ aufgenommen wurden. Unter Zusammenfassung beider Fälle merken wir den Satz an: Bei Ausübung der Substitution $T$ transformieren sich die $X_{\lambda}$ nach dem Gesetze:

$$
\begin{cases}i^{\frac{n-1}{2}} \sqrt{n} X_{\lambda}\left(u \mid \omega_{2},-\omega_{1}\right)=\sum_{x=0}^{n-1} \varepsilon^{* \lambda} X_{x}\left(u \mid \omega_{1}, \omega_{2}\right), & n \equiv 1(\bmod 2), \\ -\sqrt{n} X_{\lambda}\left(u \mid \omega_{2},-\omega_{1}\right)=\sum_{x=0}^{n-1} \varepsilon^{\varkappa \lambda} X_{\varkappa}\left(u \mid \omega_{1}, \omega_{2}\right), & n \equiv 0(\bmod 2),\end{cases}
$$

wo $\sqrt{n}$ beide Male positiv zu nehmen ist.

Da die Gruppe $\Gamma^{(\omega)}$ aus den Substitutionen $S$ und $T$ erzeugbar ist, so erfahren die $n$ Funktionen $X_{\lambda}$ jedes Systems gegenüber irgendeiner Substitution der $\Gamma^{(\omega)}$ und also im Sinne von $I, 184$ bei irgendeiner "linearen" Transformation der Perioden selbst eine homogene lineare Substitution mit konstanten Koeffizienten. Da ferner die zum Grade $n$ gehörenden $X_{\lambda}$ bei den Substitutionen einer gewissen Kongruenzgruppe unverändert bleiben, so erhalten wir, dem Index dieser Untergruppe entsprechend, nur endlich viele $X_{\lambda}$-Substitutionen, die ihrerseits eine endliche Gruppe $G$ bilden.

Um dies wenigstens in dem wichtigeren Falle eines ungeraden $n$, wo die $X_{\lambda}$ den Charakter von Modulformen $n^{\text {ter }}$ Stufe haben, näher auszuführen, stellen wir auch noch die Wirkung einer die Bedingungen $\boldsymbol{\beta} \equiv 0, \gamma \equiv 0 .(\bmod n)$ befriedigenden Substitution fest. Bei einer solchen Substitution $\left(\begin{array}{l}\alpha, \beta \\ \gamma, \delta\end{array}\right)$ ist $\alpha$ mit einer $\operatorname{der} \varphi(n) \bmod n$ inkongruenten, gegen $n$ teilerfremden Zahlen kongruent, und es gilt $\delta \equiv \alpha^{-1}(\bmod n)$; insofern die Substitution $\bmod n$ durch $\alpha$ bereits bestimmt ist, möge sie $U_{\alpha}$ genannt werden. Für die durch (2) S. 298 gegebenen transformierten Perioden $\omega_{1}^{\prime}, \omega_{2}^{\prime}$ rechnet sich die Substitution $U_{\alpha}$ auf $\left(\begin{array}{c}\alpha, \beta n \\ \gamma n^{-1}, \delta\end{array}\right)$ um. Nach (3) in I, 451 hat man nun zunächst:

$$
\sigma_{\lambda, 0}\left(u \mid \alpha \omega_{1}^{\prime}+\beta n \omega_{2}^{\prime}, \gamma n^{-1} \omega_{1}^{\prime}+\delta \omega_{2}^{\prime}\right)=\sigma_{\alpha \lambda, \beta \lambda n}\left(u \mid \omega_{1}^{\prime}, \omega_{2}^{\prime}\right) .
$$

Weiter folgt aus (4) a. a. O.:

$$
\sigma_{\alpha \lambda, \beta \lambda n}\left(u \mid \dot{\omega}_{1}^{\prime}, \omega_{2}^{\prime}\right)=(-1)^{\beta \lambda} \cdot e^{\frac{\pi i}{n} \alpha \beta^{\prime} \lambda^{2}} \sigma_{\alpha \lambda, 0}\left(u \mid \omega_{1}^{\prime}, \omega_{2}^{\prime}\right) .
$$


Da $\beta$ durch $n$ teilbar ist und $n$ ungerade sein sollte, so gilt für den rechts auftretenden Faktor:

$$
(-1)^{\beta \lambda+\alpha \frac{\beta}{n} \hat{\lambda}^{2}}=(-1)^{\beta \lambda(1+\alpha)}=(-1)^{\lambda(1+\alpha)} ;
$$

man beachte hierbei, daB $\beta(1+\alpha) \equiv 1+\alpha(\bmod 2)$ gilt, da $\alpha$ und $\beta$ nie zugleich gerade sind. Hiernach gilt:

$$
(-1)^{\lambda} \sigma_{\lambda, 0}\left(u \mid \alpha \omega_{1}+\beta \omega_{2}, \frac{\gamma \omega_{1}+\delta \omega_{2}}{n}\right)=(-1)^{\alpha \lambda} \sigma_{\alpha \lambda, 0}\left(u \omega_{1}, \frac{\omega_{2}}{n}\right) .
$$

Die Modulform $G_{1}$ ist gegenüber $U_{\alpha}$ invariant, der Faktor

$$
\sqrt[8]{\Delta^{\prime} \Delta}(\sqrt[4]{\Delta})^{n_{0}}
$$

aber zeigt dasselbe Verhalten wie die Modulform (5) S. 299, d. h. sie nimmt den Faktor $\left(\frac{\delta}{n}\right)$ an (vgl. (12) S. 303), für den wir wegen $\alpha \cdot \delta \equiv 1$ $(\bmod n)$ auch $\left(\frac{\alpha}{n}\right)$ schreiben können. Es gilt also der Satz: Gegeniiber einer die Bedingung $U_{\alpha} \equiv\left(\begin{array}{c}\alpha, 0 \\ 0, \alpha^{-1}\end{array}\right)(\bmod n)$ erfiillenden Substitution zeigen die $X_{2}$ eines ungeraden $n$ das Verhalten:

$$
X_{\lambda}\left(u \mid \alpha \omega_{1}+\beta \omega_{2}, \gamma \omega_{1}+\delta \omega_{2}\right)=\left(\frac{\alpha}{n}\right) X_{\alpha \lambda}\left(u \mid \omega_{1}, \omega_{2}\right),
$$

wo $\left(\frac{\alpha}{n}\right)$ das Legendre-Jacobische Zeichen ist. Die erste Regel (18) S. 308 ist hierin als besonderer Fall enthalten.

Die $X_{\lambda}$ eines ungeraden $n$ bleiben nun bei den Substitutionen der Hauptkongruenzgruppe $n^{\text {ter }}$ Stufe $\Gamma_{n \chi_{(n)}}$ unverändert. Es ist jetzt leicht zu zeigen, daß sie insgesamt auch nur bei den Substitutionen der $\Gamma_{n \chi(n)}$ unverändert bleiben. Soll nämlich zunächst $X_{0}\left(u \omega_{1}, \omega_{2}\right) \operatorname{durch}\left(\begin{array}{l}\alpha, \beta \\ \gamma, \delta\end{array}\right)$ in sich transformiert werden, so muß $\sigma\left(u \mid \omega_{1}^{\prime}, \omega_{2}^{\prime}\right)$ durch die Substitution $\left(\begin{array}{c}\alpha, \beta n \\ \gamma n^{-1}, \delta\end{array}\right)$ in sich transformiert werden, was ein ganzzahliges $\gamma n^{-1}, \mathrm{~d}$. h. ein durch $n$ teilbares $\gamma$ voraussetzt. Wir haben also nur noch die mit:

$$
S^{\alpha \beta} \cdot U_{\alpha} \equiv\left(\begin{array}{c}
\alpha, \beta \\
0, \alpha^{-1}
\end{array}\right) \quad(\bmod n)
$$

kongruenten Substitutionen einer unserer von S. 252 her bekannten Kongruenzgruppen $\Gamma_{\psi(n)}$ zuzulassen. Die Substitution (18) transformiert $X_{\lambda}$ in:

$$
\varepsilon^{-\alpha \beta \frac{\lambda(n-\lambda)}{2}}\left(\frac{\alpha}{n}\right) X_{\alpha \lambda}\left(u \quad \omega_{1}, \omega_{2}\right) .
$$

Hieraus ziehen wir zunächst den später anzuwendenden Satz: Die Funktion $X_{0}\left(u \mid \omega_{1}, \omega_{2}\right)$ eines ungeraden $n$ bleibt bei den Substitutionen der durch $\gamma \equiv 0(\bmod n)$ erklärten Kongruenzgruppe $n^{\text {ter }}$ Stufe $\Gamma_{\psi(n)}$ unverändert oder erfährt mur einen Zeichenvechsel, entsprechend der Regel: 
314 II, 2. Systeme ganzer elliptischer Funktionen dritter Art $n^{\text {ter }}$ Stufe

$$
X_{0}\left(u \mid \alpha \omega_{1}+\beta \omega_{2}, \gamma \omega_{1}+\delta \omega_{2}\right)=\left(\frac{\alpha}{n}\right) X_{0}\left(u \mid \omega_{1}, \omega_{2}\right), \quad \gamma \equiv 0(\bmod n) .
$$

Soll weiter $X_{1}$ durch die Substitution (18) in sich transformiert werden, so muß zufolge $(19)$ notwendig $\alpha \equiv 1, \beta \equiv 0(\bmod n)$ zutreffen. Damit ist die Behauptung, daB die $X_{\lambda}$ insgesamt nur bei den Substitutionen der $\Gamma_{n_{\chi}(n)}$ unverändert bleiben, bewiesen.

Zwei mod $n$ inkongruente Substitutionen $V, V^{\prime}$ liefern hiernach stets zwei verschiedene $X_{\lambda}$-Transformationen, da sonst $V^{\prime} \cdot V^{-1}$ die „identische“ $X_{\lambda}$-Transformation ergeben würde. Damit aber ist der folgende Satz bewiesen: Aus den beiden gleich wieder durch $S$ und $T z u$ bezeichnenden linearen $X_{\lambda}$-Transformationen:

$$
\begin{gathered}
X_{\lambda}^{\prime}=\varepsilon^{-\frac{\lambda(n-\lambda)}{2}} X_{\lambda}, \\
i^{\frac{n-1}{2}} \sqrt{n} X_{\lambda}^{\prime}=\sum_{\nu=0}^{n-1} \varepsilon^{\mu \lambda} X_{\kappa}
\end{gathered}
$$

erzeugt man im Falle eines ungeraden $n$ durch Wiederholung und Kombination eine endliche Gruppe $G_{n_{\chi}(n)}$ der Ordnung $n_{\chi}(n)$ solcher Transformationen, die isomorph mit der mod $n$ reduzierten homogenen Modulgruppe ist. Den bei der Transformation $T$ links auftretenden Faktor kann man nach der letzten Gleichung in I, 494 als "Gaußsche Summe" darstellen:

$$
i^{\frac{n-1}{2}} \sqrt{n}=(-1)^{\frac{n-1}{2}} \sum_{v=0}^{n-1} \varepsilon^{2 v^{2}} \text {. }
$$

Wir merken daraufhin noch den Satz an: Bei ungeradem $n$ sind die Koeffizienten der $n \chi(n)$ linearen Transformationen der $X_{\lambda}$ Zahlen des zum Teilungsgrade $n$ gehörenden Kreisteilungskörpers vom Grade $\varphi(n)$.

\section{Systeme von Modulformen für ungerade Stufen.}

Für die Transformationstheorie wichtige Größensysteme gewinnen wir in den Modulformen, welche aus den $X_{\lambda}\left(u \mid \omega_{1}, \omega_{2}\right)$ und ihren ersten nach $u$ genommenen Ableitungen $X_{\lambda}^{\prime}\left(u \mid \omega_{1}, \omega_{2}\right)$ für $u=0$ hervorgehen. Indem wir $n$ als ungerade voraussetzen, entnehmen wir für den Zeichenwechsel des ersten Argumentes $u$ aus (16) S. 307:

$$
X_{\lambda}(-u)=-X_{n-\lambda}(u), \quad X_{\lambda}^{\prime}(-u)=+X_{n-\lambda}^{\prime}(u),
$$

wo für $\lambda=0$ statt des Index $n$ rechts $0 \mathrm{zu}$ setzen ist. Schreiben wir nun abkürzend:

$$
X_{\lambda}\left(0 \mid \omega_{1}, \omega_{2}\right)=x_{\lambda}\left(\omega_{1}, \omega_{2}\right), \quad X_{\lambda}^{\prime}\left(0 \mid \omega_{1}, \omega_{2}\right)=\xi_{2}\left(\omega_{1}, \omega_{2}\right),
$$

so ergibt sich aus (1):

$$
x_{n-\lambda}\left(\omega_{1}, \omega_{2}\right)=-x_{\lambda}\left(\omega_{1}, \omega_{2}\right), \quad \xi_{n-\lambda}\left(\omega_{1}, \omega_{2}\right)=\xi_{\lambda}\left(\omega_{1}, \omega_{2}\right),
$$


Die Systeme der Modulformen $x_{\lambda}\left(\omega_{1}, \omega_{2}\right)$ und $\xi_{\lambda}\left(\omega_{1}, \omega_{2}\right)$

während $x_{0}\left(\omega_{1}, \omega_{2}\right)$ identisch verschwindet. Für jedes ungerade $n$ erhalten wir somit ein System von $\frac{n-1}{2}$ Modulformen $x_{1}\left(\omega_{1}, \omega_{2}\right), x_{2}\left(\omega_{1}, \omega_{2}\right), \ldots$, $x_{\frac{n-1}{2}}\left(\omega_{1}, \omega_{2}\right)$ der $n^{\text {ten }}$ Stufe und ein System von $\frac{n+1}{2}$ Modulformen $\xi_{0}\left(\omega_{1}, \omega_{2}\right)$, $\xi_{1}\left(\omega_{1}, \omega_{2}\right), \ldots, \xi_{\frac{n-1}{2}}\left(\omega_{1}, \omega_{2}\right)$ der gleichen Stufe.

Aus den Gleichungen (10) und (12) S. 306 ergeben sich folgende Darstellungen der $\frac{n-1}{2}$ Modulformen $x_{\lambda}\left(\omega_{1}, \omega_{2}\right)$ :

$$
\begin{gathered}
x_{\lambda}\left(\omega_{1}, \omega_{2}\right)=(-1)^{\lambda+1} \sqrt{\frac{2 \pi}{\omega_{2}}} \sqrt[8]{\Delta}(\sqrt[4]{\Delta})^{n_{0}} q^{\frac{\lambda^{2}}{n}} \vartheta_{1}\left(\lambda \omega, q^{n}\right), \\
x_{\lambda}\left(\omega_{1}, \omega_{2}\right)=\frac{(-1)^{\lambda}}{i} \sqrt{\frac{2 \pi}{\omega_{2}}} \sqrt[8]{\Delta}(\sqrt[4]{\Delta})^{n_{0}} \sum_{v=-\infty}^{+\infty}(-1)^{v} q^{\frac{((2 v+1) n-2 \lambda)^{2}}{4 n}} .
\end{gathered}
$$

Weiter folgt aus (12) S. 306 für die $\frac{n+1}{2}$ Modulformen $\xi_{\lambda}\left(\omega_{1}, \omega_{2}\right)$ :

$$
\begin{aligned}
\xi_{\lambda}\left(\omega_{1}, \omega_{2}\right)=(-1)^{\lambda} \frac{\pi}{\omega_{2}} \sqrt{\frac{2 \pi}{\omega_{2}}} \sqrt[8]{\Delta}(\sqrt[4]{\Delta})^{n_{0}} \\
\\
\cdot \sum_{v=-\infty}^{+\infty}(-1)^{v}((2 \nu+1) n-2 \lambda) q^{\frac{(2 v+1) n-2 \lambda)^{2}}{4 n}} .
\end{aligned}
$$

Für $\xi_{0}\left(\omega_{1}, \omega_{2}\right)$ haben wir auch die schon in (6) S. 310 genannte Darstellung:

$$
\xi_{0}\left(\omega_{1}, \omega_{2}\right)=\frac{n}{\omega_{2}} \sqrt{\frac{2 \pi}{\omega_{2}}} \sqrt[8]{\Delta}(\sqrt[4]{\Delta})^{n_{0}} \vartheta_{1}^{\prime}\left(q^{n}\right)
$$

die wir mit Hilfe von (25) in I, 420 auch noch umkleiden in:

$$
\xi_{0}\left(\omega_{1}, \omega_{2}\right)=\frac{1}{\sqrt{n}} \sqrt[8]{\Delta}(\sqrt[4]{\Delta})^{n_{0}} \sqrt[8]{\Delta}\left(\omega_{1}, \frac{\omega_{2}}{n}\right)
$$

Aus den Rechnungen von S. $308 \mathrm{ff}$. geht folgender Satz hervor: Die $\frac{n-1}{2}$ Modulformen $x_{\lambda}$ substituieren sich gegenüber den Substitutionen der homogenen Modulgruppe $\Gamma^{(\omega)}$ selbst linear und homogen, und insbesondere entsprechen den erzeugenden Substitutionen $S$ und $T$ der $\Gamma^{(\omega)}$ die Transformationen:

$$
\begin{aligned}
x_{\lambda}^{\prime} & =\varepsilon^{-\frac{\lambda(n-\lambda)}{2}} x_{\lambda}, \\
i^{\frac{n-1}{2}} \sqrt{n} x_{\lambda}^{\prime} & =\sum_{x=1}^{\frac{n-1}{2}}\left(\varepsilon^{x \lambda}-\varepsilon^{-x \lambda}\right) x_{x} .
\end{aligned}
$$

Derselbe Satz gilt für die $\frac{n+1}{2}$ Modulformen $\xi_{\lambda}$, bei denen die Transformationen $S$ und $T$ die Gestalten haben: 


$$
\begin{gathered}
\xi_{\lambda}^{\prime}=\varepsilon^{-\frac{\lambda(n-\lambda)}{2}} \xi_{\lambda}, \\
i^{\frac{n-1}{2}} \sqrt{n} \xi_{\lambda}^{\prime}=\xi_{0}+\sum_{x=1}^{\frac{n-1}{2}}\left(\varepsilon^{x \lambda}+\varepsilon^{-x \lambda}\right) \xi_{x} .
\end{gathered}
$$

Für $\xi_{0}\left(\omega_{1}, \omega_{2}\right)$ insbesondere notieren wir zu späterem Gebrauche die Regeln:

$$
\begin{gathered}
\xi_{0}^{\prime}=\xi_{0}, \\
i^{\frac{n-1}{2}} \sqrt{n} \xi_{0}^{\prime}=\xi_{0}+2 \xi_{1}+2 \xi_{2}+\cdots+2 \frac{\xi_{n-1}}{2}, \\
\xi_{0}^{\prime}=\left(\frac{\alpha}{n}\right) \xi_{0} .
\end{gathered}
$$

Die Reihen (5) und (6) sind für $|q|<1$ konvergent und weisen keine negativen Exponenten der Entwicklungsgröße $q$ auf. Da $\Delta$ eine „ganze“ Modulform erster Stufe ist (vgl. I, 305), so sind die Modulformen $x_{\lambda}$ und $\xi_{\lambda}$ im Innern der $\omega$-Halbebene polfrei und bleiben auch für $q=0$, a.h. in der nach $\omega=i \infty$ ziehenden Spitze der Kreisbogendreiecke der $\omega$-Halbebene endlich. Der eben erkannte Satz, daß sich die $x_{\lambda}$ und auch die $\xi_{\lambda}$ gegenüber den Substitutionen der $\Gamma^{(\omega)}$ linear und homogen substituieren, führt von hieraus noch zu einer wichtigen Folgerung. Schreiben wir die einzelne Substitution der $\Gamma^{(\omega)}$ nicht-homogen in der Gestalt:

$$
\omega^{\prime}=\frac{\alpha \omega+\beta}{\gamma \omega+\delta},
$$

so nähert sich $\omega^{\prime}$ vom Innern des Dreiecksnetzes dem rationalen Punkte $\frac{\alpha}{\gamma}$, falls $\omega$ in die Spitze $i \infty$ wandert. Da sich nun die $x_{\lambda}$ und ebenso die $\xi_{\lambda}$ bei Ausübung der Substitution (9) linear und ganz substituieren, so werden diese Größen, auch wenn wir uns im Innern eines einzelnen Dreiecks der w-Halbebene einem rationalen Punkte $\frac{\alpha}{\gamma}$ annähern, endlich bleiben. Im AnschluB an die in I, 305 eingeführte Bezeichnung nennen wir dieserhalb die $x_{\lambda}$ und $\xi_{\lambda}$,ganze" Modulformen $n^{\text {ter Stufe. Insbesondere }}$ erweisen sie sich in einem Diskontinuitätsbereiche der Hauptkongruenzgruppe $n^{\text {ter }}$ Stufe $\Gamma_{n \chi(n)}$ überall als polfrei, eine Eigenschaft, die späterhin $\mathrm{zu}$ wichtigen Folgerungen den Grund legt.

\section{Ein weiteres System von Modulformen für ungerade Stufen.}

Da weiterhin Funktionssysteme $X_{\lambda}$ für verschiedene Grade $\boldsymbol{n}$ miteinander kombiniert werden sollen, so erscheint es nötig, den Grad $n$ als aberen Index am $X_{\lambda}$ anzubringen. Wir ziehen nun die drei Funktionen $X_{\lambda}^{(3)}\left(u_{1} \mid \omega_{1}, \omega_{2}\right)$ und die $3 n$ Funktionen $X_{\lambda}^{(3 n)}\left(u_{2} \mid \omega_{1}, \omega_{2}\right)$ heran, unter $n$ 
eine ungerade, gegen 3 teilerfremde Zahl verstanden; jedes System sei für ein besonderes erstes Argument $u_{1}$ bzw. $u_{2}$ gebildet, während das Periodenpaar in beiden Funktionen das gleiche sein soll. Aus beiden Funktionssystemen setzen wir die folgenden dreigliedrigen Ausdrücke zusammen:

(1) $\quad B_{\lambda}\left(u_{1}, u_{2} \mid \omega_{1}, \omega_{2}\right)=X_{0}^{(3)} X_{3 \lambda}^{(3 n)}+X_{1}^{(3)} X_{3 \lambda+n}^{(3 n)}+X_{2}^{(3)} X_{3 \lambda+2 n}^{(3 n)}$,

die wir "bilinear" nennen und dieserhalb durch das Symbol $B$ bezeichnen, weil sie in den $X$ jedes der beiden Systeme linear aufgebaut sind. Ändert man $\lambda$ um $n$, so ändert sich der untere Index der einzelnen Funktion $X^{\left({ }^{3} n\right)}$ um $3 n$, wobei sie nach der Regel (9) S. 305 unverändert bleibt. Aus dem Ansatze (1) entstehen also im ganzen höchstens $n$ verschiedene bilineare Verbindungen $B_{0}, B_{1}, \ldots, B_{n-1}$.

Dieser Ansatz soll in $\$ 6$ auf mehrgliedrige bilineare Ausdrücke der $X_{\lambda}$ verallgemeinert werden. Im vorliegenden besonderen Falle setzen wir sogleich $u_{1}=0$ und können dann bei $u_{2}$ den Index 2 fortlassen. Da $X_{0}^{(3)}(0)$ identisch verschwindet und $X_{1}^{(3)}(0)=-X_{2}^{(3)}(0)=x_{1}^{(3)}$ ist, so gelangen wir zu den $n$ Funktionen:

$$
B_{\lambda}\left(u \mid \omega_{1}, \omega_{2}\right)=x_{1}^{(3)}\left(X_{3 \lambda+n}^{(3 n)}-X_{3 \lambda-n}^{(3 n)}\right) .
$$

Für $x_{1}^{(3)}$ folgt aus (5) S. 315 die Darstellung:

$$
x_{1}^{(3)}=i \sqrt{\frac{2 \pi}{\omega_{2}}} \sqrt[8]{\Delta^{5}} \sum_{v=-\infty}^{+\infty}(-1)^{v} q^{\frac{(6 v+1)^{2}}{12}},
$$

so daB man zufolge (9) in I, 433 findet:

$$
x_{1}^{(3)}=i(\sqrt[3]{\Delta})^{2} .
$$

An Stelle vọ̣ (2) können wir also auch schreiben:

$$
B_{\lambda}\left(u \mid \omega_{1}, \omega_{2}\right)=i(\sqrt[3]{\Delta})^{2}\left(X_{3 \lambda+n}^{(3 n)}-X_{3 \lambda-n}^{(3 n)}\right) .
$$

Bei Ausübung der Substitution $S$ nimmt $\sqrt[3]{\Delta}$ den Faktor $\varrho=e^{\frac{2 i \pi}{3}}$ an (vgl. (13) in I, 455), während das Verhalten der $X^{(3 n)}$ aus der ersten Regel (1) S. $308 \mathrm{zu}$ bestimmen ist. Man findet:

$$
B_{\lambda}\left(u \mid \omega_{1}+\omega_{2}, \omega_{2}\right)=\rho^{2-n} \varepsilon^{-3 \frac{\lambda(n-\lambda)}{2}} B_{\lambda}\left(u \mid \omega_{1}, \omega_{2}\right) .
$$

Gegenüber $T$ bleibt $\sqrt[3]{\Delta}$ und also $x_{1}^{(3)}$ unverändert. Das aus der ersten Gleichung (16) S. 312 abzuleitende Verhalten $\operatorname{der} X^{(3 n)}$ ergibt:

$$
\begin{aligned}
& i^{\frac{3 n-1}{2}} \sqrt{3 n} B_{\lambda}\left(u \mid \omega_{2},-\omega_{1}\right) \\
& =x_{1}^{(3)} \sum_{x=0}^{3 n-1}\left(e^{\frac{2 \pi i}{3 n} \times(3 \lambda+n)}-e^{\frac{2 \pi i}{3 n} *(3 \lambda-n)}\right) X_{x}^{(3 n)} .
\end{aligned}
$$


Der Faktor von $X_{x}^{(3 n)}$ unter dem Summenzeichen läßt sich kürzer in die Gestalt $\left(\varrho^{x}-\varrho^{-x}\right) \varepsilon^{x \lambda}$ setzen. Da er verschwindet, so oft $x$ durch 3 teilbar ist, so genügt es, $x=3 x^{\prime}+n$ und $x=3 x^{\prime}-n$ zu schreiben, wo dann beide Male $x^{\prime}$ die Zahlen $0,1,2, \ldots, n-1$ zu durchlaufen hat. Man erhält dabei:

$$
\left(\varrho^{x}-\varrho^{-x}\right) \varepsilon^{x \lambda}=\left(\varrho^{ \pm n}-\varrho^{\mp n}\right) \varepsilon^{3 x^{\prime} \lambda}= \pm\left(\frac{n}{3}\right) i \sqrt{3} \varepsilon^{3 x^{\prime} \lambda},
$$

wo $\left(\frac{n}{3}\right)$ das Legendresche Zeichen ist. Somit findet sich:

$$
i^{\frac{3 n-1}{2}} \sqrt{3 n} B_{\lambda}\left(u \mid \omega_{2},-\omega_{1}\right)=i\left(\frac{n}{3}\right) \sqrt{3} \sum_{x=0}^{n-1} \varepsilon^{3 x \lambda} x_{1}^{(3)}\left(X_{3 x+n}^{(3 n)}-X_{3 x-n}^{(3 n)}\right) .
$$

Nimmt man die vor dem Summenzeichen stehenden Faktoren nach links hinüber; so ziehen sie sich mit den Faktoren von $B_{\lambda} \mathrm{zu}$ :

$$
i^{\frac{3 n-3}{2}}\left(\frac{n}{3}\right) \sqrt{n}=(-1)^{\frac{n-1}{2}}\left(\frac{n}{3}\right) \cdot i^{\frac{n-1}{2}} \sqrt{n}=i^{\frac{n-1}{2}}\left(\frac{3}{n}\right) \sqrt{n}
$$

zusammen, wie man mit Hilfe des auf das Legendre-Jacobische Zeichen verallgemeinerten Reziprozitätsgesetzes findet. ${ }^{1}$ ) Als Wirkung von $T$ hat man also:

$$
i^{\frac{n-1}{2}}\left(\frac{3}{n}\right) \sqrt{n} B_{\lambda}\left(u \mid \omega_{2},-\omega_{1}\right)=\sum_{x=0}^{n-1} \varepsilon^{3 \times \lambda} B_{x}\left(u \mid \omega_{1}, \omega_{2}\right) .
$$

Ist $n \equiv 2(\bmod 3)$, so liegen in $(5)$ und $(6)$ genau wieder die $X_{\lambda^{-}}$ Substitutionen $S$ und $T$ von S. 314 vor, nur ist an Stelle von $\varepsilon$ die gleichfalls primitive $n^{\text {te }}$ Einheitswurzel $\varepsilon_{3}=\varepsilon^{3}$ getreten. Zum Zwecke dieses Ersatzes hat man $i^{\frac{n-1}{2}} \sqrt{n}$ in Gestalt der GauBschen Summe (21) S. 314 darzustellen, worauf dann nach der letzten Gleichung in I, 494 beim Ersatze von $\varepsilon$ durch $\varepsilon_{3}$ sich der Faktor $\left(\frac{3}{n}\right)$ einfindet. Im Falle $n \equiv 1$ (mod 3) benutzen wir nochmals den Unstand, daß $\sqrt[3]{\Delta}$ gegenüber $S$ den Faktor $\varrho$ annimmt und durch $T$ in sich transformiert wird. Also wird im Falle $n \equiv 1(\bmod 3)$ der Quotient $B_{\lambda}: \sqrt[3]{\Delta}$ die $X_{\lambda}$-Substitutionen unter Ersatz von $\varepsilon$ durch $\varepsilon_{3}$ erfahren.

Das zu erklärende System von Modulformen gewinnt man nun aus den $B_{\lambda}$ durch Einsetzung von $u=0$. Aus der Regel (16) S. 307 leitet man leicht:

$$
B_{\lambda}\left(-u \mid \omega_{1}, \omega_{2}\right)=B_{n-\lambda}\left(u \mid \omega_{1}, \omega_{2}\right)
$$

ab, so daB man für $u=0 \mathrm{zu}$ einem System von $\frac{n+1}{2}$ Modulformen $n^{\text {ter }}$ 4. Aufl.

1) Vgl. Dirichlet-Dedekind, „Vorles. über Zahlentheorie“, S. $104 \mathrm{ff}$. der 
Stufe gelangt, wenn man im Falle $n \equiv 1(\bmod 3)$ statt der Nullwerte von $B_{\lambda}$ diejenigen der Quotienten $B_{\lambda}: \sqrt[3]{\Delta}$ benutzt. Zur Vereinfachung einer sogleich anzugebenden Darstellung des Nullwertes von $B_{0}$ versehen wir alle Nullwerte noch mit dem gemeinsamen Faktor $-\frac{1}{2}$ und bezeichnen die damit gewonnenen Modulformen durch $z_{\lambda}\left(\omega_{1}, \omega_{2}\right)$. In den S. 314 eingeführten Formen $x$ gelten also die Darstellungen:

$$
\begin{cases}z_{\lambda}\left(\omega_{1}, \omega_{2}\right)=-\frac{1}{2} i \sqrt[3]{\Delta}\left(x_{3 \lambda+n}^{(3 n)}-x_{3 \lambda-n}^{(3 n)}\right), & n \equiv 1(\bmod 3) \\ z_{\lambda}\left(\omega_{1}, \omega_{2}\right)=-\frac{1}{2} i \sqrt[3]{\Delta^{2}}\left(x_{3 \lambda+n}^{(3 n)}-x_{3 \lambda-n}^{(3 n)}\right), & n \equiv 2(\bmod 3)\end{cases}
$$

Als Ergebnis merken wir an: Für jede gegen 6 teilerfremde Stufe $n$ existiert ein System von $\frac{n+1}{2}$ durch (7) gegebener ganzer Modulformen $n^{\text {ter }}$ Stufe, die sich bei Ausïbung der Substitutionen $S$ und $T$ in folgender Art substituieren:

$$
\begin{aligned}
& z_{\lambda}^{\prime}=\varepsilon_{3}^{-\frac{\lambda(n-\lambda)}{2}} z_{\lambda}, \\
& i^{\frac{n-1}{2}}\left(\frac{3}{n}\right) \sqrt{n} z_{\lambda}^{\prime}=z_{0}+\sum_{x=1}^{\frac{n-1}{2}}\left(\varepsilon_{3}^{x \lambda}+\varepsilon_{3}^{-x^{\lambda}}\right) z_{x} \text {, }
\end{aligned}
$$

unter $\varepsilon_{3}$ die primitive $n^{\text {te }}$ Einheitswurzel $\varepsilon^{3}$ verstanden.

Da die beiden Modulformen $x_{n}^{(3 n)}$ und $x_{-n}^{(3 n)}=x_{2 n}^{(3 n)}$ sich nur im Vorzeichen unterscheiden, so gilt speziell für die Form $z_{0}$ :

$$
z_{0}=-i \sqrt[3]{\Delta} x_{n}^{(3 n)} \quad \text { bzw. } \quad z_{0}=-i \sqrt[3]{\Delta^{2}} x_{n}^{(3 n)} .
$$

Aus der Reihendarstellung (5) S. 315 der $x$ aber ergibt sich:

$$
x_{n}^{(3 n)}=i \sqrt{\frac{2 \pi}{\omega_{2}}} \sqrt[8]{\Delta}(\sqrt[4]{\Delta})^{(3 n)_{0}} \sum_{\nu=-\infty}^{+\infty}(-1)^{\nu} q^{\frac{(6 v+1)^{2} n}{12}} .
$$

Damit sind wir zur Reihe (9) in I, 433 für die $24^{\text {ste }}$ Wurzel der Diskriminante geführt. Verstehen wir unter $\Delta^{\prime}$ die transformierte Diskriminante $\Delta^{\prime}=\Delta\left(n \omega_{1}, \omega_{2}\right)$, so ergibt sich der folgende Satz: Die Modulform $n^{\text {ter }}$ Stufe $z_{0}\left(\omega_{1}, \omega_{2}\right)$ stellt sich in der Diskriminante wie folgt dar:

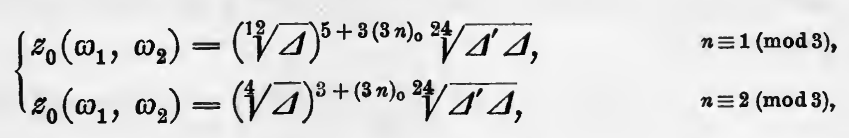

$n$ ist eine beliebige gegen 6 teilerfremde Stufe, $\Delta^{\prime}$ hat die Bedeutung $\Delta\left(n \omega_{1}, \omega_{2}\right)$ und $(3 n)_{0}$ ist die kleinste, nicht negative ganze Zahl, die der Kongruenz genügt:

$$
(3 n)_{0} \equiv-\frac{3 n+1}{2}(\bmod 4) .
$$




\section{$\$$ 6. Mehrgliedrige Bilinearverbindungen der $\boldsymbol{X}_{2}$ und ihre lineare Transformation.}

Es soll jetzt der Ansatz (1) S. 317 der dreigliedrigen Bilinearverbindungen der $X_{\lambda}$ auf entsprechende Ausdrücke mit einer beliebigen Gliederanzahl $l$ verallgemeinert werden. Wir denken $n$ nach wie vor als ungerade Zabl gewählt und setzen $l$ (wie oben 3) als teilerfremd gegen $n$ voraus. Die zu untersuchende Bilinearverbindung der Funktionen $X^{(n)}$ und $X^{(l n)}$ erklären wir durch:

$$
B_{\lambda}\left(u_{1}, u_{2} \mid \omega_{1}, \omega_{2}\right)=\sum_{x=0}^{l-1} X_{\psi}^{(l)}\left(u_{1}\right) X_{l \lambda+n k \%}^{(l n)}\left(u_{2}\right),
$$

die für $l=3, k=1$ den Ansatz (1) S. 317 wieder liefert. Um diesen Ausdrücken bei beliebigem $l$ möglichst einfache Eigenschaften zu erteilen, verstehen wir unter $k$ eine der Kongruenz:

$$
n k^{2}+1 \equiv 0 \quad(\bmod 2 l) \quad \text { bzw. } \quad(\bmod l)
$$

genügende ganze Zahl, je nachdem $l$ gerade oder ungerade ist. Wir können dieser Kongruenz entsprechend auch setzen:

$$
n k^{2}+1=2 l m \quad \text { bzw. } n k^{2}+1=l m,
$$

unter $m$ eine weitere ganze Zahl verstanden, die wie $l$ offenbar positiv ist. Im Falle eines geraden $l$ gilt die erste Gleichung (3), aus der hervorgeht, daB $k$ in diesem Falle ungerade ist. Dann ist $k^{2} \equiv 1(\bmod 4)$, und da $2 l m$ durch 4 teilbar ist, so ergibt sich, daß im Falle eines geraden $l$ die ungerade Zahl $n$ der Beschränkung $n \equiv 3(\bmod 4)$ unterliegt. Indem wir der Reihe nach $\lambda=0,1,2, \ldots, n-1$ in (1) einsetzen, erhalten wir ein System von $n$ bilinearen Ausdrücken. Das Periodenpaar $\omega_{1}, \omega_{2}$ soll in allen Funktionen $X^{(l)}$ und $X^{(l n)}$ dasselbe sein. Es ist zunächst festzustellen, wie sich unser Größensystem $B_{0}, B_{1}, \ldots, B_{n-1}$ bei linearer Transformation der Perioden verhält.

Ist erstlich $l$ und also $l n$ gerade, so nimmt das Produkt:

$$
X_{\%}^{(l)}\left(u_{1}\right) X_{l i+n k \%}^{(l n)}\left(u_{2}\right)
$$

zufolge der zweiten Formel (1) S. 308 gegenüber der Substitution $S$ den Faktor an:

$$
e^{\frac{2 i \pi}{l}\left(\frac{l}{8}+\frac{x^{2}}{2}\right)+\frac{2 i \pi}{l n}\left(\frac{l n}{8}+\frac{(l \lambda+n k x)^{2}}{2}\right)}=i e^{\frac{i \pi}{l n}\left(n x^{2}+l^{2} \lambda^{2}+n^{2} k^{2} x^{2}\right)} .
$$

Mit Hilfe der ersten Gleichung (3) führt man den Ausdruck dieses Faktors leicht über in:

$$
i e^{\frac{i \pi}{l n}\left(\eta^{2} \lambda^{2}+2 l m n \varkappa^{2}\right)}=i e^{\frac{l i \pi}{n} \lambda^{2}}=i \varepsilon_{l}^{-\frac{\lambda(n-\lambda)}{2}},
$$


wo $\varepsilon_{l}$ die primitive $n^{\text {to }}$ Einheitswurzel $\varepsilon^{l}$ ist. Ist $l$ ungerade, so nimmt das Produkt (4) gegenüber $S$ den Faktor an:

$$
e^{\frac{\pi i}{l}\left(x^{2}-l x\right)+\frac{\pi i}{l n}\left((l \lambda+n k x)^{2}-l n(l \lambda+n k x)\right)}=(-1)^{2+x+k x} e^{\frac{\pi i}{l n}\left(n x^{2}+l^{2} z^{2}+n^{2} k^{2} x^{2}\right)},
$$

wie aus der ersten Gleichung (1) S. 308 folgt. Mit Hilfe der zweiten Gleichung (3) und der aus ihr folgenden Kongruenz $1+k+m \equiv 0(\bmod 2)$ wandelt man die Gestalt des eben erhaltenen Faktors in:

$$
(-1)^{\lambda} e^{\frac{\pi i}{n} l \lambda^{2}}=\varepsilon_{l}^{-\frac{2(n-\lambda)}{2}}
$$

um. Hiernach erweist sich der Faktor in beiden Fällen als von $x$ unabhängig, so daB er für alle Glieder der Summe (1) ein und derselbe ist. Die Bilinearverbindung (1) nimmt also gegenüber $S$ den Faktor:

$$
i \varepsilon_{l}^{-\frac{\lambda(n-\lambda)}{2}} \text { bzw. } \varepsilon_{l}^{-\frac{\lambda(n-\lambda)}{2}}
$$

an, je nachdem $l$ gerade oder ungerade ist.

Um die Wirkung der Substitution $T$ festzustellen, schreiben wir die Faktoren von $X_{\lambda}$ auf den linken Seiten der Gleichungen (16) S. 312 kurz $C_{n}$, so daB:

$$
C_{n}=i^{\frac{n-1}{2}} \sqrt{n} \text { oder }=-\sqrt{n}
$$

gilt, je nachdem $n$ ungerade oder gerade ist. Aus den eben genannten Gleichungen findet man dann:

$$
C_{l} C_{l n} B_{\lambda}\left(u_{1}, u_{2} \mid \omega_{2},-\omega_{1}\right)=\sum_{x=0}^{l-1} \sum_{\mu=0}^{l-1} \sum_{\nu=0}^{l n-1} e^{\frac{2 i \pi}{l n}(n \times \mu+l 2 v+n k \times v)} X_{\mu}^{(l)} X_{\nu}^{(l n)}
$$

wo die rechts stehenden Funktionen $X$ für die ursprünglichen Perioden $\omega_{1}, \omega_{2}$ gebildet sind. Nimmt man die Summation in bezug auf $x \mathrm{zu}$ nächst vor, so folgt:

$$
C_{l} C_{l n} B_{2}\left(u_{1}, u_{2} \mid \omega_{2},-\omega_{1}\right)=\sum_{\mu, v}\left(\varepsilon^{\lambda v} X_{\mu}^{(l)} X_{v}^{(l n)} \sum_{x=0}^{l-1} e^{\frac{2 i \pi}{l} x(\mu+k v)}\right) .
$$

Die innere Summe hat für die einzelne Zahlkombination $\mu, \nu$ stets den Wert 0 , falls $\mu+k \nu \neq 0(\bmod l)$ ist, dagegen den Wert $l$, wenn $\mu+k \nu$ $\equiv 0(\bmod l)$ zutrifft. Die letzte Gleichung vereinfacht sich demnach zu:

$$
C_{l} C_{l n} B_{\lambda}\left(u_{1}, u_{2} \mid \omega_{2}^{\prime},-\omega_{1}\right)=l \sum_{v=0}^{l n-1}\left(\varepsilon^{\lambda v} X_{-k v}^{(l)} X_{v}^{(l n)}\right) .
$$

Über die frei gewordenen Summationsbuchstaben $x$ und $\mu$ können wir jetzt im neuen Sinne verfügen. Wir setzen $\nu=l x+n \mu$ und erhalten ein System $\bmod l n$ inkongruenter Zahlen $\nu$, falls $x$ die Zahlen $0,1, \ldots, n-1$ und $\mu$ die Zahlen $0,1, \ldots, l-1$ durchlaufen: 


$$
C_{l} C_{l n} B_{\lambda}\left(u_{1}, u_{2} \mid \omega_{2},-\omega_{1}\right)=l \sum_{x=0}^{n-1}\left(\varepsilon^{l \times \lambda} \sum_{\mu=0}^{l-1} X_{-k n \mu}^{(l)} X_{l x+n \mu}^{(l n)}\right) .
$$

Da $k n$ zufolge (3) teilerfremd gegen $l$ ist, so durchläuft, falls man $-k n \mu \equiv \nu(\bmod l)$ setzt und auf diese Wejse auch $\nu$ in neuer Bedeutung gebraucht, mit $\mu$ auch $\nu$ ein Restsystem $\bmod l$. Durch Multiplikation der letzten Kongruenz mit $k$ und weiter mit $n$ folgt aber wegen (2):

$$
-k^{2} n \mu \equiv \mu \equiv k \nu \quad(\bmod l), \quad n \mu \equiv k n \nu \quad(\bmod l n) .
$$

Die innere Summe der letzten Gleichung läßt sich also auch so schreiben:

$$
\sum_{v=0}^{l-1} X_{v}^{(l)} X_{l x+n k v}^{(l n)}
$$

und erweist sich also zufolge (1) als mit $B_{\varkappa}$ identisch:

$$
C_{l} C_{l n} B_{\lambda}\left(u_{1}, u_{2} \mid \omega_{2},-\omega_{1}\right)=l \sum_{z=0}^{n-1} \varepsilon_{l}^{* \lambda} B_{x}\left(u_{1}, u_{2} \mid \omega_{1}, \omega_{2}\right) .
$$

Ist nun erstlich $l$ gerade, so ist $C_{l} \cdot C_{l n}=l \sqrt{n}$. Es läßt sich zeigen, daB in diesem Falle das Legendre-Jacobische Zeichen $\left(\frac{l}{n}\right)$ der Gleichung:

$$
\left(\frac{l}{n}\right)=i^{\frac{n+1}{2}}
$$

genügt. Schreibt man nämlich $l=2^{h} \cdot l^{\prime}$, unter $l^{\prime}$ den gröBten ungeraden Teiler von $l$ verstanden, so gilt, da zufolge (2) die Zahl $-n$.quadratischer Rest von $l^{\prime}$ ist, zufolge des verallgemeinerten Reziprozitätsgesetzes (s. die Note S. 318):

$$
1=\left(\frac{-n}{l^{\prime}}\right)=(-1)^{\frac{l^{\prime}-1}{2} \cdot \frac{n+1}{2}}\left(\frac{l^{\prime}}{n}\right)=\left(\frac{l^{\prime}}{n}\right),
$$

da jetzt $n \equiv \mathbf{3}(\bmod 4)$ ist. Man findet also:

$$
\left(\frac{l}{n}\right)=\left(\frac{2}{n}\right)^{h}\left(\frac{l^{\prime}}{n}\right)=\left(\frac{2}{n}\right)^{h}=(-1)^{n^{n^{2}-1}} 8 .
$$

Ist $h=1$, so ist $\left(\frac{l}{n}\right)=+1$ oder -1 , je nachdem $n \equiv 7$ oder $\equiv 3$ (mod 8) gilt. In beiden Fällen erweist sich die Gleichung (7) als richtig. Ist $h>1$, so folgt $n \equiv 7(\bmod 8)$ aus (2). Nun ist $\left(\frac{l}{n}\right)$ zufolge gleich 1, so daB die Gleichung (7) auch jetzt sich als richtig erweist. Trägt man in (6) für $C_{l} C_{l n}$ seinen Wert $l \sqrt{n}$ ein und benutzt die Gleichung (7), so folgt: Im Falle eines geraden $l$ zeigen die $n$ Bilinearverbindungen (1) bei Ausiibung der Substitution T das Verhalten:

$$
i^{\frac{n-1}{2}}\left(\frac{l}{n}\right) \sqrt{n} B_{\lambda}\left(u_{1}, u_{2} \mid \omega_{2},-\omega_{1}\right)=-i \sum_{x=0}^{n-1} \varepsilon_{l}^{* \lambda} B_{x}\left(u_{1}, u_{2} \mid \omega_{1}, \omega_{2}\right) \text {. }
$$


Im Falle eines ungeraden $l$ gilt:

$$
C_{l} C_{l n}=i^{\frac{l-1}{2}} \sqrt{l} \cdot i^{\frac{l n-1}{2}} \sqrt{l n}=(-1)^{\frac{l-1}{2} \cdot \frac{n+1}{2}} \cdot i^{\frac{n-1}{2}} l \sqrt{n} .
$$

$\mathrm{Da}-n$ zufolge (2) quadratischer Rest von $l$ ist, so gilt:

$$
1=\left(\frac{-n}{l}\right)=(-1)^{\frac{l-1}{2} \cdot \frac{n+1}{2}}\left(\frac{l}{n}\right), \quad(-1)^{\frac{l-1}{2} \cdot \frac{n+1}{2}}=\left(\frac{l}{n}\right)
$$

Im Falle eines ungeraden $l$ transformieren sich die $n$ Ausdrïcke $B_{\lambda}$ gegenïber der Substitution $T$ nach der Regel:

$$
i^{\frac{n-1}{2}}\left(\frac{l}{n}\right) \sqrt{n} B_{\lambda}\left(u_{1}, u_{2} \mid \omega_{2},-\omega_{1}\right)=\sum_{x=0}^{n-1} \varepsilon_{l}^{x \lambda} B_{x}\left(u_{1}, u_{2} \mid \omega_{1}, \omega_{2}\right) .
$$

Nach (10) in I, 454 zeigt die Modulform vierter Stufe $\sqrt[4]{\Delta}$ gegenüber $S$ und $T$ das Verhalten:

$$
\sqrt[4]{\Delta}\left(\omega_{1}+\omega_{2}, \omega_{2}\right)=i \sqrt[4]{\Delta}\left(\omega_{1}, \omega_{2}\right), \quad \sqrt[4]{\Delta}\left(\omega_{2},-\omega_{1}\right)=-i \sqrt[4]{\Delta}\left(\omega_{1}, \omega_{2}\right) .
$$

Im Falle eines geraden $l$ zeigen demnach die $n$ Quotienten $B_{\lambda}: \sqrt[4]{\Delta}$ gegenüber linearen Transformationen der Perioden genau das gleiche Verhalten wie die $B_{\lambda}$ mit ungeradem $l$.

Nun gehen die Koeffizienten der linearen Transformationen $S$ und $T$ der $B_{2}: \sqrt[4]{\Delta}$ bzw. der $B_{\lambda}$ aus den Koeffizienten der entsprechenden $X_{\lambda}$-Transformationen dadurch hervor, daß man die primitive $n^{\text {te }}$ Einheitswurzel $\varepsilon$ durch die gleichfalls primitive $n^{\text {te }}$ Einheitswurzel $\varepsilon_{l}$ ersetzt.

Der Ausdruck $i^{\frac{n-1}{2}} \sqrt{n}$ ist dabei als ganze Zahl des Kreisteilungskörpers $(\Re, \varepsilon)$ in der Gestalt (21) S. 314 darzustellen. Die SchluBformel in I, 494 lehrt dann eben, daB $i^{\frac{n-1}{2}} \sqrt{n}$ beim Ersatz von $\varepsilon$ durch $\varepsilon_{l}$ das Vorzeichen $\left(\frac{l}{n}\right)$ als Faktor annimmt. Im Anschluß an die Ergebnisse von S. 314 finden wir den Satz: Gegenüber der Gesamtgruppe $\Gamma^{(\omega)}$ aller linearen Periodentransformationen erfahren die n Funktionen $B_{\lambda}: \sqrt[4]{\Delta}$ bzw. $B_{\lambda}$ eine Gruppe $G_{n \chi(n)}$ von $n \chi(n)$ linearen Transformationen, welche mit den entsprechenden $X_{\lambda}$-Transformationen als, „konjugiert" $z u$ bezeichnen sind, insofern sie aus jenen durch Ersatz von $\varepsilon$ durch die gleichfalls primitive $n^{\text {te }}$ Einheitswurzel $\varepsilon_{l}$ hervorgehen. Hieraus folgt insbesondere: Die $n$ Funktio-

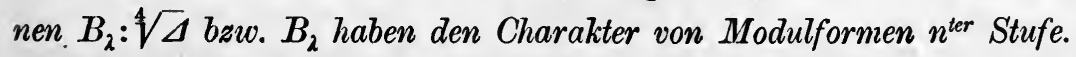

Eine rationale Zahl des Kreisteilungskörpers $(\Re, \varepsilon)$ ist nur mit sich selbst konjugiert. Rational aber sind die Koeffizienten aller $X_{\lambda}$-Transformationen, die den Substitutionen mit $\beta \equiv \gamma \equiv 0(\bmod n)$ entsprechen: Für alle der Kongruenz $\beta \equiv \gamma \equiv 0(\bmod n)$ genügenden Substitutionen sind die Transformationen der $B_{2}: \sqrt[4]{\Delta}$ bzw. der $B_{\lambda}$ genau so gebaut wic diejenigen der $X_{\lambda}$. 


\section{§ \%. Die Systeme der Funktionen $\boldsymbol{Y}_{\lambda}$ und der Modulformen $y_{\lambda}$.}

Für den Fall eines geraden $l$ und also einer der Kongruenz $n \equiv 3$ (mod 4) genügenden Zahl $n$ sollen jetzt Reihendarstellungen der bilinearen Ausdrücke entwickelt werden: Indem man für die $X_{\lambda}$ ihre Reihen (13) S. 306 einträgt, gewinnt man folgenden Ansatz:

$$
\frac{B_{\lambda}}{\sqrt[4]{\Delta}}=\frac{2 \pi}{\omega_{2}} e^{\frac{l \eta_{2}}{2 \omega_{2}}\left(u_{1}^{2}+n u_{2}^{2}\right)} \sum_{x, v_{1}, v_{2}} q^{\frac{n v^{2}+N^{2}}{l n}} e^{\frac{2 \pi i}{\omega_{2}}\left(v u_{1}+N u_{2}\right)}
$$

dabei haben $\nu$ und $N$ die Bedeutungen:

$$
\nu=\nu_{1} l-x, \quad N=\nu_{2} n l-l \lambda-n k x,
$$

von den Summationsbuchstaben durchläuft $x$ die Zahlen $0,1, \ldots, l-1$, während $\nu_{1}$ und $\nu_{2}$ unabhängig voneinander alle ganzen Zahlen durchlaufen. Die Zahl $\nu$ nimmt somit gerade einmal jeden ganzzahligen Wert an. Für das einzelne $\nu$ ist $\varkappa$ der kleinste, nicht-negative Rest von $-\nu$ $\bmod l$, und weiter berechnet sich $\nu_{1}$ aus der ersten Gleichung (1). Die ganze Zahl $N$ läßt sich in die Gestalt setzen:

$$
N=n k \nu+l \mu, \quad \mu=\nu_{2} n-\lambda-\nu_{1} k n .
$$

Bei gegebenem $\nu$ und also bestimmtem $\nu_{1}$ durchläuft die durch die zweite Gleichung erklärte ganze Zahl $\mu$ wegen der Bedeutung von $\nu_{2}$ gerade einmal alle $\bmod n$ mit $-\lambda$ kongruenten ganzen Zahlen. Bei Benutzung der ersten Gleichung (3) S. 320 findet man nun:

$$
n \nu^{2}+N^{2}=n \nu^{2}+(n k \nu+l \mu)^{2}=2 l\left(\frac{l}{2} \mu^{2}+k n \mu \nu+m n \nu^{2}\right) .
$$

Den hier rechts stehenden Klammerausdruck fassen wir als eine in den variablen ganzen Zahlen $\mu, \nu$ geschriebene ganzzahlige binäre quadratische Form:

$$
f(\mu, \nu)=\frac{l}{2} \mu^{2}+k n \mu \nu+m n \nu^{2}
$$

auf, für die wir nach früherem Brauche auch die symbolische Bezeichnung $\left(\frac{l}{2}, k n, m n\right)$ benutzen (vgl.S. 137 ff.). Die quadratische Form $f(\mu, v)$ hat die negative Diskriminante:

$$
D=k^{2} n^{2}-2 \operatorname{ll} n n=-n,
$$

sie ist positiv und ursprünglich, da zufolge (3) S. 320 die beiden ersten Koeffizienten $\frac{l}{2}, k n$ teilerfremd sind.

An Stelle der bisherigen Variablen $u_{1}, u_{2}$ führt man jetzt zweckmäBig zwei neue Variable $u, v$ durch:

$$
u_{1}=u+\frac{n k}{l} v, \quad u_{2}=-\frac{v}{l}
$$


Die Funktionen $Y_{\lambda}\left(u, v \mid \omega_{1}, \omega_{2}\right)$ und die Modulformen $y_{\lambda}\left(\omega_{1}, \omega_{2}\right)$

ein. Dann wird nämlich einerseits:

$$
l\left(u_{1}^{2}+n u_{\Omega}^{2}\right)=l\left(u^{2}+2 \frac{n k}{l} u v+\frac{n^{2} k^{2}+n}{l^{2}}+v^{2}\right)=2 f(u, v),
$$

und andererseits gilt:

$$
\nu u_{1}+N u_{2}=\nu\left(u+\frac{n k}{l} v\right)-(n k \nu+l \mu) \frac{v}{l}=\nu u-\mu v .
$$

In Abhängigkeit von $u$ und $v$ mögen nun die Quotienten der Bilinearverbindungen $B_{\lambda}$ und der Modulform $\sqrt{\Delta}$ durch $Y_{\lambda}(u, v)$ bezeichnet werden:

$$
\frac{B_{\lambda}\left(u+\frac{n k}{l} v,-\frac{v}{l}\right)}{\sqrt[4]{\Delta}}=Y_{\lambda}\left(u, v \mid \omega_{1}, \omega_{2}\right) .
$$

Als Reihendarstellung dieser Funktionen $Y_{\lambda}$ gewinnt man:

$$
Y_{\lambda}\left(u, v \mid \omega_{1}, \omega_{2}\right)=\frac{2 \pi}{\omega_{2}} e^{\frac{\eta_{2}}{\omega_{2}} f(u, v)} \sum_{\mu, v} q^{\frac{2 f(\mu, v)}{n}} e^{\frac{2 \pi i}{\omega_{2}}(v u-\mu v)},
$$

wo $f$ die quadratische Form (2) ist, $\mu$ alle der Kongruenz $\mu \equiv-\lambda(\bmod n)$ genügenden ganzen Zahlen und $\nu$ alle ganzen Zahlen durchlaufen.

Aus (5) folgt, daB die Funktion $Y_{0}(u, v)$ bei gleichzeitigem Zeichenwechsel von $u$ und $v$ unverändert bleibt, während die $(n-1)$ übrigen Funktionen $Y$ das Verhalten zeigen:

$$
Y_{\lambda}\left(-u,-v \mid \omega_{1}, \omega_{2}\right)=Y_{n-\lambda}\left(u, v \mid \omega_{1}, \omega_{2}\right) \text {. }
$$

Für $u=0, v=0$ liefern demgemä $\beta$ die Funktionen $Y_{\lambda}$ ein System von $\frac{n+1}{2}$ ganzen Modulformen der $n^{\text {ten }}$ Stufe $y_{0}, y_{1}, \ldots, y_{\frac{n-1}{2}}$, welche durch die Potenzreihen:

$$
y_{\lambda}\left(\omega_{1}, \omega_{2}\right)=\frac{2 \pi}{\omega_{2}} \sum_{\mu, v} q^{\frac{8 f(\mu, v)}{n}}, \quad \quad \mu \equiv-\lambda(\bmod n)
$$

darstellbar sind und gegenüber den linearen Periodentransformationen die S. 316 angegebenen $\xi_{\lambda}$-Substitutionen unter Ersatz von $\varepsilon$ durch $\varepsilon_{l}$ erfahren.

Von diesen Modulformen werden wir später insbesondere die erste $y_{0}\left(\omega_{1}, \omega_{2}\right)$ gebrauchen. Schreiben wir hier $\mu=\mu^{\prime} n$, so durchläuft $\mu^{\prime}$ alle ganzen Zahlen, und man hat $f(\mu, \nu)=n f^{\prime}\left(\mu^{\prime}, \nu\right)$, wo $f^{\prime}$ die quadratische Form $\left(\frac{1}{2} l n, k n, m\right)$ der Diskriminante $-n$ ist. Auch diese Form ist positiv und ursprünglich, da nach (3) S. 320 die ganzen Zahlen $m$ und in teilerfremd sind. Als Reihenentwicklung der Modulform $y_{0}$ folgt:

$$
y_{0}\left(\omega_{1}, \omega_{2}\right)=\frac{2 \pi}{\omega_{2}} \sum_{\mu, v} q^{2 f^{\prime}(\mu, v),}
$$

wo $\mu, \nu$ alle Paare ganzer Zahlen durchlaufen. Est ist nun in jeder Klasse 
ursprünglicher positiver Formen der Diskriminante $-n$ eine Form der Gestalt $\left(\frac{l}{2} n, k n, m\right)$ nachweisbar. Nach S. 141 können wir nämlich zunächst einer beliebig vorgelegten Klasse eine Form $(a, b, c)$ entnehmen, deren dritter Koeffizient $c$ eine gegen $n$ teilerfremde Zahl $m$ ist. Gehen wir sodann mittelst einer Transformation (4) S. 138 zu einer äquivalenten Form $\left(a^{\prime}, b^{\prime}, c^{\prime}\right)$, indem wir $\alpha=\delta=1, \beta=0$, wählen, so sind die Koeffizienten der neuen Form durch:

$$
a^{\prime}=a-\gamma b+\gamma^{2} m, \quad b^{\prime}=b-2 \gamma m, \quad c^{\prime}=m
$$

gegeben, und man kann wegen teilerfremder Zahlen $2 m$ und $n$ über $\gamma$ so verfügen, daß $b^{\prime}$ ein Vielfaches $k n$ von $n$ wird. Da die Diskriminante nach wie vor gleich $-n$ ist, so gilt:

$$
k^{2} n^{2}-4 a^{\prime} m=-n,
$$

so daß $a^{\prime}$ durch $n$ teilbar ist. Schreiben wir $2 \frac{a^{\prime}}{n}=l$, so haben wir in der vorgelegten Klasse eine Form $\left(\frac{1}{2} l n, k n, m\right)$ der gew ünschten Gestalt erreicht. Endlich können wir aber von der Form $f^{\prime}$ unserer Klasse durch eine ganzzahlige Substitution der Determinante 1:

$$
\mu=\alpha \mu^{\prime}+\beta \nu^{\prime}, \quad \nu=\gamma \mu^{\prime}+\delta \nu^{\prime}
$$

zu jeder beliebigen Form $(a, b, c)$ der Klasse zurückgehen. Es gilt:

$$
f^{\prime}(\mu, \nu)=a \mu^{\prime 2}+b \mu^{\prime} \nu^{\prime}+c \nu^{\prime 2}
$$

und da mit $\mu, \nu$ zufolge (8) auch $\mu^{\prime}, \nu^{\prime}$ genau alle Paare ganzer Zahlen durchlaufen, so haben wir bei Fortlassung der oberen Indizes an den neuen Summationsbuchstaben $\mu^{\prime}, \nu^{\prime}$ an Stelle von (7) die Reihendarstellung:

$$
y_{0}\left(\omega_{1}, \omega_{2}\right)=\frac{2 \pi}{\omega_{2}} \sum_{\mu, \nu} q^{2\left(a \mu^{2}+b \mu \nu+c \nu^{2}\right)} .
$$

Da mit $\mu, \nu$ auch immer das Zahlenpaar $\mu,-\nu$ auftritt, so ergeben zwei "entgegengesetzte" Formen $(a, b, c)$ und $(a,-b, c)$ eine und dieselbe Modulform $y_{0}\left(\omega_{1}, \omega_{2}\right)$. Unter Erinnerung an die Substitutionen $S$ und $U_{\alpha}$, deren Wirkung auf die $\xi_{\lambda}$ oben (S. 316) festgestellt wurde, und unter Zusammenfassung aller Ergebnisse haben wir folgenden für später grundlegenden Satz gewonnen: Im Falle einer die Kongruenz $n \equiv 3(\bmod 4)$ befriedigenden Stufe $n$ liefert jede zueiseitige Klasse ursprünglicher positiver quadratischer Formen der Diskriminante $-n$ und ebenso jedes Paar entgégengesetzter solcher Klassen eine ganze Modulform $n^{\text {ter }}$ Stufe (9) der Dimension -1 , die gegenüber den Substitutionen der durch $\gamma \equiv 0(\bmod n)$ erklärten Kongruenzyruppe $\Gamma_{\psi(n)}$ das Verhalien:

$$
y_{0}\left(\alpha \omega_{1}+\beta \omega_{2}, \gamma \omega_{1}+\delta \omega_{2}\right)=\left(\frac{\alpha}{n}\right) y_{0}\left(\omega_{1}, \omega_{2}\right)
$$


seigt; die quadratische Form $(a, b, c)$ in (9) kann der Klasse willkïrlich entnommen werden, die $\mu, \nu$ durchlaufen alle Paare ganzer Zahlen.

In den später zu betrachtenden Einzelfällen ordnen wir die Reihen (9) stets nach ansteigenden Potenzen von $q$ um. Die einzelne Modulform $y_{0}$ ist dann in der Gestalt gegeben:

$$
y_{0}\left(\omega_{1}, \omega_{2}\right)=\frac{2 \pi}{\omega_{2}} \sum_{i=0}^{\infty} A_{i} q^{2 i},
$$

wo nach der Sprechweise von S. 152 die nicht-negative ganze $Z$ ahl $A_{i}$ die Anzahl der „Darstellungen" der ganzen Zahl $i$ durch die quadratische Form $(a, b, c)$ angibt.

\section{$\$ 8$. Die Systeme der Funktionen $Z_{\lambda}$ und der Modulformen $\approx_{\lambda}$.}

$\mathrm{Zu}$ ähnlichen wenn auch nicht so einfachen Ergebnissen gelangt man im Falle eines ungeraden $l$. Wir dürfen hier, wie aus dem Ansatze (1) S. 320 hervorgeht, ohne Änderung der Funktion $B_{\lambda}$ die Zahl $k$ um $l$ vermehren und wollen von diesem Umstande in der Weise Gebrauch machen, daß wir $k$ stets als ungerade gewählt denken. Dann gilt:

$$
x+l \lambda+n k x \equiv \lambda(\bmod 2),
$$

so daß wir durch Eintragen der aus (12) S. $306 \mathrm{zu}$ entnehmenden Reihenentwicklungen der $X$ in die Gleichung (1) S. 320 für die Entwicklung des bilinearen Ausdrucks $B_{2}$ den Ansatz haben:

$$
\begin{aligned}
B_{\lambda}=-(\sqrt[4]{\Delta})^{1+l_{0}+(n l)_{0}} & \cdot \frac{2 \pi}{\omega_{2}} e^{\frac{l \eta_{2}}{2 \omega_{2}}\left(u_{1}^{2}+n u_{2}^{2}\right)} \\
& \cdot \sum_{x, v_{1}, v_{2}}(-1)^{\lambda+v_{1}+v_{2}} q^{\frac{n v^{2}+N^{2}}{4 l u}} e^{\frac{\pi i}{\omega_{2}}\left(v u_{1}+N u_{2}\right)} ;
\end{aligned}
$$

$\nu$ und $N$ sind Abkürzungen für folgende Ausdrücke:

$$
\nu=\left(2 \nu_{1}+1\right) l-2 \varkappa, \quad N=\left(2 \nu_{9}+1\right) l n-2 l \lambda-2 n k x,
$$

$*$ durchläuft die Zahlen $0,1,2, \ldots, l-1$, während $\nu_{1}$ und $\nu_{2}$ alle ganzen Zahlen durchlaufen.

Hier hat nun $v$ gerade genau einmal alle ungeraden ganzen Zahlen zu durchlaufen. Für das einzelne $\nu$ ist $2 x$ als kleinste, nicht-negative gerade Zahl, die $\bmod l \operatorname{mit}-\boldsymbol{\nu}$ kongruent ist, bestimmt, worauf sich dann weiter $\nu_{1}$ aus (2) eindeutig berechnet. Für $N$ können wir statt des Ausdrucks (2) auch den folgenden setzen:

$$
N=n k \nu+2 l \mu, \quad \mu=\nu_{2} n-\nu_{1} n k+n \frac{1-k}{2}-\lambda .
$$

Die damit eingeführte ganze Zahl $\mu$ hat dann bei stehendem $\nu_{1}$ wegen 
328 II, 2. Systeme ganzer elliptischer Funktionen dritter Art $n^{\text {ter }}$ Stufe

der Bedeutung von $\nu_{2}$ gerade einmal alle $\bmod n \operatorname{mit}-\lambda$ kongruenten Zahlen zu durchlaufen.

Es sind jetzt die einzelnen Bestandteile auf der rechten Seite des Ansatzes (1) auf den Summationsbuchstaben $\mu$ umzurechnen. Man findet: erstlich bei Benutzung der zweiten Gleichnng (3) S. 320:

$$
n \nu^{2}+N^{2}=2 l\left(2 l \mu^{2}+2 k n \mu \nu+\frac{m}{2} n \nu^{2}\right),
$$

wobei in Betracht kommt, daB wegen der ungeraden $k$ die ganze Zahl $m$ gerade ist. Es stellt sich also hier die ganzzahlige binäre quadratische Form ein:

$$
f(\mu, \nu)=2 l \mu^{2}+2 k n \mu \nu+\frac{m}{2} n \nu^{2}
$$

Diese Form $\left(2 l, 2 k n, \frac{m}{2} n\right)$ ist wieder positiv und hat die negative Diskriminante:

$$
D=4 k^{2} n^{2}-4 \operatorname{lm} n=-4 n .
$$

Die beiden ersten Koeffizienten $2 l$ und $2 k n$ haben den gröBten gคmeinsamen Teiler 2. Da $m$ zufolge der zweiten Gleichung (3) S. 320 die Kongruenz $m \equiv n+1(\bmod 4)$ befriedigt, so ist $m$ das Doppelte einer ungeraden Zahl oder durch 4 teilbar, je nachdem $n \equiv 1$ oder $\equiv 3(\bmod 4)$ ist. Die quadratische Form (4) ist also ursprünglich, oder sie hat den Teiler 2 , je nachdem $n \equiv 1$ oder $\equiv 3(\bmod 4)$ gilt.

Entsprechend den Gleichungen (3) S. 324 führen wir hier an Stelle der bisherigen Variablen $u_{1}$ und $u_{2}$ die neuen Variablen $u$ und $v$ durch die Gleichungen ein:

$$
u_{1}=u+\frac{n k}{2 l} v, \quad u_{2}=-\frac{v}{2 l} .
$$

Dann gilt erstlich:

$$
2 l\left(u_{1}^{2}+n u_{2}^{2}\right)=2 l\left(u^{2}+\frac{n k}{l} u v+\frac{n^{2} k^{2}+n}{4 l^{2}} v^{2}\right)=f(u, v),
$$

und man findet andrerseits:

$$
\nu u_{1}+N u_{2}=\nu\left(u+\frac{n k}{2 l} v\right)-(n k \nu+2 l \mu) \frac{v}{2 l}=\nu u-\mu v .
$$

Endlich ist noch das Vorzeichen unter der Summe des Ansatzes (1) umzugestalten. Dies geschieht auf Grund der aus der zweiten Gleichung (3) folgenden Kongruenz:

$$
\lambda+\nu_{1}+\nu_{2} \equiv \mu+\frac{k-1}{2}(\bmod 2)
$$

Wir erklären nun im AnschluB an die Gleichung (4) S. 325 ein System von Funktionen $Z_{\lambda}(u, v)$ durch die Festsetzung:

$$
(-1)^{\frac{k+1}{2}} \frac{B_{\lambda}\left(u+\frac{n k}{2 l} v,-\frac{v}{2 l}\right)}{(\sqrt[4]{\Delta})^{1+l_{0}+(n l)_{0}}}=Z_{2}\left(u, v \mid \omega_{1}, \omega_{2}\right) \text {. }
$$


Die Funktionen $Z_{\lambda}\left(u, v \mid \omega_{1}, \omega_{2}\right)$ und die Modulformen $z_{\lambda}\left(\omega_{1}, \omega_{2}\right)$ Als Reihenentricklungen dieser Funktion hat man:

$$
Z_{\lambda}\left(u, v \mid \omega_{1}, \omega_{2}\right)=\frac{2 \pi}{\omega_{2}} e^{\frac{\eta_{2}}{4 \omega_{2}} f(u, v)} \sum_{\mu, v}(-1)^{\mu} q^{\frac{f(\mu, v)}{2 n}} \frac{\pi i}{e^{\omega_{2}}(v u-\mu v)},
$$

wo $f$ die quadratische Form $(4)$ ist, $\mu$ alle der Kongruenz $\mu \equiv-\lambda(\bmod n)$ genügenden ganzen Zahlen und $\nu$ alle ungeraden ganzen Zahlen durchlaufen. Will man nur mit ursprünglichen quadratischen Formen arbeiten, so mag man den vorstehenden Satz nur auf die der Kongruenz $n \equiv 1(\bmod 4)$ genügenden Stufen beziehen, im Falle $n \equiv 3(\bmod 4)$ aber den 'Teiler 2 der Form fortheben. Es folgt dann der Satz: Ist $n \equiv 3(\bmod 4)$, so haben wir als Reiliendarstellung der $Z_{\lambda}$ :

$$
Z_{\lambda}\left(u, v \mid \omega_{1}, \omega_{2}\right)=\frac{2 \pi}{\omega_{2}} e^{\frac{\eta_{2}}{2 \omega_{2}} f(u, v)} \sum_{\mu, v}(-1)^{\mu} q^{\frac{f(\mu, v)}{n}} e^{\frac{\pi i}{\omega_{2}}(v u-\mu v)},
$$

unter $f$ jetzt die ursprïngliche quadratische Form:

$$
f=\left(l, k n, \frac{m}{4} n\right)
$$

der negativen Diskriminante $D=-n$ verstanden; die Summationsbedingungen für $\mu$ und $\nu$ sind die bisherigen. Den Charakter von Modulformen $n^{\text {ter }}$ Stufe haben, wie wir in Erinnerung bringen, erst die Produkte der $Z_{\lambda}$ und der Modulform $(\sqrt[4]{\Delta})^{1}+l_{0}+(n l)_{0}$, wo $l_{0}$ und $(n l)_{0}$ die kleinsten, nichtnegativen ganzen Zahlen sind, die die Kongruenzen befriedigen:

$$
i_{0} \equiv-\frac{l+1}{2}, \quad(n l)_{0} \equiv-\frac{n l+1}{2} \quad(\bmod 4) .
$$

Diese Produkte erfahren dann gegenüber den linearen Transformationen der Perioden die $X_{\lambda}$-Substitutionen, jedoch unter Ersatz von $\varepsilon$ durch $\varepsilon_{\boldsymbol{l}}$,

Aus den Reihenentwicklungen (7) und (8) ergibt sich, daB bei gleichzeitigem Zeichenwechsel von $u$ und $v$ die Funktion $Z_{0}(u, v)$ in sich übergeht, während sich die übrigen Funktionen $Z_{\lambda}(u, v)$ des einzelnen Systems nach dem Gesetze:

$$
Z_{\lambda}\left(-u,-v \mid \omega_{1}, \omega_{2}\right)=Z_{n-\lambda}\left(u, v \mid \omega_{1}, \omega_{2}\right)
$$

transformieren. Für $u=0, v=0$ erhalten wir demnach aus den $Z_{\lambda}$ des einzelnen Systems ein System von $\frac{n+1}{2}$ ganzen Modulformen $(-1)^{\text {ter }}$ Dimension $z_{0}, z_{1}, \ldots, z_{\frac{n-1}{2}}$, welche durch Multiplikation mit $(\sqrt[4]{\Delta})^{1+l_{0}+(n)_{0}} z u$

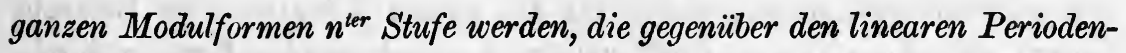
transformationen dic $\xi_{\lambda}$-Substitutionen unter Ersatz von $\varepsilon$ durch $\varepsilon_{l}$ erfahren. Als Reihendarstellungen der $z_{\lambda}$ haben wir im Falle einer mod 4 mit 1 kongruenten Zahl n:

$$
z_{2}\left(\omega_{1}, \omega_{2}\right)=\frac{2 \pi}{\omega_{2}} \sum_{\mu, 2}(-1)^{\mu} q^{\frac{f(\mu, v)}{2 n}}
$$


wo $f(\mu, \nu)$ die ursprüngliche quadratische Form (4) der negativen Diskriminante $-4 n$ ist; im Falle $n \equiv 3(\bmod 4)$ aber gilt:

$$
z_{\lambda}\left(\omega_{1}, \omega_{2}\right)=\frac{2 \pi}{\omega_{2}} \sum_{\mu, v}(-1)^{\mu} q^{\frac{f(\mu, v)}{n}},
$$

unter $f$ die ursprüngliche Form (9) der negativen Diskriminante - $n$ verstanden. Die Summationsbuchstaben $\mu, \nu$ sind natürlich den bisherigen Bedingungen unterworfen.

Vom einzelnen Systeme der $z_{\lambda}$ wird später insbesondere wieder die erste Modulform $z_{0}\left(\omega_{1}, \omega_{2}\right)$ benutzt, die wir bei Wiederholung der S. $325 \mathrm{ff}$. durchgeführten Überlegung so dargestellt finden:

$$
\begin{cases}z_{0}=\frac{2 \pi}{\omega_{2}} \sum_{\mu, v}(-1)^{\mu} q^{\frac{f^{\prime}(\mu, v)}{2},} & n \equiv 1(\bmod 4) \\ z_{0}=\frac{2 \pi}{\omega_{2}} \sum_{\mu, v}(-1)^{\mu} q^{f^{\prime}(\mu, v)}, & n \equiv 3(\bmod 4)\end{cases}
$$

Die quadratische Form $f^{\prime}$ ist durch:

$$
f^{\prime}=\left(2 \ln , 2 k n, \frac{m}{2}\right) \quad \text { bzw. } \quad f^{\prime}=\left(\ln , k n, \frac{m}{4}\right)
$$

gegeben, während $\mu$ jetzt alle ganzen Zahlen und $\nu$ alle ungeraden ganzen Zahlen zu durchlaufen haben. Auch die Form $f^{\prime}$ hat die Diskriminante $D=-4 n$ bzw. $-n$ und ist positiv und ursprünglich.

Es ist nun zu untersuchen, ob in jeder Klasse ursprünglicher positiver Formen der Diskriminante $-4 n$ bzw. $-n$ mindestens eine Form der Gestalt (14) nachweisbar ist. Ist erstlich $n \equiv 1(\bmod 4)$ und also $D=-4 n$, so entnehmen wir der einzelnen vorgelegten Klasse eine erste Form $(a, b, c)$ mit einem gegen $2 n$ teilerfremden $c$ und setzen $2 c=m$, so daB $m$ das Doppelte einer ungeraden Zahl ist. Wir gehen sodann zu einer äquivalenten Form $\left(a^{\prime}, b^{\prime}, c^{\prime}\right)$ mit den Koeffizienten:

$$
a^{\prime}=a-\gamma b+\gamma^{2} c, \quad b^{\prime}=b-2 \gamma c, \quad c^{\prime}=c
$$

und können die ganze Zahl $\gamma$ den beiden Kongruenzen:

$$
b^{\prime}=b-2 \gamma c \equiv 0 \quad(\bmod n), \quad b^{\prime}=b-2 \gamma c \equiv 2 \quad(\bmod 4)
$$

entsprechend bestimmen, wobei für die zweite Kongruenz in Betracht kommt, daB die mittleren Koeffizienten der vorliegenden Formen durchweg gerade Zahlen sind. Setzt man $b^{\prime}=2 k n$, so erweist sich $k$ als ungerade ganze Zahl. Für $a^{\prime}$ folgt aus:

$$
n+k^{2} n^{2}=a^{\prime} \frac{m}{2}
$$


daB diese ZahJ gerade und durch $n$ teilbar ist. Setzt man dementsprechend $a^{\prime}=2 l n$, so folgt $1+k^{2} n=l m$ und damit wegen $m \equiv 2(\bmod 4)$ :

$$
2 l \equiv l m=1+k^{2} n \equiv n+1 \equiv 2 \quad(\bmod 4),
$$

so daB $l$ eine ungerade Zahl ist. Also haben wir in der Tat eine brauchbare Form (14) in der Klasse nachgewiesen.

Ist zweitens $n \equiv 3(\bmod 4)$, so entnehmen wir der vorgelegten Klasse eine Form $(a, b, c)$ mit einem gegen $n$ teilerfremden $c$ und setzen $4 c=m$. Jetzt sind die mittleren Koeffizienten ungerade. Gehen wir also durch die Transformation (15) zu einer äquivalenten Form $\left(a^{\prime}, b^{\prime}, c^{\prime}\right)$ mit $b^{\prime} \equiv 0$ $(\bmod n)$, so ist $b^{\prime}=k n$ mit einer ungeraden Zahl $k$ zu setzen. Da $D=-n$ ist, so ist $a^{\prime}$ durch $n$ teilbar. Setzt man also $a^{\prime}=l n$, so ist nur noch zu untersuchen, ob man hierbei ein ungerades $l$ erreichen kann. Dies ist, falls $n \equiv 3(\bmod 8)$ ist, selbstrerständlich; dann gilt nämlich:

$$
4 \equiv 1+n \equiv 1+k^{2} n=l m=4 c l \quad(\bmod 8),
$$

woraus $l \equiv 1(\bmod 2)$ folgt. Im Falle $n \equiv 7(\bmod 8)$ kann zunächst aber auch ein grades $l$ auftreten. Doch ist dies, wenn $c$ gerade ist, stets vermeidbar, indem man die in der Transformation (15) auftretende ganze Zahl $\gamma$ nötigenfalls um $n$ gröBer wählt. Ist aber $c$ ungerade, so gehe man von der eben erhaltenen Form $(a, b, c)=(l n, k n, c)$ zunächst durch die Transformation:

$$
a^{\prime}=a, \quad b^{\prime}=-2 a+b, \quad c^{\prime}=a-b+c=(l-k) n+c
$$

zu einer äquivalenten Form $\left(a^{\prime}, b^{\prime}, c^{\prime}\right)$ mit einem geraden, gegen $n$ teilerfremden $c^{\prime}$. Von dieser Form aus gelangt man dann wie soeben leicht zu einer allen Anforderungen genügenden äquivalenten Form. Also ist auch für $n \equiv 3(\bmod 4)$ in jeder ursprünglichen Klasse positiver Formen der Diskriminante $-n$ eine brauchbare Form (14) enthalten.

Wir fragen jetzt zunächst im Falle $n \equiv 1(\bmod 4)$, ob zwei äquivalente Formen (14) verschiedene Modulformen $z_{0}$ zu liefern vermögen. Transformieren wir durch die Substitution:

$$
\mu^{\prime}=\alpha \mu+\beta \nu, \quad \nu^{\prime}=\gamma \mu+\delta \nu,
$$

so erhalten wir von der Form (14) aus die Form $(a, b, c)$ der Koeffizienten:

$$
\left\{\begin{array}{l}
a=\delta^{2} 2 \ln -\gamma \delta 2 k n+\gamma^{2} \frac{m}{2}, \\
b=-2 \beta \delta 2 \ln +(\alpha \delta+\beta \gamma) 2 k n-2 \alpha \gamma \frac{m}{2}, \\
c=\beta^{2} 2 \ln -\alpha \beta 2 k n+\alpha^{2} \frac{m}{2} .
\end{array}\right.
$$

Die Kongruen' $\gamma \equiv 0(\bmod 2 n)$ ist hinreichend und notwendig, damit $(a, b, c)$ wieder die Gestalt (14) hat. Zu letzterem Zwecke müssen nämlich erstlich $\gamma^{2}$ und $\alpha \gamma$ und also $\gamma$ durch $n$ teilbar sein. Außerdem aber muB 
332 II, 2. Systeme ganzer elliptischer Funktionen dritter Art $n^{\text {ter }}$ Stufe $\gamma$ gerade sein. Gilt andrerseits $\gamma \equiv 0(\bmod 2 n)$, so ist $\alpha$ und also $c$ teilerfremd gegen $2 n$, und es sind $a$ und $b$ durch $2 n$ teilbar. Übrigens gilt:

$$
a \equiv \delta^{2} \cdot 2 \ln \equiv 2, \quad b \equiv \alpha \delta \cdot 2 k n \equiv 2(\bmod 4),
$$

so dab in der Tat eine Form (14) vorliegt.

Nennen wir die transformierte Form $\left(2 l^{\prime} n, 2 k^{\prime} n, \frac{m^{\prime}}{2}\right)$, so ist einerseits

$$
2 \ln \mu^{2}+2 k n \mu \nu+\frac{m}{2} \nu^{2}=2 l^{\prime} n \mu^{\prime 2}+2 k^{\prime} n \mu^{\prime} \nu^{\prime}+\frac{m^{\prime}}{2} \nu^{\prime 2},
$$

und andrerseits ergibt sich aus $\nu \equiv 1, \alpha \equiv \delta \equiv 1, \gamma \equiv 0(\bmod 2)$ :

$$
(-1)^{\mu}=(-1)^{\beta} \cdot(-1)^{\mu^{\prime}}, \quad \nu^{\prime} \equiv \nu \equiv 1 \quad(\bmod 2) ;
$$

$\mu^{\prime}$ durchläuft wieder alle ganzen Zahlen, $\nu^{\prime}$ alle ungeraden ganzen Zahlen. Die für die äquivalente Form hergestellte Modulform $z_{0}$ ist also, abgesehen vom Faktor $(-1)^{\beta}$, mit der ersten Modulform identisch.

Übrigens können wir an Stelle der Form (14) jede beliebige Form der Klasse mit geradem ersten Koeffizienten $a$ benutzen. Für eine solche Form ergibt sich aus:

$$
\left(\frac{b}{2}\right)^{2}-a c=-n \equiv 3 \quad(\bmod 4),
$$

daB $b$ das Doppelte einer ungeraden Zahl sein muB. Weiter folgt dann $a c \equiv 2(\bmod 4)$, so daB $c$ ungerade und $a$ das Doppelte einer ungeraden $\mathrm{Zahl}$ ist. Um in (17) eine solche Form zu erreichen, ist in (16) die Bedingung $\gamma \equiv 0(\bmod 2)$ hinreichend und notwendig. Dann aber gelten wieder die Bedingungen (18) Man findet von hieraus leicht folgenden abschließenden Satz: Jede zweiseitige Klasse und jedes Paar entgegengesetzter Klassen ursprünglicher positiver Formen der Diskriminante $D=-4 n$ liefert im wesentlichen nur eine Modulform:

$$
z_{0}\left(\omega_{1}, \omega_{2}\right)=\frac{2 \pi}{\omega_{2}} \sum_{\mu, v}(-1)^{\mu} q^{\frac{a \mu^{2}+b \mu \nu+c v^{2}}{2}},
$$

wo $\mu$ alle ganzen Zahlen und $\nu$ alle ungeraden ganzen Zahlen durchlaufen und übrigens eine beliebige Form $(a, b, c)$ der Klasse mit geradem a zu wählen ist. Das Produkt von $z_{0}$ und einer richtig gewählten Potenz von $\sqrt[4]{\Delta}$ gehört zur $n^{\text {ten }}$ Stufe und bleibt gegenüber $S$ unverändert, hat also eine Potenzreihenentwicklung nach ganzen Potenzen von $q^{2}$. Aus dem Charakter der Zahlen $a, b, c$ mod 2 folgt demnach: Zur $n^{\text {ten }}$ Stufe gehört das Produkt von $z_{0}$ mit $(\sqrt[4]{\Delta})^{3}$ oder $\sqrt[4]{\Delta}$, je nachdem $c \equiv 1$ oder $\equiv 3$ (mod 4) zutrifft.

Im Falle $n \equiv 3(\bmod 4)$ transformieren wir die zweite Form (14). 
mittelst der Substitution (16) und gewinnen eine äquivalente Form $(a, b, c)$ mit den Koeffizienten:

$$
\left\{\begin{array}{l}
a=\delta^{2} \ln -\gamma \delta k n+\gamma^{2} \frac{m}{4}, \\
b=-2 \beta \delta l n+(\alpha \delta+\beta \gamma) k n-2 \alpha \gamma \frac{m}{4}, \\
c=\beta^{2} \ln -\alpha \beta k n+\alpha^{2} \frac{m}{4} .
\end{array}\right.
$$

Damit hier wieder eine Form $(14)$ vorliegt, $\operatorname{muB}$ erstlich $\gamma \equiv 0(\bmod n)$ zutreffen; dann sind auch sicher $a$ und $b$ durch $n$ teilbar, $c$ aber ist teilerfremd gegen $n$. Da $b$ jetzt stets ungerade ist, haben wir nur noch die Forderung $a \equiv 1(\bmod 2)$ zu untersuchen. Nun folgt aus der zweiten Gleichung (3) S. 320:

$$
l m \equiv n+1 \quad(\bmod 8), \quad \frac{m}{4} \equiv \frac{m}{4} n \equiv \ln \frac{n+1}{4} \equiv \frac{n+1}{4} \quad(\bmod 2),
$$

so daB sich die gestellte Forderung in die Gestalt kleidet:

$$
a \equiv \delta^{2}+\gamma \delta+\frac{n+1}{4} \gamma^{2} \equiv 1 \quad(\bmod 2) .
$$

Diese Kongruenz aber ist für $n \equiv 3(\bmod 8)$ stets erfüllt, da $\gamma$ und $\delta$ nicht zugleich gerade sind. Für $n \equiv 7(\bmod 8)$ ist indes $\gamma \equiv 0(\bmod 2)$ erforderlich und hinreichend. Damit die zweite Form (14) durch eine Substitution (16) wieder in eine ebenso gebaute Form übergeht, ist hinreichend und notwendig die Kongruenz $\gamma \equiv 0(\bmod n)$, falls $n \equiv 3(\bmod 8)$ gilt, und die Kongruenz $\gamma \equiv 0(\bmod 2 n)$, falls $n \equiv 7(\bmod 8)$ ist.

Im Falle $n \equiv 7(\bmod 8)$ kommt daraufhin die Untersuchung leicht wie bei $n \equiv 1(\bmod 4)$ zum AbschluB. Man kann auch wieder eine beliebige Form $(a, b, c)$ der Klasse mit ungeradem $a$ zum Gebrauche heranziehen, da beim Übergange zu einer solchen Form die Bedingung $\gamma \equiv 0$ (mod 2$)$ besteht und also die Bedingungen (18) erfüllt sind. Wir finden den Satz: Ist $n \equiv 7(\bmod 8)$, so liefert jede zweiseitige Klasse und jedes Paar entgegengesetzter Klassen ursprünglicher positiver Formen der Diskriminante $D=-n$ im wesentlichen nur eine Modulform:

$$
z_{0}\left(\omega_{1}, \omega_{2}\right)=\frac{2 \pi}{\omega_{2}} \sum_{\mu, \nu}(-1)^{\mu} q^{a \mu^{2}+b \mu \nu+c v^{2}},
$$

wo die quadratische Form $(a, b, c)$ mit ungeradem a der Klasse beliebig zu entnehmen ist, während $\mu$ alle ganzen Zahlen und $\nu$ alle ungeraden ganzen Zahlen durchlaufen. Da im vorliegenden Falle $c$ notwendig gerade ist, während $a$ und $b$ ungerade sind, so gehört die Form (21) unmittelbar der $n^{\text {ten }}$ Stufe an. 
334 II, 2. Systeme ganzer elliptischer Funktionen dritter Art $n^{\text {ter }}$ Stufe

Ist endlich $n \equiv 3(\bmod 8)$, so sind die Substitutionen $(16)$, die eine Form (14) wieder in eine ebenso gebaute Form überführen, durch $\gamma \equiv 0$ $(\bmod n)$ charakterisiert und $\bmod 2$ keiner weiteren Beschränkung unterworfen. Übt man eine Substitution (16) aus, so gilt:

$$
(-1)^{\mu}=(-1)^{\delta \mu^{\prime}-\beta \nu^{\prime}}, \quad \nu=-\gamma \mu^{\prime}+\alpha \nu^{\prime} \equiv 1 \quad(\bmod 2)
$$

und es sind diese Formeln gegenüber den sechs mod 2 inkongruenten Substitutionen näher zu untersuchen. Ist erstlich $\gamma \equiv 0(\bmod 2)$, so kommen wir auf die Bedingungen (18) zurück, so daß wir im wesentlichen die Modulform $z_{0}$ für die transformierte quadratische Form wieder gewinnen. Ist zweitens $\alpha \equiv \gamma(\bmod 2)$, wo dann natürlich beide Zahlen $\alpha, \gamma$ ungerade sind, so folgt $\mu^{\prime} \neq \nu^{\prime}(\bmod 2)$, und für $(-1)^{\mu}$ ergibt sich entweder $(-1)^{\mu^{\prime}}$, nämlich wenn $\beta \equiv 0(\bmod 2)$ ist, oder aber $(-1)^{v^{\prime}}=-(-1)^{\mu^{\prime}}$, nämlich wenn $\delta \equiv 0(\bmod 2)$ gilt. Wir kommen also im wesentlichen zum Ansatze (13) zurück, jetzt jedoch mit der Summationsbedingung $\mu \neq \nu$ $(\bmod 2)$. Ist endlich $\alpha \equiv 0(\bmod 2)$, so folgt:

$$
(-1)^{\mu}=(-1)^{\delta} \cdot(-1)^{\nu^{\prime}}, \quad \nu \equiv \mu^{\prime} \equiv 1 \quad(\bmod 2) .
$$

Man überzeuge sich noch, daB man stets zu den gesamten Paaren ganzer Zahlen $\mu^{\prime}, \nu^{\prime}$ gelangt, die den neuen Bedingungen genïgen.

Auch hier können wir an Stelle der Form (14) sogleich eine beliebige Form der Klasse benutzen. Mit Rücksicht auf die soeben abgeleiteten Ergebnisse gelangen wir zu dem Satze: Ist $n \equiv 3(\bmod 8)$, so liefert jede zweiseitige Klasse und jedes Paar entgegengesetzter Klassen ursprünglicher positiver Formen der Diskriminante $D=-n$ im wesentlichen drei Ansätze von Modulformen $z_{0}$, nämlich zunächst zwei Ansätze der Gestalt (21), und zwar den ersten Ansatz mit der Summationsbedingung $\nu \equiv 1$ $(\bmod 2)$, den zweiten mit der Bedingung $\mu \neq \nu(\bmod 2)$, sodann den dritten Ansatz:

$$
z_{0}\left(\omega_{1}, \omega_{2}\right)=\frac{2 \pi}{\omega_{2}} \sum_{\mu, v}(-1)^{v} q^{a \mu^{2}+b \mu \nu+c v^{2}}
$$

mit der Bedingung $\mu \equiv 1(\bmod 2) ;$ die Form $(a, b, c)$ ist irgendeine willkïrlich der Klasse entnommene. Da man aus:

$$
b^{2}-4 a c=-n \equiv 5 \quad(\bmod 8)
$$

leicht folgert, daß $a, b, c$ durchweg ungerade sind, so gehört jetzt in jedem Falle das Produkt $z_{0} \sqrt{\Delta}$ zur $n^{\text {ten }}$ Stufe: 


\section{Drittes Kapitel.}

\section{Die speziellen Transformationsgleichungen erster Stufe.}

Nach der S. 297 vereinbarten Sprechweise sollten die algebraischen Gleichungen, denen die bei Transformation $n^{\text {ten }}$ Grades aus $g_{2}, g_{3}, \Delta$ und $J$ entstehenden Größen genügen, als "spezielle Transformationsgleichungen" bezeichnet werden. Insbesondere bezeichnen wir sie als spezielle Transformationsgleichungen "erster Stufe" und wollen hierzu auch noch diejenigen Gleichungen rechnen, die wir für die Wurzeln $8^{\text {ten }}, 12^{\text {ten }}$ und $24^{\text {sten }}$ Grades der Diskriminante $\Delta$ finden werden. Die speziellen Transformationsgleichungen entsprechen, wie oben bemerkt, den speziellen Teilungsgleichungen, denen sie als Resolventen zugehören. Es soll zunächst die allgemeine Theorie dieser Transformationsgleichungen erster Stufe entwickelt werden. Abschließende Einzeluntersuchungen über niedere Transformationsgrade folgen im nächsten Kapitel.

\section{$\S$ 1. Die speziellen Transformationsgleichungen als Resolventen der speziellen Teilungsgleichungen.}

Nach S.273ff. erhalten wir aus einer Modulform erster Stufe $\left.f\left(\omega_{1}, \omega_{2}\right)^{1}\right)$ durch Transformation $n^{\text {ten }}$ Grades im ganzen $\Phi(n)$ verschiedene transformierte Funktionen $f\left(a \omega_{1}+b \omega_{2}, c \omega_{1}+d \omega_{2}\right)$, unter $\Phi(n)$ die Teilersumme des Transformationsgrades $n$ verstanden. Nach der Lehre von den „Repräsentanten" der $\Phi(n)$ „Klassen“ von Transformationen $n^{\text {ten }}$ Grades (vgl. S. 276) können wir die $\Phi(n)$ transformierten Formen in der Gestalt:

$$
f\left(A \omega_{1}+B \omega_{2}, D \omega_{2}\right), \quad A \cdot D=n, \quad 0 \leqq B<D
$$

schreiben, wo $A, B, D$ positive ganze Zahlen sind, die den in (1) hinzugefügten Bedingungen genügen. Haben die Zahlen $A, B, D$ keinen gemeinsamen Teiler, der $>1$ ist, so liegt ,eigentlich" Transformation $n^{\text {ten }}$ Grades vor. Nach den Überlegungen von S. $276 \mathrm{ff}$. können wir uns auf eigentliche Transformationen $n^{\text {ten }}$ Grades beschränken. Die Anzahl der Klassen solcher eigentlicher Transformationen $n^{\text {ten }}$ Grades ist $\psi(n)$, dieses Symbol $\psi(n)$ in der bekannten Bedeutung (4) S. 220 gebraucht. Zu den eigentlichen Transformationen $n^{\text {ten }}$ Grades gehören insbesondere die beiden Haupttransformationen (vgl.S.278), von denen die erste durch $A=n, B=0$, $D=1$, die zweite durch $A=1, B=0, D=n$ gegeben ist.

Die Existenz der Transformationsgleichungen erster Stufe, vorerst freilich unter AusschluB derjenigen Gleichungen, die etwa für die ge-

1) Ist die Dimension dieser Form gleich 0, so haben wir eine Modulfunktion erster Stufe. 
nannten Wurzeln der Diskriminante $\Delta$ bestehen mögen, ergibt sich nun aus folgender Überlegung: Aus der Modulform erster Stufe geht durch die erste Haupttransformation $n^{\text {ten }}$ Grades eine Modulform $n^{\text {ter }}$ Stufe:

$$
f^{\prime}\left(\omega_{1}, \omega_{2}\right)=f\left(n \omega_{1}, \omega_{2}\right)
$$

hervor. In der Tat zeigt man sofort, da $\beta$ die transformierte Form (2) bei den Substitutionen derjenigen Kongruenzgruppe $n^{\text {ter }}$ Stufe unverändert bleibt, die durch $\gamma \equiv 0(\bmod n)$ erklärt ist. Durch diese Kongruenz ist aber eine unserer $\psi(n)$ gleichberechtigten Untergruppen $\Gamma_{\psi(n)}$ des Index $\psi(n)$ erklärt.

Wir zerlegen nun die Gesamtgruppe $\Gamma^{(\omega)}$ entsprechend dieser $\Gamma_{\psi(n)}$ in $\psi(n)$ Nebengruppen:

$$
\Gamma^{(\omega)}=\Gamma_{\psi}+\Gamma_{\psi} \cdot V_{1}+\Gamma_{\psi} \cdot V_{2}+\cdots+\Gamma_{\psi} \cdot V_{\psi-1}
$$

und schreiben $V_{v}=\left(\begin{array}{l}\alpha_{v}, \beta_{v} \\ \gamma_{v},\end{array} \delta_{v}\right)$ und speziell $V_{0}=1$. Die Form (2) geht dann durch die Substitutionen der $\Gamma^{(\omega)}$ im ganzen in $\psi(n)$ verschiedene Formen über, die wir unter Benutzung der eben eingeführten Substitutionen $V_{v}$ in den Gestalten anschreiben können:

$$
\begin{array}{r}
f^{(v+1)}\left(\omega_{1}, \omega_{2}\right)=f^{\prime}\left(\alpha_{v} \omega_{1}+\beta_{v} \omega_{2}, \gamma_{v} \omega_{1}+\delta_{v} \omega_{2}\right)= \\
=f\left(n \alpha_{v} \omega_{1}+n \beta_{v} \omega_{2}, \gamma_{v} \omega_{1}+\delta_{v} \omega_{2}\right) .
\end{array}
$$

Diese $\psi(n)$ Formen $f^{\prime}, f^{\prime \prime}, \ldots, f^{(\psi)}$, die zu den $\psi(n)$ mit $\Gamma_{\psi(n)}$ gleichberechtigten Untergruppen gehören, sind dann nach S. 279 ff. die $\psi(n)$ durch eigentliche Transformation $n^{\text {ten }}$ Grades aus $f\left(\omega_{1}, \omega_{2}\right)$ herstellbaren transformierten Formen.

Übt man nun auf die Argumente $\omega_{1}, \omega_{2} \operatorname{der} \psi(n)$ transformierten Formen (4) irgendeine Substitution der $\Gamma^{(\omega)}$ aus, so permutieren sich diese Formen. Daraus folgt, daß die symmetrischen Grundfunktionen der $\psi(n)$ Formen (4) gegenüber jeder Substitution der $\Gamma^{(\omega)}$ unverändert bleiben, also Modulformen „erster" Stufe darstellen und als solche rationale Funktionen von $g_{2}$ und $g_{3}$ sind. Wir gelangen zu dem Satze: Die $\psi(n)$ durch eigentliche Transformation $n^{\text {ten }}$ Grades aus einer Modulform erster Stufe entstehenden Modulformen $n^{\text {ter }}$ Stufe sind die Lösungen einer Gleichung $\psi^{\text {ten }}$ Grades:

$$
f^{\psi}+R_{1}\left(g_{2}, g_{3}\right) f^{\psi-1}+R_{2}\left(g_{2}, g_{3}\right) f^{\psi-2}+\cdots+R_{\psi}\left(g_{2}, g_{3}\right)=0
$$

deren Koeffizienten rationale Funktionen von $g_{2}, g_{3}$ sind. In (5) haben wir den allgemeinen Ansatz für die Transformationsgleichungen erster Stufe gewonnen.

Wir wenden nun den Ansatz (5) auf die Formen $g_{2}, g_{3}, \Delta$ und die Funktion $J$ an und zeigen zunächst, daB wir hierbei in der Tat zu Resolventen der speziellen Teilungsgleichung für den $n^{\text {ten }}$ Teilungsgrad geführt werden. $\mathrm{Zu}$ diesem $\mathrm{Z}$ wecke ziehen wir die Gleichung (6) S.:285 

(unter Austausch der beiden Haupttransformationen) heran und folgern:

$$
e^{-G_{1} u^{2}} \frac{\sigma\left(u \mid \omega_{1}, \frac{\omega_{2}}{n}\right)}{\sigma\left(u \mid \omega_{1}, \omega_{2}\right)^{n}}=\prod_{\mu=1}^{n-1} \frac{\sigma_{0 \mu}(u)}{\sigma_{0 \mu} \sigma(u)}
$$

wo $G_{1}=G_{1}\left(\omega_{1}, \omega_{2}\right)$ die Modulform $n^{\text {ter }}$ Stufe:

$$
G_{1}\left(\omega_{1}, \omega_{2}\right)=\frac{1}{2} \sum_{\mu=1}^{n-1} \wp_{0 \mu}
$$

ist und bei den $\sigma_{0 \mu}$ und $\wp_{0 \mu}$ der $n^{\text {te }}$ Teilungsgrad vorliegt. Der einzelne Faktor des Produktes (6) ist bereits in (3) S. 226 eingeführt. Aus der Regel (4) daselbst ergibt sich, daß der Ausdruck (6) bei ungeradem $n$ die Perioden $\omega_{1}, \omega_{2}$ hat, bei geradem $n$ aber die Periode $\omega_{2}$ besitzt und bei Vermehrung von $u$ um $\omega_{1}$ Zeichenwechsel erfährt. Der Ausdruck hat einen Pol $(n-1)^{\text {tor }}$ Ordnung bei $u=0$ und $(n-1)$ leicht näher angebbare Nullpunkte in Periodenparallelogramm. Nach bekannten Methoden zeigt man leicht als Darstellung des Ausdrucks (6) in der $\wp$-Funktion für ungerades $n$ :

$$
e^{-G_{1} u^{2}} \frac{\sigma\left(u \mid \omega_{1} \frac{\omega_{2}}{n}\right)}{\sigma\left(u \mid \omega_{1}, \omega_{2}\right)^{n}}=\prod_{\mu=1}^{\frac{n-1}{2}}\left(\wp(u)-\wp_{0}\right),
$$

während sich im Falle eines geraden $n$ die Darstellung findet:

$$
e^{-G_{1} u^{2}} \frac{\sigma\left(u ! \omega_{1}, \frac{\omega_{2}}{n}\right)}{\sigma\left(u \mid \omega_{1}, \omega_{2}\right)^{n}}=\sqrt{\wp(u)-e_{2}} \prod_{\mu=1}^{\frac{n-2}{2}}\left(\wp(u)-\wp_{0 \mu}\right) .
$$

Die Größe $e_{2}$ gehört bei geradem $n$ zu den $\wp$-Teilwerten, nämlich als $\wp_{0, \frac{n}{2}}$. Für die transformierte $\sigma-F u n k t i o n$ ergibt sich hiernach bei ungeradem $n$ die Darstellung:

$$
\sigma\left(u \mid \omega_{1}, \frac{\omega_{2}}{n}\right)=e^{G_{1} u^{2}} \sigma\left(u \mid \omega_{1}, \omega_{2}\right)^{n} \prod_{\mu=1}^{\frac{n-1}{2}}\left(\wp(u)-\wp_{0 \mu}\right),
$$

woran sich bei geradem $n$ anschließt:

$$
\text { (9) } \sigma\left(u \mid \omega_{1}, \frac{\omega_{2}}{n}\right)=e^{\sigma_{1} u^{2}} \sigma\left(u \mid \omega_{1}, \omega_{2}\right)^{n} \cdot \sqrt{\wp(u)-e_{2}} \prod_{\mu=1}^{\frac{n-1}{2}}\left(\wp(u)-\wp_{0_{\mu}}\right) \text {. }
$$

Entwickeln wir jetzt rechts nach Potenzen von $u$, so ergibt sich in beiden Fällen eine Reihe der Gestalt:

$$
u+a_{4} u^{5}+a_{6} u^{7}+a_{8} u^{9}+\cdots,
$$

wo $a_{2 v}$ eine ganze rationale Funktion der $g_{2}, g_{3}$ und der Teilwerte $\wp_{0 \mu}$ 
(vgl. Formel (7)) von der Dimension $-2 \nu$ in $\omega_{1}, \omega_{2}$ ist. Die numerischen Koeffizienten dieser ganzen rationalen Funktion sind rationale Zahlen. Aber zufolge der linken Seiten von (8) und (9) gilt:

$$
\begin{aligned}
& a_{4}=-\frac{1}{240} g_{2}\left(\omega_{1}, \frac{\omega_{2}}{n}\right)=-\frac{n^{4}}{240} g_{2}\left(n \omega_{1}, \omega_{2}\right), \\
& a_{6}=-\frac{1}{840} g_{3}\left(\omega_{1}, \frac{\omega_{2}}{n}\right)=-\frac{n^{6}}{840} g_{3}\left(n \omega_{1}, \omega_{2}\right) .
\end{aligned}
$$

Indem wir die beiden für jeden dieser Koeffizienten gefundenen Ausdrücke einander gleich setzen, ergibt sich der Satz: Die durch die erste Haupttransformation umgeformten Modulformen $g_{2}, g_{3}$ sind in der Gestalt:

$$
g_{2}\left(n \omega_{1}, \omega_{2}\right)=G_{2}\left(g_{2}, \wp_{0 \mu}\right), \quad g_{3}\left(n \omega_{1}, \omega_{2}\right)=G_{3}\left(g_{2}, g_{3}, \wp_{0 \mu}\right)
$$

als ganze rationale Funktionen der $g_{2}, g_{3}$ und der Teilwerte $\wp_{0 \mu}$ von den Dimensionen -4 und -6 in $\omega_{1}, \omega_{2}$ mit rationalen Zahlenkoeffizienten darstellbar. Auf der rechten Seite der ersten Gleichung (10) kann wegen der Dimension -4 die ursprüngliche Form $g_{3}$ noch nicht auftreten. Die etwa noch nicht „eigentlich“ zum $n^{\text {ten }}$ Teilungsgrade gehörenden $\wp_{0 \mu}$ kann man nach früheren Sätzen in den Wurzeln der „irreduzibelen“ Teilungsgleichung ausdrücken. Man erkennt so in den transformierten Formen (10) „natürliche Irrationalitäten“ $\operatorname{der}$ zum $n^{\text {ten }}$ Teilungsgrade gehörenden irreduzibelen speziellen Teilungsgleichung der $\wp$-Funktion.

Innerhalb der Galoisschen Gruppe der speziellen Teilungsgleichung, die wir S. 261 auf Grund der damaligen Kongruenzen (20) darstellten, bleiben unsere Irrationalitäten bei den Permutationen derjenigen Untergruppe $G_{\frac{1}{2} n \varphi(n)^{2}}$ unverändert, die durch $\gamma \equiv 0(\bmod n)$ erklärt ist. Es handelt sich somit um $\psi(n)$-wertige Irrationalitäten, so daß die zugehörigen Resolventen vom Grade $\psi(n)$ sind. Da $\Delta$ und $J$ in $g_{2}$ und $g_{3}$ rational mit rationalen Zahlenkoeffizienten darstellbar sind, so folgt als abschließender Satz: Die speziellen Transformationsgleichungen $\psi(n)^{\text {ten }}$ Grades der Modulformen $g_{2}, g_{3}, \Delta$ und der Modulfunktion $J$ sind rationale Resolventen der speziellen Teilungsgleichung der $\wp$-Funktion; die Koeffizienten jener Transformationsgleichungen gehören also dem Körper $\left(\Re, g_{2}, g_{3}\right)$ an.

Entsprechend diesem Ergebnisse wollen wir es als eine wesentliche Eigenschaft einer speziellen Transformationsgleichung erster Stufe ansehen, daß sie den Grad $\psi(n)$ hat und Koeffizienten des Körpers $\left(\Re, g_{2}, g_{3}\right)$ aufweist. Diese Eigenschaft haben nun auch noch gewisse Gleichungen für die mehrfach genannten Wurzeln der Diskriminante $\Delta$. Wir gehen zunächst auf die in (9) S. 319 für ein gegen 6 teilerfremdes $n$ erklärten ganzen Modulformen $z_{0}\left(\omega_{1}, \omega_{2}\right)$ zurück. Diese Formen zeigen gegenüber einer Substitution, die die Kongruenz $\gamma \equiv 0(\bmod n)$ erfüllt, das Verhalten: 


$$
z_{0}\left(\alpha \omega_{1}+\beta \omega_{2}, \gamma \omega_{1}+\delta \omega_{2}\right)=\left(\frac{\alpha}{n}\right) z_{0}\left(\omega_{1}, \omega_{2}\right),
$$

wo $\left(\frac{\alpha}{n}\right)$ das Legendre-Jacobische Zeichen ist. Dieses Vorzeichen ist erklärt durch:

$$
\left(\frac{\alpha}{n}\right)=\prod_{p}\left(\frac{\alpha}{p}\right)
$$

wo rechts das Legendresche Zeichen gemeint ist und das Produkt sich auf alle Primteiler von $n$ bezieht, jeden so oft gezählt, als er in $n$ enthalten ist. Hat nun $n$ irgendeinen Primteiler $p$ in ungerader höchster Potenz, so kann man $\alpha$ so wählen, daB $\left(\frac{\alpha}{p}\right)=-1$ ist, daß aber $\alpha$ bezüglich aller übrigen Primteiler von $n$ quadratischer Rest ist. Dann ist das Vorzeichen (12) das negative. Ist hingegen $n$ eine gegen 6 teilerfremde Quadratzahl, so ist das Vorzeichen (12) stets das positive. Gegenüber den Substitutionen der durch $\gamma \equiv 0(\bmod n)$ erklärten Kongruenzgruppe $\Gamma_{\psi(n)}$ bleibt $z_{0}\left(\omega_{1}, \omega_{2}\right)$ unverändert, falls $n$ eine gegen 6 teilerfremde Quadratzahl ist; dagegen bleibt gegenüber der $\Gamma_{\psi(n)}$ erst das Quadrat von $z_{0}\left(\omega_{1}, \omega_{2}\right)$ unverändert, falls $n$ eine nicht-quadratische gegen 6 teilerfremde Zahl ist.

In den Darstellungen (9) S. 319 der Formen $z_{0}$ durch die Diskriminante $\Delta$ bedeutet $(3 n)_{0}$ die kleinste nicht-negative, der Kongruenz (10) daselbst genügende Zahl. Ist $n$ quadratisch, so findet man, da $B z_{0}$ gleich $\sqrt[24]{\Delta^{\prime} \Delta^{23}}$ ist. Der SchluB auf die Existenz einer Gleichung $\psi^{\text {ten }}$ Grades für diese Modulform wird durch Wiederholung der S. 336 ausgeführten Überlegung vollzogen: Im Falle einer gegen 6 teilerfremden Quadratzahl n geniigt die durch die erste Gleichung:

$$
f\left(\omega_{1}, \omega_{2}\right)=z_{0}=\sqrt[24]{\Delta^{\prime} \Delta^{23},} \quad z_{0}=\sqrt[24]{\frac{\Delta^{\prime}}{\Delta}}
$$

gegebene ganze Modulform einer Gleichung $\psi^{\text {ten }}$ Grades mit Koeffizienten, die ganze rationale Funktionen von $g_{2}, g_{3}$ sind, während der in der zweiten Gleichung dargestellte Quotient eine später zu betrachtende Modulfunktion der $\Gamma_{\psi}$ ist.

Ist $n$ nicht-quadratisch, so haben wir mit der zweiten Potenz von $z_{0} \mathrm{zu}$ arbeiten. Man bestimme die ganze Zahl $(3 n)_{0}$, indem man der Reihe nach die acht mod 24 zu unterscheidenden $n$ durchrechnet. Ohne das Verhalten der Form $z_{0}^{2}$ gegenüber der $\Gamma_{\psi(n)}$ zu ändern, kann man diese Form um eine ganze Potenz von $\Delta$ als Faktor ändern. Mit Rücksicht hierauf gelangt man zu folgendem Satze, bei dem auch die quadratischen $n$ nicht ausgeschlossen sind: Je nachdem $n \equiv 1,5,7$ oder $11(\bmod 12)$ gilt, ist die erste, zweite, dritte oder vierte der folgenden ganzen Modulformen: 


$$
f\left(\omega_{1}, \omega_{2}\right)=\sqrt[12]{\Delta^{\prime} \Delta^{11}},=\sqrt[12]{\Delta^{\prime} \Delta^{7}},=\sqrt[12]{\Delta^{\prime} \Delta^{5}},=\sqrt[12]{\Delta^{\prime} \Delta}
$$

die Wurzel einer Gleichung $\psi^{\text {ten }}$ Grades, deren Koeffizienten rationale ganze Funktionen von $g_{2}$ und $g_{3}$ sind.

Für die durch 3 teilbaren Transformationsgrade $n$ gehen wir auf die Modulformen $\xi_{0}\left(\omega_{1}, \omega_{2}\right)$ zurück, deren Zusammenhang mit der Diskriminante durch (8) S. 315 gegeben ist. Es wiederholen sich dieselben Betrachtungen wie soeben, doch ist die Anzahl der Fallunterscheidungen geringer. In den Schlußergebnissen brauchen wir die gegen 3 teilerfremden $n$ nicht auszuschließen. Wir haben erstlich den Satz: Für ungerade Quadratzahlen $n$ ist die durch die erste Gleichung:

$$
f\left(\omega_{1}, \omega_{2}\right)=\sqrt[8]{\Delta^{\prime} \Delta^{7}}, \quad \frac{f\left(\omega_{1}, \omega_{2}\right)}{\Delta}=\sqrt[8]{\frac{\Delta^{\prime}}{\Delta}}
$$

gegebene ganze Modulform die Wurzel einer Gleichung $\psi^{\text {ten }}$ Grades, deren Koeffizienten rational und ganz in $g_{2}$ und $g_{3}$ sind. Endlich aber schlieBt sich der für alle ungeraden Grade $n$ gültige Satz an: Je nachdem der ungerade Transformationsgrad $n \equiv 1$ oder $\equiv 3(\bmod 4)$ ist, genügt die erste oder zweite der folgenden ganzen Modulformen:

$$
f\left(\omega_{1}, \omega_{2}\right)=\sqrt[4]{\Delta^{\prime} \Delta^{3}}, \quad=\sqrt[4]{\Delta^{\prime} \Delta}
$$

einer Gleichung $\psi^{\text {ten }}$ Grades mit Koeffizienten, die rationale ganze Funktionen von $g_{2}, g_{3}$ sind.

Soweit vierte und zwölfte Wurzeln aus Diskriminantenausdrücken in Betracht kommen, stellen sich die betreffenden Modulformen auf Grund der Relationen (4) und (5) S. 299 als rationale Funktionen der Teilwerte $\wp_{0 \mu}$ mit rationalen Zahlenkoeffizienten dar. $\left.{ }^{1}\right)$ Für die Formen (13) und (15) kommen wegen (4) S. 299 auch noch die Teilwerte $\wp_{0_{\mu}^{\prime}}^{\prime}$ zur Benutzung. In unseren Gleichungen $\psi^{\text {ten }}$ Grades erkennen wir auf diese Weise rationale Resolventen der speziellen Teilungsgleichungen; die Koeffizienten der Gleichungen $\psi^{\text {ten }}$ Grades gehören demnach durchweg dem Körper $\left(\Re, g_{2}, g_{3}\right)$ an. Es erscheint also gerechtfertigt, die fraglichen Gleichungen den speziellen Transformationsgleichungen erster Stufe zuzurechnen.

$\mathrm{Zu}$ einer weiteren wichtigen Resolvente $\psi^{\text {ten }}$ Grades der speziellen Teilungleichung, die für alle Grade $n>1$ (auch für die geraden) existiert, gelangen wir bei der Transformation der Perioden $\eta_{1}, \eta_{2}$ des Normalintegrals zweiter Gattung. Die erste Haupttransformation führt uns in (1) S. 304 zur Größe $G_{1}\left(\omega_{1}, \omega_{2}\right)$, die infolge ihrer Darstellung (2) S. 304 eine zur Gruppe $\Gamma_{\psi(n)}$ gehörende ganze Modulform $n^{\text {ter }}$ Stufe ist. Wir entwickeln zunächst für diese Form $G_{1}\left(\omega_{1}, \omega_{2}\right)$ aus der zweiten Dar-

1) Man beachte, daB $\Delta^{\prime}$ nach S. 338 selbst eine solche Darstellung in den $\wp_{\mu}$ zuläBt. 
stellung (1) S. 304 eine später zur Benutzung kommende Potenzreihe. Aus der Reihendarstellung (15) in I, 271 findet man bei Umordnung nach ansteigenden Potenzen von $q$ für $\eta_{2}$ die Entwicklung:

$$
\frac{\eta_{2}}{2 \omega_{2}}=\left(\frac{2 \pi}{\omega_{2}}\right)^{2}\left(\frac{1}{24}-\sum_{\nu=1}^{\infty} \Phi(\nu) q^{2 v}\right)
$$

wo $\Phi(\nu)$ wie üblich die Teilersumme von $\nu$ ist. Tragen wir die durch (2) S. 298 gegebenen transformierten Perioden ein, so folgt:

$$
\frac{\eta_{2}^{\prime}}{2 \omega_{2}^{\prime}}=n\left(\frac{2 \pi}{\omega_{2}}\right)^{2}\left(\frac{n}{24}-\sum_{\nu=1}^{\infty} n \Phi(\nu) q^{2 n v}\right)
$$

Für $G_{1}\left(\omega_{1}, \omega_{2}\right)$ ergibt sich demnach:

$$
G_{1}\left(\omega_{1}, \omega_{2}\right)=n\left(\frac{2 \pi}{\omega_{2}}\right)^{2}\left(\frac{n-1}{24}+\sum_{\nu=1}^{\infty} \Phi(\nu) q^{2 \nu}-\sum_{\nu=1}^{\infty} n \Phi(\nu) q^{2 n \nu}\right) .
$$

Ordnet man rechts beide Summen nach ansteigenden Potenzen von $q$ zusammen, so erhalten alle Potenzen $q^{2 v}$ mit einem nicht durch $n$ teilbaren $\nu$ wieder $\Phi(\nu)$ als Koeffizienten. Die einzelne Potenz $q^{2 n v}$ aber bekommt den Koeffizienten $(\Phi(n \nu)-n \Phi(\nu))$, d. h. die Teilersumme $\Phi(n \nu)$ von $n \nu$, vermindert um die Summe aller Teiler von $n \nu$, die $n$ als Faktor enthalten: Die bei der ersten Haupttransformation der Perioden $\eta_{1}, \eta_{2}$ ein-

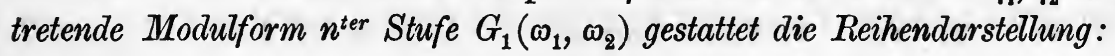

$$
G_{1}\left(\omega_{1}, \omega_{2}\right)=n\left(\frac{2 \pi}{\omega_{2}}\right)^{2}\left(\frac{n-1}{24}+\sum_{\nu=1}^{\infty} \Phi_{n}(\nu) q^{2 \nu}\right),
$$

wo $\Phi_{n}(\nu)$ die Summe aller Teiler von $\nu$ ist, die nicht selbst den Transformationsgrad $n$ als Teiler enthalten.

Der SchluB auf die Existenz der Gleichung $\psi^{\text {ten }}$ Grades für $G_{1}$ wird wie in den obigen Fällen begründet. Als Resolvente der speziellen Teilungsgleichung aber ist diese Gleichung durch die Darstellung (2) S. 304 der Form $G_{1}$ als symmetrischer Funktion der $\wp_{0 \mu}$ charakterisiert. Wir merken sogleich den Satz an: Für jeden Transformationsgrad $n>1$ genügt die ganze Modulform $n^{\text {ter }}$ Stufe $G_{1}\left(\omega_{1}, \omega_{2}\right)$ einer Transformationsgleichung erster Stufe $\psi(n)^{\text {ten }}$ Grades, deren Koeffizienten als ganze Funktionen von $g_{2}, g_{3}$ dem Körper $\left(\Re, g_{2}, g_{3}\right)$ angehören.

Übrigens ist einleuchtend, daB wir ganz entsprechend wie oben die $z_{0}$ und $\xi_{0}$ auch die S. $325 \mathrm{ff}$. für ungerade $n$ gewonnenen Formen $y_{0}$ und $z_{0}$ zum Ausgangspunkte für die Bildung von Gleichungen $\psi^{\text {ton }}$ Grades machen können. Für alle ungeraden $n$ werden die Quadrate $y_{0}^{2}, z_{0}^{2}$ Wurzeln von Gleichuingen $\psi^{\text {ten }}$ Grades, deren Koeffizienten rational und ganz in $g_{2}, g_{3}$ sind; bei ungeraden Quadratzahlen $n$ genügen die $y_{0}, z_{0}$ selbst solchen Gleichungen. 


\section{§. Ansatz der speziellen Transformationsgleichungen. Geschichtliche Notizen.}

Über die Gestalt der verschiedenen Transformationsgleichungen, die in $\S 1$ als existierend erkannt wurden, kann man auf Grund der allgemeinen Sätze in I, $299 \mathrm{ff}$. über Darstellung der Modulfunktionen und insbesondere der ganzen Modulformen erster Stufe eine Reihe allgemeiner Angaben machen. Jede ganze Modulform erster Stufe der Dimension $-2 v$ ist als rationale ganze Funktion von $g_{2}, g_{3}$ in der Gestalt (8) in I, 309 darstellbar, wo die $C_{l, m}$ konstante Koeffizienten sind. Es ist nun z. B. die Dimension des Koefizizienten von $G_{1}^{\psi-v}$ in der Gleichung $\psi^{\text {ten }}$ Grades für $G_{1}$ gleich $-2 \nu$. Wir haben also den fraglichen Koefizienten nach der eben genannten Regel (8) in I, 309 anzusetzen, wobei dann die $C_{l, m}$ rationale Zahlen werden. Die Überlegung überträgt sich sofort auch auf die Transformationsgleichungen für $g_{2}$ und $g_{3}$, deren Koeffizienten wieder "ganze" Modulformen erster Stufe sind: Als Ansütze für die speziellen Transformationsgleichungen der Formen $G_{1}, g_{2}^{\prime}, g_{3}^{\prime}$ haben wir:

$$
\begin{gathered}
G_{1}^{\psi}+\alpha_{1} g_{2} G_{1}^{\psi-2}+\alpha_{2} g_{3} G_{1}^{\psi-3}+\alpha_{3} g_{2}^{2} G_{1}^{\psi-4}+\alpha_{4} g_{2} g_{3} G_{1}^{\psi-5} \\
+\left(\alpha_{5} g_{2}^{3}+\beta_{5} g_{3}^{2}\right) G_{1}^{\psi-6}+\alpha_{6} g_{2}^{2} g_{3} G_{1}^{\psi-7}+\cdots=0 \\
g_{2}^{\prime} \psi+\alpha_{1}^{\prime} g_{2} g_{2}^{\prime} \psi-1+\alpha_{2}^{\prime} g_{2}^{2} g_{2}^{\prime} \psi-2+\left(\alpha_{3}^{\prime} g_{2}^{3}+\beta_{3}^{\prime} g_{3}^{2}\right) g_{2}^{\prime} \psi-3+\cdots=0 \\
g_{3}^{\prime} \psi+\alpha_{1}^{\prime \prime} g_{3} g_{3}^{\prime} \psi-1+\left(\alpha_{2}^{\prime \prime} g_{2}^{3}+\beta_{2}^{\prime \prime} g_{3}^{2}\right) g_{3}^{\prime} \psi-2+\left(\alpha_{3}^{\prime \prime} g_{2}^{3} g_{3}+\beta_{3}^{\prime \prime} g_{3}^{3}\right) g_{3}^{\prime} \psi-3+\cdots=0
\end{gathered}
$$

wo die $\alpha, \beta, \ldots$ durchweg rationale Zahlen sind.

Bei den Gleichungen für die Modulformen (14) S. 340 kann man den Ansatz noch ein wenig verfeinern. Nach einem in I, 309 aufgestellten Satze hat eine ganze Modulform erster Stufe der Dimension $-2 \nu$ in Diskontinuitätsbereiche der Modulgruppe $\Gamma^{(\omega)}$ Nullpunkte in der Gesamtordnung $\frac{\nu}{6}$. Liegt in der Spitze $\omega=i \infty$ jenes Bereiches ein Nullpunkt der Ordnung $m$, so ist hierfür charakteristisch, daß die nach Potenzen von $q$ fortschreitende Reihe der Modulform mit der Potenz $q^{2 m}$ beginnt. In diesem Falle ist auch noch der Quotient der Modulform und der $m^{\text {ten }}$ Potenz $\Delta^{m}$ der Diskriminante eine ganze Modulform erster Stufe, so daB die vorgelegte Form als Produkt von $\Delta^{m}$ und einer rationalen ganzen Funktion der $g_{2}, g_{3}$ darstellbar ist. In einer ganzen Modulform erster Stufe, die nur in der Spitze $\omega=i \infty$ des Diskontinuitätsbereiches verschwindet, erkennt man leicht das Produkt einer Konstanten und einer Potenz von $\Delta$. Endlich beachte man für die zu vollziehenden Schlüsse, daB eine ganze Modulform erster Stufe in der fraglichen Spitze des Diskontinuitätsbereiches jedenfalls nur einen Nullpunkt ganzzahliger Ordnung haben kann; sie ist nämlich gegenüber der Substitution $S$ invariant und dieserhalb nach ganzen Potenzen von $q^{2}$ entwickelbar. 
Die Modulformen (14) S. 340 haben nun die Dimensionen - 12, $-8,-6$ und -2 , woraus man die Dimensionen der Koeffizienten in den zugehörigen Transformationsgleichungen zu bestimmen hat. Die Potenzreihe für $f=\sqrt[12]{\Delta^{\prime} \Delta^{11}}$ sowie die Reihen für alle mit ihr gleichberechtigten Formen beginnen mit Exponenten $>\frac{11}{6}$ von $q$, woraus man leicht auf eine Mindestordnung des Nullpunktes der einzelnen symmetrischen Grundfunktion jener $\psi(n)$ Formen in der Spite $\omega=i \infty$ schlieBt. Speziell kann das Absolutglied der Transformationsgleichung als Wurzel aus einem Produkt von Diskriminanten nur in der fraglichen Spitze verschwinden und stellt demnach, abgesehen von einem konstanten Faktor, eine Potenz von $\Delta$ dar. Diese Überlegungen, die man leicht auch auf die drei anderen Formen (14) S. 340 ausdehnt, führen zu dem Satze: Für die zwölfte Wurzel der Diskriminante $\triangle$ haben wir in den vier zu unterscheidenden Fällen $n \equiv 1,5,7$ und $11(\bmod 12)$ als Ansätze der Transformationsgleichungen der Formen (14) S. 340:

$$
\begin{aligned}
& f^{\psi}+\alpha_{1} \Delta f^{\psi-1}+\left(\alpha_{2} g_{2}^{3}+\beta_{2} g_{3}^{2}\right) \Delta f^{\psi-2} \\
& \quad+\left(\alpha_{3} g_{2}^{3}+\beta_{3} g_{3}^{2}\right) \Delta^{2} f^{\psi-3}+\cdots+\alpha_{\psi} \Delta^{\psi}=0, \\
& f^{\psi}+\alpha_{2} g_{2} \Delta f^{\psi-2}+\left(\alpha_{3} g_{2}^{3}+\beta_{3} g_{3}^{2}\right) \Delta f^{\psi-3} \\
& \quad+\alpha_{4} g_{2}^{2} \Delta^{2} f^{\psi-4}+\cdots+\alpha_{\psi} \Delta^{\frac{2}{3} \psi}=0 \\
& f^{\psi}+\alpha_{2} \Delta f^{\psi-2}+\alpha_{3} g_{3} \Delta f^{\psi-3}+\left(\alpha_{4} g_{2}^{3}+\beta_{4} g_{3}^{2}\right) \Delta f^{\psi-4} \\
& \quad+\alpha_{5} g_{3} \Delta^{2} f^{\psi-5}+\cdots+\alpha_{\psi} \Delta^{\frac{1}{2} \psi}=0 \\
& f^{\psi}+\alpha_{6} \Delta f^{\psi-6}+\alpha_{8} g_{2} \Delta f^{\psi-8}+\alpha_{9} g_{3} \Delta f^{\psi-9} \\
& \quad+\alpha_{10} g_{2}^{2} \Delta f^{\psi-10}+\alpha_{11} g_{2} g_{3} \Delta f^{\psi-11}+\cdots+\alpha_{\psi} \Delta^{\frac{1}{6} \psi}=0,
\end{aligned}
$$

wo die $\alpha, \beta, \ldots$ durchweg rationale Zahlen sind. ${ }^{1}$ )

Ähnliche Betrachtungen wird man auch bei den übrigen Transformationsgleichungen erster Stufe für Modulformen durchführen. Bei den ungeraden nicht-quadratischen und durch 3 teilbaren Graden $n$ haben wir mit den beiden in (16) S. 340 gegebenen Formen $f\left(\omega_{1}, \omega_{2}\right)$ zu arbeiten, und zwar mit $\sqrt[4]{\Delta^{\prime} \Delta^{3}}$ oder $\sqrt[4]{\Delta^{\prime} \Delta}$, je nachdem $n \equiv 1$ oder $\equiv 3(\bmod 4)$ gilt. Hier besteht der Satz, bei dem übrigens auch die quadratischen $n$ nicht ausgeschlossen $\mathrm{zu}$ werden brauchen: Ist der Transformationsgrad $n$ durch 3 teilbar und $\equiv 1(\bmod 4)$, so liegt für die Modulform $f=\sqrt[4]{\Delta^{\prime} \Delta^{3}}$ eine Transformationsgleichung der Gestalt:

1) Natürlich ist nicht gemeint, daB die z. B. in den drei ersten Gleichungen übereinstimmend gebrauchte Bezeichnung $\alpha_{g}$ stets die gleiche rationale Zahl sein soll. 


$$
\begin{aligned}
& f^{\psi}+\alpha_{1} \Delta f^{\psi-1}+\left(\alpha_{2} g_{2}^{3}+\beta_{2} g_{3}^{2}\right) \Delta f^{\psi-2} \\
& \quad+\left(\alpha_{3} g_{2}^{3}+\beta_{2} g_{3}^{2}\right) \Delta^{2} f^{\psi-3}+\cdots+\alpha_{\psi} \Delta^{\psi}=0
\end{aligned}
$$

vor, während sich im Falle $n \equiv 3(\bmod 4)$ für die Form $f=\sqrt[4]{\Delta^{\prime} \Delta}$ der Ansatz anschließt:

(9) $f^{\psi}+\alpha_{2} \Delta f^{\psi-2}+\alpha_{3} g_{3} \Delta f^{\psi-3}+\alpha_{4} \Delta^{2} f^{\psi-4}+\cdots+\alpha_{\psi} \Delta^{\frac{1}{2} \psi}=0$.

Endlich reihen wir noch die besonderen Transformationsgleichungen an, die bei quadratischen Transformationsgraden $n$ auftreten: Für alle gegen 6 teilerfremden quadratischen Transformationsgeraden $n$ genügt $f=\sqrt[24]{\Delta^{\prime} \Delta^{23}}$ einer Transformationsgleichung:

$$
\begin{aligned}
& f^{\psi}+\alpha_{1} \Delta f^{\psi-1}+\left(\alpha_{2} g_{2}^{3}+\beta_{2} g_{3}^{2}\right) \Delta f^{\psi-2} \\
& \quad+\left(\alpha_{3} g_{2}^{3}+\beta_{3} g_{3}^{2}\right) \Delta^{2} f^{\psi-3}+\cdots+\alpha_{\psi} \Delta^{\psi}=0,
\end{aligned}
$$

und für die durch 3 teilbaren ungeraden Quadrate $n$ hat man bei der Form $f=\sqrt[8]{\Delta^{\prime} \Delta^{7}}$ den Ansatz:

$$
\begin{aligned}
& f^{\psi}+\alpha_{1} \Delta f^{\psi-1}+\left(\alpha_{2} g_{2}^{3}+\beta_{2} g_{3}^{2}\right) \Delta f^{\psi-2} \\
& \quad+\left(\alpha_{3} g_{2}^{3}+\beta_{3} g_{3}^{2}\right) \Delta^{2} f^{\psi-3}+\cdots+\alpha_{\psi} \Delta^{\psi}=0 .
\end{aligned}
$$

Soweit die Glieder explizite angegeben sind, stimmen die Ansätze (10) und (11) noch überein. Die $\alpha, \beta, \ldots$ sind natürlich wieder rationale Zahlen.

Die wichtigsten Transformationsgleichungen erster Stufe sind diejenigen für die Modulfunktion erster Stufe $J(\omega)$. Wir wollen übrigens hier die Gleichungen nicht für $J(\omega)$, sondern nach Vorgang von $\mathrm{H}$. W eber für die Funktion:

$$
12^{3} J(\omega)=j(\omega)
$$

anschreiben, weil dadurch in arithmetischer Hinsicht wesentliche Vorteile gewonnen werden. Es gründen sich diese Vorteile auf die Potenzreihe für $j(\omega)$, die wir zunächst aufstellen. Aus (3) in I, 274 und (9) in I, 433 folgt:

$$
\begin{gathered}
12 g_{2}=\left(\frac{2 \pi}{\omega_{2}}\right)^{4}\left(1+240 q^{2}+2160 q^{4}+6720 q^{6}+\cdots\right), \\
\frac{1}{\sqrt[3]{\Delta}}=\left(\frac{\omega_{2}}{2 \pi}\right)^{4} q^{-\frac{2}{3}}\left(1+8 q^{2}+44 q^{4}+192 q^{6}+\cdots\right),
\end{gathered}
$$

wo die in den Klammern stehenden Potenzreihen durchweg ganzzahlige Koeffizienten besitzen. Durch Multiplikation der beiden letzten Gleichungen folgt:

$$
\begin{aligned}
\frac{12 g_{2}}{\sqrt[3]{\Delta}} & =12 \sqrt[3]{J}=\sqrt[3]{j(\omega)} \\
& =q^{-\frac{2}{3}}\left(1+248 q^{2}+4124 q^{4}+34752 q^{6}+\cdots\right)
\end{aligned}
$$


Durch Erheben zur dritten Potenz ergibt sich für $j(\omega)$ selbst:

$$
j(\omega)=q^{-2}+744+196884 q^{2}+21493760 q^{4}+\cdots
$$

oder bei Spaltung der Koeffizienten in Primfaktoren:

$$
j(\omega)=q^{-2}+2^{3} \cdot 3 \cdot 31+2^{2} \cdot 3^{3} \cdot 1823 q^{2}+2^{11} \cdot 5 \cdot 2099 q^{4}+\cdots
$$

Das Wichtige ist hier, daß der Koeffizient des ersten Gliedes gleich 1 ist und die Koeffizienten aller Glieder ganze Zahlen sind.

Die $\psi(n)$ bei Transformation $n^{\text {ten }}$ Grades aus $j(\omega)$ zu gewinnenden Größen sind nun:

$$
j\left(\frac{A \omega+B}{D}\right)=e^{-\frac{2 i \pi B}{D}} q^{-\frac{2 A}{D}}+744+196884 e^{\frac{2 i \pi B}{D}} q^{\frac{2 A}{D}}+\cdots,
$$

wo $A, B, D$ im bekannten Sinne (vgl. S. 335) gebraucht sind. Liegt $\omega$ im Innern der $\omega$-Halbebene, so gilt dasselbe von allen transformierten Werten $\frac{A \omega+B}{D}$. Die symmetrischen Grundfunktionen der $\psi(n)$ transformierten Funktionen (16), in denen wir zunächst rationale Funktionen von $j(\omega)$ erkannten, sind demnach "ganze" rationale Funktionen von $j(\omega)$, da sie für keinen endlichen Wert von $j$ unendlich werden können. Für die Transformationsgleichung haben wir somit den Ansatz:

$$
j^{\prime \psi}+G_{1}(j) j^{\prime \psi-1}+G_{2}(j) j^{\prime \psi-2}+\cdots+G_{\psi}(j)=0,
$$

wo die Koeffizienten rationale ganze Funktionen von $j(\omega)$ mit rationalen Zahlenkoeffizienten sind.

Es läßt sich nun beweisen, daß die Zahlenkoeffizienten der Funktionen $G(j)$ durchweg ganz sind. Es gilt nämlich folgender Satz: Hat eine ganze Funktion:

$$
G(j)=c_{0} j^{m}+c_{1} j^{m-1}+c_{2} j^{m-2}+\cdots+c_{m}
$$

von $j$ eine Potenzreihe nach $q^{2}$ mit durchweg ganzzahligen Koeffizienten, so müssen die $c_{0}, c_{1}, \ldots, c_{m}$ selbst ganze Zahlen sein. Man kann den Satz durch den SchluB der vollständigen Induktion zeigen und nehme also an, daB er für Funktionen $(m-1)^{\text {ten }}$ Grades richtig ist. Aus (14) folgt für die $m^{\text {te }}$ Potenz von $j$ eine Entwicklung:

$$
j^{m}=q^{-2 m}+a_{-m+1} q^{-2(m-1)}+a_{-m+2} q^{-2(m-2)}+\cdots
$$

mit durchweg ganzzahligen Koeffizienten $a$. Das Anfangsglied der Potenzreihe für $G(j)$ ist also $c_{0} q^{-2 m}$, so daß $c_{0}$ der Voraussetzung nach eine ganze Zahl ist. Dann aber hat (wegen der ganzzahligen $a_{-m+1}, a_{-m+2}, \ldots$ ) auch die Funktion $(m-1)^{\text {ten }}$ Grades:

$$
G(j)-c_{0} j^{m}=c_{1} j^{m-1}+c_{2} j^{m-2}+\cdots+c_{m}
$$


eine Potenzreihe nach $q^{2}$ mit ganzzahligen Koeffizienten, so daB der Aunahme nach auch die $c_{1}, c_{2}, \ldots, c_{m}$ ganze Zahlen sind. Der Beweis wird bündig durch den Umstand, daB der Satz für Funktionen des Grades $m=0$ selbstrerständlich ist.

Die in (17) auftretenden ganzen Funktionen $G(j)$ haben nun sicher rationale Zahlenkoeffizienten, so daß mit Rücksicht auf die Ganzzahligkeit der Koeffizienten in (14) die Potenzreihen für jene Funktionen $G(j)$ rationale Koeffizienten haben. Nun sind aber die $G(j)$ die symmetrischen Grundfunktionen der $\psi(n)$ transformierten Funktionen (16). Bei der Bauart der Koeffizienten in (16) rechts folgt also, daB die Reihenkoeffizienten der $G(j)$ ganze Zahlen des Kreisteilungskörpers $(\Re, \varepsilon)$ sind, wo $\varepsilon=e^{\frac{2 i \pi}{n}}$ ist. Ganze Zahlen dieses Körpers, die rational sind, stellen aber rationale ganze Zablen dar. Also haben die Reihenentwicklungen nach Potenzen von $q^{2}$ für die in (17) auftretenden $G(j)$ durchweg ganze rationale Koeffizienten. Nach dem eben bewiesenen Satze sind demnach auch die Koeffizienten $c$ in den Ausdrücken (18) dieser Funktionen, wie zu beweisen war, ganze Zahlen.

Ein weiterer wichtiger Satz ist, daß die Transformationsgleichung (17) auch in $j$ auf den Grad $\psi(n)$ ansteigt. Unter den symmetrischen Grundfunktionen der $\psi(n)$ transformierten Funktionen (16) wird nämlich das Produkt dieser Funktionen $\pm G_{\psi}(j)$ bei $\omega=i \infty$ stärker unendlich als die übrigen Funktionen $G(j)$. Die Ordnung des Unendlichwerdens ergibt sich aus dem Anfangsgliede der Reihenentwicklung:

$$
\pm G_{\psi}(j)=e^{-2 i \pi_{0} \sum^{\frac{A \omega+B}{D}}}+\cdots,
$$

wo sich die Summe auf die $\psi(n)$ Repräsentanten für eigentliche Transformation $n^{\text {ten }}$ Grades bezieht. Ist nun $n=A \cdot D$ die einzelne Zerlegung von $n$ in zwei positive ganzzahlige Faktoren, so durchläuft für diese $A, D$ die Zahl $B$ alle diejenigen Zahlen der Reihe $0,1,2, \ldots, D-1$, welche teilerfremd gegen den größten gemeinsamen Teiler $t$ von $A$ und $D$ sind. Es sind dies $\frac{D}{t} \varphi(t)$ Zahlen $B$, so daB das einzelne Paar $A, D$ immer $\frac{D}{t} \varphi(t)$ Glieder der Summe in (20) liefert. Statt (20) können wir demnach auch schreiben:

$$
G_{\psi}(j)= \pm q^{-2 \sum_{A}^{\frac{A}{t} p(t)}}+\cdots
$$

wo sich die Summe auf alle Teiler $A$ von $n$ bezieht. Nun ist aber, da die einzelne Zerlegung $n=A \cdot D$ im ganzen $\frac{D}{t} \varphi(t)$ Repräsentanten liefert, die Anzahl $\psi(n)$ aller Repräsentanten in der Gestalt darstellbar: 


$$
\psi(n)=\sum_{D} \frac{D}{t} \varphi(t)
$$

wo die Summe sich wieder auf alle Teiler $D$ von $n$ bezieht. Also ist die Summe im Exponenten von (21) einfach gleich $\psi(n)$. Daraus folgt aber in der Tat, daB $G_{\psi}(j)$ in $j$ auf den Grad $\psi(n)$ ansteigt. Der Koeffizient des Anfangsgliedes in (21) durfte sogleich gleich \pm 1 gesetzt werden; denn er ist zufolge (20) eine Einheitswurzel, und er ist andrerseits bekanntlich rational. Das höchste Glied ron $G_{\psi}(j)$ ist demnach $\pm j^{\psi}$, während keine der übrigen Funktionen $G(j)$ im Ansatze (17) den Grad $\psi(n)$ erreicht.

Die algebraische Natur der Transformationsgleichungen betreffend notieren wir zunächst nur den folgenden gleich zu benutzenden Satz: Die gesamten Transformationsgleichungen $\psi^{\text {ten }}$ Grades sind im Körper $\left(\Re, g_{2}, g_{3}\right)$ irreduzibel und bleiben auch dann irreduzibel, wenn man den Körper durch Adjunktion irgend welcher "numerischer" Irrationalitäten erweitert. Døn Beweis kann man direkt aus der Gleichberechtigung der $\psi(n)$ Wurzeln der einzelnen Transformationsgleichung bezüglich der Modulgruppe $\Gamma^{(\omega)}$ durch dieselben Überlegungen führen, die wir S. $247 \mathrm{ff}$. für die speziellen Teilungsgleichungen ausführten.

Die auf der linken Seite der Transformationsgleichung (17) stehende ganze ganzzahlige Funktion von $j^{\prime}$ und $j$ möge kurz $F\left(j^{\prime}, j\right)$ genannt werden. Setzt man insbesondere $j^{\prime}=j(n \omega)$, der ersten Haupttransformation entsprechend, so besteht die Gleichung:

$$
F(j(n \omega), j(\omega))=0
$$

in $\omega$ identisch und bleibt also auch gültig, wenn man $\frac{\omega}{n}$ statt $\omega$ einträgt:

$$
F\left(j(\omega), j\left(\frac{\omega}{n}\right)\right)=0 \text {. }
$$

Nun ist aber $j^{\prime}=j\left(\frac{\omega}{n}\right)$ die durch die zweite Haupttransformation aus $j(\omega)$ entstehende Funktion. Wir haben somit in $F\left(j, j^{\prime}\right)=0$, wo $j^{\prime}$ wieder auf den Grad $\psi(n)$ ansteigt, gleichfalls eine Gleichung $\psi(n)^{\text {ten }}$ Grades für die transformierten Funktionen $j^{\prime}$. Wegen der schon festgestellten Irreduzibilität ist also diese Gleichung $F\left(j, j^{\prime}\right)=0$ bis auf einen konstanten Faktor mit der Gleichung $F\left(j^{\prime}, j\right)=0$ identisch. Dieser konstante Faktor aber ist gleich +1 oder -1 , da $F\left(j^{\prime}, j\right)$ als höchstes Glied in j die Potenz $\pm j^{\psi}$ hat:

$$
F\left(j, j^{\prime}\right)= \pm F\left(j^{\prime}, j\right) \text {. }
$$

Die Funktion $F\left(j^{\prime}, j\right)$ ist also entweder in $j, j^{\prime}$ symmetrisch gebaut oder wechselt bei Austausch ron $j$ und $j^{\prime}$ das Zeichen. Der zweite Fall kann aber nicht vorliegen. Würde er gelten, so wäre $F(j, j)=0$, d. h. unsere 
Gleichung $\psi^{\text {ten }}$ Grades $F\left(j^{\prime}, j\right)=0$ für $j^{\prime}$ hätte die Lösung $j^{\prime}=j$, so daB $F\left(j^{\prime}, j\right)$ den Linearfaktor $\left(j^{\prime}-j\right)$ besäße. Dies widerspricht aber der Irreduzibilität. Also ist $F\left(j^{\prime}, j\right)$ in $j^{\prime}$ und $j$ symmetrisch.

Da $G_{1}(j)$ die negativ genommene Summe der $\psi(n)$ transformierten Funktionen (16) ist und unter ihnen $j(n \omega)$ in der Spitze $i \infty$ am stärksten unendlich wird, so gilt als Anfangsglied der Potenzreihe von $G_{1}(j)$ :

$$
G_{1}(j)=-q^{-2 n}+\cdots
$$

Im Ausdrucke (18) von $G_{1}(j)$ ist also $-j^{n}$ das höchste Glied. Nun ist zufolge (22) oder auch zufolge der ursprünglichen Erklärung (4) S. 220 von $\psi(n)$ die Anzahl $\psi(n)-1$ bei primzahligem $n$ gleich $n$, bei zusammengesetztem $n$ aber stets $>n$. Das in $F\left(j^{\prime}, j\right)$ auftretende Glied $-j^{\prime} \psi-1 j^{n}$ ist also bei primzahligem $n$ sich selbst symmetrisch, während bei zusammengesetztem $n$ daneben noch das Glied $j^{\prime}{ }^{n}{ }^{\psi-1}$ auftritt. Unter Zusammenfassung aller einzelnen Ergebnisse notieren wir den Satz: Die bei Transformation $n^{\text {ten }}$ Grades eintretende irreduzible Transformationsgleichung $\psi(n)^{\text {ten }}$ Grades für $j(\omega)=12^{3} J(\omega)$ hat die Gestalt:

$$
j^{\prime} \psi+j^{\psi}+\sum_{k, l} c_{k i} j^{\prime k} j^{l}=0,
$$

wo sich die Summe auf die Kombinationen ganzer Zahlen $k, l$ der Reihe $0,1,2, \ldots, \psi-1$ bezieht; die $c$ sind der Bedingung $c_{k l}=c_{z k}$ genügende ganze Zahlen, und speziell gilt bei primzahligem $n$, wo $\psi(n)=n+1$ ist, $c_{n n}=-1$, während bei zusammengesetztem Transformationsgrade $c_{\psi-1, n}$ $=c_{n, \psi-1}=-1$ zutrifft.

Die Gleichungen (1), (2) und (3) für $G_{1}, g_{2}^{\prime}$ und $g_{3}^{\prime}$ sind von F. Müller ${ }^{1}$ ) behandelt, und zwar in der Art, daB die Transformationsgleichungen (1) für $G_{1}$ wirklich in Ansatz gebracht werden, während für $g_{2}^{\prime}$ und $g_{3}^{\prime}$ rationale Ausdrücke in $G_{1}, g_{2}, g_{3}$ notiert werden, mittels deren die Gleichungen (2) und (3) als Resolventen von (1) eingeführt werden. Die Endergebnisse sind noch sehr wenig weitgehend; es gelingt nur für die Transformationsgrade 2, 3 und 4 die Gleichungen wirklich anzugeben.

Die Transformationsgleichungen für die Wurzeln der Diskriminante $\Delta$ sind zuerst von Kiepert ${ }^{2}$ ) untersucht, und zwar unter Bevorzugung der Primzahltransformationen. Es gelingt, bis zum Grade 23 die fertigen Gestalten der Gleichungen zu gewinnen. Die Hilfsmittel zur Berechnung der Koeffizienten der Transformationsgleichungen sind in den bisher genannten Arbeiten die Potenzreihen der ursprünglichen und der transformierten Größen nach $q^{2}$.

1) „De transformatione functionum ellipticarum", Berliner Dissert. von 1867. Vgl. auch die weiteren Nachweise in „Enneper-Müller", S. $495 \mathrm{ff}$.

2) „Zur Transformationstheorie der elliptischen Funktionen", Abh. 1 und 3, Journ. f. Math., Bd. 87 (1879) und Bd. 95 (1883). 
Eine Weiterbildung von grundsätzlicher Bedeutung erfuhr die Theorie der speziellen Transformationsgleichungen durch die von Klein ausgebildete Theorie der Modulfunktionen. Gegenüber den verschiedenartigen Gattungen von Transformationsgleichungen lieferte diese Theorie zunächst die Möglichkeit der Sichtung und sachgemäßen Anordnung, indem sie ein Urteil darüber lieferte, welche unter den transformierten Größen im einzelnen Falle als die einfachste anzusehen ist, und welches die Beziehungen der übrigen zu diesen einfachsten Größen sind. Darüber hinaus benutzt Klein neben den Potenzreihen die auf gruppentheoretischer und geometrischer Grundlage erwachsenen Hilfsmittel der Riemannschen Theorie der algebraischen Funktionen zur Aufstellung der Transformationsgleichungen. Die nachfolgende Darstellung wird die großen Erfolge der Kleinschen Methoden darlegen. In den Vordergrund treten jetzt zunächst die Transformationsgleichungen für $J(\omega)$. Die Primzahlgrade bis $n=13$ behandelte Klein selbst ${ }^{1}$ ); im Anschluß hieran stellte J. Gierster ${ }^{2}$ ) für einige niedere zusammengesetzte Grade Untersuchungen über die Transformationsgleichungen von $J(\omega)$ an. Später sind diese Untersuchungen dann von Kiepert ${ }^{3}$ ) aufgenommen und wesentlich auch mit dem Hilfsmittel der Reihenentwicklungen gefördert. Eine Behandlung der Transformationsgleichungen für die Wurzeln der Diskriminante vom Standpunkte der Theorie der Modulfunktionen gab A. Hurwitz. ${ }^{4}$ )

\section{$\S$ 3. Das Transformationspolygon $T_{n}$ und die Transformationsfläche $\boldsymbol{F}_{\boldsymbol{n}}$.}

Die von Klein eingeführte Methode zur Behandlung der Transformationsgleichungen gründet sich auf die Diskontinuitätsbereiche der Kongruenzgruppen $\Gamma_{\psi(n)}$ (vgl. I, 233). Wir haben vielfach die zu den beiden Haupttransformationen $n^{\text {ten }}$ Grades gehörenden Untergruppen nebeneinander zu betrachten und unterscheiden sie dieserhalb durch die

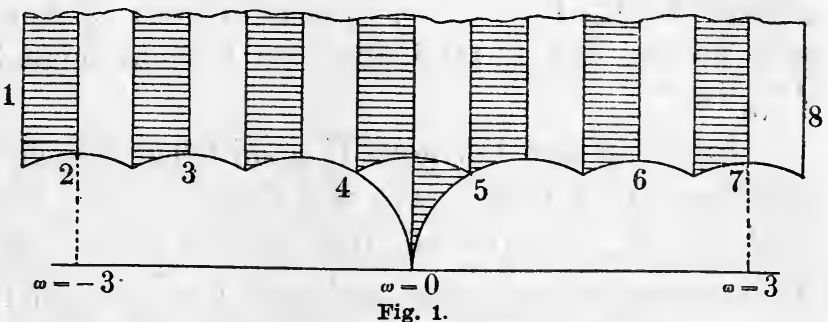

1) „Über die Transformation der elliptischen Funktionen und die Auflösung der Gleichungen fünften Grades", Math. Ann., Bd. 14 (1878).

2) „Notiz über Modulargleichungen bei zusammengesetztem Transformationsgrad", Math. Ann., Bd. 14 (1879).

3) "Über die Transformation der elliptischen Funktionen bei zusammengesetztem Transformationsgrad“, Math. Ann., Bd. 32 (1888).

4) "Grundlagen einer independenten Theorie der elliptischen Modulfunktionen und Theorie der Multiplikatorgleichungen erster Stufe", Math. Ann., Bd. 18 (1881). 
Bezeichnungen $\Gamma_{\psi(n)}$ und $\Gamma_{\psi(n)}^{\prime}$. Die zur ersten Haupttransformation gehörende $\Gamma_{\psi(n)}$ ist durch $\gamma \equiv 0(\bmod n)$ charakterisiert, die $\Gamma_{\psi(n)}^{\prime}$ aber durch $\beta \equiv 0(\bmod n)$. Die in der $\omega$-Halbebene gelegenen Diskontinuitätsbereiche dieser Gruppen können wir in der Gestalt von Kreisbogenpolygonen wählen, die wir „Transformationspolygone ${ }^{\prime \prime}$ nennen und durch $\mathbf{T}_{n}$ bzw. $\mathrm{T}_{n}^{\prime}$ bezeichnen.

Als Beispiel betrachten wir zunächst das zum Grade $n=7$ gehörende Transformationspolygon $\mathrm{T}_{7}^{\prime}$. Entsprechend dem Index $\psi(7)=8 \mathrm{der}$ Gruppe können wir $T_{7}^{\prime}$ aus acht Doppeldreiecken des Netzes der $\omega$-Halbebene aufbauen, die sich zu dem in Fig. 1, S. 349, angegebenen Polygone zusammenordnen. Wir bezeichnen diese acht Doppeldreiecke durch die Substitution $\left(\begin{array}{l}\alpha, \beta \\ \gamma, \delta\end{array}\right)$, mittels deren sie aus dem als Diskontinuitätsbereich der Gesamtgruppe $\Gamma=\Gamma^{(\omega)}$ in I, 295 ausgewählten Doppeldreiecke hervorgehen. Es handelt sich dann in Fig. 1 um die acht Doppeldreiecke:

$$
\left(\begin{array}{l}
1,0 \\
0,1
\end{array}\right), \quad\left(\begin{array}{c}
1, \pm 1 \\
0,1
\end{array}\right), \quad\left(\begin{array}{c}
1, \pm 2 \\
0,1
\end{array}\right), \quad\left(\begin{array}{c}
1, \pm 3 \\
0,1
\end{array}\right), \quad\left(\begin{array}{c}
0,-1 \\
1,0
\end{array}\right)
$$

Man stellt leicht fest, daB keine zwei unter diesen Doppeldreiecken bezüglich der $\Gamma_{\psi(7)}^{\prime}$ äquivalent sind. Die acht in (1) gegebenen Substitutionen $V_{0}=1, V_{1}, \ldots, V_{7}$ können wir demnach zur Zerlegung der Gruppe $\Gamma$ in acht Nebengruppen:

$$
\Gamma=\Gamma_{\psi(7)}^{\prime}+\Gamma_{\psi(7)}^{\prime} \cdot V_{1}+\Gamma_{\psi(7)}^{\prime} \cdot V_{2}+\cdots+\Gamma_{\psi(7)}^{\prime} \cdot V_{7}
$$

benutzen. Da hier rechts jede Substitution von $\Gamma$ einmal und nur einmal auftritt, so ist jedes Doppeldreieck des die ganze $\omega$-Halbebene bedeckenden Netzes bezüglich der $\Gamma_{\psi(7)}^{\prime}$ mit einem und nur einem der acht Doppeldreiecke $V_{0}, V_{1}, V_{2}, \ldots, V_{7}$ des Polygons $T_{7}^{\prime}$ äquivalent. Daraus aber geht hervor, daß $T_{7}^{\prime}$ tatsächlich ein Diskontinuitätsbereich der Gruppe $\Gamma_{\psi(7)}^{\prime}$ ist.

Irgendein dem Polygone $T_{7}^{\prime}$ unmittelbar benachbartes Doppeldreieck des Netzes ist natürlich auch mit einem der acht Doppeldreiecke von $T_{7}^{\prime}$ bezüglich $\Gamma_{\psi(7)}^{\prime}$ äquivalent. Dies hat zur Folge, daß die am Rande von $\mathbf{T}_{7}^{\prime}$ liegenden Dreiecksseiten zu Paaren bezüglich der $\Gamma_{\psi(7)}^{\prime}$ äquivalent sind, und zwar immer die Seite eines schraffierten Dreiecks mit der eines freien. Man zählt zunächst 16 solche am Rande liegende Seiten ab. Doch können wir mehrere zu Seitenketten zusammenfassen und haben in Fig. 1 insbesondere immer diejenigen Seiten, die einen Kreisbogen (eine Polygonseite) bilden, zusammengenommen und mit einer Nummer versehen. Die $\mathrm{Zu}$ sammenordnung dieser Polygonseiten regelt sich dann wie folgt durch Substitutionen der Gruppe $\Gamma_{\psi(7)}^{\prime}$ : 

(2) $1 \rightarrow 8,\left(\begin{array}{l}1,7 \\ 0,1\end{array}\right)$;
$2 \rightarrow 3,\left(\begin{array}{c}2,7 \\ -1,-3\end{array}\right)$
$4 \rightarrow \tilde{\partial}$,
$\left(\begin{array}{l}1,0 \\ 1,1\end{array}\right)$
$6 \rightarrow 7,\left(\begin{array}{l}3,-7 \\ 1,-2\end{array}\right)$.

Neben der Zuordnung der Seiten ist jedesmal die Substitution angegeben, welche diese Zuordnung vermittelt. Wie man sieht, gehören alle vier hier auftretenden Substitutionen in der Tat zur Gruppe $\Gamma_{\psi(7)}^{\prime}$.

Übt man auf $T_{7}^{\prime}$ der Reihe nach die acht Substitutionen (1) aus, so gèwinnt man die Diskontinuitätsbereiche der acht mit $\Gamma_{\psi(7)}$ gleichberechtigten Kongruenzgruppen. Die ersten sieben Substitutionen (1) ergeben einfach Translationen von $T_{7}^{\prime}$ in Richtung der reellen $\omega$-Achse. Eine Änderung der Gestalt wird indessen durch die in (1) an letzter Stelle genannte Substitution $T$ geliefert, die zu dem in Fig. 2 abgebildeten Transformationspolygone $T_{7}$ hinführt. Der Deutlichkeit halber ist die Längeneinheit in dieser Zeichnung weit größer als in Fig. 1 gewählt. Die Beziehung der Rand-

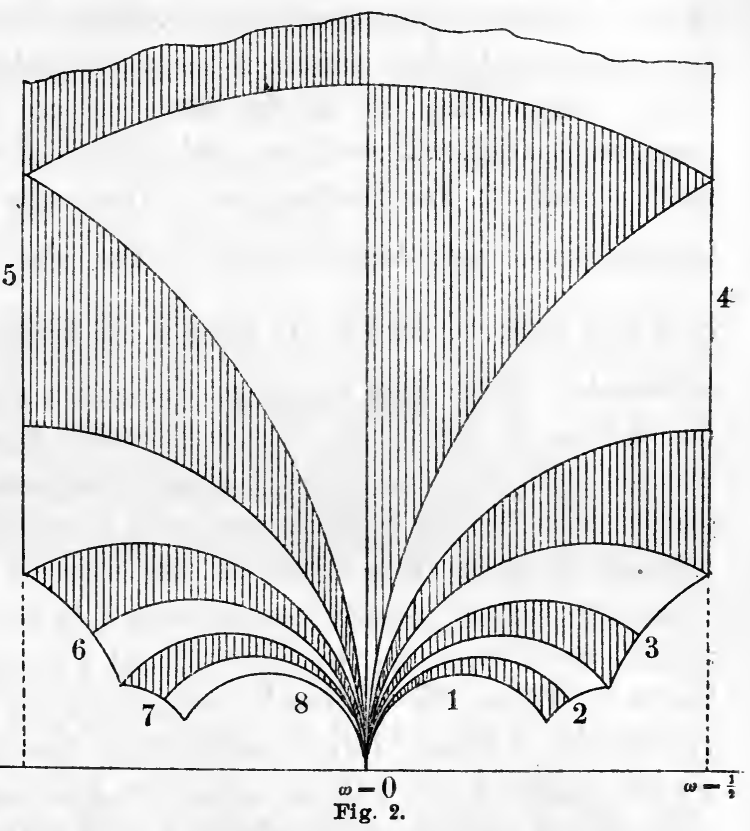
kurven aufeinander ist hier durch die Substitutionen geregelt:

$$
1 \rightarrow 8,\left(\begin{array}{c}
1,0 \\
-7,1
\end{array}\right) ; 2 \rightarrow 3,\left(\begin{array}{l}
3,-1 \\
7,-2
\end{array}\right) ; 4 \rightarrow 5,\left(\begin{array}{l}
1,-1 \\
0,
\end{array}\right) ; 6 \rightarrow 7,\left(\begin{array}{rr}
2, & 1 \\
-7,-3
\end{array}\right)
$$

Diese Substitutionen, die aus den Substitutionen (2) einfach durch Transformation mit $T$ hervorgehen, gehören in der Tat alle zur Gruppe $\Gamma_{\psi(7)}$. Die Polygone $\mathbf{T}_{n}$ stehen nun zu den Transformationsgleichungen $F\left(j^{\prime}, j\right)=0$ in nächster Beziehung, wie zunächst wieder am Beispiele$n=7$ dargelegt werden soll. Wir knüpfen an das besonders übersichtliche Polygon $T_{7}^{\prime}$ der Fig. 1 an und bilden dieses mittels der Funktion $j(\omega)$ auf die $j$-Ebene ab. Wir erhalten eine achtblättrige, zunächst noch zerschnittene Riemannsche Fläche $F$, mit einem in sich zurücklaufenden Schnitte, der vom Polygonrande herrührt. Die den Seiten 1 bis 8 des Polygons entsprechenden Schnittränder liegen aber jetzt so, daß je zwei durch eine Substitution (2) einander zugeordnete Uferpunkte übereinander liegen. Indem wir entsprechend der Zuordnung (2) die Schnittränder aneinander heften, ergibt sich eine geschlossene Riemannsche. 
Fläche über der $j$-Ebene, die wir als „Transformationsfläche" $\mathbf{F}_{\mathbf{7}}$ bezeichnen. ${ }^{1}$ )

Die Fläche $F_{7}$ kann Verzweigungspunkte nur an den drei Stellen $j=0,12^{3}, \infty$ haben, da übrigens die $\omega$-Halbebene auf die Ebene der Funktion $j(\omega)$ konform bezogen ist. Um mit $j=\infty \mathrm{zu}$ beginnen, so lesen wir aus Fig. 1 zunächst ab, daB 14 einfache Dreiecke von $T_{7}^{\prime}$ mit ihren Spitzen nach $\omega=i \infty$ laufen. Indem wir vom Rande 1 bis zum Rande" 8 diese 14 Dreiecke durchlaufen, beschreiben wir eine Linie, die auf $\boldsymbol{F}_{\mathbf{7}}$ einen geschlossenen Umlauf um einen bei $j=\infty$ gelegenen Verzweigungspunkt liefert. Also haben wir an der Stelle $j=\infty$ einen siebenblättrigen Verzweigungspunkt, während das achte Blatt der Spitze $\omega=0$ entsprechend isoliert verläuft. Die Stellen $j=0$ rühren von den Ecken unserer Kreisbogendreiecke mit den Winkeln $\frac{\pi}{3}$ her, also von den Punkten $\omega=\varrho$, $\varrho \pm 1, \varrho \pm 2, \ldots$ der Fig. 1, unter $\varrho$ die dritte Einheitswurzel $\frac{-1+i \sqrt{3}}{2}$ verstanden. Betrachten wir zuerst die beiden Punkte $\omega=\rho$ und $\omega=\rho+1$ der Seiten 4 und 5, die durch die dritte Substitution (2) aufeinander bezogen sind, so ist aus Fig. 1 einleuchtend, daß der Umlauf um die zugehörige Stelle der $F_{7}$ sich erst nach Durchschreiten von sechs $j$-Halbblättern schließt. Wir gelangen also hier zu einem dreiblättrigen Verzweigungspunkte. Anders verhält sich z. B. der Punkt $\omega=-2+\varrho$, der zufolge der Zuordnung der Seiten 2 und 3 zu einem bei $j=0$ isoliert verlaufenden Blatte führt. Durch Fortsetzung der Betrachtung gelangt man zu folgendem Satze: Unsere achtblättrige Transformationsfläche $\mathbf{F}_{\mathbf{7}}$ ist nur an den Stellen $j=0,12^{3}, \infty$ verzweigt, und zwar hat man bei $j=0$ zwei dreiblättrige Verzweigungspunkte und zwei isoliert verlaufende Blätter, bei $j=12^{3}$ vier zweiblättrige Verzweigungspunkte und bei $j=\infty$ einen siebenblättrigen Verzweigungspunkt und ein isoliert verlaufendes Blatt.

Zwischen der geschlossenen Fläche $F_{7}$ und der $\omega$-Halbebene besteht

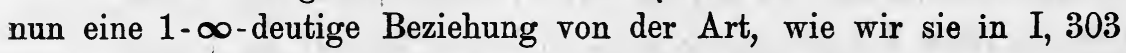
zwischen der $j$-Ebene und der $\omega$-Halbebene fanden. Der einzelnen Stelle $\operatorname{der} \mathbf{F}_{\mathbf{7}}$ entsprechen unendlich viele Punkte der $\boldsymbol{\omega}$-Halbebene, die bezüglich $\operatorname{der} \Gamma_{\psi(7)}^{\prime}$ äquivalent sind; umgekehrt liefert jedes System bezüglich der $\Gamma_{\psi(7)}^{\prime}$ äquivalenter Punkte $\omega$ eine Stelle der $\mathbf{F}_{7}$. Die Folge ist, daß die durch die zweite Haupttransformation zu gewinnende Funktion $j\left(\frac{\omega}{7}\right)$, die zur $\Gamma_{\psi(7)}^{\prime}$ gehört, von der $\omega$-Halbebene auf die $F_{7}$ verpflanzt, daselbst eine eindeutige, und zwar algebraische Funktion liefert. Somit hängen

1) Hier bedeutet also der Index 7 nicht, wie in I, $49 \mathrm{ff}$., die Blätteranzahl der Eläche, sondern den Trangformationsgrad. 
$j^{\prime}=j\left(\frac{\omega}{7}\right)$ und $j=j(\omega)$ als eindeutige algebraische Funktionen unserer $\mathbf{F}_{\boldsymbol{\gamma}}$ durch eine algebraische Relation zusammen, und eben diese Relation ist unsere Transformationsgleichung. Um dies noch etwas näher darzulegen, bemerken wir, daB durch Angabe eines Wertes $j$ acht Punkte der $\mathbf{F}_{\mathbf{7}}$ festgelegt sind, denen acht bezüglich der Gesamtgruppe $\Gamma$ äquivalente Punkte im Bereiche $\mathbf{T}_{7}^{\prime}$ zugehören. Ist unter ihnen $\boldsymbol{\omega}$ der im Diskontinuitätsbereiche der $\Gamma^{(\omega)}$ gelegene Punkt, so sind die sieben weiteren $\omega \pm 1$, $\omega \pm 2, \omega \pm 3, \frac{-1}{\omega}$. An diesen acht Stellen finden folgende Werte der transformierten Funktion statt:

$$
j\left(\frac{\omega}{7}\right), \quad j\left(\frac{\omega \pm 1}{7}\right), j\left(\frac{\omega \pm 2}{7}\right), \quad j\left(\frac{\omega \pm 3}{7}\right), \quad j\left(\frac{-1}{7 \omega}\right)=j(7 \omega) .
$$

Dies sind aber genau die acht verschiedenen durch Transformation siebenten Grades von $j(\omega)$ zu gewinnenden Funktionen, die, wie wir wissen, in der Tat die acht Lösungen der Transformationsgleichung sind.

Das Transformationspolygon $\mathbf{T}_{n}^{\prime}$ für beliebigen ungeraden Primzahl$\operatorname{grad} n$ ist, wie wir noch an weiteren Beispielen darlegen werden, entsprechend durch $n$ nebeneinander gereihte Doppeldreiecke $\left(\begin{array}{c}1,0 \\ 0,1\end{array}\right),\left(\begin{array}{c}1, \pm 1 \\ 0,1\end{array}\right),\left(\begin{array}{c}1, \pm 2 \\ 0,1\end{array}\right), \ldots$ $\left(\begin{array}{l}1, \pm \frac{1}{2}(n-1) \\ 0,1\end{array}\right)$ und das inmitten unten angehängte Doppeldreieck $\left(\begin{array}{cc}0,-1 \\ 1, & 0\end{array}\right)$ zusammensetzbar. Nicht so leicht zu übersehen ist indessen die Gestalt von $\mathrm{T}_{n}^{\prime}$ bei zusammengesetztem $n$. Als Beispiel diene der Fall $n=6$, wo wir mit einer Untergruppe des Index $\psi(6)$ $=12 \mathrm{zu}$ tun haben. Das Polygon $\mathbf{T}_{6}^{\prime}$ ist in Fig. 3 dargestellt. Hier liegt in der Tat ein aus 24 einfachen Dreiecken zusammengesetzter Bereich vor, von denen 12 schraf-

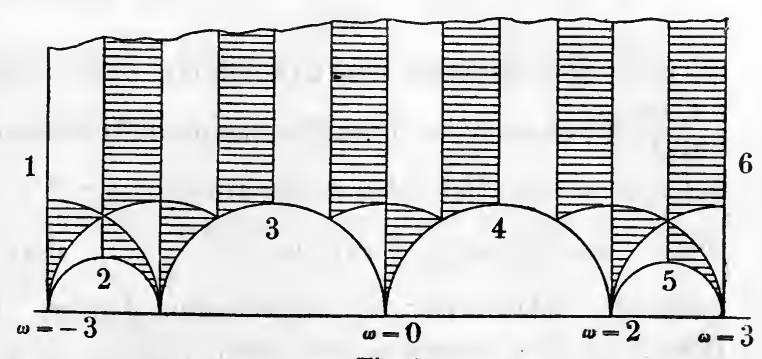

Fig. 3. fiert und 12 frei sind. Man kann zeigen, daß keine zwei gleichartige Dreiecke durch eine Substitution der $\Gamma_{\psi(6)}^{\prime}$ zusammenhängen. Die sechs mit Ziffern bezeichneten Seiten dieses Sechsecks sind nach folgender Regel einander zuigeordnet:

$$
1 \rightarrow 6,\left(\begin{array}{l}
1,6 \\
0,1
\end{array}\right) ; \quad 2 \rightarrow 5,\left(\begin{array}{l}
5,12 \\
2,5
\end{array}\right) ; \quad 3 \rightarrow 4,\left(\begin{array}{l}
1,0 \\
1,1
\end{array}\right)
$$

Diese Substitutionen gehören in der Tat der Gruppe $\Gamma_{\psi(6)}^{\prime}$ an. Die Transformation von $T_{6}^{\prime}$ mittels der Substitution $T$ liefert das Polygon $T_{6}$, das 
in Fig. 4 dargestellt ist. Der Deutlichkeit halber liegt dieser Figur wieder ein weit größerer Maßstab zugrunde als der Fig. 3.

Durch Abbildung des Transformationspolygons $\mathbf{T}_{n}$ mittels der Funk-

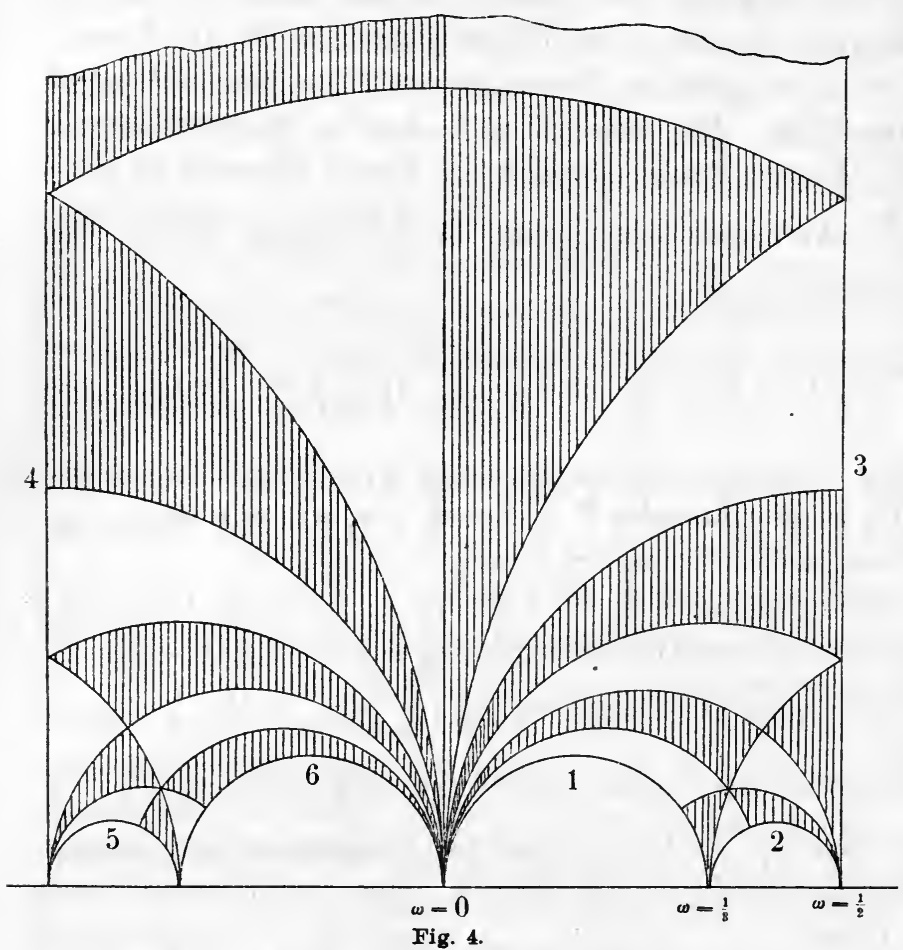
tion $j(\omega)$ gewinnen wir für jeden Grad $n$ eine geschlossene $\psi(n)$ blättrige Riemannsche Fläche über der $j$-Ebene, die wir wieder als „Transformationsfläche" $\mathbf{F}_{n}$ bezeichnen. Eine Frage von grundsätzlicher Bedeutung ist, wie groß das Geschlecht $p$ der Fläche $\mathbf{F}_{n}$ ist; wir wollen diese Zahl $p$ auch das. Geschlecht des Transformations. polygons $\mathbf{T}_{n}$ nennen und genauer durch $p(n)$ bezeichnen. Die $\psi(n)$ Blätter $\operatorname{der} F_{n}$ sind den $\psi(n)$ Repräsentanten $\left(\begin{array}{c}A, B \\ 0, D\end{array}\right)$ für eigentliche Transformation $n^{\text {ten }}$ Grades eindeutig zugeordnet. Wir nehmen $\omega$ im Diskontinuitätsbereiche der $\Gamma^{(\omega)}$ an und wählen eine einzelne transformierte Funktion $j\left(\frac{A \omega+B}{D}\right)$, deren Werte wir auf dem zugehörigen Blatte der $F_{n}$ aufgetragen denken. Umlaufen wir von jenem Blatte aus den Punkt $j=\infty$ etwa $\nu$ Male, so kommt dies darauf hinaus, daß wir durch $\nu$-malige Ausübung der Substitution $S$ nach $\omega^{\prime}=\omega+\nu$ gehen. Dabei aber wird $j\left(\frac{A \omega+B}{D}\right)$ in $j\left(\frac{A \omega+(B+v A)}{D}\right)$ übergeführt. Um nun festzustellen, wie viele Blätter in dem fraglichen Punkte $j=\infty \mathrm{zu}$ sammenhängen, hat man die kleinste positive Zahl $\dot{v}$ anzugeben, für welche die Transformation $\left(\begin{array}{cc}A, B+\nu A \\ 0, & D\end{array}\right)$ wieder durch die anfängliche $\left(\begin{array}{c}A, B \\ 0, D\end{array}\right)$ repräsentiert wird. Hierzu ist hinreichend und notwendig, daB in der Gruppe $\Gamma^{(\omega)}$ eine Substitution $V=\left(\begin{array}{l}\alpha, \beta \\ \gamma, \delta\end{array}\right)$ existiert, für die: 
gilt. Es folgt:

$$
\frac{A \omega+B+v A}{D}=V\left(\frac{A \omega+B}{D}\right)=\frac{\alpha A \omega+(\alpha B+\beta D)}{\gamma A \omega+(\gamma B+\delta D)}
$$

$$
\gamma=0, \quad \alpha=\delta=1, \quad \nu A=\beta D .
$$

Ist also $t$ der gröBte gemeinsame Teiler von $A$ und $D$, so ist:

$$
\nu=\frac{D}{t}, \quad \beta=\frac{A}{t} .
$$

Wir erhalten also einen $\frac{D}{t}$ - blättrigen Verzweigungspunkt, so daß die Blätteranzahl allein von $D$ abhängt. Bei stehendem $D$ ist $A=\frac{n}{D}$ und $B$ durchläuft, um alle zugehörigen Repräsentanten der „eigentlichen“ Transformationen $n^{\text {ten }}$ Grade für dieses Zahlenpaar $A, D$ zu gewinnen, alle gegen $t$ teilerfremden ganzen Zahlen des Intervalles $0 \leqq B<D$. Dies sind $\frac{D}{t} \varphi(t)$ Zahlen $B$, so daß wir für das einzelne $D$ im ganzen $\varphi(t)$ je $\frac{D}{t}$ - blättrige Verzweigungspunkte finden. Durchläuft $D$ alle Teiler von $n$, so erschöpfen wir alle $\psi(n)$ Blätter von $\mathbf{F}_{n}$; wir gelangen dabei zu der schon unter (22) S. 347 aufgestellten Gleichung zurück.

Von dem zu $j\left(\frac{A \omega+B}{D}\right)$ gehörenden Blatte der Fläche aus soll jetzt die Stelle $j=12^{3}$ einmal umlaufen werden, was auf die Ausübung der Transformation $T$ auf $\boldsymbol{\omega}$ hinausläuft. Zweimaliger Umlauf um diese Stelle führt stets zum ersten Blatte zurück, einmaliger Umlauf aber stets und nur dann, wenn bereits die Transformation $\frac{B \omega-A}{D \omega}$ wieder durch $\frac{A \omega+B}{D}$ repräsentiert wird, d.h. wenn es eine Substitution $V=\left(\begin{array}{l}\alpha, \beta \\ \gamma, \delta\end{array}\right)$ gibt, für die

$$
\frac{B \omega-A}{D \omega}=V\left(\frac{A \omega+B}{D}\right)=\frac{\alpha A \omega+(\alpha B+\beta D)}{\gamma A \omega+(\gamma B+\delta D)}
$$

gilt. Diese Forderung ist gleichbedeutend mit den vier Bedingungen:

$$
\alpha A=B, \quad \alpha B+\beta D=-A, \quad \gamma A=D, \quad \gamma B+\delta D=0 .
$$

Wegen der dritten Gleichung gilt $\gamma \neq 0$. Wäre $\alpha=0$, so wäre $\gamma=1$, $B=0, A=D=\sqrt{n}$, was aber selbst bei quadratischem $n$ unzulässig ist, da keine eigentliche Transformation $n^{\text {ten }}$ Grades vorliegen würde. Somit gilt $\alpha \neq 0, \gamma \neq 0$, so daB $A$ in $B$ und $D$ zugleich aufgeht und also gleich 1 ist. Man gewinnt $A=1, D=n$ und damit

$$
\alpha=B, \quad \gamma=n, \quad \delta=-B, \quad \beta=-\frac{B^{2}+1}{n} .
$$

Damit $\beta$ ganzzahlig ausfällt, haben wir nur diejenigen Zahlen $B$ des Intervalls $0 \leqq B<n$ zuzulassen, für welche die Kongruenz:

$$
B^{2}+1 \equiv 0 \quad(\bmod n)
$$


erfüllt ist. Die Anzahl inkongruenter Iı̈sungen dieser Kongruenz möge durch $\mu_{1}(n)$ bezeichnet werden. Dann gilt der Satz: Bei $j=12^{3}$ verlaufen $\mu_{1}(n)$ Blätter der Fläche $\mathbf{F}_{n}$ isoliert, während die übrigen zu Paaren in $\frac{1}{2}\left(\psi(n)-\mu_{1}(n)\right)$ Verzweigungspunkten zusammenhängen.

Für die Stelle $j=0$ kommt an Stelle von $S$ und $T$ die Substitution $U(\omega)=\frac{\omega+1}{-\omega}$ zur Benutzung. Nach dreimaligem Umlaufe um die Stelle $j=0$ gelangt man vom Blatte der Funktion $j\left(\frac{A \omega+B}{D}\right)$ stets zu diesem Blatte zurück. Das Blatt aber verläuft isoliert, so oft es eine Substitution $V=\left(\begin{array}{l}\alpha, \beta \\ \gamma, \delta\end{array}\right)$ gibt, für die die Gleichung zutrifft:

$$
\frac{(A-B) \omega+A}{-D \omega}=V\left(\frac{A \omega+B}{D}\right)=\frac{\alpha A \omega+(\alpha B+\beta D)}{\gamma A \omega+(\gamma B+\delta D)} .
$$

Dies führt auf die vier Bedingungen:

$$
\alpha A=A-B, \quad \alpha B+\beta D=A, \quad \gamma A=-D, \quad \gamma B+\delta D=0 .
$$

Aus der dritten Gleichung folgt wieder $\gamma \neq 0$. Durch Elimination von $D$ aus der zweiten und vierten Gleichung gewinnt man $B=\delta A$. Wäre nun $\delta=0$, so wäre $B=0, \gamma=-1, A=D=\sqrt{n}$, was ausgeschlossen ist. Also gilt $\delta \neq 0$, so $\operatorname{da} B A$ als gemeinsamer Teiler von $B$ und $D$ gleich 1 ist. Man hat demnach $A=1, D=n$ und findet:

$$
\alpha=1-B, \quad \gamma=-D, \quad \delta=B, \quad \beta=\frac{B^{2}-B+1}{n} .
$$

Jetzt handelt es sich also um diejenigen $B$ des Intervalles $0 \leqq B<n$, für welche die Kongruenz:

$$
B^{2}-B+1 \equiv 0 \quad(\bmod n)
$$

erfüllt ist. Bezeichnet man die Anzahl inkongruenter Lösungen dieser Kongruenz durch $\mu_{0}(n)$, so hat man den Satz: Bei $j=0$ verlaufen $\mu_{0}(n)$ Blätter der Fläche $F_{n}$ isoliert, während die übrigen $z u$ je dreien in $\frac{1}{3}\left(\psi(n)-\mu_{0}(n)\right)$ Verzweigungspunkten zusammenhängen.

Das Geschlecht $p(n)$ der $\mathbf{F}_{n}$ berechnen wir jetzt nach (4) in I, 88 und finden:

$p(n)=-\psi(n)+1+\frac{1}{4}\left(\psi(n)-\mu_{1}(n)\right)+\frac{1}{3}\left(\psi(n)-\mu_{0}(n)\right)+\frac{1}{2} \sum_{D} \varphi(t)\left(\frac{D}{t}-1\right)$

Mit Hilfe der Relation (22) S. 347 läbt sich die rechte Seite dieser Gleichung noch wesentlich zusammenziehen: Das Geschlecht $p(n)$ der zum Grade $n$ gehörenden Transformationsfläche $\mathbf{F}_{n}$ und damit des Transformationspolygons $\mathrm{T}_{n}$ ist:

$$
p(n)=1+\frac{1}{12} \psi(n)-\frac{1}{3} \mu_{0}(n)-\frac{1}{4} \mu_{1}(n)-\frac{1}{2} \sum_{D} \varphi(t) .
$$


Die Transformationsgrade bis $n=72$ verteilen sich auf die Geschlechter $p=0$ bis $p=9$ in folgender Art:

$$
\begin{aligned}
& p=0, \quad n=2,3,4,5,6,7,8,9,10,12,13,16,18,25, \\
& p=1, \quad n=11,14,15,17,19,20,21,24,27,32,36,49, \\
& p=2, \quad n=22,23,26,28,29,31,37,50, \\
& p=3, \quad n=30,33,34,35,39,40,41,43,45,48,64, \\
& p=4, \quad n=38,44,47,53,54,61, \\
& p=5, \quad n=42,46,51,52,55,56,57,59,63,65,67,72, \\
& p=6, \quad n=58,71, \\
& p=7, \quad n=60,62,68,69, \\
& p=9, \quad n=66,70 .
\end{aligned}
$$

\section{\$4. Die erweiterte Gruppe $\Gamma^{(n)}$ und das Klassenpolygon $\mathbf{K}_{\boldsymbol{n}}$.}

Unter $W$ und $W^{\prime}$ verstehen wir die linearen Substitutionen der Periode 2 und der Determinante $n$ :

$$
\omega^{\prime}=W(\omega)=\frac{-1}{n \omega}, \quad \omega^{\prime}=W^{\prime}(\omega)=-\frac{n}{\omega} .
$$

Die folgende Entwicklung gründet sich auf die Tatsache, daß die zu den Haupttransformationen $n^{\text {ten }}$ Grades gehörenden Gruppen $\Gamma_{\psi(n)}$ und $\Gamma_{\psi(n)}^{\prime}$ durch $W$ bzw. $W^{\prime}$ in sich transformiert werden:

$$
W^{-1} \cdot \Gamma_{\psi(n)} \cdot W=\Gamma_{\psi(n)}, \quad W^{\prime-1} \cdot \Gamma_{\psi(n)}^{\prime} \cdot W^{\prime}=\Gamma_{\psi(n)}^{\prime} .
$$

Eine Substitution $V=\left(\begin{array}{l}\alpha, \beta \\ \gamma, \delta\end{array}\right)$ wird nämlich durch $W$ in:

$$
V^{\prime}=W^{-1} \cdot V \cdot W=\left(\begin{array}{c}
\delta,-\gamma n^{-1} \\
-n \beta, \alpha
\end{array}\right)
$$

transformiert. Ist $V$ in $\Gamma_{\psi(n)}$ enthalten, so gilt $\gamma \equiv 0(\bmod n)$, so daB auch $V^{\prime}$ eine ganzzahlige Substitution der Determinante 1 mit einem durch $n$ teilbaren dritten Koeffizienten ist. Also ist $V^{\prime}$ in $\Gamma_{\psi(n)}$ enthalten. Auch ist leicht einzusehen, daß man in den $W^{-1} \cdot V \cdot W$ wieder die ganze $\Gamma_{\psi(n)}$ gewinnt, wenn $V$ diese Gruppe durchläuft. Damit ist die erste Gleichung (2) bewiesen; die zweite ergibt sich entsprechend.

Die Gleichungen (2) kann man auch in die Gestalten setzen:

$$
\Gamma_{\psi(n)} \cdot W=W \cdot \Gamma_{\psi(n)}, \quad \Gamma_{\psi(n)}^{\prime} \cdot W^{\prime}=W^{\prime} \cdot \Gamma_{\psi(n)}^{\prime},
$$

aus denen hervorgeht, daß die Gruppen $\Gamma_{\psi(n)}$ und $\Gamma_{\psi(n)}^{\prime}$ mit $W$ bzw. $W^{\prime}$ vertauschbar sind. Hieraus aber folgt weiter, $d a \beta$ die Gruppen $\Gamma_{\psi(n)}$ und $\Gamma_{\psi(n)}^{\prime}$ durch Zusatz $W$ bzw. $W^{\prime} z u$ zwei Gruppen:

$$
\Gamma^{(n)}=\Gamma_{\psi(n)}+\Gamma_{\psi(n)} \cdot W, \quad \Gamma^{\prime(n)}=\Gamma_{\psi(n)}^{\prime}+\Gamma_{\psi(n)}^{\prime} \cdot W^{\prime}
$$


erweitert werden, in denen die ursprünglichen $\Gamma_{\psi(n)}$ und $\Gamma_{\psi(n)}^{\prime}$ ausgezeichnete Untergruppen des Index 2 sind.

Wie in I, 296 verstehen wir unter $\bar{U}$ die Substitution „zweiter Art“ $\omega^{\prime}=\bar{U}(\omega)=-\bar{\omega}$, wo $\bar{\omega}$ der zu $\omega$ konjugiert komplexe Wert ist. Diese Substitution, die geometrisch die Spiegelung an der imaginären $\omega$-Achse bedeutet, übt auf eine beliebige Substitution $V=\left(\begin{array}{l}\alpha, \beta \\ \gamma, \delta\end{array}\right)$ die Wirkung aus:

$$
\bar{U}^{-1} \cdot V \cdot \bar{U}=\left(\begin{array}{rr}
\alpha,-\beta \\
-\gamma, & \delta
\end{array}\right) \text {. }
$$

Jede der vier Gruppen $\Gamma_{\psi(n)}, \Gamma_{\psi(n)}^{\prime}, \Gamma^{(n)}, \Gamma^{\prime(n)}$ ist also mit $\bar{U}$ vertauschbar und wird entsprechend durch Zusatz von $\bar{U}$ zu einer erweiterten Gruppe ausgestaltet, in der die ursprüngliche wieder eine ausgezeichnete Untergruppe des Index 2 ist. Wir bezeichnen diese Gruppen durch $\bar{\Gamma}_{\psi(n)}, \bar{\Gamma}_{\psi(n)}^{\prime}, \bar{\Gamma}^{(n)}, \bar{\Gamma}_{(n ;}^{\prime}$; für $\bar{\Gamma}^{(n)}$ haben wir die Darstellung:

$$
\overline{\Gamma^{(n)}}=\Gamma_{\psi(n)}+\Gamma_{\psi(n)} \cdot W+\Gamma_{\psi(n)} \cdot \bar{U}+\Gamma_{\psi(n)} \cdot W \cdot \bar{U}
$$

und eine entsprechende Darstellung gilt für $\bar{\Gamma}^{\prime}(n)$.

Die Substitutionen zweiter Art $W \cdot \bar{U}$ und $W^{\prime} \cdot \bar{U}$ mögen durch $\bar{W}$ und $\bar{W}^{\prime}$ bezeichnet werden:

$$
\omega^{\prime}=\bar{W}(\omega)=\frac{1}{n \bar{\omega}}, \quad \omega^{\prime}=\bar{W}^{\prime}(\omega)=\frac{n}{\bar{\omega}} ;
$$

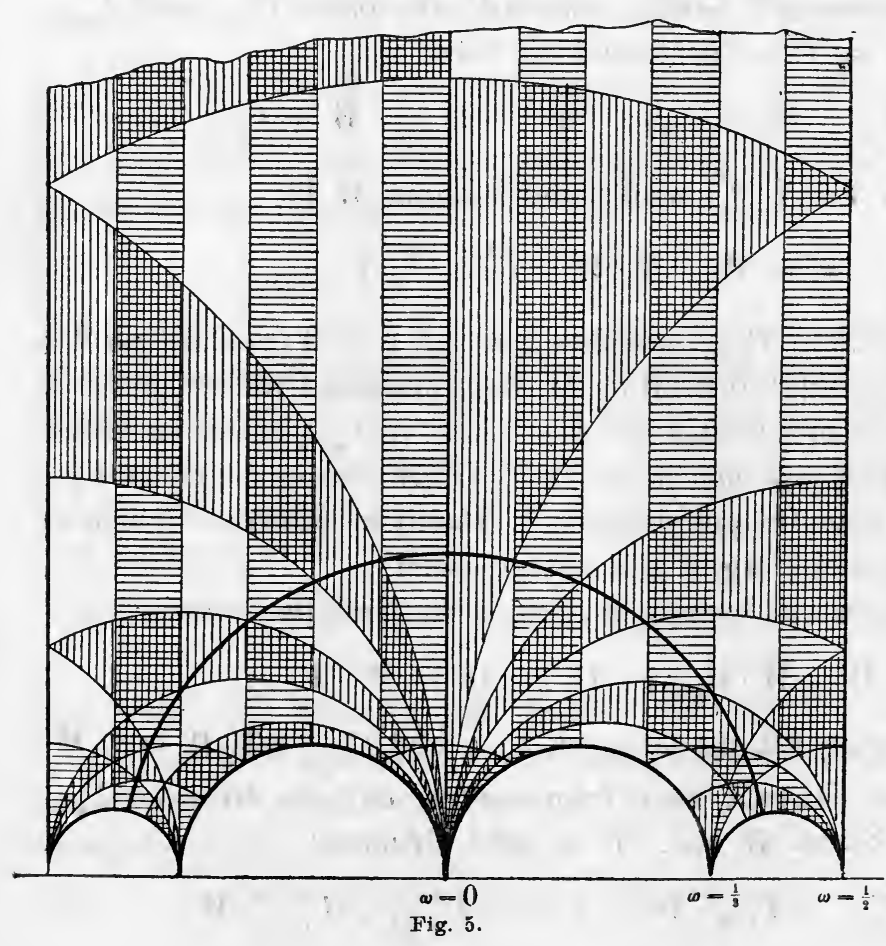

sie stellen Spiegelungen an den Kreisen derRadien $\frac{1}{\sqrt{n}}$ und $\sqrt{n}$ um den Nullpunkt $\omega=0$ dar. Die Substitutionen $W$ und $W^{\prime}$ erster Art könnien dann auch aus den Spiegelungen $\bar{U}, \bar{W}, \bar{W}^{\prime}$ in den Gestalten hergestellt werden:

$$
\begin{aligned}
W & =\bar{W} \cdot \bar{U}, \\
W^{\prime} & =\overline{W^{\prime}} \cdot \bar{U} .
\end{aligned}
$$

Indem mannun auf den Diskontinuitätsbereich $\mathbf{T}_{n}$ $\operatorname{der} \Gamma_{\psi(n)}$ die Sub- 
stitution $W$ ausübt, gewinnt man entsprechend der ersten Gleichung (2) wieder einen Diskontinuitätsbereich dieser Gruppe. Die Transformation von $\mathbf{T}_{n}$ durch $W$ können wir aber durch die beiden hintereinander auszuübenden Spiegelungen $\bar{W}, \bar{U}$ ersetzen. Im Falle $n=6$ wird das in Fig. 4 dargestellte Polygon $\mathrm{T}_{6}$ durch $W$ bzw. durch $\bar{W}$ und $\bar{U}$ unmittelbar in sich transformiert, wie Fig. 5 erläutert. In dieser Figur sind zwei Dreiecksnetze übereinander getragen. Das Netz mit der Vertikalschraffierung stellt das Polygon $\mathbf{F}_{6}$ wie in Fig. 4 dar. Durch die Spiegelung $\bar{U}$ geht dies Netz in sich über. Durch die Spiegelung $\bar{W}$ an dem in der Figur stark ausgezogenen Kreise des Radius $\frac{1}{\sqrt{6}}$ gelangt man zu dem Netze mit der Horizontalschraffierung. In dieses Netz geht also das ursprüngliche Netz durch die Substitution $W$ über.

Nicht immer kommt das durch $W$ oder $\bar{U}$ und $\bar{W}$ transformierte Polygon $\mathbf{T}_{n}$ unmittelbar mit seiner ursprünglichen Gestalt zur Deckung. So erkennt man z. B. in der den Fall $n=7$ erläuternden Fig. 6, dab die beiden ${ }_{\mathrm{s}}^{\circ N e t z e ~ n i c h t ~ g l a t t ~ z u r ~ D e c k u n g ~ k o m m e n, ~ s o n d e r n ~ e i n ~ w e n i g ~ u ̈ b e r-~}$ einander hinweggreifen. Je ein überschießender Bestandteil des einen Netzes ist dann natürlich mit einem solchen des anderen Netzes bezüglich der $\Gamma_{\psi(n)}$ äquivalent. Man könnte freilich die Anordnung stets so treffen, daB der Bereich $\mathbf{T}_{n}$ bei Ausübung von $W$ genau in sich selbst übergeht. Nur müBte man dann die Randkurven von $T_{n}$ gelegentlich durch das Innere von Dreiecken führen,was man sich an Fig. 6 näher veranschaulichen wolle.

In den beiden Figuren 5 und 6 tritt noch eine weitere Tatsache hervor. Das Dreiecksnetz des Polygons $T_{n}$ geht bei Ausübung der Transformation $W$ in das im Verhältnis von $n$ zu 1 verkleinerte Dreiecksnetz des Transformationspolygons $T_{n}^{\prime} \operatorname{der} \Gamma_{\psi(n)}^{\prime}$ über. Dies ist rechnerisch unmittelbar

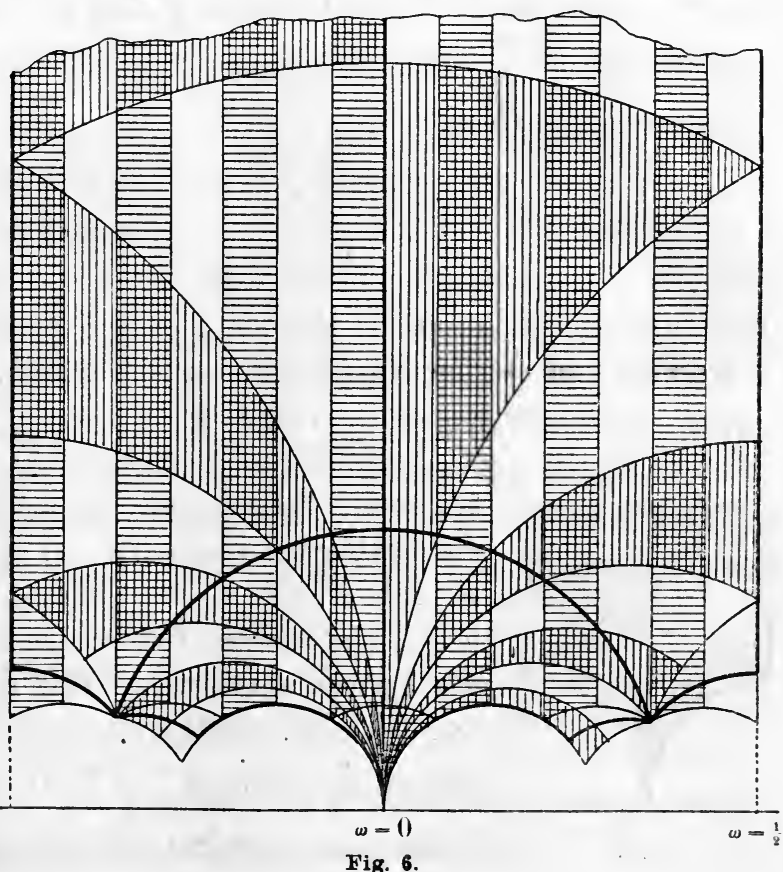

Fig. 6. 
einleuchtend. Setzen wir nämlich

$$
n \omega=\Omega,
$$

so trifft im Punkte $\omega=\frac{1}{n}$ bereits $\Omega=1 \mathrm{zu}$, so daB das zu $\Omega$ gehörende Dreiecksnetz gegenüber dem ursprünglichen auf $\frac{1}{n}$ verkleinert erscheint. Nun wird durch (5) die Substitution:

$$
\omega^{\prime}=\frac{\alpha \omega+\beta}{\gamma \omega+\delta} \quad \text { in } \quad \Omega^{\prime}=\frac{\alpha \Omega+n \beta}{\gamma n^{-1} \Omega+\delta}
$$

transformiert, so daß wir, wenn $\gamma \equiv 0(\bmod n)$ gilt, rechts in der Tat eine ganzzahlige Substitution der Determinante 1 gewinnen, deren "zweiter" Koeffizient durch $n$ teilbar ist. Durch (5) wird also die Gruppe $\Gamma_{\psi(n)}$ in $\Gamma_{\psi(n)}^{\prime}$ transformiert.

Wir gehen nun genauer auf die unter (3) gewonnene Gruppe $\Gamma^{(n)}$ ein. Die Nebengruppe $\Gamma_{\psi(n)} \cdot W$ besteht aus allen ganzzahligen Substitutionen $\left(\begin{array}{l}\alpha, \beta \\ \gamma, \delta\end{array}\right)$, die die Bedingungen:

$$
\alpha \delta-\beta \gamma=n, \quad \alpha \equiv \gamma \equiv \delta \equiv 0 \quad(\bmod n)
$$

befriedigen. Es soll festgestellt werden, welche unter diesen Substitutionen elliptisch oder parabolisch sind. Da die zweite Potenz jeder Substitution der Nebengruppe $\Gamma_{\psi(n)} \cdot W$ in der $\Gamma_{\psi(n)}$ enthalten ist, so hat eine elliptische Substitution unter ihnen eine der Perioden 2, 4 oder 6 und erzeugt dann eine zyklische Untergruppe $G_{2}, G_{4}$ oder $G_{6}$. Wir untersuchen zunächst die Möglichkeit der Perioden 4 oder 6. Die zweite Potenz einer Substitution $\left(\begin{array}{l}\alpha, \beta \\ \gamma, \delta\end{array}\right)$ der Nebengruppe $\Gamma_{\psi(n)} \cdot W$ hat, auf die Determinante 1 reduziert, die Gestalt:

$$
\left(\begin{array}{ll}
\frac{\alpha^{2}+\beta \gamma}{n}, & \frac{(\alpha+\delta) \beta}{n} \\
\frac{(\alpha+\delta) \gamma}{n}, & \frac{\beta \gamma+\delta^{2}}{n}
\end{array}\right) .
$$

Soll diese Substitution die Periode 2 oder 3 haben, so muß die Summe des ersten und vierten Koeffizienten gleich 0 bzw. \pm 1 sein:

$$
\frac{\alpha^{2}+2 \beta \gamma+\delta^{2}}{n}=\frac{(\alpha+\delta)^{2}-2 n}{n}=0 \quad \text { bzw. }= \pm 1 \text {. }
$$

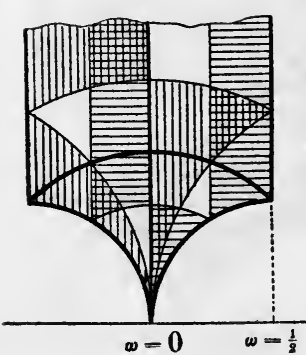

Im ersten Falle ist also $(\alpha+\delta)^{2}=2 n$. Da nun $(\alpha+\delta)$ ein Vielfaches von $n$ ist, so ist $2 n$ durch $n^{2}$ teilbar, so daB einzig $n=2$ zulässig ist. Allein in der bei $n=2$ eintretenden $\bar{\Gamma}^{(2)}$ können elliptische Substitutionen der Periode 4 auftreten. Wie man in Fig. 7 sieht, ist der Diskontinuitätsbereich der $\bar{\Gamma}^{(2)}$ ein Kreisbogendreieck der Winkel $\frac{\pi}{2}, \frac{\pi}{4}, 0$, so daB sich in $\operatorname{der} \Gamma^{(2)}$ Fig. 7. tatsächlich ein System gleichberechtigter zyklischer $G_{4}$ 
Elliptische und parabolische Substitutionen in der Nebengruppe $\Gamma_{\psi} \cdot W \quad 361$ findet. Für die Periode 6 ist nur $(\alpha+\delta)^{2}=3 n$ brauchbar. Man gelangt wie soeben leicht zu dem Satze: Allein in der bei $n=3$ eintretenden $\Gamma^{(3)}$ können elliptische Substitutionen der Periode 6 auftreten. Der in Fig. 8 dargestellte Diskontinuitätsbereich der $\bar{\Gamma}^{(3)}$ ist ein Kreisbogendreieck der Winkel $\frac{\pi}{2}, \frac{\pi}{6}, 0$ und zeigt somit, daß in der $\Gamma^{(3)}$ ein System gleichberechtigter zyklischer $G_{6}$ auftritt.

Ehe wir die Periode 2 betrachten, soll die Möglichkeit parabolischer Substitutionen in der Nebengruppe $\Gamma_{\psi(n)} \cdot W$ untersucht werden. Hier muB der in (8) links stehende Ausdruck gleich \pm 2 sein. Doch ist nur

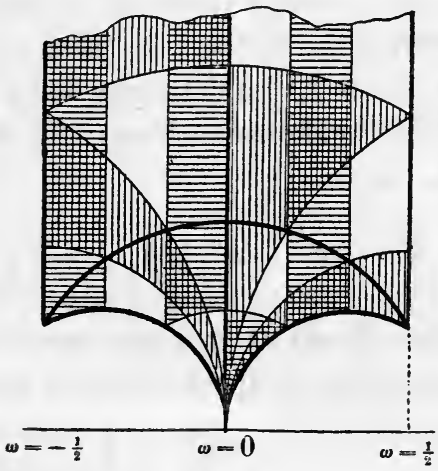

Fig. 8.

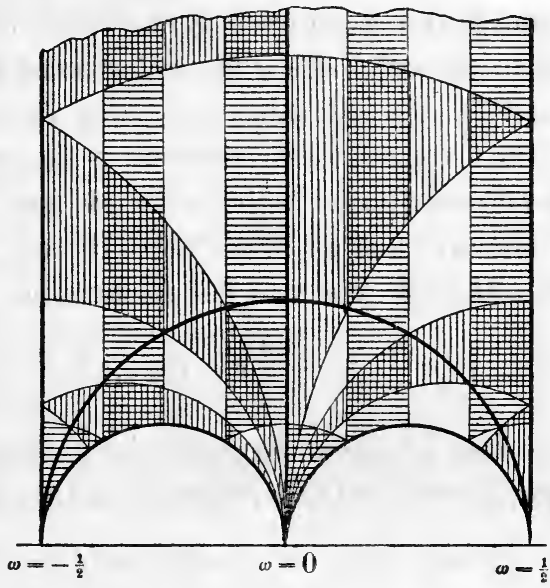

Fig. 9.

das obere Zeichen brauchbar, da das untere zu $\alpha+\delta=0$ und also zu den elliptischen Substitutionen der Periode 2 führt. Aus $(\alpha+\delta)^{2}=4 n$ folgt wie oben der Satz: Nur in der $z u n=4$ gehörenden $\Gamma^{(4)}$ können parabolische Substitutionen innerhalb der Nebengruppe $\Gamma_{\psi(4)} \cdot W$ auftreten. $\mathrm{DaB}$ sie wirklich auftreten, zeigt der in Fig. 9 dargestellte Diskontinuitätsbereich der $\bar{\Gamma}^{(4)}$, der ein Kreisbogendreieck der Winkel $\frac{\pi}{2}, 0,0$ ist; zur Spitze $\omega=-\frac{1}{2}$ gehört die der $\Gamma^{(4)}$ angehörende parabolische Substitution $\left(\begin{array}{r}4,1 \\ -4,0\end{array}\right)$, die nocb nicht in der $\Gamma_{\psi(4)}$ enthalten ist.

Es bleibt der Fall der elliptischen Substitutionen der Periode 2, der zu einem wichtigen Ergebnisse hinführen wird. Soll die Substitution $\left(\begin{array}{l}\alpha, \beta \\ \gamma, \delta\end{array}\right)$ der Nebengruppe $\Gamma_{\psi(n)} \cdot W$ die Periode 2 haben, so ist hierfür $\alpha+\delta=0$ und also $\delta=-\alpha$ charakteristisch, so dab der in der positiven ๓-Halbebene gelegene Fixpunkt der Substitution der Gleichung genügt:

$$
\gamma \omega^{2}-2 \alpha \omega-\beta=0 \text {. }
$$

Aus den Bedingungen (7) folgt leicht, daB $\gamma$ nicht verschwinden kann; man kann also nötigenfalls durch gleichzeitigen Zeichenwechsel von 
$\alpha, \beta, \gamma, \delta$ stets $\gamma>0$ erreichen. Dann aber ist durch:

$$
(\gamma,-2 \alpha,-\beta)=(a, b, c)
$$

eine positive ganzzahlige binäre quadratische Form der Diskriminante $-4 n$ :

$$
D=b^{2}-4 a c=4 \alpha^{2}+4 \beta \gamma=-4 n
$$

gegeben. Da für alle Substitutionen der Nebengruppe $\Gamma_{\psi(n)} \cdot W$ die Zahlen $\gamma$ und $\delta$ durch $n$ teilbar sind, so sind $\alpha$ und $\beta$ zufolge (7) teilerfremd. Die Form (10) kann also nur dann eine ,abgeleitete“ sein (S. 137), wenn $\beta$ und $\gamma$ zugleich gerade sind, und zwar hat sie dann den Teiler 2. Da in diesem Falle $\alpha$ als teilerfremd gegen $\beta$ ungerade ist, so gilt $n \equiv 3$ $(\bmod 4)$. Es gilt also der Satz: Ist $n \equiv 0,1$ oder $2(\bmod 4)$, so ist die Form (10) der Diskriminante $-4 n$ ursprünglich; für $n \equiv 3(\bmod 4)$ ist sie entweder ursprïnglich oder sie hat den Teiler 2. Im letzteren Falle bedienen wir uns an Stelle von (10) der durch 2 gehobenen Form, die dann ursprünglich und von der Diskriminante $-n$ ist:

$$
\left(\frac{\gamma}{2},-\alpha,-\frac{\beta}{2}\right)=(a, b, c), \quad D=b^{2}-4 a c=\alpha^{2}+\beta \gamma=-n .
$$

Das erhaltene Ergebnis ist umkehrbar. Es sei $(a, b, c)$ eine ursprüngliche Form der Diskriminante $-4 n$, und es seien $a$ durch $n$ und $b$ durch $2 n$ teilbar. Dann ist $c$ teilerfremd gegen $n$, und man erhält in $\left(\begin{array}{c}-\frac{1}{2} b,-c \\ a, \frac{1}{2} b\end{array}\right)$ eine elliptische Substitution der Periode 2, die der Nebengruppe $\Gamma_{\psi(n)} \cdot W$ angehört. Weiter sei im Falle $n \equiv 3(\bmod 4)$ eine ursprüngliche Form $(a, b, c)$ der Diskriminante $-n$ vorgelegt, deren Koeffizienten $a, b$ durch $n$ teilbar seien. Dann ist wieder $c$ teilerfremd gegen $n$, und man hat in $\left(\begin{array}{cc}-b, & -2 c \\ 2 a, & b\end{array}\right)$ eine der Nebengruppe $\Gamma_{\psi(n)} \cdot W$ angehörende Substitution der Periode 2.

Es läßt sich weiter leicht zeigen, daß sich in jeder Klasse ursprünglicher positiver Formen der Diskriminante $-4 n$ bzw. $-n$ eine zur Bildung einer elliptischen Substitution der Periode 2 im vorstehenden Sinne brauchbare Form nachweisen läßt. Man entnehme der Klasse zunächst eine Form $\left(a_{0}, b_{0}, c\right)$ mit einem gegen $n$ teilerfremden $c$ und gehe von ihr mittels einer Substitution $\left(\begin{array}{l}1,0 \\ \gamma, 1\end{array}\right)$ zur äquivalenten Form $(a, b, c)$. Liegt die Diskriminante $-4 n$ vor, so sind die mittleren Koeffizienten der Formen gerade, und man hat $\frac{1}{2} b=\frac{1}{8} b_{0}-\gamma c$. Da $c$ teilerfremd gegen $n$ ist, so kann man $\gamma$ so wählen, daB $\frac{1}{2} b$ durch $n$ und also $b$ durch $2 n$ teilbar ist, worauf aus $b^{2}-4 a c=-4 n$ sich $a \equiv 0(\bmod n)$ ergibt. Haben wir im Falle $n \equiv 3(\bmod 4)$ die Diskriminante $-n$, so ist, da jetzt $2 c$ teilerfremd gegen $n$ ist, $\gamma$ so wählbar daB $b=b_{0}-2 \gamma c$ durch $n$ teilbar ist; aus 
$b^{2}-4 a c=-n$ folgt dann wieder $a \equiv 0(\bmod n)$. Es ist also in jeder zulässigen Klasse eine brauchbare Form nachgewiesen.

Es seien nun $(a, b, c)$ und $\left(a^{\prime}, b^{\prime}, c^{\prime}\right)$ zwei äquivalente Formen, die beide zur Bildung elliptischer Substitutionen der Periode 2 von $\Gamma_{\psi(n)} \cdot W$ brauchbar sind. Im Falle der Diskriminante $D=-4 n$ seien also $a$ und $a^{\prime}$ durch $n, b$ und $b^{\prime}$ durch $2 n$ teilbar, während für $D=-n$ (im Falle $n \equiv 3(\bmod 4))$ die Zahlen $a, a^{\prime}, b$ und $b^{\prime}$ durch $n$ teilbar sind. Die Äquivalenz beider Formen sei durch die Substitution $\left(\begin{array}{l}\alpha, \beta \\ \gamma, \delta\end{array}\right)$ vermittelt, so daß nach (4) S. 138 die Gleichungen gelten:

$$
\left\{\begin{array}{l}
a^{\prime}=\delta^{2} a-\gamma \delta b+\gamma^{2} c \\
b^{\prime}=-2 \beta \delta a+(\alpha \delta+\beta \gamma) b-2 \alpha \gamma c, \\
c^{\prime}=\beta^{2} a-\alpha \beta b+\alpha^{2} c .
\end{array}\right.
$$

Im Falle $D=-4 n$ folgt durch Reduktion der ersten Gleichung (12) $\bmod n$ und der zweiten $\bmod 2 n$ :

$$
\gamma^{2} c \equiv 0(\bmod n), \quad 2 \alpha \gamma c \equiv 0(\bmod 2 n),
$$

so daB $\gamma^{2} c$ und $\alpha \gamma c$ durch $n$ teilbar sind. Da aber $\alpha$ und $\gamma$ teilerfremde Zahlen sind und $c$ teilerfremd gegen $n$ ist, so ergibt $\operatorname{sich} \gamma \equiv 0(\bmod n)$. $\mathrm{Zu}$ demselben Ergebnis gelangt man leicht auch im Falle $D=-n$. Geht man andrerseits von einer brauchbaren Form $(a, b, c)$ mittels einer Substitution mit $\gamma \equiv 0(\bmod n)$ zur äquivalenten Form $\left(a^{\prime}, b^{\prime}, c^{\prime}\right)$, so ist erstlich $\alpha$ und also zufolge der dritten Gleichung (12) auch $c^{\prime}$ teilerfremd gegen $n$. Weiter aber folgt aus den beiden ersten Gleichungen (12) sofort $a^{\prime} \equiv 0$ $(\bmod n)$ und $b^{\prime} \equiv 0(\bmod 2 n$ bzw. $n)$. Also ist auch $\left(a^{\prime}, b^{\prime}, c^{\prime}\right)$ eine zur Bildung einer unserer elliptischen Substitutionen brauchbare Form.

Diese brauchbaren Formen der einzelnen Klasse fassen wir nun zu einer "Unterklasse“ zusammen. Die Formen der einzelnen Unterklasse sind bezüglich der $\Gamma_{\psi(n)}$ äquivalent und mögen kurz „relativ äquivalent" heißen; die einzelne Unterklasse besteht dann aus allen mit einer unter ihnen relativ äquivalenten Formen. Die „Nullpunkte“ $\omega$ dieser Formen (vgl. S. 139), die die Fixpunkte der zugehörigen elliptischen Substitutionen liefern, sind also selbst bezüglich der $\Gamma_{\psi(n)}$ äquivalent, so daB im Transformationspolygone $\mathbf{T}_{n}$ für jede Unterklasse ein und nur ein Nullpunkt gelegen ist. Wir haben damit das wichtige Ergebnis gewonnen: Die Anzahl der im Transformationspolygone $\mathrm{T}_{n}$ gelegenen Fixpunkte elliptischer Substitutionen der Periode 2 der Nebengruppe $\Gamma_{\psi(n)} \cdot W$ ist gleich der Anzahl der Klassen ursprünglicher positiver. Formen der Diskriminante $D=-4 n$, falls $n \equiv 0,1$ oder $2(\bmod 4)$ gilt, und gleich der Summe der Klassenzahlen solcher Formen der Diskriminanten $D=-4 n$ und $D=-n$, falls $n \equiv 3(\bmod 4)$ ist; die Fixpunkte werden dabei unmittelbar von den 
Nullpunkten der „bezüglich der $\Gamma_{\psi(n)}$ reduzierten“ Formen der Unterklassen geliefert.

Als Beispiel betrachten wir den Fall $n=11$. Die Anzahl der Klassen ursprünglicher positiver Formen der negativen Diskriminante $D$ wurde S. 147 ff. durch $h(|D|)$ bezeichnet. Nach einer S. 148 aufgestellten Regel ist $h(44)=3 h(11)$. In der Tat gilt $h(11)=1, h(44)=3$. Man hat nämlich für $D=-11$ nur eine zweiseitige Klasse mit der im ursprünglichen Sinne von S. 140 reduzierten Form $(1,1,3)$, während für $D=-44$ eine zweiseitige Klasse und zwei entgegengesetzte Klassen mit den reduzierten Formen $(1,0,11)$ und $(3, \pm 2,4)$ vorliegen. Mit den vier genannten Formen sind bzw. die folgenden äquivalent:

$$
(11,11,3), \quad(11,0,1), \quad(33, \mp 22,4) ;
$$

wir können diese Formen als die repräsentierenden Formen der vier Unterklassen wählen und haben als ihre Nullpunkte:

$$
\omega=\frac{-\sqrt{11}+i}{2 \sqrt{11}}, \quad \omega=\frac{i}{\sqrt{11}}, \quad \omega=\frac{ \pm \sqrt{11}+i}{3 \sqrt{11}},
$$

sowie als die zugehörigen elliptischen Substitutionen:

$$
\left(\begin{array}{c}
11,6 \\
-22,-11
\end{array}\right),\left(\begin{array}{c}
0,-1 \\
11,0
\end{array}\right),\left(\begin{array}{c}
11, \mp 4 \\
\pm 33,-11
\end{array}\right)
$$

Die geometrischen Verhältnisse in der $\omega$-Halbebene werden durch Fig. 10 erläutert. Das Transformationspolygon ist mit der $\Omega$-Teilung ge-

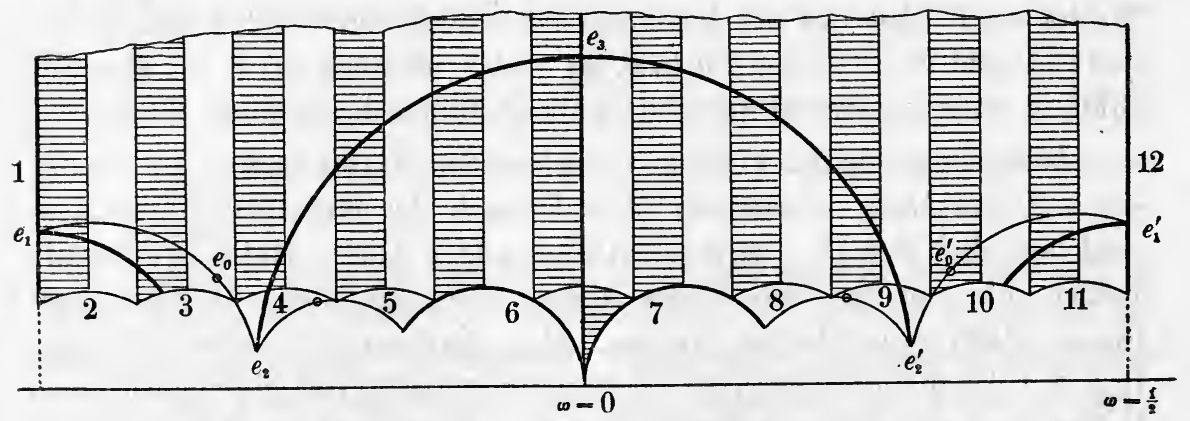

Fig. 10.

zeichnet, so daß die Figur das Aussehen des Polygons $T_{11}^{\prime}$ mit dem auf $\frac{1}{11}$ verkleinerten Maßstabe hat. Die Zusammenordnung der mit Nummern 1 bis 12 versehenen Randkreise geschieht durch folgende, auf $\Omega$ auszuübende Substitutionen, die also der $\Gamma_{\psi(11)}^{\prime}$ angehören:

$$
\begin{aligned}
& 1 \rightarrow 12,\left(\begin{array}{c}
1,11 \\
0,1
\end{array}\right) ; 2 \rightarrow 5,\left(\begin{array}{c}
2,11 \\
-1,-5
\end{array}\right) ; 3 \rightarrow 9,\left(\begin{array}{c}
3,11 \\
1,4
\end{array}\right) ; \\
& 4 \rightarrow 10,\left(\begin{array}{c}
4,11 \\
1,3
\end{array}\right) ; 6 \rightarrow 7,\left(\begin{array}{l}
1,0 \\
1,1
\end{array}\right) ; 8 \rightarrow 11,\left(\begin{array}{c}
5,-11 \\
1,-2
\end{array}\right)
\end{aligned}
$$


Die Umrechnung auf $\omega$ geschieht einfach entsprechend dem Rückgange von der zweiten Substitution (6) zur ersten. Die vier Punkte (13) liegen an den in der Figur mit $e_{1}, e_{3}, e_{0}, e_{0}^{\prime}$ bezeichneten Stellen.

Der Diskontinuitätsbereich der durch $W$ erweiterten Gruppe $\Gamma^{(11)}$ setzt sich aus zwei bezüglich der imaginären $\omega$-Achse symmetrischen Hälften zusammen. Die einzelne, etwa linke Hälfte, die für sich einen Diskontinuitätsbereich der $\bar{\Gamma}^{(11)}$ bildet, fassen wir als ein Kreisbogenfünfeck der Ecken $i \infty, e_{1}, e_{0}, e_{2}, e_{3}$ auf, obschon in der Ecke $e_{0}$ der Winkel $\pi$ vorliegt. Die stark ausgezogenen Kreisbogen sind Symmetriekreise von Spiegelungen der erweiterten Gruppe $\bar{\Gamma}^{(11)}$. Die Seite $e_{0}, e_{1}$ ist durch die vierte Substitution (14) auf die Seite $e_{0}, e_{2}$ bezogen. Entsprechend wird die Seite $e_{0}^{\prime}, e_{1}^{\prime}$ durch die dritte Substitution (14) in $e_{0}^{\prime}, e_{2}^{\prime}$ transformiert, und endlich wird die Seite $e_{2}, e_{3}$ der linken Hälfte des Diskontinuitätsbereiches der $\Gamma^{(11)}$ durch die zweite Substitution (14) in die symmetrische Seite $e_{2}^{\prime}, e_{3}$ der rechten Hälfte übergeführt.

Der aus den beiden symmetrischen Kreisbogenfünfecken zusammengesetzte Diskontinuitätsbereich der $\Gamma^{(11)}$ möge das zum Grade 11 gehörende „Rlassenpolygon" genannt und durch $K_{11}$ bezeichnet werden. Der Name rechtfertigt sich durch die Beziehung der Ecken von $K_{\mathbf{1 1}} \mathbf{z u}$ den Formklassen der Diskriminanten $-4 \cdot 11$ und -11 . Von diesen Ecken gehört die bei $\omega=i \infty$ bereits zum Transformationspolygon $\mathbf{T}_{11}$. Weiter sind die vier Ecken $e_{1}, e_{2}, e_{1}^{\prime}, e_{2}^{\prime}$ bezüglich der $\Gamma^{(11)}$ äquivalent und bilden, wie man sagt, einen Zyklus zusammengehöriger Ecken. ${ }^{1}$ ) Die übrigen drei Ecken $e_{0}, e_{0}^{\prime}, e_{3}$ aber sind bezüglich der $\Gamma^{(11)}$ inäquivalent. Lassen wir von den vier äquivalenten Ecken des Zyklus nur eine als den Zyklus repräsentierend zu, so sind nun eben die noch nicht dem Transformationspolygon $\mathbf{T}_{11}$ zugehörigen Ecken des Klassenpolygons eindeutig den Klassen ursprünglicher positiver quadratischer Formen der Diskriminanten $-4 \cdot 11$ und -11 zugeordnet.

Dieselben Verhältnisse kehren entsprechend bei jedem Grade $n$ wieder, wo wir den Diskontinuitätsbereich der $\Gamma^{(n)}$ wieder als „Klassenpolygon “ $\mathrm{K}_{n}$ bezeichnen. Auch die oben in den Figuren 7,8 und 9 erläuterten niedersten Fälle $n=2,3$ und 4 bilden keine Ausnahmen. Wie oben festgestellt wurde, kommen für $n \equiv 0,1,2(\bmod 4)$ nur die Formklassen der Diskriminante $D=-4 n$ in Betracht, während für $n \equiv 3(\bmod 4)$ auch noch die Klassen der Diskriminante $D=-n$ dazutreten. ${ }^{2}$ )

1) Vgl. die allgemeine Theorie der Diskontinuitätsbereiche von Gruppen linearer Substitutionen einer Variablen in den „Vorlesungen über die Theorie der antomorphen Funktionen" ron F. Klein und R. Fricke, Bd. 1, S. 159 ff. (Leipzig 1897).

2) Der Name „Klassenpolygon" für $K_{n}$ erscheint um so mehr gerechtfertigt, als die unter den Randkurven von $\boldsymbol{K}_{n}$ befindlichen Symmetriekreise von Spiege- 
Bilden wir das Klassenpolygon $K_{n}$ mittelst einer zur $\Gamma^{(n)}$ gehörenden Funktion auf die Ebene dieser Funktion ab, so entsteht eine Riemannsche Fläche $\mathbf{F}^{(n)}$, deren Geschlecht wir durch $p_{0}(n)$ bezeichnen und zugleich als das Geschlecht des Klassenpolygons $\mathrm{K}_{n}$ ansehen wollen. $\mathrm{Um} p_{0}(n) \mathrm{zu}$ bestimmen, wenden wir die Formel (4) in I, 88 an:

$$
p_{0}(n)=-m+1+\sum \frac{v-1}{2},
$$

wo $m$ die Blätterzahl der $\mathbf{F}^{(n)}$ ist und die Summe sich auf die Verzweigungspunkte der $\boldsymbol{F}^{(n)}$ bezieht, deren einzelner $\boldsymbol{\nu}$-blättrig gedacht ist. Wenn wir nun das Transformationspolygon $\mathbf{T}_{n}$ durch dieselbe Funktion abbilden, so erhalten wir eine Fläche, deren Geschlecht $p(n)$ wir bereits in (5) $\mathrm{S} .356$ berechnet haben. Sie erscheint hier aus zwei übereinander gelagerten Exemplaren der Fläche $F^{(n)}$ bestehend, die in einer Anzahl zweiblättriger Verzweigungspunkte aneinander geheftet sind. Diese Anzahl ist aber, wenn wir von den drei Fällen $n=2,3,4$, wo $p_{0}=0$ ist; absehen, gleich der Klassenanzahl $h(4 n)$ oder gleich der Summe $h(4 n)+h(n)$, je nachdem $n \equiv 0,1,2$ oder $\equiv 3(\bmod 4)$ ist. Nach der Regel von S. 148 können wir auch sagen, jene Anzahl sei gleich $\varepsilon_{n} h(4 n)$, wo $\varepsilon_{n}=1$ ist, wenn $n \equiv 0,1$ oder $2(\bmod 4)$ ist, dagegen $\varepsilon_{n}=2$ oder $=\frac{4}{3}$ gilt, je nachdem $n \equiv 7$ oder $\equiv 3(\bmod 8)$ zutrifft.

Stellen wir nun das Geschlecht $p(n)$ der $2 m$-blättrigen Fläche auf Grund der Regel (4) in I, 88 dar, so kommt für $n>4$ :

$$
p(n)=-2 m+1+2 \sum \frac{v-1}{2}+\frac{1}{2} \varepsilon_{n} h(4 n),
$$

wofür wir mit Rücksicht auf (15) auch schreiben können:

$$
p(n)=2 p_{0}(n)-1+\frac{1}{2} \varepsilon_{n} h(4 n) .
$$

Es ergibt sich hieraus der Satz: Das Geschlecht $p_{0}(n)$ des Klassenpolygons $\mathbf{K}_{n}$ berechnet sich nach der Regel:

$$
p_{0}(n)=\frac{1}{2} p(n)+\frac{1}{2}-\frac{1}{4} \varepsilon_{n} h(4 n),
$$

wo $p(n)$ das durch (5) S. 356 gegebene Geschlecht des Transformationspolygons $\mathbf{T}_{n}$ ist, $h(4 n)$ die Klassenanzahl ursprünglicher positiver Formen der Diskriminante $D=-4 n$ ist und $\varepsilon_{n}$ für $n \equiv 7(\bmod 8)$ den Wert 2, für $n \equiv 3(\bmod 8)$ den Wert $\frac{4}{3}$ und sonst den Wert 1 hat. Man kann hieraus noch den speziellen Satz ableiten: Die mit $\varepsilon_{n}$ multiplizierte Klassen-

lungen der Gruppe $\vec{\Gamma}^{(n)}$, die noch nicht der Gruppe $\bar{\Gamma}_{\psi(n)}$ angehören, für $n \equiv 0$, 2 und $3(\bmod 4)$ eindentig den ursprünglichen Formklassen der positiven Diskriminante $4 n$ zugeordnet sind, im Falle $n \equiv 1(\bmod 4)$ aber ebenso den ursprünglichen Formklassen der beiden positiven Diskriminanten $n$ und $4 n$. Vgl. R. Fricke „Über Transformations- und Klassenpolygone“, Gött. Nachr. von 1919. 
zahl $h(4 n)$ liefert ein Produkt $\varepsilon_{n} h(4 n)$, das stets $\leqq 2 p(n)+2$ ist; erreicht die Anzahl $\varepsilon_{n} h(4 n)$ ihre obere Schranke $2 p(n)+2$, so ist das Geschlecht des Klassenpolygons gleich 0 . Unter den 71 Graden $n$, für welche oben S. 357 die Gescblechter $p(n)$ angegeben sind, liefern 36 Klassenpolygone des Geschlechtes $p_{0}=0$, nämlich:

$$
\begin{gathered}
n=2,3, \ldots, 21,23,24,25,26,27,29,31,32,35,36,39,41, \\
47,49,50,71 .
\end{gathered}
$$

\section{§ 5. Algebraische Methode zur Aufstellung der speziellen Transformationsgleichungen.}

Wie schon oben (S. 349) angedeutet wurde, gründet sich die von Klein entwickelte algebraische Methode zur Aufstellung der speziellen Transformationsgleichungen auf den Gebrauch der Transformationspolygone. $\left.{ }^{1}\right)$ Durch die Funktion $j(\omega)$ bildeten wir das einzelne $\mathbf{T}_{n}$ auf die Transformationsfläche $F_{n}$ ab, die $\psi(n)$-blättrig die $j$-Ebene überlagerte, und deren Verzweigung, wie oben (S. 352) im Falle $n=7$ geschildert wurde, aus dem mit dem Dreiecksnetze der $\omega$-Halbebene ausgefüllten Polygone $\mathbf{T}_{n}$ abgelesen werden kann. Auf dieser Fläche $\mathbf{F}_{n}$ ist daun $j^{\prime}$ eine $\psi(n)$-wertige algebraische Funktion, deren Zusammenhang mit $j$ eben durch die Transformationsgleichung dargestellt wird. Die von Klein entwickelte Theorie der Transformationsgleichungen beruht nun auf der Verwertung der Hilfsmittel von Riemanns Theorie der algebraischen Funktionen. Der Grundgedanke ist, auf der Riemannschen Fläche $\mathbf{F}_{n}$ nicht sogleich die Funktionen $j$ ' und $j$ einer verhältnismäBig hohen Wertigkeit zu betrachten, sondern sich zunächst geeignete Funktionen einer möglichst niedrigen Wertigkeit $z u$ verschaffen und sodann $j$ und $j$ in ihnen rational darzustellen.

Bei den 14 S. 357 genannten Graden $n$, für welche die Flächen $\mathbf{F}_{n}$ das Geschlecht $p=0$ haben, gibt es einwertige Funktionen. Eine geeignete Funktion dieser Art, die $\tau(\omega)$ genannt werden möge, ist im Einzelfalle auszuwählen, und sodann sind $j$ und $j^{\prime}$ rational in $\tau$ darzustellen. Für die Gewinnung dieser rationalen Darstellungen benutzte Klein eine rein algebraische Methode, die sich auf die Verzweigung der $\psi(n)$-blättrigen Fläche $F_{n}$ über der $j$-Ebene gründet. Es gelang ohne Be-

1) Das Polygon $T_{n}$ gibt uns, wenn wir über die $\omega$-Teilung noch die nach dem Maßstabe $\frac{1}{n}$ gezeichnete $\Omega$-Teilung getragen denken (s. die Figuren 5,6,.. S. $358 \mathrm{ff}$.), ein anschauliches Bild für die zum $n^{\text {ten }}$ Grade gehörende spezielle Transformationsgleichung $F\left(j^{\prime}, j\right)=0$. Ein beliebig vorgeschriebener komplexer Wert $j$ tritt in $\psi(n)$ Punkten von $\mathbf{T}_{n}$ auf, die äquivalent im Netze der $\omega$-Teilung sind. Sie sind in der $\Omega$-Teilung $\psi(n)$ Punkte, deren zugehörige Funktionswerte $j^{\prime}(\omega)=j(\Omega)$ die $\psi(n)$ zugehörigen Wurzeln der Gleichung $F\left(j^{\prime}, j\right)=0$ sind. 
nutzung der Potenzreihen die gewünschten Darstellungen für $j^{\prime}$ und $j$ in allen 14 Fällen in Erfahrung zu bringen. Der Transformation $W$ wird dabei eine wichtige Rolle zuerteilt. $\left.{ }^{1}\right)$ Die Transformationsgleichung selbst ergibt sich durch Elimination von $\tau$ aus den beiden Gleichungen für $j$ und $j^{\prime}$. Zur Weiterführung dieser Entwicklungen zog Fricke ${ }^{2}$ ) neben den Polygonen $\mathbf{T}_{n}$ auch noch die Klassenpolygone $\mathbf{K}_{n}$ und die zugehörigen Flächen $F^{(n)}$ heran und benutzte überdies bei den algebraischen Rechnungen formentheoretische Methoden und Potenzreihen. Auf diese Weise werden die $36 \mathrm{Fälle}$, in denen das Klassenpolygon $\mathbf{K}_{n}$ das Geschlecht 0 hat, ziemlich leicht zugänglich.

Die vorliegende. Darstellung entwickelt insbesondere die letzte $\mathrm{Me}-$ thode, und zwar in einer Reihe von Fällen, in denen das Geschlecht $p_{0}(n)=0$ ist. Wir bezeichnen mit $\tau(\omega)$ eine geeignet gewählte einwertige Funktion der dem Klassenpolygone $\mathbf{K}_{n}$ entsprechenden Fläche $\mathbf{F}^{(n)}$ in ihrer $\mathbf{A b}$ hängigkeit von $\omega$. Diese Funktion ist dann gegenüber den Substitutionen der erweiterten Gruppe $\Gamma^{(n)}$ invariant. Durch Abbildung des Transformationspolygons $T_{n}$ vermittelst $\tau(\omega)$ gewinnt man eine zweiblättrige Fläche über der $\tau$-Ebene mit $(2 p+2)$ Verzweigungspunkten, wo $p$ das Geschlecht des Transformationspolygons $T_{n}$ ist. Wir denken $\tau$ so gewählt, daß der Unendlichkeitspunkt dieser Funktion nicht gerade in einen jener Verzweigungspunkte fällt. Ist dann $f_{2 p+2}(\tau)$ diejenige rationale ganze Funktion $(2 p+2)^{\text {ten }}$ Grades mit dem höchsten Koeffizienten 1, deren Nullpunkte die $(2 p+2)$ Verzweigungswerte $\tau$ sind, so ist:

$$
\sigma=\sqrt{f_{2 p+2}(\tau)}
$$

eine zweite Funktion der $F_{n}$ bzw. der $\Gamma_{\psi(n)}$, die gegenüber der Substitution $W$ Zeichenwechsel erfährt, und die zusammen mit $\tau$ zur rationalen Darstellung aller Funktionen der $\mathbf{F}_{n}$ ausreicht. ${ }^{3}$ )

$\mathrm{Zu}$ diesen Funktionen gehören nun insbesondere auch $j$ und $j^{\prime}$. Mit Rücksicht auf das Verhalten von $\sigma$ und $\tau$ gegenüber $W$ folgt somit der Satz: In den 36 Fällen, in denen das Geschlecht des Klassenpolygons $\mathbf{K}_{\boldsymbol{n}}$ gleich 0 ist, gibt es stets zwei Funktionen $\tau(\omega)$ und $\sigma(\omega)$ der $\Gamma_{\psi(n)}$, die

1) Vgì. die S. 349 genannten Arbeiten von Klein und Gierster. Übrigens sind die betreffenden Entwicklungen in „Modulfunktionen“, Bd. 1, S. $634 \mathrm{ff}$. ausführlich dargestellt.

2) Vgl. die Abhandlung „Neue Beiträge zur Transformationstheorie der elliptischen Funktionen", Math. Ann., Bd. 40 (1891).

3) Die Gleichung $f_{2 p+2}(\tau)=0$ hat als Wurzeln die Werte der Funktion $\tau(\omega)$ in den Nullpunkten der S. $362 \mathrm{ff}$. betrachteten $h(4 n)$ bzw. $(h(4 n)+h(n))$ die Werte repräsentierenden quadratischen Formen. Wir nennen eine Gleichung dieser Art $f_{2 p+2}(\tau)=0$ deshalb eine "Klassengleichung" und kommen bei den arithmetischen Anwendungen der elliptischen Funktionen auf deren Theorie ausführlich zurüok. 
durch eine algebraische Relation:

$$
\sigma^{2}-f_{2 p+2}(\tau)=0
$$

verbunden sind, wo $f_{2 p+2}(\tau)$ eine rationale ganze Funktion des Grades $(2 p+2)$ ist und $p$ das Geschlecht des Transformationspolygons bedeutet. In den Funktionen $\tau(\omega)$ und $\sigma(\omega)$, von denen die erste gegenüber $W$ unverändert bleibt, während die zweite Zeichenwechsel erfährt, lassen sich $j$ und $j^{\prime}$ rational in der Gestalt:

$$
j=R(\tau, \sigma), \quad j^{\prime}=R(\tau,-\sigma)
$$

darstellen. Durch Elimination von $\sigma$ und $\tau$ aus den drei Gleichungen (2) und (3) ergibt sich die spezielle Transformationsgleichung $F\left(j^{\prime}, j\right)=0$ für den $n^{\text {ten }}$ Grad.

Bei der wirklichen Herstellung der Funktionen $\tau(\omega)$ und $\sigma(\omega)$ bedienen wir uns ganzer Modulformen der $\Gamma_{\psi(n)}$. Die algebraischen Überlegungen stützen sich dabei auf einen Satz über die Anzahl der Nullpunkte solcher Formen im Transformationspolygon $T_{n}$, der zunächst aufzustellen ist. Wir verstehen unter $G_{\frac{1}{2} \delta}\left(\omega_{1}, \omega_{2}\right)$ eine ganze Modulform der $\Gamma_{\psi(n)}$ von der Dimension $-\delta$, die gegenüber den Substitutionen der $\Gamma_{\psi(n)}$ entweder unverändert bleibt oder sich nur um Einheitswurzeln als Faktoren ändert; eine hinreichend hohe, etwa $m^{\text {te }}$ Potenz dieser Form bleibt dann gegenüber den Substitutionen der $\Gamma_{\psi(n)}$ unverändert. Demnach ist der Quotient:

$$
\frac{G_{\frac{1}{2} \delta}\left(\omega_{1}, \omega_{2}\right)^{12 m}}{\Delta\left(\omega_{1}, \omega_{2}\right)^{m \delta}}
$$

eine Modulfunktion der $\Gamma_{\psi(n)}$ und also eine algebraische Funktion der Transformationsfläche $F_{n}$. Für eine solche Funktion ist aber die Summe der Ordnungen aller Nullpunkte auf der $F_{n}$ gleich der Summe der Ordnungen aller Pole. Für den Zähler des Quotienten (4) ist also die Summe aller „,im Polygone $\mathbf{T}_{n}$ gemessenen“ Nullpunkte (vergl. I, 307) gleich derjenigen des Nenners, d. h. gleich $m \delta \cdot \psi(n)$. Damit ergibt sich der Satz: Eine ganze Modulform der Dimension - $\delta$, die gegenüber den Substitutionen der $\Gamma_{\psi(n)}$, abgesehen von multiplikativen Einheitswurzeln, unverändert bleibt, hat im Transformationspolygon $\mathbf{T}_{n}$ Nullpunkte in der Gesamtordnung $\frac{\delta}{12} \psi(n)$. DaB bei Modulformen Nullpunkte gebrochener Ordnungen in einem Diskontinuitätsbereiche auftreten können, erkannten wir bereits in I, 307. Nach den damaligen Erörterungen wird man sofort folgenden Satz verstehen: In einer mit $\omega=i$ äquivalenten Ecke des Transformationspolygons $\mathbf{T}_{n}$ (deren zugehörige elliptische Substitution der Periode 2 dann in $\Gamma_{\psi(n)}$ enthalten ist) kann eine ganze Modulform der $\Gamma_{\psi(n)}$, ",im Polygone $\mathbf{T}_{n}$ gemessen“, einen Nullpunkt gebrochener Ordnung 
mit dem Nenner 2 haben, und ebenso kann in einer mit $\omega=\rho$ äquivalenten Ecke von $\mathrm{T}_{n}$ ein Nullpunkt gebrochener Ordnung mit dem Nenner 3 auftreten.

Beim einzelnen Transformationsgrade $n$ haben wir nun die Betrachtung allemal an die ganzen Modulformen möglichst niedriger Dimension anzuknüpfen. Diese aber werden uns von den Modulformen $(-1)^{\text {ter }}$ Dimension $y_{0}$ und $z_{0}$ des vorigen Kapitels geliefert, auch von den Potenzen $\operatorname{des}$ Ausdrucks $\sqrt[24]{\Delta^{\prime} \Delta}$ und den Formen $\xi_{0}$. Wie hierbei zu verfahren ist, muB den folgenden Einzelbetrachtungen überlassen bleiben. Das erste Ziel wird sein, jedesmal ein Paar von Formen $\tau_{1}\left(\omega_{1}, \omega_{2}\right), \tau_{2}\left(\omega_{1}, \omega_{2}\right)$ gleicher Dimension herzustellen, die als Quotienten $\tau_{1}: \tau_{2}=\tau$ die gewünschte einwertige Funktion der $\Gamma^{(n)}$ liefern.

Die weitere Entwicklung hat alsdann die Darstellung von $j$ und $j^{\prime}$ in $\tau$ und dem zugehörigen $\sigma$ zum Gegenstande. Wir schreiben zunächst die Transformation $W$ in homogener Gestalt:

$$
\omega_{1}^{\prime}=\frac{i \omega_{2}}{\sqrt{n}}, \quad \omega_{2}^{\prime}=\frac{\omega_{1} \sqrt{n}}{i},
$$

in der sie gleichfalls die Periode 2 hat. Die Ausdrücke:

$$
g_{2}\left(\frac{i \omega_{2}}{\sqrt{n}}, \frac{\omega_{1} \sqrt{n}}{i}\right) \pm g_{2}\left(\omega_{1}, \omega_{2}\right), \quad g_{3}\left(\frac{i \omega_{2}}{\sqrt{n}}, \frac{\omega_{1} \sqrt{n}}{i}\right) \pm g_{3}\left(\omega_{1}, \omega_{2}\right)
$$

sind dann ganze Modulformen der $\Gamma_{\psi(n)}$, die gegenüber $W$ unverändert bleiben oder Zeichenwechsel erfahren, je nachdem in (6) die oberen oder unteren Zeichen gelten. Wir werden diese Ausdrücke (6) in den $\tau_{1}, \tau_{2}, \sigma$ darzustellen versuchen und gelangen auf diese Weise schließlich zu den Ausdrücken (3) von $j$ und $j^{\prime}$. Hierbei wird dann auch noch das Hilfsmittel der Potenzreihen eine wichtige Rolle spielen.

\section{Viertes Kapitel.}

\section{Aufstellung der Transformationsgleichungen erster Stufe für niedere Grade $n$.}

Bei Entwicklung der allgemeinen Ansätze des vorigen Kapitels werden die ungeraden Transformationsgrade bevorzugt. Unter den $36 \mathrm{~S} .367 \mathrm{zu}-$ sammengestellten Graden $n$ mit $p_{0}(n)=0$ sind nun zunächst die fünf ersten Potenzen $n=2,4,8,16,32$ der Zahl 2 enthalten. Ihre Behandlung schließen wir unmittelbar an die Entwicklungen über die Funktionen zweiter Stufe in I, $434 \mathrm{ff}$. an, indem wir uns übrigens der soeben entwickelten algebraischen Methoden bedienen. Für die ungeraden Transformationsgrade ziehen wir dann die analytischen Hilfsmittel der voraufgehenden Kapitel ausführlich heran. Ist aber allgemein der Grad 
$n=2^{v} \cdot n^{\prime}$ und $n^{\prime}$ ungerade, so werden wir versuchen, die Transformation dieses Grades $n$ auf diejenigen der beiden Grade $2^{v}$ und $n^{\prime}$ zurückzuführen. Es vereinfacht die Formeln ein wenig, wenn wir uns an Stelle von $j(\omega)=12^{3} J(\omega)$ wieder der ursprünglichen Funktion $J(\omega)$ bedienen. Mit $\tau(\omega)$ und $\sigma(\omega)$ bezeichnen wir einwertige oder zweiwertige Funktionen der Gruppen $\Gamma_{\psi(n)}$; doch sei bemerkt, daß diese Bezeichnungen wenigstens anfangs nicht immer genau in dem S. 368 vereinbarten Sinne gebraucht sind.

\section{\$ 1. Die Transformationsgrade 2, 4, 8, 16 und 32.}

1. Transformation zweiten Grades von $J(\omega)$. Die beim Transformationsgrade $n=2$ auftretende Transformationsgleichung dritten Grades für $J(\omega)$ kann aus den Entwicklungen in I, $434 \mathrm{ff}$. abgeleitet werden. In I, 442 wurde die mit $\lambda(\omega)$ bezeichnete einwertige Funktion der Hauptkongruenzgruppe zweiter Stufe eingeführt, die nach der zweiten Gleichung (3) in I, 444 in:

$$
\tau(\omega)=\frac{-4}{\lambda(\omega) \lambda(\omega+1)}=4 \frac{1-\lambda(\omega)}{\lambda(\omega)^{2}}
$$

eine für unsere $\mathrm{Zwecke} \mathrm{geeignete} \mathrm{einwertige} \mathrm{Funktion} \mathrm{des} \mathrm{Transformations-}$ polygons $T_{2}$ liefert. Aus $\lambda(i \infty)=0, \lambda(0)=1, \lambda\left(\frac{1+i}{2}\right)=2$ berechnen sich als Werte von $\tau(\omega)$ in den Ecken des durch Fig. 7, S. 360, dargestellten Polygons $\mathrm{T}_{2}$ :

$$
\tau(0)=0, \tau\left(\frac{ \pm 1+i}{2}\right)=-1, \quad \tau(i \infty)=\infty .
$$

Die Substitution $W$ transformiert $\mathbf{T}_{2}$ in sich, so daB $\tau(W(\omega))$ wieder eine einwertige Funktion von $\mathbf{T}_{2}$ und als solche eine lineare Funktion von $\tau(\omega)$ ist. In der Tat gilt:

$$
\tau\left(\frac{-1}{2 \omega}\right)=\frac{1}{\tau(\omega)}
$$

da durch $W$ die Polygonspitzen 0 und $i \infty$ und also die Werte $\tau=0$ und $\tau=\infty$ ausgetauscht werden und der Eckenzyklus $\frac{ \pm 1+i}{2}$ und damit der Wert $\tau=-1$ in sich transformiert wird.

Aus (6) in I, 445 folgt bei Umrechnung auf $\tau$ :

$$
J:(J-1): 1=(\tau+4)^{3}:(\tau-8)^{2}(\tau+1): 27 \tau^{2} .
$$

Übt man die Substitution $W$ aus und schreibt zur Abkürzung:

$$
J\left(\frac{-1}{2 \omega}\right)=J(2 \omega)=J^{\prime}(\omega), \quad \tau\left(\frac{-1}{2 \omega}\right)=\tau^{\prime}(\omega),
$$

so folgt bei Fortlassung des Argumentes $\omega$ : 


$$
J^{\prime}:\left(J^{\prime}-1\right): 1=\left(\tau^{\prime}+4\right)^{3}:\left(\tau^{\prime}-8\right)^{2}\left(\tau^{\prime}+1\right): 27 \tau^{\prime 2}, \quad \tau^{\prime}=\frac{1}{\tau}
$$

oder als Darstellung der transformierten Funktion $\boldsymbol{J}^{\prime}$ durch $\tau$ :

$$
J^{\prime}:\left(J^{\prime}-1\right): 1=(4 \tau+1)^{3}:(8 \tau-1)^{2}(\tau+1): 27 \tau .
$$

Die Elimination von $\tau$ aus (4) und (6) führt zur Transformationsgleichung von $J(\omega)$ für den zweiten Grad. Benutzt man vorübergehend wieder $j(\omega)$, so folgt zunächst:

$$
\sqrt[3]{j}=4 \frac{\tau+4}{(\sqrt[3]{\tau})^{2}}, \quad \sqrt[3]{j^{\prime}}=4 \frac{\tau^{\prime}+4}{\left(\sqrt[3]{\tau^{\prime}}\right)^{2}}, \quad \tau \cdot \tau^{\prime}=1
$$

Man findet somit:

$$
\begin{aligned}
& \sqrt[3]{j j^{\prime}}=16\left(4\left(\tau+\tau^{\prime}\right)+17\right) \\
& j+j^{\prime}=64\left(64\left(\tau+\tau^{\prime}\right)^{2}+49\left(\tau+\tau^{\prime}\right)-104\right),
\end{aligned}
$$

sowie weiter durch Elimination von $\left(\tau+\tau^{\prime}\right)$ :

$$
j^{\prime}+j-\left(\sqrt[3]{j^{\prime} j}\right)^{2}+3^{2} \cdot 5 \cdot 11 \sqrt[3]{j^{\prime} j}-2^{4} \cdot 3^{3} \cdot 5^{3}=0 .
$$

Durch Fortschaffung der Kubikwurzel aus $j j$ ergibt sich als spezielle Transformationsgleichung für $j(\omega)$ beim zweiten Grade:

(8) $j^{\prime 3}+j^{3}-j^{\prime 2} j^{2}+1488 j^{\prime} j\left(j^{\prime}+j\right)-162 \cdot 10^{3}\left(j^{\prime 2}+j^{2}\right)+40773375 j^{\prime} j$

$$
+8748 \cdot 10^{6}\left(j^{\prime}+j\right)-157464 \cdot 10^{9}=0
$$

oder bei Zerlegung der Koeffizienten in ihre Primfaltoren:

$$
\begin{aligned}
& j^{\prime 3}+j^{3}-j^{2} j^{2}+2^{4} \cdot 3 \cdot 31 j^{\prime} j\left(j^{\prime}+j\right)-2^{4} \cdot 3^{4} \cdot 5^{3}\left(j^{\prime 2}+j^{2}\right) \\
& +3^{4} \cdot 5^{3} \cdot 4027 j^{\prime} j+2^{8} \cdot 3^{7} \cdot 5^{6}\left(j^{\prime}+j\right)-2^{12} \cdot 3^{9} \cdot 5^{9}=0 .
\end{aligned}
$$

Die Koeffizienten entsprechen den allgemeinen Sätzen von S. 348, sind aber bereits in diesem niedersten Falle $n=2$ außerordentlich große ganze Zahlen, wenn sie auch (abgesehen von 4027) nur aus ganz niederen Primfaktoren aufgebaut sind. Schon in den nächsten Fällen $n=3,4, \ldots$ bietet die endgültige Herstellung der Transformationsgleichungen für $j(\omega)$ große rechneri überwunden sind. Für den späteren Gebrauch wird es nun nicht nötig sein, die Gleichungen in fertiger Gestalt zu besitzen. Es wird für unsere $Z$ wecke, den Fall $n=2$ betreffend, ausreichend sein, die beiden Gleichungen (4) und (6) zu kennen, aus denen die Transformationsgleichung selbst durch Elimination von $\tau$ gewonnen wird, oder, was auf dasselbe hinausläuft, die Gleichung (4) und damit die gleichgebaute Gleichung (5) zwischen $\boldsymbol{J}$ und $\tau^{\prime}$, sowie die Relation zwischen $\tau$ und $\tau^{\prime}$ zu besitzen. Bis zu diesem Punkte werden wir demnach die Untersuchung in den folgenden Fällen $n=3,4, \ldots$ führen. ${ }^{1}$ )

1) Im Sinne späterer Entwicklungen haben wir in (7) eine „Transformations- 
2. Transformation vierten Grades von $J(\omega)$. Der Übergang $\mathrm{zu}$ den Graden 4, 8, 16, 32 wird jedesmal durch Ausziehen einer einzigen Quadratwurzel vollzogen. ${ }^{1}$ ) In Fig. 11 ist das Polygon $\mathbf{T}_{4}$ dargestellt. Die imaginäre $\omega$-Achse und die beiden von $\omega=0$ nach $\omega=\frac{ \pm 1+i}{2}$ ziehenden Kreisbogen zerlegen $\mathbf{T}_{4}$ in vier Kreisbogendreiecke. Die beiden schraffierten Dreiecke sind Abbilder der positiven- $-\frac{1}{s}$ Halbebene der eben bei $n=2$ benutzten Funktion $\tau$,

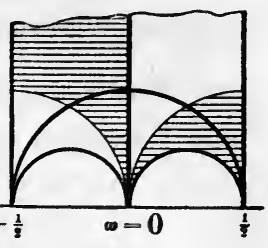

Fig. 11. die fortan genauer $\tau_{2}$ heiße. Einige Werte dieser Funktion sind:

$$
\begin{gathered}
\tau_{2}(0)=0, \quad \tau_{2}\left(\frac{ \pm 1+i}{2}\right)=-1, \quad \tau_{2}(i \infty)=\tau_{2}\left( \pm \frac{1}{2}\right)=\infty, \\
\tau_{2}\left(\frac{i}{2}\right)=\frac{1}{8}
\end{gathered}
$$

die letzte Angabe folgt aus der Gleichung (6) für $J^{\prime}=J(2 \omega)$, falls man $\omega=\frac{i}{2}$ und also $J^{\prime}=1$ einträgt.

Eine einwertige Funktion des Polygons $T_{4}$ heiße jetzt $\tau(\omega)$. Wie schon in I, 442 erörtert wurde, ist eine solche einwertige Funktion dadurch eindeutig erklärbar, daß man an drei Stellen des Polygons die drei Werte von $\tau(\omega)$ vorschreibt. Wir setzen die Gleichungen fest:

$$
\tau\left( \pm \frac{1}{2}\right)=-\frac{1}{2}, \quad \tau(0)=0, \quad \tau(i \infty)=\infty .
$$

Die zu $n=4$ gehörenden Substitutionen $W$ und $\bar{W}$ mögen genauer $W_{4}$ und $\bar{W}_{4}$ heißen. Der Symmetriekreis der Spiegelung $\bar{W}_{4}$ ist der $\mathrm{T}_{4}$ durchschneidende, die Punkte $\omega= \pm \frac{1}{2}$ verbindende Halbkreis. Durch $W_{4}$ werden die Punkte $\omega=0$ und $i \infty$ und also die Werte $\tau=0$ und $\infty$ ausgetauscht. Anderseits wird durch $W_{4}$ der Punkt $\omega=\frac{i}{2}$ in sich transformiert und ebenso der Eckenzyklus $\omega= \pm \frac{1}{2}$. Da $\tau\left( \pm \frac{1}{2}\right)=-\frac{1}{2}$ ist, so ist die Wirkung von $W_{4}$ auf $\tau(\omega)$ durcb:

gleichung dritter Stufe", nämlich eine solche für die Funktion dritter Stufe $\sqrt[3]{j}$. Diese Gleichung hat bereits viel kleinere ganzzahlige Koeffizienten. Die gleiche Erscheinung kommt um so mehr zur Geltung, je höher die Stufe der Gleichung ist. Die in ihrer fertigen Gestalt am einfachsten gebauten Transformationsgleichungen werden diejenigen sein, die wix im übernächsten Kapitel als "Jacobische" und als „Schlaeflische Modulargleichıngen" betrachten; sie gehören zu den Stufen 16 und 48.

1) Man vgl. den Satz von S. 262 über die Lösung der speziellen Teilungsgleichung für einen Grad, der eine Primzahlpotenz ist. 


$$
\tau\left(\frac{-1}{4 \omega}\right)=\frac{1}{4 \tau(\omega)}
$$

gegeben, woraus man weiter auf $\tau\left(\frac{i}{2}\right)=\frac{1}{2}$ schließt.

Nun ist $\tau_{2}(\omega)$ als zweiwertige Funktion des Polygons $T_{4}$ eine rationale Funktion zweiten Grades von $\tau$. Ihre beiden Nullpunkte fallen bei $\omega=0$ und also bei $\tau=0$ zusammen, die Pole liegen bei $\omega=i \infty$ und $\omega= \pm \frac{1}{2}$ und also bei $\tau=\infty$ und $\tau=-\frac{1}{2}$. Da endlich für $\tau=\frac{1}{9} \mathrm{der}$ Wert $\tau_{2}=\frac{1}{8}$ zutrifft, so besteht für $\tau_{2}$ die Darstellung:

$$
\tau_{2}=\frac{\tau^{2}}{2 \tau+1}
$$

in $\tau$. Trägt man diesen Ausdruck von $\tau_{2}$ in (4) für die daselbst $\tau$ genannte Funktion ein, so folgt:

$$
\begin{gathered}
J:(J-1): 1=\left(\tau^{2}+8 \tau+4\right)^{3}:\left(\tau^{3}-15 \tau^{2}-24 \tau-8\right)^{2} \\
: 27 \tau^{4}(2 \tau+1) .
\end{gathered}
$$

Diese Gleichung, die entsprechende in $J^{\prime}$ und $\tau^{\prime}$, sowie die aus (12) folgende Gleichung $4 \boldsymbol{\tau}^{\prime} \tau=1$ haben wir als Ersatz der Transformationsgleichung für $J(\omega)$ beim vierten Grade anzusehen.

3. Transformation achten Grades von $J(\omega)$. Fig. 12 stellt das Polygon $\mathbf{T}_{8}$ dar, dessen sechs mit Nummern versehene Seiten durch fol-

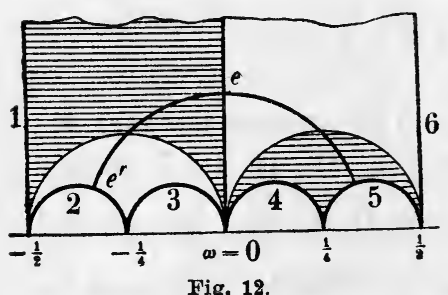

Fig. 12. gende der $\Gamma_{\psi(8)}$ angehörende Substitutionen aufeinander bezogen sind:

$$
\begin{aligned}
& 1 \rightarrow 6,\left(\begin{array}{l}
1,1 \\
0,1
\end{array}\right) ; 2 \rightarrow 5,\left(\begin{array}{l}
3,1 \\
8,3
\end{array}\right) ; \\
& 3 \rightarrow 4,\left(\begin{array}{l}
1,0 \\
8,1
\end{array}\right) .
\end{aligned}
$$

Die stark ausgezogenen Kreise sind Symmetriekreise von Spiegelungen der Gruppe $\bar{\Gamma}^{(8)}$. Der Diskontinuitätsbereich dieser $\bar{\Gamma}^{(8)}$ ist ein Kreisbogenviereck mit zwei Winkeln 0 und zwei rechten Winkeln. Die in der Figur mit $e$ und $e^{\prime}$ bezeichneten Ecken der rechten Winkel liegen bei:

$$
\omega=\frac{-2 \sqrt{2}+i}{6 \sqrt{2}} \text { und } \omega=\frac{i}{2 \sqrt{2}} .
$$

Die beiden in der Figur schraffierten Dreiecke sind Abbilder der positiven $\tau_{4}$-Halbebene. ${ }^{1}$ ) Diese in (14) mit $\tau$ bezeichnete Funktion hat die

1) In sofort verständlicher Weise bezeichnen wir mit $\tau_{4}(\omega)$ die soeben bei der Transformation vierten Grades von $J(\omega)$ benutzte Funktion $\tau(\omega)$. Einer entsprechenden Bezeichnungsweise bedienen wir uns in den nächsten Fällen. 
Spitzenwerte:

$$
\tau_{4}\left( \pm \frac{1}{2}\right)=-\frac{1}{2}, \quad \tau_{4}\left( \pm \frac{1}{4}\right)=\tau_{4}(i \infty)=\infty, \quad \tau_{4}(0)=0 .
$$

Die Abbildung von $\mathrm{T}_{8}$ durch $\tau_{4}(\omega)$ liefert, wie man aus Fig. 12 abliest, eine zweiblättrige Fläche über der $\tau_{4}$-Ebene, die zwei bei $\tau_{4}=0$ und $-\frac{1}{2}$ gelegene Verzweigungspunkte hat. Demnach haben wir in der Quadratwurzel:

$$
t(\omega)=\sqrt{\frac{2 \tau_{4}(\omega)+1}{2 \tau_{4}(\omega)}}
$$

die auf der imaginären $\omega$-Achse positiv genommen werden soll, eine einwertige Funktion von $\mathbf{T}_{8}$ mit den Spitzenwerten:

$$
t(i \infty)=1, \quad t(0)=\infty, \quad t\left( \pm \frac{1}{2}\right)=0, \quad t\left( \pm \frac{1}{4}\right)=-1 .
$$

Durch $W_{8}$ werden die Werte 1 und $\infty$ von $t$ ausgetauscht und ebenso die Werte 0 und -1 , woraus man leicht folgert:

$$
t\left(\frac{-1}{8 \omega}\right)=\frac{t(\omega)+1}{t(\omega)-1} .
$$

An die Stelle von $t(\dot{\omega})$ soll nun weiterhin die gleichfalls einwertige Funktion:

$$
\tau(\omega)=2(t(\omega)-1)
$$

treten, deren Spitzenwerte nach (18) die folgenden sind:

$$
\tau(i \infty)=0, \quad \tau(0)=\infty, \quad \tau\left( \pm \frac{1}{2}\right)=-2, \quad \tau\left( \pm \frac{1}{4}\right)=-4
$$

Die Wirkung von $W_{8}$ ist zufolge (19):

$$
\tau\left(\frac{-1}{8 \omega}\right)=\frac{8}{\tau(\omega)},
$$

woraus sich für die beiden Stellen (15) die Werte berechnen:

$$
\tau\left(\frac{-2 \sqrt{2}+i}{6 \sqrt{2}}\right)=-2 \sqrt{2}, \quad \tau\left(\frac{i}{2 \sqrt{2}}\right)=2 \sqrt{2} .
$$

Für $\tau_{4}$ ergibt sich aus (17) als rationaler Ausdruck in $\tau$ :

$$
\tau_{4}=\frac{2}{\tau^{2}+4 \tau} \text {. }
$$

Durch Eintragung dieses Ausdrucks von $\tau_{4}$ in die rechte Seite von (14) gelangt man zu dem Ergebnis: Der Ersatz der Transformationsgleichung für $J(\omega)$ beim achten Grade ist die Gleichung:

$$
\begin{aligned}
J:(J-1) & : 1=4\left(\tau^{4}+8 \tau^{3}+20 \tau^{2}+16 \tau+1\right)^{3} \\
& :\left(2 \tau^{6}+24 \tau^{5}+108 \tau^{4}+224 \tau^{3}+207 \tau^{2}+60 \tau-2\right)^{2} \\
& : 27 \tau(\tau+4)(\tau+2)^{2},
\end{aligned}
$$

die entsprechende Gleichung zwischen $J^{\prime}$ und $\tau^{\prime}$ und die Relation $\tau^{\prime} \cdot \tau=8$ 
4. Transformation $16^{\text {ten }}$ Grades von $J(\omega)$. In Fig. 13 ist das Polygon $T_{16}$ dargestellt, dessen zwölf Seiten durch folgende Substitutionen aufeinander bezogen sind:

$$
\begin{gathered}
1 \rightarrow 12,\left(\begin{array}{l}
1,1 \\
0,1
\end{array}\right) ; 2 \rightarrow 11,\left(\begin{array}{r}
7,3 \\
16,7
\end{array}\right) ; 3 \rightarrow 4,\left(\begin{array}{r}
-3,-1 \\
16,5
\end{array}\right) ; 5-8,\left(\begin{array}{r}
7,1 \\
48,7
\end{array}\right) ; \\
6 \rightarrow 7,\left(\begin{array}{r}
1,0 \\
16,1
\end{array}\right) ; 9 \rightarrow 10\left(\begin{array}{r}
5,-1 \\
16,-3
\end{array}\right) .
\end{gathered}
$$

Die stark ausgezogenen Kreise sind Symmetriekreise von Spiegelungen

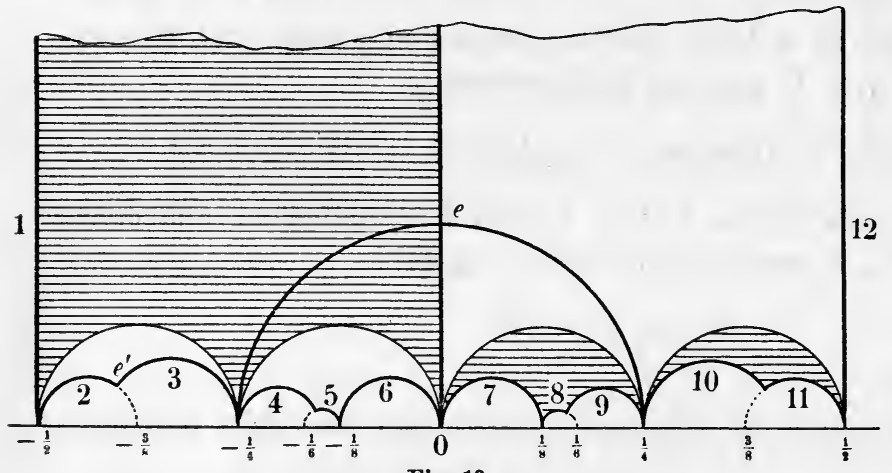

Fig. 13. der Gruppe $\bar{\Gamma}^{(16)}$. Das ganze Polygon $T_{16}$ ist hier aufgebaut aus vier Kreisbogenfünfecken, deren einzelnes drei Winkel 0 und zwei rechte Winkel hat und durchweg aus

Symmetriekreisen von Spiegelungen eingegrenzt ist. So haben z. B. die mit den Nummern 2 und 3 versehenen Kreise die Gleichungen:

$$
16\left(\xi^{2}+\eta^{2}\right)+14 \xi+3=0, \quad 48\left(\xi^{2}+\eta^{2}\right)+32 \xi+5=0 ;
$$

sie sind die Symmetriekreise der in der $\bar{\Gamma}^{(16)}$ enthaltenen Spiegelungen:

$$
\omega^{\prime}=\frac{7 \bar{\omega}+3}{-16 \bar{\omega}-7}, \quad \omega^{\prime}=\frac{16 \bar{\omega}+5}{-48 \bar{\omega}-16} .
$$

Der die beiden Punkte $\pm \frac{1}{4}$ verbindende Halbkreis ist der Symmetriekreis der Spiegelung $\bar{W}_{16}$. Die Punkte $e$ und $e^{\prime}$, gelegen bei

$$
\omega=\frac{i}{4} \quad \text { und } \quad \omega=\frac{-8+i}{20} \text {, }
$$

sind die Nullpunkte der beiden quadratischen Formen $(16,0,1)$ und $(80,64,13)$, durch die wir die beiden Formklassen der Diskriminante $D=-64$ repräsentieren können.

Das schraffierte Kreisbogenviereck der Ecken $0, i \infty,-\frac{1}{2},-\frac{1}{4}$ ist hier ein Abbild der negativen $\tau_{\mathbf{8}}$-Halbebene. Das zweite Abbild dieser Halbebene findet sich zur rechten Hand am unteren Ende des Polygons $\mathbf{T}_{16}$ und wird durch den Rand dieses Polygons in zwei Stücke zerschnitten. Die Spitzenwerte von $\tau_{8}$ sind:

$$
\begin{gathered}
\tau_{8}(0)=\infty, \quad \tau_{8}(i \infty)=\tau_{8}\left( \pm \frac{1}{8}\right)=0, \quad \tau_{8}\left( \pm \frac{1}{2}\right)=-2 \\
\tau_{8}\left( \pm \frac{1}{4}\right)=-4
\end{gathered}
$$


Mittelst der Funktion $\tau_{8}(\omega)$ wird $T_{16}$ auf eine zweiblättrige Fläche mit zwei von $\omega=0$ und dem Eckenzyklus $\pm \frac{1}{2}$ herrührenden, bei $\tau_{8}=\infty$ und $\tau_{8}=-2$ gelegenen Verzweigungspunkten abgebildet. Eine einwertige Funktion von $T_{16}$ hat man demnach in:

$$
t(\omega)=\sqrt{\frac{1}{2} \tau_{8}(\omega)+1},
$$

wo die Wurzel auf der imaginären $\omega$-Achse positiv genommen werden mag. Diese Funktion hat folgende Spitzenwerte:

$$
t(0)=\infty, \quad t(i \infty)=1, \quad t\left( \pm \frac{1}{2}\right)=0, \quad t\left( \pm \frac{1}{8}\right)=-1,
$$

aus denen man leicht als Wirkung der Substitution $W_{16}$ folgert:

$$
t\left(\frac{-1}{16 \omega}\right)=\frac{t(\omega)+1}{t(\omega)-1}
$$

An Stelle von $t(\omega)$ soll jetzt, wie im Falle $n=8$, die gleichfalls einwertige Funktion des Polygons $\mathrm{T}_{16}$ :

$$
\tau(\omega)=2(t(\omega)-1)
$$

eingeführt werden, die die folgenden Spitzenwerte hat:

$$
\tau(0)=\infty, \quad \tau(i \infty)=0, \quad \tau\left( \pm \frac{1}{2}\right)=-2, \quad \tau\left( \pm \frac{1}{8}\right)=-4
$$

und zufolge (27) gegenüber $W_{16}$ das Verhalten zeigt:

$$
\tau\left(\frac{-1}{16 \omega}\right)=\frac{8}{\tau(\omega)} .
$$

Der Zusammenhang zwischen $\tau_{8}$ und $\tau$ ist:

$$
\tau_{8}=\frac{1}{2} \tau^{2}+2 \tau \text {. }
$$

Durch Eintragung dieses Ausdrucks von $\tau_{8}$ in (24) ergibt sich der Satz: Der Ersatz der Transformationsgleichung für $J(\omega)$ beim $16^{\text {ten }}$ Grade ist die Gleichung:

$$
\begin{aligned}
& J:(J-1): 1=\left(\tau^{8}+16 \tau^{7}\right.+112 \tau^{6}+448 \tau^{5}+1104 \tau^{4}+1664 \tau^{3} \\
&\left.+1408 \tau^{2}+512 \tau+16\right)^{3} \\
&:\left(\tau^{12}+24 \tau^{11}\right.+264 \tau^{10}+1760 \tau^{9}+7896 \tau^{8} \\
&+24960 \tau^{7}+56448 \tau^{6}+90624 \tau^{5} \\
&+99960 \tau^{4}+70592 \tau^{3}+27456 \tau^{2} \\
&+3840 \tau-64)^{2} \\
&: 1728 \tau(\tau+4)\left(\tau^{2}+4 \tau+8\right)(\tau+2)^{4}
\end{aligned}
$$

die entsprechende Gleichung zwischen $J^{\prime}$ und $\tau^{\prime}$ und die Beziehung $\tau^{\prime} \tau=8$. 
II, 4. Transformationsgleichungen erster Stufe für niedere Grade $n$

5. Transformation $32^{\text {sten }}$ Grades von $J(\omega)$. Aus $T_{16}$ kann man das Polygon $T_{32}$ dadurch herstellen, daß man die beiden durch die imaginäre $\omega$-Achse ausgeschnittenen Hälften von $T_{16}$ mittels der Substitutionen $\omega^{\prime}=\frac{\omega}{ \pm 16 \omega+1}$ transformiert und die entstehenden Bereiche längs der Seiten 7 und 6 von $T_{16}$ anfügt. Neben den Spitzen von $T_{16}$ treten dann am Polygone $T_{32}$ noch weitere Spitzen bei $\omega= \pm \frac{1}{12}, \pm \frac{1}{14}, \pm \frac{1}{16}$ auf.

Es ist indessen nicht nötig, dieses Polygon $\mathbf{T}_{32}$ wirklich herzustellen, vielmehr können wir uns zur Erledigung der Transformation $32^{\text {sten }}$ Grades von $J(\omega)$ der soeben bei $n=16$ benutzten Funktion $\tau(\omega)$ bedienen. Mittels dieser Funktion wird $\mathbf{T}_{3}$ : auf eine zweiblättrige Fläche abgebildet, die als zum Geschlechte $p=1$ gehörig vier Verzweigungspunkte hat. Diese Verzweigungspunkte rühren von den Spitzen $\omega=0,-\frac{1}{2}$ (oder $+\frac{1}{2}$ ) und $\pm \frac{1}{4}$ des Polygons $\mathbf{T}_{16}$ her, da die zu diesen Punkten gehörenden Substitutionen (vgl. die bei Fig. 13 genannten Substitutionen):

$$
\left(\begin{array}{c}
1,0 \\
16,1
\end{array}\right), \quad\left(\begin{array}{c}
7,4 \\
-16,-9
\end{array}\right), \quad\left(\begin{array}{c}
5,-1 \\
16,-3
\end{array}\right),\left(\begin{array}{c}
-3,-1 \\
16,5
\end{array}\right)
$$

noch nicht der $\Gamma_{\psi(32)}$ angehören, während ihre Quadrate der Bedingung $\gamma \equiv 0(\bmod 32)$ genügen. Die vier Verzweigungspunkte liegen also bei $\tau=\infty,-2$ und $(-2 \pm 2 i)$; die beiden letzten Punkte sind die Nullpunkte des im dritten Gliede der rechten Seite von (30) auftretenden Faktors $\left(\tau^{2}+4 \tau+8\right)$. Es ergibt sich also der Satz: Für das zum Geschlechte $p=1$ gehörende Transformationspolygon $\mathrm{T}_{32}$ hat man ein Funktionssystem in:

$$
\tau(\omega), \quad \sigma(\omega)=\sqrt{\tau^{3}+6 \tau^{2}+16 \tau+16,}
$$

wo $\tau(\omega)$ die bei $n=16$ so benannte Funktion ist und die Quadratwurzel auf der imaginären $\omega$-Achse positiv genommen werden mag. ${ }^{1}$ )

Mit $\tau(\omega)$ ist nun auch $\tau^{\prime}(\omega)=\tau\left(\frac{-1}{32 \omega}\right)$ eine zweiwertige Funktion von $\mathbf{T}_{32}$, so daß $\tau$ und $\boldsymbol{\tau}^{\prime}$ durch eine algebraische Relation der Gestalt:

$$
\tau^{\prime 2}\left(a_{0} \tau^{2}+b_{0} \tau+c_{0}\right)+\tau^{\prime}\left(a_{1} \tau^{2}+b_{1} \tau+c_{1}\right)+\left(a_{2} \tau^{2}+b_{2} \tau+c_{2}\right)=0
$$

aneinander gebunden sind. Die beiden Stellen $\tau^{\prime}=\infty$ fallen in der Spitze $\omega=i \infty$, d. h. bei $\tau=0$ zusammen, so dab $\left(a_{0} \tau^{2}+b_{0} \tau+c_{0}\right)$ mit $\tau^{2}$ identisch ist. Die beiden Stellen $\tau^{\prime}=0$ treten bei $\omega=0$ und $\omega= \pm \frac{1}{2}$ ein, und also bei $\tau=\infty$ und $\tau=-2$, so daB $\left(a_{2} \tau^{2}+b_{2} \tau+c_{2}\right)$ mit $b_{2}(\tau+2)$ identisch ist. Da die Relation zwischen $\tau$ und $\tau^{\prime}$ überdies symmetrisch sein muB, so hat sie die Gestalt:

$$
\tau^{\prime 2} \tau^{2}+\tau^{\prime}(b \tau+c)+c(\tau+2)=0 .
$$

1) Man hat hier ein elliptisches Gebilde des harmonischen Falles vor sich. 
Man trage hier noch ein:

$$
\omega=-\frac{1}{8}, \quad \tau^{\prime}=\tau\left(\frac{1}{4}\right)=-2+2 i, \quad \tau=\tau\left(\frac{-1}{8}\right)=-4
$$

und findet mit Rücksicht auf den Umstand, daß die Koeffizienten $b, c$ reell sein müssen, $b=-32, c=-64$. Die gesuchte Relation ist:

$$
\tau^{\prime 2} \tau^{2}-32 \tau^{\prime} \tau-64\left(\tau^{\prime}+\tau\right)-128=0 .
$$

Als Bestätigung dieses Ergebnisses kann die nach $\tau^{\prime}$ gelöste Gleichung gelten:

$$
\tau^{\prime}=\tau^{-2}\left(16 \tau+32 \pm 8 \sqrt{\tau^{3}+6 \tau^{2}+16 \tau+16}\right),
$$

insofern sich rechts, wie es sein muB, die Quadratwurzel (31) einfindet. Als Ersatz der Transformationsgleichung für $J(\omega)$ beim Grade $n=32$ hat man nun einfach die Gleichung (30), die entsprechende Gleichung zwischen $\boldsymbol{J}^{\prime}$ und $\boldsymbol{\tau}^{\prime}$ und die Beziehung (32) zwischen $\boldsymbol{\tau}^{\prime}$ und $\boldsymbol{\tau}$ anzusehen.

6. Weitere Transformationsgleichungen für den zweiten Grad. Die Transformationsgleichung für die Modulform $G_{1}\left(\omega_{1}, \omega_{2}\right)$ lautet beim ziveiten Grade einfach:

$$
2^{5} G_{1}^{3}-2 g_{2} G_{1}-g_{3}=0,
$$

da $G_{1}=\frac{1}{2} e_{2}=\frac{1}{2} \wp\left(\frac{\omega_{2}}{2}\right)$ ist. Die durch $W_{2}$ transformierten Formen:

$$
g_{2}^{\prime}=g_{2}\left(\frac{i \omega_{2}}{\sqrt{2}}, \frac{\omega_{1} \sqrt{2}}{i}\right), \quad g_{3}^{\prime}=g_{3}\left(\frac{i \omega_{2}}{\sqrt{2}}, \frac{\omega_{1} \sqrt{2}}{i}\right), \quad \Delta^{\prime}=\Delta\left(\frac{i \omega_{2}}{\sqrt{2}}, \frac{\omega_{1} \sqrt{2}}{i}\right)
$$

gestatten folgende Behandlung. Nach einem Satze von S. 369 hat $g_{2}^{\prime}$ in $\mathbf{T}_{2}$ einen Nullpunkt, der zufolge (6) S. 372 bei $\tau=-\frac{1}{4}$ liegt; derjenige von $g_{2}$ liegt bei $\left.\tau=-4 .{ }^{1}\right)$ Der Quotient $g_{2}^{\prime}: g_{2}$ ist demnach als einwertige Funktion in $T_{2}$ linear durch $\tau$ darstellbar, und zwar in der Gestalt:

$$
\frac{g_{9}^{\prime}}{g_{2}}=c \frac{4 \tau+1}{\tau+4}
$$

wo $c$ eine Konstante ist. Da der links stehende Quotient gegenüber $W_{2}$ in seinen reziproken Wert übergeht und dasselbe zufolge (3) von $\tau(\omega)$ gilt, so kann $c$ nur gleich +1 oder -1 sein. Da auf der imaginären $\omega$-Achse der links stehende Quotient und auch $\tau$ reell und positiv sind, so ist $c=1$. In ähnlicher Weise findet man auch die zweite und dritte der folgenden Gleichungen:

$$
\frac{g_{2}^{\prime}}{g_{2}}=\frac{4 \tau+1}{\tau+4}, \quad \frac{g_{3}^{\prime}}{g_{3}}=-\frac{8 \tau-1}{\tau-8}, \quad \frac{\Delta^{\prime}}{\Delta}=\frac{1}{\tau} .
$$

1) Vgl. auch Fig. 7, S. 360, die das mit den beiden Netzen versehene Polygon $T_{2}$ darstellt. Hier sind die von sechs Dreiecken umlagerten Punkte $\omega=\frac{-1+i \sqrt{3}}{2}$ bzw. $\omega=\frac{-1+i \sqrt{3}}{4}$ die Nullpunkte von $g_{2}$ und $g_{2}^{\prime}$. 
Durch Elimination von $\tau$ aus (4) und der ersten oder zweiten dieser Gleichungen gewinnt man als Transformationsgleichungen für $g_{2}$ und $g_{3}$ beim zweiten Grade:

$$
\left\{\begin{array}{l}
2^{4} g_{2}^{\prime 3}-2^{3} \cdot 3^{2} g_{2} g_{2}^{\prime 2}+3 \cdot 11 g_{2}^{2} g_{2}^{\prime}+\left(11^{2} g_{2}^{3}-3^{3} \cdot 5^{3} g_{3}^{2}\right)=0 \\
2^{6} g_{3}^{\prime 3}+2^{4} \cdot 3 \cdot 11 g_{3} g_{3}^{\prime 2}+\left(2^{4} \cdot 5 g_{2}^{3}+3^{3} \cdot 7^{2} g_{3}^{2}\right) g_{3}^{\prime}-\left(7^{2} g_{2}^{3} g_{3}-11^{3} g_{3}^{3}\right)=0 .
\end{array}\right.
$$

Aus der dritten Gleichung (33) und der Gleichung (4) folgt:

$$
\frac{3 g_{2}}{\sqrt[3]{\Delta}}=\frac{\tau+4}{(\sqrt[3]{\tau})^{2}}=\frac{4 \Delta^{\prime}+\Delta}{\sqrt[3]{\Delta^{\prime}}(\sqrt[3]{\Delta})^{2}}, \quad 4 \Delta^{\prime}-3 g_{2} \sqrt[3]{\Delta^{\prime} \Delta}+\Delta=0
$$

Durch Zusatz des Faktors $\Delta$ ergibt sich für die Modulform zweiter Stufe $f\left(\omega_{1}, \omega_{2}\right)=\sqrt[3]{\Lambda^{\prime} \Delta}$ beim zweiten Grade die Transformationsgleichung:

$$
4 f^{3}-3 g_{2} \Delta f+\Delta^{2}=0 \text {. }
$$

7. Weitere Transformationsgleichungen für den vierten Grad. Auch beim vierten Transformationsgrade sind noch einige weitere Gleichungen bekannt. Gehen wir zunächst auf die Darstellungen (1) S. 304 von $G_{1}\left(\omega_{1}, \omega_{2}\right)$ für irgendein $n$ zurück und üben die in (5) S. 370 gegebene homogene Substitution $W_{n}$ aus, so finden wir mit Rücksicht auf den Umstand, daB die Perioden des Integrals zweiter Gattung mit den $\omega_{1}, \omega_{2}$ kogredient sind und übrigens in den $\omega_{1}, \omega_{2}$ die Dimension -1 haben:

$$
G_{1}\left(\frac{i \omega_{2}}{\sqrt{n}}, \frac{\omega_{1} \sqrt{n}}{i}\right)=G_{1}\left(\omega_{1}, \omega_{2}\right)
$$

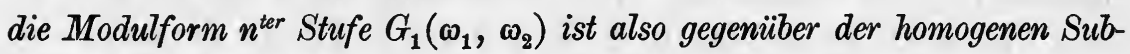
stitution $W_{n}$ invariant.

Wir stellen nun die Modulform vierter Stufe $G_{1}\left(\omega_{1}, \omega_{2}\right)$ mit der zur zweiten und also auch zur vierten Stufe gehörenden Form $e_{2}\left(\omega_{1}, \omega_{2}\right) \mathrm{zu}$ sammen und notieren die Anfangsglieder:

$$
G_{1}=\left(\frac{2 \pi}{\omega_{2}}\right)^{2}\left(\frac{1}{2}+4 q^{2}+\cdots\right), \quad e_{2}=\left(\frac{2 \pi}{\omega_{2}}\right)^{2}\left(\frac{1}{6}+4 q^{2}+\cdots\right) .
$$

Nach dem Satze von S. 369 hat jede dieser Formen im Polygone $\mathrm{T}_{4}$ einen Nullpunkt erster Ordnung. Derjenige von $G_{1}$ wird wegen (36) durch $W_{4}$ in sich transformiert, liegt also entweder bei $\omega=\frac{i}{2}$ oder $\omega= \pm \frac{1}{2}$. Die erste Möglichkeit ist indessen ausgeschlossen, da $G_{1}$ wegen der positiven Reihenkoeffizienten auf der imaginären $\omega$-Achse nicht verschwindet. Also liegt der Nullpunkt von $G_{1}$ bei $\omega= \pm \frac{1}{2}$ und damit bei $\tau_{4}=\tau=-\frac{1}{2}$. Die Form $e_{2}$ hat in $T_{2}$ einen Nullpunkt der Ordnung $\frac{1}{2}$, der nur bei $\omega=\frac{ \pm 1+i}{2}$ und damit bei $\tau_{4}=\tau=-1$ liegen kann. Hieraus entsteht der Ansatz:

$$
\frac{G_{1}}{e_{2}}=c \frac{2 \tau+1}{\tau+1} \text {. }
$$


Die Konstante $c$ ergibt sich für $\omega=i \infty$ aus (16) und (37) zu $\frac{3}{2}$ :

$$
\frac{G_{1}}{e_{2}}=\frac{6 \tau+3}{2 \tau+2}, \quad \tau=\frac{2 G_{1}-3 e_{2}}{-2 G_{1}+6 e_{2}} .
$$

Wir bilden jetzt, unter $a$ und $b$ zwei endliche nicht zugleich verschwindende Koeffizienten verstanden, die ,lineare Formenschar" $\left(a G_{1}+b e_{2}\right)$ der Dimension - 2. Die einzelne Form dieser Schar hat in $\mathbf{T}_{4}$ dann wieder nur einen Nullpunkt erster Ordnung, der durch geeignete Wahl von $a, b$ an eine beliebig vorgeschriebene Stelle von $T_{4}$ gebracht werden kann. Haben wir dann irgendeine ganze Form $g_{\delta}\left(\omega_{1}, \omega_{2}\right)$ des Polygons $\mathbf{T}_{4}$ von der Dimension $-2 \delta$, die nach dem Satze von S. $369 \delta$ einfache Nullpunkte in $\mathbf{T}_{4}$ hat, so entsteht die Möglichkeit, diese Form in der Gestalt:

$$
g_{\delta}=\left(a_{1} G_{1}+b_{1} e_{2}\right)\left(a_{2} G_{1}+b_{2} e_{2}\right) \cdots\left(a_{\delta} G_{1}+b_{\delta} e_{2}\right)
$$

als Produkt von $\delta$ Formen der Schar darzustellen. Man kann nämlich ein solches Produkt bilden, das dieselben Nullstellen wie $g_{\delta}$ hat; der Quotient von $g_{\delta}$ und diesem Produkte ist dann als eine von Polen und Nullpunkten freie Funktion der $\Gamma_{\psi(4)}$ mit einer Konstanten identisch. Insbesondere findet man für $g_{2}$ und $g_{3}$ die Darstellungen:

$$
g_{2}=-4 G_{1}^{2}+12 G_{1} e_{2}+3 e_{2}^{2}, \quad g_{3}=4 G_{1}^{2} e_{2}-12 G_{1} e_{2}^{2}+e_{2}^{3} .
$$

Die beiden Nullpunkte von $g_{2}$ sind nämlich die Wurzeln der quadratischen Gleichung $\tau^{2}+8 \tau+4=0$, so daß man mit Benutzung von (38) den Ansatz hat:

$$
g_{2}=C\left(4 G_{1}^{2}-12 G_{1} e_{2}-3 e_{2}^{2}\right) \text {. }
$$

Die Konstante $C$ bestimmt man aus den Anfangsgliedern der Reihenentwicklungen. Entsprechend findet man die zweite Gleichung (40).

In der mit 4 multiplizierten zweiten Gleichung (40) setze man noch $\left(g_{2} e_{2}+g_{3}\right)$ für $4 e_{2}^{3}$ ein und ordne beide Gleichungen uach Potenzen von $e_{2}$ :

$$
\left\{\begin{array}{l}
3 e_{2}^{2}+12 e_{2} G_{1}-\left(4 G_{1}^{2}+g_{2}\right)=0, \\
48 e_{2}^{2} G_{1}-\left(16 G_{1}^{2}+g_{2}\right) e_{2}+3 g_{3}=0 .
\end{array}\right.
$$

Durch Elimination von $e_{2}$ aus diesen beiden Gleichungen erhält man als Gleichung für $G_{1}$ beim vierten Transformationsgrade:

$$
\begin{aligned}
2^{10} G_{1}^{6}-2^{7} \cdot 3 \cdot 5 g_{2} G_{1}^{4}-2^{6} \cdot 3^{3} \cdot 5 g_{3} G_{1}^{3}-2^{2} \cdot 3^{3} \cdot 5 g_{2}^{2} G_{1}^{2} \\
-2^{2} \cdot 3^{4} g_{2} g_{3} G_{1}+\Delta=0 .
\end{aligned}
$$

Der Nullpunkt von $e_{2}$ liegt bei $\tau=-1$. Die durch $W_{4}$ transformierte Form $e_{2}^{\prime}$ verschwindet also bei $\tau=-\frac{1}{4}$ und damit wegen (38) für $G_{1}=e_{2}$. Die Wirkung von $W_{4}$ auf $e_{2}$ ist somit:

$$
e_{2}^{\prime}=G_{1}-e_{2}
$$


382 II, 4. Transformationsgleichungen erster Stufe für niedere Grade $n$ da die zweimalige Ausübung von $W_{4}$ zu $e_{2}$ zurückführen muB. Aus (40) berechnen sich demnach weiter die Darstellungen:

$$
g_{2}^{\prime}=11 G_{1}^{2}-18 G_{1} e_{2}+3 e_{2}^{2}, \quad g_{3}^{\prime}=-7 G_{1}^{3}+17 G_{1}^{2} e_{2}-9 G_{1} e_{2}^{2}-e_{2}^{3}
$$

für die transformierten $g_{2}^{\prime}, g_{3}^{\prime}$, sowie damit die Gleichungen:

$$
g_{2}^{\prime}-g_{2}=15 G_{1}\left(G_{1}-2 e_{2}\right), \quad g_{3}^{\prime}+g_{3}=-7\left(G_{1}^{3}-3 G_{1}^{2} e_{2}+3 G_{1} e_{2}^{2}\right)
$$

Aus jeder dieser Gleichungen und den beiden Gleichungen (41) wolle man nun $e_{2}$ eliminieren. Es entstehen zwei Ergebnisse, die nach $g_{8}^{\prime}$ und $g_{3}^{\prime}$ aufgelöst folgende Darstellungen der transformierten $g_{2}^{\prime}, g_{3}^{\prime}$ in $G_{1}, g_{2}$, $g_{3}$ liefern:

$$
\left\{\begin{array}{l}
g_{2}^{\prime}=\frac{2^{4} \cdot 3 \cdot 5^{2} G_{1}^{4}-257 G_{1}^{2} g_{2}-2 \cdot 3^{2} \cdot 5 G_{1} g_{3}+g_{2}^{2}}{2^{4} \cdot 13 G_{1}^{2}+g_{2}}, \\
g_{3}^{\prime}=-\frac{2^{4} \cdot 5 \cdot 7 G_{1}^{5}-3^{3} \cdot 7 G_{1}^{3} g_{2}-107 G_{1}^{2} g_{3}+7 G_{1} g_{2}^{2}+g_{2} g_{3}}{2^{4} \cdot 13 G_{1}^{2}+g_{2}} .
\end{array}\right.
$$

Auf dieser Grundlage kann man die Transformationsgleichungen für $g_{2}$ und $g_{3}$ als Resolventen der Gleichung (42) einführen.

Aus (44) und (40) folgt durch Rückgang zur Funktion $\tau$ :

$$
\frac{g_{2}^{\prime}}{g_{2}}=\frac{64 \tau^{2}+32 \tau+1}{4\left(\tau^{2}+8 \tau+4\right)}
$$

Andrerseits ergibt sich aus (14) und Ausübung von $W_{\mathbf{1}}$ :

$$
\frac{27 g_{2}^{3}}{\Delta}=\frac{\left(\tau^{2}+8 \tau+4\right)^{3}}{\tau^{4}(2 \tau+1)}, \quad \frac{27 g_{2}^{\prime 3}}{\Delta^{\prime}}=\frac{\left(64 \tau^{2}+32 \tau+1\right)^{3}}{8 \tau(2 \tau+1)} .
$$

Dividiert man die zweite dieser Gleichungen durch die erste, so folgt bei Benutzung von (47):

$$
\frac{\Delta}{\Delta^{\prime}}=8 \tau^{3}, \quad \sqrt[3]{\frac{\Delta}{\Delta^{\prime}}}=2 \tau .
$$

Erklären wir daraufhin $f\left(\omega_{1}, \omega_{2}\right)$ als ganze Modulform vierter Stufe durch:

$$
f\left(\omega_{1}, \omega_{2}\right)=4 \sqrt[3]{\Delta^{\prime} \Delta^{2}}=\frac{2 \Delta}{\tau}
$$

so ergibt sich durch Elimination von $\tau$ zwischen dieser Gleichung und der aus (14) folgenden Relation:

$$
\Delta\left(\tau^{2}+8 \tau+4\right)^{3}-27 g_{2}^{3} \tau^{4}(2 \tau+1)=0
$$

für $f\left(\omega_{1}, \omega_{2}\right)$ die zum vierten Grade gehörende Transformationsgleichung der Diskriminante $\Delta$ :

$$
\begin{aligned}
2^{2} f^{6} & +2^{4} \cdot 3 \Delta f^{5}+2^{2} \cdot 3 \cdot 17 \Delta^{2} f^{4}+2^{5} \cdot 11 \Delta^{3} f^{3}+\left(3 \cdot 59 g_{2}^{3}\right. \\
& \left.-2^{2} \cdot 3^{4} \cdot 17 g_{3}^{2}\right) \Delta^{3} f^{2}-\left(2^{2} \cdot 3 \cdot 5 g_{2}^{3}+2^{4} \cdot 3^{4} g_{3}^{2}\right) \Delta^{4} f+2^{2} \Delta^{6}=0 .
\end{aligned}
$$




\section{§ 2. Die Transformationsgrade 3, 9 und $2 \%$.}

1. Transformation dritten Grades. Das Transformationspolygon $\mathbf{T}_{3}$ ist in Fig. 8, S. 361, dargestellt; das Klassenpolygon $K_{3}$ läßt sich aus zwei symmetrischen Kreisbogendreiecken der Winkel $\frac{\pi}{2}, \frac{\pi}{6}, 0$ und der Ecken $\omega=\frac{i}{\sqrt{3}}, \frac{ \pm 3+i \sqrt{3}}{6}, i \infty$ zusammensetzen.

Die Form $G_{1}$ ist hier einfach $\wp\left(\frac{\omega_{2}}{3}\right)$, so daß die beim dritten.Grade eintretende Transformationsgleichung für $G_{1}$ die zu diesem Teilungsgrade gehörende spezielle Teilungsgleichung:

$$
48 G_{1}^{4}-24 g_{2} G_{1}^{2}-48 g_{3} G_{1}-g_{2}^{2}=0
$$

der §-Funktion ist (vgl. den Ausdruck von $\psi^{(3)}(u)$ S. 185).

Eine einwertige Funktion $\tau(\omega)$ für $\mathrm{T}_{3}$ führen wir durch die Festsetzung:

$$
\tau(i \infty)=0, \quad \tau\left(\frac{i}{\sqrt{3}}\right)=1, \quad \tau(0)=\infty
$$

ein. Da $W_{3}$ die Punkte $\omega=0$ und $i \infty$ austauscht und $\omega=\frac{i}{\sqrt{3}}$ zum Fixpunkte hat, so gilt:

$$
\tau\left(\frac{-1}{3 \omega}\right)=\frac{1}{\tau(\omega)} .
$$

Die Funktion $\tau(\omega)$ ist auf der imaginären $\omega$-Achse reell und positiv, auf dem äußeren Rande von $T_{3}$ reell und negativ, und speziell gilt:

$$
\tau\left(\frac{ \pm 3+i \sqrt{3}}{6}\right)=-1 \text {. }
$$

Die Formen $g_{2}, g_{2}^{\prime}$ der Dimension -4 haben in $\mathrm{T}_{3}$ Nullpunkte je in der Gesamtordnung $\frac{4}{3}$. Ein gemeinsamer Nullpunkt der Ordnung $\frac{1}{3}$ liegt im Eckenzyklus $\frac{ \pm 3+i \sqrt{3}}{6}$; die Lagen der beiden anderen Nullpunkte sind aus den beiden Netzen der Fig. 8 sofort abzulesen, sie treten für zwei reelle negative, einander reziproke Werte $\tau$ ein. Hieraus folgt der Ansatz:

$$
\frac{g_{2}^{\prime}}{g_{2}}= \pm \frac{\tau+a}{a \tau+1}
$$

wo $a$ reell und positiv ist. Das rechts nicht noch ein von \pm 1 verschiedener Faktor auftreten kann, folgt aus dem Umstande, da $B$ sowobl der links stehende Quotient wie auch $\tau$ durch $W_{3}$ in ihre reziproken Werte transformiert werden. Für $\omega=i \infty$ wird $\tau=0$ und die linke Seite gleich 9 ; also ist $a=9$, und es gilt das obere Zeichen:

$$
\frac{g_{2}^{\prime}}{g_{2}}=\frac{\tau+9}{9 \tau+1} \text {. }
$$


Die Form $G_{1}$ der Dimension -2 hat in $\mathbf{T}_{3}$ einen Nullpunkt der Ordnung $\frac{8}{3}$, der nur im Eckenzyklus $\frac{ \pm 3+i \sqrt{3}}{6}$ und also bei $\tau=-1$ liegen kann. Demnach gilt der Ansatz:

$$
\frac{g_{2}}{G_{1}^{2}}=c \frac{9 \tau+1}{\tau+1} .
$$

Die Konstante $c$ bestimmt man für $\tau=0$ mit Hilfe der Anfangsglieder der Reihen für $G_{1}$ und $g_{2}$. Es gilt:

$$
\frac{g_{2}}{G_{1}^{2}}=\frac{4}{3} \frac{9 \tau+1}{\tau+1}, \quad \frac{g_{2}^{\prime}}{G_{1}^{2}}=\frac{4}{3} \frac{\tau+9}{\tau+1},
$$

wo die zweite Formel aus der ersten durch $W_{3}$ oder auch mit Hilfe von (5) hergeleitet wird. Eliminiert man $g_{2}$ aus (6) und (1), so folgt:

$$
\frac{g_{3}}{G_{1}^{3}}=-\frac{8}{27} \frac{27 \tau^{2}+18 \tau-1}{(\tau+1)^{2}}, \quad \frac{g_{3}^{\prime}}{G_{1}^{3}}=+\frac{8}{27} \frac{\tau^{2}-18 \tau-27}{(\tau+1)^{2}},
$$

wo die zweite Gleichung aus der ersten wieder durch $W_{3}$ hervorgeht.

Da $\Delta$ eine Form erster Stufe mit einer durch 4 teilbaren Dimension ist, so können wir an Stelle der durch die homogene Substitution $W_{3}$ umgeformten Diskriminante $\Delta$ auch:

$$
\Delta^{\prime}=\Delta\left(\omega_{1} \sqrt{3}, \frac{\omega_{2}}{\sqrt{3}}\right)
$$

treten lassen, was mit Rücksicht auf die vorzunehmenden Radizierungen vorzuziehen ist. Es gilt nun einfach:

$$
\frac{\Delta^{\prime}}{\Delta}=\tau^{2}, \quad \sqrt{\frac{\Delta^{\prime}}{\Delta}}=\tau,
$$

wo die links stehende Wurzel auf der imaginären $\omega$-Achse positiv zu nehmen ist. Der Quotient der Diskriminanten ist nämlich eine Funktion des Polygons $\mathbf{T}_{3}$, die nur in den beiden Spitzen $i \infty$ und 0 verschwinden oder unendlich werden kann. Da sie (wegen des Anfangsgliedes der Reihe) bei $i \infty$ einen Nullpunkt zweiter Ordnung hat, so liegt bei $\omega=0$ ein Pol der gleichen Ordnung. In dem daraus sich ergebenden Ansatze $\Delta^{\prime}: \Delta=c \tau^{2}$ muB aber $c= \pm 1$ sein, da der Quotient $\Delta^{\prime}: \Delta$ und $\tau$ bei $W_{3}$ in ihre reziproken Werte übergehen. Endlich ist $c=1$, da sowohl $\Delta^{\prime}: \Delta$ als $\tau$ auf der imaginären $\omega$-Achse reell und positiv sind.

Die weiteren zum dritten Transformationsgrade gehörenden Gleichungen folgen nun einfach durch Eliminationen. Wir entnehmen zunächst aus (6):

$$
\frac{3 g_{2}-20 G_{1}^{2}}{16 G_{7}^{2}}=\frac{\tau-1}{\tau+1}
$$

und finden sodann durch Addition der Gleichungen (6) und weiter durch Subtraktion der Gleichungen (7) bei Benutzung von (10): 


$$
\left\{\begin{array}{l}
3 g_{2}^{\prime}=40 G_{1}^{2}-3 g_{2}, \\
27 g_{3}^{\prime}=-280 G_{1}^{3}+42 g_{2} G_{1}+27 g_{3} .
\end{array}\right.
$$

Mittelst dieser Gleichungen sind die Transformationsgleichungen für $g_{2}$ und $g_{3}$ beim dritten Grade als Tschirnhausenresolventen der Gleichung (1) erklärbar.

Weiter folgt aus (6), und (7):

$$
\frac{g_{2}^{3}-27 g_{3}^{2}}{G_{1}^{6}}=\frac{\Delta}{G_{1}^{6}}=\frac{4096 \tau}{27(\tau+1)^{2}}<?_{4}
$$

Aus dieser Gleichung und den eben genannten Gleichungen (6) und (7) ergibt sich bei Einführung von $J(\omega)$ :

$$
J:(J-1): 1=(\tau+1)(9 \tau+1)^{3}:\left(27 \tau^{2}+18 \tau-1\right)^{2}: 64 \tau .
$$

Diese Gleichung, die entsprechende für $J^{\prime}$ und $\tau^{\prime}$ und die Beziehung $\tau^{\prime} \cdot \tau=1$ bilden den Ersatz der Transformationsgleichung für $J$ beim dritten Grade. $\left.{ }^{1}\right)$

Nach S. 340 existiert beim dritten Grade eine Transformationsgleichung für $\sqrt[4]{\Delta^{\prime} \Delta}$. Man schreibt zweckmäBig:

$$
f\left(\omega_{1}, \omega_{2}\right)=\sqrt{3} \sqrt[4]{\Delta^{\prime} \Delta}=\sqrt{3 \Delta \tau}
$$

und findet aus dem zweiten und dritten Gliede von (12) beim Ausziehen der Quadratwurzel unter richtiger Bestimmung des Vorzeichens:

$$
-\frac{3 \sqrt{3} g_{3}}{\Delta}=\frac{27 \tau^{2}+18 \tau-1}{8 \sqrt{\tau}}
$$

Indem man den aus (13) folgenden Wert von $\tau$ hier einträgt, ergibt sich

$$
3 f^{4}+6 \Delta f^{2}-24 g_{3} \Delta f-\Delta^{2}=0
$$

als die zum dritten Grade gehörende Transformationsgleichung der Diskriminante.

2. Transformation neunten Grades. Das Transformationspolygon $T_{9}$ ist in Fig. 14 (S. 386) dargestellt; seine acht Seiten sind durch folgende vier Substitutionen einander zugewiesen:

$1 \rightarrow 8,\left(\begin{array}{l}1,1 \\ 0,1\end{array}\right) ; 2 \rightarrow 3,\left(\begin{array}{c}2,1 \\ -9,-4\end{array}\right) ; 4 \rightarrow 5,\left(\begin{array}{l}1,0 \\ 9,1\end{array}\right) ; 6 \rightarrow 7,\left(\begin{array}{l}4,-1 \\ 9,-2\end{array}\right)$, die sämtlich der Gruppe $\Gamma_{\psi(9)}$ angehören. Das Klassenpolygon $K_{9}$ besteht

1) Die fertige Transformationsgleichung ist von St. Smith in den „Proceedings" der Londoner mathematischen Gesellschaft von 1878 (S. 242) und 1879 (S. 87) mitgeteilt; sie lautet auf $j$ und $j$ umgerechnet:

$$
\begin{gathered}
j^{\prime}\left(j^{\prime}+2^{31} \cdot 3 \cdot 5^{3}\right)^{3}+j\left(j+2^{31} \cdot 3 \cdot 5^{3}\right)^{3}-j^{\prime} j+2^{3} \cdot 3^{2} \cdot 31 j^{\prime 2} j^{2}\left(j^{\prime}+j\right) \\
-2^{2} \cdot 3^{3} \cdot 9907 j^{\prime} j\left(j^{\prime 2}+j^{2}\right)+2 \cdot 3^{4} \cdot 13 \cdot 193 \cdot 6367 j^{2} j^{2} \\
+2^{16} \cdot 3^{5} \cdot 5^{3} \cdot 17 \cdot 263 j^{\prime} j\left(j^{\prime}+j\right)-2^{31} \cdot 5^{6} \cdot 22973 j^{\prime} j=0 .
\end{gathered}
$$


aus zwei symmetrischen Kreisbogenvierecken mit zwei Winkeln 0 und zwei rechten Winkeln. Die Seiten des einzelnen Vierecks sind durchweg

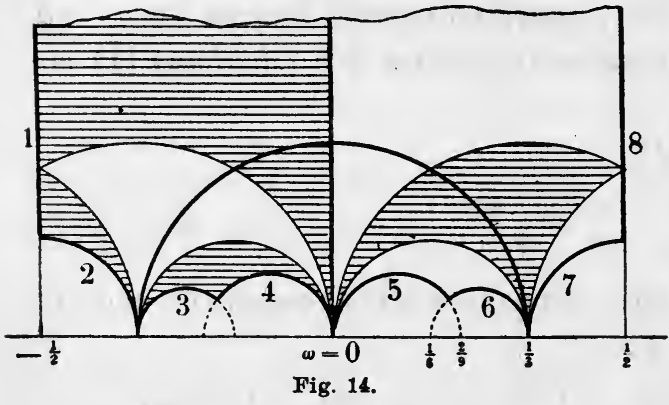
Symmetriekreise von Spiegelungen der Gruppe $\bar{\Gamma}^{(9)}$. Das zur Linken der imaginären $\omega$-Achse gelegene Viereck hat die beiden Spitzen $\omega=i \infty$ und $-\frac{1}{3}$. Die Ecken der beiden rechten Winkel dieses Vierecks liegen bei $\omega=\frac{i}{3}$ und $\omega=\frac{-3+i}{6}$, die die Null-

punkte der die beiden Formklassen der Diskriminante $D=-36$ repräsentierenden Formen $(9,0,1)$ und $(18,18,5)$ sind. Die Seite 2 ist der Symmetriekreis der in der $\bar{\Gamma}^{(9)}$ enthaltenen Spiegelung:

$$
\omega^{\prime}=\frac{-9 \bar{\omega}-4}{18 \bar{\omega}+9} .
$$

Die Abbilder der drei negativen $\tau_{\mathbf{3}}$-Halbebenen sind in Fig. 14 schraffiert. Diese Abbilder sind von Symmetriekreisen der $\bar{\Gamma}^{(9)}$ durchzogen; eines von ihnen (in Fig. 14, links unten) ist in zwei getrennte Teile zerlegt. Durch $\tau_{3}(\omega)$ wird das Polygon $\mathbf{T}_{9}$ auf eine dreiblättrige Riemannsche Fläche über der $\tau_{3}$-Ebene abgebildet, die (den Stellen $\omega=0$ und $\omega=\frac{ \pm 3+i \sqrt{3}}{6}$ entsprechend) zwei dreiblättrige Verzweigungspunkte bei $\tau_{3}=\infty$ und -1 hat. Man kann demnach $\sqrt[3]{\tau_{3}+1}$ oder noch zweckmäBiger:

$$
\tau(\omega)=-1+\sqrt[3]{\tau_{3}+1}
$$

mit der Bestimmung, daß die Kubikwurzel auf der imaginären $\omega$-Achse reell genommen werden soll, als einwertige Funktion des Polygons $\mathbf{T}_{9}$ benutzen. Die Spitzenwerte dieser Funktion sind:

$$
\tau(i \infty)=0, \quad \tau(0)=\infty, \quad \tau\left( \pm \frac{1}{3}\right)=\frac{-3 \pm i \sqrt{3}}{2} .
$$

Die Wirkung der Substitution $W_{9}$ auf $\tau(\omega)$ ist:

$$
\tau\left(\frac{-1}{9 \omega}\right)=\frac{3}{\tau(\omega)},
$$

da durch $W_{9}$ die beiden Werte $\tau=0$ und $\tau=\infty$ sowie andrerseits die beiden Spitzenwerte $\tau\left( \pm \frac{1}{3}\right)$ ausgetauscht werden.

Trägt man nun den aus (15) folgenden Ausdruck:

$$
\tau_{3}(\omega)=\tau\left(\tau^{2}+3 \tau+3\right)
$$

von $\tau_{3}$ im jetzigen $\tau$ in die Relation (12) zwischen $J$ und $\tau_{3}$ ein, so folgt: 


$$
\begin{aligned}
J:(J-1) & : 1=\left(9 \tau^{4}+36 \tau^{3}+54 \tau^{2}+28 \tau+1\right)^{3} \\
& :\left(27 \tau^{6}+162 \tau^{5}+405 \tau^{4}+504 \tau^{3}+297 \tau^{2}+54 \tau-1\right)^{2} \\
& : 64 \tau\left(\tau^{2}+3 \tau+3\right) .
\end{aligned}
$$

Diese Relation, die entsprechende zwischen $J^{\prime}$ und $\boldsymbol{t}^{\prime}$ und die Gleichung $\tau^{\prime} \tau=3$ bilden den Ersatz der Transformationsgleichung für $J$ beim neunten Grade.

Nach S. 340 muß beim neunten Grade für die Form $\sqrt[8]{\Delta^{\prime} \Delta^{7}}$ eine Transformationsgleichung zwölften Grades bestehen. Um sie zu gewinnen, üben wir $W_{9}$ auf die Gleichung (18) aus und finden bei Benutzung von (3) und (17):

$$
\tau_{3}\left(\frac{-1}{9 \omega}\right)=\frac{1}{\tau_{3}(3 \omega)}=\frac{3}{\tau}\left(\frac{9}{\tau^{2}}+\frac{9}{\tau}+3\right),
$$

wo bei den Funktionen $\tau$, die ohne Argument geschrieben sind, $\omega$ als solches zu denken ist. Für die zur Gruppe $\Gamma_{\psi(9)}$ gehörende Funktion $\tau_{3}(3 \omega)$ berechnet sich hieraus:

Nun folgt aus (9) und (18):

$$
9 \tau_{3}(3 \omega)=\frac{\tau^{3}}{\tau^{2}+3 \tau+3}
$$

$$
\sqrt{\frac{\Delta\left(\omega \sqrt{3}, \frac{\omega_{2}}{\sqrt{3}}\right)}{\Delta\left(\omega_{1}, \omega_{2}\right)}}=\tau_{3}(\omega)=\tau(\omega)\left(\tau(\omega)^{2}+3 \tau(\omega)+3\right) .
$$

Setzt man $\omega_{1} \sqrt{3}, \frac{\omega_{2}}{\sqrt{3}}$ an Stelle von $\omega_{1}, \omega_{2}$ und also $3 \omega$ an Stelle von $\omega$ ein, so folgt bei Benutzung von (20):

$$
\sqrt{\frac{\Delta\left(3 \omega_{1}, \frac{\omega_{q}}{3}\right)}{\Delta\left(\omega_{1} \sqrt{3}, \frac{\omega_{q}}{\sqrt{3}}\right)}}=\tau_{3}(3 \omega)=\frac{\tau(\omega)^{3}}{9\left(\tau(\omega)^{2}+3 \tau(\omega)+3\right)} .
$$

Durch Multiplikation der beiden letzten Gleichungen und Ausziehen der vierten Wurzel ergibt sich:

$$
\sqrt{3} \sqrt[8]{\frac{\Delta\left(3 \omega_{1}, \frac{\omega_{2}}{3}\right)}{\Delta\left(\omega_{1}, \omega_{2}\right)}}=\sqrt{3} \sqrt[8]{\frac{\Delta^{\prime}}{\Delta}}=\tau(\omega) .
$$

Erklären wir demnach eine zur $\Gamma_{\psi(9)}$ gehörende Modulform $f\left(\omega_{1}, \omega_{2}\right)$ durch die Gleichung:

$$
f\left(\omega_{1}, \omega_{2}\right)=\sqrt{3} \sqrt[8]{\Delta\left(3 \omega_{1}, \frac{\omega_{2}}{3}\right) \Delta\left(\omega_{1}, \omega_{2}\right)^{7}}=\sqrt{3} \sqrt[8]{\Delta^{\prime} \Delta^{7}}=\Delta \tau,
$$

so ergibt sich für diese Form aus (19) die gesuchte Transformationsgleichung in der Gestalt:

$$
\begin{gathered}
\left(9 f^{4}+36 \Delta f^{3}+54 \Delta^{2} f^{2}+28 \Delta^{3} f+\Delta^{4}\right)^{3} \\
-64 g_{2}^{3} \Delta^{8} f\left(f^{2}+3 \Delta f+3 \Delta^{2}\right)=0
\end{gathered}
$$


388 II, 4. Transformationsgleichungen erster Stufe für niedere Grade $n$

3. Tran sformation $27^{\text {sten }}$ Grades. Beim Grade 27 schlagen wir einen ähnlichen Weg ein wie oben (S. 378) beim Grade 32. Unter $\tau(\omega)$ verstehe man die bei $n=9$ benutzte Funktion, unter $\tau^{\prime}(\omega)$ die aus ihr durch Ausübung von $W_{27}$ hervorgehende Funktion. Man kann alsdann $\tau(\omega)$ und $\tau^{\prime}(\omega)$ als ein Funktionssystem des zum Geschlechte 1 gehörenden Polygons $T_{27}$ benutzen. Um die zwischen $\tau$ und $\tau^{\prime}$ bestehende Relation zu finden, übe man $W_{27}$ auf die Gleichung (20) aus und findet:

$$
9 \tau_{3}\left(\frac{-1}{9 \omega}\right)=\frac{\tau^{\prime 3}}{\tau^{\prime 2}+3 \tau^{\prime}+3}
$$

Andrerseits folgt aus (18) durch Ausübung von $W_{9}$ mit Benutzung von (17):

$$
\tau_{3}\left(\frac{-1}{9 \omega}\right)=\frac{3}{\tau}\left(\frac{9}{\tau^{2}}+\frac{9}{\tau}+3\right)=\frac{9\left(\tau^{2}+3 \tau+3\right)}{\tau^{3}} .
$$

Aus den beiden letzten Gleichungen ergibt sich als Relation zwischen den beiden dreiwertigen Funktionen $\tau$ und $\tau^{\prime}$ des Transformationspolygons $\mathbf{T}_{27}$ :

$$
\tau^{\prime 3} \tau^{3}=81\left(\tau^{\prime 2}+3 \tau^{\prime}+3\right)\left(\tau^{2}+3 \tau+3\right) .
$$

Statt $\tau$ und $\boldsymbol{\tau}^{\prime}$ kann man auch $\tau$ mit einer geeigneten zweiwertigen Funktion $\sigma \mathrm{zu}$ einem Funktionssysteme der Fläche $\mathbf{F}_{27}$ zusammenstellen. Man formt nämlich die Gleichung (24) leicht in die Gestalt:

$$
\left(\frac{\tau^{\prime}(\tau+3)}{\tau^{\prime}+3}\right)^{3}=9\left(\tau^{2}+3 \tau+3\right)
$$

um und findet demnach eine geeignete Funktion $\sigma$ in:

$$
\sigma=\sqrt[3]{9\left(\tau^{2}+3 \tau+3\right)}=\frac{\tau^{\prime}(\tau+3)}{\tau^{\prime}+3} .
$$

Die beiden durch die Relation:

$$
9\left(\tau^{2}+3 \tau+3\right)=\sigma^{3} \quad \text { oder } \quad\left(6\left(\tau+\frac{3}{2}\right)\right)^{2}=4 \sigma^{3}-2 \tau
$$

aneinander gebundenen Funktionen zeigen, daß wir hier mit einem elliptischen Gebilde des "äquianharmonischen" Falles zu tun haben (vgl. I, 136).

Geht $\sigma$ durch Ausübung von $W_{27}$ in $\sigma^{\prime}$ über, so folgt aus (25):

$$
\sigma^{\prime}=\frac{\tau\left(\tau^{\prime}+3\right)}{\tau+3}
$$

Durch Auflösung dieser Gleichung und der Gleichung (25) nach $\tau^{\prime}$ und $\sigma^{\prime}$ ergibt sich der Satz: Die Wirkung der Substitution $W_{27}$ auf das Funktionssystem $\tau$, б der $\Gamma_{\psi(27)}$ ist:

$$
\tau^{\prime}=\frac{3 \sigma}{\tau-\sigma+3}, \quad \sigma^{\prime}=\frac{3 \tau}{\tau-\sigma+3} .
$$

Für die späteren $\mathrm{Z}_{w e c k e}$ ist es etwas vorteilhafter, mit einer zweiwertigen Funktion zu arbeiten, die gegenüber $W_{27}$ unverändert bleibt und ihr eine zweite Funktion anzureihen, die bei Ausübung von $W_{97}$ 
Zeichenwechsel erfährt. Von der Funktion:

$$
\tau=\frac{\sigma-3}{\tau+3}
$$

zeigt man auf Grund von (27), daB sie bei $W_{27}$ unverändert bleibt. Durch Elimination von $\sigma$ aus (26) und (28) folgt:

$\tau^{3} \bar{\tau}^{3}+9 \tau^{2}\left(\bar{\tau}^{3}+\bar{\tau}^{2}-1\right)+27 \tau\left(\bar{\tau}^{3}+2 \bar{\tau}^{2}+\bar{\tau}-1\right)+27\left(\bar{\tau}^{3}+3 \bar{\tau}^{2}+3 \bar{\tau}\right)=0$.

Diese Gleichung gestattet die Absonderung des Linearfaktors $(\tau+3)$ und liefert dann als Beziehung zwischen $\tau$ und $\overline{\boldsymbol{\tau}}$;

$$
\tau^{2} \bar{\tau}^{3}+3 \tau\left(2 \bar{\tau}^{3}+3 \bar{\tau}^{2}-3\right)+9\left(\bar{\tau}^{3}+3 \bar{\tau}^{2}+3 \bar{\tau}\right)=0 .
$$

Die Diskriminante dieser für $\tau$ quadratischen Gleichung ist nach Fortlassung eines numerischen Faktors gleich $\left(\bar{\tau}^{4}+4 \bar{\tau}^{3}+6 \bar{\tau}^{2}-3\right)$. Durch Ausziehen der Quadratwurzel folgt der Satz: Als ein Funktionssystem der $\Gamma_{\psi(27)}$ kann man:

$$
\bar{\tau}, \quad \bar{\sigma}=\sqrt{\bar{\tau}^{4}+4 \bar{\tau}^{3}+6 \bar{\tau}^{2}-3}
$$

gebrauchen $\left.^{1}\right) ; \bar{\tau}$ ist zweiwertig und $\bar{\sigma}$ vierwertig, gegenïber $W_{27}$ zeigen diese Funktionen das einfache Verhalten:

$$
\bar{\tau}^{\prime}=\bar{\tau}, \quad \bar{\sigma}^{\prime}=-\bar{\sigma} .
$$

Die Funktion $\bar{\tau}$ verschwindet für $\omega=i \infty$; $\bar{\sigma}$ sei dadurch eindeutig erklärt, daB $\sigma(i \infty)=+i \sqrt{3}$ zutrifft. Die Funktionen $\tau$ und $\sigma$ stellen sich in $\overline{\boldsymbol{\tau}}$ und $\bar{\sigma}$ umgekehrt in der Gestalt:

$$
\tau=\frac{-6 \bar{\tau}^{3}-9 \bar{\tau}^{2}+9+3 i \sqrt{3} \bar{\sigma}}{2 \bar{\tau}^{3}}, \quad \sigma=\frac{\bar{\tau}^{3}+3+i \sqrt{3} \bar{\sigma}}{\bar{\tau}^{2}}
$$

dar. Was endlich die Transformationsgleichung für $J(\omega)$ beim Grade 27 angeht, so ist es am kürzesten, die Gleichung (19), die entsprechende in $J^{\prime}$ und $\tau^{\prime}$ und die Relation (24) zwischen $\tau$ und $\boldsymbol{\tau}^{\prime}$ als Ersatz jener Gleichung anzusehen.

\section{\$. Die Transformationsgrade $5,25,7$ und 49.}

1. Transformation fünften Grades. Fig. 15 (S. 319) zeigt das Transformationspolygon $T_{5}$ mit den beiden übereinander getragenen Dreiecksnetzen; es zerfällt in vier Kreisbogenvierecke je mit drei rechten Winkeln und einem Winkel 0, die durch die stark ausgezogenen Symmetriekreise von Spiegelungen der Gruppe $\bar{\Gamma}^{(5)}$ geliefert werden. Die acht mit Nummern versehenen Seiten sind durch folgende Substitutionen einander zugewiesen:

1) Nach I, $137 \mathrm{muB}$ die auf Grund von (10) in I, 122 zu berechnende Invariante $g_{2}$ für die unter der Quadratwurzel (29) stehende Funktion natürlich wieder verschwinden, was in der Tat zutrifft. 
$1 \rightarrow 8,\left(\begin{array}{l}1,1 \\ 0,1\end{array}\right) ; 2 \rightarrow 3,\left(\begin{array}{c}2,1 \\ -5,-2\end{array}\right) ; 4 \rightarrow 5,\left(\begin{array}{l}1,0 \\ 5,1\end{array}\right) ; 6 \rightarrow 7,\left(\begin{array}{l}2,-1 \\ 5,-2\end{array}\right)$.

Das Kreisbogenviereck der Ecken $i \infty, \frac{i}{\sqrt{5}}, \frac{-2+i}{5}, \frac{-5+i \sqrt{5}}{10}$ und sein zur rechten Seite der imaginären $\omega$-Achse gelegenes Spiegelbild mögen das Klassenpolygon $K_{5}$ zusammensetzen; die beiden Ecken $\frac{\mp 2+i}{5}$ bilden dann einen Zyklus und ebenfalls die beiden Ecken $\frac{\mp 5+i \sqrt{5}}{10}$.

Die zum fünften Grade gehörende Form $G_{1}$ hat in $T_{5}$ einen Null-

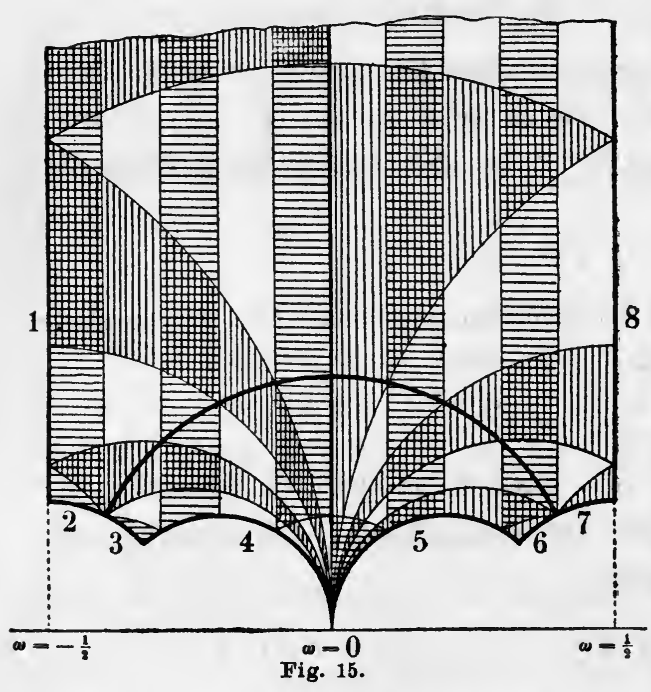
punkt der Ordnung 1 und also in $\boldsymbol{K}_{5}$ einen solchen der Ordnung $\frac{1}{2}$, der in einem der beiden eben genannten Eckenzyklen liegen muß. Der Quotient von $G_{1}^{12}$ und $\Delta^{\prime} \cdot \Delta$ liefert eine sechswertige Funktion von $K_{5}$ mit einem

8 Nullpunkte sechster Ordnung im Nullpunkte von $G_{1}$ und einem Pole der gleichen Ordnung bei $\omega=i \infty$. Also ist jener Quotient die sechste Potenz einer einwertigen Funktion von $K_{5}$, die selbst als Quotient von $G_{1}^{2}$ und $\sqrt[6]{\Delta^{\prime} \Delta}$ darstellbar ist.

Da nun $G_{1}^{2}$ gegenüber den Substitutionen der $\Gamma^{(5)}$ unverändert bleibt, so gilt dasselbe von $\sqrt[6]{\Delta^{\prime} \Delta}$. Wir verstehen hier unter $\Delta^{\prime}$ zweckmäBig die Form $\Delta\left(\omega_{1}, \frac{\omega_{2}}{5}\right)$ und haben dann folgende Anfangsglieder der Reihen:

$$
\left\{\begin{array}{l}
G_{1}^{2}=25\left(\frac{2 \pi}{\omega_{2}}\right)^{4}\left(\frac{1}{36}+\frac{1}{3} q^{2}+2 q^{4}+\frac{22}{3} q^{6}+\cdots\right), \\
\sqrt[6]{\Delta^{\prime} \Delta}=25\left(\frac{2 \pi}{\omega_{2}}\right)^{4}\left(q^{2}-4 q^{4}+2 q^{6}+\cdots\right) .
\end{array}\right.
$$

Mit $G_{1}^{2}$ und $\sqrt[6]{\Delta^{\prime} \Delta}$ bilden wir nun wieder eine lineare $\operatorname{Schar}\left(a G_{1}^{2}+b \sqrt[6]{\Delta^{\prime} \Delta}\right)$ von Formen mit einem im Polygone $K_{5}$ beweglichen Nullpunkte und können dann die übrigen ganzen Formen von $K_{5}$ als Produkte von $G_{1}$ und von Formen jener Schar oder auch nur aus Formen der Schar herstellen.

Für die mittelst der homogenen Substitution $W_{5}$ umgestaltete Form $g_{2}$ gilt zunächst der Ansatz:

$$
g_{2}^{\prime}+g_{2}=a G_{1}^{2}+b \sqrt[6]{\Delta^{\prime} \Delta}, \quad g_{2}^{\prime} \cdot g_{2}=a^{\prime} G_{1}^{4}+b^{\prime} G_{1}^{2} \sqrt[6]{\Delta^{\prime} \Delta}+c^{\prime} \sqrt[3]{\Delta^{\prime} \Delta}
$$


Durch Heranziehung der Potenzreihen findet man die Koeffizienten:

$$
\left\{\begin{array}{l}
25\left(g_{2}^{\prime}+g_{2}\right)=78 G_{1}^{2}-6 \sqrt[6]{\Delta^{\prime} \Delta}, \\
25 g_{2}^{\prime} \cdot g_{2}=9 G_{1}^{4}+54 G_{1}^{2} \sqrt[6]{\Delta^{\prime} \Delta}+\sqrt[3]{\Delta^{\prime} \Delta} .
\end{array}\right.
$$

Bei Berechnung von $g_{2}, g_{2}^{\prime}$ einzeln stellt sich die Quadratwurzel:

$$
G_{2}\left(\omega_{1}, \omega_{2}\right)=\sqrt{81 G_{1}^{4}-99 G_{1}^{2} \sqrt[6]{\Delta^{\prime} \Delta}-\sqrt[3]{\Delta^{\prime} \Delta}}
$$

ein, die durch Angabe des Anfangsgliedes $\frac{25}{4}\left(\frac{2 \pi}{\omega_{2}}\right)^{4}$ der Reihenentwicklung als eindeutige Modulform $(-4)^{\text {ter }}$ Dimension erklärt sein mag. Sie erfährt gegenüber $W_{5}$ Zeichenwechsel, ihre beiden Nullpunkte in $\mathbf{T}_{5}$ sind die Fixpunkte von $W_{5}$, d. h. die Stellen $\frac{i}{\sqrt{5}}$ und $\frac{ \pm 5+i \sqrt{5}}{10}$. Man folgert, aus (3), daß $G_{1}$ für $\frac{ \pm 5+i \sqrt{5}}{10}$ nicht verschwindet: Die Form $G_{1}$ hat ihren Nullpunkt im Eckenzyklus $\omega=\frac{ \pm 2+i}{5}$. Aus (2) und (3) berechnet man als Darstellungen für $g_{2}$ und $g_{2}^{\prime}$ :

$$
\left\{\begin{array}{l}
25 g_{2}=39 G_{1}^{2}-3 \sqrt[6]{\Delta^{\prime} \Delta}-4 G_{2}, \\
25 g_{2}^{\prime}=39 G_{1}^{2}-3 \sqrt[6]{\Delta^{\prime} \Delta}+4 G_{2} .
\end{array}\right.
$$

Da $g_{3}$ und $g_{3}^{\prime}$ im Nullpunkte von $G_{1}$ zugleich verschwinden (vgl. Fig. 15), so sind die Quotienten $g_{3}: G_{1}$ und $g_{3}^{\prime}: G_{1}$ ganze Formen $(-4)^{\text {ter }}$ Dimension des Polygons $T_{5}$. Es gelten demnach die Ansätze:

$$
g_{3}^{\prime}+g_{3}=G_{1}\left(a G_{1}^{2}+b \sqrt[6]{\Delta^{\prime} \Delta}\right), \quad g_{3}^{\prime}-g_{3}=c G_{1} G_{2},
$$

wo man wegen der zweiten Gleichung beachten wolle, daß eine ganze Modulform des Polygons $\mathbf{T}_{5}$, die bei $W_{5}$ Zeichenwechsel erfährt, bis auf einen konstanten Faktor mit $G_{2}$ identisch ist. $\left.{ }^{1}\right)$ Die Koeffizienten $a, b, c$ bestimmt man aus den Anfangsgliedern der Reihen; es findet sich für. $g_{3}$ und $g_{3}^{\prime}$ :

$$
\left\{\begin{array}{l}
125 g_{3}=-G_{1}\left(62 G_{1}^{2}-24 \sqrt[6]{\Delta^{\prime} \Delta}-7 G_{2}\right) \\
125 g_{3}^{\prime}=-G_{1}\left(62 G_{1}^{2}-24 \sqrt[6]{\Delta^{\prime} \Delta}+7 G_{2}\right)
\end{array}\right.
$$

$\mathrm{Zu}$ entsprechenden Formeln für $\Delta$ führt folgende Überlegung: $T_{5}$ hat die beiden Spitzen $i \infty$ und 0 und gehört zum Geschlechte 0 . Eine Hauptfunktion $\tau(\omega)$ werde so ausgesucht, daB $\tau(i \infty)=0$ und $\tau(0)=\infty$ ist, wodurch $\tau(\omega)$ bis auf einen konstanten Faktor festgelegt ist. Der Quotient von $\Delta^{\prime}=\Delta\left(\omega_{1}, \frac{\omega_{2}}{5}\right)$ und $\Delta=\Delta\left(\omega_{1}, \omega_{2}\right)$ ist eine vierwertige

1) Die fragliche Form muB nämlich dieselben Nullpunkte wie $G_{2}$ haben, da andernfalls der Quotient der Form mit $G_{2}$ eine „Funktion" des Klassenpolygons $K_{5}$ wäre, die an den Nullstellen von $G_{2}$ Nullpunkte oder Pole, ,gebrochener" Ordnung bätte. 
392 II, 4. Transformationsgleichungen erster Stufe für niedere Grade $n$

Funktion von $\mathrm{T}_{5}$, die bei $\omega=i \infty$ einen Nullpunkt vierter Ordnung und also bei $\omega=0$ einen Pol der gleichen Ordnung hat. Wir können demnach $\tau(\omega)$ eindeutig durch die Festsetzung:

$$
\tau(\omega)=\sqrt[4]{\frac{\Delta^{\prime}}{\Delta}}=5 \sqrt{5} \sqrt[4]{\frac{\Delta\left(\omega_{1} \sqrt{5}, \frac{\omega_{2}}{\sqrt{5}}\right)}{\Delta\left(\omega_{1}, \omega_{2}\right)}}
$$

erklären, wobei auf der imaginären $\omega$-Achse reelle positive Werte $\tau(\omega)$ vorliegen sollen. Es gilt dann:

$$
\tau\left(\frac{-1}{\omega \omega}\right)=\frac{125}{\tau(\omega)} .
$$

Man hat nun in:

$$
\left(\tau-\frac{125}{\tau}\right) \sqrt[6]{\Delta^{\prime} \Delta}=\frac{\sqrt{\Delta^{\prime}}-125 \sqrt{\Delta}}{\sqrt[12]{\Delta^{\prime} \Delta}}
$$

eine ganze Form $(-4)^{\text {ter }}$ Dimension von $\mathbf{T}_{5}$, die gegenüber $W_{5}$ Zeicherwechsel erfährt und also bis auf einen konstanten Faktor mit $G_{2}$ identisch ist. Den Faktor bestimmt man aus dem Anfangsgliede der Reihe:

$$
\sqrt{\Delta^{\prime}}-125 \sqrt{\Delta}=-4 \sqrt[12]{\Delta^{\prime} \Delta} \cdot G_{2}
$$

Mit Rücksicht auf (3) folgert man hieraus:

$$
\left(\sqrt{\Delta^{\prime}}+125 \sqrt{\Delta}\right)^{2}=4 \sqrt[6]{\Delta^{\prime} \Delta}\left(324 G_{1}^{4}-396 G_{1}^{2} \sqrt[6]{\Delta^{\prime} \Delta}+121 \sqrt[3]{\Delta^{\prime} \Delta}\right)
$$

sowie durch Wurzelziehung bei richtiger Bestimmung des Zeichens:

$$
\sqrt{\Delta^{\prime}}+125 \sqrt{\Delta}=\sqrt[12]{\Delta^{\prime} \Delta}\left(36 G_{1}^{2}-22 \sqrt[6]{\Delta^{\prime} \Delta}\right)
$$

Aus (8) und (9) ergeben sich für $\sqrt{\Delta}$ und $\sqrt{\Delta^{\prime}}$ die Darstellungen:

$$
\left\{\begin{aligned}
125 \sqrt{\Delta} & =\left(18 G_{1}^{2}-11 \sqrt[6]{\Delta^{\prime} \Delta}+2 G_{2}\right) \sqrt[12]{\Delta^{\prime} \Delta} \\
\sqrt{\Delta^{\prime}} & =\left(18 G_{1}^{2}-11 \sqrt[6]{\Delta^{\prime} \Delta}-2 G_{2}\right) \sqrt[12]{\Delta^{\prime} \Delta}
\end{aligned}\right.
$$

Die Transformationsgleichungen beim fünften Grade ergeben sich nun einfach durch algebraische Umgestaltungen der gewonnenen Formeln. Erstlich folgen aus (6) und (9), sowie, weiter aus (6) und (8) die Darstellungen:

$$
G_{1}^{2}=\frac{\tau^{2}+22 \tau+125}{36 \tau} \sqrt[6]{\Delta^{\prime} \Delta}, \quad G_{2}=-\frac{\tau^{2}-125}{4 \tau} \sqrt[6]{\Delta^{\prime} \Delta}
$$

von $G_{1}^{2}$ und $G_{2}$ in $\tau$ und $\sqrt[6]{\Delta^{\prime} \Delta}$. Durch Eintragung dieser Ausdrücke in die ersten Gleichungen (4) und (5) folgt:

$$
g_{2}=\frac{\tau^{2}+10 \tau+5}{12 \tau} \sqrt[6]{\Delta^{\prime} \Delta}, \quad g_{3}=-G_{1} \frac{\tau^{2}+4 \tau-1}{36 \tau} \sqrt[6]{\Delta^{\prime} \Delta}
$$

woran wir noch die aus (6) fließende Gleichung $\Delta=\tau^{-2} \sqrt{\Delta^{\prime} \Delta}$ reihen. 
Bei Einführung von $J(\omega)$ ergibt sich aus (11) und (12):

$$
\begin{aligned}
J:(J-1): 1= & \left(\tau^{2}+10 \tau+5\right)^{3} \\
& :\left(\tau^{2}+22 \tau+125\right)\left(\tau^{2}+4 \tau+1\right)^{2} \\
& : 1728 \tau,
\end{aligned}
$$

eine Gleichung, die uns in bekannter Weise im Verein mit der Relation $\boldsymbol{\tau}^{\prime} \cdot \tau=125$ die beim fünften Grade eintretende Transformationsgleichung für $J(\omega)$ ersetzt.

Durch geeignete Verbindung der Gleichungen (12) beweist man:

$$
\begin{aligned}
& 2 g_{2}-\frac{30 g_{3}}{G_{1}}-5 \sqrt[6]{\Delta^{\prime} \Delta}=\tau \sqrt[6]{\Delta^{\prime} \Delta}, \\
& 2 g_{2}+\frac{6 g_{3}}{G_{1}}-\sqrt[6]{\Delta^{\prime} \Delta}=\frac{\sqrt[6]{\Delta^{\prime} \Delta}}{\tau},
\end{aligned}
$$

ebenso mit Benutzung der ersten Gleichung (11):

$$
7 g_{2}+\frac{20 g_{3}}{G_{1}}-G_{1}^{2}=3 \sqrt[6]{\Delta^{\prime} \Delta} .
$$

Trägt man den hier gewonnenen Ausdruck von $\sqrt[6]{\Delta^{\prime} \Delta}$ in die voraufgehenden Gleichungen ein, so folgt:

$$
\begin{array}{r}
5 G_{1}^{2}-29 g_{2}-190 \frac{g_{3}}{G_{1}}=-\tau\left(G_{1}^{2}-7 g_{2}-\frac{20 g_{3}}{G_{1}}\right), \\
G_{1}^{2}-g_{2}-\frac{2 g_{3}}{G_{1}}=-\frac{1}{\tau}\left(G_{1}^{2}-7 g_{2}-\frac{20 g_{3}}{G_{1}}\right) .
\end{array}
$$

Durch Multiplikation dieser Gleichungen findet man als Transformationsgleichung sechsten Grades für $G_{1}$ beim fünften Transformationsgrade:

$$
G_{1}^{6}-5 g_{2} G_{1}^{4}-40 g_{3} G_{1}^{3}-5 g_{2}^{2} G_{1}^{2}-8 g_{2} g_{3} G_{1}-5 g_{3}^{2}=0
$$

Durch Elimination von $\sqrt[6]{\Delta^{\prime} \Delta}$ aus (14) und der ersten Gleichung (2), sowie weiter durch Elimination von $\sqrt[6]{\Delta^{\prime} \Delta}$ und $G_{2}$ aus (5) und (14) gewinnt man:

$$
\left\{\begin{aligned}
25 g_{8}^{\prime} & =80 G_{1}^{2}-39 g_{2}-\frac{40 g_{3}}{G_{1}}, \\
125 g_{3}^{\prime} & =-140 G_{1}^{3}+112 g_{2} G_{1}+195 g_{3} .
\end{aligned}\right.
$$

Durch diese Darstellungen der transformierten Formen $g_{2}^{\prime}, g_{3}^{\prime}$ sind die Transformationsgleichungen der $g_{2}, g_{3}$ beim fünften Grade als Resolventen der Gleichung (15) erklärt.

Nach S. 339 ff. besteht hier endlich noch für die Form:

$$
f\left(\omega_{1}, \omega_{2}\right)=\sqrt[12]{\Delta^{\prime} \Delta^{7}}=\sqrt[3]{\tau} \sqrt[3]{\Delta^{2}}
$$

eine 'Transformationsgleichung sechsten Grades, die unter (5) S. 343 allgemein angesetzt wurde. Diese Gleichung ergibt sich aus (13), indem 
394 II, 4. Transformationsgleichungen erster Stufe für niedere Grade $n$ $\operatorname{man} \tau=f^{3} \cdot \Delta^{-2}$ einträgt:

$$
\frac{12^{3} g_{2}^{3}}{\Delta}=\frac{\left(\tau^{2}+10 \tau+5\right)^{3}}{\tau}=\frac{\left(f^{6}+10 f^{3} \Delta^{2}+5 \Delta^{4}\right)^{3}}{f^{3} \cdot \Delta^{10}}
$$

Man multipliziere mit $\Delta$ und ziehe die Kubikwurzel, womit man als Transformationsgleichung fïr die Form (17) findet:

$$
f^{6}+10 \Delta^{2} f^{3}-12 g_{2} \Delta^{3} f+5 \Delta^{4}=0 .
$$

2. Transformation $25^{\text {sten }}$ Grades. Das Polygon $\mathbf{T}_{25}$ entsteht aus dem in Fig. 15 abgebildeten $\mathbf{T}_{5}$, indem man auf diesen Bereich die vier Substitutionen $\left(\begin{array}{c}1,0 \\ \pm 5,1\end{array}\right)$ und $\left(\begin{array}{c}1,0 \\ \pm 10,1\end{array}\right)$ ausübt und die vier so entstehenden Bereiche dem Polygone $\mathbf{T}_{5}$ anfügt. Polygonspitzen von $\mathbf{T}_{25}$ liegen bei $\omega=i \infty, 0, \pm \frac{1}{5}, \pm \frac{1}{10}$. Je die beiden von der einzelnen dieser sechs Spitzen ausziehenden Polygonseiten sind durch Substitutionen der Gruppe $\Gamma_{\psi(25)}$ aufeinander bezogen, und zwar der Reihe nach durch

$\left(\begin{array}{l}1,1 \\ 0,1\end{array}\right), \quad\left(\begin{array}{r}1,0 \\ 25,1\end{array}\right), \quad\left(\begin{array}{r}4,-1 \\ 25,-6\end{array}\right), \quad\left(\begin{array}{r}6, \quad 1 \\ -25,-4\end{array}\right), \quad\left(\begin{array}{r}9,-1 \\ 100,-11\end{array}\right), \quad\left(\begin{array}{rr}11, & 1 \\ -100,-9\end{array}\right)$.

Auch $\mathbf{T}_{25}$ hat das Geschlecht 0. Eine zugehörige einwertige Funktion $\tau(\omega)$ sei durch die Festsetzungen:

$$
\tau(i \infty)=0, \tau(0)=\infty, \tau\left(\frac{i}{5}\right)=\sqrt{5}
$$

näher erklärt; sie zeigt gegenüber $W_{\mathbf{2 5}}$ das Verhalten:

$$
\tau\left(\frac{-1}{25 \omega}\right)=\frac{5}{\tau(\omega)}
$$

Die vier Spitzenwerte $\tau\left( \pm \frac{1}{5}\right), \tau\left( \pm \frac{1}{10}\right)$ sind zu Paaren konjugiert komplex und genügen also einer biquadratischen Gleichung mit reellen Koeffizienten:

$$
\tau^{4}+a \tau^{3}+b \tau^{2}+c \tau+d=0
$$

von denen $d$ positiv ist. Durch $W_{5}$ werden die fraglichen vier Spitzen permutiert, so daß wegen (20) die letzte Gleichung auch:

$$
\tau^{4}+\frac{5 c}{d} \tau^{3}+\frac{25 b}{d} \tau^{2}+\frac{125 a}{d} \tau+\frac{625}{d}=0
$$

geschrieben werden kann. Also ist $d=25, c=5 a$, und unsere Gleichung lautet:

$$
\tau^{4}+a \tau^{3}+b \tau^{2}+5 a \tau+25=0
$$

Die Funktion $\tau_{5}(\omega)$ ist in $T_{25}$ fünfwertig und zwar durch $\tau$ als ganze Funktion fünften Grades darstellbar, da die fünf Pole von $\tau_{5}$ bei $\omega=0$ zusammenfallen. Die fünf Nullpunkte dieser Funktion liegen in den übrigen fünf Polygonspitzen, so daB der Ansatz gilt:

$$
\tau_{5}(\omega)=C \cdot \tau\left(\tau^{4}+a \tau^{3}+b \tau^{2}+5 a \tau+25\right),
$$

wo rechts $\omega$ als Argument zu denken ist. Übt man die Substitution $W_{25}$ 
aus, so folgt mit Benutzung von (7) und (20):

$$
\frac{1}{\tau_{5}(5 \omega)}=\frac{C}{\tau^{5}}\left(\tau^{4}+a \tau^{3}+b \tau^{2}+5 a \tau+25\right) \text {. }
$$

Mit Rücksicht auf (6) findet man aus den beiden letzten Gleichungen:

$$
\tau(\omega)=\sqrt[6]{\tau_{5}(5 \omega) \tau_{5}(\omega)}=\sqrt[24]{\frac{\Delta\left(\omega_{1}, \frac{\omega_{2}}{25}\right)}{\Delta\left(\omega_{1}, \omega_{2}\right)}} .
$$

Aus der Reihe für $\sqrt[24]{\Delta}$ (vgl. I, 433) stellt man leicht folgende Anfangsglieder der Reihen von $\tau_{5}(\omega)$ und $\tau(\omega)$ fest:

$$
\begin{aligned}
& \tau_{5}(\omega)=125 q^{2}\left(1+6 q^{2}+27 q^{4}+\cdots\right), \\
& \tau(\omega)=5 q^{2}\left(1+q^{2}+2 q^{4}+\cdots\right) .
\end{aligned}
$$

Trägt man diese Reihen in (21) ein, so folgt durch Vergleichung der Koeffizienten gleich hoher Potenzen von $q$ rechts und links $C=1, a=5$, $b=15$. Die Darstellung von $\tau_{5}$ als ganze Funktion von $\tau$ ist somit:

$$
\tau_{5}=\tau\left(\tau^{4}+5 \tau^{3}+15 \tau^{2}+25 \tau+25\right) .
$$

Diesen Ausdruck von $\tau_{5}$ setze man nun in der rechten Seite von (13) für $\tau$ ein. Es ergibt sich als Darstellung von $J(\omega)$ in der $z u n=25$ gehörenden Funktion $\tau$ :

$$
\begin{gathered}
J:(J-1): 1=\left(\tau^{10}+10 \tau^{9}+55 \tau^{8}+200 \tau^{7}+525 \tau^{6}+1010 \tau^{5}\right. \\
\left.+1425 \tau^{4}+1400 \tau^{3}+875 \tau^{2}+250 \tau+5\right)^{3} \\
:\left(\tau^{2}+2 \tau+5\right)\left(\tau^{4}+4 \tau^{3}+9 \tau^{2}+10 \tau+5\right)^{2}\left(\tau^{10}+10 \tau^{9}\right. \\
+55 \tau^{8}+200 \tau^{7}+525 \tau^{6}+1004 \tau^{5}+1395 \tau^{4} \\
\left.+1310 \tau^{3}+725 \tau^{2}+100 \tau-1\right)^{2} \\
: 1728 \tau\left(\tau^{4}+5 \tau^{3}+15 \tau^{2}+25 \tau+25\right) .
\end{gathered}
$$

Nach S. 339 genügt beim quadratischen Transformationsgrade $n=25$ die Form:

$$
f\left(\omega_{1}, \omega_{2}\right)=\sqrt[24]{\Delta^{\prime} \Delta^{23}}=\tau \cdot \Delta
$$

einer Transformationsgleichung $30^{\text {sten }}$ Grades. Diese Gleichung läßt sich aus (24) unmittelbar abschreiben:

$$
\begin{aligned}
& \left(f^{10}+10 \Delta f^{9}+55 \Delta^{2} f^{8}+200 \Delta^{3} f^{7}+525 \Delta^{4} f^{6}+1010 \Delta^{5} f^{5}\right. \\
& \left.+1425 \Delta^{6} f^{4}+1400 \Delta^{7} f^{3}+875 \Delta^{8} f^{2}+250 \Delta^{9} f+5 \Delta^{10}\right)^{3} \\
& -1728 g_{2}^{3} \Delta^{24} f\left(f^{4}+5 \Delta f^{3}+15 \Delta^{2} f^{2}+25 \Delta^{3} f+25 \Delta^{4}\right)=0 .
\end{aligned}
$$

3. Transformation siebenten Grades. Die Polygone $T_{7}$ und $K_{7}$ sind in Fig. 2, S. 351, und Fig. 6, S. 359, figürlich dargestellt und daselbst näher besprochen. Als eine erste zur $\Gamma_{\psi(7)}$ gehörende Modulform ziehen 
wir das in (9) S. 326 gegebene $y_{0}$ der quadratischen Form $(1,1,2)$ der Diskriminante $D=-7$ heran:

$$
y_{0}=\frac{2 \pi}{\omega_{2}} \sum_{\mu, v} q^{2\left(\mu^{2}+\mu v+4 \nu^{2}\right)}=\frac{2 \pi}{\omega_{2}}\left(1+2 q^{2}+4 q^{4}+*+6 q^{8}+\cdots\right)
$$

Durch den Stern soll darauf aufmerksam gemacht werden, daB das Glied mit $q^{6}$ ausfällt. Bei den Substitutionen der $\Gamma_{\psi(7)}$ bleibt $y_{0}$ unverändert oder erleidet nur einen Zeichenwechsel. In $\mathbf{T}_{7}$ hat $y_{0}$ Nullpunkte in der Gesamtordnung $\frac{2}{3}$. Nullpunkte gebrochener Ordnung können nur in den Ecken $\frac{ \pm 5+i \sqrt{3}}{14}$ von $T_{7}$ auftreten. Da in zwei bezüglich der imaginären $\omega$-Achse symmetrischen Punkten die in (26) rechts stehende Potenzreihe konjugierte Werte hat, so liegt in jeder der beiden genannten Ecken ein Nullpunkt der Ordnung $\frac{1}{3}$. Durch die homogene Substitution $W_{7}$ wird demnach $y_{0}$, vielleicht vom Vorzeichen abgesehen, reproduziert. Setzen wir aber in:

$$
y_{0}\left(\frac{i \omega_{2}}{\sqrt{7}},-i \omega_{1} \sqrt{7}\right)= \pm y_{0}\left(\omega_{1}, \omega_{2}\right)
$$

dem Fixpunkte von $W_{7}$ entsprechend, $\omega_{1}=i, \omega_{2}=\sqrt{7}$ ein, so folgt, da $y_{0}(i, \sqrt{7})$ nicht gleich 0 ist, die Gültigkeit des oberen Zeichens in (27).

Von der zu $n=7$ gehörenden Form $G_{1}$ stellt man fest, daB sie in $T_{7}$ nur zwei Nullpunkte je der Ordnung $\frac{2}{3}$ in den Ecken $\frac{ \pm 5+i \sqrt{3}}{14}$ hat; sie ist demnach bis auf einen konstanten Faktor mit $y_{0}^{2}$ identisch. Die Reihenentwicklung:

$$
G_{1}=7\left(\frac{2 \pi}{\omega_{2}}\right)^{2}\left(\frac{1}{4}+q^{2}+3 q^{4}+4 q^{6}+7 q^{8}+\cdots\right)
$$

bestätigt dies und liefert:

$$
4 G_{1}=7 y_{0}^{2}
$$

Für $K_{7}$ bilden die beiden Ecken $\frac{ \pm 5+i \sqrt{3}}{14}$ einen Zyklus, in dem die Form $y_{0}^{3}$ einen Nullpunkt erster Ordnung hat. Unter $\Delta^{\prime}$ verstehen wir hier zweckmäBig ' $\Delta\left(7 \omega_{1}, \omega_{2}\right)$. Im Quotienten von $y_{0}^{24}$ und $\Delta^{\prime} \Delta$ erkennen wir dann die achte Potenz einer einwertigen Funktion von $K_{7}$, so daB der Quotient von $y_{0}^{3}$ und:

$$
\sqrt[8]{\Delta^{\prime} \Delta}=\left(\frac{2 \pi}{\omega_{2}}\right)^{3}\left(*+q^{2}-3 q^{4}+*+5 q^{8}-\cdots\right)
$$

eine einwertige Funktion von $K_{7}$ ist, deren Pol in der Spitze $i \infty$ liegt. Aus den beiden Formen $y_{0}^{3}$ und $\sqrt[8]{\Delta^{\prime} \Delta}$, die gegenüber den Substitutionen von $\Gamma^{(7)}$ immer zugleich unverändert bleiben oder Zeichenwechsel erfahren, bilden wir nun wieder eine lineare Schar $\left(a y_{0}^{3}+b \sqrt[8]{\Delta^{\prime} \Delta}\right)$ mit einem in 
$K_{\mathbf{7}}$ beweglichen Nullpunkte. Die übrigen ganzen Formen von $\mathbf{K}_{\mathbf{7}}$, die wie $y_{0}$ und die Formen der Schar bei $W_{7}$ unverändert bleiben, lassen sich dann als Produkte aus Faktoren $y_{0}$ und Formen der Schar darstellen.

Die Entwicklung geht nun auch weiter genau denselben Weg wie bei $n=5$. Man gewinnt mit der Benutzung der Potenzreihe für $g_{2} \mathbf{z u}$ nächst die Darstellungen:

$$
\left\{\begin{aligned}
6\left(g_{2}^{\prime}+g_{2}\right) & =y_{0}\left(25 y_{0}^{3}-80 \sqrt[8]{\Delta^{\prime} \Delta}\right) \\
144 g_{2}^{\prime} g_{2} & =49 y_{0}^{2}\left(y_{0}^{6}+224 y_{0}^{3} \sqrt[8]{\Delta^{\prime} \Delta}+448 \sqrt[4]{\Delta^{\prime} \Delta}\right) .
\end{aligned}\right.
$$

Bei Berechnung von $g_{2}^{\prime}$ und $g_{2}$ stellt sich eine Quadratwurzel ein, die die Modulform der Dimension - 3:

$$
G_{\frac{3}{2}}\left(\omega_{1}, \omega_{2}\right)=\sqrt{y_{0}^{6}-26 y_{0}^{3} \sqrt[8]{\Delta^{\prime} \Delta}-27 \sqrt[4]{\Delta^{\prime} \Delta}}
$$

liefert und durch das Anfangsglied der Potenzreihe $\left(\frac{2 \pi}{\omega_{2}}\right)^{3}$ eindeutig erklärt sei. Diese Form hat im Klassenpolygon $\mathbf{K}_{\mathbf{7}}$ zwei Nullpunkte je der Ordnung $\frac{1}{2}$ bei $\omega=\frac{i}{\sqrt{7}}$ und im Eckenzyklus $\frac{ \pm 7+i \sqrt{7}}{14} ;$ gegenüber $W_{7}$ erfährt $G_{\frac{3}{9}}$ Zeichenwechsel. Für $g_{2}$ und $g_{2}^{\prime}$ einzeln finden wir die Darstellungen:

$$
12 g_{2}, 12 g_{2}^{\prime}=y_{0}\left(25 y_{0}^{3}-80 \sqrt[8]{\Delta^{\prime} \Delta} \mp 24 G_{\frac{3}{2}}\right) .
$$

Zur Berechnung von $g_{3}$ und $g_{3}^{\prime}$ ist es etwas bequemer, neben $\left(g_{3}^{\prime}+g_{3}\right)$ die Differenz $\left(g_{3}^{\prime}-g_{3}\right)$ darzustellen, nämlich als Produkt von $G_{\frac{3}{2}}$ und einer Form der Schar. Die Rechnung führt zu:

$$
\begin{aligned}
216 g_{3}, 216 g_{3}^{\prime} & =-9\left(19 y_{0}^{6}-200 y_{0}^{3} \sqrt[8]{\Delta^{\prime} \Delta}-72 \sqrt[4]{\Delta^{\prime} \Delta}\right) \\
& \pm 4\left(43 y_{0}^{3}-20 \sqrt[8]{\Delta^{\prime} \Delta}\right) G_{\frac{3}{2}}
\end{aligned}
$$

Im Falle $n=7$ erkennt man aus den Anfangsgliedern der Reihen im Quotienten von $\Delta^{\prime}$ und $\Delta$ die sechste Potenz einer einwertigen Funktion von $\mathbf{T}_{7}$. Im Anschluß daran erklären wir eine solche einwertige Funktion $\tau(\omega)$ selbst durch:

$$
\tau(\omega)=49 \sqrt[6]{\frac{\Delta^{\prime}}{\Delta}}=7 \sqrt[6]{\frac{\Delta\left(\omega_{1} \sqrt{7}, \frac{\omega_{2}}{\sqrt{7}}\right)}{\Delta\left(\omega_{1}, \omega_{2}\right)}}
$$

mit der Bestimmung, daß diese Funktion auf der imaginären $\omega$-Achse reell und positiv sein soll. Die Wirkung von $W_{7}$ ist:

$$
\tau\left(\frac{-1}{7 \omega}\right)=\frac{49}{\tau(\omega)} \text {. }
$$


398 II, 4. Transformationsgleichungen erster Stufe für niedere Grade $n$

Das Produkt von $\left(\tau-49 \tau^{-1}\right)$ und $\sqrt[8]{\Delta^{\prime} \Delta}$ ist nun eine ganze Form $(-3)^{\text {tor }}$ Dimension, die die Nullpunkte von $G_{\frac{3}{2}}$ hat und also mit dieser Form bis auf einen konstanten Faktor übereinstimmt. Die Anfangsglieder der Reihen liefern diesen Faktor und führen zur Gleichung:

$$
\sqrt[3]{\Delta}-49 \sqrt[3]{\Delta^{\prime}}=G_{\frac{3}{2}} \sqrt[24]{\Delta^{\prime} \Delta}
$$

Zur Bestätigung dieses Ergebnisses leite man aus ihm mit Benutzung von (31) einen Ausdruck von $\left(\sqrt[3]{\Delta}+49 \sqrt[3]{\Delta^{\prime}}\right)$ ab. Es muB sich das Produkt von $\sqrt[24]{\Delta^{\prime} \Delta}$ mit einer Form der Schar finden; in der Tat ergibt sich bei richtiger Bestimmung eines Vorzeichens:

$$
\sqrt[3]{\Delta}+49 \sqrt[3]{\Delta^{\prime}}=\left(y_{0}^{3}-13 \sqrt[8]{\Delta^{\prime} \Delta}\right) \sqrt[24]{\Delta^{\prime} \Delta}
$$

Durch Kombination der letzten Gleichungen gewinnt man für die Diskriminante $\Delta$ die Gleichungen:

$$
\left\{\begin{array}{c}
2 \sqrt[3]{\Delta}=\left(y_{0}^{3}-13 \sqrt[8]{\Delta^{\prime} \Delta}+G_{\frac{3}{2}}\right) \sqrt[24]{\Delta^{\prime} \Delta} \\
98 \sqrt[3]{\Delta^{\prime}}=\left(y_{0}^{3}-13 \sqrt[8]{\Delta^{\prime} \Delta}-G_{\frac{3}{2}}\right) \sqrt[24]{\Delta^{\prime} \Delta}
\end{array}\right.
$$

Um nun die Transformationsgleichungen zu gewinnen, entnehmen wir zunächst aus (37) und (36) mit Rücksicht auf (34) die Folgerungen:

$$
y_{0}^{3}=\frac{\tau^{2}+13 \tau+49}{\tau} \sqrt[8]{\Delta^{\prime} \Delta,} \quad G_{\frac{3}{2}}=-\frac{\tau^{2}-49}{\tau} \sqrt[8]{\Delta^{\prime} \Delta}
$$

Durch Eintragung dieser Ausdrücke von $y_{0}^{3}$ und $G_{\frac{3}{2}}$ in die ersten Gleichungen (32) und (33) ergeben sich für $g_{2}$ und $g_{3}$ die Darstellungen:

$$
\left\{\begin{array}{c}
12 g_{2}=7^{2} \sqrt[8]{\Delta^{\prime} \Delta} \cdot y_{0} \frac{\tau^{2}+5 \tau+1}{\tau}, \\
216 g_{3}=-7^{3} \sqrt[4]{\Delta^{\prime} \Delta} \frac{\tau^{4}+14 \tau^{3}+63 \tau^{2}+70 \tau-7}{\tau^{2}} .
\end{array}\right.
$$

Nimmt man die aus (34) folgende Gleichung $\Delta=7^{6} \tau^{-3} \cdot \sqrt{\Delta^{\prime} \Delta}$ hinzu, so gelangt man zu folgender Darstellung von $J(\omega)$ als rationale Funktion achten Grades von $\tau$ :

$$
\begin{aligned}
J:(J-1): 1= & \left(\tau^{2}+13 \tau+49\right)\left(\tau^{2}+5 \tau+1\right)^{3} \\
& :\left(\tau^{4}+14 \tau^{3}+63 \tau^{2}+70 \tau-7\right)^{2} \\
& : 1728 \tau,
\end{aligned}
$$

eine Gleichung, die uns in bekannter Weise im Verein mit $\tau^{\prime} \tau=49$ die Transformationsgleichung für $J(\omega)$ beim siebenten Grade ersetzt.

Nach S. $339 \mathrm{ff}$. besteht beim siebenten Grade eine Transformationsgleichung für die Form: 


$$
f\left(\omega_{1}, \omega_{2}\right)=7 \sqrt[12]{\Delta^{\prime} \Delta^{5}}=\sqrt{\Delta \tau},
$$

die durch ihr Anfangsglied $\left(\frac{2 \pi}{\omega_{2}}\right)^{6} 7 q^{2}$ eindeutig erklärt sei. Sie läßt sich fast unmittelbar aus der Gleichung (41) abschreiben, der wir zunächst die Gestalt geben:

$$
\left(\tau^{4}+14 \tau^{3}+63 \tau^{2}+70 \tau-7\right)^{2} \Delta^{2}=6^{6} g_{3}^{2} \tau \Delta=\left(216 g_{3} f\right)^{2} .
$$

Nach Ausziehen der Quadratwurzel (unter richtiger Bestimmung des Vorzeichens) und Multiplikation mit $\Delta^{3}$ ergibt sich als Transformationsgleichung der Diskriminante beim siebenten Grade:

$$
f^{8}+14 \Delta f^{6}+63 \Delta^{2} f^{4}+70 \Delta^{3} f^{2}+216 g_{3} \Delta^{3} f-7 \Delta^{4}=0 .
$$

Zur Gewinnung der Gleichung achten Grades für $G_{1}$ ist folgender Weg am kürzesten. Die fragliche Gleichung hat nach S. 342 die Gestalt:

$$
\begin{aligned}
G_{1}^{8}+\alpha_{1} g_{2} G_{1}^{6}+\alpha_{2} g_{3} G_{1}^{5}+\alpha_{3} g_{2}^{2} G_{1}^{4} & +\alpha_{4} g_{2} g_{3} G_{1}^{3}+\left(\alpha_{5} g_{2}^{3}+\beta_{5} \Delta\right) G_{1}^{2} \\
& +\alpha_{6} g_{2}^{2} g_{3} G_{1}+\alpha_{7} g_{3}^{4}=0,
\end{aligned}
$$

wo die $\alpha, \beta$ rationale Zahlen sind. Das Absolutglied ist bis auf einen numerischen Faktor gleich $g_{2}^{4}$, da $G_{1}$ und damit die mit $G_{1}$ gleichberechtigten Formen, d. h. alle acht Wurzeln der Gleichung (44) nur in Nultstellen von $g_{2}$ verschwinden.

Es ist nun zunächst möglich, mit einem Schlage alle sieben Koeffizienten $\alpha$ zu bestimmen, und zwar dadurch, daB man $q=0$ einträgt. Hierbei reduzieren sich $g_{2}, g_{3}, \Delta$ auf ihre Anfangsglieder:

$$
g_{8}=\frac{1}{12}\left(\frac{2 \pi}{\omega_{2}}\right)^{4}, \quad g_{3}=\frac{1}{216}\left(\frac{2 \pi}{\omega_{2}}\right)^{6}, \quad \Delta=0 ;
$$

von den acht Wurzeln $G_{1}$ wird aber die eine $\frac{7}{4}\left(\frac{2 \pi}{\omega_{2}}\right)^{2}$, während die sieben anderen einander gleich und gleich $-\frac{1}{4}\left(\frac{2 \pi}{\omega_{2}}\right)^{2}$ werden. Aus der Invarianz von $G_{1}$ gegenüber $W_{7}$ folgt nämlich:

$$
G_{1}\left(\omega_{2},-\omega_{1}\right)=G_{1}\left(-\frac{i \omega_{1}}{\sqrt{7}},-i \omega_{2} \sqrt{7}\right)=-\frac{1}{4}\left(\frac{2 \pi}{\omega_{2}}\right)^{2},
$$

ein Ausdruck, der gegenüber der Substitution $\omega_{1}^{\prime}=\omega_{1}+\omega_{2}, \omega_{2}^{\prime}=\omega_{2}$ unverändert bleibt und also den gemeinsamen Wert der sieben Wurzeln $G_{1}\left(\omega_{2},-\omega_{1}\right), G_{1}\left(\omega_{2},-\omega_{1}-\omega_{2}\right), \ldots$ für $q=0$ liefert. Man trage nun die Ausdrücke (45) in (44) ein und fordere, daß die entstehende Gleichung die acht angegebenen Wurzeln hat. Man findet die $\alpha$ und schreibt die Gleichung am bequemsten als solche für $2 G_{1}$ :

$$
\begin{gathered}
\left(2 G_{1}\right)^{8}-84 g_{2}\left(2 G_{1}\right)^{6}-3024 g_{3}\left(2 G_{1}\right)^{5}-1890 g_{2}^{2}\left(2 G_{1}\right)^{4}-18144 g_{2} g_{3}\left(2 G_{1}\right)^{3} \\
-\left(3780 g_{2}^{3}+a \Delta\right)\left(2 G_{1}\right)^{2}-1664 g_{2}^{2} g_{3}\left(2 G_{1}\right)-567 g_{2}^{4}=0
\end{gathered}
$$


Zur Bestimmung des einzigen noch unbekannten Koeffizienten $a$ stetzen wir:

$$
\tau=\frac{-5+\sqrt{21}}{2}, \quad \tau^{-1}=\frac{-5-\sqrt{21}}{2}
$$

ein und finden $g_{2}=0$ aus (40), sowie weiter aus (28), (39) und (40):

$$
\frac{\left(2 G_{1}\right)^{3}}{g_{3}}=3 \cdot 56 \sqrt{3}(3 \sqrt{3}+2 \sqrt{7}) .
$$

Andrerseits folgt aus der Gleichung für $G_{1}$ im Falle $g_{2}=0$ :

$$
\frac{\left(2 G_{1}\right)^{6}}{g_{3}^{2}}-3024 \frac{\left(2 G_{1}\right)^{3}}{g_{3}}+27 a=0 .
$$

Da diese Gleichung durch den Wert (46) befriedigt werden muß, so folgt $a=-2^{6} \cdot 49$. Die Gleichung achten Grades für $G_{1}$ beim siebenten Transformationsgrade ist hiernach:

$$
\begin{gathered}
\left(2 G_{1}\right)^{8}-84 g_{2}\left(2 G_{1}\right)^{6}-3024 g_{3}\left(2 G_{1}\right)^{5}-1890 g_{2}^{2}\left(2 G_{1}\right)^{4} \\
-18144 g_{2} g_{3}\left(2 G_{1}\right)^{3}-\left(3780 g_{2}^{3}-3136 \Delta\right)\left(2 G_{1}\right)^{2} \\
-1664 g_{2}^{2} g_{3}\left(2 G_{1}\right)-567 g_{2}^{4}=0
\end{gathered}
$$

4. Transformation $49^{\text {sten }}$ Grades. Das Polygon $\mathbf{T}_{49}$ ist aus dem in Fig. 2, S. 351, dargestellten Polygone $T_{7}$ dadurch gewinnbar, daß man auf das letztere Polygon die Substitutionen $\left(\begin{array}{cc}1, & 0 \\ \pm 7,1\end{array}\right),\left(\begin{array}{cc}1, & 0 \\ \pm 14,1\end{array}\right),\left(\begin{array}{cc}1, & 0 \\ \pm 21,1\end{array}\right)$ ausübt und die sechs so entspringenden Bereiche dem Polygone $T_{7}$ anfügt. $T_{49}$ ragt mit acht Spitzen an die Punkte $\omega=i \infty, 0, \pm \frac{1}{7}, \pm \frac{1}{14}, \pm \frac{1}{21}$ heran, wobei je die beiden von der einzelnen dieser Spitzen auslaufenden Polygonseiten aufeinander bezogen sind und zwar durch die Substitutionen:

$$
\begin{aligned}
& \left(\begin{array}{l}
1,1 \\
0,1
\end{array}\right), \quad\left(\begin{array}{r}
1,0 \\
49,1
\end{array}\right), \quad\left(\begin{array}{r}
6,-1 \\
49,-8
\end{array}\right), \quad\left(\begin{array}{r}
8, \\
-49,-6
\end{array}\right), \quad\left(\begin{array}{c}
13,-1 \\
196,-15
\end{array}\right),\left(\begin{array}{rr}
15, & 1 \\
-196,-13
\end{array}\right), \\
& \left(\begin{array}{c}
20,-1 \\
441,-22
\end{array}\right), \quad\left(\begin{array}{cc}
22, & 1 \\
-441,-20
\end{array}\right) \text {. }
\end{aligned}
$$

Die in (34) gegebene Funktion $\tau(\omega)$ ist im Polygone $T_{49}$ siebenwertig. Die sieben Pole fallen in der Spitze $\omega=0$ zusammen, während in den anderen sieben Spitzen Nullpunkte je erster Ordnung liegen. Durch die Transformation $W_{49}$ des Polygons $\mathbf{T}_{49}$ in sich werden die beiden Spitzen 0 und $i \infty$ ausgetauscht, und ebenso werden die sechs weiteren Spitzen untereinander permutiert. Demnach ist $\tau^{\prime}(\omega)=\tau\left(W_{49}(\omega)\right)$ eine siebenwertige Funktion von $\mathrm{T}_{49}$, die in der Spitze $i \infty$ einen Pol siebenter Ordnung hat und in den übrigen sieben Spitzen je in der ersten Ordnung verschwindet. Der Quotient $\frac{\tau}{\tau^{\prime}}$ ist demnach achtwertig mit einem Pole achter Ordnung bei $\omega=0$ und einem Nullpunkte gleicher Ordnung bei $i \infty$. 
$\mathrm{Da} \mathbf{T}_{49}$ zum Geschlechte 1 gehört, so kann die achte Wurzel des eben genannten Quotienten, da sie auf $\mathbf{T}_{49}$ einwertig sein würde, nicht mehr eine Funktion der $\Gamma_{\psi(49)}$ sein. Wohl aber gilt dies von der vierten Wurzel des Quotienten. Aus (34) und (35) folgt nämlich:

Setzen wir nun:

$$
\tau^{\prime}(\omega)=\tau\left(\frac{-1}{49 \omega}\right)=\frac{49}{\tau(7 \omega)}=\sqrt[6]{\frac{\Delta\left(7 \omega_{1}, \omega_{2}\right)}{\Delta\left(49 \omega_{1}, \omega_{2}\right)}} .
$$

$$
\sigma(\omega)=\sqrt[4]{\frac{\tau}{49 \tau^{\prime}}}=\sqrt[24]{\frac{\Delta\left(49 \omega_{1}, \omega_{2}\right)}{\Delta\left(\omega_{1}, \omega_{2}\right)}},
$$

so gelangen wir zu einer Funktion, die nach S. 339 in der Tat zur Gruppe $\Gamma_{\psi(49)}$ gehört. Ihre eindeutige Erklärung gehe aus der Reihenentwicklung hervor:

$$
\sigma(\omega)=q^{4}+q^{6}+2 q^{8}+3 q^{10}+5 q^{12}+7 q^{14}+11 q^{16}+15 q^{18}+\cdots
$$

Gegenüber der Substitution $W_{49}$ zeigt $\sigma(\omega)$ das Verhalten:

$$
\sigma^{\prime}(\omega)=\sigma\left(\frac{-1}{49 \omega}\right)=\frac{1}{7 \sigma(\omega)} .
$$

Andrerseits folgt aus (48) für $\tau(\omega)$ das Verhalten:

$$
\tau^{\prime}(\omega)=\tau\left(\frac{-1}{49 \omega}\right)=\frac{\tau(\omega)}{49 \sigma(\omega)^{4}} .
$$

Zwischen $\tau$ und $\sigma$ besteht eine algebraische Relation, die entsprechend der Wertigkeit dieser Funktionen in $\tau$ vom zweiten und in $\sigma$ vom siebenten Grade ist. Bei Anordnung nach Potenzen von $\tau$ ist der Koeffizient von $\tau^{2}$ gleich 1 , da die sieben Pole von $\tau$ bei $\omega=0$ und also $\sigma=\infty \mathrm{zu}$ sammenfallen. Man setze demnach an:

$$
\tau^{2}+g_{1}(\sigma) \tau+g_{2}(\sigma)=0,
$$

wo die $g$ ganze Funktionen höchstens siebenten Grades von $\sigma$ sind. Die Absolutglieder von $g_{1}$ und $g_{2}$ verschwinden, da die beiden Nullpunkte von $\sigma$ bei $\omega=i \infty$, d.h. in einem Nullpunkte von $\tau$ zusammenfallen. Übt man $W_{49}$ aus, so geht der Ansatz wegen (50) und (51) über in:

$$
\tau^{2}+7^{2} \sigma^{4} g_{1}\left(\frac{1}{7 \sigma}\right) \tau+7^{4} \sigma^{8} g_{2}\left(\frac{1}{7 \sigma}\right)=0,
$$

so daß man für $g_{1}$ und $g_{2}$ noch die Bedingungen hat:

$$
7^{2} \sigma^{4} g_{1}\left(\frac{1}{7 \sigma}\right)=g_{1}(\sigma), \quad 7^{4} \sigma^{8} g_{2}\left(\frac{1}{7 \sigma}\right)=g_{2}(\sigma) .
$$

Diese Funktionen haben also die Gestalten:

$$
\begin{aligned}
& g_{1}(\sigma)=a_{1} \sigma+a_{2} \sigma^{2}+7 a_{1} \sigma^{3}, \\
& g_{2}(\sigma)=b_{1} \sigma+b_{2} \sigma^{2}+b_{3} \sigma^{3}+b_{4} \sigma^{4}+7 b_{3} \sigma^{5}+7^{2} b_{2} \sigma^{6}+7^{3} b_{1} \sigma^{7} .
\end{aligned}
$$


Die sechs jetzt noch unbekannten Koeffizienten bestimmt man mittelst der Reihe (49) und derjenigen für $\tau$ :

$$
\begin{aligned}
\tau(\omega)=7^{2}\left(q^{2}+4 q^{4}+14 q^{6}+40 q^{8}+\right. & 105 q^{10}+252 q^{12} \\
& \left.+574 q^{14}+1236 q^{16}+\cdots\right) .
\end{aligned}
$$

Zwischen den Funktionen $\tau$ und $\sigma$ des Polygons $\mathbf{T}_{49}$ besteht die algebraische Relation:

$$
\begin{array}{r}
\tau^{2}-7^{3} \tau\left(\sigma+5 \sigma^{2}+7 \sigma^{3}\right)-7^{4}\left(\sigma+7 \sigma^{2}+3 \cdot 7 \sigma^{3}+7^{2} \sigma^{4}+3 \cdot 7^{2} \sigma^{5}\right. \\
\left.+7^{3} \sigma^{6}+7^{3} \sigma^{7}\right)=0
\end{array}
$$

Als Ersatz der Transformationsgleichung von $J(\omega)$ für den $49^{\text {sten }}$ Grad können wir nun die Gleichung (41), die entsprechende Gleichung zwischen $J^{*}$ und $\tau^{\prime}$, die Relation $49 \sigma^{4} \tau^{\prime}=\tau$ und die Gleichung (53) ansehen. Durch Elimination von $\tau, \tau^{\prime}$ und $\sigma$ würde die fragliche Gleichung gewinnbar sein.

Übrigens hat man in $\sigma$ und $\tau$ noch nicht die einfachsten Funktionen der Polygone $\mathbf{T}_{49}$ und $\mathbf{K}_{49}$ erhalten. Aus (50) und (51) folgt, daB:

$$
\bar{\tau}=\frac{\tau}{7^{3} \sigma^{2}}, \quad \bar{\sigma}=\sigma+\frac{1}{7 \sigma}
$$

Funktionen von $K_{49}$ sind, und zwar ist $\bar{\tau}$ dreiwertig mit einem Pole dritter Ordnung bei $i \infty$ und $\bar{\sigma}$ zweiwertig mit einem Pole zweiter Ordnung ebenda. Aus (53) folgt als Relation zwischen $\bar{\sigma}$ und $\bar{\tau}$ :

$$
\bar{\tau}^{2}-\bar{\tau}(7 \bar{\sigma}+5)-7 \bar{\sigma}^{2}(\bar{\sigma}+1)+1=0,
$$

eine Gleichung, die man auch in die Gestalt kleiden kann:

$$
7(\bar{\sigma}-1)(\bar{\sigma}+1)^{2}=(\bar{\tau}+1)^{2}-7(\bar{\tau}+1)(\bar{\sigma}+1) .
$$

Erklärt man nun eine neue Funktion $\tau_{0}(\omega)$ des Klassenpolygons $K_{49}$ durch:

$$
\tau_{0}=\frac{\bar{\tau}+1}{\bar{\sigma}+1}
$$

die in der Spitze $i \infty$ einen Pol erster Ordnung hat, so folgt aus (55) und (56):

$$
\left\{\begin{array}{l}
7 \bar{\sigma}=\tau_{0}^{2}-7 \tau_{0}+7 \\
7 \bar{\tau}=\tau_{0}^{3}-7 \tau_{0}^{2}+14 \tau_{0}-7 .
\end{array}\right.
$$

$\mathrm{Da}$ hieraus hervorgeht, daB $\tau_{0}$ keinen weiteren $\mathrm{Pol}$ in $\mathbf{K}_{49}$ hat, so haben wir in $\tau_{0}(\omega)$ eine einwertige Funktion des Klassenpolygons $\mathbf{K}_{49}$ gewonnen. Bei Auflösung der Gleichung:

$$
7 \sigma^{2}-\left(\tau_{0}^{2}-7 \tau_{0}+7\right) \sigma+1=0
$$

nach $\sigma$ stellt sich, dem Geschlechte 1 von $\mathbf{T}_{49}$ entsprechend, die Quadratwurzel einer ganzen Funktion vierten Grades von $\tau_{0}$ ein. Diese Wurzel liefert uns in der Gestalt: 


$$
\sigma_{0}=\sqrt{\tau_{0}^{4}-14 \tau_{0}^{3}+63 \tau_{0}^{2}-98 \tau_{0}+21}
$$

eine Funktion der $\Gamma_{\psi(49)}$, die durch die Festsetzung $\lim _{\omega=i \infty}\left(\sigma_{0} \tau_{0}^{-2}\right)=1$ eindeutig erklärt sein mag. In $\tau_{0}$ und $\sigma_{0}$ haben wir dann die einfachsten Funktionen des Polygons $\mathrm{T}_{49}$ vom Geschlechte 1 erhalten. Die Berechnung der zugehörigen Invarianten (nach I, 121) zeigt, dab hier ein elliptisches Gebilde der absoluten Invariante $J=-\frac{5^{8}}{2^{6}}$ vorliegt.

Die Funktionen $\sigma$ und $\tau$ berechnen sich aus $\tau_{0}$ und $\sigma_{0}$ so:

$$
\left\{\begin{aligned}
14 \sigma & =\tau_{0}^{2}-7 \tau_{0}+7+\sigma_{0}, \\
4 \tau & =\left(\tau_{0}^{3}-7 \tau_{0}^{2}+14 \tau_{0}-7\right)\left(\tau_{0}^{2}-7 \tau_{0}+7+\sigma_{0}\right)^{2} .
\end{aligned}\right.
$$

Die vier Nullpunkte von $\sigma_{0}$ in $T_{49}$ liefern die Nullpunkte der repräsentierenden Formen für die vier Formklassen der Diskriminante $D=-196$.

\section{\$4. Primzahlige Transformationsgrade der Gestalt $n=4 h+3$.}

1. Transformation elften Grades. Die eine bei der Diskriminante $D=-11$ auftretende Formklasse kann durch die reduzierte Form $(1,1,3)$ repräsentiert werden. Man hat also für die Gruppe $\Gamma_{\psi(11)}$, dem Ansatze (9) S. 326 entsprechend, eine Modulform $y_{0}$, die kurz $y$ heiße und die Reihenentwicklung zuläBt:

$$
y=\frac{2 \pi}{\omega_{2}}\left(1+2 q^{2}+4 q^{6}+2 q^{8}+4 q^{10}+\cdots\right) .
$$

Von den drei nach S. 334 anzusetzenden Formen $z_{0}$ verschwindet die erste identisch, während die beiden anderen sich nur im Vorzeichen unterscheiden. Die durch 2 geteilte zweite Form $z_{0}$ heiße kurz $z$; sie hat die Reihenentwicklung:

$$
z=\frac{2 \pi}{\omega_{2}}\left(q-q^{3}-q^{5}+q^{11}+q^{15}-q^{23}-\cdots\right) .
$$

Die Formen $y$ und $z \sqrt{\Delta}$ gehören zur elften Stufe und nehmen gegenüber einer Substitution der $\Gamma_{\psi(11)}$ den Faktor $\left(\frac{\alpha}{11}\right)$ an.

Die Gesamtordnung des Verschwindens sowohl von $y$ als von $z$ im Polygone $T_{11}$ ist 1. Da $\Gamma_{\psi(11)}$ keine elliptische Substitution enthält und $y$ in der einen bei $\omega=i \infty$ gelegenen Spitze nicht verschwindet, so hat $y$ an einer von dieser Spitze verschiedenen Stelle von $T_{11}$ einen Nullpunkt erster Ordnung. Dagegen verschwindet $z$ zufolge (2) in der Spitze $i \infty$ in der Ordnung $\frac{1}{2}$; es bleibt dann nur noch ein Nullpunkt in der gleichen Ordnung $\frac{1}{2}$ über, der nur in der zweiten Spitze von $T_{11}$, d. h. bei $\omega=0$ liegen kann. 
404 II, 4. Transformationsgleichungen erster Stufe für niedere Grade $n$

Die Substitution $W_{11}$ transformiere $y$ und $z$ in $y^{\prime}$ und $z^{\prime}$. Die Formen $y^{\prime}$ und $z^{\prime} \sqrt{\Delta}$ nehmen gegenüber einer Substitution der $\Gamma_{\psi(11)}$ den Faktor $\left(\frac{\delta}{11}\right)$ an, der wegen $\alpha \delta \equiv 1(\bmod 11)$ gleich $\left(\frac{\alpha}{11}\right)$ ist. Somit ergibt sich bei der Lage der Nullpunkte von $z$ im Quotienten $\frac{z^{\prime}}{z}$ eine von Nullpunkten und Polen freie Funktion der $\Gamma_{\psi(11)}$, die also eine Konstante ist. Aber auch der Quotient $\frac{y^{\prime}}{y}$ ist eine Konstante, da dieser Quotient höchstens eine einwertige Funktion auf dem Polygone $\mathbf{T}_{11}$ des Geschlechtes 1 sein könnte. Da $W_{11}$ die Periode 2 hat, so schließen wir auf das Bestehen der Gleichungen $y^{\prime}= \pm y, z^{\prime}= \pm z$. Indem wir dem auf der imaginären $\omega$-Achse liegenden Fixpunkte von $W_{11}$ entsprechend $\omega_{1}=i, \omega_{2}=\sqrt{11}$ eintragen, ergibt sich die Gültigkeit des oberen Zeichens, da weder $y$ (zufolge der Reihe (1)) noch $z$ in diesem Fixpunkte verschwindet. Die beiden Formen $(-1)^{\text {ter }}$ Dimension $y$ und $z$ sind gegenïber $W_{11}$ invariant:

(3) $y\left(\frac{i \omega_{2}}{\sqrt{11}},-i \omega_{1} \sqrt{11}\right)=y\left(\omega_{1}, \omega_{2}\right), z\left(\frac{i \omega_{2}}{\sqrt{11}},-i \omega_{1} \sqrt{11}\right)=z\left(\omega_{1}, \omega_{2}\right)$.

Die Form $y$ hat notwendig ihren Nullpunkt in einem Fixpunkte von $W_{11}$. Die beiden in Fig. 10, S. 364, mit $e_{0}$ und $e_{0}^{\prime}$ bezeichneten, symmetrisch liegenden Punkte können hierbei nicht in Betracht kommen, da $y$ (wegen (1)) entweder in beiden Punkten zugleich oder in keinem von beiden verschwindet. Also folgt der Satz: Der Nullpunkt der Modulform $y$ ist der in Fig. 10 mit $e_{1}$ bezeichnete Nullpunkt $\omega=\frac{-\sqrt{11}+i}{2 \sqrt{11}}$ der quadratischen Form $(11,11,3)$.

Man hat nun in $\left(a y^{2}+b z^{2}\right)$ eine zum Klassenpolygone $\mathbf{K}_{11}$ gehörende Formenschar mit einem beweglichen Nullpunkte, die der Darstellung der übrigen Formen zugrunde zu legen ist. Dies mag zunächst für die zu $n=11$ gehörende Form:

$$
G_{1}=\frac{11}{12}\left(\frac{2 \pi}{\omega_{2}}\right)^{2}\left(5+12 q^{2}+36 q^{4}+48 q^{6}+84 q^{8}+72 q^{10}+\cdots\right)
$$

geprüft werden. Die beiden Anfangsglieder der Reihen liefern:

$$
12 G_{1}=11\left(5 y^{2}-8 z^{2}\right) \text {. }
$$

Dieses Ergebnis kann zu einer Bestätigung der bisher entwickelten Schlüsse dienen. Indem man nämlich rechts für $y$ und $z$ die Reihen (1) und (2) einträgt, müssen sich auch die weiter in der Reihe für $G_{1}$ angegebenen Koeffizienten wiederfinden, was in der Tat der Fall ist.

Weiter ist $\left(g_{2}^{\prime}+g_{2}\right)$ eine ganze homogene Funktion zweiten Grades von $y^{2}$ und $z^{2}$, sowie $\left(g_{2}^{\prime}-g_{2}\right)^{2}$ eine ebensolche Funktion vierten Grades. Die vier Nullpunkte der letzteren in $K_{11}$ sind die vier unter (13) S. 364 
genannten, in Fig. 10 daselbst mit $e_{0}, e_{0}^{\prime}, e_{1}, e_{3}$ bezeichneten Ecken des Polygons $K_{11}$. Da $y$ in der Ecke $e_{1}$ verschwindet, so hat die fragliche Funktion vierten Grades den Faktor $y^{2}$. Die Reihenentwicklungen ergeben die Koeffizienten:

$$
\left\{\begin{array}{l}
6\left(g_{2}^{\prime}+g_{2}\right)=61 y^{4}-2^{4} \cdot 23 y^{2} z^{2}+2^{5} \cdot 11 z^{4} \\
\left(g_{2}^{\prime}-g_{2}\right)^{2}=100 y^{2}\left(y^{6}-2^{2} \cdot 5 y^{4} z^{2}+2^{3} \cdot 7 y^{2} z^{4}-2^{2} \cdot 11 z^{6}\right) .
\end{array}\right.
$$

Die Quadratwurzel des in der letzten Gleichung rechts stehenden Ausdrucks, der die „Verzweigungsform“ (vgl. I,119) für eine unten zu nennende Riemannsche Fläche mit vier Verzweigungspunkten liefert, hat in den vier Punkten $e_{0}, e_{0}^{\prime}, e_{1}, e_{3}$ des Polygons $\mathrm{T}_{11}$ einfache Nullpunkte und liefert eine Modulform $(-4)^{\text {ter }}$ Dimension:

$$
f_{2}=y \sqrt{y^{6}-2^{2} \cdot 5 y^{4} z^{2}+2^{3} \cdot 7 y^{2} z^{4}-2^{2} \cdot 11 z^{6}}
$$

der Gruppe $\Gamma_{\psi(11)}$, die gegenüber $W_{11}$ Zeichenwechsel erfährt. Die Potenzreihe von $f_{2}$ ist:

$$
f_{2}=\left(\frac{2 \pi}{\omega_{2}}\right)^{4}\left(1-2 q^{2}-18 q^{4}-56 q^{6}-146 q^{8}-252 q^{10}-\cdots\right) .
$$

Die Darstellungen von $g_{2}, g_{2}^{\prime}$ durch $y^{2}, z^{2}, f_{2}$ fassen wir zusammen in:

$$
12 g_{2}, 12 g_{2}^{\prime}=61 y^{4}-2^{4} \cdot 23 y^{2} z^{2}+2^{5} \cdot 11 z^{4} \mp 2^{2} \cdot 3 \cdot 5 f_{2} \text {. }
$$

Auf entsprechendem Wege gewinnt man für $g_{3}$ und $g_{3}^{\prime}$ wieder unter Zusammenfassung beider Formeln:

$$
\begin{array}{r}
216 g_{3}, 216 g_{3}^{\prime}=-5 \cdot 7 \cdot 19 y^{6}+2^{4} \cdot 3 \cdot 7 \cdot 23 y^{4} z^{2}-2^{6} \cdot 3 \cdot 7 \cdot 11 y^{2} z^{4} \\
+2^{3} \cdot 7 \cdot 11^{2} z^{6} \pm 2 \cdot 3^{2} f_{2}\left(37 y^{2}-2^{3} \cdot 11 z^{2}\right) .
\end{array}
$$

Nach S. 339 ist $\sqrt[12]{\Delta^{\prime} \Delta}=\sqrt[12]{\Delta\left(11 \omega_{1}, \omega_{2}\right) \Delta\left(\omega_{1}, \omega_{2}\right)}$ gegenüber den Substitutionen der $\Gamma_{\psi(11)}$ invariant. Da man in den beiden Spitzen von $\mathbf{T}_{11}$ je einfache Nullpunkte dieser Form feststellt, so ist sie bis auf einen konstanten Faktor gleich $z^{2}$. Es gilt aber einfach:

$$
\sqrt[24]{\Delta^{\prime} \Delta}=z
$$

wie die Potenzreihen bestätigen. Aus der Invarianz von $\sqrt{\Delta^{\prime} \Delta}$ folgt, daB $\sqrt{\Delta}$ und $\sqrt{\Delta^{\prime}}$ bei den Substitutionen der $\Gamma_{\psi(11)}$ entweder zugleich unverändert bleiben oder zugleich Zeichenwechsel erfahren. Aus der zweiten Formel (9) in I, 453 folgt aber:

$$
\sqrt{\Delta}\left(\frac{i \omega_{2}}{\sqrt{11}},-i \omega_{1} \sqrt{11}\right)=-\sqrt{\Delta}\left(i \omega_{1} \sqrt{11}, \frac{i \omega_{2}}{\sqrt{11}}\right)=11^{3} \cdot \sqrt{\Delta^{\prime}}
$$

Umgekehrt gilt also:

$$
\sqrt{\Delta^{\prime}}\left(\frac{i \omega_{2}}{\sqrt{11}},-i \omega_{1} \sqrt{11}\right)=11^{-3} \sqrt{\Delta}
$$


Jede der beiden Formen $\left(1331 \sqrt{\Delta^{\prime}} \pm \sqrt{\Delta}\right)$ wird also durch die Substitutionen der $\Gamma_{\psi(1)}$, vielleicht vom Zeichen abgesehen, in sich transformiert. Bei Ausübung von $W_{11}$ aber bleibt die erste Form unverändert, während die zweite Zeichenwechsel erfährt. Im Polygone $K_{11}$ hat jede dieser Formen Nullpunkte der Gesamtordnung 3. Je ein Nullpunkt der Ordnung $\frac{1}{2}$ liegt in der Spitze $i \infty$ von $K_{11}$, so daß noch Nullpunkte je in der Ordnung $\frac{5}{2}$ übrig bleiben. Nun verschwindet $\left(1331 \sqrt{\Delta^{\prime}}-\sqrt{\Delta}\right)$ im Punkte $\omega=\frac{i}{\sqrt{11}}\left(\right.$ Punkt $e_{3}$ der Fig. 10, S. 364). Das Quadrat:

$$
\left(1331 \sqrt{\Delta^{\prime}}-\sqrt{\Delta}\right)^{2}=11^{6} \Delta^{\prime}+\Delta-2 \cdot 11^{3} z^{12},
$$

das als homogene ganze Funktion sechsten Grades von $y^{2}$ und $z^{2}$ mit rationalen Zahlenkoeffizienten darstellbar ist, hat hiernach mit der im rationalen Körper irreduzibelen Funktion:

$$
f_{2}^{2} \cdot y^{-2}=y^{6}-2^{2} \cdot 5 y^{4} z^{2}+2^{3} \cdot 7 y^{2} z^{4}-2^{2} \cdot 11 z^{6}
$$

einen Nullpunkt gemein und enthält demnach diese Funktion als Faktor. Da überdies der Faktor $z$ (wegen des Nullpunktes in der Spitze $i \infty$ ) vorliegt, so gilt der Ansatz:

$$
y\left(1331 \sqrt{\Delta^{\prime}}-\sqrt{\Delta}\right)=z f_{2}\left(a y^{2}+b z^{2}\right)
$$

Die Koeffizienten bestimmt man mittelst der ersten Reihenglieder; es gilt:

$$
y\left(\sqrt{\Delta}-1331 \sqrt{\Delta^{\prime}}\right)=z f_{2}\left(y^{2}-11 z^{2}\right) .
$$

Zur Prüfung dieses Ergebnisses berechne man aus ihm den Ausdruck für $y\left(\sqrt{\Delta}+1331 \sqrt{\Delta^{\prime}}\right)$, wobei sich die im Laufe der Rechnung auftretende Quadratwurzel rational ausziehen lassen muB. Dies bestätigt sich in der Tat; man findet:

$$
\sqrt{\Delta}+1331 \sqrt{\Delta^{\prime}}=y z\left(y^{4}-3 \cdot 7 y^{2} z^{2}+2^{3} \cdot 11 z^{4}\right) .
$$

Durch Kombination der beiden letzten Gleichungen folgt:

$$
\left\{\begin{aligned}
2 y \sqrt{\Delta} & =z\left(y^{2}\left(y^{4}-3 \cdot 7 y^{2} z^{2}+2^{3} \cdot 11 z^{4}\right)+f_{2}\left(y^{2}-11 z^{2}\right)\right) \\
2 \cdot 11^{3} \cdot y \sqrt{\Delta^{\prime}} & =z\left(y^{2}\left(y^{4}-3 \cdot 7 y^{2} z^{2}+2^{3} \cdot 11 z^{4}\right)-f_{2}\left(y^{2}-11 z^{2}\right)\right)
\end{aligned}\right.
$$

Man bilde nun die beiden Quotienten:

$$
\tau(\omega)=\frac{y^{2}}{z^{2}}, \quad \sigma(\omega)=\frac{f_{2}}{z^{4}},
$$

von denen der erste eine einwertige Funktion des Klassenpolygons darstellt. $\mathbf{T}_{11}$ wird durch $\tau$ auf eine zweiblättrige Fläche des Geschlechtes 1 abgebildet, für die wir ein Funktionssystem in:

$$
\tau \quad \text { und } \sigma=\sqrt{\tau\left(\tau^{3}-20 \tau^{2}+56 \tau-44\right)}
$$


besitzen. Hier liegt ein elliptisches Gebilde von der absoluten Invariante $J=-\frac{2^{6} \cdot 31^{3}}{3^{3} \cdot 11^{6}}$ vor.

Aus (7), (8) und (11) berechnet sich folgende Darstellung von $J(\omega)$ im Funktionssystem $\sigma, \tau$ :

$$
\begin{aligned}
J:(J-1): 1= & \tau\left(61 \tau^{2}-2^{4} \cdot 23 \tau+2^{5} \cdot 11-2^{2} \cdot 3 \cdot 5 \sigma\right)^{3} \\
& : \tau\left(5 \cdot 7 \cdot 19 \tau^{3}-2^{4} \cdot 3 \cdot 7 \cdot 23 \tau^{2}+2^{6} \cdot 3 \cdot 7 \cdot 11 \tau\right. \\
& \left.-2^{3} \cdot 7 \cdot 11^{2}-2 \cdot 3^{2} \sigma\left(37 \tau-2^{3} \cdot 11\right)\right)^{2} \\
& : 2^{4} \cdot 3^{3}\left(\tau\left(\tau^{2}-3 \cdot 7 \tau+2^{3} \cdot 11\right)+\sigma(\tau-11)\right)^{2} .
\end{aligned}
$$

Die Gleichung für die transformierte Funktion $J^{\prime}$ geht hieraus durch Zeichenwechsel von $\sigma$ hervor. Beide Gleichungen im Verein mit der Relation (13) ersetzen uns die Transformationsgleichung für $J(\omega)$.

Beim elften Grade gibt es eine Transformationsgleichung für:

$$
f=11 z^{2}=11 \sqrt[12]{\Delta\left(11 \omega_{1}, \omega_{2}\right) \cdot \Delta\left(\omega_{1}, \omega_{3}\right)}
$$

deren Gestalt unter (7) S. 343 angesetzt ist. Man könnte diese Gleichung durch Eliminationen aus den entwickelten Relationen gewinnen. Doch ist es leichter, direkt an den eben genannten allgemeinen Ansatz anzuknüpfen und die noch unbekannten numerischen Koeffizienten aus den Reihenentwicklungen zu bestimmen. Für die Form (15) hat man zunächst die Reihe:

$$
f\left(\omega_{1}, \omega_{2}\right)=11\left(\frac{2 \pi}{\omega_{2}}\right)^{2}\left(q^{2}-2 q^{4}-q^{6}+2 q^{8}+q^{10}+2 q^{12}-\cdots\right) .
$$

Aus der Invarianz von $z$ gegenüber $W_{11}$ folgt:

$$
f\left(\frac{i \omega_{2}}{\sqrt{11}},-i \omega_{1} \sqrt{11}\right)=11 z\left(\omega_{1}, \omega_{2}\right)^{2}
$$

sowie, falls $\operatorname{man} \frac{\omega_{1}}{i \sqrt{11}}, \frac{\sqrt{11} \omega_{2}}{i}$ an Stelle von $\omega_{1}, \omega_{2}$ einträgt:

$$
f\left(\omega_{2},-\omega_{1}\right)=11 z\left(\frac{\omega_{1}}{i \sqrt{11}}, \frac{\sqrt{11}}{i} \omega_{2}\right)^{2}=-z\left(\frac{\omega_{1}}{11}, \omega_{2}\right)^{2}
$$

Die Form $f\left(\omega_{2},-\omega_{1}\right)$, die gleichfalls eine Lösung der gesuchten Transformationsgleichung ist, hat hiernach die Potenzreihe:

$$
f\left(\omega_{2},-\omega_{1}\right)=-\left(\frac{2 \pi}{\omega_{2}}\right)^{2}\left(q^{\frac{2}{11}}-2 q^{\frac{4}{11}}-q^{\frac{6}{11}}+2 q^{\frac{8}{11}}+q^{\frac{10}{11}}+2 q^{\frac{12}{11}}-\cdots\right) .
$$

Man trägt nun in den mehrfach genanuten A.nsatz (7) (S. 343) zweckmäBig $12 g_{2}$ und $216 g_{3}$ an Stelle von $g_{2}$ und $g_{3}$ ein, damit die Anfangskoeffizienten der Reihen für diese Produkte gleich 1 sind. Das vorletzte Glied der gesuchten Gleichung bestimmt sich dann aus dem Anfangsgliede der Reihe (17), die übrigen Glieder findet man aber leicht durch 
408 II, 4. Transformationsgleichungen erster Stufe für niedere Grade $n$

Vermittlung der Reihe (16).: Die beim elften Grade auftretende Transformationsgleichung für die Form (15) ist:

$$
\begin{array}{r}
f^{12}-2 \cdot 3^{2} \cdot 5 \cdot 11 \Delta f^{6}+2^{3} \cdot 5 \cdot 11\left(12 g_{2}\right) \Delta f^{4}-3 \cdot 5 \cdot 11\left(216 g_{3}\right) \Delta f^{3} \\
+2 \cdot 3 \cdot 11\left(12 g_{2}\right)^{2} \Delta f^{2}+\left(12 g_{2}\right)\left(216 g_{3}\right) \Delta f-11 \Delta^{2}=0
\end{array}
$$

2. Transformation $19^{\text {ten }}$ Grades. Die zur linken Seite der ima-

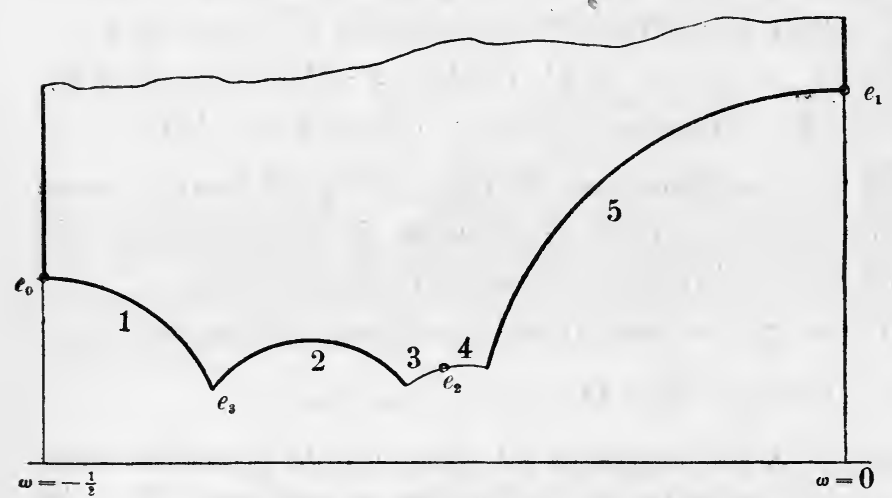

Fig. 16.

ginären $\omega$-Achse liegende Hälfte des Klassenpolygons $K_{19}$ ist in Fig. 16 abgebildet. Neben den beiden geradlinigen Seiten, die auch schon Symmetrielinien des Transformationspolygons

$\mathbf{T}_{19}$ sind, kommen noch die drei in Fig. 16 mit 1, 2 und 5 bezeichneten Symmetriekreise hinzu, von denen der letzte zur Spiegelung $\bar{W}_{19}$ gehört, während die beiden ersten die Gleichungen:

$$
38\left(\xi^{2}+\eta^{2}\right)+38 \xi+9=0, \quad 57\left(\xi^{2}+\eta^{2}\right)+38 \xi+6=0
$$

haben und zu den in der $\bar{\Gamma}^{(19)}$ enthaltenen Spiegelungen gehören:

$$
\omega^{\prime}=\frac{-19 \bar{\omega}-9}{38 \bar{\omega}+19}, \quad \omega^{\prime}=\frac{-19 \bar{\omega}-6}{57 \bar{\omega}+19} .
$$

Bei der Diskriminante $D=-19$ gibt es nur eine Formklasse mit der reduzierten Form $(1,1,5)$. Die nach Vorschrift von S. 362 der Klasse entnommene Form $(19,19,5)$ hat als Nullpunkt die in Fig. 16 mit $e_{0}$ bezeichnete, bei $\omega=\frac{-\sqrt{19}+i}{2 \sqrt{19}}$ gelegene Ecke. Nach S. 148 gehören zur Diskriminante $D=-76$ drei Formklassen, und zwar neben der Hauptklasse zwei entgegengesetze Klassen mit den reduzierten Formen $(4, \pm 2,5)$. Der in Fig. 16 mit $e_{1}$ bezeichnete Eckpunkt ist der Nullpunkt der in der Hauptklasse enthaltenen Form $(19,0,1)$. Der bei $\omega=\frac{-\sqrt{19}+i}{4 \sqrt{19}}$ gelegene Punkt $e_{2}$ ist der Fixpunkt der elliptischen Substitution der Periode zwei $\left(\begin{array}{rr}-19, & -5 \\ 76 & 19\end{array}\right)$ der $\Gamma^{(19)}$ und zugleich der Nullpunkt der in der einen der beiden entgegengesetzten Klassen enthaltenen Form $(76,38,5)$; durch jene Substitution werden die Seiten 3 und 4 der Fig. 16 ineinander transformiert. Der bezüglich der imaginären $\omega$-Achse mit $e_{2}$ symmetrische 
Punkt $e_{8}^{\prime}$ gehört der anderen der beiden entgegengesetzten Klassen an. Der Punkt $e_{3}$, der bei $\omega=\frac{-15+i \sqrt{3}}{38}$ gelegen ist, bildet mit dem symmetrischen Punkte $e_{3}^{\prime}$ einen Eckenzyklus des Klassenpolygons $\mathrm{K}_{19}$. Am Transformationspolygon aber stehen diese Ecken je für sich und sind die Fixpunkte zweier in der $\boldsymbol{\Gamma}_{\psi(19)}$ enthaltenen elliptischen Substitutionen der Periode drei $\left(\begin{array}{c}7, \pm 1 \\ \mp 57,-8\end{array}\right)$. Sie liefern für die über der $J$-Ebene lagernden Transformationsfläche $F_{19}$ die beiden bei $J=0$ isoliert verlaufenden Blätter, deren Auftreten aus der Abzählung von S. 356 hervorgeht.

Die analytischen Ansätze von S. 326 ff. gestalten sich gerade so wie bei $n=11$. Die quadratische Form $(1,1,5)$ der Diskriminante $D=-19$ liefert die Modulform $(-1)^{\text {tor }}$ Dimension:

$$
y=\frac{2 \pi}{\omega_{2}}\left(1+2 q^{2}+2 q^{8}+4 q^{10}+4 q^{14}+2 q^{18}+\cdots\right) .
$$

Von den drei Formen $z_{0}$ verschwindet eine identisch ${ }^{1}$ ), während die beiden anderen sich nur im Vorzeichen unterscheiden. Eine dieser Formen, von dem gemeinsamen Faktor 2 ihrer Reihenkoeffizienten befreit, ist:

$$
z=\frac{2 \pi}{\omega_{q}}\left(q-q^{5}-q^{7}+q^{9}-q^{11}-q^{17}+q^{19}+\cdots\right) .
$$

Dieses $z$ liefert erst im Produkte $z \sqrt{\Delta}$ eine zur $\Gamma_{\psi(19)}$ gehörende Modulform. Gegenüber der einzelnen Substitution $\operatorname{der} \Gamma_{\psi(19)}$ nehmen die beiden Formen $y$ und $z \sqrt{\Delta}$ den Faktor $\left(\frac{\alpha}{19}\right)$ an.

Die Formen $y$ und $z$ haben in $\mathbf{T}_{19}$ Nullpunkte in der Gesamtordnung $\frac{5}{3}$. Die Lage der Nullpunkte von $z$ ist leicht feststellbar. Infolge (20) liegt in der Spitze $i \infty$ im Nullpunkt der Ordnung $\frac{1}{2}$. $\mathrm{Da} z$ in den beiden symmetrischen Punkten $e_{3}, e_{3}^{\prime}$ (wegen der reellen Reihenkoeffizienten) Nullpunkte gleicher Ordnung hat, so muB in jeder dieser Ecken, damit die Gesamtordnung $\frac{5}{3}$ herauskommt, ein Nullpunkt der Ordnung $\frac{1}{3}$ liegen. Der rückständige Nullpunkt der Ordnung $\frac{1}{2}$ liegt in der Spitze $\omega=0$, was durch den Umstand bestätigt wird, daB $z \cdot \sqrt{\Delta}$ gegenüber der Substitution $\left(\begin{array}{l}1,0 \\ 19,1\end{array}\right)$ unverändert bleibt. Die Form $y$ ist bei $\omega=i \infty$ von 0 verschieden und kann (wegen ihrer Invarianz gegenüber der eben genannten Substitution) bei $\omega=0$ höchstens in ganzzahliger Ordnung ver-

1) Das identische Verschwinden steht bereits fest, wenn in der Potenzreihe von $z_{0}$ kein Glied mit einem Exponenten $<4$ von $q$ auftritt. In diesem Falle würde nämlich, falls $z_{0}$ nicht identisch verschwände, bei $\omega=i \infty$ ein Nullpunkt einer Ordnung $\geqq 2$ auftreten, während doch $z_{0}$, als von der Dimension - 1 , in $T_{10}$ nur Nullpunkte in der Gesamtordnung $\frac{5}{3}$ hat. 
schwinden. Damit die Gesamtordnung $\frac{5}{3}$ herauskommt, muß $y$ in $e_{3}$ und $e_{3}^{\prime}$ Nullpunkte der Ordnung $\frac{1}{3}$ haben, so daB noch ein einziger Nullpunkt der Ordnung 1 übrig bleibt. Die funktionentheoretische Überlegung, die schon im Falle $n=11$ ausgeübt wurde, zeigt, daß dieser Nullpunkt notwendig in dem bei $\omega=\frac{-\sqrt{19}+i}{2 \sqrt{19}}$ gelegenen Punkte $e_{0}$ von $T_{19}$ liegt.

Aus der Lage der Nullpunkte folgt weiter, $d a \beta$ y und $z$ gegenüber $W_{19}$ invariant sind. Ein Zeichenwechsel gegenüber $W_{19}$ ist deshalb ausgeschlossen, weil diese Formen im Punkte $e_{1}$ nïht verschwinden.

Wir haben nun wieder in $\left(a y^{2}+b z^{2}\right)$ eine lineare Formenschar mit einem beweglichen Nullpunkte erster Ordnung im Klassenpolygone $\mathbf{K}_{19}$ und übrigens je zwei festen Nullpunkten der Ordnung $\frac{1}{3}$ in $e_{3}$ und $e_{3}^{\prime}$. Die zu $n=19$ gehörende Form $G_{1}$ gehört der Schar an; es gilt nämlich:

$$
4 G_{1}=19\left(3 y^{2}-8 z^{2}\right) \text {. }
$$

Zur Gewinnung derjenigen ganzen homogenen Funktion dritten Grades von $y^{2}$ und $z^{2}$, deren drei Nullpunkte erster Ordnung in $K_{19}$ die zu den drei Formklassen mit $D=-76$ gehörenden Ecken $e_{1}, e_{2}, e_{2}^{\prime}$ sind, kann man so vorgehen: Im Quotienten:

$$
\tau(\omega)=\frac{y^{2}}{z^{2}}=q^{-2}+4+6 q^{2}+\cdots
$$

haben wir eine einwertige Funktion von $K_{19}$, die ihren Pol bei $\omega=i \infty$ hat. Wir verstehen nun unter $(\omega, d \omega)$ das schon in I, 318 eingeführte homogene Differential:

$$
(\omega, d \omega)=\omega_{1} \cdot d \omega_{2}-\omega_{2} \cdot d \omega_{1}=-\omega_{2}^{2} \cdot d \omega,
$$

das gegenüber den homogenen Substitutionen der $\Gamma_{\psi(19)}$ invariant ist und bei $W_{19}$ Zeichenwechsel erfährt. Nennen wir die in (19) und (20) rechts in Klammern stehenden Potenzreiben $\operatorname{kurz} y^{\prime}$ und $z^{\prime}$, so haben wir in:

$$
\frac{d \tau}{(\omega, d \omega)}=-\frac{2 \pi i}{\omega_{2}^{2}} \frac{q y^{\prime}}{z^{\prime 2}}\left(\frac{d y^{\prime}}{d q}-y^{\prime} \frac{d \log z^{\prime}}{d q}\right)
$$

eine Form $(-2)^{\text {ter }}$ Dimension, die gegenüber $W_{19}$ Zeichenwechsel erfährt, und die in den beiden Polygonspitzen $\omega=i \infty$ und 0 je einen Pol erster Ordnung hat. Demnach wird in:

$$
2 i \pi z^{2} \frac{d \tau}{(\omega, d \omega)}=\left(\frac{2 \pi}{\omega_{2}}\right)^{3} q y\left(\frac{d y^{\prime}}{d q}-y^{\prime} \frac{d \log z^{\prime}}{d q}\right)
$$

eine ganze Form $(-4)^{\text {ter }}$ Dimension von $\mathbf{T}_{19}$ gewonnen sein, deren Nullpunkte in der Gesamtordnung $\frac{20}{3}$ nicht in den beiden Spitzen von $T_{19}$ liegen. Die Lage und Ordnung dieser Nullpunkte läBt sich sofort angeben, da die von $z^{2}$ bekannt sind und die des Differentialquotienten in (22) links sich nur an jenen Stellen finden, wo die konforme Beziehung zwischen der 
$\tau$-Ebene und der $\omega$-Halbebene unterbrochen ist, d.h. an den Stellen $e_{0}, e_{1}$, $e_{2}, e_{3}^{\prime}, e_{3}, e_{3}^{\prime}$. In $\mathrm{T}_{19}$ gemessen hat die Form (22) je einen Nullpunkt erster Ordnung in den vier Punkten $e_{0}, e_{1}, e_{2}, e_{2}^{\prime}$ und je einen Nullpunkt der Ordnung $\frac{4}{3}$ an den Stellen $e_{3}, e_{3}^{\prime}$. Demnach hat die Form:

$$
v\left(\omega_{1}, \omega_{2}\right)=-2 i \pi \frac{z^{2}}{y} \frac{d \tau}{(\omega, d \omega)}=\left(\frac{2 \pi}{\omega_{2}}\right)^{3} q\left(y^{\prime} \frac{d \log z^{\prime}}{d q}-\frac{d y^{\prime}}{d q}\right)
$$

in $\mathbf{T}_{19}$ fünf einfache Nullpunkte bei $e_{1}, e_{2}, e_{2}^{\prime}, e_{3}, e_{3}^{\prime}$. Die Bezeichnung $v$ möge darauf hinweisen, daB diese Form mit der Verzweigungsform der dem Polygone $T_{19}$ entsprechenden zweiblättrigen Riemannschen Fläche über der $\tau$-Ebene eng zusammenhängt; die Potenzreihe von $v$ ist:

$$
v=\left(\frac{2 \pi}{\omega_{2}}\right)^{3}\left(1-2 q^{2}-4 q^{4}-14 q^{6}-22 q^{8}-48 q^{10}-\cdots\right) .
$$

Das Quadrat von $v$ ist nun eine Form des Klassenpolygons $\mathbf{K}_{19}$, die drei einfache Nullpunkte in den Ecken $e_{1}, e_{2}, e_{2}^{\prime}$ und einen Nullpunkt zweiter Ordnung im Eckenzyklus $e_{3}, e_{3}^{\prime}$ hat. Somit ist $v^{2}$ als ganze homogene Funktion dritten Grades von $y^{2}$ und $z^{2}$ darstellbar, und zwar, wie die Reihenentwicklungen zeigen, in der Gestalt:

$$
v^{2}=y^{6}-2^{4} y^{4} z^{2}+2^{6} y^{2} z^{4}-2^{2} \cdot 19 z^{6} .
$$

Das Produkt $y^{2} v^{2}$ liefert die Verzweigungsform der eben genannten zweiblättrigen Fläche, auf die $\mathbf{T}_{19}$ durch $\tau(\omega)$ abgebildet wird. Die Quadratwurzel $y v$ dieser Verzweigungsform heiße als ganze Modulform $(-4)^{\text {ter }}$ Dimension $f_{2}\left(\omega_{1}, \omega_{2}\right)$. Wir haben dann in:

$$
\tau \quad \text { und } \quad \sigma=\frac{f_{2}}{z^{4}}=\sqrt{\tau\left(\tau^{3}-2^{4} \tau^{2}+2^{6} \tau-2^{2} \cdot 19\right)}
$$

ein Funktionssystem der fraglichen Fläche. Hier liegt, wie man durch Berechnung der Invarianten feststellt, ein elliptisches Gebilde der absoluten Invariante $J=-\frac{2^{12} \cdot 7^{3}}{3^{3} \cdot 19^{3}}$ vor.

Ehe wir $g_{2}, g_{2}^{\prime}, g_{3}, \ldots$ in $y, z$ und $f_{2}$ darstellen, soll ein bisher schon wiederholt hervorgetretener Satz allgemein bewiesen werden. Die Ecken oder Eckenzyklen von $\mathrm{K}_{n}$, die noch nicht als solche am Polygone $\mathrm{T}_{n}$ auftreten, wurden von den Fixpunkten der elliptischen Substitutionen der Periode 2 in der Nebengruppe $\Gamma_{\psi(n)} \cdot W_{n}$ geliefert und waren übrigens den Formklassen der Diskriminante $D=-4 n$ bzw. der Diskriminanten $D=-n$ und $D=-4 n$ zugeordnet. Es gilt der Satz, daß eine Form $G_{v}$ der geraden Dimension $-2 \nu$, die gegenüber der $\Gamma_{\psi(n)}$ absolut invariant ist, aber gegenüber $W_{n}$ Zeichenwechsel erfährt, in jedem der eben genannten Punkte von $\mathbf{T}_{n}$ einen Nullpunkt hat. Durchläuft $\left(\begin{array}{l}\alpha, \beta \\ \gamma, \delta\end{array}\right)$ die $\Gamma_{\psi(n)}$, d. h. ist $\gamma$ durch $n$ teilbar, und benutzen wir für $W_{n}$ die homogene Schreibweise 
(5) S. 370 der Periode 2, so erhalten wir für $\gamma=n \beta$ die elliptischen Substitutionen von der Periode 2 der Nebengruppe $\Gamma_{\psi(n)} \cdot W_{n}$ in der Gestalt:

$$
\left(\begin{array}{l}
i \beta \sqrt{\bar{n}},-\frac{i \alpha}{\sqrt{n}} \\
i \delta \sqrt{n},-i \beta \sqrt{n}
\end{array}\right), \quad \alpha \delta-n \beta^{2}=1
$$

Der Fixpunkt der einzelnen dieser Substitutionen ist $\frac{\beta \sqrt{n}+i}{\delta \sqrt{n}}$, wo $\delta$ als positiv gelten darf. Der Annahme gemäß besteht nun die Gleichung:

$$
G_{\nu}\left(i \beta \omega_{1} \sqrt{n}-\frac{i \alpha \omega_{2}}{\sqrt{n}}, \quad i \delta \omega_{1} \sqrt{n}-i \beta \omega_{2} \sqrt{n}\right)=-G_{\nu}\left(\omega_{1}, \omega_{2}\right) .
$$

Setzt man hier, dem eben genannten Fixpunkte entsprechend, $\omega_{1}=\beta \sqrt{n}+i$, $\omega_{2}=\delta \sqrt{n}$ ein, so folgt:

$$
G_{v}(-\beta \sqrt{n}-i,-\delta \sqrt{n})=-G_{v}(\beta \sqrt{n}+i, \delta \sqrt{n}),
$$

woraus sich wegen der geraden Dimension von $G_{v}$ das Verschwinden dieser Form an der fraglichen Stelle ergibt.

Das Polygon $\mathbf{T}_{19}$ hat an Punkten der fraglichen Art die vier mit $e_{0}, e_{1}, e_{2}, e_{2}^{\prime}$ bezeichneten. Hier verschwindet die Form $f_{2}$ je einfach. Wenden wir den eben bewiesenen Satz auf $\left(g_{2}^{\prime}-g_{2}\right)$ an, so heben sich die vier Nullpunkte bei $e_{0}, e_{1}, e_{2}, e_{2}^{\prime}$ aus dem Quotienten $\left(g_{2}^{\prime}-g_{2}\right): f_{2}$ fort. Nun hat $f_{2}$ außerdem noch im Polygone $\mathbf{T}_{19}$ in den beiden Ecken $e_{3}, e_{3}^{\prime}$ je einen Nullpunkt der Ordnung $\frac{4}{3}$ und ist übrigens allenthalben von 0 verschieden. Für $\left(g_{2}^{\prime}-g_{2}\right)$ können wir in $e_{3}$ und $e_{3}^{\prime}$ zunächst nur je einen Nullpunkt der Ordnung $\frac{1}{3}$ feststellen. Demnach ist der gegenüber $W_{19}$ invariante Quotient $\left(g_{2}^{\prime}-g_{2}\right): f_{2}$ eine einwertige Funktion von $\mathbf{K}_{19}$ oder eine Konstante, nämlich letzteres, falls der noch übrig bleibende Nullpunkt von $\left(g_{2}^{\prime}-g_{2}\right)$ etwa auch im Eckenzyklus $e_{3}, e_{3}^{\prime}$ liegen sollte. In jedem Falle gilt der Ansatz:

$$
\left(a y^{2}+b z^{2}\right)\left(g_{2}^{\prime}-g_{2}\right)=f_{2}\left(c y^{2}+d z^{2}\right) .
$$

Der erste Faktor links ist diejenige Form unserer linearen Schar, die den beweglichen Nullpunkt im Eckenzyklus $e_{3}, e_{3}^{\prime}$ von $\mathbf{K}_{19}$ hat. Es ist also weder $a$ noch $b$ gleich 0 , und also kann einer dieser Koeffizienten, etwa $a$, gleich 1 gesetzt werden. Die drei anderen Koeffizienten ergeben sich leicht aus den Potenzreihen; man findet:

$$
\left(y^{2}-z^{2}\right)\left(g_{2}^{\prime}-g_{2}\right)=10 f_{2}\left(3 y^{2}-5 z^{2}\right),
$$

so daB $\left(y^{2}-z^{2}\right)$ die Form der Schar ist, die im Eckenzyklus $e_{3}, e_{3}^{\prime}$ von $K_{19}$ einen Nullpunkt der Ordnung $\frac{5}{3}$ hat.

Einfacher ist die Darstellung von $\left(g_{2}^{\prime}+g_{2}\right)$. Man erkennt leicht, daB $\left(y^{2}-z^{2}\right)\left(g_{2}^{\prime}+g_{2}\right)$ eine homogene Funktion dritten Grades von $y^{2}$ 
und $z^{2}$ sein muB, deren Koeffizienten wie üblich aus den Potenzreihen bestimmt werden. Durch Kombination beider Ergebnisse folgen für $g_{2}$ und $g_{2}^{\prime}$ die Darstellungen:

$$
\begin{aligned}
12\left(y^{2}-z^{2}\right) g_{2}, \quad 12\left(y^{2}-z^{2}\right) g_{2}^{\prime} & =181 y^{6}-3 \cdot 503 y^{4} z^{2}+2^{4} \cdot 211 y^{2} z^{4} \\
& -2^{5} \cdot 3 \cdot 19 z^{6} \mp 60 f_{2}\left(3 y^{2}-5 z^{2}\right)
\end{aligned}
$$

Für die Darstellung von $J$ in $\sigma$ und $\tau$ ist es ausreichend, wenn wir neben (28) noch die Ausdrücke von $\Delta$ und $\Delta^{\prime}=\Delta\left(19 \omega_{1}, \omega_{2}\right)$ in $y, z$ und $f_{2}$ kennen. Diesem Zwecke dient folgende Schlußweise. In $\Delta^{\prime} \cdot \Delta$ haben wir eine Form des Polygons $K_{19}$ mit einem Nullpunkte $20^{\text {ster }}$ Ordnung in der Spitze $i \infty$, die sich infolge ihres Anfangskoeffizienten sofort als mit dem Quotienten von $z^{40}$ und $\left(y^{2}-z^{2}\right)^{8}$ identisch erweist, welcher in der Tat in derselben Weise verschwindet. Wir finden bei richtiger Bestimmung der Wurzeln:

$$
\sqrt[8]{\Delta^{\prime} \Delta}=\frac{z^{5}}{y^{2}-z^{2}}, \quad \sqrt[6]{\Delta^{\prime} \Delta}=z^{6} \sqrt[3]{\frac{z^{2}}{\left(y^{2}-z^{2}\right)^{4}}} .
$$

Weiter ist nach den Sätzen von S. $339 \mathrm{ff}$. die sechste Wurzel des Quotienten $\Delta^{\prime}: \Delta$ eine Funktion der $\Gamma_{\psi(19)}$. Hieraus folgt, daß jeder der beiden Ausdrücke $\left(\sqrt[6]{\Delta} \pm 19 \sqrt[6]{\Delta^{\prime}}\right)$ gegenüber den Substitutionen der $\Gamma^{(19)}$ bis auf multiplikative sechste Einheitswurzeln invariant ist. Nun liegt zunächst für die Form $\left(\sqrt[6]{\Delta}-19 \sqrt[6]{\Delta^{\prime}}\right)$ ein Nullpunkt der Ordnung $\frac{1}{6}$ in der Spitze $i \infty$. Es bleiben Nullpunkte der Gesamtordnung $\frac{3}{2}$ übrig, und da die fragliche Form im Punkte $e_{1}$ verschwindet, während andrerseits die Funktion dritten Grades (25) von $y^{2}$ und $z^{2}$, deren einer Nullpunkt $e_{1}$ ist, im rationalen Körper irreduzibel ist, so sind die beiden anderen Nullpunkte je der Ordnung $\frac{1}{2}$ von $\left(\sqrt[6]{\Delta}-19 \sqrt[6]{\Delta^{\prime}}\right)$ die Punkte $\left.e_{2}, e_{2}^{\prime} \cdot{ }^{1}\right)$ Hiernach ist der Quotient von $z^{2} v^{6}$ und $\left(\sqrt[6]{\Delta}-19 \sqrt[6]{\Delta^{\prime}}\right)^{6}$ eine ganze Form des Polygons $K_{19}$, die nur noch im Eckenzyklus $e_{3}, e_{3}^{\prime}$ verschwindet. Infolge des Anfangsgliedes der Potenzreihe ist sie einfach gleich $\left(y^{2}-z^{2}\right)^{4}$. Wir haben also wieder bei richtiger Bestimmung der Wurzel das Ergebnis:

$$
\sqrt[6]{\Delta}-19 \sqrt[6]{\Delta^{\prime}}=v \sqrt[3]{\frac{z}{\left(y^{2}-z^{2}\right)^{2}}}
$$

Die Schlußweise gestattet eine Prüfung, indem man aus (30) und der Jweiten Formel (29) den Ausdruck für $\left(\sqrt[6]{\Delta}+19 \sqrt[6]{\Delta^{\prime}}\right)$ berechnet. Es muB sich nämlich die Quadratwurzel aus $\left(v^{2}+76 z^{6}\right)$ in $y$ und $z$ rational darstellen. Dies ist in der Tat der Fall, man gewinnt:

1) Man beachte, daB das Produkt von $\left(y^{2}-z^{2}\right)^{4}$ und der sechsten Potenz von $\left(\sqrt[6]{\Delta}-19 \sqrt[6]{\Delta^{\prime}}\right)$ ganz und numerisch rational in $y^{2}$ und $z^{2}$ darstellbar ist. 
414 II, 4. Transformationsgleichungen erster Stufe für nıeaere Grade $n$

$$
\sqrt[6]{\Delta}+19 \sqrt[6]{\Delta^{\prime}}=y\left(y^{2}-8 z^{2}\right) \sqrt[3]{\frac{z}{\left(y^{2}-z^{2}\right)^{2}}} .
$$

Für $\Delta$ selbst ergibt sich endlich der Ausdruck:

$$
2^{6}\left(y^{2}-z^{2}\right)^{4} \Delta=z^{2}\left(y^{3}-8 y z^{2}+v\right)^{6} .
$$

Geht man jetzt zum Funktionssystem $\sigma, \tau$ von $T_{19}$ zurück, so findet man aus (28) und (32) folgenden Ausdruck für $J(\omega)$ als rationale Funktion von $\sigma$ und $\tau$ :

$$
J=\frac{(\tau-1) \tau^{3}\left(181 \tau^{3}-3 \cdot 503 \tau^{2}+2^{4} \cdot 211 \tau-2^{5} \cdot 3 \cdot 19-60 \sigma(3 \tau-5)\right)^{8}}{27\left(\tau^{2}-8 \tau+\sigma\right)^{6}} .
$$

Die Gleichung für $J^{\prime}$ ergibt sich hieraus durch Zeichenwechsel von б. Beide Gleichungen ersetzen uns im Verein mit der Relation (26) die beim $19^{\text {ten }}$

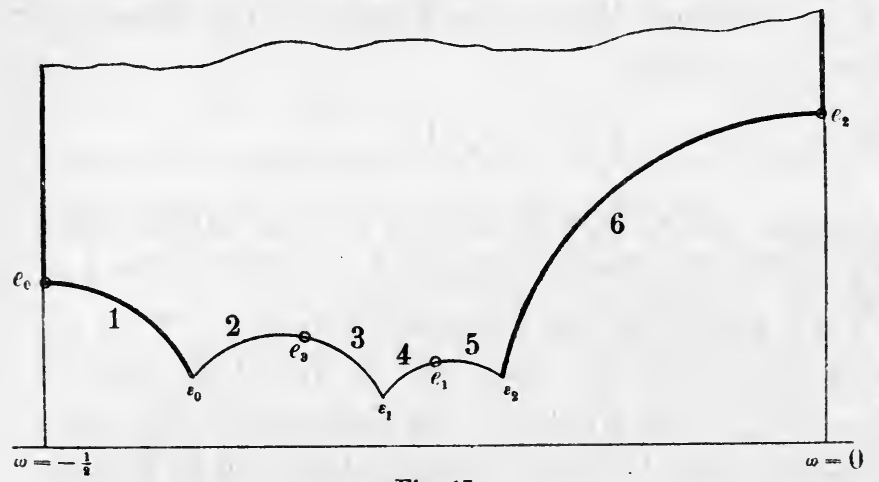

Fig. 17.

Grade auftretende Transformationsgleichungfür $\left.J(\omega){ }^{1}{ }^{1}\right\rangle$

3. Transformation $23^{\text {ten }} \mathrm{Gra-}$ des. Die zur linken Seite der imaginären $\omega$-Achse gelegene Hälfte des Klassenpolygons $K_{23}$ ist in

Fig. 17 dargestellt. Die mit 1 und 6 bezeichneten Kreisbogen sind die Symmetriekreise der Spiegelungen:

$$
\omega^{\prime}=\frac{-23 \bar{\omega}-11}{46 \bar{\omega}+23}, \quad \omega^{\prime}=\frac{1}{23 \bar{\omega}} .
$$

Die Seiten 2 und 3 sind aufeinander bezogen, ebenso 4 und 5 , nämlich durch:

$$
\omega^{\prime}=\frac{-23 \omega-8}{69 \omega+23} \quad \text { bzw. } \quad \omega^{\prime}=\frac{-23 \omega-6}{92 \omega+23},
$$

deren Fixpunkte $e_{3}$ und $e_{1}$ gelegen sind bei:

$$
\omega=\frac{-\sqrt{23}+i}{3 \sqrt{23}} \text { und } \omega=\frac{-\sqrt{23}+i}{4 \sqrt{23}}
$$

Den drei Formklassen der Diskriminante $D=-23$ gehören die Ecken $e_{0}$ (bei $\omega=\frac{-\sqrt{23}+i}{2 \sqrt{23}}$ gelegen), $e_{1}$ und die bezüglich der imaginären $\omega$-Achse symmetrische Ecke $e_{1}^{\prime}$ an. Ebenso entsprechen die Ecken $e_{2}\left(\right.$ bei $\omega=\frac{i}{\sqrt{23}}$

1) Die Transformationsgleichung für die $12^{\text {te }}$ Wurzel der Diskriminante $\Delta$ ist beim $19^{\text {ten }}$ Grade von Kiepert im Journ. f. Math. Bd. 87, S. 216 angegeben. 
gelegen), $e_{3}$ und die symmetrische Ecke $e_{3}^{\prime}$ den drei Formklassen mit $D=-92$. Die drei Ecken $\varepsilon_{0}, \varepsilon_{1}, \varepsilon_{2}$, gelegen bei:

$$
\omega=\frac{-93+i \sqrt{91}}{230}, \frac{-13 \sqrt{7}+i \sqrt{13}}{46 \sqrt{7}}, \frac{-47+i \sqrt{91}}{230},
$$

und die symmetrischen Ecken $\varepsilon_{0}^{\prime}, \varepsilon_{1}^{\prime}$, $\varepsilon_{2}^{\prime}$ bilden für das ganze Polygon $K_{23}$ einen Zyklus. ${ }^{1}$ )

Die drei reduzierten Formen der Diskriminante $D=-23$ sind die Hauptform $(1,1,6)$ und die entgegengesetzten Formen $(2, \pm 1,3)$. Der Ansatz (9) S. 326 liefert zwei verschiedene Modulformen:

$$
\left\{\begin{array}{l}
y=\frac{2 \pi}{\omega_{2}}\left(1+2 q^{2}+2 q^{8}+4 q^{12}+4 q^{16}+2 q^{18}+\cdots\right), \\
y_{1}=\frac{2 \pi}{\omega_{2}}\left(1+2 q^{4}+2 q^{6}+2 q^{8}+2 q^{12}+2 q^{16}+2 q^{18}+\cdots\right) .
\end{array}\right.
$$

Der Ansatz (21) S. 333 liefert für die Hauptform eine identisch verschwindende Reihe und für die beiden entgegengesetzten Formen $(3, \pm 1,2)$ eine Modulform:

$$
z=\frac{2 \pi}{\omega_{\mathrm{z}}}\left(q^{2}-q^{4}-q^{6}+q^{12}+q^{16}-q^{26}-\cdots\right),
$$

die mit $\frac{1}{2}\left(y-y_{1}^{\prime}\right)$ identisch ist. Übrigens gilt einfach:

$$
z=\sqrt[24]{\Delta^{\prime} \Delta}=\sqrt[24]{\Delta\left(23 \omega_{1}, \omega_{2}\right) \cdot \Delta\left(\omega_{1}, \omega_{2}\right)}
$$

wie aus der Gleichheit der beiden ersten Glieder der beiderseitigen Reihenentwicklungen folgt. $\left.{ }^{2}\right)$ Gegenüber einer Substitution der $\Gamma_{\psi(23)}$ nehmen die Formen $y, y_{1}, z$ den Faktor $\left(\frac{\alpha}{23}\right)$ an.

Die Formen $y$ und $z$ haben auf dem Polygone $\mathbf{T}_{23}$ Nullpunkte je in der Gesamtordnung 2. Ihr Quotient:

$$
\tau(\omega)=\frac{y}{z}=q^{-2}+3+4 q^{3}+\cdots
$$

ist demnach eine zweiwertige Funktion von $\mathbf{T}_{23}$, da er nicht konstant ist. und einwertige Funktionen auf diesem Polygone des Geschlechtes 2 nicht vorkommen. Nun gibt es auf einer hyperelliptischen Fläche im wesentlichen, $\mathrm{d}$. $\mathrm{h}$. von linearer Transformation abgesehen, nur eine zweiwertige Funktion. ${ }^{3}$ ) In unserem Falle haben wir einerseits in $\tau(\omega)$, andrerseits.

1) Es handelt sich hier um sogenannte „zufällige“ Ecken (vgl. die „Vorlesungen über automorphe Funktionen", I, 110), die nicht Fixpunkte elliptischer Substitutionen sind; entsprechend ist die Summe der Eckenwinkel gleich $2 \pi$.

2) Vgl. die Note S. 409.

3) Existieren auf einer Riemannschen Fläche zwei zweiwertige Funktionen, die nicht linear miteinander zusammenhängen, so gehen diese beiden Funktionen eine algebraische Beziehung ein, die man leicht als zum Geschlecht $p=0$ oder $p=1$ gehörig erkennt. 
II, 4. Transformationsgleichungen erster Stufe für niedere Grade $n$

in jeder einwertigen Funktion des Klassenpolygons $\mathbf{K}_{23}$ eine zweiwertige Funktion von $\mathbf{T}_{23}$. Also ist $\tau(\boldsymbol{\omega})$ eine einwertige Funktion von $\mathbf{K}_{23}$, und $d a z$ zufolge (36) gegenïber $W_{23}$ invariant ist, so gilt dasselbe von $y$ und also auch von $y_{1}$.

Das Polygon $T_{23}$ wird durch $\tau(\omega)$ auf eine zweiblättrige Riemannsche Fläche mit sechs Verzweigungspunkten abgebildet, die von den Punkten $e_{0}, e_{1}, e_{1}^{\prime}, e_{2}, e_{3}, e_{3}^{\prime}$ herrühren. Die zu dieser hyperelliptischen Fläche gehörende "Verzweigungsform" gewinnen wir wieder durch einen Differentiationsprozeß. Es handelt sich hierbei einfach um eine Wiederholung der Überlegung von S. $410 \mathrm{ff}$. Wir bilden die zur $\Gamma_{\psi(23)}$ gehörende, gegenüber $W_{23}$ Zeichenwechsel erfahrende Form $(-1)^{\text {ter }}$ Dimension:

$$
v=-2 i \pi z \frac{d \tau}{(\omega, d \omega)}=-\frac{1}{2}\left(\frac{2 \pi}{\omega_{z}}\right)^{3} q\left(\frac{d y^{\prime}}{d q}-y^{\prime} \frac{d \log z^{\prime}}{d q}\right),
$$

wo $y^{\prime}$ und $z^{\prime}$ die in der ersten Gleichung (34) und in der Gleichung (35) rechts in Klammern stehenden Potenzreihen sind. Für die Form $v$ findet man die Reihenentwicklung:

$$
\begin{aligned}
v=\left(\frac{2 \pi}{\omega_{2}}\right)^{3}\left(1-q^{2}-5 q^{4}-10 q^{6}\right. & -21 q^{8}-22 q^{10} \\
& \left.-50 q^{12}-44 q^{14}-85 q^{16}-\cdots\right) .
\end{aligned}
$$

Die Ableitung von $\tau$ nach $\omega$ hat zwei Pole erster Ordnung in den Spitzen von $T_{23}$, die aber durch die daselbst liegenden Nullpunkte des Faktors $z$ fortgehoben werden. Andrerseits sind die sechs Nullpunkte erster Ordnung der ganzen Form $v$ die sechs Punkte $e_{0}, e_{1}, e_{1}^{\prime}, e_{2}, e_{3}, e_{3}^{\prime}$, die die Verzweigungspunkte unserer zweiblättrigen Fläche liefern.

Die Verzweigungsform wird durch das Quadrat von $v$ geliefert, das gegenüber $W_{23}$ invariant ist. Man beachte, $d a \beta$ wir für $K_{23}$ jetzt in $(a y+b z)$ eine lineare Formenschar mit einem beweglichen Nullpunkte haben. Demnach ist $v^{2}$ als homogene Funktion sechsten Grades von $y$ und $z$ darstellbar, die dann die gewünschte Verzweigungsform liefert. Die Reihenentwicklungen ergeben:

$$
v^{2}=y^{6}-2 \cdot 7 y^{5} z+3 \cdot 19 y^{4} z^{2}-2 \cdot 53 y^{3} z^{3}+2 \cdot 3^{2} \cdot 5 y^{2} z^{4}-2^{4} y z^{5}-19 z^{6} .
$$

Als Funktionssystem für das Transformationspolygon $\mathbf{T}_{23}$ führen wir nun:

$$
\tau=\frac{y}{z}, \quad \sigma=\frac{v}{z^{3}}
$$

ein, wobei sich die zweite Funktion in der ersten in Gestalt der folgenden Quadratwurzel darstellt:

$$
\sigma=\sqrt{\tau^{6}-14 \tau^{5}+57 \tau^{4}-106 \tau^{3}+90 \tau^{2}-16 \tau-19} .
$$

Dieses Ergebnis ist einer bemerkenswerten Prüfung zugänglich. Durch Nullsetzen der unter dem Wurzelzeichen stehenden Funktion gewinnt man eine Gleichung, deren Lösungen die drei zu den Formklassen 
der Diskriminante $D=-23$ gehörenden Werte $\tau\left(\frac{-\sqrt{23}+i}{2 \sqrt{23}}\right), \tau\left(\frac{\mp \sqrt{23}+i}{4 \sqrt{23}}\right)$ und die drei entsprechend zu $D=-92$ gehörenden $\tau\left(\frac{i}{\sqrt{23}}\right), \tau\left(\frac{\mp \sqrt{23}+i}{3 \sqrt{23}}\right)$ sind. Aus den späteren arithmetischen Anwendungen der elliptischen Funktionen folgt nun, daß jedes dieser Systeme zu je drei Werten selbst einer Gleichung mit rationalen Koeffizienten genügt. Die Gleichung sechsten Grades muB also im rationalen Körper reduzibel sein und zwar in zwei kubische Gleichungen zerfallen. Dies ist in der Tat der Fall; die beiden kubischen Gleichungen sind:

$$
\begin{aligned}
& D=-23, \quad \tau^{3}-3 \tau^{2}+2 \tau+1=0, \\
& D=-92, \quad \tau^{3}-11 \tau^{2}+22 \tau-19=0 .
\end{aligned}
$$

DaB die zweite Gleichung zur Diskriminante - 92 gehört, folgert man aus der Tatsache, daB sie. (aber nicht die erste Gleichung) eine reelle positive Lösung hat (die den Wert $\tau\left(\frac{i}{\sqrt{23}}\right)$ liefert).

Um eine gleich auszuführende Rechnung zu erleichtern, bilden wir uns in:

$$
\left\{\begin{array}{l}
v_{1}=\sqrt{y^{3} z-3 y^{2} z^{2}+2 y z^{3}+z^{4}} \\
v_{2}=\sqrt{y^{3} z-11 y^{2} z^{2}+22 y z^{3}-19 z^{4}}
\end{array}\right.
$$

zwei Modulformen $(-2)^{\text {ter }}$ Dimension, von denen die erste drei Nullpunkte erster Ordnung an den Stellen $e_{0}, e_{1}, e_{1}^{\prime}$ von $\mathrm{T}_{23}$, die zweite ebensolche in den Punkten $e_{2}, e_{3}, e_{3}^{\prime}$ hat. Außerdem hat jede dieser Formen noch einen Nullpunkt der Ordnung $\frac{1}{2}$ in jeder der beiden Polygonspitzen. Durch Eintragung der Reihen für $y$ und $z$ in (43) ergeben sich für $v_{1}$ und $v_{2}$ die beiden Potenzreihen:

$$
\left\{\begin{array}{l}
v_{1}=\left(\frac{2 \pi}{\omega_{2}}\right)^{2}\left(q+q^{3}+2 q^{7}-2 q^{9}+*+\cdots\right), \\
v_{2}=\left(\frac{2 \pi}{\omega_{2}}\right)^{2}\left(q-3 q^{3}-2 q^{5}-4 q^{7}+6 q^{9}+2 q^{11}+\cdots\right),
\end{array}\right.
$$

wo der Stern in der ersten Reihe andeuten soll, daB ein Glied mit $q^{11}$ nicht auftritt. Da die Nullpunkte jeder Form $v_{1}, v_{2}$ durch $W_{23}$ in sich transformiert werden, so wird jede dieser Formen durch $W_{23}$ bis auf einen konstanten Faktor in sich transformiert. Die dabei auftretenden Faktoren können aber wegen der Periode 2 von $W_{93}$ nur gleich \pm 1 sein. Bei $v_{1}$ kann der Faktor -1 nicht auftreten, da $v_{1}$ im Punkte $e_{2}$ nicht verschwindet. Nehmen wir noch hinzu, daB $v_{1} \cdot v_{2}=z v$ gegenüber $W_{23}$ Zeichenwechsel erfährt, so folgt: Gegenüber $W_{23}$ ist $v_{1}$ invariant und $v_{2}$ erfährt Zeichenwechsel. 
Die Darstellung von $J$ durch $\sigma$ und $\tau$ bahnen wir jetzt wieder dadurch an, daß wir $g_{2}$ und $\Delta$ durch die Formen $y, z, \ldots$ darstellen. $\mathrm{Zu}$ nächst bietet $g_{2}$ keine Schwierigkeit; $\left(g_{2}^{\prime}+g_{2}\right)$ ist eine homogene Funktion vierten Grades von $y$ und $z,\left(g_{2}^{\prime}-g_{2}\right)$ ist das Produkt von $v$ und einer linearen Funktion von $y$ und $z$. Die Reihenentwicklungen liefern die Darstellungen:

$$
\left\{\begin{array}{l}
6\left(g_{2}^{\prime}+g_{2}\right)=5\left(53 y^{4}-2^{4} \cdot 5^{2} y^{3} z+2^{4} \cdot 59 y^{2} z^{2}-2^{4} \cdot 3 \cdot 19 y z^{3}+2^{5} \cdot 3^{2} z^{4}\right) \\
6\left(g_{2}^{\prime}-g_{2}\right)=2^{5} \cdot 3^{2} v\left(11 y-2^{4} z\right)
\end{array}\right.
$$

Da $\sqrt{\Delta^{\prime} \Delta}$ gegenüber der $\Gamma_{\psi(23)}$ invariant ist, so bleiben $\sqrt{\Delta}$ und $\sqrt{\Delta^{\prime}}$ bei der einzelnen Substitution dieser Gruppe stets zugleich unverändert oder erleiden zugleich Zeichenwechsel. Demnach sind $\left(\sqrt{\Delta} \pm 12167 \sqrt{\Delta^{\prime}}\right)$ Formen, die gegenüber der $\Gamma_{\psi(23)}$ bis auf Zeichenwechsel invariant sind; gegenüber $W_{23}$ ist die erste Form invariant, die zweite erfährt Zeichenwechsel. Das Produkt der beiden Formen enthält den Faktor $v$ (vgl. S. 411) und auch den Faktor $z$ (wegen der Nullpunkte in den Polygonspitzen); man kann demnach auch sagen, es enthalte den Faktor $v_{1} \cdot v_{2}$. Nun hat $\left(\sqrt{\Delta}-12167 \sqrt{\Delta^{\prime}}\right)$ mit der Form $v_{2}$ den Nullpunkt $e_{2}$ gemein. Da aber $\left(\sqrt{\Delta}-12167 \sqrt{\Delta^{\prime}}\right)^{2}$ eine ganze Funktion der $y$, $z$ mit rationalen Koeffizienten ist und $v_{2}^{2}$ eine im rationalen Körper irreduzible ganze Funktion von $y$ und $z$ ist, so enthält $\left(\sqrt{\Delta}-12167 \sqrt{\Delta^{\prime}}\right)$ den Faktor $v_{2}$. Den Faktor $v_{1}$ kann sie nicht auch noch enthalten, da sonst $\left(\sqrt{\Delta}-12167 \sqrt{\Delta^{\prime}}\right)$ in $y$ und $z$ rational wäre, was wegen des Nullpunktes der Ordnung $\frac{1}{2}$ bei $\omega=i \infty$ nicht möglich ist. Somit sind die Formen $\left(\sqrt{\Delta} \pm 12167 \sqrt{\Delta^{\prime}}\right)$ als Produkte von $v_{1}$ und $v_{2}$ mit ganzen homogenen Funktionen vierten Grades von $y$ und $z$ darstellbar. Die Potenzreihen ergeben:

$$
\left\{\begin{aligned}
\sqrt{\Delta}+12167 \sqrt{\Delta^{\prime}}= & v_{1}\left(y^{4}-3 \cdot 7 y^{3} z+2^{2} \cdot 37 y^{2} z^{2}\right. \\
& \left.-2^{2} \cdot 5 \cdot 19 y z^{3}+2^{2} \cdot 53 z^{4}\right) \\
\sqrt{\Delta}-12167 \sqrt{\Delta^{\prime}}= & v_{2}\left(y^{4}-17 y^{3} z+2 \cdot 3^{2} \cdot 5 y^{2} z^{2}-2 \cdot 71 y z^{3}-2 \cdot 7 z^{4}\right)
\end{aligned}\right.
$$

Eine Prüfung der vollzogenen Schlußweise kann man dadurch anstellen, daB man die Differenz der Quadrate der hier rechts stehenden Ausdrücke bildet, die sich zufolge (36) auf $4 \cdot 23^{3} z^{12}$ zusammenziehen muß.

An die Stelle der etwas umständlichen Gleichung für $J$ als rationale Funktion von $\sigma$ und $\tau$ lassen wir die beiden Gleichungen treten:

$$
\left\{\begin{array}{r}
12 g_{2} z^{-4}=5\left(53 \tau^{4}-400 \tau^{3}+944 \tau^{2}-912 \tau+288\right)-288 \sigma(11 \tau-16) \\
2 \Delta z^{-12}-2 \cdot 23^{3}=\left(\tau^{4}-17 \tau^{3}+90 \tau^{2}-142 \tau-14\right) \\
\left(\left(\tau^{3}-11 \tau^{2}+22 \tau-19\right)\left(\tau^{4}-17 \tau^{3}+90 \tau^{2}-142 \tau-14\right)\right. \\
\left.+\sigma\left(\tau^{4}-21 \tau^{3}+148 \tau^{2}-380 \tau+212\right)\right)
\end{array}\right.
$$


Sie bilden mit der Gleichung (42) in bekannter Weise den Ersatz der Transformationsgleichung für $J(\omega)$ beim 23. Grade. ${ }^{1}$ )

4. Transformation $31^{\text {sten }}$ Grades. Die zur linken Seite der imaginären $\omega$-Achse gelegene Hälfte des Klassenpolygons $K_{31}$ hat die in

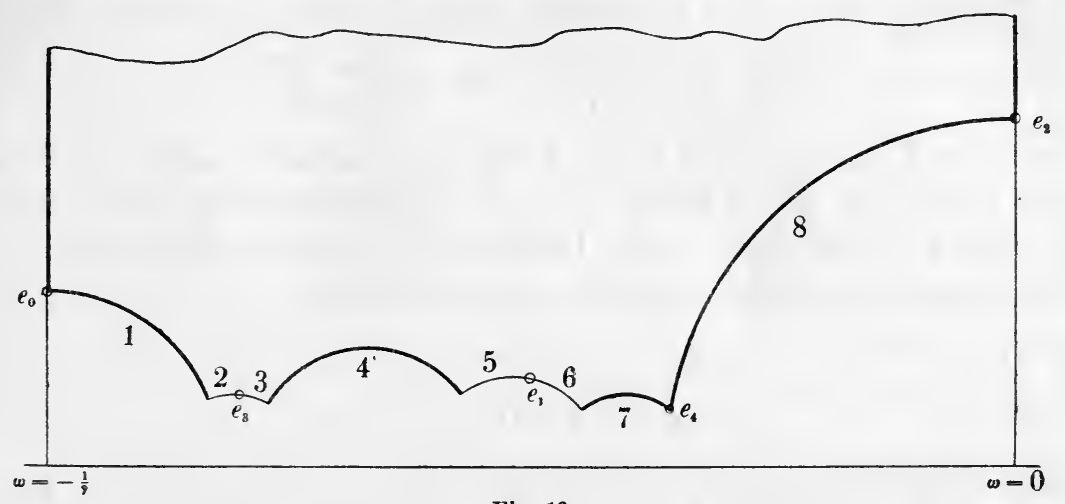

Fig. 18.

Fig. 18 gegebene Gestalt. Die mit den Nummern 1, 4, 7 und 8 bezeichneten Symmetriekreise nebst zugehörigen Spiegelungen der $\bar{\Gamma}^{(31)}$ sind der Reihe nach gegeben durch:

$$
\begin{aligned}
2 \cdot 31\left(\xi^{2}+\eta^{2}\right)+2 \cdot 31 \xi+15 & =0, \quad \omega^{\prime}=\frac{-31 \bar{\omega}-15}{62 \bar{\omega}+31} \\
3 \cdot 31\left(\xi^{2}+\eta^{2}\right)+2 \cdot 31 \xi+10 & =0, \quad \omega^{\prime}=\frac{-31 \bar{\omega}-10}{93 \bar{\omega}+31} \\
5 \cdot 31\left(\xi^{2}+\eta^{2}\right)+2 \cdot 31 \xi+6 & =0, \quad \omega^{\prime}=\frac{-31 \bar{\omega}-6}{155 \bar{\omega}+31} \\
31\left(\xi^{2}+\eta^{2}\right)-1 & =0, \quad \omega^{\prime}=\frac{1}{31 \bar{\omega}} .
\end{aligned}
$$

Die zu $e_{0}, e_{1}, e_{3}$ und $e_{4}$ bezüglich der imaginären Achse symmetrischen Eckpunkte der anderen Hälfte von $K_{31}$ mögen wieder $e_{0}^{\prime}, e_{1}^{\prime}, e_{3}^{\prime}$ und $e_{4}^{\prime}$ heißen. Der Eckenzyklus $e_{0}, e_{0}^{\prime}$ und die beiden Ecken $e_{1} e_{1}^{\prime}$, gelegen bei:

$$
\omega=\frac{\mp \sqrt{31}+i}{2 \sqrt{31}}, \quad \omega=\frac{-\sqrt{31}+i}{4 \sqrt{31}}, \quad \omega=\frac{\sqrt{31}+i}{4 \sqrt{31}}
$$

gehören den drei Formklassen der Diskriminante $D=-31$ bzw. den diese Klassen repräsentierenden Formen $(31,31,8),(62, \pm 31,4)$ an, von denen die erste zweiseitig, die beiden anderen entgegengesetzt sind. Ebenso gehören die Punkte $e_{2}, e_{3}$ und $e_{3}^{\prime}$, gelegen bei:

1) Auch für den Grad 23 ist die Transformationsgleichung der zwölften Wurzel der Diskriminante $\Delta$ durch Kiepert berechnet; s. Journ. f. Math., Bd. 95, S. 230. 


$$
\omega=\frac{i}{\sqrt{31}}, \quad \omega=\frac{-2 \sqrt{31}+i}{5 \sqrt{31}}, \quad \omega=\frac{2 \sqrt{31}+i}{5 \sqrt{31}}
$$

zu den drei Formklassen der Diskriminante $D=-124$, repräsentiert durch die Formen $(31,0,1),(155, \pm 124,25)$. Die Punkte $e_{1}$ und $e_{3}$ sind die Fixpunkte der beiden elliptischen Substitutionen der Periode 2:

$$
\omega^{\prime}=\frac{-31 \omega-8}{124 \omega+31}, \quad \omega^{\prime}=\frac{-62 \omega-25}{155 \omega+62},
$$

die in der $\Gamma^{(31)}$, aber noch nicht in der $\Gamma_{\psi(31)}$ enthalten sind. Durch die erste werden die beiden Seiten 5 und 6 ineinander transformiert, durch die zweite die Seiten 2 und 3. Die beiden durch diese Seitenpaare zusammengesetzten Kreisbogen haben die Gleichungen:

$$
\begin{aligned}
& 31\left(\xi^{2}+\eta^{2}\right)+25 \xi+5=0, \\
& 31\left(\xi^{2}+\eta^{2}\right)+16 \xi+2=0 .
\end{aligned}
$$

Diese beiden Kreise schneiden die in der Figur stark ausgezogenen Symmetriekreise 1, 4, 7 überall unter rechten Winkeln. Der Punkt $e_{4}$ endlich bildet mit dem symmetrischen Eckpunkte $e_{4}^{\prime}$ am Polygone $\mathbf{K}_{31}$ einen Eckenzyklus. Diese Punkte, die bei $\omega=\frac{\mp 11+i \sqrt{3}}{62}$ gelegen sind, stehen am Transformationspolygone $T_{31}$ für sich und bilden die Fixpunkte der beiden in der $\Gamma_{\psi(31)}$ enthaltenen elliptischen Substitutionen der Periode 3:

$$
\omega^{\prime}=\frac{-6 \omega-1}{31 \omega+5}, \quad \omega^{\prime}=\frac{6 \omega-1}{31 \omega-5} ;
$$

sie liefern für die über der $J$-Ebene gedachten Fläche $F_{31}$ die Stellen mit $J=0$ in den beiden hier unverzweigt verlaufenden Blättern.

Die bekannten Ansätze (S. $326 \mathrm{ff}$.) liefern für $n=31$ zwei Modulformen $y$ und eine Form $z$ :

$$
\left\{\begin{array}{l}
y=\frac{2 \pi}{\omega_{2}}\left(1+2 q^{2}+2 q^{8}+4 q^{16}+2 q^{18}+\cdots\right), \\
y_{1}=\frac{2 \pi}{\omega_{2}}\left(1+2 q^{4}+2 q^{8}+2 q^{30}+2 q^{14}+2 q^{16}+\cdots\right), \\
z=\frac{2 \pi}{\omega_{2}}\left(q^{2}-q^{4}-q^{10}-q^{14}+q^{16}+q^{18}+\cdots\right),
\end{array}\right.
$$

die auch hier wieder in der Beziehung $2 z=y-y_{1}$ stehen. Alle drei Formen nehmen wieder gegenüber einer Substitution der $\Gamma_{\psi(31)}$ den Faktor $\left(\frac{\alpha}{31}\right)$ an.

Jede der drei Modulformen $y, y_{1}, z$ hat im Polygone $\mathbf{T}_{31}$ Nullpunkte der Gesamtordnung $\frac{8}{3}$. Hieraus folgt leicht, daß $y, y_{1}$ und $z$ in $e_{4}$ und $e_{4}^{\prime}$ je einen Nullpunkt der Ordnung $\frac{1}{3}$ haben; außerdem besitzt jede Form 
noch zwei Nullpunkte erster Ordnung in $\mathbf{T}_{31}$, die auch zusammenfallen können, vielleicht auch in den Ecken $e_{4}, e_{4}^{\prime}$ gelegen sein mögen.

Im Quotienten:

$$
\tau(\omega)=\frac{y}{z}=q^{-2}+3+3 q^{2}+\cdots
$$

heben sich die genannten Nullpunkte in $e_{4}$ und $e_{4}^{\prime}$ fort, so daß wir auch hier in $\tau(\omega)$ eine zweiwertige Funktion des zum Geschlechte 2 gehörenden Polygons $\mathbf{T}_{31}$ und also eine einwertige Funktion von $\boldsymbol{K}_{31}$ erkennen, die übrigens ihren Pol in der Spitze $i \infty$ hat. Da diese Spitze durch $W_{31}$ nach $\omega=0$ verlegt wird, so liegen die beiden Nullpunkte erster Ordnung von $z$ in den Spitzen $i \infty$ und 0 von $\mathbf{T}_{31}$. Hieraus aber ergibt sich leicht, daß $z$ und damit auch $y$ und $y_{1}$ gegenüber $W_{31}$ invariant sind. Wie bisher stellen wir aus $y$ und $z$ in $(a y+b z)$ eine lineare Formenschar des Klassenpolygons $\mathbf{K}_{31}$ her mit einem beweglichen Nullpunkte erster Ordnung und einem festen der Ordnung $\frac{1}{3} \mathrm{im}$ Eckenzyklus $e_{4}, e_{4}^{\prime}$.

Zur Gewinnung der „Verzweigungsform" der zweiblättrigen hyperelliptischen Fläche über der $\tau$-Ebene, die das Abbild von $T_{31}$ ist, benutzen wir wieder den Ansatz (38), der uns hier mit der Modulform versieht:

$$
\begin{aligned}
v=\left(\frac{2 \pi}{\omega_{2}}\right)^{3}\left(1-q^{2}-3 q^{4}-3 q^{6}-13 q^{8}-18 q^{10}-27 q^{12}\right. \\
\left.-36 q^{14}-59 q^{16}-\cdots\right) .
\end{aligned}
$$

Diese Form hat in den sechs Punkten $e_{0}, e_{1}, e_{1}^{\prime}, e_{2}, e_{3}, e_{3}^{\prime}$ von $\mathrm{T}_{31}$ je Nullpunkte erster Ordnung; außerdem tritt je ein Nullpunkt erster Ordnung in $e_{4}$ und $e_{4}^{\prime}$ auf, da hier die Ableitung von $\tau$ nach $\omega$ in der Ordnung ${ }_{3}^{2}$ und $z$ in der Ordnung $\frac{1}{3}$ verschwindet. Hiernach ist das Quadrat von $v$ als homogene Funktion sechsten Grades in $y$ und $z$ darstellbar, und zwar ergeben die Potenzreihen als Gestalt dieser Funktion:

$$
v^{2}=y^{6}-2 \cdot 7 y^{5} z+61 y^{4} z^{2}-2 \cdot 53 y^{3} z^{3}+2 \cdot 3 \cdot 11 y^{2} z^{4}-2^{3} y z^{5}-3 z^{6}
$$

in dem hier rechts stehenden Ausdrucke haben wir die Verzweigungsform der zweiblättrigen Fläche über der $\tau$-Ebene gewonnen. Als Funktionssystem des Transformationspolygons führen wir ein:

$$
\tau(\omega)=\frac{y}{z}, \quad \sigma(\omega)=\frac{v}{z^{3}},
$$

wobei sich $\sigma$ in $\tau$ mittels der Quadratwurzel darstellt:

$$
\sigma=\sqrt{\tau^{6}-14 \tau^{5}+61 \tau^{4}-106 \tau^{3}+66 \tau^{2}-8 \tau-3} .
$$

Die gewonnenen Ergebnisse sind wieder wie bei $n=23$ einer Prüfung zugänglich. Die durch Nullsetzen der unter der Quadratwurzel (51) stehenden Funktion entstehende Gleichung sechsten Grades muB wieder im rationalen Körper reduzibel sein und in zwei kubische Gleichungen 
zerfallen. Die Wurzeln der einen kubischen Gleichung sind die Werte $\tau$ in den drei Nullpunkten $e_{0}, e_{1}, e_{1}^{\prime}$ der quadratischen Formen mit $D=-31$, die andere Gleichung gehört entsprechend zu $D=-124$. Diese Zerfällung der Gleichung sechsten Grades ist in der Tat möglich; wir gelangen zu den beiden kubischen Gleichungen:

$$
\begin{aligned}
& D=-31, \quad \tau^{3}-5 \tau^{2}+6 \tau+1=0, \\
& D=-124, \quad \tau^{3}-9 \tau^{2}+10 \tau-3=0,
\end{aligned}
$$

die ihrerseits leicht als im rationalen Körper irreduzibel erkannt werden. ${ }^{1}$ ) Die Verteilung der beiden kubischen Gleichungen auf die Diskriminanten $D=-31$ und -124 entspricht dem Umstande, daB die zweite Gleichung die reelle positive Lösung $\tau\left(\frac{i}{\sqrt{31}}\right)$, die erste die reelle negative $\tau\left(\frac{-\sqrt{31}+i}{2 \sqrt{31}}\right)$ hat.

Wie bei $n=23$ führen wir in:

$$
\left\{\begin{array}{l}
v_{1}=\sqrt{y^{3} z-5 y^{2} z^{2}+6 y z^{3}+z^{4}} \\
v_{2}=\sqrt{y^{3} z-9 y^{2} z^{2}+10 y z^{3}-3 z^{4}}
\end{array}\right.
$$

zwei Modulformen $(-2)^{\text {ter }}$ Dimension ein, deren Produkt gleich $z v$ ist. Die Nullpunkte der Gesamtordnung $\frac{16}{3}$ jeder dieser Formen $v_{1}, v_{2}$ im Polygone $\boldsymbol{T}_{31}$ verteilen sich so: Erstlich hat $v_{1}$ je drei einfache Nullpunkte in $e_{0}, e_{1}, e_{1}^{\prime}$ und $v_{2}$ in $e_{2}, e_{3}, e_{3}^{\prime}$. Ferner haben die Formen gemeinsam je einen Nullpunkt der Ordnung $\frac{2}{3}$ in den Punkten $e_{4}$ und $e_{4}^{\prime}$ und je einen solchen der Ordnung $\frac{1}{2}$ in den Spitzen $i \infty$ und 0 von $\mathrm{T}_{31}$. Durch dieselbe Überlegung wie bei $n=23$ findet man, $d a \beta v_{1}$ gegenüber $W_{31}$ invariant ist, während $v_{2}$ Zeichenwechsel erfährt. Endlich gelten die Potenzreihen der $v_{1}, v_{2}$ :

$$
\left\{\begin{array}{l}
v_{1}=\left(\frac{2 \pi}{\omega_{2}}\right)^{2}\left(q+*+q^{5}+3 q^{7}-3 q^{9}+6 q^{11}+\cdots\right), \\
v_{2}=\left(\frac{2 \pi}{\omega_{2}}\right)^{2}\left(q-2 q^{3}-3 q^{5}-q^{7}+q^{9}-6 q^{11}+\cdots\right) .
\end{array}\right.
$$

Es soll nun zunächst diejenige Form $(a y+b z)$ der linearen Schar festgestellt werden, für welche der bewegliche Nullpunkt der Schar mit dem festen Nullpunkte im Eckenzyklus $e_{4}, e_{4}^{\prime}$ von $K_{31}$ zusammenfällt. Die gesuchte Form $(a y+b z)$ hat im genannten Eckenzyklus einen Nullpunkt der Ordnung $\frac{4}{3}$ und kann in folgender Art berechnet werden. Das Produkt:

$$
\Delta^{\prime} \cdot \Delta=\Delta\left(31 \omega_{1}, \omega_{2}\right) \cdot \Delta\left(\omega_{1}, \omega_{2}\right)
$$

ist eine Form von $K_{31}$ mit einem einzigen Nullpunkte $32^{\text {ster }}$ Ordnung in

1) Keine der beiden kubischen Gleichungen hat nämlich eine ganzzahlige Lösung. 
der Spitze $i \infty$. Die Potenz $z^{32}$ hat ebenda gleichfalls einen Nullpunkt $32^{\text {ster }}$ Ordnung, außerdem aber einen Nullpunkt der Ordnung $\frac{32}{3} \mathrm{im} \mathrm{Zyk-}$ lus $e_{4}, e_{4}^{\prime}$. Demnach ist $z^{32}$ mit dem Produkte $(a y+b z)^{8} \cdot \Delta^{\prime} \Delta$ identisch, und man findet durch Ausziehen der achten Wurzel den Ansatz:

$$
z^{4}=(a y+b z) \sqrt[8]{\Delta^{\prime} \Delta} .
$$

Die beiden ersten Glieder der Potenzreihen liefern $a$ und $b$ :

$$
z^{4}=(y-3 z) \sqrt[8]{\Delta^{\prime} \Delta},
$$

so $\mathrm{daB}(y-3 z)$ die gesuchte Form ist.

Die beiden Formen $\left(g_{2}^{\prime} \pm g_{2}\right)$ haben in $K_{31}$ Nullpunkte der Gesamtordnung $\frac{16}{3}$, so daß zunächst für den Eckenzyklus $e_{4}, e_{4}^{\prime}$ je ein Nullpunkt der Ordnung $\frac{1}{3}$ anzusetzen ist. Weiter hat $\left(g_{2}^{\prime}-g_{2}\right)$ je einen Nullpunkt der Ordnung $\frac{1}{2}$ in den sechs Ecken $e_{0}, e_{1}, e_{1}^{\prime}, e_{2}, e_{3}, e_{3}^{\prime}$. Für $\left(g_{2}^{\prime}-g_{2}\right)$ restieren also noch zwei, für $\left(g_{2}^{\prime}+g_{2}\right)$ noch fünf einfache Nullpunkte. Das Produkt von $v$ mit einer ganzen Funktion zweiten Grades von $y$ und $z$ und ebenso eine ganze Funktion fünften Grades von $y$ und $z$ haben aber die Dimension -5 und je einen Nullpunkt der Ordnung $\frac{5}{3}$ im Zyklus $e_{4}, e_{4}^{\prime}$. Hier also haben wir nicht $\left(g_{2}^{\prime} \pm g_{2}\right)$, sondern die Produkte $\left(g_{2}^{\prime} \pm g_{2}\right)(y-3 z)$ dazustellen. Die Potenzreihen fuihren auf folgende Ausdrücke $\left(g_{2}^{\prime} \pm g_{2}\right)$ in $y, z$, v:

$$
\left\{\begin{array}{c}
6\left(g_{2}^{\prime}+g_{2}\right)(y-3 z)=13 \cdot 37 y^{5}-5171 y^{4} z+2^{5} \cdot 5 \cdot 11^{2} y^{3} z^{2} \\
-2^{4} \cdot 23 \cdot 83 y^{2} z^{3}+2^{4} \cdot 3^{2} \cdot 131 y z^{4}-2^{5} \cdot 5 \cdot 23 z^{5} \\
\left(g_{2}^{\prime}-g_{2}\right)(y-3 z)=2^{2} \cdot 5 v\left(2^{2} y^{2}-17 y z+2^{4} z^{2}\right)
\end{array}\right.
$$

Aus den Sätzen von S. $339 \mathrm{ff}$. folgert man, daß beim $31^{\text {sten }}$ Grade die beiden Formen $(-2)^{\text {ter }}$ Dimension $\left(\sqrt[6]{\Delta} \pm 31 \sqrt[6]{\Delta^{\prime}}\right)$ gegenüber den Substitutionen der $\Gamma_{\psi(31)}$ bis auf eine multiplikative sechste Einheitswurzel invariant sind. Gegenüber $W_{31}$ bleibt die erste Form unverändert, die zweite erfährt Zeichenwechsel. Jede dieser Formen hat auf $\mathbf{K}_{\mathbf{3 1}}$ Nullpunkte der Gesamtordnung $\frac{8}{3}$. Da in der Spitze $i \infty$ beide Male ein Nullpunkt der Ordnung $\frac{1}{6}$ liegt, so bleiben Nullpunkte der Gesamtordnung $\frac{5}{2}$ übrig. Es treten also bei beiden Formen Nullpunkte in den Ecken $e_{0}$, $e_{1}, \ldots, e_{3}^{\prime}$ auf, und zwar folgt aus dem Verhalten gegenüber $W_{31}$ und der Irreduzibilität der beiden oben genannten kubischen Gleichungen für $\tau$, daß $\left(\sqrt[6]{\Delta}+31 \sqrt[6]{\Delta^{\prime}}\right)$ drei bei $e_{0}, e_{1}, e_{1}^{\prime}$ gelegene Nullpunkte der Ordnung $\frac{1}{2}$ hat, $\left(\sqrt[6]{\Delta}-31 \sqrt[6]{\Delta^{\prime}}\right)$ aber ebensolche Nullpunkte bei $e_{2}, e_{3}, e_{3}^{\prime}$. Jetzt restiert für jede unserer Formen noch ein Nullpunkt erster Ordnung. Bilden wir aber demnach für diese Formen die Ansätze $v_{1}(a y+b z)$, $v_{2}(c y+d z)$, so ist zu beachten, daB diese Produkte im Eckenzyklus $e_{4}, e_{4}^{\prime}$ Nullpunkte erster Ordnung haben. Wir dividieren also noch durch $\sqrt[3]{z(y-3 z)^{2}}$ und erreichen so, daß die Quotienten nicht nur die richtigen 
Nullpunkte haben, sondern auch die Dimension -2 besitzen. Die noch unbekannten Koeffizienten bestimmt man aus den beiden ersten Reihengliedern und gelangt zu dem Ergebnis: Die Darstellung der ursprünglichen und der transformierten Diskriminante in den $y, z, v_{1}, v_{2}$ ist beim Grade $n=31$ geleistet durch:

$$
\left\{\begin{array}{l}
\left.\left(\sqrt[6]{\Delta}+31 \sqrt[6]{\Delta^{\prime}}\right) \sqrt[3]{z(y-3 z}\right)^{2}=v_{1}(y-7 z) \\
\left(\sqrt[6]{\Delta}-31 \sqrt[6]{\Delta^{\prime}}\right) \sqrt[3]{z(y-3 z)^{2}}=v_{2}(y-5 z)
\end{array}\right.
$$

$\mathrm{Zu}$ einer Bestätigung der vollzogenen Schlußweise berechne man $\sqrt[6]{\Delta \Delta^{\prime}}$ durch Quadrieren und Subtrahieren aus den beiden Gleichungen (57). Dabei zieht sich, wie es sein muB, die Differenz der Quadrate der rechten Seiten auf das einzige Glied $124 z^{6}$ zusammen, und man gelangt zur Gleichung (55) zurück.

Als Ersatz der Transformationsgleichung für $J(\omega)$ beim Grade 31 benutzen wir wie im voraufgehenden Falle die beiden Gleichungen:

$$
\left\{\begin{array}{r}
12 g_{2}(\tau-3) z^{-4}=481 \tau^{5}-5171 \tau^{4}+19360 \tau^{3}-30544 \tau^{2}+18864 \tau \\
-3680-120 \sigma\left(4 \tau^{2}-17 \tau+16\right) \\
2 \sqrt[3]{\Delta}(\tau-3) \sqrt[3]{\tau-3} z^{-4}=\tau^{5}-19 \tau^{4}+125 \tau^{3}-328 \tau^{2}+280 \tau-13 \\
+\sigma\left(\tau^{2}-12 \tau+35\right)
\end{array}\right.
$$

im Verein mit der Gleichung (51). ${ }^{1}$ )

\section{§ 5. Primzahlige Transformationsgrade der Gestalt $n=4 h+1$.}

1. Transformation $13^{\text {ten }}$ Grades. Die zur linken Seite der imaginären $\omega$-Achse liegende Hälfte des Klassenpolygons $K_{13}$ ist in Fig. 19 dargestellt. Sie ist ein aus lauter Symmetriekreisen der $\bar{\Gamma}^{(13)}$ begrenztes Kreisbogenfünfeck mit drei rechten Winkeln, einem Winkel $\frac{\pi}{3}$ und einem Winkel 0. Die mit den Nummern 1 und 2 bezeichneten Symmetriekreise nebst zugehörigen Spiegelungen sind:

$$
\begin{aligned}
& 13\left(\xi^{2}+\eta^{2}\right)+13 \xi+3=0, \quad \omega^{\prime}=\frac{-13 \bar{\omega}-6}{26 \bar{\omega}+13}, \\
& 39\left(\xi^{2}+\eta^{2}\right)+26 \xi+4=0, \quad \omega^{\prime}=\frac{-13 \bar{\omega}-4}{39 \bar{\omega}+13},
\end{aligned}
$$

mit 3 ist der Symmetriekreis der Spiegelung $\bar{W}_{13}$ bezeichnet. Die Ecken $e_{0}$ und $e_{1}$, gelegen bei $\omega=\frac{i}{\sqrt{13}}$ und $\frac{-\sqrt{13}+i}{2 \sqrt{13}}$, sind die Fixpunkte der in der $\Gamma^{(13)}$ enthaltenen Substitutionen $W_{13}$ und $\left(\begin{array}{rr}-13, & -7 \\ 26, & 13\end{array}\right)$; sie ent-

1) Über die Transformationsgrade 47 und 71 , für die auch noch das Geschlecht des Klassenpolygons 0 ist, vgl. man „Modulfunktionen“, Bd. 2, S. $463 \mathrm{ff}$. 
sprechen den beiden (zweiseitigen) Formklassen der Diskriminante $D=-52$ bzw. den diese Klassen repräsentierenden Formen $(13,0,1),(26,26,7)$. Der Eckpunkt $e_{\mathbf{2}}$ bildet am Polygone $\boldsymbol{K}_{\mathbf{1 3}}$ mit dem symmetrischen $e_{2}^{\prime}$ einen Zyklus, ebenso die Eckpunkte $e_{3}, e_{3}^{\prime}$. Am Transformationspolygon stehen diese vier Ecken je für sich und liefern die Fixpunkte der schon in der $\Gamma_{\psi(13)}$ enthaltenen Substitutionen der Perioden 2 bzw. 3:

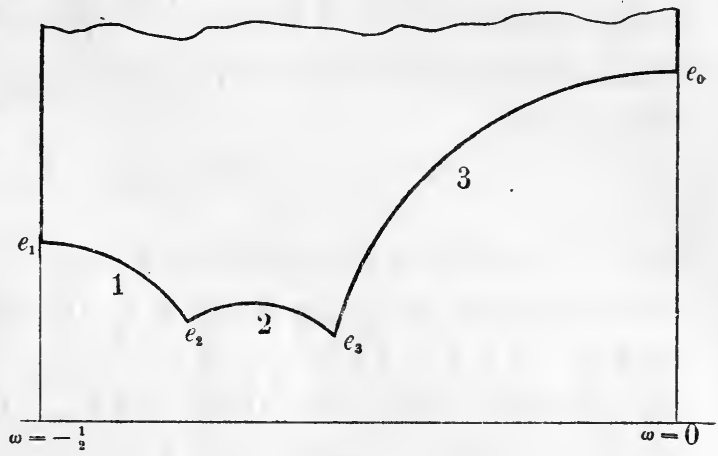

Fig. 19.

$$
\omega^{\prime}=\frac{-5 \omega \mp 2}{ \pm 13 \omega+5}, \quad \omega^{\prime}=\frac{-3 \omega \mp 1}{ \pm 13 \omega+4} .
$$

Auf der über der $J$-Ebene gedachten Fläche $\boldsymbol{F}_{13}$ sind $e_{2}, e_{2}^{\prime}$ die beiden Stellen bei $J=1$ in den daselbst unverzweigten Blättern, ebenso sind $e_{3}, e_{3}^{\prime}$ die beiden Stellen bei $J=0$ in den hier isoliert verlaufenden Blättern.

Der Ansatz (19) S. 332 liefert nur für die Hauptklasse der Diskriminante $D=-52$ eine nicht identisch verschwindende Reihe. Man gelangt zur Moduilform:

$$
z=\frac{2 \pi}{\omega_{2}} q^{\frac{1}{2}}\left(1+q^{4}-q^{6}-2 q^{8}+q^{12}-2 q^{14}-\cdots\right),
$$

die mit $(\sqrt[4]{\Delta})^{3}$ multipliziert eine Form der $\Gamma_{\psi(13)}$ liefert und also ihrerseits gegenüber den Substitutionen der $\Gamma_{\psi(13)}$ bis auf multiplikative vierte Einheitswurzeln unverändert bleibt. Die Form $z$ hat auf dem Polygone $T_{13}$ Nullpunkte der Gesamtordnung $\frac{7}{6}$. Zunächst liegt zufolge (2) in der Spitze $i \infty$ ein Nullpunkt der Ordnung $\frac{1}{4}$. Da ferner in $e_{3}$ und $e_{3}^{\prime}$ Nullpunkte gleicher Ordnung auftreten, so kann diese Ordnung nur $\frac{1}{3}$ sein. Endlich kann der übrigbleibende Nullpunkt der Ordnung $\frac{1}{4}$ nur in der Spitze $\omega=0$ gelegen sein. Da die Nullpunkte von $z$ durch $W_{13}$ in sich transformiert werden und keiner dieser Nullpunkte bei $e_{0}$ liegt, so ist die Modulform z gegenüber $W_{13}$ invariant.

Zwei weitere Formen $(-1)^{\text {ter }}$ Dimension des Klassenpolygons $\mathbf{K}_{13}$ gewinnt man auf folgende Art. Nach S. $339 \mathrm{ff}$. ist, falls wir:

$$
\sqrt[12]{\Delta^{\prime}}=\sqrt[12]{\Delta}\left(13 \omega_{1}, \omega_{2}\right)=\frac{1}{\sqrt{13}} \sqrt[12]{\Delta}\left(\omega_{1} \sqrt{13}, \frac{\omega_{2}}{\sqrt{13}}\right)
$$

setzen, die zwölfte Wurzel des Quotienten $\Delta^{\prime}: \Delta$ eine Funktion der $\Gamma_{\psi(13)}$. Da man für diese Funktion leicht einen einzigen Nullpunkt erster Ordnung in $\mathbf{T}_{13}$, gelegen bei $\omega=i \infty$, und einen Pol erster Ordnung bei $\omega=0$ feststellt, so ist: 


$$
\tau(\omega)=13 \sqrt[12]{\frac{\Delta^{\prime}}{\Delta}}=\sqrt{13} \sqrt{\frac{\Delta\left(\omega_{1} \sqrt{13}, \frac{\omega_{2}}{\sqrt{13}}\right)}{\Delta\left(\omega_{1}, \omega_{2}\right)}}
$$

eine einwertige Funktion von $\mathbf{T}_{13}$, die zufolge (3) gegenüber $W_{13}$ das Verhalten zeigt:

$$
\tau\left(\frac{-1}{13 \omega}\right)=\frac{13}{\tau(\omega)} .
$$

Durch $W_{13}$ werden nur die beiden Punkte $e_{0}$ und $e_{1}$ von $\mathbf{T}_{13}$ in sich transformiert, denen somit die Werte $\tau=\sqrt{\overline{13}}$ und $-\sqrt{13}$ zugehören. Hiernach haben wir in $\left(\sqrt[12]{\Delta} \pm \sqrt{13} \sqrt[12]{\Delta^{\prime}}\right)$ zwei Formen der Dimension -1 , die gegenüber den Substitutionen der $\Gamma_{\psi(13)}$ bis auf multiplikative zwölfte Einheitswurzeln invariant sind und gegenüber $W_{13}$ unverändert bzw. bis auf einen Zeichenwechsel unverändert bleiben. Im Klassenpolygon $K_{13}$ haben beide Formen einen Nullpunkt der Ordnung $\frac{1}{12}$ in der Spitze $i \infty$. Weiter hat $\left(\sqrt[12]{\Delta}+\sqrt{13} \sqrt[12]{\Delta^{\prime}}\right)$ einen Nullpunkt der Ordnung $\frac{1}{2}$ im Eckenzyklus $e_{1}, e_{1}^{\prime}$ und $\left(\sqrt[12]{\Delta}-\sqrt{13} \sqrt[12]{\Delta^{\prime}}\right)$ einen ebensolchen Nulpunkt in der Ecke $e_{0}$. Diese beiden Modulformen haben zwar nicht mehr selbst rationale Entwicklungskoeffizienten, wohl aber gilt dies von ihrem Produkte:

(5) $\sqrt[6]{\Delta}-13 \sqrt[6]{\Delta^{\prime}}=\left(\frac{2 \pi}{\omega_{2}}\right)^{6} q^{\frac{1}{3}}\left(1-4 q^{2}-11 q^{4}+8 q^{6}-5 q^{8}-4 q^{10}+\cdots\right)$.

Eine einwertige Funktion des Klassenpolygons $\mathbf{K}_{13}$ hat man in:

$$
\tau(\omega)+\frac{13}{\tau(\omega)}=\frac{\sqrt[6]{\Delta}+13 \sqrt[6]{\Delta^{\prime}}}{\sqrt[12]{\Delta^{\prime} \Delta}}
$$

Zähler und Nenner des rechts stehenden Quotienten liefern einzeln zwei Formen:

$$
\left\{\begin{array}{c}
\sqrt[6]{\Delta}+13 \sqrt[6]{\Delta^{\prime}}=\left(\frac{2 \pi}{\omega_{2}}\right)^{2} q^{\frac{1}{3}}\left(1-4 q^{2}+15 q^{4}+8 q^{6}-5 q^{8}-4 q^{10}+\cdots\right) \\
\sqrt[12]{\Delta^{\prime} \Delta}=\left(\frac{2 \pi}{\omega_{2}}\right)^{2} q^{\frac{1}{3}}\left(q^{2}-2 q^{4}-q^{6}+2 q^{8}+q^{10}-\cdots\right)
\end{array}\right.
$$

die gegenüber $W_{13}$ invariant und gegenüber den Substitutionen der $\Gamma_{\psi(13)}$ bis auf multiplikative sechste Einheitswurzeln invariant sind. Aus beiden Formen gewinnt man in:

$$
a\left(\sqrt[6]{\Delta}+13 \sqrt[6]{\Delta^{\prime}}\right)+b \sqrt[12]{\Delta^{\prime} \Delta}
$$

eine Formenschar des Klassenpolygons $K_{13}$ mit einem festen Nullpunkte der Ordnung $\frac{1}{6}$ in der Spitze $i \infty$ und einem beweglichen Nullpunkte erster Ordnung.

Um die Beziehung der Form $z$ zu den Formen (7) herzustellen, be- 
merke man, daß der Quotient von $z^{3}$ und $\sqrt[24]{\Delta^{\prime} \Delta}$ diejenige Form der Schar (8) ist, für welche der bewegliche Nullpunkt in den Eckenzyklus $e_{3}, e_{3}^{\prime}$ fällt, wie aus der Betrachtung der Nullpunkte hervorgeht. Die Potenzreihen liefern für die fragliche Form (8) die Koeffizienten $a=1, b=5$, woraus sich für $z$ die Darstellung ergibt:

$$
z=\sqrt[3]{\left(\sqrt[6]{\Delta}+13 \sqrt[6]{\Delta^{\prime}}+5 \sqrt[12]{\Delta^{\prime} \Delta}\right) \sqrt[24]{\Delta^{\prime} \Delta}}
$$

Weiter stellt man mit Hilfe der zum Polygone $\mathbb{K}_{13}$ gehörenden Form:

$$
G_{1}=\frac{13}{2}\left(\frac{2 \pi}{\omega_{2}}\right)^{2}\left(1+2 q^{2}+6 q^{4}+8 q^{6}+14 q^{8}+12 q^{10}+\cdots\right)
$$

leicht fest, für welche Form (8) der bewegliche Nullpunkt in den Eckenzyklus $e_{2}, e_{2}^{\prime}$ fällt. Von den Nullpunkten der Gesamtordnung $\frac{7}{6}$, die $G_{1}$ in $K_{13}$ hat, liegt nämlich zunächst ein solcher der Ordnung $\frac{2}{3}$ im Eckenzyklus $e_{3}, e_{3}^{\prime}$. Der rückständige Nullpunkt der Ordnung $\frac{1}{2}$ kann nur in einem der Eckenzyklen $e_{1}, e_{1}^{\prime}$ oder $e_{2}, e_{2}^{\prime}$ liegen. Nun besteht wegen der ersten Substitution (1) die Gleichung:

$$
G_{1}\left(-5 \omega_{1}-2 \omega_{2}, 13 \omega_{1}+5 \omega_{2}\right)=G_{1}\left(\omega_{1}, \omega_{2}\right) .
$$

Trägt man hier der Ecke $e_{2}$ entsprechend $\omega_{1}=-5+i, \omega_{2}=13$ ein, so folgt mit Rücksicht auf die Dimension von $G_{1}$ :

$$
G_{1}(-1-5 i, 13 i)=-G_{1}(-5+i, 13)=G_{1}(-5+i, 13),
$$

so daß der fragliche Nullpunkt im Eckenzyklus $e_{2}, e_{2}^{\prime}$ liegt. Man hat nun, wie wieder aus der Lage der Nullpunkte folgt, im Quotienten $G_{1}^{2}: z^{4}$ eine einwertige Funktion von $\mathbf{K}_{13}$ mit dem Nullpunkte im Zyklus $e_{2}, e_{2}^{\prime}$ und dem Pole in der Spitze $i \infty$, die demnach eine lineare ganze Funktion der in (6) eingeführten einwertigen Funktion von $K_{13}$ ist. Der sich hieraus ergebende Ansatz liefert unter Zuhilfenahme der Potenzreihen:

$$
2 G_{1} \sqrt[24]{\Delta^{\prime} \Delta}=13 z^{2} \sqrt{\sqrt[6]{\Delta}}+13 \sqrt[6]{\Delta^{\prime}}+6 \sqrt[12]{\Delta^{\prime} \Delta}
$$

Somit haben wir in:

$$
\sqrt{\sqrt[6]{\Delta}+13 \sqrt[6]{\Delta^{\prime}}+6 \sqrt[12]{\Delta^{\prime} \Delta}}=\frac{2 \pi}{\omega_{2}} q^{\frac{1}{6}}\left(1+q^{2}+q^{4}+*+3 q^{8}+\cdots\right)
$$

eine Form $(-1)^{\text {ter }}$ Dimension, die neben einem Nullpunkte der Ordnung $\frac{1}{12}$ in der Spitze $i \infty$ einen solchen der Ordnung $\frac{1}{2}$ im Eckenzyklus $e_{2}, e_{2}^{\prime}$ von $K_{13}$ hat.

Die Formen $\left(g_{2}^{\prime} \pm g_{2}\right)$ der Dimension -4 haben auf $K_{18}$ Nullpunkte der Gesamtordnung $\frac{7}{3}$. Für beide Formen liegt ein Nullpunkt der Ordnung $\frac{1}{3}$ im Zyklus $e_{3}, e_{3}^{\prime}$, und $\left(g_{2}^{\prime}-g_{2}\right)$ hat zwei Nullpunkte der Ordnung $\frac{1}{2}$ bei $e_{0}$ und $e_{1}$; es bleiben dann noch zwei Nullpunkte erster Ordnung für $\left(g_{2}^{\prime}+g_{2}\right)$, und ein solcher für $\left(g_{2}^{\prime}-g_{2}\right)$ übrig. Setzen wir aber für 
$\left(g_{2}^{\prime}+g_{2}\right)$ das Produkt von $z$ mit einer homogenen Funktion zweiten Grades der Formen (7) an und für $\left(g_{2}^{\prime}-g_{2}\right)$ das Produkt von $z\left(\sqrt[6]{\Delta}-13 \sqrt[6]{\Delta^{\prime}}\right)$ und einer Form (8), so ist zu beachten, dab diese Produkte in der Spitze $i \infty$ in der Ordnung $\frac{7}{12}$ verschwinden. Diese Nullpunkte sind also durch Division mit $\sqrt[24]{\Delta^{\prime} \angle}$ wieder fortzuheben. Die entstehenden Ansätze werden leicht mit Hilfe der Potenzreihen durchgebildet. Es ergibt sich der Satz: Beim 13 $3^{\text {ten }}$ Transformationsgrade bestehen für $g_{2}$ und $g_{2}^{\prime}$ die Gleichungen:

$$
\left\{\begin{array}{l}
6\left(g_{2}^{\prime}+g_{2}\right) \sqrt[24]{\Delta^{\prime} \Delta}=5 z\left(17\left(\sqrt[6]{\Delta}+13 \sqrt[6]{\Delta^{\prime}}\right)^{2}\right. \\
\left.\quad+11 \cdot 13\left(\sqrt[6]{\Delta}+13 \sqrt[6]{\Delta^{\prime}}\right) \sqrt[12]{\Delta^{\prime} \Delta}+2 \cdot 3^{2} \cdot 17 \sqrt[6]{\Delta^{\prime} \Delta}\right) \\
\left(g_{2}^{\prime}-g_{2}\right) \sqrt[24]{\Delta^{\prime} \Delta}=2 z\left(\sqrt[6]{\Delta}-13 \sqrt[6]{\Delta^{\prime}}\right)\left(7\left(\sqrt[6]{\Delta}+13 \sqrt[6]{\Delta^{\prime}}\right)+3 \cdot 13 \sqrt[12]{\Delta^{\prime} \Delta}\right) .
\end{array}\right.
$$

Eine entsprechende Überlegung führt zur Darstellung von $g_{3}$ und $g_{3}^{\prime}$. Die Rolle der Form $z$ übernimmt dabei die Form (12). Man gelangt zu dem Satze: Für die beiden Formen $g_{3}$ und $g_{3}^{\prime}$ gelten beim Grade $n=13$ die Gleichungen:

$$
\left\{\begin{array}{c}
6\left(g_{3}+g_{3}^{\prime}\right) \sqrt[24]{\Delta^{\prime} \Delta}=-\sqrt{\sqrt[6]{\Delta}+13 \sqrt[6]{\Delta^{\prime}}+6 \sqrt[12]{\Delta^{\prime} \Delta}}\left(61\left(\sqrt[6]{\Delta}+13 \sqrt[6]{\Delta^{\prime}}\right)^{3}\right. \\
+2^{4} \cdot 3 \cdot 13\left(\sqrt[6]{\Delta}+13 \sqrt[6]{\Delta^{\prime}}\right) \sqrt[21]{\Delta^{\prime} \Delta}+7 \cdot 11 \cdot 13\left(\sqrt[6]{\Delta}+13 \sqrt[6]{\Delta^{\prime}}\right) \sqrt[6]{\Delta^{\prime} \Delta} \\
\left.+2 \cdot 9 \cdot 13 \sqrt[2]{\Delta^{\prime} \Delta}\right) \\
108\left(g_{3}-g_{3}^{\prime}\right) \sqrt[24]{\Delta^{\prime} \Delta}=7 \sqrt{\sqrt[6]{\Delta}+13 \sqrt[6]{\Delta^{\prime}}+6 \sqrt[12]{\Delta^{\prime} \Delta}}\left(\sqrt[6]{\Delta}-13 \sqrt[6]{\Delta^{\prime}}\right) \\
\left(157\left(\sqrt[6]{\Delta}+13 \sqrt[6]{\Delta^{\prime}}\right)^{2}+2 \cdot 13 \cdot 59\left(\sqrt[6]{\Delta}+13 \sqrt[6]{\Delta^{\prime}}\right) \sqrt[12]{\Delta^{\prime} \Delta}\right. \\
\left.+3 \cdot 5 \cdot 13 \cdot 19 \sqrt[6]{\Delta^{\prime} \Delta}\right)
\end{array}\right.
$$

Berechnet man $g_{2}$ und $g_{3}$ einzeln aus (13) und (14), so gelangt man bei Einführung der einwertigen Funktion $\tau$ von $T_{13}$ zu folgenden Gleichungen:

$$
\left\{\begin{array}{l}
\frac{12}{\sqrt[3]{3}}=\sqrt[3]{\tau+5+\frac{13}{\tau}}\left(\tau^{4}+7 \tau^{3}+20 \tau^{2}+19 \tau+1\right) \\
\frac{216 g_{3}}{\sqrt{\Delta}}=\sqrt{\tau+6+\frac{13}{\tau}}\left(\tau^{6}+10 \tau^{5}+46 \tau^{4}+108 \tau^{3}+122 \tau^{2}+38 \tau-1\right)
\end{array}\right.
$$

Die Darstellung von $J$ als rationale Funktion $14^{\text {ten }}$ Grades von $\tau$ kleidet sich hiernach wieder in die für die Fälle des Geschlechtes 0 charakteristische Gestalt:

$$
\begin{aligned}
& J:(J-1): 1=\left(\tau^{2}+5 \tau+13\right)\left(\tau^{4}+7 \tau^{3}+20 \tau^{2}+19 \tau+1\right)^{3} \\
& \quad:\left(\tau^{2}+6 \tau+13\right)\left(\tau^{6}+10 \tau^{5}+46 \tau^{4}+108 \tau^{3}+122 \tau^{2}+38 \tau-1\right)^{2} \\
& \quad: 1728 \tau .
\end{aligned}
$$


Diese Gleichung, die entsprechende für $J^{\prime}$ und $\tau^{\prime}$ und die Relation $\tau^{\prime} \cdot \tau=13$ ersetzen uns die Transformationsgleichung für $J(\omega)$ beim $13^{\text {ten }}$ Grade.

Nach S. $339 \mathrm{ff}$. besteht beim Grade 13 eine Transformationsgleichung für die Form:

$$
f\left(\omega_{1}, \omega_{2}\right)=13 \sqrt[12]{\Delta^{\prime} \cdot \Delta^{11}}=\tau \cdot \Delta .
$$

Die Gestalt dieser Gleichung ergibt sich sofort aus der ersten Gleichung (15) durch Erheben zur dritten Potenz und Einführung von $f$ anstelle von $\tau$ :

$$
\begin{gathered}
\left(f^{2}+5 \Delta f+13 \Delta^{2}\right)\left(f^{4}+7 \Delta f^{3}+20 \Delta^{2} f^{2}+19 \Delta^{3} f+\Delta^{4}\right)^{3} \\
-1728 g_{2}^{3} \Delta^{12} f=0 .
\end{gathered}
$$

2. Transformation $17^{\text {ten }}$ Grades. In Fig. 20 hat man die zur linken Seite der imaginären $\omega$-Achse gelegene Hälfte des Klassenpolygons $K_{17}$ vor sich. Wie immer sind die stark ausgezogenen Seiten Symmetriekreise von Spiegelungen der Gruppe $\bar{\Gamma}^{(17)}$, und zwar sind die Gleichungen der Kreise 1 und $4 \frac{1}{\omega=-\frac{1}{2}}$ und die zugehörigen Spiegelungen:

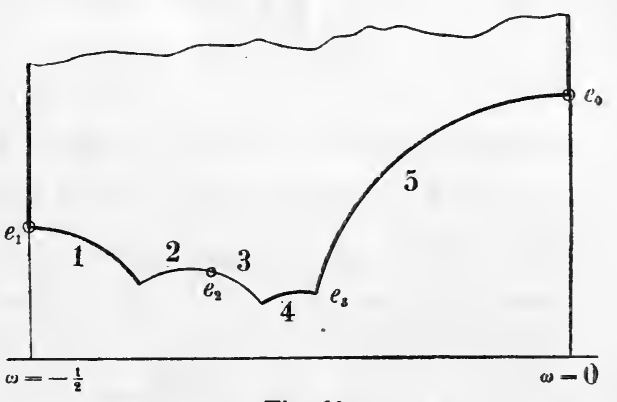

$$
\begin{array}{ll}
17\left(\xi^{2}+\eta^{2}\right)+17 \xi+4=0, & \omega^{\prime}=\frac{-17 \bar{\omega}-8}{34 \bar{\omega}+17}, \\
34\left(\xi^{2}+\eta^{2}\right)+17 \xi+2=0, & \omega^{\prime}=\frac{-17 \bar{\omega}-4}{68 \bar{\omega}+17},
\end{array}
$$

während der Symmetriekreis 5 der Spiegelung $\bar{W}_{17}$ angehört. Die mit den Eckpunkten $e_{1}, e_{2}, e_{3}$ symmetrischen Punkte der rechten Hälfte des Polygons $K_{17}$ mögen wieder $e_{1}^{\prime}, e_{2}^{\prime}, e_{3}^{\prime}$ heißen. Die Punkte $e_{0}, e_{1}, e_{2}, e_{2}^{\prime}$, gelegen bei:

$$
\omega=\frac{i}{\sqrt{17}}, \frac{-\sqrt{17}+i}{2 \sqrt{17}}, \frac{\mp \sqrt{17}+i}{3 \sqrt{17}},
$$

sind die Fixpunkte der Substitutionen $W_{17},\left(\begin{array}{c}-17,-9 \\ 34,17\end{array}\right),\left(\begin{array}{c}\mp 17,-6 \\ 51, \pm 17\end{array}\right)$ und die Nullpunkte von quadratischen Formen, durch die wir die vier Klassen der Diskriminante $D=-68$ repräsentieren können. Durch die dritte dieser Substitutionen werden die Seiten 2 und 3 von $K_{17}$ ineinander transformiert. Diese Seiten setzen den Kreisbogen der Gleichung:

$$
17\left(\xi^{2}+\eta^{2}\right)+12 \xi+2=0
$$

zusammen, der die beiden Seiten 1 und 4 orthogonal trifft. Die Punkte $e_{3}$ und $e_{3}^{\prime}$ endlich, gelegen bei $\omega=\frac{\mp 4+i}{17}$ sind Fixpunkte der elliptischen Substitutionen $\left(\begin{array}{c}\mp 4,-1 \\ 17, \pm 4\end{array}\right)$ der Periode 2 , die auch bereits in der $\Gamma_{\psi(17)}$ 
II, 4. Transformationsgleichungen erster Stufe für niedere Grade $n$

enthalten sind. Diese Punkte liefern auf der über der $J$-Ebene lagernden Fläche $F_{17}$ die beiden Stellen mit $J=1$ in den daselbst isoliert verlaufenden Blättern. Am Polygone $\mathbf{K}_{17}$ bilden sie einen Eckenzyklus.

Von den drei Reihen, die wir für $n=17$ dem Ansatze (19) S. 332 entnehmen, verschwindet eine identisch. Von den beiden übrigen Reihen liefert eine die Modulform:

$$
\sqrt[24]{\Delta^{\prime} \Delta}=\frac{2 \pi}{\omega_{2}} q^{\frac{3}{2}}\left(1-q^{2}-q^{4}+q^{10}+q^{14}-q^{24}-\cdots\right),
$$

wo $\Delta^{\prime}=\Delta\left(17 \omega_{1}, \omega_{2}\right)$ ist, die andere Reihe ergibt die Form:

$$
z=\frac{2 \pi}{\omega_{2}} q^{\frac{1}{2}}\left(1+q^{4}-q^{8}-2 q^{10}+q^{12}-2 q^{16}+q^{24}+\cdots\right) .
$$

Zur $\Gamma_{\psi(17)}$ gehören nach S. 332 die Produkte $z(\sqrt[4]{\Delta})^{3}, \sqrt[24]{\Delta^{\prime} \Delta} \cdot \sqrt[4]{\Delta}$; sie nehmen gegenüber einer Substitution dieser Gruppe den Faktor $\left(\frac{\alpha}{17}\right)$ an.

In $T_{17}$ gemessen haben die Formen $z$ und $\sqrt[24]{\Delta^{\prime} \Delta}$ Nullpunkte je der Gesamtordnung $\frac{3}{2}$. Im einzelnen hat $\sqrt[24]{\Delta^{\prime} \Delta}$ zwei Nullpunkte der Ordnung $\frac{3}{4}$ in den beiden Polygonspitzen $i \infty$ und 0 ; gegenüber $W_{17}$ bleibt $\sqrt[24]{\Delta^{\prime} \Delta}$ invariant. Die Form $z$ hat zunächst einen Nullpunkt der Ordnung $\frac{1}{4}$ bei $\omega=i \infty$ und muß demnach, da ein Nullpunkt gebrochener Ordnung des Nenners 4 nur noch in der Spitze $\omega=0$ auftreten kann, hier einen Nullpunkt mindestens der Ordnung $\frac{1}{4}$ haben. Die übrig bleibenden Nullpunkte der Gesamtordnung 1 liegen in den Punkten $e_{3}$ und $e_{3}^{\prime}$, wo $z$ je in der Ordnung $\frac{1}{2}$ verschwindet. Da nämlich $\left(\frac{-4}{17}\right)=+1$ ist, so gilt für das Produkt $z \cdot \sqrt[4]{\Delta^{3}}$ :

$$
z \sqrt[4]{\Delta^{3}}\left(-4 \omega_{1}-\omega_{2}, 17 \omega_{1}+4 \omega_{2}\right)=z \sqrt[4]{\Delta^{3}}\left(\omega_{1}, \omega_{2}\right) .
$$

Tragen wir, dem Punkte $e_{3}$ entsprechend, $\omega_{1}=-4+i, \omega_{2}=17$ ein, so folgt mit Rücksicht auf die Dimension -10 :

$$
-z \sqrt[4]{\Delta^{3}}(-4+i, 17)=z \sqrt[4]{\Delta^{3}}(-4+i, 17) .
$$

Demnach verschwindet $z$ im Punkte $e_{3}$ und also auch in $e_{3}^{\prime}$. Gegenüber der homogenen Substitution $W_{17}$ bleibt $z$, wie jetzt leicht folgt, invariant. Wir notieren die beiden Potenzreihen:

(20) $\left\{\begin{array}{l}z^{2}=\left(\frac{2 \pi}{\omega_{2}}\right)^{2} q\left(1+2 q^{4}-q^{8}-4 q^{10}-4 q^{14}-q^{16}+4 q^{18}+\cdots\right), \\ \sqrt[12]{\Delta^{\prime} \Delta}=\left(\frac{2 \pi}{\omega_{2}}\right)^{2} q^{3}\left(1-2 q^{2}-q^{4}+2 q^{6}+q^{8}+2 q^{10}-2 q^{12}-2 q^{16}-\cdots\right)\end{array}\right.$

und bilden aus diesen beiden Formen die Schar $\left(a z^{2}+b \sqrt[12]{\Delta^{\prime} \Delta}\right)$ mit einem festen Nullpunkte der Ordnung $\frac{1}{2}$ in der Spitze $i \infty$ und einem beweglichen Nullpunkte erster Ordnung im Klassenpolygone $K_{17}$. Daneben 
führen wir als einwertige Funktion dieses Polygons $K_{17}$ :

$$
\tau(\omega)=\frac{z^{2}}{\sqrt[12]{\Delta^{\prime} \Delta}}=q^{-2}+2+7 q^{2}+12 q^{4}+\cdots
$$

ein, die ihren Pol erster Ordnung in der Spitze $i \infty$ hat.

Durch $\tau(\omega)$ wird das dem Geschlechte 1 angehörende Transformationspolygon $T_{17}$ auf eine zweiblättrige Riemannsche Fläche mit vier Verzweigungspunkten abgebildet. Um deren Verzweigungsform zu gewinnen, ziehen wir wie üblich einen Differentiationsprozeß heran. Die Form $(-2)^{\text {ter }}$ Dimension $\frac{d \tau}{(\omega, d \omega)}$, die gegenüber $W_{17}$ Zeichenwechsel erfährt, hat im Polygone $K_{17}$ gemessen fünf Nullpunkte der Ordnung $\frac{1}{2}$ in den Ecken $e_{0}$, $e_{2}, e_{2}^{\prime}$ und den Zyklen $e_{1}, e_{1}^{\prime}$ und $e_{3}, e_{3}^{\prime}$, sowie einen Pol erster Ordnung in der Spitze $i \infty$. Aus ihr gewinnnen wir in:

$$
v\left(\omega_{1}, \omega_{2}\right)=-2 \pi i \frac{\sqrt[8]{\Delta^{\prime} \Delta}}{z} \frac{d \tau}{(\omega, d \omega)}
$$

eine Form $(-4)^{\text {ter }}$ Dimension mit der Reihenentwicklung:

$$
\text { (23) } v=\left(\frac{2 \pi}{\omega_{2}}\right)^{4} q^{2}\left(1-3 q^{2}-8 q^{4}+q^{6}+6 q^{8}+24 q^{10}-28 q^{12}+21 q^{14}+\cdots\right) \text {, }
$$

die in der Spitze $i \infty$ von $\mathbf{K}_{17}$ einen Nullpunkt erster Ordnung und in den Punkten $e_{0}, e_{2}, e_{2}^{\prime}$ sowie im Zyklus $e_{1}, e_{1}^{\prime}$ je einen solchen der Ordnung $\frac{1}{2}$ hat. Gegenüber $W_{17}$ erfährt diese Form Zeichenwechsel. Das Quadrat von $v$ ist als homogene ganze Funktion vierten Grades von $z^{2}$ und $\sqrt[12]{\Delta^{\prime} \Delta}$ darstellbar und liefert in dieser Gestalt die Verzweigungsform der eben genannten Riemannschen Fläche über der $\tau$-Ebene. Die fragliche Darstellung von $v^{2}$ ist:

$$
v^{2}=z^{8}-6 z^{6} \sqrt[12]{\Delta^{\prime} \Delta}-27 z^{4} \sqrt[6]{\Delta^{\prime} \Delta}-28 z^{2} \sqrt[4]{\Delta^{\prime} \Delta}-16 \sqrt[3]{\Delta^{\prime} \Delta}
$$

Die Reihenentwicklungen liefern in bekannter Art die Koeffizienten dieses Ausdrucks. Als ein Funktionssystem für die $\Gamma_{\psi(17)}$ gewinnen wir endlich:

$$
\tau(\omega)=\frac{z^{2}}{\sqrt[12]{\Delta^{\prime} \Delta}}, \quad \sigma(\omega)=\frac{v}{\sqrt[6]{\Delta^{\prime} \Delta}},
$$

wobei sich $\sigma$ als Funktion von $\tau$ mittelst der Quadratwurzel darstellt:

$$
\sigma=\sqrt{\tau^{4}-6 \tau^{3}-27 \tau^{2}-28 \tau-16} .
$$

Es liegt hier ein elliptisches Gebilde mit der absoluten Invariante $J=-\frac{11^{3}}{2^{6} \cdot 17^{8}}$ vor.

Auf Grund der damit gewonnenen Ergebnisse lassen sich nun wieder die ursprünglichen und die transformierten Formen $g_{2}, g_{3}$ und $\Delta$ mittels formentheoretischer Überlegungen und der Potenzreihen darstellen. Für 
432 II, 4. Transformationsgleichungen erster Stufe für niedere Grade $n$ die Gewinnung des Ausdrucks von $J$ in $\sigma$ und $\tau$ ist es ausreichend, $g_{2}$ und $\Delta \mathrm{zu}$ betrachten. Wir finden erstlich für $g_{2}$ und $g_{2}^{\prime}$ :

(27) $\left\{\begin{array}{l}6\left(g_{2}^{\prime}+g_{2}\right) \sqrt[12]{\Delta^{\prime} \Delta}=5\left(29 z^{6}-34 z^{4} \sqrt[12]{\Delta^{\prime} \Delta}-103 z^{2} \sqrt[6]{\Delta^{\prime} \Delta}-36 \sqrt[4]{\Delta^{\prime} \Delta}\right), \\ \left(g_{2}^{\prime}-g_{2}\right) \sqrt[12]{\Delta^{\prime} \Delta}=4 v\left(6 z^{2}+\sqrt[12]{\Delta^{\prime} \Delta}\right) .\end{array}\right.$

Zur Darstellung von $\Delta$ beachte man, daß $\sqrt[4]{\Delta} \sqrt[84]{\Lambda^{\prime} \Delta}$ gegenüber den Substitutionen der $\Gamma_{\psi(17)}$ abgesehen vom Vorzeichen invariant ist; $\sqrt{\Delta} \sqrt[12]{\Delta^{\prime} \Delta}$ ist also absolut invariant. In:

$$
\left(\sqrt{\Delta}-17^{3} \sqrt{\Delta^{\prime}}\right) \sqrt[12]{\Delta^{\prime} \Delta}
$$

hat man demnach eine Form der $\Gamma_{\psi(17)}$, die gegenüber $W_{17}$ Zeichenwechsel erfährt und also als Produkt von $v$ und einer ganzen homogenen Funktion zweiten Grades von $z^{2}$ und $\sqrt[12]{\Delta^{\prime} \Delta}$ darstellbar ist. Die Potenzreihen liefern:

$$
\left(\sqrt{\Delta}-17^{3} \sqrt{\Delta^{\prime}}\right) \sqrt[12]{\Delta^{\prime} \Delta}=v\left(z^{4}-11 z^{2} \sqrt[12]{\Delta^{\prime} \Delta}+26 \sqrt[6]{\Delta^{\prime} \Delta}\right)
$$

Zur Prüfung dieses Ergebnisses berechne man durch Quadrieren usw. $\left(\sqrt{\Delta}+17^{3} \sqrt{\Delta^{\prime}}\right)^{2} \cdot \sqrt[6]{\Delta^{\prime} \Delta}$. Für diese Form muB sich, indem man für $v^{2}$ den Ausdruck (24) einträgt, das Quadrat einer homogenen Funktion vierten Grades von $z^{2}$ und $\sqrt[12]{\Delta^{\prime} \Delta}$ ergeben. Die Rechnung bestätigt dies und führt auf:

$$
\begin{aligned}
& \left(\sqrt{\Delta}+17^{3} \sqrt{\Delta^{\prime}}\right) \sqrt[12]{\Delta^{\prime} \Delta}=z^{8}-14 z^{6} \sqrt[12]{\Delta^{\prime} \Delta}+41 z^{4} \sqrt[6]{\Delta^{\prime} \Delta} \\
& +52 z^{2} \sqrt[4]{\Delta^{\prime} \Delta}-94 \sqrt[3]{\Delta^{\prime} \Delta}
\end{aligned}
$$

Durch Kombination der vorstehenden Formeln und Einführung der Funktionen $\sigma$ und $\tau$ findet man:

$$
\left\{\begin{array}{l}
\frac{12 g_{2}}{\sqrt[6]{\Delta^{\prime} \Delta}}=5\left(29 \tau^{3}-2 \cdot 17 \tau^{2}-103 \tau-2^{2} \cdot 3^{2}\right)-2^{3} \cdot 3 \sigma(2 \cdot 3 \tau+1) \\
\frac{2 \sqrt{\Delta}}{\sqrt[4]{\Delta^{\prime} \Delta}}=\tau^{4}-2 \cdot 7 \tau^{3}+41 \tau^{2}+2^{2} \cdot 13 \tau-2 \cdot 47+\sigma\left(\tau^{2}-11 \tau+2 \cdot 13\right) .
\end{array}\right.
$$

Hieraus ist der Ausdruck von $J$ als einer rationalen Funktion von $\sigma$ und $\tau$ leicht gewinnbar. Der entsprechende Ausdruck von $J^{\prime}$ folgt durch Zeichenwechsel von б. Man kann auch bereits die Gleichungen (30) im Verein mit der Gleichung (26) als Ersatz der Transformationsgleichung für $J$ beim 17ten Grade ansehen. ${ }^{1}$ )

3. Transformation $29^{\text {sten }}$ Grades. Die zur linken Seite der imaginären $\omega$-Achse gelegene Hälfte des Klassenpolygons $\mathbf{K}_{29}$ ist in Fig. 21

1) Die Transformationsgleichung für die Diskriminante $\Delta$ beim Grade 17 ist von Kiepert im Journ. f. Math., Bd. 87, S. 215 mitgeteilt. 
abgebildet. Der mit 8 bezeichnete Symmetriekreis gehört zur Spiegelung $\bar{W}_{29}$; die Gleichungen der Kreise 1, 2 und 5 und die zugehörigen Spiegelungen sind:
1) $29\left(\xi^{2}+\eta^{2}\right)+29 \xi+7=0$,
$\omega^{\prime}=\frac{-29 \bar{\omega}-14}{58 \bar{\omega}+29}$
2) $145\left(\xi^{2}+\eta^{2}\right)+116 \xi+23=0$,
$\omega^{\prime}=\frac{-58 \bar{\omega}-23}{145 \bar{\omega}+58}$,
5) $116\left(\xi^{2}+\eta^{2}\right)+58 \xi+7=0$,
$\omega^{\prime}=\frac{-29 \bar{\omega}-7}{116 \bar{\omega}+29}$.

Von den sechs Formklassen der Diskriminante $D=-116$ sind zwei

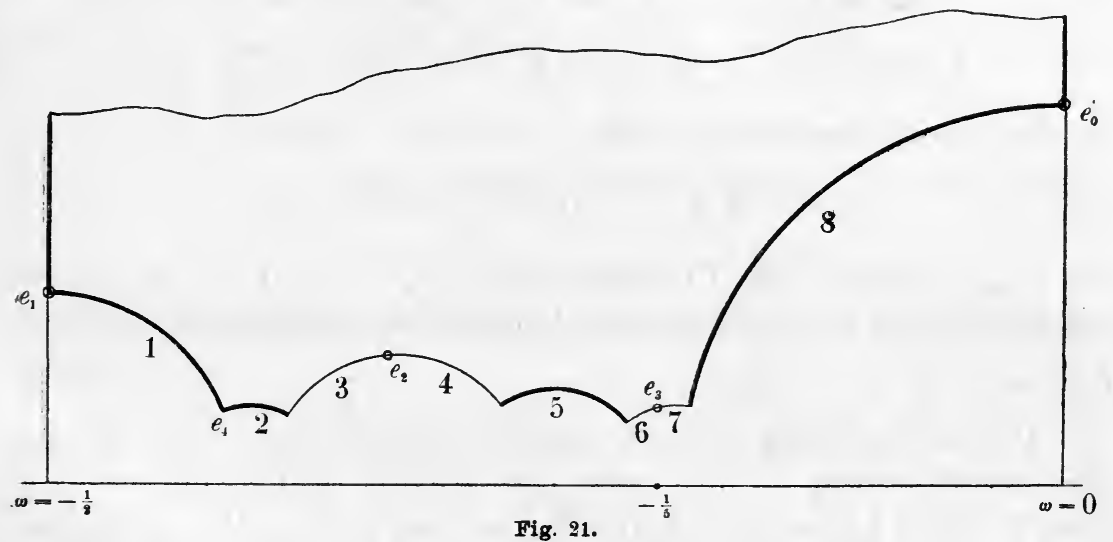

ambig; man kann sie durch die Formen $(29,0,1)$ und $(58,58,15)$ repräsentieren, deren Nullpunkte die in der Figur mit $e_{0}$ und $e_{1}$ bezeichneten, bei $\omega=\frac{i}{\sqrt{29}}$ und $\frac{-\sqrt{29}+i}{2 \sqrt{29}}$ gelegenen Fixpunkte der Substitutionen $W_{29}$ und $\left(\begin{array}{r}-29,-15 \\ 58,\end{array} 29\right)$ sind. Die vier übrigen Klassen sind zu Paaren entgegengesetzt und repräsentierbar durch $(87, \pm 58,10),(145, \pm 58,6)$. Von ihren Nullpunkten $e_{2}, e_{2}^{\prime}$ und $e_{3}, e_{3}^{\prime}$ sind $e_{2}$ und $e_{3}$ in Fig. 21 angegeben und bei $\omega=\frac{-\sqrt{29}+i}{3 \sqrt{29}}, \frac{-\sqrt{29}+i}{5 \sqrt{29}}$ gelegen; sie sind die Fixpunkte der in

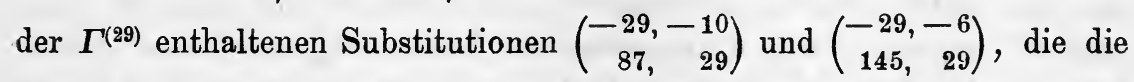
Seiten 3 und 4 bzw. 6 und 7 von $K_{29}$ ineinander transformieren. Die von diesen Seitenpaaren gebildeten Kreisbogen haben die Gleichungen:

$$
29\left(\xi^{2}+\eta^{2}\right)+19 \xi+3=0, \quad 29\left(\xi^{2}+\eta^{2}\right)+11 \xi+1=0 .
$$

Endlich sind $e_{1}$ und der bezüglich der imaginären $\omega$-Achse symmetrische Punkt $e_{4}^{\prime}$ die Fixpunkte der schon in der $\Gamma_{\psi(29)}$ auftretenden Substitutionen $\left(\begin{array}{c}\mp 12,-5 \\ 29, \pm 12\end{array}\right)$; sie liefern auf der über der $J$-Ebene gelegenen Fläche $F_{29}$ 
II, 4. Transformationsgleichungen erster Stufe für niedere Grade $n$ zwei Stellen $J=1$ in den daselbst isoliert verlaufenden Blättern. Am Klassenpolygon $\mathrm{K}_{29}$ vereinigen sich $e_{4}$ und $e_{4}^{\prime} \mathrm{zu}$ einem Eckenzyklus.

Der Ansatz (19) S. 332 liefert für die Hauptklasse der Diskriminante $D=-116$ und für die beiden Paare entgegengesetzter Klassen nicht identisch verschwindende Reihen. Wir gelangen zur Kenntnis dreier Modulformen der $\Gamma_{\psi(29)}$, die wir durch die Bezeichnungen $z_{0}, z_{1}, z_{2}$ unterscheiden:

$$
\left\{\begin{array}{l}
z_{0}=\frac{2 \pi}{\omega_{2}} q^{\frac{1}{2}}\left(1+q^{4}+q^{12}-q^{14}-2 q^{16}-2 q^{22}+\cdots\right), \\
z_{1}=\frac{2 \pi}{\omega_{2}} q^{\frac{5}{2}}\left(1-q^{2}-q^{4}+q^{10}+q^{14}-q^{24}-\cdots\right), \\
z_{2}=\frac{2 \pi}{\omega_{2}} q^{\frac{3}{2}}\left(1-q^{4}-q^{6}+q^{12}-q^{14}+q^{18}-q^{20}+\cdots\right)
\end{array}\right.
$$

Für die zweite dieser Formen gilt:

$$
z_{1}=\sqrt[24]{\Delta^{\prime} \Delta}=\sqrt[24]{\Delta\left(29 \omega_{1}, \omega_{2}\right) \Delta\left(\omega_{1}, \omega_{2}\right)}
$$

Zur $\Gamma_{\psi(29)}$ gehören die Produkte $z_{0} \sqrt[4]{\Delta^{3}}, z_{1} \sqrt[4]{\Delta^{3}}, z_{2} \sqrt[4]{\Delta} ;$ sie nehmen gegenüber einer Substitution jener Gruppe übereinstimmend den Faktor $\left(\frac{\alpha}{29}\right)$ an.

Die einzelne Form $z$ hat im Transformationspolygone $\mathbf{T}_{29}$ Nullpunkte der Gesamtordnung $\frac{5}{2}$, und zwar hat $z_{1}$ zwei Nullpunkte der Ordnung $\frac{5}{4}$ in den beiden Spitzen von $\mathrm{T}_{29}$, woraus man leicht die Invarianz von $z_{1}$ gegenüber $W_{29}$ folgert, die sich ja auch aus (32) ergibt. Die Form. $z_{0}$ hat in der Spitze $i \infty$ einen Nullpunkt der Ordnung $\frac{1}{4}$ und muß demnach (da die Ordnung $\frac{9}{4}$ restiert) bei $\omega=0$ mindestens einen Nullpunkt der Ordnung $\frac{1}{4}$ haben. Der Quotient:

$$
\tau(\omega)=\frac{z_{0}}{z_{1}}=q^{-2}+1+3 q^{2}+4 q^{4}+\cdots,
$$

der eine Funktion der $\Gamma_{\psi(29)}$ ist, stellt demgemäß eine zweiwertige Funktion des zum Geschlechte $p=2$ gehörenden Polygons $\mathbf{T}_{29}$ und also eine einwertige Funktion des Klassenpolygons $\boldsymbol{K}_{29}$ dar. Hieraus folgt wiederum, daß mit $z_{1}$ auch $z_{0}$ gegenüber $W_{29}$ invariant ist, und daß man demnach in $\left(a z_{0}+b z_{1}\right)$ eine Formenschar des Polygons $\mathbf{K}_{29}$ mit „einem" beweglichen Nullpunkte erster Ordnung und einem festen der Ordnung $\frac{1}{4}$ in der Spitze $i \infty$ hat.

Die Form $z_{2}$ hat in der Spitze $i \infty$ zunächst einen Nullpunkt der Ordnung $\frac{3}{4}$. Gegenüber der Substitution $\left(\begin{array}{r}-12,-5 \\ 29,12\end{array}\right)$ zeigt das Produkt $z_{2} \sqrt[4]{\Delta}$, da $\left(\frac{-12}{29}\right)=-1$ ist, das Verhalten:

$$
z_{2} \sqrt[4]{\Delta}\left(-12 \omega_{1}-5 \omega_{2}, 29 \omega_{1}+12 \omega_{2}\right)=-z_{2} \sqrt[4]{\Delta}\left(\omega_{1}, \omega_{2}\right)
$$


Trägt man, dem Eckpunkt $e_{4}$ entsprechend, $\omega_{1}=-12+i, \omega_{2}=29$ ein, so folgt:

$$
z_{2} \sqrt[4]{\Delta}(-1-12 i, 29 i)=-z_{2} \sqrt[4]{\Delta}(-12+i, 29)
$$

und also, da $z_{2} \sqrt[4]{\Delta}$ der Dimension -4 angehört:

$$
z_{2} \sqrt[4]{\Delta}(-12+i, 29)=-z_{2} \sqrt[4]{\Delta}(-12+i, 29) .
$$

Die Form $z_{2}$ verschwindet also im Punkte $e_{4}$ und damit auch in $e_{4}^{\prime}$, und zwar kann hier je nur noch die Ordnung $\frac{1}{2}$ vorliegen. Der zurückbleibende Nullpunkt der Ordnung $\frac{3}{4}$ kann nur noch im Punkte $\omega=0$ liegen. Hieraus ergibt sich, daß auch $z_{2}$ gegenüber $W_{29}$ invariant ist.

Die vorstehenden Darlegungen zeigen, daß der Quotient $z_{2}^{2}: z_{1}$ diejenige Form $\left(a z_{0}+b z_{1}\right)$ der Schar ist, für welche der bewegliche Nullpunkt in den Eckenzyklus $e_{4}, e_{4}^{\prime}$ fällt. Es muß sich also $z_{2}^{2}$ in der Gestalt $\left(a z_{0}+b z_{1}\right) z_{1}$ darstellen lassen. Die beiden Anfangsglieder der Reihen liefern $a=b=1$, so daß zwischen den drei Formen (31) die Gleichung besteht:

$$
\left(z_{0}+z_{1}\right) z_{1}=z_{2}^{2} .
$$

In der Tat stimmen für die beiderseitigen Reihen auch die weiteren Glieder überein.

Das Transformationspolygon $\mathbf{T}_{29}$ wird durch $\tau(\omega)$ auf eine zweiblättrige Riemannsche Fläche abgebildet, die sechs, den Punkten $e_{0}, e_{1}$, $e_{2}, e_{2}^{\prime}, e_{3}, e_{3}^{\prime}$ entsprechende Verzweigungspunkte hat. Die zugehörige Verzweigungsform machen wir in üblicher Weise zugänglich, indem wir zunächst eine der $\Gamma_{\psi(29)}$ zugehörige Modulform (-3) ter $^{\text {temension: }}$

$$
v\left(\omega_{1}, \omega_{2}\right)=-2 \pi i \frac{z_{1}^{2}}{z_{2}} \frac{d \tau}{(\omega, d \omega)}
$$

erklären. Es handelt sich um eine ganze Modulform, die gegenüber der $W_{27}$ Zeichenwechsel erfährt, die die Reihenentwicklung besitzt:

$$
v=\left(\frac{2 \pi}{\omega_{2}}\right)^{3} q^{\frac{3}{2}}\left(1-2 q^{2}-3 q^{4}-q^{6}-6 q^{8}+2 q^{10}-13 q^{12}+21 q^{14}+2 q^{16}+\cdots\right)
$$

und die neben einem Nullpunkte der Ordnung $\frac{3}{4}$ in der Spitze $i \infty$ von $\mathbf{K}_{29}$ sechs Nullpunkte der Ordnung $\frac{1}{2}$ in den genannten sechs Punkten $e_{0}, e_{1}, \ldots$ aufweist. Das Quadrat von $v$ muß nun gleich einer ganzen Funktion sechsten Grades von $z_{0}$ und $z_{1}$ sein und liefert in dieser Funktion die gesuchte Verzweigungsform. Man findet in bekannter Art mittelst der Potenzreihen:

$$
v^{2}=z_{0}^{6}-4 z_{0}^{5} z_{1}-12 z_{0}^{4} z_{1}^{2}+2 z_{0}^{3} z_{1}^{3}+8 z_{0}^{2} z_{1}^{4}+8 z_{0} z_{1}^{5}-7 z_{1}^{6}
$$

wo rechts die Verzweigungsform der genannten zweiblättrigen Riemannschen Fläche mit sechs Verzweigungspunkten gewonnen ist. ${ }^{1}$ )

1) Diese Verzweigungsform muß (nach späteren Sätzen) im rationalen Körper 
II, 4. Transformationsgleichungen erster Stufe für niedere Grade $n$

Als ein Funktionssystem für die $\Gamma_{\psi(29)}$ kann man nun:

$$
\tau(\omega)=\frac{z_{0}}{z_{1}}, \quad \sigma(\omega)=\frac{v}{z_{1}^{3}}
$$

benutzen, wobei sich die zweite, gegenüber $W_{29}$ Zeichenwechsel erfahrende Funktion in der ersten mittels der Quadratwurzel darstellt:

$$
\sigma=\sqrt{\tau^{6}-4 \tau^{5}-12 \tau^{4}+2 \tau^{3}+8 \tau^{2}+8 \tau-7} .
$$

Für die Gewinnung von $J$ in Gestalt einer rationalen Funktion von $\tau$ und $\sigma$ ist es wieder ausreichend, $g_{2}, g_{2}^{\prime}, \Delta$ und $\Delta^{\prime}$ in $z_{0}, z_{1}$ und $v$ darzustellen. Die formentheoretischen Überlegungen gestalten sich wie üblich; wir erhalten zunächst für $\left(g_{2}^{\prime} \pm g_{2}\right)$ die Ausdrücke:

$$
\left\{\begin{array}{l}
6\left(g_{2}^{\prime}+g_{2}\right) z_{1}=421 z_{0}^{5}-301 z_{0}^{4} z_{1}-1867 z_{0}^{3} z_{1}^{2}-671 z_{0}^{2} z_{1}^{3} \\
\left(g_{3}^{\prime}-g_{2}\right) z_{1}=10 v\left(7 z_{0}^{2}+5 z_{0} z_{1}-z_{1}^{2}\right) .627 z_{0} z_{1}^{4}+405 z_{1}^{5}
\end{array}\right.
$$

Die Untersuchung von $\Delta$ und $\Delta^{\prime}$ beginnt man am einfachsten mit der Differenz $\left(\sqrt{\Delta}-29^{3} \cdot \sqrt{\Delta^{\prime}}\right)$, für welche die formentheoretische Überlegung im Verein mit den Potenzreihen die Darstellung ergibt:

$$
\left(\sqrt{\Delta}-29^{3} \cdot \sqrt{\Delta^{\prime}}\right) \sqrt[24]{\Delta^{\prime} \Delta}=v\left(z_{0}^{4}-11 z_{0}^{3} z_{1}+31 z_{0}^{2} z_{1}^{3}+14 z_{0} z_{1}^{9}-100 z_{1}^{4}\right)
$$

Dies Ergebnis ist wieder einer Prüfung zugänglich. Berechnet man nämlich aus (40) unter Benutzung von (36) den Ausdruck von:

$\left(\sqrt{\Delta}-29^{3} \cdot \sqrt{\Delta^{\prime}}\right)^{2} \cdot \sqrt[12]{\Delta^{\prime} \Delta}+4 \cdot 29^{3}\left(\sqrt[12]{\Delta^{\prime} \Delta}\right)^{7}=\left(\sqrt{\Delta}+29^{3} \cdot \sqrt{\Delta^{\prime}}\right)^{2} \cdot \sqrt[12]{\Delta^{\prime} \Delta}$ in $z_{0}$ und $z_{1}$, so muB sich das Quadrat einer homogenen Funktion $7^{\text {ten }}$ Grades von $z_{0}$ und $z_{1}$ ergeben. Dies bestätigt sich in der Tat; durch Ausziehen der Quadratwurzel gelangt man zu:

$$
\begin{aligned}
\left(\sqrt{\Delta}+29^{3} \cdot \sqrt{\Delta^{\prime}}\right) \sqrt[24]{\Delta^{\prime} \Delta} & =z_{0}^{7}-13 z_{0}^{6} z_{1}+45 z_{0}^{5} z_{1}^{2}+25 z_{0}^{4} z_{1}^{3} \\
& -269 z_{0}^{3} z_{1}^{4}+29 z_{0}^{2} z_{1}^{5}+300 z_{0} z_{1}^{6}+166 z_{1}^{7} .
\end{aligned}
$$

Der fertige Ausdruck von $J$ als rationale Funktion von $\sigma$ und $\tau$ geht nun leicht aus den beiden Gleichungen hervor:

irrednzibel sein. Dagegen muB sie (entsprechend den beiden Geschlechtern zu je drei Formklassen bei der Diskriminante $D=-116)$ nach Adjunktion von $\sqrt{29}$ in zwei kubische Faktoren zerlegbar sein. Dies ist in der Tat der Fall; die beiden kubischen Faktoren sind:

$$
z_{0}^{3}-(2 \pm \sqrt{29}) z_{0}^{2} z_{1}+\frac{13 \pm \sqrt{39}}{2} z_{0} z_{1}^{2}-\frac{1 \pm \sqrt{29}}{2} z_{1}^{3}
$$




$$
\begin{aligned}
& \mid \begin{aligned}
\frac{12 g_{2}}{\sqrt[6]{\Delta^{\prime} \Delta}}=421 \tau^{5}-7 \cdot 43 \tau^{4}-1867 \tau^{3}-11 \cdot 61 \tau^{2}+3 \cdot 11 \cdot 19 \tau+3^{4} \cdot 5 \\
-2^{2} \cdot 3 \cdot 5 \sigma\left(7 \tau^{2}+5 \tau-1\right)
\end{aligned} \\
& \left\{\begin{aligned}
\frac{2 \sqrt{\Delta}}{\sqrt[4]{\Delta^{\prime} \Delta}}=\tau^{7}-13 \tau^{6} & +3^{2} \cdot 5 \tau^{5}+5^{2} \tau^{4}-269 \tau^{3}+29 \tau^{2}+2^{2} \cdot 3 \cdot 5^{2} \tau \\
& +2 \cdot 83+\sigma\left(\tau^{4}-11 \tau^{3}+31 \tau^{2}+2 \cdot 7 \tau-2^{2} \cdot 5^{2}\right) .
\end{aligned}\right.
\end{aligned}
$$

Die entsprechenden transformierten Modulformen ergeben sich durch Zeichenwechsel von $\sigma$. Diese Gleichungen im Verein mit der Relation (38) ersetzen uns in bekannter Weise die Transformationsgleichung für $J$.

\section{§ 6. Zusammengesetzte ungerade Transformationsgrade.}

1. Transfor mation $15^{\text {ten }}$ Grades. Die zur linken Seite der imaginären $\omega$-Achse gelegene Hälfte des Klassenpolygons $K_{15}$ ist das in Fig. 22 dargestellte, von sechs Symmetriekreisen begrenzte Kreisbogensechseck. Die beiden Ecken $e_{0}$ und $e_{1}$, bei

$$
\omega=\frac{-\sqrt{15}+i}{2 \sqrt{15}}
$$

und $\omega=\frac{-\sqrt{15}+i}{4 \sqrt{15}}$

gelegen, sind die Fixpunkte der Substitutionen $\left(\begin{array}{r}-15,-8 \\ 30,15\end{array}\right)$ und $\left(\begin{array}{r}-15,-4 \\ 60,15\end{array}\right)$; sie sind die Nullpunkte der beiden qua- $\omega=-\frac{1}{2}$ dratischen Formen $(15,15,4)$,

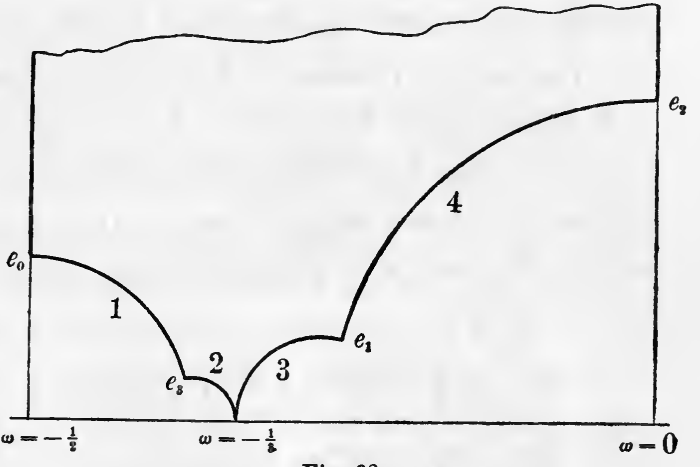

Fig. 22. $(30,15,2)$, durch die wir die beiden Klassen der Diskriminante $D=-15$ repräsentieren können. Der Fixpunkt $e_{2}$ von $W_{15}$ gehört zur Hauptklasse der Diskriminante $D=-60$; und der bei $\omega=\frac{-3 \sqrt{15}+i}{8 \sqrt{15}}$ gelegene Fixpunkt der Substitution $\left(\begin{array}{r}-45,-17 \\ 120,\end{array}\right)$ ist der Nullpunkt der quadratischen Form $(120,90,17)$, die die zweite Formklasse mit $D=-60$ repräsentiert. Die drei mit den Nummern 1, 2, 3 versehenen Seiten entsprechen den Gleichungen und Spiegelungen:

$$
\begin{array}{lll}
\text { 1. } & 30\left(\xi^{2}+\eta^{2}\right)+30 \xi+7=0, & \omega^{\prime}=\frac{-15 \bar{\omega}-7}{30 \bar{\omega}+15}, \\
\text { 2. } & 15\left(\xi^{2}+\eta^{2}\right)+11 \xi+2=0, & \omega^{\prime}=\frac{-11 \bar{\omega}-4}{30 \bar{\omega}+11}, \\
\text { 3. } & 15\left(\xi^{2}+\eta^{2}\right)+8 \xi+1=0, & \omega^{\prime}=\frac{-4 \bar{\omega}-1}{15 \bar{\omega}+4},
\end{array}
$$

während der Kreis 4 der Symmetriekreis der Spiegelung $\bar{W}_{15}$ ist. Die 
zweite und dritte Spiegelung sind bereits in der $\Gamma_{\psi(15)}$ enthalten. Neben der Spitze $i \infty$ ragt $K_{15}$ noch mit dem Spitzenzyklus $\pm \frac{1}{3}$ an die reelle $\omega$-Achse heran.

Funktionentheoretisch sind die zusammengesetzten Transformationsgrade besonders leicht zugänglich. Man setze zur Abkürzung:

$$
\Delta\left(\nu \omega_{1}, \omega_{2}\right)=\Delta_{v}
$$

und beachte, daß sowohl $\Delta_{3} \cdot \Delta_{5}$ wie $\Delta_{1} \cdot \Delta_{15}$ gegenüber den Substitutionen der $\Gamma_{15}$ invariant sind. Der Quotient $\left(\Delta_{3} \Delta_{5}\right):\left(\Delta_{1} \Delta_{15}\right)$ ist also eine Funktion des Polygons $K_{15}$, und zwar kann diese Funktion Pole und Nullpunkte nur in der Spitze $i \infty$ und im Spitzenzyklus $\pm \frac{1}{3}$ haben. Da aber in der Spitze $i \infty$ ein Pol achter Ordnung liegt, so findet sich im Zyklus $\pm \frac{1}{3}$ ein Nullpunkt der gleichen Ordnung, so daB in:

$$
\tau(\omega)=\sqrt[8]{\frac{\Delta_{3} \Delta_{5}}{\Delta_{1} \Delta_{15}}}=q^{-2}+3+9 q^{2}+\cdots
$$

bereits eine einwertige Funktion von $K_{15}$ gewonnen ist.

Die beiden Formen $\sqrt[8]{\Delta_{3} \Delta_{5}}$ und $\sqrt[8]{\Delta_{1} \Delta_{15}}$ als solche der Dimension -3 haben beide Nullpunkte der Gesamtordnung 3 auf $K_{15}$. Dabei hat $\sqrt[8]{\Delta_{3} \Delta_{5}}$ in der Spitze $i \infty$ einen Nullpunkt erster Ordnung und also im Zyklus $\pm \frac{1}{3}$ einen solchen zweiter Ordnung, während $\sqrt[8]{\Delta_{1} \Delta_{15}}$ an diesen beiden Stellen bzw. Nullpunkte der Ordnung 2 und 1 hat. Hiernach haben wir in:

$$
\left\{\begin{array}{l}
z_{0}=\sqrt[24]{\frac{\Delta_{3}^{2} \Delta_{5}^{2}}{\Delta_{1} \Delta_{15}}}=\frac{2 \pi}{\omega_{2}}\left(1+q^{2}+2 q^{4}+q^{6}+3 q^{8}+q^{10}+\cdots\right), \\
z_{1}=\sqrt[\sqrt[24]{\Delta_{1}^{2} \Delta_{15}^{2}}]{\Delta_{3} \Delta_{5}}=\frac{2 \pi}{\omega_{2}}\left(q^{2}-2 q^{4}-q^{6}+3 q^{8}-q^{10}+\cdots\right)
\end{array}\right.
$$

zwei ganze Formen mit je einem Nullpunkte erster Ordnung im Zyklus $\pm \frac{1}{3} \mathrm{bzw}$. in der Spitze $i \infty$, deren Quotient $z_{0}: z_{1}$ die in (1) erklärte Funktion $\tau$ ist. In $\left(a z_{0}+b z_{1}\right)$ aber haben wir eine Formenschar mit einem beweglichen Nullpunkte erster Ordnung auf $\mathbf{K}_{15}$.

Das Transformationspolygon $T_{15}$ wird durch $\tau(\omega)$ auf eine zweiblättrige Riemannsche Fläche des Geschlechtes 1 abgebildet, deren Verzweigungsform wir in üblicher Art herstellen. Die nähere formentheoretische Diskussion zeigt, daß die zur $\Gamma_{\psi(15)}$ gehörende ganze Modulform $(-2)^{\text {ter }}$ Dimension:

(3) $v=-2 \pi i \frac{z_{1}}{z_{0}} \frac{d \tau}{(\omega, d \omega)}=\left(\frac{2 \pi}{\omega_{2}}\right)^{2}\left(1-3 q^{2}-9 q^{4}-3 q^{6}-21 q^{8}-\cdots\right)$,

die gegenüber $W_{15}$ Zeichenwechsel erfährt, ihre vier einfachen Nullstellen in den Punkten hat, die die Verzweigungspunkte jener zweiblättrigen Fläche liefern. Die Potenzreihen ergeben dann für $v^{2}$ die Darstellung: 


$$
v^{2}=z_{0}^{4}-10 z_{0}^{3} z_{1}-13 z_{0}^{2} z_{1}^{2}+10 z_{0} z_{1}^{3}+z_{1}^{4},
$$

womit die Verzweigungsform gewonnen ist. Als Funktionssystem des Transformationspolygons $\mathbf{T}_{15}$ haben wir daraufhin:

$$
\tau(\omega)=\frac{z_{0}}{z_{1}}, \quad \sigma(\omega)=\frac{v}{z_{1}^{z}}=-\frac{2 \pi i}{z_{0} z_{1}} \frac{d \tau}{(\omega, d \omega)},
$$

wobei sich $\sigma$ in $\tau$ durch die Quadratwurzel darstellt:

$$
\sigma=\sqrt{\tau^{4}-10 \tau^{3}-13 \tau^{2}+10 \tau+1}
$$

so daß wir hier mit einem elliptischen Gebilde der absoluten Invariante $\frac{1^{3} \cdot 37^{3}}{2^{6} \cdot 3^{7} \cdot 5^{4}}$ zu tun haben.

Zur Darstellung yon $J$ als rationale Funktion von $\sigma$ und $\tau$ bedienen wir uns der in (6) S. 392 bei der Transformation fünften Grades eingeführten Funktion $\tau$, die hier mit $\tau_{5}$ bezeichnet werden möge, und in der sich $J$ in der Gestalt (13) S. 393 darstellt. Es ist hinreichend, die auf $\mathrm{T}_{15}$ vierwertige Funktion $\tau_{5}$ in $\sigma$ und $\tau$ darzustellen. Wird $\tau_{5}$ durch $W_{15}$ in $\tau_{\tilde{5}}^{\prime}$ transformiert, so hat man:

$$
\tau_{5}=125 \sqrt[4]{\frac{\Delta_{5}}{\Delta_{1}}}, \quad \tau_{5}^{\prime}=\sqrt[4]{\frac{\Delta_{3}}{\Delta_{15}}}, \quad \tau_{5}^{\prime}-\tau_{5}=\frac{\sqrt[4]{\Delta_{1} \Delta_{3}}-125 \sqrt[4]{\Delta_{5} \Delta_{15}}}{\sqrt[4]{\Delta_{1} \Delta_{15}}} .
$$

Der rechts stehende Zähler ist nun eine ganze Form $(-6)^{\text {ter }}$ Dimension von $\mathrm{K}_{15}$, die gegenüber $W_{15}$ Zeichenwechsel erfährt und also den Faktor $v$ enthält. Hierdurch werden Nullpunkte der Gesamtordnung 2 auf $\mathbf{K}_{\mathbf{1 5}}$ erledigt, so daß noch solche der Gesamtordnung 4 übrigbleiben. Ein Nullpunkt erster Ordnung liegt in der Spitze $i \infty$, so dab die Ordnung 3 verbleibt. Da diese Ordnung ganzzahlig ist, so muß im Zyklus $\pm \frac{1}{3}$, wo unser Ausdruck sicher verschwindet, mindestens ein Nullpunkt erster Ordnung liegen. Zwei weitere Nullpunkte dieser Ordnung sind dann noch zu bestimmen. Dieser Überlegung entspricht der Ansatz:

$$
\sqrt[4]{\Delta_{1} \Delta_{3}}-125 \sqrt[4]{\Delta_{5} \Delta_{15}}=v z_{0} z_{1}\left(a z_{0}^{2}+b z_{0} z_{1}+c z_{1}^{2}\right) .
$$

Die Potenzreihen ergeben:

$$
\sqrt[4]{\Delta_{1} \Delta_{3}}-125 \sqrt[4]{\Delta_{5} \Delta_{15}}=v z_{0} z_{1}\left(z_{0}^{2}-4 z_{0} z_{1}-z_{1}^{2}\right)
$$

Zur Prüfung dieses Ergebnisses berechne man mit Hilfe von (4) den Ausdruck von $\left(\sqrt[4]{\Delta_{1} \Delta_{3}}+125 \sqrt[4]{\Delta_{5} \Delta_{15}}\right)^{2}$ in $z_{0}, z_{1}$, wobei sich das Quadrat einer homogenen Funktion sechsten Grades ergeben muß. Dies bestätigt sich, man findet durch Ausziehen der Quadratwurzel:

$$
\sqrt[4]{\Delta_{1} \Delta_{3}}+125 \sqrt[4]{\Delta_{5} \Delta_{15}}=z_{0} z_{1}\left(z_{0}^{4}-9 z_{0}^{3} z_{1}-9 z_{0} z_{1}^{3}-z_{1}^{4}\right)
$$

Von (7) und (8) aus gelangt man nun leicht zum Ziele: Der gesuchte 
440 II, 4. Transformationsgleichungen erster Stufe für nieđere Grade $n$ Ausdruck der beim Grade 5 auftretenden Funktion $\tau_{5}$ in den jetzigen $\sigma$ und $\boldsymbol{\tau}$ ist:

$$
\tau_{5}=\frac{\tau^{4}-9 \tau^{3}-9 \tau-1-\sigma\left(\tau^{2}-4 \tau-1\right)}{2 \tau}
$$

2. Transformation $21^{\text {sten }}$ Grades. Das halbe KJassenpolygon $\mathbf{K}_{21}$

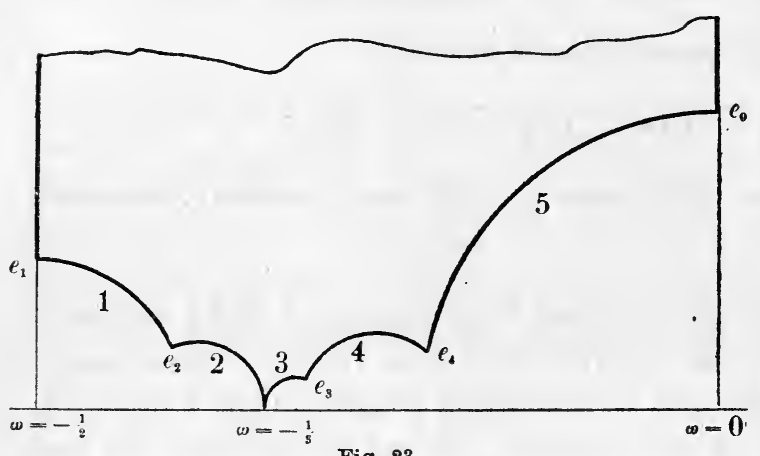

Fig. 23.

$$
\begin{array}{llll}
e_{0}, \quad \omega=\frac{i}{\sqrt{21}}, & (21,0,1), & \omega^{\prime}=\frac{-1}{21 \omega}, \\
e_{1}, \quad \omega=\frac{-\sqrt{21}+i}{2 \sqrt{21}}, & (42,42,11), & \omega^{\prime}=\frac{-21 \omega-11}{42 \omega+21}, \\
e_{2}, \quad \omega=\frac{-2 \sqrt{21}+i}{5 \sqrt{21}}, & (105,84,17), & \omega^{\prime}=\frac{-42 \omega-17}{105 \omega+42}, \\
e_{3}, \quad \omega=\frac{-3 \sqrt{21}+i}{10 \sqrt{21}}, & (210,126,19), & \omega^{\prime}=\frac{-63 \omega-19}{210 \omega+63} .
\end{array}
$$
ist ein von lauter Symmetriekreisen begrenztes Kreisbogensiebeneck (Fig. 23). Die vier Ecken $e_{0}, e_{1}, e_{2}, e_{3}$ gehören zu den vier Formklassen der Diskriminante $D=$ - 84. Die näheren Angaben gehen aus folgender Zusammenstellung hervor:

Die Ecke $e_{4}$ ist bei $\omega=\frac{-9+i \sqrt{3}}{42}$ gelegen und stellt den Fixpunkt der auch schon in der $\Gamma_{\psi(21)}$ enthaltenen Substitution $\left(\begin{array}{rr}-5,-1 \\ 21, & 4\end{array}\right)$ der Periode 3 dar. Für die mit den Nummern 1 bis 4 versehenen Seiten gelten die Angaben:

$$
\begin{aligned}
& \text { 1. } 21\left(\xi^{2}+\eta^{2}\right)+21 \xi+5=0, \quad \omega^{\prime}=\frac{-21 \bar{\omega}-10}{42 \bar{\omega}+21}, \\
& \text { 2. } 21\left(\xi^{2}+\eta^{2}\right)+16 \xi+3=0, \quad \omega^{\prime}=\frac{-8 \bar{\omega}-3}{21 \bar{\omega}+8}, \\
& \text { 3. } 42\left(\xi^{2}+\eta^{2}\right)+26 \xi+4=0, \cdot \omega^{\prime}=\frac{-13 \bar{\omega}-4}{42 \bar{\omega}+13} \\
& \text { 4. } \quad 84\left(\xi^{2}+\eta^{2}\right)+42 \xi+5=0, \quad \omega^{\prime}=\frac{-21 \bar{\omega}-5}{84 \bar{\omega}+21}
\end{aligned}
$$

Die zweite und dritte Spiegelung sind auch bereits in $\operatorname{der} \Gamma_{\psi(\mathbf{2 1})}$ enthalten. Die Seite 5 des Polygons $\mathbf{K}_{21}$ gehört natürlich zur Spiegelung $\bar{W}_{21}$. Gebraucht man die Abkürzung $\Delta_{v}$ wie soeben, so hat man in: 


$$
\left\{\begin{array}{c}
x_{0}=\sqrt[12]{\Delta_{3} \Delta_{7}}=\left(\frac{2 \pi}{\omega_{2}}\right)^{2} q^{\frac{5}{3}}\left(1-2 q^{6}-q^{12}-2 q^{14}+2 q^{18}+\cdots\right) \\
x_{1}=\sqrt[12]{\Delta_{1} \Delta_{21}}=\left(\frac{2 \pi}{\omega_{2}}\right)^{2} q^{\frac{11}{3}}\left(1-2 q^{2}-q^{4}+2 q^{6}+q^{8}+2 q^{10}-2 q^{12}\right. \\
\left.-2 q^{16}-2 q^{18}+\cdots\right)
\end{array}\right.
$$

zwei Formen, die auf $\mathbf{K}_{21}$ Nullpunkte der Gesamtordnung $\frac{8}{3}$ haben. Da diese Nullpunkte nur in der Spitze $i \infty$ und im Zyklus $\pm \frac{1}{3}$ auftreten, so hat zufolge (10) $x_{0}$ an diesen beiden Stellen Nullpunkte der Ordnungen $\frac{5}{6}$ und $\frac{11}{6}, x_{1}$ umgekehrt solche der Ordnungen $\frac{11}{6}$ und $\frac{5}{6}$. Hiernach haben wir in:

$$
\tau=\frac{x_{0}}{x_{1}}=\sqrt[12]{\frac{\Delta_{3} \Delta_{7}}{\Delta_{1} \Delta_{21}}}=q^{-2}+2+\check{5} q^{2}+\cdots
$$

bereits eine einwertige Funktion von $\mathbf{K}_{21}$.

Es ist hier auch noch der Ansatz (19) S. 332 heranzuziehen. Zwei unter den vier Formklassen der Diskriminante - 84 liefern identisch verschwindende Reihen, die beiden anderen ergeben die Reihen:

$$
\left\{\begin{array}{l}
z_{0}=\frac{2 \pi}{\omega_{2}} q^{\frac{1}{2}}\left(1+q^{4}-q^{10}-q^{12}-2 q^{18}+\cdots\right), \\
\left.z_{1}=\frac{2 \pi}{\omega_{2}} q^{\frac{3}{2}}\left(1-q^{2}-2 q^{8}+q^{12}+2 q^{14}+*+*+\cdots\right) \cdot{ }^{1}\right)
\end{array}\right.
$$

Die Produkte $z_{0}(\sqrt[4]{\Delta})^{3}$ und $z_{1} \sqrt[4]{\Delta}$ nehmen gegenüber den Substitutionen der $\Gamma_{\psi(21)}$ den Faktor $\left(\frac{\alpha}{21}\right)$ an. Hiernach können Nullpunkte gebrochener Ordnung des Nenners 3 nur in den beiden Ecken $e_{4}, e_{4}^{\prime}$ von $\mathrm{T}_{21}$ auftreten; man zählt leicht ab, daß sowohl $z_{0}$ als $z_{1}$ an jeder dieser Stellen einen Nullpunkt der Ordnung $\frac{1}{3}$ hat. Für beide Formen bleiben Nullpunkte der Gesamtordnung 2 übrig. Werden nun $z_{0}, z_{1}$ durch $W_{21}$ in $z_{0}^{\prime}, z_{1}^{\prime}$ transformiert, so sind die Quotienten $z_{0}^{\prime}: z_{0}$ und $z_{1}^{\prime}: z_{1}$ entweder Konstante oder Funktionen der $\Gamma_{\psi(21)}$. Da aber wegen der in der Spitze $i \infty$ sich forthebenden Nullpunkte in beiden Fällen die Wertigkeit unter 2 herabsinkt, so folgt mit Rücksicht auf das Geschlecht 1 von $T_{21}$, daB beide Quotienten mit Konstanten identisch sind. Hiernach sind $z_{0}$ und $z_{1}$ gegenüber $W_{21}$ bis auf Faktoren, die nur gleich \pm 1 sein können, invariant.

Die Form $z_{1}$ hat nun auf $K_{21}$ einen Nullpunkt der Ordnung $\frac{3}{4}$ in der Spitze $i \infty$ und einen solchen der Ordnung $\frac{1}{3}$ im Zyklus $e_{4}$, $e_{4}^{\prime}$, so daB nur noch ein Nullpunkt der Ordnung $\frac{1}{4}$ übrig bleibt, der nur im Zyklus $\pm \frac{1}{3}$ liegen kann. Demgegenüber hat $z_{0}$ einen Nullpunkt der Ordnung $\frac{1}{4}$ in der Spitze $i \infty$ und einen solchen der Ordnung $\frac{1}{3}$ im Zyklus $e_{4}, e_{4}^{\prime}$; es

1) Die beiden Sterne sollen andeuten, daB Glieder mit $q^{16}$ und $q^{18}$ nicht anftreten. 
II, 4. Transformationsgleichungen erster Stufe für niedere Grade $n$

bleibt also noch die Alternative, daß entweder ein Nullpunkt der Ordnung $\frac{3}{4}$ im Zyklus $\pm \frac{1}{3}$ liegt, oder daß sich daselbst ein Nullpunkt der Ordnung $\frac{1}{4}$ und dann ein weiterer Nullpunkt der Ordnung $\frac{1}{2}$ in einem der Punkte $e_{0}, \ldots, e_{3}$ findet. In beiden Fällen ist $z_{0}^{2}: z_{1}^{2}$ eine einwertige Funktion von $K_{21}$ mit dem Pole in der Spitze $i \infty$ und also eine lineare ganze Funktion des in (11) erklärten $\tau$. Die Reihenentwicklungen liefern aber sofort das Bestehen der Gleichung:

$$
\frac{z_{0}^{2}}{z_{1}^{2}}=\frac{x_{0}}{x_{1}}
$$

so daB $z_{0}$.im Zyklus $\pm \frac{1}{3}$ einen Nullpunkt der Ordnung $\frac{3}{4}$ hat.

Zufolge dieser Überlegung haben wir in:

$$
z_{0} z_{1}=\left(\frac{2 \pi}{\omega_{2}}\right)^{2} q^{2}\left(1-q^{2}+q^{4}-q^{6}-2 q^{8}-q^{10}-q^{12}+3 q^{14}+\cdots\right)
$$

eine Form von $K_{21}$, welche Nullpunkte erster Ordnung in der Spitze $i \infty$ und im Zyklus $\pm \frac{1}{3}$ hat und einen solchen der Ordnung $\frac{2}{3}$ im Zyklus $e_{4}$, $e_{4}^{\prime}$ aufweist. Dieser Form bedürfen wir zur Herstellung der Verzweigungsform der zweiblättrigen Riemannschen Fläche, auf die $T_{21}$ durch $\tau(\omega)$ abgebildet wird. Wir haben zu setzen:

$$
v=-2 \pi i \frac{\sqrt[24]{\Delta_{1} \Delta_{3} \Delta_{7} \Delta_{21}}}{z_{0} z_{1}} \frac{d \log \tau}{(\omega, d \omega)}
$$

und finden die Potenzreihe:

$$
v=\left(\frac{2 \pi}{\omega_{2}}\right)^{2} q^{\frac{2}{3}}\left(1-2 q^{2}-8 q^{4}+*+5 q^{8}+4 q^{10}-7 q^{12}+*+\cdots\right) .
$$

Diese Form hat im Klassenpolygon Nullpunkte je der Ordnung $\frac{1}{3}$ an den Stellen $i \infty$ und $\pm \frac{1}{3}$, sowie vier Nullpunkte der Ordnung $\frac{1}{2}$ in $e_{0}$ und den Zyklen $\left(e_{1}, e_{1}^{\prime}\right),\left(e_{2}, e_{2}^{\prime}\right),\left(e_{3}, e_{3}^{\prime}\right)$. Das Produkt $v^{2} \sqrt[12]{\Delta_{1} \Delta_{3} \Delta_{7} \Delta_{21}}$ muB als homogene Funktion vierten Grades von $x_{0}$ und $x_{1}$ darstellbar sein. Die Reihen liefern:

$$
v^{2} x_{0} x_{1}=x_{0}^{4}-6 x_{0}^{3} x_{1}-17 x_{0}^{2} x_{1}^{2}-6 x_{0} x_{1}^{3}+x_{1}^{4}, \quad .
$$

womit die Verzweigungsform der vorhin genannten Fläche gewonnen ist. Als Funktionssystem der $\Gamma_{\psi(21)}$ haben wir damit erhalten:

$$
\tau(\omega)=\frac{x_{0}}{x_{1}}=\frac{z_{0}^{2}}{z_{1}^{2}}, \quad \sigma(\omega)=\frac{v \sqrt[24]{\Delta_{1} \Delta_{3} \Delta_{7} \Delta_{21}}}{x_{1}^{2}}=\frac{-2 \pi i}{z_{0} z_{1}} \frac{d \tau}{(\omega, d \omega)},
$$

wobei sich $\sigma$ als Funlition von $\tau$ mittels der Wurzel:

$$
\sigma=\sqrt{\tau^{4}-6 \tau^{3}-17 \tau^{2}-6 \tau+1}
$$


darstellt. $^{1}$ ) Es liegt hier also ein elliptisches Gebilde der absoluten Invariante $-\frac{193}{2^{3} \cdot 3^{4}}$ vor.

Zur Darstellung von $J$ als rationale Funktion von $\sigma$ und $\tau$ bedienen wir uns der Vermittlung der in (34) S. 397 gegebenen, beim siebenten Grade benutzten Funktion $\tau_{7}$. Aus der leicht beweisbaren Gleichung:

$$
\sqrt[12]{\Delta_{1} \Delta_{3}}-7 \sqrt[12]{\Delta_{7} \Delta_{21}}=\sqrt[12]{\Delta_{7} \Delta_{21}} \frac{49 \tau-7 \tau_{7}}{\tau_{7}}
$$

folgt, daß die links stehende ganze Modulform gegenüber den Substitutionen der $\Gamma_{\psi(21)}$ bis auf multiplikative Einheitswurzeln invariant ist; gegenüber $W_{21}$ erfährt sie Zeichenwechsel. Die Betrachtung der Nullpunkte zeigt, daß sie bis auf einen konstanten Faktor gleich $v$ ist. Sie ist unmittelbar gleich $v$, was die Potenzreihen bestätigen. Eine weitere Prüfung des Ergebnisses liefert die Berechnung von $\left(\sqrt[12]{\Delta_{1} \Delta_{3}}+7 \sqrt[12]{\Delta_{7} \Delta_{21}}\right)$, wobei sich der Ausdruck in (16) rechts, vermehrt um $28 x_{0}^{2} x_{1}^{2}$, als Quadrat einer homogenen Funktion zweiten Grades von $x_{0}, x_{1}$ ergeben muß. Dies bestätigt sich; man findet:

$$
\sqrt[24]{\Delta_{1} \Delta_{3} \Delta_{7} \Delta_{21}}\left(\sqrt[18]{\Delta_{1} \Delta_{3}}+7 \sqrt[12]{\Delta_{7} \Delta_{21}}\right)=x_{0}^{2}-3 x_{0} x_{1}+x_{1}^{2}
$$

Durch Fortsetzung der Rechnung gelangt man zu dem Ergebnis: Die beim siebenten Grade benutzte Funktion $\tau_{7}$ stellt sich in den jetzigen $\sigma, \tau$ so dar:

$$
\tau_{7}=\frac{\left(\tau^{2}-3 \tau+1-\sigma\right)^{2}}{4 \tau} .
$$

3. Transformation $35^{\text {sten }} \mathrm{Grades}$. Das halbe Klassenpolygon $\mathrm{K}_{\mathbf{3 5}}$ ist in Fig. 24 (S. 444) abgebildet. Von den Ecken $e$ gehören $e_{0}$ und $e_{1}$ zu den beiden Formklassen der Diskriminante $D=-35$, die beide zweiseitig sind. Die Lage dieser Ecken, die repräsentierenden Formen und die zugehörigen Substitutionen der $\Gamma_{35}$ sind:

$$
\begin{aligned}
& e_{0}, \quad \omega=\frac{-\sqrt{35}+i}{2 \sqrt{35}}, \quad(35,35,9), \quad \omega^{\prime}=\frac{-35 \omega-18}{70 \omega+35}, \\
& e_{1}, \quad \omega=\frac{-\sqrt{35}+i}{6 \sqrt{35}}, \quad(105,35,3), \quad \omega^{\prime}=\frac{-35 \omega-6}{210 \omega+35} .
\end{aligned}
$$

Die Ecke $e_{2}$ gehört zur Hauptklasse der Diskriminante $D=-140$ und damit zur Substitution $W_{35}$. Endlich gehören $e_{3}, e_{4}, e_{5}$ und die zu den

1) Die Funktion unter der Wurzel (18) muB nach Adjunktion von $\sqrt{\mathbf{2 1}}$ reduzibel sein und in das Produkt zweier Funktionen zweiten Grades zerfallen. Dies bestätigt sich; die Funktionen zweiten Grades sind:

$$
\tau^{2}-(3 \pm \sqrt{21}) \tau-\frac{5 \pm \sqrt{21}}{2} .
$$


444 II, 4. Transformationsgleichungen erster Stufe für niedere Grade $n$ beiden letzten symmetrischen Ecken $e_{4}^{\prime}, e_{5}^{\prime}$ zu den fünf restierenden Formklassen mit $D=-140$. Die näheren Angaben gehen aus der Zusammenstellung hervor:

$$
\begin{array}{llll}
e_{3}, & \omega=\frac{-5 \sqrt{35}+i}{12 \sqrt{35}}, & (420,350,73), & \omega^{\prime}=\frac{-175 \omega-73}{420 \omega+175}, \\
e_{4}, & \omega=\frac{-\sqrt{35}+i}{3 \sqrt{35}}, & (105,70,12), & \omega^{\prime}=\frac{-35 \omega-12}{105 \omega+35} \\
e_{5}, & \omega=\frac{-\sqrt{35}+i}{4 \sqrt{35}}, & (140,70,9), & \omega^{\prime}=\frac{-35 \omega-9}{140 \omega+35}
\end{array}
$$

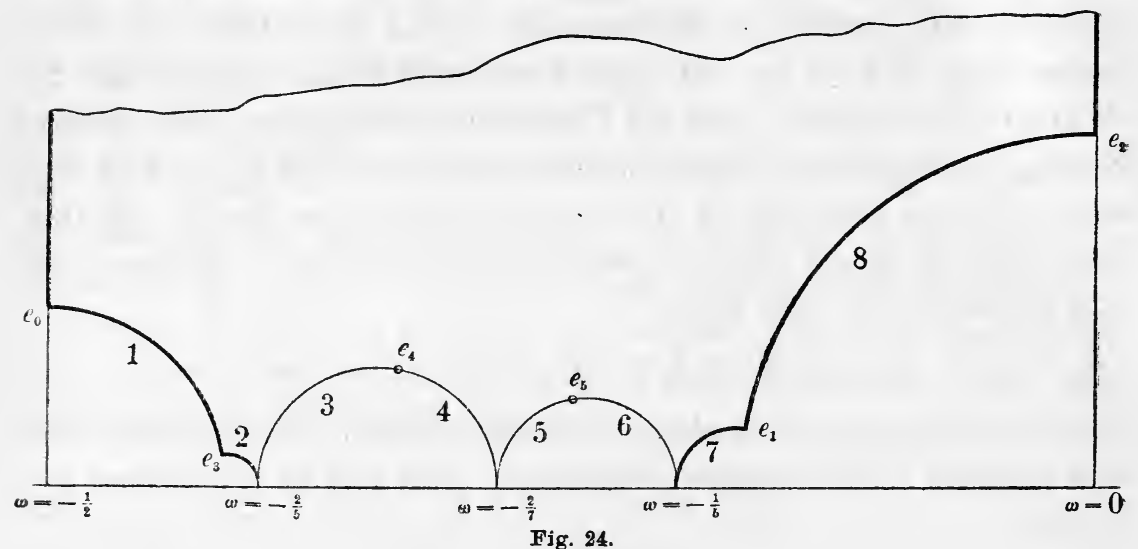

Durch die beiden letzten Substitutionen werden die Seiten 3 und 4 bzw. 5 und 6 von $K_{35}$ in einander transformiert. Auf der reellen $\omega$-Achse liefern diese Seitenpaare die Polygonspitzen $\omega=-\frac{2}{5},-\frac{2}{7},-\frac{1}{5}$, die mit den symmetrischen $\frac{2}{5}, \frac{2}{7}, \frac{1}{5}$ zu einem einzigen Spitzenzyklus zusammengehören. Die Angaben, die Kreise 1, 2 und 7 betreffend, sind:

$$
\begin{aligned}
& \text { 1. } 70\left(\xi^{2}+\eta^{2}\right)+70 \xi+17=0, \quad \omega^{\prime}=\frac{-35 \bar{\omega}-17}{70 \bar{\omega}+35}, \\
& \text { 2. } 35\left(\xi^{2}+\eta^{2}\right)+29 \xi+6=0, \quad \omega^{\prime}=\frac{-29 \bar{\omega}-12}{70 \bar{\omega}+29}, \\
& \text { 7. } 35\left(\xi^{2}+\eta^{2}\right)+12 \xi+1=0, \quad \omega^{\prime}=\frac{-6 \bar{\omega}-1}{35 \bar{\omega}+6} .
\end{aligned}
$$

Die beiden letzten Spiegelungen sind auch bereits in der $\Gamma_{\psi(35)}$ enthalten.

Die funktionentheoretische Behandlung des Grades 35 kann man auf die beiden Formen gründen:

$$
\left\{\begin{array}{l}
z_{0}=\sqrt[24]{\Delta_{5} \Delta_{7}}=\frac{2 \pi}{\omega_{2}} q\left(1-q^{10}-q^{14}-q^{20}+q^{24}+\cdots\right) \\
z_{1}=\sqrt[24]{\Delta_{1} \Delta_{35}}=\frac{2 \pi}{\omega_{2}} q^{3}\left(1-q^{2}-q^{4}+q^{10}+q^{14}-q^{24}-\cdots\right),
\end{array}\right.
$$

deren erste in der Spitze $i \infty$ und im Spitzenzyklus von $\mathbf{K}_{35}$ Nullpunkte 
der Ordnungen $\frac{1}{2}$ und $\frac{3}{2}$ hat, während $z_{1}$ an diesen Stellen umgekehrt in den Ordnungen $\frac{3}{2}$ und $\frac{1}{2}$ verschwindet. Folglich haben wir in:

$$
\tau=\frac{z_{0}}{z_{1}}=\sqrt[24]{\frac{\Delta_{5} \Delta_{7}}{\Delta_{1} \Delta_{35}}}=q^{-2}+1+2 q^{2}+\cdots
$$

bereits eine einwertige Funktion des Klassenpolygons $\mathbf{K}_{35}$ erhalten.

Zur Berechnung der Verzweigungsform derjenigen zweiblättrigen Fläche, auf die $T_{35}$ durch $\tau(\omega)$ abgebildet wird, erklären wir zunächst die Modulform $(-2)^{\text {ter }}$ Dimension:

$$
v=-2 \pi i \frac{d \log \tau}{(\omega, d \omega)}
$$

die zur $\Gamma_{\psi(35)}$ gehört und gegenüber $W_{35}$ Zeichenwechsel erfährt. Diese Form hat auf $K_{35}$ gemessen acht Nullpunkte der Ordnung $\frac{1}{2}$ in den acht Ecken $e$, die die Verzweigungspunkte der genannten zweiblättrigen Fläche liefern; ihre Reihenentwicklung ist:

$$
\begin{aligned}
v=\left(\frac{2 \pi}{\omega_{2}}\right)^{2}\left(1-q^{2}-3 q^{4}-4 q^{6}-7 q^{8}-q^{10}-12 q^{12}-q^{14}\right. \\
\left.-15 q^{16}-13 q^{18}-3 q^{20}-\cdots\right) .
\end{aligned}
$$

Das Produkt von $v^{2}$ und $\left(z_{0} z_{1}\right)^{2}$ ist als homogene Funktion achten Grades in $z_{0}, z_{1}$ darstellbar:

$$
\begin{gathered}
\left(v z_{0} z_{1}\right)^{2}=z_{0}^{8}-4 z_{0}^{7} z_{1}-6 z_{0}^{6} z_{1}^{2}-4 z_{0}^{5} z_{1}^{3}-9 z_{0}^{4} z_{1}^{4}+4 z_{0}^{3} z_{1}^{5}-6 z_{0}^{2} z_{1}^{6} \\
+4 z_{0} z_{1}^{7}+z_{1}^{8}
\end{gathered}
$$

und liefert in dieser Gestalt die gesuchte Verzweigungsform.

Als Funktionssystem der $\Gamma_{35}$ haben wir nunmehr:

$$
\tau(\omega)=\frac{z_{0}}{z_{1}}=\sqrt[24]{\frac{\Delta_{5} \Delta_{7}}{\Delta_{1}} \frac{\Delta_{35}}{s_{5}}}, \quad \sigma(\omega)=\frac{v z_{0}}{z_{1}^{3}}=-\frac{2 \pi i}{z_{1}^{2}} \frac{d \tau}{(\omega, d \omega)},
$$

wobei sich $\sigma$ als Funktion von $\tau$ mittelst der Quadratwurzel ausdrïckt:

$$
\left.\sigma=\sqrt{\tau^{8}-4 \tau^{7}-6 \tau^{6}-4 \tau^{5}-9 \tau^{4}+4 \tau^{3}-6 \tau^{2}+4 \tau+1}{ }^{1}\right)
$$

Zur Darstellung von $J$ durch $\sigma$ und $\tau$ bedienen wir uns wieder der Vermittlung der in (34) S. 397 gegebenen Funktion $\tau_{7}$, durch die sich $J$ in der Gestalt (41) S. 398 ausdrückt. Es ist demnach ausreichend $\tau_{7}$ in $\sigma$ und $\tau$ darzustellen. $\mathrm{Zu}$ diesem $\mathrm{Zwecke}$ knüpfen wir an die leicht beweisbare Gleichung:

1) Eine Prüfung des Ergebnisses kann man aus dem Umstande herleiten, dab der Ausdruck unter der Wurzel (27) im rationalen Körper reduzibel ist, nämlich das Produkt zweier ganzzahliger Faktoren der Grade 2 und 6 sein muB, entsprechend den zwei Formklassen mit $D=-3$ and den sechs mit $D=-140$. Die Zerlegung ist:

$$
\left(\tau^{2}+\tau-1\right)\left(\tau^{6}-5 \tau^{5}-9 \tau^{3}-5 \tau-1\right)
$$


446 II, 4. Transformationsgleichungen erster Stufe für niedere Grade $n$

$$
\sqrt[6]{\Delta_{1} \Delta_{5}}-49 \sqrt[6]{\Delta_{7} \Delta_{35}}=\left(\tau_{7}^{\prime}-\tau_{7}\right) \sqrt[6]{\Delta_{1} \Delta_{35}}
$$

wo $\tau_{7}^{\prime}$ die durch $W_{35}$ aus $\tau_{7}$ hervorgehende Funktion ist. Die hier links stehende Form hat acht Nullpunkte auf $K_{35}$. Erstens hat sie die acht Nullpunkte der Ordnung $\frac{1}{2}$ mit $v$ gemein, sodann kommen zwei einfache Nullpunkte in der Spitze $i \infty$ und im Spitzenzyklus dazu und endlich bleiben noch zwei Nullpunkte erster Ordnung übrig. Jene Form ist demnach als das Produkt von $v$ und einer homogenen Funktion zweiten Grades von $z_{0}, z_{1}$ darstellbar; die Potenzreihen ergeben:

$$
\sqrt[6]{\Delta_{1} \Delta_{5}}-49 \sqrt[6]{\Delta_{7} \Delta_{35}}=v\left(z_{0}^{2}-3 z_{0} z_{1}-z_{1}^{2}\right)
$$

Die übliche Probe dieses Ergebnisses führt man bei der Berechnung von $\left(\sqrt[6]{\Delta_{1} \Delta_{5}}+49 \sqrt[6]{\Delta_{7} \Delta_{35}}\right)$ durch. Man findet die Gleichung (28) bestätigt und erhält:

$$
\left(\sqrt[6]{\Delta_{1} \Delta_{5}}+49 \sqrt[6]{\Delta_{7} \Delta_{35}}\right) z_{0} z_{1}=z_{0}^{6}-5 z_{0:}^{5} z_{1}+5 z_{0}^{3} z_{1}^{3}-5 z_{0} z_{1}^{5}-z_{1}^{6} .
$$

Die Fortsetzung der Rechnung liefert für $\tau_{7}$ den Ausdruck:

$$
\tau_{7}=\frac{\tau^{6}-5 \tau^{5}+5 \tau^{3}-5 \tau-1-\sigma\left(\tau^{2}-3 \tau-1\right)}{2 \tau} .
$$

\section{$\$$ \%. Zusammengesetzte gerade Transformationsgrade.}

1. Transformation sechsten Grades. Die Verhältnisse liegen hier noch so einfach, daß man der Betrachtung unmittelbar das Transformationspolygon $\mathbf{T}_{6}$ zugrunde legen kann, das in Fig. 4, S. 354, abgebildet ist und das Geschlecht 0 hat. Neben den beiden Spitzen bei $\omega=i \infty$ und $\omega=0$ hat $\mathrm{T}_{6}$ die beiden Spitzenzyklen $\pm \frac{1}{2}$ und $\pm \frac{1}{3}$, die durch $W_{6}$ in einander transformiert werden. Eine einwertige Funktion $\tau(\omega)$ von $\mathrm{T}_{6}$ möge so erklärt werden, daß die Werte $\tau(i \infty)=0, \tau(0)=\infty$, $\tau\left(\frac{i}{\sqrt{6}}\right)=3 \sqrt{2}$ zutreffen. Schreibt man abkürzend $\tau\left(W_{6}(\omega)\right)=\tau^{\prime}(\omega)$, so hat man zufolge der letzten Festsetzung:

$$
\tau^{\prime}=\frac{18}{\tau}
$$

als Verhalten von $\tau(\omega)$ gegenüber $W_{6}$.

Zum Polygone $\mathbf{T}_{6}$ gehören auch die bei den Graden 2 und 3 benutzten Funktionen:

$$
\tau_{2}=64 \frac{\Delta_{2}}{\Delta_{1}}, \quad \tau_{3}=27 \sqrt{\frac{\Delta_{3}}{\Delta_{1}}},
$$

unter $\Delta_{v}$ wie bisher $\Delta\left(v \omega_{1}, \omega_{2}\right)$ verstanden. Die erste dieser Funktionen ist reziprok zu der in (33) S. 379 bei $n=2$ dargestellten Funktion $\tau$, die zweite ist direkt die in (9) S. 384 erklärte Funktion $\tau$ des dritten Grades. Die Lage der Nullpunkte und Pole von $\tau_{2}$ und $\tau_{3}$ in $\mathbf{T}_{6}$ stellt man da- 
durch fest, daß man erstlich $T_{6}$ in vier mit $T_{2}$ äquivalente Teilbereiche zerlegt, sodann in drei mit $\mathbf{T}_{3}$ äquivalente Teilbereiche und auf die Lage der Nullpunkte und Pole von $\tau_{2}$ und $\tau_{3}$ in den Teilbereichen Rücksicht nimmt. Der Wert von $\tau$ im Spitzenzyklus $\pm \frac{1}{9}$ heiße $-a$, so daß wegen (1) für $\tau\left( \pm \frac{1}{3}\right)$ der Wert $-18 a^{-1}$ folgt. Dann ist die Werteverteilung: von $\tau, \tau_{2}$ und $\tau_{3}$ in den Spitzen von $\mathrm{T}_{6}$ aus folgender tabellarischen $\mathrm{Zu}$ sammenstellung zu ersehen:

\begin{tabular}{c|c|c|c|c} 
& $i \infty$ & 0 & $\pm \frac{1}{2}$ & $\pm \frac{1}{3}$ \\
\hline$\tau$ & $0^{1}$ & $\infty^{1}$ & $-a$ & $-18 a^{-1}$ \\
\hline$\tau_{2}$ & $0^{1}$ & $\infty^{3}$ & $0^{3}$ & $\infty^{1}$ \\
\hline$\tau_{3}$ & $0^{1}$ & $\infty^{2}$ & $\infty^{1}$ & $0^{2}$
\end{tabular}

d. h. $\tau_{2}$ hat in der Spitze $i \infty$ einen Nullpunkt erster Ordnung, $\tau_{3}$ in der Spitze 0 einen Pol zweiter Ordnung usw.

Aus diesen Angaben entnimmt man folgende Ansätze von $\tau_{2}$ und $\tau_{3}$. als rationale Funktionen von $\tau$ :

$$
\tau_{2}=64 \frac{\Delta_{2}}{\Delta_{1}}=\frac{\tau(\tau+a)^{3}}{b(a \tau+18)}, \quad \tau_{3}=27 \sqrt{\frac{\Delta_{3}}{\Delta_{1}}}=\frac{\tau(a \tau+18)^{2}}{c(\tau+a)},
$$

wo $b$ und $c$ Konstante sind. Durch $W_{6}$ gehen $\tau_{2}, \tau_{3}$ in $\tau_{2}^{\prime}, \tau_{3}^{\prime}$ über; mit. Rücksicht auf (1) folgt:

$$
\tau_{2}^{\prime}=\frac{1}{64} \frac{\Delta_{3}}{\Delta_{6}}=\frac{(a \tau+18)^{3}}{b \tau^{3}(\tau+a)}, \quad \tau_{3}^{\prime}=\frac{1}{27} \sqrt{\frac{\Delta_{2}}{\Delta_{6}}}=\frac{18^{3}(\tau+a)^{2}}{c \tau^{2}(a \tau+18)}
$$

Durch Division der Gleichungen (3) und (4) durch einander und Ausziehen der vierten bzw. dritten Wurzel folgt weiter:

$$
8 \sqrt[4]{\frac{\Delta_{2} \Delta_{6}}{\Delta_{1} \Delta_{3}}}=\frac{\tau(\tau+a)}{a \tau+18}, \quad 162 \sqrt[6]{\frac{\Delta_{8} \Delta_{6}}{\Delta_{1} \Delta_{2}}}=\frac{\tau(a \tau+18)}{\tau+a} .
$$

Indem man diese beiden Gleichungen mit einander multipliziert und die Quadratwurzel zieht, findet man als Darstellung der einwertigen Funktion. $\tau(\omega)$ von $\mathrm{T}_{6}$ durch $\Delta$ :

$$
\tau=36 \sqrt[24]{\frac{\Delta_{2} \Delta_{6}^{5}}{\Delta_{1}^{5} \Delta_{3}}}
$$

Hiernach ist das Anfangsglied der Potenzreihe von $\tau$ durch $36 q^{2}$ gegeben. Nimmt man nun in der ersten Gleichung (5) rechts und links die Anfangsglieder, so folgt:

$$
8 q^{2}=\frac{a}{18} \tau=2 a q^{2}
$$

so daB $a=4$ ist. Indem man entsprechend für die Gleichungen (3) die 
448 II, 4. 'Transformationsgleichungen erster Stufe für niedere Grade $n$ Anfangsglieder berechnet, folgt weiter $b=2, c=108$. Die Darstellungen von $\tau_{2}$ und $\tau_{3}$ als rationale. Funktionen von $\tau$ sind somit:

$$
\tau_{2}=\frac{\tau(\tau+4)^{3}}{4(2 \tau+9)}, \quad \tau_{3}=\frac{\tau(2 \tau+9)^{2}}{27(\tau+4)} .
$$

Endlich ergibt sich der Ausdruck von $J$ mit $\tau$ einfach dadurch, daß man den in der zweiten Gleichung (7) gegebenen Ausdruck von $\tau_{3}$ in (12) S. 385 für das damalige $\tau$ einträgt.

2. Transformation zehnten Grades. Auch die Behandlung dieses Falles kann man unmittelbar auf das Transformationspolygon $\mathbf{T}_{10}$

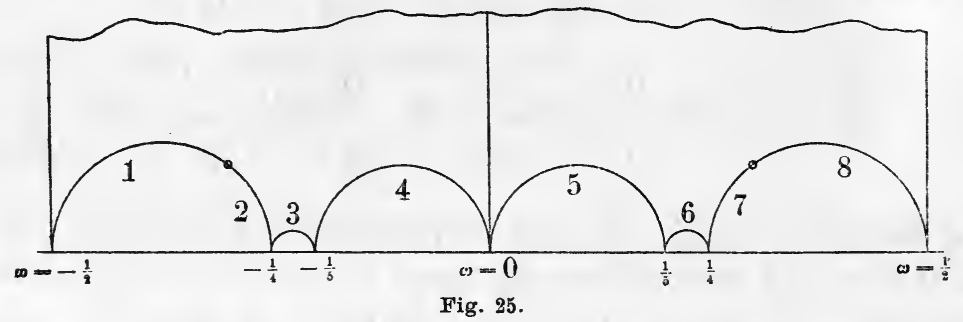

gründen, das in Fig. 25 dargestellt ist und wieder zum Geschlechte 0 gehört. Die Seiten 1 und 2 und ebenso 7 und 8 werden in einander transformiert durch die elliptischen Substitutionen $\left(\begin{array}{c}-3, \mp 1 \\ \pm 10,3\end{array}\right)$ der Periode 2, deren Fixpunkte in der Figur kenntlich gemacht sind. Die Seite 3 geht durch die Substitution $\left(\begin{array}{c}9,2 \\ 40,9\end{array}\right)$ in 6 über, die Seite $4 \operatorname{durch}\left(\begin{array}{c}1,0 \\ 10,1\end{array}\right)$ in 5 . Alle diese Substitutionen sind in der Tat in der $\Gamma_{\psi(10)}$ enthalten. Die vier Spitzen $\pm \frac{1}{2}, \pm \frac{1}{4}$ gehören zu einem Zyklus zusammen, der kurz der Zyklus $\pm \frac{1}{2}$ heiße; ebenso gehören die Spitzen $\pm \frac{1}{5}$ zu einem Zyklus zusammen.

Trägt man die Dreiecke des ursprünglichen Netzes der Modulgruppe $\Gamma^{(\omega)}$ in $\mathbf{T}_{10}$ ein, so erkennt man leicht, daß beim Umlaufen des Zyklus $\pm \frac{1}{2}$ fiinf Doppeldreiecke jenes Netzes durchschritten werden, beim Umlaufen des Zyklus $\pm \frac{1}{5}$ aber deren zwei. Dasselbe kann man durch Rechnung auf folgende Art feststellen: Die in der $\Gamma^{(\omega)}$ enthaltene zyklische Untergruppe parabolischer Substitutionen des Fixpunktes $\omega=-\frac{1}{2}$ wird aus $\left(\begin{array}{r}-1,-1 \\ 4,3\end{array}\right)$ erzeugt; von dieser Substitution ist erst die fünfte Potenz $\left(\begin{array}{r}-9,-5 \\ 20,11\end{array}\right)$ in der $\Gamma_{\psi(10)}$ enthalten. Die erzeugende Substitution der entsprechend zum Fixpunkte - $\frac{1}{5}$ gehörenden zyklischen Untergruppe ist aber $\left(\begin{array}{r}-4,-1 \\ 25,\end{array}\right)$, und von dieser Substitution ist schon die zweite Potenz $\left(\begin{array}{r}-9,-2 \\ 50,11\end{array}\right)$ in $\Gamma_{\psi(10)}$ enthalten.

Diese Angaben sind wichtig, wenn man die Polygone $T_{2}$ und $T_{5}$ in 
$T_{10}$ einträgt und die auf $T_{10}$ gemessenen Ordnungen der Nullpunkte und Pole der beiden Funktionen:

$$
\tau_{2}=64 \frac{\Delta_{2}}{\Delta_{1}}, \quad \tau_{5}=125 \sqrt[4]{\frac{\Delta_{5}}{\Delta_{1}}}
$$

feststellt. Man findet diese Ordnungen sogleich tabellarisch zusammengestellt. Vorerst möge noch eine einwertige Funktion $\tau(\omega)$ von $T_{10}$ durch die Festsetzungen $\tau(i \infty)=0, \tau(0)=\infty, \tau\left(\frac{i}{\sqrt{\overline{10}}}\right)=\sqrt{5}$ erklärt werden. Diese Funktion wird dann durch $W_{10}$ in:

$$
\tau^{\prime}=\frac{5}{\tau}
$$

transformiert. Die beiden Zyklen $\pm \frac{1}{2}$ und $\pm \frac{1}{5}$ werden durch $W_{10}$ permutiert. Wird also $\tau\left( \pm \frac{1}{2}\right)=-a$ gesetzt, so ist $\tau\left( \pm \frac{1}{5}\right)=-5 a^{-1}$. Die Tabelle der Spitzenwerte von $\tau, \tau_{2}, \tau_{5}$ ist dann:

\begin{tabular}{c|c|c|c|c} 
& $i \infty$ & 0 & $\pm \frac{1}{2}$ & $\pm \frac{1}{5}$ \\
\hline$\tau$ & $0^{1}$ & $\infty^{1}$ & $-a$ & $-5 a^{-1}$ \\
\hline$\tau_{2}$ & $0^{1}$ & $\infty^{5}$ & $0^{5}$ & $\infty^{1}$ \\
\hline$\tau_{5}$ & $0^{1}$ & $\infty^{2}$ & $\infty^{1}$ & $0^{2}$
\end{tabular}

Diesen Angaben entsprechen folgende Ansätze für $\tau_{9}$ und $\tau_{5}$ in Gestalt rationaler Funktionen von $\tau$ :

$$
\tau_{2}=64 \frac{\Delta_{2}}{\Delta_{1}}=\frac{\tau(\tau+a)^{5}}{b(a \tau+5)}, \quad \tau_{5}=125 \sqrt{\frac{4}{\Delta_{5}}}=\frac{\tau(a \tau+5)^{2}}{c(\tau+a)},
$$

wo $b$ und $c$ zwei Konstante sind. Wir verfahren nun genau wie in Falle der Transformation sechsten Grades. Durch Ausübung der Transformation $W_{10}$ gehen die Gleichungen (10) über in:

$$
\tau_{2}^{\prime}=\frac{1}{64} \frac{\Delta_{5}}{\Delta_{10}}=\frac{(a \tau+5)^{5}}{b \tau^{5}(\tau+a)}, \quad \tau_{5}^{\prime}=\sqrt[4]{\frac{\Delta_{2}}{\Delta_{10}}}=\frac{125(\tau+a)^{2}}{c \tau^{2}(a \tau+5)} .
$$

Dividiert man die Gleichungen (10) und (11) durcheinander und zieht die sechste bzw. dritte Wurzel, so folgt weiter:

$$
4 \sqrt[6]{\frac{\Delta_{2} \Delta_{10}}{\Delta_{1} \Delta_{5}}}=\frac{\tau(\tau+a)}{a \tau+5}, \quad 25 \sqrt[12]{\frac{\Delta_{5} \Delta_{10}}{\Delta_{1} \Delta_{2}}}=\frac{\tau(a \tau+5)}{\tau+a} .
$$

Durch Multiplikation dieser Gleichungen miteinander und Ausziehen der Quadratwurzel findet man als Ausdruck der einwertigen Funktion $\tau$ in der Diskriminante $\Delta$ :

$$
\tau=10 \sqrt[24]{\frac{\Delta_{2} \Delta_{10}^{3}}{\Delta_{1}^{3} \Delta_{5}}}
$$


Das Anfangsglied der Reihe für $\tau$ ist hiernach $10 q^{2}$. Die Bestimmung von $a, b, c$ aus der ersten Gleichung (12) und den Gleichungen (11) geschieht nun wie im vorigen Falle. Es findet sich $a=2, b=c=1$. Die Darstellung der bei den Transformationsgraden 2 und 5 benutzten Funktionen $\tau_{2}$ und $\tau_{5}$ als rationale Funktionen des $z u \mathrm{~T}_{10}$ gehörenden $\tau$ ist:

$$
\tau_{2}=\frac{\tau(\tau+2)^{5}}{2 \tau+5}, \quad \tau_{5}=\frac{\tau(2 \tau+5)^{2}}{\tau+2} .
$$

Die Darstellung von $J$ durch $\tau$ erhält man endlich durch Eintragen des in der zweiten Gleichung (14) gegebenen Ausdrucks für $\tau_{5}$ in die Gleichung (13) S. 393 an Stelle des damaligen $\tau$.

3. Transformation zwölften Grades. Das in Fig. 26 dargestellte Polygon $T_{12}$ ist ein Zehneck, dessen untere mit Nummern bezeichnete Seiten so zusammenhängen:

$$
1 \rightarrow 8,\left(\begin{array}{c}
5,2 \\
12,5
\end{array}\right) ; 2 \rightarrow 7,\left(\begin{array}{c}
7,2 \\
24,7
\end{array}\right) ; 3 \rightarrow 6,\left(\begin{array}{c}
5,1 \\
24,5
\end{array}\right) ; 4 \rightarrow 5,\left(\begin{array}{c}
1,0 \\
12,1
\end{array}\right) .
$$

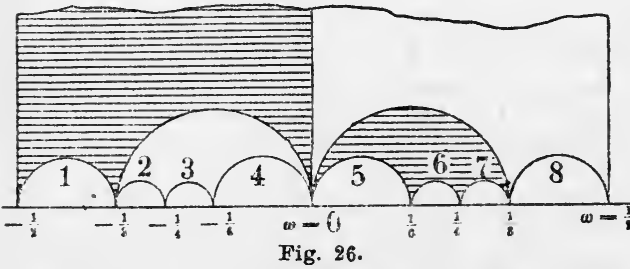

Diese Substitutionen gehören in der Tat alle vier der $\Gamma_{\psi(12)}$ an. Neben den Spitzen bei $\omega=i \infty$ und $\omega=0$ haben wir noch vier Spitzenzyklen $\pm \frac{1}{2}, \pm \frac{1}{3}, \pm \frac{1}{4}$ $\pm \frac{1}{6}$, die durch $W_{12}$ zu Paaren permutiert werden.

Die beiden schraffierten Teilbereiche sind Abbilder der negativen $\tau_{6}$-Halbebene, die beiden freien Bereiche solche der positiven $\tau_{6}$-Halbebene. Wie man unmittelbar aus der Figur abliest, wird $T_{12}$ durch $\tau_{6}$ auf eine zweiblättrige Riemannsche Fläche abgebildet, die zwei der Spitze 0 und dem Zyklus $\pm \frac{1}{3}$ entsprechende Verzweigungspunkte bei $\tau_{6}=\infty$ und $\tau_{6}=-\frac{9}{2}$ hat. Eine einwertige Funktion von $\mathrm{T}_{12}$ können wir demnach aus $\tau_{6}$ mit einer einzigen Quadratwurzel in der Gestalt berechnen:

$$
\tau=-3+\sqrt{2 \tau_{6}+9}
$$

mit der Festsetzung, daß die Quadratwurzel auf der imaginären $\omega$-Achse positiv genommen werden soll. Wir erzielen hierdurch die Spitzenwerte $\tau(i \infty)=0, \tau(0)=\infty$. Auf dem äuBeren Rande von $\mathrm{T}_{12}$ wird $\tau$ reell und negativ, und es gelten insbesondere folgende Spitzenwerte:

$$
\tau\left( \pm \frac{1}{2}\right)=-2, \quad \tau\left( \pm \frac{1}{3}\right)=-3, \quad \tau\left( \pm \frac{1}{4}\right)=-4, \quad \tau\left( \pm \frac{1}{6}\right)=-6 .
$$

Da durch $W_{12}$ die beiden Zyklen $\pm \frac{1}{3}, \pm \frac{1}{4}$ ausgetauscht werden, so findet man als Wirkung der Transformation $W_{12}$ auf $\tau$ :

$$
\tau^{\prime}=\frac{12}{\tau} \text {. }
$$


Indem man in die Darstellung von $J$ durch $\tau_{6}$ für $\tau_{6}$ den Ausdruck $\frac{1}{2} \tau(\tau+6)$ einsetzt, erhält man den Ausdruck von $J$ in dem zu $n=12$ gehörenden $\tau$. Diese Angaben über den Grad $n=12$ sind für später ausreichend.

4. Trans formation $14^{\text {ten }}$ Grades. Man knüpft hier zweckmäBig an das Klassenpolygon $K_{14}$ an, dessen eine Hälfte in Fig. 27 dargestellt ist. Den vier Formklassen der Diskriminante $D=-56$ entsprechen die vier Punkte $e_{0}, e_{1}, e_{2}, e_{2}^{\prime}$, wo $e_{2}^{\prime}$ zu $e_{2}$ symmetrisch liegt. Die näheren Angaben sind:

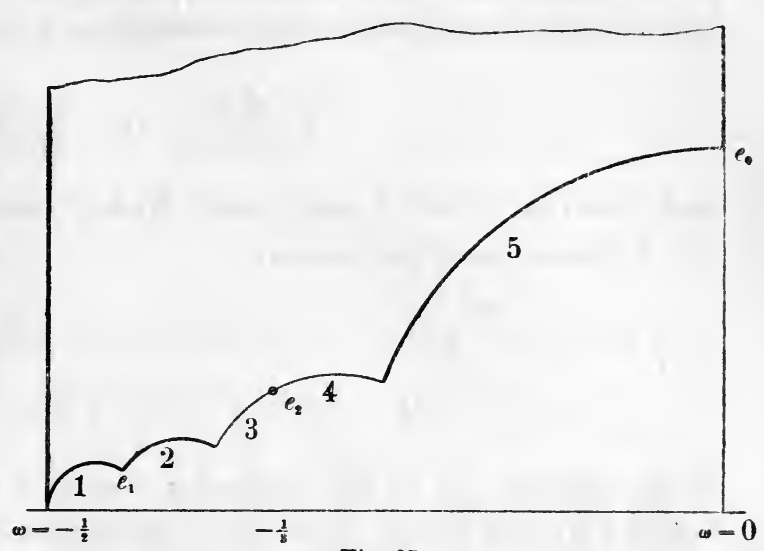

$$
\begin{aligned}
& e_{0}, \omega=\frac{i}{\sqrt{14}}, \\
& (14,0,1) \text {, } \\
& \left(\begin{array}{rr}
0, & -1 \\
14, & 0
\end{array}\right) \\
& e_{1}, \quad \omega=\frac{-4 \sqrt{14}+i}{9 \sqrt{14}} \\
& (126,112,25), \quad\left(\begin{array}{rr}
-56,-25 \\
126, & 56
\end{array}\right) \\
& e_{2}, \omega=\frac{-\sqrt{14}+i}{3 \sqrt{14}},
\end{aligned}
$$

Durch die letzte Substitution werden die Kreisbogen 3 und 4 ineinander transformiert, die den Kreis der Gleichung:

$$
14\left(\xi^{2}+\eta^{2}\right)+8 \xi+1=0
$$

zusammensetzen. Der Kreis 5 ist der Symmetriekreis der Spiegelung $\bar{W}_{14}$; für die Kreise 1 und 2 gelten die Angaben:

$$
\begin{aligned}
\text { 1. } 14\left(\xi^{2}+\eta^{2}\right)+13 \xi+3=0, & \omega^{\prime}=\frac{-13 \bar{\omega}-6}{28 \bar{\omega}+13}, \\
\text { 2. } 70\left(\xi^{2}+\eta^{2}\right)+56 \xi+11=0, & \omega^{\prime}=\frac{-28 \bar{\omega}-11}{70 \bar{\omega}+28} .
\end{aligned}
$$

Das Klassenpolygon hat nur die Spitze $i \infty$ und den Spitzenzyklus $\pm \frac{1}{2}$, so dab die formentheoretische Behandlung des Grades 14 sehr einfach ist.

Man erkennt zunächst im Quotienten $\Delta_{2} \Delta_{7}: \Delta_{1} \Delta_{14}$ durch Abzählung der Nullpunkte des Zählers und Nenners die sechste Potenz einer einwertigen Funktion $\tau(\omega)$ von $K_{14}$, die demnach selbst durch:

$$
\tau(\omega)=\sqrt[6]{\frac{\Delta_{2} \Delta_{7}}{\Delta_{1} \Delta_{14}}}=q^{-2}+4+10 q^{2}+\cdots
$$

zu erklären ist. Die Formen $\sqrt[6]{\Delta_{2} \Delta_{7}}$ und $\sqrt[6]{\Delta_{1} \Delta_{14}}$ haben Nullpunkte der Gesamtordnung 4 , und zwar hat $\sqrt[6]{\Delta_{2} \Delta_{7}}$ in der Spitze $i \infty$ einen Null- 
452 II, 4. Transformationsgleichungen erster Stufe für niedere Grade $n$ punkt der Ordnung $\frac{3}{2}$ und im Zyklus $\pm \frac{1}{2}$ einen solchen der Ordnung $\frac{5}{2}$, während $\sqrt[6]{\Delta_{1} \Delta_{14}}$ an diesen Stellen umgekehrt Nullpunkte der Ordnungen $\frac{5}{2}$ und $\frac{3}{2}$ hat. Demnach hat $\sqrt[24]{\Delta_{1} \Delta_{2} \Delta_{7} \Delta_{14}}$ in der Spitze $i \infty$ und im Zyklus $\pm \frac{1}{2}$ je einen Nullpunkt der Ordnung 1 . Wir setzen nun:

$$
z_{0}:=\frac{\sqrt[6]{\Delta_{2} \Delta_{7}}}{\sqrt[24]{\Delta_{1} \Delta_{2} \Delta_{7} \Delta_{14}}}=\sqrt[24]{\frac{\Delta_{2}^{3} \Delta_{7}^{3}}{\Delta_{1} \Delta_{14}}}, \quad z_{1}=\frac{\sqrt[6]{\Delta_{1} \Delta_{14}}}{\sqrt[24]{\Delta_{1} \Delta_{2} \Delta_{7} \Delta_{14}}}=\sqrt[24]{\frac{\Delta_{1}^{3} \Delta_{14}^{3}}{\Delta_{2} \Delta_{7}}}
$$

und haben in $z_{0}$ und $z_{1}$ zwei ganze Modulformen der Dimension -2 mit den Potenzreihenentwicklungen:

$$
\left\{\begin{array}{l}
z_{0}=\left(\frac{2 \pi}{\omega_{2}}\right)^{2} q\left(1+q^{2}-q^{4}+*-q^{8}-2 q^{10}+q^{12}-9 q^{14}+\cdots\right) \\
z_{1}=\left(\frac{2 \pi}{\omega_{2}}\right)^{2} q^{3}\left(1-3 q^{2}+q^{4}+2 q^{6}+2 q^{8}-q^{10}-4 q^{12}+2 q^{14}+\cdots\right)
\end{array}\right.
$$

deren Quotient $z_{0}: z_{1}$ die einwertige Funktion $\tau$ ist. Die Formenschar $\left(a z_{0}+b z_{1}\right)$ hat neben zwei festen Nullpunkten der Ordnung $\frac{1}{2}$ in den Spitzen einen beweglichen Nullpunkt erster Ordnung auf $\mathbb{K}_{14}$.

Die Verzweigungsform der zweiblättrigen Fläche des Geschlechtes 1, auf die das Polygon $\mathrm{T}_{14}$ durch $\tau$ abgebildet wird, kann wie bisher durch einen Differentiationsproze $B$ leicht bestimmt werden. Doch ist es noch etwas kürzer, diese Bestimmung sogleich mit der Darstellung der bei $n=7$ benutzten Funktion $\tau_{7}$ zu verknüpfen. Wir haben zunächst, wenn wieder $\tau_{7}\left(W_{14}(\omega)\right)=\tau_{7}^{\prime}(\omega)$ geschrieben wird:

$$
\tau_{7}=49 \sqrt[6]{\frac{\overline{\Delta_{7}}}{\Delta_{1}}}, \quad \tau_{7}^{\prime}=\sqrt[6]{\frac{\Delta_{2}}{\Delta_{14}}},
$$

woraus durch Addition hervorgeht:

$$
\tau_{7}^{\prime}+\tau_{7}=\frac{\sqrt[6]{\Delta_{1} \Delta_{2}}+49 \sqrt[6]{\Delta_{7} \Delta_{14}}}{\sqrt[6]{\Delta_{1} \Delta_{14}}}
$$

Die hier im Zähler stehende Form hat Nullpunkte der Gesamtordnung 4 auf $K_{14}$. Da in der Spitze $i \infty$ ein Nullpunkt der Ordnung $\frac{1}{2}$ auftritt und die Form in den Ecken $e$ nicht verschwindet, so liegt auch im Zyklus $\pm \frac{1}{2}$ ein Nullpunkt der Ordnung $\frac{1}{2}$, so daß das Produkt jener Form mit $\sqrt[24]{\Delta_{1} \Delta_{2} \Delta_{7} \Delta_{14}}$ als homogene ganze Funktion dritten Grades von $z_{0}$ und $z_{1}$ darstellbar ist. Die Potenzreihen ergeben:

$$
\sqrt[24]{\Delta_{1} \Delta_{2} \Delta_{7} \Delta_{14}}\left(\sqrt[6]{\Delta_{1} \Delta_{2}}+49 \sqrt[6]{\Delta_{7} \Delta_{14}}\right)=z_{0}^{3}-8 z_{0}^{2} z_{1}-8 z_{0} z_{1}^{2}+z_{1}^{3}
$$

Indem man diese Gleichung quadriert und $196 \sqrt[4]{\Delta_{1} \Delta_{2} \Delta_{7} \Delta_{14}}=196 z_{0}^{3} z_{1}^{3}$ abzieht, findet man:

$$
\begin{gathered}
\sqrt[12]{\Delta_{1} \Delta_{2} \Delta_{7} \Delta_{14}}\left(\sqrt[6]{\Delta_{1} \Delta_{2}}-49 \sqrt[6]{\Delta_{7} \Delta_{14}}\right)^{2}=z_{0}^{6}-16 z_{0}^{5} z_{1}+48 z_{0}^{4} z_{1}^{2}-66 z_{0}^{3} z_{1}^{3} \\
+48 z_{0}^{2} z_{1}^{4}-16 z_{0} z_{1}^{5}+z_{1}^{6} .
\end{gathered}
$$


Dies Ergebnis ist einer Prüfung fähig. Es muß nämlich die rechts stehende Funktion reduzibel sein und in das Produkt der oben genannten Verzweigungsform vierten Grades und des Quadrates einer linearen Funktion zerfallen. Dies bestätigt sich; man findet durch Ausziehen der Quadratwurzel:

$$
\begin{aligned}
\sqrt[24]{\Delta_{1} \Delta_{2} \Delta_{7} \Delta_{14}}\left(\sqrt[6]{\Delta_{1} \Delta_{2}}-49 \sqrt[6]{\Delta_{7} \Delta_{14}}\right) \\
=\left(z_{0}-z_{1}\right) \sqrt{z_{0}^{4}-14 z_{0}^{3} z_{1}+19 z_{0}^{2} z_{1}^{2}-14 z_{0} z_{1}^{3}+z_{1}^{4}}
\end{aligned}
$$

wo unter der Wurzel rechter Hand die Verzweigungsform gewonnen ist.

Neben $\tau(\omega)$ führen wir nun als zweite Funktion $\sigma(\omega)$ der $\Gamma_{\psi(14)}$ den Quotienten der in (21) rechts stehenden Wurzel mit $z_{1}^{2}$ ein: Für das Funktionssystem $\sigma, \tau$ der $\Gamma_{\psi(14)}$ besteht dann die Relation:

$$
\sigma=\sqrt{\tau^{4}-14 \tau^{3}+19 \tau^{2}-14 \tau+1},
$$

so daB wir mit einem elliptischen Gebilde der absoluten Invariante $\frac{5^{3} \cdot 43^{3}}{2^{12} \cdot 3^{3} \cdot 7^{3}}$ zu tun haben. Durch Subtraktion der Gleichung (21) von (20) findet man nach kurzer Zwischenrechnung den Satz: Die beim siebenten Grade benutzte Funktion $\tau_{7}$ stellt sich als rationale Funktion von $\sigma$ und $\tau$ in der Gestalt dar:

$$
\tau_{7}=\frac{(\tau+1)\left(\tau^{2}-9 \tau+1\right)-(\tau-1) \sigma}{2 \tau} .
$$

5. Transformation $18^{\text {ten }}$ Grades. Das Transformationspolygon $T_{18}$ ist in Fig. 28 dargestellt, die man sich auf der rechten Seite der imagi-

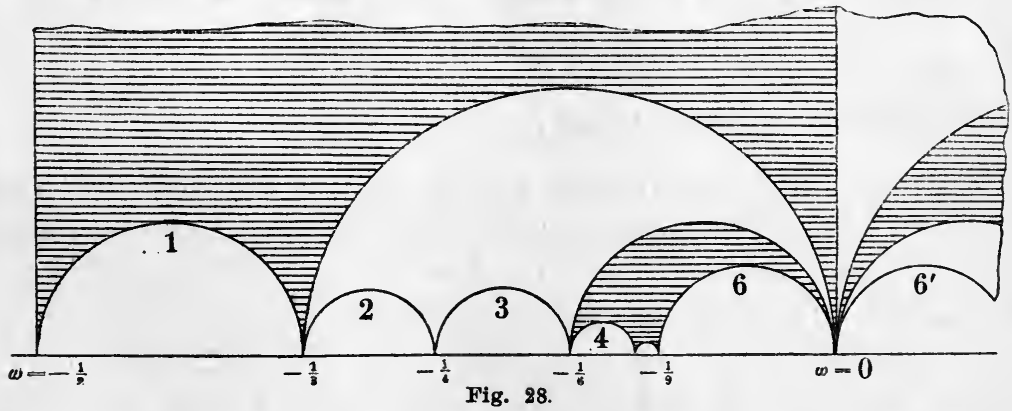

nären $\omega$-Achse symmetrisch fortgesetzt zu denken hat. Die sechs Halbkreise, die $T_{18}$ links von $\omega=0$ nach unten begrenzen, bezeichne man der Reihe nach mit $1,2, \ldots, 6$, die ihnen symmetrischen Halbkreise seien etwa durch $6^{\prime}, 5^{\prime}, \ldots, 1^{\prime}$ bezeichnet. Dann gelten folgende Zuordnungen der Polygonseiten durch erzeugende Substitutionen $\operatorname{der} \Gamma_{\psi(18)}$ :

$$
\begin{aligned}
1 \rightarrow 2,1^{\prime} \rightarrow 2^{\prime},\left(\begin{array}{c}
-5, \mp 2 \\
\pm 18,7
\end{array}\right) ; 3 \rightarrow 4,3^{\prime} \rightarrow 4^{\prime},\left(\begin{array}{c}
-5, \mp 1 \\
\pm 36,7
\end{array}\right) \\
5 \rightarrow 5^{\prime},\left(\begin{array}{c}
17,2 \\
144,17
\end{array}\right) ; 6 \rightarrow 6^{\prime} ;\left(\begin{array}{c}
1,0 \\
18,1
\end{array}\right) .
\end{aligned}
$$


454 II, 4. Transformationsgleichungen erster Stufe für niedere Grade $n$

An Polygonspitzen hat man erstlich die sechs bei $\omega=i \infty, 0, \frac{1}{3}, \frac{1}{6},-\frac{1}{3},-\frac{1}{6}$ gelegenen; von den übrigen Spitzen bilden die sechs $\pm \frac{1}{2}, \pm \frac{1}{4}, \pm \frac{1}{8}$ einen Zyklus, die beiden $\pm \frac{1}{9}$ einen zweiten.

Die drei schraffierten Teilbereiche sind Bilder der negativen $\tau_{6}$-Halbebene, die drei freien solche der positiven $\tau_{6}$-Halbebene. Man liest aus der Figur leicht den Satz ab: $T_{18}$ wird durch $\tau_{6}$ auf eine dreiblättrige Riemannsche Fläche mit zwei dreiblättrigen Verzweigungspunkten abgebildet, die der Spitze $\omega=0$ und dem Zyklus $\left( \pm \frac{1}{2}, \pm \frac{1}{4}, \pm \frac{1}{8}\right)$ entsprechen und also bei $\tau_{6}=-4$ und $\tau_{6}=\infty$ liegen. Eine einwertige Funktion von $\mathrm{T}_{18}$ ist also in:

$$
\tau=-2+\sqrt[3]{2 \tau_{6}+8}
$$

hergestellt, wo die Bestimmung gelte, daß die Kubikwurzel auf dẻr imaginären $\omega$-Achse reell ist.

Als Spitzenwerte dieser Funktion berechnet man aus denen von $\tau_{6}$ :

$$
\left\{\begin{array}{l}
\tau(i \infty)=0, \quad \tau(0)=\infty, \quad \tau\left( \pm \frac{1}{2}\right)=\tau\left( \pm \frac{1}{4}\right)=\tau\left( \pm \frac{1}{8}\right)=-2, \\
\tau\left( \pm \frac{1}{9}\right)=-3, \quad \tau\left( \pm \frac{1}{6}\right)=-3 \pm i \sqrt{3}, \quad \tau\left( \pm \frac{1}{3}\right)=\frac{-3 \pm i \sqrt{3}}{2} .
\end{array}\right.
$$

Da durch die Transformation $W_{18}$ die Spitzen $\frac{1}{2}$ und $-\frac{1}{9}$ ausgetauscht werden, so ist die Wirkung von $W_{18}$ auf $\tau$ :

$$
\tau^{\prime}=\frac{6}{\tau} \text {. }
$$

Den Ausdruck von $J$ in $\tau$ erhält man einfach durch Eintragung von:

$$
\tau_{6}=\frac{1}{2} \tau\left(\tau^{2}+6 \tau+12\right)
$$

in die Gleichung zwischen $J$ und $\tau_{6}$.

6. Die Transformationsgrade 20, 24 und 36. Diese drei Grade, für welche die Transformationspolygone übereinstimmend zum Geschlechte 1 gehören, lassen sich sehr leicht im AnschluB an die Grade 10, 12 und 18 behandeln. Man verstehe unter $\tau$ der Reihe nach die Funktionen $\tau_{10}$, $\tau_{12}, \tau_{18}$, bezeichne mit $\tau^{\prime}$ die bzw. durch $W_{20}, W_{24}, W_{36}$ transformierte Funktion und beachte, daB in jedem Falle $\tau$ und $\tau^{\prime}$ zwei zweiwertige Funktionen von $T$ sind, die demnach durch eine in jeder Funktion $\tau, \tau^{\prime}$ auf den zweiten Grad ansteigende Gleichung verknüpft sind. Die Spitzenwerte genügen bereits, diese in jedem Falle in $\tau$ und $\tau^{\prime}$ symmetrischen Relationen fertig anzuschreiben ${ }^{1}$ ):

1) Man verfährt am zweckmäBigsten so, daß man in jedem Falle zunächst die Relation zwischen $\tau(\omega)$ und $\tau(2 \omega)$ aufstellt und dann die Substitution $W$ ausübt, wobei $\tau(\omega)$ in $\tau^{\prime}(\omega)$ übergeht und $\tau(2 \omega)$ auf Grund der Gleichungen (9) bzw. (16) oder (26) durc̀ $\tau(\omega)$ auszudrücken ist. 


$$
\begin{cases}\tau=\tau_{10}, & \tau^{\prime 2} \tau^{2}-30 \tau^{\prime} \tau-50\left(\tau^{\prime}+\tau\right)-100=0 \\ \tau=\tau_{12}, & \tau^{\prime 2} \tau^{2}-72 \tau^{\prime} \tau-144\left(\tau^{\prime}+\tau\right)-288=0 \\ \tau=\tau_{18}, & \tau^{\prime 2} \tau^{2}-24 \tau^{\prime} \tau-36\left(\tau^{\prime}+\tau\right)-72=0 .\end{cases}
$$

Indem man diese Gleichungen nach $\tau^{\prime}$ löst, stellt sich in jedem Falle die Quadratwurzel einer ganzen Funktion dritten Grades von $\tau$ ein, die als Funktion von $\omega$ durch $\sigma(\omega)$ bezeichnet sei und mit $\tau(\omega)$ zu einem Funktionssystem der $\Gamma_{\psi}$ vereint werden soll. Für die drei Transformationspolygone $\mathrm{T}_{20}, \mathrm{~T}_{24}$ und $\mathrm{T}_{36}$ erhalten wir so die drei Funktionssysteme:

$$
\begin{cases}\tau=\tau_{10}, & \sigma=\sqrt{2 \tau^{3}+13 \tau^{2}+30 \tau+25} \\ \tau=\tau_{12}, & \sigma=\sqrt{\tau^{3}+11 \tau^{2}+36 \tau+36} \\ \tau=\tau_{18}, & \sigma=\sqrt{\tau^{3}+6 \tau^{2}+12 \tau+9}\end{cases}
$$

so dab wir mit drei elliptischen Gebilden der absoluten Invarianten $\frac{11^{3}}{2^{2} \cdot 3^{3} \cdot 5^{2}}$, $\frac{1^{3}}{2^{2} \cdot 3^{5}}, 0 \mathrm{zu}$ tun haben. Übrigens seien die Wurzeln in (29) so bestimmt, $\mathrm{da} B$ die Funktionen $\sigma(\omega)$ in der Spitze $i \infty$ die Werte $+5,+6$ und +3 annehmen.

Bei Ausübung der Substitutionen $W$ gehen die $\tau(\omega)$ über in $\tau^{\prime}(\omega)$, die man aus (28) durch Auflösung nach $\tau^{\prime}$ unter richtiger Bestimmung des Vorzeichens der Quadratwurzel in folgenden Gestalten berechnet:

$$
\begin{cases}n=20, & \tau^{\prime}=\frac{5}{\tau^{2}}(3 \tau+5+\sigma), \\ n=24, & \tau^{\prime}=\frac{12}{\tau^{2}}(3 \tau+6+\sigma), \\ n=36, & \tau^{\prime}=\frac{6}{\tau^{2}}(2 \tau+3+\sigma) .\end{cases}
$$

Wie man sieht, haben wir hier noch nicht mit Funktionenpaaren $\tau, \sigma \mathrm{zu}$ tun, die gegenüber $W$ invariant sind, bzw. Zeichenwechsel erfahren. Doch ist es leicht, aus den $\sigma, \tau$ solche Paare herzustellen. In jedem Falle ist nämlich $\left(\tau^{\prime}+\tau\right)$ eine zweiwertige Funktion des Klassenpolygons K mit einem Pole zweiter Ordnung in der Spitze $i \infty$. Es muB demnach möglich sein, $\boldsymbol{\tau}^{\prime}+\tau$ durch Zusatz einer additiven Konstanten zum Quadrate einer einwertigen Funktion $\bar{\tau}$ von $\mathrm{K}$ zu machen. In der Tat führt dieser Ansatz in den drei Fällen 20, 24 und 36 zu folgenden Funktionen $\bar{\tau}$ :

$$
\left\{\begin{array}{l}
\bar{\tau}=\sqrt{2 \tau+2 \tau^{\prime}+13}=\frac{5+\sigma}{\tau}, \\
\bar{\tau}=\sqrt{\tau+\tau^{\prime}+11}=\frac{6+\sigma}{\tau}, \\
\bar{\tau}=\sqrt{\tau+\tau^{\prime}+6}=\frac{3+\sigma}{\tau} .
\end{array}\right.
$$


Tragen wir die hieraus sich ergebenden Ausdrücke $\tau \bar{\tau}-5, \ldots$ von $\sigma$ in die zugehörigen Gleichungen (29) ein und lösen noch $\tau$ auf, so stellen sich folgende Quadratwurzeln ein:

$$
\begin{cases}n=20, & \bar{\sigma}=\sqrt{\bar{\tau}^{4}-26 \bar{\tau}^{2}-80 \bar{\tau}-71,} \\ n=24, & \bar{\sigma}=\sqrt{\bar{\tau}^{4}-22 \bar{\tau}^{2}-48 \bar{\tau}-23,} \\ n=36, & \bar{\sigma}=\sqrt{\bar{\tau}^{4}-12 \bar{\tau}^{2}-24 \bar{\tau}-12 .}\end{cases}
$$

In $\bar{\tau}, \bar{\sigma}$ haben wir nun jedesmal ein System von Funktionen der $\Gamma_{\psi}$, von denen die erste gegenüber $W$ invariant ist, während die zweite Zeichenwechsel erfährt.

Natürlich sind in jedem Falle die beiden Funktionssysteme $\sigma, \tau$ und $\bar{\sigma}, \bar{\tau}$ gegenseitig rational ineinander ausdrückbar. Speziell ergeben sich bei den eben angedeuteten Rechnungen folgende Ausdrücke der $\tau$ in den Funktionen $\bar{\sigma}, \bar{\tau}$ :

$$
4 \tau=\bar{\tau}^{2}-13-\bar{\sigma}, \quad 2 \tau=\bar{\tau}^{2}-11-\bar{\sigma}, \quad 2 \tau=\bar{\tau}^{2}-6-\bar{\sigma},
$$

die sich wieder der Reihe nach auf $n=20,24$ und 36 beziehen. Durch Eintragen dieser Ausdrücke von $\tau_{10}, \tau_{12}$ und $\tau_{18}$ in die zwischen diesen Funktionen und $J$ bestehenden Gleichungen gelangt man zu den Darstellungen von $J$ durch unsere drei Funktionssysteme $\bar{\sigma}, \bar{\tau}$.

7. Transformation $26^{\text {sten }}$ Grades. Die Klassenanzahl der quadratischen Formen der Diskriminante $D=-104$ ist 6; die reduzierten Formen sind:

$$
(1,0,26), \quad(2,0,13), \quad(3, \pm 2,9), \quad(5, \pm 4,6),
$$

von denen die beiden ersten ambig sind. Das halbe Klassenpolygon $\mathbf{K}_{\mathbf{2 6}}$ ist in Fig. 29 gegeben. Die Kreise 1, 6 und 7 sind Symmetriekreise,

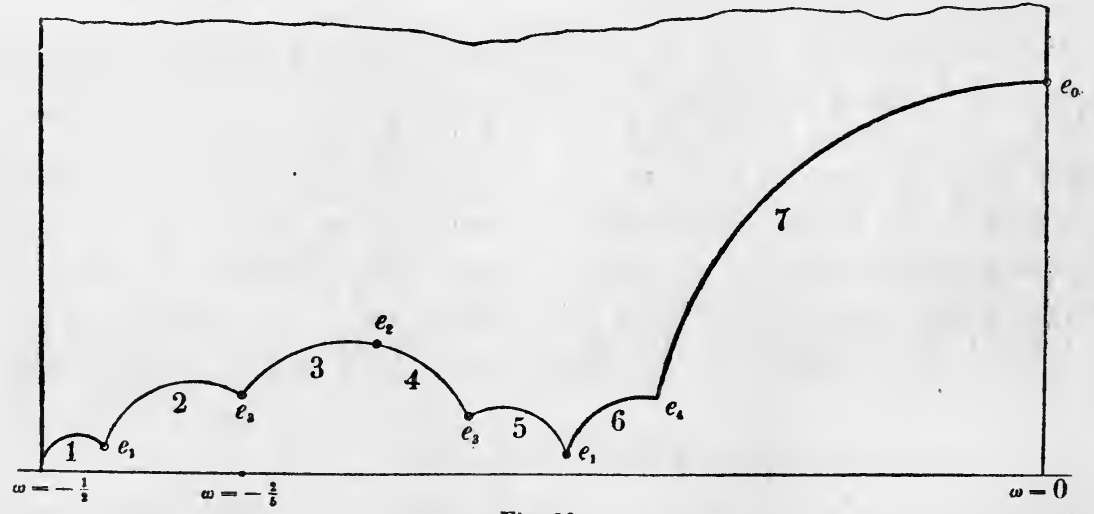

Fig. 29.

nämlich 7 derjenige der Spiegelung $\bar{W}_{26}$, während für die beiden anderen Kreise die Angaben gelten: 

1. $26\left(\xi^{2}+\eta^{2}\right)+25 \xi+6=0, \quad \omega^{\prime}=\frac{-25 \bar{\omega}-12}{52 \bar{\omega}+25}$,
6. $130\left(\xi^{2}+\eta^{2}\right)+\check{2} 2 \xi+5=0, \quad \omega^{\prime}=\frac{-26 \bar{\omega}-5}{130 \bar{\omega}+26}$.

Die zum Kreise 1. gehörende Spiegelung ist bereits in der $\Gamma_{\psi(26)}$ enthalten. Die Seiten 2, 3, 4, 5 sind aufeinander bezogen durch folgende Substitutionen:

$$
2 \rightarrow 5,\left(\begin{array}{r}
-7,-3 \\
26,11
\end{array}\right) ; 3 \rightarrow 4,\left(\begin{array}{r}
26,-9 \\
78,26
\end{array}\right),
$$

deren erste bereits in der $\Gamma_{\psi(\mathbf{2 6})}$ enthalten ist.

Die Ecke $e_{0}$ gehört zur Hauptklasse der Diskriminante -104 . Die beiden mit $e_{1}$ bezeichneten Ecken bilden mit den beiden symmetrischen Ecken auf der rechten Seite der imaginären Achse einen Zyklus. Diese beiden Ecken $e_{1}$ liegen bei:

$$
\omega=\frac{-7 \sqrt{26}+i}{15 \sqrt{26}} \text { und } \omega=\frac{-5 \sqrt{26}+i}{21 \sqrt{26}},
$$

sie sind die Nullpunkte der beiden äquivalenten Formen $(390,364,85)$ und (546, 260, 31), die der zweiten ambigen Klasse angehören. Der Punkt $e_{2}$ liegt bei $\omega=\frac{-\sqrt{26}+i}{3 \sqrt{26}}$ und stellt den Nullpunkt der Form $(78,52,9)$ dar, die mit $(3,-2,9)$ äquivalent ist. Die beiden Ecken $e_{3}$ liegen bei:

$$
\omega=\frac{-2 \sqrt{26}+i}{5 \sqrt{26}} \text { und } \omega=\frac{-2 \sqrt{26}+i}{7 \sqrt{26}},
$$

sie sind die Nullpunkte der äquivalenten Formen $(130,104,21)$ und $(182,104,15)$, die der Klasse der reduzierten Form $(5,4,6)$ angehören. Der Eckpunkt $e_{4}$ bei $\omega=\frac{-5+i}{26}$ ist der Fixpunkt der auch schon in der $\Gamma_{\psi(26)}$ auftretenden Substitution $\omega^{\prime}=\frac{-5 \omega-1}{26 \omega+5}$.

Die funktionentheoretische Behandlung des Falles $n=26$ bietet: keine Schwierigkeit dar. Durch Abzählung der Nullpunkte der Produkte $\Delta_{2} \Delta_{13}$ und $\Delta_{1} \Delta_{26}$ in den beiden in Betracht kommenden Spitzen stellt man leicht fest, daB:

$$
\tau=\sqrt[12]{\frac{\Delta_{2} \Delta_{13}}{\Delta_{1} \Delta_{26}}}=q^{-2}+2+3 q^{2}+\cdots
$$

eine einwertige Funktion des Klassenpolygons ist; ihr Pol liegt in der Spitze $i \infty$, der Nullpunkt im Zyklus $\pm \frac{1}{2}$. Aus den beiden Formen:

$$
\left\{\begin{aligned}
z_{0}=\sqrt[12]{\Delta_{2} \Delta_{13}}=\left(\frac{2 \pi}{\omega_{2}}\right)^{2} q^{\frac{5}{2}}\left(1-2 q^{4}-q^{8}+2 q^{12}+\cdots\right) \\
z_{1}=\sqrt[12]{\Delta_{1} \Delta_{26}}=\left(\frac{2 \pi}{\omega_{2}}\right)^{2} q^{\frac{9}{2}}\left(1-2 q^{2}-q^{4}+2 q^{6}\right. \\
\left.+q^{8}+2 q^{10}-2 q^{12}+\cdots\right)
\end{aligned}\right.
$$


II, 5. Die Gruppen der speziellen Transformationsgleichungen usw.

setzt man in üblicher Art die Schar von Formen $\left(a z_{0}+b z_{1}\right)$ zusammen, die einen beweglichen Nullpunkterster Ordnung auf $K_{26}$ haben und in der Spitze $i \infty$ sowie im Zyklus $\pm \frac{1}{2}$ je in der Ordnung $\frac{5}{4}$ verschwinden.

Die Gewinnung einer zweiten Funktion $\sigma$ neben $\tau$ für $\Gamma_{\psi(26)}$ und die Darstellung von $\tau_{13}$ und dadurch mittelbar auch von $J$ in $\sigma$ und $\tau$ werden zweckmäßig wie im Falle $n=14$ durchgeführt. Die in (3) S. 426 eingeführte Funktion $\tau_{13}$ möge durch $W_{26}$ in $\tau_{13}^{\prime}$ transformiert werden. Dann gilt:

$$
\tau_{13}^{\prime}+\tau_{13}=\frac{\sqrt[12]{\Delta_{1} \Delta_{2}}+13 \sqrt[12]{\Delta_{13} \Delta_{26}}}{\sqrt[12]{\Delta_{1} \Delta_{26}}}
$$

Die im Zähler stehende Form wolle man auf Zahl und Lage ihrer Nullpunkte untersuchen. Es stellt sich heraus, daB das Produkt dieser Form mit $z_{0} z_{1}=\sqrt[12]{\Delta_{1} \Delta_{2} \Delta_{13} \Delta_{26}}$ als homogene ganze Funktion dritten Grades von $z_{0}$ und $z_{1}$ darstellbar ist. Mittels der Potenzreihen findet man in üblicher Weise:

$$
z_{0} z_{1}\left(\sqrt[12]{\Delta_{1} \Delta_{2}}+13 \sqrt[12]{\Delta_{13} \Delta_{26}}\right)=z_{0}^{3}-4 z_{0}^{2} z_{1}-4 z_{0} z_{1}^{2}+z_{1}^{3}
$$

Bei der Berechnung von $\left(\sqrt[12]{\Delta_{1} \Delta_{2}}-13 \sqrt[12]{\Delta_{13} \Delta_{26}}\right)$ stellt sich die Quadratwurzel ein:

$$
\sqrt{z_{0}^{6}-8 z_{0}^{5} z_{1}+8 z_{0}^{4} z_{1}^{2}-18 z_{0}^{3} z_{1}^{3}+8 z_{0}^{2} z_{1}^{4}-8 z_{0} z_{1}^{5}+z_{1}^{6}} .
$$

Der unter dieser Wurzel stehende Ausdruck ist die Verzweigungsform der zweiblättrigen Fläche, auf die das Transformationspolygon $T_{26}$ durch $\tau(\omega)$ abgebildet wird, und die in der Tat das Geschlecht 2 hat. Wir notieren den Satz: Als Funktionssystem der $\Gamma_{\psi(26)}$ kann die in (34) erklärte Funktion $\tau$ und die in ihr durch die Quadratwurzel:

$$
\sigma=\sqrt{\tau^{6}-8 \tau^{5}+8 \tau^{4}-18 \tau^{3}+8 \tau^{2}-8 \tau+1}
$$

dargestellte Funktion $\sigma$ benutzen. Als Ausdruck für die Funktion $\tau_{13}$ in $\sigma$ und $\tau$ findet man:

$$
\tau_{13}=\frac{\tau^{3}-4 \tau^{2}-4 \tau+1-\sigma}{2 \tau} .
$$

Die Darstellung von $J$ in $\sigma$ und $\tau$ ist durch Vermittlung der Gleichung (16) S. 428 zu gewinnen.

Fünftes Kapitel.

Die Gruppen der speziellen Transformationsgleichungen und die drei Resolventen der Grade 5, 7 and 11.

Die folgenden Ausführungen schließen sich an die Entwicklungen von S. $261 \mathrm{ff}$. über die Lösung der speziellen Teilungsgleichungen der $\wp$-Funktion an und sollen die Fortsetzung dieser Entwicklungen für die 
speziellen Transformationsgleichungen geben. Nach S. 262 besteht der Satz, daB, wenn die Teilwerte der primzahligen Teilungsgrade $n$ bekannt sind, die Teilwerte aller weiteren Grade allein durch rationale Rechnungen und Wurzelziehungen berechenbar sind. Jedoch waren in allen Primzahlfällen $n>3$ die speziellen Teilungsgleichungen der $\rho$-Funktion nicht mehr durch Wurzelziehungen allein lösbar, so daß sich gerade diesen Fällen $n$ das weitere Interesse zuwendet.

Wir haben inzwischen die speziellen Transformationsgleichungen als Resolventen der speziellen Teilungsgleichungen kennen gelernt. Es wird sich jetzt darum handeln, genauer die algebraische Theorie dieser Gleichungen zu entwickeln, wobei wir uns nach dem Gesagten auf die primzahligen Fälle $n>3$ beschränken können. Die erste Frage wird die nach der Galoisschen Gruppe der speziellen Transformationsgleichung sein. Indem wir sodann auf die Struktur dieser Gruppe näher eingehen, behandeln wir die Frage, ob die speziellen Transformationsgleichungen, die beim einzelnen $n$ den Grad $(n+1)$ haben, die Resolventen niedersten Grades der speziellen Teilungsgleichung sind oder nicht. Hierauf antwortet ein berühmter von Galois entdeckter Satz, nach dem zwar für $n>11$ die Transformationsgleichungen die niedersten Resolventen sind, daß aber in den drei ersten Fällen $n=5,7$ und 11 Resolventen $n^{\text {ten }}$ Grades existieren. ${ }^{1}$ )

\section{§ 1. Die Galoisschen Gruppen der speziellen Transformationsgleichungen.}

Der Übergang von den Teilungsgleichungen zu den Transformationsgleichungen wurde durch die Überlegungen von S. $335 \mathrm{ff}$. vollzogen. Die $\frac{1}{2}\left(n^{2}-1\right)$ Teilwerte $\wp_{\lambda_{\mu}}$ des primzahligen Grades $n$ ordnen wir in $(n+1)$ Systeme:

$$
\wp_{\lambda, \mu}, \wp_{2 \lambda, 2 \mu}, \wp_{3 \lambda, 3 \mu}, \ldots, \wp_{\frac{n-1}{2} \lambda, \frac{n-1}{2} \mu}
$$

zu je $\frac{1}{2}(n-1)$ an, die gegenüber den Substitutionen der Galoisschen Gruppe $G_{\frac{1}{2} n(n-1)\left(n^{2}-1\right)}$ der Teilungsgleichung invariant sind. Diese Gruppe konnten wir nämlich aus allen auf die Indizes $\lambda, \mu$ auszuübenden inkońgruenten Substitutionen:

$$
\lambda^{\prime} \equiv \alpha \lambda+\gamma \mu, \quad \mu^{\prime} \equiv \beta \lambda+\delta \mu, \quad(\bmod n)
$$

aufbauen, deren Determinanten $(\alpha \delta-\beta \gamma)$. teilerfremd gegen $n$ waren, und bei denen zwei durch gleichzeitigen Zeichenwechsel von $\alpha, \beta, \gamma, \delta$

1) Der Satz ist von Galois in seinem Briefe an A. Chevalier vom 29. Mai 1832 mitgeteilt; man vgl. die Sammlung der Galoisschen Arbeiten im Journ. de Math., Bd. 11 (1846). 
II, 5. Die Gruppen der speziellen Transformationsgleichungen usw.

ineinander übergehende Substitutionen als nicht verschieden galten (S.261). Durch die einzelne dieser Substitutionen werden die Systeme (1), abgesehen von Umstellungen der $\wp$-Teilwerte im einzelnen Systeme, in der Tat nur untereinander permutiert. Das einzelne System (1) mit $\lambda \neq 0$ können wir durch diejenige Zahl $x$ der Reihe $0,1,2, \ldots, n-1$ charakterisieren, die der Kongruenz $x \lambda \equiv \mu(\bmod n)$ genügt, eine Zahl, die wir auch in bekannter Weise durch $\frac{\mu}{\lambda}$ oder $\mu \cdot \lambda^{-1}$ bezeichnen dürfen. Für die Bezeichnung des Systems (1) mit $\lambda=0$ benutzen wir entsprechend das Symbol $x=\infty$.

Als wichtigstes Beispiel der Transformationsgleichungen ziehen wir nun diejenige von $j(\omega)=12^{3} J(\omega)$ heran. Nach S. 338 ist die bei der ersten Haupttransformation eintretende transformierte Funktion $j(n \boldsymbol{\omega})$ eine symmetrische Funktion der $\frac{1}{2}(n-1)$ Teilwerte des zu $x=\infty$ gehörenden Systems (1) mit Koeffizienten des Körpers $\left(\Re, g_{2}, g_{3}\right)$. Wir schreiben unter Aufnahme von $x$ als Index:

$$
j_{\infty}=j(n \omega)=R\left(\wp_{01}, \wp_{02}, \ldots, \wp_{\left.0, \frac{n-1}{2}\right)} .\right.
$$

Daran reihen sich die weiteren $n$ unter sich und mit $j_{\infty}$ gleichberechtigten, den übrigen Systemen (1) entsprechenden Funktionen:

$$
j_{x}=j\left(\frac{\omega+x}{n}\right)=R\left(\wp_{1, x}, \wp_{2,2 x}, \wp_{3,3 x}, \ldots, \wp_{\frac{n-1}{2}, \frac{n-1}{2} x}\right)
$$

mit $x=0,1,2, \ldots, n-1$, wo $R$ dieselbe Bedeutung wie in (3) hat. Insbesondere entspricht $j_{0}$ der zweiten Haupttransformation $n^{\text {ten }}$ Grades.

In $j_{\infty}, j_{0}, j_{1}, \ldots, j_{n-1}$ haben wir nun die Wurzeln der Transformationsgleichung vor uns, auf welche jetzt die allgemeinen Grundsätze der Galoisschen Theorie betreffend die Gruppen der Resolventen in Anwendung zu bringen sind (vgl. S. 52 ff.). Wir haben zu dem Zwecke zunächst die Untergruppe derjenigen Substitutionen (2) festzustellen, die die identische Permutation der $j_{\infty}, j_{0}, j_{1}, \ldots, j_{n-1}$ bewirken. Nun wird $j_{\infty}$ durch die Substitutionen $(2)$ mit $\gamma \equiv 0(\bmod n)$ in sich übergeführt, $j_{0}$ aber durch die Substitutionen $\operatorname{mit} \beta \equiv 0(\bmod n)$. Ebenso finden wir als Bedingung dafür, daB $j_{1}$ in sich übergeht, $\alpha \equiv \delta(\bmod n)$. Die Substitutionen (2), die diesen drei Bedingungen genügen, transformieren aber bereits. alle Wurzeln (3) und (4) in sich und liefern also den Durchschnitt aller $(n+1)$ zu den einzelnen Wurzeln gehörenden Untergruppen: Die Substitutionen der Galoisschen Gruppe $G_{\frac{1}{2} n(n-1)\left(n^{2}-1\right)}$ der Teilungsgleichung, die die identische Permutation der $j_{\infty}, j_{0}, j_{1}, \ldots, j_{n-1}$ liefern, bilden die ausgezeichnete Untergruppe $G_{\frac{1}{2}(n-1)}$ der $\frac{1}{2}(n-1)$ Substitutionen:

$$
\lambda^{\prime} \equiv \alpha \lambda, \quad \mu^{\prime} \equiv \alpha \mu \quad(\bmod n) .
$$


Bei Fortgang zur Transformationsgleichung tritt nun nach S. 53 die Reduktion der Galoisschen Gruppe auf die Quotientengruppe $G_{\frac{1}{2} n(n-1)\left(n^{2}-1\right)} / G_{\frac{1}{2}(n-1)}$ ein, so daß die Galoissche Gruppe der speziellen Transformationsgleichung eine $G_{n\left(n^{2}-1\right)}$ der Ordnung $n\left(n^{2}-1\right)$ ist. Wir können sie aus der Gruppe (2) einfach dadurch herstellen, daß wir alle Substitutionen (2) mit proportionalen Zahlenquadrupeln $\alpha, \beta, \gamma, \delta$ als nicht voneinander verschieden ansehen. Es genügt also, alle $\frac{1}{2} n\left(n^{2}-1\right)$ Substitutionen (2) mit der Determinante 1 und außerdem alle Substitutionen (2) zuzulassen, deren Determinante gleich einem beliebig zu wählenden quadratischen Nichtreste von $n$ ist, also im Falle $n=4 h+3$ etwa gleich dem Nichtreste -1 . Indem wir die Indizes $x \equiv \mu \lambda^{-1}$ einführen, können wir den gewonnenen Satz auch so ausdrücken: Die Galoissche Gruppe der speziellen Transformationsgleichung besteht aus allen $n\left(n^{2}-1\right)$ Permutationen der $j_{\infty}, j_{0}, j_{1}, \ldots, j_{n-1}$, welche durch die auf den Index $x$ auszuïbenden Substitutionen:

$$
x^{\prime} \equiv \frac{\delta x+\beta}{\gamma x+\alpha} \quad(\bmod n)
$$

erhalten werden, wobei $\alpha \delta-\beta \gamma \equiv 1$ und kongruent einem beliebig zu wählenden quadratischen Nichtreste von $n$ zu nehmen ist und von zwei durch Zeichenwechsel ineinander übergehenden Quadrupeln $\alpha, \beta, \gamma, \delta$ natürlich wieder nur eines zuzulassen ist.

In der Galoisschen Gruppe $G_{n\left(n^{2}-1\right)}$ ist als ausgezeichnete Untergruppe des Index 2 die Monodromiegruppe $G_{\frac{1}{2} n\left(n^{2}-1\right)}$ enthalten $\left.^{1}\right)$, die ihrerseits in allen hier in Frage kommenden Fällen $n>3$ nach S. 262 einfach ist. Die $G_{\frac{1}{2} n\left(n^{2}-1\right)}$ ist isomorph mit der $\bmod n$ reduzierten nichthomogenen Modulgruppe $\Gamma^{(\omega)}$ und kann demnach erzeugt werden aus zwei Permutationen, welche den beiden Substitutionen $S=\left(\begin{array}{l}1,1 \\ 0,1\end{array}\right)$ und $T=\left(\begin{array}{c}0,1 \\ -1,0\end{array}\right)$ entsprechen. Diese beiden Permutationen sind, wie man leicht feststellt:

$$
\begin{gathered}
j_{\infty}^{\prime}=j_{\infty}, \quad j_{x}^{\prime \prime}=j_{x+1}, \\
j_{\infty}^{\prime}=j_{0}, \quad j_{0}^{\prime}=j_{\infty}, \quad j_{x}^{\prime}=j_{-x^{-1}},
\end{gathered}
$$

wo bei der Permutation $S$ natürlich $j_{n}=j_{0}$ zu nehmen ist. ${ }^{2}$ )

Für die Reduktion der Galoisschen Gruppe auf die Monodromie-

1) Das ist die Gruppe aller Substitutionen (2) mit $\alpha \delta-\beta \gamma \equiv 1(\bmod n)$.

2) Für die Galoissche Gruppe $G_{n\left(n^{2}-1\right)}$ kommt dann als eine dritte erzeugende Permutation:

$$
j_{\infty}^{\prime}=j_{\infty}, \quad j_{0}^{\prime}=j_{0}, \quad j_{x}^{\prime}=j_{\nu x}
$$

hinzu, wo $v$ irgendein quadratischer Nichtrest von $n$ ist. 
gruppe ist die Adjunktion der Einheitswurzel $\varepsilon=e^{\frac{2 i \pi}{n}}$ hinreichend, aber nicht notwendig. Da der Index der Monodromiegruppe in $\operatorname{der} G_{n\left(n^{2}-1\right)}$ gleich 2 ist, so genügt bereits die Adjunktion einer einzelnen numerischen Irrationalität zweiten Grades. Da sie dem Kreisteilungskörper $(\Re, \varepsilon)$ angehört, so handelt es sich um die Wurzel der quadratischen Resolvente der Kreisteilungsgleichung für den $n^{\text {ten }}$ Teilungsgrad. Diese quadratische Resolvente ist die Gleichung für die $\frac{1}{2}(n-1)$-gliedrige Summe:

$$
\varepsilon+\varepsilon^{4}+\varepsilon^{9}+\cdots+\varepsilon^{\left(\frac{n-1}{2}\right)^{2}}=\frac{1}{2}\left(-1+i^{\frac{n-1}{2}} \sqrt{n}\right),
$$

wo links in den Exponenten die $\frac{1}{2}(n-1)$ quadratischen Reste von $n$ stehen. ${ }^{1}$ )

Nach S. 260 ist $\varepsilon$ eine natürliche Irrationalität der . $\diamond$-Teilungsgleichung, also als rationale Funktion der $\wp_{\lambda \mu}$ mit Koeffizienten des Körpers $\left(\Re, g_{2}, g_{3}\right)$ darstellbar. Diese Funktion bleibt unverändert bei den Substitutionen der Monodromiegruppe und geht bei der einzelnen Substitution (2) der Determinante $\alpha \delta-\beta \gamma \equiv d$ in $\varepsilon^{d}$ über. Die Summe (7) ist demnach eine Größe, die gegenüber der $G_{\frac{1}{2}(n-1)}$ der Substitutionen

invariant ist, d.h. sie gehört nach S. $49 \mathrm{ff}$. als natürliche Irrationalität zur Transformationsgleichung: Die Galoissche Gruppe $G_{n\left(n^{2}-1\right)}$ der speziellen Transformationsgleichung reduziert sich nach Adjunktion der zu ihr natürlichen Irrationalität $i^{\frac{n-1}{2}} \sqrt{n}$ auf die Monodromiegruppe $G_{\frac{1}{2} n\left(n^{2}-1\right)}$ die mit der $\bmod n$ reduzierten nicht-homogenen Modulgruppe isomorph ist.

\section{$\$$ 2. Die Galoisschen imaginären Zahlen und die imaginäre Gestalt $\operatorname{der} G_{\frac{1}{2} n\left(n^{2}-1\right)^{\circ}}$}

Um die Untergruppen der $G_{\frac{1}{2} n\left(n^{2}-1\right)}$ aller mod $n$ inkongruenten nichthomogenen Substitutionen $\left(\begin{array}{l}\alpha, \beta \\ \gamma, \delta\end{array}\right)^{\frac{1}{2} n\left(n^{2}-1\right)}$ der $\Gamma^{(\omega)}$ bequem aufstellen zu können, müssen wir diese Substitutionen noch auf eine neue Gestalt transformieren und zwar mit Benutzung der von Galois in die Zahlentheorie eingeführten imaginären Zahlen. Ist $N$ irgendein bestimmt gewählter quadratischer Nichtrest von $n$, so ist die Kongruenz $x^{2} \equiv N(\bmod n)$ durch keine der Zahlen $0,1,2, \ldots, n-1 \mathrm{zu}$ befriedigen. Man führt demnach genau wie in der Algebra eine neue imaginäre Zahl $\iota$ ein, die die Eigenschaft besitzt, daB $\iota^{2} \equiv N(\bmod n)$ sein soll, und knüpft an diese Einführung entin $I, 494$.

1) Die Berechnung der Summe (7) geschieht auf Grund der letzten Gleichung 
sprechende Folgerungen, wie an die Einführung der gewöhnlichen imaginären Einheit $i$ in die Algebra. Man bildet also mittelst der reellen ganzen Zahlen $a, b$ und der neuen imaginären Zahl $\iota$ die $n^{2} \bmod n$ inkongruenten komplexen ganzen Zahlen $(a+b \iota)$ und findet dann zunächst den Satz, daB jede bisher irreduzibele Kongruenz $A x^{2}+B x+C \equiv 0$ $(\bmod n)$ mit gewöhnlichen ganzzahligen Koeffizienten ein Paar konjugiert komplexer Lösungen $(a \pm b \iota)$ erhält. Hat eine Kongruenz beliebigen Grades mit reellen Koeffizienten eine komplexe Lösung $(a+b \iota)$, so hat sie stets auch die konjugierte Lösung $(a-b \iota)$. Durch bekannte algebraische Überlegungen ${ }^{1}$ ) zeigt man, da $B$ eine solche Kongruenz, wenn $m$ ihr Grad ist, auch im erweiterten Zahlengebiete niemals mehr als $m$ verschiedene Lösungen haben kann.

Erhebt man irgendeine der $\left(n^{2}-1\right)$ inkongruenten, durch $n$ nicht teilbaren Zahlen $(a+b \iota)$ in die $n^{\text {te }}$ Potenz, so folgt bei Fortlassung aller durch $n$ teilbaren Glieder:

$$
(a+b \iota)^{n} \equiv a^{n}+b^{n} \iota^{n} \equiv a^{n}+b^{n} N^{\frac{n-1}{2}} \iota \quad(\bmod n) .
$$

Nach dem Fermatschen Satze gilt $a^{n} \equiv a, b^{n} \equiv b$. Ferner gilt $N^{\frac{n-1}{2}} \equiv-1$, da $N$ quadratischer Nichtrest von $n$ ist. Es folgt also:

$$
(a+b \iota)^{n} \equiv a-b \iota \quad(\bmod n),
$$

und man findet durch nochmaliges Erheben zur $n^{\text {ten }}$ Potenz:

$$
(a+b \iota)^{n^{2}} \equiv a+b \iota, \quad(a+b \iota)^{n^{2}-1} \equiv 1 \quad(\bmod n)
$$

als Verallgemeinerung des Fermatschen Satzes. Die Kongruenz $x^{n^{2}-1} \equiv 1$ $(\bmod n)$ hat hiernach die Höchstzahl zulässiger Lösungen, nämlich $\left(n^{2}-1\right)$.

Ist $n^{2}-1=\mu \cdot \nu$ irgendeine Faktorenzerlegung von $\left(n^{2}-1\right)$, so folgt aus:

$$
x^{n^{2}-1}-1=\left(x^{\mu}-1\right)\left(x^{\mu(\nu-1)}+x^{\mu(\nu-2)}+\cdots+x^{\mu}+1\right),
$$

daß eine Lösung von $x^{n^{2}-1} \equiv 1(\bmod n)$ mindestens eine der Kongruenzen:

$$
x^{\mu} \equiv 1, \quad x^{\mu(v-1)}+x^{\mu(\nu-2)}+\cdots+x^{\mu}+1 \equiv 0 \quad(\bmod n)
$$

befriedigt. Da aber die zweite höchstens $\mu(\nu-1)$ Lösungen hat, so besitzt die erste sicher $\mu$ verschiedene Lösungen. Ist also $\mu$ irgendein Teiler von $\left(n^{2}-1\right)$, so hat auch $x^{\mu} \equiv 1(\bmod n)$ notwendig $\mu$ verschiedene Lösungen.

Ist $(a+b \iota)$ eine dieser Lösungen, die nicht bereits einer Kongruenz $x^{\lambda} \equiv 1(\bmod n)$ mit $\lambda<\mu$ genügt, so sagt man,$(a+b \iota)$ gehöre zum Exponenten $\mu^{\prime \prime}$. Eine zum Exponenten $\left(n^{2}-1\right)$ gehörende Zahl wird im vorliegenden erweiterten Zahlgebiete als eine "primitive Wurzel“ der

1) Vgl. Dirichlet-Dedekind, „Vorles. über Zahlentheorie“ (4. Aufl.), S. 58. 
464 II, 5. Die Gruppen der speziellen Transformationsgleichungen usw.

Primzahl $n$ bezeichnet. Die Existenz primitiver Wurzeln geht aus folgender Betrachtung hervor:

Es seien $\mu$ und $\mu^{\prime}$ zwei gegeneinander teilerfremde Divisoren von $\left(n^{2}-1\right)$, und es mögen zwei zu den Exponenten $\mu$ und $\mu^{\prime}$ gehörende Zahlen $(a+b \iota)$ und $\left(a^{\prime}+b^{\prime} \iota\right)$ existieren. Dann ist sicher die $\left(\mu \cdot \mu^{\prime}\right)^{\text {te }}$ Potenz von:

$$
c+d \iota=(a+b \iota)\left(a^{\prime}+b^{\prime} \iota\right)^{\mu^{\prime}-1}
$$

mit 1 kongruent. Soll aber genauer $(c+d \iota)$ zum Exponenten $m$ gehören:

$$
(c+d \iota)^{m} \equiv(a+b \iota)^{m}\left(a^{\prime}+b^{\prime} \iota\right)^{m \mu^{\prime}-m} \equiv 1 \quad(\bmod n),
$$

so folgt:

$$
(a+b \iota)^{m} \equiv\left(a^{\prime}+b^{\prime} \iota\right)^{m} \quad(\bmod n) \text {. }
$$

Die hier links stehende Zahl gehört als Potenz von $(a+b \iota)$ notwendig $\mathrm{zu}$ einem in $\mu$ aufgehenden Exponenten, die rechts stehende entsprechend zu einem in $\mu^{\prime}$ aufgehenden. Da aber beide Zahlen als mod $n$ kongruent zum gleichen Exponenten gehören und $\mu, \mu^{\prime}$ teilerfremd sind, also den größten gemeinsamen Teiler 1 haben, so gehören beide Zahlen zum Exponenten 1:

$$
(a+b \iota)^{m} \equiv\left(a^{\prime}+b^{\prime} \iota\right)^{m} \equiv 1 \quad(\bmod n) .
$$

Hiernach ist $m$ ein Vielfaches sowohl von $\mu$ als $\mu^{\prime}$ und also von $\mu \cdot \mu^{\prime}$. Aus der schon festgestellten Kongruenz $(c+d \iota)^{\mu \cdot \mu^{\prime}} \equiv 1$ folgt schließlich $m=\mu \cdot \mu^{\prime}$, so daß wir aus den beiden zu $\mu$ und $\mu^{\prime}$ gehörenden Zahlen $(a+b \iota)$ und $\left(a^{\prime}+b^{\prime} \iota\right)$ stets eine zum Exponenten $\mu \cdot \mu^{\prime}$ gehörende Zahl $(c+d \iota)$ herstellen können.

Mit Hilfe dieses Satzes können wir durch eine bekannte Schlußweise die Existenz von primitiven Wurzeln der Primzahl $n$ nachweisen, wenn wir nur sicher sind, daß es zu jeder höchsten in $\left(n^{2}-1\right)$ enthaltenen Primzahlpotenz $p^{v}$ Zahlen $(a+b \iota)$ gibt. Würden solche Zahlen aber nicht vorkommen, so müßten alle $p^{v}$ Lösungen der Kongruenz $x^{p^{v}} \equiv 1$ schon der Kongruenz $x^{p^{v-1}} \equiv 1$ genügen, was unmöglich ist. Die Existenz der primitiven Wurzeln steht also fest.

Wir bilden nun die $(n-1)^{\text {te }}$ Potenz irgendeiner primitiven Wurzel $(g+h \iota)$ :

$$
(g+h \iota)^{n-1} \equiv a+b \iota \quad(\bmod n)
$$

und haben damit eine zum Exponenten $(n+1)$ gehörende Zahl gewonnen. Diese Zahl $(a+b \iota)$ genügt der Kongruenz:

$$
(a+b \iota)^{\frac{n+1}{2}} \equiv-1 \quad(\bmod n)
$$

während alle voraufgehenden Potenzen $(a+b \iota),(a+b \iota)^{2}, \ldots,(a+b \iota)^{\frac{n-1}{2}}$ untereinander und gegen +1 und -1 inkongruent sind. Da übrigens $(a+b \iota)^{n} \equiv a-b \iota$ gilt, so folgt aus $(a+b \iota)^{n+1} \equiv 1$ noch:

$$
(a+b \iota)(a-b \iota) \equiv 1 \quad(\bmod n) \text {. }
$$


Wir schreiben nun die Substitutionen der $G_{\frac{1}{2} n\left(n^{2}-1\right)}$, indem wir in (6) S. 461 statt des Index $\varkappa$ zum besseren AnschluB an die Gruppe $\Gamma^{(\omega)}$ die Variable $\omega$ einführen:

$$
\omega^{\prime} \equiv \frac{\alpha \omega+\beta}{\gamma \omega+\delta}, \quad \alpha \delta-\beta \gamma \equiv 1 \quad(\bmod n) .
$$

Die Kongruenzzeichen beziehen sich darauf, daß die Koeffizienten $\alpha, \beta$ $\gamma, \delta \operatorname{nur} \bmod n$ zu nehmen sind. Zwei Substitutionen, die durch gleichzeitigen Zeichenwechsel der vier Koeffizienten $\alpha, \beta, \gamma, \delta$ ineinander übergehen, gelten als nicht verschieden.

Neben dieser „reellen Gestalt" der $G_{\frac{1}{2} n\left(n^{2}-1\right)}$ führen wir jetzt eine „imaginäre Gestalt unserer Gruppe“ ein, indem wir die Variable $\omega$ durch eine neue Variable $\zeta$ vermöge der Transformation:

$$
\omega=\frac{-\zeta+1}{\iota \zeta+\iota}
$$

ersetzen. Die Substitution (2) geht über in:

$$
\zeta^{\prime} \equiv \frac{(a+b \iota) \xi+(c+d \iota)}{(c-d \iota) \xi+(a-b \iota)} \quad(\bmod n)
$$

wo $a, b, c, d$ vier reelle ganze Zahlen sind, die sich aus:

$$
\begin{cases}2^{-1}(\alpha+\delta) \equiv a, & -2^{-1}\left(\beta+N^{-1} \gamma\right) \equiv b \\ 2^{-1}(-\alpha+\delta) \equiv c, & -2^{-1}\left(\beta-N^{-1} \gamma\right) \equiv d\end{cases}
$$

berechnen, und die, wie man leicht feststellt, die Kongruenz:

$$
(a+b \iota)(a-b \iota)-(c+d \iota)(c-d \iota) \equiv 1 \quad(\bmod n)
$$

befriedigen. Da übrigens aus (4) umgekehrt:

$$
\alpha \equiv a-c, \quad \beta \equiv-b-d, \quad \gamma \equiv(d-b) N, \quad \delta \equiv a+c \quad(\bmod n)
$$

folgt und die Kongruenz (5) sich auf $\alpha \delta-\beta \gamma \equiv 1(\bmod n)$ umrechnet, so entspricht auch jeder Substitution (3) eine solche der Gestalt (2). In der imaginären Gestalt besteht somit die $G_{\frac{1}{2} n\left(n^{2}-1\right)}$ aus allen Substitutionen (3) der Determinante 1, wobei natürlich wieder zwei durch gleichzeitigen Zeichenwechsel der vier Koeffizienten ineinander übergehende Substitutionen (3) als nicht verschieden gelten.

\section{\$3. Zyklische Gruppen, metazyklische Gruppen und Diedergruppen in der $G_{1}$}

Die Substitution $S \equiv\left(\begin{array}{ll}1 & 1 \\ 0, & 1\end{array}\right)$ hat die Periode $n$ und erzeugt somit eine zyklische Untergruppe $G_{n}$ der Ordnung $n$, bestehend ans allen Sub- 
II, 5. Die Gruppen der speziellen Transformationsgleichungen usw.

stitutionen $\left(\begin{array}{l}1, \beta \\ 0,1\end{array}\right)$ der $G_{\frac{1}{2} n\left(n^{2}-1\right)}$. Bei der Abzählung der mit dieser $G_{n}$ gleichberechtigten Untergruppen gelangen wir zu den Betrachtungen von S. $251 \mathrm{ff}$. zurück, die sich jedoch damals auf homogene Substitutionen bezogen. Alle Substitutionen $\operatorname{der} G_{\frac{1}{2} n\left(n^{2}-1\right)}$, die mit der $G_{n}$ vertauschbar sind und also die $G_{n}$ in sich transformieren, bilden wieder eine Untergruppe, in der die $G_{n}$ ausgezeichnet enthalten ist. Ist die Ordnung dieser Untergruppe $k n$, so zerfällt die Gesamtgruppe ihr entsprechend in $\frac{n^{2}-1}{2 k}$ Nebengruppen. Die Substitutionen der einzelnen Nebengruppe transformieren die $G_{n}$ in eine und dieselbe gleichberechtigte $G_{n}^{\prime}$, Substitutionen verschiedener Nebengruppen liefern aber verschiedene $G_{n}^{\prime}, G_{n}^{\prime \prime}$. Die Anzahl der mit der $G_{n}$ gleichberechtigten Gruppen ist also gleich $\frac{n^{2}-1}{2 k}$.

Soll nun aber $\left(\begin{array}{l}\alpha, \beta \\ \gamma, \delta\end{array}\right)$ die aus $S$ zu erzeugende $G_{n}$ in sich transformieren, so muB:

$$
V^{-1} \cdot S \cdot V \equiv\left(\begin{array}{c}
1+\gamma \delta, \delta^{2} \\
-\gamma^{2}, 1-\gamma \delta
\end{array}\right) \equiv\left(\begin{array}{c}
1, \beta^{\prime} \\
0,1
\end{array}\right) \quad(\bmod n)
$$

gelten. Hierzu ist hinreichend und notwendig, daB $\gamma \equiv 0(\bmod n)$ gilt, was uns zu allen Substitutionen der Gestalt $\left(\begin{array}{c}\alpha, \beta \\ 0, \alpha^{-1}\end{array}\right)$ hinführt. Da $\alpha \neq 0$ $(\bmod n) \operatorname{sein} \operatorname{mu} B$ und gleichzeitiger Zeichenwechsel von $\alpha, \beta$ statthaft ist, so haben wir mit $\frac{1}{2} n(n-1)$ eine $G_{\frac{1}{2} n(n-1)}$ bildenden Substitutionen zu tun. Wir gelangen zu den Gruppen zurück, die in homogener Gestalt als „metazyklische" Gruppen bezeichnet wurden (vgl. S.252), eine Bezeichnung, die wir auch auf die $G_{\frac{1}{2} n(n-1)}$ übertragen. $\left.{ }^{1}\right)$ Als Ergebnis aber folgt: In $\operatorname{der} G_{\frac{1}{2} n\left(n^{2}-1\right)} \operatorname{treten}(n+1)$ gleichberechtigte zyklische Untergruppen $G_{n}$ der Ordnung $n$ auf und ihnen entsprechend ebenso viele gleichberechtigte metazyklische Untergruppen $G_{\frac{1}{2} n(n-1)}$ der Ordnung $\frac{1}{2} n(n-1)$.

Die einzelne $G_{n}$ als Gruppe von Primzahlordnung ist aus jeder ihrer Substitutionen, abgesehen von der identischen Substitution 1, erzeugbar. Hieraus folgt, daB zwei verschiedene $G_{n}$, abgesehen von der Substitution 1, keine gemeinsamen Substitutionen enthalten können. Im ganzen finden wir demnach $(n+1)(n-1)=n^{2}-1$ Substitutionen der Periode $n$.

Es besteht der Satz: Abgesehen von den etwa in den $G_{\frac{1}{2} n(n-1)}$ noch enthaltenen Untergruppen und der Gesamtgruppe $G_{\frac{1}{2} n\left(n^{2}-1\right)}$ sind die metazy-

1) Sie heißen auch wohl in der nicht-homogenen Gestalt „halbmetazyklische“ Gruppen. 
Zyklische und metazyklische Untergruppen der $G_{\frac{1}{2} n\left(n^{2}-1\right)}$

klischen $G_{\frac{1}{2} n(n-1)}$ die einzigen Gruppen, an denen die zyklischen $G_{n}$ beteiligt sind. Andernfalls gäbe es nämlich insbesondere eine von der Gesamtgruppe verschiedene Gruppe $G$, die die aus $S$ zu erzeugende $G_{n}$ und mindestens noch eine Substitution $V \equiv\left(\begin{array}{l}\alpha, \beta \\ \gamma, \delta\end{array}\right)$ mit einem nicht durch $n$ teilbaren $\gamma$ enthielte. Mit $S$ und $V$ fände sich in $G$ auch:

$$
\left.S^{(1-\alpha) \gamma^{-1}} \cdot V \cdot S^{(1-\delta) \gamma^{-1}} \equiv\left(\begin{array}{l}
1,0 \\
\gamma, 1
\end{array}\right) \quad(\bmod n)^{1}\right)
$$

und damit auch $\left(\begin{array}{c}1,0 \\ -1,1\end{array}\right)$, sowie endlich:

$$
S \cdot\left(\begin{array}{c}
1,0 \\
-1,1
\end{array}\right) \cdot S=\left(\begin{array}{cr}
0,-1 \\
1, & 0
\end{array}\right)=T .
$$

Aus $S$ und $T$ aber wird die Gesamtgruppe $G_{\frac{1}{2} n\left(n^{2}-1\right)}$ erzeugt, so daB $G$ entgegen der Annahme die $G_{\frac{1}{2} n\left(n^{2}-1\right)}$ wäre.

Ist $g$ eine primitive Wurzel der Primzahl $n$ im gewöhnlichen Sinne, so ist $g^{\frac{1}{2}(n-1)} \equiv-1$ (mod. $\left.n\right)$, während die $\frac{1}{2}(n-3)$ Zahlen $g, g^{2}, g^{3}, \ldots$, $g^{\frac{1}{2}(n-3)}$ untereinander und gegen +1 und -1 inkongruent sind. Die Substitution $\left(\begin{array}{c}g, 0 \\ 0, g^{-1}\end{array}\right)$ hat demnach die Periode $\frac{1}{2}(n-1)$ und erzeugt eine zyklische $G_{\frac{1}{2}(n-1)}$, die aus den Substitutionen:

$$
\left(\begin{array}{c}
g, 0 \\
0, g^{-1}
\end{array}\right),\left(\begin{array}{c}
g^{2}, 0 \\
0, g^{-2}
\end{array}\right),\left(\begin{array}{c}
g^{3}, 0 \\
0, g^{-3}
\end{array}\right), \ldots,\left(\begin{array}{c}
g^{\frac{1}{2}(n-1)}, 0 \\
0, g^{-\frac{1}{2}(n-1)}
\end{array}\right) \equiv 1
$$

besteht. Transformiert man $\left(\begin{array}{c}g^{v}, 0 \\ 0, g^{-v}\end{array}\right)$, unter $\nu$ eine der Zahlen 1, 2, 3,..., $\frac{1}{2}(n-3)$ verstanden, durch $V \equiv\left(\begin{array}{l}\alpha, \beta \\ \gamma, \delta\end{array}\right)$, so folgt:

$$
V^{-1} \cdot\left(\begin{array}{c}
g^{v}, 0 \\
0, g^{-v}
\end{array}\right) \cdot V \equiv\left(\begin{array}{c}
\alpha \delta g^{v}-\beta \gamma g^{-v} \\
\left.\alpha \gamma\left(g^{-v}-g^{v}\right), \alpha \delta g^{v}-g^{-v}\right) \\
\alpha \delta g^{-v}-\beta \gamma g^{v}
\end{array}\right) \text {. }
$$

Nun ist $g^{v}-g^{-v} \neq 0(\bmod n)$, so daB die transformierte Substitution (2) stets und nur dann wieder der $G_{\frac{1}{2}(n-1)}$ der Substitutionen (1) angehört, wenn $\alpha \gamma \equiv 0, \beta \delta \equiv 0(\bmod n)$ gilt. Die Untergruppe aller diese Kongruenzen befriedigenden Substitutionen besteht aus den Substitutionen (1) und den $\frac{1}{2}(n-1)$ Substitutionen der Periode zwei:

$$
\left(\begin{array}{c}
0,-g \\
g^{-1}, 0
\end{array}\right),\left(\begin{array}{c}
0,-g^{2} \\
g^{-2}, 0
\end{array}\right), \ldots,\left(\begin{array}{c}
0,-g^{\frac{1}{2}(n-1)} \\
g^{-\frac{1}{2}(n-1)}, 0
\end{array}\right) \equiv\left(\begin{array}{c}
0,1 \\
-1,0
\end{array}\right) \text {. }
$$

1) Es sei daran erinnert, daß unter $\gamma^{-1}$ die Lösung der Kongruenz $\gamma x \equiv 1$ mod. $n$ ) zu verstehen ist. 
II, ธ. Die Gruppen der speziellen Transformationsgleichungen usw.

Auf Grund der vorhin bei den $G_{n}$ benutzten Überlegung gelangen wir zu dem Satze: Die zyklische $G_{\frac{1}{2}(n-1)}$ ist also ausgezeichnet in einer umfassendsten $G_{n-1}$ vom Diedertypus (vgl. I, 132) enthalten, so daß wir im ganzen $\frac{1}{2} n(n+1)$ zyklische Untergruppen $G_{\frac{1}{2}(n-1)}$ der Ordnung $\frac{1}{2}(n-1)$ gewinnen, von denen eine aus den Substitutionen (1) besteht.

Es gilt wieder der Satz: Keine zwei unter den zyklischen Gruppen der Ordnung $\frac{1}{2}(n-1)$ können, abgesehen von der Substitution 1, eine Substitution gemein haben. Andernfalls müßte es auch eine von der Gruppe (1) verschiedene, aber mit ihr gleichberechtigte $G_{\frac{1}{2}(n-1)}^{\prime}$ geben, die eine von 1 verschiedene Substitution $\left(\begin{array}{c}g^{\mu}, 0 \\ 0, g^{-\mu}\end{array}\right)$ der $G_{\frac{1}{2}(n-1)}$ enthielte. Ist diese Substitution mit der von 1 verschiedenen Substitution $\left(\begin{array}{c}g^{v}, 0 \\ 0, g^{-v}\end{array}\right)$ der $G_{\frac{1}{2}(n-1)}$ gleichberechtigt, so gäbe es eine Substitution $V \equiv\left(\begin{array}{l}\alpha, \beta \\ \gamma, \delta\end{array}\right)$, für welche die in (2) rechts stehende Substitution die $\left(\begin{array}{c}g^{\mu}, 0 \\ 0, g^{-\mu}\end{array}\right)$ wäre. Dies aber würde zu den Bedingungen $\alpha \gamma \equiv 0, \beta \delta \equiv 0(\bmod n)$ zurückführen, so daß $V$ unter den Substitutionen (1) und (3) enthalten wäre. Dann aber wäre $G_{\frac{1}{2}(n-1)}^{\prime}$ wieder mit $G_{\frac{1}{2}(n-1)}$ identisch. Wir stellen noch den Satz fest: Die $\frac{1}{2} n(n+1)$ zyklischen Untergruppen $G_{\frac{1}{2}(n-1)}$ enthalten außer 1 im ganzen $\frac{1}{4} n(n+1)(n-3)$ verschiedene Substitutionen.

Jede der zyklischen $G_{\frac{1}{2}(n-1)}$ liefert eine Diedergruppe $G_{n-1}$, in der sie ausgezeichnet enthalten ist. Zwei dieser Diedergruppen können, sobald $n>5$ ist, nicht gleich sein; denn sie enthalten zwei verschiedene zyklische Gruppen eiues Ordnung $>2$ und außerdem nur Substitutionen der Periode zwei. Für $* 5$ finden wir $\frac{1}{2} n(n+1)$ gleichberechtigte Diedergruppen $G_{n-1}$, von denen eine aus den Substitutionen (1) und (3) besteht. Für $n=5$ liegt ein Ausnahmefall vor. Hier werden die 15 Gruppen $G_{4}$, die den „Vierertypus" zeigen (vgl. I, 130), zu je dreien einander gleich, so daß in der zu $n=5$ gehörenden $G_{60}$ nur ,fünf" gleichberechtigte Vierergruppen $G_{4}$ auftreten. Die von den Substitutionen (1) und (3) gelieferte $G_{4}$ besteht nämlich aus:

$$
V_{0} \equiv 1, \quad V_{1} \equiv\left(\begin{array}{ll}
2, & 0 \\
0,3
\end{array}\right), \quad V_{2} \equiv\left(\begin{array}{ll}
0, & 2 \\
2, & 0
\end{array}\right), \quad V_{3} \equiv\left(\begin{array}{ll}
0, & 1 \\
4, & 0
\end{array}\right)
$$

In ihr ist aber nicht nur $V_{1}$, sondern sind auch $V_{2}$ und $V_{3}$ ausgezeichnet enthalten. In der $G_{60}$ kommen außer den 15 gleichberechtigten $G_{2}$, wie sich gleich zeigen wird, keine weiteren Substitutionen der Periode 2 vor. Also sind die Substitutionen $V_{2}$ und $V_{3}$ mit $V_{1}$ gleichberechtigt, und die 
Zyklische Gruppen und Diedergruppen in $\operatorname{der} G_{\frac{1}{2} n\left(n^{2}-1\right)}$

Gruppe (4) ist auch die von $V_{2}$ und ebenso die von $V_{3}$ gelieferte Diedergruppe.

Für eine noch fehlende Art zyklischer Untergruppen haben wir die imaginäre Gestalt der $G_{\frac{1}{2} n\left(n^{2}-1\right)}$ zu benutzen. Wir kürzen deren Substitutionen in der Gestalt $\left(\frac{A}{\bar{B}}, \frac{B}{A}\right)$ ab, wo $A$ und $B$ zwei komplexe Zahlen der S. 463 eingeführten Art sind, $\bar{A}$ und $\bar{B}$ die zu ihnen konjugierten Zahlen bedeuten und $A \bar{A}-B \bar{B} \equiv 1(\bmod n)$ gilt. Unter $(a+b \iota)$ verstehen wir eine zum Exponenten $(n+1)$ gehörende Zahl. Dann gilt $(a+b \iota)^{\frac{1}{2}(n+1)} \equiv-1(\bmod n)$, die Potenzen $(a+b \iota),(a+b \iota)^{2}, \ldots$, $(a+b \iota)^{\frac{1}{2}(n-1)}$ sind untereinander und gegen +1 und -1 inkongruent, und die folgenden Potenzen $(a+b \iota)^{\frac{1}{2}(n+3)},(a+b \iota)^{\frac{1}{2}(n+5)}, \ldots,(a+b \iota)^{n}$ sind den vorgenannten Potenzen, negativ genommen, kongruent. Zugleich liefern die $(n+1)$ Potenzen $(a+b \iota)^{0}=1,(a+b \iota),(a+b \iota)^{2}, \ldots,(a+b \iota)^{n}$ alle Lösungen der Kongruenz $x^{n+1} \equiv 1(\bmod n)$ oder der mit ihr gleichwertigen Kongruenz $x \bar{x} \equiv 1(\bmod n)$, unter $\bar{x}$ die mit $x$ konjugierte Zahl verstanden.

Nach diesen Darlegungen erzeugt die in der $G_{\frac{1}{2} n\left(n^{2}-1\right)}$ enthaltene Substitution $\left(\begin{array}{l}a+b \iota, 0 \\ 0, a-b \iota\end{array}\right)$ eine zyklische $G_{\frac{1}{2}(n+1)}$ der Ordnung $\frac{1}{2}(n+1)$, die aus allen Substitutionen der $G_{\frac{1}{2} n\left(n^{2}-1\right)}$ mit verschwindenden zweiten und dritten Koeffizienten besteht. Verstehen wir unter $\left(\begin{array}{c}a^{\prime}+b^{\prime} \iota, \\ 0, a^{\prime}-b^{\prime} \iota\end{array}\right)$ irgendeine von 1 verschiedene Substitution $\operatorname{der} G_{\frac{1}{2}(n+1)}$, so liefert deren Transformation mit $V \equiv\left(\begin{array}{l}A, B \\ \bar{B}, \frac{B}{A}\end{array}\right)$ :

$$
V^{-1} \cdot\left(\begin{array}{c}
a^{\prime}+l^{\prime} \iota, 0 \\
0, a^{\prime}-b^{\prime} \iota
\end{array}\right) \cdot V \equiv\left(\begin{array}{c}
a^{\prime}+(A \bar{A}+B \bar{B}) b^{\prime} \iota, 2 \bar{A} B b^{\prime} \iota \\
-2 A \bar{B} b^{\prime} \iota, a^{\prime}-(A \bar{A}+B \bar{B}) b^{\prime} \iota
\end{array}\right) \text {. }
$$

Soll diese Substitution wieder der $G_{\frac{1}{2}(n+1)}$ angehören, so ist, da $b \neq 0$ ist, hierzu das Bestehen der Kongruenz $A \bar{B} \equiv 0(\bmod n)$ hinreichend und notwendig; es muB also entweder $B \equiv 0$ oder $A \equiv 0(\bmod n)$ gelten. Die erste Kongruenz führt zu den $\frac{1}{2}(n+1)$ Substitutionen $V$ der $G_{\frac{1}{2}(n+1)}$; die zweite ergibt $\frac{1}{2}(n+1)$ Substitutionen der Periode 2 von der Gestalt $\left(\begin{array}{l}0, c+d \iota \\ c-d \iota, 0\end{array}\right)$, die mit der $G_{\frac{1}{2}(n+1)}$ eine Diedergruppe $G_{n+1}$ liefern. Eine erste dieser neuen Substitutionen bilden wir mit der Zahl $(c+d \iota)$, die wir vermittelst der oben schon gebrauchten primitiven Wurzel $(g+h \iota)$ von $n$ im erweiterten Zahlgebiete aus:

$$
(g+h \iota)^{\frac{n-1}{2}} \equiv c+d \iota \quad(\bmod n)
$$


berechnen. Hieraus folgt in der Tat leicht:

$$
(g+h \iota)^{\frac{n^{2}-1}{2}} \equiv(c+d \iota)^{n+1} \equiv(c+d \iota)(c-d \iota) \equiv-1 \quad(\bmod n) .
$$

Die übrigen $\frac{1}{2}(n-1)$ Substitutionen der Periode 2 werden aus der ersten durch Kombination mit den Substitutionen der zyklischen Untergruppe $G_{\frac{1}{2}(n+1)}$ hergestellt.

Wie man sieht, gestalten sich hier die Verhältnisse ähnlich wie bei den Gruppen $G_{\frac{1}{2}(n-1)}$. Wir finden $\frac{1}{2} n(n-1)$ gleichberechtigte zyklische Gruppen $G_{\frac{1}{2}(n+1)}$ der Ordnung $\frac{1}{2}(n+1)$ und ihnen entsprechend ebenso viele gleichberechtigte Diedergruppen $G_{n+1}$, deren einzelne die umfassendste Gruppe ist, in der die zugehörige $G_{\frac{1}{2}(n+1)}$ ausgezeichnet enthalten ist. Auch hier besteht wieder der Satz: Zwei zyklische $G_{\frac{1}{2}(n+1)}$ können außer der identischen Substitution 1 keine Substitution gemeinsam haben. Es müBte sonst auch die besondere $G_{\frac{1}{2}(n+1)}$ der Substitutionen $\left(\begin{array}{l}a+b \iota, 0 \\ 0, a-b \iota\end{array}\right)$ mit einer zweiten $G_{\frac{1}{2}(n+1)}^{\prime}$ eine von 1 verschiedene Substitution gemein haben. Die Transformation von $G_{\frac{1}{2}(n+1)}$ in $G_{\frac{1}{2}(n+1)}^{\prime}$ ist indessen durch eine Substitution mit $\bar{A} B \neq 0 \mathrm{zu}$ vollziehen, und hierbei gehen alle von 1 verschiedenen Substitutionen $\left(\begin{array}{l}a+b \iota, 0 \\ 0, a-b \iota\end{array}\right)$ in Substitutionen über, deren zweite und dritte Koeffizienten $\neq 0(\bmod n)$ sind. Wir zählen daraufhin $a b, d a \beta$ in den $G_{\frac{1}{2}(n+1)}$, abgesehen von der identischen Substitution, im ganzen $\frac{1}{4} n(n-1)^{2}$ verschiedene Substitutionen enthalten sind.

Es sind nunmehr unter EinschluB der identischen Substitution im ganzen:

$$
1+\left(n^{2}-1\right)+\frac{1}{4} n(n+1)(n-3)+\frac{1}{4} n(n-1)^{2}=\frac{1}{2} n\left(n^{2}-1\right)
$$

verschiedene Substitutionen, d. h. die gesamten Substitutionen unserer $G_{\frac{1}{2} n\left(n^{2}-1\right)}$, in zyklische Gruppen eingeordnet. Nehmen wir also noch die in den $G_{\frac{1}{2}(n-1)}$ und $G_{\frac{1}{2}(n+1)}$ enthaltenen zyklischen Untergruppen hinzu, so sind damit alle in $\operatorname{der} G_{\frac{1}{2} n\left(n^{2}-1\right)}$ überhaupt enthaltenen zyklischen Untergruppen gewonnen. Die in den $G_{\frac{1}{2}(n-1)}$ und $G_{\frac{1}{2}(n+1)}$ enthaltenen zyklischen Untergruppen geben auch wieder zur Bildung von Diedergruppen Anlab, indem man sie mit Substitutionen der Periode 2 aus den zugehörigen diedrischen $G_{n-1}$ bzw. $G_{n+1}$ kombiniert. Auf diese Weise gelangt man dann auch zu allen Untergruppen vom Diedertypus, die in der $G_{\frac{1}{2} n\left(n^{2}-1\right)}$ enthalten sind. 


\section{\$ 4. Ansatz zur Aufstellung aller Untergruppen der $G_{\frac{1}{2} n\left(n^{2}-1\right)}$}

Die etwa noch fehlenden Untergruppen $\operatorname{der} G_{\frac{1}{2} n\left(n^{2}-1\right)}$ kann man auf Grund einer von C. Jordan entwickelten Überlegung aufstellen. ${ }^{1}$ ) Die in den metazyklischen $G_{\frac{1}{2} n(n-1)}$ noch enthaltenen Untergruppen, die ohne Mühe von den zyklischen Untergruppen $\operatorname{der} G_{\frac{1}{2}(n-1)}$ aus gewonnen werden können, mögen beiseite gelassen werden, da es sich weiterhin nur um „umfassendste" Untergruppen handeln wird. Wir haben demnach überhaupt nur noch nach solchen Untergruppen zu suchen, an denen die zyklischen $G_{n}$ nicht beteiligt sind.

Es sei nun $G_{m}$ irgendeine dieser Untergruppen und $G_{\mu_{1}}$ eine erste in $G_{m}$ enthaltene umfassendste zyklische Untergruppe. Diese $G_{\mu_{1}}$ ist in $G_{m}$ entweder nur mit ihren eigenen Substitutionen vertauschbar oder darüber hinaus noch mit $\mu_{1}$ Substitutionen der Periode 2, die dann mit der $G_{\mu_{2}}$ eine in $G_{m}$ enthaltene Diedergruppe $G_{2_{\mu_{2}}}$ bilden. $\left.{ }^{2}\right)$ Um beide Fälle zusammenzufassen, verstehen wir unter $e_{1}$ eine der Zahlen 1 oder 2 und sagen, $G_{\mu_{1}}$ sei innerhalb $G_{m}$ mit $e_{1} \mu_{1}$ eine $G_{e_{1} \mu_{2}}$ bildenden Substitutionen vertauschbar. Den $\frac{m}{e_{1} \mu_{1}}$ zu $G_{e_{1} \mu_{1}}$ gehörenden Nebengruppen entsprechend wird die $G_{\mu_{1}}$ in $G_{m}$ im ganzen in $\frac{m}{e_{1} \mu_{1}}$ gleichberechtigte zyklische $G_{\mu_{1}}$ transformiert, in denen abgesehen von der identischen Substitution im ganzen $\frac{m}{e_{1} \mu_{1}}\left(\mu_{1}-1\right)$ verschiedene Substitutionen auftreten.

Enthält die $G_{m}$ noch weitere Substitutionen, so können wir auch eine neue umfassendste zyklische Untergruppe $G_{\mu_{2}}$ bilden und an sie dieselbe Betrachtung wie an $G_{\mu_{1}}$ anknüpfen. Wir finden ihr entsprechend $\frac{m}{e_{2} \mu_{2}}\left(\mu_{2}-1\right)$ weitere Substitutionen in $G_{m}$ und können in derselben Art fortfahren, bis alle Substitutionen $\operatorname{der} G_{m}$ erschöpft sind. Es geht hieraus hervor, daß die ganzen Zahlen $m, \mu, e$ durch die Gleichung verbunden sind:

$$
m=1+\frac{m}{e_{1} \mu_{1}}\left(\mu_{1}-1\right)+\frac{m}{e_{2} \mu_{2}}\left(\mu_{2}-1\right)+\cdots+\frac{m}{e_{k} \mu_{k}}\left(\mu_{k}-1\right),
$$

die wir auch in die Gestalt setzen können:

$$
\frac{m-1}{m}=\frac{\mu_{1}-1}{e_{1} \mu_{1}}+\frac{\mu_{2}-1}{e_{2} \mu_{2}}+\cdots+\frac{\mu_{k}-1}{e_{k} \mu_{k}} .
$$

1) Vgl. dessen Abhandlung „Mémoire sur les équations différentielles linéaires à intégrale algébrique", Journ. f. Math., Bd. 84 (1878).

2) Wäre $G_{\mu_{1}}$ noch mit mehr als $\mu_{1}$ Substitutionen der Periode 2 vertauschbar, so würde man leicht den SchluB ziehen, daB $G_{\mu_{1}}$ keine "umfassendste" zyklische Untergruppe von $G_{m}$ wäre. 
472 II, 5. Die Gruppen der speziellen Transformationsgleichungen usw.

Das einzelne Glied rechts ist $\geqq \frac{1}{4}$. Da die links stehende Zahl $<1$ ist, so gilt für die Anzahl der Glieder die Ungleichung $k<4$.

Diese für jede $G_{m}$ gültige Gleichung (1) versuchen wir nun für die drei zulässigen Fälle $k=1,2$ und 3 in ganzen Zahlen $m, \mu, e$ zu lösen und beginnen mit dem Falle $k=1$ :

$$
\frac{m-1}{m}=\frac{\mu_{1}-1}{e_{1} \mu_{1}} \text {. }
$$

Hier ist $e_{1}=2$ unbrauchbar, da in diesem Falle die rechte Seite $<\frac{1}{2}$ ist, während $\frac{m-1}{m} \geqq \frac{1}{2}$ gilt. Also bleibt nur $e_{1}=1, m=\mu_{1}$, was zu den zyklischen Gruppen zurückführt. .

Für $k=2$ haben wir die Gleichung:

$$
\frac{m-1}{m}=\frac{\mu_{1}-1}{e_{1} \mu_{1}}+\frac{\mu_{2}-1}{e_{2} \mu_{2}} .
$$

zu lösen. Die Kombination $e_{1}=e_{2}=1$ ist unbrauchbar, da dann die rechts stehende Summe $\geqq 1$ ist. Gilt $e_{1}=e_{2}=2$, so formt sich die Gleichung um in:

$$
\frac{1}{m}=\frac{1}{2 \mu_{1}}+\frac{1}{2 \mu_{2}} \text {. }
$$

Auch dieser Fall ist unbrauchbar, da die $G_{m}$ die Diedergruppe $G_{2 \mu_{1}}$ enthält, also $m$ ein Vielfaches von $2 \mu_{1}$ ist. Also bleibt nur der Fall, daB eine der Zahlen $e$ gleich 2, die andere gleich 1 ist; wir setzen also $e_{1}=2$, $e_{2}=1$ und haben:

$$
\frac{1}{2}+\frac{1}{m}=\frac{1}{2 \mu_{1}}+\frac{1}{\mu_{2}}
$$

zu lösen. Ist $\mu_{2} \geqq 4$, so folgt:

$$
\frac{1}{2 \mu_{1}}-\frac{1}{m}=\frac{1}{2}-\frac{1}{\mu_{2}} \geqq \frac{1}{4},
$$

was wieder unmöglich ist, da die links stehende Zahl selbst für den kleinsten Wert $\mu_{1}=2$ kleiner als $\frac{1}{4}$ ist. Also bleiben nur die Fälle $\mu_{2}=2$ und 3 übrig. Im ersten Falle folgt $m=2 \mu_{1}$, so daB die $G_{m}$ wieder eine Diedergruppe $G_{2 \mu_{1}}$ ist. Dagegen führt $\mu_{2}=3$ zu einer ersten neuen Lösung hin:

$$
e_{1}=2, \quad e_{2}=1, \quad \mu_{1}=2, \quad \mu_{2}=3, \quad m=12 .
$$

Ist endlich $k=3$, so $\mathrm{muB} e_{1}=e_{2}=e_{3}=2$ gelten, da für alle übrigen Kombinationen die in (1) rechts stehende Summe $\geqq 1$ ist. Wir haben also jetzt die Gleichung:

$$
\frac{1}{\mu_{1}}+\frac{1}{\mu_{2}}+\frac{1}{\mu_{3}}-1=\frac{2}{m}
$$

zu lösen. Da offenbar mindestens eine der Zahlen $\mu$ gleich 2 sein muB, so setzen wir $\mu_{1}=2$ und haben weiter: 
Allgemeine Bedingungen für die Untergruppen der $G_{\frac{1}{2} n\left(n^{2}-1\right)}$

$$
\frac{1}{\mu_{2}}+\frac{1}{\mu_{3}}-\frac{1}{2}=\frac{2}{m}
$$

zu lösen. Weiter muß mindestens eine der Zahlen $\mu_{2}, \mu_{3}$ kleiner als 4 sein, so daß nur die Möglichkeiten $\mu_{2}=2$ und $\mu_{2}=3$ übrig bleiben. Für $\mu_{\mathrm{g}}=2$ folgt $m=2 \mu_{3}$ und also wieder nur eine Diedergruppe $G_{m}$. Also bleibt nur $\mu_{2}=3$ und damit die Gleichung:

$$
\frac{1}{\mu_{3}}-\frac{1}{6}=\frac{2}{m} \text {. }
$$

Hieraus ergibt sich $\mu_{3} \leqq 5$. Für $\mu_{3}=2$ würde wieder eine Diedergruppe $G_{6}$ eintreten. Eine der Lösung:

$$
e_{1}=e_{2}=e_{3}=2, \quad \mu_{1}=2, \quad \mu_{2}=\mu_{3}=3, \quad m=12
$$

entsprechende $G_{12}$ gibt es nicht. Diese $G_{12}$ enthielte nämlich nur drei Substitutionen der Periode 2 und vier Diedergruppen $G_{6}$. In allen vier $G_{6}$ müBten also dieselben drei Substitutionen der Periode 2 vorkommen. Indessen ist eine diedrische $G_{6}$ aus ihren drei Substitutionen der Periode 2 erzeugbar, so daB die eben festgestellte Folgerung unhaltbar ist. Hiernach bleiben nur noch die beiden Werte $\mu_{3}=4$ und $\mu_{3}=5$ übrig, die in der Tat zu zwei neuen brauchbaren Lösungen hinführen. Unter Zusammenfassung mit der schon oben notierten Lösung (2) finden wir das Ergebnis: Außer den in $\S 3$ genannten Gruppen können in der $G_{\frac{1}{2} n\left(n^{2}-1\right)}$ nur noch drei Arten von Untergruppen $G_{12}, G_{24}$ und $G_{60}$ der Ordnungen 12, 24 und 60 auftreten, die den folgenden Lösungen der Gleichung (1) entsprechen:

$$
\begin{aligned}
& G_{12}, \quad k=2, \quad e_{1}=2, \quad e_{2}=1, \quad \mu_{1}=2, \quad \mu_{2}=3, \quad m=\dot{1} 2, \\
& G_{24}, \quad k=3, \quad e_{1}=e_{2}=e_{3}=2, \quad \mu_{1}=2, \quad \mu_{2}=3, \quad \mu_{3}=4, \quad m=24, \\
& G_{60}, \quad k=3, \quad e_{1}=e_{2}=e_{3}=2, \quad \mu_{1}=2, \quad \mu_{2}=3, \quad \mu_{3}=5, \quad m=60 .
\end{aligned}
$$

In der $G_{12}$ sind, da $e_{2}=1$ ist, vier gleichberechtigte zyklische Gruppen der Ordnung 3 enthalten. Wir nennen diese Gruppen $G_{3}, G_{3}^{\prime}, G_{3}^{\prime \prime}, G_{3}^{\prime \prime \prime}$ und bemerken, daB sie durch die einzelne Substitution $V$ der $G_{12}$ in vier Gruppen $V^{-1} \cdot G_{3} \cdot V, V^{-1} \cdot G_{3}^{\prime} \cdot V, \ldots$ transformiert werden, die abgesehen von der Anordnung wieder die $G_{3}, G_{3}^{\prime}, \ldots$ sind. Jeder Substitution $V$ ordnen wir so eine Permutation der vier Gruppen $G_{3}, G_{3}^{\prime}, G_{3}^{\prime \prime}, G_{3}^{\prime \prime \prime}$ zu, der gesamten $G_{12}$ aber eine Permutationsgruppe der $G_{3}, G_{3}^{\prime}, \ldots$ Die Ordnung dieser Permutationsgruppe aber ist $\frac{\mathbf{1 2}}{\boldsymbol{\nu}}$, falls im ganzen $\nu$ Substitutionen $V$ jede der Gruppen $G_{3}, G_{3}^{\prime}, \ldots$ einzeln in sich transformieren und also der identischen Permutation entsprechen. Nun ist die einzelne $G_{3}$ wegen $e_{2}=1$ nur mit ihren eigenen Substitutionen vertauschbar. Da aber die vier Gruppen $G_{3}, G_{3}^{\prime}, \ldots$ nur die identische Substitution gemein 
474 II, 5. Die Gruppen der speziellen Transformationsgleichungen usw.

haben, so ist $\nu=1$, so daß die Permutationsgruppe der $G_{3}, G_{3}^{\prime}, \ldots$ gleichfalls die Ordnung 12 hat. Die einzige Gruppe dieser Art ist die Gruppe der geraden Vertauschungen $\operatorname{der} G_{3}, G_{3}^{\prime}, G_{3}^{\prime \prime}, G_{3}^{\prime \prime \prime}$. In der $G_{24}$ sind wegen $e_{2}=2$ vier gleichberechtigte Diedergruppen $G_{6}$ enthalten, deren einzelne nur mit ihren eigenen Substitutionen vertauschbar ist. Die einzelne der 6 gleichberechtigten Substitutionen der Periode 2 ist immer an zwei Diedergruppen $G_{6}$ beteiligt, woraus sich ergibt, daB der Durchschnitt der vier $G_{6}$ nur aus der Substitution 1 besteht. Bilden wir demnach wieder die mit der $G_{24}$ homomorphe Permutationsgruppe der vier $G_{6}$, so gilt aufs neue $\nu=1$, d. h. die Permutationsgruppe ist gleichfalls eine $G_{24}$ und umfaßt also alle 24 Permutationen der vier $G_{6}$. Endlich haben wir in $\operatorname{der} G_{60}$ fünfzehn gleichberechtigte Substitutionen der Periode 2, während die übrigen Substitutionen andere Perioden haben. Jede Substitution der Periode 2 ist ausgezeichnet in einer Vierergruppe $G_{4}$ enthalten, und da wir die einzelne $G_{4}$ immer von drei Substitutionen der Periode 2 aus erhalten, so haben wir im ganzen fünf gleichberechtigte Vierergruppen $G_{4}$. Wir können demnach die $G_{60}$ homomorph auf eine Permutationsgruppe $G_{\frac{60}{v}}$ der fünf $G_{4}$ beziehen. Nun ist die einzelne $G_{4}$ als eine unter fünf gleichberechtigten Gruppen mit 12, eine $G_{12}$ bildenden Substitutionen vertauschbar. Wir finden so 5 gleichberechtigte $G_{12}$, deren Durchschnitt notwendig aus der identischen Substitution 1 allein besteht. ${ }^{1}$ ) Also ist auch hier $\nu=1$, und die Permutationsgruppe ist eine $G_{60}, d$. h. die Gruppe aller geraden Permutationen der fünf $G_{4}$.

Die drei gewonnenen Permutationsgruppen sind nun bekanntlich isomorph mit der Tetraeder-, der Oktaeder- und der Ikosaedergruppe. ${ }^{2}$ ) Da übrigens bei Auftreten von Untergruppen $G_{24}$ und $G_{60}$ die Ordnung $\frac{1}{2} n\left(n^{2}-1\right)$ der Gesamtgruppe $G_{\frac{1}{2} n\left(n^{2}-1\right)}$ selbstrerständlich durch 24 bzw. 60 teilbar sein $\mathrm{muB}$, so folgt als abschließender Satz: Außer den in $\S 3$ bereits genannten Gruppen liönnen in der $G_{\frac{1}{2} n\left(n^{2}-1\right)}$ nur noch Tetruedergruppen $G_{12}$, Oltaedergruppen $G_{24}$ und Ikosaedergruppen $G_{60}$ auftreten, wobei das Vorkommen von Oktaedergruppen Primzahlen $n$ der Gestalt $n=8 h \pm 1$ zur Voraussetzung hat, dasjenige von Ikosaedergruppen aber Primzahlen der Gestalt $n=10 h \pm 1$. Eine Ausnahme von der letzten, die $G_{60}$ betreffenden Angabe liegt nur in niedersten Falle $n=5$ vor, insofern die zu $n=5$ gehörende Gesamtgruppe $G_{\frac{1}{2} n\left(n^{2}-1\right)}$ selbst cine Ilosaedergruppe ist. Man

1) Der Durchschnitt ist nämlich eine ausgezeichnete Untergruppe, deren Ordnung ein Teiler von 12 ist. Diese Untergruppe kann demnach weder die $15 \mathrm{zy}-$ klischen $G_{2}$, noch die 10 zyklischen $G_{3}$ enthalten, stellt also die $G_{1}$ dar.

2) Vgl. Klein, "Vorlesungen über das Ikosaeder usw.", S. $14 \mathrm{ff}$. 
Tetraeder-, Oktaeder- und Ikosaedergruppen in der $G_{\frac{1}{2} n\left(n^{2}-1\right)}$ folgert dies genau wie oben leicht aus dem Vorkommen der zyklischen Gruppen und Vierergruppen in $\operatorname{der} G_{60} \cdot{ }^{1}$ )

\section{§ 5. Der Satz von Galois.}

Die Galoissche Gruppe der Transformationsgleichung war im Primzahlfalle $n$ eine S. 461 näher bestimmte $G_{n\left(n^{2}-1\right)}$, die sich nach Adjunktion der Quadratwurzel $i^{\frac{n-1}{2}} \sqrt{n}$ auf die in $\S 3$ ff. zerlegte $G_{\frac{1}{2} n\left(n^{2}-1\right)}$ reduzierte. Die Transformationsgleichung war dabei diejenige Resolvente, die zu den $(n+1)$ metazyklischen $G_{\frac{1}{2} n(n-1)}$ gehörte. Dabei lieferte der Index $(n+1)$ dieser Gruppen in der Gesamtgruppe $G_{\frac{1}{2} n\left(n^{2}-1\right)}$ den Grad $(n+1)$ der Transformationsgleichung.

Wir halten zunächst an der Adjunktion von $i^{\frac{n-1}{2}} \sqrt{n}$ fest, so dab die Gruppe jeder Resolvente wieder die $G_{\frac{1}{2} n\left(n^{2}-1\right)}$ ist, da diese Gruppe einfach ist. $\left.{ }^{2}\right)$ Eine Gleichung mit einem Grade $<n$ kann keine Gruppe haben, deren Ordnung durch die Primzahl $n$ teilbar ist. Soll demnach die Möglichkeit erörtert werden, ob Resolventen eines Grades $<n+1$ auftreten, so kann es sich höchstens noch um Resolventen des $n^{\text {ten }}$ Grades selbst handeln. Die Antwort auf die aufgeworfene Frage ist im Satze von Galo is enthalten, nach dem zwar in den drei niedersten Fällen $n=5$, 7 und 11, aber bei keiner weiteren Primzahl $n$ Resolventen $n^{\text {ten }}$ Grades auftreten.

Der negative Teil des Satzes geht sofort aus den Ergebnissen von $\S 3$ und 4 hervor. Die zyklischen Untergruppen und die Diedergruppen der $G_{\frac{1}{2} n\left(n^{2}-1\right)}$ erreichen in keinem Falle die Ordnung der metazyklischen $G_{\frac{1}{3} n(n-1)}$. Sollen demnach sogar Untergruppen vorkommen, deren Ordnung $>\frac{1}{2} n(n-1)$ ist, so müBten sie zu den in $\S 4$ aufgestellten $G_{12}, G_{24}$ oder $G_{60}$ gehören. Ist $n \geqq 13$, so gilt $\frac{1}{2} n(n-1) \geqq 78$, so daß dann sicher die $G_{\frac{1}{2} n(n-1)}$ die Untergruppen gröBter Ordnung sind. Dagegen ist für $n=5$ die Ordnung 12 , für $n=7$ die Ordnung 24 und für $n=11$ die Ordnung 60 gröBer als die jeweilige Ordnung 10, 21 und 55 der metazyklischen Gruppen.

Im niedersten Falle $n=5$ ist das wirkliche Auftreten von Gruppen $G_{12}$ leicht beweisbar. Wir stellten bereits ein System von fünf gleichberechtigten Vierergruppen $G_{4}$ in der $G_{60}$ fest, zu denen sich die 15 in der $G_{60}$ enthaltenen Substitutionen der Periode 2 zu je dreien zusammen-

1) Das Auftreten von 5 gleichberechtigten Vierergruppen wurde bereits S. 468 festgestellt.

2) Vgl. S. 262; es gilt $n \geq 5$. 
476 II, 5. Die Gruppen der speziellen Transformationsgleichungen usw.

schließen. Als eine unter 5 gleichberechtigten Gruppen ist die einzelne $G_{4}$ mit 12 eine $G_{12}$ bildenden Substitutionen vertauschbar. Es treten also in der $G_{60}$ tatsächlich fünf gleichberechtigte $G_{12}$ auf, die nach $\S 4$ Tetraedergruppen sind.

Diesen $G_{12}$ entsprechen nach den S. 250 allgemein entwickelten Sätzen fünf gleichberechtigte Kongruenzgruppen fünfter Stufe $\Gamma_{5}$ des Index 5, deren einzelne, mod 5 reduziert, sich auf die zugehörige $G_{12}$ zusammenzieht. Wir greifen etwa diejenige $G_{12}$ heraus, der die Substitution $T \equiv\left(\begin{array}{c}0,-1 \\ 1,0\end{array}\right)$ (mod 5) der Periode 2 angehört, und wollen den Diskontinuitätsbereich der zugehörigen $\Gamma_{5}$ herstellen. Dieser Bereich läßt sich, da die aus $S \equiv\left(\begin{array}{l}1,1 \\ 0,1\end{array}\right)$

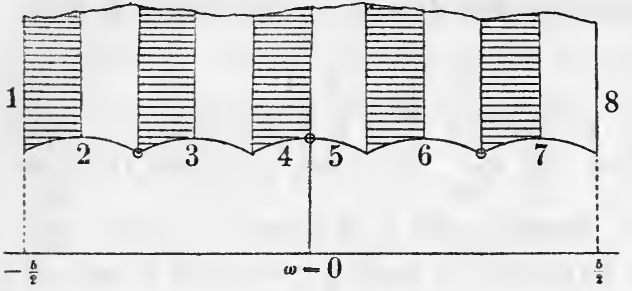

Fig. 30. zu erzeugende zyklische $G_{5}$ in $\operatorname{der} G_{12}$ nicht enthalten ist, aus den fünf in Fig. 30 nebeneinander geordneten Dreieckspaaren zusammensetzen, die den Substitutionen 1, $S \pm 1, S \pm 2$ entsprechen. Die mit Nummern ver-

sehenen Seiten hängen durch folgende Substitutionen zusammen:

$$
1 \rightarrow 8,\left(\begin{array}{l}
1,5 \\
0,1
\end{array}\right) ; 2 \rightarrow 3,\left(\begin{array}{r}
1, \\
-1,-2
\end{array}\right) ; 4 \rightarrow 5,\left(\begin{array}{c}
0,-1 \\
1,0
\end{array}\right) ; 6 \rightarrow 7,\left(\begin{array}{l}
2,-3 \\
1,-1
\end{array}\right) .
$$

Die zur Substitution $T$ gehörende Vierergruppe $G_{4}$ besteht nämlich aus den Substitutionen:

$$
1,\left(\begin{array}{c}
0,-1 \\
1,0
\end{array}\right),\left(\begin{array}{l}
2,0 \\
0,3
\end{array}\right),\left(\begin{array}{l}
0,2 \\
2,0
\end{array}\right)(\bmod 5)
$$

Diese $G_{4}$ wird aber in der Tat sowohl durch $\left(\begin{array}{r}1, \\ -1,-2\end{array}\right)$ als durch $\left(\begin{array}{l}2,-3 \\ 1,-1\end{array}\right)$ in sich transformiert. Am Diskontinuitätsbereiche sind diejenigen Ecken, die Fixpunkte elliptischer Substitutionen der $\Gamma_{5}$ sind, durch kleine Kreise kenntlich gemacht. Die Diskontinuitätsbereiche der weiteren vier gleichberechtigten $\Gamma_{5}$ gehen aus dem in Fig. 30 dargestellten Bereiche durch Ausübung der Substitutionen $S=\left(\begin{array}{l}1, \\ 0,1\end{array}\right), S^{2}, S^{3}, S^{4}$ hervor, entsprechend dem Umstande, daß auch die fünf $G_{12}$ aus einer ersten unter ihnen durch Transformation mit $1, S, S^{2}, S^{3}, S^{4}$ entstehen.

In der zu $n=7$ gehörenden $G_{168}$ sind nach S. $470 \mathrm{im}$ ganzen $21 \mathrm{zy}-$ klische $G_{4}$ enthalten, die alle gleichberechtigt sind, und die 21 ebensolche $G_{2}$ in sich enthalten. Jede solche $G_{2}$ ist mit 4 anderen Substitutionen der Periode 2 vertauschbar und bildet, mit ihnen zusammengesetzt, zwei Vierergruppen $G_{4}$. Da aber dieselbe $G_{4}$ immer von 3 Substitutionen der Periode 2 aus gewonnen wird, so haben wir nicht zweimal 21, sondern 
nur zweimal 7 Vierergruppen $G_{4}$. Es ist nun zunächst die Alternative $\mathrm{zu}$ entscheiden, ob diese $14 G_{4}$ alle untereinander gleichberechtigt sind, oder ob sie zwei Systeme zu je 7 gleichberechtigten $G_{4}$ bilden. Im ersten Falle ist die einzelne $G_{4}$ in der $G_{168}$ im ganzen mit 12 , eine Tetraedergruppe bildenden Substitutionen vertauschbar, im zweiten Falle aber mit 24, eine Oktaedergruppe bildenden Substitutionen. Bezeichnen wir aber mit $S$ die erzeugende Substitution einer zyklischen $G_{4}$ und durch $T$ eine von $S^{2}$ verschiedene Substitution der Periode 2, die mit dieser $G_{4}$ vertauschbar ist, so gilt:

$$
T^{-1} \cdot S \cdot T \equiv T \cdot S \cdot T \equiv S^{3} \text { oder } \equiv S \quad(\bmod 7) .
$$

Es folgt demnach $S^{-1} \cdot T \cdot S \equiv S^{2} T$ oder $\equiv T$, so daB in beiden Fällen die aus $1, S^{2}, T, S^{2} T$ bestehende Vierergruppe $G_{4}$ durch die Substitution $S$ der Periode vier in sich transformiert wird. Eine Tetraeder- $G_{12}$ enthält aber keine Substitution der Periode 4, so daß der zweite der beiden in Rede stehenden Fälle vorliegt. Die bei $n=7$ eintretende $G_{168}$ enthält demgemäß zwei Systeme zu je sieben gleichberechtigten Vierergruppen $G_{4}$. Da nun die einzelne $G_{4}$ mit 24, eine Oktaeder- $G_{24}$ bildenden Substitutionen vertiluschbar ist, so enthält die $G_{168}$ in der Tat auch zwei Systeme von je sieben gleichberechtigten Olitaedergruppen $G_{24}$.

Diesen Gruppen entsprechen zwei Systeme von je sieben gleichberechtigten Kongruenzgruppen $\gamma^{\text {ter }}$ Stufe $\Gamma_{7}$ vom Index 7, deren Diskontinuitätsbereiche wir aufstellen wollen. Wir wählen zunächst die $G_{24}$, die die aus $S \equiv\left(\begin{array}{l}1,2 \\ 1,3\end{array}\right) \cdot(\bmod 7)$ zu erzeugende zyklische $G_{4}$ enthält. Diese $G_{4}$ ist mit $T \equiv\left(\begin{array}{l}3,4 \\ 1,4\end{array}\right)$ vertauschbar, so daB die aus $S^{2}$ und $T$ zu erzeugende Vierergruppe $G_{4}$ aus den Substitutionen:

$$
1, \quad S^{2} \equiv\left(\begin{array}{l}
3,1 \\
4,4
\end{array}\right), \quad T \equiv\left(\begin{array}{l}
3,4 \\
1,4
\end{array}\right), \quad S^{2} \cdot T \equiv\left(\begin{array}{l}
3,2 \\
2,4
\end{array}\right) \quad(\bmod 7)
$$

besteht. Für die zugehörige $\Gamma_{7}$ läßt sich ein Diskontinuitätsbereich aus den 7 in Fig. 31 aneinander gereihtenDreieckspaaren aufbauen, da keine zwei dieser Paare bezüglich der $\Gamma_{7}$ äquivalent sein

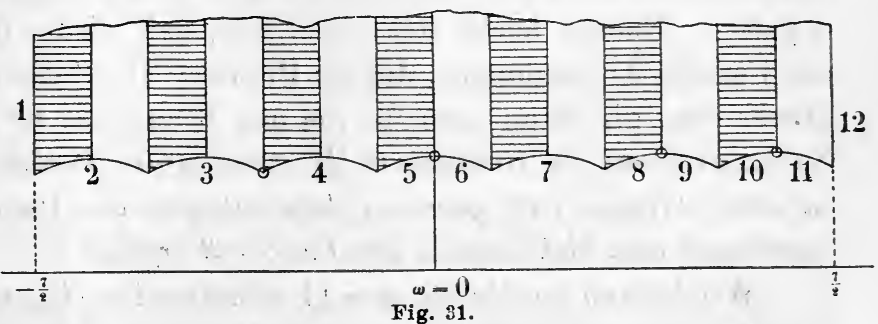
können. Die Zusammenordnung der Randkurven aber geschieht nach folgendem Gesetze: 


$$
\begin{gathered}
1 \rightarrow 12,\left(\begin{array}{l}
1,7 \\
0,1
\end{array}\right) ; 2 \rightarrow 7,\left(\begin{array}{l}
1,2 \\
1,3
\end{array}\right) ; 3 \rightarrow 4,\left(\begin{array}{c}
1,3 \\
-1,-2
\end{array}\right) ; 5 \rightarrow 6,\left(\begin{array}{c}
0,-1 \\
1,0
\end{array}\right) ; \\
8 \rightarrow 9,\left(\begin{array}{l}
2,-5 \\
1,-2
\end{array}\right) ; 10 \rightarrow 11,\left(\begin{array}{c}
3,-10 \\
1,-3
\end{array}\right) .
\end{gathered}
$$

Die erste dieser Substitutionen ist $\equiv 1(\bmod 7)$, die zweite ist die soeben mit $S$ bezeichnete Substitution, und die letzte ist mod 7 mit der eben $T$ genannten Substitution kongruent. Auch die übrigen drei Substitutionen transformieren die Vierergruppe (1) in sich und gehören also der $\Gamma_{7}$ an. Die am Rande des Diskontinuitätsbereiches gelegenen Fixpunkte elliptischer Substitutionen der $\Gamma_{7}$ sind wieder durch kleine Kreise kenntlich gemacht. Durch Ausübung der Substitutionen $\left(\begin{array}{l}1,1 \\ 0,1\end{array}\right),\left(\begin{array}{l}1,2 \\ 0,1\end{array}\right), \ldots,\left(\begin{array}{l}1,6 \\ 0,1\end{array}\right)$ auf den Bereich der Fig. 31 entstehen die Diskontinuitätsbereiche der sechs weiteren mit $\Gamma_{7}$ gleichberechtigten Gruppen. Wie aus der Zuordnung der Randkurven hervorgeht, sind die Bereiche unsymmetrisch. Durch Spiegelung an der imaginären $\omega$-Achse entstehen die Diskontinuitätsbereiche des zweiten Systems der 7 gleichberechtigten Gruppen $\Gamma_{7}$.

Der Aufsuchung etwaiger Ikosaeder- $G_{60}$ in der zu $n=11$ gehörenden $G_{660}$ ist folgende Überlegung betreffs der Erzeugung der $G_{60}$ vorauszuschicken, bei der wir die $G_{60}$ in der Gestalt der $60 \bmod 5$ inkongruenten Substitutionen $\left(\begin{array}{l}\alpha, \beta \\ \gamma, \delta\end{array}\right)$ der Determinante 1 benutzen (vgl. (2) S.465). Die einzelne dieser Substitutionen hat die Periode 2, 3 oder 5 , je nachdem $\alpha+\delta \equiv 0$, $\equiv \pm 1$ oder $\equiv \pm 2(\bmod 5)$ ist; nur findet sich unter den Substitutionen mit $\alpha+\delta \equiv \pm 2$ auch die identische Substitution. Die Substitutionen der Periode 5 zerfallen in 2 Systeme zu je 12 gleichberechtigten; diese beiden Systeme können wir durch $S \equiv\left(\begin{array}{ll}1, & 1 \\ 0, & 1\end{array}\right)$ und $S^{2} \equiv\left(\begin{array}{ll}1 & 2 \\ 0 & 1\end{array}\right)$ repräsentieren. Ist $T \equiv\left(\begin{array}{c}\alpha, \beta \\ \gamma,-\alpha\end{array}\right)$ irgendeine Substitution der Periode 2, so hat $S \cdot T \equiv\left(\begin{array}{c}\alpha+\gamma, \beta-\alpha \\ \gamma,-\alpha\end{array}\right)$ dann und nur dann die Periode 3, wenn $\gamma \equiv \pm 1$ $(\bmod 5)$ gilt. Man zählt leicht ab, daB es in der $G_{60}$ im ganzen 5 Substitutionen $T$ der Periode 2 gibt, die in $S \cdot T$ eine Substitution der Periode 3 liefern. Ebenso leicht stellt man fest, daB wieder 5 Substitutionen $T$ der Periode 2 vorkommen, die ein Produkt $S^{2} \cdot T$ der Periode 3 ergeben. Dieser Satz gilt dann auch für die mit $S$ und mit $S^{2}$ gleichberechtigten Substitutionen: $Z u$ irgendeiner Substitution der Periode 5 der $G_{60}$ gibt es in dieser Gruppe stets genau 5 Substitutionen der Periode 2, die mit ihr liombiniert eine Substitution der Periode 3 ergeben.

Wir kehren zu der bei $n=11$ eintretenden $G_{660}$ zurück und machen zunächst folgende Angaben über die Perioden ihrer Substitutionen: Die 55 Substitutionen der Periode 2 sind durch $\alpha+\delta \equiv 0(\bmod 11)$ charakterisiert und ebenso die 2.55 Substitutionen der Periode 3 durch $\alpha+\delta$ 
$\equiv \pm 1(\bmod 11)$. Ferner ist ein erstes System von 2.66 gleichberechtigten Substitutionen der Periode 5 durch $\alpha+\delta \equiv \pm 3$ und ein zweites durch $\alpha+\delta \equiv \pm 4(\bmod 11)$ gegeben; das erste System mag durch $S \equiv\left(\begin{array}{l}2,0 \\ 0,6\end{array}\right)$, das zweite durch $S^{2}=\left(\begin{array}{l}4,0 \\ 0,3\end{array}\right)$ repräsentiert werden.

Eine etwa in der $G_{660}$ vorkommende $G_{60}$ ist nur mit ihren eigenen Substitutionen vertauschbar, da sie nicht ausgezeichnet sein kann und auch keine umfassendere Untergruppe auftritt. Somit ist die einzelne $G_{60}$ immer eine von elf gleichberechtigten Untergruppen. Da nun die $G_{60}$ sechs zyklische $G_{5}$ enthält und in der $G_{660}$ im ganzen 66 zyklische $G_{5}$ vorkommen, so ist die einzelne $G_{5}$ stets nur in einer unter den elf $G_{60}$ enthalten. Wir suchen nun nach denjenigen $G_{60}$, welche die aus $S \equiv\left(\begin{array}{l}2,0 \\ 0,6\end{array}\right)$ zu erzeugende $G_{5}$ enthalten. Jede derselben müBte 5 Substitutionen $T \equiv\left(\begin{array}{c}\alpha, \beta \\ \gamma,-\alpha\end{array}\right)$ enthalten, die in $S \cdot T \equiv\left(\begin{array}{c}2 \alpha, 2 \beta \\ 6 \gamma,-6 \alpha\end{array}\right)$ Substitutionen der Periode 3 liefern. Hierfür ist $-4 \alpha \equiv \pm 1(\bmod 11)$ charakteristisch, so $\mathrm{daB}$ wir $\alpha \equiv 3$, $\gamma \equiv \beta^{-1}(\bmod 11) \mathrm{zu}$ setzen haben. $\left.{ }^{1}\right)$ Da hier $\beta$ einen beliebigen der Reste $1,2,3, \ldots, 10$ bedeuten darf, so haben wir im ganzen 10 brauchbare Substitutionen $T$. Also folgt: Wenn ïberhaupt Ikosaeder- $G_{60}$ in $\operatorname{der} G_{660}$ auftreten, so gibt es entweder ein System von elf gleichberechtigten $G_{60}$ oder zwei solche Systeme.

Wir halten nun an $S \equiv\left(\begin{array}{l}2,0 \\ 0,6\end{array}\right)$ fest und wählen $T \equiv\left(\begin{array}{rr}3, & 1 \\ 1,-3\end{array}\right) . \quad$ Da $S \cdot T$ die Periode 3 hat, so wird aus $S$ und $T$ eine Gruppe erzeugt, deren Ordnung durch 30 teilbar ist, also (da es keine $G_{30}$ in der $G_{660}$ gibt) entweder eine $G_{60}$ oder die Gesamtgruppe $G_{660}$. Eine der Vierergruppen $G_{4}$, an denen die gewählte Substitution $T$ beteiligt ist, besteht aus:

$$
\left(\begin{array}{c}
3,1 \\
1,-3
\end{array}\right),\left(\begin{array}{c}
0,-1 \\
1,0
\end{array}\right),\left(\begin{array}{c}
-1,3 \\
3,1
\end{array}\right), 1
$$

Diese $G_{4}$ wird durch $S, S^{2}, S^{3}, S^{4}$ transformiert in:

$$
\begin{aligned}
& G_{4}^{(1)},\left(\begin{array}{c}
3,4 \\
3,-1
\end{array}\right),\left(\begin{array}{c}
0,-4 \\
3,0
\end{array}\right),\left(\begin{array}{c}
1,-1 \\
2,-1
\end{array}\right), 1, \\
& G_{4}^{(2)},\left(\begin{array}{c}
3,3 \\
4,-3
\end{array}\right),\left(\begin{array}{c}
0,-3 \\
4,0
\end{array}\right),\left(\begin{array}{c}
1,2 \\
-1,-1
\end{array}\right), 1, \\
& G_{4}^{(3)},\left(\begin{array}{l}
3,-2 \\
5,-3
\end{array}\right),\left(\begin{array}{c}
0,2 \\
5,0
\end{array}\right),\left(\begin{array}{c}
-1,5 \\
4,1
\end{array}\right), 1, \\
& G_{4}^{(4)},\left(\begin{array}{c}
3,5 \\
-2,-3
\end{array}\right),\left(\begin{array}{l}
0,5 \\
2,0
\end{array}\right),\left(\begin{array}{c}
-1,4 \\
5,1
\end{array}\right), 1 .
\end{aligned}
$$

Das System der fünf Gruppen $G_{5}, G_{5}^{(1)}, \ldots$ wird durch $S$ zyklisch per-

1) Man beachte, daB ein gleichzeitiger Zeichenwechsel von $\alpha, \beta, \gamma, \delta$ statthaft ist. 
mutiert. Aber auch bei Transformation durch $T$ gehen die fünf Gruppen ineinander über, da $G_{4}$ mit $T$ vertauschbar ist, während sich die anderen vier Gruppen wie folgt permutieren:

$$
\begin{aligned}
T^{-1} \cdot G_{4}^{(1)} \cdot T=G_{4}^{(4)}, & T^{-1} \cdot G_{4}^{(2)} \cdot T=G_{4}^{(3)}, \quad T^{-1} \cdot G_{4}^{(3)} \cdot T=G_{4}^{(2)}, \\
& T^{-1} \cdot G_{4}^{(4)} \cdot T=G_{4}^{(1)} .
\end{aligned}
$$

Somit transformieren auch alle aus $S$ und $T$ erzeugbaren Substitutionen das System der fünf $G_{4}$ stets nur wieder in sich, so daB aus $S$ und $T$ noch nicht die Gesamtgruppe, sondern vielmehr eine $G_{60}$ erzeugt wird.

Nun wird aus $S \equiv\left(\begin{array}{l}2,0 \\ 0,6\end{array}\right)$ und $T^{\prime} \equiv\left(\begin{array}{c}3,-1 \\ -1,-3\end{array}\right)$ eine zweite $G_{60}$ erzeugt, deren Substitutionen aus denen der ersten $G_{60}$ durch gleichzeitigen Zeichenwechsel von $\beta$ und $\gamma$ in allen Substitutionen entstehen. Die zweite $G_{60}$ enthält gleichfalls die aus $S$ zu erzeugende $G_{5}$ und ist deshalb mit der ersten nicht gleichberechtigt. Damit sind in der $G_{660}$ in der Tat zwei Systeme von je elf gleichberechtigten Ikosaedergruppen $G_{60}$ nachgewiesen.

Den $G_{60}$ entsprechen zwei Systeme von je elf gleichberechtigten Kongruenzgruppen $\Gamma_{11}$ elfter Stufe vom Index 11. Für diejenige $\Gamma_{11}$, die der

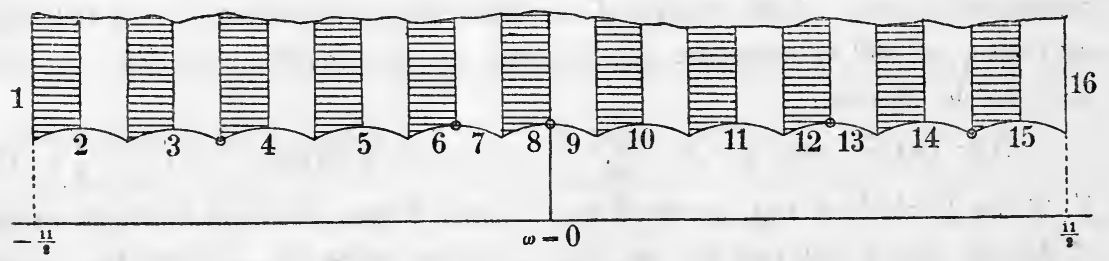

Fig. 32.

ersten der obigen $G_{60}$ entspricht, ist der Diskontinuitätsbereich in Fig. 32 angegeben, dessen Seiten, wie folgt, zusammengehören:

$$
\left\{\begin{aligned}
1 \rightarrow 16,\left(\begin{array}{c}
1,11 \\
0,1
\end{array}\right) ; 2 \rightarrow 11,\left(\begin{array}{c}
2,9 \\
1,5
\end{array}\right) ; 3 \rightarrow 4 ;\left(\begin{array}{c}
3,13 \\
-1,-4
\end{array}\right) ; \\
5 \rightarrow 10,\left(\begin{array}{c}
1,1 \\
1,2
\end{array}\right) ; 6 \rightarrow 7,\left(\begin{array}{c}
1,2 \\
-1,-1
\end{array}\right) ; 8 \rightarrow 9,\left(\begin{array}{c}
0,-1 \\
1,0
\end{array}\right) ; \\
12 \rightarrow 13,\left(\begin{array}{c}
3,-10 \\
1,-3
\end{array}\right) ; 14 \rightarrow 15,\left(\begin{array}{c}
5,-21 \\
1,-4
\end{array}\right) .
\end{aligned}\right.
$$

Die $G_{60}$ enthält nämlich mit $S$ und $T$ auch die Substitution:

$$
S^{2} \cdot T \cdot S^{3} \cdot T \cdot S^{2} \cdot T \equiv\left(\begin{array}{c}
0,-1 \\
1,0
\end{array}\right) \quad(\bmod 11),
$$

also alle vier Substitutionen (2) und damit überhaupt alle 5 Vierergruppen $G_{4}, G_{4}^{(1)}, \ldots, G_{4}^{(4)}$. Es erweisen sich also nicht nur die erste Substitution der Zusammenstellung (6), sondern auch die $5^{\text {te }}, 6^{\text {to }}$ und $7^{\text {te }}$ unmittelbar als in der aus $S$ und $T$ zu erzeugenden $\Gamma_{11}$ enthalten. Die $3^{\text {te }}$ 
und $8^{\text {te }}$ Substitution (3), die elliptisch von der Periode 3 sind, lassen sich zufolge der Kongruenzen:

$$
\begin{gathered}
S^{3} \cdot\left(\begin{array}{c}
-1,3 \\
3,1
\end{array}\right) \equiv\left(\begin{array}{l}
3,0 \\
0,4
\end{array}\right) \cdot\left(\begin{array}{c}
-1,3 \\
3,1
\end{array}\right) \equiv\left(\begin{array}{c}
3,13 \\
-1,-4
\end{array}\right) \\
S \cdot\left(\begin{array}{c}
3,5 \\
-2,-3
\end{array}\right) \equiv\left(\begin{array}{l}
2,0 \\
0,6
\end{array}\right) \cdot\left(\begin{array}{c}
3,5 \\
-2,-3
\end{array}\right) \equiv\left(\begin{array}{c}
5,-21 \\
1,-4
\end{array}\right)
\end{gathered}
$$

aus Substitutionen herstellen, die bereits in der $G_{60}$ nachgewiesen sind. Aber auch die beiden noch fehlenden Substitutionen $\left(\begin{array}{l}2,9 \\ 1,5\end{array}\right)$ und $\left(\begin{array}{l}1,1 \\ 1,2\end{array}\right)$ lassen sich zufolge der Gleichungen:

$$
\left(\begin{array}{c}
0,-1 \\
1,0
\end{array}\right) \cdot\left(\begin{array}{c}
1,2 \\
-1,-1
\end{array}\right)=\left(\begin{array}{l}
1,1 \\
1,2
\end{array}\right), \quad\left(\begin{array}{c}
0,-1 \\
1,0
\end{array}\right) \cdot\left(\begin{array}{c}
1,2 \\
-1,-1
\end{array}\right) \cdot\left(\begin{array}{c}
3,13 \\
-1,-4
\end{array}\right)=\left(\begin{array}{l}
2,9 \\
1,5
\end{array}\right)
$$

aus Substitutionen erzeugen, die wir bereits als in der $\Gamma_{11}$ enthalten erkannten. Aus dem gewonnenen Bereiche gehen durch Ausübung der Substitutionen $\left(\begin{array}{ll}1 & 1 \\ 0,1\end{array}\right),\left(\begin{array}{l}1, \\ 0,1 \\ 0,1\end{array}\right), \ldots,\left(\begin{array}{c}1,10 \\ 0,1\end{array}\right)$ die Diskontinuitätsbereiche der übrigen 10 mit $\Gamma_{11}$ gleichberechtigten Gruppen $\Gamma_{11}$ hervor. Diese Bereiche sind wieder unsymmetrisch; durch Spiegelung an der imaginären $\omega$-Achse gelangt man zu den Diskontinuitätsbereichen für das zweite System der elf gleichberechtigten $\Gamma_{11}$.

Innerhalb der auf den rationalen Zahlkörper bezogenen Galoisschen Gruppe $G_{120}$ der zu $n=\check{5}$ gehörenden Transformationsgleichung sind die 5 Tetraedergruppen $G_{12}$ wieder nur unter sich gleichberechtigt. Die einzelne $G_{12}$ ist demnach in der $G_{120}$ mit 24 eine $G_{24}$ (Oktaedergruppe) bildenden Substitutionen vertauschbar. Man findet also in der $G_{120}$ fünf gleichberechtigte Untergruppen $G_{24}$ des Index 5 .

Anders liegen die Verhältnisse bei $n=7$ und $n=11$. Da -1 quadratischer Nichtrest sowohl von 7 als von 11 ist, so kann man die auf den rationalen Körper bezogenen Galoisschen Gruppen beide Male dadurch zur Darstellung bringen, daß man den bisherigen Substitutionen $\left(\begin{array}{l}\alpha, \beta \\ \gamma, \delta\end{array}\right)$ noch die mit:

$$
\alpha \delta-\beta \gamma \equiv-1 \quad(\bmod n)
$$

hinzufügt. Insbesondere ist in der Galoisschen $G_{n\left(n^{2}-1\right)}$ jedesmal die Substitution $U \equiv\left(\begin{array}{r}-1,0 \\ 0,1\end{array}\right)$ enthalten. Bei Transformation der einzelnen Substitution $\left(\begin{array}{l}\alpha, \beta \\ \gamma, \delta\end{array}\right)$ durch $U$ aber gilt:

$$
U^{-1} \cdot\left(\begin{array}{l}
\alpha, \beta \\
\gamma, \delta
\end{array}\right) \cdot U \equiv\left(\begin{array}{l}
\alpha,-\beta \\
-\gamma, \delta
\end{array}\right) \quad(\bmod n) .
$$

Die Transformation durch $U$ läuft also für die $\Gamma_{7}$ und $\Gamma_{11}$ darauf hinaus, dab diese Gruppen durch die Spiegelung $\omega^{\prime}=-\bar{\omega}$ an der imaginären 
$\omega$-Achse transformiert.werden. Hierdurch aber werden jedesmal die beiden innerhalb der ursprünglichen Modulgruppe noch nicht gleichberechtigten Systeme von Gruppen $\Gamma_{n}$ ineinander transformiert. Wir gelangen zu dem Satze, den wir sogleich wieder für die Galoisschen $G_{n\left(n^{2}-1\right)}$ aussprechen: Innerhalb der Galoisschen Gruppe $G_{336}$ tritt ein System von 14 gleichberechtigten Oktaedergruppen $G_{24}$ des Index 14 auf, und ebenso findet sich innerhalb der Galoisschen $G_{1320}$ ein System von 22 gleichberechtigten Ikosaedergruppen $G_{60}$ des Index 22.

Wir ziehen nun entsprechend den allgemeinen Sätzen der Galoisschen Gleichungstheorie hieraus sogleich die algebraischen Folgerungen: Erstlich gelangen wir im Falle $n=5$ zu einer numerisch rationalen Resolvente fünten Grades der Transformationsgleichung sechsten Grades. Sieht man bei $n=7$ und 11 vorerst noch von der Adjunktion der Irrationalität $i \sqrt{n}$ ab, so folgt weiter: Bei den Transformationsgraden $n=7$ und 11 tritt je eine Resolvente des Grades 14 bzw. 22 mit rationalen Zahlenkoeffizienten auf. Bei Fortgang zu den Monodromiegruppen und also nach Adjunktion von $i \sqrt{7}$ bzw. $i \sqrt{11}$ aber werden diese Resolventen reduzibel, und man gelangt zu dem Satze: Bei den Transformationsgraden $n=7$ und $n=11$ existieren je zwei Resolventen der Grade 7 bzw. 11, in deren Koeffizienten die Irrationalitäten $i \sqrt{7}$ bzw. $i \sqrt{11}$ auftreten.

\section{\$ 6. Die Resolventen fünften und siebenten Grades.}

Um die Resolvente fünften Grades zu gewinnen, die im Falle der Transformation fünften Grades existiert, hätte man, den allgemeinen Ansätzen der Galoisschen Theorie folgend, zunächst den Galoisschen Körper der Transformationsgleichung zu bilden und für eine einzelne der Gruppen $G_{12}$ eine geeignete Funktion jenes Körpers zu wählen, die dann die Wurzel der gesuchten Gleichung fünften Grades wäre. Es handelt sich hierbei um ausgedehnte Entwicklungen, welche erschöpfend von Klein in den „Vorlesungen über das Ikosaeder" zur Darstellung gebracht sind. ${ }^{1}$ ) Die entsprechende Untersuchung für den Fall der Transformation siebenten Grades ist von Klein in der Abhandlung „Über die Transformation siebenter Ordnung der elliptischen Funktionen“"2) gegeben. Nun kann 'man aber wenigstens in den beiden genannten niedersten Fällen $n=5$ und $n=7$ zur wirklichen Kenntnis der Resolventen mit Umgehung der Galoisschen Körper unmittelbar von den Diskontinuitätsbereichen der Gruppen $\Gamma_{5}$ und $\Gamma_{7}$ aus gelangen, wie dies durch Klein in einer beson-

1) S. auch die Abhandlung von Klein „Über die Transformation der elliptischen Funktionen und die Auflösung der Gleichungen fünften Grades", Math. Ann., Bd. 14, S. 111.

2) Math. Ann., Bd. 14, S. 428 ff. Vgl. auch „Modulfunktionen“ Bd. 1, S. $692 \mathrm{ff}$. 
deren Abhandlung „Über die Erniedrigung der Modulargleichungen “1) gezeigt wurde. Man hat sich hierbei jener rein algebraischen Methode zu bedienen, die Klein auch bereits bei den. Transformationsgleichungen selbst zur Verwendung brachte. Indem wir betreffs der Beziehung der Resolventen fünften und siebenten Grades zu den Galoisschen Körpern und den Transformationsgleichungen auf die genannten Darstellungen vèrweisen, wollen wir im folgenden nur noch die wirkliche Aufstellung der Resolventen nach der algebraischen Methode Kleins geben.

Es möge nun diejenige $G_{12}$ herangezogen werden, deren zugehörige $\Gamma_{5}$ den in Fig. 30, S. 476 dargestellten Diskontinuitätsbereich hat. Eine zur $G_{12}$ gehörende Funktion des Galoisschen Körpers ist dann in Abhängigkeit von $\omega$ eine Modulfunktion der $\Gamma_{5}$. Um unter diesen Funktionen eine geeignete auszusuchen, bilden wir den genannten Bereich durch $J(\omega)$ ab. Als Abbild ergibt sich eine fünfblättrige Riemannsche Fläche über der $J$-Ebene, die nur bei $J=0,1$ und $\infty$ verzweigt ist; und zwar tritt bei $J=0$ ein dreiblättriger Verzweigungspunkt auf, bei $J=1$ zwei zweiblättrige und bei $J=\infty$ ein fünfblättriger. Diese Fläche hat das Geschlecht 0, so daß wir zum Aufbau der Resolvente eine einwertige Funktion verwenden können. Wir nennen diese Funktion $\xi(\omega)$ und erklären sie eindeutig durch die Festsetzung, daß:

$$
\xi\left(\frac{ \pm 1+i \sqrt{3}}{2}\right)=0, \quad \xi(i)=3, \quad \xi(i \infty)=\infty
$$

sein soll. Der Wert $\xi=0$ findet also im dreiblättrigen Verzweigungspunkte der Riemannschen Fläche statt, der bei $J=0$ liegt; ferner wird $\xi=3$ bei $J=1$ in dem daselbst isoliert verlaufenden Blatte, und man hat $\xi=\infty$ im fünfblättrigen Verzweigungspunkte bei $J=\infty$.

Umgekehrt ist $J$ oder, was hier zweckmäBiger ist, $j=12^{3} J$ als fünfwertige Funktion der Fläche eine rationale Funktion $5^{\text {ten }}$ Grades von $\xi$. Da $j$ nur mit $\xi$ selbst unendlich wird, so handelt es sich um eine ganze Funktion $5^{\text {ten }}$ Grades. Aus der soeben bei $J=0$ und $J=1$ angegebenen Werteverteilung von $\xi$ aber gewinnt man die beiden Ansätze:

$$
j=a \xi^{3}\left(\xi^{2}+b \xi+c\right), j-12^{3}=a(\xi-3)\left(\xi^{2}+d \xi+e\right)^{2},
$$

wo $a$ in beiden Gleichungen dieselbe Bedeutung hat.

Zur Bestimmung der Koeffizienten berechne man aus beiden Ansätzen (1) die Ableitung $\frac{d j}{d \zeta}$ und setze die entstehenden Ausdrücke gleich. Bei Fortlassung des Faktors $5 a$ ergibt sich die identische Gleichung:

$$
\left(\xi^{2}+\frac{4}{5} b \xi+\frac{3}{5} c\right) \xi^{2}=\left(\xi^{2}+d \xi+e\right)\left(\xi^{2}+\frac{1}{5}(3 d-12) \xi+\frac{1}{5}(e-6 d)\right) .
$$

Nun ist jedenfalls $e \neq 0$, da für $\xi=0$ die Funktion $j=0$ und also nicht

1) Math. Ann., Bd. 14, S. $417 \mathrm{ff}$. 
484 II, 5. Die Gruppen der speziellen Transformationsgleıchungen usw.

gleich $12^{3}$ ist. Der Ausdruck in der zweiten Klammer der rechten Seite der letzten Gleichung ist also mit $\xi^{2}$ identisch, und man findet durch Koeffizientenvergleichung:

$$
3 d-12=0, \quad e-6 d=0, \quad 4 b=5 d, \quad 3 c=5 e,
$$

woraus sich $d=4, e=24, b=5, c=40$ ergibt. Den Faktor $a$ findet man aus dem ersten Ansatz (1), indem man $\xi=3, j=12^{3}$ einträgt, zu $a=1$. Der Ausdruck von $j$ als rationale Funktion von $\xi$ kann in die Gestalten:

$$
j=\zeta^{3}\left(\xi^{2}+5 \zeta+40\right), \quad j-12^{3}=(\xi-3)\left(\xi^{2}+4 \xi+24\right)^{2}
$$

gekleidet werden, zwei Gleichungen, die uns als solche für $\zeta$ nun unmittelbar unsere gesuchte Resolvente fünften Grades in zwei Gestalten liefern.

Die beiden Gleichungen (2) kann man auch in solche für die einfachsten Modulformen der $\Gamma_{5}$ umschreiben. Zunächst folgt aus der ersten Gleichung bei Division durch $\xi^{5}$ und Multiplikation mit $12^{2} g_{2}^{2} \Delta$ :

$$
\left(\frac{12 g_{2}}{\zeta}\right)^{5}=\Delta\left(40\left(\frac{12 g_{2}}{\zeta}\right)^{2}+60 g_{2}\left(\frac{12 g_{2}}{\zeta}\right)+144 g_{2}^{2}\right) .
$$

Für die zur $\Gamma_{5}$ gehörende ganze Modulform $(-4)^{\text {ter }}$ Dimension:

$$
f\left(\varpi_{1}, \omega_{2}\right)=\frac{12 g_{2}}{\zeta}
$$

ergibt sich somit die Gleichung $5^{\text {ten }}$ Grades:

$$
f^{5}-40 \Delta f^{2}-60 g_{2} \Delta f-144 g_{2}^{2} \Delta=0 \text {. }
$$

Andrerseits finden wir aus der zweiten Gleichung (2) nach Multiplikation mit $\Delta$ und Ausziehung der Quadratwurzel:

$$
216 g_{3}=\sqrt{(\xi-3) \Delta}\left(\xi^{2}+4 \xi+24\right) \text {. }
$$

Hieraus folgt der Satz: Auch noch die Quadratwurzel:

$$
\varphi\left(\omega_{1}, \omega_{2}\right)=\sqrt{(\xi-3) \Delta}=\frac{216 g_{3}}{\xi^{2}+4 \xi+24}
$$

ist eine eindeutige und zwar ganze Modulform $(-6)^{\text {ter }}$ Dimension der $\Gamma_{5}$, die der folgenden Gleichung fünften Grades genügt:

$$
\varphi^{5}+10 \Delta \varphi^{3}+45 \Delta^{2} \varphi-216 g_{3} \Delta^{2}=0 .
$$

Die gleiche Methode ist auch im Falle $n=7$ zur Gewinnung der beiden Resolventen $7^{\text {ten }}$ Grades ausreichend. Der in Fig. 31, S. 477, dargestellte Diskontinuitätsbereich einer der Gruppen $\Gamma_{7}$ wird durch $J(\omega)$ auf eine siebenblättrige Riemannsche Fläche über der $J$-Ebene abgebildet, die wieder nur bei $J=0,1$ und $\infty$ verzweigt ist; und zwar liegen jetzt bei $J=0$ zwei dreiblättrige Verzweigungspunkte, bei $J=1$ zwei zweiblättrige und bei $J=\infty$ ein siebenblättriger. Auch diese Fläche gehört 
zum Geschlechte 0, so daß wir uns wieder einer einwertigen Funktion $\zeta(\omega)$ bedienen können. Es gelte:

$$
\xi\left(\frac{-3+i \sqrt{3}}{2}\right)=0, \quad \xi(i \infty)=\infty,
$$

so daß $\xi$ bei $J=0$ in dem daselbst isoliert verlaufenden Blatte verschwindet und im siebenblätt):igen Verzweigungspunkte bei $J=\infty$ unendlich wird. Durch diese beiden Bedingungen ist dann $\xi$ nur erst bis auf einen konstanten Faktor bestimmt; eine weitere Festsetzung bleibe vorbehalten.

In $\zeta$ ist $J$ eine ganze Funktion $7^{\text {ten }}$ Grades, und zwar leitet man aus der Werteverteilung von $\xi$ bei $J=0$ und $J=1$ die Ansätze ab:

(7) $J=a \xi\left(\xi^{2}+b \xi+7 c\right)^{3}, \quad J-1=a\left(\xi^{3}+d \xi^{2}+e \xi+f\right)\left(\xi^{2}+g \xi+h\right)^{2}$,

wo zur Kürzung der Rechnung im Absolutgliede der ersten Klammer der Faktor 7 aufgenommen ist. Durch Gleichsetzung der beiden für $\frac{d J}{d \xi} \mathrm{zu}$ gewinnenden Ausdrücke ergibt sich bei Fortlassung überflüssiger Faktoren die identische Gleichung:

$$
\begin{gathered}
\left(\xi^{2}+\frac{4}{7} b \xi+c\right)\left(\xi^{2}+b \xi+7 c\right)^{2}=\left(\xi^{2}+g \xi+h\right)\left(\xi^{4}+\frac{6 d+5 g}{7} \xi^{3}\right. \\
\left.+\frac{4 d g+5 e+3 h}{7} \xi^{2}+\frac{2 d h+3 e g+4 f}{7} \xi+\frac{e h+2 f g}{7}\right) .
\end{gathered}
$$

Nun folgt aus (7), daB die Funktionen $\left(\xi^{2}+b \xi+7 c\right)$ und $\left(\xi^{2}+g \xi+h\right)$ keinen Linearfaktor gemein haben. Somit sind die Funktionen $\left(\xi^{2}+\frac{4}{7} b \xi+c\right)$ und $\left(\xi^{2}+g \xi+h\right)$ identisch, und man findet durch Koeffizientenvergleichung insgesamt:

$$
\begin{array}{cl}
7 g=4 b, & h=c, \quad 6 d+5 g=14 b, \quad 4 d g+5 e+3 h=7 b^{2}+98 c \\
2 d h+3 e g+4 f=98 b c, \quad e h+2 f g=343 c^{2} .
\end{array}
$$

Wäre nun $b=0$, so würde $g=0, d=0$ und wegen der vorletzten Gleichung $f=0$ folgen. Aber es ist $f \neq 0$, da für $\xi=0$ auch $J=0$ und nicht $J=1$. Hiernach ist $b \neq 0$. Dann aber können wir den zur eindeutigen Bestimmung von $\xi$ noch verfügbaren Faktor so festsetzen, daß $b=7$ wird. Die aufzulösenden Gleichungen kürzen sich nun zu:

$$
\begin{gathered}
b=7, \quad g=4, \quad h=c, \quad d=13, \quad e=27+19 c, \quad 3 e+f=165 c, \\
c e+8 f=343 c^{2} .
\end{gathered}
$$

Durch Elimination von $e$ und $f$ aus den drei letzten Gleichungen folgt für $c$ die quadratische Gleichung:

$$
4 c^{2}-11 c+8=0,
$$

bei deren Lösung sich, wie es sein muß, die Irrationalität $i \sqrt{7}$ einfindet. Wir gewinnen, unseren beiden Resolventen entsprechend, die beiden 
486 II, 5. Die Gruppen der speziellen Transformationsgleichungen usw.

Koeffizientensysteme:

$$
c=h=\frac{11 \pm i \sqrt{7}}{8}, \quad e=\frac{425 \pm 19 i \sqrt{7}}{8}, f=\frac{135 \pm 27 i \sqrt{7}}{2},
$$

während die Koeffizienten $b, g, d$ die schon angegebenen rationalen Werte haben. ${ }^{1}$ ) Endlich ergibt sich der Wert $a$, indem man in die zweite Gleichung (7) $\xi=0$ und $J=0$ einträgt; man findet:

$$
-1=a \cdot f \cdot h^{2}=a \frac{135 \pm 27 i \sqrt{7}}{2}\left(\frac{11 \pm i \sqrt{7}}{8}\right)^{2},-a^{-1}=\frac{351 \pm 189 i \sqrt{7}}{4} .
$$

Setzt man die berechneten Werte der Koeffizienten in die Ansätze (7) ein, so lassen sich diese in die folgende Proportion zusammenziehen:

(8) $J:(J-1): 1=\xi\left(\xi^{2}+7 \xi+\frac{77 \pm 7 i \sqrt{7}}{8}\right)^{3}$

$$
\begin{aligned}
& :\left(\xi^{3}+13 \xi^{2}+\frac{425 \pm 19 i \sqrt{7}}{8} \zeta+\frac{135 \pm 27 i \sqrt{7}}{2}\right)\left(\zeta^{2}+4 \xi\right. \\
+ & \left.\frac{11 \pm i \sqrt{7}}{8}\right)^{2}:-\frac{351 \pm 189 i \sqrt{7}}{4} .
\end{aligned}
$$

Als Gleichungen $7^{\text {ten }}$ Grades für $\xi$ aufgefa $\beta$, haben wir hier die beiden Resolventen $7^{\text {ten }}$ Grades in einer ersten Gestalt vor uns.

Aus (8) kann man auch leicht Gleichungen für die einfachsten Modulformen der $\Gamma_{7}$ herleiten. Man berechne zunächst aus dem ersten und dritten Gliede der Proportion (8):

$$
\left(12 g_{2}\right)^{3}=\left(\frac{1 \pm i \sqrt{7}}{2}\right)^{7} \Delta \xi\left(\zeta^{2}+7 \xi+\frac{77 \pm 7 i \sqrt{7}}{8}\right)^{3} .
$$

Hieraus geht hervor, $d a \beta$ auch noch die Kubikwurzcln:

$$
f\left(\omega_{1}, \omega_{2}\right)=\sqrt[3]{\frac{1 \pm i \sqrt{7}}{2} \cdot \Delta \xi}
$$

eindeutige und zwar ganze Modulformen $(-4)^{\text {ter }}$ Dimension der $\Gamma_{7}$ sind. Führt man $f$ statt $\xi$ als Unbekannte in die vorletzte Gleichung ein, so ergibt sich nach Ausziehen der Kubikwurzel als neue Gestalt unserer Resolventen yten $^{t}$ Grades:

$$
f^{7}+7 \frac{1 \pm i \sqrt{7}}{2} \Delta f^{4}-7 \frac{5 \mp i \sqrt{7}}{2} \Delta^{2} f-12 g_{2} \Delta^{2}=0 .
$$

\section{$\$$ \%. Die beiden Resolventen elften Grades.}

Für die Gewinnung der Resolvente fünften Grades genügte die Angabe, daß die fünfblättrige Riemannsche Fläche über der $J$-Ebene bei

1) Man beachte, daß die zur zweiten Resolvente $7^{\text {ten }}$ Grades führende Riemannsche Fläche über der $J$-Ebene, die zur ersten Fläche symmetrisch ist, Verzweigungspunkte der gleichen Blätterzahl bei $J=0,1$ und $\infty$ hat. Die durchgeführte Überlegung muBte also zugleich zur zweiten Resolvente hinführen. 
$J=0$ einen dreiblättrigen Verzweigungspunkt, bei $J=1$ zwei zweiblättrige und bei $J=\infty$ einen fünfblättrigen Verzweigungspunkt hatte, sowie daß weitere Verzweigungspunkte nicht auftraten. Welche Blätter bei $J=0$ und $J=1$ miteinander zusammenhängen, brauchte indessen nicht näher festgestellt zu werden. Es gibt demnach nur eine einzige fünfblättrige Fläche, die die fragliche Verzweigung besitzt. Entsprechend gibt es im Falle $n=7$ nur zwei (einander symmetrische) Flächen, die die für diesen Fall oben näher bezeichnete Verzweigung besitzen. Demgegenüber fand Klein bei der Ausdehnung seiner Untersuchungen auf den Fall $n=11^{1}$ ), $\mathrm{daB}$ es nicht nur zwei, sondern im ganzen zchn elfblättrige Riemannsche Flächen über der $J$-Ebene gibt, welche die durch den Bereich der Fig. 32, S. 480, gegebenen Anzahlen von Verzweigungspunkte bei $J=0,1$ und $\infty$ haben. Dierein algebraische Methode des vorigen Paragraphen läßt sich zwar, da wieder das Geschlecht 0 vorliegt, mit einer einwertigen Funktion $\xi$ geradeso wie oben ansetzen, müßte aber hier zu zehn verschiedenen Koeffizientensystemen hinführen, und es würde demnach erst noch näher zu untersuchen sein, welche unter den zehn Gleichungen die beiden gesuchten Resolventen sind. Es erscheint demnach hier zweckmäBiger, entsprechend der am Anfange des vorigen Paragraphen ausgeführten Überlegung an den Galoisschen Körper anzuknüpfen und von ihm aus eine geeignete Funktion für die einzelne Ikosaedergruppe $G_{60}$ in der $G_{660}$ herzustellen.

Zum Aufbau des Galoisschen Körpers benutzt Klein das zu $n=11$ gehörende System der fünf Modulformen $x_{\lambda}$, die durch (5) S. 315 gegeben sind. $\left.{ }^{2}\right)$ Bei Fortlassung des gemeinsamen Faktors $i$ und Benutzung der fünf quadratischen Reste von 11 als Indizes hat man für diese der Dimension (-8) angehörenden Größen die Darstellungen:

$$
\left\{\begin{array}{l}
x_{1}=\sqrt{\frac{2 \pi}{\omega_{2}}} \sqrt[8]{\Delta^{5}} q^{\frac{81}{44}}\left(1-q^{2}-q^{20}+\cdots\right), \\
x_{3}=\sqrt{\frac{2 \pi}{\omega_{2}}} \sqrt[8]{\Delta^{5}} q^{\frac{25}{44}}\left(1-q^{6}-q^{16}+\cdots\right) \\
x_{9}=\sqrt{\frac{2 \pi}{\omega_{2}}} \sqrt[8]{\Delta^{5}} q^{\frac{49}{44}}\left(1-q^{4}-q^{18}+\cdots\right) \\
x_{5}=\sqrt{\frac{2 \pi}{\omega_{2}}} \sqrt[8]{\Delta^{5}} q^{\frac{1}{44}}\left(1-q^{10}-q^{12}+\cdots\right) \\
x_{4}=-\sqrt{\frac{2 \pi}{\omega_{2}}} \sqrt[8]{\Delta^{5}} q^{\frac{9}{44}}\left(1-q^{8}-q^{14}+\cdots\right)
\end{array}\right.
$$

1) „Über die Transformation elfter Ordnung der elliptischen Funktionen", Math. Ann. Bd. 15, S. 533. S. auch "Modulfunktionen" Bd. 2, S. $401 \mathrm{ff}$.

2) Über die Beziehung dieses Systems zur Transformationsgleichung vgl. man $\$ 11$ und 12 der eben genannten Abhandlung von Klein. 
488 II, 5. Die Gruppen der speziellen Transformationsgleichungen usw.

Gegenüber den Substitutionen $S=\left(\begin{array}{l}1,1 \\ 0,1\end{array}\right)$ und $T=\left(\begin{array}{r}0,1 \\ -1,0\end{array}\right)$ erfahren die $x_{\lambda}$ nach S. 315 die linearen Transformationen:

$$
\left\{\begin{aligned}
(S) \quad x_{\lambda}^{\prime} & =\varepsilon^{-\frac{\lambda(11-\lambda)}{2}} x_{\lambda}, \\
(T) \quad i \sqrt{11} x_{\lambda}^{\prime} & =\sum_{\chi}\left(\varepsilon^{* \lambda}-\varepsilon^{-\chi \lambda}\right) x_{x},
\end{aligned}\right.
$$

wo sich die auf $x$ bezogene Summe hier und weiterhin auf die fünf quadratischen Reste von 11 bezieht und $\varepsilon=e^{\frac{2 i \pi}{11}}$ ist. Die $G_{660}$ der Transformationen des Galoisschen Körpers in sich stellt sich hier als eine Gruppe linearer $x_{2}$-Substitutionen dar, die aus den beiden Substitutionen (2) erzeugbar ist.

Wir ziehen nun die zum Diskontinuitätsbereiche der Fig. 32, S. 480 gehörende Ikosaedergruppe $G_{60}$ heran und haben zugehörige Funktionen der $x_{2}$ zu bilden. In der $G_{60}$ ist die mit $S^{\prime}$ zu bezeichnende Substitution der Periode fünf $S^{\prime} \equiv\left(\begin{array}{l}3,0 \\ 0,4\end{array}\right)(\bmod 11)$ enthalten, bei deren Ausübung sich die $x_{\lambda}$ nach (18) und (19) S. 313 permutieren:

$$
\text { (S') } \quad x_{\lambda}^{\prime}=x_{3 \lambda} \text {. }
$$

Die abgekürzt mit $\sigma_{\infty} \mathrm{zu}$ bezeichnende Summe der $x_{\lambda}$ :

$$
\sigma_{\infty}=x_{1}+x_{3}+x_{9}+x_{5}+x_{4}
$$

stellt demnach eine Modulform dar, die gegenüber $S^{\prime}$ invariant ist. Die Summe ist aber, wie man mit Hilfe der Relation:

$$
\varepsilon+\varepsilon^{3}+\varepsilon^{9}+\varepsilon^{5}+\varepsilon^{4}-\varepsilon^{10}-\varepsilon^{8}-\varepsilon^{2}-\varepsilon^{6}-\varepsilon^{7}=i \sqrt{11}
$$

zeigt, auch gegenüber $T$ invariant, gehört also zu der aus $S^{\prime}$ und $T$ zu erzeugenden metazyklischen Untergruppe $G_{10}$ der $G_{60^{\circ}}$. Durch die gesamten Substitutionen der $G_{60}$ wird demnach die Modulform (4) im ganzen in sechs gleichberechtigte Formen transformiert, die durch $\sigma_{\infty}, \sigma_{0}, \sigma_{1}, \ldots, \sigma_{4}$ bezeichnet werden mögen. Insbesondere gehe $\sigma_{0}$ aus $\sigma_{\infty}$ durch die der $G_{60}$ angehörenden Substitution der Periode zwei $S^{-1} \cdot T \cdot S=\left(\begin{array}{rr}1, & 2 \\ -1, & -1\end{array}\right)$ hervor. Die Wirkung dieser Substitution auf die $x_{\lambda}$ ist:

$$
i \sqrt{11} x_{\lambda}^{\prime}=\sum_{\nu}\left(\varepsilon^{x \lambda}-\varepsilon^{-x \lambda}\right) \varepsilon^{6\left(x^{2}-\lambda^{2}\right)} x_{x^{\circ}}
$$

Hieraus berechnet man für $\sigma_{0}$ die Gleichung:

$$
i \sqrt{11} \sigma_{0}=\sum_{x}\left(\varepsilon^{\varkappa^{2}}+2 \varepsilon^{2 \varkappa^{2}}-2 \varepsilon^{5 \varkappa^{2}}-\varepsilon^{6 \varkappa^{2}}\right) x_{\varkappa^{*}}
$$

Die übrigen vier Formen $\sigma_{1}, \sigma_{2}, \ldots$ folgen aus $\sigma_{0}$ durch wiederholte 
Ausübung der Substitution $S^{\prime}$; man findet:

$$
i \sqrt{11} \sigma_{v}=\sum_{x}\left(\varepsilon^{\varkappa^{2}}+2 \varepsilon^{2 \varkappa^{2}}-2 \varepsilon^{5 \varkappa^{2}}-\varepsilon^{6 \varkappa^{2}}\right) x_{x \cdot 3^{y}}, \quad v=0,1, \ldots, 4 .
$$

Als Funktionen der $G_{60}$ benutzen wir nun symmetrische Ausdrücke der sechs 6 . Da ihre Summe identisch verschwindet, so sind die beiden einfachsten Ausdrücke die zweite und die dritte Potenzsumme, für die man in den $x_{2}$ die Darstellungen findet:

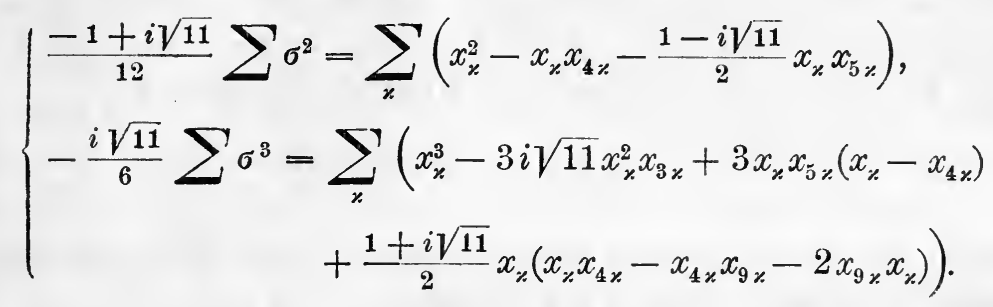

Da die $x_{\lambda}$ der Dimension (-8) angehören, so haben wir in den hier rechts stehenden Ausdrücken ganze Modulformen der Dimensionen -16 und -24 der zur $G_{60}$ gehörenden $\Gamma_{11}$. Zufolge (1) ist auch noch der Quotient der ersten dieser Formen und der Diskriminante $\Delta$ eine ganze Modulform der $\Gamma_{11}$, und zwar von der Dimension -4 . In dieser durch $f\left(\omega_{1}, \omega_{2}\right) \mathrm{zu}$ bezeichnenden Form:

$$
f\left(\omega_{1}, \omega_{2}\right)=\Delta^{-1} \cdot \sum_{*}\left(x_{\varkappa}^{2}-x_{*} x_{4 \%}-\frac{1-i \sqrt{11}}{2} x_{\varkappa} x_{5 \%}\right),
$$

deren Reihendarstellung sich aus (1) zu:

(8) $f\left(\omega_{1}, \omega_{2}\right)=\left(\frac{2 \pi}{\omega_{2}}\right)^{4} q^{\frac{6}{11}}\left(1+q^{\frac{2}{11}}+q^{\frac{4}{11}}-\frac{1-i \sqrt{11}}{2} q^{\frac{6}{11}}+\frac{1-i \sqrt{11}}{2} q^{\frac{8}{11}}+\cdots\right)$ berechnet, haben wir eine besonders einfache Modulform der $\Gamma_{11}$ gewonnen.

Die auf der rechten Seite der zweiten Gleichung (6) stehende Form ergibt, durch $\Lambda^{2}$ geteilt, eine Modulfunktion der $\Gamma_{11}$, die nur in der Spitze $\omega=i \infty$ des Diskontinuitätsbereiches unendlich wird. Da man aus (1) für diesen Quotienten als Anfangsglied der Potenzreihe $q^{-\frac{2}{11}}$ berechnet, so liegt in jener Spitze ein Pol erster Ordnung, so daß wir im Quotienten eine einwertige Funktion der $\Gamma_{11}$ gewonnen haben. Zur Vereinfachung der folgenden Formeln führen wir den um $3 i \sqrt{11}$ verminderten Quotienten als einwertige Funktion $\xi(\omega)$ der $\Gamma_{11}$ ein:

(9) $\xi(\omega)=-3 i \sqrt{11}+\Delta^{-2} \sum_{\varkappa}\left(x_{\varkappa}^{3}-3 i \sqrt{11} x_{\varkappa}^{2} x_{3 \varkappa}+3 x_{\varkappa} x_{5 \varkappa}\left(x_{\varkappa}-x_{4 \varkappa}\right)+\cdots\right)$.

Die Potenzreihe dieser Funktion ist dann:

$$
\text { (10) } \xi(\omega)=q^{-\frac{2}{11}}\left(1+*+\frac{1+i \sqrt{11}}{2} q^{\frac{4}{11}}+2 q^{\frac{6}{11}}+\frac{1+i \sqrt{11}}{2} q^{\frac{8}{11}}+\cdots\right) \text {, }
$$

wo durch den Stern hervorgehoben wird, daß das Glied mit $q^{\frac{2}{11}}$ ausfällt. 
Die Form $f$ als von der Dimension (-4) hat im Diskontinuitätsbereiche Nullpunkte der Gasamtordnung $\frac{11}{3}$. Da zufolge (8) in der Spitze $i \infty$ ein Nullpunkt der Ordnung 3 vorliegt, so restiert noch die Ordnung $\frac{2}{3}$. Es wird also entweder in den beiden Bereichecken bei $\omega=\frac{-7+i \sqrt{3}}{2}$ und $\frac{9+i \sqrt{3}}{2}$ je ein Nullpunkt der Ordnung $\frac{1}{3}$ liegen oder an einer dieser Stellen ein Nullpunkt der Ordnung $\frac{2}{3}$. In jedem Falle erkennt man im Quotienten von $f^{3}$ und $\Delta$ eine ganze Funktion zweiten Grades von $\xi$, für welche die Reihenentwicklungen ohne Mühe die Darstellung:

$$
\frac{f^{3}}{\Delta}=\xi^{2}+3 \xi+(5-i \sqrt{11})
$$

ergeben. Da hier rechts kein Quadrat steht, so liegen in den beiden genannten Ecken Nullpunkte je der Ordnung $\frac{1}{3}$ von $f$ vor.

Wir bilden ferner den Quotienten von $g_{2}$ und $f$, der gleichfalls eine Funktion der $\Gamma_{11}$ darstellt. Die beiden eben genannten Nullpunkte heben sich in diesem Quotienten fort. Es verbleiben, vom Zähler $g_{2}$ herrührend, drei Nullpunkte erster Ordnung in den drei Eckenzyklen des Bereiches, die sich aus den Ecken $\frac{ \pm 1+i \sqrt{3}}{2}, \frac{ \pm 3+i \sqrt{3}}{2}, \ldots$ zusammensetzen, und ein Pol dritter Ordnung in der Spitze $\omega=i \infty$, vom Nenner $f$ herrührend. Der Quotient ist also eine ganze Funktion dritten Grades von $\xi$, für die die Potenzreihen die Darstellung ergeben:

$$
\frac{g_{2}}{f}=\frac{1}{12}\left(\xi^{3}-\xi^{2}-3 \frac{1+i \sqrt{11}}{2} \xi-\frac{7-i \sqrt{11}}{2}\right) .
$$

Die Elimination von $\xi$ aus (11) und (12) führt zu dem Ergebnis: Die eine der beiden gesuchten Resolventen $11^{\text {ten }}$ Grades hat als Gleichung für die Modulform $f$ der $\Gamma_{11}$ die Gestalt:

$$
\begin{gathered}
f^{11}-22 \Delta f^{8}+11(9+2 i \sqrt{11}) \Delta^{2} f^{5}-132 g_{2} \Delta^{2} f^{4}+88 i \sqrt{11} \Delta^{3} f^{2} \\
+66(3-i \sqrt{11}) g_{2} \Delta^{3} f-144 g_{2}^{2} \Delta^{3}=0
\end{gathered}
$$

die zweite Resolvente ergibt sich hieraus durch Zeichenwechsel von $\sqrt{11}$.

Eine zweite Gestalt der Resolvente erhalten wir durch Multiplikation der Gleichung (11) mit der dritten Potenz der Gleichung (12):

$$
\text { (14) } 12^{3} J=\left(\xi^{2}+3 \xi+(5-i \sqrt{11})\right)\left(\xi^{3}-\xi^{2}-3 \frac{1+i \sqrt{11}}{2} \xi-\frac{7-i \sqrt{11}}{2}\right)^{3} \text {. }
$$

Dieses Ergebnis kann man einer Prüfung unterziehen. Bilden wir den Diskontinuitätsbereich der $\Gamma_{11}$ auf die $J$-Ebene ab, so entsteht eine Riemannsche Fläche, die neben anderen Verzweigungspunkten (bei $J=0$ 
und $J=\infty$ ) vier zweiblättrige Verzweigungspunkte bei $J=1$ hat. Demnach muB $(J-1)$ gleich dem Produkte einer ganzen Funktion dritten Grades und des Quadrats einer ganzen Funktion vierten Grades von $\xi$ sein, so dab eine in $\zeta$ identische Gleichung der folgenden Gestalt besteht:

$$
\begin{gathered}
\left(\xi^{2}+3 \xi+(5-i \sqrt{11})\right)\left(\xi^{3}-\xi^{2}-3 \frac{1+i \sqrt{11}}{2} \xi-\frac{7-i \sqrt{11}}{2}\right)^{3}-12^{3} \\
=\left(\xi^{3}+a \xi^{2}+b \xi+c\right)\left(\xi^{4}+d \xi^{3}+e \xi^{2}+f \xi+g\right)^{2} .
\end{gathered}
$$

Die Rechnung bestätigt dies in der Tat. Indem man die vorstehende Gleichung nach $\xi$ differenziert und wie im vorigen Paragraphen verfährt, findet man leicht die Koeffizienten $a, b, c, \ldots$ Als zweite Gestalt der beiden Resolventen elften Grades notieren wir die beiden folgenden Gleichungen elften Grades für $\xi$ :

$$
\text { (15) } \begin{aligned}
J:(J-1): 1=\left(\xi^{2}+3 \xi+(5 \mp i \sqrt{11})\right)\left(\xi^{3}-\xi^{2}-3 \frac{1 \pm i \sqrt{11}}{2} \xi-\frac{7 \mp i \sqrt{11}}{2}\right)^{3} \\
:\left(\xi^{3}-4 \xi^{2}+\frac{7 \mp 5 i \sqrt{11}}{2} \xi-(4 \mp 6 i \sqrt{11})\right)\left(\xi^{4}+2 \xi^{3}+3 \frac{1 \mp i \sqrt{11}}{2} \xi^{2}\right. \\
\left.-(5 \pm i \sqrt{11}) \xi-3 \frac{5 \pm i \sqrt{11}}{2}\right)^{2}: 12^{3} .
\end{aligned}
$$

Sechstes Kapitel.

\section{Die speziellen Transformationsgleichungen höherer Stufen.}

Eine allgemeine Theorie der Transformationsgleichungen für Modulfunktionen und Modulformen höherer Stufen ist unter Heranziehung weitergehender gruppentheoretischer Hilfsmittel in "Modulfunktionen“, Bd. 2, S. $83 \mathrm{ff}$. durchgeführt. Auch sind dort, S. $147 \mathrm{ff}$., ausführliche literarische Nachweise über ältere und neuere Arbeiten betreffs der Transformationsgleichungen für Funktionen höherer Stufen gegeben. Vor der Entwicklung der Weierstrabschen Theorie waren es die von Jacobi und im AnschluB an ihn von Sohnke aufgestellten Transformationsgleichungen für $\sqrt[4]{k}$, die achte Wurzel des Integralmoduls $k^{2}$, die das Hauptinteresse hatten. Neben diese Jacobi-Sohnkeschen „Modulargleichungen“ traten später die „Schlaeflischen Modulargleichungen“, d. i. die Transformationsgleichungen für die Modulfunktion $48^{\text {ster }}$ Stufe $\sqrt[12]{k_{k^{\prime}}}$. Der Transformationsgrad $n$ wird als teilerfremd gegen die Stufe der transformierten Funktion vorausgesetzt, um die allgemeine Theorie der fraglichen Gleichungen zu vereinfachen. Es kommen also insbesondere nur ungerade Transformationsgrade zur Behandlung. Die Theorie dieser Modulargleichungen soll im vorliegenden Kapitel entwickelt werden. 
Für die Transformationsgrade $n=2^{v}$ möge am Anfang dieses Kapitels eine auf die Formeln (2) S. 291 der Landenschen Transformation gegründete Entwicklung nachgetragen werden, welche die Wirkung wiederholter Landenscher Transformation auf die Integralmoduln, Thetanullwerte usw. betrifft.

Von den eben behandelten besonderen Resolventen $n^{\text {ten }}$ Grades, die bei $n=5,7$ und 11 eintreten, hat in der älteren Theorie insbesondere die Resolvente fünften Grades in ihren verschiedenen Gestalten wegen ihrer Beziehung zur allgemeinen Theorie der Gleichungen fünften Grades das Interesse für sich gehabt. Einige hierauf bezügliche Ausführungen sollen sich an die Modulargleichungen anschließen.

Endlich sind seit lange gewisse sehr einfache irrationale Gestalten einiger Modulargleichungen bekannt, die in der von Klein aufgestellten Theorie der Modularkorrespondenzen ibre Aufklärung gefunden haben. Im Zusammenhange mit diesen „irrationalen Modulargleichungen" stehen die schon gelegentlich (S. 296) erwähnten „Thetarelationen“. Über diese Gegenstände folgen am Schlusse des Kapitels einige Andeutungen.

\section{$\S 1$. Wiederholte Landensche Transformation.}

Die erste Haupttransformation zweiten Grades der doppeltperiodischen Funktionen mit den Argumenten $u, \omega_{1}, \omega_{2}$ besteht in dem Ersatze von $\omega_{1}$ durch $2 \omega_{1}$ bei unveränderten $u, \omega_{2}$. Die Ausübung dieser Operation auf die Funktionen zweiter Stufe führte uns nach S. 292 zu den Formeln der "Landenschen Transformation". Wir nennen die erste Haupttransformation zweiten Grades deshalb kurz "Landensche Transformation" und können die erste Haupttransformation des Grades $n=2^{v}$ durch $v$-malige Wiederholung der Landenschen Transformation erzielen.

Die vierten Wurzeln aus den Integralmoduln $k^{2}$ und $k^{2}$ sind durch die Gleichungen (1) S. 291 als eindeutige Modulfunktionen dargestellt. Durch $v$-malige Ausübung der Landenschen Transformation mögen diese beiden Funktionen $\sqrt{k}$ und $\sqrt{k_{k}^{\prime}}$ übergeben in:

$$
\sqrt{k_{v}}=\sqrt{k_{i}}\left(2^{v} \omega\right), \quad \sqrt{k_{v}^{\prime}}=\sqrt{k^{\prime}}\left(2^{v} \omega\right) \text {. }
$$

Zufolge (2) S. 291 gilt dann insbesondere für $\nu=1$ :

$$
k_{1}=\frac{1-k^{\prime}}{1+k^{\prime}}, \quad k_{1}^{\prime}=\frac{2 \sqrt{k^{\prime}}}{1+k^{\prime}}, \quad 1+k_{1}=\frac{2}{1+k^{\prime}} .
$$

Das Legendresche Integral erster Gattung $w$, das das erste Argument der Jacobischen Funktionen $s n, c n, d n$ ist, hängt mit dem Integrale $u$ durch die Gleichung (5) S. 292 zusammen. Bei $\nu$-maliger Ausübung der Landenschen Transformation gehe $w$ in $w_{v}$ über, wo wir dann insbesondere für $\nu=1$ zufolge (6) S. 292 die Darstellung haben: 


$$
w_{1}=\left(1+k^{\prime}\right) w, \quad w=\frac{1+k_{1}}{2} w_{1} \text {. }
$$

Die vollständigen Integrale $K$ und $K^{\prime}$ Legendres, die in der Jacobischen Theorie an die Stelle der $\omega_{1}, \omega_{2}$ treten, sind nach (5) in I, 388 auch durch:

(4) $2 K=\omega_{2} \sqrt{e_{2}-e_{1}}=\pi \vartheta_{3}(q)^{2}, \quad 2 i K^{\prime}=\omega_{1} \sqrt{e_{2}-e_{1}}=\omega \pi \vartheta_{3}(q)^{2}$

erklärbar. Bei $\boldsymbol{\nu}$-maliger Ausübung der Landenschen Transformation mögen sie in $K_{v}$ und $K_{v}^{\prime}$ übergehen. Aus der letzten Gleichung (9) S.289 und der zweiten Gleichung (1) S. 291 ergibt sich dann für $v=1$ :

(5) $K_{1}=K \frac{1+k^{\prime}}{2}, \quad K=\left(1+k_{1}\right) K_{1}, \quad K_{1}^{\prime}=\left(1+k^{\prime}\right) K^{\prime}, \quad K^{\prime}=\frac{1+k_{1}}{2} K_{1}^{\prime}$.

Führt man nach I, 388 im Sinne Jacobis $\varphi=$ am $(w, k)$ als „Amplitude von $w^{\text {" }}$ ein und schreibt $\varphi_{v}=$ am $\left(w_{v}, k_{v}\right)$ als Ergebnis $\nu$-maliger Ausübung der Landenschen Transformation, so folgt bei Division der beiden ersten Gleichungen (7) S. 292 durch einander wegen $\operatorname{sn} w=\sin \varphi$ und en $w=\cos \varphi$ speziell für $\nu=1$ die einfache Beziehung:

$$
\operatorname{tg} \varphi_{1}=\frac{\left(1+k^{\prime}\right) \operatorname{tg} \varphi}{1-k^{\prime} \operatorname{tg}^{2} \varphi}, \quad \operatorname{tg}\left(\varphi_{1}-\varphi\right)=k^{\prime} \operatorname{tg} \varphi .
$$

Bezieht man die Formeln der Landenschen Transformation auf die Quadratwurzeln $k, k^{\prime}$ aus den Integralmoduln, so ist zur Berechnung der transformierten Größen neben rationalen Rechnungen die Quadratwurzel $\sqrt{k_{i}^{\prime}}$ zu bestimmen. Der Wert dieser Wurzel ist eindeutig durch den Ausdruck von $\sqrt{k^{\prime}}$ als Quotient von $\vartheta_{0}(q)$ und $\vartheta_{3}(q)$ gegeben. In dem für spätere numerische Rechnungen wichtigen Falle, daB der Periodenquotient $\omega$ im Dreiecksnetze der Modulgruppe einem der nach dem Punkte $\omega=i \infty$ hinziehenden Dreiecke angehört, läßt sich aber auch direkt leicht angeben, wie $\sqrt{k^{\prime}}$ zu bestimmen ist. Man stelle die Werteverteilung von $\sqrt{k^{\prime}}$ in jenen Dreiecken fest, wozu in I, $440 \mathrm{ff}$. alle Mittel gegeben sind, und findet die Regel: Gehört $\omega$ einem der nach $i \infty$ hinziehenden Dreiecke der $\omega$-Halbebene an, so muß die in der zweiten Formel (2) stehende Quadratwurzel einen "positiven" reellen Bestandteil haben.

Bei wiederholter Ausübung der Landenschen Transformation erhält man den Formeln (2) ff. entsprechend die Rekursionsformeln:

$$
\begin{gathered}
k_{v}=\frac{1-k_{v-1}^{\prime}}{1+k_{v-1}^{\prime}}, \quad k_{v}^{\prime}=\frac{2 \sqrt{k_{v-1}^{\prime}}}{1+k_{v-1}^{\prime}}, \quad 1+k_{v}=\frac{2}{1+k_{v-1}^{\prime}}, \\
K_{v}=K_{v-1} \frac{1+k_{v-1}}{2}, \quad K_{v}^{\prime}=K_{v-1}^{\prime}\left(1+k_{v-1}^{\prime}\right), \\
w_{v}=\left(1+k_{v-1}^{\prime}\right) w_{v-1}, \quad \operatorname{tg}\left(\varphi_{v}-\varphi_{v-1}\right)=k_{v-1}^{\prime} \operatorname{tg} \varphi_{v-1} .
\end{gathered}
$$

Liegt, wie wir annehmen wollen, $\omega$ in einem der nach $i \infty$ ziehenden Dreiecke der $\omega$-Halbebene, so gilt dasselbe von allen Werten $2^{v} \omega$. Die Quadrat- 
wurzel $\sqrt{k_{v-1}^{\prime}}$ ist dann für jedes $\nu$ mit positivem reellen Bestandteile zu nehmen.

An die vorstehenden Rekursionsformeln knüpft $\mathrm{Jacobi}^{1}$ ) eine bemerkenswerte Darstellung von $K$ in Gestalt eines unendlichen Produktes. Durch Multiplikation der letzten Gleichung (7) und der ersten Gleichung: (8) folgt $K_{v-1}=\left(1+k_{v}\right) K_{v}$. Bildet man diese Gleichung für $\nu=1,2,3, \ldots, v$, so ergibt die Multiplikation aller $\nu$ Gleichungen nach Fortheben überflüssiger Faktoren:

$$
K=\left(1+k_{1}\right)\left(1+k_{2}\right)\left(1+k_{3}\right) \ldots\left(1+k_{v}\right) K_{v} .
$$

Nun gilt für $\lim v=\infty$ bekanntlich $\lim q^{2^{\nu}}=0$, und also folgt aus (10) in I, 464:

$$
\lim k_{v}=0, \quad \lim k_{v}^{\prime}=1, \quad \lim K_{v}=\frac{\pi}{2} .
$$

Die Gleichung (10) führt demnach beim Grenzübergange für $\nu=\infty \mathrm{zu}$ folgender Darstellung von $K$ durch ein unendliches Produkt:

$$
K=\frac{\pi}{2}\left(1+k_{1}\right)\left(1+k_{2}\right)\left(1+k_{3}\right) \cdots
$$

Da zufolge der Gleichungen (7):

$$
1+k_{v}=\frac{1}{\sqrt{k_{v-1}^{\prime}}} \cdot \frac{2 \sqrt{k_{v-1}^{\prime}}}{1+k_{v-1}^{\prime}}=\frac{k_{v}^{\prime}}{\sqrt{k_{v-1}^{\prime}}}
$$

gilt, so kann man der Gleichung (10) auch die Gestalt geben:

$$
K=\sqrt{k_{1}^{\prime}} \sqrt{k_{2}^{\prime}} \sqrt{\overline{k_{3}^{\prime}}} \ldots \sqrt{\overline{k_{v-1}^{\prime}}} \cdot \frac{k_{v}^{\prime}}{\sqrt{k^{\prime}}} \cdot K_{v}
$$

Beim Grenzübergange findet man an Stelle von (12) für $K$ das unendliche Produkt:

$$
K \cdot \sqrt{k^{\prime}}=\frac{\pi}{2} \sqrt{\overline{k_{1}^{\prime}}} \sqrt{k_{2}^{\prime}} \sqrt{k_{3}^{\prime}} \cdots
$$

Die Gleichungen der wiederholten Landenschen Transformation, auf die Quadrate der Thetanullwerte $\vartheta_{0}(q)$ und $\vartheta_{3}(q)$ angewandt, führen zum Algorithmus des GauBschen arithmetrisch-geometrischen Mittels. ${ }^{2}$ ) Aus. (9) S. 289 ergibt sich, wenn man $v=0$ einträgt:

$$
\left\{\begin{array}{c}
\vartheta_{3}\left(q^{2}\right)^{2}=\frac{1}{2}\left(\vartheta_{3}(q)^{2}+\vartheta_{0}(q)^{2}\right) \\
\vartheta_{0}\left(q^{2}\right)^{2}=\vartheta_{3}(q) \vartheta_{0}(q)
\end{array}\right.
$$

Schreibt man zur Abkürzung:

$$
\vartheta_{3}\left(q^{2 \eta}\right)^{2}=a_{v}, \quad \vartheta_{0}\left(q^{2 \eta}\right)^{2}=b_{v},
$$

1) In Art. 38 der „Fundamenta nova“, Jacobis Werke, Bd. 1, S. 149.

2) Vgl. GauB' Werke, Bd. 3, S. 361 ff. und die Ausführungen Jacobis in Art. 38 : der „Fundamenta nova". 
Wiederholte Landensche Transformation und arithm.-geom. Mittel

so ergibt sich aus (15) bei Wiederholung der Landenschen Transformation die Formelkette:

$$
\begin{cases}a_{1}=\frac{1}{2}\left(a_{0}+b_{0}\right), & b_{1}=\sqrt{a_{0} b_{0}}, \\ a_{2}=\frac{1}{2}\left(a_{1}+b_{1}\right), & b_{2}=\sqrt{a_{1} b_{1}}, \\ a_{3}=\frac{1}{2}\left(a_{2}+b_{2}\right), & b_{3}=\sqrt{a_{2} b_{2}}, \\ \cdot \cdot \cdot \cdot \cdot \cdot \cdot \cdot \cdot \cdot\end{cases}
$$

die den fraglichen Algorithmus darstellt.

GauB beweist unter der Annahme reeller positiver $a_{0}, b_{0}$ und positiv genommener Wurzeln, daß die beiden Zahlenreihen $a_{1}, a_{2}, a_{3}, \ldots$ und $b_{1}, b_{2}, b_{3}, \ldots$ je einer bestimmten Grenze zustreben, sowie da $B$ diese beiden Grenzen einander gleich sind. Den gemeinsamen Wert der Grenzen nennt Gauß das „arithmetisch-geometrische Mittel“ von $a_{0}$ und $b_{0}$ und bezeichnet. dieses Mittel durch:

$$
M\left(a_{0}, b_{0}\right)=\lim a_{v}=\lim b_{v}
$$

Aus der vorliegenden Bedeutung (16) unserer GröBenreihen $a_{v}, b_{v}$ ist auch bei beliebigem $\omega$ die Konvergenz des Algorithmus (17) im GauBschen Sinne selbstverständlich, da:

$$
\lim a_{v}=\lim b_{v}=M\left(\vartheta_{3}(q)^{2}, \vartheta_{0}(q)^{2}\right)=1 \quad \text { gilt. }
$$

Ändert man $a_{0}$ und $b_{0}$ um einen gemeinsamen Faktor, so ändern sich alle $a_{v}$ und $b_{v}$ und also auch $M\left(a_{0}, b_{0}\right)$ um denselben Faktor. Nun ist aber nach der obigen Gleichung (4) sowie nach (1) S. 291:

$$
\frac{\pi}{2 K} \vartheta_{3}(q)^{2}=1, \quad \frac{\pi}{2 K} \vartheta_{0}(q)^{2}=k^{\prime}
$$

Es ergibt sich demnach: $\quad M\left(1, k^{\prime}\right)=\frac{\pi}{2 K}$,

so daß sich das vollständige Integral $K$ durch das arithmetisch-geometrische Mittel aus 1 und $k^{\prime}$ so darstellt:

$$
K=\frac{\pi}{2 M\left(1, k^{\prime}\right)}
$$

\section{§ 2. Die Jacobi-Sohnkeschen Modulargleichungen.}

Jacobi bezeichnet die achte Wurzel des Integralmoduls $\sqrt[4]{\bar{k}}$ abgekürzt durch $u$. Da eine Verwechselung mit dem weiterhin nicht mehr auftretenden Integral erster Gattung $u$ ausgeschlossen ist, so nehmen wir die Bezeichnung Jacobis auf und notieren aus (10) in I, 464 als Produktentwicklung von $u(\omega)=\sqrt[4]{k}(\omega)$ : 


$$
u(\omega)=\sqrt[4]{k}(\omega)=\sqrt{2} q^{\frac{1}{8}} \prod_{m=1}^{\infty}\left(\frac{1+q^{2 m}}{1+q^{2 \overline{m-1}}}\right),
$$

woraus zugleich die Reihenentwicklung folgt:

$$
u(\omega)=\sqrt{2} q^{\frac{1}{8}}\left(1-q+2 q^{2}-3 q^{3}+4 q^{4}-6 q^{5}+9 q^{6}-\cdots\right) .
$$

Nach der Tabelle in I, 459 bleibt $\sqrt[4]{\bar{k}}(\omega)$ bei allen denjenigen Substitutionen der Hauptkongruenzgruppe zweiter Stufe unverändert, für die:

$$
e^{\frac{\pi i \alpha \beta}{8}}=\left(\frac{2}{\alpha}\right)=(-1)^{\frac{\alpha^{2}-1}{8}}
$$

gilt. Für ungerade $\alpha$ der Gestalt $(8 h \pm 1) \operatorname{mu}$ also $\beta \equiv 0(\bmod 16)$ zutreffen, für $\alpha=8 h \pm 3$ aber $\beta \equiv 8(\bmod 16)$. Unter Zusammenfassung beider Fälle kann man sagen, $\sqrt[4]{k}$ gehöre zu derjenigen Kongruenzgruppe $16^{\text {ter }}$ Stufe, deren Substitutionen mit:

$$
\left(\begin{array}{cc}
\alpha, & 4\left(1-\left(\frac{2}{\alpha}\right)\right) \\
\gamma, & \alpha^{-1}
\end{array}\right) \quad(\bmod 16)
$$

kongruent sind, wo $\gamma$ gerade und $\alpha$ ungerade ist. Im ganzen hat man 32 mod 16 inkongruente Substitutionen dieser Art. Da die Hauptkongruenzgruppe $16^{\text {ter }}$ Stufe innerhalb der nicht-homogenen Modulgruppe den Index 1536 hat, so bilden die Substitutionen, die $\sqrt[4]{k}$ in sich transformieren, eine Kongruenzgruppe $16^{\text {ter }}$ Stufe $\Gamma_{48}$ des Index 48.

Innerhalb der Hauptkongruenzgruppe zweiter Stufe $\Gamma_{6}$, deren Diskontinuitätsbereich durch Fig. 81 in I, 440 angegeben ist, stellt die $\Gamma_{48}$ eine Untergruppe des Index 8 dar. Entsprechend läßt sich aus acht neben einander gereihten Bereichen der eben genannten Art ein Diskontinuitätsbereich der $\Gamma_{48}$ aufbauen. Dieser Bereich ist in Fig. 33 angedeutet; die

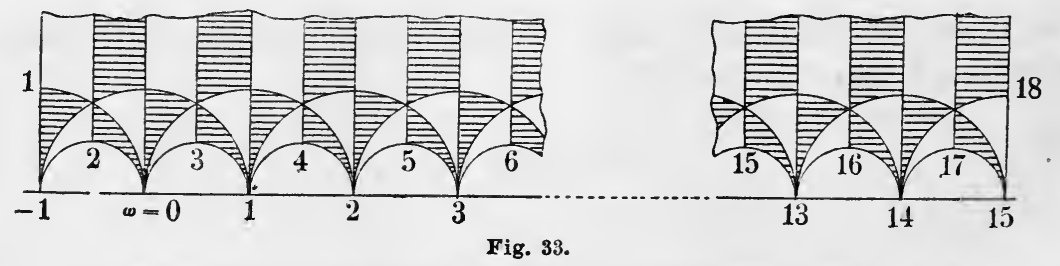

Seiten sind zu Paare nebeneinander liegend in folgender Art einander zugeordet:

$$
\begin{gathered}
1 \rightarrow 18,\left(\begin{array}{c}
1,16 \\
0,1
\end{array}\right) ; 2 \rightarrow 3,\left(\begin{array}{l}
1,0 \\
2,1
\end{array}\right) ; 4 \rightarrow 5,\left(\begin{array}{c}
5,-8 \\
2,-3
\end{array}\right) ; 6 \rightarrow 7,\left(\begin{array}{c}
9,-32 \\
2,-7
\end{array}\right) ; \\
8 \rightarrow 9,\left(\begin{array}{c}
13,-72 \\
2,-11
\end{array}\right) ; \quad \ldots ; 16 \rightarrow 17,\left(\begin{array}{c}
29,-392 \\
2,-27
\end{array}\right) .
\end{gathered}
$$


Der Diskontinuitätsbereich der $\Gamma_{48}$ ist vom Geschlechte 0 und hat $u(\omega)=\sqrt[V]{k}(\omega)$ $z u$ einer einwertigen Funktion. Die Spitze bei $\omega=i \infty$ des Bereiches trägt den Nullpunkt der Funktion, der Pol ist in dem aus den Spitzen bei $\omega=-1,1,3,5, \ldots, 15$ bestehenden Zyklus gelegen, während in den acht weiteren Spitzen bei $\omega=0,2,4, \ldots, 14$ die Funktionswerte vorliegen:

(4) $u(0)=1, u(2)=\frac{1+i}{\sqrt{2}}, u(4)=i, u(6)=\frac{-1+i}{\sqrt{2}}, \ldots, u(14)=\frac{1-i}{\sqrt{2}}$.

Entsprechend dem Brauche bei den älteren Untersuchungen sei der Transformationsgrad $n$ eine ungerade Primzahl. Die erste Haupttransformation $n^{\text {ten }}$ Grades ergibt als transformierte Funktion $u(n \omega)=\sqrt[4]{k}(n \omega)$, deren zugehörige Gruppe zunächst festgestellt werden soll. Damit aber $u(n \omega)$ gegenüber $\left(\begin{array}{l}\alpha, \beta \\ \gamma, \delta\end{array}\right)$ unverändert ist:

$$
u\left(n \frac{\alpha \omega+\beta}{\gamma \omega+\delta}\right)=u\left(\frac{\alpha \cdot n \omega+n \beta}{\gamma n^{-1} \cdot n \omega+\delta}\right)=u(n \omega),
$$

ist hinreichend und notwendig, daB $\left(\begin{array}{c}\alpha, n \beta \\ n^{-1} \gamma, \delta\end{array}\right)$ der $\Gamma_{48}$ angehört. Die Substitution $\left(\begin{array}{l}\alpha, \beta \\ \gamma, \delta\end{array}\right) \mathrm{muB}$ also selbst der $\Gamma_{48}$ angehören und außerdem der Kongruenz $\gamma \equiv 0(\bmod n)$ genügen. Da $n$ teilerfremd gegen 16 ist und also in der $\Gamma_{48}$ noch alle $\frac{1}{2} n\left(n^{2}-1\right) \bmod n$ inkongruenten Substitutionen vorkommen (S. $222 \mathrm{ff}$.), so wird durch die Forderung $\gamma \equiv 0(\bmod n)$ in $\operatorname{der} \Gamma_{48}$, ebenso wie in der Gesamtgruppe, eine Untergruppe des Index $(n+1)$ ausgesondert. Die Substitutionen, welche $u(\omega)$ und $u(n \omega)$ zugleich unverändert lassen, bilden hiernach in der Gesamtgruppe eine Kongruenzuntergruppe $\Gamma_{48(n+1)}$ des Index $48(n+1)$ und der Stufe $16 n$.

Im Diskontinuitätsbereiche der $\Gamma_{48(n+1)}$ ist $u(\omega)$ eine $(n+1)$-wertige Funktion. Als transformierte Funktion wollen wir übrigens nur für $n \equiv \pm 1$ (mod 8) unmittelbar $u(n \omega)$ gebrauchen, für $n \equiv \pm 3(\bmod 8)$ aber $-u(n \omega)$. Mit Jacobi bezeichnen wir, beide Fälle zusammenfassend, die transformierte Funktion durch:

$$
v(\omega)=\left(\frac{2}{n}\right) u(n \omega)=(-1)^{\frac{n^{2}-1}{8}} u(n \omega) .
$$

Dann sind nach den allgemeinen Sätzen über Modulfunktionen gleicher Gruppe $u$ und $v$ durch eine algebraische Relation:

$$
F(v, u)=0
$$

verbunden, die jedenfalls für $v$ auf den Grad $(n+1)$ ansteigt. Die so in Ansatz gebrachte Relation ist die Modulargleichung für den $n^{\text {ten }}$ Transformationsgrad. Es gilt nun, eine Reihe von Eigenschaften der Modulargleichung zu entwickeln, die uns in den Stand setzen sollen, für niedere Grade $n$ die Gleichungen wirklich anzugeben. 
Zunächst soll die Gestalt aller $(n+1)$ Lösungen $v$ der Gleichung (6) festgestellt werden. Wir schreiben ausführlich:

$$
F\left(\left(\frac{2}{n}\right) \sqrt[4]{k}(n \omega), \sqrt[4]{\bar{k}}(\omega)\right)=0
$$

und üben auf diese in $\omega$ identisch bestehende Gleichung eine Substitution aus, welche den Bedingungen:

$$
\alpha \equiv 1(\bmod 8), \quad \beta \equiv \gamma \equiv 0(\bmod 16), \quad \delta=n^{2}
$$

genügt. Die Funktion $u=\sqrt{k}$ bleibt unverändert, für $v$ liefert die Tabelle I, 459:

$$
v\left(\frac{\alpha \omega+\beta}{\gamma \omega+\delta}\right)=\left(\frac{2}{n}\right) \sqrt{k}\left(\frac{n \alpha \frac{\omega}{n}+\beta}{\gamma \frac{\omega}{n}+\frac{\delta}{n}}\right)=\sqrt[4]{k}\left(\frac{\omega}{n}\right),
$$

so daB die Gleichung (7) übergeht in:

$$
F\left(\sqrt[4]{k}\left(\frac{\omega}{n}\right), \sqrt[4]{\sqrt{k}}(\omega)\right)=0 .
$$

Übt man weiter die Substitution $\left(\begin{array}{c}1,16 \nu \\ 0,1\end{array}\right)$ aus, bei der $\sqrt[4]{k}$ wieder unverändert bleibt, so folgt aus (8):

$$
F\left(\sqrt[4]{k}\left(\frac{\omega+16 v}{n}\right), \sqrt[4]{k}(\omega)\right)=0, \quad v=0,1,2, \ldots, n-1 .
$$

Die $(n+1)$ Lösungen der Modulargleichung (6), die wir durch $v_{\infty}, v_{0}, v_{1}$, $v_{2}, \ldots, v_{n-1}$ bezeichnen, sind demnach:

$$
v_{\infty}=\left(\frac{2}{n}\right) \sqrt[4]{\sqrt{k}}(n \omega), \quad v_{v}=\sqrt[4]{k}\left(\frac{\omega+16 v}{n}\right), \quad v=0,1,2, \ldots, n-1 .
$$

Im Anschluß hieran nennt man die Zusammenstellung der $(n+1)$ Transformationen:

$$
\left(\begin{array}{l}
n, 0 \\
0,1
\end{array}\right),\left(\begin{array}{l}
1,0 \\
0, n
\end{array}\right),\left(\begin{array}{c}
1,16 \\
0, n
\end{array}\right),\left(\begin{array}{c}
1,32 \\
0, n
\end{array}\right), \ldots,\left(\begin{array}{c}
1,16(n-1) \\
0,
\end{array}\right)
$$

ein "Repräsentantensystem $16^{\text {ter }}$ Stufe für die Primzahltransformation $n^{\text {". }}$ Setzt man in (8) für $\omega$ das Produkt $n \omega$ ein, so folgt:

$$
F\left(u,\left(\frac{2}{n}\right) v_{\infty}\right)=0 \text {. }
$$

Da die Gleichung (6) irreduzibel ist, so müssen wir sie in (11) bis auf einen konstanten Faktor $c$ wieder gewonnen haben. Für die Funktion $F(v, u)$ der als unabhängig gedachten $u, v$ gilt also:

$$
F\left(u,\left(\frac{2}{n}\right) v\right)=c F(v, u),
$$

wo die Bestimmung der Konstanten $c$ vorbehalten bleibt. Auch in $u$ erreicht $F(v, u)$ den $\operatorname{Grad}(n+1)$. 
Nähert sich $\omega$ einem rationalen Punkte $\frac{p}{q}$, so sind drei Fälle zu unterscheiden, je nachdem $p$ gerade oder $q$ gerade oder $p$ und $q$ ungerade sind. Im ersten Falle wird $|u|=1$, im zweiten $u=0$, im dritten $u=\infty$. Mit $\omega$ nähern sich auch die $(n+1)$ transformierten Argumente $\frac{n p}{q}$, $\frac{p+16 v q}{n q}$ rationalen Punkten, und zwar liegt bei allen diesen Argumenten immer auch derselbe der drei eben unterschiedenen Fälle vor wie bei $\omega$. Aus dem zweiten und dritten Falle folgern wir, daß eine Lösung $v$ der Modulargleichung stets und nur dann verschwindet oder unendlich wird, wenn auch $u=0$ bzw. $=\infty$ ist. Die Modulargleichung hat also die Gestalt:

$$
v^{n+1}+g_{1}(u) v^{n}+g_{2}(u) v^{n-1}+\cdots+g_{n+1}(u)=0,
$$

wo die Koeffizienten ganze Funktionen von $u$ sind, die mit $u$ verschwinden. Insbesondere $\mathrm{muB} g_{n+1}(u)$ abgesehen von einem konstanten Faktor mit $u^{n+1}$ identisch sein. Indem man aber:

$$
g_{n+1}(u)=v_{\infty} v_{0} v_{1} \ldots v_{n-1}=\left(\frac{2}{n}\right) \sqrt[4]{k}(n \omega) \prod_{\nu=0}^{n-1} \sqrt[4]{k}\left(\frac{\omega+16 \nu}{n}\right)
$$

setzt und die Reihenentwicklung (2) heranzieht, ergibt sich jener Faktor zu $\left(\frac{2}{n}\right)$. Wir verbinden mit diesen Ergebnissen noch den vorläufigen Satz (12) und finden, daB die $n$ Funktionen $g_{1}(u), g_{2}(u), \ldots, g_{n}(u)$ den Grad $(n+1)$ nicht erreichen. Die Modulargleichung läßt sich demnach in die Gestalt kleiden:

$$
v^{n+1}+\left(\frac{2}{n}\right) u^{n+1}+u v\left(a v^{n-1} u^{n-1}+\cdots\right)=0,
$$

und an Stelle von (12) haben wir genauer:

$$
F\left(u,\left(\frac{2}{n}\right) v\right)=\left(\frac{2}{n}\right) F(v, u)
$$

Speziell für $\omega=0$ hat man zufolge (4) und (9):

$$
u=1, \quad v_{\infty}=\left(\frac{2}{n}\right), \quad v_{0}=1, \quad v_{v}=\sqrt[4]{k}\left(\frac{16 v}{n}\right), \quad v=1,2, \ldots, n-1
$$

Da für $\nu=1,2, \ldots, n-1$ die beiden Zahlen $16 \nu$ und $n$ teilerfremd sind, so ist eine Substitution $\left(\begin{array}{l}\alpha, \beta \\ \gamma, \delta\end{array}\right)$ angebbar, die die Bedingungen befriedigt:

$$
\beta=16 \nu, \delta=n, \quad \gamma \equiv 0(\bmod 2), \quad \alpha \equiv n(\bmod 8) .
$$

Die Tabelle in I, 459 ergibt:

$$
\sqrt[4]{k}\left(\frac{\alpha \omega+\beta}{\gamma \omega+\delta}\right)=\left(\frac{2}{n}\right)^{4} \sqrt{k}(\omega),
$$

woraus wir für $\omega=0$ als gemeinsamen Wert der letzten $(n-1)$ Lösungen 
(15) sofort $\left(\frac{2}{n}\right)$ ablesen. Wir haben damit den Satz gewonnen: Für $u=1$ reduziert sich die Modulargleichung auf:

$$
(v-1)^{n+1}=0 \text { oder }(v-1)(v+1)^{n}=0,
$$

je nachdem $n \equiv \pm 1$ oder $\equiv \pm 3(\bmod 8)$ gilt.

Übt man auf $\omega$ in der Gleichung (7) die Substitution $\left(\begin{array}{l}1,2 \\ 0,1\end{array}\right)$ aus, so nehmen nach der Tabelle in I, 459 die beiden Argumente der linken Seite jener Gleichung die Faktoren $e^{\frac{n \pi i}{4}}$ und $e^{\frac{\pi i}{4}}$ an. Mit Rücksicht auf (13) folgt: Die Funktion $F(v, u)$ der beiden unabhängig gedachten $v, u$ befriedigt die Bedingung:

$$
F\left(e^{\frac{n \pi i}{4}} v, e^{\frac{\pi i}{4}} u\right)=i^{\frac{n+1}{2}} F(v, u) .
$$

Übt man zweitens in (7) auf $\omega$ die Substitution $\left(\begin{array}{l}1,0 \\ n, 1\end{array}\right)$ aus, so gehen nach der wiederholt genannten Tabelle beide Argumente in ihre reziproken Werte über. Hieraus folgt mit Rücksicht auf (13): Die Funktion $F(u, v)$ der unabhängigen $u, v$ befriedigt die Bedingung:

$$
F\left(\frac{1}{v}, \frac{1}{u}\right)=\left(\frac{2}{n}\right) v^{-n-1} \cdot u^{-n-1} \cdot F(v, u) .
$$

Bei der in (17) auf $v$ und $u$ ausgeübten Transformation nehmen $v^{n+1}$, $u u^{n+1}$ und $u v$ übereinstimmend den Faktor $i^{\frac{n+1}{2}}$ an, so daß bei dieser Transformation der in (13) auftretende Klammerausdruck invariant ist. Man kann somit statt (13) genauer schreiben:

$$
v^{n+1}+\left(\frac{2}{n}\right) u^{n+1}+v u \sum_{\mu, v} a_{\mu v} v^{u} u^{v}=0
$$

wo sich die Summe auf alle nicht negativen ganzzahligen Exponentenpaare $\mu, \nu$ bezieht, die den Bedingungen:

$$
\mu \leqq n-1, \quad \nu \leqq n-1, \quad \mu n+\nu \equiv 0(\bmod 8)
$$

genügen. Zufolge der Regel (18) bestehen zwischen den numerischen Koeffizienten $a_{\mu, v}$ die Beziehungen:

$$
a_{n-1-\mu, n-1-v}=\left(\frac{2}{n}\right) a_{\mu, v} .
$$

Übrigens ist allgemein der Koeffizient $a_{00}$ leicht angebbar. Setzen wir nämlich $v=v_{\infty}$ und entwickeln $F\left(v_{\infty}, u\right)$ nach Potenzen von $q$, so ergeben die beiden Glieder $\left(\frac{2}{n}\right) u^{n+1}$ und $a_{00} v u$ die niedersten Potenzen der Reihe:

$$
F\left(v_{\infty}, u\right)=\left(\frac{2}{n}\right)\left(2^{\frac{n+1}{2}}+2 a_{00}\right) q^{\frac{n+1}{8}}+\cdots
$$


Die Modulargleichung für Primzahlgrad $n$. Beispiele $n=3,5,7,11$

Da $F\left(v_{\infty}, u\right)$ als Funktion von $\omega$ identisch verschwindet, so findet man unter Heranziehung der Relation (21):

$$
a_{00}=-2^{\frac{n-1}{2}}, \quad a_{n-1, n-1}=-\left(\frac{2}{n}\right) 2^{\frac{n-1}{2}} .
$$

Auf Grund der entwickelten Regeln sollen nun für die vier niedersten Grade $n=3,5,7$ und 11 die Modulargleichungen wirklich hergestellt werden. Die Gleichung (19) liefert mit Benutzung von (21) und (22) für $n=3,5$ und 7 die Ansätze

$$
v^{4}-u^{4}+v u\left(2 v^{2} u^{2}-2\right)=0
$$

$$
\left\{\begin{array}{l}
v^{6}-u^{6}+v u\left(4 v^{4} u^{4}+a v^{3} u-a v u^{3}-4\right)=0 \\
v^{8}+u^{8}-v u\left(8 v^{6} u^{6}+a v^{5} u^{5}+b v^{4} u^{4}+c v^{3} u^{3}+b v^{2} u^{2}+a v u+8\right)=0 .
\end{array}\right.
$$

Die Gleichung (23) für $n=3$ ist bereits fertig. In die beiden für $n=5$ und 7 angesetzten Gleichungen (24) tragen wir $u=1$ ein, worauf die erste Gleichung die Gestalt $(v-1)(v+1)^{5}=0$ annehmen muß, die zweite aber die Gestalt $(v-1)^{8}=0$. Die noch unbekannten Koeffizienten bestimmen sich hierdurch, und man findet für $n=5$ und 7 die Modulargleichungen:

$$
\left\{\begin{array}{r}
v^{6}-u^{6}+v u\left(4 v^{4} u^{4}+5 v^{3} u-5 v u^{3}-4\right)=0 \\
v^{8}+u^{8}-v u\left(8 v^{6} u^{6}-28 v^{5} u^{5}+56 v^{4} u^{4}-70 v^{3} u^{3}+56 v^{2} u^{2}\right. \\
-28 v u+8)=0
\end{array}\right.
$$

Im Falle $n=11$ verwerten wir zur Ausgestaltung des Ansatzes (19) nicht nur die Formeln (21) und (22), sondern ziehen auch die Regel (14) heran. Der Ansatz gewinnt dadurch die Gestalt:

$$
\begin{aligned}
& v^{12}-u^{12}+v u\left(32 v^{10} u^{10}+a v^{10} u^{2}+b v^{9} u^{5}+c v^{8} u^{8}-a v^{8}+d v^{7} u^{3}+e v^{6} u^{6}\right. \\
& \left.+b v^{5} u-b v^{5} u^{9}-e v^{4} u^{4}-d v^{3} u^{7}+a v^{2} u^{10}-c v^{2} u^{2}-b v u^{5}-a u^{8}-32\right)=0
\end{aligned}
$$

mit fünf noch unbekannten Koeffizienten. Für $u=1$ gewinnen wir:

$$
v^{12}+(32+a) v^{11}+b v^{10}+(c-a) v^{9}+d v^{8}+e v^{7}-e v^{5}-\cdots=0 .
$$

Hier müssen wir aber links die Entwicklung von $(v-1)(v+1)^{11}$ haben, woraus alle fünf unbekannten Koefizienten $a, b, \ldots$ hervorgehen. Die fertige Modulargleichung für den elften Grad ist hiernach:

$$
\begin{gathered}
v^{12}-u^{12}+v u\left(32 v^{10} u^{10}-22 v^{10} u^{2}+44 v^{9} u^{5}+88 v^{8} u^{8}+22 v^{8}\right. \\
+165 v^{7} u^{3}+132 v^{6} u^{6}-44 v^{5} u^{9}+44 v^{5} u-132 v^{4} u^{4}-165 v^{3} u^{7} \\
\left.-22 v^{2} u^{10}-88 v^{2} u^{2}-44 v u^{5}+22 u^{8}-32\right)=0
\end{gathered}
$$

Die Modulargleichungen der Grade 3 und 5 sind von Jacobi in Art. 13 ff. der „Fundamenta nova“ aufgestellt und zwar durch Elimina- 
tionen bei Gelegenheit der algebraischen Durchführung der Transformationen dieser beiden Grade. Unter Zuhilfenahme der transzendenten Theorie hat alsdann Sohnke ${ }^{1}$ im Anschluß an Jacobi die Modulargleichungen behandelt und in den Fällen $n=7,11,13,17$ und 19 fertig berechnet. In dem nächsten hier nicht abschließend behandelten Falle $n=13$ bleibt bei Anwendung der eben benutzten Hilfsmittel noch ein Koeffizient unbestimmt. Hier und in den weiteren Fällen sind dann auch noch die Potenzreihen nach $q$ heranzuziehen.

\section{§ 3. Die Schlaeflischen Modulargleichungen.}

Während die Transformationsgleichungen für $J(\omega)$, abgesehen vom niedersten Falle $n=2$, oben nicht endgültig aufgestellt werden konnten, gelang es soeben, diejenigen für $\sqrt[4]{k}(\omega)$ ziemlich leicht in den Fällen $n=3$, 5, 7 und 11 anzugeben, da sich diese Gleichungen sowohl durch geringe Anzahl der Glieder wie auch durch niedrige Zahlenkoeffizienten vor denen von $J(\omega)$ auszeichnen. Der gleiche Vorteil tritt noch in erhöhtem MaBe bei den zuerst von Schlaefli ${ }^{2}$ ) betrachteten Transformationsgleichungen für die Modulfunktion $48^{\text {ster }}$ Stufe $\sqrt[12]{k k^{\prime}}$ ein.

Mit Schlaefli bedienen wir uns der abkürzenden Bezeichnung:

$$
\sqrt[3]{2} \sqrt[12]{k k^{\prime}}(\omega)=s(\omega)
$$

und leiten aus den Produktdarstellungen in I, 460 für $s(\omega)$ die Potenzreihe ab:

(2) $s(\omega)=\sqrt{2} q^{\frac{1}{24}}\left(1-q+q^{2}-2 q^{3}+2 q^{4}-3 q^{5}+4 q^{6}-5 q^{7}+6 q^{8}-\cdots\right)$. Aus der Gleichung (23) in I, 460 zieht man die Folgerung, daß $s(\omega)$ gegenüber einer beliebigen Substitution der Hauptkongruenzgruppe zweiter Stufe den Faktor:

$$
\left(\frac{2}{\alpha \delta}\right) e^{\frac{3 \pi i}{8}(\alpha \beta-\gamma \delta)+\frac{2 \pi i}{3}\left(\alpha^{2}+\gamma^{2}\right)(\alpha \beta+\gamma \delta)}
$$

annimmt. Außerdem aber bleibt $s$, wie man aus (17) in I, 456 leicht folgert, gegenüber der Substitution $\left(\begin{array}{rr}0,-1 \\ 1, & 0\end{array}\right)$ unverändert:

$$
s\left(\frac{-1}{\omega}\right)=s(\omega) \text {. }
$$

Auf Grund dieser Angaben stellt man fest, $d a \beta s(\omega)$ zu einer Kongruenzgruppe $48^{\text {ster }}$ Stufe $\Gamma_{72}$ vom Index 72 gehört, deren Diskontinuitätsbereich

1) „Aequationes modulares pro transformatione functionum ellipticarum", Journ. f. Math., Bd. 36, S. $97 \mathrm{ff}$.

2) „Beweis der Hermiteschen Verwandlungstafeln für die elliptischen Modularfunktionen", Journ. f. Math., Bd. 72. 
in Fig. 34 dargestellt ist. Es sind hier die Seiten 1 und 50, sowie außerdem immer die beiden Quadranten des einzelnen unteren Halbkreises aufeinander bezogen:

$$
1 \rightarrow 50,\left(\begin{array}{c}
1,48 \\
0,1
\end{array}\right) ; \quad(2 v+2) \rightarrow(2 v+3),\left(\begin{array}{c}
2 v,-4 v^{2}-1 \\
1,-2 v
\end{array}\right), \quad v=0,1,2, \ldots, 23 .
$$

Der Diskontinuitätsbereich hat das Geschlecht 0 , und $s(\omega)$ ist eine zugehörige einwertige Funktion. Der Nullpunkt von $s(\omega)$ liegt in der Polygonspitze

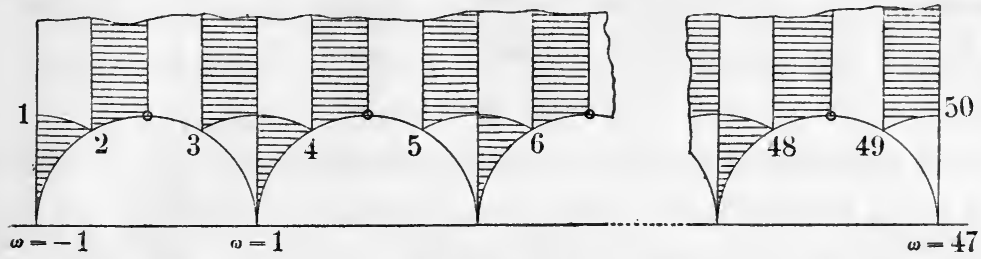

Fig. 34.

$i \infty$, der Pol in dem von den unteren Spitzen des Bereiches gelieferten Zyklus. Im Punkte $\omega=i$ liegt der Wert $s=\sqrt[4]{\overline{2}}$ vor.

Der Transformationsgrad $n$ sei eine Primzahl und teilerfremd gegen 6. Wie bei den Jacobischen Modulargleichungen zeigt man dann, daB die transformierte Funktion $s(n \omega)=t(\omega)$ bei denjenigen in der $\Gamma_{72}$ enthaltenen Substitution unverändert bleibt, die die Kongruenz $\gamma \equiv 0(\bmod n)$ erfüllen, und daß die Gesamtheit dieser Substitutionen eine Kongruenzgruppe $\Gamma_{72(n+1)}$ der Stufe $48 n$ bildet, die in der $\Gamma_{72}$ den Index $(n+1)$ und in der Gesamtgruppe den Index $72(n+1)$ hat. Die entsprechend zwischen $s$ und $t$ bestehende irreduzible algebraische Relation:

$$
F(t, s)=0,
$$

in der $t$ auf den Grad $(n+1)$ ansteigt, ist? die zu untersuchende Schlaeflische Modulargleichung für den $n^{\text {ten }}$ Transformationsgrad.

Um die Gestalt der $(n+1)$ Lösungen der Gleichung (5) zu gewinnen, schreiben wir die in $\omega$ identisch bestehende Gleichung an:

$$
F(s(n \omega), s(\omega))=0 \text {. }
$$

Übt man die Substitution $\left(\begin{array}{rr}0, & -1 \\ 1, & 0\end{array}\right)$ und darauf $\left(\begin{array}{cc}1, & 48 v \\ 0, & 1\end{array}\right)$ aus, so folgt mit Benutzung von (4):

$$
F\left(s\left(\frac{\omega}{n}\right), s(\omega)\right)=0, \quad F\left(s\left(\frac{\omega+48 \nu}{n}\right), s(\omega)\right)=0 .
$$

Die $(n+1)$ Lösungen der Gleichung $(6)$, die wir durch $t_{\infty}, t_{0}, t_{1}, t_{2}, \ldots, t_{n-1}$ bezeichnen, sind hiernach:

(8) $t_{\infty}=s(n \omega), \quad t_{0}=s\left(\frac{\omega}{n}\right), \quad t_{1}=s\left(\frac{\omega+48}{n}\right), \ldots, t_{n-1}=s\left(\frac{\omega+48(n-1)}{n}\right)$.

Im AnschluB daran nennt man die Zusammenstellung der $(n+1)$ Trans- 
formationen:

$$
\left(\begin{array}{l}
n, 0 \\
0,1
\end{array}\right),\left(\begin{array}{l}
1,0 \\
0, n
\end{array}\right),\left(\begin{array}{cc}
1,48 \\
0, & n
\end{array}\right),\left(\begin{array}{c}
1,96 \\
0, n
\end{array}\right), \ldots,\left(\begin{array}{c}
1,48(n-1) \\
0,1
\end{array}\right)
$$

ein "Repräsentantensystem $48^{\text {ster }}$ Stufe" für die Primzahltransformation $n^{\text {ten }}$ Grades.

Ersetzt man in der ersten Gleichung (7) das Argument $\omega$ durch $n \omega$, so ergibt sich $F(s, t)=0$. Wegen der Irreduzibilität der Gleichung folgt hieraus genau so wie S. 347 bei den Transformationsgleichungen für $J(\omega)$, $d a \beta F(s, t)$ eine symmetrische Funktion von $s$ und $t$ sein muß:

$$
F(s, t)=F(t, s) \text {. }
$$

Die rationalen Punkte $\omega$ zerfallen bezüglich der $\Gamma_{72}$ in nur zwei Klassen (vgl. Fig. 34); die eine Klasse enthält die Punkte $\frac{p}{q}$ mit ungeraden $p$ und $q$, die andere Klasse die übrigen rationalen Punkte und die Stelle $\omega=i \infty$. In den Punkten der ersten Klasse wird $s=\infty$, in denen der zweiten aber $=0$. Nähert sich $\omega$ einem Punkte der einzelnen Klasse, so nähern sich zufolge (8) die sämtlichen transformierten Argumente Punkten der gleichen Klasse. Irgendeine Lösung $t$ der Gleichung (5) wird stets und nur dann gleich 0 oder $\infty$, wenn auch $s$ gleich 0 oder $\infty$ ist. Die Gleichung (5) hat demnach die Gestalt:

$$
t^{n+1}+g_{1}(s) t^{n}+g_{2}(s) t^{n-1}+\cdots+g_{n+1}(s)=0,
$$

wo die $g$ ganze Funktionen von $s$ sind, die wegen (10) höchstens auf den Grad $(n+1)$ ansteigen. Insbesondere enthält $g_{n+1}(s)$ nur das höchste Glied mit der Potenz $s^{n+1}$, so daß wieder wegen (10) die Funktion $g_{n+1}(s)$ einfach gleich $s^{n+1}$ ist. Zufolge der Symmetrie von $F(s, t)$ in $s$ und $t$ schließen wir demnach, wie bei den Jacobischen Modulargleichungen, auf die Gestalt:

$$
t^{n+1}+s^{n+1}+t s\left(a t^{n-1} s^{n-1}+\cdots\right)=0
$$

der Gleichung (5).

Gegenüber der Substitution $\left(\begin{array}{ll}1 & 2 \\ 0,1\end{array}\right)$ erfahren die Funktionen $s(\omega)$ und $t(\omega)=s(n \omega)$ gleichzeitig Abänderung um die Faktoren $e^{\frac{\pi i}{12}}$ und $e^{\frac{n \pi i}{12}}$. Da $n$ teilerfremd gegen 6 ist, so nehmen $t^{n+1}, s^{n+1}$ und $t s$ übereinstimmende Faktoren an, und also bleibt gegenüber $\left(\begin{array}{l}1,2 \\ 0,1\end{array}\right)$ der Ausdruck in der Klammer (11) unverändert. Schreibt man demnach die Gleichung (11) ausführlicher:

$$
t^{n+1}+s^{n+1}+t s \sum_{\mu, v} a_{\mu, v} t^{\mu} s^{v}=0,
$$

so unterliegen die Exponenten $\mu, \nu$ den Bedingungen:

$$
\mu \leqq n-1, \quad \nu \leqq n-1, \quad \mu n+\nu \equiv 0(\bmod 24) .
$$


Zur Modulfunktion zweiter Stufe $k^{2} k^{\prime 2}(\omega)$ gehört die Kongruenzgruppe $\Gamma_{3}$ zweiter Stufe des Index 3 , die durch $\alpha \equiv \delta, \beta \equiv \gamma(\bmod 2)$ erklärt ist. Diese $\Gamma_{3}$ wird durch die nicht in der Modulgruppe $\Gamma^{(\omega)}$ enthaltene Substitution $W=\left(\begin{array}{c}1,-1 \\ 1,1\end{array}\right)$ in sich transformiert. Es ist nämlich:

$$
W \cdot\left(\begin{array}{l}
\alpha, \beta \\
\gamma, \delta
\end{array}\right) \cdot W^{-1}=\left(\begin{array}{l}
\frac{1}{2}(\alpha-\beta-\gamma+\delta), \frac{1}{2}(\alpha+\beta-\gamma-\delta) \\
\frac{1}{2}(\alpha-\beta+\gamma-\delta), \frac{1}{2}(\alpha+\beta+\gamma+\delta)
\end{array}\right),
$$

und die rechts stehende Substitution gehört wieder der $\Gamma_{3}$ an, kann auch bei zweckmäBiger Auswahl von $\left(\begin{array}{l}\alpha, \beta \\ \gamma, \delta\end{array}\right)$ mit jeder vorgeschriebenen Substitution der $\Gamma_{3}$ gleich gemacht werden. Mit $k^{2} k^{2}(\omega)$ ist somit auch $k^{2} k^{\prime 2}(W(\omega))$ eine einwertige Funktion der $\Gamma_{3}$ und als solche eine lineare Funktion von $k^{2} k^{\prime 2}(\omega)$. Da aber die Substitution $W$ den Nullpunkt und den Pol von $k^{2} k^{\prime 2}$ austauscht und der Wert dieser Funktion im Fixpunkte$\omega=i$ von $W$ gleich $\frac{1}{4}$ ist, so gilt:

$$
k^{2} k^{\prime 2}\left(\frac{\omega-1}{\omega+1}\right)=\frac{1}{16 k^{2} k^{\prime 2}(\omega)} .
$$

Kehren wir durch Ausziehen der $24^{\text {ston }}$ Wurzel zur Funktion $s(\omega)$ zurück und beachten, $\mathrm{daB} s(i)=\sqrt[4]{\sqrt{2}}$ ist, so folgt weiter:

$$
s\left(\frac{\omega-1}{\omega+1}\right)=\frac{\sqrt{2}}{s(\omega)} .
$$

Um die Wirkung von $W$ auf $t_{\infty}(\omega)=s(n \omega)$ festzustellen, folgern wir aus (14):

$$
s\left(\frac{n \omega+n-2}{\omega+1}\right)=s\left(\frac{\omega-1}{\omega+1}+n-1\right)=e^{\frac{(n-1) \pi i}{24}} \frac{\sqrt{2}}{s(\omega)} .
$$

Ist $m$ diejenige unter den Zahlen 1, 5, 7, 11, ., 47, die der Kongruenz. $m n \equiv 1(\bmod 48)$ genügt, so folgt durch Ausübung der Substitution. $\left(\begin{array}{c}1, m-1 \\ 0,1\end{array}\right)$ auf $\omega$ aus der letzten Gleichung:

$$
s\left(\frac{n \omega+m n-2}{\omega+m}\right)=e^{\frac{(n-m) \pi i}{24}} \frac{\sqrt{2}}{s(\omega)} .
$$

Nun ist aber, wie man leicht feststellt, $n-m \equiv 0$ oder $\equiv 24(\bmod 48)$, je nachdem $n \equiv \pm 1$ oder $\equiv \pm 3(\bmod 8)$ gilt. Also folgt:

$$
s\left(\frac{n \omega+m n-2}{\omega+m}\right)=\left(\frac{2}{n}\right) \frac{\sqrt{2}}{s(\omega)} \text {. }
$$

Für $t_{\infty}(\omega)$ bilden wir nun den Ansatz:

$$
t_{\infty}\left(\frac{\omega-1}{\omega+1}\right)=s\left(\frac{n \omega-n}{\omega+1}\right)=s\left(\frac{n \frac{\omega+48 v}{n}-(1+48 v)}{\frac{\omega+48 v}{n}+\frac{1-48 v}{n}}\right),
$$


wo $\nu$ diejenige unter den Zahlen $1,2,3, \ldots, n-1$ sein soll, die der Kongruenz $1-48 v \equiv 0(\bmod n)$ genügt. Dann ist $\frac{1-48 v}{n}$ eine ganze Zahl, die offenbar mod 48 mit $m$ kongruent ist. Zufolge (15) ist also, da $s(\omega)$ gegenüber $\left(\begin{array}{ll}1, & 48 \\ 0, & 1\end{array}\right)$ invariant ist, die in (16) rechts stehende Funktion gleich:

$$
\left(\frac{2}{n}\right) \frac{\sqrt{2}}{s\left(\frac{\omega+48 v}{n}\right)} \text {. }
$$

Die Wirkung der Substitution $W$ auf $t_{\infty}$ ist demnach:

$$
t_{\infty}\left(\frac{\omega-1}{\omega+1}\right)=\left(\frac{2}{n}\right) \frac{\sqrt{2}}{t_{v}(\omega)} .
$$

Zufolge der Irreduzibilität der Schlaeflischen Modulargleichung haben wir hiernach folgenden Satz: Die Modulargleichung (5) wird durch folgende Substitution der Periode 2 in sich transformiert:

$$
s^{\prime}=\frac{\sqrt{2}}{s}, \quad t^{\prime}=\left(\frac{2}{n}\right) \frac{\sqrt{2}}{t} .
$$

Die zur Gleichung (5) gehörende $(n+1)$-blättrige Riemannsche Fläche über der $s$-Ebene kann an der dem Werte $\omega=i$ entsprechenden Stelle $s=\sqrt[4]{2}$ höchstens zweiblättrige Verzweigungspunkte haben. Da die Substitution $\left(\begin{array}{l}0,-1 \\ 1,\end{array}\right)$, die einem Umlaufe um die fragliche Stelle entspricht, die beiden Lösungen $t_{\infty}$ und $t_{0}$ permutiert, so hängen jedenfalls daselbst die diesen beiden Lösungen entsprechenden Blätter zusammen. Ist ferner $\nu$ ein Index aus der Reihe $1,2,3, \ldots, n-1$, so folgt wegen (4):

$t_{v}\left(\frac{-1}{\omega}\right)=s\left(\frac{48 v \omega-1}{n \omega}\right)=s\left(\frac{n \omega}{-48 v \omega+1}\right)=s\left(\frac{n \frac{\omega+48 \mu}{n}-48 \mu}{-48 v \frac{\omega+48 \mu}{n}+\frac{1+48^{2} \mu \nu}{n}}\right)$, wo $\mu$ der aus der Kongruenz:

$$
48^{2} \mu \nu+1 \equiv 0(\bmod n)
$$

eindeutig bestimmte Index sein soll. Wie aus (3) hervorgeht, haben wir dann in:

$$
\left(\begin{array}{c}
n,-48 \mu \\
-48 v,\left(1+48^{2} \mu \nu\right) n^{-1}
\end{array}\right)
$$

eine Substitution der $\Gamma_{72}$, und also folgt:

$$
t_{v}\left(\frac{-1}{\omega}\right)=t_{\mu}(\omega)
$$

Das zu $t_{v}$ gehörende Blatt der Riemannschen Fläche wird also an der Stelle $s=\sqrt[4]{2}$ dann und nur dann isoliert verlaufen, wenn $\mu=\nu$ wird und also $v$ eine Lösung der Kongruenz: 


$$
48^{2} \nu^{2}+1 \equiv 0(\bmod n)
$$

ist. Diese Kongruenz hat aber keine oder zwei inkongruente Lösungen, je nachdem $n \equiv-1$ oder $\equiv+1(\bmod 4)$ ist. Im ersten Falle hat man $\frac{1}{2}(n+1)$ zweiblättrige Verzweigungspunkte, im zweiten $\frac{1}{2}(n-1)$ und zwei isoliert verlaufende Blätter. Für die Gleichung (5) folgt: Setzt man in die Schlaeflische Modulargleichung $s=\sqrt[4]{2}$ ein, so gcht sie im Falle $n \equiv-1(\bmod 4)$ in das Quadrat einer ganzen Funktion des Grades $\frac{1}{2}(n+1)$ in $t$ über, für $n \equiv+1(\bmod 4)$ aber liefert ihre linke Seite fïr $s=\sqrt[4]{2}$ das Produkt einer Funktion zweiten Grades und des Quadrates einer ganzen Funktion des Grades $\frac{1}{2}(n-1)$.

Setzt man in (12) insbesondere $t=t_{\infty}=s(n \omega)$ ein, so muß diese Gleichung in $\omega$ identisch bestehen. Entwickelt man aber die linke Seite auf Grund von (2) nach Potenzen von $q$, so liefern die beiden Glieder $s^{n+1}$ und $a_{00} t s$ die niederste Potenz:

$$
\left(2^{\frac{n+1}{2}}+2 a_{00}\right) q^{\frac{n+1}{12}} \text {. }
$$

Da der Koeffizient verschwindet, so ergibt sich $a_{00}$ sowie nach dem auf Grund von (18) folgenden allgemeinen Gesetze:

$$
a_{n-1-\mu, n-1-v}=\left(\frac{2}{n}\right)^{\mu+1} 2^{\frac{\mu+v-n+1}{2}} a_{\mu, v}
$$

auch $a_{n-1, n-1}$ :

$$
a_{00}=-2^{\frac{n-1}{2}}, \quad a_{n-1, n-1}=-\left(\frac{2}{n}\right) .
$$

Im niedersten Falle $n=5$ erhält man auf Grund des Ansatzes (12) und den Regeln (22) als fertige Modulargleichung:

$$
t^{6}+s^{6}+t s\left(t^{4} s^{4}-4\right)=0 .
$$

Im nächsten Falle $n=7$ findet man den Ansatz:

$$
t^{8}+s^{8}-t s\left(t^{6} s^{6}+a t^{3} s^{3}+8\right)=0,
$$

sowie weiter für den Grad $n=11$ :

$$
t^{12}+s^{12}+t s\left(t^{10} s^{10}+a\left(t^{8} s^{8}-8 t^{2} s^{2}\right)+b\left(t^{6} s^{6}-2 t^{4} s^{4}\right)-32\right)=0 .
$$

Für $s=\sqrt[4]{2}$ müssen die linken Seiten dieser Gleichungen Quadrate von Ausdrücken vierten bzw. sechsten Grades werden. Daraus bestimmen sich die noch unbekannten Koeffizienten ohne Mühe; man findet für die Grade 7 und 11:

$$
\left\{\begin{array}{c}
t^{8}+s^{8}-s t\left(t^{6} s^{6}-7 t^{3} s^{3}+8\right)=0 \\
t^{12}+s^{12}+t s\left(t^{10} s^{10}-11 t^{8} s^{8}+44 t^{6} s^{6}-88 t^{4} s^{4}+88 t^{2} s^{2}-32\right)=0
\end{array}\right.
$$

Benutzt man weiterhin auch noch die Symmetrie in $s$ und $t$, so bleiben 
bei $n=13$ noch drei, bei $n=17$ und 19 aber noch fünf unbestimmte Koeffizienten im Ansatze. Zur Berechnung dieser Koeffizienten zieht man zweckmäBig die Reihenentwicklungen heran. Die Schlaeflischen Modulargleichungen der Grade 13, 17 und 19 sind:

$$
\left\{\begin{array}{c}
t^{14}+s^{14}+t s\left(t^{12} s^{12}-64\right)+13 t^{2} s^{2}\left(t^{10}+s^{10}\right)+52 t^{4} s^{4}\left(t^{6}+s^{6}\right) \\
+78 t^{6} s^{6}\left(t^{2}+s^{2}\right)=0 \\
t^{18}+s^{18}-t s\left(t^{16} s^{16}+256\right)+17 t^{2} s^{2}\left(t^{6}+s^{6}\right)\left(t^{8} s^{8}+16\right) \\
-34 t^{3} s^{3}\left(t^{12}+s^{12}\right)+34 t^{5} s^{5}\left(t^{8} s^{8}+16\right)+119 t^{6} s^{6}\left(t^{6}+s^{6}\right) \\
+340 t^{9} s^{9}=0 \\
t^{20}+s^{20}+t s\left(t^{18} s^{18}-512\right)+19 t^{2} s^{2}\left(t^{4}+s^{4}\right)\left(t^{12} s^{12}+64\right) \\
+95 t^{3} s^{3}\left(t^{8}+s^{8}\right)\left(t^{6} s^{6}-8\right)+114 t^{4} s^{4}\left(t^{12}+s^{12}\right) \\
+38 t^{7} s^{7}\left(t^{6} s^{6}-8\right)-95 t^{8} s^{8}\left(t^{4}+s^{4}\right)=0 .
\end{array}\right.
$$

\section{§. Die Jacobischen Multiplikatorgleichnngen.}

Das Integral erster Gattung $w$ nimmt bei Ausübung der ersten Haupttransformation $n^{\text {ten }}$ Grades einen in (7) S. 296 berechneten Faktor an. Den mit $(-1)^{\frac{n-1}{2}}$ multiplizierten Faktor bezeichnen wir mit Jacobi durch $M$ :

$$
M=(-1)^{\frac{n-1}{2}} n \frac{\vartheta_{s}\left(q^{n}\right)^{2}}{\vartheta_{3}(q)^{2}}
$$

und nennen ihn den „Multiplikator" des Integrals erster Gattung $w$. Beschränken wir uns sogleich wieder auf den Fall einer ungeraden Primzahl $n$, so beweist Jacobi, daß der Multiplikator $M$ einer algebraischen Gleichung $(n+1)^{\text {ten }}$ Grades genügt, deren Koeffizienten rationale Funktionen von $k^{2}$ sind.

Diese sogenannten Multiplikatorgleichungen haben das Interesse, dab sie in unserem Sinne die ersten Transformationsgleichungen für Modulformen sind, die in der Literatur auftreten. Führen wir nämlich (vgl. (21) in I, 419) die Modulform vierter Stufe $(-1)^{\text {ter }}$ Dimension:

$$
f\left(\omega_{1}, \omega_{2}\right)=\sqrt{e_{2}-e_{1}}=\frac{\pi}{\omega_{2}} \vartheta_{3}(q)^{2}
$$

ein, so stellt sich der Multiplikator $M$ in der Gestalt dar:

$$
M=(-1)^{\frac{n-1}{2}} n \frac{f\left(n \omega_{1}, \omega_{2}\right)}{f\left(\omega_{1}, \omega_{2}\right)}
$$

so daß die Jacobischen Multiplikatorgleichungen einfach die Transformationsgleichungen der Modulform vierter Stufe $f=\sqrt{e_{2}-e_{1}}$ sind. Aus der $\boldsymbol{\vartheta}_{\mathbf{3}}$-Reihe folgt für $f$ die Darstellung: 
Schlaeflische Modulargleichungen für $n=13,17,19$. Jacobis Multiplikator 509

$$
\begin{aligned}
f\left(\omega_{1}, \omega_{2}\right)=\frac{\pi}{\omega_{2}}\left(1+4 q+4 q^{2}+4 q^{4}+8 q^{5}+\right. & 4 q^{8}+4 q^{9}+8 q^{10} \\
& \left.+8 q^{13}+4 q^{16}+\cdots\right)
\end{aligned}
$$

Das Verhalten von $f$ gegenüber den Substitutionen der $\Gamma^{(\omega)}$ ist in I, 478 angegeben. Die Gruppe der Form $f$ ist die in I, $474 \mathrm{ff}$. vielfach hervorgetretene homogene $\Gamma_{19}$, die durch:

$$
\alpha \equiv \delta \equiv 1, \quad 2 \beta \equiv 2 \gamma \equiv 0 \quad(\bmod 4)
$$

erklärt ist. Man beachte, da $B$ in dieser $\Gamma_{12}$ die Substitution $\omega_{1}^{\prime}=-\omega_{1}$, $\omega_{2}^{\prime}=-\omega_{2}$ nicht enthalten ist.

Die mittelst der ersten Haupttransformation $n^{\text {ton }}$ Grades entstehende Form $f\left(n \omega_{1}, \omega_{2}\right)$ gehört zur Gruppe $\Gamma_{12(n+1)}$ des Index $12(n+1)$ und der Stufe $4 n$, die durch $(5)$ und die Kongruenz $\gamma \equiv 0(\bmod n)$ erklärt ist. Der Quotient (3) aber ist eine Modulfunktion, die als solche auch bei der Substitution $\omega_{1}^{\prime}=-\omega_{1}, \omega_{2}^{\prime}=-\omega_{2}$ unverändert bleibt und zu der durch:

$$
\alpha \equiv \delta \equiv 1, \quad \beta \equiv \gamma \equiv 0 \quad(\bmod 2), \quad \gamma \equiv 0 \quad(\bmod n)
$$

erklärten Kongruenzgruppe $\Gamma_{6(n+1)}$ des Index $6(n+1)$ und der Stufe $2 n$ gehört. Innerhalb der Hauptkongruenzgruppe $\Gamma_{6}$ zweiter Stufe ist die Gruppe $\Gamma_{6(n+1)}$ vom Index $(n+1)$. Folglich ist $M$ die Lösung einer Gleichung:

$$
M^{n+1}+R_{1}\left(k^{2}\right) M^{n}+R_{2}\left(k^{2}\right) M^{n-1}+\cdots+R_{n+1}\left(k^{2}\right)=0
$$

$(n+1)^{\text {ten }}$ Grades, deren Koeffizienten rationale Funktionen von $k^{2}$ sind. In (7) ist die Jacobische Multiplikatorgleichung für den $n^{\text {ten }}$ Transformationsgrad in Ansatz gebracht.

Die in (3) vorliegende erste Lösung von (7) möge jetzt insbesondere durch $M_{\infty}$ bezeichnet werden. Um die übrigen $n$ Lösungen darzustellen, üben wir auf die für $M=M_{\infty}$ in $\omega$ identisch gültige Gleichung (7) erstlich die Substitution $\left(\begin{array}{l}n, n+1 \\ n-1, n\end{array}\right)$ aus. Mit Benutzung von I, 478 findet man für die ursprüngliche Form $f\left(\omega_{1}, \omega_{2}\right)$ :

$$
f\left(n \omega_{1}+(n+1) \omega_{2},(n-1) \omega_{1}+n \omega_{2}\right)=(-1)^{\frac{n-1}{2}} f\left(\omega_{1}, \omega_{2}\right),
$$

für die transformierte Form $f\left(n \omega_{1}, \omega_{2}\right)$ aber folgt bei Ausübung der eben genannten Substitution mit Rücksicht auf ihre Dimension -1 in $\omega_{1}, \omega_{2}$ :

$$
n^{-1} f\left(n^{2} \frac{\omega_{1}}{n}+(n+1) \omega_{2},(n-1) \frac{\omega_{1}}{n}+\omega_{2}\right)=n^{-1} f\left(\frac{\omega_{1}}{n}, \omega_{2}\right) .
$$

Also haben wir eine zweite Lösung von (7) in:

$$
M_{0}=M_{\infty}\left(\frac{n \omega+n+1}{(n-1) \omega+n}\right)=\frac{f\left(\frac{\omega_{1}}{n}, \omega_{2}\right)}{f\left(\omega_{1}, \omega_{2}\right)},
$$


wie aus der Unveränderlichkeit von $k^{2}$ gegenüber der ausgeübten Substitution und der Irreduzibilität der Gleichung (7) folgt. Den Rest der Lösungen gewinnen wir auf entsprechendem Wege durch wiederholte Ausübung der Substitution $\left(\begin{array}{l}1,2 \\ 0,1\end{array}\right)$. Unter Zusammenfassung folgt: Die $(n+1)$ Lösungen der Multiplikatorgleichung sind:

(8) $M_{\infty}=(-1)^{\frac{n-1}{2}} n \frac{f\left(n \omega_{1}, \omega_{2}\right)}{f\left(\omega_{1}, \omega_{2}\right)}, \quad M_{\nu}=\frac{f\left(\frac{\omega_{1}+2 \nu \omega_{2}}{n}, \omega_{2}\right)}{f\left(\omega_{1}, \omega_{2}\right)} \cdot v=0,1,2, \ldots, n-1$.

In $f\left(\omega_{1}, \omega_{2}\right)$ hat man eine ganze Modulform, die im Diskontinuitätsbereiche der Hauptkongruenzgruppe $\Gamma_{6}$ zweiter Stufe einen einzigen Nullpunkt der Ordnung $\frac{1}{2}$ hat, gelegen im Spitzenzyklus $\omega= \pm 1$ (vgl. Fig. 81 in I, 440). Die $(n+1)$ transformierten Formen:

$$
(-1)^{\frac{n-1}{2}} n f\left(n \omega_{1}, \omega_{2}\right), \quad f\left(\frac{\omega_{1}+2 \nu \omega_{2}}{n}, \omega_{2}\right)
$$

sind demnach gleichfalls ganze Modulformen; ihre Nullpunkte liegen ausschlieblich in rationalen Punkten $\omega=\frac{p}{q}$ mit ungeraden $p, q$. Auch die symmetrischen Grundfunktionen der Formen (9) sind also ganze Modulformen, die sich in den Gestalten $R_{v}\left(k^{2}\right) f\left(\omega_{1}, \omega_{2}\right)^{\nu}$ darstellen. Sie bleiben gegenüber den Substitutionen der homogenen $\Gamma_{6}$ vielleicht von einem Zeichenwechsel abgesehen unverändert und verschwinden im Spitzenzyklus $\omega= \pm 1$, dem Pole von $k^{2}$. Da $f^{\nu}$ nur in diesem Spitzenzyklus: verschwindet, und zwar in der Ordnung $\frac{\nu}{2}$, während $R_{v}\left(k^{2}\right) f^{v}$ sicher überall polfrei ist, so folgt, $d a \beta R_{v}\left(k^{2}\right)$ eine ganze Funktion von $k^{2}$ eines Grades $<\frac{v}{2}$ ist. Speziell ist $R_{n+1}\left(k^{2}\right) f^{n+1}$ als Produkt der Formen (9), abgesehen vielleicht vom Zyklus $\omega= \pm 1$, sicher frei von Nullpunkten. Aber nur an dieser Stelle könnte sich der Pol von $R_{n+1}\left(k^{2}\right)$ finden. Also ist $R_{n+1}\left(k^{2}\right)$ als eine ganze Funktion ohne Nullpunkte mit einer Konstanten identisch.

Nach I, 478 gilt $f\left(\omega_{2},-\omega_{1}\right)=i f\left(\omega_{1}, \omega_{2}\right)$. Mit Rücksicht auf die Dimension -1 von $f\left(\omega_{1}, \omega_{2}\right)$ folgt hieraus leicht:

$$
M_{\infty}\left(\frac{-1}{\omega}\right)=(-1)^{\frac{n-1}{2}} M_{0}(\omega)
$$

während andrerseits $k^{2}(\omega)$ bei Ausübung der Substitution $\omega^{\prime}=\frac{-1}{\omega}$ in den komplementären Modul $k^{\prime 2}=1-k^{2}$ übergeht. Wegen der Irreduzibilität der Gleichung (7) ist also ihre linke Seite absolut invariant bei Ersatz von $M$ und $k^{2}$ durch $(-1)^{\frac{n-1}{2}} M$ und $k^{\prime 2}=1-k^{2}$. Für $n \equiv 1$ 
(mod 4) sind also alle Koeffizienten $R_{v}\left(k^{2}\right)$ invariant beim Ersatze von $k^{2}$ durch $k^{2}=1-k^{2}:$ Im Falle $n \equiv 1(\bmod 4)$ ist $R_{v}\left(k^{2}\right)$ eine ganze Funktion von $k^{2} k^{\prime 2}$ eines Grades $<\frac{v}{4}$. Im Falle $n \equiv-1(\bmod 4)$ sind die $R_{v}\left(k^{2}\right)$ mit geradem $\nu$ invariant gegenüber $\omega^{\prime}=\frac{-1}{\omega}$, während die $R_{v}\left(k^{2}\right)$ mit ungeradem $\nu$ Zeichenwechsel erfahren. Diese $R_{v}\left(k^{2}\right)$ verschwinden somit im Punkte $\omega=i$ und haben demgemäB den Faktor $\left(1-2 k^{2}\right)$. Man gelangt zu dem Satze: Für $n \equiv-1(\bmod 4)$ sind die Koeffizienten $R_{v}\left(k^{2}\right)$ mit geradem $\nu$ ganze Funktionen von $k^{2} k^{\prime 2}$ eines Grades $<\frac{v}{4}$, diejenigen mit ungeradem $\nu$ aber Produkte von $\left(1-2 k^{2}\right)$ mit . ganzen Funktionen von $k^{2} k^{\prime 2}$ eines Grades $<\frac{\nu-1}{4}$.

Für $\omega=i \infty$ hat man $k^{2}=0$ und zufolge (8) und (4):

$$
M_{\infty}=(-1)^{\frac{n-1}{2}} n, \quad M_{0}=M_{1}=\cdots=M_{n-1}=1 .
$$

Für $k^{2}=0$ nimmt die Multiplikatorgleichung also die Gestalt an:

$$
\left(M-(-1)^{\frac{n-1}{2}} n\right)(M-1)^{n}=0 .
$$

Hieraus folgt: Die Absolutglieder $a_{1}, a_{2}, \ldots$ der ganzen Funktionen $R_{1}\left(k^{2}\right), R_{2}\left(k^{2}\right), \ldots \sin d$ :

$$
\left\{\begin{array}{l}
a_{1}=-n\left(1+(-1)^{\frac{n-1}{2}}\right), \quad a_{2}=\left(\begin{array}{l}
n \\
1
\end{array}\right)\left(\frac{n-1}{2}+(-1)^{\frac{n-1}{2}} n\right), \\
a_{3}=-\left(\begin{array}{l}
n \\
2
\end{array}\right)\left(\frac{n-2}{3}+(-1)^{\frac{n-1}{2}} n\right) \\
a_{4}=\left(\begin{array}{l}
n \\
3
\end{array}\right)\left(\frac{n-3}{4}+(-1)^{\frac{n-1}{2}} n\right), \cdots, a_{n+1}=(-1)^{\frac{n-1}{2}} n .
\end{array}\right.
$$

Nimmt man die Regeln über Bauart und Grad der Funktionen $R_{v}\left(k^{2}\right)$ hinzu, so findet man eine Anzahl Anfangskoeffizienten, sowie den letzten Koeffizienten endgültig bestimmt. Im Falle $n \equiv 1(\bmod 4)$ gilt:

$$
\begin{gathered}
R_{1}=-2 n, \quad R_{2}=\frac{1}{2} n(3 n-1), \quad R_{3}=-\frac{1}{3} n(n-1)(2 n-1), \\
R_{4}=\frac{1}{24} n(n-1)(n-2)(5 n-3), \quad R_{n+1}=n,
\end{gathered}
$$

während für $n \equiv-1(\bmod 4)$ folgende Koeffizienten der Multiplikatorgleichung endgültig bestimmt sind:

$$
\left\{\begin{array}{l}
R_{1}=0, \quad R_{2}=-\frac{1}{2} n(n+1), \quad R_{3}=\frac{1}{3} n\left(n^{2}-1\right)\left(1-2 k^{2}\right) \\
R_{4}=-\frac{1}{8} n\left(n^{2}-1\right)(n-2), \\
R_{5}=\frac{1}{30} n\left(n^{2}-1\right)(n-2)(n-3)\left(1-2 k^{2}\right), \quad R_{n+1}=-n
\end{array}\right.
$$


Auf Grund der entwickelten Regeln kann man die Multiplikatorgleichung für $n=3$ :

$$
M^{4}-6 M^{2}+8\left(1-2 k^{2}\right) M-3=0
$$

sofort fertig hinschreiben. In den beiden nächsten Fällen $n=5$ und 7 bleiben im Ansatze vorerst noch eine bzw. zwei Konstante unbekannt. Man bestimmt diese leicht mit Hilfe der Potenzreihen. Die Multiplikatorgleichungen der Transformationsgrade 5 und 7 sind:

$$
\begin{gathered}
M^{6}-10 M^{5}+35 M^{4}-60 M^{3}+55 M^{2} \\
-\left(26-256 k^{2} k^{2}\right) M+5=0
\end{gathered}
$$

$$
-\left(140+5376 k^{2} k^{\prime 2}\right) M^{2}+\left(1-2 k^{2}\right)\left(48-2048 k^{2} k^{\prime 2}\right) M-7=0 \text {. }
$$

Jacobi hat in Art. 32 der „Fundamenta nova" eine Beziehung zwischen den Wurzeln der Multiplikatorgleichung und denen der Modulargleichung abgeleitet, welche man leicht auf folgendem Wege gewinnt. Nach der ersten Gleichung (5) in I, 449 gilt:

$$
e_{2}-e_{1}=f\left(\omega_{1}, \omega_{2}\right)^{2}=\frac{1}{2} D_{\eta}\left(\log \frac{\lambda}{\lambda-1}\right)
$$

Durch Entwicklung des Differentialausdrucks folgt:

$$
f\left(\omega_{1}, \omega_{2}\right)^{2}=\frac{1}{2 \lambda(1-\lambda)} D_{\eta}(\lambda)=-\frac{\pi i}{\omega_{2}^{2}} \frac{1}{\lambda(1-\lambda)} \frac{d \lambda}{d \omega}
$$

oder bei Übergang zu den Jacobischen Bezeichnungen:

$$
f\left(\omega_{1}, \omega_{2}\right)^{2}=-\frac{2 \pi i}{\omega_{2}^{2}} \frac{1}{k k^{\prime 2}} \cdot \frac{d k}{d \omega} .
$$

Übt man die erste Haupttransformation $n^{\text {ten }}$ Grades aus und bezeichnet die aus $k$ und $k^{\prime}$ hervorgehenden Größen durch $l$ und $l^{\prime}$, so findet man weiter:

$$
f\left(n \omega_{1}, \omega_{2}\right)^{2}=-\frac{1}{n} \frac{2 \pi i}{\omega_{2}^{2}} \frac{1}{l l^{\prime 2}} \frac{d l}{d \omega} .
$$

Die Division dieser Gleichung durch die voraufgehende ergibt zufolge (3) die fragliche Beziehung in der Gestalt:

$$
M^{2}=n \frac{k k^{\prime 2}}{l l^{\prime 2}} \frac{d l}{d k}
$$

wo $M, l$ und $l^{\prime}$ die zur ersten Haupttransformation gehörenden Größen sind. Durch Ausïbung geeigneter Substitutionen der $\Gamma^{(\omega)}$ zeigt man, daß die Relation (16) unverändert auch für die übrigen Klassen der Transformationen $n^{\text {ten }}$ Grades gültig bleibt. 


\section{\$5. Gruppentheoretische Grundlagen für die Resolventen füinften Grades zweiter Stufe.}

Der Galoissche Körper der Jacobischen Modulargleichung für Primzahltransformation $n$ wird gewonnen, indem man zur Funktion $u(\omega)$ die $(n+1)$ transformierten Funktionen $v_{\infty}(\omega), v_{0}(\omega), v_{1}(\omega), \ldots, v_{n-1}(\omega)$ adjungiert. Die zugehörige Untergruppe der Modulgruppe $\Gamma^{(\omega)}$ ist diejenige Kongruenzgruppe $16 n^{\text {ter }}$ Stufe des Index $24 n\left(n^{2}-1\right)$, die innerhalb der $\Gamma_{48}$ der Funktion $u(\omega)$ durch die Forderung $\alpha \equiv \delta \equiv \pm 1$, $\beta \equiv \gamma \equiv 0(\bmod n)$ ausgesondert wird. Sie ist innerhalb der $\Gamma_{48}$ eine ausgezeichnete Untergruppe des Index $\frac{1}{2} n\left(n^{2}-1\right)$, und der letzteren entspricht die Monodromiegruppe $G_{\frac{1}{2} n\left(n^{2}-1\right)}$ der Jacobischen Modulargleichung, die mit der Monodromiegruppe der Transformationsgleichung für $J(\boldsymbol{\omega})$ isomorph ist.

Es existieren somit auch für die Jacobischen Modulargleichungen bei Primzahltransformation nur in drei Fällen $n=5,7$ und 11 Resolventen eines Grades $<n+1$, nämlich $n^{\text {ten }}$ Grades. Es soll jedoch hier nur noch die Resolvente fünften Grades in ihren verschiedenen Gestalten besprochen werden. Wir nennen diese Gleichungen die „Resolventen fünften Grades zweiter Stufe", wogegen die Gleichungen (2), (4) und (6) S. 484 als "Resolventen fünften Grades erster Stufe" bezeichnet werden sollen

Die Unbekannten der zuletzt genannten Gleichungen waren Modulfunktionen einer Kongruenzgruppe $\Gamma_{5}$ fünfter Stufe, die sich mod 5 auf die 12, eine Tetraedergruppe $G_{12}$ bildende Substitutionen:

$$
\left\{\begin{array}{l}
\left.\left(\begin{array}{l}
1,0 \\
0,1
\end{array}\right),\left(\begin{array}{l}
0,1 \\
4,0
\end{array}\right),\left(\begin{array}{l}
2,0 \\
0,3
\end{array}\right),\left(\begin{array}{l}
0,2 \\
2,0
\end{array}\right),\left(\begin{array}{l}
1,1 \\
2,3
\end{array}\right),\left(\begin{array}{l}
1,2 \\
1,3
\end{array}\right)\right\} \\
\left(\begin{array}{l}
1,3 \\
4,3
\end{array}\right),\left(\begin{array}{l}
1,4 \\
3,3
\end{array}\right),\left(\begin{array}{l}
2,1 \\
2,4
\end{array}\right),\left(\begin{array}{l}
2,2 \\
1,4
\end{array}\right),\left(\begin{array}{l}
2,3 \\
4,4
\end{array}\right),\left(\begin{array}{l}
2,4 \\
3,4
\end{array}\right)
\end{array}\right\} \quad(\bmod 5)
$$

reduzierte. Man wird nun zu den verschiedenen Resolventen fünften Grades zweiter Stufe geführt, indem man die Durchschnitte dieser $\Gamma_{5}$ mit den einzelnen Kongruenzgruppen zweiter Stufe bildet und für die so entstehenden Kongruenzgruppen zehnter Stufe geeignete Funktionen auswählt, die dann die „Unbekannten“ der zu bildenden Gleichungen fünften Grades sind. Einige Feststellungen über die fraglichen Gruppen zehnter Stufe sind zunächst vorauszuschicken.

Erstlich stellen wir die $\Gamma_{5}$ mit der ausgezeichneten Gruppe $\Gamma_{2}$ zweiter Stufe zusammen, die sich mod 2 auf die Substitutionen:

$$
\left(\begin{array}{ll}
1, & 0 \\
0, & 1
\end{array}\right),\left(\begin{array}{l}
1,1 \\
1,
\end{array}\right),\left(\begin{array}{l}
0,1 \\
1,1
\end{array}\right) \quad(\bmod 2)
$$

reduziert. Die gemeinsame Untergruppe (der Durchschnitt) ist eine Gruppe zehnter Stufe $\Gamma_{10}$ des Index 10, deren Diskontinuitätsbereich in 
Fig. 35 gegeben ist. Die zwölf Seiten sind in folgender Anordnung aufeinander bezogen:

$$
\begin{aligned}
& 1 \rightarrow 12,\left(\begin{array}{l}
1,10 \\
0,1
\end{array}\right) ; \quad 2 \rightarrow 3,\left(\begin{array}{r}
1,3 \\
-1,-2
\end{array}\right) ; 4 \rightarrow 9,\left(\begin{array}{l}
5,-1 \\
1,
\end{array}\right) ; \\
& 5 \rightarrow 6,\left(\begin{array}{l}
2,-3 \\
1,-1
\end{array}\right) ; \quad 7 \rightarrow 8,\left(\begin{array}{l}
4,-13 \\
1,-3
\end{array}\right) ; 10 \rightarrow 11,\left(\begin{array}{l}
7,-43 \\
1,-6
\end{array}\right) ;
\end{aligned}
$$

In der Tat reduzieren sich alle diese Substitutionen mod 5 auf Substitu-tionen (1) und mod 2 auf Substitutionen (2).

Wir kombinieren zweitens die $\Gamma_{5}$ mit einer der drei gleichberechtigten Gruppen $\Gamma_{3}$ zweiter Stufe, etwa mit der durch $\beta \equiv 0(\bmod 2)$ er-

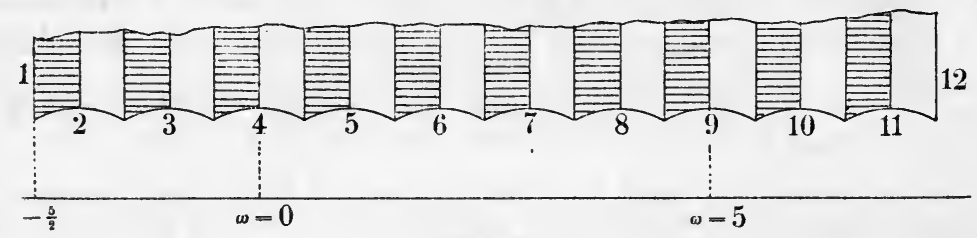

Fig. 35.

klärten. Der Durchschnitt ist eine Kongruenzgruppe zehnter Stufe $\Gamma_{15}$. des Index 15, deren Diskontinuitätsbereich in Fig. 36 dargestellt ist. Die Seiten sind in folgender Art einander zugeordnet:

$$
1 \rightarrow 8,\left(\begin{array}{rr}
1, & 10 \\
0, & 1
\end{array}\right) ; 2 \rightarrow 7,\left(\begin{array}{rr}
5,24 \\
1, & 5
\end{array}\right) ; \quad 3 \rightarrow 5,\left(\begin{array}{l}
1,2 \\
1,3
\end{array}\right) ; \quad 4 \rightarrow 6,\left(\begin{array}{l}
3,2 \\
1,1
\end{array}\right) .
$$

Diese Substitutionen reduzieren sich nämlich mod 5 wieder auf Substitutionen (1) und befriedigen außerdem die Kongruenz $\beta \equiv 0(\bmod 2)$.

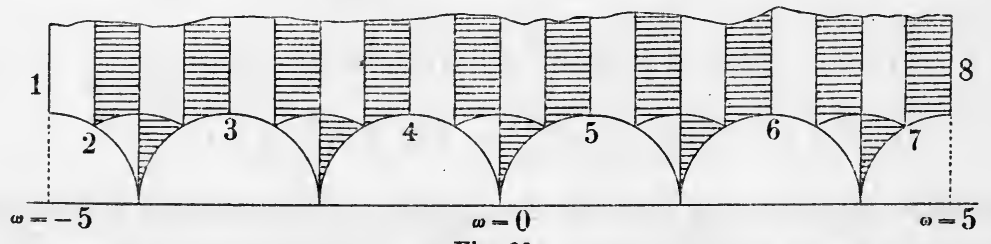

Fig. 36.

Die $\Gamma_{15}$ ist innerhalb der $\Gamma_{3}$ eine Untergruppe des Index 5 . Nun ist (vgl. S. 360) die $\Gamma_{\mathbf{3}}$ mit der Substitution:

$$
\omega^{\prime}=W(\omega)=-\frac{2}{\omega}
$$

vertauschbar und wird also durch Zusatz von $W$ zu einer Gruppe:

$$
\Gamma^{\prime}=\Gamma_{3}+\Gamma_{3} \cdot W
$$

erweitert, in der sie eine ausgezeichnete Untergruppe $\Gamma_{2}^{\prime}$ des Index 2 ist. Der Diskontinuitätsbereich der $\Gamma^{\prime}$ ist ein Paar von Kreisbogendreieeken der Winkel $\frac{\pi}{2}, \frac{\pi}{4}, 0$, wie aus Fig. 7, S. 360, hervorgeht. Die $\Gamma_{15}$ ist dann als Untergruppe der $\Gamma^{\prime}$ eine $\Gamma_{10}^{\prime}$ des Index 10, und dementsprechend 
läBt sich ihr Diskontinuitätsbereich aus zehn Dreieckspaaren der Winkel $\frac{\pi}{2}, \frac{\pi}{4}, 0$ aufbauen, wie in Fig. 37 zur Darstellung kommt.

Besonders wichtig ist nun aber die Tatsache, daß auch die $\Gamma_{10}^{\prime} d u r c h$ $W$ in sich transformiert wird. Nennt man nämlich die vier Substitutionen (3) der Reihe nach $V_{1}, V_{2}, V_{3}, V_{4}$, so bestätigt man leicht die Glei- $\omega=-5$ chungen:

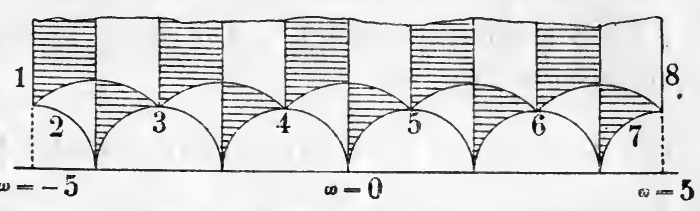

Fig. 37.

$$
\begin{gathered}
W \cdot V_{1} \cdot W^{-1}=V_{4}^{-1} \cdot V_{3} \cdot V_{2}^{-1} \cdot V_{4} \cdot V_{3}^{-1}, \\
W \cdot V_{2} \cdot W^{-1}=V_{4}^{-1} \cdot V_{3} \cdot V_{1}^{-1} \cdot V_{4} \cdot V_{3}^{-1}, \\
W \cdot V_{3} \cdot W^{-1}=V_{3}^{-1}, \quad W \cdot V_{4} \cdot W^{-1}=V_{4}^{-1} .
\end{gathered}
$$

Also ist $W \cdot \Gamma_{10}^{\prime} \cdot W^{-1}$ jedenfalls in $\Gamma_{10}^{\prime}$ enthalten, muß aber mit $\Gamma_{10}^{\prime}$ identisch sein, da die nochmalige Transformation durch $W$ zur $\Gamma_{10}^{\prime}$ zurückführt. Die $\Gamma_{10}^{\prime}$ ist demnach durch Zusatz von $W$ zu einer Gruppe $\Gamma_{5}^{\prime}$ erweiterungsfähig, die in der $\Gamma^{\prime}$ eine Untergruppe des Index 5 ist, und in der die $\Gamma_{10}^{\prime}$ eine ausgezeichnete Untergruppe des Index 2 ist. Der in Fig. 38 gegebene Diskontinuitätsbereich der $\Gamma_{5}^{\prime}$

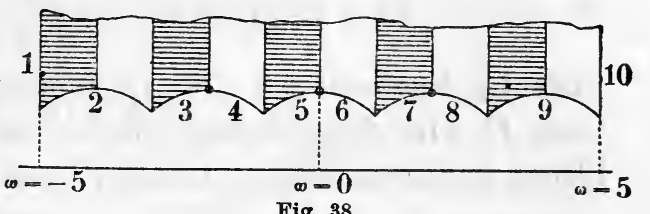

Fig. 38. besteht aus fünf Paaren von Kreisbogendreiecken der Winkel $\frac{\pi}{2}, \frac{\pi}{4}, 0$. Die Seiten sind in folgender Art einander zugeordnet:

$$
\begin{gathered}
1 \rightarrow 10,\left(\begin{array}{ll}
1, & 10 \\
0, & 1
\end{array}\right) ; 2 \rightarrow 9,\left(\begin{array}{l}
4,14 \\
1,
\end{array}\right) ; 3 \rightarrow 4,\left(\begin{array}{rr}
2, & 6 \\
-1,-2
\end{array}\right) ; \\
5 \rightarrow 6,\left(\begin{array}{r}
0,2 \\
-1,0
\end{array}\right) ; \quad 7 \rightarrow 8,\left(\begin{array}{l}
2,-6 \\
1,-2
\end{array}\right) .
\end{gathered}
$$

Die zweite, dritte und fünfte dieser Substitutionen stellen sich in den eben mit $V_{3}, V_{4}$ und $W$ bezeichneten Substitutionen so dar:

$$
\left(\begin{array}{lr}
4, & 14 \\
1, & 4
\end{array}\right)=W \cdot V_{4}^{-1} \cdot V_{3}, \quad\left(\begin{array}{r}
2, \\
-1,-2
\end{array}\right)=W \cdot V_{3}, \quad\left(\begin{array}{l}
2,-6 \\
1,-2
\end{array}\right)=W \cdot V_{4}^{-1}
$$

\section{\$ 6. Aufstellung der Resolventen fünften Grades zweiter Stufe.}

Eine erste Resolvente fünften Grades zweiter Stufe wird von der Gruppe $\Gamma_{10}$ geliefert. Mittelst der zur $\Gamma_{5}$ gehörenden einwertigen Funktion $\zeta(\omega)$, die S. 483 näher erklärt ist, wird der in Fig. 35 dargestellte Diskontinuitätsbereich der $\Gamma_{10}$ auf eine zweiblättrige Riemannsche Fläche abgebildet, die, wie der Vergleich mit dem Diskontinuitätsbereiche der $\Gamma_{5}$ (Fig. 30, S. 476) zeigt, nur bei $\xi=\infty$ (dem Punkte $\omega=i \infty$ entsprechend) und bei $\xi=3$ (den Punkten $\omega=i$ und $5+i$ der Fig. 35 ent- 
sprechend) verzweigt ist. Demnach ist $y=\sqrt{\xi-3}$ eine einwertige Funktion der $\Gamma_{10}$. Die Beziehung zur einwertigen Funktion $\sqrt{J-1}$ der $\Gamma_{2}$ geht unmittelbar aus der zweiten Gleichung (2) S. 484 hervor, indem man die Quadratwurzel zieht und durch Wahl des Vorzeichens über $\sqrt{\xi-3}$ eindeutig verfügt. $\left.{ }^{1}\right)$ Die zur $\Gamma_{10}$ gehörende Resolvente fünften Grades zweiter Stufe ist:

$$
y^{5}+10 y^{3}+45 y+24 \sqrt{3} \sqrt{J-1}=0 .
$$

Gleichungen fünften Grades dieser Gestalt, in denen also die Glieder vierten und zweiten Grades ausfallen, bezeichnet Klein ${ }^{2}$ ) als „Diagonalgleichungen".

Die Gruppe $\Gamma^{\prime}$, der die Einteilung der $\omega$-Halbebene in Kreisbogendreiecke der Winkel $\frac{\pi}{2}, \frac{\pi}{4}, 0$ zugehört, habe als einwertige Funktion $L(\omega)$, die durch die Festsetzung der Eckenwerte:

$$
L(-1+i)=0, \quad L(i \sqrt{2})=1, \quad L(i \infty)=\infty
$$

eindeutig bestimmt sei. Der Diskontinuitätsbereich der Gruppe zweiter Stufe $\Gamma_{3}$ wird durch diese Funktion auf eine zweiblättrige Riemannsche Fläche mit zwei bei $L=0$ und $L=1$ gelegenen Verzweigungspunkten abgebildet. Also ist $\sqrt{\frac{L-1}{L}}$ eine einwertige Funktion der $\Gamma_{3}$. Andrerseits ist, da die $\Gamma_{3}$ durch Zusatz von $\omega^{\prime}=\frac{\omega}{\omega+1}$ aus der Hauptkongruenzgruppe $\Gamma_{6}$ zweiter Stufe entsteht, auch:

$$
k^{2}(\omega)+k^{2}\left(\frac{\omega}{\omega+1}\right)=k^{2}+\frac{1}{k^{2}}=\frac{1+k^{4}}{k^{2}}
$$

eine einwertige Funktion der $\Gamma_{3}$, so daß wir auf eine Relation:

$$
\sqrt{\frac{L-1}{L}}=\frac{a\left(1+k^{4}\right)+b k^{2}}{c\left(1+k^{4}\right)+d k^{2}}
$$

schließen. Die Koeffizienten $a, b, c, d$ bestimmen sich aus der Tatsache, dab den Werten $k^{2}=0,1,-1$ die Werte $1,-1, \infty$ der in der letzten Gleichung links stehenden Funktion entsprechen. Man findet von hier aus leicht den Satz: Die einwertige Funktion $L(\omega)$ der Gruppe $\Gamma^{\prime}$ stellt

1) Die Funktion $\sqrt{J-1}$ sei auf der imaginären $\omega$-Achse positiv.

2) Vgl. die „Vorlesungen über das Ikosaeder und die Auflösung der Gleichungen vom fünften Grade", S. 166. Wegen der Beziehung der Gleichung (1) zu der von Brioschi aufgestellten Resolvente fünften Grades der „allgemeinen Jacobischen Gleichung sechsten Grades" vgl, man ebenda S. 150 ff. 
sich in dem Integralmodul wie folgt dar:

$$
L=\frac{\left(1+k^{2}\right)^{4}}{16 k^{2} k^{\prime}} \text {. }
$$

Der Diskontinuitätsbereich der $\Gamma_{5}^{\prime}$ wird durch $L(\omega)$ auf eine fünfblättrige Riemannsche Fläche abgebildet, die nur bei $L=0,1$ und $\infty$ verzweigt ist. Bei $L=0$ hat. man einen vierblättrigen Verzweigungspunkt, von dem Zyklus der Ecken $\pm 1+i, \pm 3+i$ herrührend, bei $L=1$ einen zweiblättrigen, dem Zyklus der Ecken $\pm 4+i \sqrt{2}$ entsprechend, während bei $L=\infty$ ein fünfblättriger Verzweigungspunkt liegt. Als Geschlecht dieser Fläche berechnet sich 0, so dab eine einwertige Funktion der $\Gamma_{5}^{\prime}$ existiert. Sie werde $\sigma(\omega)$ genannt und durch die Festsetzungen:

$$
\sigma( \pm 1+i)=\sigma( \pm 3+i)=0, \quad \sigma( \pm 5+i)=5, \quad \sigma(i \infty)=\infty
$$

eindeutig erklärt. Dann ist zufolge der eben angegebenen Verzweigung der fünfblättrigen Fläche $L$ bis auf einen konstanten Faktor gleich der ganzen Funktion fünften Grades $\sigma^{4}(\sigma-5)$ :

$$
\sigma^{4}(\sigma-5)=a L \text {. }
$$

Der Faktor $a$ bestimmt sich aus der Forderung, daB diese Gleichung für $L=1$ eine von 0 verschiedene Doppelwurzel haben muB. Man berechnet diese Wurzel zu $\sigma=4$ und findet $a=-256$. Zwischen den einwertigen Funktionen $\sigma(\omega)$ und $L(\omega)$ der Gruppen $\Gamma_{5}^{\prime}$ und $\Gamma^{\prime}$ besteht die Beziehung:

$$
\sigma^{5}-5 \sigma^{4}+256 L=0,
$$

in der wir eine zweite Resolvente fünften Grades zweiter Stufe gewonnen haben. Diese Gleichung fünften Grades ist dadurch ausgezeichnet, daB in ihr die Glieder dritten, zweiten und ersten Grades fehlen.

Es besteht nun folgender wichtige Satz: Während die Monodromiegruppe der Resolvente (1), ebenso wie die der Resolvente fünften Grades erster Stufe, die $G_{60}$ der geraden Vertauschungen der Wurzeln (Ikosaedergruppe) ist, hat die Gleichung (4) als Monodromiegruppe die $G_{120}$ aller 120 Permutationen der fünf Wurzeln. Der Durchschnitt der fünf mit der $\Gamma_{5}$ gleichberechtigten Gruppen ist nämlich die Hauptkongruenzgruppe fünfter Stufe $\Gamma_{60}$, die mit der $\Gamma_{2}$ eine ausgezeichnete Kongruenzgruppe zehnter Stufe $\Gamma_{120}$ gemein hat. Die $\Gamma_{120}$ ist ihrerseits in der $\Gamma_{2}$ eine ausgezeichnete Untergruppe des Index 60, und ihr entspricht die Monodromiegruppe $G_{60}$ der Gleichung (1). Ebenso ist der Durchschnitt der $\Gamma_{60}$ und der $\Gamma_{3}$ eine (nicht ausgezeichnete) Kongruenzgruppe $\Gamma_{180}$ zehnter Stufe, die aber in der $\Gamma_{3}$ ausgezeichnet vom Index 60 ist. Aus ihr gewinnt man durch Zusatz von $W$ eine in der Gruppe $\Gamma^{\prime}$ ausgezeichnete Untergruppe $\Gamma_{120}^{\prime}$ des Index 120, und eben dieser Untergruppe gehört 
die Monodromiegruppe $G_{120}$ der Gleichung (4) zu. Bezeichnen wir die fünf Wurzeln der Gleichung (4) durch $\sigma_{\nu}(\omega)=\sigma_{0}(\omega+2 \nu)$, wo $\nu$ die Zahlen 0, 1, 2, 3, 4 durchläuft, so entspricht zwar der Substitution $\left(\begin{array}{l}1,2 \\ 0,1\end{array}\right)$ die zyklische, also gerade Permutation $\sigma_{v}^{\prime}=\sigma_{v+1}$, aber die Substitution $W$ liefert, wie man leicht feststellt, die ungerade Permutation:

$$
\sigma_{0}^{\prime}=\sigma_{0}, \quad \sigma_{1}^{\prime}=\sigma_{1}, \quad \sigma_{2}^{\prime}=\sigma_{3}, \quad \sigma_{3}^{\prime}=\sigma_{2}, \quad \sigma_{4}^{\prime}=\sigma_{4}^{\prime} .
$$

Mit der Gleichung (4) ist die von Hermite ${ }^{1}$ ) aufgestellte Resolvente der Modulargleichung sechsten Grades (25) S. 501 nahe verwandt. Die sechs Wurzeln dieser Modulargleichung waren:

$$
v_{\infty}=-\sqrt[4]{k}(5 \omega), \quad v_{v}=\sqrt[4]{k}\left(\frac{\omega+16 \nu}{5}\right),
$$$$
v=0,1, \ldots, 4
$$

deren Produkt nach (25) S. 501 gleich $-u^{6}$ ist:

$$
v_{\infty} \cdot v_{0} \cdot v_{1} \cdot v_{2} \cdot v_{3} \cdot v_{4}=-u^{6} \text {. }
$$

Die Wirkung der Substitutionen $\omega^{\prime}=\omega+2$ und $\omega^{\prime}=\frac{\omega}{\omega+1}$ auf die $v$ stellen wir tabellarisch zusammen; ist $\varepsilon=e^{\frac{\pi i}{4}}$, so gilt:

\begin{tabular}{c|c|c|c|c|c|c} 
& $v_{\infty}$ & $v_{0}$ & $v_{1}$ & $v_{2}$ & $v_{3}$ & $v_{4}$ \\
\hline$\left(\begin{array}{l}1,2 \\
0,1\end{array}\right)$ & $\varepsilon^{5} v_{\infty}$ & $\varepsilon^{5} v_{2}$ & $\varepsilon^{5} v_{3}$ & $\varepsilon^{5} v_{4}$ & $\varepsilon^{5} v_{0}$ & $\varepsilon^{5} v_{1}$ \\
\hline$\left(\begin{array}{c}1,0 \\
1,1\end{array}\right)$ & $\frac{1}{v_{1}}$ & $\frac{1}{v_{0}}$ & $\frac{1}{v_{3}}$ & $\frac{1}{v_{4}}$ & $\frac{1}{v_{2}}$ & $\frac{1}{v_{\infty}}$
\end{tabular}

Die Richtigkeit dieser Angaben ist mit Hilfe der Tabelle in I, 459 leicht zu bestätigen. Man hat z. B.:

$$
v_{0}(\omega+2)=\sqrt[4]{k}\left(\frac{\omega+2}{5}\right)=\sqrt[4]{k}\left(\frac{\omega+2 \cdot 16}{5}-6\right)=\varepsilon^{5} v_{2}(\omega)
$$

oder für die zweite Substitution:

$$
v_{2}\left(\frac{\omega}{\omega+1}\right)=\sqrt[4]{k}\left(\frac{\frac{\omega}{\omega+1}+32}{5}\right)=\sqrt[4]{k}\left(\frac{33 \omega+32}{5 \omega+5}\right)=\sqrt[4]{k}\left(\frac{33 \frac{\omega+4 \cdot 16}{5}-416}{5 \frac{\omega+4 \cdot 16}{5}-63}\right),
$$

wofür man aus der eben genannten Tabelle $\frac{1}{v_{4}}$ gewinnt. Durch Wiederholung und Zusammensetzung der beiden ausgeübten Substitutionen stellt man die Wirkung der S. 515 mit $V_{1}, V_{2}, V_{3}, V_{4}$ bezeichneten Substitutionen fest:

1) Sur la résolution de l'équation du cinquième degré", Compt. Rend., Bd. 46 (1859). 


\begin{tabular}{c|c|c|c|c|c|c|c} 
& $v_{\infty}$ & $v_{0}$ & $v_{1}$ & $v_{2}$ & $v_{3}$ & $v_{4}$ & $u$ \\
\hline$V_{1}$ & $\varepsilon v_{\infty}$ & $\varepsilon v_{0}$ & $\varepsilon v_{1}$ & $\varepsilon v_{2}$ & $\varepsilon v_{3}$ & $\varepsilon v_{4}$ & $\varepsilon^{5} u$ \\
\hline$V_{2}$ & $\frac{1}{v_{0}}$ & $\frac{1}{v_{\infty}}$ & $\frac{1}{v_{4}}$ & $\frac{1}{v_{2}}$ & $\frac{1}{v_{3}}$ & $\frac{1}{v_{1}}$ & $\frac{1}{u}$ \\
\hline$V_{3}$ & $\frac{\varepsilon^{3}}{v_{3}}$ & $\frac{\varepsilon^{3}}{v_{2}}$ & $\frac{\varepsilon^{3}}{v_{0}}$ & $\frac{\varepsilon^{3}}{v_{1}}$ & $\frac{\varepsilon^{3}}{v_{4}}$ & $\frac{\varepsilon^{3}}{v_{\infty}}$ & $\frac{\varepsilon^{7}}{u}$ \\
\hline$V_{4}$ & $\frac{\varepsilon^{5}}{v_{1}}$ & $\frac{\varepsilon^{5}}{v_{4}}$ & $\frac{\varepsilon^{5}}{v_{2}}$ & $\frac{\varepsilon^{5}}{v_{\infty}}$ & $\frac{\varepsilon^{5}}{v_{0}}$ & $\frac{\varepsilon^{5}}{v_{3}}$ & $\frac{\varepsilon}{u}$
\end{tabular}

In der letzten Spalte ist die Wirkung der $V$ auf $u(\omega)$ angegeben.

Im Anschluß an Hermite bilden wir nun den Ausdruck:

$$
w(\omega)=\left(v_{\infty}-v_{0}\right)\left(v_{1}-v_{4}\right)\left(v_{2}-v_{3}\right) \cdot u
$$

und stellen sogleich als Anfangsglied seiner Reihenentwicklung:

$$
w(\omega)=4 \sqrt{5} q^{\frac{1}{5}}+\cdots
$$

fest. Aus der letzten Tabelle folgt, daß $w$ gegenüber $V_{1}$ invariant ist, bei Ausübung von $V_{2}, V_{3}$ und $V_{4}$ aber übereinstimmend in $w^{\prime}=\frac{w}{k^{2}}$ übergeht. Da $k^{2}$ gegenüber den $V_{2}, V_{3}, V_{4}$ in $\frac{1}{k^{2}}$ übergeht, so erweist sich:

$$
\frac{w}{1+k^{2}}
$$

als eine Funktion der Gruppe $\Gamma_{15}=\Gamma_{10}^{\prime}$.

Der in Fig. 36 dargestellte Diskontinuitätsbereich dieser Gruppe hat nun das Geschlecht $p=1$ und besitzt an Spitzen erstlich die bei $\omega=i \infty$ und weiter nur noch den Spitzenzyklus $\omega=0, \pm 2, \pm 4$. In der ersten Spitze hat die Funktion (8) zufolge (7) einen Nullpunkt erster Ordnung. Im Spitzenzyklus liegt gleichfalls ein Nullpunkt der Funktion (8), da für $\omega=0$ der Nenner $1+k^{2}=2$ und $u=1$ ist, während von den sechs $v$ fünf gleich -1 und eine gleich +1 ist (vgl. S. 500). Auch in allen übrigen Punkten des genannten Bereiches ist $w$ endlich. Der Nenner $\left(1+k^{2}\right)$ aber hat zwei Nullpunkte erster Ordnung in den beiden Eckenzyklen $\omega=-1+i, 3+i$ und $\omega=1+i,-3+i$, sowie weiter nur noch einen Nullpunkt der Ordnung $\frac{1}{2}$, im Bereiche gemessen, im Eckenzyklus $\omega= \pm 5+i$. Die Funktion (8) hat demnach nur zwei Pole erster Ordnung in den Zyklen $\omega=-1+i, 3+i$ und $\omega=1+i$, $-3+i$, da im dritten Zyklus nur noch ein Pol der Ordnung $\frac{1}{2}$ auftreten könnte, der aber bei einer Modulfunktion der $\Gamma_{10}^{\prime}$ ausgeschlossen ist. Da wir somit in (8) eine zweiwertige Funktion der $\Gamma_{10}^{\prime}$ erkannt haben, 
muB neben dem Nullpunkte erster Ordnung bei $\omega=i \infty$ ein zweiter Nullpunkt erster Ordnung im Spitzenzyklus $\omega=0, \pm 2, \pm 4$ auftreten.

Nun haben wir in $\frac{1}{\sigma(\omega)}$ eine zweite zweiwertige Funktion der $\Gamma_{10}^{\prime}$, die in der Lage und Ordnung der Nullpunkte und Pole mit der Funktion (8) übereinstimmt und also von ihr nur durch einen konstanten Faktor verschieden ist. Man berechnet aus (3) und (4) als Anfangsglied der Potenzreihe von $\sigma(\omega)$ leicht $-q^{-\frac{1}{5}}$. Damit ergibt sich bei Benutzung von (7) der Satz: Die Funktionen $\sigma(\omega)$ und $w(\omega)$ stehen in der einfachen Beziehung:

$$
w \cdot \sigma=-4 \sqrt{5}\left(1+k^{2}\right),
$$

so daß sich die Gleichung (4) mit Rücksicht auf (3) in folgende Gleichung fünften Grades für $w$ umrechnet:

$$
w^{5}-2^{4} \cdot 5^{3} k^{2} k^{\prime 4} w-2^{6} \cdot 5^{2} \gamma^{\prime} \overline{5} k^{2} k^{\prime 4}\left(1+k^{2}\right)=0 .
$$

Hier haben wir die von Hermite angegebene Resolvente fünften Grades gewonnen. Sie steht gegenüber der Gleichung (4) insofern zurück, als die Unbekannte $w$ der Gleichung (10) als Modulfunktion zehnter Stufe erst zu derjenigen $\Gamma_{30}$ gehört, die der Durchschnitt der $\Gamma_{5}$ mit der Hauptkongruenzgruppe zweiter Stufe $\Gamma_{6}$ ist. Will man an Stelle von (4) mit einer Gleichung arbeiten, in der die Glieder vierten, dritten und zweiten Grades fehlen ${ }^{1}$, so führe man $z=-5 \sigma^{-1}$ als Unbekannte ein. Wir gelangen so zur Gestalt:

$$
z^{5}-\frac{5^{5}}{2^{8} L}(z+1)=0
$$

der Resolvente fünften Grades, die natürlich gleichfalls die Eigenschaft derGleichung (4) besitzt, daß ihre Monodromiegruppe die $G_{120}$ aller 120 Permutationen der fünf Wurzeln ist. ${ }^{2}$ )

\section{§ 7. Notizen über die Lösung der allgemeinen Gleichung fünften Grades durch elliptische Funktionen.}

Auf der Resolvente fünften Grades der Modulargleichung für den Transformationsgrad $n=5$ beruht die Verwendbarkeit der elliptischen Funktionen zur Lösung der allgemeinen Gleichung fünften Grades. Gemeinsam ist den verschiedenen Gestalten jener Resolvente die Eigenschaft, daß neben der jeweiligen Unbekannten der einzelnen Gleichung in ihren

1) Gleichungen dieser Art nennt Klein „Bringsche Gleicbungen"; sie wurden früher auch nach Jerrard benannt. Vgl. die „Vorles. über das Ikosaeder usw.", S. 143.

2) Diese Eigenschaft büßt die Hermitesche Gleichung (10) ein, weil in ihr der Integralmodul $k^{2}$ den „Parameter" (im Sinne von § 7) darstellt. 
Koeffizienten stets nur eine GröBe auftritt, die als komplexe Variable willkürlich wählbar ist. Es handelt sich, wie man sagt, um Gleichungen fünften Grades mit einem „Parameter". In der Gleichung (1) S. 516 ist dieser Parameter. $\sqrt{J-1}$, in der Hermiteschen Gleichung $k^{2}$, in den Gleichungen (4) und (11) $\S 6$ aber $L$.

Gegenüber diesen Gleichungen hat die „allgemeine Gleichung fünften Grades":

$$
x^{5}+a_{1} x^{4}+a_{2} x^{3}+a_{3} x^{2}+a_{4} x+a_{5}=0
$$

mit beliebigen komplexen Koeffizienten $a$ zunächst fünf Parameter. Soll es möglich sein, die elliptischen Funktionen unter Vermittlung einer unserer Resolventen zu einer „transzendenten" Lösung der Gleichung (1) zu verwerten, so müßte die Gleichung (1) zunächst in eine neue Gestalt transformierbar sein, in der nur ein Parameter auftritt. Das Mittel, dessen man sich hierbei zu bedienen hat, ist die Tschirnhausen-Transformation (vgl. S. 26). Man führt als neue Unbekannte $y$ eine ganze Funktion vierten Grades von $x$ ein:

$$
y=p_{0}+p_{1} x+p_{2} x^{2}+p_{3} x^{3}+p_{4} x^{4}
$$

wobei die Berechnung von $x$ aus $y$ nur noch die Lösung einer Gleichung vom vierten Grade erfordert. Ist die Gleichung für $y$ :

$$
y^{5}+b_{1} y^{4}+b_{2} y^{3}+b_{3} y^{2}+b_{4} y+b_{5}=0
$$

so ist $b_{\nu}$ als symmetrische Grundfunktion $\nu^{\text {ten }}$ Grades der fünf Ausdrücke:

$$
y_{i}=p_{0}+p_{1} x_{i}+p_{2} x_{i}^{2}+p_{3} x_{i}^{3}+p_{4} x_{i}^{4}
$$$$
i=1,3 \ldots, 5
$$

unter den $x_{i}$ die Lösungen von (1) verstanden, eine ganze homogene Funktion $\nu^{\text {ten }}$ Grades der $p_{0}, p_{1}, \ldots, p_{4}$, deren Koeffizienten als symmetrisch in den $x_{i}$ sich rational in den Koeffizienten $a$ darstellen. Man gibt nun über die Koeffizienten $b$ möglichst weitgehende Vorschriften und sucht diesen Vorschriften durch zweckmäßige Auswahl der zur Verfügung stehenden Größen $p \mathrm{zu}$ genügen.

Die sich dabei einstellenden algebraischen Entwicklungen sind sehr ausgedehnt, so daB es hier nur möglich ist, einige Hauptgesichtspunkte zu notieren. ${ }^{1)}$ Zunächst kann man aus der in den $p$ linearen Gleichung $b_{1}=0$ eine der Größen $p$, etwa $p_{0}$, rational in den $p_{1}, p_{2}, p_{3}, p_{4}$ und den Koeffizienten $a$ berechnen, und zwar linear und homogen in den $p_{1}, p_{3}, p_{3}, p_{4}$. Trägt man diesen Ausdruck von $p_{0}$ in $b_{2}, b_{3}, \ldots$ ein, so werden diese $b_{\nu}$ homogene ganze Funktionen $\nu^{\text {ten }}$ Grades in den vier restierenden $p$. Die Funktion zweiten Grades $b_{2}$ der vier $p$ läßt sich dann nach einem

1) Eine elementare Darstellung findet man bei Serret, „Handbuch der Algebra". 2. Aufl. (Leipzig 1878), Bd. 2, S. $350 \mathrm{ff.}$ 
bekannten Satze (s. Serret a. a. 0.) als Summe von vier Quadraten linearer Ausdrücke in $p$ schreiben:

$$
b_{2}=q_{1}^{2}+q_{2}^{2}+q_{3}^{2}+q_{4}^{2},
$$

wobei die $q_{i}$ in den $p$ von der Gestalt:

$$
q_{i}=\alpha_{i 1} p_{1}+\alpha_{i 2} p_{2}+\alpha_{i 3} p_{3}+\alpha_{i 4} p_{4}
$$

sind. Bei der Herstellung dieser linearen Ausdrücke sind aber bereits Quadratwurzeln zu ziehen, also „Irrationalitäten“ zu adjungieren. Hierbei hat man $\mathrm{zu}$ entscheiden, ob die einzelne zu adjungierende Irrationalität eine „natürliche“ oder eine „akzessorische“ ist (vgl. S. 51 ff.). Die nähere Untersuchung hat gezeigt, daß die eben erwähnten Quadratwurzeln bereits alizessorisch sind.

Soll jetzt auch $b_{2}=0$ sein, so kann dies unter vielen Möglichkeiten z. B. so erreicht werden, dab:

$$
q_{1}+i q_{2}=0, \quad q_{3}+i q_{4}=0
$$

gesetzt wird. Damit hat man zwei lineare homogene Gleichungen für die $p_{1}, p_{2}, p_{3}, p_{4}$ gewonnen, aus denen man etwa $p_{1}$ und $p_{2}$ linear und homogen in $p_{3}$ und $p_{4}$ berechnet. Nun wird $b_{3}$ nach Eintragung dieser Ausdrücke von $p_{1}$ und $p_{2}$ zu einer ganzen homogenen Funktion dritten Grades in $p_{3}$ und $p_{4}$, so daB die weitere Forderung $b_{3}=0$ durch Lösung einer kubischen Gleichung für den Quotienten $p_{3}: p_{4}$ zu befriedigen ist. Hiernach ist es möglich, durch Tschirnhausen-Transformation die allgemeine Gleichung fünften Grades auf die Bringsche Gestalt zu transformieren:

$$
y^{5}+b_{4} y+b_{5}=0
$$

wobei die einzuführenden Irrationalitäten Quadratwurzeln und Kubikwurzeln sind. Die Gleichung (6) aber ist sofort durch die weitere Transformation $y=\frac{b_{5}}{b_{4}} z$ auf eine Gleichung:

$$
z^{5}+\frac{b_{4}^{5}}{b_{5}^{4}}(z+1)=0
$$

mit nur einem Parameter zu reduzieren, die die Gestalt unserer Resolvente (11) S. 520 hat.

Die führenden Untersuchungen von Hermite (s. die Note S. 518) über die Verwendung der elliptischen Funktionen schließen sich an die Bringsche Gleichung an. Die weiteren Entwicklungen von Brioschi ${ }^{1}$ ), Kronecker ${ }^{2}$ ) und Klein ${ }^{3}$ ) sind hiervon nicht nur abgewichen, indem

1) „Sulla risoluzione delle equazione di quinti grado", Annali di matem., ser. 1, Bd. 1 (1858).

2) Brief an Hermite vom Juni 1858, Compt. Rend., Bd. 46.

3) "Weitere Untersuchungen über das Ikosaeder", Math. Ann. Bd. 12 und 13 (1878). 
sie andere, den elliptischen Funktionen gleichfalls zugängliche Gestalten von Gleichungen fünften Grades mit einem Parameter bevorzugten, sondern sie haben vor allem auch die algebraische Seite der Theorie wesentlich vervollständigt und vertieft. $\left.{ }^{1}\right) \mathrm{Es} \mathrm{muB}$ hier genügen, in letzterer Hinsicht die beiden folgenden wichtigsten Theoreme zu nennen. Erstlich ist der Satz, daß es unmöglich ist, die allgemeine Gleichung fünften Grades allein bei Gebrauch „natürlicher" Irrationalitäten in eine Gleichung mit nur „einem" Parameter zu transformieren, von Kronecker entdeckt und von Klein zuerst öffentlich bewiesen. Zweitens hat Klein gezeigt, daß man eine "Hauptgleichung" fünften Grades (d.h. eine Gleichung mit ausfallenden Gliedern vierten und dritten Grades) allein vermöge der Quadratwurzel der Diskriminante der Gleichung, also einer „natürlichen" Irrationalität, in eine Gleichung mit nur einem Parameter ïberführen kann. Hieraus ging die grundlegende Tatsache hervor, daB die früher benutzte Kubikwurzel, welche zur Bringschen Gleichung hinführte, bei Bevorzugung anderer Gleichungsformen mit nur einem Parameter entbehrlich ist; denn die Hauptgleichung ist, wie oben gesagt, durch akzessorische Quadratwurzeln erreichbar.

Das Eingreifen der elliptischen Funktionen in den LösungsprozeB der Gleichungen fünften Grades ist ziemlich naheliegend. Wir erläutern die Verhältnisse etwa im AnschluB an die Gleichung (4) S. 517, die mit der Bringschen Gleichung unmittelbar verwandt ist. Eine vorgelegte Gleichung fünften Grades wird man zunächst durch Tschirnhausen-Transformation auf die Gestalt bringen:

$$
y^{5}-5 y^{4}+256 L=0 \text {. }
$$

Zum vorliegenden Werte $L$ gehört dann ein eindeutig bestimmter Wert $\omega$ im Diskontinuitätsbereiche der Gruppe $\Gamma^{\prime}$. Man wird dabei zweckmäBig diesen Bereich aus dem Dreiecke der Ecken $\omega=-1+i, i \sqrt{2}$, $i \infty$ und seinem Spiegelbilde an der imaginären Achse aufbauen. Für die Berechnung des fraglichen Wertes $\omega$ aus $L$ gibt es eine Gleichung, die sich genau an die Darstellung von $\omega$ durch $J$, wie sie in der Gleichung (10) in I, 336 gegeben ist, anschlieBt. Die fragliche Formel ist:

$$
\pi i \omega=-\log L-8 \log 2+\frac{F_{1}\left(\frac{1}{8}, \frac{3}{8} ; \frac{1}{L}\right)}{F\left(\frac{1}{8}, \frac{3}{8}, 1 ; \frac{1}{L}\right)},
$$

wo rechts im Nenner die hypergeometrische Reihe und im Zähler die

1) Eine ausführliche Darstellung in geometrischem Gewande gibt der $\mathrm{Ab}$ schnitt II der „Vorles. über das Ikosaeder usw." von Klein; eine weitere Darstellung, die sich auf Hermites invariantentheoretische Behandlung der Tschirnhausen-Transformation gründet, findet man in Webers "Lehrbuch der Algebra", Bd. 1, S. $210 \mathrm{ff}$. 
mit ihr verwandte Reihe (15) in I, 114 gemeint ist. Die Gleichung folgt. in derselben Art aus der Theorie der hypergeometrischen Differentialgleichung, wie dies für die genannte Gleichung (10) in I, 336 a. a 0. ausführlich entwickelt ist. Hat man $\omega$ und damit $q$ gewonnen, so sind die fünf Lösungen der Gleichung (7) durch:

$$
y_{0}=\sigma(\omega), y_{1}=\sigma(\omega+2), \ldots, y_{4}=\sigma(\omega+8)
$$

gegeben, wo $\sigma(\omega)$ die S. 517 erklärte einwertige Funktion der $\Gamma_{5}^{\prime}$ ist. Die Potenzreihe für $\sigma(\omega)$ kann man auf Grund von (6) und (9) $\S 6$ aus den Reihen für $u, v_{\infty}, v_{0}, v_{1}, \ldots$ ableiten; die Anfangsglieder sind:

$$
\sigma(\omega)=-q^{-\frac{1}{5}}\left(1-q^{\frac{1}{5}}+2 q^{\frac{2}{5}}-4 q^{\frac{3}{5}}+7 q^{\frac{4}{5}}+12 q+\cdots\right) .
$$

Man kann sich auch zur Berechnung von $\omega$ aus $L$ der Vermittlung des Integralmoduls $k^{2}$ bedienen, indem man aus der Gleichung (3) S. 517 mittelst zweier Quadratwurzeln $k^{2}$ berechnet. Die Wurzeln sind dabei so zu wählen, dab $\omega$ im oben bezeichneten Diskontinuitätsbereiche der Gruppe $\Gamma^{\prime}$ liegt. Für die Berechnung von $\omega$ aus $k^{2}$ hat man dann nach S. $494 \mathrm{ff}$. den schnell konvergenten Prozeß des arithmetisch-geometrischen Mittels zur Hand. Auch bei der Berechnung von $\sigma$ für gegebenes $\omega$ kann man sich der Vermittlung der Größen $v_{\infty}, v_{0}, \ldots$ bedienen, die wegen der guten Konvergenz der Thetareihen besonders brauchbar erscheinen.

\section{\$ 8. Notizen über irrationale Modulargleichungen.}

Es ist schon sehr früh bemerkt, daß sich die Modulargleichungen gelegentlich in sehr einfache Gestalten zusammenziehen lassen, wenn man neben der achten Wurzel des Integralmoduls $u=\sqrt[4]{V} \bar{k}$ auch noch die des komplementären Moduls $\sqrt[4]{k^{\prime}}=\sqrt[8]{1-u^{8}}$ und natürlich die aus ihnen durch Transformation entstehenden Größen zuläßt. Die fraglichen Gleichungen würden dann, in $u$ und $v$ geschrieben, noch Wurzelausdrücke enthalten und heißen dieserhalb „irrationale Modulargleichungen".

Die nach (23) S. 501 für den dritten Grad bestehende Gleichung:

$$
\left(v^{4}-u^{4}\right)^{2}=4 v^{2} u^{2}\left(v^{2} u^{2}-1\right)^{2}
$$

schreibt man leicht in die Gestalt um:

$$
\left(1-u^{8}\right)\left(1-v^{8}\right)=\left(1-u^{2} v^{2}\right)^{4} .
$$

Man beziehe diese Gleichung zunächst nur auf die erste Haupttransformation und bediene sich für die transformierten Moduln der schon S. 512 benutzten Bezeichnung $l^{2}$ und $l^{\prime 2}$. Dann kleidet sich die letzte Gleichung in die Gestalt:

$$
k^{\prime 2} l^{\prime 2}=(1-\sqrt{k} \sqrt{l})^{4} .
$$


Zieht man die vierte Wurzel und berücksichtigt, daB für $\omega=i \infty$ die Werte $\sqrt{k}=0, \sqrt{\imath}=0, \sqrt{k^{\prime}}=1, \sqrt{l^{\prime}}=1$ zutreffen, so folgt als irrationale Modulargleichung fuir den dritten Grad ${ }^{1}$ ):

$$
\sqrt{k} \sqrt{l}+\sqrt{k^{\prime}} \sqrt{l^{\prime}}=1 \text {. }
$$

Die zweite Gleichung (25) S. 501 für den siebenten Grad kleidet sich leicht in die Gestalt:

$$
\left(1-u^{8}\right)\left(1-v^{8}\right)=(1-u v)^{8}
$$

Nach Ausziehen der achten Wurzel findet man gleichfalls zunächst für die erste Haupttransformation als irrationale Modulargleichung für den siebenten Grad ${ }^{2}$ ):

$$
\sqrt[4]{k} \sqrt[4]{\imath}+\sqrt[4]{k^{\prime}} \sqrt{l^{\prime}}=1
$$

Für den fünften Grad hat Jacobi in Art. 30 der „Fundamenta nova“ eine irrationale Modulargleichung angegeben, die jedoch nicht die sogleich bei $n=5$ aufzustellende Gleichung ist. Eine Reihe weiterer Gleichungen der fraglichen Art sind dann später von Schroeter ${ }^{3}$ ) aufgestellt worden.

Es sollen hier noch drei irrationale Modulargleichungen übereinstimmender Bauart für $n=5,11$ und 23 abgeleitet werden und zwar nicht durch algebraische Umformungen der betreffenden Modulargleichungen, sondern mittelst einer funktionentheoretischen Überlegung, die wir etwa am Falle $n=11$ erläutern. Man bilde für die erste Haupttransformation elften Grades den Ausdruck:

$$
\boldsymbol{\Phi}(\boldsymbol{\omega})=\sqrt{k} \sqrt{\imath}+\sqrt{k^{\prime}} \sqrt{l^{\prime}}-1
$$

und untersuche die Wirkung der durch $\gamma \equiv 0(\bmod 11)$ erklärten Kongruenzgruppe $\Gamma_{12}$ elfter Stufe auf $\Phi(\omega)$. Schreibt man $\omega^{\prime}=11 \omega$, so gilt:

$$
\sqrt{l}(\omega)=\sqrt{k}\left(\omega^{\prime}\right), \quad \sqrt{\bar{l}^{\prime}}(\omega)=\sqrt{k^{\prime}}\left(\omega^{\prime}\right)
$$

und es ist, falls auf $\omega$ die Substitution $V=\left(\begin{array}{l}\alpha, \beta \\ \gamma, \delta\end{array}\right)$ ausgeübt wird, auf $\omega^{\prime}$ die Substitution $\left(\begin{array}{c}\alpha, 11 \beta \\ 11^{-1} \gamma, \delta\end{array}\right)$ anzuwenden. Diese beiden Substitutionen sind mod 2 einander kongruent, so daß bei Feststellung der Wirkung von $\left(\begin{array}{l}\alpha, \beta \\ \gamma, \delta\end{array}\right)$ auf die einzelnen Faktoren in (3) rechts immer dieselbe Zeile der Tabelle in I, 459 zur Geltung kommt. Gilt nun erstlich:

1) Die Gleichung (1), die bereits Legendre bekannt war, findet man bei $\mathrm{Jacobi}$ in Art. 30 der „Fundamenta nova" entwickelt.

2) Die Gleichung (2) ist von Gützlaff in der Abhandlung "Aequatio modularis pro transformatione functionum ellipticarum septimi ordinis", Journ. f. Math., Bd. 12 (1832) entwickelt.

3) In der Schrift „De aequationibus modularibus", Regiomonti, 1854. 


$$
V=\left(\begin{array}{l}
\alpha, \beta \\
\gamma, \delta
\end{array}\right) \equiv\left(\begin{array}{ll}
1, & 1 \\
0, & 1
\end{array}\right) \quad(\bmod 2),
$$

so folgt aus der letzten Zeile der genannten Tabelle ${ }^{1}$ ):

Gilt aber zweitens

$$
\Phi(V(\omega))=-\frac{\sqrt{k} \sqrt{l}}{\sqrt{k^{\prime}} \sqrt{l^{\prime}}}+\frac{1}{\sqrt{k^{\prime}} \sqrt{l^{\prime}}}-1=-\frac{\Phi(\omega)}{\sqrt{k^{\prime}} \sqrt{l^{\prime}}} .
$$

$$
V=\left(\begin{array}{l}
\alpha, \beta \\
\gamma, \delta
\end{array}\right) \equiv\left(\begin{array}{l}
0,1 \\
1,0
\end{array}\right) \quad(\bmod 2),
$$

so folgt aus der vierten Zeile der Tabelle noch einfacher:

$$
\Phi(V(\omega))=\Phi(\omega) \text {. }
$$

Man übe weiter auf den Ausdruck $\sqrt{k \bar{k}} \sqrt{l l^{\prime}}$ die gleichen Substitutionen aus und findet, je nachdem der Fall (4) oder (6) vorliegt, als transformierten Ausdruck:

$$
-\frac{\sqrt{k k^{\prime}} \sqrt{l l^{\prime}}}{\left(\sqrt{k^{\prime}} \sqrt{l^{\prime}}\right)^{3}} \quad \text { bzw. } \quad \sqrt{k k^{\prime}} \sqrt{l l^{\prime}}
$$

Unter Heranziehung von (5) und (7) ergibt sich, daß in beiden Fällen:

$$
\frac{\Phi(\omega)^{3}}{\sqrt{\overline{k k^{\prime}} \sqrt{l l^{\prime}}}}=\frac{\left(\sqrt{k} \sqrt{l}+\sqrt{k^{\prime}} \sqrt{l^{\prime}}-1\right)^{3}}{\sqrt{\overline{k k^{\prime}}} \sqrt{\overline{l l^{\prime}}}}
$$

invariant ist. $\mathrm{Da}$ man nun durch Kombination der Substitutionen (4) und (6) alle sechs mod 2 inkongruenten Typen von Substitutionen gewinnt, so erweist sich der Quotient (8) gegenüber allen Substitutionen der $\Gamma_{12}$ als invariant und stellt also eine Funktion dieser Gruppe dar. Aber diese Funktion ist auch invariant gegenüber der Substitution $\boldsymbol{\omega}^{\prime}=\frac{-1}{11 \omega}$, bei welcher sich zufolge (17) in I, 456 die Funktionen $\sqrt{k}$, $\sqrt{l^{\prime}}$ und ebenso die $\sqrt{k^{\prime}}, \sqrt{l}$ austauschen. Die Funktion (8) ist also invariant sogar gegenüber der zum Klassenpolygon elften Grades gehörenden Gruppe $\Gamma_{11}$. Dieses Polygon hat nur eine einzige an den Rand der $\omega$-Halbebene heranragende Spitze, nämlich die bei $\omega=i \infty$, und nur an dieser Stelle kann somit die Funktion (8) einen Pol haben. Doch zeigen die Reihenentwicklungen, daB auch hier kein Pol vorliegt. Die Funktion ist also mit einer Konstanten identisch; und dasselbe gilt demnach auch von ihrer dritten Wurzel, für welche die Potenzreihen den Wert $-2 \sqrt[3]{2}$ liefern. Als irrationale Modulargleichung für den elften Grad gewinnt man so:

$$
\sqrt{k} \sqrt{l}+\sqrt{k^{\prime}} \sqrt{l^{\prime}}+2 \sqrt[3]{2} \sqrt[6]{k k^{\prime}} \sqrt[6]{l l^{\prime}}=1,
$$

woran sich für die Grade 5 und 23 die auf ganz entsprechenden Wegen

1) Im Zähler des daselbst angegebenen transformierten Ausdrucks von $\sqrt[4]{k}$ muB $\sqrt[4]{k}$ statt $\sqrt{k}$ gesetzt werden. 
aufzustellenden Gleichungen anschließen:

$$
\left\{\begin{array}{l}
k l+k^{\prime} l^{\prime}+2 \sqrt[3]{4} \sqrt[3]{k k^{\prime}} \sqrt[3]{l l^{\prime}}=1 \\
\sqrt[4]{k} \sqrt[4]{\imath}+\sqrt[4]{k^{\prime}} \sqrt[4]{l^{\prime}}+\sqrt[3]{4} \sqrt[12]{k k^{\prime}} \sqrt[12]{l l^{\prime}}=1
\end{array}\right.
$$

Alle fünf aufgestellten irrationalen Modulargleichungen sind hier zunächst auf die ersten Haupttransformationen der betreffenden Grade n bezogen. Schreibt $\operatorname{man} \frac{-1}{\omega}$ an Stelle von $\omega$, benutzt die Gleichungen (4) S. 502 und (17) in I, 456 und berücksichtigt die Symmetrie der Gleichungen (1) usw. in dem ursprünglichen und dem komplementären Modul, so zeigt sich, daß die fraglichen Gleichungen auch dann gelten, wenn wir unter $l$ und $l^{\prime}$ die vermittelst der zweiten Haupttransformation umgeformten Größen verstehen. Nun sind die ursprünglichen Größen $\sqrt{k}, \sqrt{k^{\prime}}$ der Gleichung (1) Modulfunktionen achter Stufe, und ebenso gehören die ursprünglichen Größen der für die Grade $n=5,7,11$ und 23 aufgestellten Gleichungen den Stufen $\nu=12,16,24$ und 48 an. Im AnschluB an die Erklärungen von S. 498 und 504 verstehen wir aber unter einem „Repräsentantensystem $v^{\text {ter }}$ Stufe $e^{\text {" }}$ für Primzahltransformation $n$ das System der $(n+1)$ Transformationen:

$$
\begin{gathered}
\omega^{\prime}=n \omega, \quad \omega^{\prime}=\frac{\omega}{n}, \quad \omega^{\prime}=\frac{\omega+\nu}{n}, \quad \omega^{\prime}=\frac{\omega+2 v}{n}, \cdots, \\
\omega^{\prime}=\frac{\omega+(n-1) \nu}{n} .
\end{gathered}
$$

$\mathrm{Da}$ in jedem Falle die ursprünglichen Größen gegenüber der Substitution $\left(\begin{array}{l}1, v \\ 0,1\end{array}\right)$ invariant sind, so gelangen wir zu dem Satze: Die einzelne der fünf aufgestellten irrationalen Modulargleichungen gilt für alle $(n+1)$ Repräsentanten eines Systems $\boldsymbol{\nu}^{\text {ter }}$ Stufe, wo $v$ den Graden $n=3,5,7,11$ und 23 entsprechend die Stufen $\nu=8,12,16,24$ und 48 bedeutet.

\section{\$. Notizen über Modularkorrespondenzen.}

Die Natur der irrationalen Modulargleichungen ist von Klein ${ }^{1}$ ) aufgeklärt. Sie wurzelt im Begriffe der „Modularkorrespondenzen“, die dann insbesondere durch die Arbeiten von A. Hurwitz ${ }^{2}$ zu einer ausgedehnten Theorie entwickelt wurden. Die grundlegende Betrachtung möge etwa am Beispiele der $16^{\text {ten }}$ Stufe erläutert werden.

Die zu $\sqrt[4]{k}$ gehörende Gruppe $\Gamma_{48}$ der $16^{\text {ten }}$ Stufe war durch die Kon-

1) „Zur Theorie der elliptischen Modulfunktionen“ Math. Ann. Bd. 17 (1879).

2) „Zur Theorie der Modulargleichungen“, Gött. Nachr. von 1883, „Über algebraische Korrespondenzen und das Korrespondenzprinzip", Math. Ann. Bd. 28, "Über Klassenzahlrelationen und Modularkorrespondenzen primzahliger Stufe", Leipz. Ber. von 1885. 
gruenz (3) S. 496 erklärt. Da $\sqrt[4]{k}$ durch die Substitution $T=\left(\begin{array}{c}0,1 \\ -1,0\end{array}\right)$ in $\sqrt[4]{k^{\prime}}$ transformiert wird, so gehört $\sqrt[4]{k^{\prime}}$ zu der mit $\Gamma_{\mathbf{4}}$ gleichberechtigten Gruppe $\Gamma_{48}^{\prime}=T \cdot \Gamma_{48} \cdot T^{-1}$. Der Durchschnitt beider Gruppen besteht aus allen die Bedingungen:

$$
V \equiv\left(\begin{array}{c}
1,0 \\
0,1
\end{array}\right),\left(\begin{array}{c}
5,8 \\
8,13
\end{array}\right),\left(\begin{array}{c}
9,0 \\
0,9
\end{array}\right),\left(\begin{array}{c}
13,8 \\
8,5
\end{array}\right)(\bmod 16)
$$

befriedigenden Substitutionen und stellt eine ausgezeichnete Kongruenzgruppe $16^{\text {ter }}$ Stufe $\Gamma_{384}$ vom Index 384 dar. Für diese Gruppe hat man dann eben in $\sqrt{k}, \sqrt[4]{k^{\prime}}$ ein Funktionssystem, daß an die Bedingung:

$$
(\sqrt[4]{k})^{8}+\left(\sqrt[4]{k^{\prime}}\right)^{8}=1
$$

gebunden ist. Der Gewobnheit halber denken wir den Diskontinuitätsbereich der $\Gamma_{384}$ mittelst der Funktion $J(\omega)$ auf eine „regulär verzweigte“ Riemannsche Fläçhe $F_{384}$ über der $J$-Ebene abgebildet.

Es ist nun möglich, die Grundsätze der Transformation $n^{\text {ten }}$ Grades, wie wir sie früher auf Funktionen anwandten, unmittelbar auf die Gruppe $\Gamma_{384}$ und die Fläche $F_{384}$ auszuüben. Der Grad $n$ sei als ungerade und also als teilerfremd gegen die Stufe 16 vorausgesetzt: Wir ordnen dem Punkte $\omega$ des Diskontinuitätsbereiches der $\Gamma_{384}$ den Punkt $\omega^{\prime}=n \omega z u$, übertragen diese Zuordnung auf die Fläche $\mathbf{F}_{384}$ und untersuchen, wie sich diese Beziehung bei Umläufen auf der Fläche ausgestaltet.

Die geschlossenen Umläufe auf der Fläche werden in der $\omega$-Halbebene, die wir zugleich mit in Betracht ziehen, von den Substitutionen der $\Gamma_{384}$ geliefert. Bei Fortsetzung der begründeten Beziehung über die

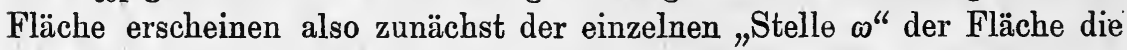
unendlich vielen Punkte der $\omega$-Halbebene:

$$
\omega^{\prime}=\frac{n \alpha \omega+n \beta}{\gamma \omega+\delta}
$$

zugeordnet, wo $\left(\begin{array}{l}\alpha, \beta \\ \gamma, \delta\end{array}\right)$ die $\Gamma_{384}$ durchläuft. Diese Werte liefern aber nur $\psi(n)$ verschiedene Stellen der Fläche, wo $\psi(n)$ in der bekannten Bedeutung (4) S. 220 gebraucht ist. In der Tat ergeben alle die Werte $\omega^{\prime}$, welche bezüglich der $\Gamma_{384}$ äquivalent sind, ein und dieselbe Stelle der Fläche. Ein System bezüglich der $\Gamma_{384}$ äquivalenter Werte $\omega^{\prime}$ repräsentieren wir aber durch einen unter ihnen und verfahren zu diesem Zwecke so:

Die Ausübung einer Substitution $V^{\prime}=\left(\begin{array}{l}\alpha^{\prime}, \\ \beta^{\prime} \\ \gamma^{\prime},\end{array}\right)$ der $\Gamma_{384}$ auf das in (3) berechnete $\omega^{\prime}$ führt zu:

$$
V^{\prime}\left(\frac{n \alpha \omega+n \beta}{\gamma \omega+\delta}\right)=\frac{\left(n \alpha^{\prime} \alpha+\beta^{\prime} \gamma\right) \omega+\left(n \alpha^{\prime} \beta+\beta^{\prime} \delta\right)}{\left(n \gamma^{\prime} \alpha+\delta^{\prime} \gamma\right) \omega+\left(n \gamma^{\prime} \beta+\delta^{\prime} \delta\right)} .
$$


Indem wir die Substitution $\left(\begin{array}{l}\alpha, \beta \\ \gamma, \delta\end{array}\right)$ der $\Gamma_{384}$ beliebig entnommen voraussetzen, wählen wir, um die rechte Seite der Gleichung (4) möglichst einfach zu gestalten, $V^{\prime}$ in folgender Weise: Wir verstehen unter $A$ den größten gemeinsamen Teiler von $n$ und $\gamma$, setzen $n=A \cdot D$ und wählen zunächst:

$$
\gamma^{\prime}=-\frac{\gamma}{A}, \quad \delta^{\prime}=\frac{n}{A} \alpha=D \alpha .
$$

Dann gilt $\gamma^{\prime} \equiv \gamma \equiv 0$ oder $\equiv 8(\bmod 16), \delta^{\prime}$ ist ungerade und $\gamma^{\prime}$ und $\delta^{\prime}$ sind teilerfremd. Es gibt also eine Reihe von Zahlenpaaren:

$$
\left\{\begin{array}{l}
\alpha^{\prime}=\alpha_{0}^{\prime}+\nu \gamma^{\prime}=\alpha_{0}^{\prime}-\nu \frac{\gamma}{A}, \\
\beta^{\prime}=\beta_{0}^{\prime}+\nu \delta^{\prime}=\beta_{0}^{\prime}+\nu D \alpha,
\end{array}\right.
$$

die alle die Gleichung $\alpha^{\prime} \delta^{\prime}-\beta^{\prime} \gamma^{\prime}=1$ befriedigen, unter $\alpha_{0}^{\prime}, \beta_{0}^{\prime}$ ein erstes solches Paar verstanden. Die Gleichung (4) aber nimmt die Gestalt an:

$$
V^{\prime}\left(\frac{n \alpha \omega+n \beta}{\gamma \omega+\delta}\right)=\frac{A \omega+\left(n \alpha_{0}^{\prime} \beta+\beta_{0}^{\prime} \delta\right)+\nu D}{D} .
$$

Unter den Zahlen $\beta^{\prime}$ kommen solche vor, die $\bmod 16$ mit $\beta$ kongruent sind. $\left.{ }^{1}\right)$ Man kann demnach auch sogleich $\beta_{0}^{\prime} \equiv \beta(\bmod 16)$ als erfüllt voraussetzen. LäBt man sodann nur noch alle durch 16 teilbaren $\nu \mathrm{zu}$, indem man etwa $\nu=16 \nu^{\prime}$ setzt, so folgt für alle Zahlen $\beta^{\prime}$ die Kongruenz $\beta^{\prime} \equiv \beta(\bmod 16)$, und man hat zusammenfassend:

$$
\beta^{\prime} \equiv \beta \equiv \gamma \equiv \gamma^{\prime} \equiv 8 \text { oder } \equiv 0 \quad(\bmod 16) .
$$

Da $n \alpha_{0}^{\prime}$ und $\delta$ ungerade sind, so wird $\left(n \dot{\alpha}_{0}^{\prime} \beta+\beta_{0}^{\prime} \delta\right)$ eine durch 16 teilbare Zahl. Setzt man daraufhin:

$$
\left(n \alpha_{0}^{\prime} \beta+\beta_{0}^{\prime} \delta\right)+16 \nu^{\prime} D=16 B,
$$

so kann man die noch verfügbare Zahl $\nu^{\prime}$ so bestimmen, daB $0 \leqq B<D$ zutrifft. Die Gleichung (4) hat damit die Gestalt:

$$
V^{\prime}\left(\frac{n \alpha \omega+n \beta}{\gamma \omega+\delta}\right)=\frac{A \omega+16 B}{D}, \quad(A \cdot D=n, \quad 0 \leqq B<D)
$$

angenommen. Aus der Gleichung:

$$
V^{\prime-1}\left(\frac{(A \omega+16 B)}{D}\right)=\frac{n \alpha \omega+n \beta}{\gamma \omega+\delta}
$$

aber stellt man leicht noch fest, daB die Zahlen $A, B, D$ keinen gemeinsamen Faktor $>1$ enthalten. Wir haben somit in (7) rechts einen der $\psi(n)$ Repräsentanten eines „Systems $16^{\text {ter }}$ Stufe" für Transformation $n^{\text {ten }}$ Grades gewonnen.

1) Man kann nämlich $\nu$ entsprechend der Kongruenz $\nu D \alpha \equiv \beta-\beta_{0}^{\prime}(\bmod 16)$ auswählen. 
Indessen ist $V^{\prime}$ noch nicht in allen Fällen eine Substitution der $\Gamma_{384}$. Damit $\nabla^{\prime}$ einer der Kongruenzen (1) genügt, ist nämlich, da $\left(\begin{array}{l}\alpha, \beta \\ \gamma, \delta\end{array}\right)$ der $\Gamma_{384}$ angehört und die Kongruenzen (6) bereits feststehen, noch erforderlich und hinreichend, daB $\delta^{\prime} \equiv \pm \alpha(\bmod 8)$ zutrifft. Dies ist aber wegen $\delta^{\prime}=D \alpha$ nur für $D \equiv \pm 1(\bmod 8)$ erfüllt. $\left.{ }^{1}\right)$ Doch genügt es, falls $D \equiv+3(\bmod 8)$ gilt, noch die Substitution $\left(\begin{array}{rr}5, & 16 \\ -16, & -51\end{array}\right)$ zu $V^{\prime}$ hinzuzusetzen, um eine Substitution der $\Gamma_{384} \mathrm{zu}$ erhalten. Um beide Fälle zusammenzufassen, verstehen wir unter $V_{D}$ die Substitution $\left(\begin{array}{l}1, \\ 0,1\end{array}\right)$ oder $\left(\begin{array}{rr}5, & 16 \\ -16,-51\end{array}\right)$, je nachdem $D \equiv \pm 1$ oder $\pm 3(\bmod 8)$ zutrifft, und lassen an Stelle der Gleichung (7) die folgende treten:

$$
V_{D} \cdot V^{\prime}\left(\frac{n \alpha \omega+n \beta}{\gamma \omega+\delta}\right)=V_{D}\left(\frac{A \omega+16 B}{D}\right), \quad(A \cdot D=n, \quad 0 \leqq B<D) .
$$

Entsprechend werden wir die $\psi(n)$ hier rechts auftretenden Transformationen zu einem „Repräsentantensysteme 16. Stufe" für Transformation des ungeraden Grades n wählen.

Die durch die Zuordnung der Werte $\omega$ und $\omega^{\prime}=n \omega$ begründete $\mathrm{Be}-$ ziehung zweier Stellen der Fläche $\mathbf{F}_{\mathbf{3 8 4}}$ aufeinander ist also so beschaffen, daß bei geschlossenen Umläufen der Stelle $\omega$ auf der Fläche als jener Stelle zugeordnet höchstens $\psi(n)$ Stellen gewonnen werden, die durch (9) gegeben sind. DaB man der einzelnen Stelle aber auch nicht weniger als $\psi(n)$ Stellen zugeordnet findet, ist gruppentheoretisch einleuchtend. Die beiden Werte:

$$
n \omega, \quad \frac{n \alpha \omega+n \beta}{\gamma \omega+\delta}=\frac{\alpha(n \omega)+n \beta}{n^{-1} \gamma(n \omega)+\delta}
$$

sind nämlich (bei variablem $\omega$ ) stets und nur dann bezüglich der $\Gamma_{384}$ äquivalent, wenn $n^{-1} \gamma$ ganzzahlig, also $\gamma \equiv 0(\bmod n)$ ist. Es muB also $\left(\begin{array}{l}\alpha, \beta \\ \gamma, \delta\end{array}\right)$ der durch $\gamma \equiv 0(\bmod n)$ und die Kongruenzen (1) erklärten Kongruenzgruppe der Stufe $16 n$ und des Index $384 \psi(n)$ angehören, die innerhalb der $\Gamma_{384}$ eine Untergruppe des Index $\psi(n)$ ist. Es finden sich also unter den Werten (3), falls $\left(\begin{array}{l}\alpha, \beta \\ \gamma, \delta\end{array}\right)$ die $\Gamma_{384}$ durchläuft, in der Tat $\psi(n)$ bezüglich dieser Gruppe inäquivalente Werte. Die auf der Fläche $\mathbf{F}_{384}$ begründete und ïber sie fortgesetzte Punktzuordnung ist also so beschaffen, daß der einzelnen Stelle der Fläche stets $\psi(n)$ Stellen zugeordnet sind.

Die Inversion der auf der Fläche $F_{384}$ konstruierten Zuordnung würde der Stelle $\omega$ die Stelle $\frac{\omega}{n}$ zuweisen, eine Zuordnung, die dann

1) Für den als Beispiel in Betracht kommenden Fall $n=7$ gehört also $V^{n}$ bereits der $\Gamma_{\mathbf{s i 4}}$ an. 
wieder über die Fläche hin fortzusetzen sein würde. Aber im Falle $n \equiv \pm 1(\bmod 8)$ ist bereits bei der ursprünglichen Zuordnung $\frac{\omega}{n}$ eine $\operatorname{der} \psi(n)$ der Stelle $\omega$ zugeordneten Stellen. Um im Falle $n \equiv \pm 3(\bmod 8)$ die Inversion $\mathrm{zu}$ vollziehen, bemerken wir zunächst, $d a \beta$ für alle $n$ die konstruierte Zuordnung nur erst eine unter 384 gleichberechtigte ist. Die $\Gamma_{384}$ ist nämlich eine ausgezeichnete Untergruppe, so daB die Fläche $\boldsymbol{F}_{384}$ 384 eindeutige Transformationen in sich zuläBt, entsprechend den 384 bezüglich der $\Gamma_{384}$ inäquivalenten Substitutionen $1, V_{1}, V_{2}, \ldots, V_{383}$ der Gesamtgruppe $\Gamma^{(\omega)}$. Ist $V_{i}$ irgendeine dieser Substitutionen, so erhalten wir stets wieder eine Zuordnung unserer Art, wenn wir der Stelle $\omega$ die $\psi(n)$ Stellen:

$$
V_{i} \cdot V_{D}\left(\frac{A \omega+16 B}{D}\right)
$$

zuweisen. Es gilt dann einfach der Satz: Im Falle $n \equiv \pm 1(\bmod 8)$ ist die durch $\omega^{\prime}=n \omega$ begrïndete Zuordnung sich selbst invers, bei $n \equiv \pm 3$ (mod 8) führt ihre Inversion zu einer anderen unter den 384 gleichberechtigten Anordnungen.

Punktzuordnungen der fraglichen Art sind zuerst auf algebraischen Kurven betrachtet und als „Korrespondenzen" bezeichnet. Von den Kurven aber überträgt man sie sofort auf Riemannsche Flächen. Die hier auf der $\mathbf{F}_{384}$ hergestellten Zuordnungen heiBen insbesondere . „Modularkorrespondenzen". Da die einzelne Zuordnung, sowie auch ihre Umkehrung jedem Flächenpunkte $\psi(n)$ Punkte zuweist, so wird die Modularkorrespondenz,$\psi(n)-\psi(n)$-deutig" genannt.

Die vorstehende Betrachtung ist auch auf andere ausgezeichnete Kongruenzuntergruppen der Modulgruppe $\Gamma^{(\omega)}$ übertragbar, von denen wir sogleich einige zu nennen haben werden. Ist die Untergruppe vom Geschlechte 0, so gibt es eine zugehörige einwertige Modulfunktion. Diese ist zur algebraischen Darstellung der Korrespondenz besonders geeignet: Wir gelangen einfach zur "Transformationsgleichung" oder "Modulargleichung" der fraglichen Funktion. Haben wir eine Gruppe mit $p>0$, so kommen zweckmäBig die Methoden der Geometrie bei Darstellung der Korrespondenzen zur Geltung, und gerade hier erhalten wir die Aufklärung ïber die Natur der irrationalen Modulargleichungen.

Ehe dies weiter ausgeführt wird, sollen die fünf ausgezeichneten Kongruenzuntergruppen und ihre Funktionssysteme zusammengestellt werden, die für die in $\S 8$ aufgestellten irrationalen Modulargleichungen in Betracht kommen. Zunächst ist eine ausgezeichnete Gruppe achter Stufe $\Gamma_{96}$ des Index $96 \mathrm{zu}$ nennen, bestehend aus allen die Kongruenzen:

$$
V \equiv\left(\begin{array}{ll}
1, & 0 \\
0,1
\end{array}\right),\left(\begin{array}{ll}
5, & 0 \\
0, & 5
\end{array}\right) \quad(\bmod 8)
$$


befriedigenden Substitutionen. Zu ihr gehört das System der Funktionen $\sqrt{k}, \sqrt{k^{\prime}}$, verbunden durch die Relation:

$$
(\sqrt{k})^{4}+\left(\sqrt{k^{\prime}}\right)^{4}=1
$$

An zweiter Stelle ordnen wir unsere obige $\Gamma_{384}$ ein. Um drei weitere Gruppen der Stufen 12, 24 und 48 zu erklären, verstehen wir unter $\Gamma_{3}$ die durch:

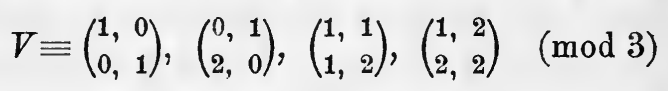

erklärte ausgezeichnete Kongruenzgruppe dritter Stufe (die der ausgezeichneten Vierergruppe $G_{4}$ in der Tetraedergruppe $G_{12}$ entspricht). Es handelt sich un die zur dritten Wurzel $\sqrt[3]{J}$ gehörende Gruppe, eine Funktion, die zum Integralmodul in der Beziehung steht:

$$
\sqrt[3]{J}=\frac{\sqrt[3]{4}\left(1-k^{2}+k^{4}\right)}{3 k k^{\prime} \sqrt[3]{k k^{\prime}}}
$$

An dritter Stelle nennen wir jetzt den Durchschnitt der $\Gamma_{3}$ und der Hauptkongruenzgruppe vierter Stufe $\Gamma_{24}$, der eine ausgezeichnete Kongruenzgruppe zwölfter Stufe $\Gamma_{72}$ des Index 72 ist. Als Funktionssystem haben wir $k, k^{\prime}$, $\sqrt[3]{J}$ oder auch (wegen (11)) die Funktionen $k, k^{\prime}, \sqrt[3]{k k^{\prime}}$, verbunden durch die beiden Relationen:

$$
k^{2}+k^{\prime 2}=1, \quad k \cdot k^{\prime}-\left(\sqrt[3]{k k^{\prime}}\right)^{3}=0 .
$$

Endlich folgen an vierter und fünfter Stelle die Durchschnitte der $\Gamma_{3}$ mit den beiden ersten Gruppen $\Gamma_{96}$ und $\Gamma_{384}$, die Kongrienzgruppen $\Gamma_{288}$ und $\Gamma_{152}$ der Stufen 24 und 48 sowie der Indizes 288 und 1152 sind. Ein System von Funktionen der $\Gamma_{288}$ ist $\sqrt{k}, \sqrt{k^{\prime}}, \sqrt[6]{k k^{\prime}}$, verbunden durch:

$$
(\sqrt{k})^{4}+\left(\sqrt{k^{\prime}}\right)^{4}=1, \quad \sqrt{k} \cdot \sqrt{k^{\prime}}-\left(\sqrt[6]{k k^{\prime}}\right)^{3}=0 ;
$$

ein solches der $\Gamma_{1152}$ aber haben wir in $\sqrt[4]{k}, \sqrt[4]{k^{\prime}}, \sqrt[12]{k k^{\prime}}$, verbunden durch die beiden Beziehungen:

$$
(\sqrt[4]{k})^{8}+\left(\sqrt[4]{k^{\prime}}\right)^{8}=1, \quad \sqrt[4]{k} \sqrt[4]{k^{\prime}}-\left(\sqrt[12]{k k^{\prime}}\right)^{3}=0
$$

Um die geometrische Sprechweise bequem einführen zu können, nennen wir die Funktionen des einzelnen Systems $x, y$ bzw. $x, y, z$ und deuten sie als rechtwinklige Koordinaten. in der Ebene bzw. im Raume. Wir haben dann unseren fünf Gruppen entsprechend, folgende Bedeutungen der $x, y, z$ und folgende Relationen:

$$
\begin{aligned}
& \Gamma_{96}, \quad x=\sqrt{k}, y=\sqrt{k^{\prime}}, \quad x^{4}+y^{4}=1, \\
& \Gamma_{384}, \quad x=\sqrt[4]{k}, \quad y=\sqrt[4]{k^{\prime}}, \quad x^{8}+y^{8}=1, \\
& \Gamma_{72}, \quad x=k, \quad y=k^{\prime}, \quad z=\sqrt[3]{k k^{\prime}} ; \quad x^{2}+y^{2}=1, \quad x y-z^{3}=0, \\
& \Gamma_{288}, \quad x=\sqrt{k}, \quad y=\sqrt{k^{\prime}}, \quad z=\sqrt[6]{k k^{\prime}}, \quad x^{4}+y^{4}=1, \quad x y-z^{3}=0 . \\
& \Gamma_{1152}, \quad x=\sqrt[4]{k}, \quad y=\sqrt[4]{k^{\prime}}, \quad z=\sqrt[12]{k k^{\prime}}, \quad x^{8}+y^{8}=1, \quad x y-z^{3}=0 .
\end{aligned}
$$


Die ersten beiden Relationen deuten wir als ebene Kurven vierten bzw. achten Grades, die drei weiteren Relationenpaare ergeben entsprechend Raumkurven sechsten, zwölften und $24^{\text {ten }}$ Grades.

Es gilt nun der Satz: Die irrationalen Modulargleichungen sind aufzufassen als die algebraischen Darstellungen der Modularkorrespondenzen auf den fraglichen Kurven. In den in $\S 8$ betrachteten Fällen sind diese Darstellungen besonders einfach, sie haben nämlich die Gestalten bilinearer Gleichungen zwischen den Koordinaten der beiden zugeordneten Punkte. Bezeichnet man die transformierten Funktionen durch $x^{\prime}, y^{\prime}$ bzw. $x^{\prime}, y^{\prime}, z^{\prime}$, so nehmen die beiden Gleichungen (1) und (2) S. 525 übereinstimmend die Gestalt an:

$$
x x^{\prime}+y y^{\prime}=1 \text {, }
$$

während die drei Gleichungen (9) und (10) S. 527 sich so schreiben:

$$
x x^{\prime}+y y^{\prime}+c z z^{\prime}=1,
$$

wo $c$ den Transformationsgraden 5, 11 und 23 entsprechend bzw. gleich $2 \sqrt[3]{4}, 2 \sqrt[3]{2}$ und $\sqrt[3]{4}$ ist. Die fünf in Rede stehenden Modularkorrespondenzen werden also auf den betreffenden Kurven durch Gerade bzw. durch Ebenen ausgeschnitten.

Die zur $\Gamma_{\mathbf{3 8 4}}$ gehörenden Modularkorrespondenzen sind ausführlich von E. Fiedler mit invariantentheoretischen Hilfsmitteln bebandelt. ${ }^{1}$ ) Die allgemeine Hurwitzsche Theorie der Modularkorrespondenzen, die auf transzendenter Grundlage ruht, ist in "Modulfunktionen" Bd. 2 ausführlich behandelt, worauf hier verwiesen sein mag.

\section{\$10. System der Modulfunktionen sechster Stufe.}

Nahe verwandt mit den irrationalen Modulargleichungen sind die schon S. 296 erwähnten "Thetarelationen“, die zahlreich in der älteren und neueren Literatur auftreten. Die algebraische Natur dieser Relationen und ihr Zusammenhang untereinander haben durch die Theorie der Modulfunktionen Aufklärung gewonnen. Um dies hier wenigstens für die bei dem dritten Transformationsgrade auftretenden Thetarelationen näher darzulegen, haben wir eine Zusammenstellung der Modulfunktionen sechster Stufe voraufzusenden.

Die Modulgruppe $\Gamma^{(\omega)}$ reduziert sich mod 6 auf eine Gruppe $G_{72}$ der Ordnung 72, in der die mod 3 mit 1 kongruenten Substitutionen eine $G_{6}$ vom Diedertypus, die mod 2 mit 1 kongruenten aber eine $G_{12}$ vom Tetraedertypus bilden. In der Diedergruppe $G_{6}$ ist bekanntlich eine aus-

1) In der Leipziger Dissertation „ர̈ber eine Klasse irrationaler Modulargleichungen der elliptischen Funktionen", veröffentlicht in der Züricher Vierteljahrsschrift, Bd. 30 (1886). 
gezeichnete zyklische $G_{3}$ enthalten; sie wird bei der vorliegenden $G_{6}$ von den folgenden Substitutionen der $G_{72}$ gebildet:

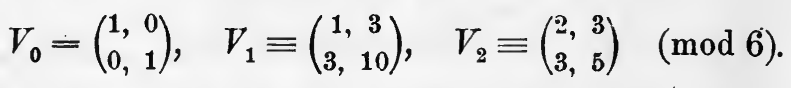

Die Tetraedergruppe enthält eine ausgezeichnete Vierergruppe $G_{4}$, die innerhalb der eben genannten Untergruppe $G_{12}$ der $G_{72}$ von den Substitutionen gebildet wird:

Für die drei letzten Substitutionen gilt, dem Typus der Vierergruppe entsprechend:

$$
\begin{gathered}
V_{2}^{\prime} \cdot V_{3}^{\prime} \equiv V_{3}^{\prime} \cdot V_{2}^{\prime} \equiv V_{1}^{\prime}, \quad V_{3}^{\prime} \cdot V_{1}^{\prime} \equiv V_{1}^{\prime} \cdot V_{3}^{\prime} \equiv V_{2}^{\prime}, \\
V_{1}^{\prime} \cdot V_{2}^{\prime} \equiv V_{2}^{\prime} \cdot V_{1}^{\prime} \equiv V_{3}^{\prime},
\end{gathered}
$$

wo sich die Kongruenzen hier und weiterhin auf den Modul 6 beziehen. Für die Transformation der vorstehenden Substitutionen mittelst der Substitution $S \equiv\left(\begin{array}{ll}1 & 1 \\ 0, & 1\end{array}\right)$ merken wir gleich die Regeln an:

$$
\left\{\begin{array}{c}
S \cdot V_{1} \cdot S^{-1} \equiv V_{2}, \quad S \cdot V_{2} \cdot S^{-1} \equiv V_{1}, \\
S \cdot V_{1}^{\prime} \cdot S^{-1} \equiv V_{3}^{\prime}, \quad S \cdot V_{2}^{\prime} \cdot S^{-1} \equiv V_{1}^{\prime}, \quad S \cdot V_{3}^{\prime} \cdot S^{-1} \equiv V_{2}^{\prime} .
\end{array}\right.
$$

Es erzeugt nun die Substitution $S$ innerhalb der $G_{72}$ eine zyklische $G_{6}$, in der die zyklische $G_{3}$ der Substitutionen $1, S^{2}, S^{4}$ und die zyklische $G_{2}$ der Substitutionen $1, S^{3}$ enthalten sind. Da aus (4) die Kon-

$$
V_{1} \cdot S^{2} \cdot V_{1}^{-1} \equiv S^{2}, \quad V_{2} \cdot S^{2} \cdot V_{2}^{-1} \equiv S^{2}
$$

folgen, so ist die zyklische $G_{3}$ nicht nur innerhalb der $G_{6}$, sondern innerhalb der Gruppe:

$$
G_{18}=G_{6}+G_{6} \cdot V_{1}+G_{6} \cdot V_{2}
$$

ausgezeichnet. Die $G_{3}$ ist demnach höchstens eine der unter $72: 18=4$ gleichberechtigten Gruppen. Da weiter aus (4) und (3) die Kongruenzen: $V_{1}^{\prime} \cdot S^{2} \cdot V_{1}^{\prime-1} \equiv S^{2} \cdot V_{2}^{\prime}, \quad V_{2}^{\prime} \cdot S^{2} \cdot V_{2}^{\prime-1} \equiv S^{2} \cdot V_{3}^{\prime}, \quad V_{3}^{\prime} \cdot S^{2} \cdot V_{3}^{\prime-1} \equiv S^{2} \cdot V_{1}^{\prime}$ folgen, so erhalten wir tatsächlich vier gleichberechtigte Gruppen:

$$
G_{3}, \quad V_{1}^{\prime} \cdot G_{3} \cdot V_{1}^{\prime-1}, \quad V_{2}^{\prime} \cdot G_{3} \cdot V_{2}^{\prime-1}, \quad V_{3}^{\prime} \cdot G_{3} \cdot V_{3}^{\prime-1} \text {. }
$$

In ähnlicher Weise zeigt man: Es gibt in der $G_{72}$ drei gleichberechtigte zyklische Untergruppen:

$$
G_{2}, \quad V_{1} \cdot G_{2} \cdot V_{1}^{-1}, \quad V_{2} \cdot G_{2} \cdot V_{2}^{-1} .
$$

Der $G_{6}$ entspricht die durch $\gamma \equiv 0(\bmod 6)$ zu erklärende Kongruenzgruppe sechster Stufe $\Gamma_{12}$, deren Diskontinuitätsbereich das in Fig. 4, S. 354, dargestellte Transformationspolygon für den sechsten Grad 
ist. Dieses Polygon hatte das Geschlecht 0. Zur $G_{3}$ gehört entsprechend die durch $\gamma \equiv 0(\bmod 6), \beta \equiv 0(\bmod 2)$ erklärte Kongruenzgruppe $\Gamma_{\mathbf{9 4}}$,

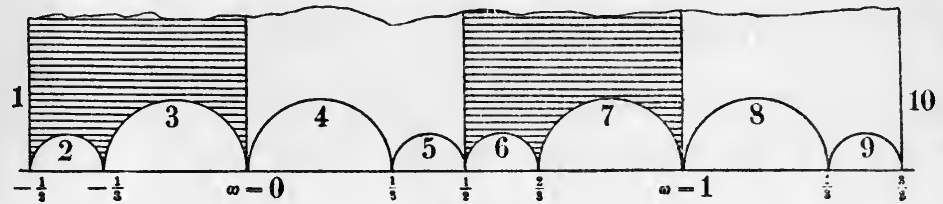

Fig. 39.

deren Diskontinuitätsbereich die in Fig. 39 dargestellte Gestalt hat. Aus der Zusammenordnung der mit Nummern versehenen Seiten:

$$
\begin{gathered}
1 \rightarrow 10,\left(\begin{array}{l}
1,2 \\
0,1
\end{array}\right) ; 2 \rightarrow 5,\left(\begin{array}{c}
5,2 \\
12,5
\end{array}\right) ; 3 \rightarrow 4,\left(\begin{array}{l}
1,0 \\
6,1
\end{array}\right) ; 6 \rightarrow 9,\left(\begin{array}{c}
17,-10 \\
12,-7
\end{array}\right) ; \\
7 \rightarrow 8,\left(\begin{array}{l}
7,-6 \\
6,-5
\end{array}\right)
\end{gathered}
$$

ergibt sich als Geschlecht dieses Bereiches gleichfalls 0. Zur $G_{2}$ gehört endlich die durch $\gamma \equiv 0(\bmod 6), \beta \equiv 0(\bmod 3)$ erklärte Kongruenz-

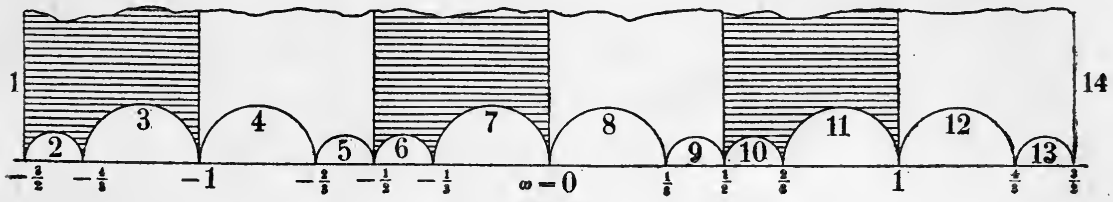

Fig. 40.

gruppe $\Gamma_{36}$, deren Diskontinuitätsbereich in Fig. 40 abgebildet ist. Die Seitenzuordnung:

$$
\begin{gathered}
1 \rightarrow 14,\left(\begin{array}{l}
1,3 \\
0,1
\end{array}\right) ; 2 \rightarrow 13,\left(\begin{array}{l}
17,24 \\
12,17
\end{array}\right) ; 3 \rightarrow 4,\left(\begin{array}{c}
5,6 \\
-6,-7
\end{array}\right) ; \\
5 \rightarrow 6,\left(\begin{array}{c}
5,3 \\
-12,-7
\end{array}\right) ; 7 \rightarrow 8,\left(\begin{array}{l}
1,0 \\
6,1
\end{array}\right) ; 9 \rightarrow 10,\left(\begin{array}{c}
7,-3 \\
12,-5
\end{array}\right) ; \\
11 \rightarrow 12,\left(\begin{array}{l}
7,-6 \\
6,-5
\end{array}\right)
\end{gathered}
$$

ergibt auch für diesen Bereich wieder das Geschlecht 0 .

An Stelle der S. $446 \mathrm{ff}$. benutzten einwertigen Funktion $\tau$ der $\Gamma_{12}$ soll hier die Funktion:

$$
z(\omega)=9\left(1+\frac{4}{\tau(\omega)}\right)
$$

eingeführt werden, deren Spitzenwerte sich aus denen von $\tau(\omega)$ so berechnen:

$$
z(i \infty)=\infty, \quad z(0)=9, \quad z\left( \pm \frac{1}{3}\right)=1, \quad z\left( \pm \frac{1}{2}\right)=0 .
$$

Mittelst $z(\omega)$ werden die Bereiche der Fig. 39 und 40 auf Riemannsche Flächen mit 2 bzw. 3 Blättern abgebildet; und zwar hat die erste Fläche zwei Verzweigungspunkte bei $z=\infty$ und $z=0$, die zweite aber zwei 
dreiblättrige Verzweigungspunkte bei $z=\infty$ und $z=1$. Als einwertigè Funktionen der beiden Gruppen $\Gamma_{24}$ und $\Gamma_{36}$ kann man demnach:

$$
y(\omega)=\sqrt{z(\omega)}, \quad x(\omega)=\sqrt[3]{z(\omega)-1}
$$

benutzen, wo die erste Wurzel auf der imaginären $\omega$-Achse positiv und die zweite reell gewählt werden mag. Die Spitzenwerte der Funktion y sind dann:

$$
\left\{\begin{array}{l}
y(i \infty)=\infty, \quad y\left( \pm \frac{1}{2}\right)=y\left(\frac{3}{2}\right)=0, \quad y\left( \pm \frac{1}{3}\right)=1 \\
y\left(\frac{2}{3}\right)=y\left(\frac{4}{3}\right)=-1, \quad y(0)=3, \quad y(1)=-3
\end{array}\right.
$$

diejenigen der Funktion $x$ aber:

$$
\left\{\begin{array}{l}
x(i \infty)=\infty, \quad x(0)=2, \quad x( \pm 1)=2 \varepsilon^{\mp 1}, \quad x\left( \pm \frac{3}{2}\right)=-1 \\
x\left( \pm \frac{1}{2}\right)=-\varepsilon^{ \pm 1}, \quad x\left( \pm \frac{1}{3}\right)=x\left( \pm \frac{2}{3}\right)=x\left( \pm \frac{4}{3}\right)=0
\end{array}\right.
$$

wo $\varepsilon$ die dritte Einheitswurzel $e^{\frac{2 i \pi}{3}}$ bedeutet. Die Wirkung der Substitution $S$ auf $y$ und $x$ ist:

$$
y(\omega+1)=-y(\omega), \quad x(\omega+1)=\varepsilon^{-1} x(\omega) .
$$

Gegenüber $V_{1}=\left(\begin{array}{c}1,3 \\ 3,10\end{array}\right)$ und $V_{2}=\left(\begin{array}{l}2,3 \\ 3,5\end{array}\right)$ substituiert sich $y(\omega)$ linear, da diese Substitutionen die $\Gamma_{24}$ in sich transformieren. Durch $V_{1}$ werden die Spitzen $\omega=i \infty,-3,-\frac{7}{2}$ bzw. in $\omega=\frac{1}{3}, 0,1$ übergeführt, und also die Werte $y=\infty,-3,0$ bzw. in $y=1,+3,-3$. Hieraus schließt man leicht auf die erste der beiden Gleichungen:

$$
y\left(V_{1}(\omega)\right)=\frac{y(\omega)-3}{y(\omega)+1}, \quad y\left(V_{2}(\omega)\right)=\frac{y(\omega)+3}{-y(\omega)+1},
$$

während die zweite entsprechend folgt. Durch Kombination mit der ersten Substitution (12) ergibt sich für $y$ eine aus den sechs Substitutionen:

$$
y^{\prime}= \pm y, \quad y^{\prime}= \pm \frac{y-3}{y+1}, \quad y^{\prime}=\mp \frac{y+3}{y-1}
$$

bestehende Diedergruppe $G_{6}$.

Entsprechendes gilt für $x(\omega)$. Hier ist die Wirkung der obigen Substitutionen $V_{1}^{\prime}, V_{2}^{\prime}, V_{3}^{\prime}$ :

$$
\begin{gathered}
x\left(V_{1}^{\prime}(\omega)\right)=-\frac{x(\omega)-2}{x(\omega)+1}, \quad x\left(V_{2}^{\prime}(\omega)\right)=-\frac{x(\omega)-2 \varepsilon}{\varepsilon^{2} x(\omega)+1}, \\
x\left(V_{3}^{\prime}(\omega)\right)=-\frac{x(\omega)-2 \varepsilon^{2}}{\varepsilon x(\omega)+1},
\end{gathered}
$$

woraus man durch Kombination mit der zweiten Substitution (12) zwölf, eine Tetraedergruppe bildende Substitutionen erhält:

$$
x^{\prime}=\varepsilon^{v} x, \quad x^{\prime}=-\varepsilon^{v} \frac{x-2}{x+1}, \quad x^{\prime}=-\varepsilon^{v} \frac{x-2 \varepsilon^{\perp-1}}{\varepsilon^{\overline{+}} x+1},
$$


Die Funktionen $x$ und $y$, die zufolge (9) in der Beziehung:

$$
\dot{y}^{2}=x^{3}+1
$$

stehen, bilden zusammengenommen ein einfachstes System von Funktionen für die Hauptkongruenzgruppe sechster Stufe $\Gamma_{72}$. Alle Funktionen dieser $\Gamma_{72}$ sind dann rational in $x$ und $y$ darstellbar. Diese Darstellungen sollen insbesondere für die mit $y$ und $x$ gleichberechtigten Funktionen angegeben werden. Zufolge (5) können wir für die vier mit der $\Gamma_{24}$ gleichberechtigten Gruppen als einwertige Funktionen:

$$
\begin{gathered}
y_{0}(\omega)=y(\omega), \quad y_{1}(\omega)=y\left(V_{1}^{\prime}(\omega)\right), \quad y_{2}(\omega)=y\left(V_{2}^{\prime}(\omega)\right), \\
y_{3}(\omega)=y\left(V_{3}^{\prime}(\omega)\right)
\end{gathered}
$$

benutzen. Hieran reihen sich zufolge (6) für die drei mit $\Gamma_{36}$ gleichberechtigten Gruppen die Funktionen:

$$
x_{0}(\omega)=x(\omega), \quad x_{1}(\omega)=x\left(V_{1}(\omega)\right), \quad x_{2}(\omega)=x\left(V_{2}(\omega)\right) .
$$

Nun ergibt sich mit Benutzung von (15) und (17):

$$
y_{1}^{2}=x\left(V_{1}^{\prime}(\omega)\right)^{3}+1=1+\left(\frac{2-x(\omega)}{1+x(\omega)}\right)^{3} .
$$

Der rechts stehende Ausdruck muB sich mit Hilfe von (17) in das Quadrat einer rationalen Funktion von $x$ und $y$ umwandeln lassen. In der Tat findet man:

$$
1+\left(\frac{2-x}{1+x}\right)^{3}=9 \frac{1-x+x^{2}}{(1+x)^{3}}=9 \frac{1+x^{3}}{(1+x)^{4}}=\left(\frac{3 y}{(1+x)^{2}}\right)^{2},
$$

womit der Ausdruck von $y_{1}$ in $x$ und $y$ bis auf das Vorzeichen gegeben ist. Das Vorzeichen aber bestimmt man leicht durch Eintragen des Wertes $\omega=0$. Entsprechend findet man die Ausdrücke für $y_{2}$ und $y_{3}$. Es gilt der Satz: Die drei mit y gleichberechtigten Funktionen $y_{1}, y_{2}, y_{3}$ stellen sich in $x$ und $y$ wie folgt dar:

$$
y_{1}=-\frac{3 y}{(1+x)^{2}}, \quad y_{2}=-\frac{3 y}{\left(1+\varepsilon^{2} x\right)^{2}}, \quad y_{3}=-\frac{3 y}{(1+\varepsilon x)^{2}} .
$$

Eine ähnliche Rechnung wird man für die $x_{1}, x_{2}$ leicht ausführen. Die mit $x$ gleichberechtigten Funktionen $x_{1}$ und $x_{2}$ stellen sich in $x$ und $y$ so dar:

$$
x_{1}=-\frac{2 x}{1+y}, \quad x_{2}=-\frac{2 x}{1-y}
$$

Ein paar naheliegende Folgerungen aus (17) und (20) sind:

$$
\left\{\begin{array}{c}
\frac{1}{y}+\frac{1}{y_{1}}+\frac{1}{y_{2}}+\frac{1}{y_{3}}=0 \\
\frac{1}{y^{2}}+\frac{1}{y_{1}^{2}}+\frac{1}{y_{2}^{2}}+\frac{1}{y_{3}^{2}}=\frac{4}{3} \\
y \cdot y_{1} \cdot y_{2} \cdot y_{3}=-27
\end{array}\right.
$$


Ebenso ergibt sich aus (17) und (21):

$$
\left\{\begin{array}{c}
\frac{1}{x}+\frac{1}{x_{1}}+\frac{1}{x_{2}}=0 \\
\frac{1}{x^{3}}+\frac{1}{x_{1}^{3}}+\frac{1}{x_{2}^{3}}=-\frac{3}{4} \\
x \cdot x_{1} \cdot x_{2}=-4 .
\end{array}\right.
$$

Um die folgenden Rechnungen nicht unterbrechen zu müssen, stellen wir noch die Wirkung der Substitution $T=\left(\begin{array}{c}0,1 \\ -1,0\end{array}\right)$ auf $y(\omega)$ fest. Die Gruppe $T \cdot \Gamma_{24} \cdot T^{-1}$ ist durch $\beta \equiv 0(\bmod 6), \gamma \equiv 0(\bmod 2)$ charakterisiert. Den gleichen Kongruenzen genügt aber die Gruppe $V_{1}^{\prime} \cdot \Gamma_{24} \cdot V_{1}^{\prime-1}$, so daB die beiden Funktionen $y\left(\frac{-1}{\omega}\right)$ und $y_{1}(\omega)$ linear zusammenhängen:

$$
y\left(\frac{-1}{\omega}\right)=\frac{a y_{1}(\omega)+b}{c y_{1}(\omega)+d} \text {. }
$$

Man setze nacheinander die drei Werte $\omega=i \infty, 0$ und -1 ein und findet, daB den Werten $y_{1}(\omega)=0,-1,-3$ bzw. die Werte $y\left(\frac{-1}{\omega}\right)=3$, $\infty,-3$ entsprechen. Hieraus bestimmen sich die Koeffizienten $a, b, c, d$ :

$$
y\left(\frac{-1}{\omega}\right)=-\frac{y_{1}(\omega)-3}{y_{1}(\omega)+1} .
$$

\section{\$ 11. Die Thetarelationen des dritten Transformationsgrades.}

Die drei Nullwerte der geraden Thetafunktionen bezeichnen wir wie üblich kurz durch $\vartheta_{v}$ für $\nu=0,2,3$ an Stelle der ausführlichen Schreibweise $\vartheta_{v}(q)$. Sie gehen durch die erste Haupttransformation dritten Grades über in $\vartheta_{v}\left(q^{3}\right)$, wofür wir kurz $\theta_{v}$ schreiben. Für die übrigen drei Transformationen dritten Grades werden wir unten die Bezeichnungen $\theta_{v}^{(1)}, \theta_{v}^{(2)}, \theta_{v}^{(3)}$ näher erklären. Diese Größen stehen nun in nächster Beziehung zu den in $\S 10$ betrachteten Funktionen sechster Stufe, und umgekehrt werden wir die zum dritten Transformationsgrade gehörenden "Thetarelationen" aus den grundlegenden algebraischen Relationen des vorigen Paragraphen ableiten können.

Zunächst sind der Integralmodul $k^{2}(\omega)$ und die durch die erste Haupttransformation entstehende Funktion $k^{2}(3 \omega)$ gegenüber der $\Gamma_{24}$ invariant und also rational in $y$ darstellbar. Man stellt sehr leicht die Werteverteilung jener beiden Funktionen im Bereiche der Fig. 39 fest, indem man einmal die ursprüngliche $\omega$-Teilung (für $k^{2}(\omega)$ ), sodann die auf ein Drittel reduzierte $\omega$-Teilung (für $k^{2}(3 \omega)$ ) einträgt. Es ergeben sich daraus die Darstellungen:

(1) $\quad k^{2}(\omega)=\left(\frac{\vartheta_{q}}{\vartheta_{3}}\right)^{4}=\frac{16 y^{3}}{(y-1)(y+3)^{3}}, \quad k^{2}(3 \omega)=\left(\frac{\theta_{2}}{\theta_{3}}\right)^{4}=\frac{16 y}{(y-1)^{3}(y+3)}$. 
Mit Benutzung von (23) in I, 419 folgt hieraus weiter:

$$
\left(\frac{\vartheta_{0}}{\vartheta_{3}}\right)^{4}=\frac{(y+1)(y-3)^{3}}{(y-1)(y+3)^{3}}, \quad\left(\frac{\theta_{0}}{\theta_{3}}\right)^{4}=\frac{(y+1)^{3}(y-3)}{(y-1)^{3}(y+3)} .
$$

Für den zum zweiten Teilungsgrade gehörenden Teilwert $\sigma_{01}$ der Sigmafunktion ergibt sich aus einer in I, 452 aufgestellten Gleichung bei wiederholter Benutzung der Produktdarstellung der Diskriminante $\Delta$ :

$$
\sigma_{01}\left(\omega_{1}, \omega_{2}\right) \sqrt[12]{\Delta}=-2 q^{\frac{1}{6}} \prod_{m=1}^{\infty}\left(1+q^{2 m}\right)^{2},
$$

(3) $\sigma_{01}\left(\omega_{1}, \omega_{2}\right) \sqrt[6]{\Delta}=-2 \sqrt[12]{\Delta_{2}}, \quad \sigma_{01}\left(3 \omega_{1}, \omega_{2}\right) \sqrt[6]{\Delta_{3}}=-2 \sqrt[12]{\Delta_{6}}$, wo $\Delta_{v}$ im Sinne von S. 438 gebraucht ist. Den Übergang zur $\vartheta_{2}$-Funktion vermitteln die Gleichungen:

$$
\sqrt{\frac{\omega_{2}}{2 \pi}} \sqrt[8]{\Delta} \sigma_{01}\left(\omega_{1}, \omega_{2}\right)=\vartheta_{2}, \quad \sqrt{\frac{\omega_{z}}{2 \pi}} \sqrt[8]{\Delta_{3}} \sigma_{01}\left(3 \omega_{1}, \omega_{2}\right)=\theta_{2}
$$

Aus (3) ergibt sich daraufhin leicht:

$$
\left(\frac{\vartheta_{2}}{\theta_{2}}\right)^{2}=\sqrt[4]{\frac{\Delta}{\Delta_{3}}}\left(\frac{\sigma_{01}\left(\omega_{1}, \omega_{2}\right)}{\sigma_{01}\left(3 \omega_{1}, \omega_{2}\right)}\right)^{2}=\sqrt[12]{\frac{\Delta_{3} \Delta_{2}^{2}}{\Delta \Delta_{6}^{2}}}
$$

Nun folgt aus den S. $447 \mathrm{ff}$. entwickelten Gleichungen der Transformation sechsten Grades:

$$
\frac{\Delta_{2}}{\Delta}=\frac{\tau(\tau+4)^{3}}{2^{8}(2 \tau+9)}, \quad \frac{\Delta_{3}}{\Delta}=\frac{\tau^{2}(2 \tau+9)^{4}}{3^{12}(\tau+4)^{2}}, \quad \frac{\Delta_{6}}{\Delta}=\frac{\tau^{5}(2 \tau+9)}{2^{8} \cdot 3^{12}(\tau+4)} .
$$

Bei Zusammenfassung dieser Gleichungen ergibt sich unter Einführung der Funktionen $z$ und $y$ von $\S 10$ :

$$
\frac{\Delta_{3} \Delta_{2}^{2}}{\Delta \Delta_{6}^{2}}=3^{12} \frac{(\tau+4)^{6}}{\tau^{6}}=\left(9\left(1+\frac{4}{\tau}\right)\right)^{6}=z^{6}=y^{12} .
$$

Man wird also zur ersten der drei folgenden Gleichungen geführt:

$$
\left(\frac{\vartheta_{2}}{\theta_{2}}\right)^{2}=y, \quad\left(\frac{\vartheta_{3}}{\theta_{3}}\right)^{2}=\frac{y+3}{y-1}, \quad\left(\frac{\vartheta_{0}}{\theta_{0}}\right)^{2}=\frac{y-3}{y+1},
$$

während sich die zweite und dritte durch Vermittlung von (1) und (2) berechnen. ${ }^{1}$ )

Auf die Gleichungen (5) übe man die Substitution $T=\left(\begin{array}{r}0,1 \\ -1,0\end{array}\right)$ aus, deren Wirkung auf $y$ in (24) S. 538 berechnet ist. Die ursprünglichen $\vartheta$-Nullwerte transformieren sich zufolge (4) in I, 482 so:

$$
\begin{gathered}
\vartheta_{0}\left(e^{\left.-\frac{\pi i}{\omega}\right)^{2}=}-i \omega \vartheta_{2}(q)^{2}, \quad \vartheta_{2}\left(e^{\left.-\frac{\pi i}{\omega}\right)^{2}}=-i \omega \vartheta_{0}(q)^{2},\right.\right. \\
\vartheta_{3}\left(e^{\left.-\frac{\pi i}{\omega}\right)^{2}}=-i \omega \vartheta_{3}(q)^{2} .\right.
\end{gathered}
$$

Für die transformierten Thetanullwerte ergibt sich entsprechend:

1) Bei Wurzelziehungen wolle man die zutreffenden Einheitswurzeln stets durch Betrachtung der Werte unserer Funktionen auf der imaginären $\omega$-Achse bestimmen. 


$$
\begin{aligned}
& \theta_{0}\left(e^{-\frac{\pi i}{\omega}}\right)^{2}=\vartheta_{0}\left(e^{-\frac{3 \pi i}{\omega}}\right)^{2}=-i \frac{\omega}{3} \vartheta_{2}\left(q^{\frac{1}{3}}\right)^{2}, \\
& \theta_{2}\left(e^{-\frac{\pi i}{\omega}}\right)^{2}=\vartheta_{2}\left(e^{-\frac{3 \pi i}{\omega}}\right)^{2}=-i \frac{\omega}{3} \vartheta_{0}\left(q^{\frac{1}{3}}\right)^{2} . \\
& \theta_{3}\left(e^{-\frac{\pi i}{\omega}}\right)^{2}=\vartheta_{3}\left(e^{-\frac{3 \pi i}{\omega}}\right)^{2}=-i \frac{\omega}{3} \vartheta_{3}\left(q^{\frac{1}{3}}\right)^{2} .
\end{aligned}
$$

Hier liegt rechts die zweite Haupttransformation dritten Grades vor, für die wir folgende Abkürzungen einführen:

$$
\vartheta_{v}\left(q^{\frac{1}{3}}\right)=i \sqrt{3} \theta_{v}^{(1)}
$$

Die Gleichungen (5) rechnen sich damit um auf:

$$
\left(\frac{\vartheta_{2}}{\theta_{2}^{(1)}}\right)^{2}=y_{1}, \quad\left(\frac{\vartheta_{3}}{\theta_{3}^{(1)}}\right)^{2}=\frac{y_{1}+3}{y_{1}-1}, \quad\left(\frac{\vartheta_{0}}{\theta_{0}^{(1)}}\right)^{2}=\frac{y_{1}-3}{y_{1}+1} .
$$

Um die beiden letzten Repräsentanten zu gewinnen, üben wir auf (6) die Substitutionen $\left(\begin{array}{l}1,8 \\ 0,1\end{array}\right)$ und $\left(\begin{array}{c}1,16 \\ 0,1\end{array}\right)$ aus, wobei $y_{1}$ in $y_{2}$ bzw. $y_{3}$ übergeht, die $\vartheta_{v}$ unverändert bleiben und die $\theta_{v}^{(1)}$ die transformierten Größen $\theta_{v}^{(2)}$ bzw. $\theta_{v}^{(3)}$ liefern mögen. Unter Hinzunahme der Gleichungen (6) findet man:

$$
\left(\frac{\vartheta_{2}}{\theta_{2}^{(i)}}\right)^{2}=y_{i}, \quad\left(\frac{\vartheta_{3}}{\theta_{3}^{(i)}}\right)^{2}=\frac{y_{i}+3}{y_{i}-1}, \quad\left(\frac{\vartheta_{0}}{\theta_{0}^{(i)}}\right)^{2}=\frac{y_{i}-3}{y_{i}+1}, \quad i=1,2,3 .
$$

Aus (20) S. 537 folgt:

$$
1+x=i \sqrt{3} \sqrt{\frac{y}{y_{1}}} .
$$

Mittelst (5) und (6) folgt hieraus die erste der Gleichungen:

$$
1+x=i \sqrt{3} \frac{\theta_{2}^{(1)}}{\theta_{2}}, \quad 1+x_{1}=i \sqrt{3} \frac{\theta_{0}^{(1)}}{\theta_{0}}, \quad 1+x_{2}=i \sqrt{3} \frac{\theta_{3}^{(1)}}{\theta_{3}} .
$$

Die zweite und dritte Gleichung kann man aus der ersten etwa durch Ausübung der S. 536 erklärten Substitutionen $V_{1}, V_{2}$ gewinnen, denen gegenüber $y$ und $y_{1}$ gleiche lineare Substitutionen erfahren. Durch Multiplikation je zweier Gleichungen (8) lassen sich bei Benutzung der Relationen (23) S. 538 hieraus noch die Formeln:

herstellen.

$$
\left\{\begin{array}{l}
1-\frac{2}{x}=i \sqrt{3} \sqrt{\frac{\theta_{0}^{(1)} \theta_{3}^{(1)}}{\theta_{0} \theta_{3}}} \\
1-\frac{2}{x_{1}}=i \sqrt{3} \sqrt{\frac{\theta_{2}^{(1)} \theta_{3}^{(1)}}{\theta_{2} \theta_{3}}} \\
1-\frac{2}{x_{2}}=i \sqrt{3} \sqrt{\frac{\theta_{0}^{(1)} \theta_{2}^{(1)}}{\theta_{0} \theta_{2}}}
\end{array}\right.
$$

Schließlich notieren wir noch die aus (1) und (2) leicht gewinnbaren Gleichungen: 


$$
\left\{\begin{array}{c}
\frac{2}{y+1}=\sqrt{\frac{\vartheta_{0} \theta_{2}^{3}}{\vartheta_{2} \theta_{0}^{3}}}, \quad \frac{2}{y-1}=\sqrt{\frac{\vartheta_{s} \theta_{2}^{3}}{\vartheta_{2} \theta_{3}^{3}}}, \\
\frac{2 y}{y-3}=\sqrt{\frac{\vartheta_{2}^{3} \theta_{0}}{\vartheta_{0}^{3} \theta_{2}}}, \quad \frac{2 y}{y+3}=\sqrt{\frac{\vartheta_{2}^{3} \theta_{3}}{\vartheta_{3}^{3} \theta_{2}}} .
\end{array}\right.
$$

Die Thetarelationen des dritten Transformationsgrades sind nun einfach die Ergebnisse der Elimination der $x$ und $y$ aus den vorstehenden Gleichungen, wobei die in $\$ 10$ aufgestellten Beziehungen zwischen den $x, y$ heranzuziehen sind. Setzt man z. B. den aus (5) folgenden Wert von $y$ der Reihe nach in die vier Gleichungen (10) ein, so entstehen die Relationen:

$$
\begin{gathered}
\frac{\vartheta_{2}^{2}+\theta_{z}^{2}}{2 \sqrt{\vartheta_{2} \theta_{2}}}=\sqrt{\frac{\theta_{0}^{3}}{\vartheta_{0}}}, \quad \frac{\vartheta_{z}^{2}-\theta_{2}^{2}}{2 \sqrt{\vartheta_{2} \theta_{2}}}=\sqrt{\frac{\theta_{3}^{3}}{\vartheta_{3}}}, \\
\vartheta_{2}^{2}-3 \theta_{2}^{2}=2 \sqrt{\vartheta_{2} \theta_{2}} \sqrt{\frac{\vartheta_{0}^{3}}{\theta_{0}^{3}}}, \quad \vartheta_{2}^{2}+3 \theta_{2}^{2}=2 \sqrt{\vartheta_{2} \theta_{2}} \sqrt{\frac{\vartheta_{3}^{3}}{\theta_{3}}} .
\end{gathered}
$$

Aus den beiden ersten Gleichungen (5) folgt:

$$
\left(\vartheta_{2}^{2}-\theta_{2}^{2}\right) \vartheta_{3}^{2}=\left(\vartheta_{2}^{2}+3 \theta_{2}^{2}\right) \theta_{3}^{2},
$$

aus der zweiten und vierten Gleichung (10):

$$
\vartheta_{2}^{2} \theta_{3}^{2}-\vartheta_{3}^{2} \theta_{2}^{2}=2 \vartheta_{0} \theta_{0} \sqrt{\vartheta_{2} \vartheta_{3}} \sqrt{\theta_{2} \theta_{3}}
$$

aus den beiden letzten Gleichungen (10):

$$
\sqrt{\frac{\hat{\vartheta}_{s}^{s}}{\theta_{2}}}-\sqrt{\frac{\hat{\vartheta}_{0}^{3}}{\theta_{0}}}-\sqrt{\frac{\hat{\vartheta}_{s}^{s}}{\theta_{s}}}=0
$$

aus dreien unter ihnen:

$$
\sqrt{\frac{\theta_{3}^{3}}{\vartheta_{3}}}+\sqrt{\frac{\theta_{0}^{3}}{\vartheta_{0}}}-\sqrt{\frac{\vartheta_{2}^{3}}{\theta_{2}}}=0
$$

Die erste Gleichung (23) S. 538 schreibt sich mit Hilfe von (8) in:

$$
\frac{\theta_{0}}{\theta_{0}-i \sqrt{3} \theta_{0}^{(1)}}+\frac{\theta_{2}}{\theta_{2}-i \sqrt{3} \theta_{2}^{(1)}}+\frac{\theta_{3}}{\theta_{3}-i \sqrt{3} \theta_{3}^{(1)}}=0
$$

um, mittelst der Gleichungen (9) aber in:

$$
\left.\sqrt{\frac{\theta_{2}^{(1)} \theta_{3}^{(1)}}{\theta_{2} \theta_{3}}}+\sqrt{\frac{\theta_{3}^{(1)} \theta_{0}^{(1)}}{\theta_{3} \theta_{0}}}+\sqrt{\frac{\theta_{0}^{(1)} \theta_{2}^{(1)}}{\theta_{0} \theta_{2}}}+i \sqrt{3}=0 . .^{1}\right)
$$

1) Ähnliche Ausführungen für den fünften Transformationsgrad finden sich in der Dissertation des Verfassers „Über Systeme elliptischer Modulfunktionen von niederer Stufenzahl“" (Braunschweig 1885). 


\section{Sachregister.}

Die Stichworte sind gesperrt gedruckt. Wiederholungen von Stichworten sind durch Bindestrichẹ angedeutet. Die Ziffern beziehen sich auf die Seiten.

\section{A}

Abelsche Gleichungen 61 .

Abelsche Gruppe 4, $14 \mathrm{ff}$; $--G_{256}$ bei den Additionstheoremen 168.

Abelsche Relationen $240 \mathrm{ff}$.

Abgeleitete binäre quadratische Form 137.

Additionstheorem des Integrals zweiter Gattung 159, 183; -e der $\wp$ - und $\wp^{\prime}$-Funktion $160 \mathrm{ff}$, in invarianter Gestalt 162, 164; -e der Funktionen sn, cn, dn 166, 180; -e der Thetafunktionen $177 \mathrm{ff}$; $-e$ für mehrgliedrige Argumentsummen 183.

Adjunktion einer Zahl zu einem Körper 33; gleichzeitige - mehrerer Zahlen zu einem Zahlkörper $35 \mathrm{ff}$; gleichzeitige - mehrerer Funktionen zu einem Funktionenkörper $66 \mathrm{ff}$.

Algebraische Zahlen in bezug auf einen Körper 33; konjugierte - 33 ; - - allgemeiner Begriff 78; ganze - 78, $85 \mathrm{ff}$.

Algebraische Zahlkörper $n^{\text {ten }}$ Grades 83.

Allgemeine Teilungsgleichung, s. "Teilungsgleichung".

Allgemeine Transformationsgleichung,s.,"Transformationsgleichung".

Alternierende Gruppe 26.

Ambige quadratische Form 140.

Äquivalenz der Ideale 104; - der Zweigideale 132; - der quadratischen Formen 138.

Arithmetisch-geometrisches Mittel 495 .

Assoziatives Gesetz bei Gruppen 2. Assoziierte Zahlen 86 .

Auflösung einer algebraischen Gleichung $55 \mathrm{ff} ., 75$; - der allgemeinen Teilungsgleichung 225. $231 \mathrm{ff}$., der speziellen $262 \mathrm{ff}$; - der Transformationsgleichung der $\wp$-Funktion 280.
Ausgezeichnete Untergruppe 8; Sätze über - $-\mathrm{n} 9 \mathrm{ff}$; gröBte $-\mathbf{1 2}$.

\section{B}

Basis des Systems der ganzen Zahlen eines algebraischen Körpers 87; eines Ideals 91, $99 \mathrm{ff}$; - eines 7weigideals 135.

Bilinear, -e Substitutionen in der Komposition der quadratischen Formen 149; -e Verbindungen aus Funktionen $X_{\lambda}\left(u \mid \omega_{1}, \omega_{2}\right) 320 \mathrm{ff}$.

\section{C}

Charaktere der Klassen quadratischer Formen 152.

\section{D}

Darstellung von Zahlen durch quadratische Formen 151.

Determinante von Brioschi und Kiepert 186.

Differentialgleichung, deren Integral das Additionstheorem liefert 163; partielle - der Funktion $\psi^{(n)} 191$; partielle - der Funktionen $G(z)$ bei den Multiplikationssätzen 208.

Diskriminante von $n$ Zahlen eines algebraischen Körpers 84; - eines Körpers 86; - eines quadratischen Körpers 121; - einer quadratischen Form 137; transformierte $-\Delta, \mathrm{Be}-$ ziehung zu den Teilwerten 298ff.; Transformationsgleichungen für die - $\triangle 339 \mathrm{ff}$., 343, 380 usw.

Divisionstheoreme für die Funktionen $\wp, \wp^{\prime} 210 \mathrm{ff}$; - für die Funktionen zweiter Stufe $235 \mathrm{ff}$.

Durchschnitt mehrerer Gruppen 8.

\section{$\mathbf{E}$}

Eigentliche Transformation $n^{\text {ten }}$ Grades 276. 
Einfache Gruppe 8.

Einheiten in algebraischen Körpern 85.

Einheitselement einer Gruppe 3.

Einheitsideal 90.

Einheitswurzel, primitive $\boldsymbol{n}^{\text {ten }}$ Grades 57.

Element einer Gruppe 2.

Elliptische Funktionen $n^{\text {ter }}$ Stufe, $225 \mathrm{ff}$., ihre Berechnung durch Radikale 230.

Erzeugendes Element einer zyklischen Gruppe 6.

\section{$\mathbf{F}$}

Faktorenzerlegung einer Funktion in einem Körper 32 ; - eines Ideals $95 \mathrm{ff}$.

Form, s. "Quadratische Form".

Formklassen 138; Beziehung der zu den Klassen der Zweigideale 147.

Funktionenkörper 64; Funktionen und Gleichungen in einem - 65 .

\section{G}

Galoissche Gleichungstheorie $46 \mathrm{ff}$., $54 \mathrm{ff}$.

Galoissche Gruppe einer Gleichung 46,$73 ;-1$ der speziellen Teilungsgleichung 260, ihre arithmetische Darstellung 261; - - der speziellen Transformationsgleichungen $459 \mathrm{ff}$.

Galois sche imaginäre Zahlen $462 \mathrm{ff}$. Galoissche Körper 41, 72. Gruppe der Transformationen eines $-\mathrm{n}-\mathrm{s}$ in sich 46, 73; Idealtheorie der $-\mathrm{n}$ $-111 \mathrm{ff}$.

Galoissche Probleme bei primzahligen Teilungsgraden 264.

Galoissche Resolvente 42, 72; - der speziellen Teilungsgleichung $255 \mathrm{ff}$.

Galoisscher Satz über Resolventen $5^{\text {ten }}, 7^{\text {ten }}$ und $11^{\text {ten }}$ Grades der speziellen Transformationsgleichungen $475 \mathrm{ff}$.

GauBsche Transformation 293.

Geschlecht des Transformationspolygons 356, des Klassenpolygons 366.

Geschlechter der Klassen quadratischer Formen 154.

Gleichberechtigte Untergruppen 7.

Gleichungen in einem Körper 32,65 ; Kreisteilungs - 57; zyklische - 59; Abelsche - 61; algebraisch lösbare - $63 \mathrm{ff}$; - fünften Grades, allge- meine Bemerkungen 521 ft; ; s. auch „Teilungsgleichungen", „Transformationsgleichungen", „Modulargleichungen", "Multiplikatorgleichungen".

Grad einer Permutationsgruppe 19; eines Primideals 108; - eines Zweiges im quadratischen Körper 122; der Teilung 210, der Transformation. 275.

Grundzahl eines Körpers 86 .

Gruppe, allgemeiner Begriff einer endlicher Ordnung 1; Grundeigenschaften einer -2 ; einfache -8 ; zusammengesetzte - 8; kommutative oder Abelsche - 4, 14; Permutations- 18; Galoissche - einer Gleichung 46, 73; Monodromie- 77; - der Idealklassen eines Körpers 107; der allgemeinen Teilungsgleichung 214, der speziellen Transformationsgleichungen 459 .

Gützlaffsche Modulargleichung 525 .

\section{H}

Hauptform 141.

Hauptgeschlecht bei den Klassen quadratischer Formen 154.

Hauptideal 92.

Hauptklasse der Ideale 105; - der quadratischen Formen 141.

Hauptkongruenzgruppe $n^{\text {ter }}$ Stufe in der Gruppe $\Gamma^{(u)} 220 \mathrm{ff}$, in der Gruppe $\Gamma^{(\omega)} 222,254$, ihr Index 223.

Haupttransformation, erste und zweite $n^{\text {ten }}$ Grades $278 \mathrm{ff}$.

Hermitesche Resolvente fünften Grades 520.

Homomorphe Gruppen 10.

\section{I}

Idea], allgemeiner Begriff $89 \mathrm{ff}$; Darstellung eines $-\mathrm{s} 91$; Multiplikation der -e 93; Faktorenzerlegung eines $-\mathrm{s} 95 \mathrm{ff}$.; Basis eines $-\mathrm{s} 99 \mathrm{ff}$.

Idealklassen eines Körpers 105; Multiplikation der -107.

Identische Permutation 18.

Ikosaedergruppe, ihr Auftreten in $\operatorname{der} G_{\frac{1}{2} n\left(n^{2}-1\right)} 474$; Sätze über Erzeugung der -478 .

Imaginäre Gestalt der Gruppe $G_{\frac{1}{2} n\left(n^{2}-1\right)} 465$.

Imprimitive Körper 40. 
Imprimitive Permutationsgruppen 21.

Imprimitive $\mathrm{Z}$ ahlen eines Körpers $39,83$.

Imprimitivität, Systeme der - 21.

Index einer Untergruppe 4.

Indexreihe einer Gruppe 13.

Integralmodul, Transformation $2^{\text {ten }}$ Grades des - s $291 \mathrm{ff}$, wiederholte $492 \mathrm{ff}$; s. auch „Modulargleichungen“.

Intransitive Permutationsgruppe 20.

Intransitivität, Systeme der - 20; mehrfache -20.

Invariante Gestalten der Additionstheoreme $162 \mathrm{ff}$.

Inverse Elemente bei Gruppen 3.

Irrationale Modulargleichungen $525 \mathrm{ff}$.

Irrationalität, natürliche - 51, 75; akzessorische -52 ; numerische -76 .

Irreduzibilität einer Funktion in einem Körper 31, 66; - einer Gleichung in einem Körper 32, 67.

Isomorphe Gruppen 7 .

\section{K}

Kettenbruchverfahren zur Berechnung von $\wp(n u) 192 \mathrm{ff}$.

Klassenanzahl der Zweigideale und Stammideale im quadratischen Körper 134.

Klassenpolygon $357 \mathrm{ff}$., 365, Beziehung zu den quadratischen Formen 363; einfachste Funktionen des - s $367 \mathrm{ff}$.

Klasse von Idealen eines Körpers 105; -n quadratischer Formen 138; - von Transformationen $n^{\text {ten }}$ Grades 275 .

Kleinsche Funktionen $X_{\lambda}\left(u \mid \omega_{1}, \omega_{2}\right)$ $305 \mathrm{ff}$., ihre lineare Transformation $308 \mathrm{ff}$; $--x_{\lambda}\left(\omega_{1}, \omega_{2}\right), \xi_{\lambda}\left(\omega_{1}, \omega_{2}\right) 315$.

Kommutativ, -e Elemente bei Gruppen 4; -e Gruppen 4, $14 \mathrm{ff}$.

Komposition der quadratischen Formen 148.

Kompositionsreihe einer Gruppe 13.

Kongruenz ganzer Zahlen bezüglich eines Ideals 101; - der Substitutionen $V \bmod n 222$.

Kongruenzgruppen $n^{\text {ter }}$ Stufe in der Gruppe $\Gamma^{(u)} 220 \mathrm{ff}$., in der Modulgruppe $\Gamma^{(\omega)} 250 \mathrm{ff}$.

Konjugierte algebraische Zahlen 33, 83 ; - Ideale 111; - Körper 38, 71, 83.
Körper, allgemeiner Begriff 28; rationaler - 28; Funktionen in einem - 28; Gleichungen in einem 32, Reduzibilität und Irreduzibilität derselben 32; quadratische - 113; Galoissche - 41; Kreisteilungs - 56.

Kreisteilungsgleichung 57.

Kreisteilungskörper 56.

Kritische Primzahlen eines algebraischen Körpers 110.

\section{$\mathbf{L}$}

Lagrangesche Resolvente 60 .

Landensche Transformation 292; wiederholte - $-492 \mathrm{ff}$.

Legendre-Jacobisches Zeichon bei Transformation der Diskriminante $\Delta$ 303.

Linear-abhängige bzw. - unabhängige Zahlen eines Körpers $83 \mathrm{ff}$,

\section{HI}

Modulargleichungen von Jacobi und Sohnke $496 \mathrm{ff}$., von Schlaefli $502 \mathrm{ff}$; irrationale $-525 \mathrm{ff}$.

Modularkorrespondenzen $527 \mathrm{ff}$.

Modulformen, Systeme von $-n^{\text {ter }}$ Stufe $x_{\lambda}\left(\omega_{1}, \omega_{2}\right), y_{\lambda}\left(\omega_{1}, \omega_{2}\right), z_{\lambda}\left(\omega_{1}, \omega_{2}\right)$ $315,325,330$.

Modulfunktionen sechster Stufe, Spezialbetrachtung $533 \mathrm{ff}$.

Monodromiegruppe einer Gleichung 77; - der allgemeinen Teilungsgleichung $214 \mathrm{ff}$., ihre algebraische Darstellung $216 \mathrm{ff}$, ihre Struktur $218 \mathrm{ff}$; - der speziellen Teilungsgleichung 462, ihre vollständige Zerlegung $466 \mathrm{ff}$., $471 \mathrm{ff}$.

Multiplikation der Ideale 93; - der Idealklassen 107; - der Zweigideale 132.

Multiplikationstheorem der Funktion 184; -e für die Funktionen sn, cn, dn $196 \mathrm{ff}$.

Multiplikatorgleichungen von Jacobi $508 \mathrm{ff}$.

\section{$\mathbf{N}$}

Nebengruppe, Begrifi 4.

Normalgleichung 43, 72 .

Normalkörper $41,72$.

Norm einer algebraischen Zahl 85; eines Ideals 101. 


\section{0}

Oktaedergruppe, ihr Auftreten in $\operatorname{der} G_{\frac{1}{2} n\left(n^{2}-1\right)} 474$.

Ordnung einer Gruppe 1.

Orthogonale Substitutionen bei der Weierstraßschen Sigmarelation 159.

\section{$\mathbf{P}$}

Periode eines Grappenelementes 6.

Permutation 18; identische - 18; zyklische - 19.

Permutationsgruppe 18; transitive und intransitive $-\mathrm{n} 20$; primitive und imprimitive $-\mathrm{n} 21$.

Potenzsummen 25.

Primideal 95; Zerlegung rationaler Primzahlen in $-\mathrm{e} 108$, in Galoisschen Körpern 112, in quadratischen Körpern 115.

Primitiv, -e Funktionen eines Körpers 71 ; -e Körper 40 ; -e Permutationsgruppen 21 ; -e Zahlen eines Körpers 39, 83.

Primzahlen in rationalen Körpern 88; kritische - eines algebraischen Körpers 110; Zerlegung rationaler - in Primideale 108, in Galoisschen Körpern 112, in quadratischen Körpern 115.

Produkt, symbolisches - von Substitutionen 1.

Punktgitter bei den Basen der Zweigideale 136.

\section{$Q$}

Quadratische Form, ganzzahlige binüre - - 137; Teiler einer 137; ursprüngliche -137 ; abgeleitete - - 137; Diskriminante einer -n - 137; positive und negative -en 138; geometrische Deutung der $-\mathrm{n}-\mathrm{en} \mathrm{139;}$ reduzierte --140 ; entgegengesetzte - -en 140 ; zweiseitige oder ambige - -en 140; Komposition der -n -en 148.

Quadratische Zahlkörper 112, $121 \mathrm{ff}$.

Quotientengruppe 10.

\section{$\mathbf{R}$}

Rational-bekannt, Begriff einer -en Größe 43.

Reduzibilität einer Funktion oder Gleichung $31 \mathrm{ff}$, $66 \mathrm{ff}$.

Reduzierte quadratische Form 140.
Reihe der Zusammensetzung einer Gruppe 13.

Repräsentanten für Transformation $n^{\text {ten }}$ Grades 276.

Repräsentantensystem $16^{\text {tor }}$ Stufe für Transformation $n^{\text {ten }}$ Grades 529; $48^{\text {ster }}$ Stufe 504, $\nu^{\text {tor }}$ Stufe 527.

Resolvente, Tschirnhausen- - 26; rationale - einer Gleichung 52; Galoissche - einer Gleichung 53 ; - n fünften Grades beim fünften Transformationsgrade 483, zweiter Stufe $516 \mathrm{ff}$; - -n siebenten und elften Grades $486 \mathrm{ff}$.

\section{$\mathbf{S}$}

Sigmarelation von WeierstraB 158; die 256 dreigliedrigen -en 173.

Singulär, -e Periodenpaare 137; - er Periodenquotient 137.

Spezielle Teilungsgleichung, s. "Teilungsgleichung".

Spezielle Transformationsgleichung, 8. „Transformationsgleichung". Spur einer algebraischen Zahl 85.

Stammdiskriminante bei quadratischen Körpern 122.

Sta $m$ m eines quadratischen Körpers 122. Stammideal in quadratischen Körpern 124.

Stammklassen von Idealen in quadratischen Körpern 1 1s1.

Strahl in einem quadratischen Körper 125.

Symmetrische Funktion 24.

Symmetrische Grundfunktionen 24.

Symmetrische Gruppe 19.

\section{$\mathbf{T}$}

Teilerfremde Funktionen 30.

Teiler, gröBter gemeinsamer - zweier Funktionen 30, 65; - einer ganzen algebraischen Zahl 87; größter gemeinsamer - zweier Ideale 96; einer quadratischen Form 137.

Teilungsgleichung, allgemeine der $\wp$-Funktion 211, ihre Monodromiegruppe $214 \mathrm{ff}$, ihre Auflösung 225, $231 \mathrm{ff}$; spezielle - der $\wp$-Funktion 245, irreduzibele 247, ihre Galoissche Resolvente $255 \mathrm{ff}$, ihre Auflösung $262 \mathrm{ff}$. Teilwerte der Funktionen $\wp$ und $\wp^{\prime}$ 213, $244 \mathrm{ff}$., der Funktionen $\sigma$ und $\sigma^{\prime}$ 226 ; - derFunktionen sn, cn, dn $265 \mathrm{ff}$. 
Tetraedergruppe, ihr Auftreten in $\operatorname{der} G_{\frac{1}{2} n\left(n^{2}-1\right)} 474$.

Thetarelationen 296, für den dritten Grad $539 \mathrm{ff}$.

Totalcharakter einer Klasse quadratischer Formen 154.

Transformation eines Gruppenelementes 6; -en eines Galoisschen Körpers in sich 44 ; - der elliptischen Funktionen, allgemeiner Ansatz $270 \mathrm{ff}$; $-n^{\text {ten }}$ Grades der elliptischen Funktionen 275, Klassen und Repräsentanten $275 \mathrm{ff}$; eigentliche $-n^{\text {ten }}$ Grades 276; - $n^{\text {ten }}$ Grades der $\wp-$

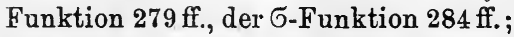
- zweiten Grades der Thetafunktionen $286 \mathrm{ff}$., des Integralmoduls 291, der Funktionen zweiter Stufe 292; Landensche - 292; Gaußsche - 293; - ungeraden Grades der Funktionen zweiter Stufe $293 \mathrm{ff}$; - der Diskriminante $\Delta$ und Teilwerte $298 \mathrm{ff}$; $-n^{\text {ten }}$ Grades der Diskriminante $\Delta 343 ;-$ $n^{\text {ten }}$ Grades von $g_{2}$ und $g_{3} 342,380$, 382 usw.; - von $J(\omega)$ der Grade $2^{v}$ $371 \mathrm{ff}$., der Grade $3^{v} 383 \mathrm{ff}$., der Grade $5^{v}$ und $7^{v} 389 \mathrm{ff}$, primzahliger Grade $403 \mathrm{ff}$., $424 \mathrm{ff}$., zusammengesetzter Grade $437 \mathrm{ff}$., $446 \mathrm{ff}$.

Transformationsfläche $351 \mathrm{ff}$, ihr Geschlecht 356.

Transformationsgleichung der $\beta$ Funktion 279, ihre Lösung 280; spozielle -en als Resolventen der Teilungsgleichungen $335 \mathrm{ff}$; spezielle - en, allgemeine Ansätze $342 \mathrm{ff}$; spezielle - für $j(\omega)=12^{3} J(\omega) 345$; algebraische Methode zur Aufstellung der -en $367 \mathrm{ff}$; spezielle -en erster Stufe für niedere Grade $371 \mathrm{ff}$; spezielle -en höherer Stufen $491 \mathrm{ff}$.
Transformationspolygon $349 \mathrm{ff}$; einfachste Funktionen des $-\mathrm{s} 367 \mathrm{ff}$, Transitive Permutationsgruppen 20.

Tschirnhausenresolvente 26.

Tschirnhausentransformation 26.

\section{$\mathbf{U}$}

Untergruppe, Begriff 4; gröBte 11 ; gleichberechtigte $-\mathrm{n} 7$; ausgezeichnete - 8 .

Ursprünglich, -e quadratische Form 137.

Vertauschbare Elemente einer Gruppe 4.

\section{W}

WeierstraBsche Sigmarelation 158.

Wertigkeit einer Funktion $g\left(z_{1}, z_{2}, \ldots, z_{n}\right)$ gegenüber der symmetrischen Gruppe 24.

\section{$\mathbf{Z}$}

Zahlkörper, allgemeiner Begriff 28.

Zahlstrahl in einem quadratischen Körper 125.

Zusammengesetzte Gruppe 8 .

Zweigdiskriminante bei quadratischen Körpern 122.

$Z$ weig eines quadratischen Körpers 122.

$\mathrm{Z}$ weigideal in einem quadratischen Körper 124.

$\mathrm{Z}$ weigklassen von Idealen in quadratischen Körpern 131.

Zwoiseitige quadratische. Formen 140.

Zyklen in Permutationsgruppen 19.

Zyklische Gleichungen 59.

Zyklische Gruppen 6.

\section{Bemerkte Versehen in Band I.}

S. 459, Tabelle, letzte Zeile, mittlere Spalte: Im Zähler muB $\sqrt[4]{k(\omega)}$ statt $\sqrt{k(\omega)}$ stehen.

S. 475, erste der drei mit $( \pm T)$ bezeichneten Gleichungen: Im Nenner der rechten Seite muB cn $\left(w, k^{2}\right)$ statt $\operatorname{dn}\left(w, k^{2}\right)$ stehen. 



RETURN Astronomy/Mathematics/Statistics Library TO $\longrightarrow 100$ Evans Hall 642-3381

\begin{tabular}{l|l|l}
\hline $\begin{array}{c}\text { LOAN PERIOD I } \\
1 \text { MONTH }\end{array}$ & 2 & 3 \\
\hline 4 & 5 & 6 \\
\hline
\end{tabular}

ALL BOOKS MAY BE RECALLED AFTER 7 DAYS

\section{DUE AS STAMPED BELOW}

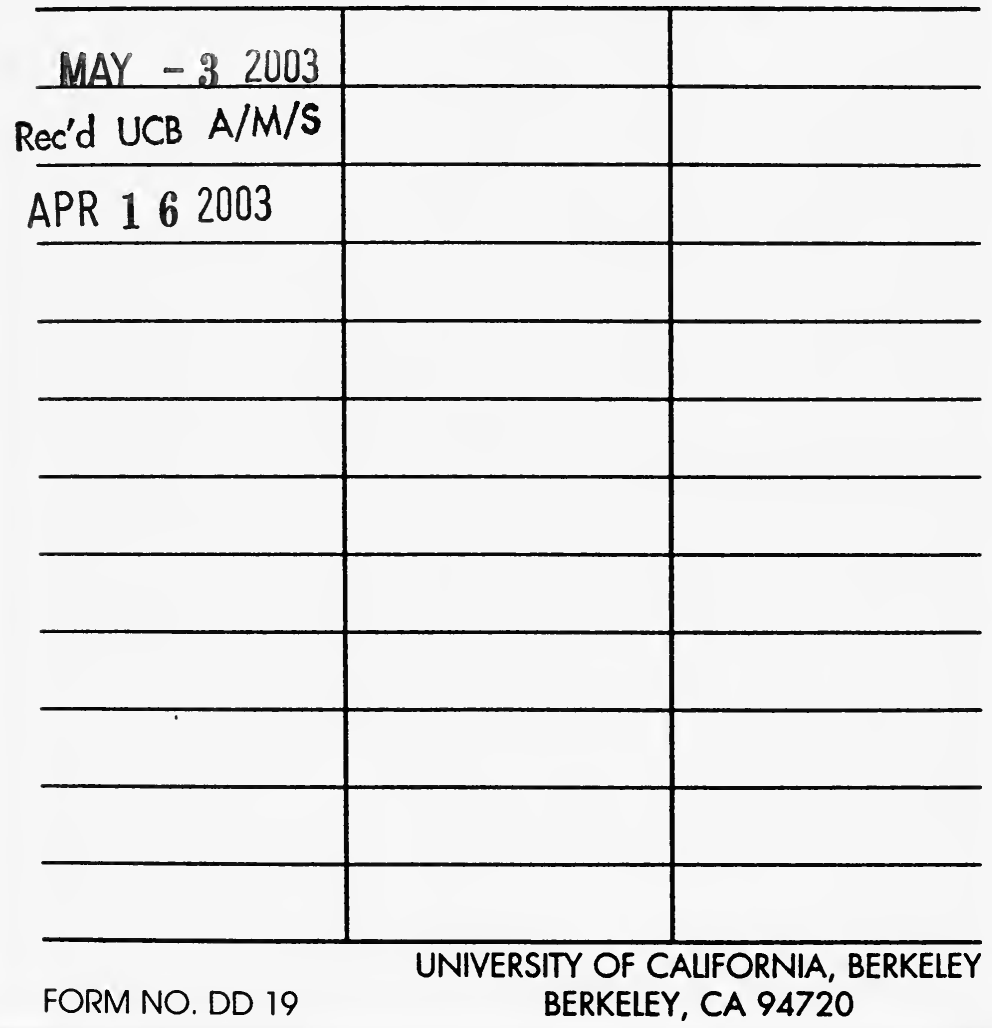


U. C. BERKELEY LIBRARIES

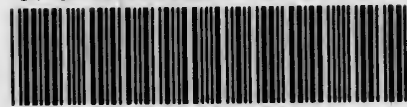

C058627037

QA
343
$F 7$
1.2

matr-

GTAT.

LIBRARY 
H. 